\title{
Economic Evaluation of Closure Cap Barrier Materials Study(U)
}

\section{Volume II}

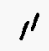

Site Geotechnical Services Department/Environmental Restoration Department

\section{UNCLASSIFIED}

Does not contain Unclassified Controlled Nuclear Information

Joginder S. Bhutani

Stephen M. Mead, P.E.

Michael G.Serrato

September 1993 
WSRC-RP-93-0878, Rev. 1,-VO/.2

\title{
Economic Evaluation of Closure CAP Barrier Materials Volume I and Volume II (U)
}

\author{
by \\ M. G. Serrato \\ Westinghouse Savannah River Company \\ Savannah River Sito \\ Aiken, South Carolina 29808 \\ J. S. Bhutani \\ S. M. Moad
}

DOE Contract No. DE-AC09-89SR18035

This paper was prepared in connection with work done under the above contract number with the U.S.

Department of Energy. By acceptance of this paper, the publisher and/or recipient acknowledges the U.S. Government's right to retain a nonexclusive, royalty-free license in and to any copyright covering this paper, along with the right to reproduce and to authorize others to reproduce all or part of the copyrighted paper. 


\section{DISCLAIMER}

This report was prepared as an account of work sponsored by an agency of the United States Government. Neither the United States Government nor any agency thereof, nor any of their employees, make any warranty, express or implied, or assumes any legal liability or responsibility for the accuracy, completeness, or usefulness of any information, apparatus, product, or process disclosed, or represents that its use would not infringe privately owned rights. Reference herein to any specific commercial product, process, or service by trade name, trademark, manufacturer, or otherwise does not necessarily constitute or imply its endorsement, recommendation, or favoring by the United States Government or any agency thereof. The views and opinions of authors expressed herein do not necessarily state or reflect those of the United States Government or any agency thereof. 


\section{DISCLAIMER}

\section{Portions of this document may be illegible in electronic image products. Images are produced from the best available original document.}


BSilnpell 4,20,94

$\mathrm{ADC}$ and Revalwing Official

B. G. Schappell, Mgr. GEE/SGS
WSRC-RP-93-0878-ReV/1-Vol.2 Rev. 1

Revision Date 9/30/93

\section{Economic Evaluation of Closure Cap Barrier Materials Study(U)}

\section{Volume II}

Site Geotechnical Services Department/Environmental Restoration Department

\section{UNCLASSIFIED}

Does not contain Unclassified Controlled Nuclear Information

Joginder S. Bhutani

Stephen M. Mead, P.E.

Michael G. Serrato

September 1993 


\section{Economic Evaluation of Closure Cap Barrier Materials (U)}

\section{Volume II}

\section{Gezuder Fifrectan. $04 / 14 / 94 /$ Joginder S. Bhutani Date Co-Author Site Geotechnical Services Department

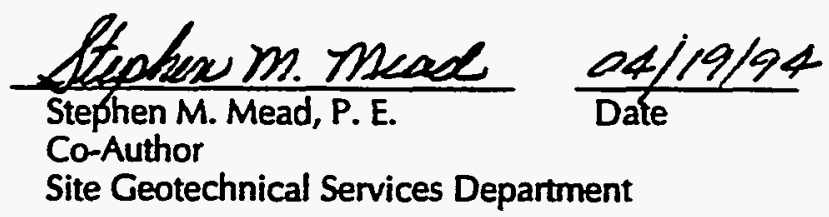

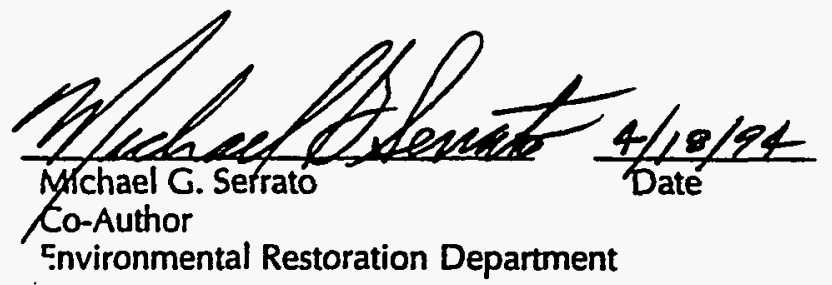

Westinghouse Savannah River Company Savannah River Site Aiken, SC 29808

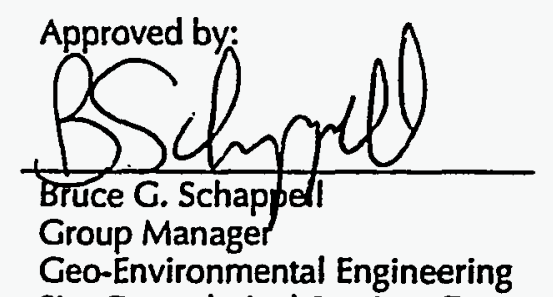

Site Geotechnical Services Department

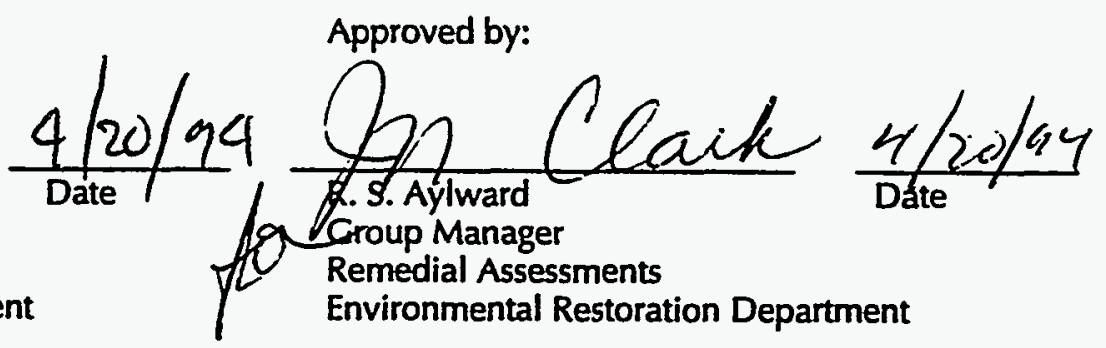

Approved by:

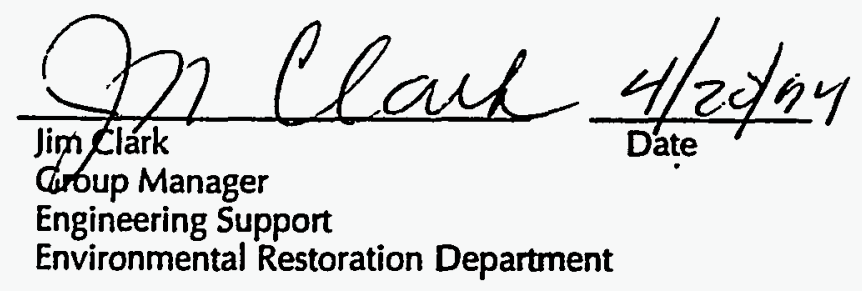




\section{DISCLAIMER}

This report was prepared as an account of work sponsored by an agency of the United States Government. Neither the United States Government nor any agency thereof, nor any of their employees, makes any warranty, express or implied, or assumes any legal liability or responsibility for the accuracy, completeness, or usefulness of any information, apparatus, product, or process disclosed, or represents that its use would not infringe privately owned rights. Reference herein to any specific commercial product, process, or service by trade name, trademark, manufacturer, or otherwise does not necessarily constitute or imply its endorsement, recommendation, or favoring by the United States Government or any agency thereof. The views and opinions of authors expressed herein do not necessarily state or reflect those of the United States Government or any agency thereof.

This report has been reproduced directly from the best available copy.

Available to DOE and DOE contractors from the Office of Scientific and Technical Information, P.O. Box 62, Oak Ridge, TN 37831; prices available from (615) 576-8401.

Available to the public from the National Technical Information Service, U.S. Department of Commerce, 5285 Port Royal Road, Springfield, VA 22161. 


\section{Table of Contents}

\section{Volume II}

Acknowledgments vii

1.0 Introduction 1

1.1 General 1

1.2 Cost Estimates 1

Appendices (Cost Estimates)

Appendix A. Cost Estimates for 0.1 Acre Site

A-1. Cost Estimate for a Cover System Utilizing Claymax and a 30-mil FML...................................... A-2

A-2. Cost Estimate for a Cover System Utilizing Bentomat ${ }^{\Phi}$ and a 30-mil FML........................................ A-8

A-3. Cost Estimate for a Cover System Utilizing Gundseal ${ }^{\otimes}$..................................................................... A-14

A-4. Cost Estimate for a Cover System Utilizing 120-mil High Density Polyethylene (HDPE) Liner

A-5. Cost Estimate for a Cover System Utilizing 1/4-inch Thick Hot Sprayed or . Emulsified Asphalt

A-6. Cost Estimate for a Cover System Utilizing Reinforced Sprayed Asphalt.

A-7 Cost Estimate for a Cover System Utilizing Compacted Kaolin (Offsite) Clay and a 30-mil FML

A-8. Cost Estimate for a Cover System Utilizing Compacted Onsite Sandy Clay a nd a 30-mil FML

A-9. Cost Estimate for a Cover System Utilizing Soil Cement and 30-mil FML

A-10. Cost Estimate for a Cover System Utilizing Bentonite Soil Additive and a 30-mil FML

A-11. Cost Estimate for a Cover System Utilizing Asphalt Concrete Hydraulic Barrier and 30-mil FML

A-12. Cost Estimate for a Cover System Utilizing Poured-in Place Concrete Slab and 30-mil FML

Appendix B - Cost Estimates for 0.2 Acre Site.

B-1. Cost Estimate for a Cover System Utilizing Claymax and a 30-mil FML

B-2. Cost Estimate for a Cover System Utilizing Bentomat and a 30-mil FML

B-3. Cost Estimate for a Cover System Utilizing Gundseal ${ }^{\oplus}$

B-4. Cost Estimate for a Cover System Utilizing 120-mil High Density Polyethylene (HDPE) Liner

B-5. Cost Estimate for a Cover System Utilizing 1/4-inch Thick Hot Sprayed or Emulsified Asphalt

B-6. Cost Estimate for a Cover System Utilizing Reinforced Sprayed Asphalt

B-7 Cost Estimate for a Cover System Utilizing Compacted Kaolin (Offsite) Clay and a 30-mil FML. 
B-8. Cost Estimate for a Cover System Utilizing Compacted Onsite Sandy Clay and a 30-mil FML

B-9. Cost Estimate for a Cover System Utilizing Soil Cement and 30-mil FML

B-10. Cost Estimate for a Cover System Utilizing Bentonite Soil Additive and 30-mil FML

B-11. Cost Estimate for a Cover System Utilizing Asphalt Concrete Hydraulic Barrier and a 30-mil FML.

B-12. Cost Estimate for a Cover System Utilizing Poured-in Place Concrete Slab and 30-mil FML

Appendix C - Cost Estimates for 0.5 Acre Site

C-1. Cost Estimate for a Cover System Utilizing Claymax and a 30-mil FML

C-2. Cost Estimate for a Cover System Utilizing Bentomat and a 30-mil FML

C-3. Cost Estimate for a Cover System Utilizing Gundseal ${ }^{\oplus}$.

C-4. Cost Estimate for a Cover System Utilizing 120-mil High Density Polyethylene (HDPE) Liner.

C-5. Cost Estimate for a Cover System Utilizing 1/4-inch Thick Hot Sprayed or Emulsified Asphalt

C-6. Cost Estimate for a Cover System Utilizing Reinforced Sprayed Asphalt C-32

C-7 Cost Estimate for a Cover System Utilizing Compacted Kaolin (Offsite) Clay and a 30-mil FML

C-8. Cost Estimate for a Cover System Utilizing Compacted Onsite Sandy Clay and a 30-mil FML

C-9. Cost Estimate for a Cover System Utilizing Soil Cement and 30-mil FML

C-10. Cost Estimate for a Cover System Utilizing Bentonite Soil Additive and 30-mil FML

C-11. Cost Estimate for a Cover System Utilizing Asphalt Concrete Hydraulic Barrier and a 30-mil FML

C-12. Cost Estimate for a Cover System Utilizing Poured-in Place Concrete Slab and 30-mil FML

Appendix D - Cost Estimates for 1-Acre Site.

D-1. Cost Estimate for a Cover System Utilizing Claymax ${ }^{\otimes}$ and a 30-mil FML

D-2. Cost Estimate for a Cover System Utilizing Bentomat and a 30-mil FML

D-3. Cost Estimate for a Cover System Utilizing Gundseal

D-4. Cost Estimate for a Cover System Utilizing 120-mil High Density Polyethylene (HDPE) Liner

D-5. Cost Estimate for a Cover System Utilizing 1/4-inch Thick Hot Sprayed or Emulsified Asphalt

D-6. Cost Estimate for a Cover System Utilizing Reinforced Sprayed Asphalt D-32 
D-7 Cost Estimate for a Cover System Utilizing Compacted Kaolin (Offsite) Clay and a 30-mil FML

D-8. Cost Estimate for a Cover System Utilizing Compacted Onsite Sandy Clay and a 30-mil FML

D-9. Cost Estimate for a Cover System Utilizing Soil Cement and 30-mil FML

D-10. Cost Estimate for a Cover System Utilizing Bentonite Soil Additive and 30-mil FML

D-11. Cost Estimate for a Cover System Utilizing Asphalt Concrete Hydraulic Barrier and a 30-mil FML

D-12. Cost Estimate for a Cover System Utilizing Poured-in Place Concrete Slab and 30-mil FML

Appendix E - Cost Estimates for 2-Acre Site

E-1. Cost Estimate for a Cover System Utilizing Claymax ${ }^{\otimes}$ and a 30-mil FML

E-2. Cost Estimate for a Cover System Utilizing Bentomat and a 30-mil FML

E-3. Cost Estimate for a Cover System Utilizing Gundsea/ ${ }^{\circ}$

E-4. Cost Estimate for a Cover System Utilizing 120-mil High Density Polyethylene (HDPE) Liner

E-5. Cost Estimate for a Cover System Utilizing 1/4-inch Thick Hot Sprayed or Emulsified Asphalt.

E-6. Cost Estimate for a Cover System Utilizing Reinforced Sprayed Asphalt

E-7 Cost Estimate for a Cover System Utilizing Compacted Kaolin (Offsite) Clay and a 30-mil FML

E-8. Cost Estimate for a Cover System Utilizing Compacted Onsite Sandy Clay and a 30-mil FML

E-9. Cost Estimate for a Cover System Utilizing Soil Cement and 30-mil FML

E-10. Cost Estimate for a Cover System Utilizing Bentonite Soil Additive and 30-mil FML

E-11. Cost Estimate for a Cover System Utilizing Asphalt Concrete Hydraulic Barrier and a 30-mil FML

E-12. Cost Estimate for a Cover System Utilizing Poured-in Place Concrete Slab and 30-mil FML

Appendix F - Cost Estimates for 5-Acre Site

F-1. Cost Estimate for a Cover System Utilizing Claymax and a 30-mil FML........................................... F-2

F-2. Cost Estimate for a Cover System Utilizing Bentomat and a 30-mil FML...................................... F-8

F-3. Cost Estimate for a Cover System Utilizing Gundseal ${ }^{\circledR}$

F-4. Cost Estimate for a Cover System Utilizing 120-mil High Density Polyethylene (HDPE) Liner

F-5. Cost Estimate for a Cover System Utilizing 1/4-inch Thick Hot Sprayed or Emulsified Asphalt

F-6. Cost Estimate for a Cover System Utilizing Reinforced Sprayed Asphalt.

F-7 Cost Estimate for a Cover System Utilizing Compacted Kaolin (Offsite) Clay and a 30-mil FML

F-8. Cost Estimate for a Cover System Utilizing Compacted Onsite Sandy Clay and a 30-mil FML 
F-9. Cost Estimate for a Cover System Utilizing Soil Cement and 30-mil FML

F-10. Cost Estimate for a Cover System Utilizing Bentonite Soil Additive and 30-mil FML

F-11. Cost Estimate for a Cover System Utilizing Asphalt Concrete Hydraulic Barrier and a 30-mil FML

F-12. Cost Estimate for a Cover System Utilizing Poured-in Place Concrete Slab and 30-mil FML

G-1. Cost Estimate for a Cover System Utilizing Claymax ${ }^{\otimes}$ and a 30-mil FML

G-2. Cost Estimate for a Cover System Utilizing Bentomat and a 30-mil FML

G-3. Cost Estimate for a Cover System Utilizing Gundseal'

G-4. Cost Estimate for a Cover System Utilizing $\mathbf{1} 20$-mil High Density Polyethylene (HDPE) Liner.

G-5. Cost Estimate for a Cover System Utilizing 1/4-inch Thick Hot Sprayed or Emulsified Asphalt

G-6. Cost Estimate for a Cover System Utilizing Reinforced Sprayed Asphalt

G-7 Cost Estimate for a Cover System Utilizing Compacted Kaolin (Ofisite) Clay and a 30-mil FML

G-8. Cost Estimate for a Cover System Utilizing Compacted Onsite Sandy Clay and a 30-mil FML

G-9. Cost Estimate for a Cover System Utilizing Soil Cement and 30-mil FML

G-10. Cost Estimate for a Cover System Utilizing Bentonite Soil Additive and 30-mil FML

G-11. Cost Estimate for a Cover System Utilizing Asphalt Concrete Hydraulic Barrier and a 30-mil FML

G-12. Cost Estimate for a Cover System Utilizing Poured-in Place Concrete Slab and 30-mil FML

Appendix H - Cost Estimates for 20-Acre Site

H-1. Cost Estimate for a Cover System Utilizing Claymax ${ }^{\otimes}$ and a 30-mil FML

H-2. Cost Estimate for a Cover System Utilizing Bentomat ${ }^{\otimes}$ and a 30-mil FML........................................H-8

H-3. Cost Estimate for a Cover System Utilizing Gundseal ${ }^{\otimes}$.

H-4. Cost Estimate for a Cover System Utilizing 120-mil High Density Polyethylene (HDPE) Liner

H-5. Cost Estimate for a Cover System Utilizing 1/4-inch Thick Hot Sprayed or Emulsified Asphalt

H-6. Cost Estimate for a Cover System Utilizing Reinforced Sprayed Asphalt

H-7 Cost Estimate for a Cover System Utilizing Compacted Kaolin (Offsite) Clay and a 30-mil FML

H-8. Cost Estimate for a Cover System Utilizing Compacted Onsite Sandy Clay and a 30-mil FML 
H-9. Cost Estimate for a Cover System Utilizing Soil Cement and 30-mil FML

H-10. Cost Estimate for a Cover System Utilizing Bentonite Soil Additive and 30-mil FML

H-11. Cost Estimate for a Cover System Utilizing Asphalt Concrete Hydraulic Barrier and a 30-mil FML

H-12. Cost Estimate for a Cover System Utilizing Poured-in Place Concrete Slab and 30-mil FML

Appendix 1 - Cost Estimates for 40-Acre Site

1-1. Cost Estimate for a Cover System Utilizing Claymax and a 30-mil FML

1-2. Cost Estimate for a Cover System Utilizing Bentomat and a 30-mil FML

1-3. Cost Estimate for a Cover System Utilizing Gundseal ${ }^{\star}$

1-4. Cost Estimate for a Cover System Utilizing 120-mil High Density Polyethylene (HDPE) Liner

1-5. Cost Estimate for a Cover System Utilizing 1/4-inch Thick Hot Sprayed or Emulsified Asphalt

1-6. Cost Estimate for a Cover System Utilizing Reinforced Sprayed Asphalt.

1-7 Cost Estimate for a Cover System Utilizing Compacted Kaolin (Offsite) Clay and a 30-mil FML

1-8. Cost Estimate for a Cover System Utilizing Compacted Onsite Sandy Clay and a 30-mil FML

1-9. Cost Estimate for a Cover System Utilizing Soil Cement and 30-mil FML

1-10. Cost Estimate for a Cover System Utilizing Bentonite Soil Additive and 30-mil FML

I-11. Cost Estimate for a Cover System Utilizing Asphalt Concrete Hydraulic Barrier and a 30-mil FML

1-12. Cost Estimate for a Cover System Utilizing Poured-in Place Concrete Slab and 30-mil FML

Appendix J - Cost Estimates for 80-Acre Site

1-1. Cost Estimate for a Cover System Utilizing Claymax and a 30-mil FML.......................................... J-2

J-2. Cost Estimate for a Cover System Utilizing Bentomat and a 30-mil FML........................................ J-8

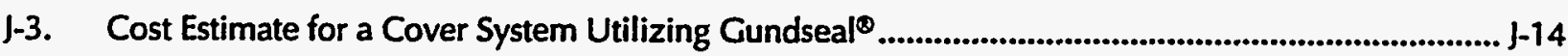

J-4. Cost Estimate for a Cover System Utilizing 120-mil High Density Polyethylene (HDPE) Liner

1-5. Cost Estimate for a Cover System Utilizing 1/4-inch Thick Hot Sprayed or Emulsified Asphalt.

1-6. Cost Estimate for a Cover System Utilizing Reinforced Sprayed Asphalt

1-7 Cost Estimate for a Cover System Utilizing Compacted Kaolin (Offsite) Clay and a 30-mil FML

J-8. Cost Estimate for a Cover System Utilizing Compacted Onsite Sandy Clay and a 30-mil FML

J-9. Cost Estimate for a Cover System Utilizing Soil Cement and 30-mil FML 
1-10. Cost Estimate for a Cover System Utilizing Bentonite Soil Additive and

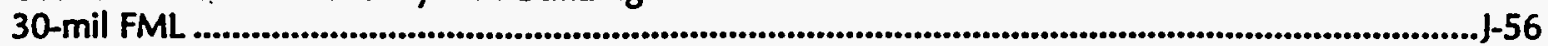

f-11. Cost Estimate for a Cover System Utilizing Asphalt Concrete Hydraulic Barrier

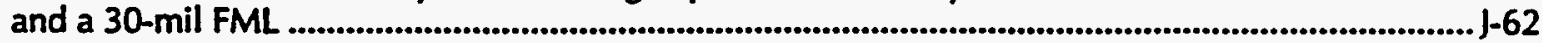

J-12. Cost Estimate for a Cover System Utilizing Poured-in Place Concrete Slab and 30-mil FML 


\section{Acknowledgments}

This study was prepared, as a part of the ongoing efforts by the Site Geotechnical Services (SGS) and Environmental Restoration (ER) departments of the Westinghouse Savannah River Company (WSRC), to select the most cost-effective and reliable barrier material for a given Savannah River Site (SRS) hazardous waste site. The study involved a major effort by the Cost Estimating, Procurement, and Materials Management departments of WSRC Engineering and Projects Division in developing unit prices and preparing cost estimates. The number of personnel who cooperated in this endeavor is too large to list here, but we have identify those individuals whose contributions were of great value.

We wish to express our thanks to James W. Iwert, manager Project Control and Cost Estimating, for his timely help and cooperation throughout the project; M. Shakoor and William Kulp for developing unit prices and for their keen interest in ensuring the estimates reflect the most acurate cost of covering a site; and Pravina Ghelani for her help in preparing and typing the cost estimates. 


\subsection{Introduction}

\subsection{General}

Volume II of the Economic Evaluation of the Closure Cap Barrier Materials, Revision 1 contains detailed cost estimates to support the economic evaluation of closure cap barrier materials. The cost estimates incorporate the life cycle costs for a generic hazardous waste seepage basin closure cap under the RCRA Post Closure Period of thirty years. The economic evaluation assessed six barrier material categories. Each of these categories consists of several composite cover system configurations, which were used to develop individual cost estimates. The information contained in this report is not intended to be used as a cost estimating manual. This information provides the decision makers with the ability to screen barrier materials, cover system configurations, and identify cost-effective materials for further consideration.

Outlined below are the composite cover system configurations evaluated:

Barrier Material Category

- Geosynthetic clay liners

- Flexible membrane liner

- Asphalt barriers

- Clays

- Soil additives

- Nonsoil materials
Composite Cover System

- Claymax ${ }^{\otimes}$ and 30-mil FML

- $\quad$ Bentomat $^{\star}$ and 30-mil FML

- Gundseal ${ }^{\oplus}$

- 120-mil high-density polyethylene (FDPE) liner

- 1/4-inch-thick hot sprayed or emulsified asphalt and 30-mil FML

- 1/4-inch-thick reinforced sprayed asphalt with geotextile fabric and 30-mil FML

- Kaolin clay (offsite) and 30-mil FML

- Onsite clay and 30-mil FML

- Soil cement and 30-mil FML

- Bentonite soil and 30-mil FMil

- 4-inch-thick asphalt concrete slab and 30-mil FML

- $\quad$ 4inch-thick poured-in-place concrete slab and 30-mil FML

Cost estimates were generated for each composite cover system configuration using ten different waste site areas. A total of 120 cost estimates were developed for waste site areas of 0.1 acre, 0.2 acre, 0.5 acre, 1.0 acre, 2.0 acres, 5.0 acres, 10 acres, 20 acres, 40 acres, and 80 acres.

\subsection{Cost Estimates}

Each cost estimate incorporates three major cost parameters:

- initial construction/installation costs

- operations and maintenance $(O / M)$ costs for post closure monitoring period (30 years)

- total life cycle costs at present worth (1994 costs) amortized over the entire project duration to include post closure monitoring period (30 years) 
Rev. 1

September 30, 1993

The initial estimated construction costs include direct construction costs (e.g.; materials, equipment and labor) and indirect costs (e.g., design, project management, industrial/health protection, and safety). The total present worth costs were calculated by projecting the annual O\&M costs over the entire postclosure monitoring period of 30 years, bringing back the projected O\&M costs to present worth cost and them adding these costs to the initial construction costs. The cost estimates generated for this economic evaluation are contained in Appendices A through J.

Note: These estimates were generated based on a generic seepage basin waste site closure cover system configuration and do not incorporate any follow-on remediation measures. 


$$
1
$$


DETAIL Ë́STIMATE

CHKD BY: 8. MEAD

LOC: : 7-03

ACTIVITY CODE: ESE260412

PROJ.NAME: ECON. EVALUATTON OF CLOSURE CAP BARRIER MATERIAL

ESTIMATOR: ROMKULP

PHONE: T-5266

DATE: OSNOASS

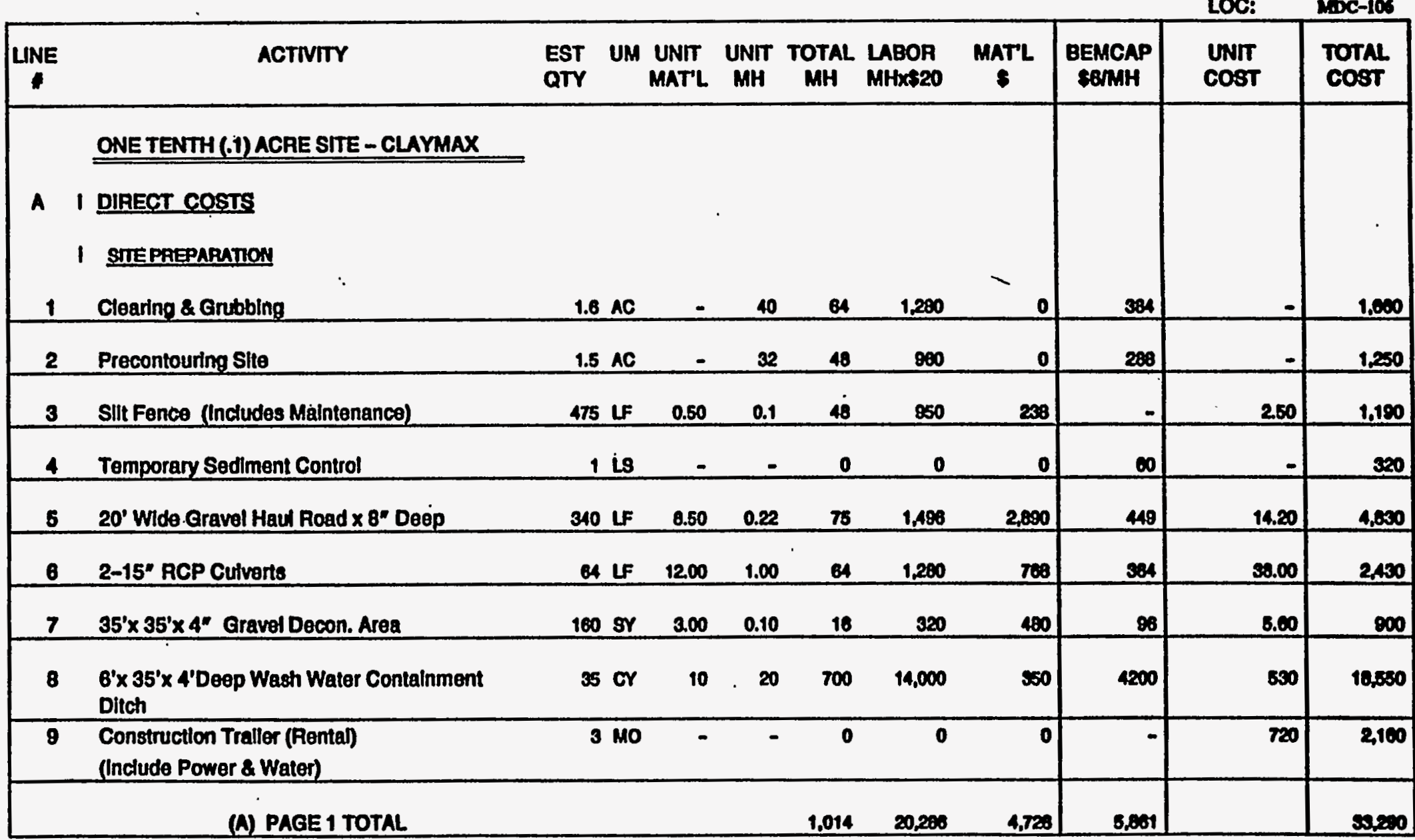

A-1. Cost Estimate for a Cover System Utilizing Claymax and a 30-mil FML 


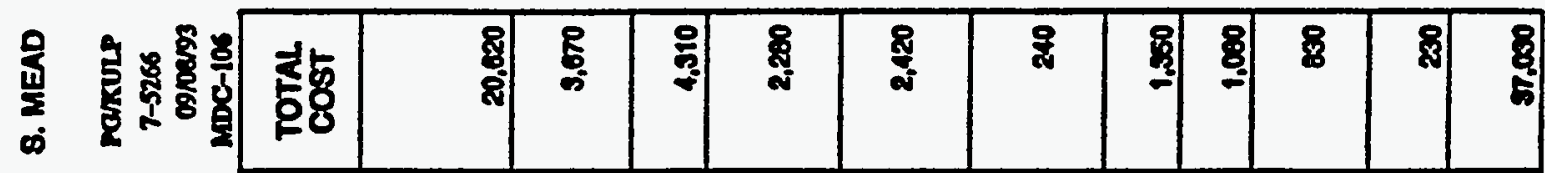

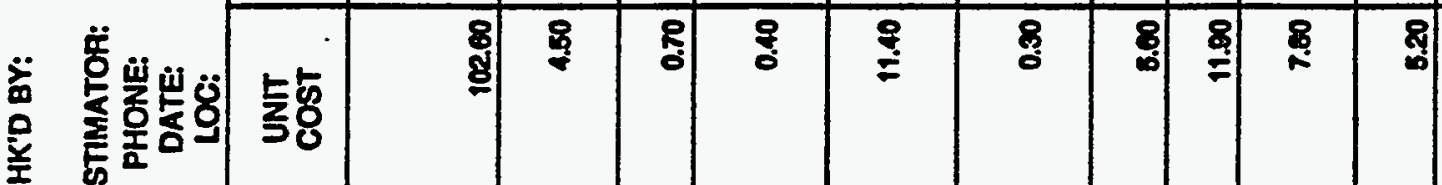

产

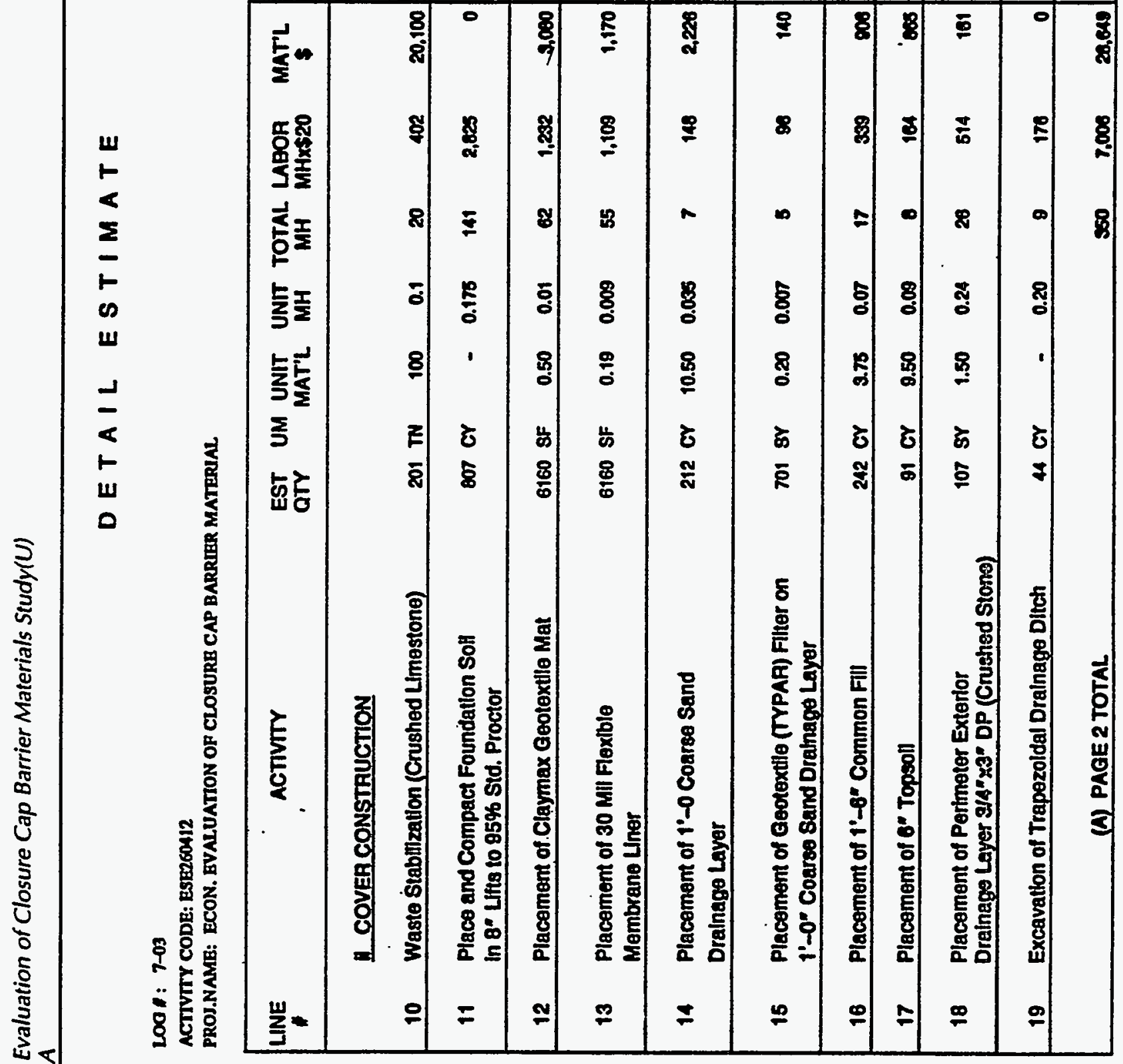

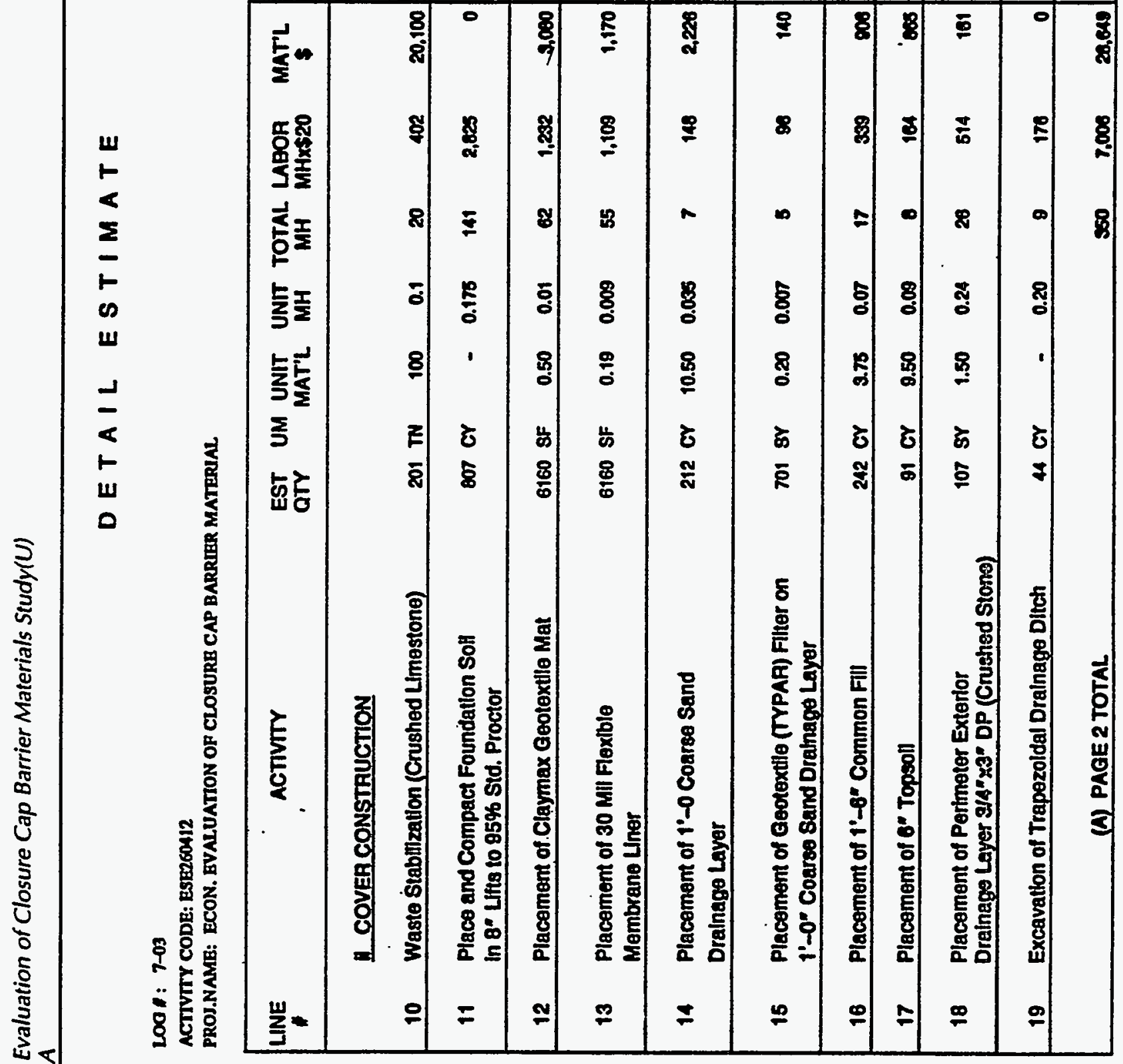
忽

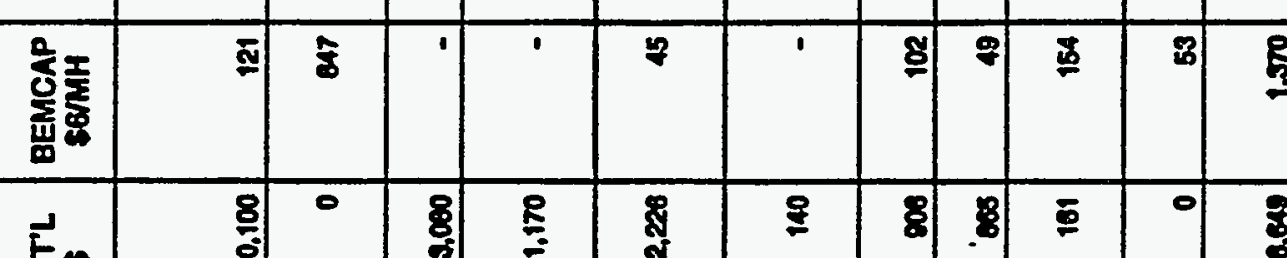

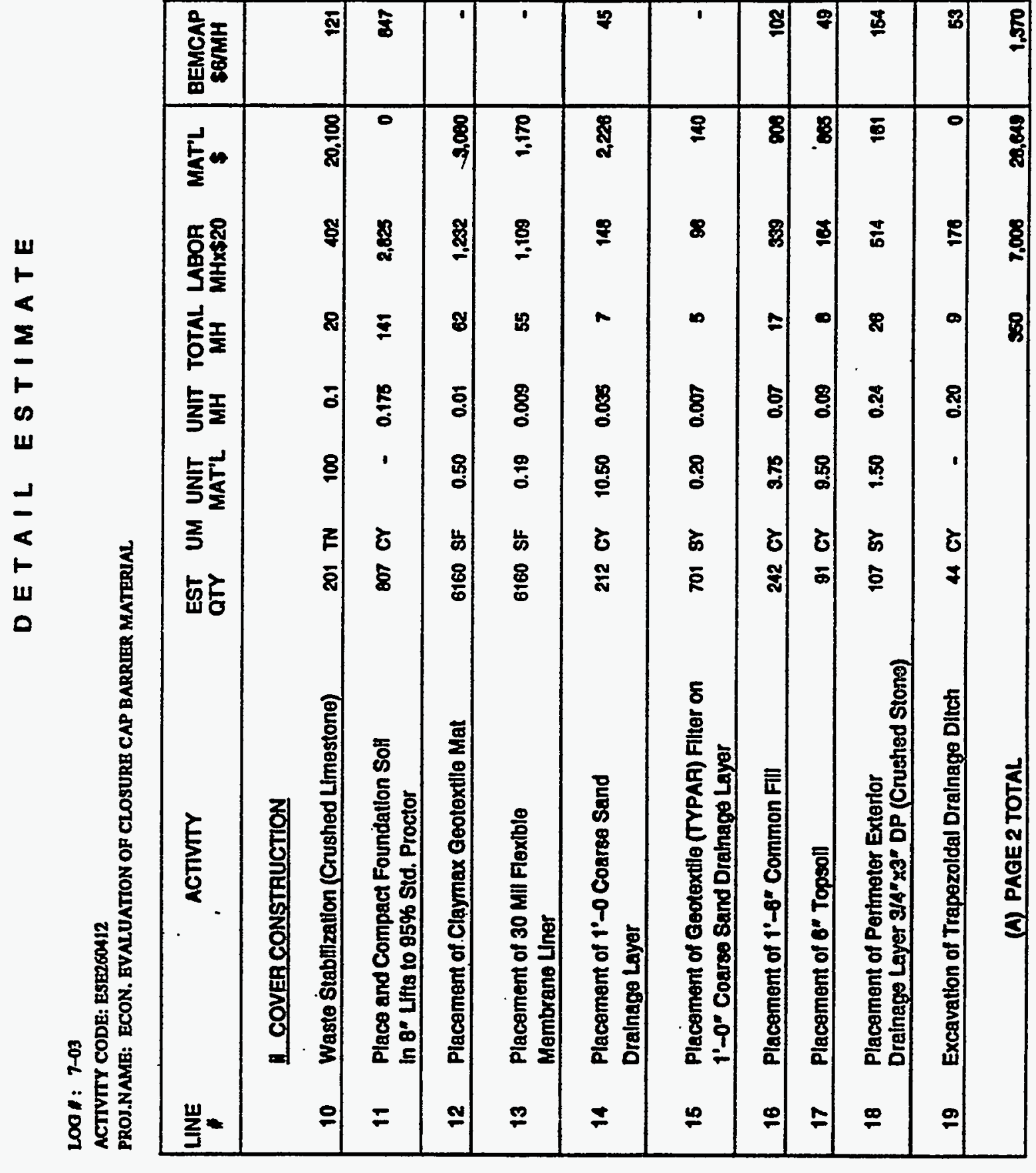

$\mid$ 
DETAILESTIMAT B

CHKD BY: 8. MEAD

Loo 1: 7-03

ACTIVITY CODE: ESE2SOH12

PROJ.NAME: ECON. EYALUATION OF CLOSURB CAP BARRTER MATERIAL

ESTIMATOR: POXULP

PHONE:

7-5260

DATE: OPRos

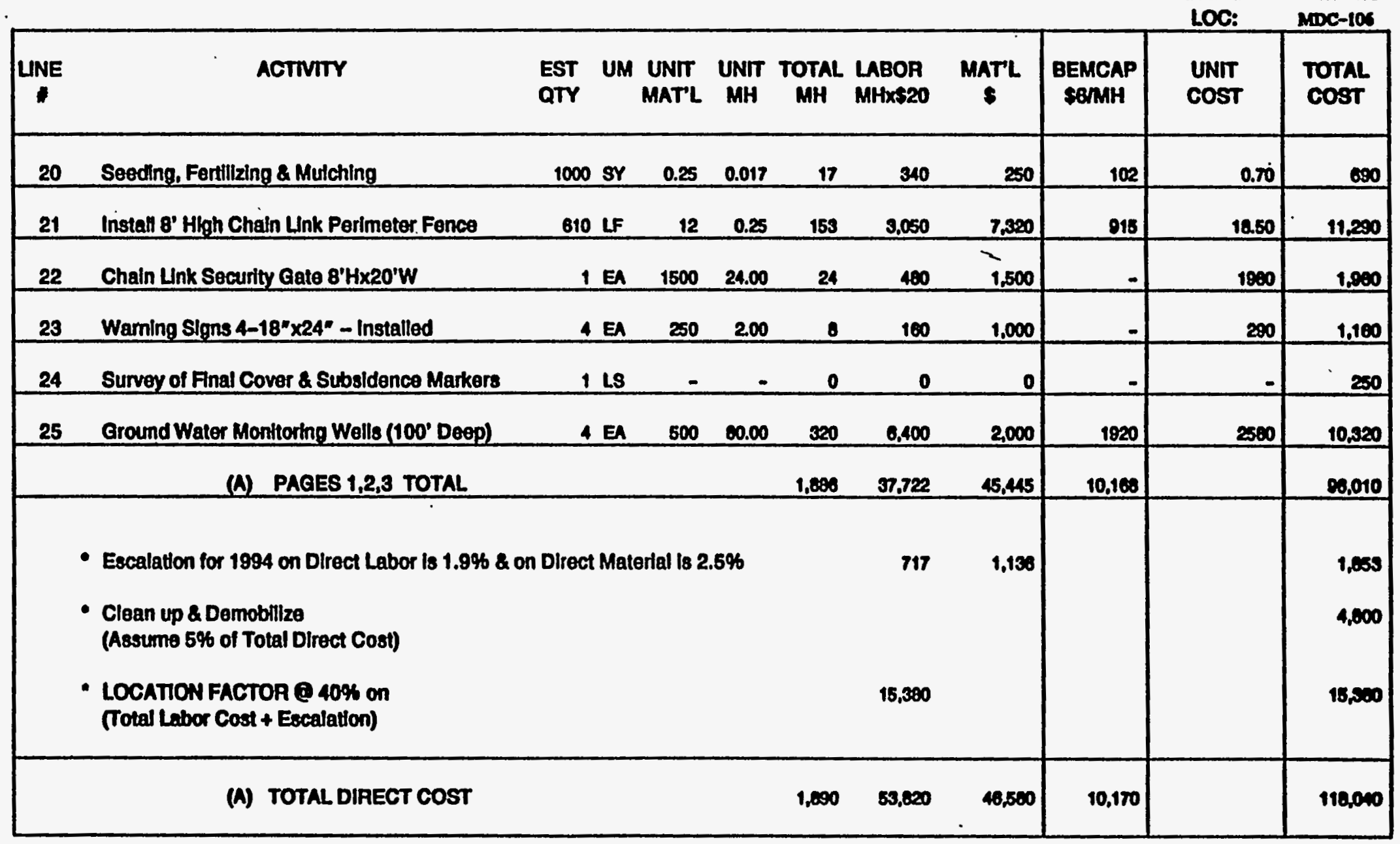

A-1. Cost Estimate for a Cover System Utilizing Claymax and a 30-mil FML (Cont'd) 
D E T A I L B S T I M A T B

CHK'D BY: $\quad$ 8. MEAD

LoO I: :-03

ACTIVITY CODE: ESE260412

PROI.NAME: ECON. BVALUATION OF CLOSURE CAP BARRIER MATERAAL

ESTIMATOR: PORULP

PHONE: 7-5206

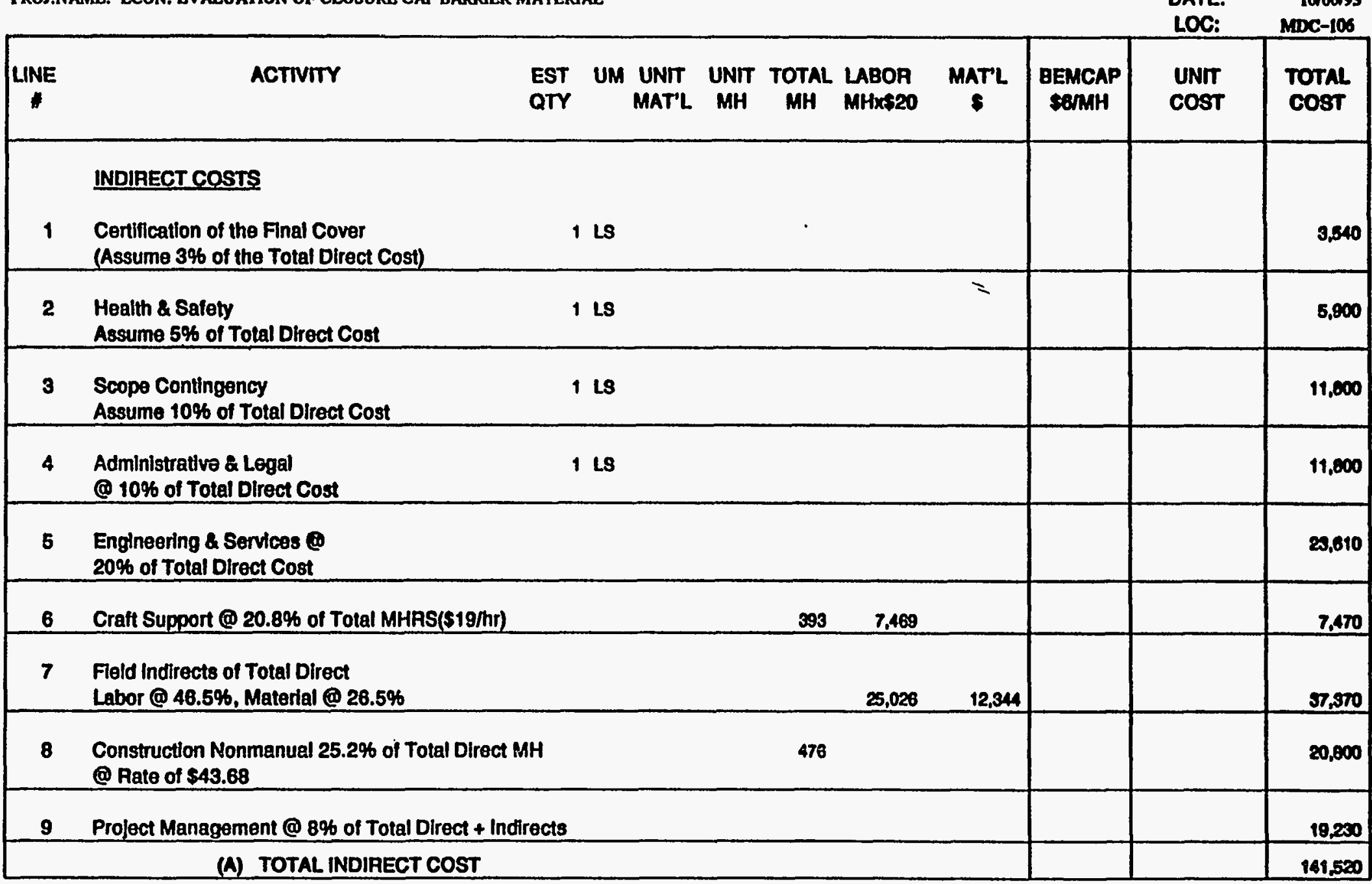

A-1. Cost Estimate for a Cover System Utilizing Claymax@ and a 30-mil FML (Cont'd) 
LO0 \&: 7-03

ACTIVITY CODE: ESE260412

PROJ.NAME: BCON. EYALUATION OF CLOSURB CAP BARRIER MATERIAL

CHKD BY: 8. MEAD

ESTIMATOR: PORULP

PHONE: 7-5260

DATE: $\quad 0910 \mathrm{M}$

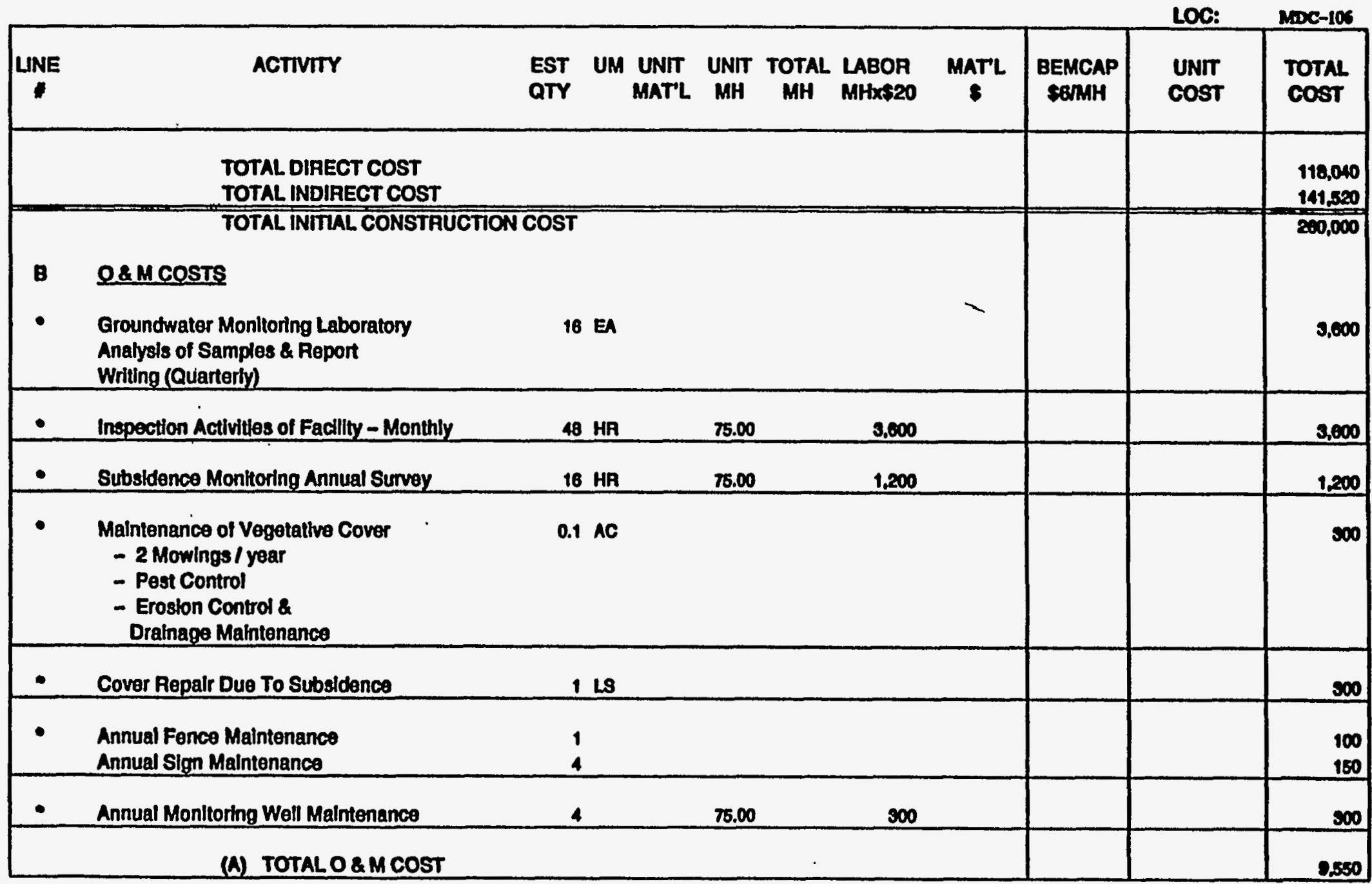

A-1. Cost Estimate for a Cover System Utilizing Claymax and a 30-mil FML (Cont'd) 
D B T A I L B S T I A A B

\begin{tabular}{|c|c|}
\hline CHK'D BY: & 8. MEAD \\
\hline $\begin{array}{l}\text { ESTIMATOR: } \\
\text { PHONE: } \\
\text { DATE: } \\
\text { LOC: }\end{array}$ & $\begin{array}{c}\text { ransuls } \\
7-5265 \\
090193 \\
\text { MDC-106 }\end{array}$ \\
\hline
\end{tabular}

ACTIVITY CODB: ESB260412

FROJ.NAME: BCON. BVALUATION OF CLOSURB CAP BARRIER MATERIAL

MDC-106

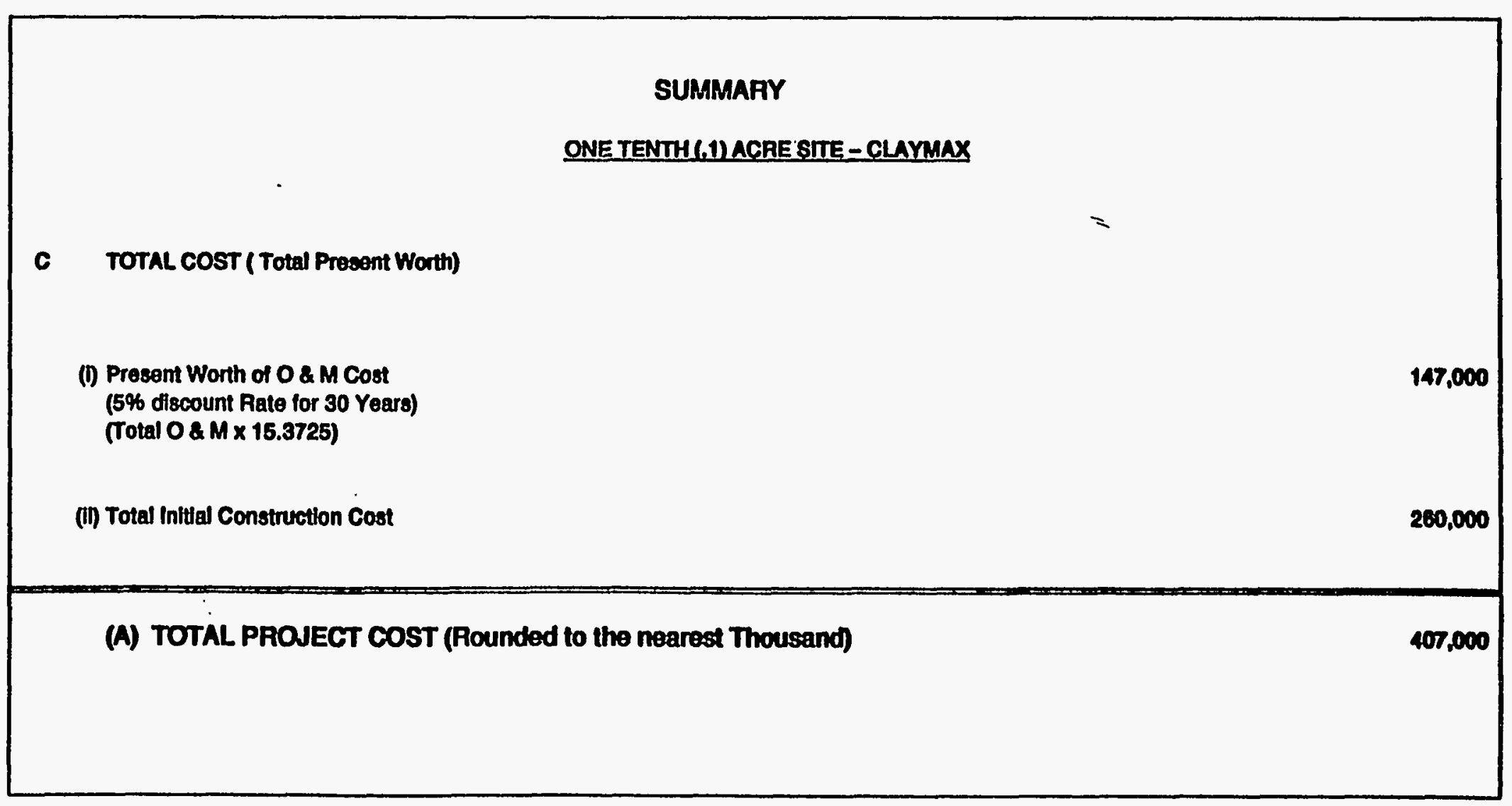

A-1. Cost Estimate for a Cover System Utilizing Claymaxid and a 30-mil FML (Cont'd) 
FIR: L.ITENTH

DETAILESTIMATE

CHKD BY: 8. MEAD

100 / : 7-03

ACTIVITY CODE: ESE2SON12

PROU.NAME: ECON. EVALUATTON OF CLOSURB CAP BARRIRR MATERIAL

ESTDEV: PamuK

T-52000

\begin{tabular}{|c|c|c|c|c|c|c|c|c|c|c|c|}
\hline LINE & ACTIVITY & $\begin{array}{l}\text { EST } \\
\text { QTY }\end{array}$ & UM & $\begin{array}{l}\text { UNIT } \\
\text { MAT'L }\end{array}$ & $\begin{array}{l}\text { UNIT } \\
\text { MH }\end{array}$ & $\underset{\text { MH }}{\text { TOTAL }}$ & $\begin{array}{l}\text { LABOA } \\
\text { MHX\$20 }\end{array}$ & $\underset{8}{\operatorname{MATL}}$ & $\begin{array}{l}\text { BEMCAP } \\
\text { SGAMH }\end{array}$ & $\begin{array}{l}\text { UNT } \\
\text { COST }\end{array}$ & $\begin{array}{l}\text { TOTAL } \\
\text { COST }\end{array}$ \\
\hline $\begin{array}{l}A \\
1 \\
\end{array}$ & $\begin{array}{l}\text { ONE TENTH (.1) ACRE STE-BENTO } \\
1 \text { DIRECT COSTS } \\
1 \text { SMIEPREPARUTION } \\
\text { Clearing \& Gnibing }\end{array}$ & 1.6 & AC & - & 40 & 64 & 1,200 & 0 & ses & - & 1.060 \\
\hline 2 & Precontouring Sile & 1.5 & $A C$ & - & 32 & 40 & 900 & 0 & 200 & $=$ & 1,250 \\
\hline 3 & Silt Fence (Includes Malntenance) & 475 & LF & 0.50 & 0.1 & 48 & 850 & 230 & - & 250 & 1.180 \\
\hline 4 & Temporary Sediment Control & 1 & L8 & - & - & 0 & $\mathbf{0}$ & 0 & $\infty$ & - & 300 \\
\hline 5 & 20' Whde Graval Haul Road x 8" Deep & 340 & LF & 0.50 & 0.22 & 75 & 1,496 & 2,000 & 49 & 14.20 & 4.030 \\
\hline 6 & 2-15" RCP Culwerts & 64 & LF & 1200 & 1.00 & 64 & 1,200 & 700 & 304 & 30,00 & 2,430 \\
\hline 7 & $35^{\prime} \times 35^{\prime} \times 4^{\prime \prime}$ Gravel Docon. Area & 160 & 8v & 3.00 & 0.10 & 16 & 320 & 400 & 86 & 8.00 & $\infty$ \\
\hline 8 & $\begin{array}{l}\text { 6'x } 35^{\prime} \times 4 \text { A'Deep } \\
\text { Wash Water Containment Ditch }\end{array}$ & 35 & cr & 10 & 20 & 700 & 14,000 & 350 & 4,200 & 800 & 18,550 \\
\hline 9 & $\begin{array}{l}\text { Construction Traller (Rental) } \\
\text { (Include Power \& Water) }\end{array}$ & s & MO & - & - & 0 & 0 & 0 & - & 720 & 2,100 \\
\hline & (ㄴ) PAGE 1 TOTAL & & & & & & 20,206 & 4,720 & 8.001 & & 30,20 \\
\hline
\end{tabular}

A-2. Cost Estimate for a Cover System Utilizing Bentomat $\left({ }^{\circ}\right.$ and a 30-mil FML 
DETAIL ESTIMATE CHKDEY: 8.MEAD

LO0 I: 7-03

ACTIVTTY CODE: BSE260112

PROJ.NAMRE: ECON. BVAL.UATION OP CLOSURR CAP BARRRER MATERUAL

EATDEY: PannK

PHONE 7-5260

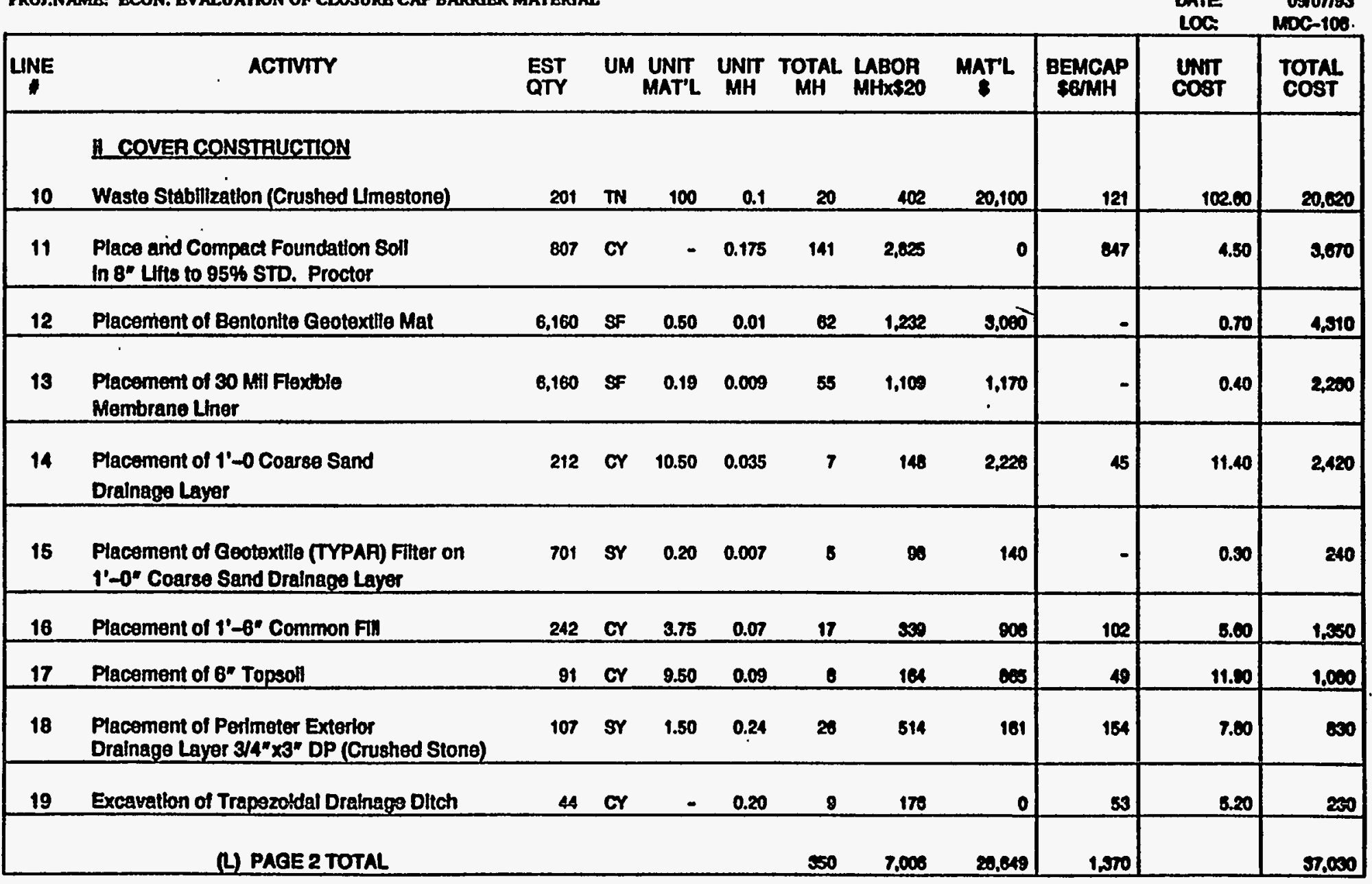

A-2. Cost Estimate for a Cover System Utilizing Bentomat and a 30-mil FML (Cont'd) 
D B T A L B S T I M A T B

\section{LOO N: $7-03$}

ACTTVITY CODE: ESE2OON12

PROI.NAME: ECON. EYALUATTON OF CLOSURE CAP BARRTER MATERIAL
CHK'D BY: S. MEAD

ESTD 8Y: PQWNK

PHONE 7-52006

DATE

100

\begin{tabular}{|c|c|c|c|c|c|c|c|c|c|c|c|}
\hline LINE & ACTIVITY & $\begin{array}{l}\text { EST } \\
\text { QTY }\end{array}$ & UM & $\begin{array}{l}\text { UNIT } \\
\text { MATLL }\end{array}$ & $\begin{array}{l}\text { UNIT } \\
\text { MH }\end{array}$ & $\begin{array}{c}\text { TOTAL } \\
\text { MH }\end{array}$ & $\begin{array}{l}\angle A B O A \\
\text { MHX\$20 }\end{array}$ & $\underset{\$}{\operatorname{MATL}}$ & $\begin{array}{l}\text { GEMCAP } \\
\text { SBMHH }\end{array}$ & $\begin{array}{l}\text { UNIT } \\
\text { COST }\end{array}$ & $\begin{array}{l}\text { TOTAL } \\
\text { COST }\end{array}$ \\
\hline 20 & Soeding, Fërtillzing \& Mulching. & 1.000 & 8Y & 0.25 & 0.017 & 17 & 340 & 250 & 102 & 0.70 & 000 \\
\hline 21 & Install 8' High Chain Link Perimeter Fence & 010 & LF & 12 & 0.25 & 159 & s,050 & 7.320 & 915 & 10.50 & 11,200 \\
\hline 22 & Chain Link Socuntiy Gate 8'H×20'W & 1 & EA & 1500 & 24.00 & 24 & 400 & 1,500 & - & 1900 & 1,000 \\
\hline 23 & Warning Signs $4-10^{\prime} \times 24^{\prime \prime}$ - Installed & 4 & EA & 250 & 200 & 8 & 100 & 1.000 & $=$ & 2000 & 1,100 \\
\hline 24 & Survey of Final Cover \& Subsidence Markers & 1 & LS & - & - & 0 & o & 0 & - & 250 & 250 \\
\hline 25 & Ground Water Monitoring Wells (100' Deep) & 4 & EA & 500 & 60.00 & 320 & 0,400 & 2,000 & 1,820 & 2580 & 10,520 \\
\hline & (ㄴ) PAGES 1,2,3 TOTAL & & & & & 1,688 & 37,722 & 45,445 & 10,100 & & 86,010 \\
\hline & $\begin{array}{l}\text { Escalation for } 1994 \text { on Direct Labor is } 1.8 \% \text { an } \\
\text { Clean up \& Demoblize } \\
\text { (Assume } 5 \% \text { of Tolal Drect Cost) } \\
\text { LOCATION FACTOR Q 40\% on } \\
\text { (Total Labor Cost + Escalation) }\end{array}$ & $2.5 \%$ on & Direc & ot Materi & & & 15,376 & 1,136 & & & $\begin{array}{l}1,050 \\
1,000\end{array}$ \\
\hline & (L) TOTAL DIRECT COST & & & & & 1,0000 & 55,010 & 46,500 & 10,170 & & 118,040 \\
\hline
\end{tabular}

A-2. Cost Estimate for a Cover System Utilizing Bentomat and a 30-mil FML (Cont'd) 
D E T A I B S TIMAT B

\section{CHK'D BY: 8. MEAD}

Lod I: 7-03

ACTIVITY CODE: ESE26OS12

PROJ.NAME: ECON. BYALUATION OF CLOSURE CAP BARRTER MATERIAL

ESTD or: Pank

Duter. 7-5200

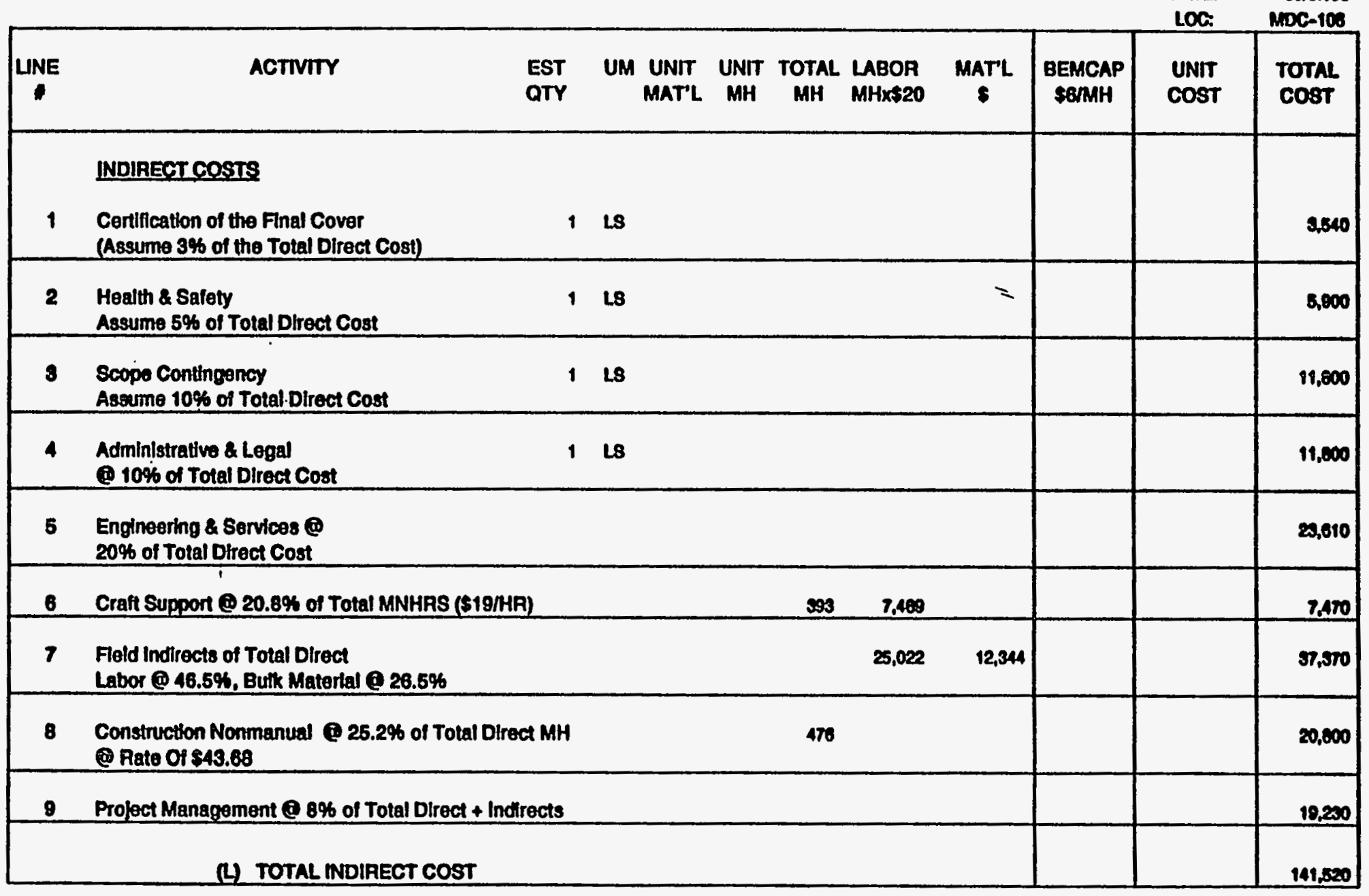

A-2. Cost Estimate for a Cover System Utilizing Bentomat@ and a 30-mil FML (Cont'd) 
D B TAIL B T TMATB

CHK'D BY: 8. MEAD

1001: 7-03

ACTIVITY CODB: ESE260412

FROJ.HAME: ECON. BVALUATFON OF CLOSURE CAP BARRIER MATERUAL

ESTD BY: POWMK

PHONE: T-5260

DATE

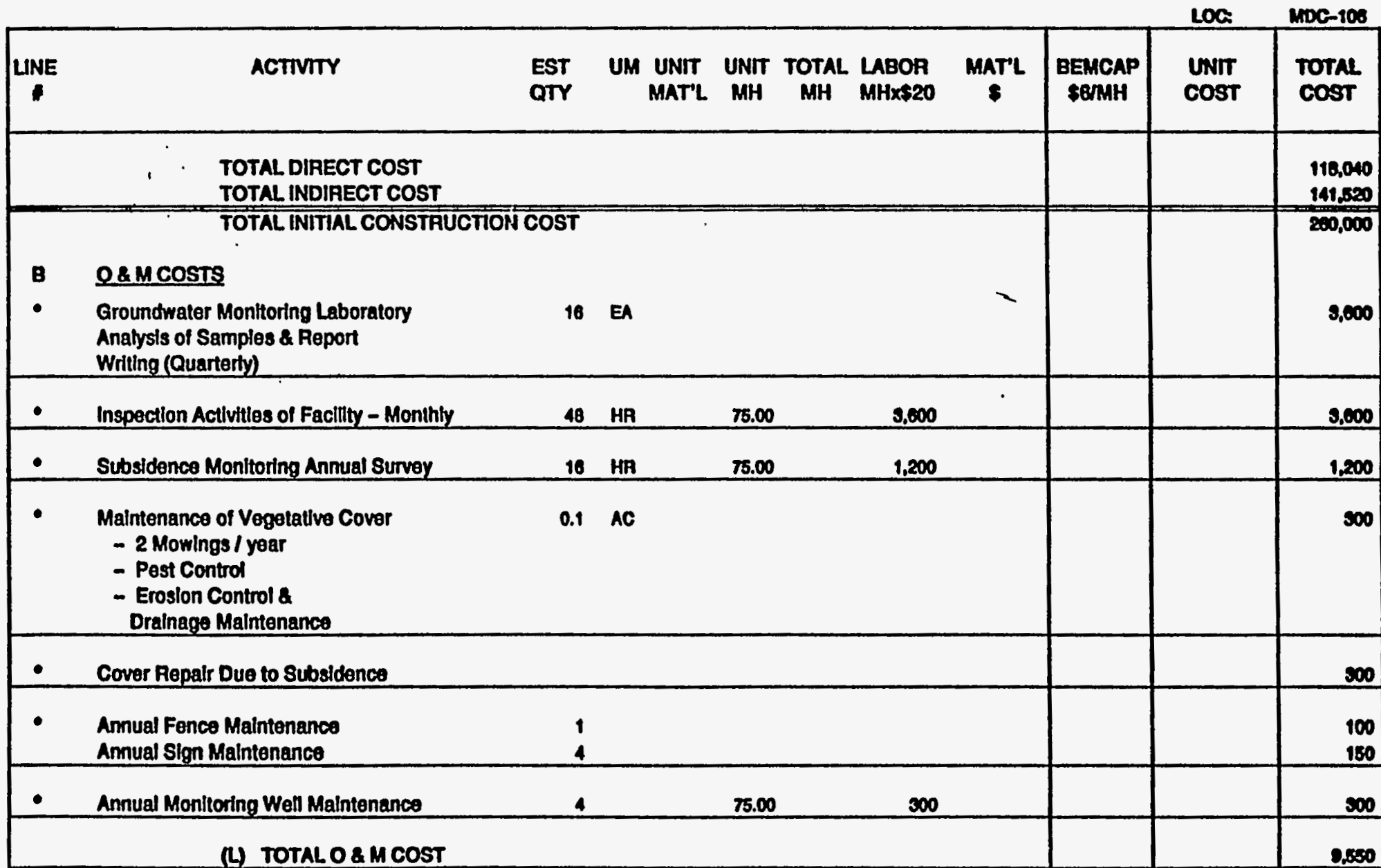

A-2. Cost Estimate for a Cover System Utilizing Bentomato and a 30-mil FML (Cont'd) 
D B T A I L B S TIMAT B

CHK'D BY: 8. MEAD

L001: $7-03$

ACTIVITY CODB: BSE260412

PROJ.NAME: ECON. EVALUATION OP CLOSURB CAP BARRRRR MATERIAL

EST'D BY:

PHONE 7-6200

DAre Derom

LOC. MOC-100

SUMMARY

ONE TENTH (.1) ACRE SITE - BENTOMAT IGBM

C TOTAL COST ( Total Prosent Worth)

(i) Present Worth of 0 \& $M$ Cost

147,000

(5\% discount Rate for 30 Years)

(Total O \& $\mathrm{MA} \times$ 15.3725)

(ii) Total Initial Construction Cost

260,000

(L) TOTAL PROJECT COST (Rounded to the nearest Thousand)

407,000

A-2. Cost Estimate for a Cover System Utilizing Bentomat $(8)$ and a 30-mil FML (Cont'd) 


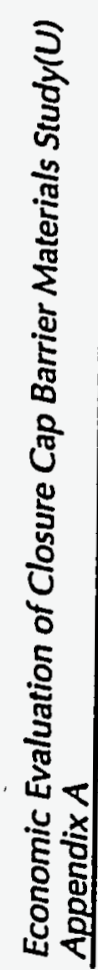

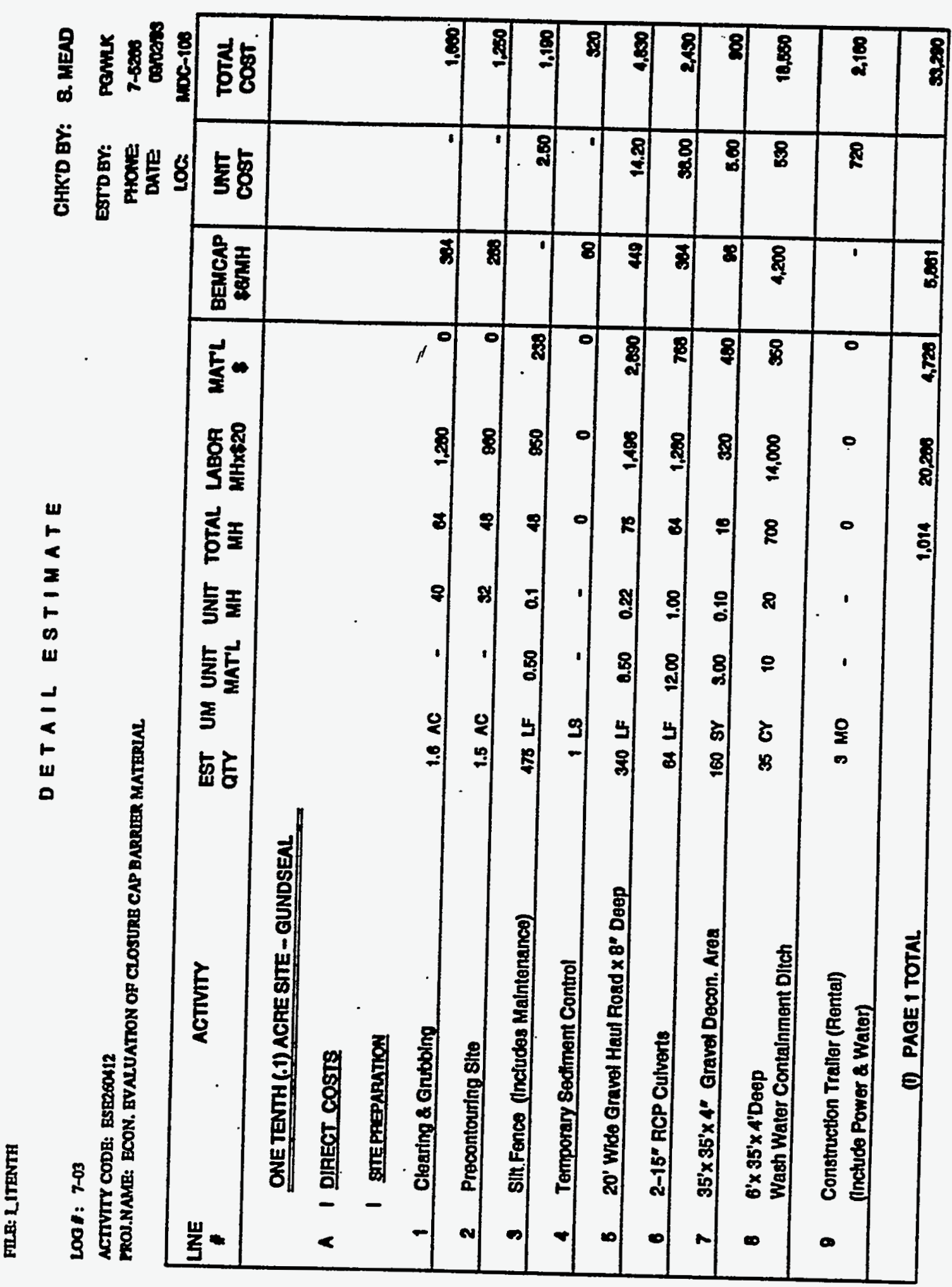

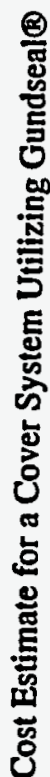


DETAIL ESTIMATE CHKDEY: 8.MEAD

1001: $7-00$

ACTIVITY CODE: BSE260012

ROSANAMB: BCON. BVNLUATION OF CLOSURB CAP BANAURR MATERHAL

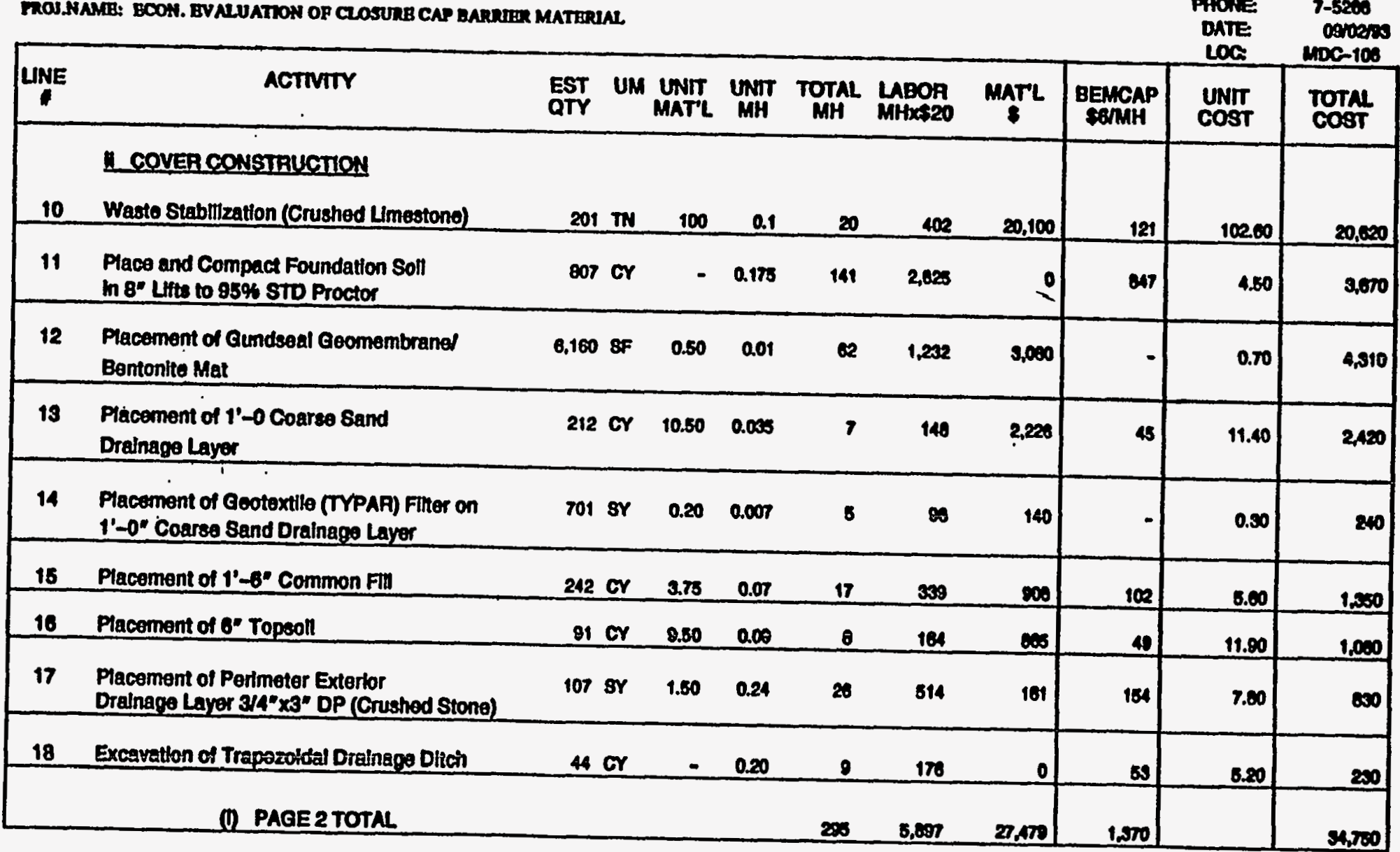

A-3. Cost Estimate for a Cover System Utilizing Gundseal (Cont'd) 
D B TAIL BSTIMATB

CHKD BY: S. MEAD
ESTD OY: POMMK

Lo01: 7-03

ACTIVIT CODE: ESE2CON12

FROS.NAME: BCON. BVALUATTON OP CLOSURB CAP BARRIER MATERHAL

PHONE 7-8206

DATE OSTros

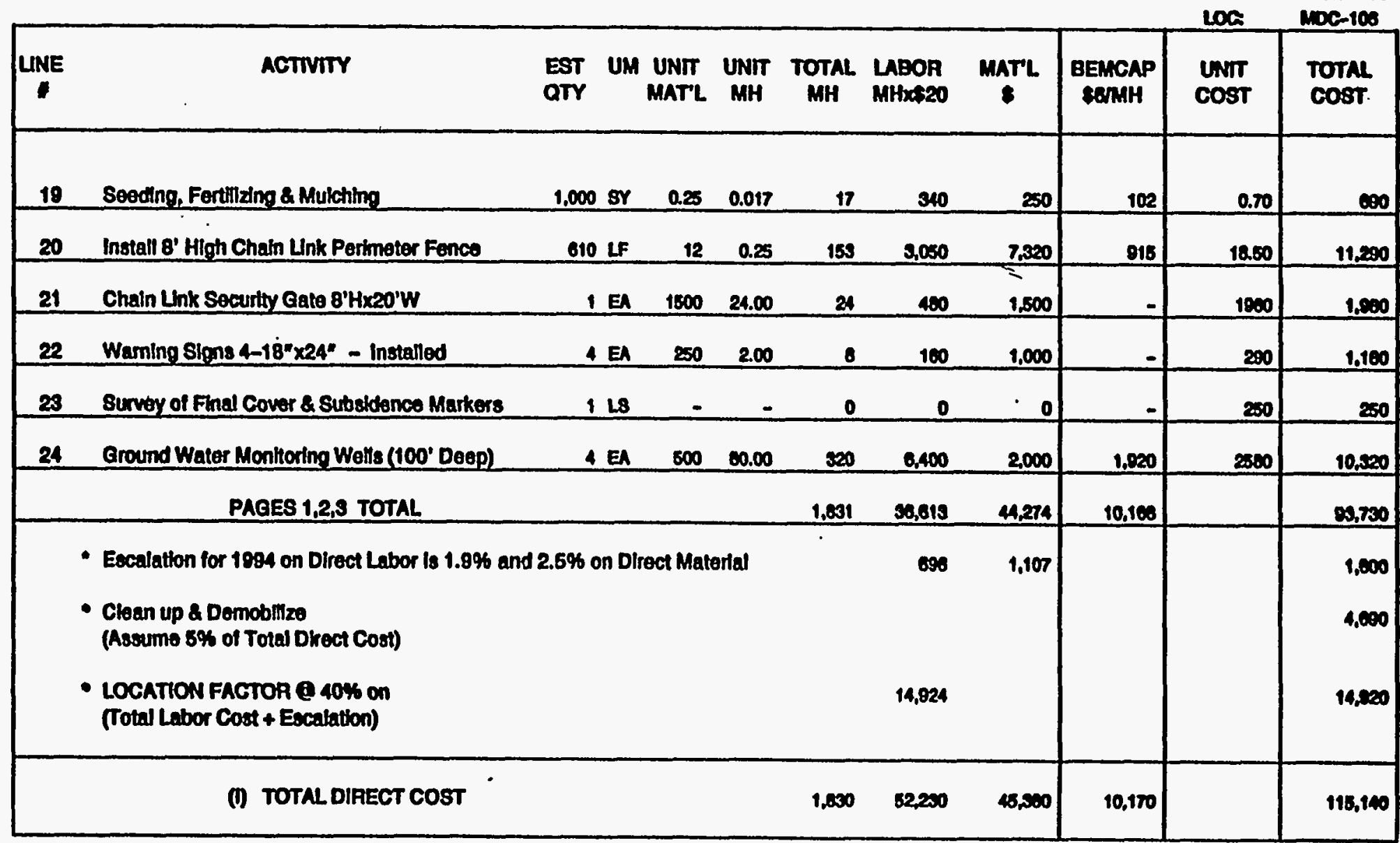

A-3. Cost Estimate for a Cover System Utilizing Gundseal@ (Cont'd) 


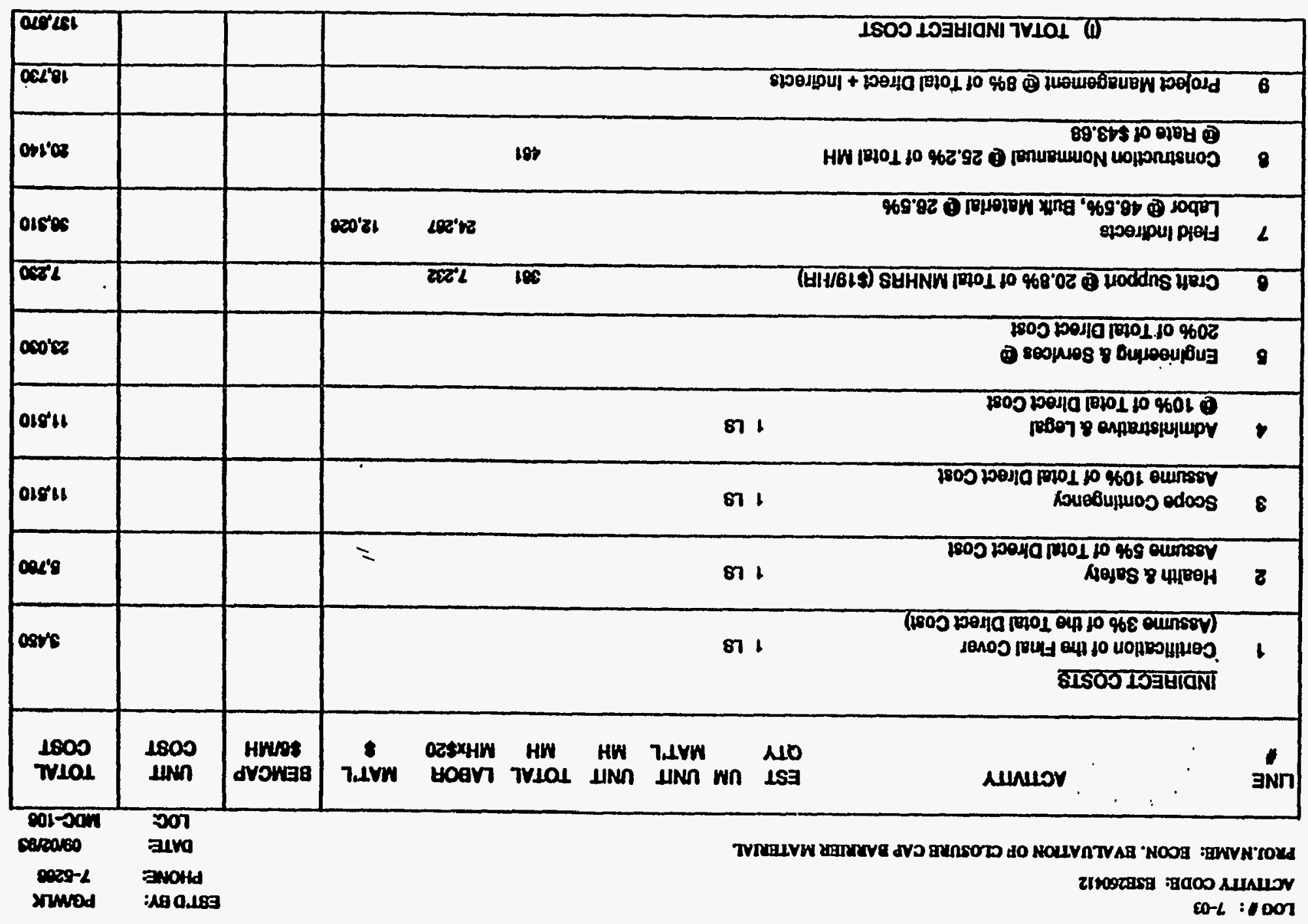

ovam '8 : 2 9 0, XHO

A I V W I S \& I I V I G 


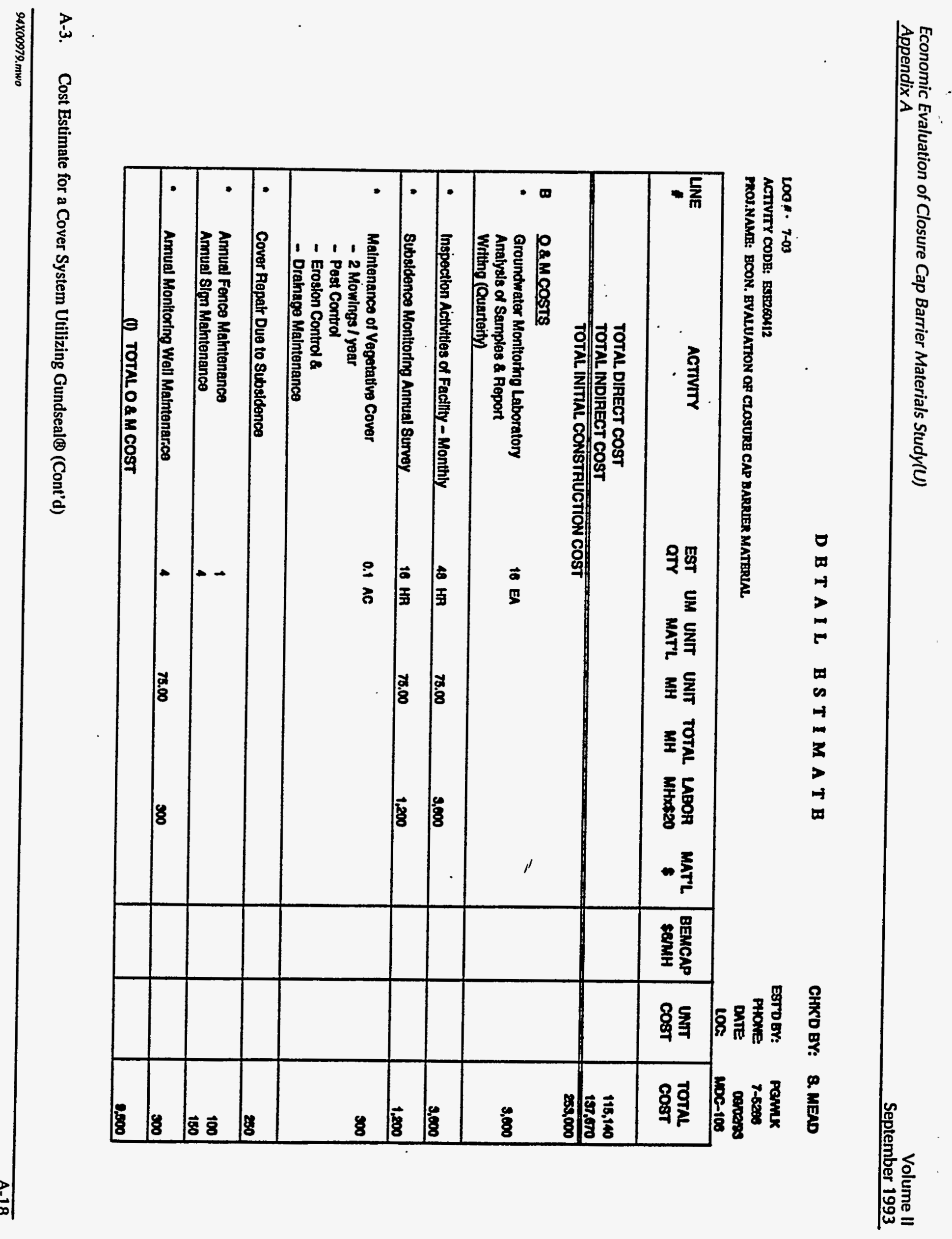


Loo1: $7-03$

ACTVITY CODB: BSE260112

Esto er: pamik

PROJ.NAMG: BCON. EVALUATION OF CLOSURB CAP BARRUER MATERUAL

PHow:

$7-6200$

OATE Davers

LOC. MOC-100

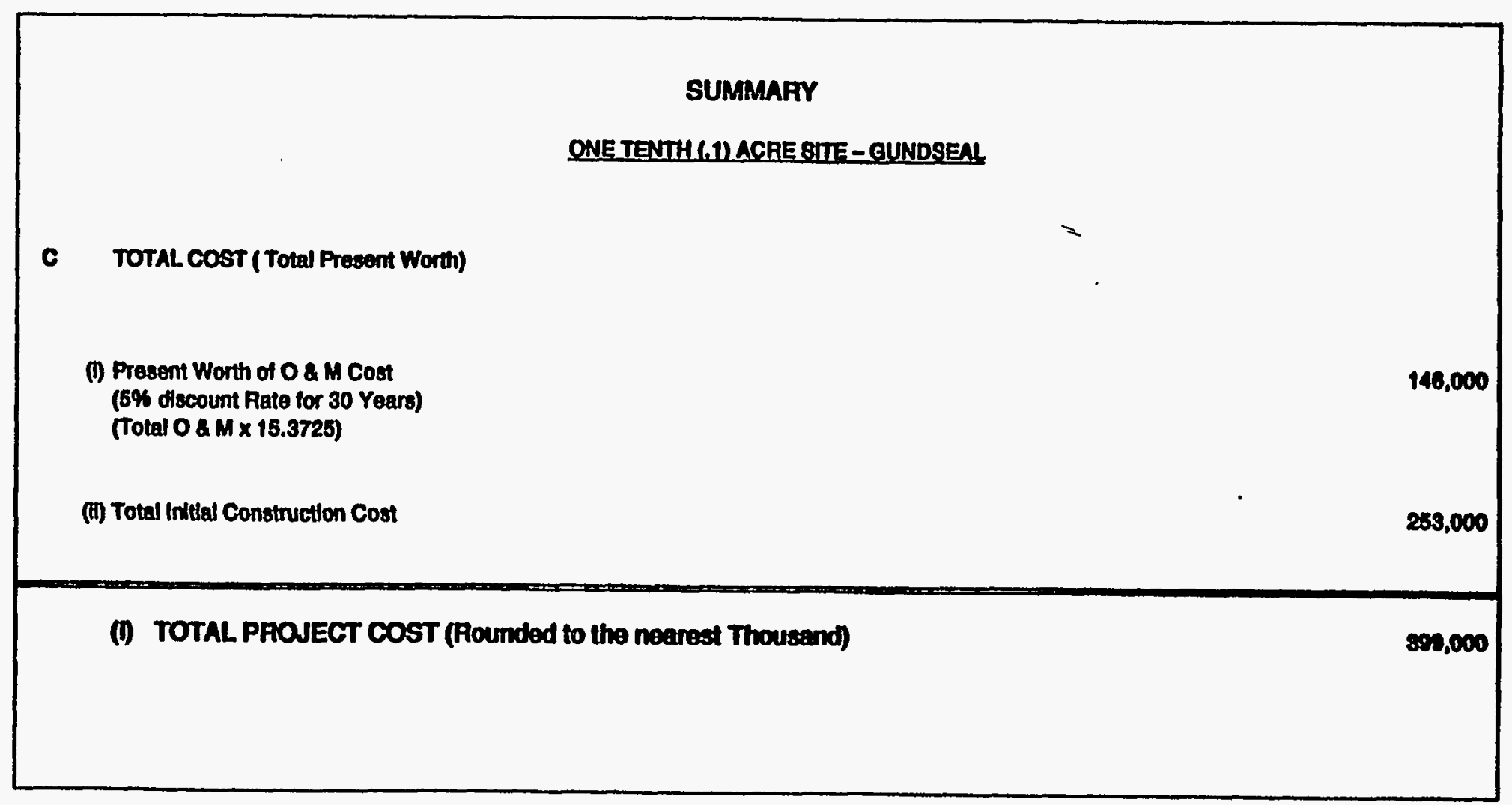

A-3. Cost Estimate for a Cover System Utilizing Gundseal@ (Cont'd) 
FILE: P_ITENTH

DETAIL ESTIMATE

CHKD BY: 8. MEAD

LOa I: 7-03

ACTIVTTY CODE: ESE2SOL12

PROY.NAMB: BCON. BVALUATION OR CLOSURE CAP BARRRER MATERUAL

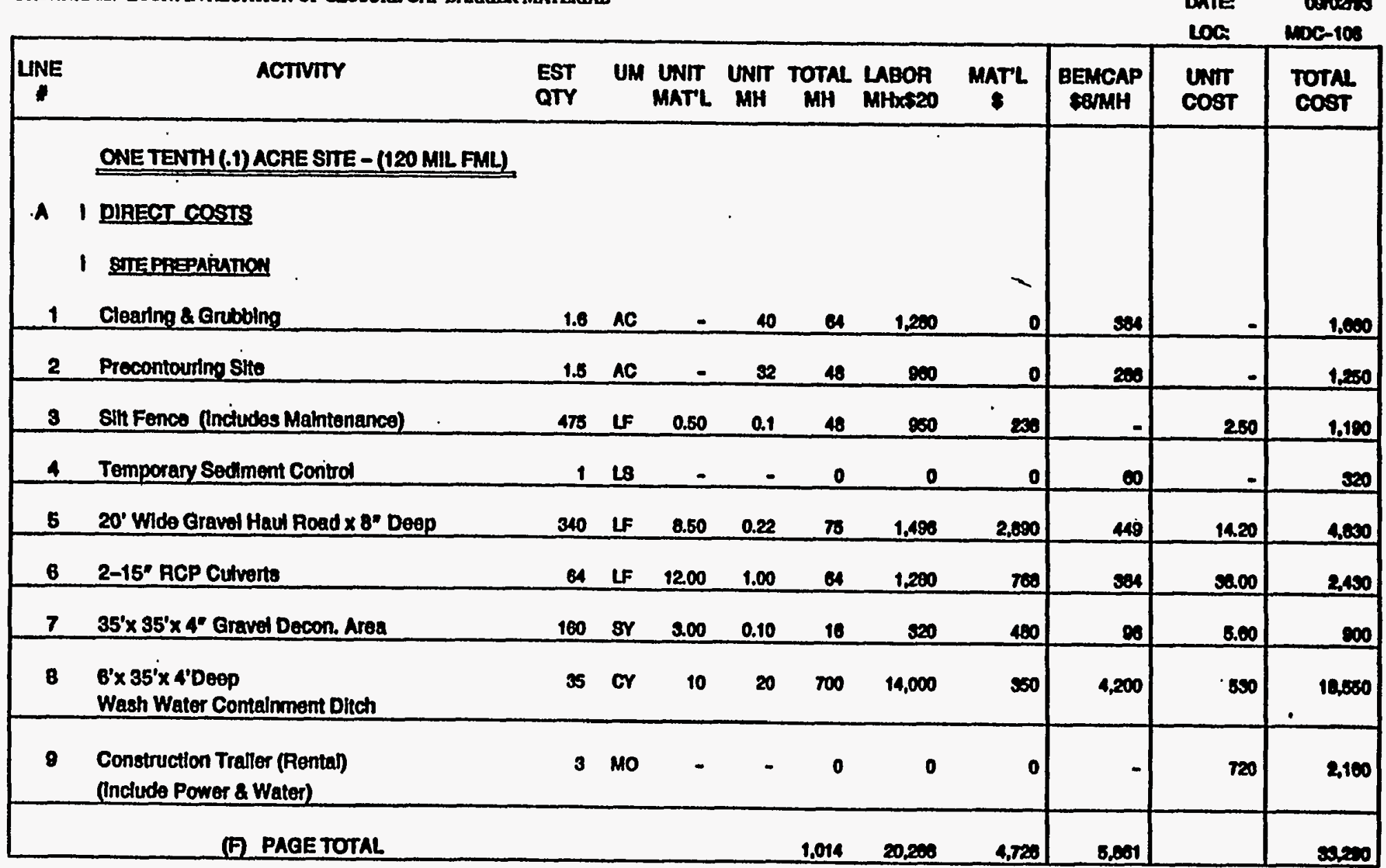

A-4. Cost Estimate for a Cover System Utilizing 120-mil High Density Polyethylene (HDPE) Liner 
DETAIL ESTIMATE

CHK'D BY: 8. MEAD

Loa : $7-03$

ACTTVITY CODE: ESE2COA12

PROJ.NAME: ECON. EVALUATION OF CLOSURE CAP BARRIER MATERIAL

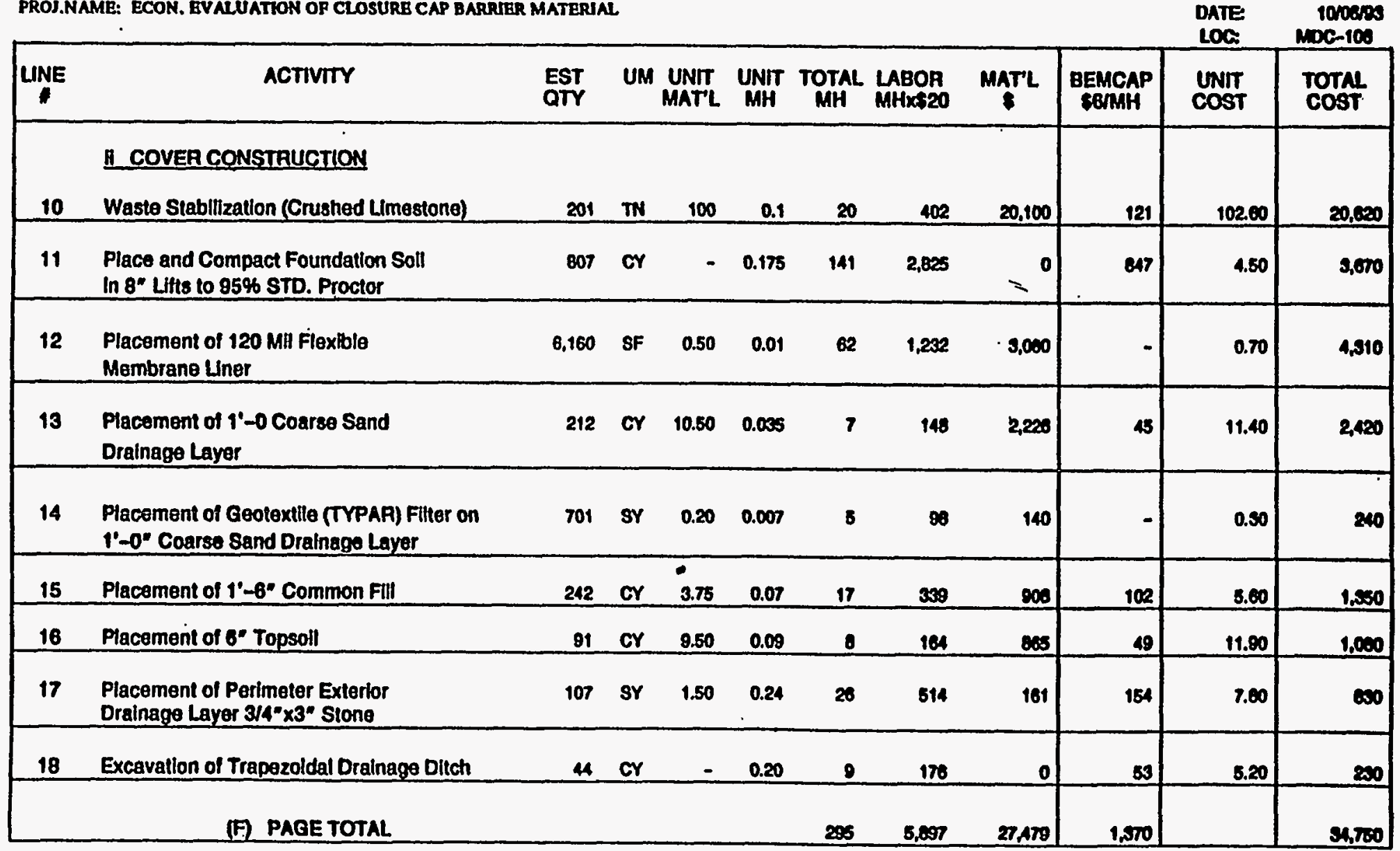

A-4. Cost Estimate for a Cover System Utilizing 120-mil High Density Polyethylene (HDPE) Liner (Cont'd) 
D B T A I B S T IMAT B

Loo: $7-03$

ACTIVITY CODE: BSE200412

PROY.NAMB: BCON. BVALUATTON OF CLOSURB CAP BARUTBR MATERURL

CHKD BY: 8. MEAD

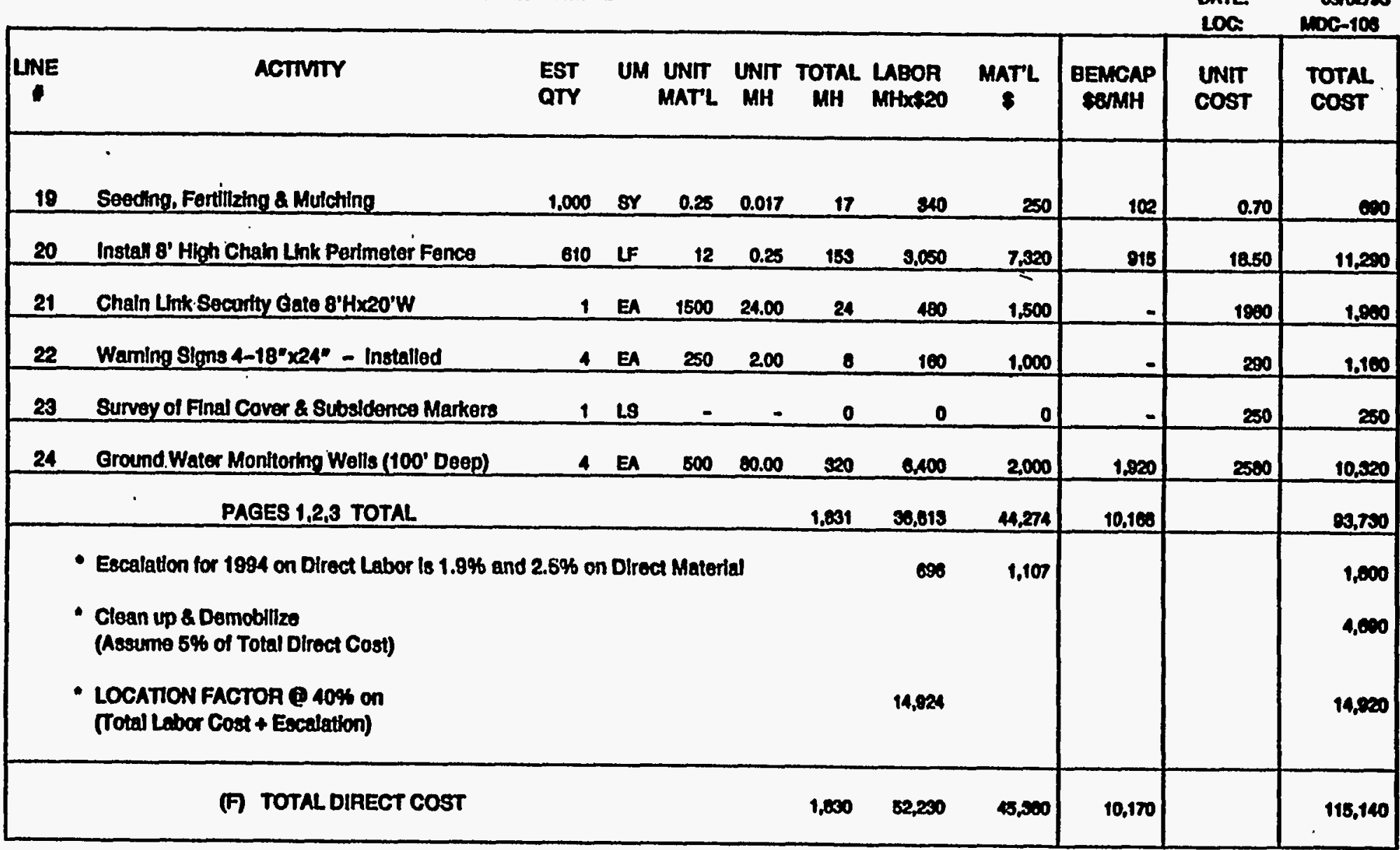

A-4. Cost Estimate for a Cover System Utilizing 120-mil High Density Polyethylene (HDPE) Liner (Cont'd) 
D BTA I L BSTIMAT B

CHK'D BY: 8. MEAD

L001: 7-03

ACTIVITY CODE: BSE200412

MOU.NAME: BCON. BVALUATION OF CLOSURB CAP BARRIER MATERIAL

ESTD EY: Pamk

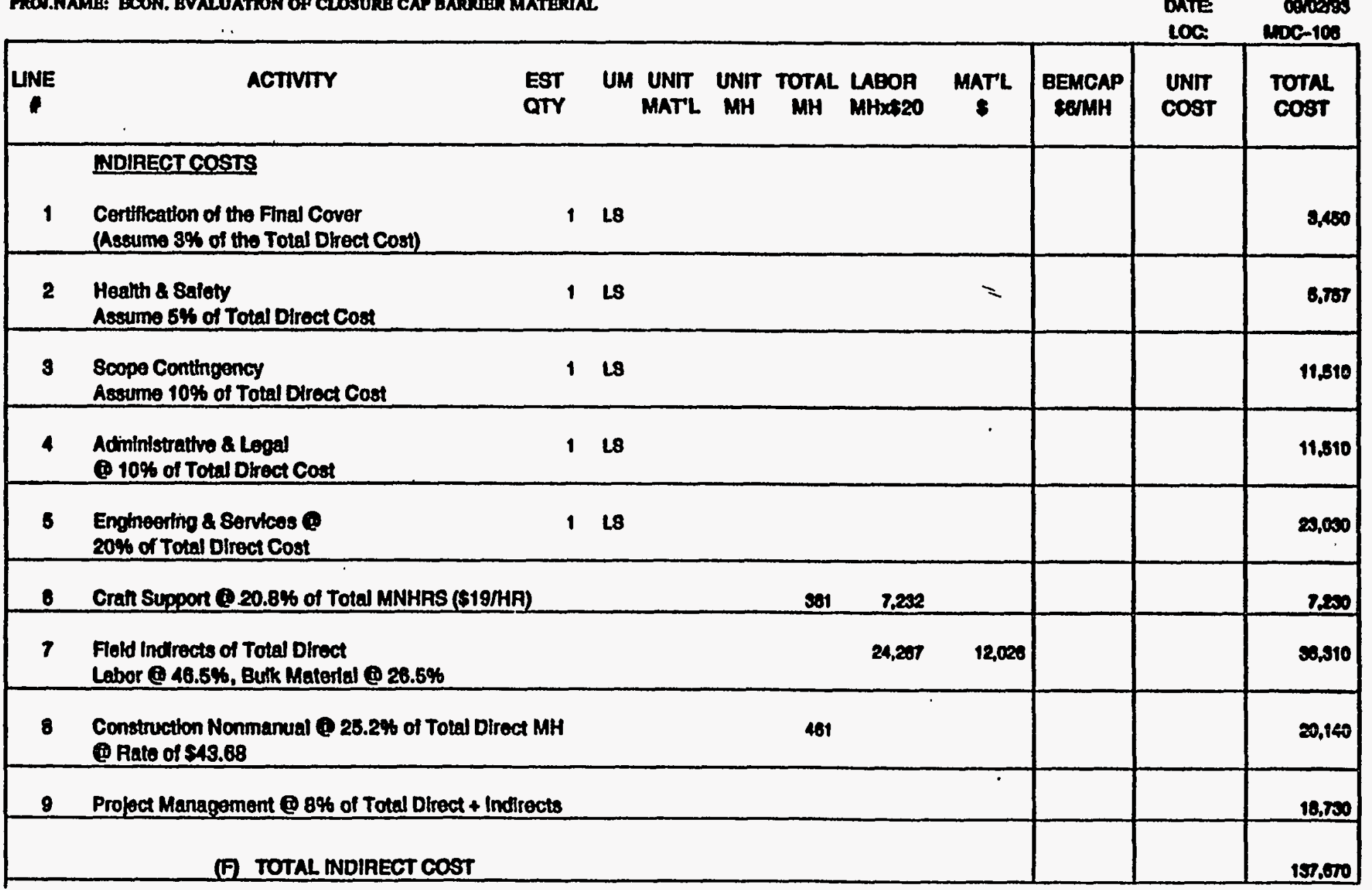

A-4. Cost Estimate for a Cover System Utilizing 120-mil High Density Polyethylene (HDPE) Liner (Cont'd) 
D BTAILABTIMATE

CHKD BY: 8. MEND

2001: 7-03

ACTIVTTY CODE: BSE2OOA12

PROJ.NAME: BCON. EYALUATION OF CLOSURB CAP BARRIER MATERIAI

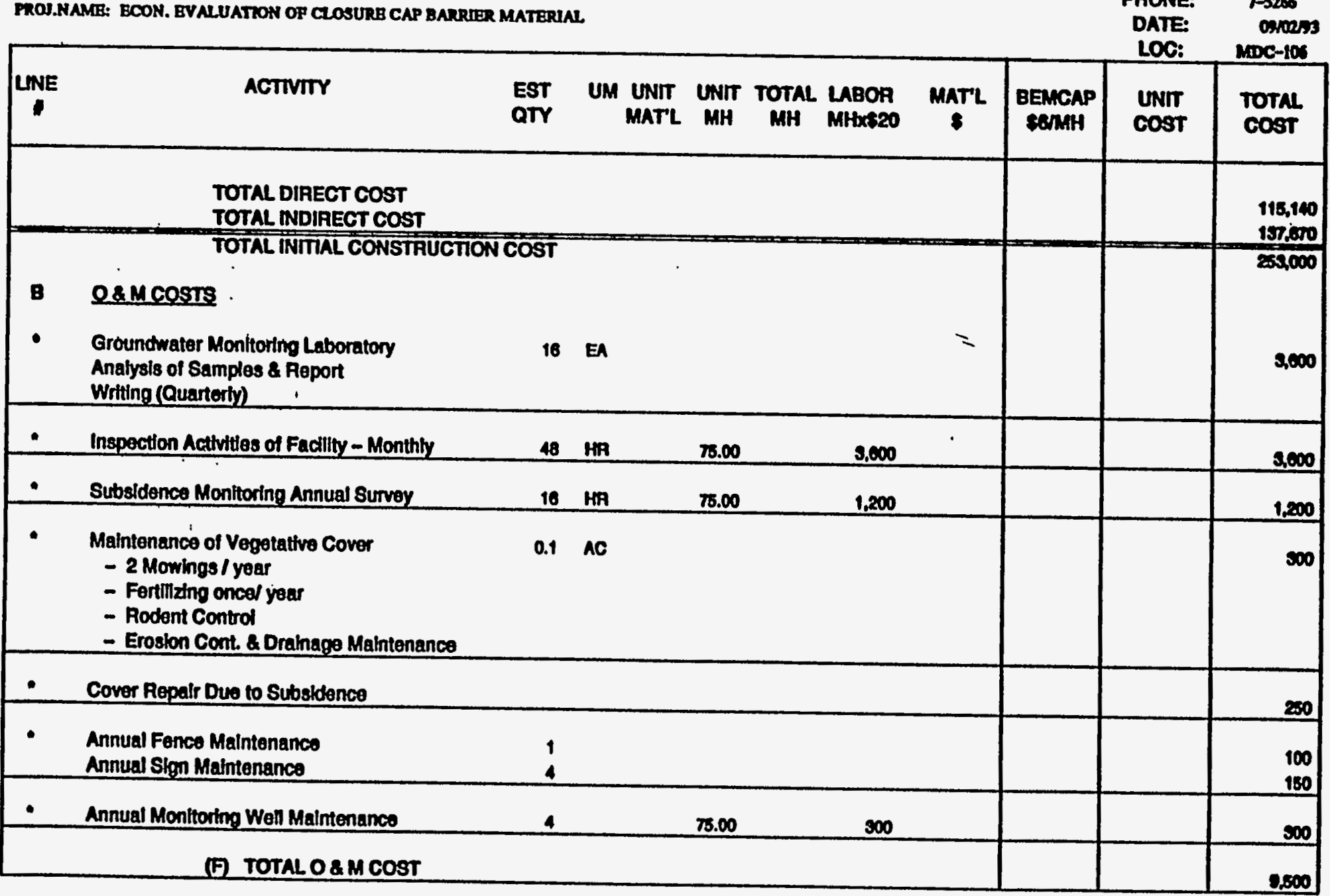

A-4. Cost Estimate for a Cover System Utilizing 120-mil High Density Polyethylene (HDPE) Liner (Cont'd) 


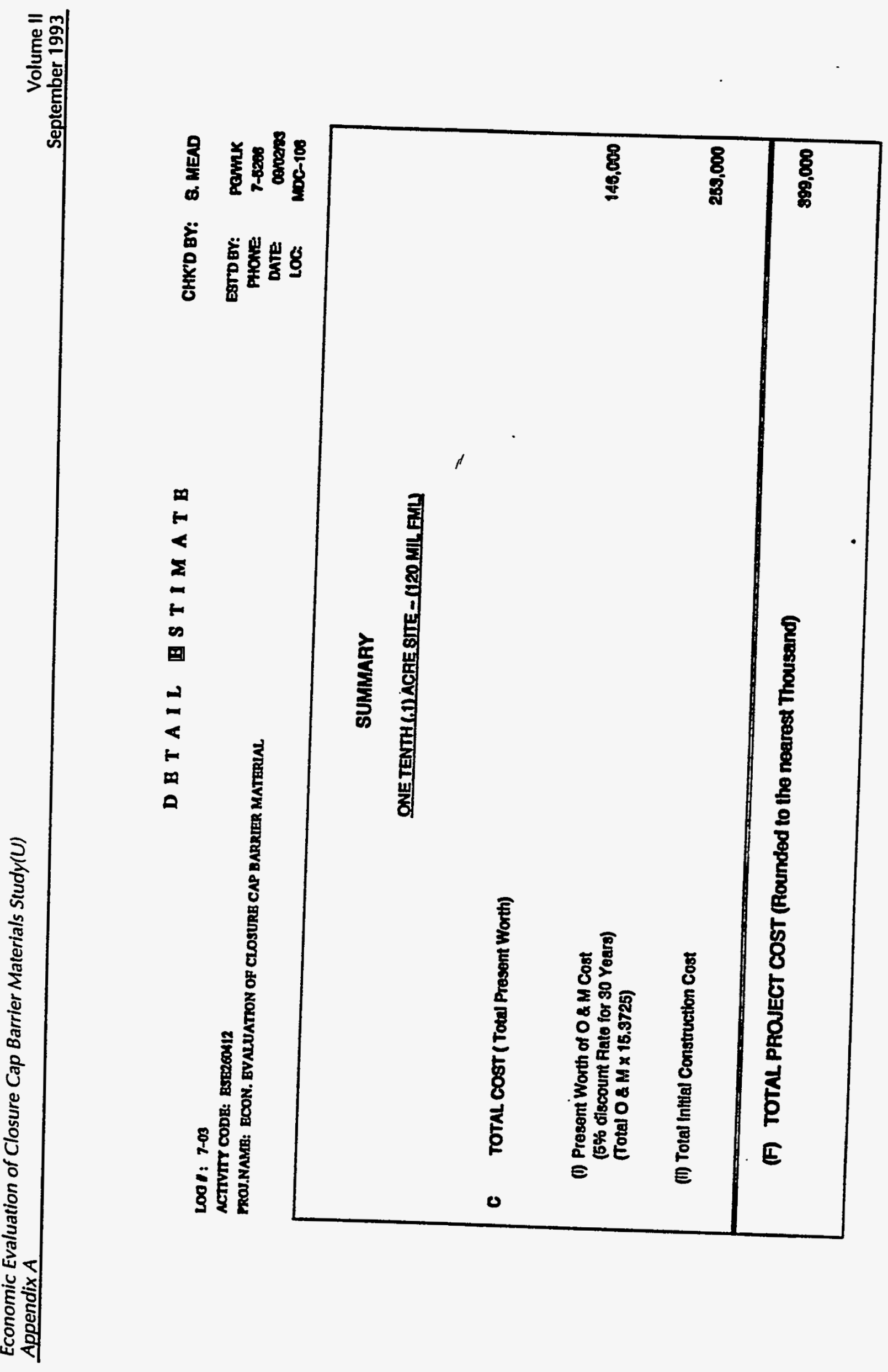


DETAILESTIMATE

Lodf: 7-03

ACTIVITY CODE: EXE250112

FROU:NAME: BCON. EVALUATION OF CLOSURR CAP BARRIER MATERIAL

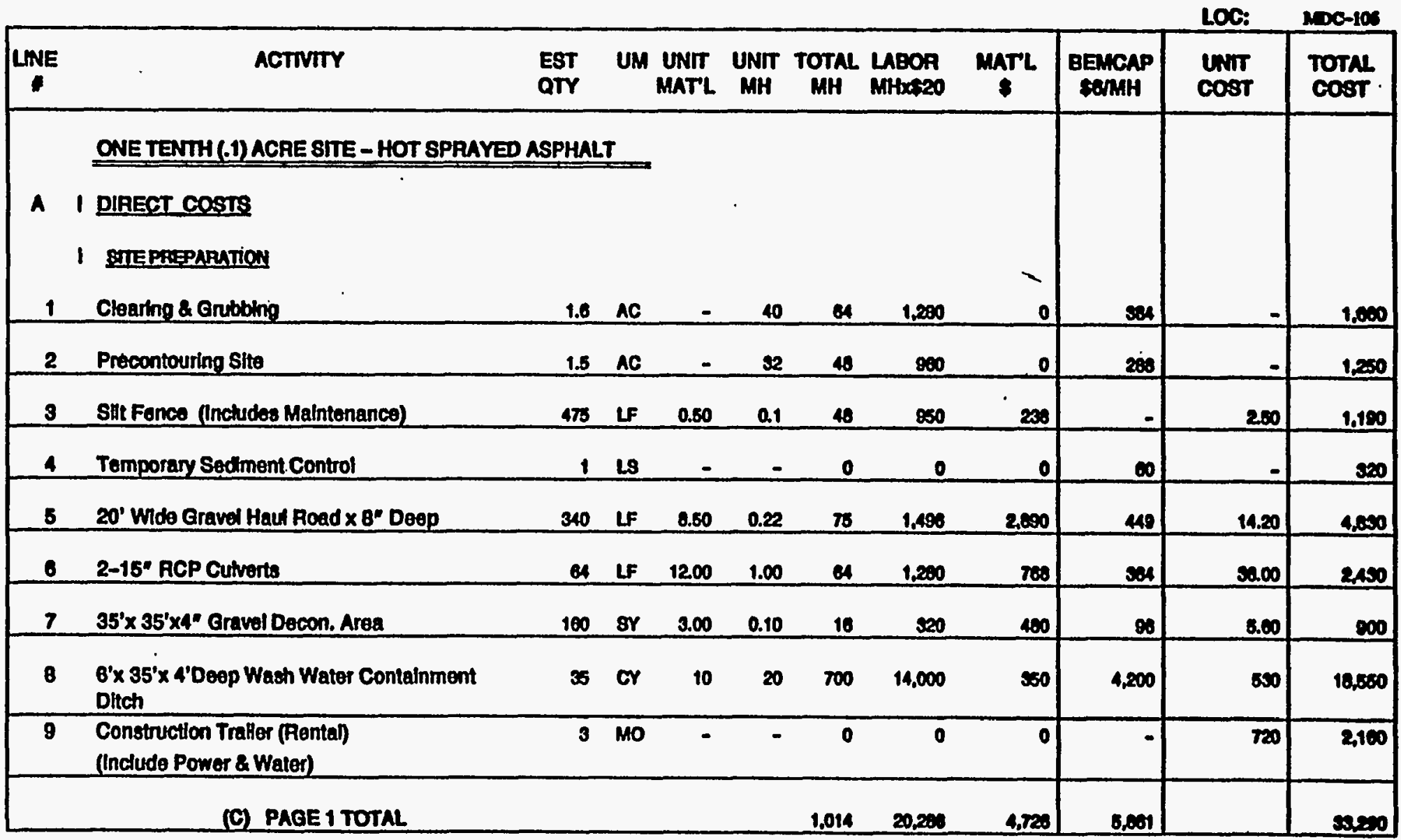

A-5. Cost Estimate for a Cover System Utilizing 1/4-inch Thick Hot Sprayed or Emulsified Asphalt 


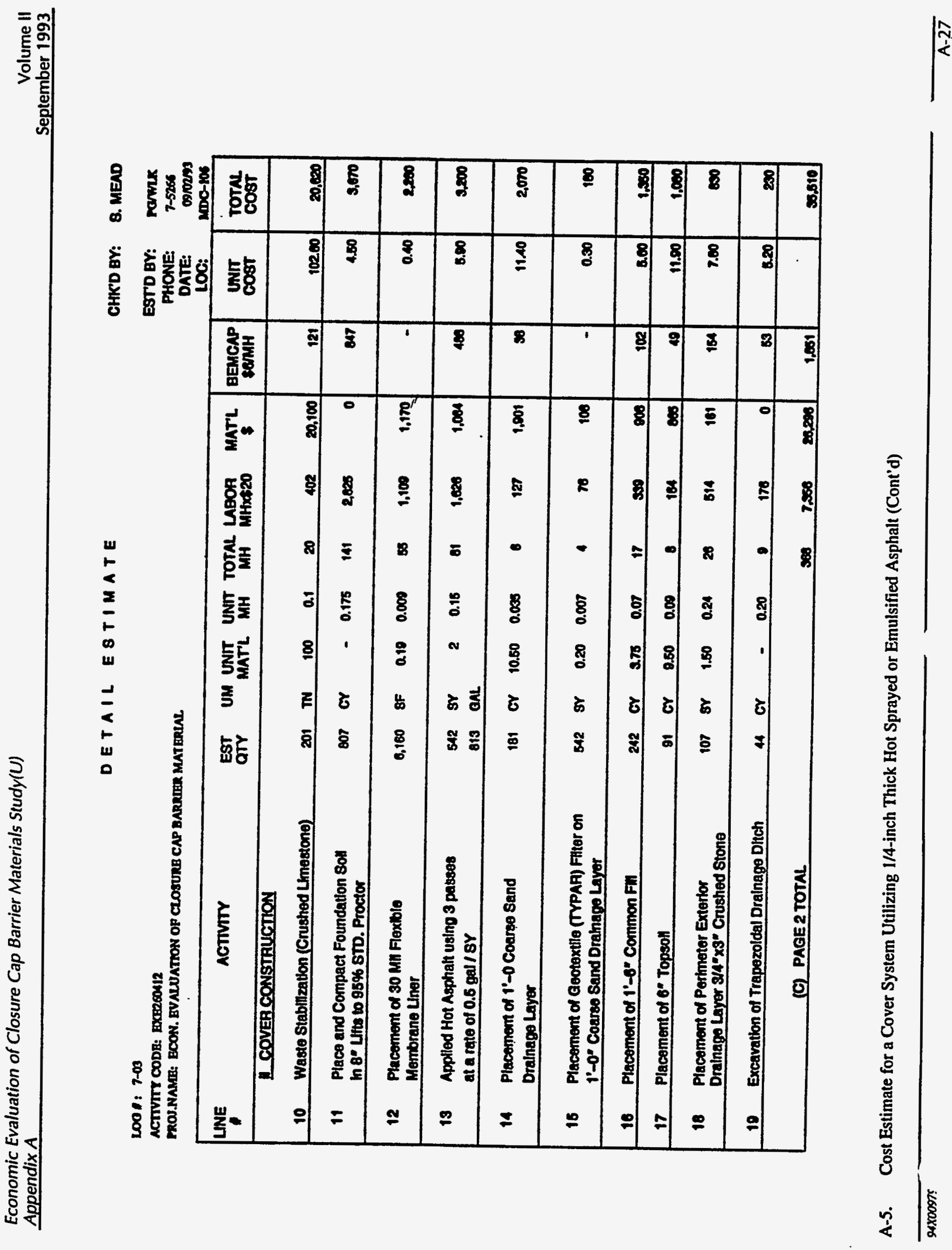


D B TAIL B S TIMAT B

Loof: $7-03$

ACTIVIY CODB: BXEROOH12

PROJ.NAMR: BCON. EVALUATION OF CLOSURB CAP BARRIER MATEIANL.

\begin{tabular}{|c|c|c|c|c|c|c|c|c|c|c|c|}
\hline LINE & ACTIVTY & $\begin{array}{l}\text { EST } \\
\text { QTY }\end{array}$ & UM & $\begin{array}{l}\text { UNIT } \\
\text { MATL }\end{array}$ & $\begin{array}{l}\text { UNIT } \\
\text { MH }\end{array}$ & $\begin{array}{c}\text { TOTAL } \\
\text { MH }\end{array}$ & $\begin{array}{l}\text { LABOR } \\
\text { MHtxi20 }\end{array}$ & $\underset{\$}{\text { MATL }}$ & $\begin{array}{c}\text { BEMCAP } \\
\text { POMHH }\end{array}$ & $\begin{array}{l}\text { unrt } \\
\text { cost }\end{array}$ & $\begin{array}{l}\text { TotAL } \\
\text { Cost }\end{array}$ \\
\hline 20 & Seading, Fortilizing \& Mulching & 1,000 & $\mathbf{8 Y}$ & 0.25 & 0.017 & 17 & 340 & 200 & 102 & 0.70 & 000 \\
\hline 21 & Install 8' High Chain Unk Perimeter Fence & 810 & LF & 12 & 0.25 & 158 & 3,050 & 7,320 & ns. & 10.50 & 11,200 \\
\hline 22 & Chain Unk Socurty Gate 8'Hx20'W & 1 & EA & 1500 & 28.00 & 24 & 400 & 1,500 & - & 1800 & 1000 \\
\hline 23 & Waming Slons $4-18^{\circ} \times 24$ - Installed & 4 & EA & 200 & 2.00 & $\mathbf{8}$ & 160 & 1,000 & - & 290 & 1.160 \\
\hline 24 & Survoy of Final Cover a subsidence Markere & 1 & 18 & - & - & 0 & 0 & $\cdot$ & - & 2050 & 200 \\
\hline 25 & Ground Water Monitoring Wells (100' Deep) & 4 & EA & 800 & 80.00 & 320 & 6.400 & 2,000 & 1.020 & 2000 & 10,500 \\
\hline & (C) PAGES 1,2,3 TOTAL & & & & & 1,004 & 30,072 & 40,001 & 10,049 & & MA:O \\
\hline & $\begin{array}{l}\text { Escalation for } 1994 \text { on Direct Labor is } 1.9 \% \text { ar } \\
\text { Clean up \& Damobmize } \\
\text { (Assume 6\% of Total Direct Cost) } \\
\text { LOCATION FACTOR P } 40 \% \text { on } \\
\text { (Total Labor Cost + Escalation) }\end{array}$ & 2.5\% on & Direc & Mater & & & 720 & 1,07 & & & $\begin{array}{l}1,000 \\
1,720\end{array}$ \\
\hline & (C) TOTAL DIRECT COST & & & & & 1,000 & 84,510 & 4,170 & 10,000 & & 11acso \\
\hline
\end{tabular}

A-5. Cost Estimate for a Cover System Utilizing 1/4-inch Thick Hot Sprayed or Emulsified Asphalt (Cont'd) 
D B T A I L B S T I M A B

CHKD BY: 8. MEAD

Lo01: 7-03

ACTTVITY CODE: EXR2OOH12

PROJ.MAME: BCON. EVALUATION OP CLOSURB CAP BARRIBR MATERIAL.

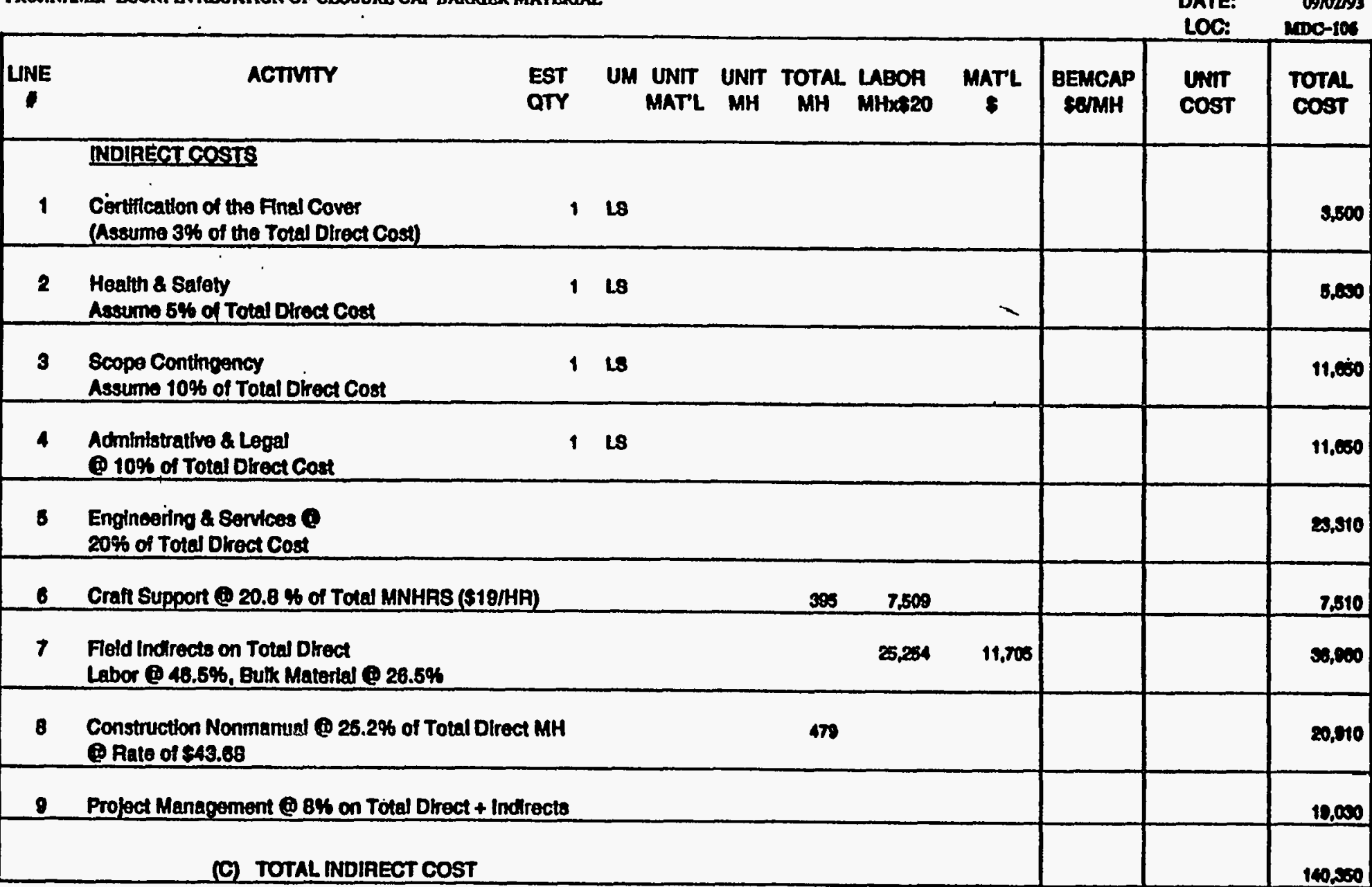

A-5. Cost Estimate for a Cover System Utilizing 1/4-inch Thick Hot Sprayed or Emulsified Asphalt (Cont'd) 
D BTAIL BSTIMATB

CHK'D BY: 8. MEAD

2001: 7-03

ACTVITY CODE: BXXEOSA1?

PROO.NAME: BCON. EYALUATTON OF CLOSURB CAP BARRIER MATERULI

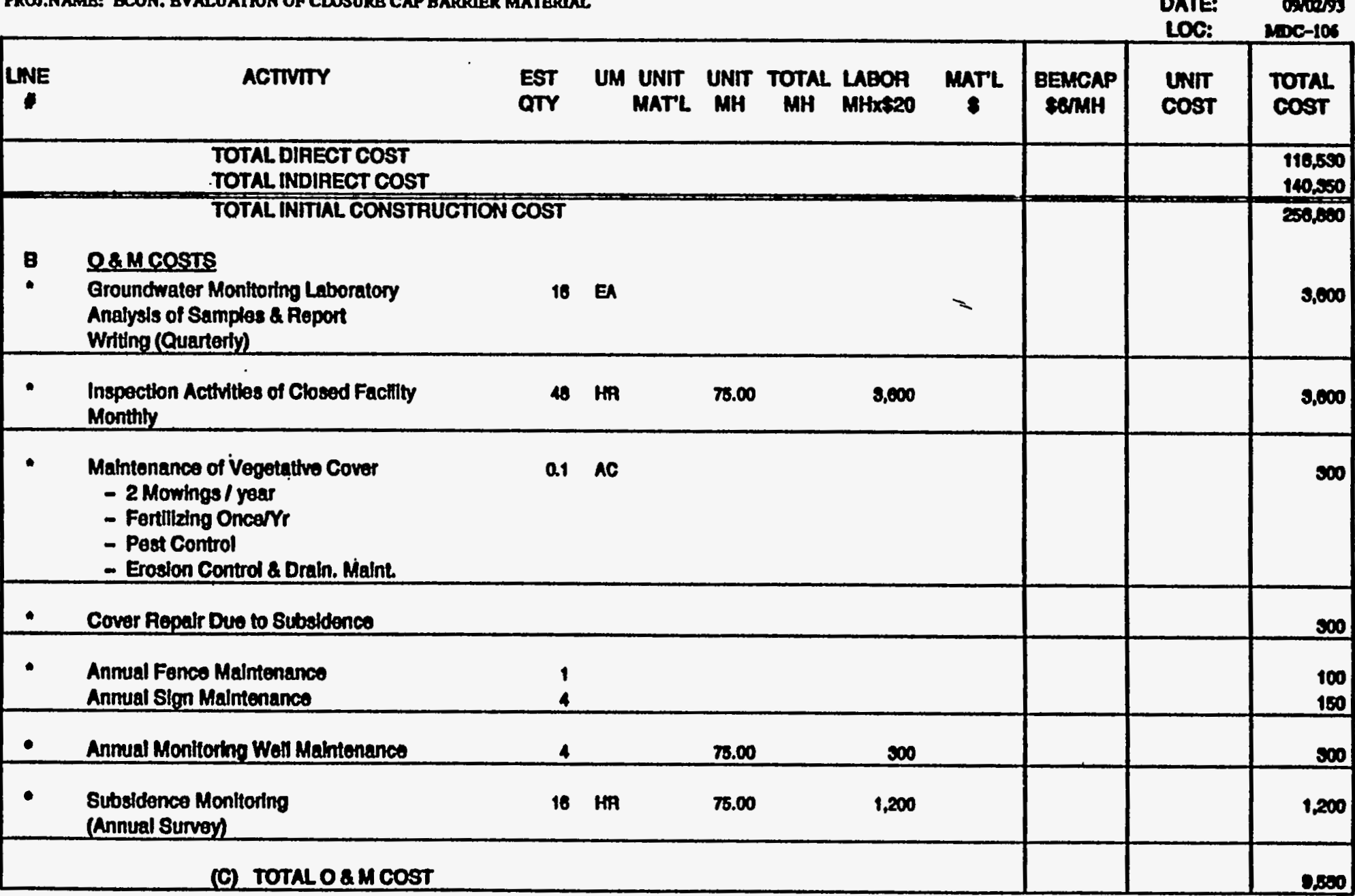

A-5. Cost Estimate for a Cover System Utilizing 1/4-inch Thick Hot Sprayed or Emulsified Asphalt (Cont'd) 
D B TAIL E S TIMATB

\begin{tabular}{|c|c|}
\hline CHK'D BY: & 8. MEAD \\
\hline $\begin{array}{l}\text { ESTO BY: } \\
\text { PHONE: } \\
\text { DATE: } \\
\text { LOC: }\end{array}$ & 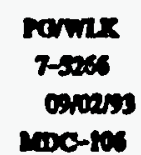 \\
\hline
\end{tabular}

L001: 7-03

ACTIVITY CODE: BXCe200412

FROU.NAME: BCON. EVALUATION OF CLOSURB CAP BARRITR MATERINL

anc-ses

\section{SUMMARY}

ONE TENTH LIIACRE 8IIE - HOT SPRAYED ASPHALT

C TOTAL COST ( Total Prosent Worth)

(1) Present Worth of 0 \& $M$ Cost

147,000

(5\% discoumt Rato for 30 Yeara)

(Total O\&M $\times$ 16.3725)

(iI) Total Capital Cost

260,800

(C) TOTAL PROJECT COST (Rounded to the nearest Thousand)

404,000

A-5. Cost Estimate for a Cover System Utilizing 1/4-inch Thick Hot Sprayed or Emulsified Asphalt (Cont'd) 
DETAIL ESTIMATE

CHK'D BY: S. MEAD

LOG II: 7-03

ACTTVTY CODE: ESE260112

PROU.NAME: ECON. EVALUATION OF CLOSURE CAP BARAIER MATERIAL

ESTOBY:

PHONE:

pamk

DATE

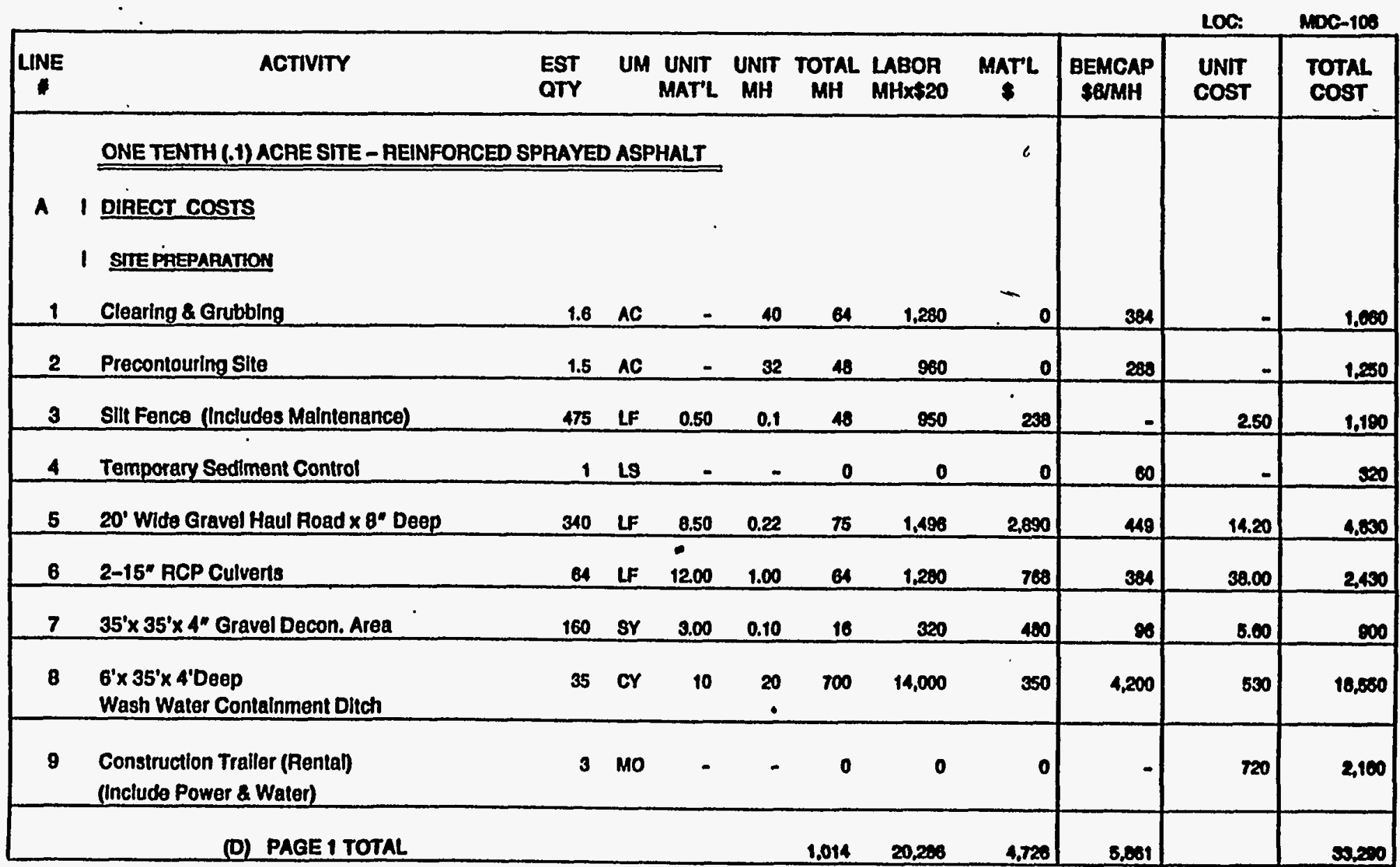

A-6. Cost Estimate for a Cover System Utilizing Reinforced Sprayed Asphalt 
DETAIL ESTIMATE CHK'DBY: 8.MEAD

LOQ : $7-03$

ACTIVIY COOE: ESE260412

PROU.NAME: ECON. EVALUATION OF CLOSURE CAP BARRAEA HATERIAL

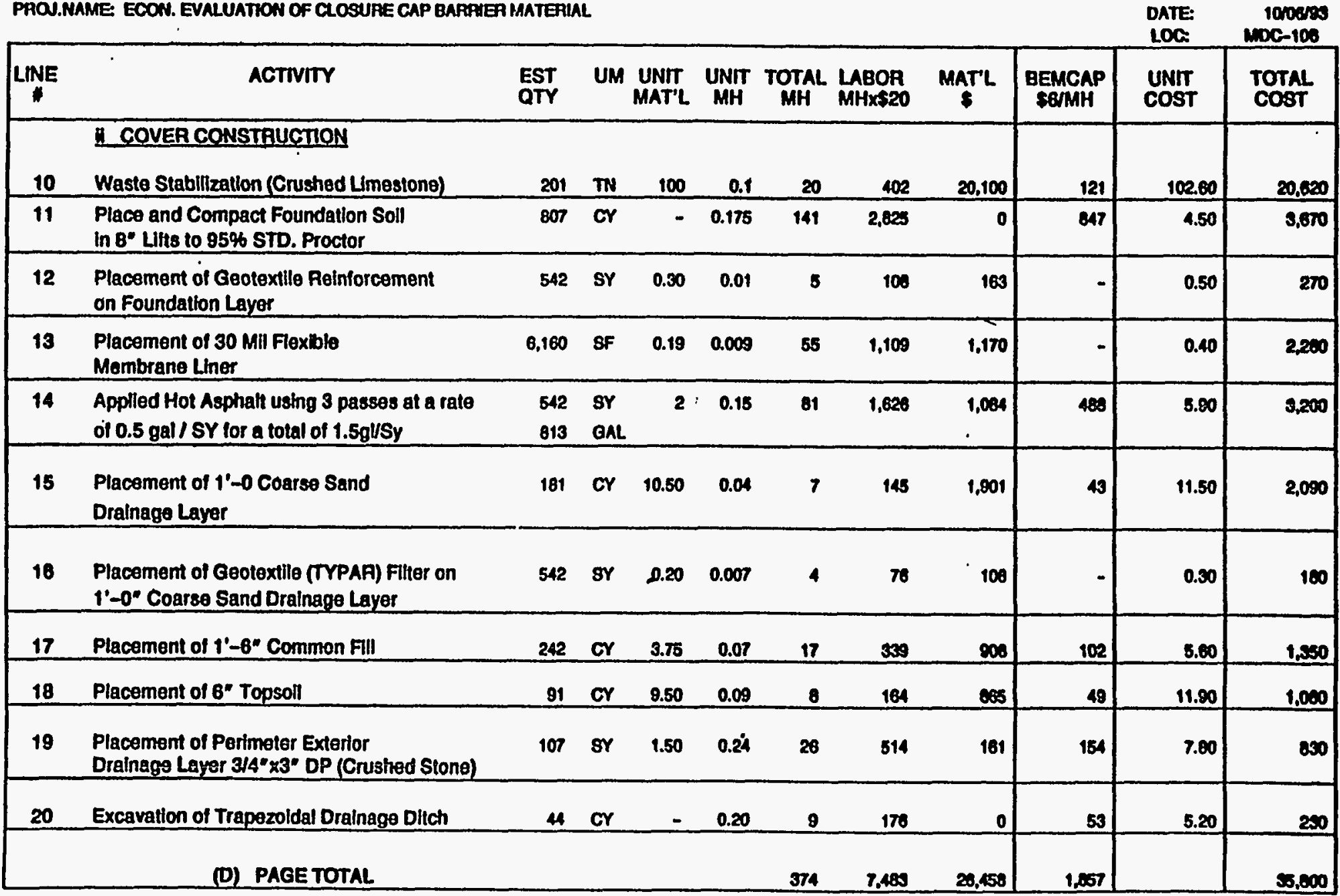

A-6. Cost Estimate for a Cover System Utilizing Reinforced Sprayed Asphalt (Cont'd) 
DETAIL ESTIMATE

LOG : : $7-03$

ACTIVTY CODE: ESE260112

PAOU.NAME: ECON. EVALUATION OF CLOSURE CAP BAPRIER MATEFAAL.

CHK'D BY: 8. MEAD

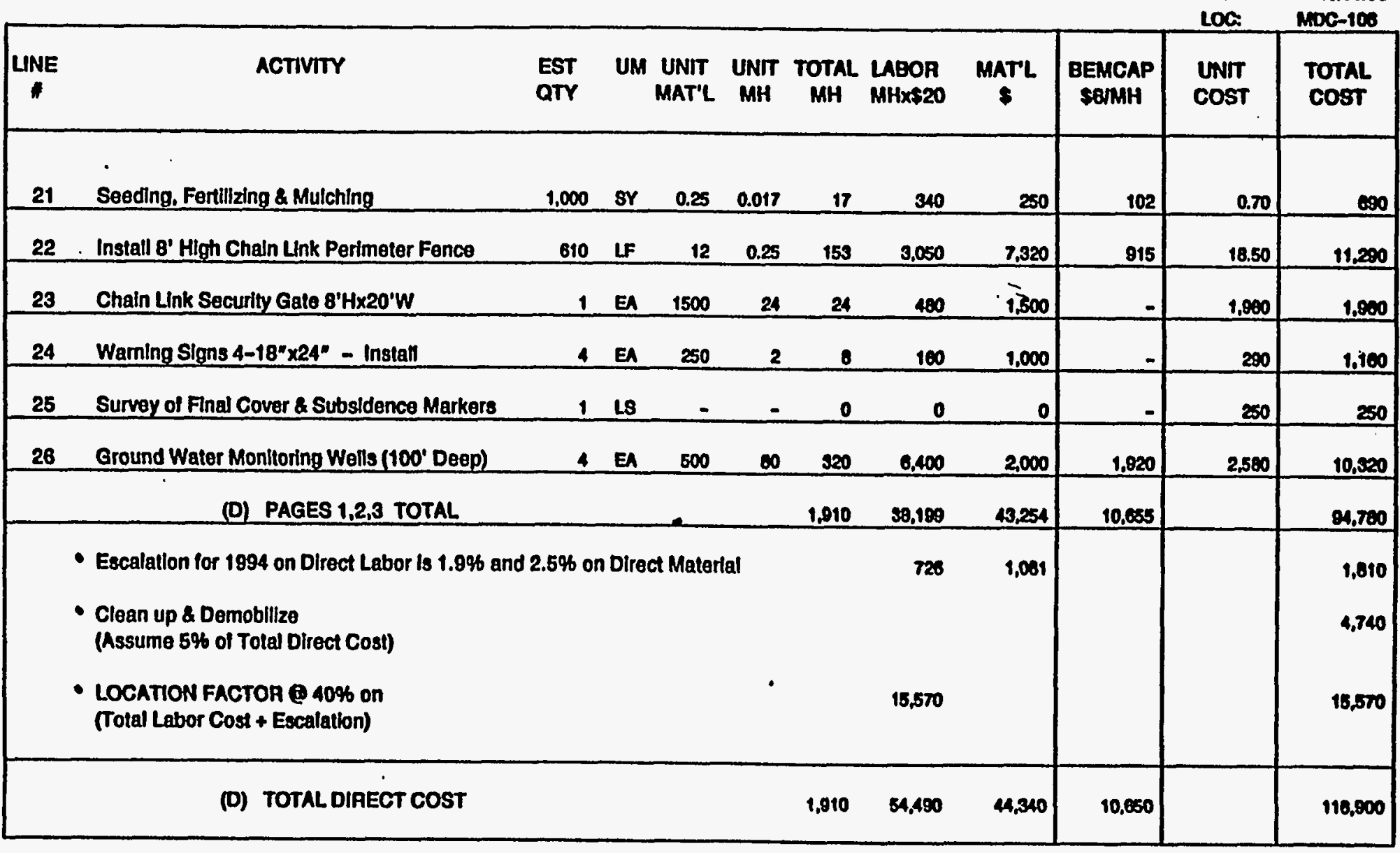

A-6. Cost Estimate for a Cover System Utilizing Reinforced Sprayed Asphalt (Cont'd) 
DETAIL ESTIMATE CHKDEY: S. MEAD

LOQ 1 : $7-03$

ACTIVIT CODE: ESE260412

PAOS.NAME ECON. EVALUATHON OF CLOSURE CAP QARFUEAMATERTAL.

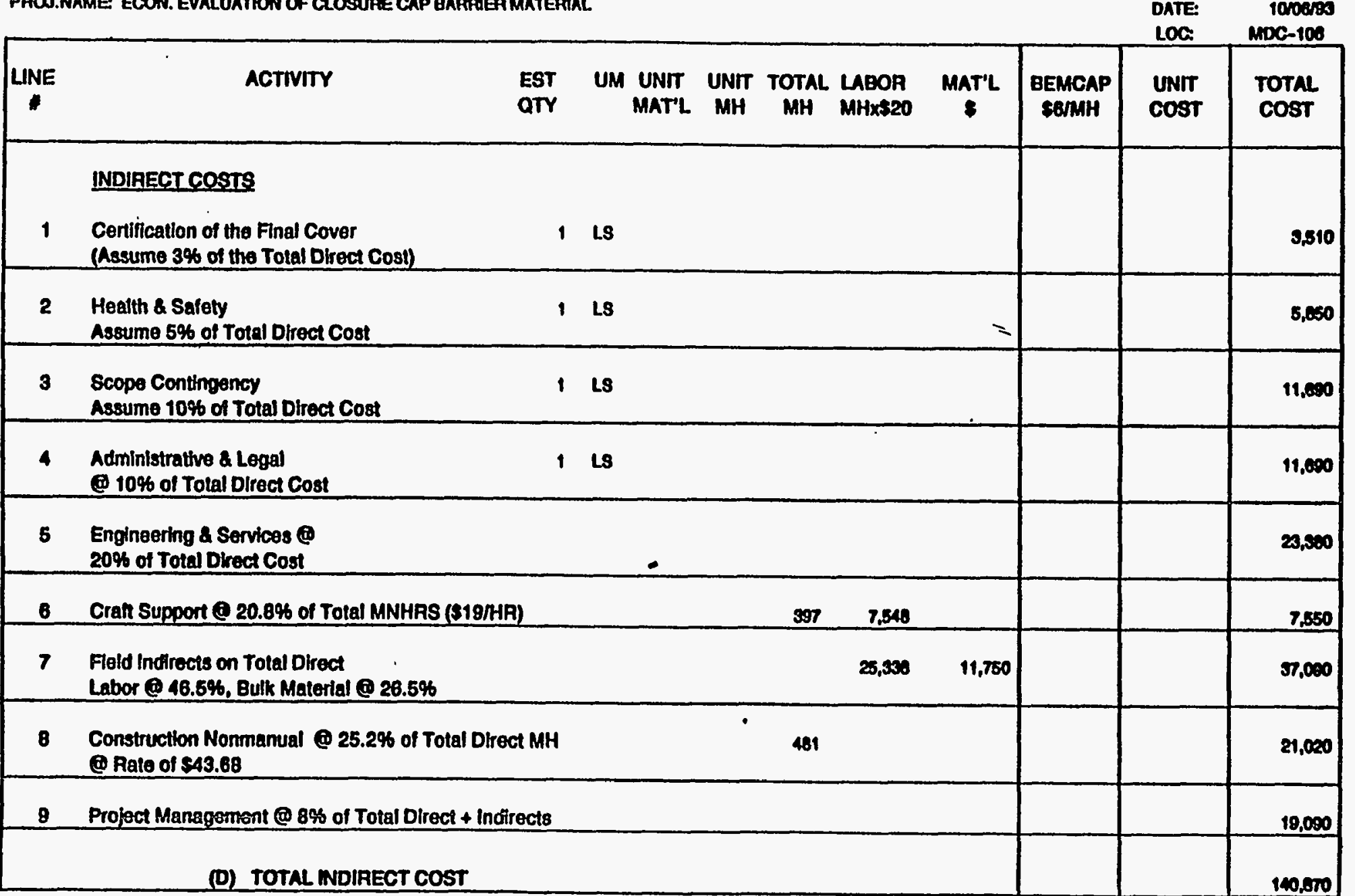

A-6. Cost Estimate for a Cover System Utilizing Reinforced Sprayed Asphalt (Cont'd) 
DETAIL ESTIMATE

CHK'D BY: S. MEAD

LOQ : : 7-03

ACTVITY CODE: ESE260412

PAOU.NAME: ECON. EVALUATION OF CLOSURE CAP BARPAEA MATERHAL

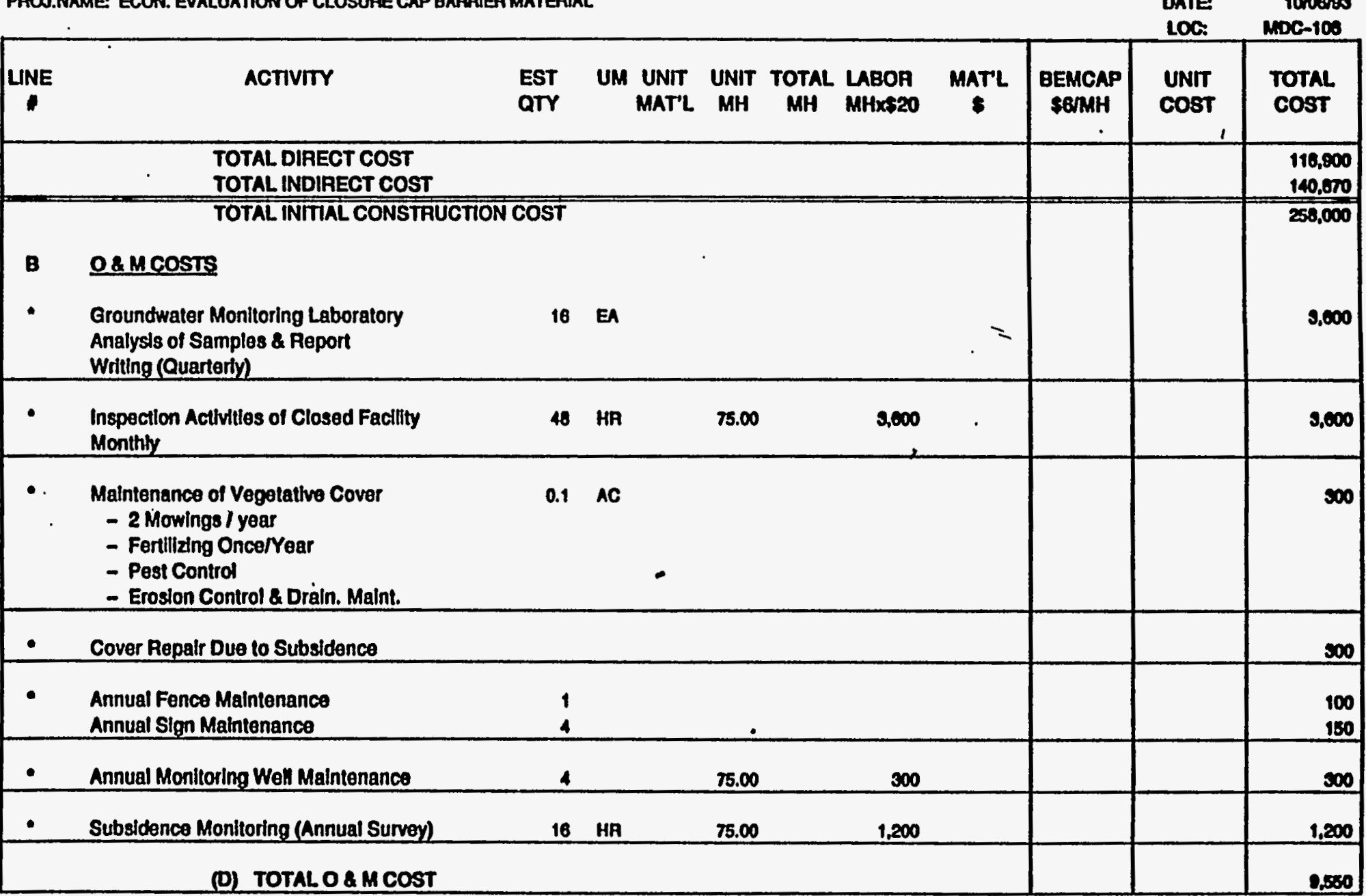

A-6. Cost Estimate for a Cover System Utilizing Reinforced Sprayed Asphalt (Cont'd) 
DETAIL ESTIMATE CHKDBY: 8.MEAD

LOOI: 7-03

ACTVITY CODE: ESE260112

PAOU.MAME: ECON. EVALUATION OF CLOSURE CAP BAFRAETMATEFALL

\begin{tabular}{|c|c|}
\hline $\begin{array}{c}\text { ESTD DV: } \\
\text { PHAONE } \\
\text { DATE: } \\
\text { LOCE }\end{array}$ & 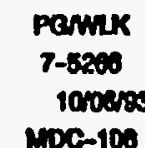 \\
\hline
\end{tabular}

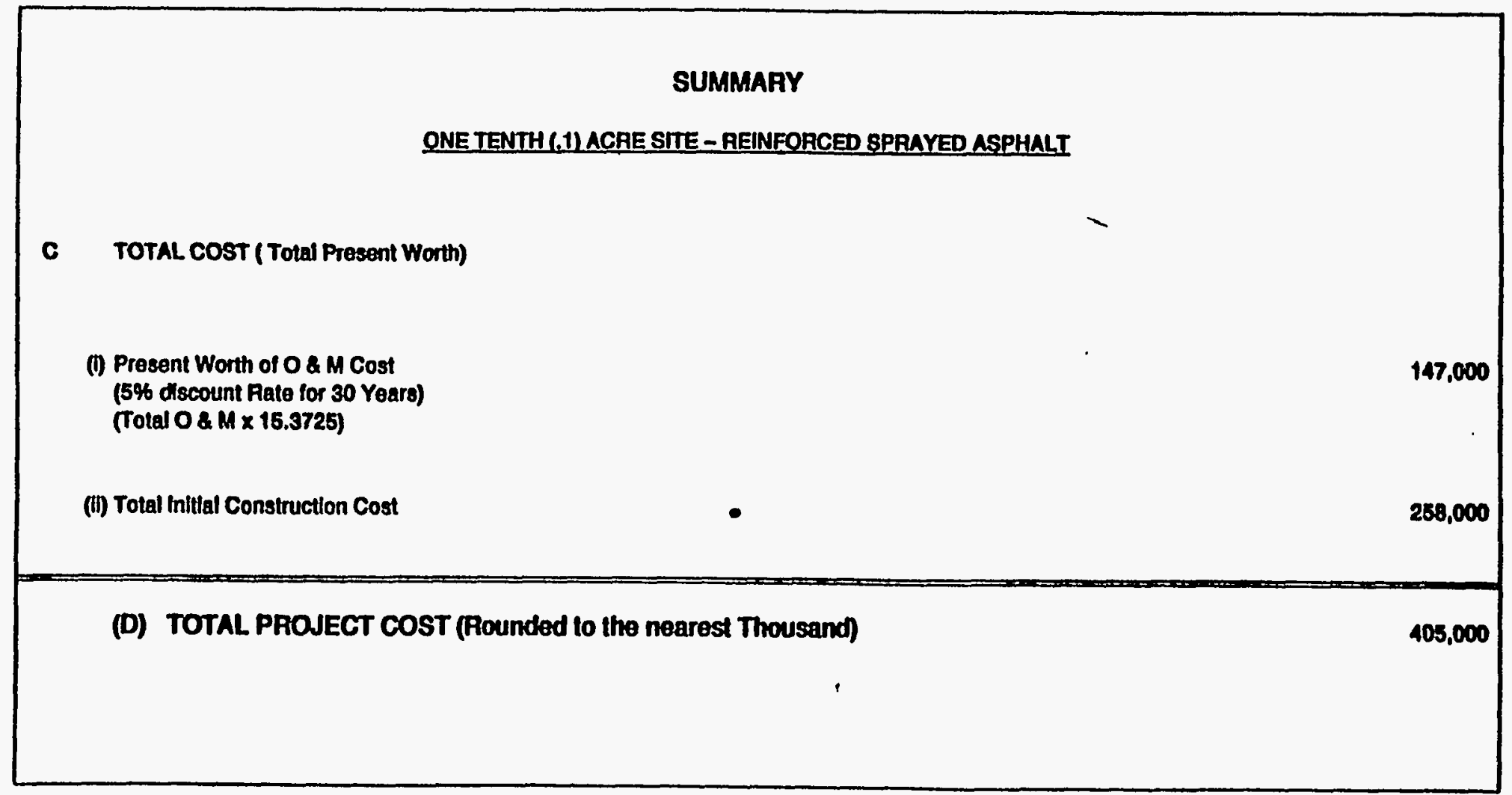

A-6. Cost Estimate for a Cover System Utilizing Reinforced Sprayed Asphalt (Cont'd) 
DETAIL ESTIMATE

CHECD BY: 8. MEAD

100 I: :-03

ACTIVTTY CODE: ESE260412

PROJ.AAME: ECON. EVALUATION OP CLOSURB CAP BARRIER MATERIAL

ESTDQY: PankK

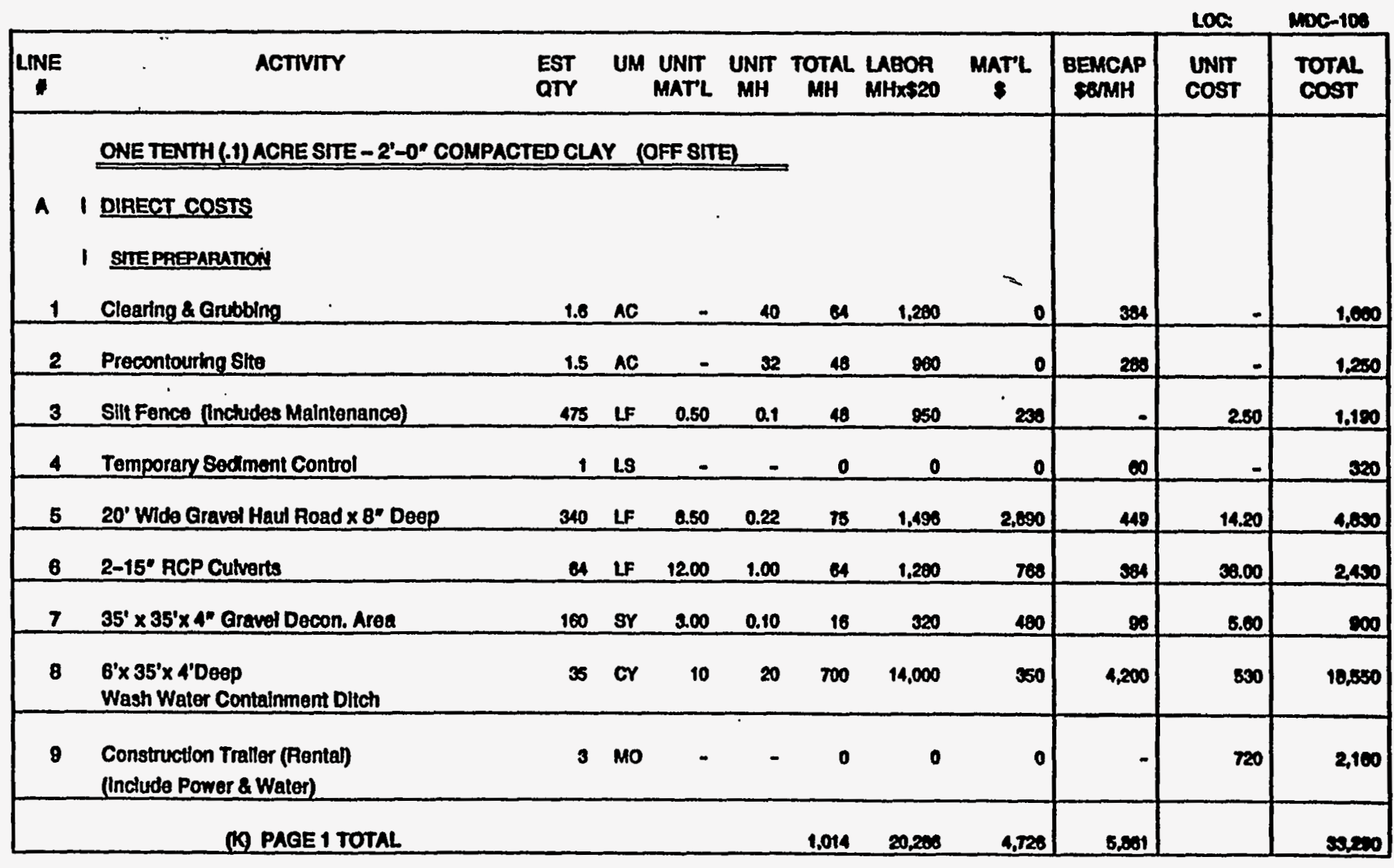

A-7. Cost Estimate for a Cover System Utilizing Compacted Kaolin (Offsite) Clay and a 30-mil FML 
DETAIL ESTIMATE

CHKD QY: 8. MEAD

\section{L001: 7-03}

ACTIVITY CODE: ESEZOOA12

PROJ:NAME: ECON. EVALUATTON OF CLOSURB CAP BARRRER MATERUAL

\begin{tabular}{|c|c|c|c|c|c|c|c|c|c|c|c|}
\hline LINE & ACTIVITY & $\begin{array}{l}\text { EST } \\
\text { QTY }\end{array}$ & & $\begin{array}{l}\text { UNIT } \\
\text { MATL }\end{array}$ & $\underset{\text { MHT }}{\text { UNT }}$ & TOTAL & $\begin{array}{l}\text { LABOR } \\
\text { MHX+\$20 }\end{array}$ & $\underset{\$}{\operatorname{MATL}}$ & $\begin{array}{l}\text { BEMCAP } \\
\text { SEMHH }\end{array}$ & $\underset{\text { Contr }}{\text { Cost }}$ & $\underset{\text { COST }}{\text { TOTAL }}$ \\
\hline & COVERCONSTAUCTION & & & & & & & & & & \\
\hline 10 & Waste Stabilization (Crushed Umestone) & 201 & $\mathbf{I N}$ & 100 & 0.1 & $\infty$ & 402 & 20,100 & 121 & 10200 & 20,020 \\
\hline 11 & $\begin{array}{l}\text { Place and Compact Foundation Soll } \\
\text { in } 8 \text { " Lints to } 95 \% \text { STD. Proctor }\end{array}$ & 807 & or & - & 0.175 & 141 & 2.825 & 0 & 847 & 4.50 & 3,670 \\
\hline 12 & $\begin{array}{l}\text { Compact 2'-0" Thick Clay Layor } \\
\text { In 6" Uitts }\end{array}$ & 339 & or & 13.00 & 0.105 & 56 & 1.118 & 4,407 & 336 & 17.30 & 8,800 \\
\hline 13 & $\begin{array}{l}\text { Placement of } 30 \text { Mill Flexiblo } \\
\text { Membrane Uner }\end{array}$ & 5,220 & SF & 0.19 & 0.009 & 47 & 940 & 692 & - & 0.40 & 1,0000 \\
\hline 14 & $\begin{array}{l}\text { Placement of } 1 \text {-0 Coarse Sand } \\
\text { Drainage Layor }\end{array}$ & 181 & or & 10.50 & 0.035 & 6 & 127 & 1,001 & $\boldsymbol{3 0}$ & 11.40 & 2,070 \\
\hline 15 & $\begin{array}{l}\text { Placement of Geotextle (TYPAR) Fiter on } \\
1^{\prime}-0^{\circ} \text { Coarse Sand Dralnage Layer }\end{array}$ & 557 & sY & 0.20 & 0.007 & 4 & 70 & 111 & - & 0.30 & 100 \\
\hline 16 & Placement of 1'-6" Common Fill & 242 & OY & 3.75 & 0.07 & 17 & 339 & 800 & 102 & 8.60 & 1.500 \\
\hline 17 & Placoment of $6^{*}$ Topsoll & 09 & $\mathbf{C r}$ & 0.50 & 0.09 & 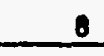 & 104 & $\infty$ & 40 & 11.80 & 1,000 \\
\hline 18 & $\begin{array}{l}\text { Placement of Perlmeter Exterior } \\
\text { Drainage Layer } 3 / 4^{*} \times 3^{n} \text { DP (Crughed Stone) }\end{array}$ & 107 & sr & 1.50 & 0.24 & 20 & 514 & 101 & 184 & 7.00 & $\infty 0$ \\
\hline 19 & Excavation of Trapezoldal Drainage Ditch & 4 & or & - & 0.20 & - & 178 & 0 & 83 & 8.20 & 200 \\
\hline & (1) PAOE 2 TOTAL & & & & & 34 & 0,062 & 20.40 & 1.000 & & 37,050 \\
\hline
\end{tabular}

A-7. Cost Estimate for a Cover System Utilizing Compacted Kaolin (Offsite) Clay and a 30-mil FML (Cont'd) 
D B TAIL B S TIMAT B

CHKD BY: 8. MEAD

200 1: $7-03$

ACTIVITY CODE: ESE2OOA12

PROS.NAME: BCON. EYALUATION OR CLOSURE CAP DATRIER MATERIAI.

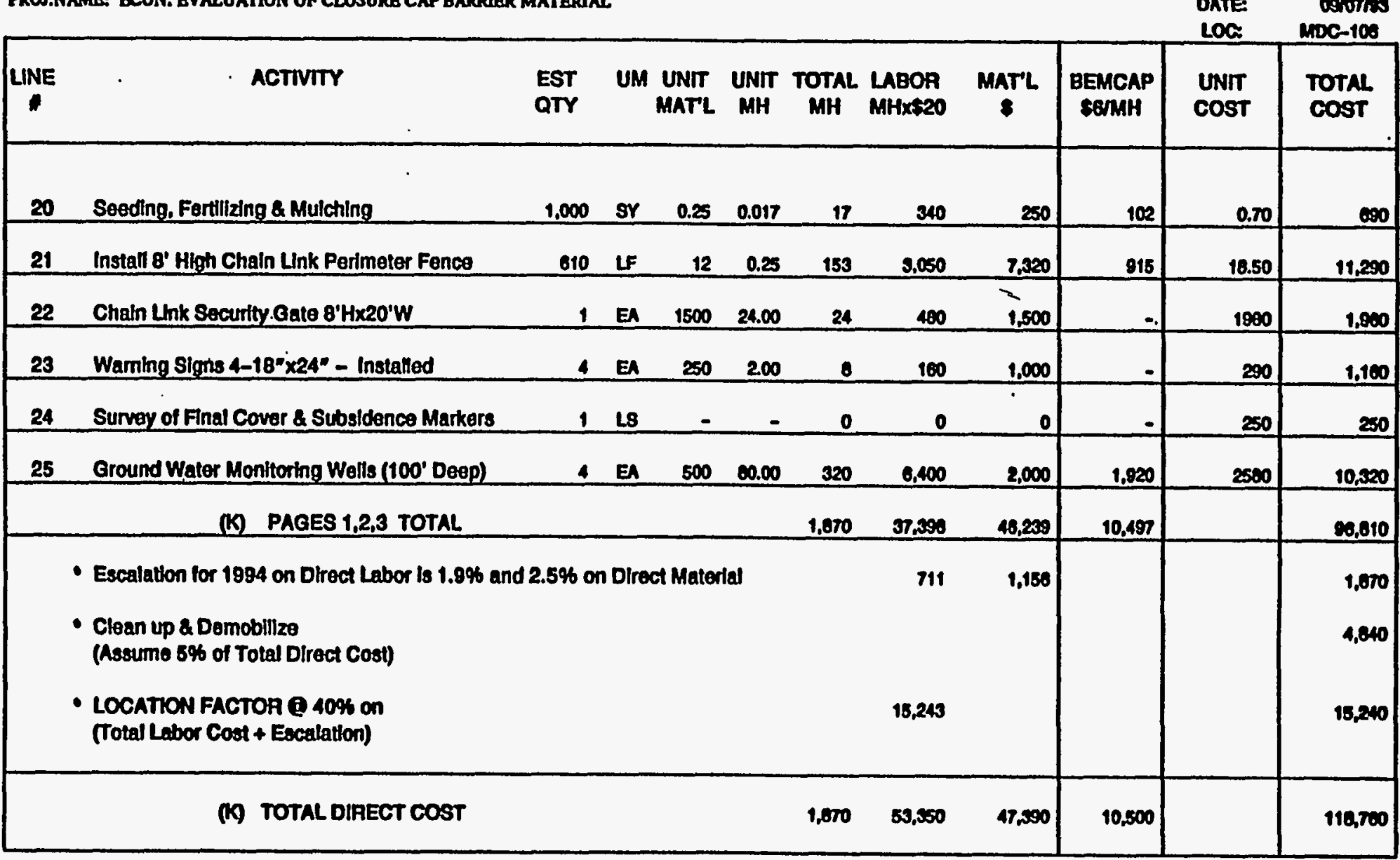

A-7. Cost Estimate for a Cover System Utilizing Compacted Kaolin (Offsite) Clay and a 30-mil FML (Cont'd) 
DETAIL BSTIMAT B

CHK'D BY: 8. MEAD

L001: 7-03

ACTIVITY CODE: ESEROOH12

PROJ.NAME: ECON. EVALUATTON OF CLOSURB CAP BARRTER MATERIAL

ESTD BY: Pank

DATE DONOTMS

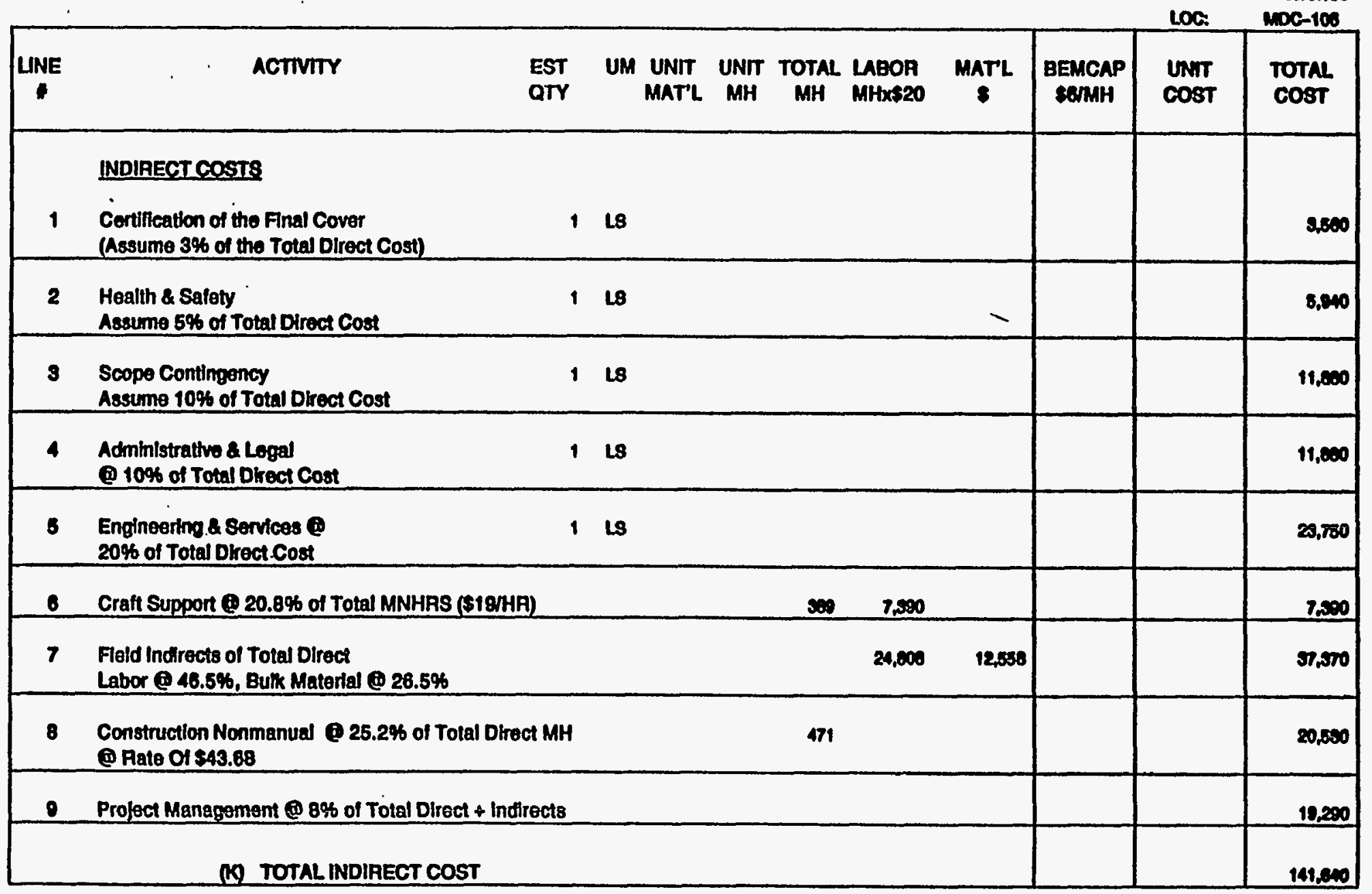

A-7. Cost Estimate for a Cover System Utilizing Compacted Kaolin (Offsite) Clay and a 30-mil FML (Cont'd) 
D B T A I B S T I M A T B

CHK'D BY: S. MEAD

Loo: : 7-03

ACTIVITY CODE: ESEZSOA12

PROI.NAME: ECON. BVALUATION OF CLOSURB CAP BARRIER MATERIAL

ESTOEY: Pamk

PHONE: 7-5260

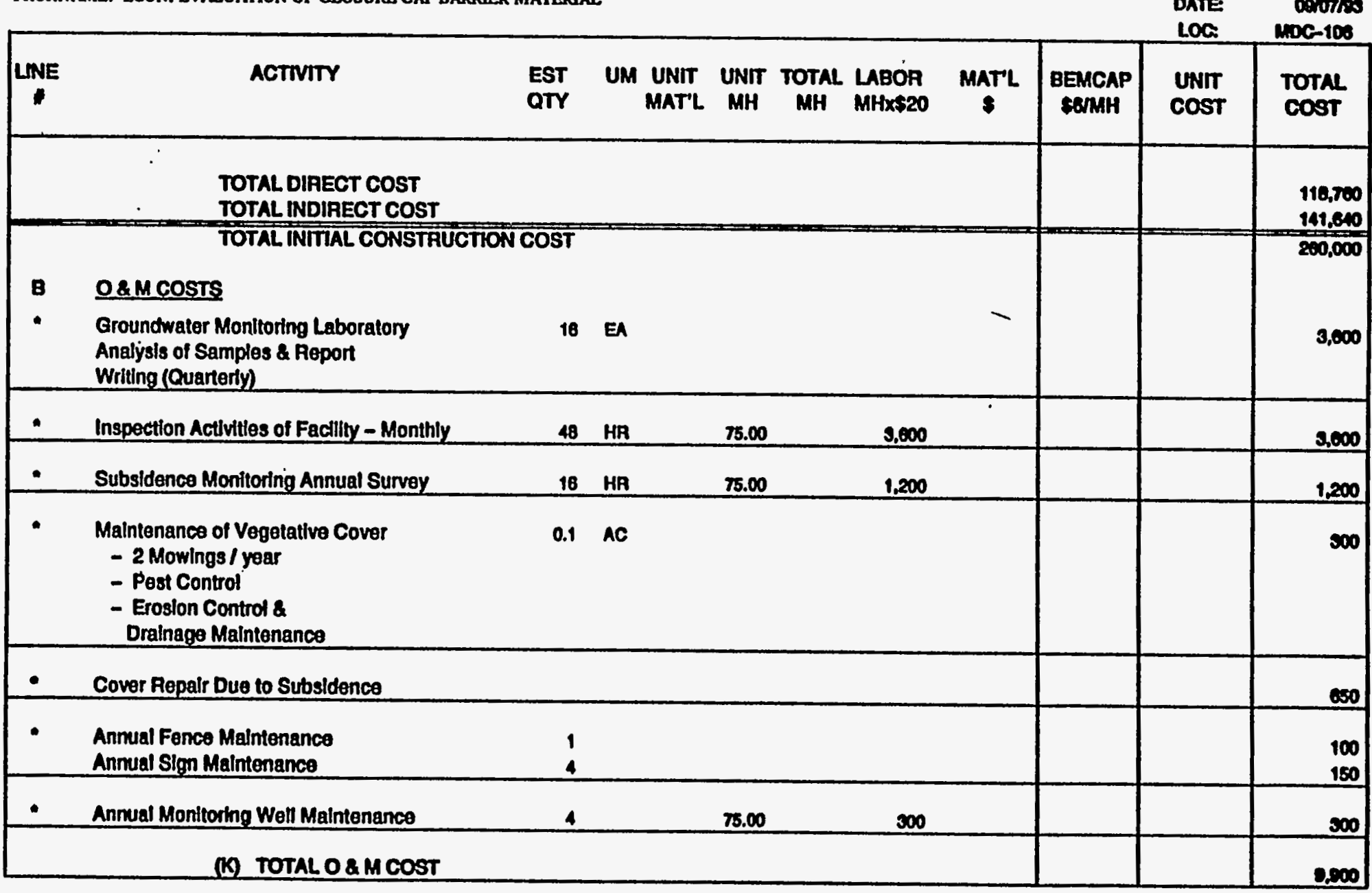

A-7. Cost Estimate for a Cover System Utilizing Compacted Kaolin (Offsite) Clay and a 30-mil FML (Cont'd) 
D BTAILESTIMATB

Lo01: $7-03$

ACTIVTY CODB: BSBZ6OA12

PROJ.NAMB: ECON. BVALUATTON OP CLOSURE CAP BARRIER MATERIAL
CHKD BY: 8. MEAD

ESTOBY:

PHONE T-5200

DATE OSTOT190

106. $\quad$ MOC-100

\section{SUMMARY}

ONE TENTH (1) 1)ACAE SITE-2'-0. COMPAGTED GLAY COFF SITE

C TOTAL COST ( Total Present Worth)

(1) Present Worth of 0 \& $M$ Cost

(5\% discount Rale for 30 Years)

182,000

(Total O \& M X 15.3725)

(ii) Total Inittal Construction Cost

260,000

(K) TOTAL PROJECT COST (Rounded to the nearest Thousand)

412,000

A-7. Cost Estimate for a Cover System Utilizing Compacted Kaolin (Offsite) Clay and a 30-mil FML (Cont'd) 
DETAIL ESTIMATE

CHKD BY: 8. MEAD

Lod: $7-03$

ACTIVITY CODB: ESE2SOA12

PROJ.NAME: ECON. BVALUATTON OF CLOSURB CAP BARRIER MATERINL

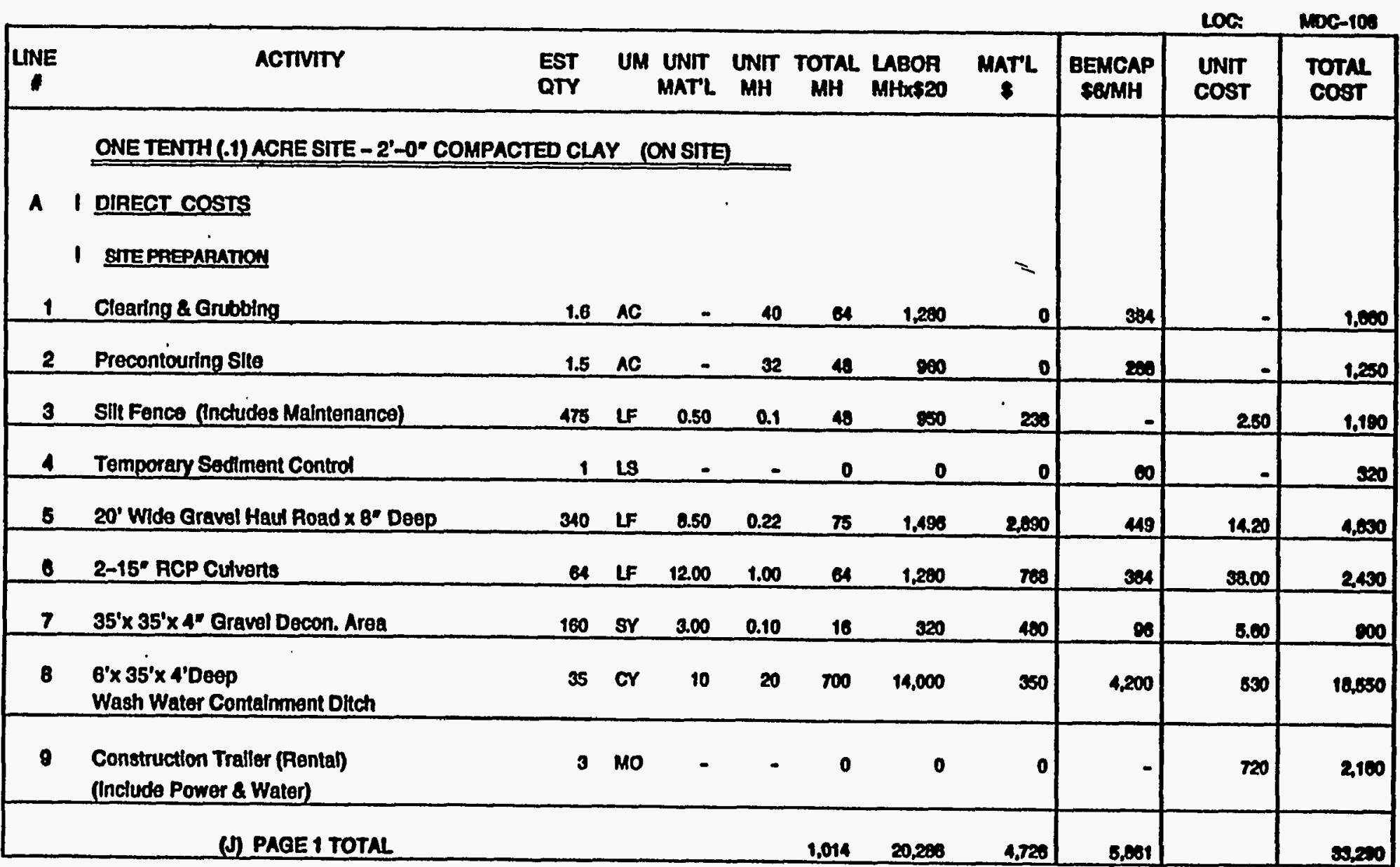

A-8. Cost Estimate a for Cover System Utilizing Compacted Onsite Sandy Clay and a 30-mil FML 
DETAIL ESTIMATE

CHK'D BY: 8. MEAD

Loof: 7-03

ACTIVTTY CODE: ESE2COA12

PROU.NAME: BCON, EYALUATTON OF CLOSURB CAP BARRIER MATERIAL

\begin{tabular}{|c|c|c|c|c|c|c|c|c|c|c|c|}
\hline LINE & ACTIVIT & $\begin{array}{l}\text { EST } \\
\text { OTY }\end{array}$ & UM & $\begin{array}{l}\text { UNIT } \\
\text { MAT'L }\end{array}$ & UNT & TOTAL & $\begin{array}{l}\text { LABOA } \\
\text { MHXSE2O }\end{array}$ & MaTL & $\begin{array}{l}\text { BEMCAP } \\
\text { SGMMH }\end{array}$ & $\begin{array}{l}\text { UNIT } \\
\text { Cost }\end{array}$ & $\begin{array}{l}\text { TOTAL } \\
\text { Cost }\end{array}$ \\
\hline 10 & $\begin{array}{l}\text { COVER CONSTRUCTION } \\
\text { Waste Stabllization (Crushed Limestone) }\end{array}$ & 201 & $\pi$ & 100 & 0.1 & 20 & 402 & 20,100 & 121 & 10200 & 20,020 \\
\hline 11 & $\begin{array}{l}\text { Place and Compact Foundation Soll } \\
\text { in 8" Uifts to } 85 \% \text { STD. Proctor }\end{array}$ & 807 & or & - & 0.175 & 141 & 2,825 & o & 847 & 4.50 & 3,070 \\
\hline 12 & $\begin{array}{l}\text { Compact 2'-0" Thick Clay Layer } \\
\text { In 6* Uns }\end{array}$ & 339 & or & - & 0.168 & 60 & 1.119 & $=$ & 330 & 4.50 & 1,400 \\
\hline 13 & $\begin{array}{l}\text { Placement of } 30 \text { Min Frexble } \\
\text { Membrane Liner }\end{array}$ & 5,220 & $8 F$ & 0.19 & 0.009 & 47 & 240 & 202 & - & 0.40 & 1,030 \\
\hline 14 & $\begin{array}{l}\text { Placement of 1'-0 Coarse Sand } \\
\text { Drainage Layor }\end{array}$ & 181 & or & 10.50 & 0.035 & 6 & 127 & 1,801 & 38 & 11.40 & 2.070 \\
\hline 15 & $\begin{array}{l}\text { Placement of Geotextile (TYPAR) Fitter on } \\
1^{1}-0^{*} \text { Coarse Sand Drainage Layer }\end{array}$ & 557 & 9y & 0.20 & 0.007 & 4 & 70 & 111 & - & 0.00 & 190 \\
\hline 16 & Placement of 1'-6" Cormmon Fill & 242 & CY & 3.75 & 0.07 & $\mathbf{n}$ & 339 & 200 & 102 & 8.00 & 1050 \\
\hline 17 & Placement of 6" Topsoll & 81 & or & 9.50 & 0.09 & - & 164 & $\infty$ & 49 & 11.00 & 1.000 \\
\hline 18 & $\begin{array}{l}\text { Placement of Permeter Exterfor } \\
\text { Drainage Layer 3/4. } \\
\text { Dra " DP (Crushed Stone) }\end{array}$ & 107 & SY & 1.00 & 0.24 & $\approx$ & 614 & 181 & 154 & 1.00 & $\mathbf{\infty 0 0}$ \\
\hline 19 & Excavation of Trapezoldal Drainage Ditch & $\mu$ & or & - & 0.20 & Q & 170 & of & 83 & 8.20 & 200 \\
\hline & (N) PAGE 2 TOTAL & & & & & 334 & 0,002 & 8,003 & 1,000 & & $\sin 20$ \\
\hline
\end{tabular}

A-8. Cost Estimate a for Cover System Utilizing Compacted Onsite Sandy Clay and a 30-mil FML (Cont'd) 
D B TAILASTIMATB

CHKC BY: 8. MEND

Lod1: 7-03

ACTIVTYY CODE: ESE260412

PROJ.MAMBE: BCON. EVALUATION OF CLOSUIRB CAP BARRIER MATERIAL

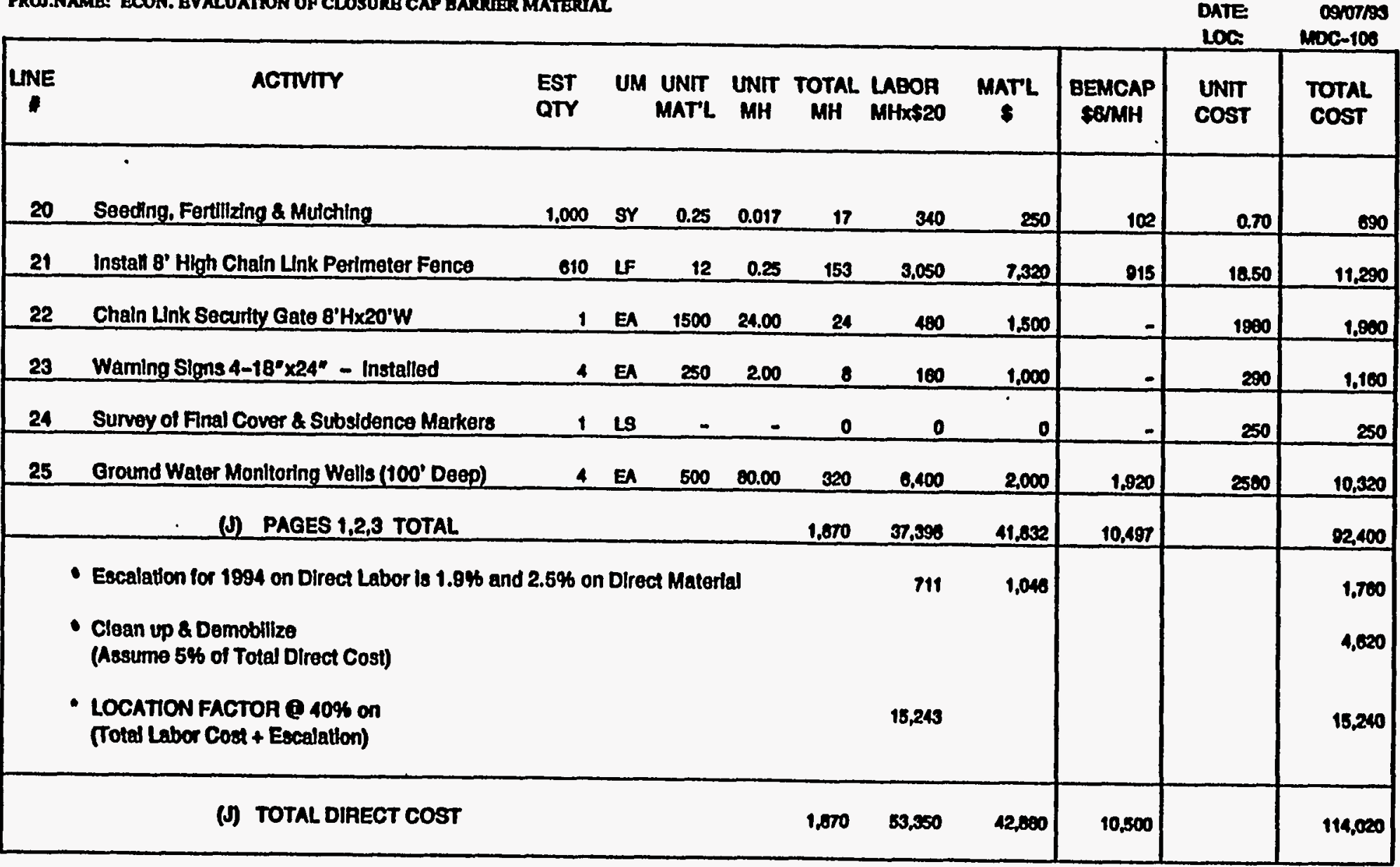

A-8. Cost Estimate a for Cover System Utilizing Compacted Onsite Sandy Clay and a 30-mil FML (Cont'd) 
D B T A I B S IMATB CHK'D BY: 8. MEAD

Lo01: $7-03$

ACTIVTYY CODE: FSEDSOA12

PROJ.NAMB: BCON. BVALUATION OP CLOSURB CAP BARRIER MATERIAL.

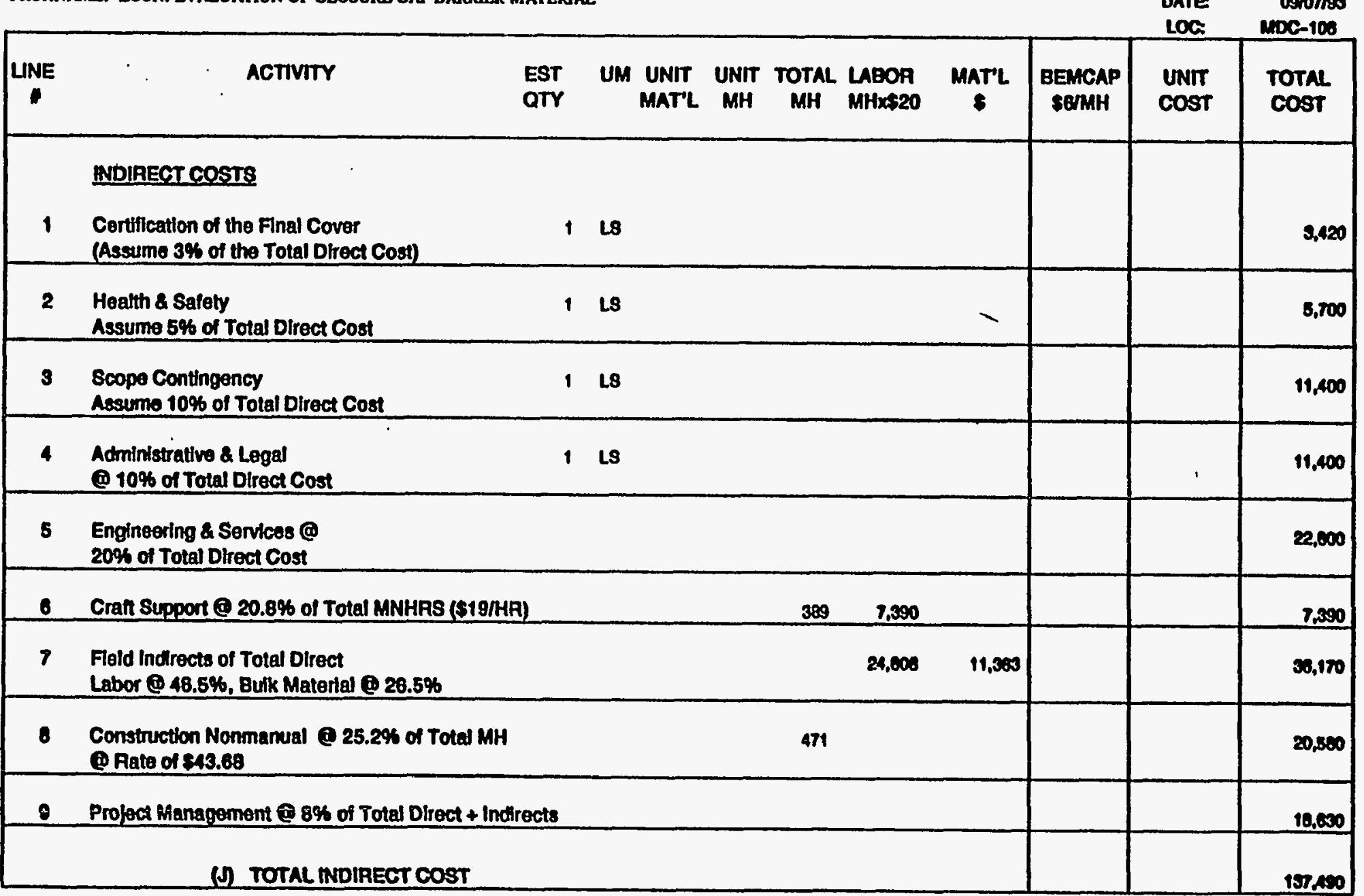

A-8. Cost Estimate a for Cover System Utilizing Compacted Onsite Sandy Clay and a 30-mil FML (Cont'd) 
$1008: 7-03$

ACTIVITY CODE: ESE260412

PROJ.NAMR: ECON. EVALUATTON OP CLOSURB CAP DARRIER MATERIAL

PTOEY: Pamk

PHONE 7-5200

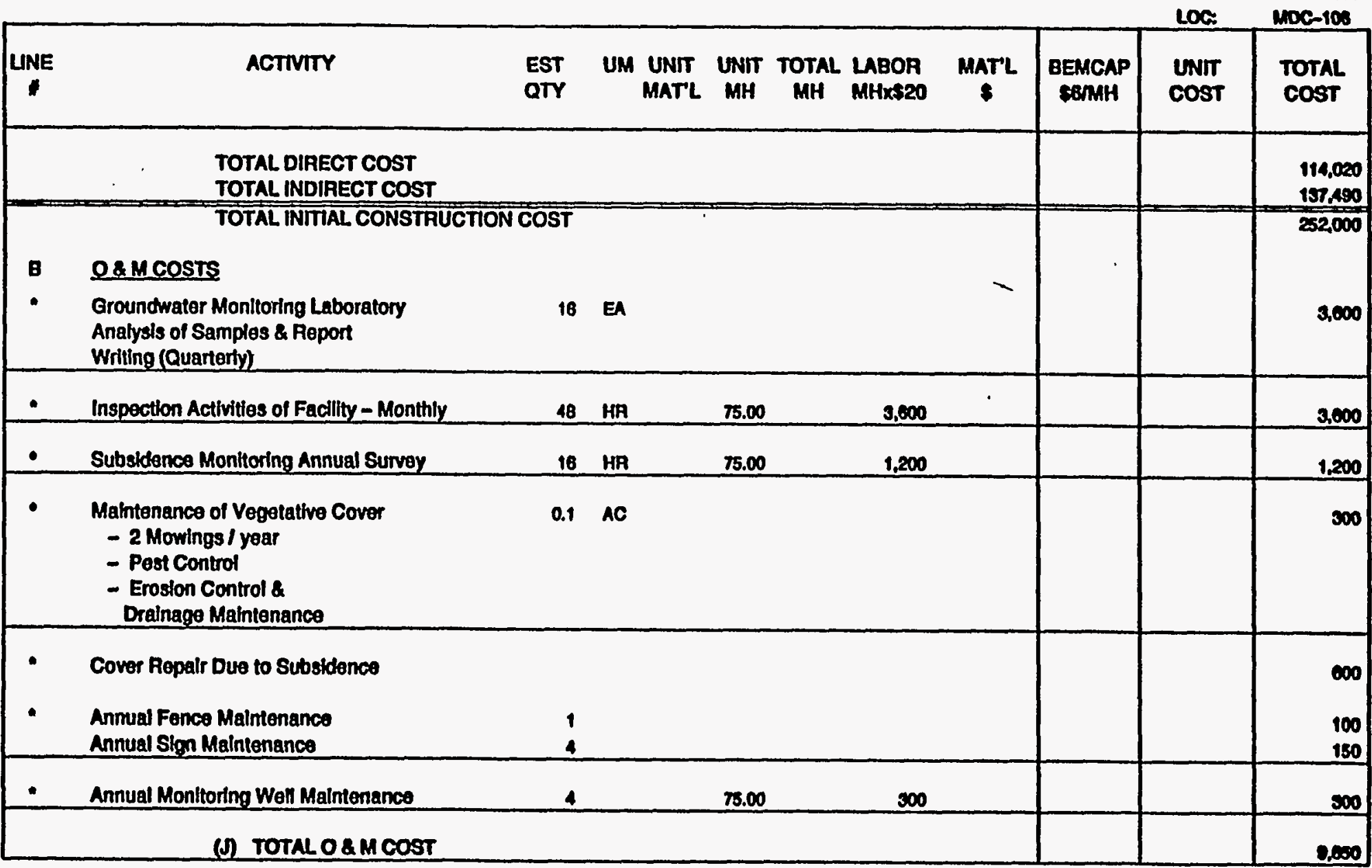

A-8. Cost Estimate a for Cover System Utilizing Compacted Onsite Sandy Clay and a 30-mil FML (Cont'd) 
D B T A L B S TIMATB

\section{Looi: $7-03$}

ACTIVITY CODB: BSB200412

FROJ.NAME: BCON. BVALUATION OF CLOSURE CAP BARRIER MATERIAL.
CHKD BY: 8. MEAD

ESTD BY:

PHONE:

DATE

Panmk

$7-5200$

0907100 MOCC-100

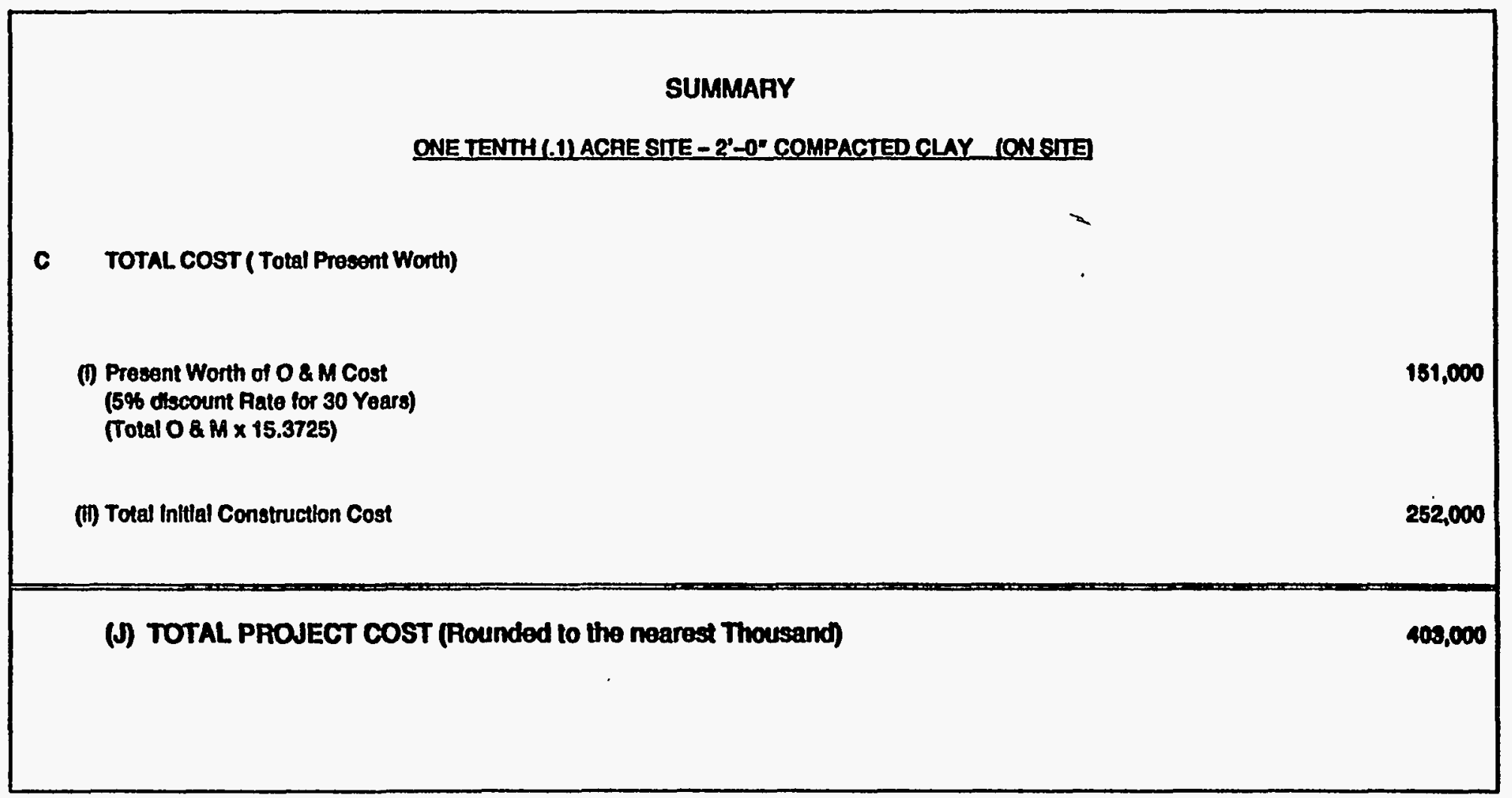

A-8. Cost Estimate a for Cover System Utilizing Compacted Onsite Sandy Clay and a 30-mil FML (Cont'd) 
DETAIL ESTIMATE

Loof: $7-03$

ACTIVITY CODE: ESE260412

PROJ.NAME: ECON. EVALUATION OP CLOSURE CAP BARRIER MATERUAL.

CHKD BY: S. MEAD

ESTDEV: PAMMK

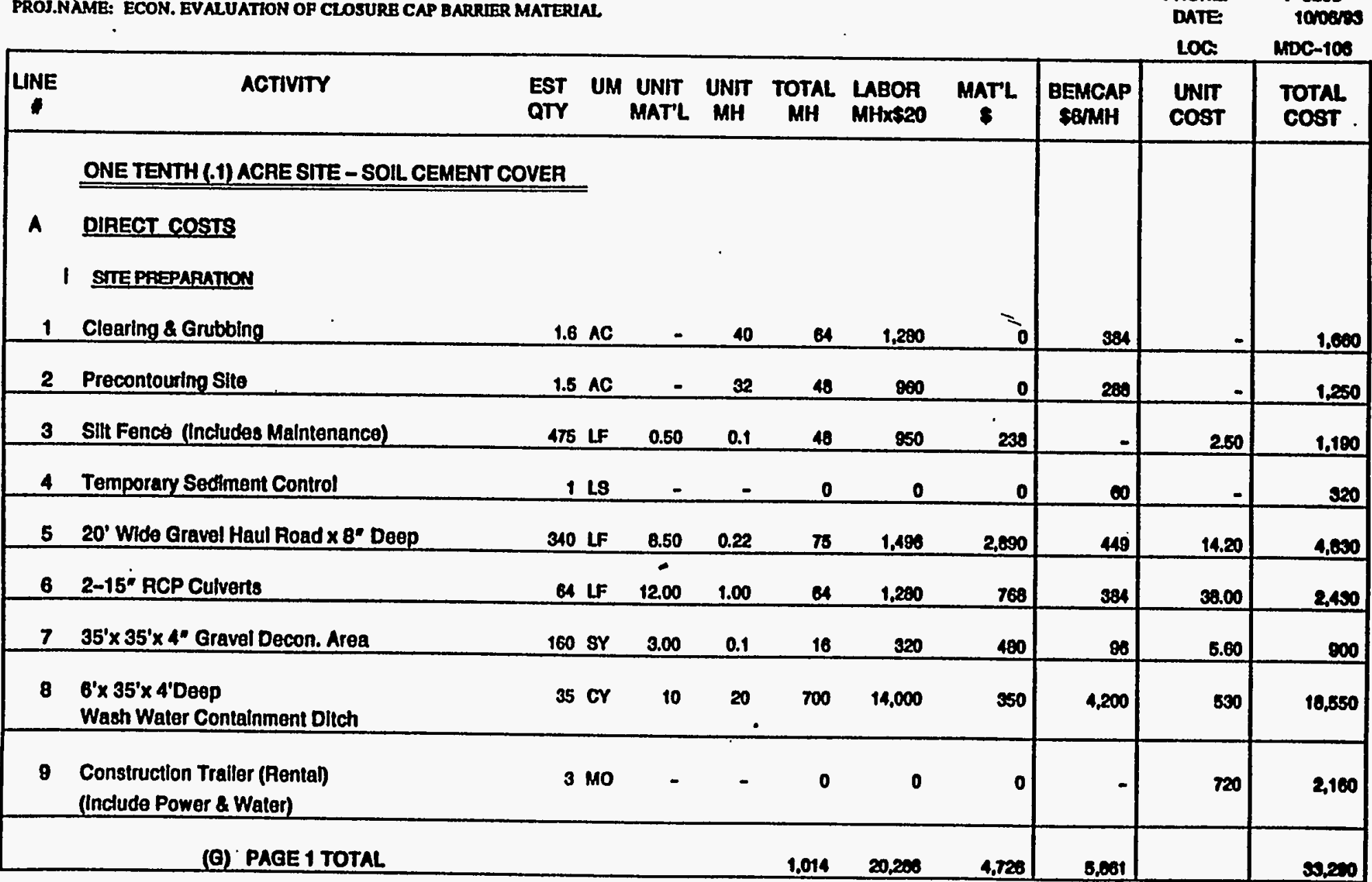

A-9. Cost Estimate for a Cover System Utilizing Soil Cement and 30-mil FML 
DETAIL ESTIMATE CHKDEY: S.MEAD

Lo01: $7-03$

ACTIVTTY CODE: ESE260412

PROS.NAME: ECON. EVALUATION OF CLOSURB CAP BARRIER MATERIAL

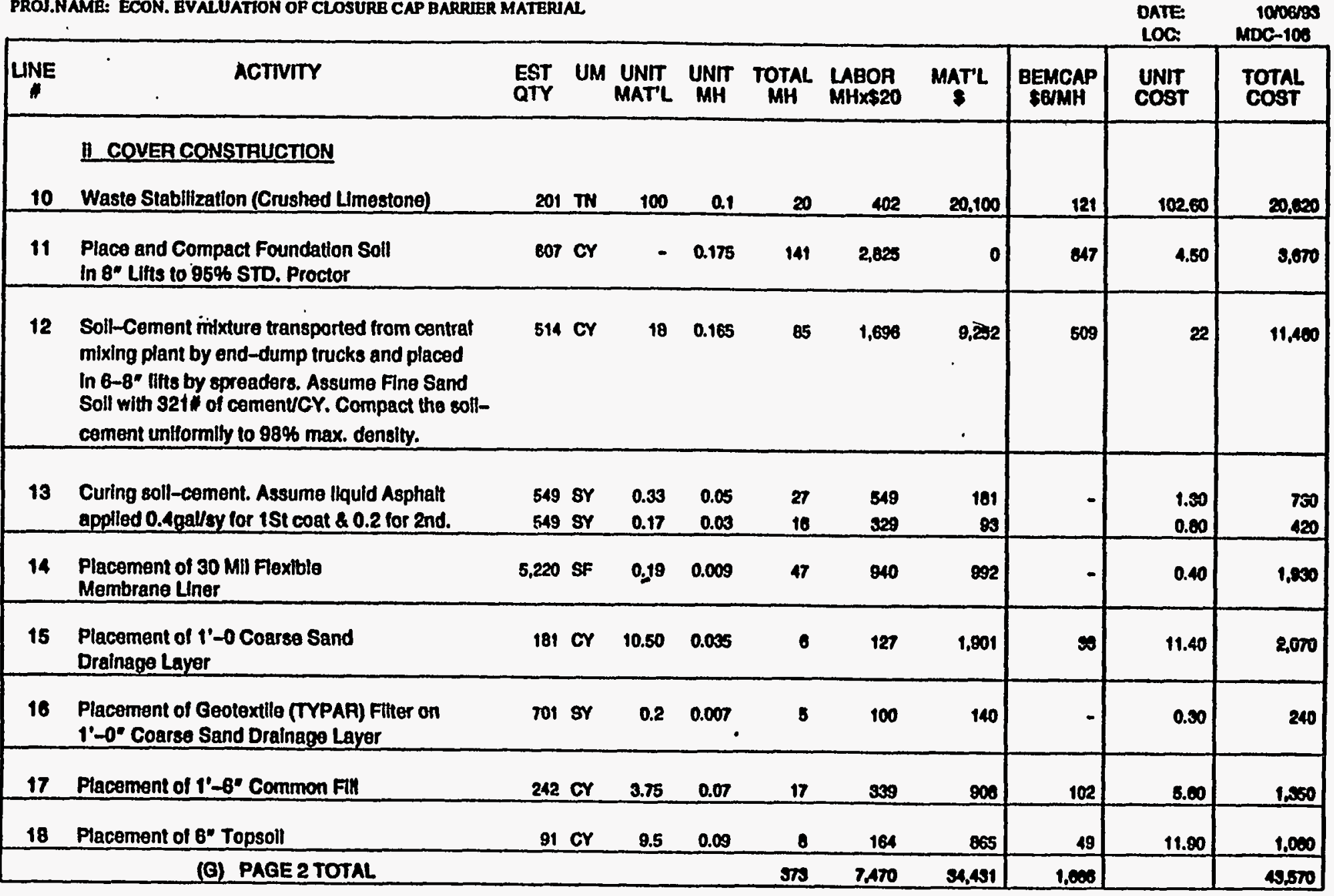

A-9. Cost Estimate for a Cover System Utilizing Soil Cement and 30-mil FML (Cont'd) 


\section{DETAIL B S T IMAT B CHK'D BY: 8. MEAD}

Lod I: $7-03$

ACTIVITY CODE: ESE260112

PROJ.NAME: ECON. BVALUATION OF CLOSURB CAP BARRIER MATERIAL.

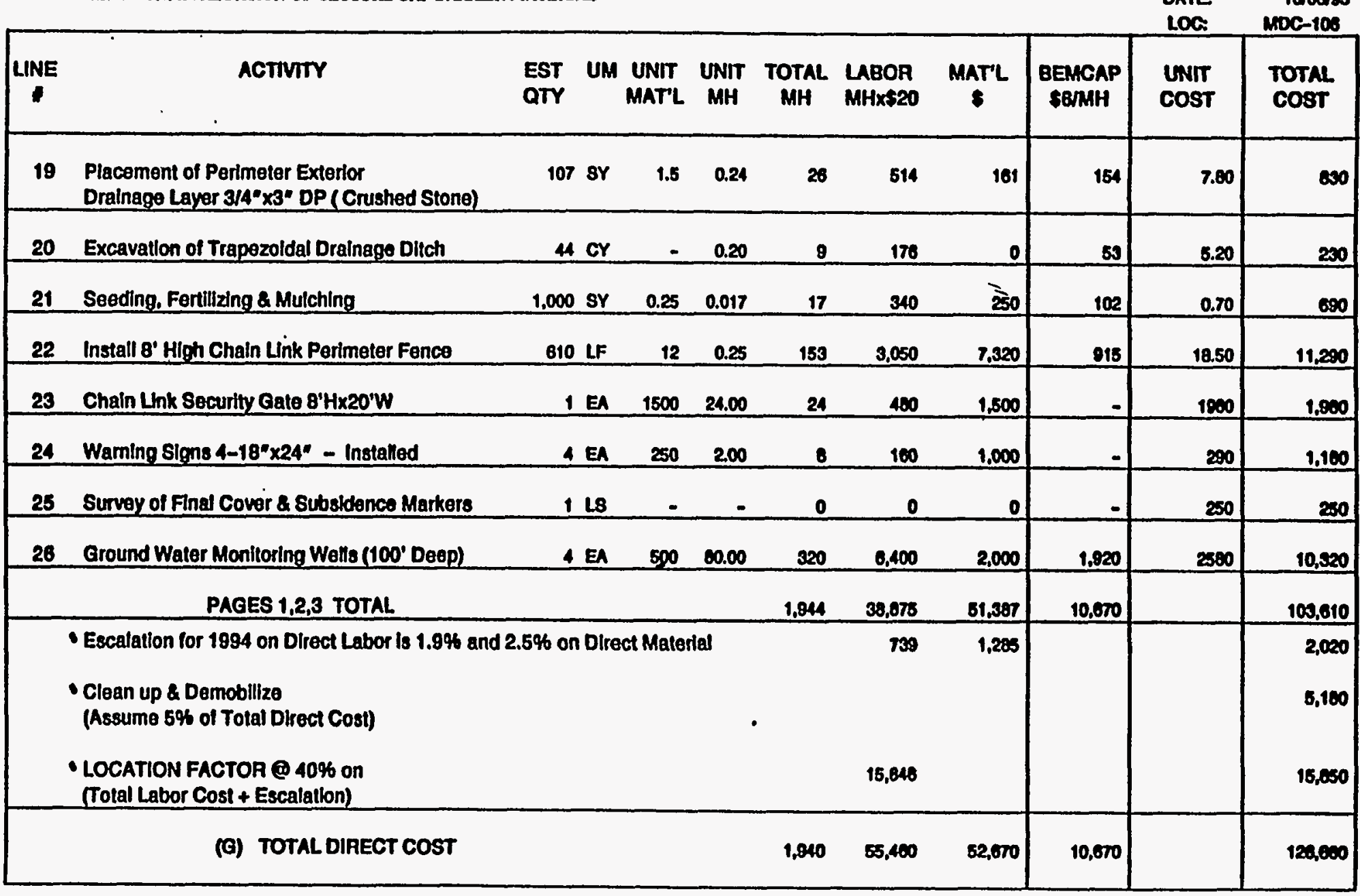

A-9. Cost Estimate for a Cover System Utilizing Soil Cement and 30-mil FML (Cont'd) 
D B T A I L B S T I M A T B

CHK'D BY: 8. MEAD

LoOf: $7-03$

ACTIVITY CODE: ESE260412

PROJ.NAME: ECON. EVALUATION OF CLOSURB CAP BARRIER MATERIAL

ESTD BV: PAMNK
PHONE: 7-5286

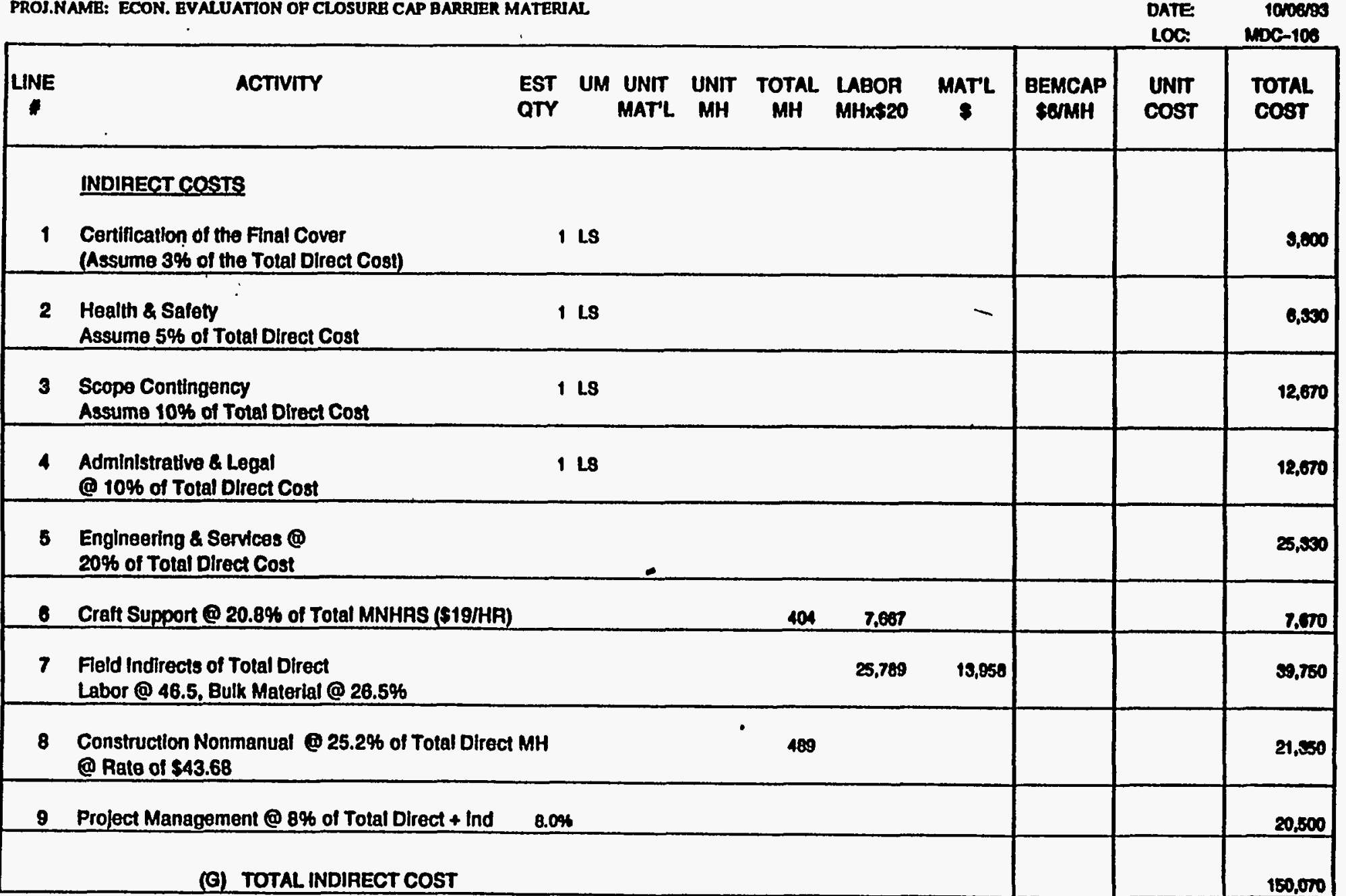

A-9. Cost Estimate for a Cover System Utilizing Soil Cement and 30-mil FML (Cont'd) 
DETAIL ESTIMATE

CHK'D BY: S. MEAD

Lo01: 7-03

ACTIVTTY CODB: ESE200412

PROJ.NAME: ECON. EVALUATION OF CLOSURE CAP BARRIER MATERIAL.

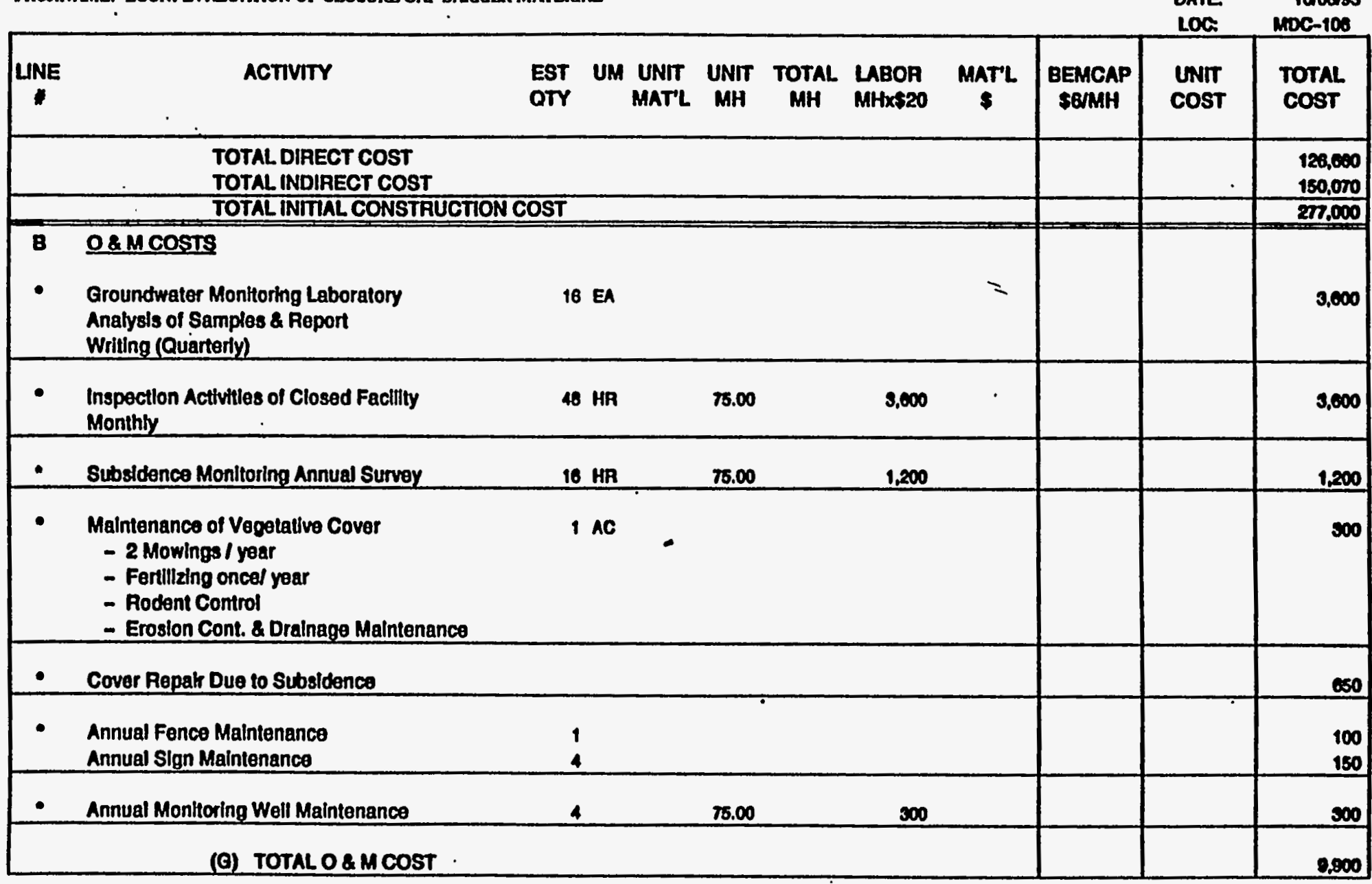

A-9. Cost Estimate for a Cover System Utilizing Soil Cement and 30-mil FML (Cont'd) 
DETAIL BSTIMAT B

$\begin{array}{ll}\text { CHK'D BY: } & \text { 8. MEAD } \\ \text { ESTD BY: } & \text { POMNK } \\ \text { PHONE: } & 7-5268 \\ \text { DATE: } & \text { 1008198 } \\ \text { LOC: } & \text { MDC-106 }\end{array}$

LOa $A: 7-03$

ACTIVITY CODE: ESE260112

PROJ.NAME: ECON. EVALUATION OF CLOSURE CAP BARRIER MATERIAL

LOC.

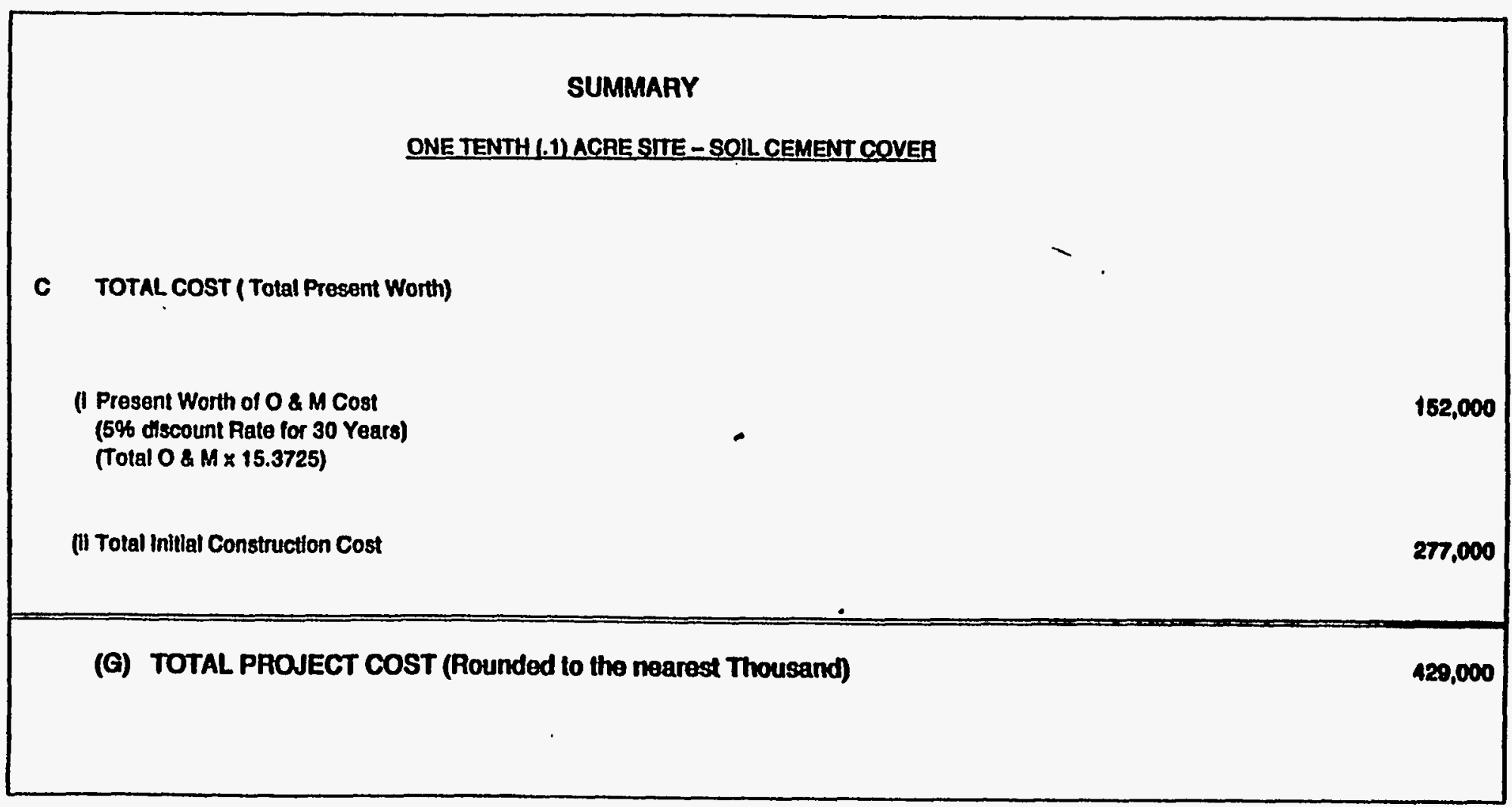

A-9. Cost Estimate for a Cover System Utilizing Soil Cement and 30-mil FML (Cont'd) 
DETAIL ESTIMATE

Loof: 2-03

ACTIVITY CODB: ESEZOOH12

PROJ.NAME: ECON. BVALUATTON OF CLOSURB CAP BANRIER MATERIAL

\begin{tabular}{|c|c|c|c|c|c|c|c|c|c|c|}
\hline UNE & ACTIVIY & $\begin{array}{l}\text { EST UM } \\
\text { QTY }\end{array}$ & $\begin{array}{l}\text { UNT } \\
\text { MATL }\end{array}$ & $\begin{array}{l}\text { UNIT } \\
\text { MH }\end{array}$ & $\underset{\text { MH }}{\text { TOTAL }}$ & $\begin{array}{l}\text { LABOA } \\
\text { MHX\$20 }\end{array}$ & $\underset{\$}{\operatorname{MATL}}$ & $\begin{array}{l}\text { BEACAP } \\
\text { SenMH }\end{array}$ & $\operatorname{UNm}_{\text {COST }}$ & $\begin{array}{l}\text { Total } \\
\cos 1\end{array}$ \\
\hline $\begin{array}{l}A \\
1 \\
\end{array}$ & $\begin{array}{l}\text { ONE TENTH (1) ACRE SITE-BENTO } \\
\text { DIRECT COSTS } \\
\text { SMEPREARATION } \\
\text { Clearing \& Onubiing }\end{array}$ & ADDITIVE & - & 40 & a & 1,200 & 0 & $\boldsymbol{s e 4}$ & $=$ & 1.000 \\
\hline 2 & Procontouring Sito & $1.5 \wedge C$ & - & 32 & 40 & 800 & 0 & 200 & - & 1,250 \\
\hline 3 & Silt Fonce (includos Maintenance) & 475 LF & 0.50 & 0.1 & 40 & 850 & 230 & - & 2.50 & 1.190 \\
\hline 4 & Temporary Sodiment Control & 118 & $=$ & - & 0 & 0 & 0 & $\infty$ & - & 320 \\
\hline 5 & 20' Wide Gravel Haul Road x 8" Doep & $340 \mathrm{LF}$ & 0.50 & 0.22 & $\pi$ & 1,490 & 2090 & 40 & 14.20 & 4,000 \\
\hline 6 & 2-15" RCP Cutverts & $64 \mathrm{LF}$ & 12,00 & 1.00 & 64 & 1,200 & 700 & $s$ & 30.00 & 2,400 \\
\hline 7 & 35'x 95' $\times 4$ " Gravel Decon. Area & $100 \mathrm{SY}$ & 3.00 & Q.10 & 16 & 320 & 400 & $\infty$ & 8.00 & 800 \\
\hline 8 & $\begin{array}{l}\text { 6'x } \times 35^{\prime} \times 4^{\prime} \text { Doep } \\
\text { Wash Water Contalnment Ditech }\end{array}$ & 35 or & 10 & 20 & 700 & 14,000 & 350 & 1,200 & 550 & 10,5800 \\
\hline 8 & $\begin{array}{l}\text { Construction Tralier (Rental) } \\
\text { (Include Power \& Water) }\end{array}$ & 3 мо & - & - & 0 & $\mathbf{0}$ & 0 & - & $\mathbf{7 2 0}$ & 2,100 \\
\hline & (H) PAGE I TOTAL & & & & 1.014 & 20,200 & 1,7200 & 8,0001 & & م:50 \\
\hline
\end{tabular}

A-10. Cost Estimate for a Cover System Utilizing Bentonite Soil Additive and a 30-mil FML 
DETAIL ESTIMATE

CHKD BY: 8. MEAD

LOO I: 7-03

ACTIVITY CODB: BSBZSON12

MROJ.NAME: BCON. EVALUATTON OF CLOSURB CAP BARRIIRR MATERIAL.

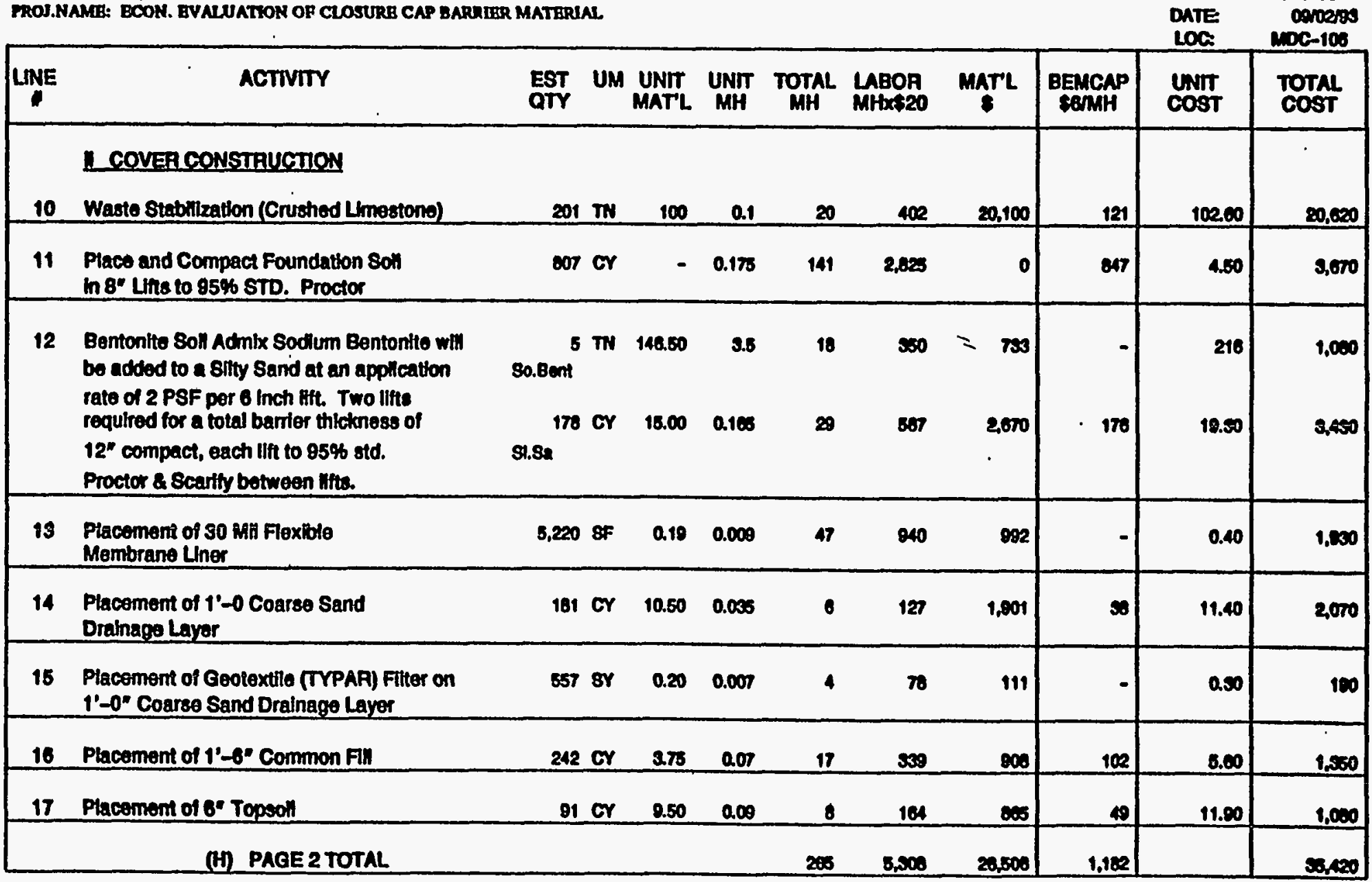

A-10. Cost Estimate for a Cover System Utilizing Bentonite Soil Additive and a 30-mil FML (Cont'd) 
DBTAIL BSTIMATB

\section{CHK'D BY: S. MEAD}

Loa 1: 7-03

ACTIVTY CODE: BSEZSOOA12

FROJ.NAME: BCON. EYALUATON OF CLOSURB CAP BARRIER MATERIAL

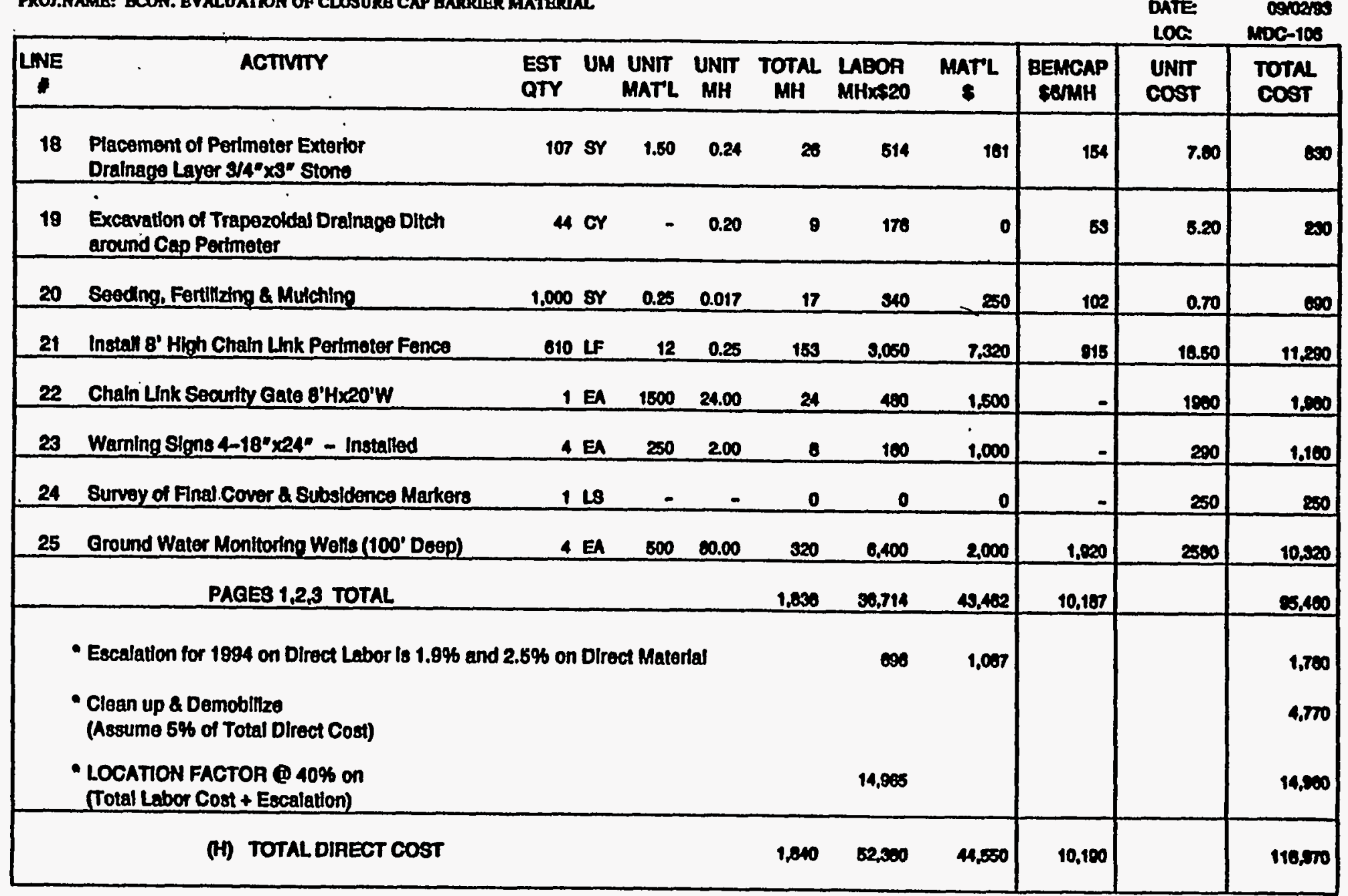

A-10. Cost Estimate for a Cover System Utilizing Bentonite Soil Additive and a 30-mil FML (Cont'd) 
D B T A I L B S T I A T B

\section{CHK'D BY: 8. MEAD}

$1001: 7-03$

ACTIVITY CODE: BSEROOH12

MOS.NAME: BCON. EVALUATTON OF CLOSURB CAP BARUIER MATERUAL

ESTOBY: Pank

PHONE T-6000

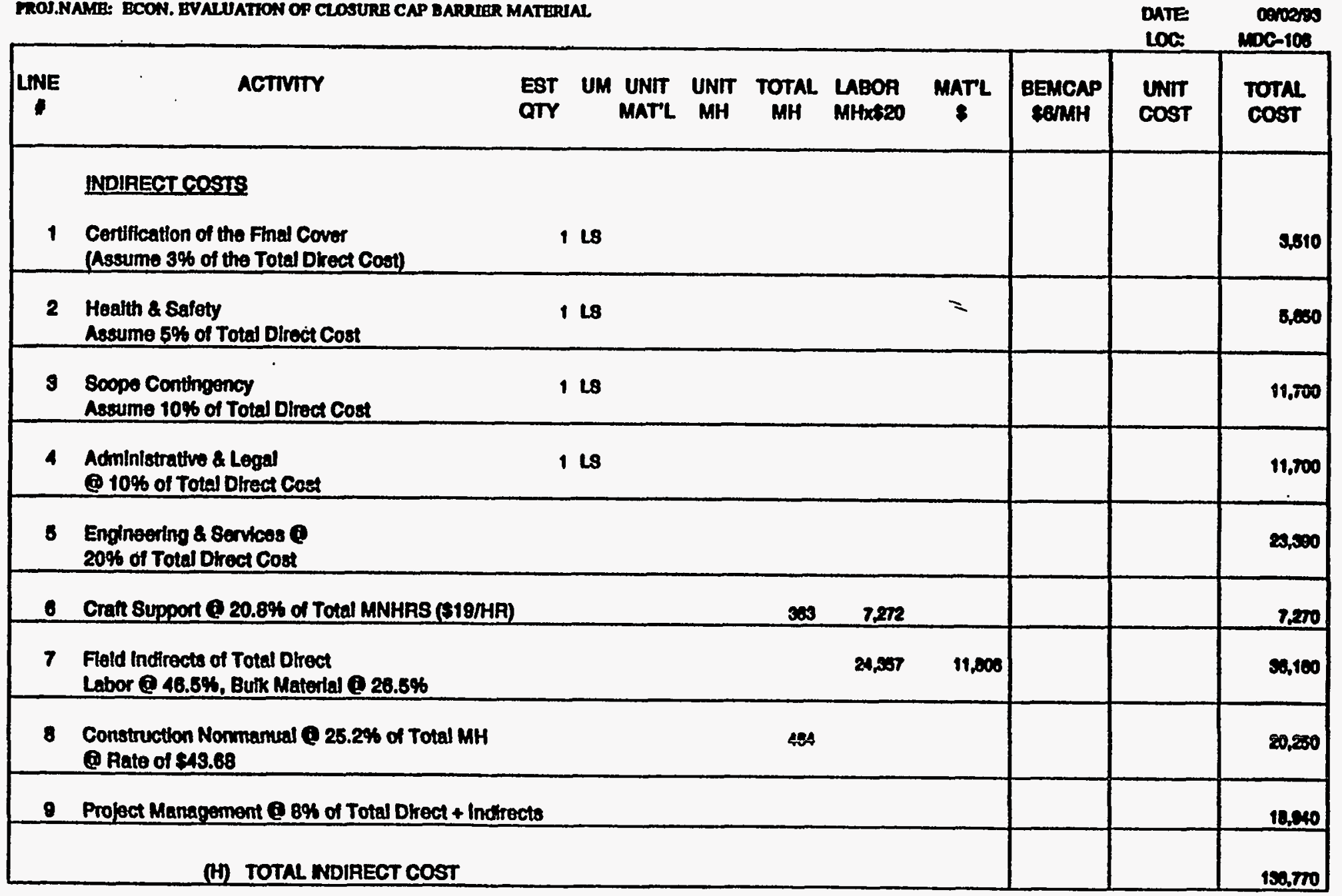

A-10. Cost Estimate for a Cover System Utilizing Bentonite Soil Additive and a 30-mil FML (Cont'd) 
CHKD BY: 8. MEAD

L00 : : 7-03

ACTIVITY CODE: ESE2SOO12

PROJ.NAME: BCON. EVALUATION OP CLOSURE CAP BARRIER MATRRIAL

PTD BY: Pank

PHONE 7-5260

DATE

LOC MDC-10

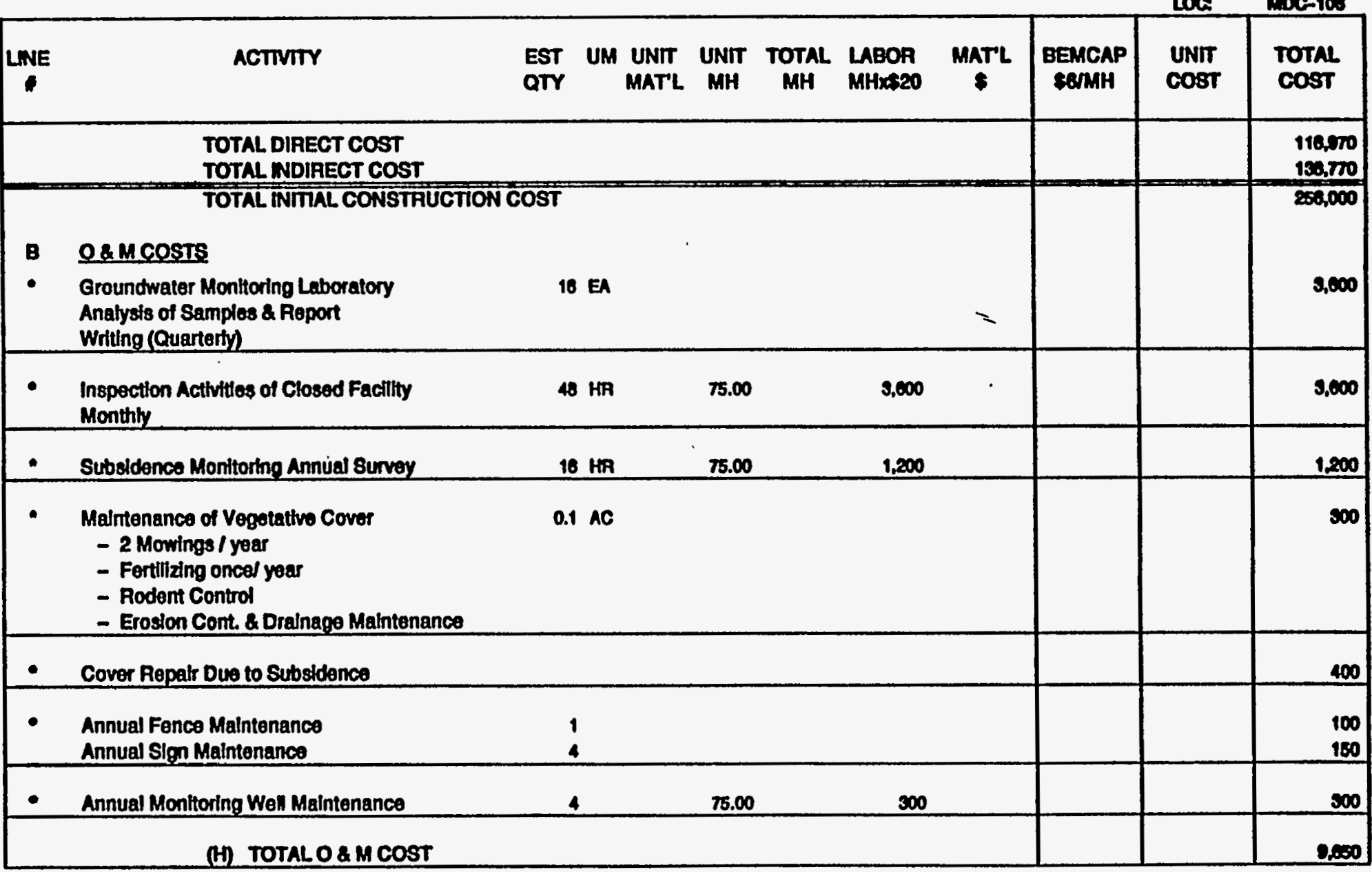

A-10. Cost Estimate for a Cover System Utilizing Bentonite Soil Additive and a 30-mil FML (Cont'd) 
DBTAILESTIMATB

Loof: 1-03

ACTIVITY CODE: ESE200412

MOJ.NAME: BCON. EVAWATION OF CLOSURB CAP BARRIER MATERLAL.
CHKO BY: 8. MEAD

EAro Br:

Phoive

DATE

LOC.
Penux

$7-6200$

onseres

ance-10s

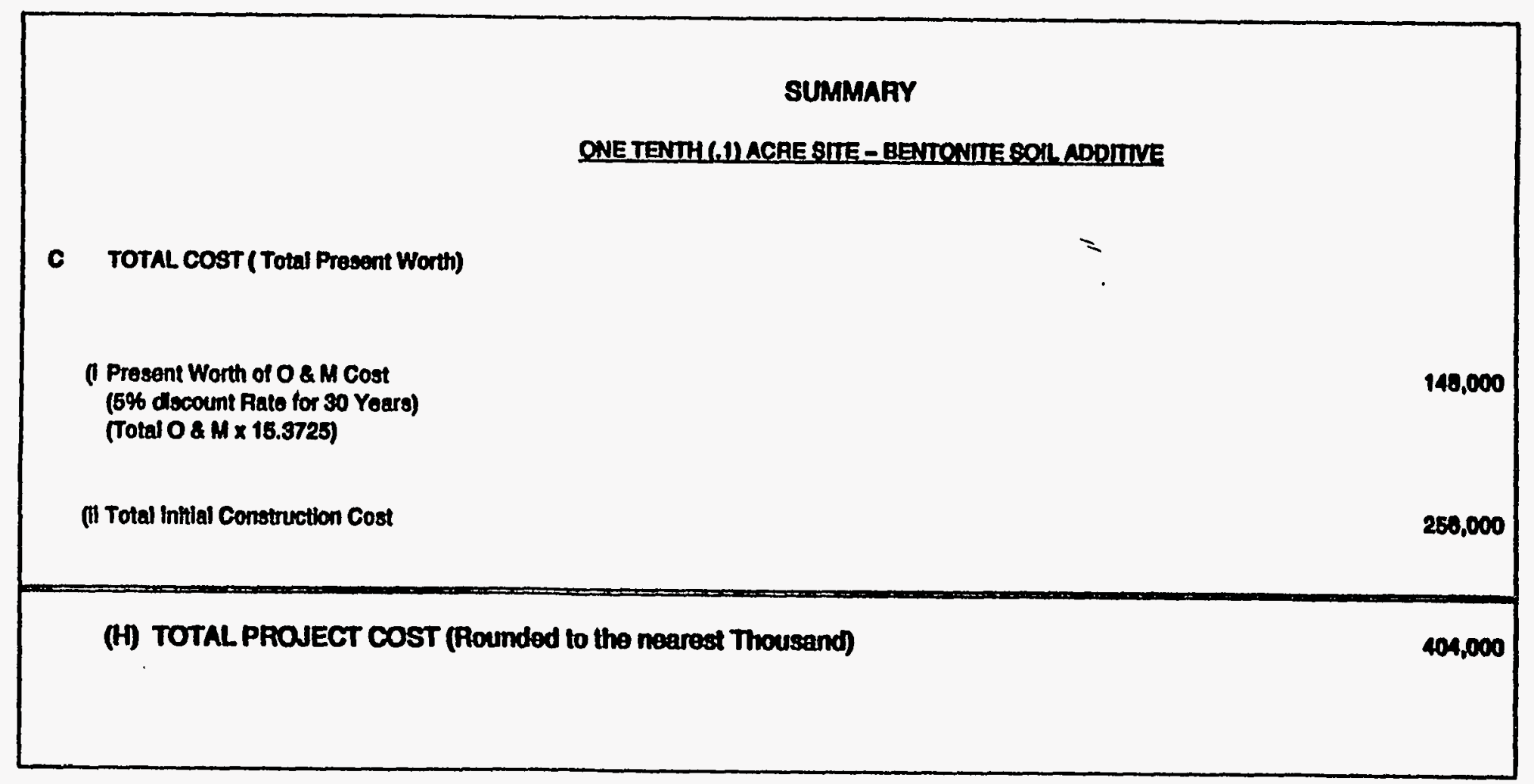

A-10. Cost Estimate for a Cover System Utilizing Bentonite Soil Additive and a 30-mil FML (Cont'd) 
DETAIL ESTIMATE

\section{CHK'D BY: 8. MEAD}

LOa : $: 7-03$

ACTIVITY CODB: ESE260412

PROI.NAME: ECON. BVALUATION OF CLOSURB CAP BARRIER MATERIAL.

ESTD BV: romL

PHONE 7-SWS

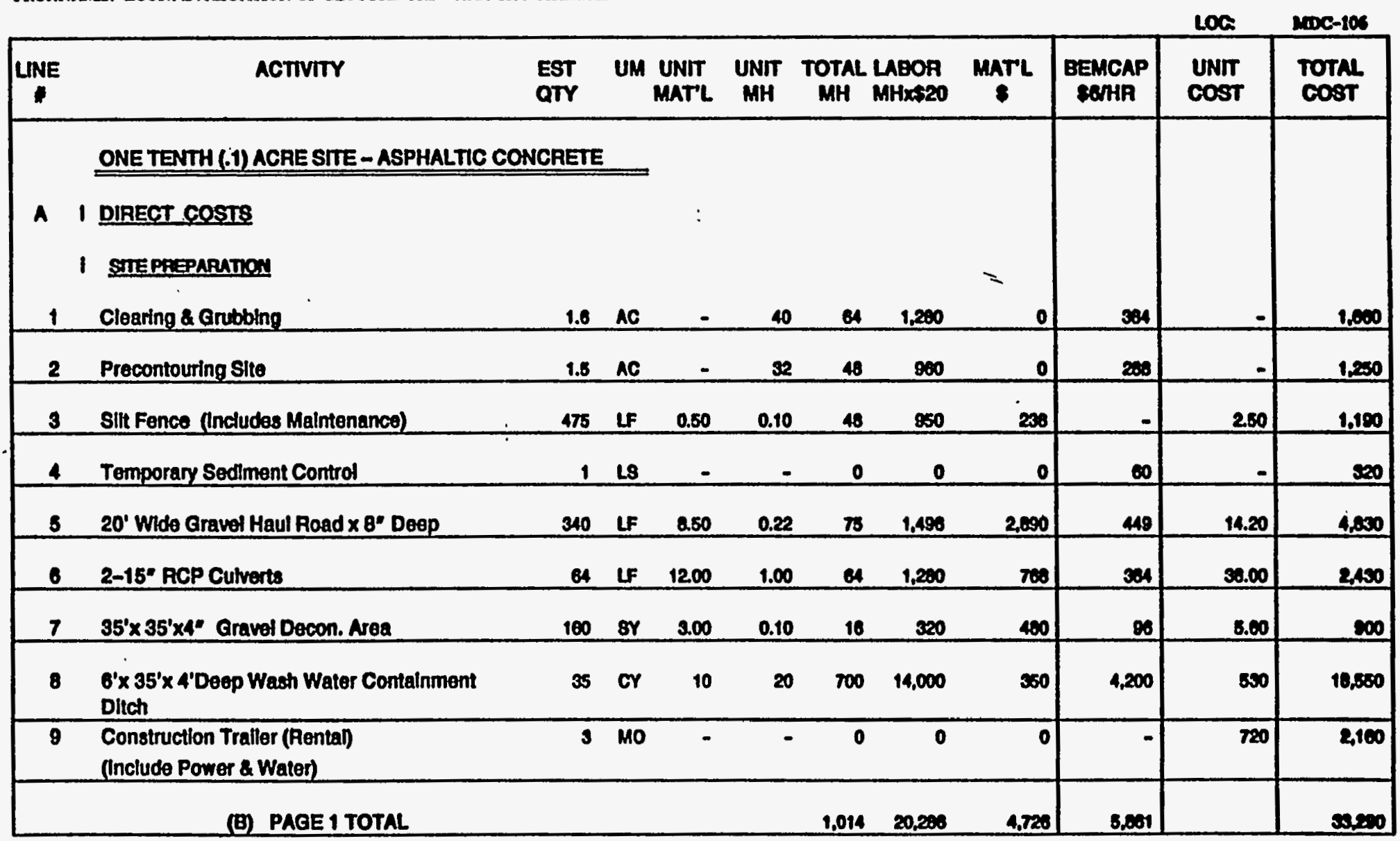

A-11. Cost Estimate for a Cover System Asphalt Concrete Hydraulic Barrier and a 30-mil FML 


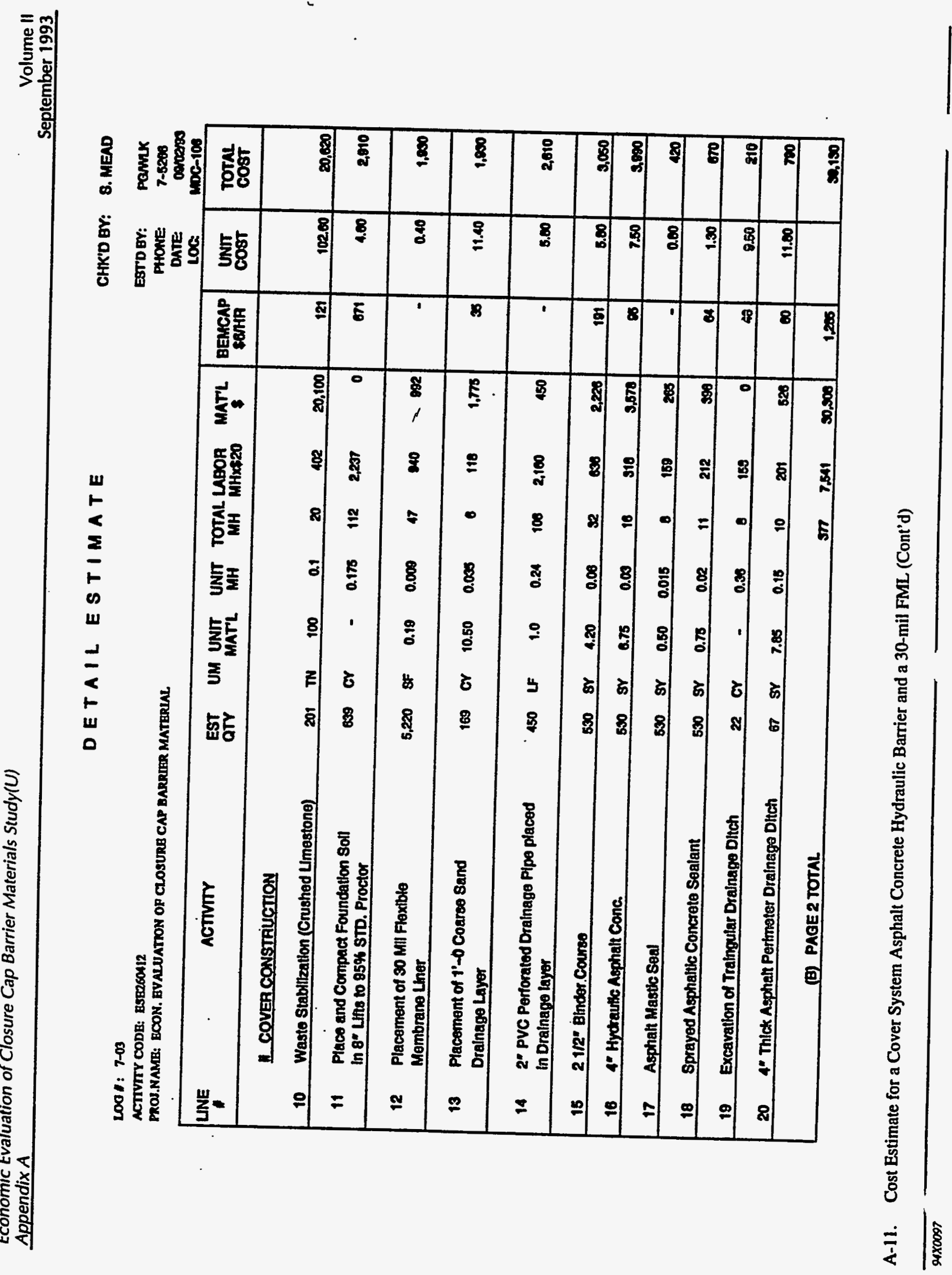


D B T A I L B S T I M A T B

\section{CHK'D QY: 8. MEAD}

Lo0 I: $7-03$

ACTIVITY CODE: ESE2SON12

RROJ.NAMB: BCON. EVALUATTON OP CLOSURB CAP BARRIER MATERJAL

ESTD BY: Pamk

PHONE: 7-5200

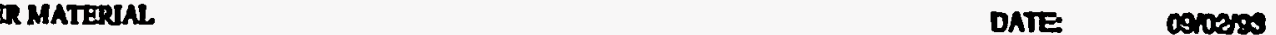

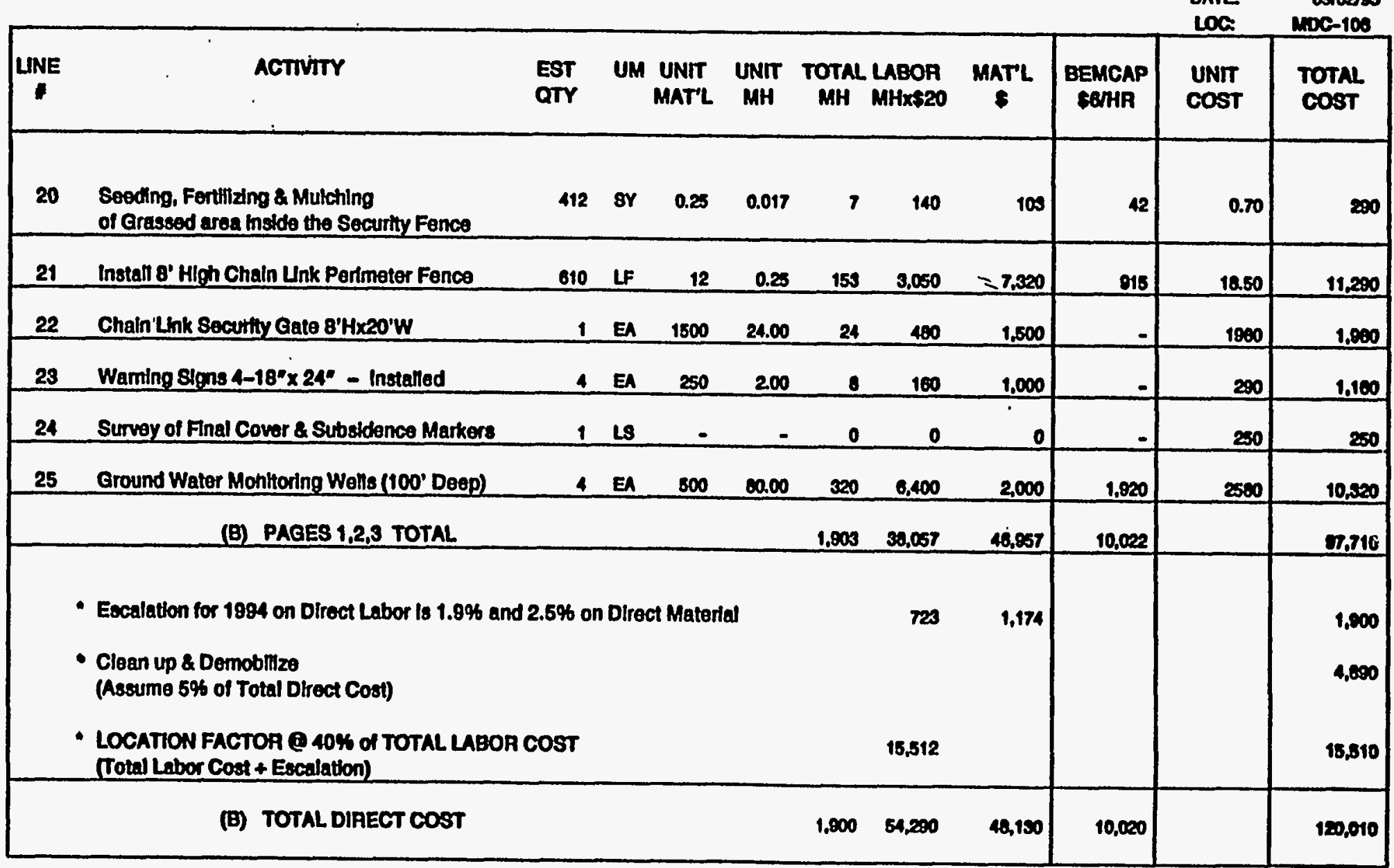

A-11. Cost Estimate for a Cover System Asphalt Concrete Hydraulic Barrier and a 30-mil FML (Cont'd) 
D B T A I L B S TIMATB

CHK' EY: 8. MEAD

\section{1: 7-03}

ACTIVITY CODE: BSEQSOS12

PROJ.NAMB: BCON. BVALUATTON OF CLOSURE CAP DARRTER MATERIAL

\begin{tabular}{|c|c|c|c|c|c|c|c|c|}
\hline LNE & ACTIVITY & $\begin{array}{l}\text { UM UNIT } \\
\text { MATL. }\end{array}$ & $\begin{array}{l}\text { UNIT } \\
\text { MH }\end{array}$ & $\begin{array}{l}\text { TOTAL LABOR } \\
\text { MH MHXS2O }\end{array}$ & $\underset{8}{\text { MATL }}$ & $\begin{array}{l}\text { BEMCAP } \\
\text { SBHA }\end{array}$ & $\begin{array}{l}\text { UNIT } \\
\text { COST }\end{array}$ & $\begin{array}{l}\text { TOTAL } \\
\text { COST }\end{array}$ \\
\hline & INDIRECT COSTS & & & & & & & \\
\hline 1 & $\begin{array}{l}\text { Cortification of the Final Cover } \\
\text { (Assume 3\% of the Total Direct Cost) }\end{array}$ & L8 & & & & & & 3,000 \\
\hline 2 & $\begin{array}{l}\text { Health \& Safoty } \\
\text { Assume 5\% of Total Direct Cost }\end{array}$ & LS & & & - & & & 0,000 \\
\hline 3 & $\begin{array}{l}\text { Scope Contingency } \\
\text { Assume } 10 \% \text { of Total Direct Cost }\end{array}$ & LS & & & & & & 12,000 \\
\hline 4 & $\begin{array}{l}\text { Administrative \& Legal } \\
\text { a } 10 \% \text { of Total Direct Cost }\end{array}$ & LS & & & & & & 12,000 \\
\hline 5 & $\begin{array}{l}\text { Enginoiring \& Servicos eil } \\
20 \% \text { af Total Direct Cost }\end{array}$ & & & & & & & 24,000 \\
\hline 6 & Craft Suppont @ 20.8\% of Total MNHAS (S19/HA) & & & 7,500 & & & & 7,810 \\
\hline 7 & $\begin{array}{l}\text { Fleld Inctrects of Total Direct } \\
\text { Labor @ 46.5. Bulk Material @ 28.5\% }\end{array}$ & & & 25,245 & 12,734 & & & 50,000 \\
\hline 8 & $\begin{array}{l}\text { Construction Nonmanual } 25.2 \% \text { of Total Dhect MH } \\
\text { Pate of } \$ 43.88\end{array}$ & & & 479 & & & & 20,910 \\
\hline 9 & Project Management @ $8 \%$ of Total Direct + Indirects & & & & & & & 10,520 \\
\hline & (B) TOTAL INDIAECT COST & & & & & & & 143960 \\
\hline
\end{tabular}

A-11. Cost Estimate for a Cover System Asphalt Concrete Hydraulic Barrier and a 30-mil FML (Cont'd) 


\section{I}

D B T A I L B S TIMATB

CHKD BY: 8. MEAD

200 ?-03

ACTIVIT CODE: BSE260112

PROS.NAME: BCON. BVALUATHON OF CLOSURB CAP BARRIFR MATBRIAL

ESTDEY: Pank

PHONE T-5200

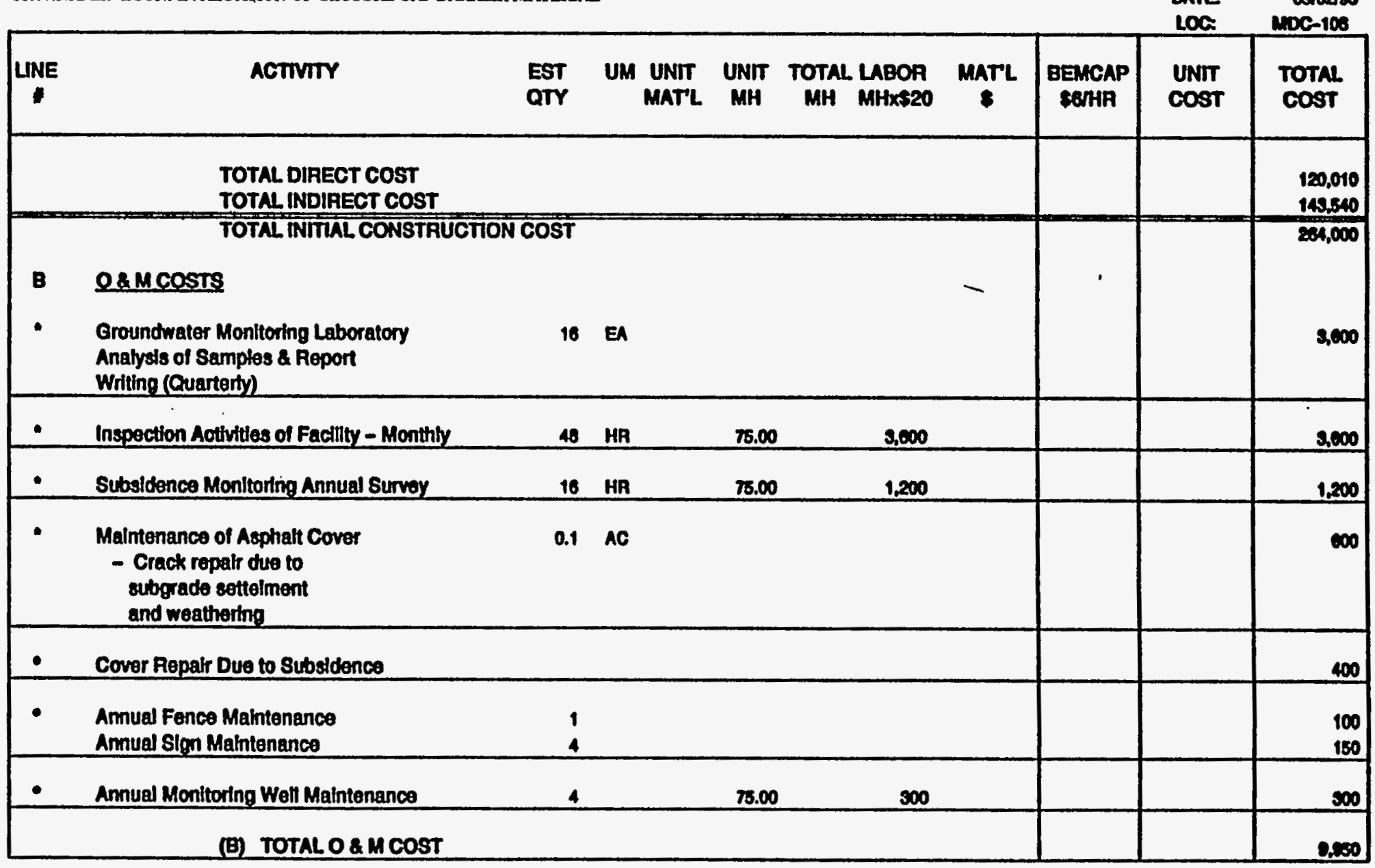

A-11. Cost Estimate for a Cover System Asphalt Concrete Hydraulic Barrier and a 30-mil FML (Cont'd) 
Lod I: 1-03

ACTIVTY CODE: ESB260412

EgTogr:

PHOME

Pannk

OATE:

PROJ.NAMBE: ECON. BVALUATION OP CLOSURB CAP BARRIER MATERJAL

Loc:

$\cos 0$

MOC-106

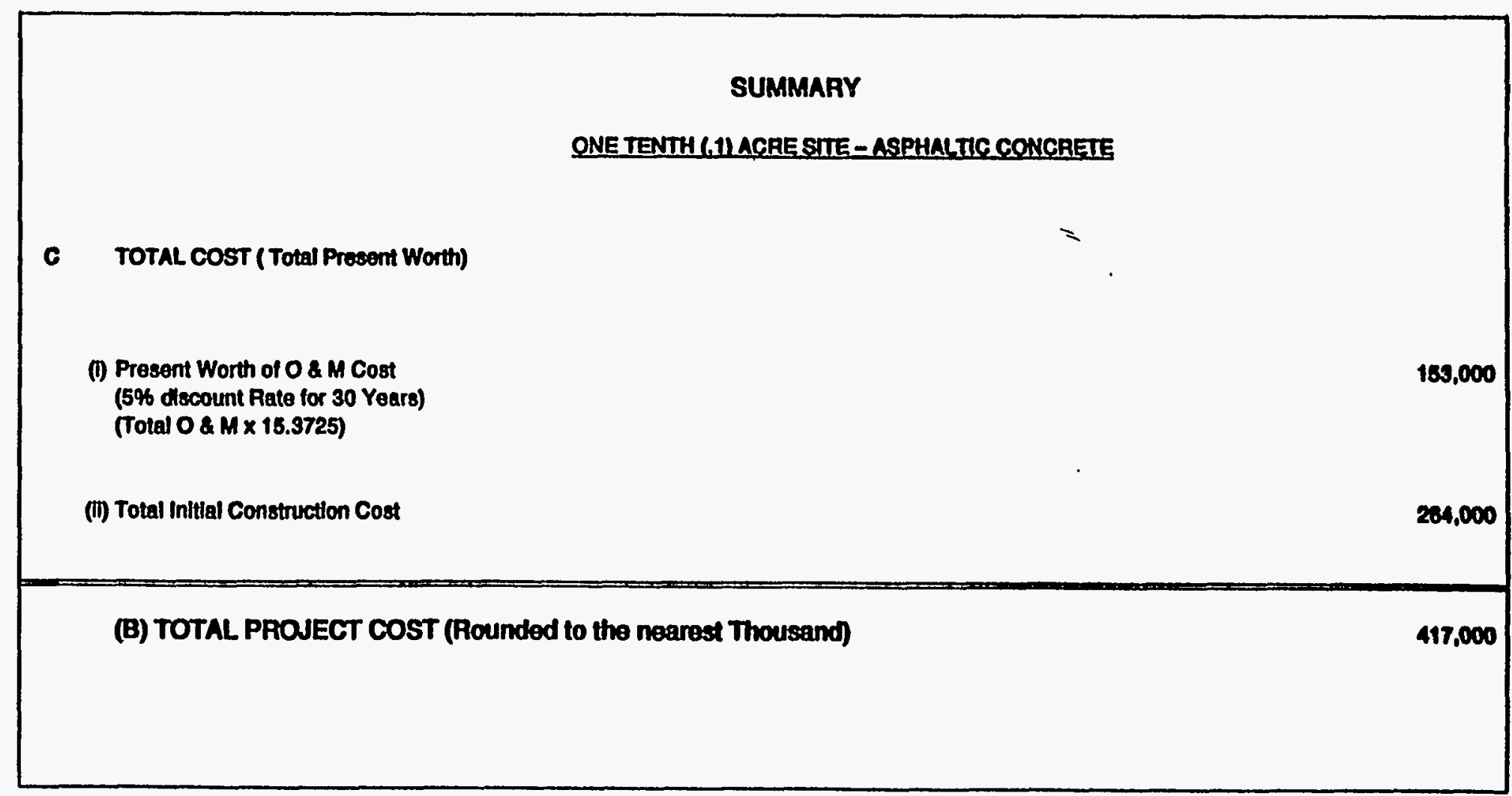

A-11. Cost Estimate for a Cover System Asphalt Concrete Hydraulic Barrier and a 30-mil FML (Cont'd) 
DETAIL ESTIMATE

LOa : $7-00$

ACTITIY CODE ESER 60412

PPOI.NAME ECON. EVALUATION OF CLOSURE CAP BAPRAER MATERUL

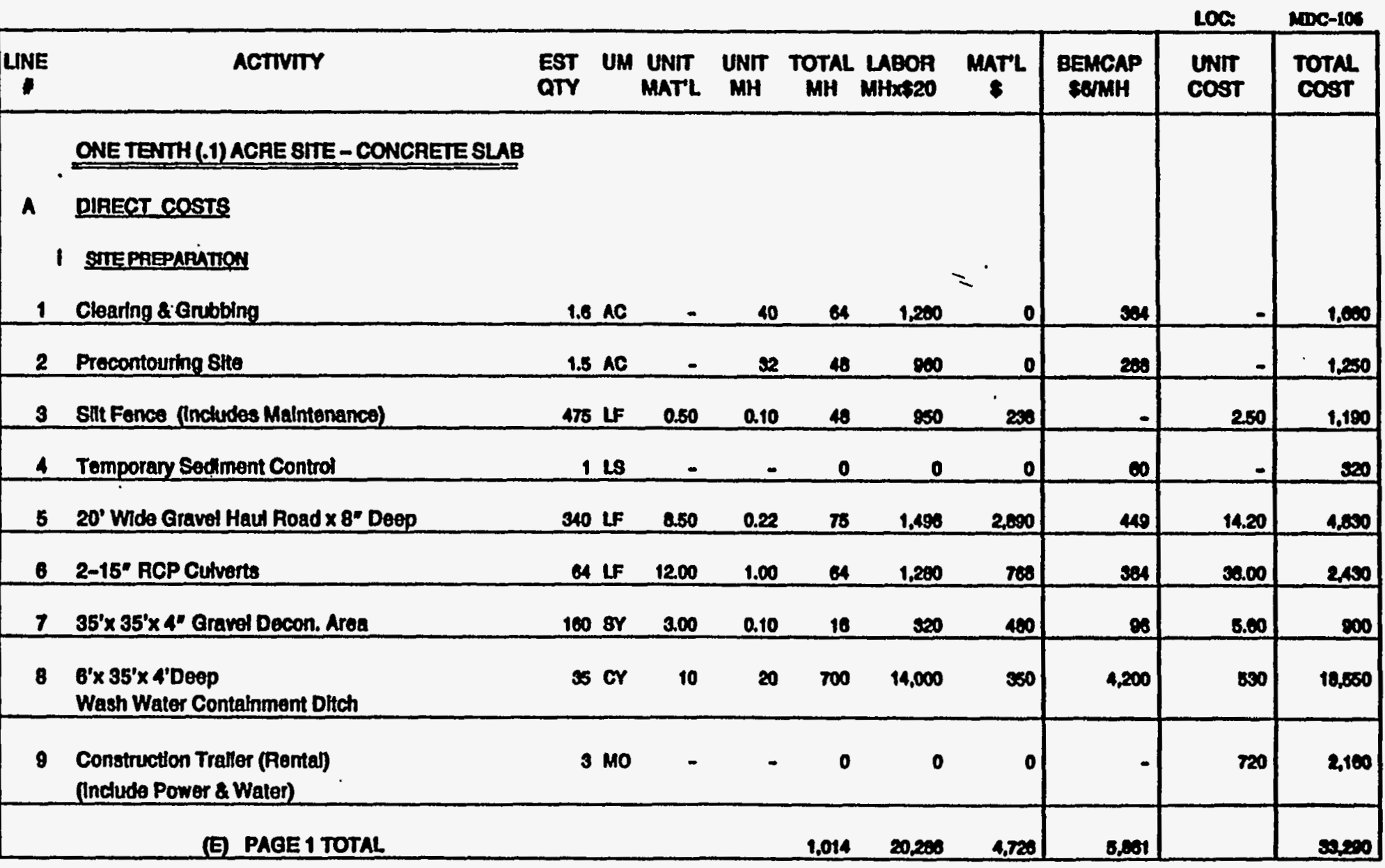

CHKO EY: 8. MEND

ESTOEY: ramx

PHONE 7-5265

100. Mmc-10

A-12. Cost Estimate for a Cover System Utilizing Poured-in Place Concrete Slab and a 30-mil FML 
DETAIL ESTIMATE

CHK'D 8Y: 8. MEAD

L001: 7-03

ACTIVITY CODE: .BSE200412

PROJ.NAMB: BCON. BVALUATEON OF CLOSURB CAP BARRRER MATERIAL.

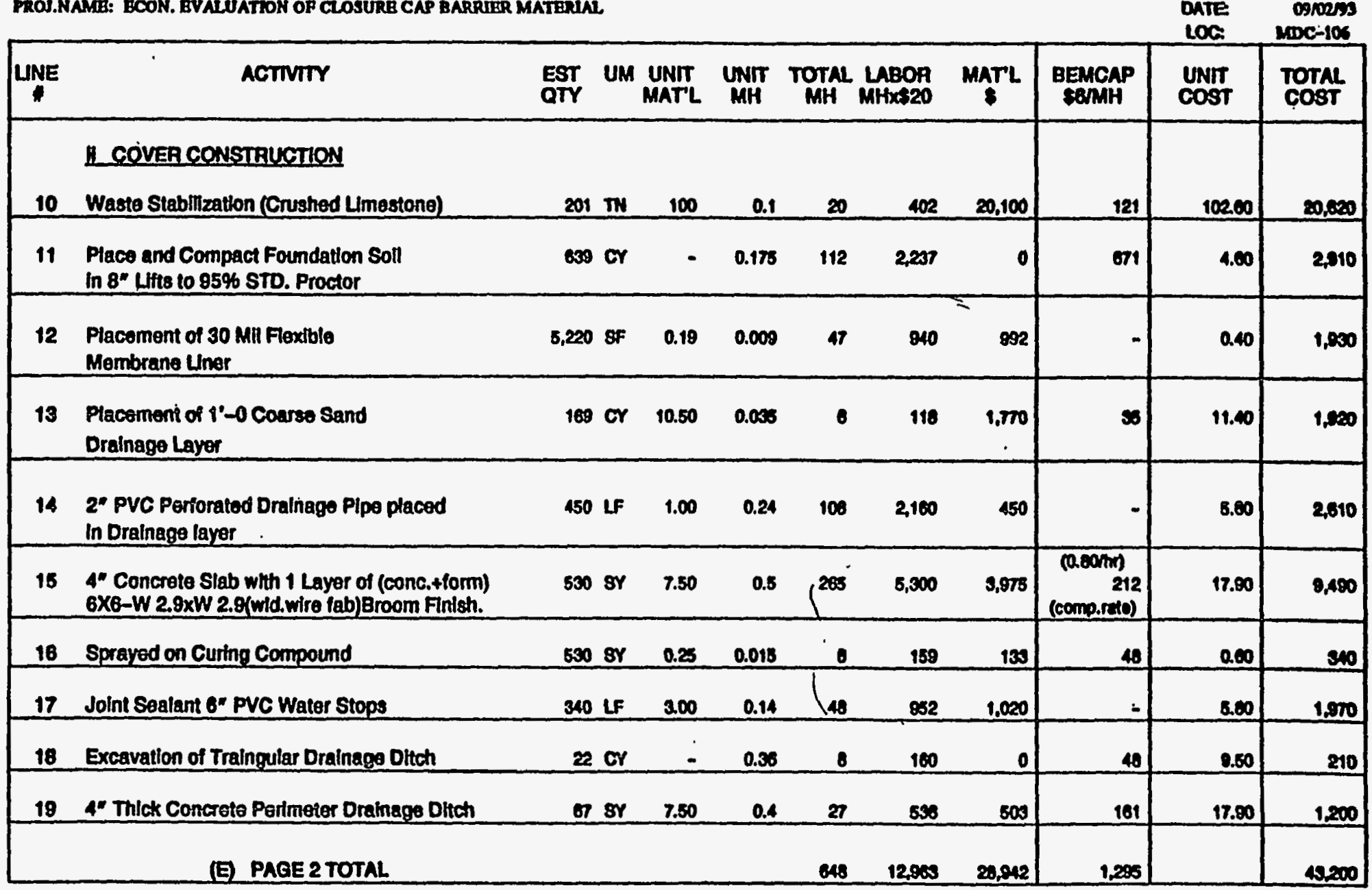

A-12. Cost Estimate for a Cover System Utilizing Poured-in Place Concrete Slab and a 30-mil FML (Cont'd) 
D B T A I B S TIMATB

\section{CHK'D BY: 8. MEAD}

Lod: $2-03$

ACTIVITY CODB: ESE200412

FROJ.NAME: BCON. BVALUATION OF CLOSURB CAP BARRIER MATBRRAL.

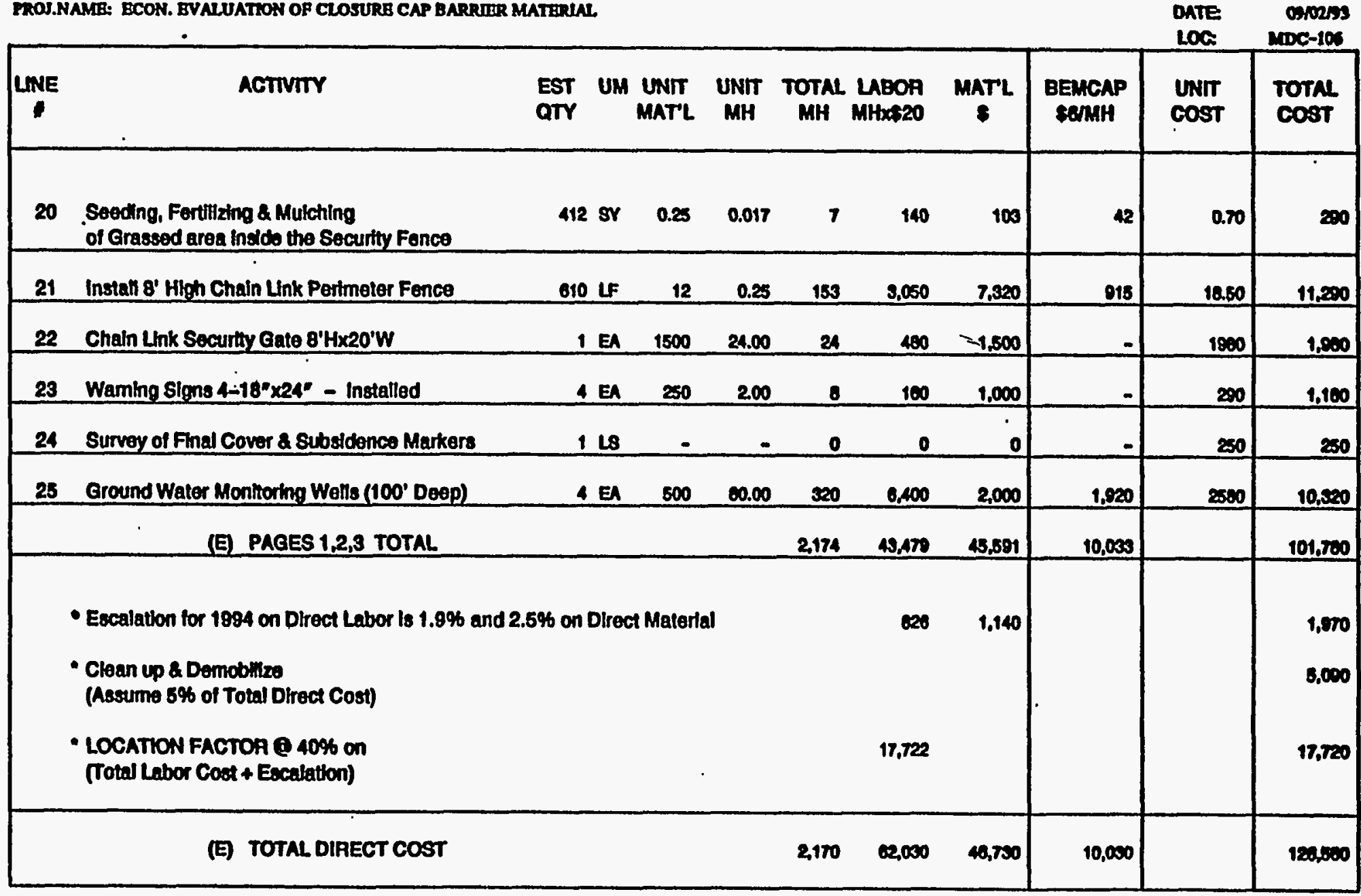

A-12. Cost Estimate for a Cover System Utilizing Poured-in Place Concrete Slab and a 30-mil FML (Cont'd) 
D B TAIL B S T I A T B

CHKD BY: 8. MEAD

L00 I: 7-03

ACTVITY CODE: BSE260112

PROU.NAME: BCON. EVALUATION OP CLOSURB CAP BARRIER MATERIAL.

\begin{tabular}{|c|c|c|c|c|c|c|c|c|}
\hline LWE & ACTIMTY & $\begin{array}{l}\text { UM UNIT } \\
\text { MATL }\end{array}$ & $\begin{array}{l}\text { UNTT } \\
\text { MH }\end{array}$ & $\begin{array}{l}\text { TOTAL LABOR } \\
\text { MH MHX\$20 }\end{array}$ & $\underset{8}{\operatorname{MATL}}$ & $\begin{array}{l}\text { BEACAP } \\
\text { SOMAH }\end{array}$ & $\begin{array}{l}\text { UNIT } \\
\text { COST }\end{array}$ & $\begin{array}{l}\text { TOTAL } \\
\text { COST }\end{array}$ \\
\hline & MNDIRECT CQSTS & & & & & & & \\
\hline 1 & $\begin{array}{l}\text { Certification of the Final Cover } \\
\text { (Assume 3\% of the Total Dlrect Cost) }\end{array}$ & L8 & & & & & & 5,000 \\
\hline 2 & $\begin{array}{l}\text { Health \& Safoty } \\
\text { Assume } 5 \% \text { of Total Direct Cost }\end{array}$ & LS & & & - & & & 0.500 \\
\hline 9 & $\begin{array}{l}\text { Scope Contingency } \\
\text { Assume 10\% of Total Direct Cost }\end{array}$ & LS & & & & & & 12,000 \\
\hline 4 & $\begin{array}{l}\text { Administrative \& Logal } \\
\text { @ 10\% of Total Direct Cast }\end{array}$ & LS & & & & & & 12000 \\
\hline 5 & $\begin{array}{l}\text { Engineering \& Sorvices } \\
20 \% \text { of Total Direct Cost }\end{array}$ & LS & & & & & & 25,510 \\
\hline 8 & Craft Suppon Q 20.8\% of Total MNHRS (\$19/HA) & & & 8.578 & & & & 8.600 \\
\hline 7 & $\begin{array}{l}\text { Feld Indirects of Total Direct } \\
\text { Labor @ 48.5\%, Bulk Matrial @ 26.5\% }\end{array}$ & & & 20,844 & 12,303 & & & 41,200 \\
\hline 8 & $\begin{array}{l}\text { Construction Nonmanual } 25.24 \text { of Total Direct MH } \\
\text { Ratio of } \$ 49.68\end{array}$ & & & 847 & & & & 20,000 \\
\hline 8 & Prolect Management @ 8\% of Total Direct + Indirects & & & & & & & 20,000 \\
\hline & (E) TOTAL INDIRECT COST & & & & & & & 180,200 \\
\hline
\end{tabular}

A-12. Cost Estimate for a Cover System Utilizing Poured-in Place Concrete Slab and a 30-mil FML (Cont'd) 
D B T A I L B S T I MAT B

CHK'D BY: 8. MEAD

Loaf: 7-03

ACTTVITY CODE: BSE200412

PROJ.NAMB: ECON. EVALUATION OR CLOSURB CAP BARRTBR MATERIAL

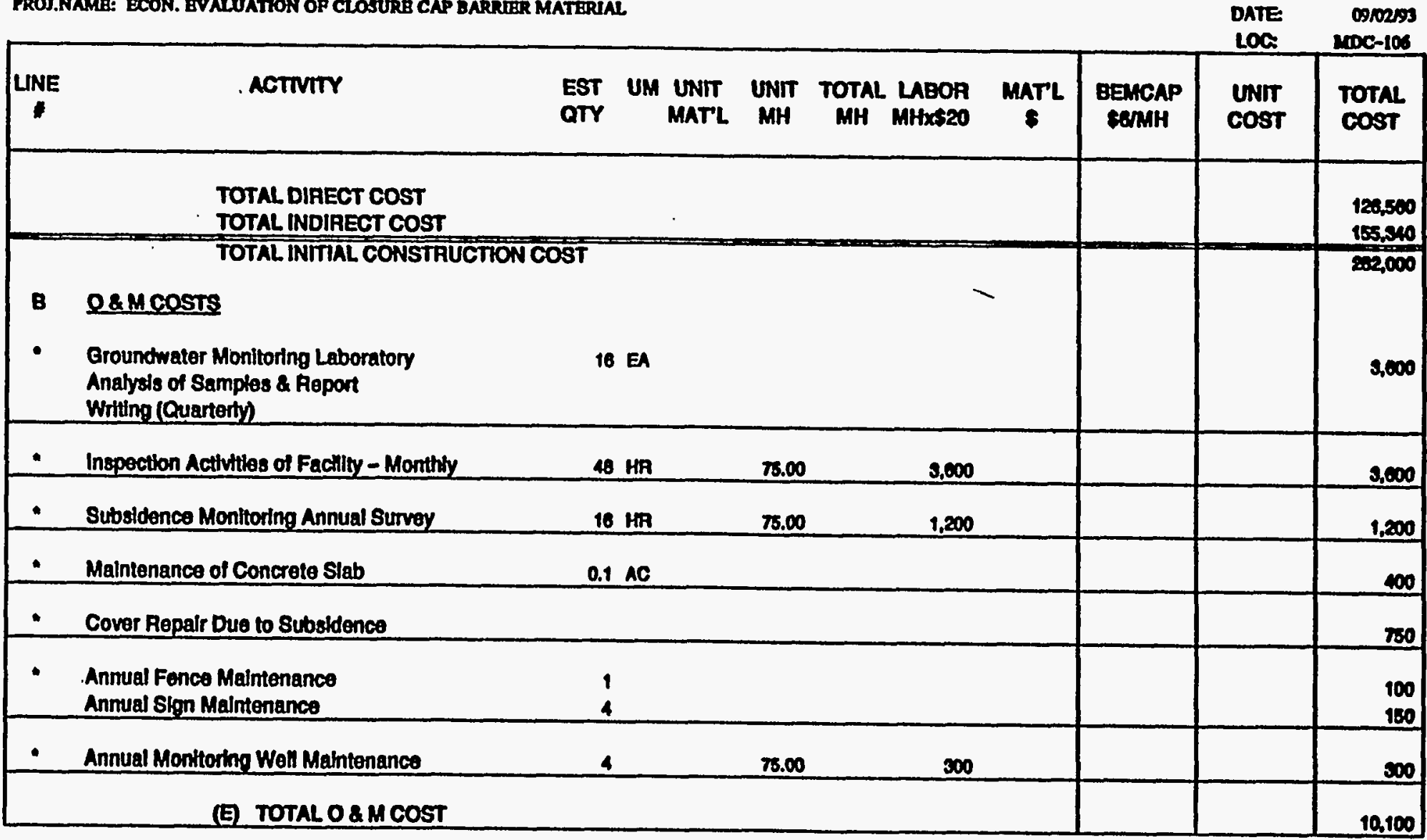

A-12. Cost Estimate for a Cover System Utilizing Poured-in Place Concrete Slab and a 30-mil FML (Cont'd) 


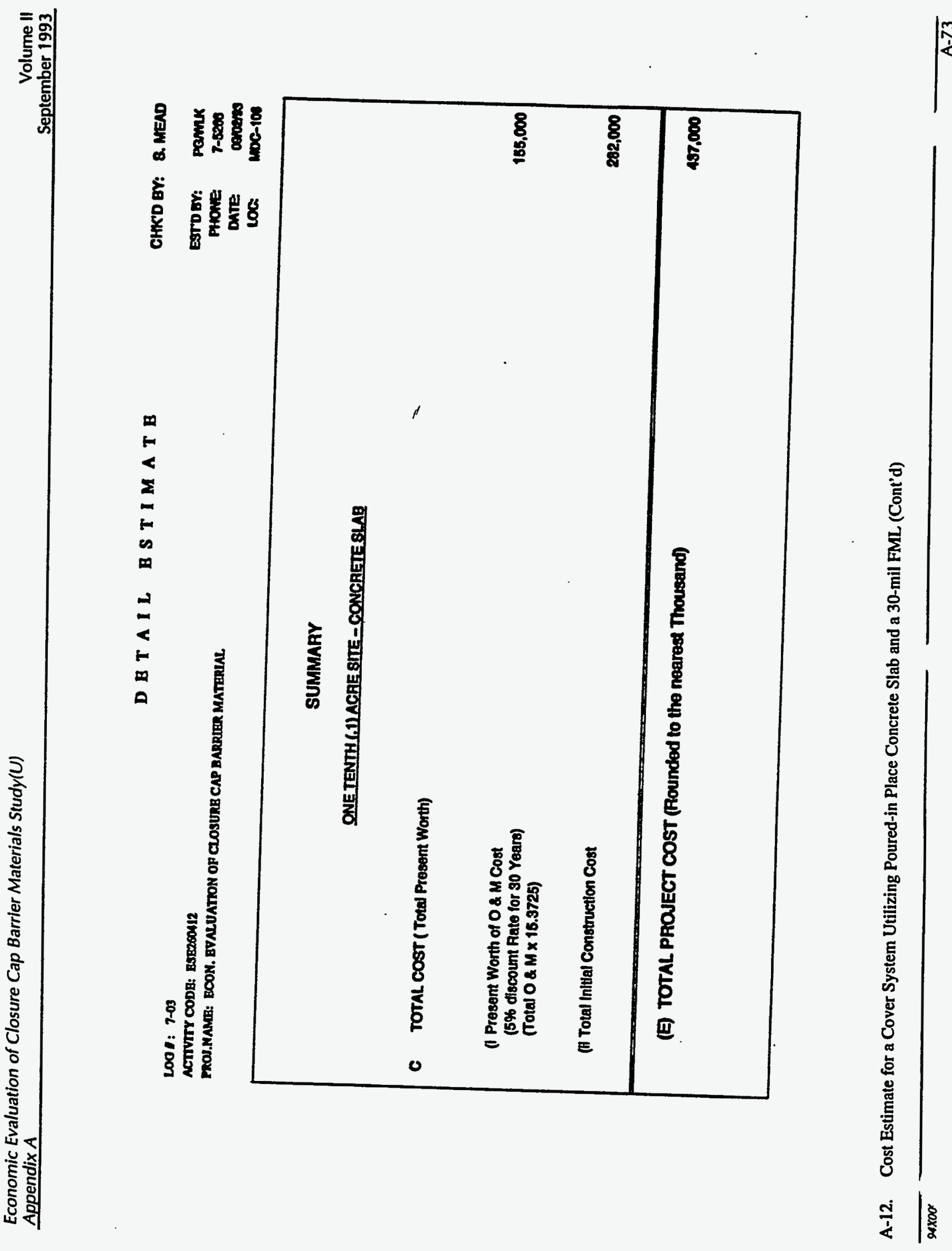




\section{Appendix B}

\section{Cost Estimates for 0.2 Acre Site}


DETAIL ESTIMATE

\section{Loa : : 7-03}

ACTIVITY CODE: ESE200412

PROS.NAME: ECON. EVALUATION OF CLOSURB CAP BARRIER MATERLAL.

CHKD BY: 8. MEAD

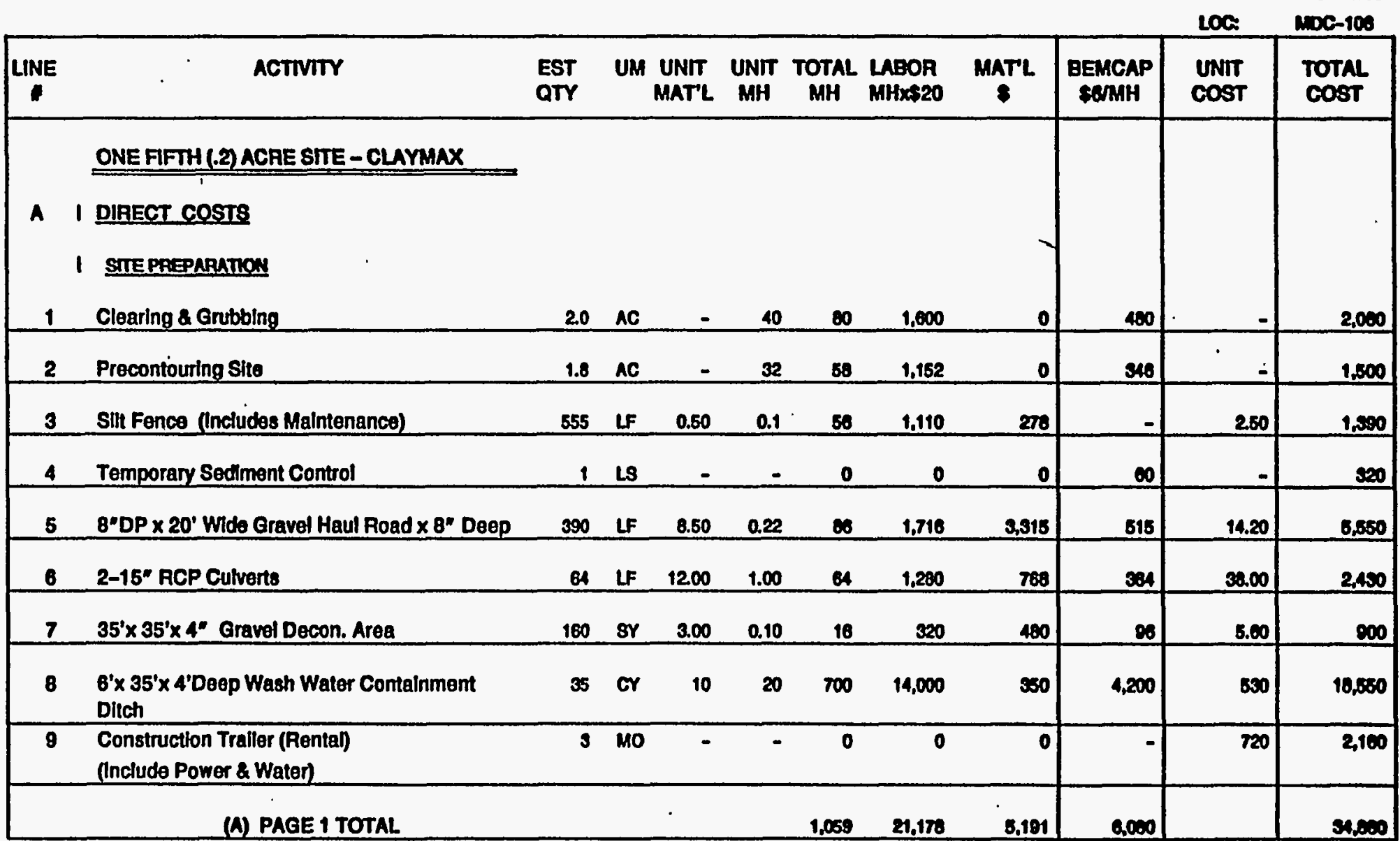

B-1. Cost Estimate for a Cover System Utilizing Claymax and a 30-mil FML 
DETAIL ESTIMATE

CHKO BY: 8. MEAD

LOA : 7-03

ACTIVITY CODE: ESEECOA12

PROJ.NAME: ECON. BVALUATON OP CLOSURB CAP BARRIER MATERIAL.

\begin{tabular}{|c|c|c|c|c|c|c|c|c|c|c|c|}
\hline LNE & ACTIVTr & $\begin{array}{l}\text { EST } \\
\text { QTY }\end{array}$ & UM & $\begin{array}{l}\text { UNIT } \\
\text { MAT'L }\end{array}$ & UNT & $\begin{array}{l}\text { TOTAL } \\
\text { MH }\end{array}$ & $\begin{array}{l}\text { UBOA } \\
\text { MHx:20 }\end{array}$ & MATL & $\begin{array}{l}\text { BEMCAP } \\
\text { BBMH }\end{array}$ & $\begin{array}{l}\text { UNIT } \\
\text { COST }\end{array}$ & $\begin{array}{l}\text { Total } \\
\text { Cost }\end{array}$ \\
\hline & 1 CONEA CONSTRUCTION & & & & & & & & & & \\
\hline 10 & Waste Stabilization (Crushed Limestone) & 400 & $T N$ & 100 & 0.1 & 40 & 800 & 40,000 & 240 & 102.00 & 41,040 \\
\hline 11 & $\begin{array}{l}\text { Place and Compact Foundation Soll } \\
\text { in 8" Lfits to } 85 \% \text { Sid. Proctor }\end{array}$ & 1,614 & Cr & - & 0.175 & 282 & 5,849 & $=0$ & 1,695 & 4.50 & 7,340 \\
\hline 12 & Placement of Claymax Geotextile Mat & 12,320 & SF & 0.50 & 0.01 & 123 & 2,464 & 6,160 & - & 0.70 & 0,020 \\
\hline 13 & $\begin{array}{l}\text { Placement of } 30 \text { Mil Flexible } \\
\text { Membrane Lirer }\end{array}$ & 12,320 & SF & 0.19 & 0.009 & 111 & 2,210 & 2,341 & - & .0 .40 & 4,500 \\
\hline 14 & $\begin{array}{l}\text { Placement of } 1 \text { ' }-0 \text { Coarse Sand } \\
\text { Dralnage Layer }\end{array}$ & 425 & or & 10.50 & 0.035 & 18 & 290 & 4,460 & $\mathbf{9}$ & 11.40 & 4,000 \\
\hline 16 & 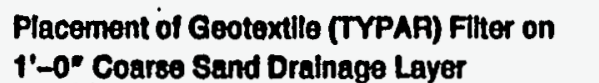 & 1,402 & sr & 0.20 & 0.007 & 10 & 180 & 200 & - & 0.30 & 400 \\
\hline 16 & Placement of 1'-6" Common Fill & 485 & or & 3.75 & 0.07 & 34 & 679 & 1,010 & 204 & 5.60 & 2,700 \\
\hline 17 & Placement of 6" Topsoil & 182 & CY & 9.50 & 0.09 & 16 & 320 & 1.720 & 86 & 11.60 & 2,180 \\
\hline 18 & $\begin{array}{l}\text { Piacement of Perlmeter Exterlor } \\
\text { Drainage Layer } 34^{*} \times 3^{*} \text { DP (Crushed Stone) }\end{array}$ & 143 & SY & 1.80 & 0.24 & 34 & 60 & 215 & 208 & 7.00 & 1.110 \\
\hline 19 & Excavation of Trapezoldal Dralnage Dltch & 88 & Cr & - & 0.2 & 10 & 352 & 0 & 108 & 8.20 & 400 \\
\hline & (A) PAGE 2 TOTAL & & & & & $\infty 00$ & 13,600 & 57,000 & 2,057 & & 70,510 \\
\hline
\end{tabular}

B-1. Cost Estimate for a Cover System Utilizing Claymax $\$$ and a 30-mil FML (Cont'd) 
DBTAILASTIMATB

CHKD BY: S. MEAD

L00 I: 7-03

ACTIVITY CODB: BSE2OOM12

PROJ.NAME: BCON. EVALUATTON OP CLOSURB CAP BARRIER MATHRIAI

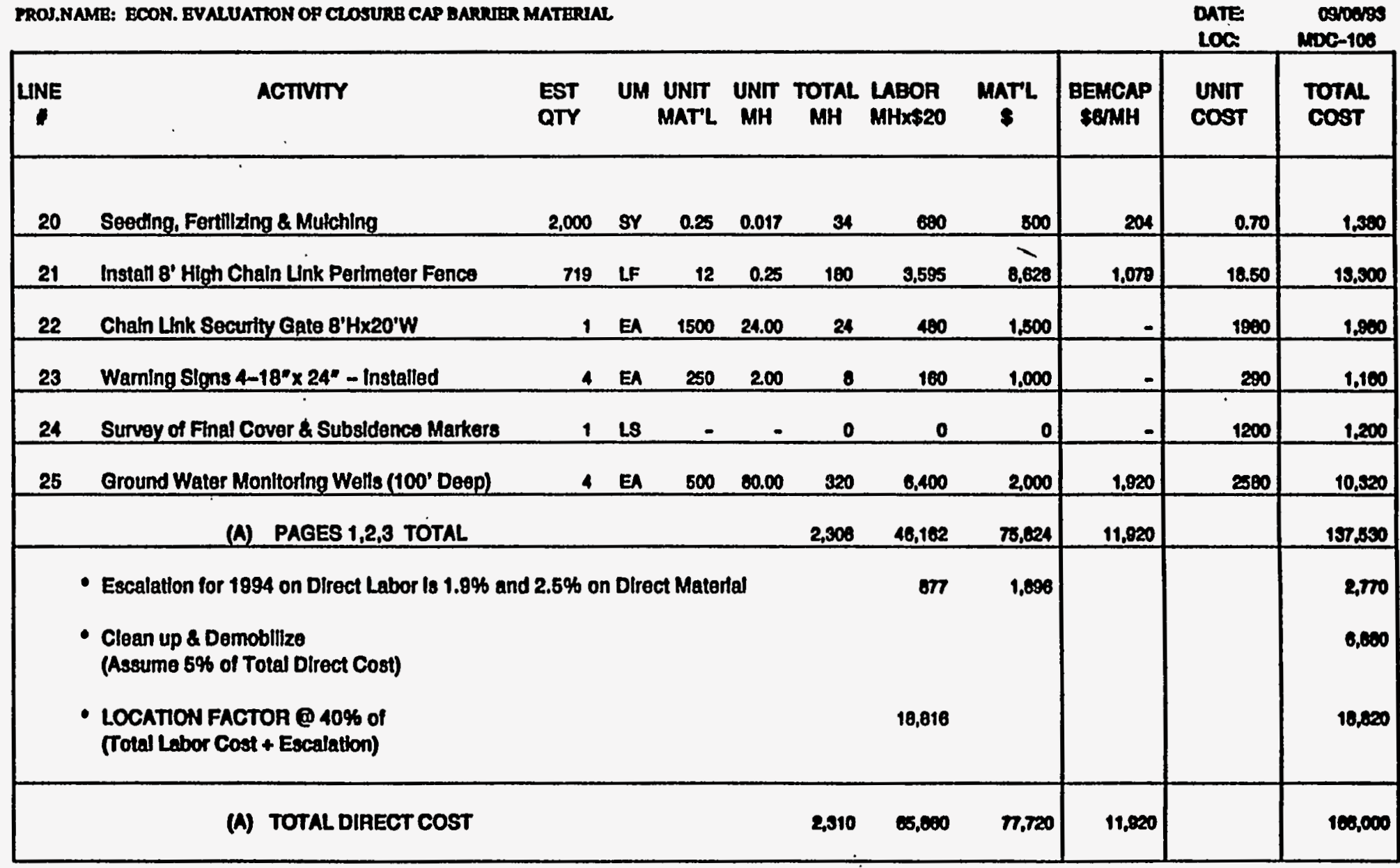

B-1. Cost Estimate for a Cover System Utilizing Claymax and a 30-mil FML (Cont'd) 
CHK'D BY: 8. MEAD

$1008: 7-03$

ACTIVITY CODE: ESE2SOA12

PROJ.NAME: BCON. BVALUATION OF CLOSURB CAP BARRTER MATERIAY

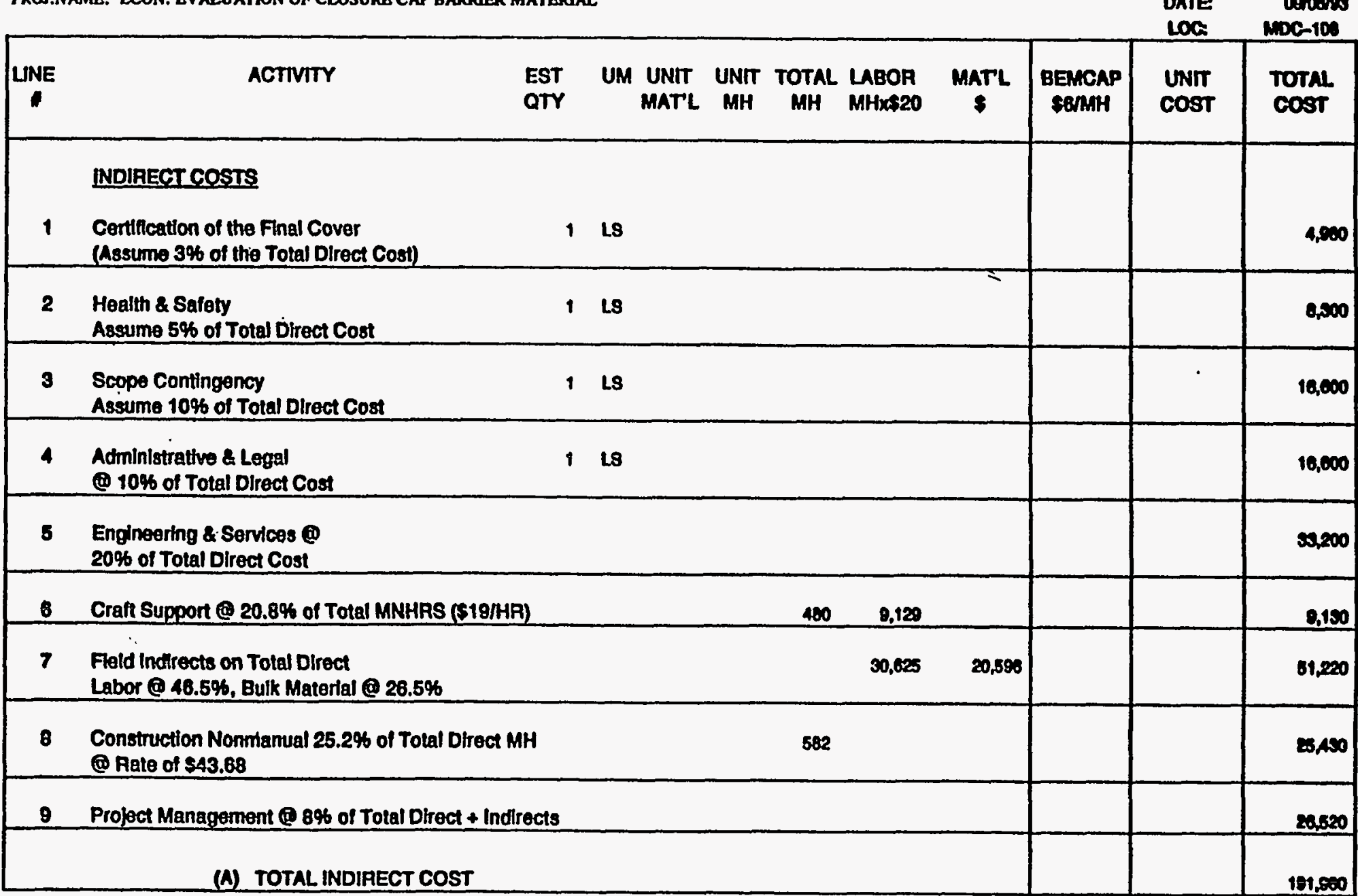

B-1. Cost Estimate for a Cover System Utilizing Claymax@ and a 30-mil FML (Cont'd) 
1

D B T A L B S TIMAT B

CHKO BY: 8. MEAD

L001: 7-03

ACTVITY CODB: ESE260412

PROU.NAMB: ECON. EVALUATTON OR CLOSURB CAP BARRIER MATERIAL.

ESTD BY: PAMLK

\begin{tabular}{|c|c|c|c|c|c|c|c|c|c|}
\hline LNE & ACTIVIT & $\begin{array}{l}\text { EST } \\
\text { QTY }\end{array}$ & $\begin{array}{l}\text { UM UNTT } \\
\text { MAT'L }\end{array}$ & $\begin{array}{l}\text { UNIT TOTAL } \\
\text { MH }\end{array}$ & $\begin{array}{l}\text { LABOR } \\
\text { MHX:20 }\end{array}$ & $\underset{8}{\operatorname{MaTL}}$ & $\begin{array}{l}\text { BEMCAP } \\
\$ 6 / M H\end{array}$ & $\begin{array}{l}\text { UNIT } \\
\text { COST }\end{array}$ & $\begin{array}{l}\text { TotAL } \\
\text { COST }\end{array}$ \\
\hline & $\begin{array}{l}\text { TOTAL DIRECT COST } \\
\text { TOTAL INDIRECT COST } \\
\end{array}$ & & & & & & & & $\begin{array}{l}168,000 \\
191,000\end{array}$ \\
\hline : & $\begin{array}{l}\text { TOTAL INITIAL CONSTAU } \\
\text { Q\&M COSTS } \\
\text { Groundwater Monitoring Laboratory } \\
\text { Analyols of Samples \& Report } \\
\text { Writing (Quarterty) }\end{array}$ & $\begin{array}{r}\text { COST } \\
16\end{array}$ & EA & & & $=$ & & - & $\begin{array}{r}360,000 \\
3,000\end{array}$ \\
\hline$\bullet$ & Inspection Activities of Facilly - Monthly & 48 & HP & 75.00 & 9,600 & & & & 0,000 \\
\hline$\bullet$ & Subsidenco Monitoring Annual Survey & 16 & HP & 75.00 & 1,200 & & & & 1,200 \\
\hline & $\begin{array}{l}\text { Maintenance of Vegetative Cover } \\
\text { - } 2 \text { Mowings / year } \\
\text { - Pest Control } \\
\text { - Eroston Control \& } \\
\text { Drainage Malntenance }\end{array}$ & 0.2 & $A C$ & & & & & & 400 \\
\hline 8 & Cover Repalr Due to Subsidence & & & & & & & & 350 \\
\hline & $\begin{array}{l}\text { Annual Fence Maintenance } \\
\text { Annual Sign Malntenance }\end{array}$ & $\begin{array}{l}1 \\
4 \\
\end{array}$ & & & & & & & $\begin{array}{l}100 \\
150 \\
\end{array}$ \\
\hline - & Annual Monitoring Well Maintenance & 4 & & 75.00 & 300 & & & & 500 \\
\hline & (A) TOTAL O \& M COST & & & & & & & & 0,700 \\
\hline
\end{tabular}

B-1. Cost Estimate for a Cover System Utilizing Claymax and a 30-mil FML (Cont'd) 
D B TAIL B S T IMA T

$1001: 7-\infty$

ACTIVTYY CODE: BSE2SOH12

PROS.NAME: BCON. BYALUATION OF CLOSURB CAP BARRIBR MATERAAL.
CHKD BY: 8. MEAD

ESTDEV: PamuK

PHOHE T-5206

DATE DOTOMOS

LOG: MDC-100

\begin{tabular}{|l|c|}
\hline & SUMMARY \\
ONE FIFTH (1.2) AGRE SITE-CLAYMAX \\
C TOTAL COST ( Total Present Worth) \\
(i) Present Worth of O\& M Cost \\
(5\% discount Rate for 30 Years) \\
(Total O \& M $\times$ 15.3725) \\
(ii) Total Initial Construction Cost \\
\hline (A) TOTAL PROJECT COST (Rounded to the nearest Thousand) \\
\hline
\end{tabular}

B-1. Cost Estimate for a Cover System Utilizing Claymax and a 30-mil FML (Cont'd) 


\section{0 : :-03}

ACTIVTTY CODE: ESE260112

PROJ.NAME: ECON. BVALUATHON OF CLOSURB CAP BARUIER MATERIALL

ESTDEY: PamuK

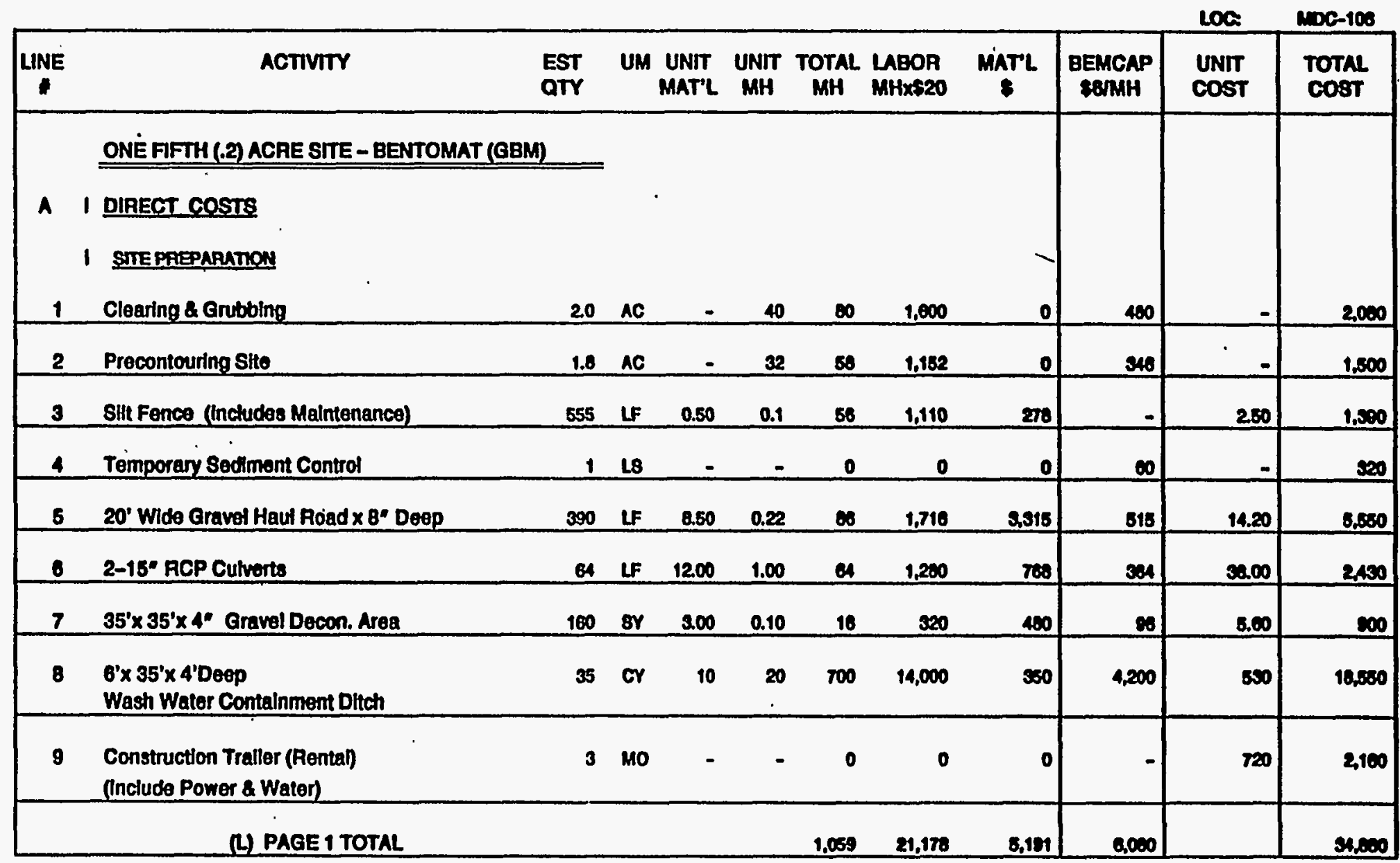

B-2. Cost Estimate for a Cover System Utilizing Bentomat@ and a 30-mil FML 
DETAIL ESTIMATE

\section{CHKD BY: 8. MEAD}

LO0 : : 7-03

ACTIVITY CODE: BSE2SOH12

PROJ.NAME: ECON. EVALUATIOH OF CLOSURB CAP BARRUER MATERAAL

\begin{tabular}{|c|c|c|c|c|c|c|c|c|c|c|c|}
\hline & & & & & & & & & & LOG & $\operatorname{MDC}-100$ \\
\hline LNE & ACTIVIV & $\begin{array}{l}\text { EST } \\
\text { QTY }\end{array}$ & UM & $\begin{array}{l}\text { UNIT } \\
\text { MAT'L }\end{array}$ & $\underset{M H}{\mathrm{UNT}}$ & TOTAL & $\begin{array}{l}\text { LABOA } \\
\text { MHX } \$ 20\end{array}$ & $\underset{\&}{\operatorname{MATL}}$ & $\begin{array}{l}\text { BEMCAP } \\
\text { SBMMH }\end{array}$ & $\begin{array}{l}\text { UNIT } \\
\text { COST }\end{array}$ & $\begin{array}{l}\text { Total } \\
\text { cost }\end{array}$ \\
\hline & L COVER CONSTRUCTION & & & & & & & & & & \\
\hline 10 & Waste Stabllization (Crushed LImestone) & 100 & $\pi$ & 100 & 0.1 & 40 & 800 & 40,000 & 240 & 102.60 & $\$ 1,040$ \\
\hline 11 & $\begin{array}{l}\text { Place and Compact Foundation Soll } \\
\text { In 8" Ufits to } 85 \% \text { STD. Proctor }\end{array}$ & 1,614 & cr & - & 0.175 & 282 & 5,640 & $=0$ & 1,095 & 4.50 & 7940 \\
\hline 12 & Placement of Bentonite Geotextile Mat & 12,320 & SF & 0.50 & 0.01 & 123 & 2,464 & 0,100 & - & a.ro & 0,620 \\
\hline 13 & $\begin{array}{l}\text { Placement of } 30 \text { MN Flexible } \\
\text { Membrant Liner }\end{array}$ & 12,320 & sf & 0.10 & 0.009 & 111 & 2,218 & 2,341 & .1 & 0.40 & 4800 \\
\hline 14 & $\begin{array}{l}\text { Placement of } 1 \text { '-O Coarse Sand } \\
\text { Drainage Lays }\end{array}$ & 425 & or & 10.5 & 0.035 & 15 & 200 & 4,400 & 69 & 11.40 & 4,850 \\
\hline 16 & $\begin{array}{l}\text { Placement of Geotoxtlie (TYPAR) Fitter on } \\
1^{\prime}-0^{*} \text { Coarso Sand Drainage Layer }\end{array}$ & 1.402 & sr & 0.20 & 0.007 & 10 & 180 & 200 & - & 0.50 & 400 \\
\hline 16 & Placement of 1'-6" Common Fill & 405 & or & 3.75 & 0.07 & $\mathbf{m}$ & one & 1,810 & 204 & 8.60 & 2,700 \\
\hline 17 & Placement of 6" Topsoil & 182 & or & 9.50 & 0.09 & 16 & 320 & 1.720 & $\boldsymbol{8}$ & 11.00 & 2.160 \\
\hline 18 & $\begin{array}{l}\text { Placsment of Perimeter Exierior } \\
\text { Drainage Layer } 3 / 4^{*} \times 3^{\circ} \text { DP (Crushed Stone) }\end{array}$ & 143 & sr & 1.50 & 0.24 & 34 & 668 & 216 & 200 & 7.00 & 1.110 \\
\hline 19 & Excavation of Trapezoldal Drainage Ditch & 8 & or. & - & 0.20 & 16 & 352 & o & 108 & 8.20 & $\$ \infty 0$ \\
\hline & (L) PAGE 2 TOTAL & & & & & 600 & 19,000 & 57,000 & 2.607 & & rasio \\
\hline
\end{tabular}

B-2. Cost Estimate for a Cover System Utilizing Bentomat $\$$ and a 30-mil FML (Cont'd) 
D BTAIL BSTIMATB

CHKD BY: S. MEAD

Lo01: 7-03

ACTIVITY CODB: BSE260012

PROJ.NAME: ECON. BVALUATION OF CLOSURB CAP BARRIRR MATERIAL

Panck

PHOWE: 7-52006.

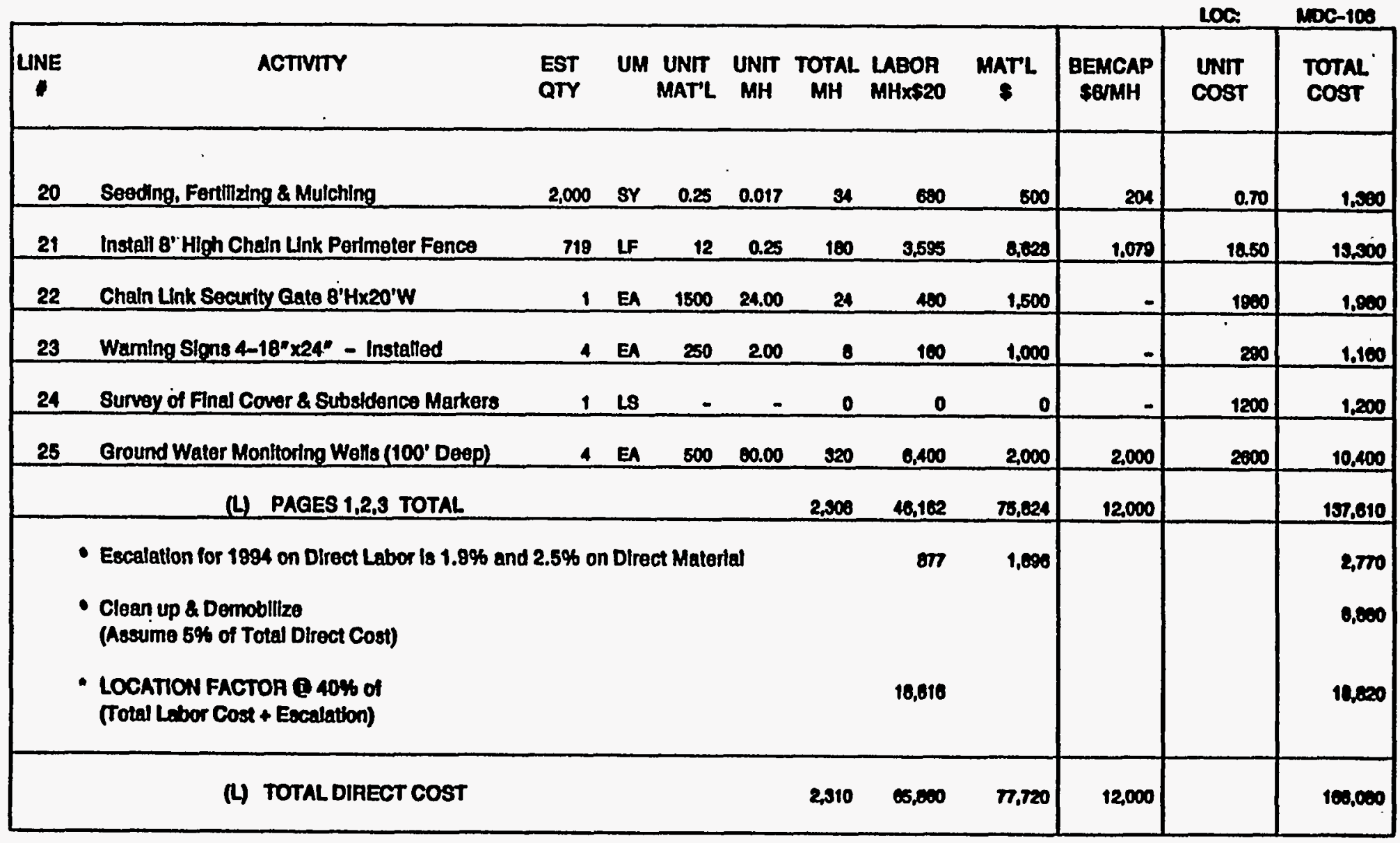

B-2. Cost Estimate for a Cover System Utilizing Bentomat@ and a 30-mil FML (Cont'd) 
D B T A I L B S T I M A T B

CHK'D BY: 8. MEAD

Loo 1: 7-03

ACTIVITY CODB: ESE200412

PROJ.NAMB: RCON. EVALUATION OF CLOSURB CAP BARRIER MATERIAL

ESTDQY: PaMnK

DATE

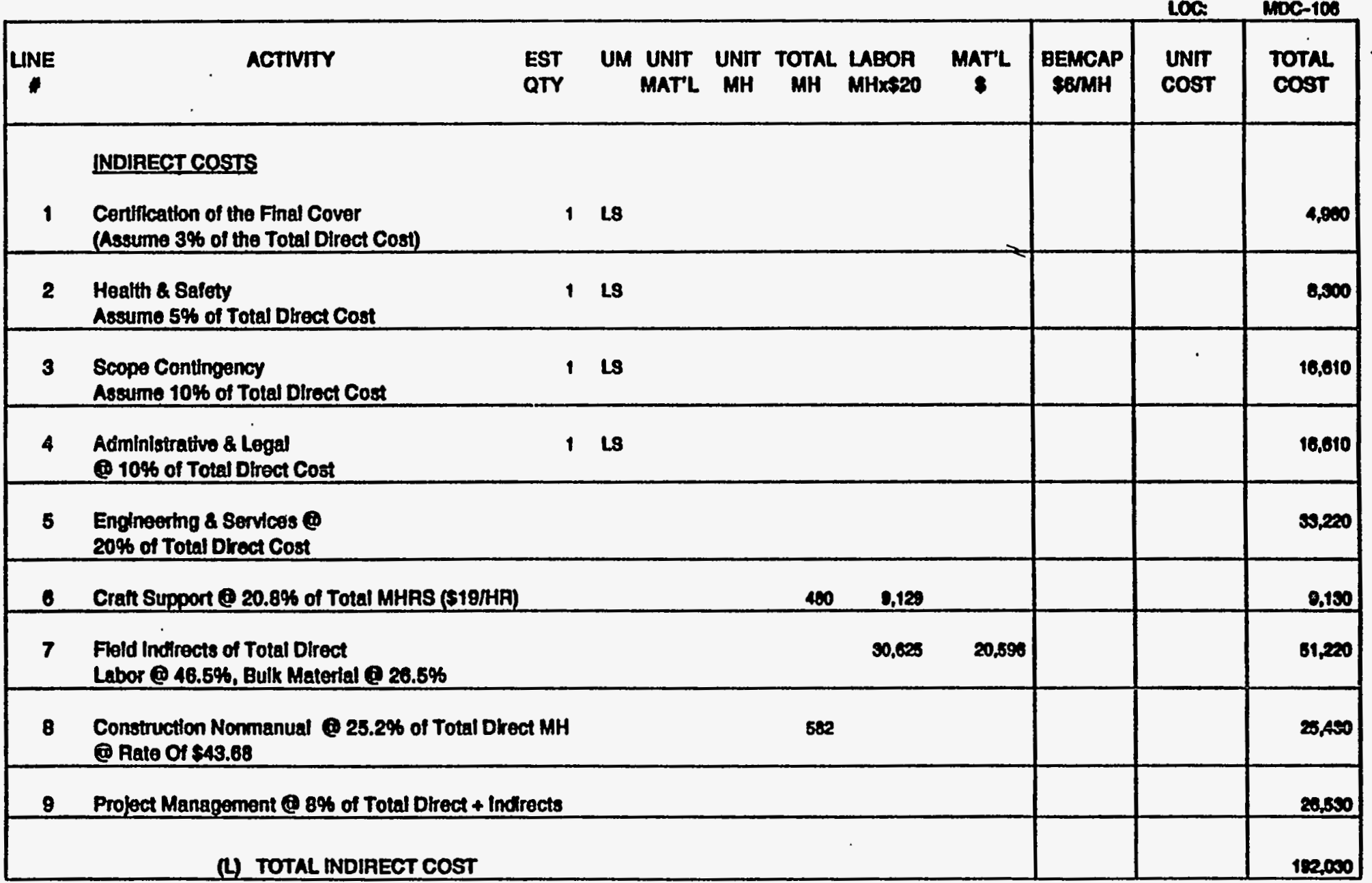

B-2. Cost Estimate for a Cover System Utilizing Bentomat@ and a 30-mil FML (Cont'd) 


\section{B T A I L B S TIMAT B}

CHKD BY: 8. MEAD

LOO I: 7-03

ACTIVITY CODB: ESEZ6ON12

PROJ.NAME: ECON. EVALUATION OF CLOSURB CAP BARRIER MATERIAL

ESTDEV: Pamk

PATONE: T-5260

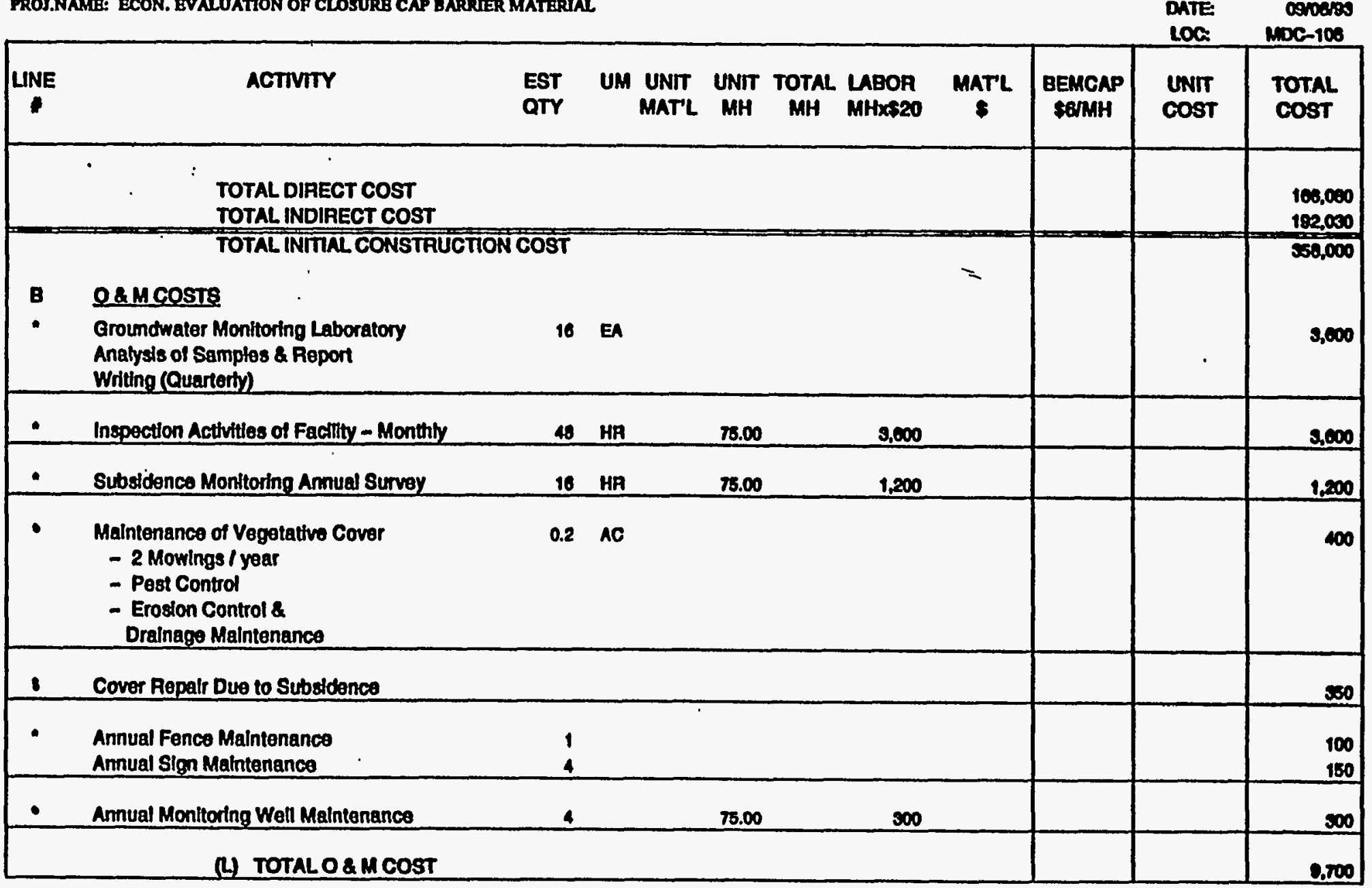

B-2. Cost Estimate for a Cover System Utilizing Bentomata and a 30-mil FML (Cont'd) 
D BTAIL BSTIMATB

CHK'D BY: 8. MEAD

L001: 7-03

ACTIVITY CODE: EST2SOS12

MROJ.MAME: BCON. BVALUATTON OF CLOSURE CAP BARRIER MATERIAL

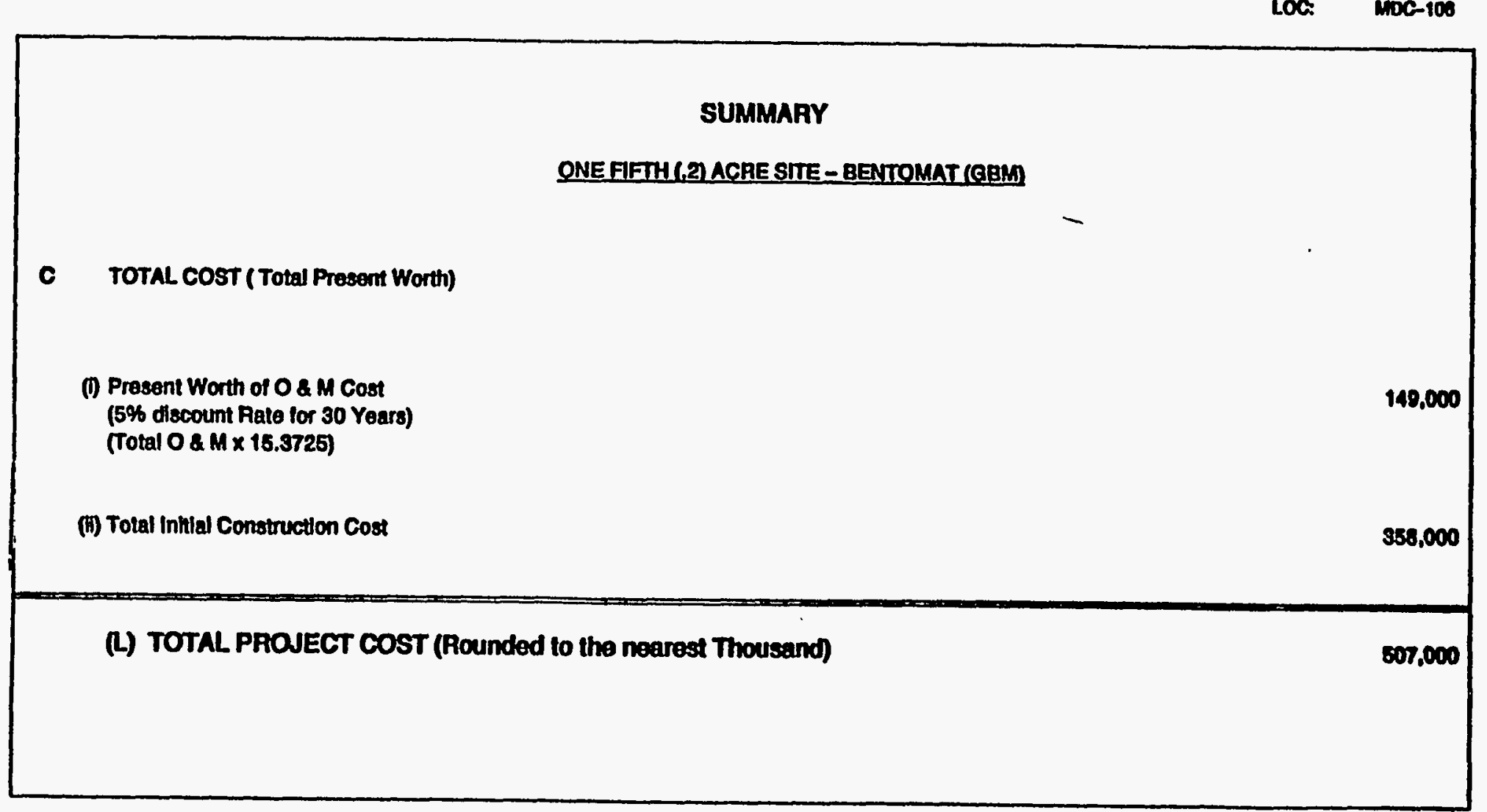

B-2. Cost Estimate for a Cover System Utilizing Bentomat $($ and a 30-mil FML (Cont'd) 
DETAIL ESTIMATE

$1001: 7-03$

ACTIVITY CODE: ESE260412

PROJ.NAME: ECON. EVALUATION OF CLOSURB CAP BARRIRR MATERIAL.

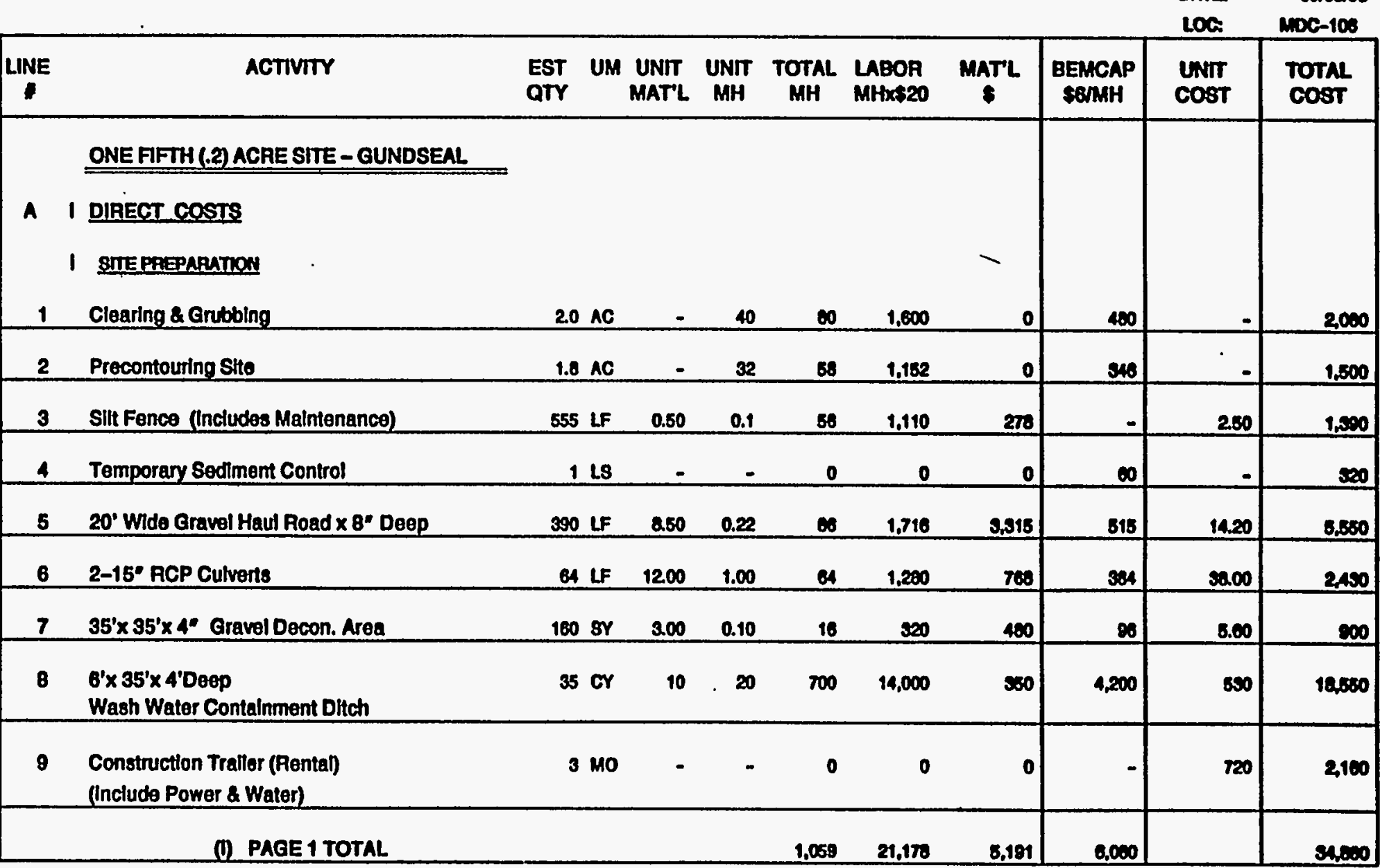

B-3. Cost Estimate for a Cover System Utilizing Gundseal@e 
DETAIL ESTIMATE

L001: 7-03

ACTIVITY CODB: ESE260112

PROJ.NAME: ECON. BVALUATTON OP CLOSURB CAP BARRRER MATENUAL

\section{CHKD BY: 8. MEAD}

EgTOQY: Pannk

PHONE 7-5200

DATE Dorowsos

LOC. MDC-100

\begin{tabular}{|c|c|c|c|c|c|c|c|c|c|c|c|}
\hline LNE & ACTIVITY & $\begin{array}{l}\text { EST } \\
\text { QTY }\end{array}$ & UM & UNIT & UNT & TOTAL & $\begin{array}{l}\text { LABOR } \\
\text { MHX } \$ 20\end{array}$ & $\underset{\xi}{\operatorname{MaTL}}$ & $\underset{B \in R M H}{\text { BEMCAP }}$ & $\begin{array}{l}\text { UNIT } \\
\text { COST }\end{array}$ & $\begin{array}{l}\text { TOTAL } \\
\text { Cost }\end{array}$ \\
\hline & I COVER CONSTRUCTION & & & & & & & & & & \\
\hline 10 & Waste Stabillzation (Crushed Limestone) & 400 & $\mathrm{TN}$ & 100 & 0.1 & 40 & 600 & 40,000 & 240 & 102.60 & 41,040 \\
\hline 11 & $\begin{array}{l}\text { Place and Compact Foundation Soll } \\
\text { in } 8^{\circ} \text { Lifts to } 95 \% \text { STD Proctor }\end{array}$ & 1.614 & or & - & 0.175 & 282 & 5,649 & $=0$ & 1,605 & 4.50 & 7,340 \\
\hline 12 & $\begin{array}{l}\text { Placement of Gundseal Geomembrane/ } \\
\text { Bentonite Mat }\end{array}$ & 12,320 & SF & 0.50 & 0.01 & 128 & 2,484 & 0,100 & - & 0.70 & 8,600 \\
\hline 19 & $\begin{array}{l}\text { Placement of 1'-0 Coarse Sand } \\
\text { Drainage Layer. }\end{array}$ & 425 & or & 10.50 & 0.035 & 15 & 200 & 4,400 & $\boldsymbol{\theta}$ & 11.40 & 4.600 \\
\hline 14 & $\begin{array}{l}\text { Placement of Geotextile (TYPAP) Filter on } \\
\text { I'-0" Coarse Sand Drainage Layer }\end{array}$ & 1,402 & sy & 0.20 & 0.007 & 10 & 198 & 280 & - & 0.30 & 400 \\
\hline 15 & Placement of 1'-6" Cormmon Fill & 405 & or & 3.75 & 0.07 & 34 & 679 & 1.010 & 2001 & 8.00 & 2,700 \\
\hline 16 & Placement of $6^{*}$ Topsoil & 162 & or & 0.50 & 0.09 & 10 & 320 & 1.720 & $\phi$ & 11.00 & 2.150 \\
\hline 17 & $\begin{array}{l}\text { Placement of Pertmeter Exterlor } \\
\text { Oralnaga Layer } 3 / 4^{*} \times 3^{*} \text { OP (Crushed Stone) }\end{array}$ & 143 & sy & 1.50 & 0.24 & 34 & 608 & 215 & 200 & 7.80 & 1,110 \\
\hline 18 & Excavation of Trapezoldal Drainage Ditch & 88 & or & - & 0.20 & 18 & 352 & 0 & 100 & 8.20 & 400 \\
\hline & (1) PAGE 2 TOTAL & & & & & 575 & 11,452 & 54,005 & 2,607 & & $\infty, \pi$ \\
\hline
\end{tabular}

B-3. Cost Estimate for a Cover System Utilizing Gundseal@ (Cont'd) 
D B T A I L B TIMAT B

CHKD BY: 8. MEAD

Lod : $7-03$

ACTIVITY CODE: BSE260412

PROJ.NAME: ECON. EVALUATION OF CLOSURE CAP BARUIRR MATERIAL.

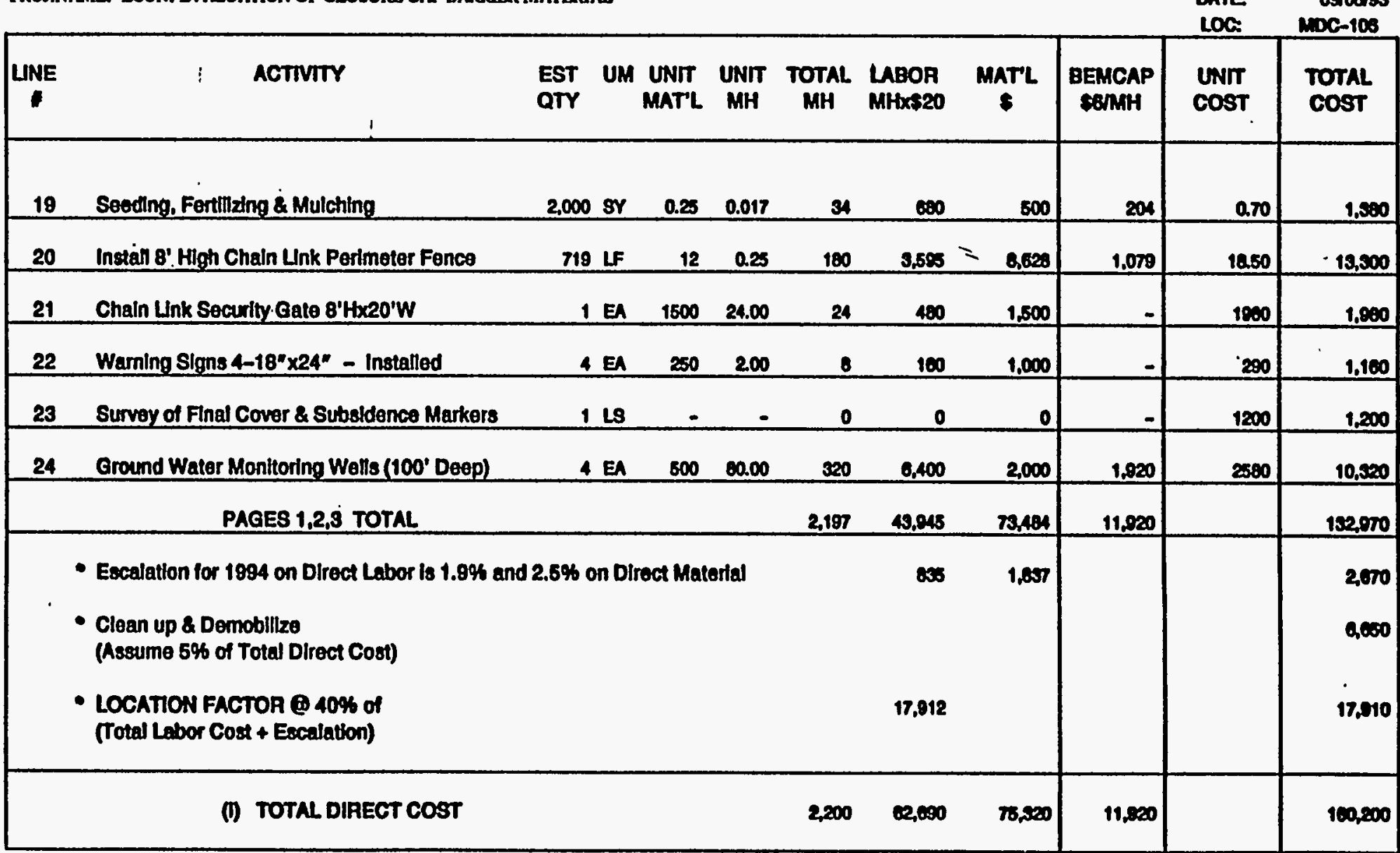

B-3. Cost Estimate for a Cover System Utilizing Gundseal(Cont'd) 
D B T A I L B S TIMATE

CHK'D BY: 8. MEAD

Loof : $7-03$

ACTIVITY CODB: ESE260112

PROJ.NAME: ECON. EVALUATION OF CLOSURB CAP BARRRER MATERHAL

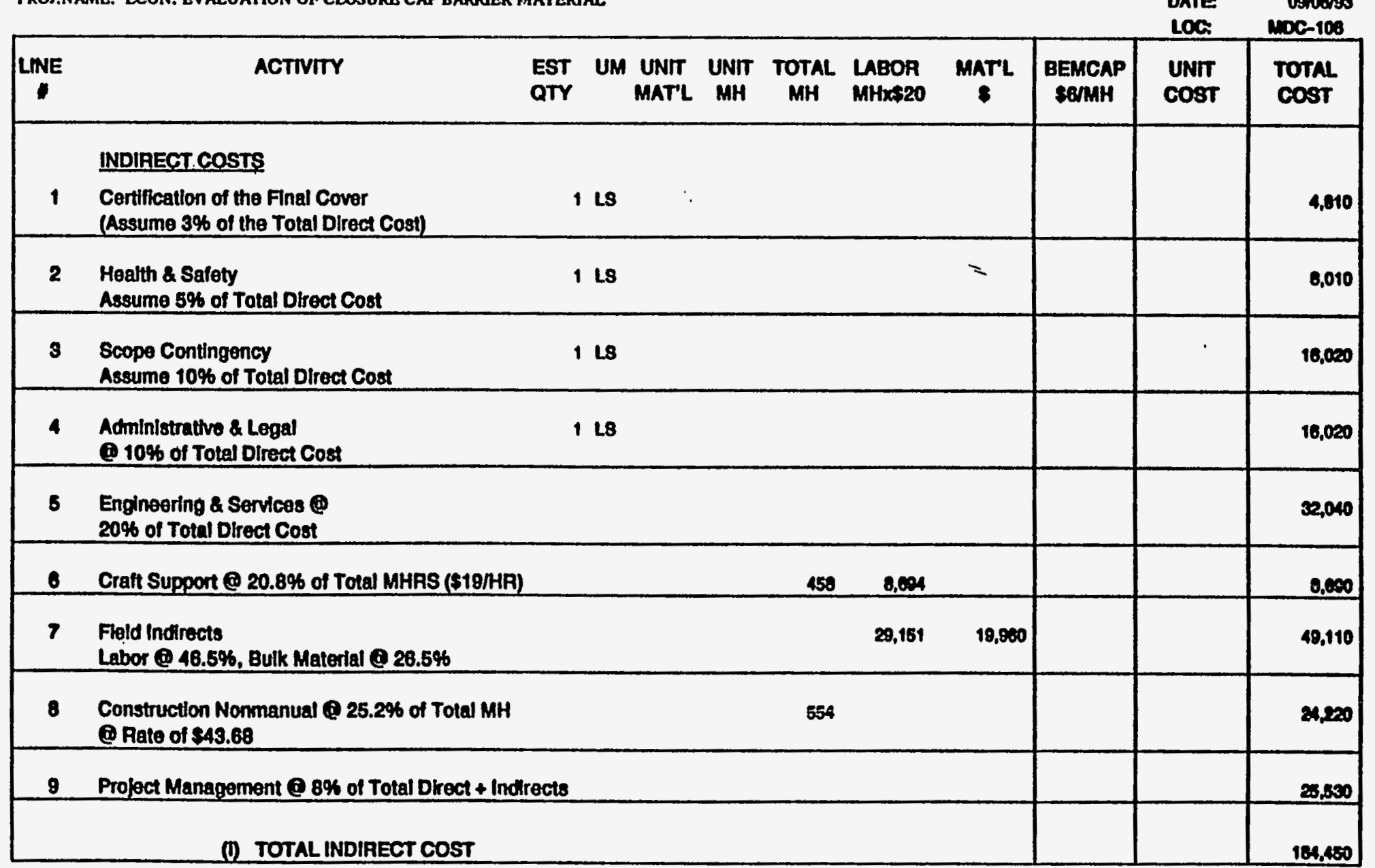

B-3. Cost Estimate for a Cover System Utilizing Gundseal@ (Cont'd) 
Loo I: $7-03$

ACTIVITY CODB: ESE260412

PROJ.NAME: BCON. EVALUATION OF CLOSURB CAP BARRIER MATERIAL.

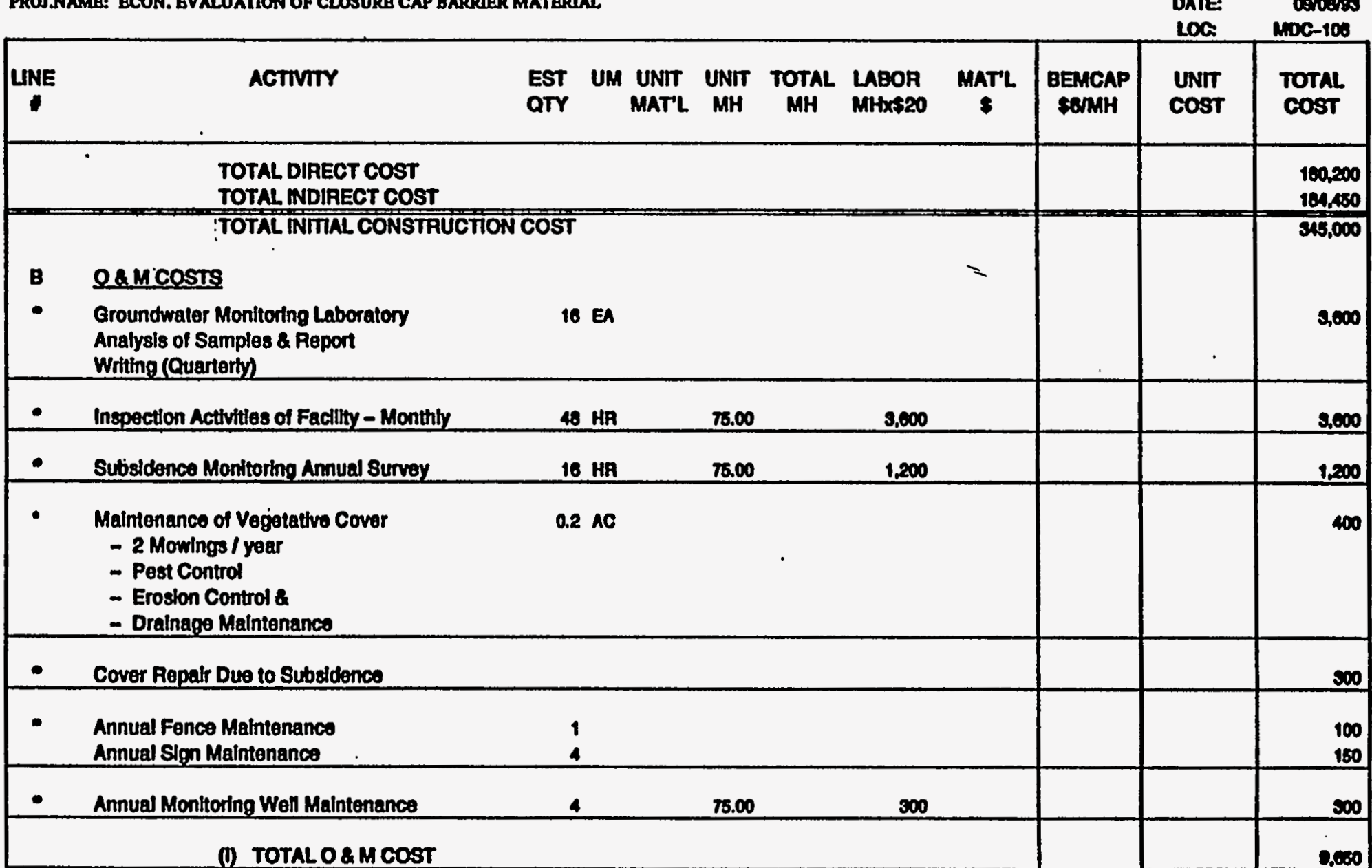

B-3. Cost Estimate for a Cover System Utilizing Gundsealo (Cont'd) 
D B TAIL BSTIMATB

L001: 7-03

ACTIVTY CODE: ESE26ON12

TROJ.NAME: ECON. BVALUATTON OR CLOSURB CAP BARRIER MATERIAL
CHK'D BY: 8. MEAD

ESTOBY:

PHONE:

DATE

Perme

$7-5200$

0000000

LOC: MDC-100

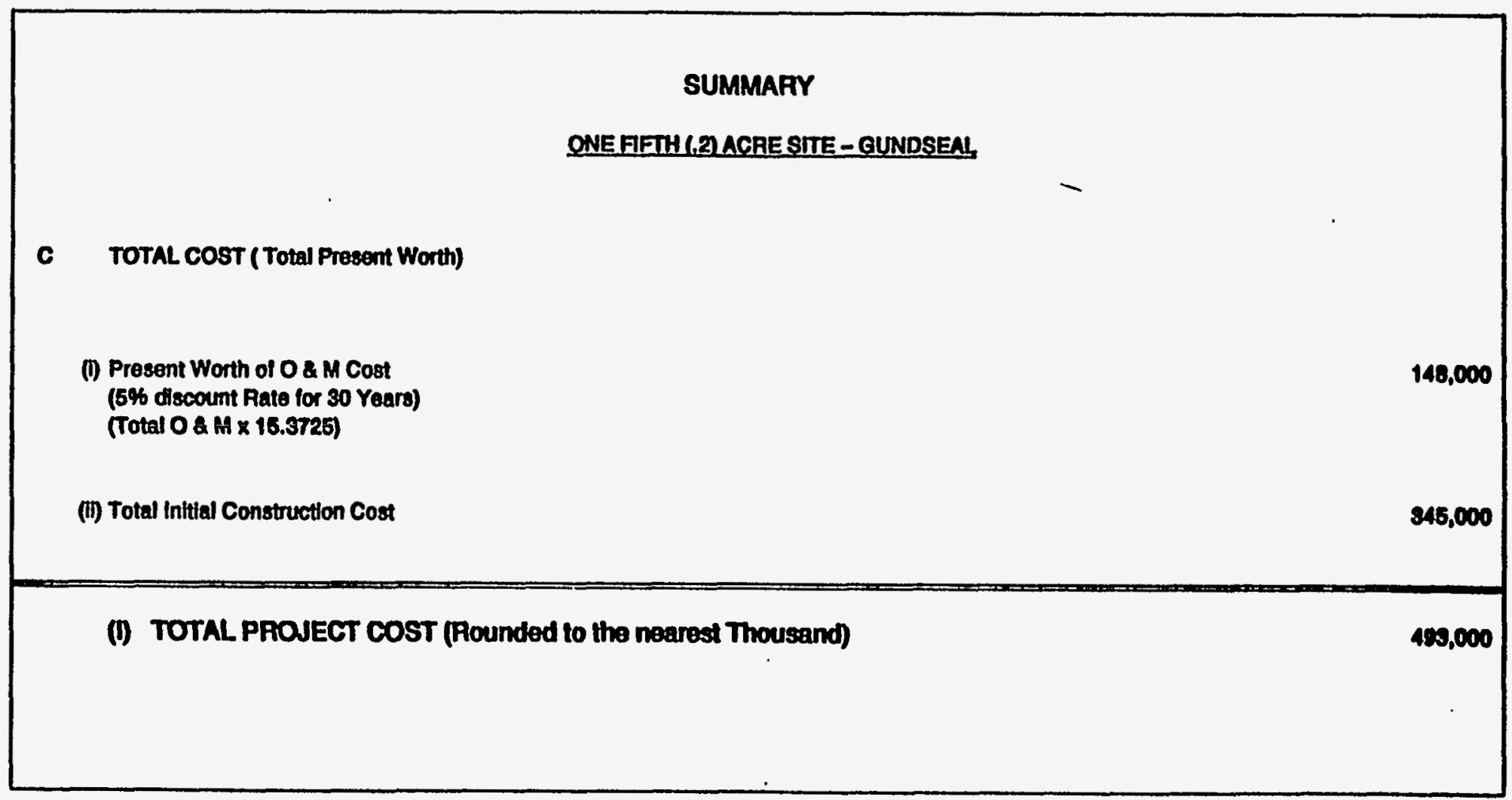

B-3. Cost Estimate for a Cover System Utilizing Gundseal@ (Cont'd) 
DETAIL ESTIMATE

CHKD BY: 8. MEAD

$200 \%: 7-03$

ACTIVTYY CODB: ESB260412

PROS.NAME: ECON. EVALUATTON OF CLOSURE CAP BARRIER MATERIAL

sto or: Pemux

PHONe 7-5200

DATE Covens

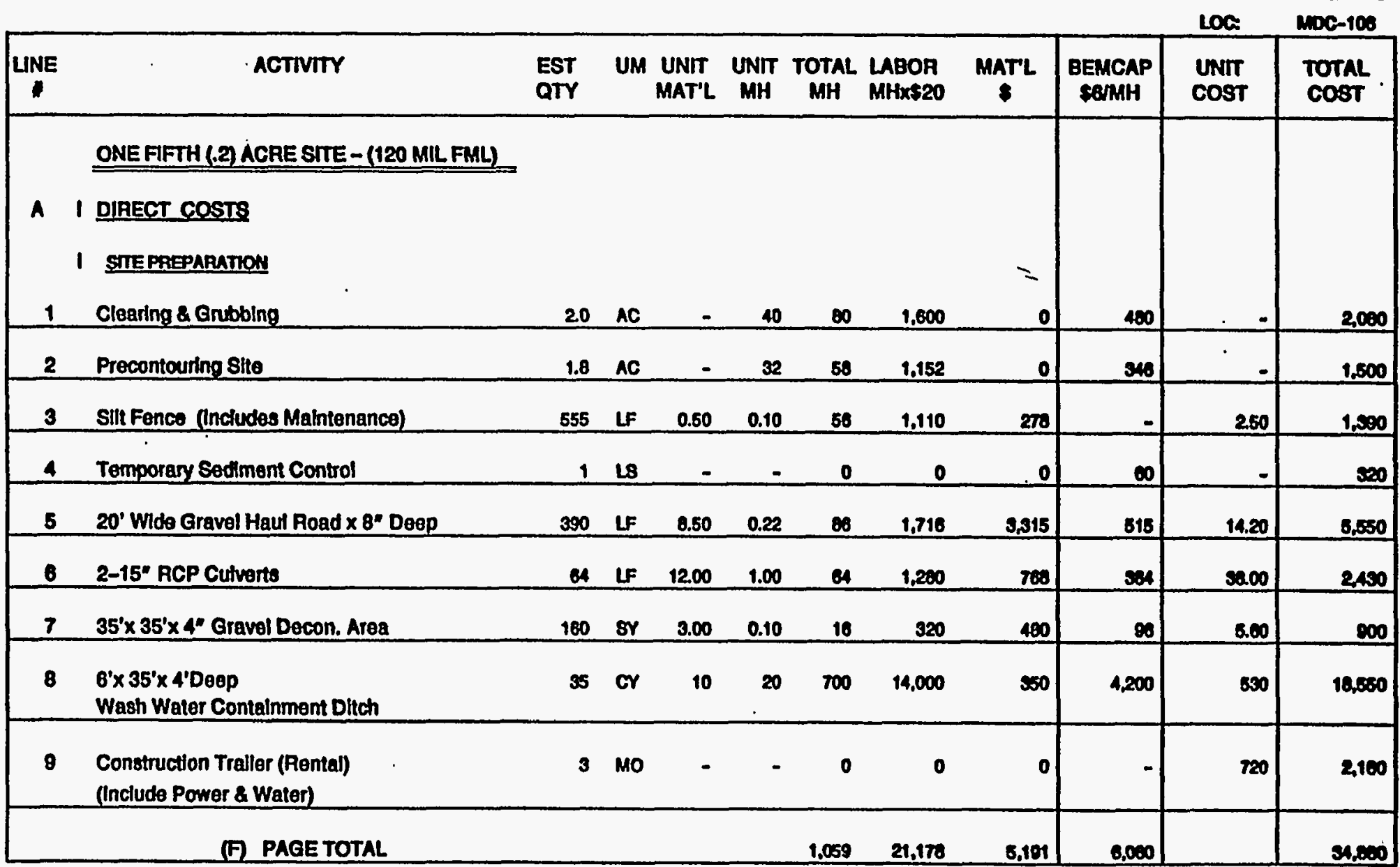

B-4. Cost Estimate for a Cover System Utilizing 120-mil High Density Polyethylene (HDPE) Liner 


\section{DETAIL ESTIMATE CHKDBY: 8.MEAD}

Lo01: $7-03$

ACTIVITY CODB: BSE200N12

PROJ.NAME: BCON. EYALUATTON OF CLOSURB CAP BARRIER MATERAAL

ESTOEY: PankK

PHONE: 7-5260

\begin{tabular}{|c|c|c|c|c|c|c|c|c|c|c|c|}
\hline & & & & & & & & & & LOG. & MDC-100 \\
\hline LINE & ACTIVITY & $\begin{array}{l}\text { EST } \\
\text { QTY }\end{array}$ & UM & $\begin{array}{l}\text { UNIT } \\
\text { MAT'L }\end{array}$ & $\underset{M H}{\text { UNIT }}$ & $\underset{\text { MH }}{\text { TOTAL }}$ & $\begin{array}{l}\text { LABOR } \\
\text { MHX\$20 }\end{array}$ & $\underset{8}{\operatorname{MATL}}$ & $\begin{array}{l}\text { BEMCAP } \\
\text { \$6/MH }\end{array}$ & $\begin{array}{l}\text { UNIT } \\
\text { COST }\end{array}$ & $\begin{array}{l}\text { TOTAL } \\
\cos \end{array}$ \\
\hline & COVER CONSTRUCTON & & & & & & & & & & \\
\hline 10 & Waste Stabilization (Crushed UImestone) & 100 & $\mathrm{TN}$ & 100 & 0.1 & 40 & 800 & 40,000 & 240 & 102.00 & 41,040 \\
\hline 11 & $\begin{array}{l}\text { Piace and Compact Foundation Soll } \\
\text { in 8" Utits to 95\% STD. Proctor }\end{array}$ & 1,614 & or & - & 0.175 & 282 & 5,649 & 0 & 1,005 & 4.50 & 7,340 \\
\hline 12 & $\begin{array}{l}\text { Placement of } 120 \text { Mil Fiexible } \\
\text { Membrane Liner }\end{array}$ & 12,320 & $\mathbf{S F}$ & 0.50 & 0.01 & 123 & 2,464 & 0,100 & - & 0.70 & 8,620 \\
\hline 13 & $\begin{array}{l}\text { Placement of 1'-0 Coarse Sand } \\
\text { Drainege Layer }\end{array}$ & 425 & or & 10.50 & 0.035 & 15 & 290 & 4,460 & $\boldsymbol{\infty}$ & 11.40 & 4,050 \\
\hline 14 & $\begin{array}{l}\text { Placement of Geotextlle (TYPAR) Filter on } \\
1^{1}-0^{*} \text { Coarse Sand Drainage Layer }\end{array}$ & 1,402 & 8r & 0.20 & 0.007 & 10 & 198 & 200 & - & 0.30 & 400 \\
\hline 15 & Placement of 1'-6" Common Fill & 485 & or & 3.75 & 0.07 & 34 & 679 & 1,010 & 2004 & 5.00 & 2,700 \\
\hline 16 & Placement of 6" Topsoll & 182 & Cr & 0.50 & 0.00 & 16 & 320 & 1,720 & $\infty$ & 18.00 & 2.100 \\
\hline 17 & $\begin{array}{l}\text { Placement of Perimeter Exterlor } \\
\text { Dralnage Layer } 34^{*} \times 3^{\prime \prime} \text { Stone }\end{array}$ & 149 & $\underset{g Y}{s y}$ & 1.50 & 0.24 & 34 & 600 & 216 & 200 & 7.00 & 8.110 \\
\hline 18 & Excavation of Trapozoldal Drainage Ditch & 80 & or & - & 0.2 & 16 & 302 & 0 & 100 & 5.20 & 400 \\
\hline & (1) PAOE TOTAL & & & & & 573 & 11.452 & 84,000 & 2007 & & $\infty, \pi \infty 0$ \\
\hline
\end{tabular}

B-4. Cost Estimate for a Cover System Utilizing 120-mil High Density Polyethylene (HDPE) Liner (Cont'd) 
D B T A I L B S T I M A T B

CHK'D BY: S. MEAD

Loof: $7-03$

ACTIVITY CODE: BSE2SO412

PROJ.NAME: ECON. EVALUATTON OF CLOSURB CAP BARRIFR MATERIAL

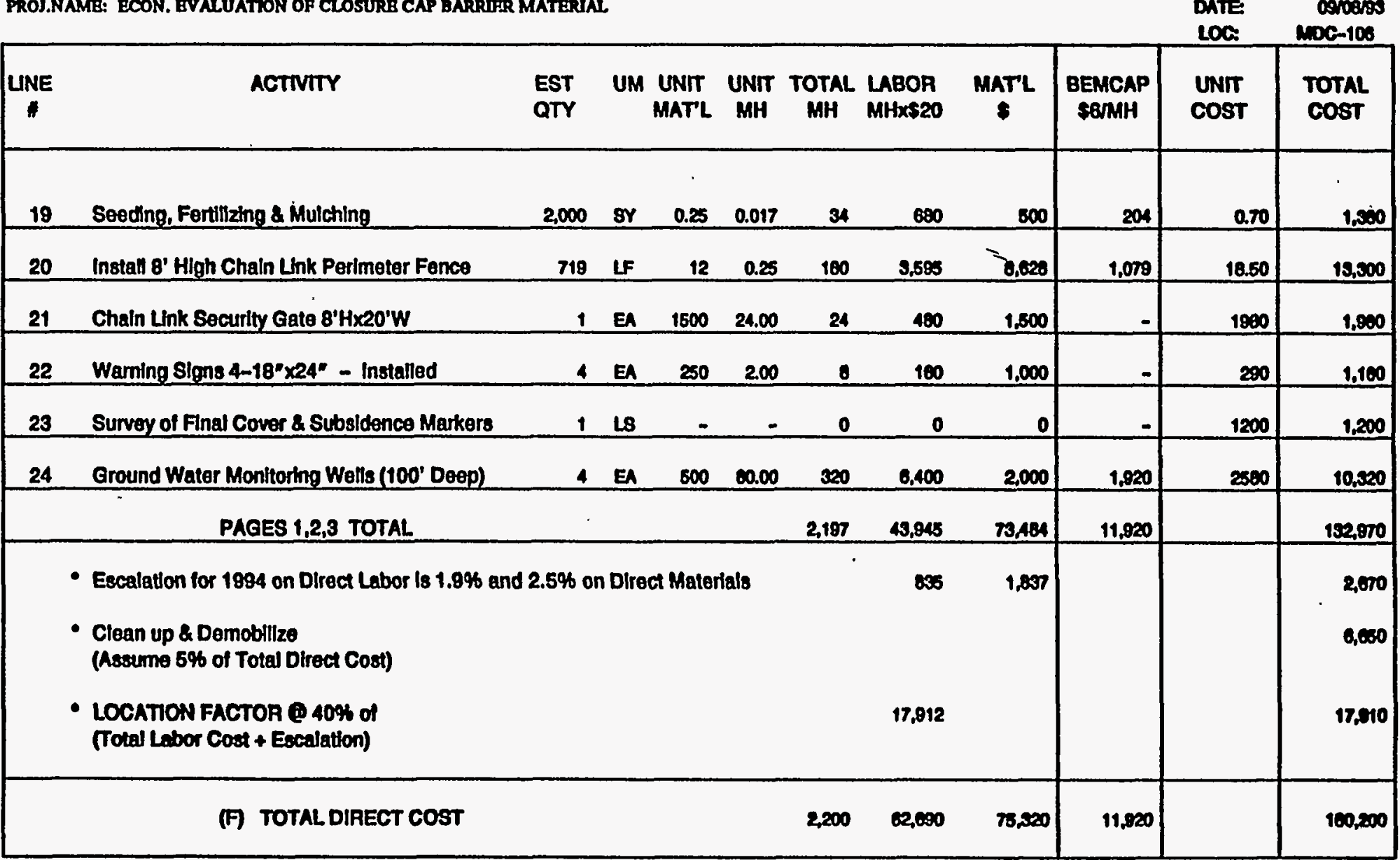

B-4. Cost Estimate for a Cover System Utilizing 120-mil High Density Polyethylene (HDPE) Liner (Cont'd) 
D B T A I L B S T I M A B

CHK'D BY: 8. MEAD

Loa I: $7-03$

ACTIVITY CODE: BSE26ON12

PROJ.NAME: ECON. EVALUATION OF CLOSURB CAP BARRRER MATERIAL

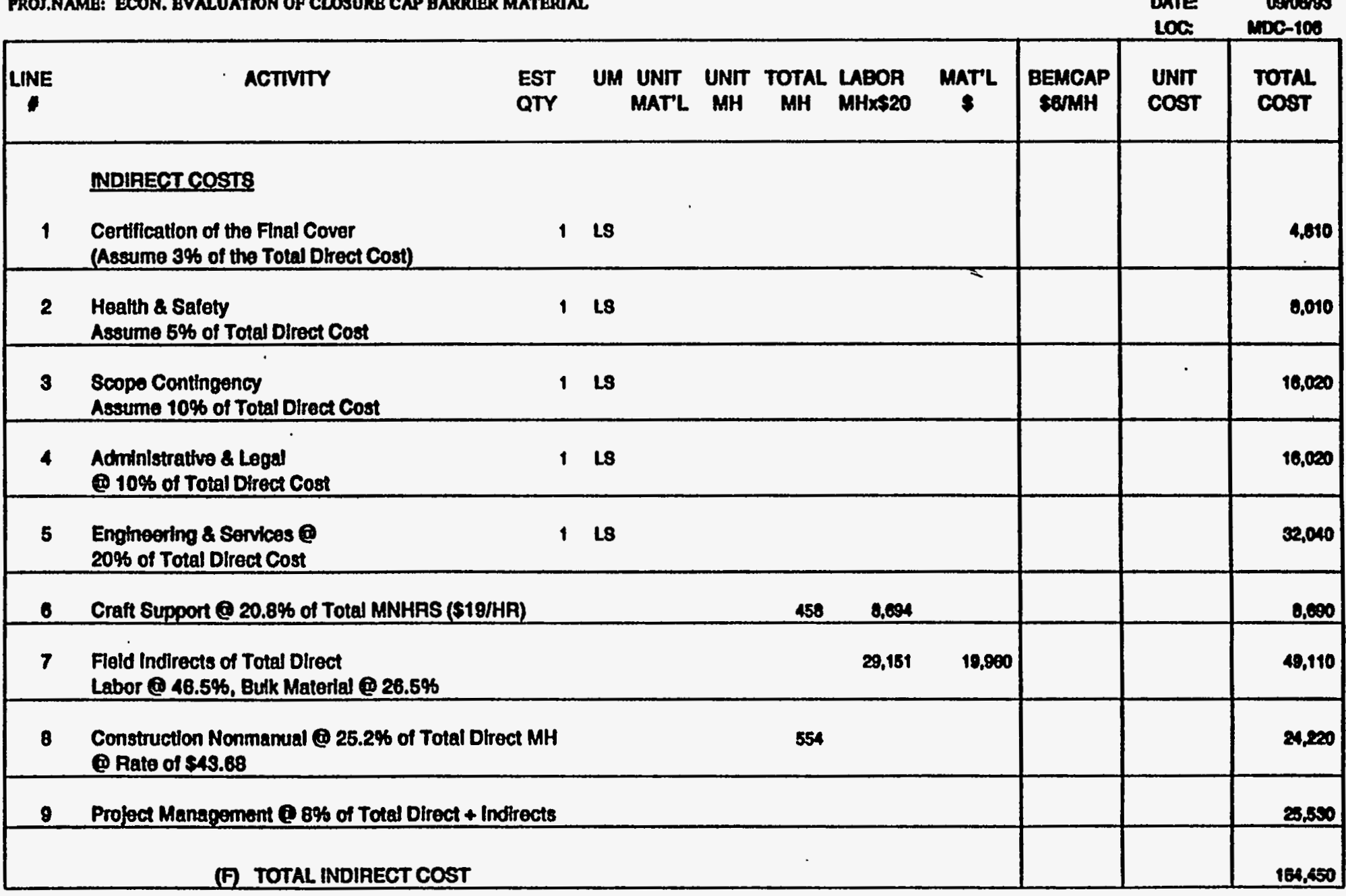

B-4. Cost Estimate for a Cover System Utilizing 120-mil High Density Polyethylene (HDPE) Liner (Cont’d) 
D B T A L B S T IMAT B

CHKD BY: 8. MEAD

$100 *$ : 7-0s

ACTIVITY CODE: BSE26O112

PROJ.NAME: ECON. EVALUATION OF CLOSURB CAP BARRIER MATERIAL.

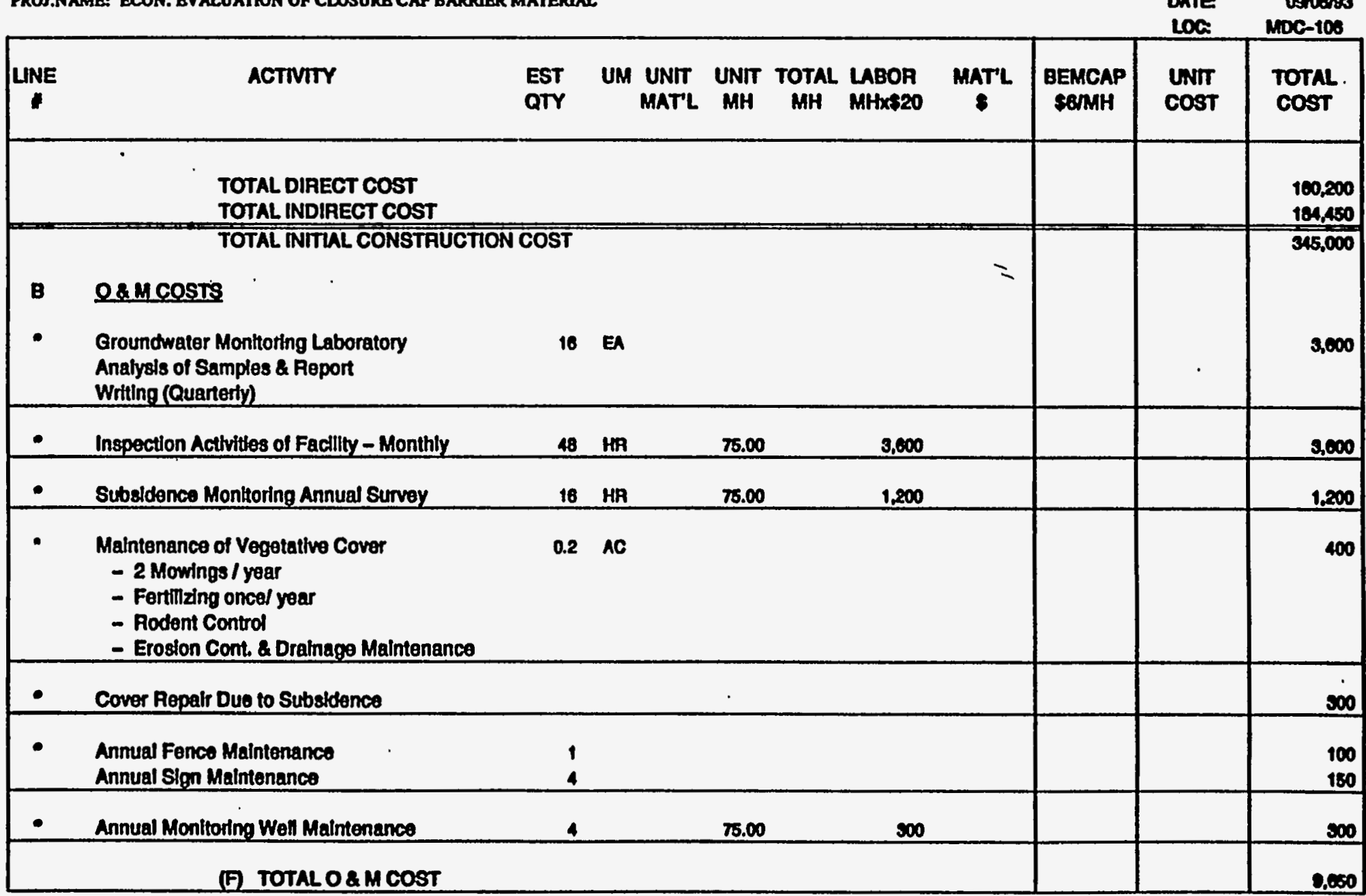

B-4. Cost Estimate for a Cover System Utilizing 120-mil High Density Polyethylene (HDPE) Liner (Cont'd) 
D BTAIL BSTIMAT B

Lool: $7-03$

ACTIVITY CODB: BSE200412

PROS.MAME: BCON. EVALUATTON OP CLOSURB CAP BARRIER MATERIAL
CHKD BY: 8. MEAD

EsToBr:

PHONE:

DATE

LOC.

Pank

$7-6200$

menoms

MDC-100

\section{SUMMARY}

ONE FIFTH (1.2) ACRE SITE - (120 MIL, FML)

C TOTAL COST (Total Present Worth)

(i) Present Worth of $0 \& \mathrm{M}$ Cost

(5\% discount Rato for 30 Yoars)

(Total O \& M X 16.3725)

(ii) Total Inttial Construetion Cose

348,000

(F) TOTAL PROJECT COST (Rounded to tha nearest Thousentit)

409,000

B-4. Cost Estimate for a Cover System Utilizing 120-mil High Density Polyethylene (HDPE) Liner (Cont'd) 
DETAIL ESTIMATE

Lo0: $7-03$

ACTIVITY CODB: ESER6ON12

PROJ.NAME: BCON. BVALUATION OR CLOSURB CAP DARRIER MATERIAL

CHKO BY: 8. MENO

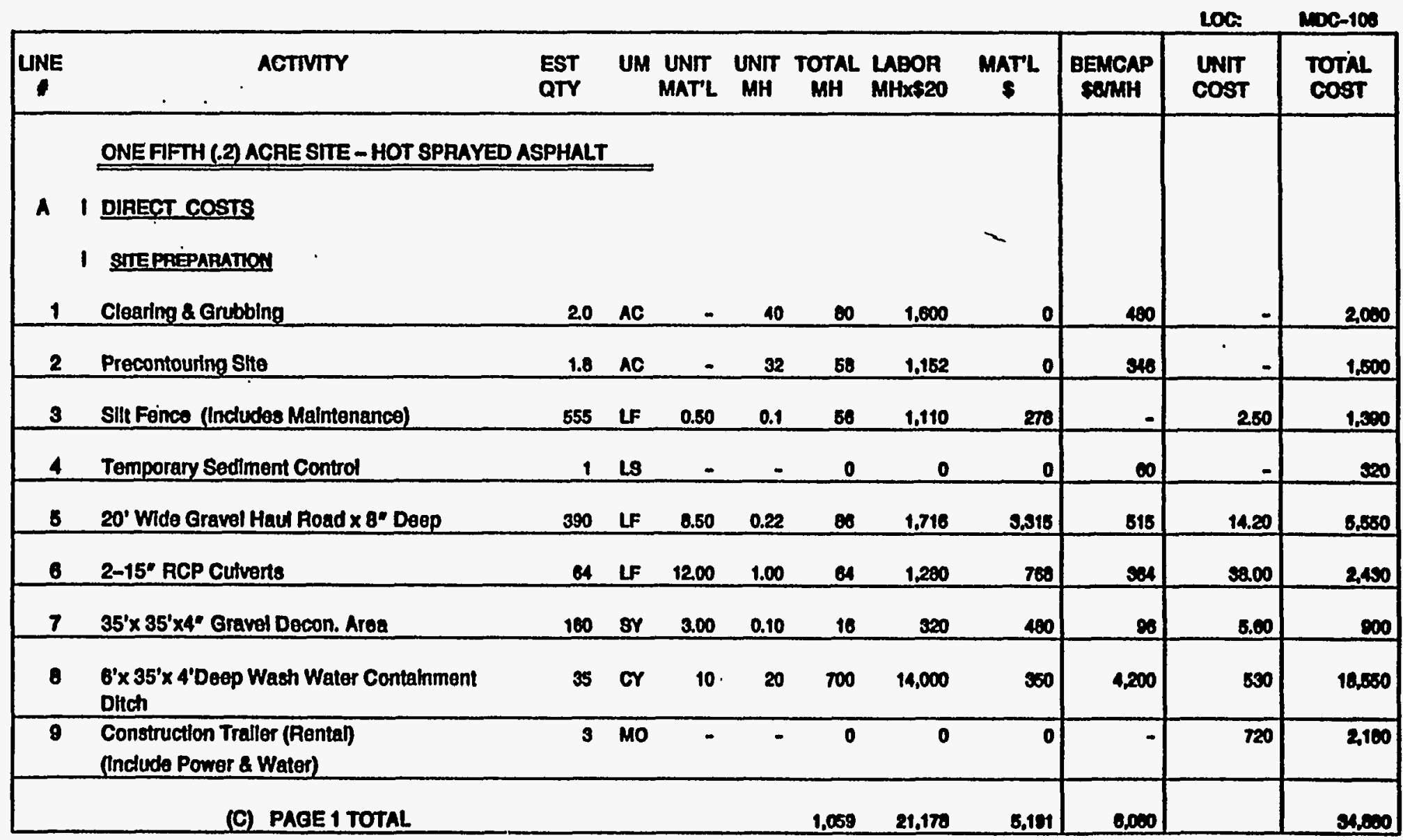

B-5. Cost Estimate for a Cover System Utilizing 1/4-inch Thick Hot Sprayed or Emulsified Asphalt 
Loof: 7-03

ACTIVITY CODE: ESE2SOA12

PROJ.NAMB: ECON. EVALUATTON OF CLOSURE CAP BARRIER MATERIAL

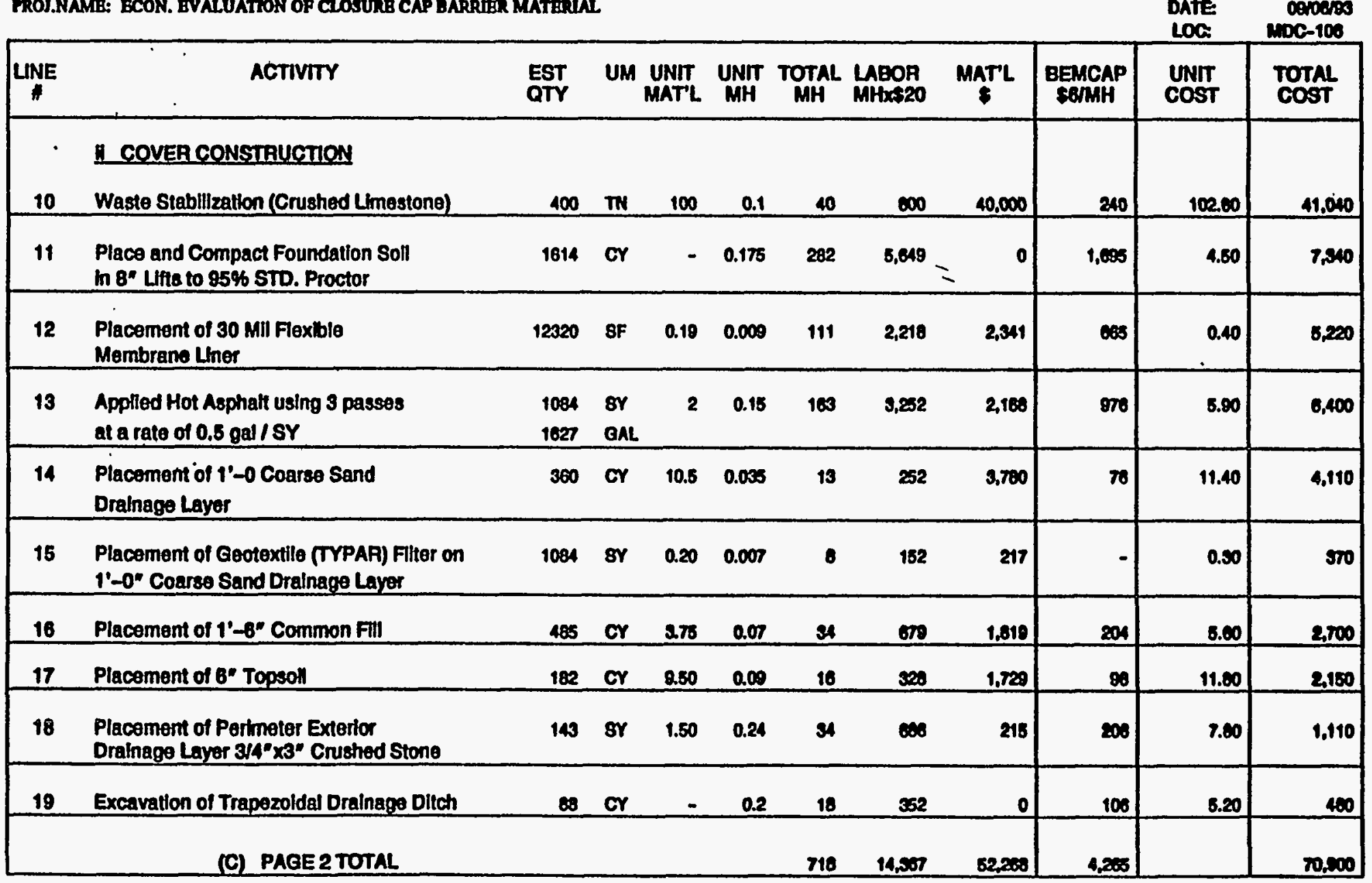

B-5. Cost Estimate for a Cover System Utilizing 1/4-inch Thick Hot Sprayed or Emulsified Asphalt (Cont'd) 


\section{Lo0 $1: 7-\infty$}

ACTIVITY CODE: ESE2CON12

PROJ.NAME: ECON. BVALUATTON OF CLOSURB CAP BARRUPR MATRRIAL

ETrD QY: PQRULP

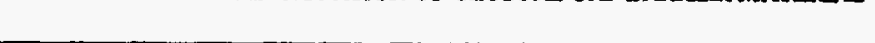

PATE 7-5200

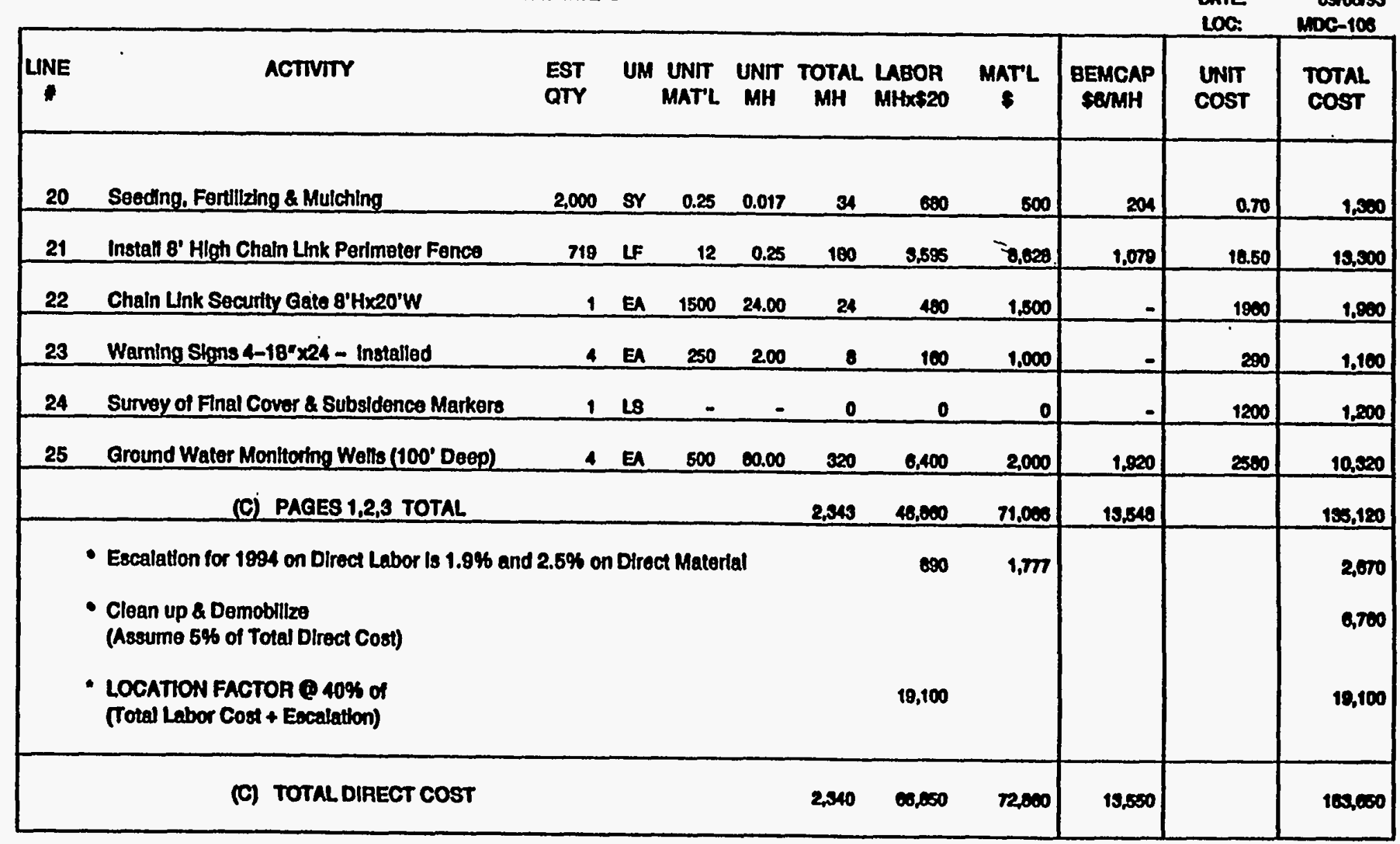

B-5. Cost Estimate for a Cover System Utilizing 1/4-inch Thick Hot Sprayed or Emulsified Asphalt (Cont'd) 
D B T A L B S T IMAT B

CHKD BY: 8. MEAD

Loo I: 7-03

ACTIVTTY CODE: BSE2SOM12

PROJ.NAME: ECON. BYALUATION OF CLOSURB CAP BARRIER MATERUAL.

EsTogr: Panare

PHowe: 7-5806

DATE DONOPS

LOC. MDC-100

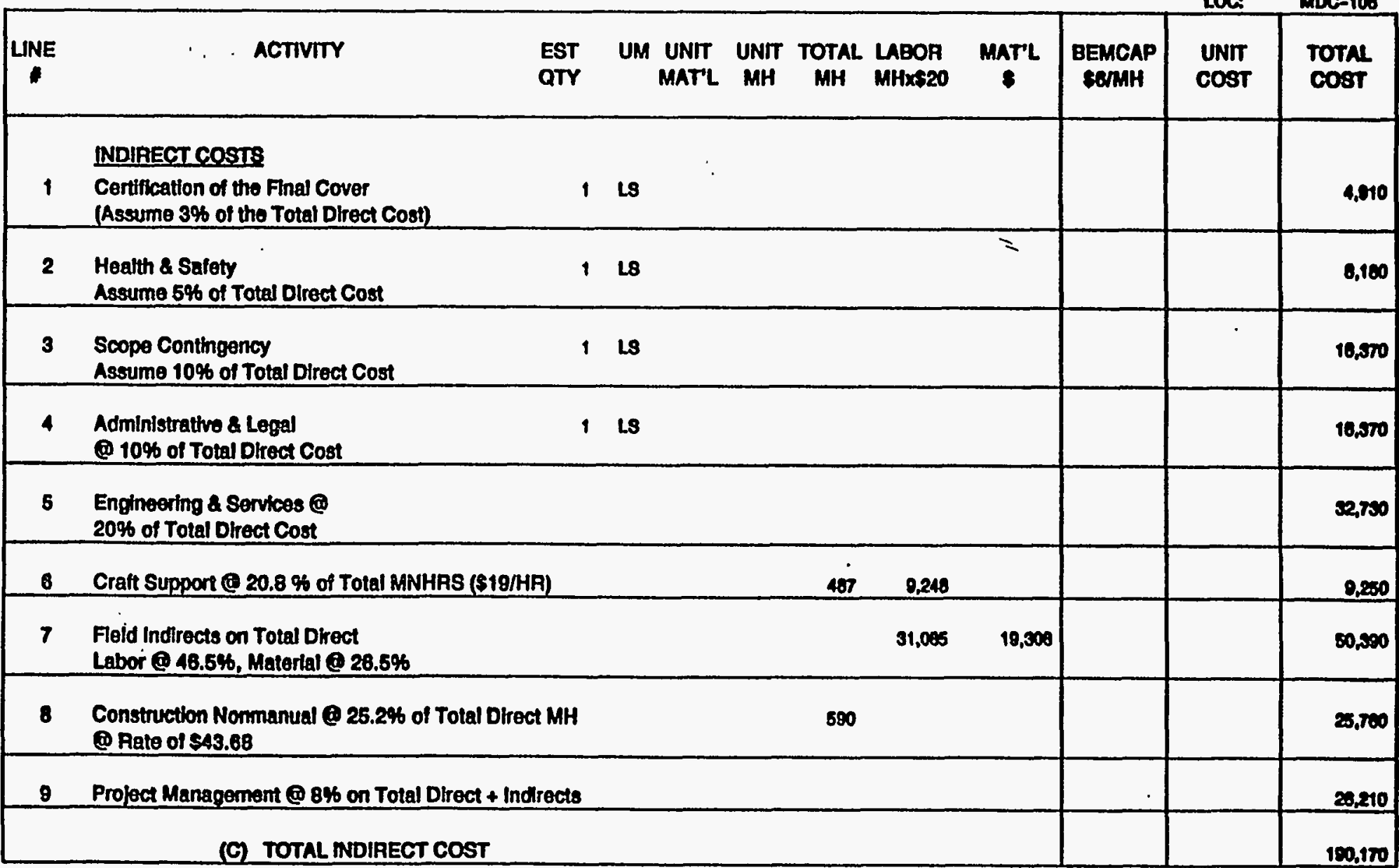

B-5. Cost Estimate for a Cover System Utilizing 1/4-inch Thick Hot Sprayed or Emulsified Asphalt (Cont'd) 
D B T A I L B S T M A T

CHKD BY: 8. MEAD

200 I: $7-03$

ACTIVITY CODE: BSE260412

PROJ.NAME: ECON. EVALUATTON OP CLOSURB CAP BARRIER MATERIAI

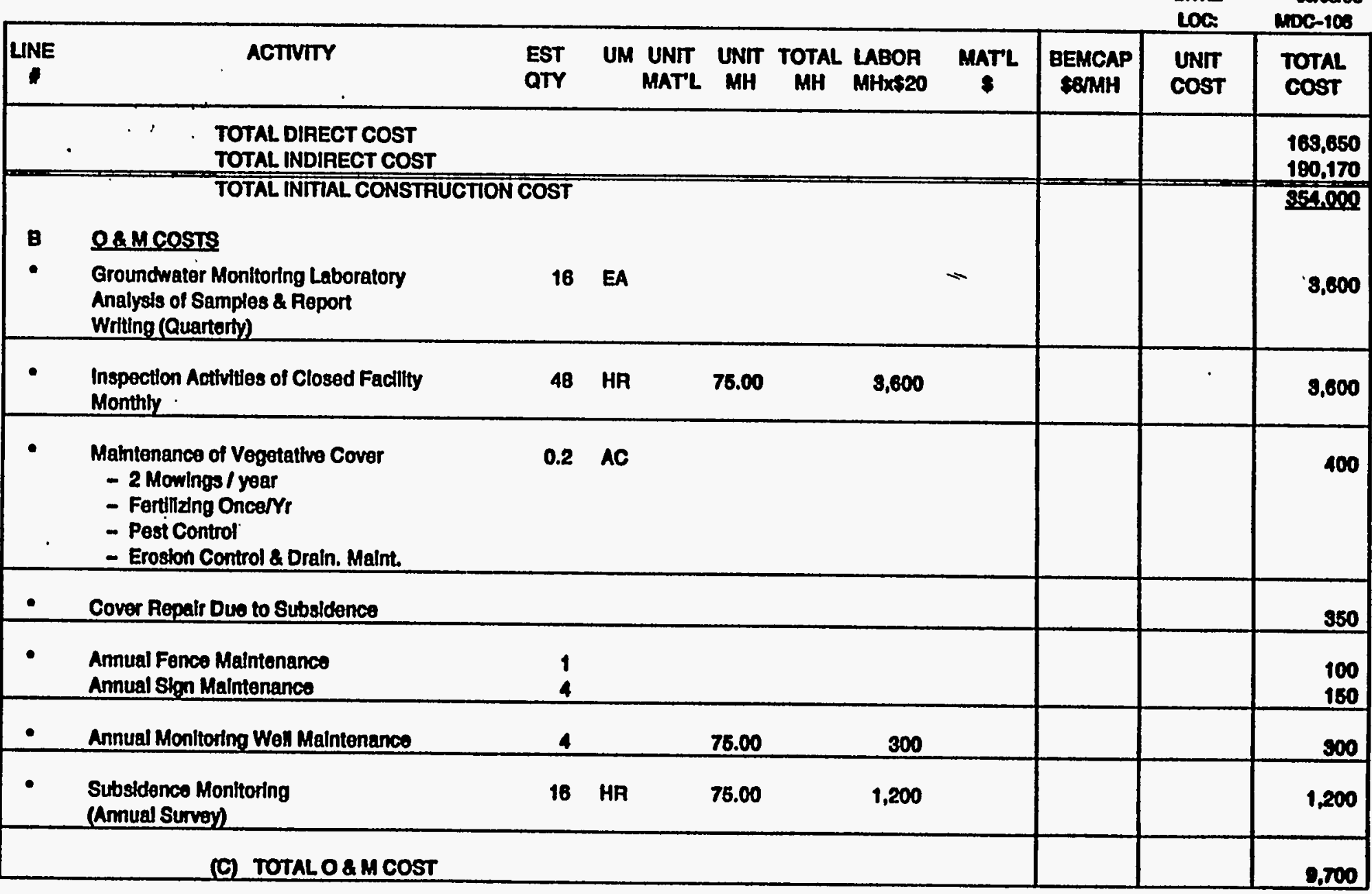

B-5. Cost Estimate for a Cover System Utilizing 1/4-inch Thick Hot Sprayed or Emulsified Asphalt (Cont'd) 
D B T A L B S T I M T B

CHK'D BY: 8. MEND

1001: $7-03$

ACTIVITY CODB: BSE2SOA12

MROU.NAMB: ECON. BVALUATTON OF CLOSURE CAP BARRIER MATERUAL

ESTD BY: TOMSURP
PHONE: $7-2565$
DATE:

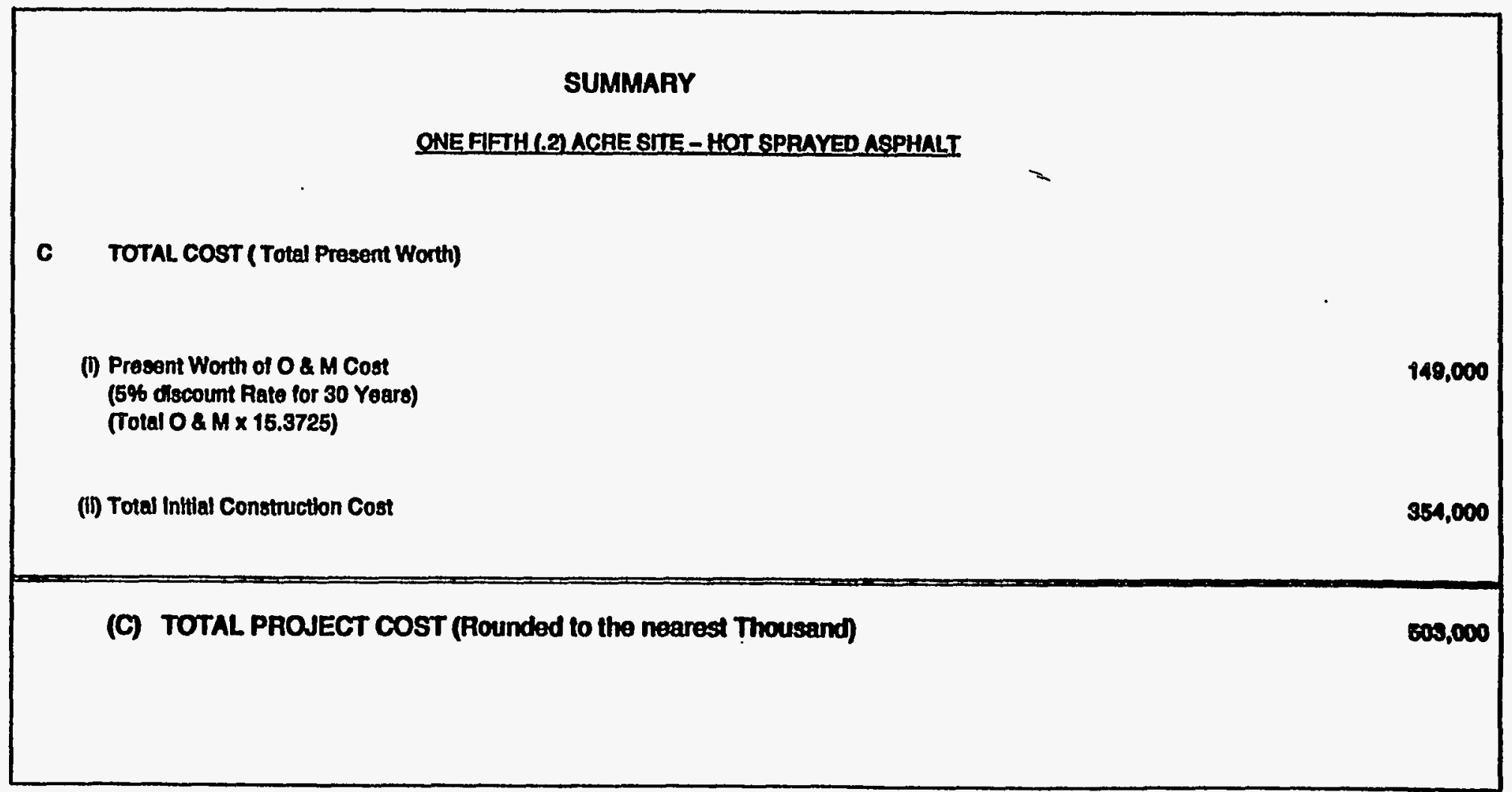

B-5. Cost Estimate for a Cover System Utilizing 1/4-inch Thick Hot Sprayed or Emulsified Asphalt (Cont'd) 
DETAIL ESTIMATE

CHKD BY: 8. MEAD

LOQ : : 7-03

ACTIVITY CODE: ESE260412

PROS.NAME: ECON. OF EVALUATION CLOSURE CAP BARAIER MATERIAL.

EST'D BV:

PHONE

Parma.

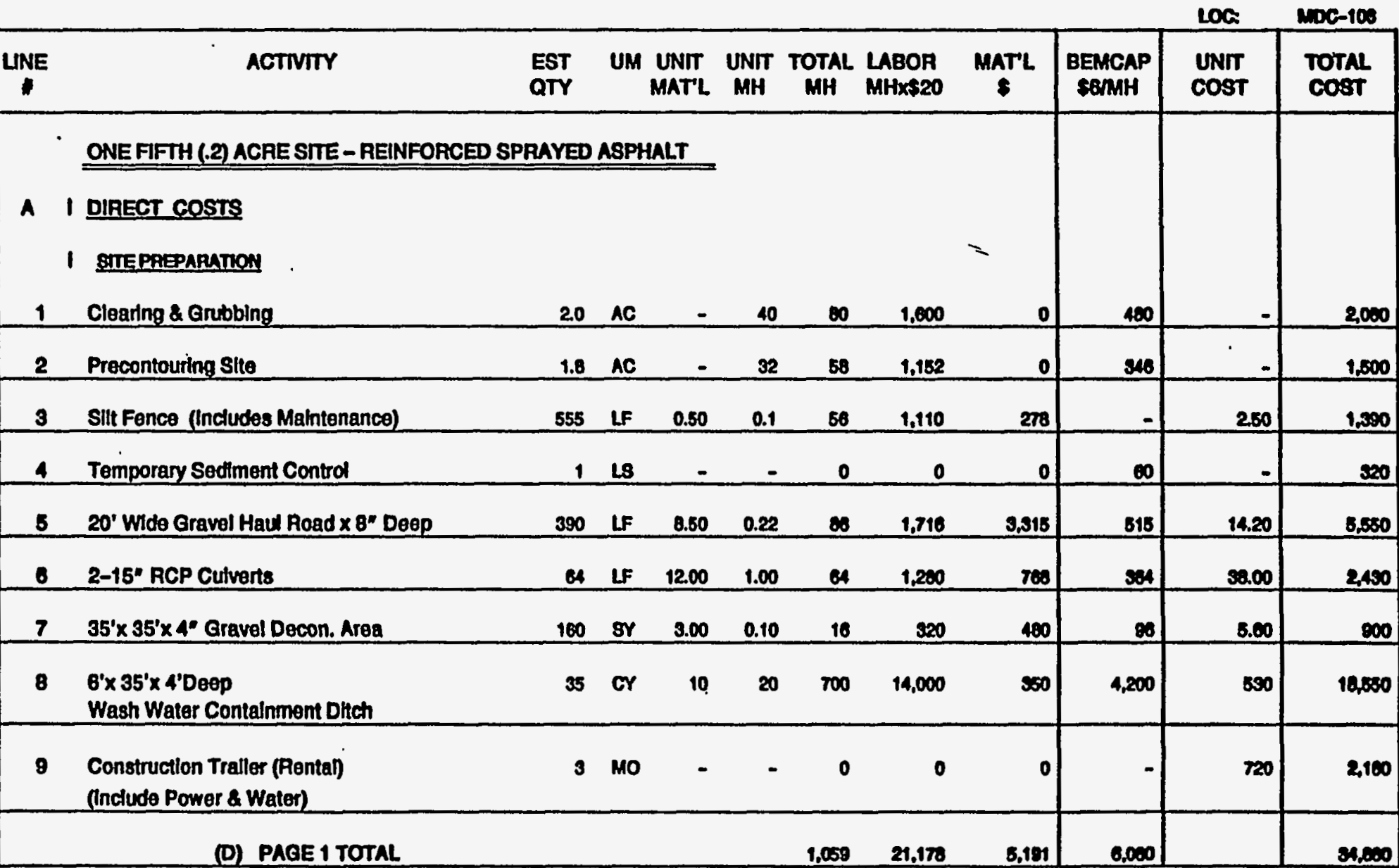

B-6. Cost Estimate for a Cover System Utilizing Reinforced Sprayed Asphalt 


\section{DETAIL ESTIMATE CHKD BY: 8. MEAD}

LOG : : 7-03

ACTIVTY COOE: ESE260412

PAOJ.NAME: ECON. OF EVALUATION CLOSUAE CAP BARAIER MATERIAL

\begin{tabular}{|c|c|c|c|c|c|c|c|c|c|c|c|}
\hline & & & & & & & & & & Loa. & $200-100$ \\
\hline LINE & ACTIVITY & $\begin{array}{l}\text { EST } \\
\text { QTY }\end{array}$ & UM & $\begin{array}{l}\text { UNIT } \\
\text { MAT'L }\end{array}$ & UNIT & $\underset{\text { MHAL }}{\text { TOTAL }}$ & $\begin{array}{l}\text { LABOR } \\
\text { MHXST2O }\end{array}$ & MATL & $\underset{\text { BEMAH }}{\text { BEMCAP }}$ & $\begin{array}{l}\text { UNIT } \\
\text { cost }\end{array}$ & $\begin{array}{l}\text { TOTAL } \\
\text { COST }\end{array}$ \\
\hline & CONER CONSTRUCTION & & & & & & & & & & \\
\hline 10 & Waste Stablization (Crushed Limestone) & 400 & $\mathbf{m}$ & 100 & 0.1 & 40 & 800 & 40.000 & 240 & 102.60 & 41,040 \\
\hline 11 & $\begin{array}{l}\text { Place and Compact Foundation Soll } \\
\text { in } 8 \text { " Lits to } 95 \% \text { STD. Proctor }\end{array}$ & 1,614 & Cr & - & 0.175 & 202 & 8,049 & of & 1,005 & 4.50 & 7,540 \\
\hline 12 & $\begin{array}{l}\text { Placement of Geotextlle Peinforcement } \\
\text { on Foundation Layer. }\end{array}$ & 1,084 & sr & 0.30 & 0.01 & 11 & 217 & $\overline{s e s}$ & - & 0.50 & 840 \\
\hline 13 & $\begin{array}{l}\text { Placement of } 30 \text { Mil Flexible } \\
\text { Membrane Liner }\end{array}$ & 12,320 & SF & 0.18 & 0.009 & 111 & 2,218 & 2,341 & - & 0.40 & 4,500 \\
\hline 14 & $\begin{array}{l}\text { Applled Hot Asphalt using } 3 \text { passes at a rate } \\
\text { of } 0.5 \text { gal / SY for a total of } 1.5 \mathrm{~g} / \mathrm{Sy}\end{array}$ & $\begin{array}{l}1,084 \\
1,627 \\
\end{array}$ & $\begin{array}{l}\text { SY } \\
\text { OAL }\end{array}$ & 2.00 & 0.18 & 103 & 3,252 & 2,168 & 970 & 8.80 & 0,400 \\
\hline 16 & $\begin{array}{l}\text { Placement of 1'-0 Coarse Sand } \\
\text { Drainage Layer }\end{array}$ & 361 & or & 10.50 & 0.035 & 13 & 253 & 0,791 & 76 & 11.40 & 4,120 \\
\hline 16 & $\begin{array}{l}\text { Placement of Geotextile (TYPAR) Filter on } \\
\text { I'-0" Coarse Sand Drainago Layer }\end{array}$ & 1,004 & 8Y & 0.20 & 0.007 & - & 162 & 217 & - & 0.50 & 370 \\
\hline 17 & Placoment of $1^{\prime}-6^{\prime}$ Common FIII & 485 & cr & 3.75 & 0.07 & 34 & 678 & 1,819 & 2004 & 8.60 & 2,700 \\
\hline 18 & Placement of 6" Topsoll & 182 & or & 0.50 & 0.09 & 16 & 220 & 1.720 & $\approx$ & 11.00 & $\mathbf{2 , 1 8 0}$ \\
\hline 18 & $\begin{array}{l}\text { Placement of Perlmeter Exterlor } \\
\text { Drainage Layer } 3 / 4^{4} \times 3^{*} \text { DP (Crushed Stone) }\end{array}$ & 143 & or & 1.50 & 0.24 & 34 & $\infty$ & 215 & 200 & 7.00 & 1,110 \\
\hline 20 & Excavation of Trapezoldal Drainage Ditch & $\infty$ & or & - & 0.20 & 18 & 332 & of & 108 & 8.20 & 400 \\
\hline & (D) PAGETOTAL & & & & & 729 & 14,525 & 52,604 & 3,000 & & 70,700 \\
\hline
\end{tabular}

B-6. Cost Estimate for a Cover System Utilizing Reinforced Sprayed Asphalt (Cont'd) 


\section{DETAIL ESTIMATE}

CHKD BY: 8. MEAD

LOG : : 7-03

ACTIVITY CODE: ESE260412

PROS.NAME: ECON. OF EYALUATION CLOSURE CAP BARAIER MATERIAL

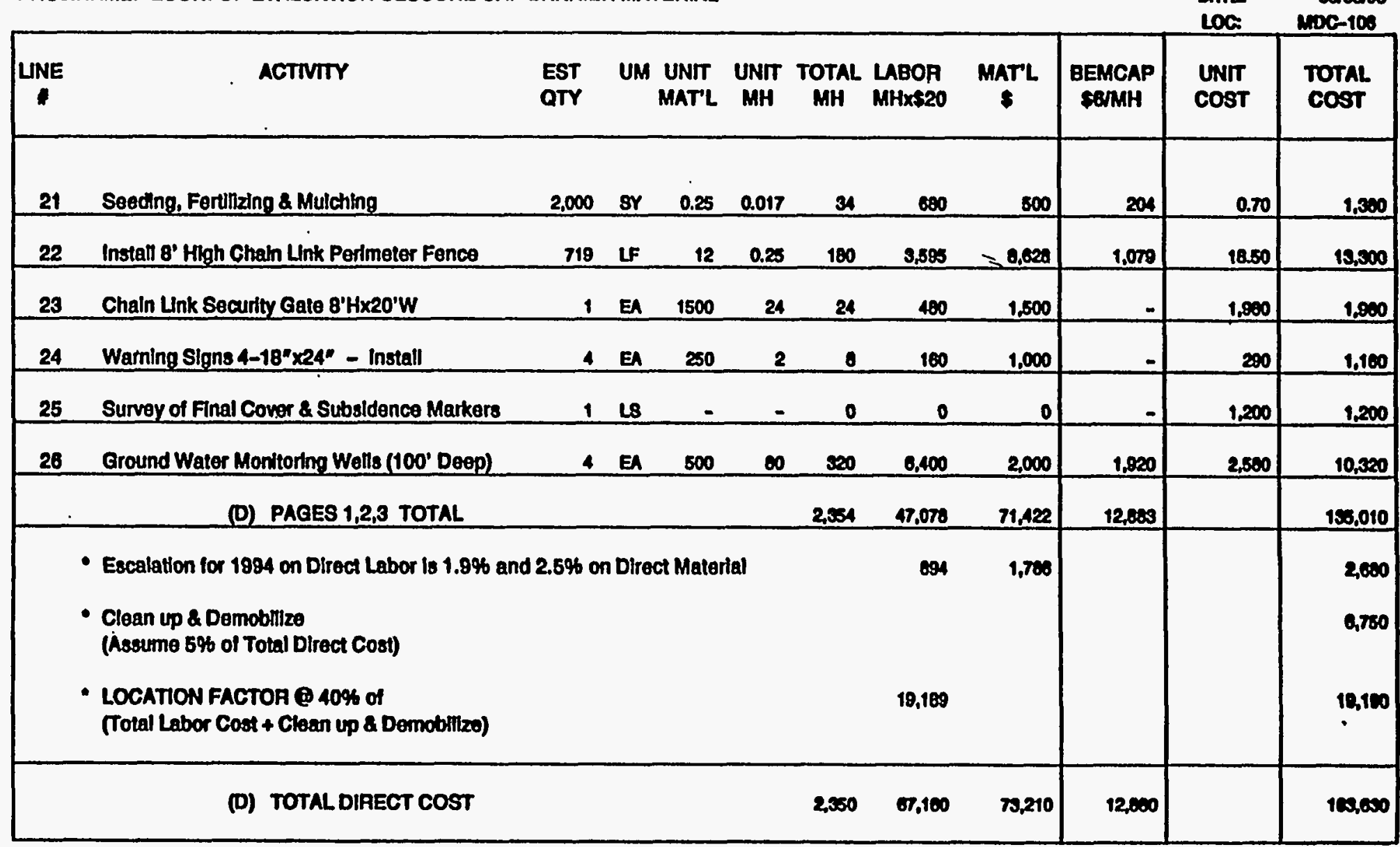

B-6. Cost Estimate for a Cover System Utilizing Reinforced Sprayed Asphalt (Cont'd) 
DETAIL ESTIMATE

CHK'D BY: 8. MEAD

LOG : 7-03

ACTIVITY CODE: ESE260412

PROJ.NAME: ECON. OF EVALUATION CLOSURE CAP BARPIER MATERIAL

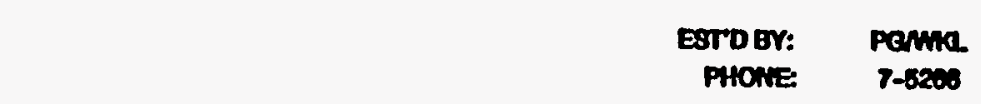

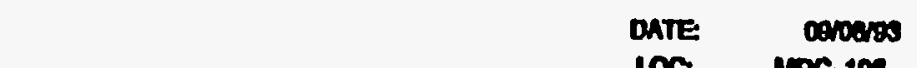

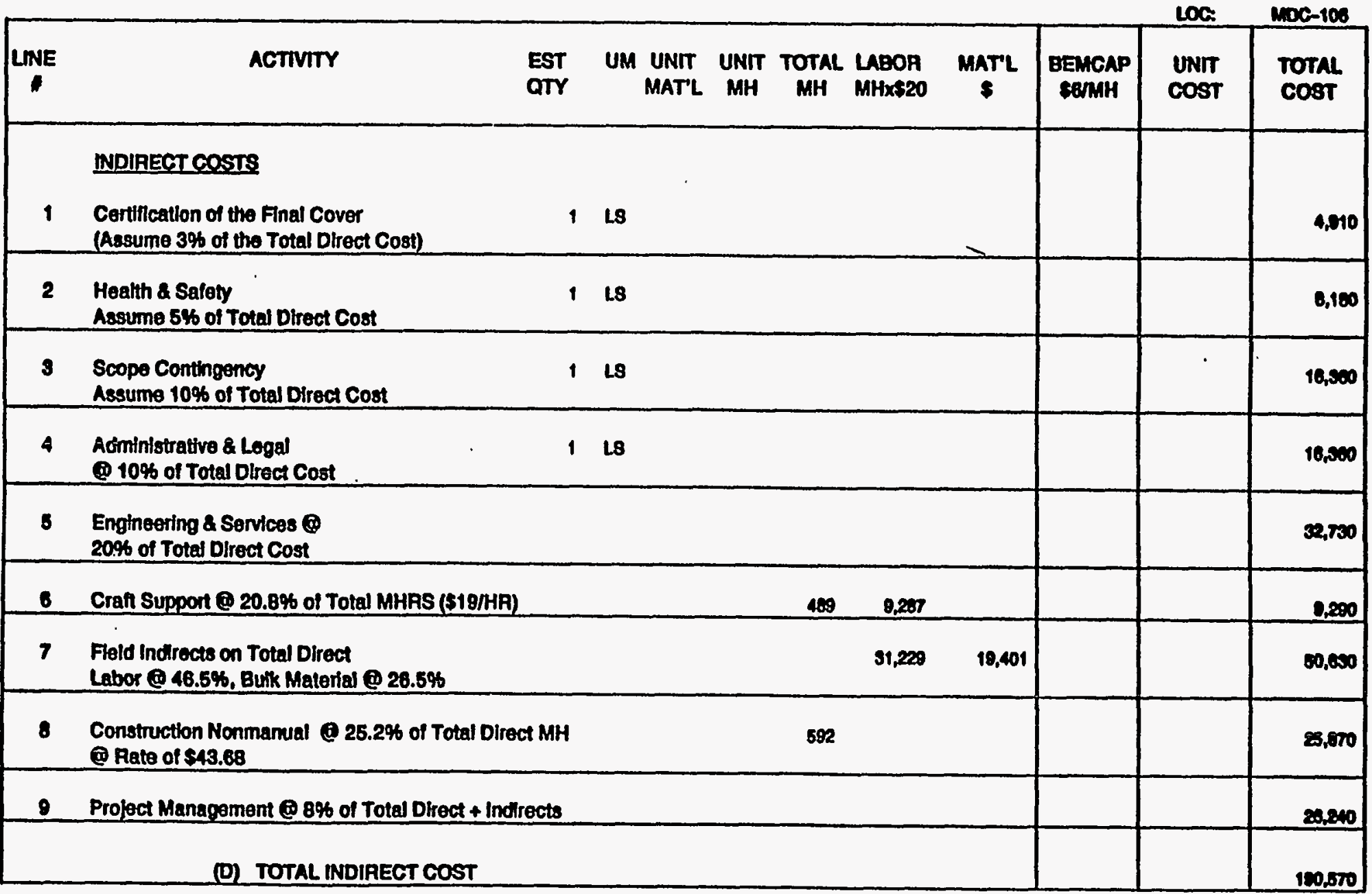

B-6. Cost Estimate for a Cover System Utilizing Reinforced Sprayed Asphalt (Cont'd) 


\section{DETAIL ESTIMATE}

LOG : : 7-03

ACTIVTYY CODE: ESE260412

PROJ.NAME: ECON. OF EVALUATION CLOSURE CAP BARAIER MATERIAL

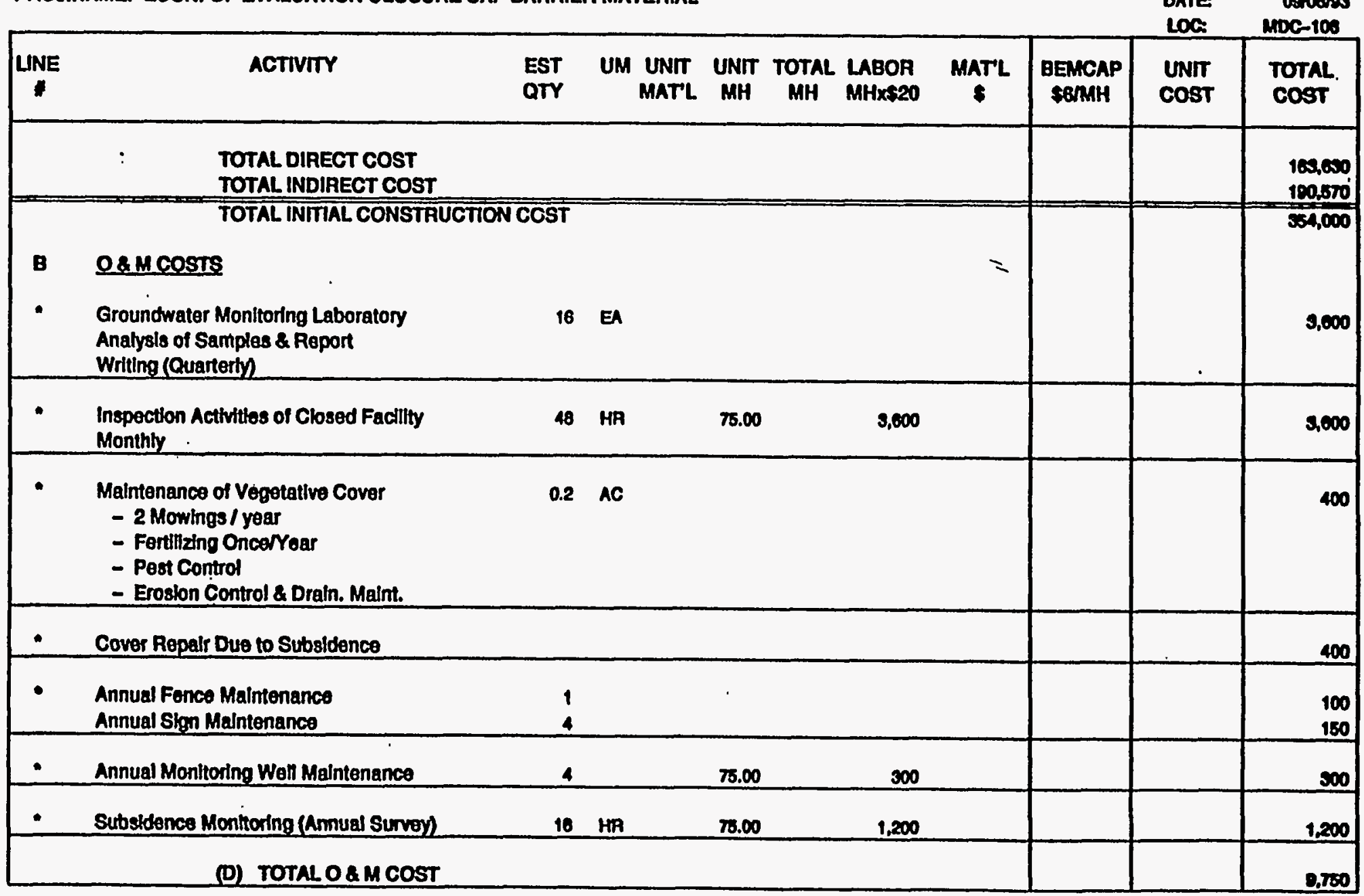

B-6. Cost Estimate for a Cover System Utilizing Reinforced Sprayed Asphalt (Cont'd) 
DETAIL ESTIMATE

LOG : : 7-03

ACTIVTY CODE: ESE260412

PAOJ.NAME: ECON. OF EVALUATION CLOSURE CAP BARRIER MATERIAL
CHKD BY: 8. MEAD

ESTOBY:

PHONE

Panke

$7-8260$

DATE DONOS

LOC: $\quad 1000-100$

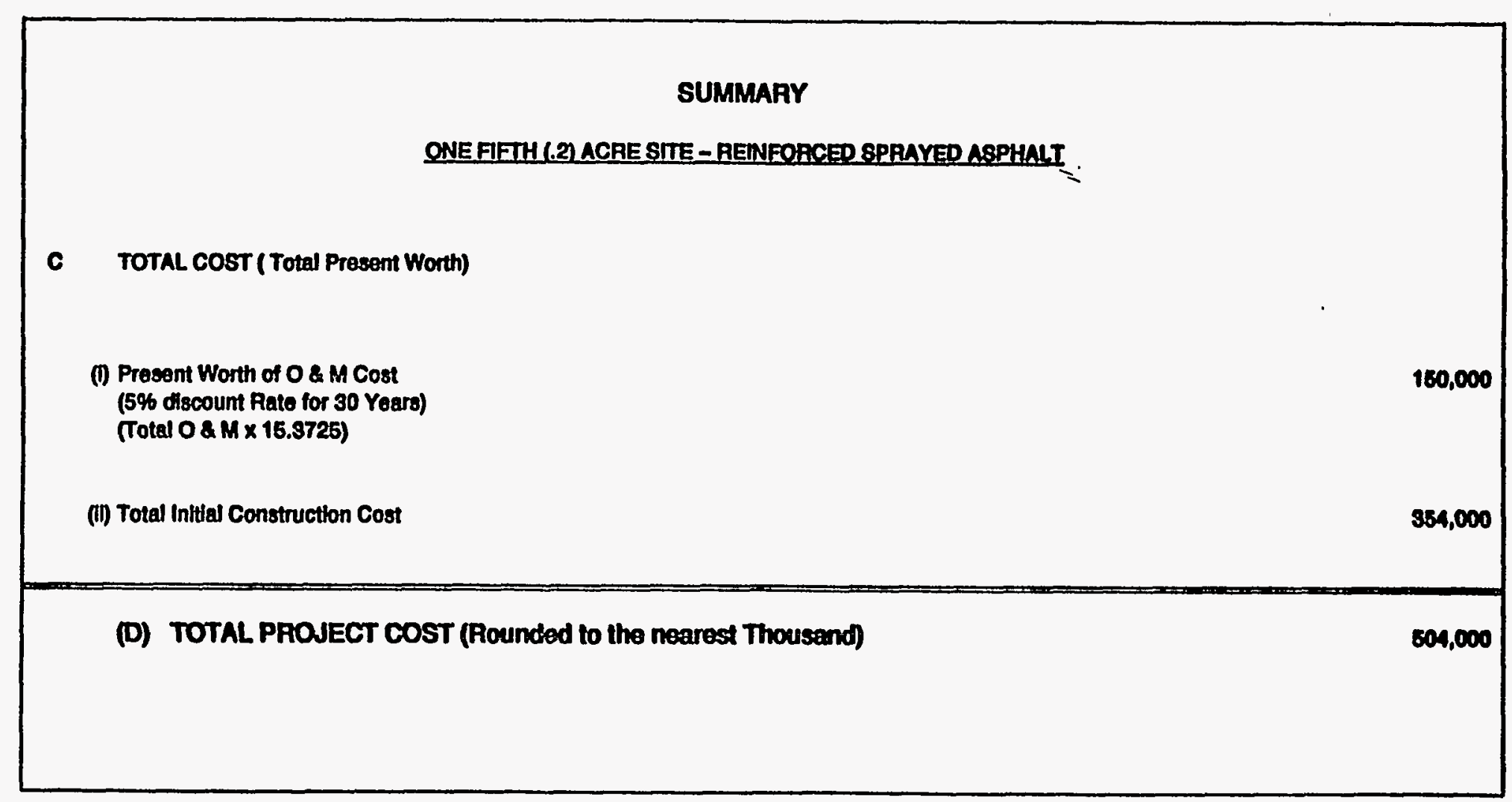

B-6. Cost Estimate for a Cover System Utilizing Reinforced Sprayed Asphalt (Cont'd) 


\section{DETAIL ESTIMATE}

\section{Lo01: 7-03}

ACTIVITY CODE: BSTE200412

FROS.NAMB: BCOK. BVALUATON OF CLOSURE CAS BARRIER MATERUAL

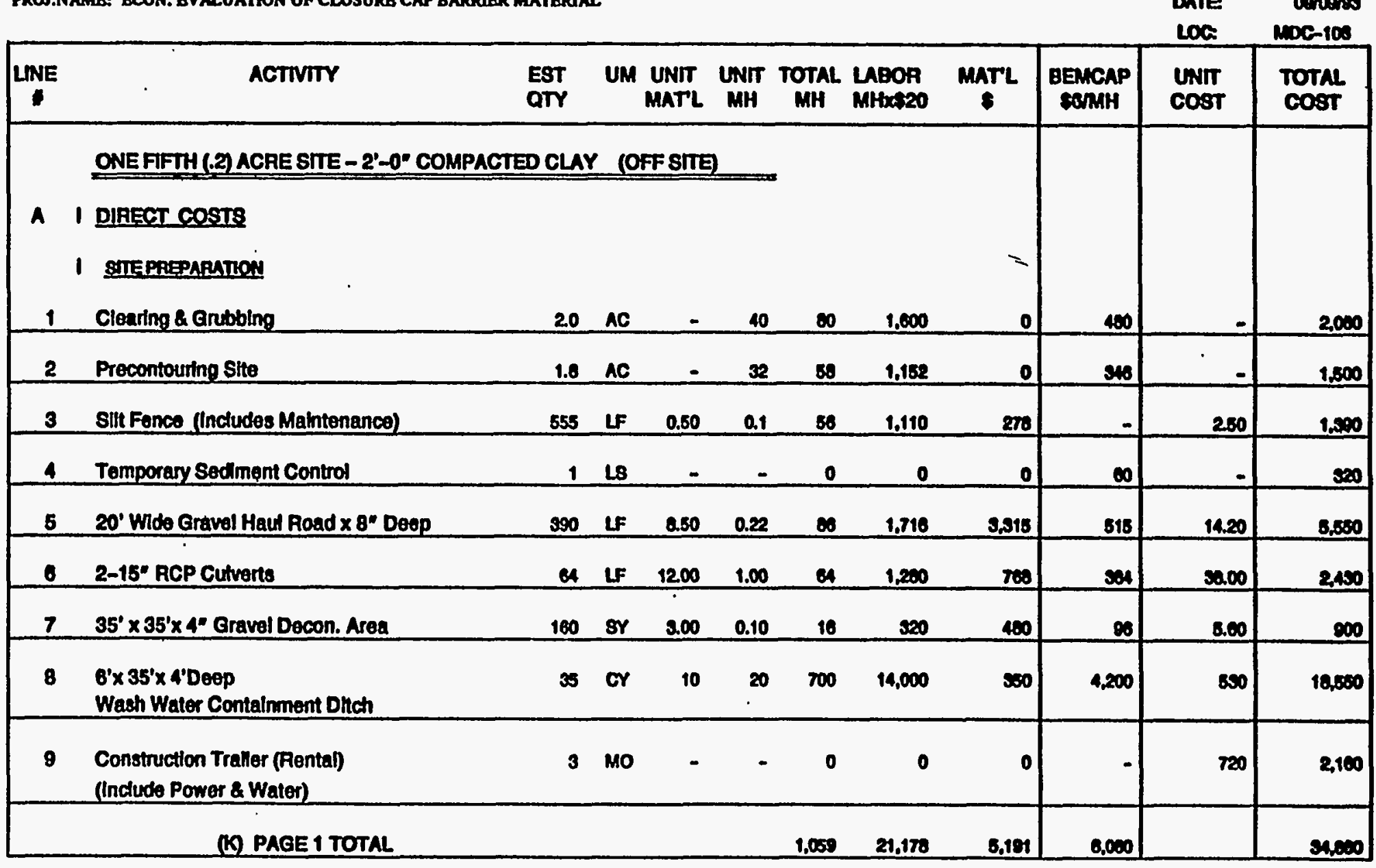

B-7. Cost Estimate for a Cover System Utilizing Compacted Kaolin (Offsite) Clay and a 30-mil FML 
DETAILESTIMATE

\section{CHK'D EY: 8. MEAD}

Lo0 $:$ : 7-03

ACTTVIT CODE: EsE200412

FOJ.NAME: BCON. EVALUATION OF CLOSURE CAP BARRIER MATERLAL

\begin{tabular}{|c|c|c|c|c|c|c|c|c|c|c|c|}
\hline LNE & ACTIVTr & $\begin{array}{l}\text { EST } \\
\text { QTY }\end{array}$ & UM & $\begin{array}{l}\text { UNIT } \\
\text { MATL }\end{array}$ & $\begin{array}{l}\text { UNTI } \\
\text { MH }\end{array}$ & TOTAL & $\begin{array}{l}\text { LABOR } \\
\text { MHax20 }\end{array}$ & $\underset{8}{\operatorname{MnT}}$ & $\begin{array}{l}\text { BEMCNP } \\
\text { PanH }\end{array}$ & $\begin{array}{l}\text { UNT } \\
\text { COST }\end{array}$ & $\begin{array}{l}\text { Total } \\
\operatorname{cost}\end{array}$ \\
\hline & - LOVVERCONSTRUCTION & & & & & & & & & & \\
\hline 10 & Waste Stablization (Crushed Limestone) & 400 & $\pi$ & 100 & 0.1 & 40 & $\infty$ & 40,000 & 240 & 102.00 & 41,040 \\
\hline 11 & $\begin{array}{l}\text { Place and Compact Foundation Soll } \\
\text { In } 8^{\circ} \text { Lifte to } 95 \% \text { 8T0. Proctor }\end{array}$ & 1,614 & Cr & - & 0.176 & 282 & 8,649 & $=$ & 1,000 & 4.80 & 7,540 \\
\hline 12 & $\begin{array}{l}\text { Compact 2'-0" Thick Clay Layor } \\
\text { In } 0^{\circ} \text { Lifts }\end{array}$ & 678 & Cr & 19.00 & 0.160 & 112 & 2,207 & 8,814 & mit & 17.50 & 11,720 \\
\hline 19 & $\begin{array}{l}\text { Placoment of } 30 \text { MH Flexiblo } \\
\text { Membrano Liner }\end{array}$ & 10,440 & $\mathbf{s F}$ & 0.18 & 0.000 & 84 & 1,079 & 1,804 & - & 0.40 & 9,000 \\
\hline 14 & $\begin{array}{l}\text { Placement of } 1 \text { '-o Coaree Sand } \\
\text { Drainage Layer }\end{array}$ & 301 & Cr & 10.50 & 0.05 & 18 & 253 & 8,791 & 70 & 10.00 & 4,120 \\
\hline 16 & $\begin{array}{l}\text { Placement of Geotextile (TYPAR) Flter on } \\
\text { 1'-0* Coarse Sand Drainage Layer }\end{array}$ & 1,114 & $\mathbf{8 Y}$ & 0.20 & 0.007 & $\mathbf{8}$ & 150 & 220 & - & 0.50 & 200 \\
\hline 18 & Placement of 1'-6* Common FII & 485 & Cr & $3 \pi$ & 0.07 & 34 & 879 & 1,010 & 201 & 8.00 & 2,700 \\
\hline 17 & Piacement of 6" Topson & 182 & CY & 8.50 & 0.00 & 16 & 328 & 1,720 & $\boldsymbol{0}$ & 18.00 & 2,100 \\
\hline 18 & $\begin{array}{l}\text { Placement of Perimeter Exterlor } \\
\text { Drainape Layer } 3 / 4^{\prime \prime} \times 3^{*} \text { DP (Crushed Stone) }\end{array}$ & 143 & $\mathbf{s Y}$ & 1.60 & 0.24 & 34 & $\cos$ & 216 & 200 & 7.80 & 1,110 \\
\hline 19 & Excavation of Trapezoldal Drainage Ditch & 88 & $\mathbf{C Y}$ & - & 0.2 & 18 & 352 & 0 & 100 & 5.20 & 400 \\
\hline & (G) PAGE 2 TOTAL & & & & & $\infty$ & 18,019 & 58,575 & 3,205 & & 74800 \\
\hline
\end{tabular}

B-7. Cost Estimate for a Cover System Utilizing Compacted Kaolin (Offsite) Clay and a 30-mil FML (Cont'd) 
CHKD EY: 8. MEAD

LO01: 7-03

ACTIVIT CODB: BSE2SON12

MROU.NAME: BCON. BVALUATLON OP CLOSURB CAP BARRIER MATERAN

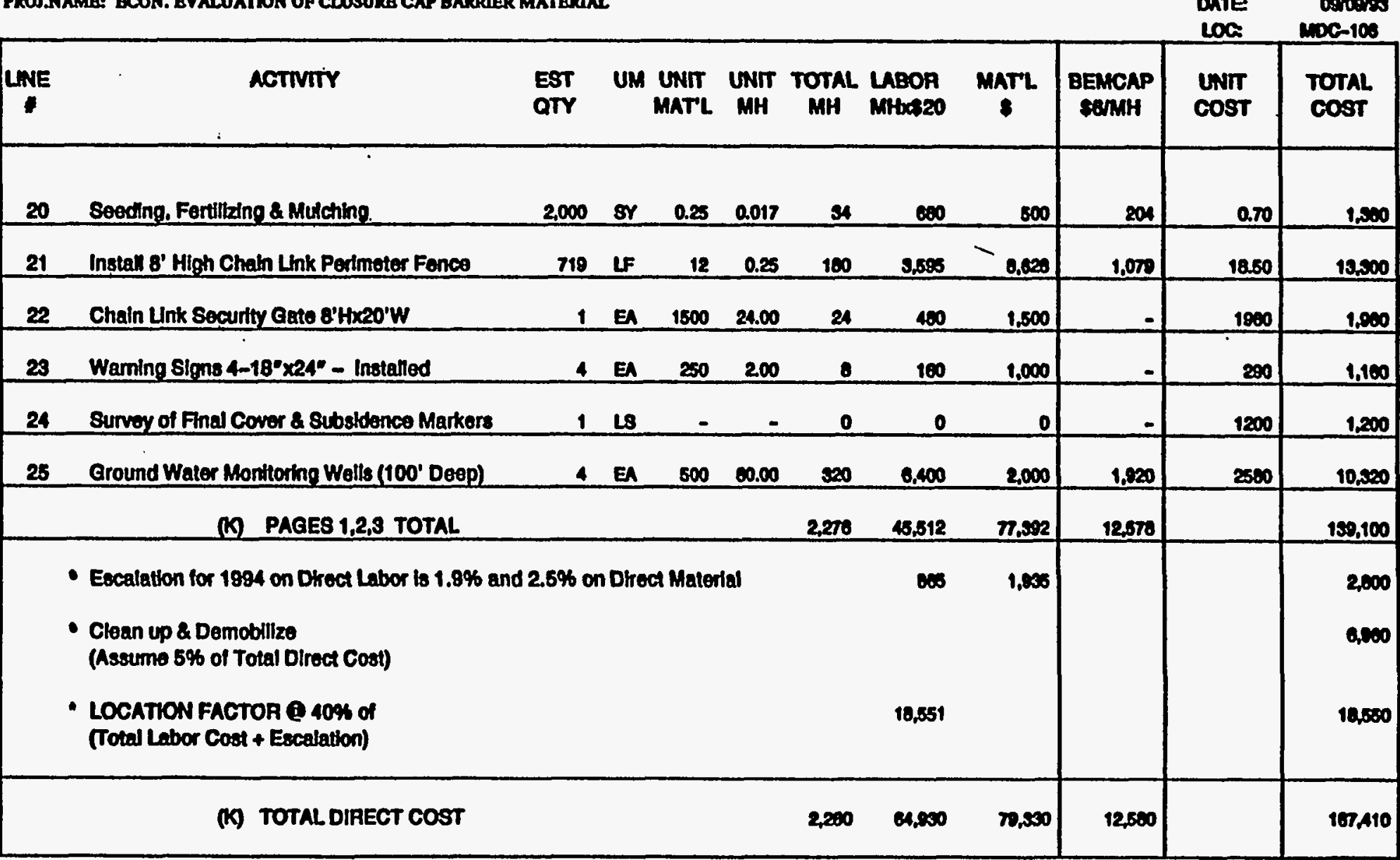

B-7. Cost Estimate for a Cover System Utilizing Compacted Kaolin (Offsite) Clay and a 30-mil FML (Cont'd) 


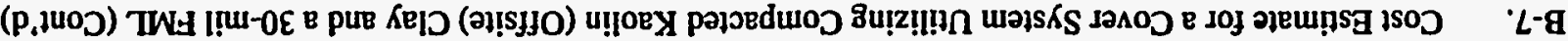

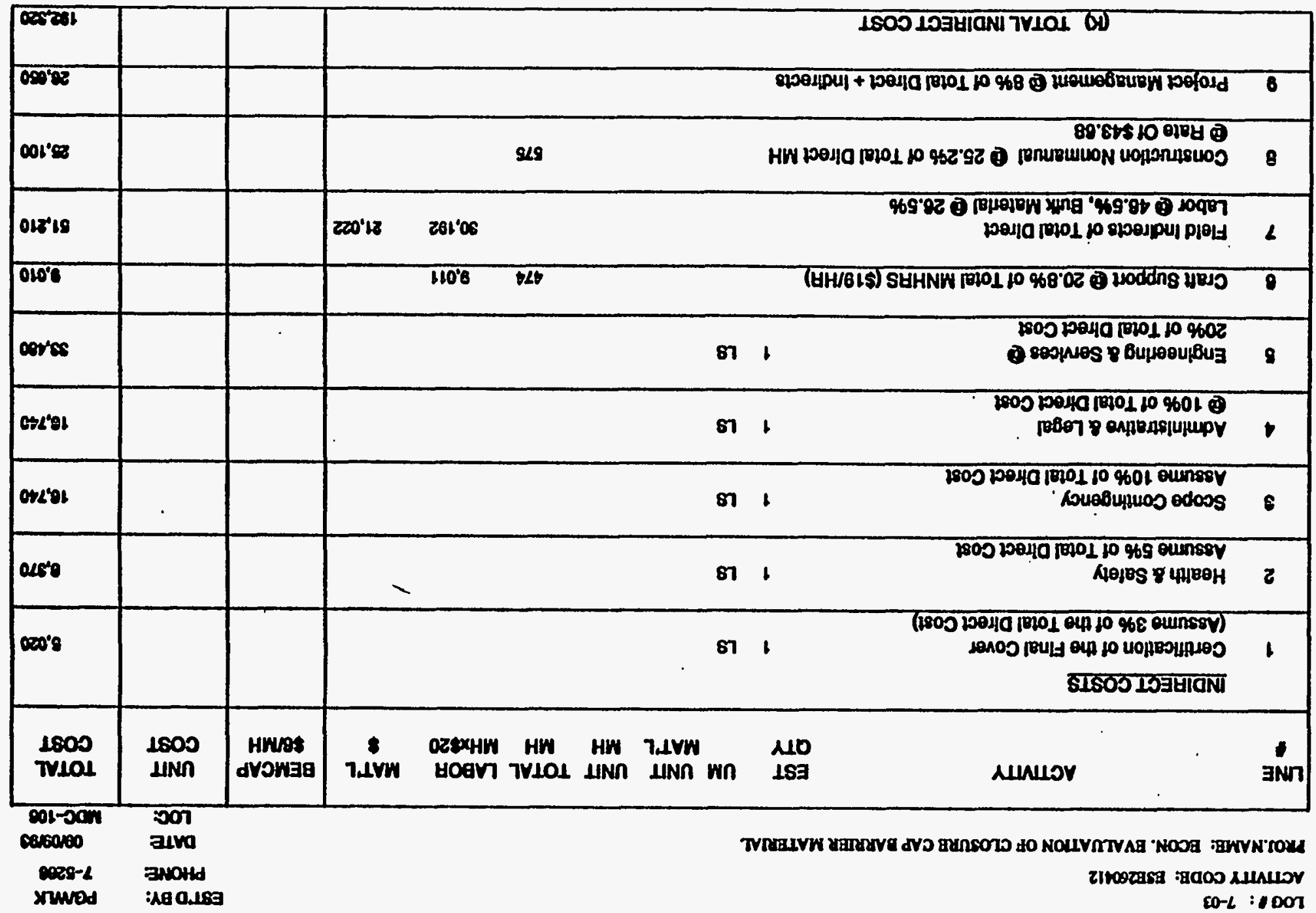

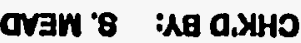

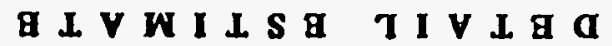


Lo0 II: 7-03

ACTIVITY CODE: BSE26O412

FROU.NAMB: BCON. BVALUATION OF CLOSUNB CAP BARRIER MATERIAL.

PTTOEY: Pamk

DATE 080000

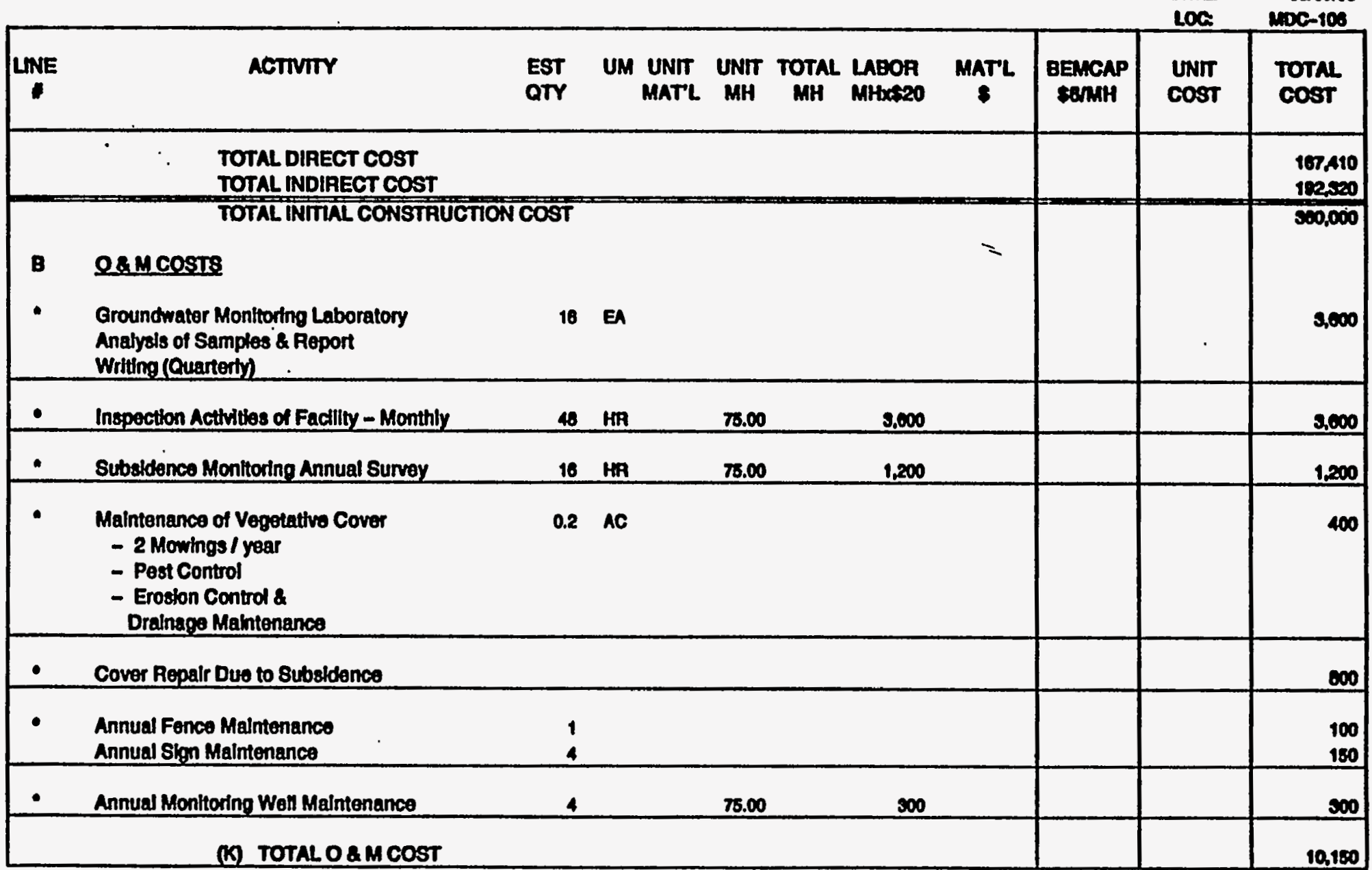

B-7. Cost Estimate for a Cover System Utilizing Compacted Kaolin (Offsite) Clay and a 30-mil FML (Cont'd) 
D B T A I L E S T I MA T B

CHKD BY: 8. MEND

Loof: $7-03$

ACTIVITY CODB: BSBROOA12

MOU.NAME: BCON. EVALUATION OF CLOSURB CAP BARRIER MATERINL

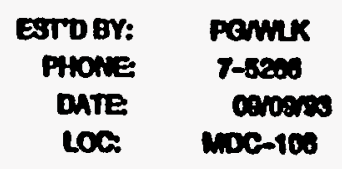

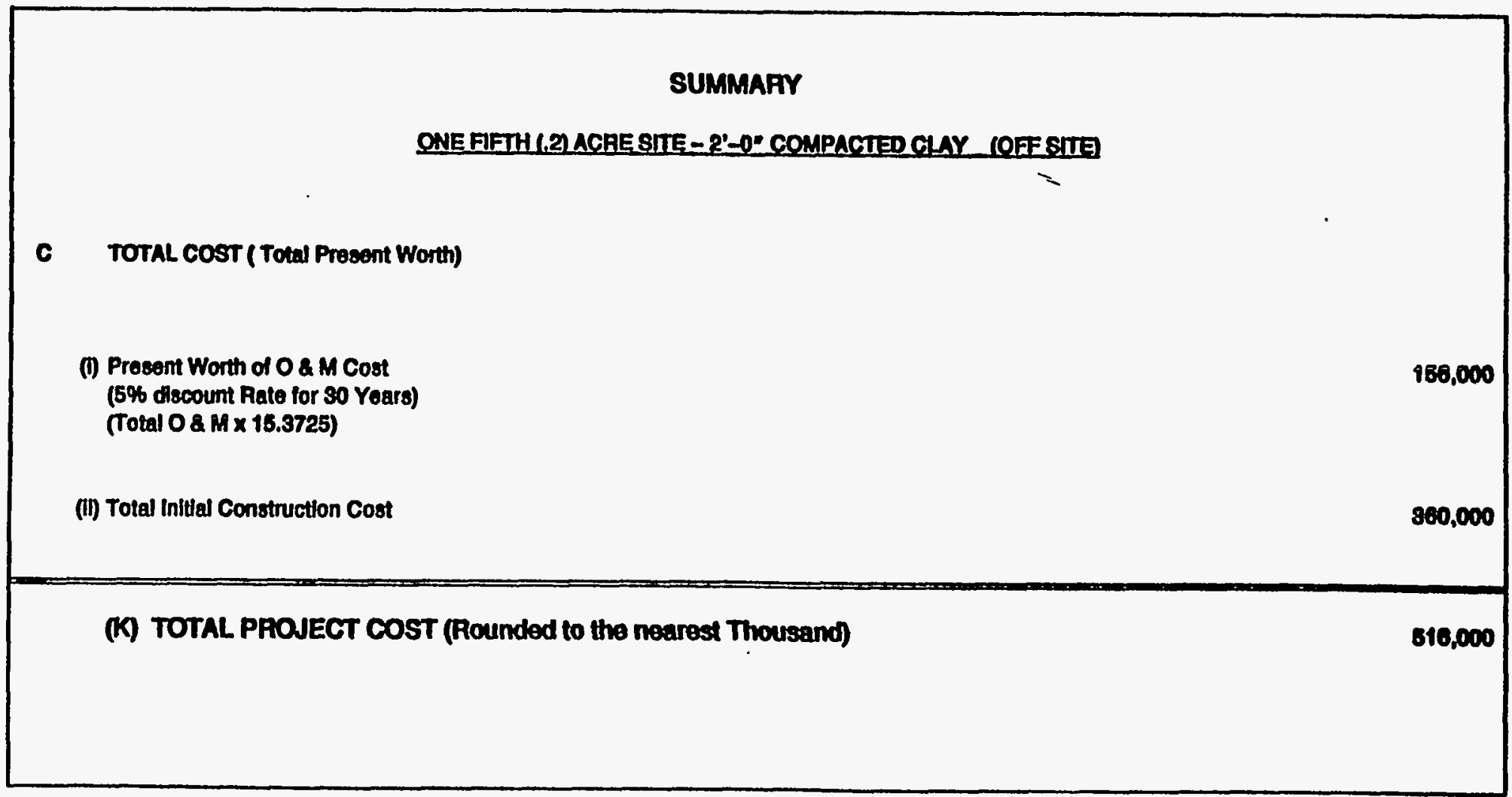

B-7. Cost Estimate for a Cover System Utilizing Compacted Kaolin (Offsite) Clay and a 30-mil FML (Cont'd) 


\section{DETAIL ESTIMATE}

100 I: $7-03$

ACTIVITY CODE: BSE260412

PROJ.NAME: ECON. EVALUATTON OF CLOSURB CAP BAJRRIER MATERIAL

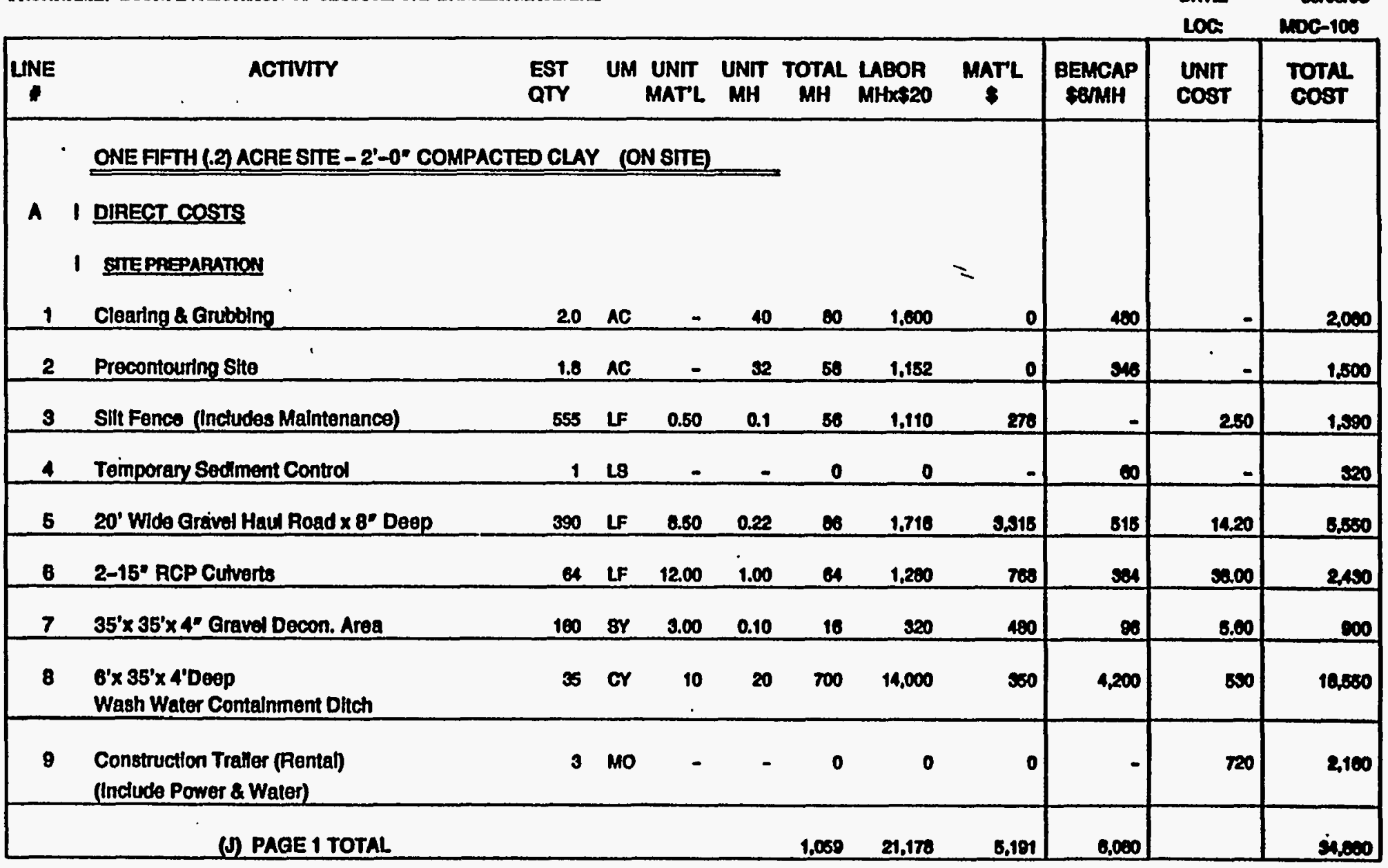

B-8. Cost Estimate a for Cover System Utilizing Compacted Onsite Sandy Clay and a 30-mil FML 


\section{DETAIL ESTIMATE}

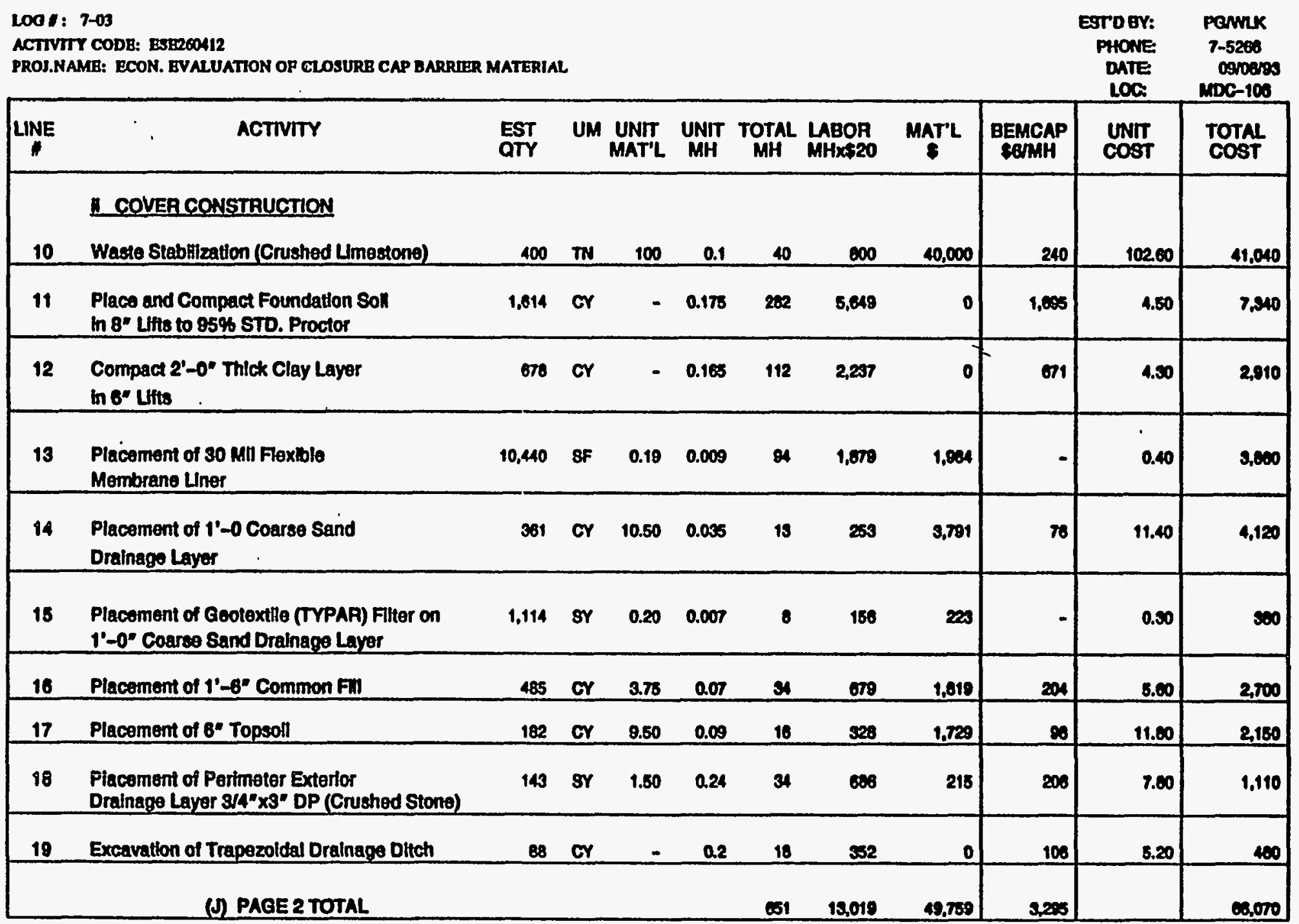

B-8. Cost Estimate a for Cover System Utilizing Compacted Onsite Sandy Clay and a 30-mil FML (Cont'd) 
D B T A I L B S T I MA T B

CHKD BY: 8. MEAD

\section{Lool: 7-03}

ACTIVITY CODE: BSE200412

PROJ.NAME: ECON. EVALUATION OF CLOSURR CAP BARRIIR MAATERIAL.

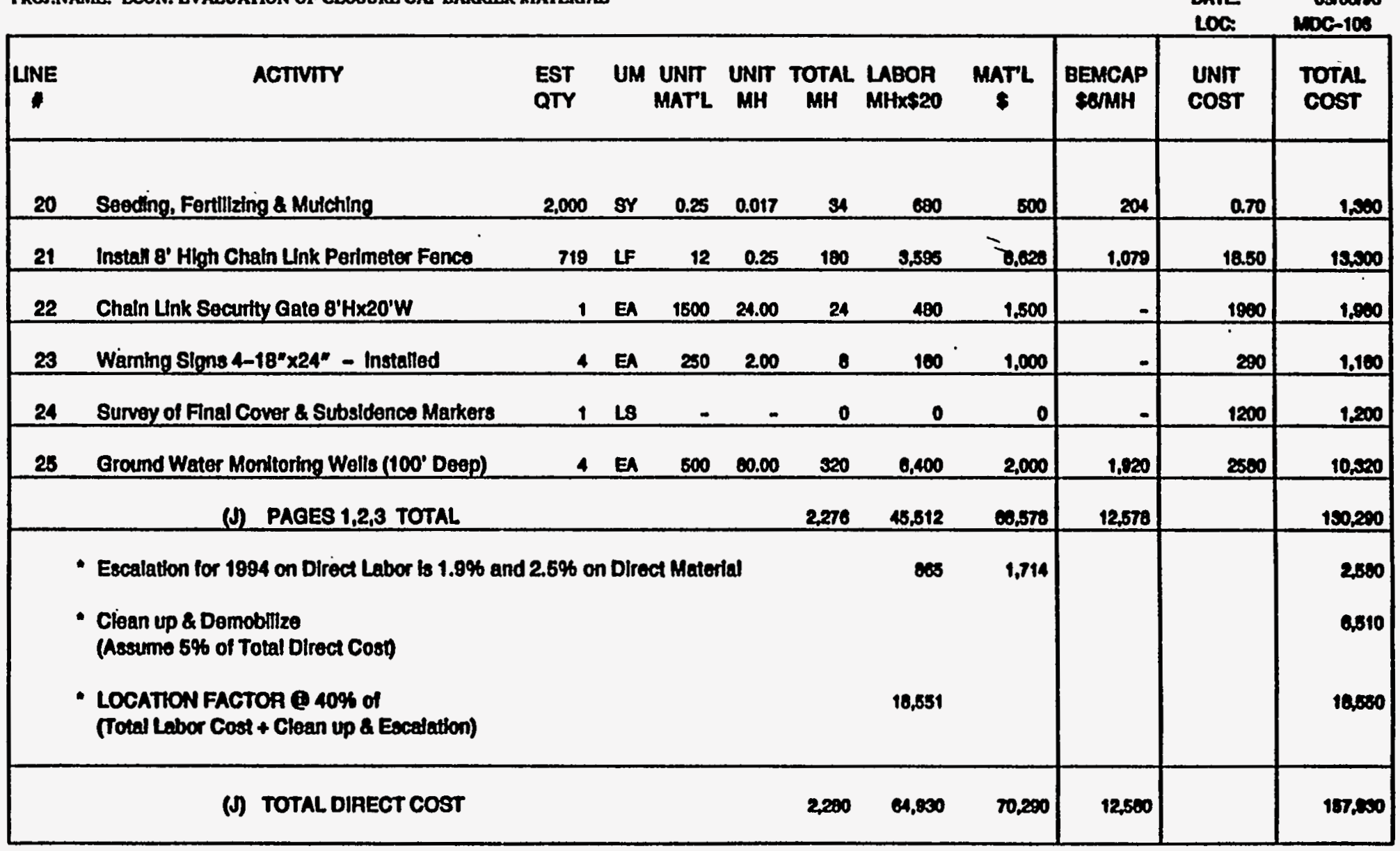

B-8. Cost Estimate a for Cover System Utilizing Compacted Onsite Sandy Clay and a 30-mil FML (Cont'd) 
D B T A L B S T IMAT B

CHK'D BY: 8. MEAD

Lo01: 7-03

ACTTVIT CODB: ESE2OON12

PROI.NAME: ECON. EVALUATTON OF CLOSURB CAP BARRIER MATERIAL

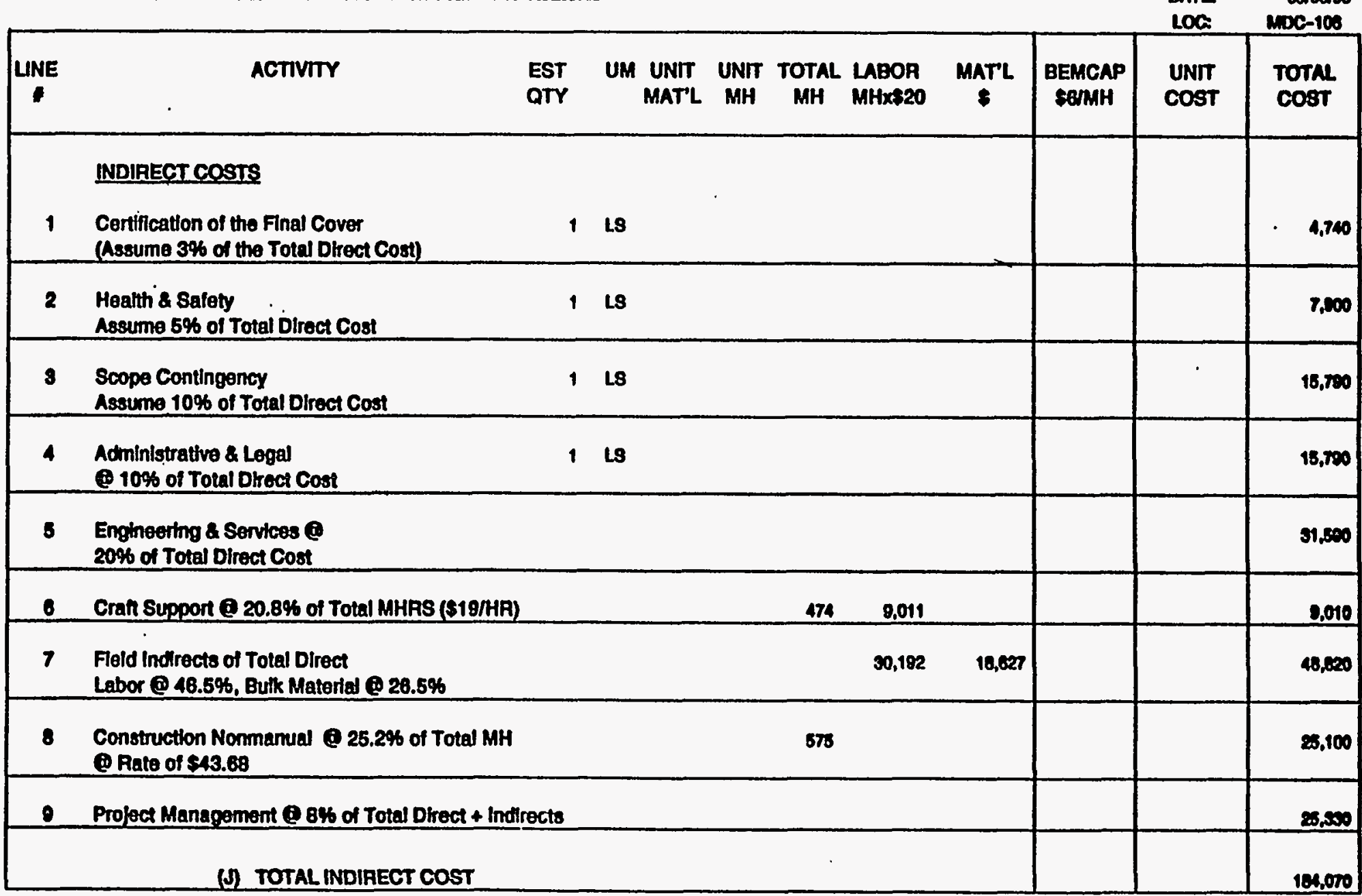

B-8. Cost Estimate a for Cover System Utilizing Compacted Onsite Sandy Clay and a 30-mil FML (Cont'd) 
D B T A I L E S T I A T B

\section{CHKD BY: 8. MEAD}

L00 I: 7-03

ACTIVITY CODE: ESE260112

PROJ.NAMB: ECON. EVALUATION OF CLOSURB CAP BARRIER MATERIN

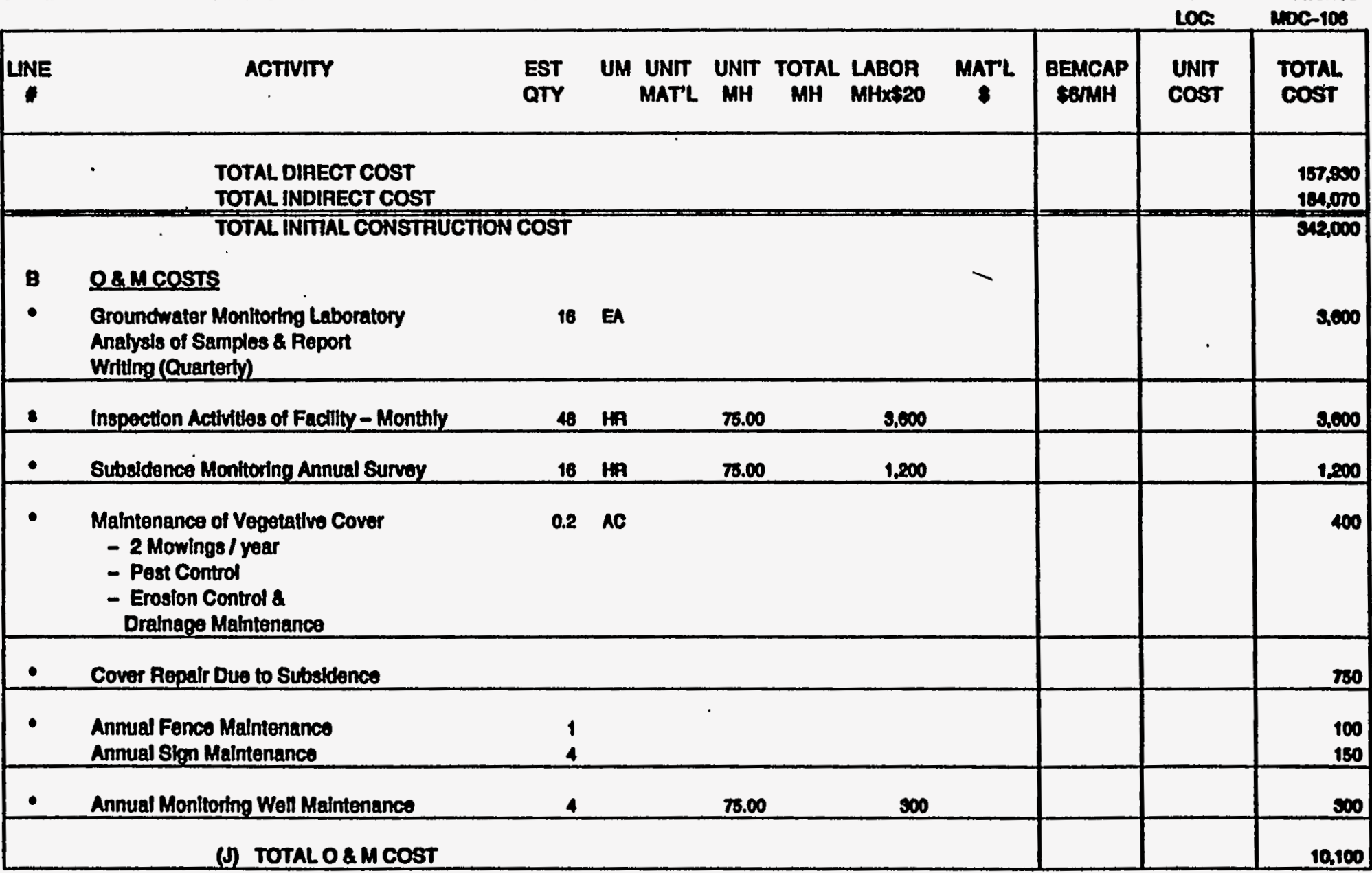

B-8. Cost Estimate a for Cover System Utilizing Compacted Onsite Sandy Clay and a 30-mil FML (Cont'd) 


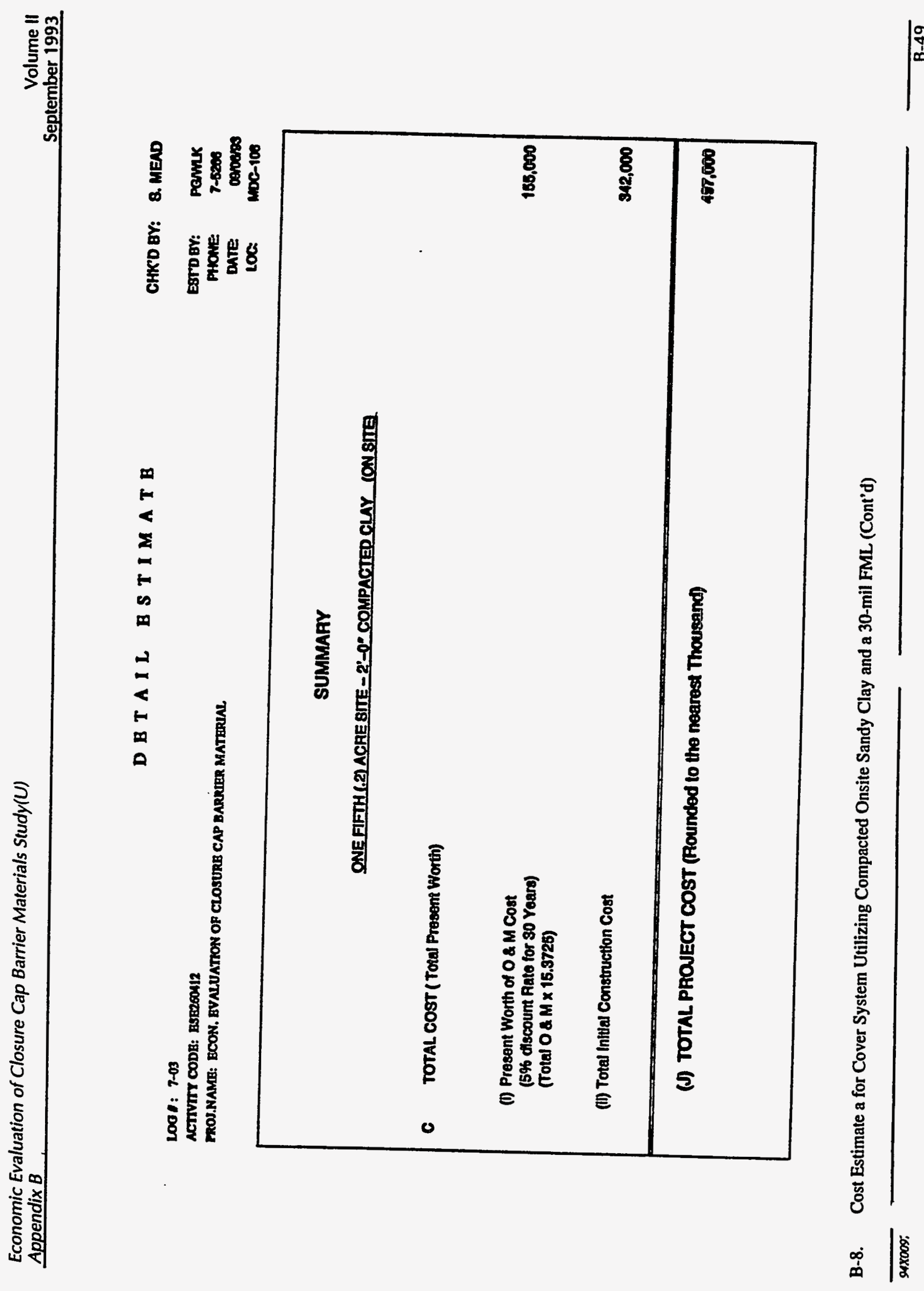




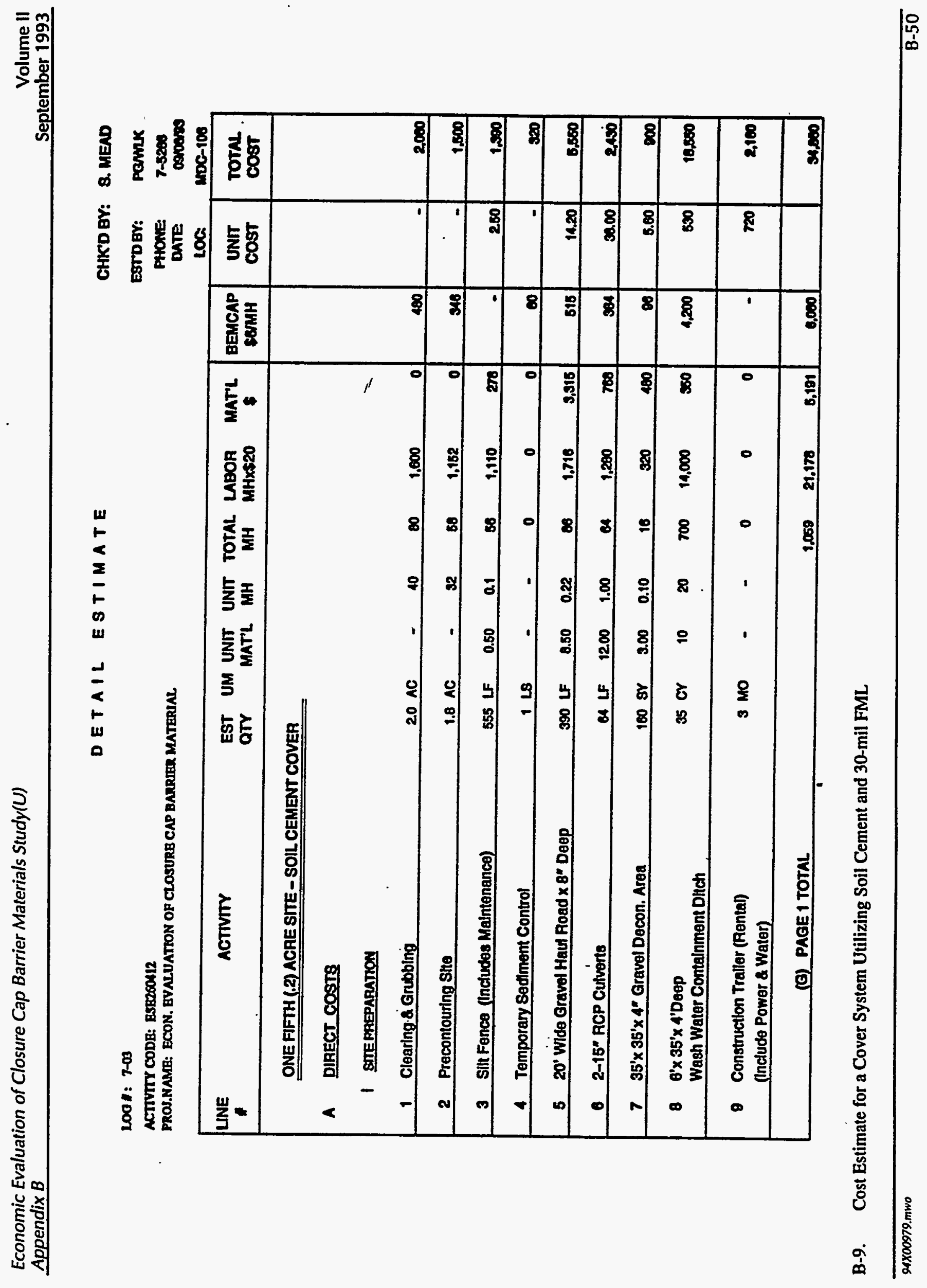


DETAIL ESTIMATE

CHK'D BY: 8. MEAD

1001: 7-03

ACTIVITY CODE: BSEZCON12

PROJ.NAME: ECON. BVALUATION OF CLOSURB CAP BARRUER MATERIAL

\begin{tabular}{|c|c|c|c|c|c|c|c|c|c|c|c|}
\hline & & & & & & & & & & LOC: & noc-100 \\
\hline LINE & ACTIVITY & $\begin{array}{l}\text { EST } \\
\text { aTY }\end{array}$ & UM & $\begin{array}{l}\text { UNTI } \\
\text { MAT'L }\end{array}$ & $\underset{M H}{\text { UNT }}$ & TOTAL & $\begin{array}{l}\text { LABOR } \\
\text { MHX } \$ 20\end{array}$ & $\underset{8}{\operatorname{MATL}}$ & $\begin{array}{l}\text { BEMCAP } \\
\text { SOMMH }\end{array}$ & $\begin{array}{l}\text { UNiT } \\
\text { Cost }\end{array}$ & $\begin{array}{l}\text { TOTAL } \\
\text { Cost }\end{array}$ \\
\hline & I COVER CONSTRUCTION & & & & & & & & & & \\
\hline 10 & Waste Stablilzation (Crushed Limestone) & 400 & $\mathbf{m}$ & 100 & 0.1 & 40 & 800 & 40,000 & 240 & 102.00 & 41.040 \\
\hline 11 & $\begin{array}{l}\text { Place and Compact Foundation Soll } \\
\text { m 8* Lits to } 95 \% \text { sto. Proctor }\end{array}$ & 1,614 & & - & 0.175 & 282 & 8,649 & $=$ & 1,685 & 4.50 & 7,340 \\
\hline 12 & 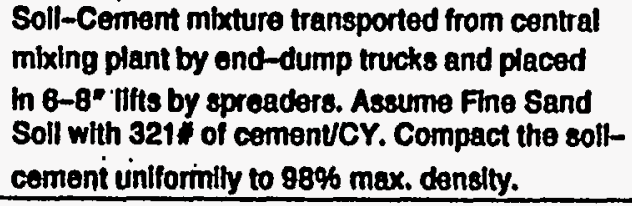 & 1,029 & or & 10 & 0.165 & 170 & 3,396 & 10,522 & 1,018 & 22.30 & 22040 \\
\hline 13 & $\begin{array}{l}\text { Curing soll-cement. Assume irquild Asphalt } \\
\text { applied 0.4gallsy for } 1 \text { St coat } 20.2 \text { for } 2 \text { 2nd: }\end{array}$ & $\begin{array}{l}1,096 \\
1,090\end{array}$ & $\begin{array}{l}\text { sy } \\
\text { or }\end{array}$ & $\begin{array}{l}0.33 \\
0.17\end{array}$ & $\begin{array}{l}0.05 \\
0.09\end{array}$ & $\begin{array}{l}65 \\
39\end{array}$ & $\begin{array}{r}1,090 \\
659\end{array}$ & $\begin{array}{l}362 \\
187\end{array}$ & - & $\begin{array}{l}1.30 \\
0.80\end{array}$ & $\begin{array}{r}1,460 \\
600\end{array}$ \\
\hline 14 & $\begin{array}{l}\text { Placement of } 30 \text { Mr Fexible } \\
\text { Membrane Liner }\end{array}$ & 10,440 & SF & 0.10 & 0.009 & o4 & 1,679 & 1,804 & - & 0.40 & 2,600 \\
\hline 15 & $\begin{array}{l}\text { Placement of 1'-o Coarse Sand } \\
\text { Drainaga Layer }\end{array}$ & 361 & or & 10.50 & 0.035 & 13 & 253 & 0,701 & 70 & 11.40 & 4,120 \\
\hline 16 & $\begin{array}{l}\text { Piacement of Ceotextile (TYPAP) Filler on } \\
1^{1} \text {-0" Coarse Sand Drainage Layer }\end{array}$ & 1,114 & Sช & 0.20 & 0.007 & • & 150 & 220 & - & 0.50 & 500 \\
\hline 17 & Placement of 1'-6" Common FIII & 405 & or & a.rs & 0.07 & su & 679 & 1,019 & 200 & 5.00 & 2,00 \\
\hline 18 & Placement of 6" Topsoll & 182 & or & 2.50 & 0.00 & 10 & 3200 & 1.720 & $\boldsymbol{9}$ & 11.00 & 2180 \\
\hline & (G) PAGE 2 TOTAL & & & & & ens & 19,000 & 06,000 & 3,000 & & 0040 \\
\hline
\end{tabular}

B-9. Cost Estimate for a Cover System Utilizing Soil Cement and 30-mil FML (Cont'd) 
D B T A I L B S T I A T B

CHK'D BY: 8. MEAD

LO0 II: 7-03

ACTIVTY CODE: ESE260412

PROJ.NAME: BCON. BVALUATTON OF CLOSURB CAP BARRIER MATERIAL.

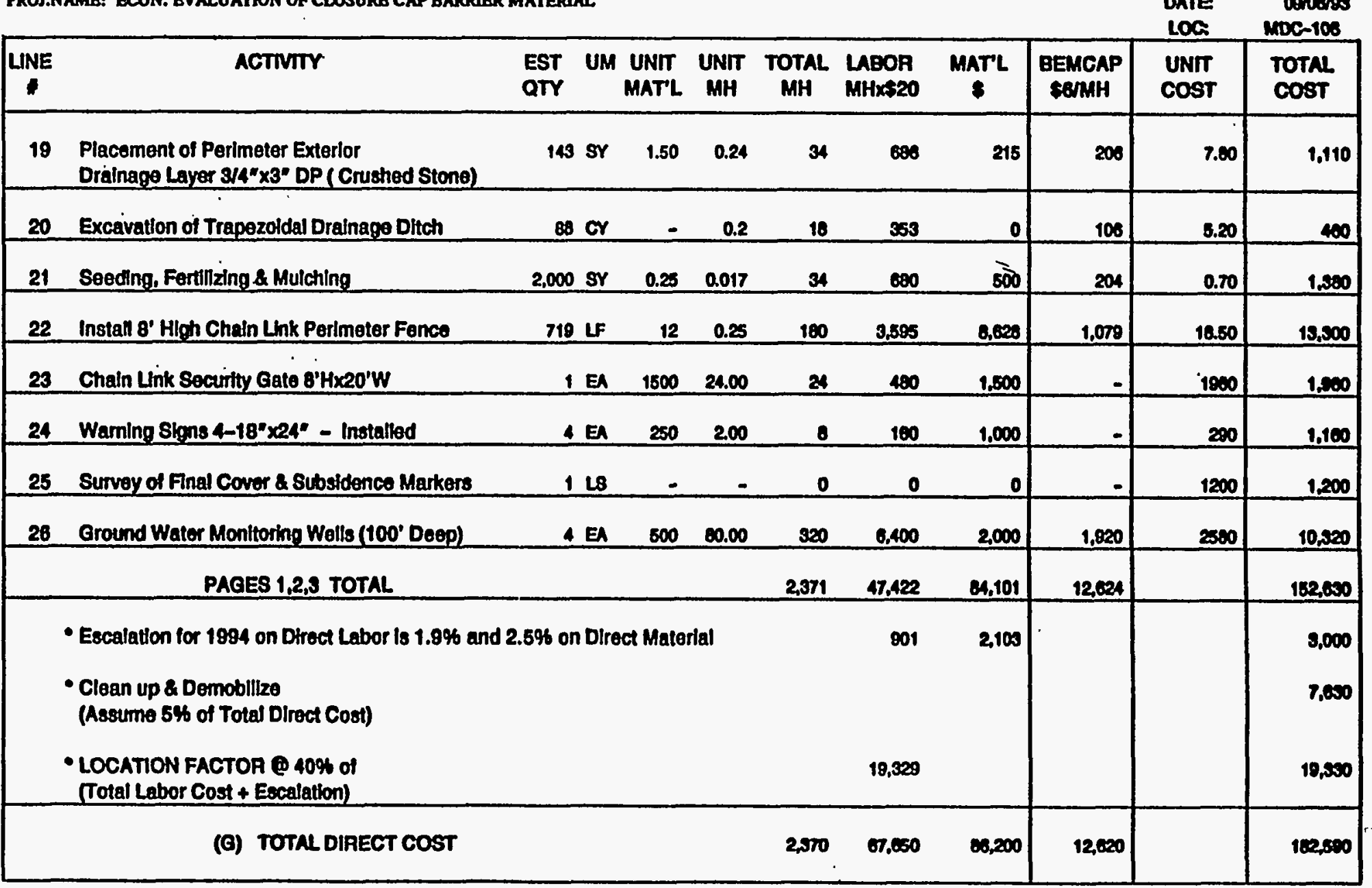

B-9. Cost Estimate for a Cover System Utilizing Soil Cement and 30-mil FML (Cont'd) 
D B T A I L B S T I M A T

CHK'D BY: 8. MEAD

2001: $7-03$

ACTTVITY CODE: ESE2SOA12

PROJ.NAMB: ECON. EYALUATROA OF CLOSURB CAP BARRIER MATERUAL

ESTOEY: Pamk

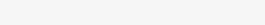

PHowe 7-62006

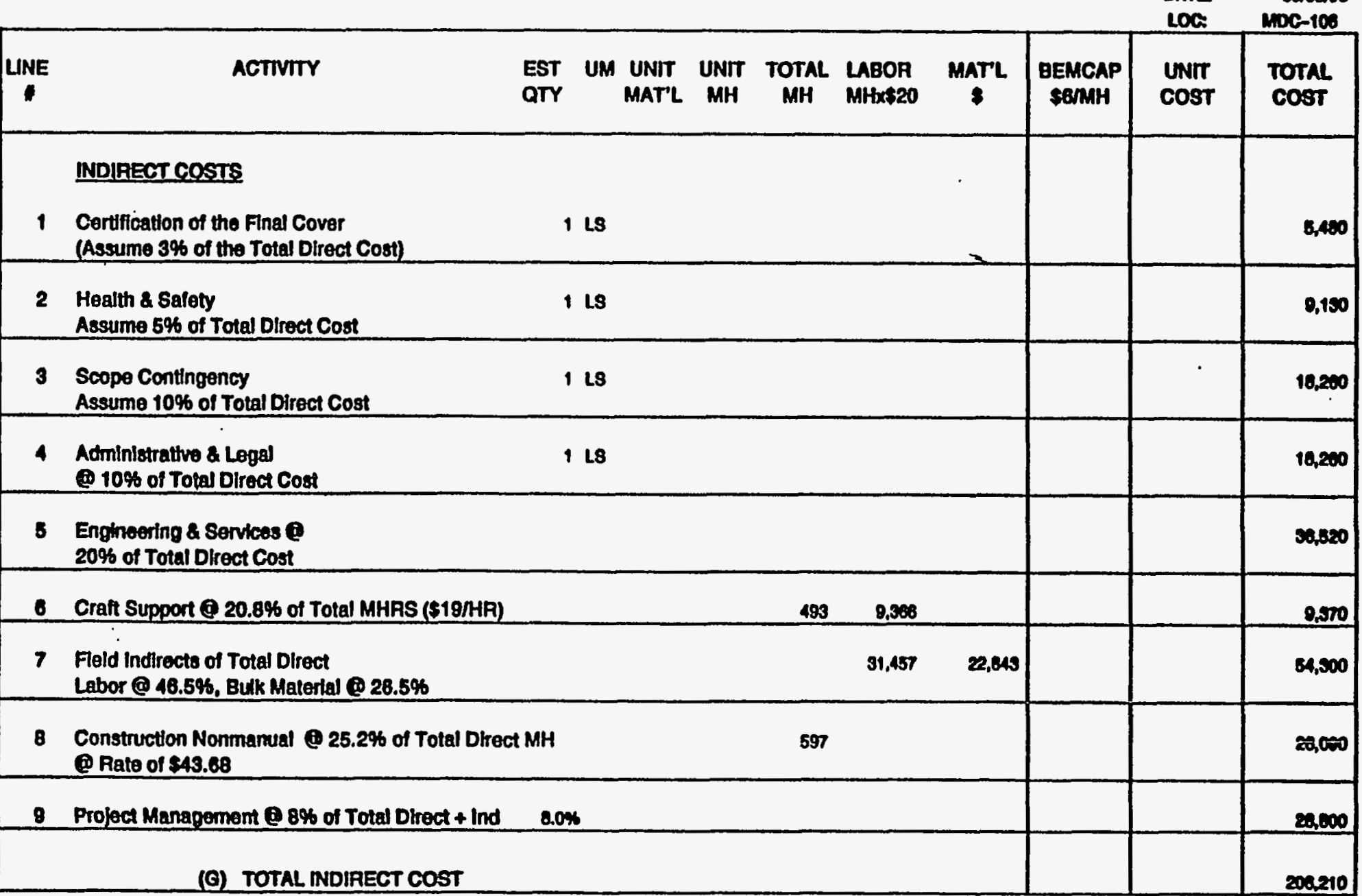

B-9. Cost Estimate for a Cover System Utilizing Soil Cement and 30-mil FML (Cont'd) 
D B TAIL E S I MAT B

CHKD BY: 8. MEAD

Lo01: 7-03

ACTIVITY CODE: ESE260112

PROJ.NAME: ECON. EVALUATION OR CLOSURB CAP BARUIER MATERALL

PTD EY: Pank

PHONE: T-5200

DATE DENos

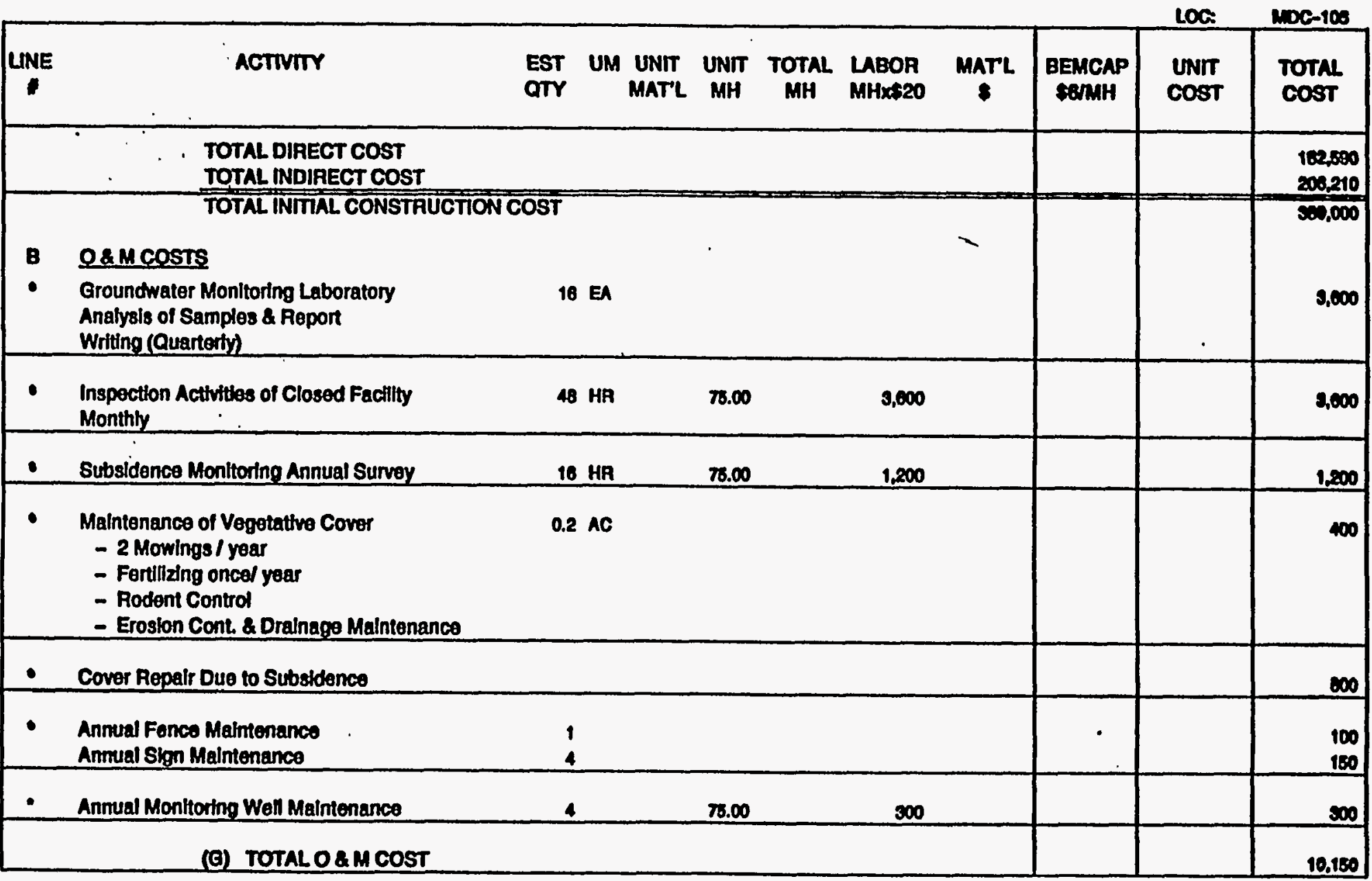

B-9. Cost Estimate for a Cover System Utilizing Soil Cement and 30-mil FML (Cont'd) 
D B T A L B S I M A T B

Loo I: 7-03

ACTIVITY CODE: ESE200412

PROJ.NAME: ECON. BVALUATION OF CLOSURB CAP BARIIER MATERIAL
CHK' BY: 8. MENO

ERTOBY:

PHowe

DATE:

Pank

$7-5260$

0000003

LOC.

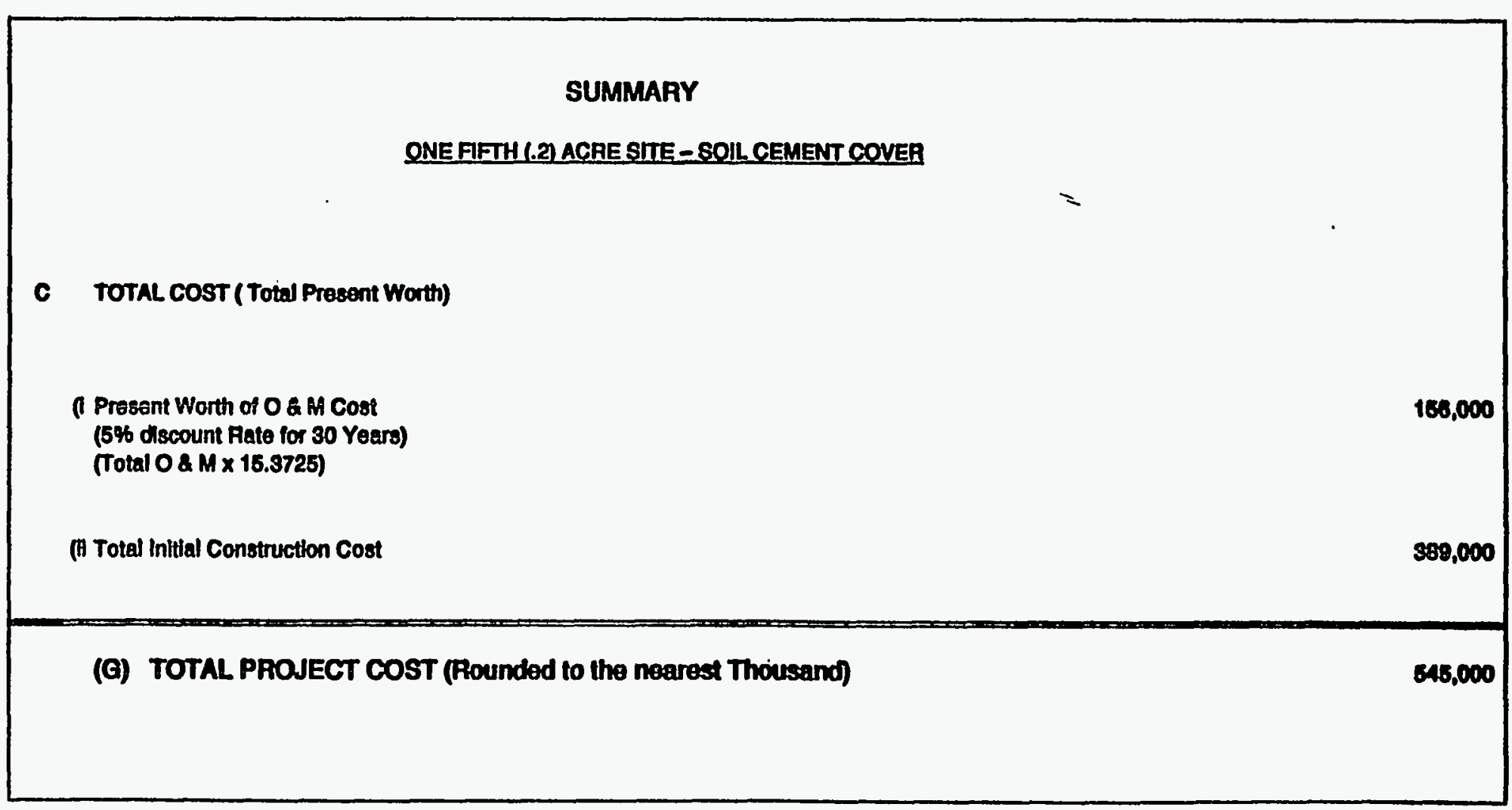

B-9. Cost Estimate for a Cover System Utilizing Soil Cement and 30-mil FML (Cont'd) 
DETAIL ESTIMATE

\section{CHK'D EY: 8. MEAD}

LoO I: 7-03

ACTIVITY CODE: ESE260412

PROJ.NAME: ECON. EVALUATION OF CLOSURB CAP BARRIER MATERIAL.

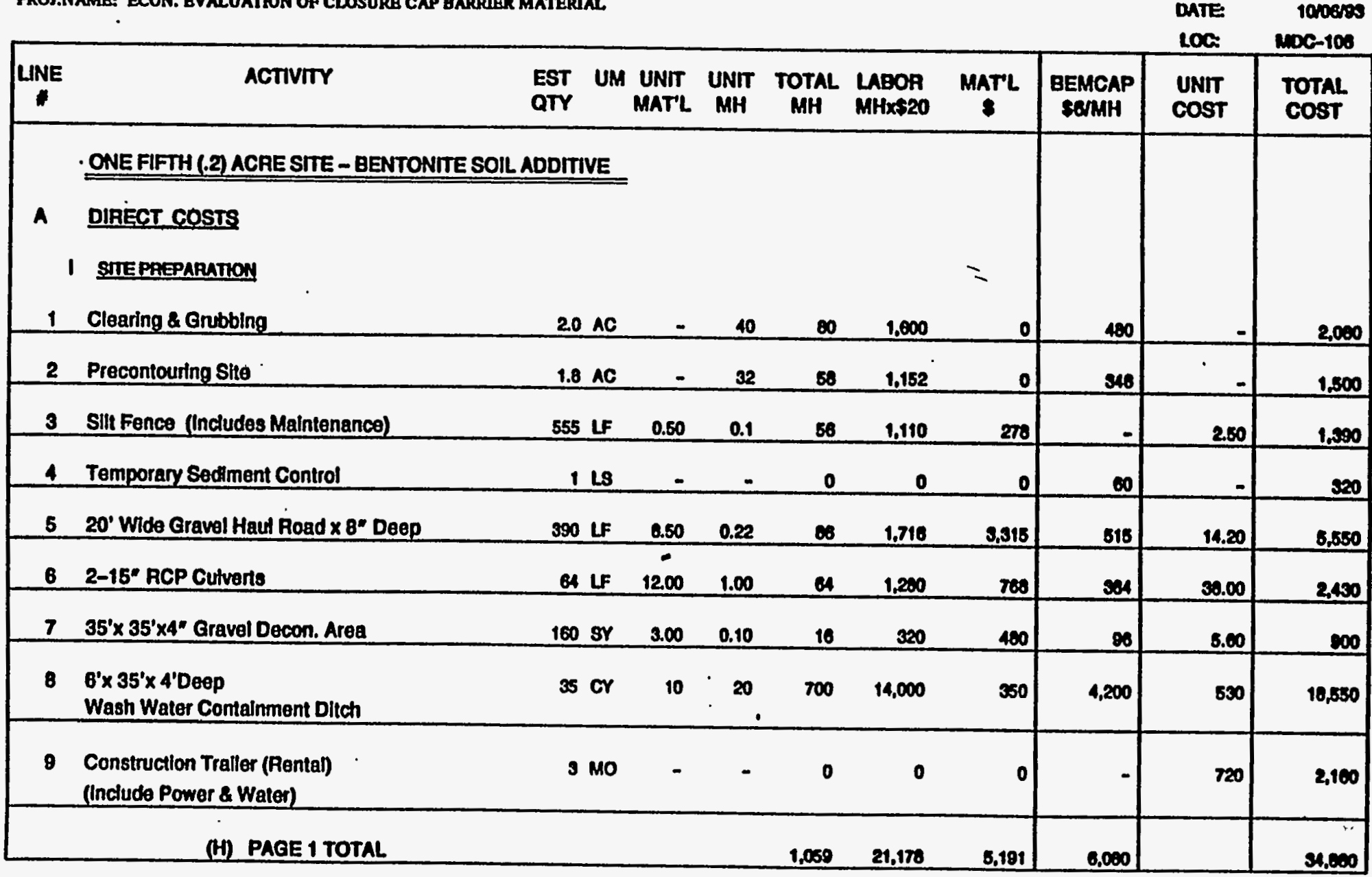

B-10. Cost Estimate for a Cover System Utilizing Bentonite Soil Additive and a 30-mil FML 
CHK'D BY: 8. MEAD

Loo A: 7-03

ACTIVITY CODE: ESE260412

PROJ.NAME: ECON. BVALUATION OF CLOSURB CAP BARRTER MATERIAL.

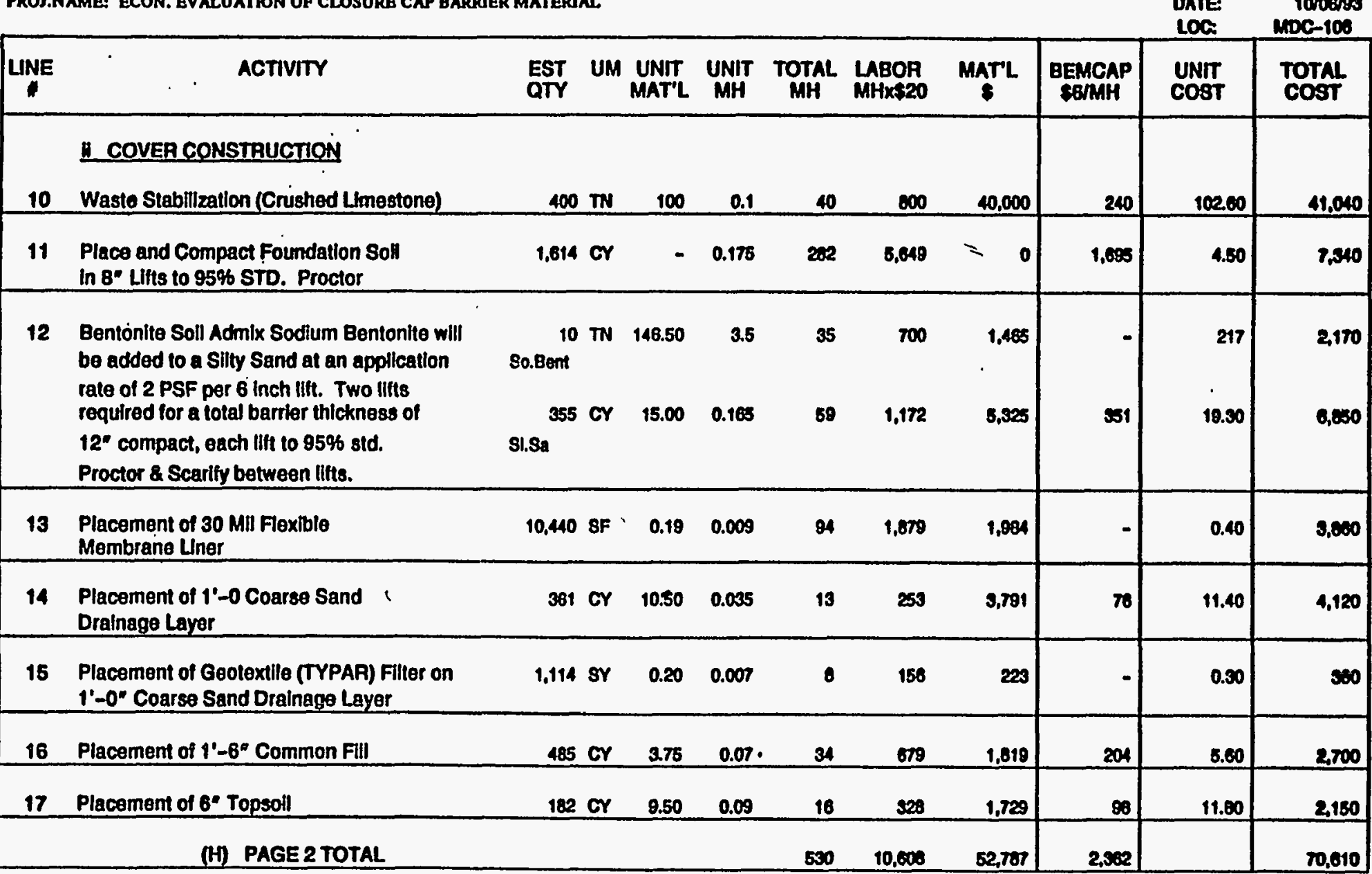

B-10. Cost Estimate for a Cover System Utilizing Bentonite Soil Additive and a 30-mil FML (Cont'd) 
D B T A I L B S T I M A T B

CHK'D BY: S. MEAD

Lod : 7-03

ACTIVITY CODE: ESE26O112

PROJ.NAME: ECON. BYALUATION OF CLOSURE CAP BARRIER MATERIAL

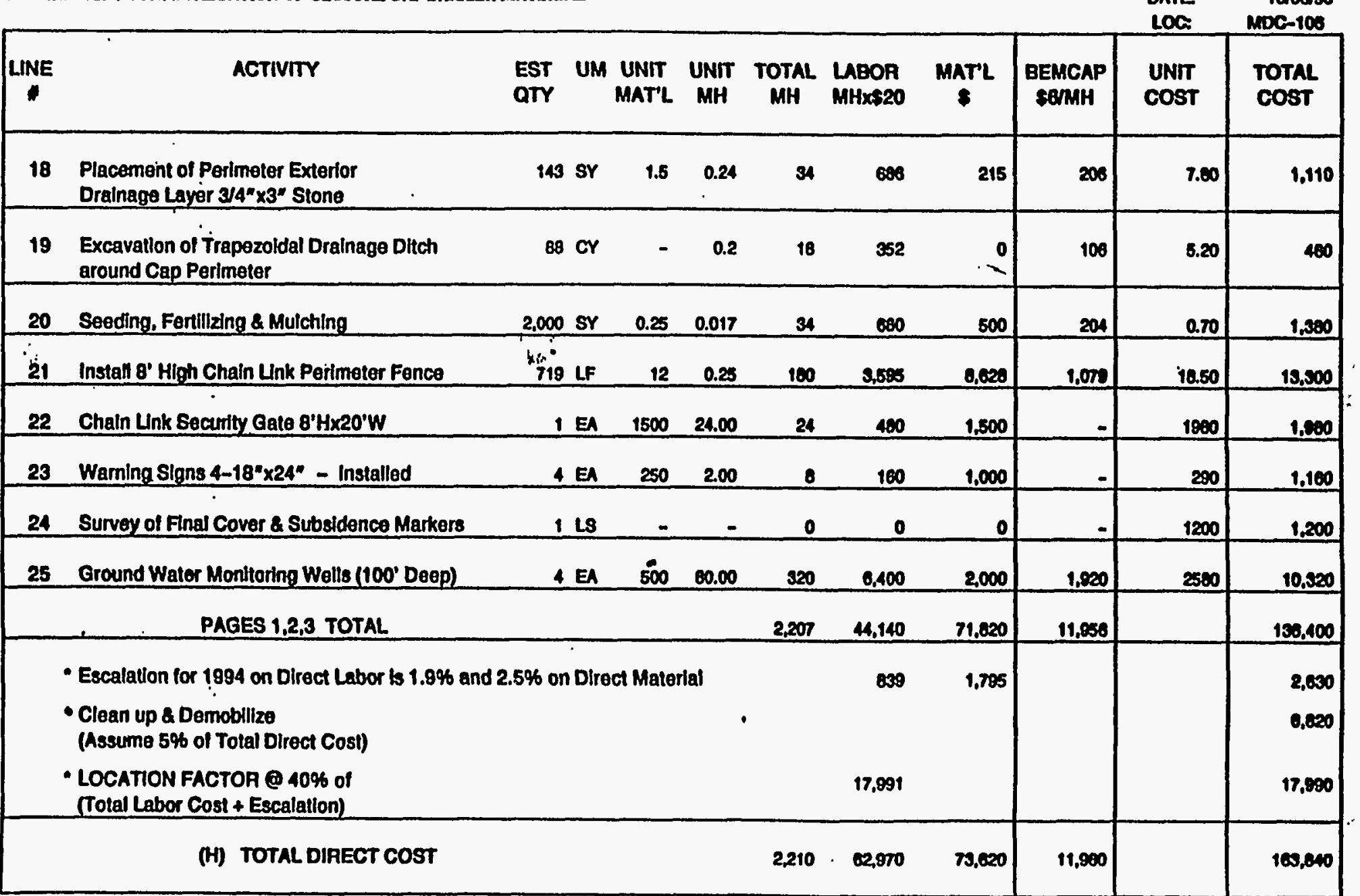

B-10. Cost Estimate for a Cover System Utilizing Bentonite Soil Additive and a 30-mil FML (Cont'd) 


\section{CHK'D BY: 8. MEAD}

$100 \%: 7-03$

ACTIVITY CODE: ESE260412

PROJ.NAME: ECON. EVALUATION OF CLOSURE CAP BARRIER MATERIAL.

\section{ESTO BY: PaMrK}

PHONE: 7-6200

DATE: $\quad$ 1000\%

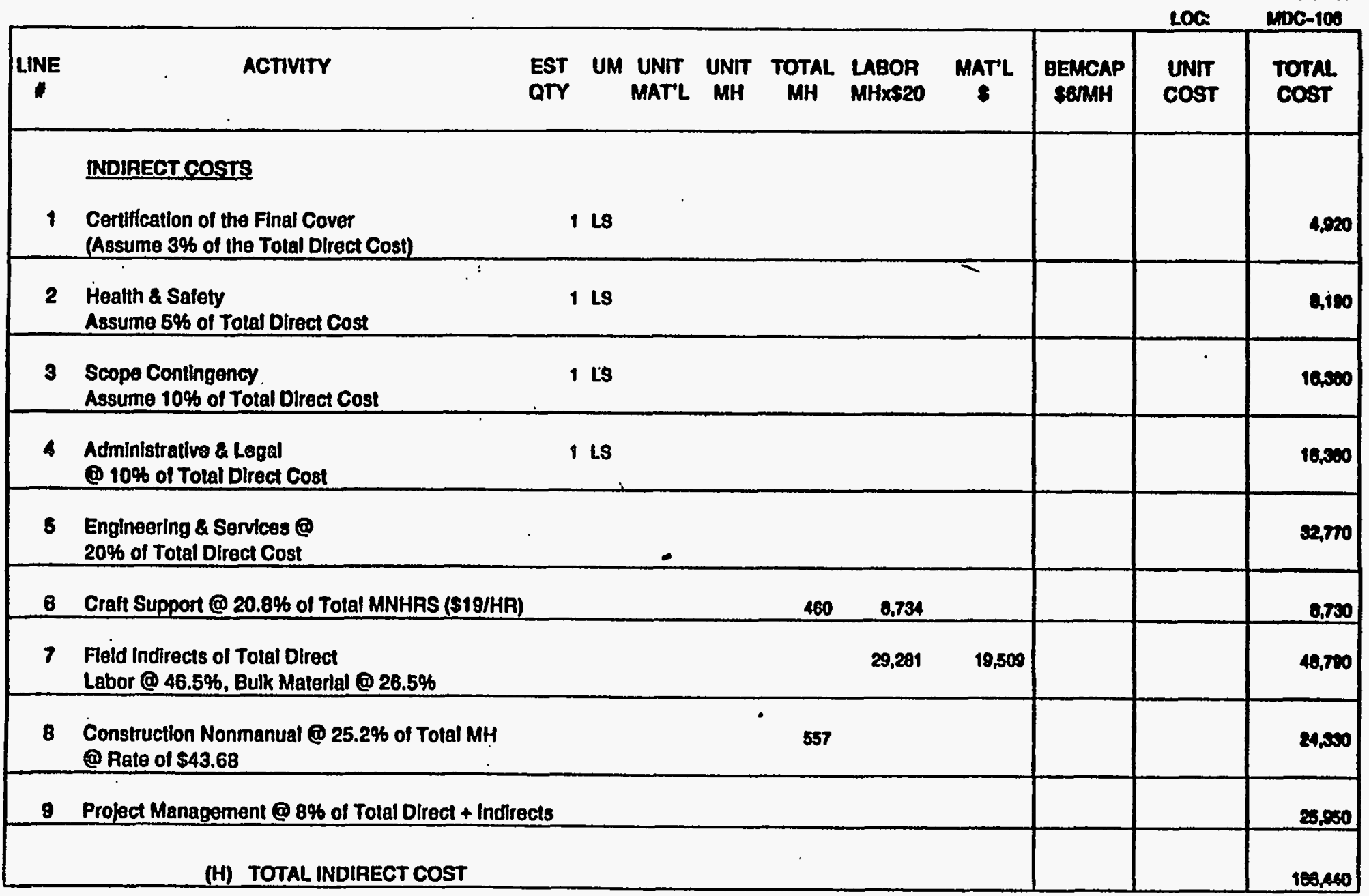

B-10. Cost Estimate for a Cover System Utilizing Bentonite Soil Additive and a 30-mil FML (Cont'd) 


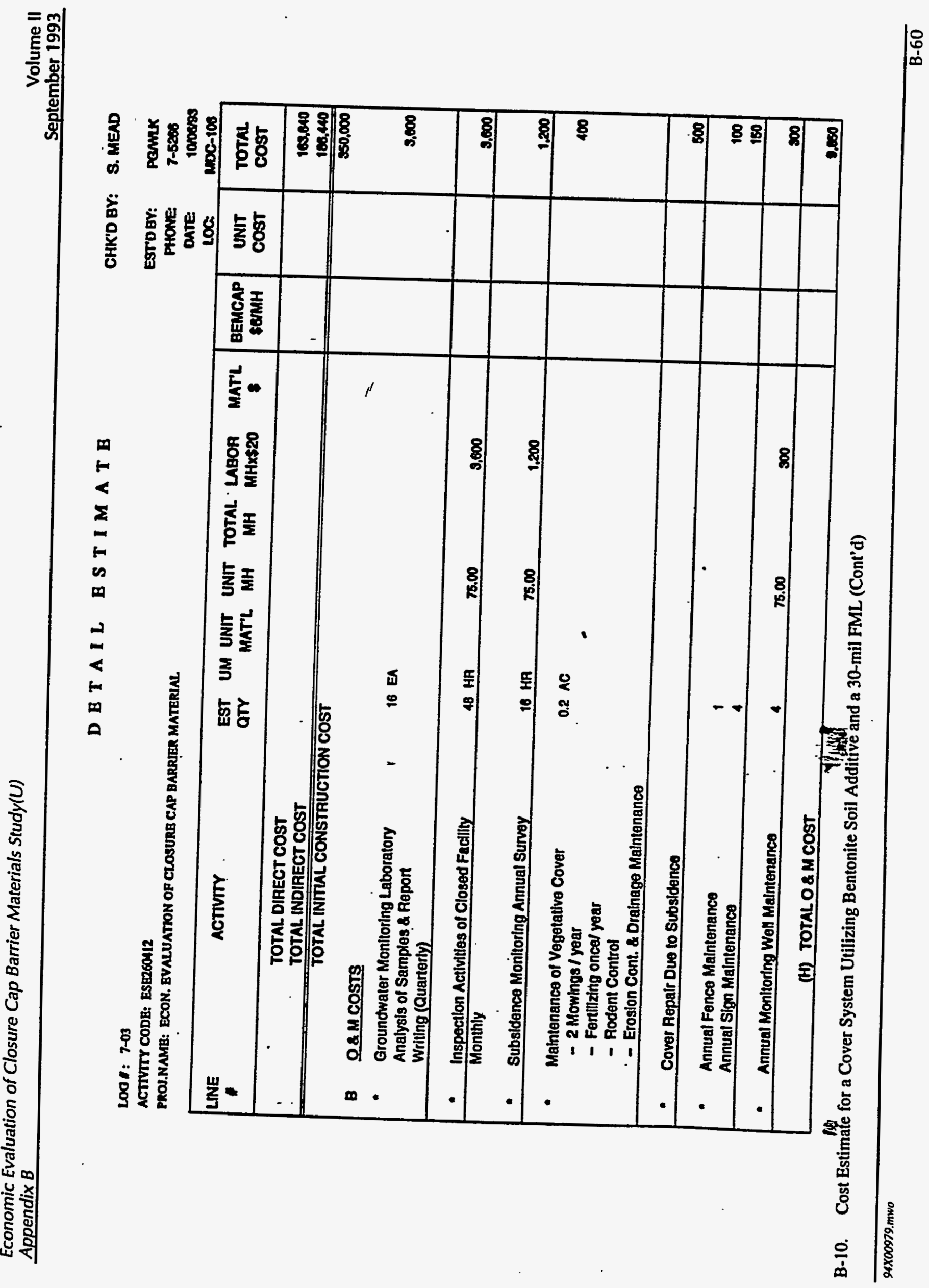




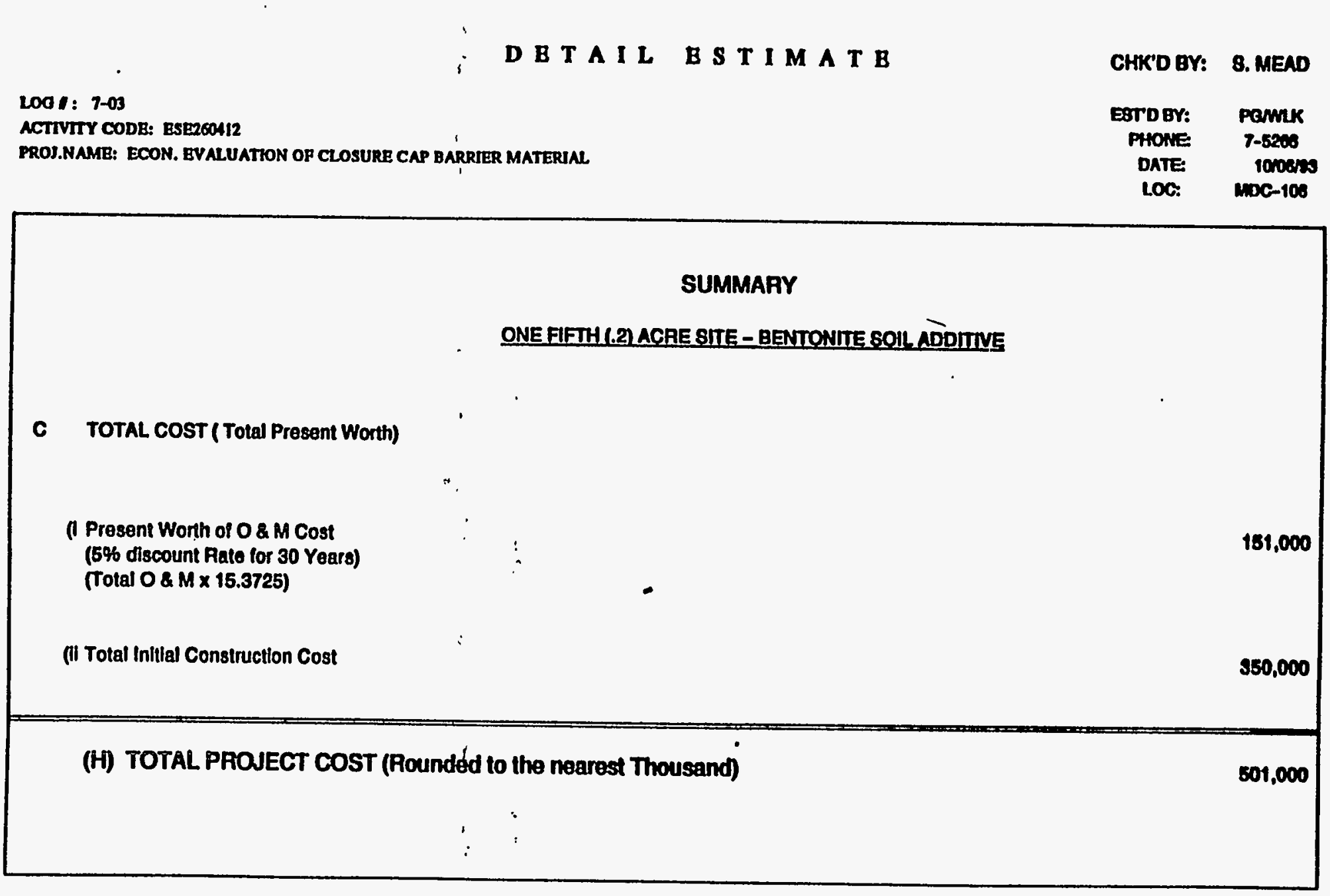

B-10. Cost Estimate for a Cover System Utilizing Bentonite Soil Additive and a 30-mil FML (Cont'd) 


\section{DETAILESTIMATE}

\section{CHK'D BY: 8. MEAD}

L00 I: $7-03$

ACTIVITY CODE: ESE200412

PROJ.NAME: ECON. EVALUATTON OR CLOSURE CAP BARRIER MATERIAL

ESTD BV:

PHONE

DATE

Panur

\begin{tabular}{|c|c|c|c|c|c|c|c|c|c|c|}
\hline LINE & ACTIVITY & $\begin{array}{l}\text { EST } \\
\text { QTY }\end{array}$ & UM & $\begin{array}{l}\text { UNIT } \\
\text { MATLL }\end{array}$ & $\underset{M H}{\text { UNT }}$ & $\begin{array}{c}\text { TOTAL LABOR } \\
\text { MH MHX\$20 }\end{array}$ & $\underset{1}{\operatorname{MATL}}$ & $\begin{array}{l}\text { BEMCAP } \\
\text { SEAMH }\end{array}$ & $\begin{array}{l}\text { UNTT } \\
\text { COST }\end{array}$ & $\begin{array}{l}\text { TOTAL } \\
\text { COST }\end{array}$ \\
\hline \multicolumn{11}{|c|}{$\begin{array}{l}\text { ONE FIFTH (.2) ACRE SITE - ASPHALTIC CONCAETE } \\
\text { A I DIRECT COSTS } \\
1 \text { SIIEPREPARATION }\end{array}$} \\
\hline 2 & Precontouring Site & 1.8 & AC & - & 32 & 1.152 & 0 & 340 & - & 1,5000 \\
\hline 3 & sili Fence (includes Maintenanco) & 855 & LF & 0.50 & 0.1 & 1.110 & 270 & - & 250 & 1,000 \\
\hline 4 & Temporary Sediment Control & 1 & LS & - & - & 0 & $\mathbf{0}$ & $\mathbf{\infty}$ & - & 200 \\
\hline 8 & 20' Wide Gravel Haut Road $\times 8^{\prime}$ Doop & 390 & LF & 2.50 & 0.22 & 1,716 & S,s16 & 816 & 14.20 & 8,500 \\
\hline 6 & 2-15" RCP Culvents & 64 & $\mathbf{L F}$ & 1200 & 1.00 & 1,280 & 700 & set & 80.00 & 2450 \\
\hline 7 & $35^{\prime} \times 35^{\prime} \times 4^{\prime \prime}$ Gravel Decon. Area & 160 & or & 3.00 & 0.10 & 320 & 400 & $\boldsymbol{\infty}$ & 8.00 & 200 \\
\hline $\boldsymbol{0}$ & $\begin{array}{l}\text { 6'x } \times 35 \text { 'x 4'Deep Wash Water Containment } \\
\text { Dilch }\end{array}$ & 35 & or & $10^{\prime}$ & 20 & $700 \quad 14,000$ & 350 & 4,200 & 650 & 10,000 \\
\hline 9 & $\begin{array}{l}\text { Construction Traller (Ronta) } \\
\text { (Include Power \& Water) }\end{array}$ & 3 & MO & - & - & 0 & 0 & - & 720 & 2,100 \\
\hline & (B) PAGE 1 TOTAL & & & & & 21,170 & 8,191 & 0,000 & & 34,800 \\
\hline
\end{tabular}

B-11. Cost Estimate for a Cover System Asphalt Concrete Hydraulic Barrier and a 30-mil FML 
DETAIL ESTIMATE

\section{0 : $7-03$}

ACTIVITY CODB: BSE2CO412

PROS.NAMR: ECON. EVALUATON OF CLOSURB CAP BARRIFR MATERUAL.

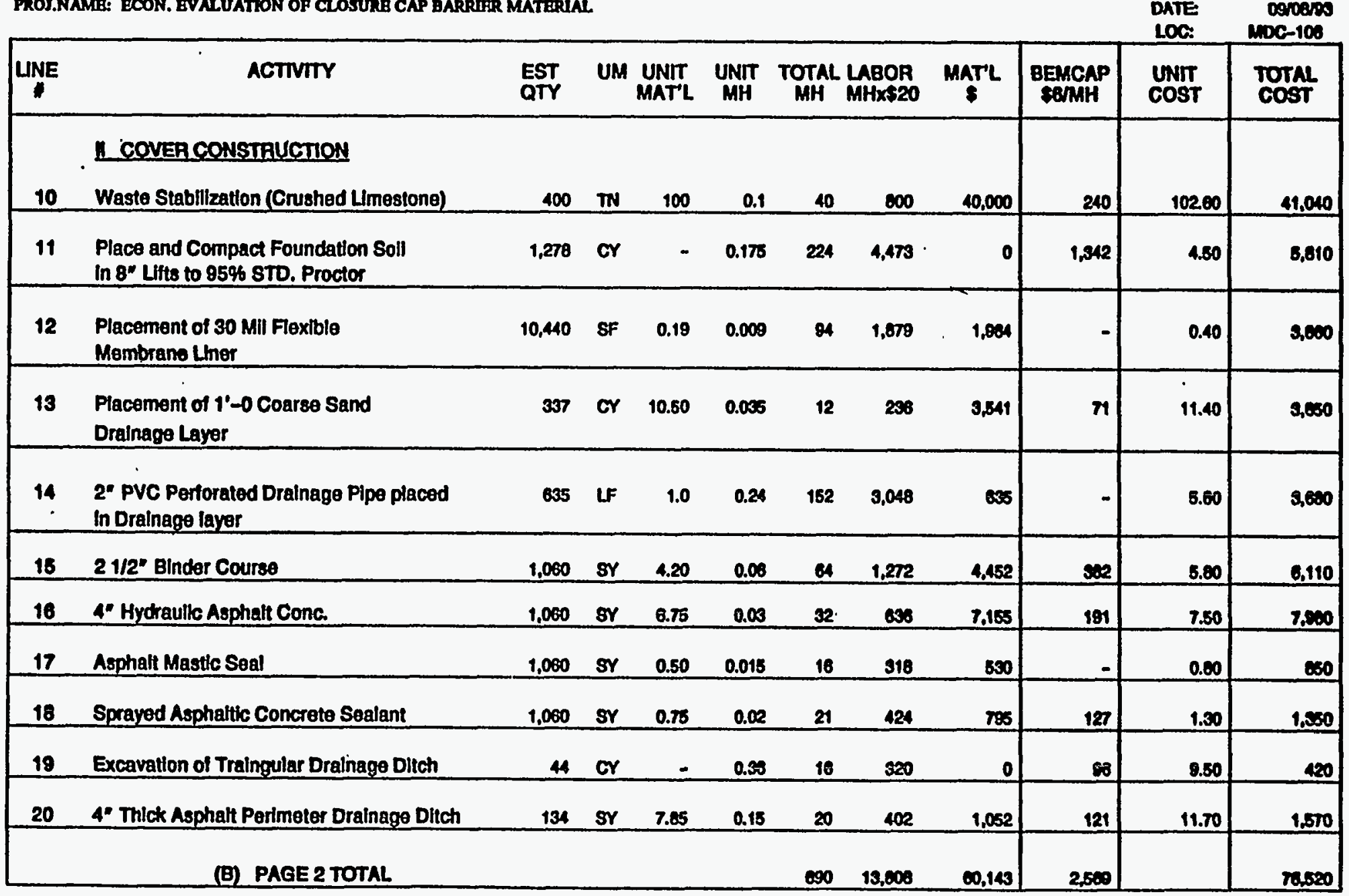

B-11. Cost Estimate for a Cover System Asphalt Concrete Hydraulic Barrier and a 30-mil FML (Cont'd) 
D B T A I L BSTIMAT B

1001: $7-03$

ACTIVITY CODB: ESE2OOH12

MROJ.NAMB: BCON. BVALUATION OF CLOSURB CAP GARRIER MATERLAL

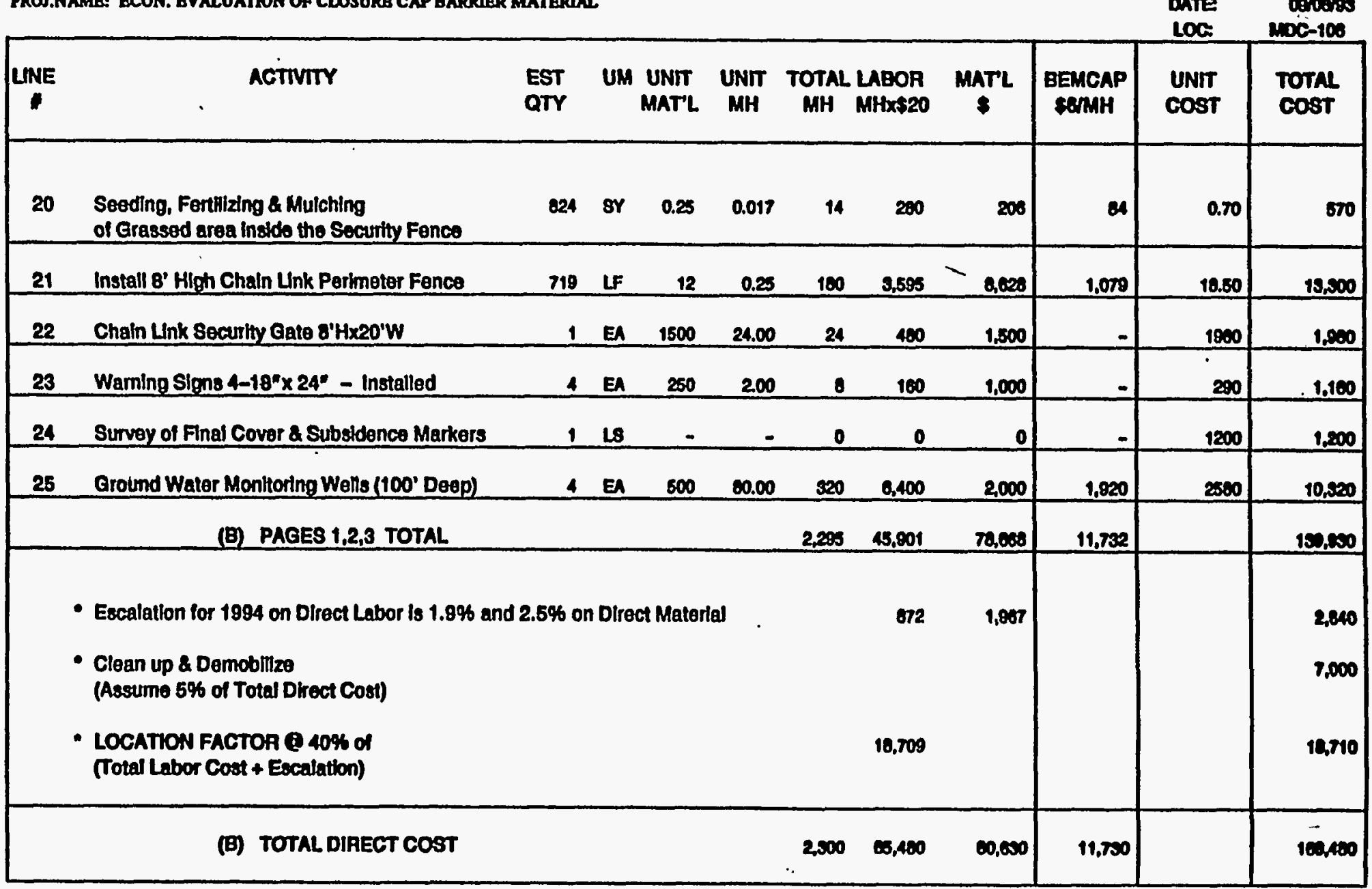

B-11. Cost Estimate for a Cover System Asphalt Concrete Hydraulic Barrier and a 30-mil FML (Cont'd) 
D E T A I L B S T I M A T B

Lo01: 7-03

ACTIVTTY CODB: BSEZSOA12

PROI.NAME: ECON. EVALUATTON OP CLOSURB CAP BARRIRR MATERJAL

\begin{tabular}{|c|c|c|c|c|c|c|c|c|}
\hline LNE & ACTIVITY & $\begin{array}{l}\text { UM UNIT } \\
\text { MAT'L }\end{array}$ & $\begin{array}{l}\text { UNIT } \\
\text { MH }\end{array}$ & $\begin{array}{l}\text { TOTAL LABOR } \\
\text { MH MHX\$20 }\end{array}$ & $\underset{\$}{\text { MATL }}$ & $\begin{array}{l}\text { BEACAP } \\
\text { SEMMH }\end{array}$ & $\begin{array}{l}\text { UNIT } \\
\text { COST }\end{array}$ & $\begin{array}{l}\text { TOTAL } \\
\text { Cost }\end{array}$ \\
\hline 1 & $\begin{array}{l}\text { INDIRECT COSTS } \\
\text { Certification of the Final Cover } \\
\text { (Assume } 3 \% \text { of the Total Direct Cost) }\end{array}$ & 28 & & & & & & 8,000 \\
\hline 2 & $\begin{array}{l}\text { Health \& Safety } \\
\text { Assume 6\% of Total Direct Cost }\end{array}$ & LS & & & $=$ & & & 0,420 \\
\hline 3 & $\begin{array}{l}\text { Scope Contingency } \\
\text { Assume } 10 \% \text { of Total Direct Cost }\end{array}$ & LS & & & & & . & 10,600 \\
\hline 4 & $\begin{array}{l}\text { Administrative \& Legal } \\
\text { (i) 10\% of Total Drect Cost }\end{array}$ & LS & & & & & & 18,850 \\
\hline B & $\begin{array}{l}\text { Engineering } 8 \text { Services @ } \\
20 \% \text { of Total Dlrect Cost }\end{array}$ & & & & & & & 58,700 \\
\hline 6 & Craft Support Q $20.8 \%$ of Total MNHAS (\$18/HA) & & & 9.090 & & & & 2.000 \\
\hline 7 & $\begin{array}{l}\text { Fleid Indirects of Total Dhect } \\
\text { Labor @ 48.5, Bukk Material @ 26.5\% }\end{array}$ & & & 30,440 & 21,307 & & & 81,020 \\
\hline 8 & $\begin{array}{l}\text { Consinucition Nonmanual @ } 25.2 \% \text { of Total Direct MH } \\
@ \text { Rate of } \$ 43.68\end{array}$ & & & 580 & & & & 20,520 \\
\hline 8 & Project Management @ 8\% of Total Direct + Indirects & & & & & & & 20,000 \\
\hline
\end{tabular}

B-11. Cost Estimate for a Cover System Asphalt Concrete Hydraulic Barrier and a 30-mil FML (Cont'd) 
CHKD BY: S. MEAD

200 I: 7-03

ACTIVITY CODB: ESEPOON12

PROJ.NAMR: BCON. GUALUATION OP CLOSURB CAP BARRIER MATERIAL

PTOQY: Pamk

PAONE $7-5000$

DATE

Loa. noc-10s

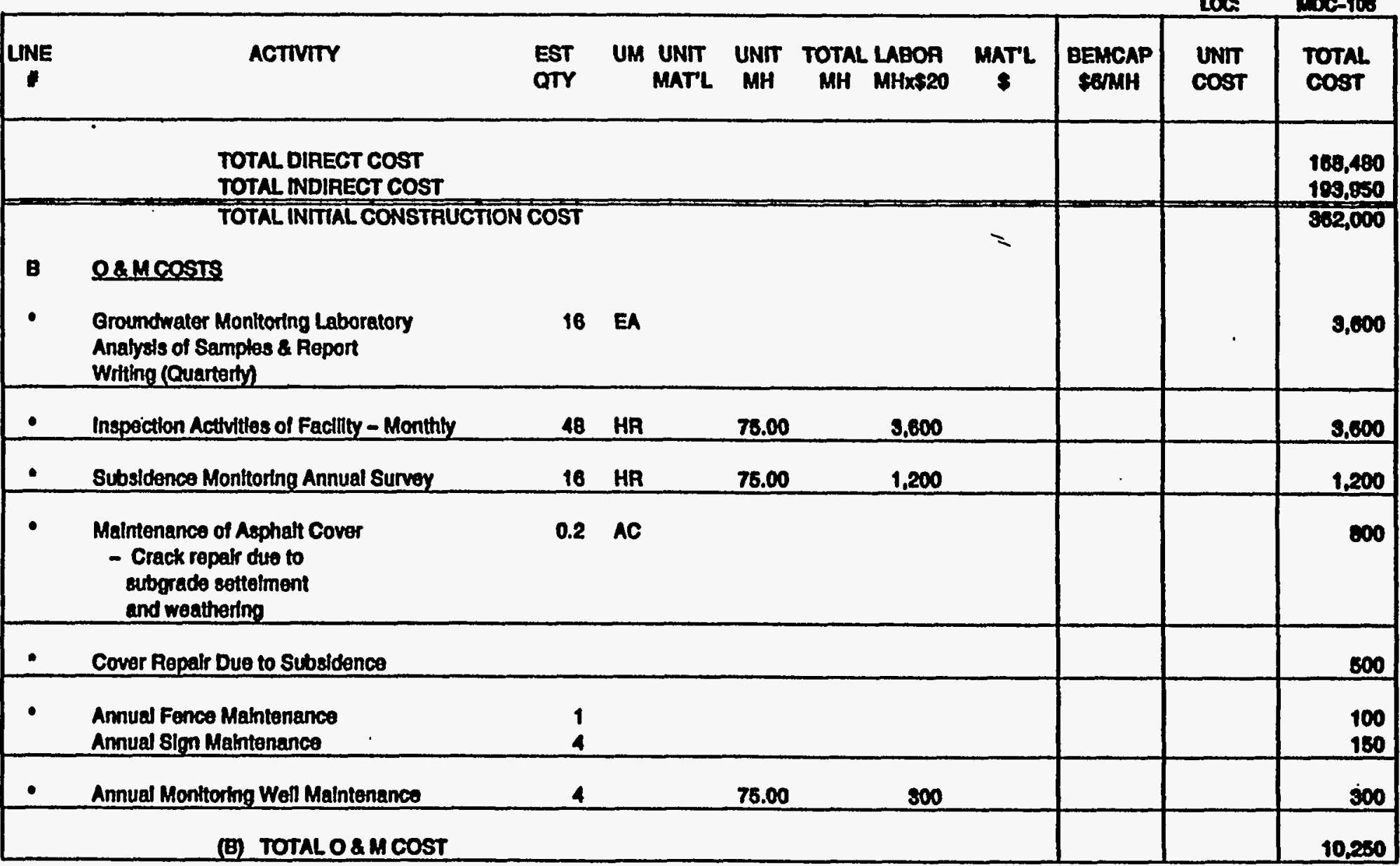

B-11. Cost Estimate for a Cover System Asphalt Concrete Hydraulic Barrier and a 30-mil FML (Cont'd) 
D B T A IL B S TIMAT B

\begin{tabular}{|c|c|}
\hline Cik & 8. MEAD \\
\hline $\begin{array}{l}\text { EST'D BY: } \\
\text { PHONE: } \\
\text { DATE: } \\
\text { LOC: }\end{array}$ & 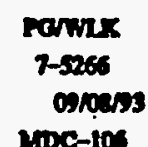 \\
\hline
\end{tabular}

Loof: $7-00$

ACTIVTY CODB: ESE200412

PROJ.NAMB: ECON. BVALUATION OP CLOSURB CAP BARRIER MATERUAL

LOC:

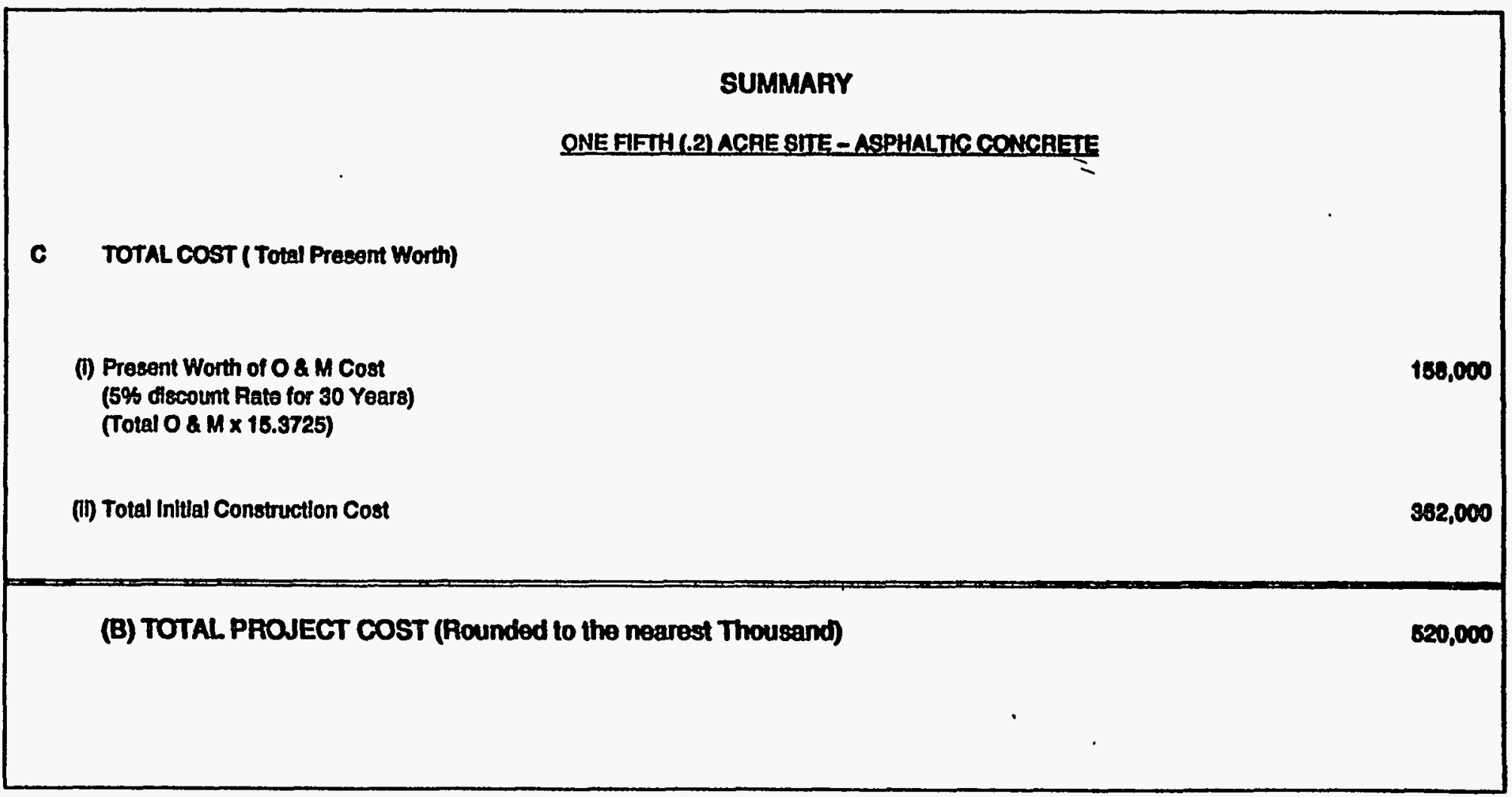

B-11. Cost Estimate for a Cover System Asphalt Concrete Hydraulic Barrier and a 30-mil FML (Cont'd) 
DETAIL ESTIMATE

LO01: 7-03

ACTINTY CODE ESE2COA12

PFOU.NAME: ECON. EVALUATION OF GLOSURE CAP BAFRAER MATERAN

CHK'D EY: 8. MEAD

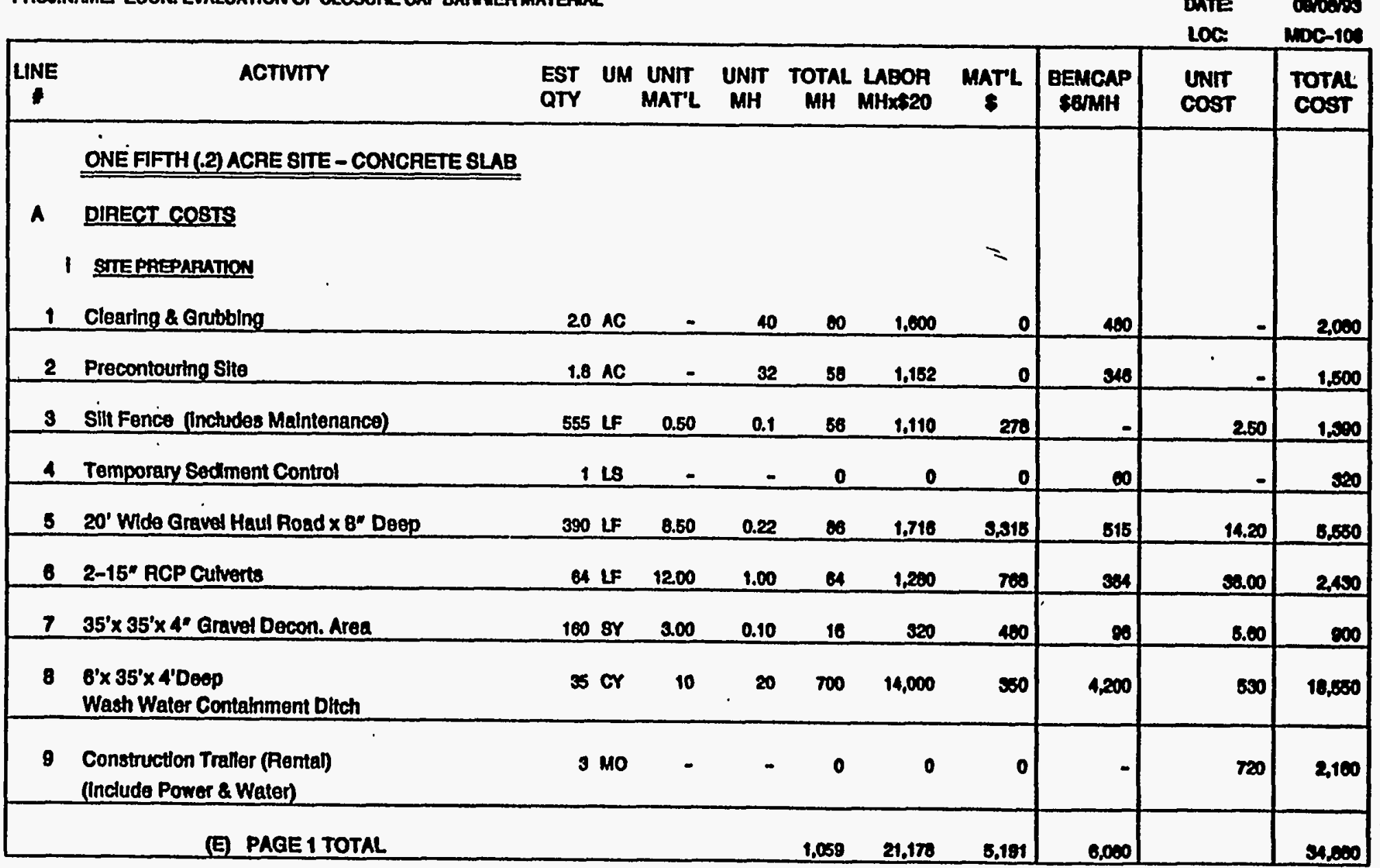

B-12. Cost Estimate for a Cover System Utilizing Poured-in Place Concrete Slab and a 30-mil FML 


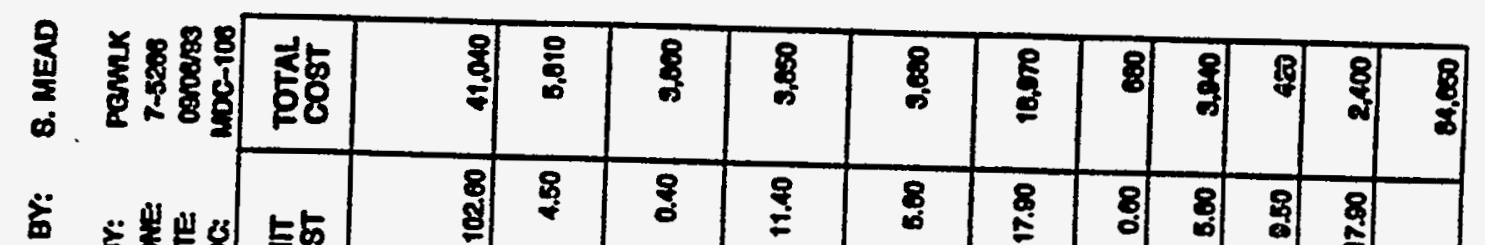

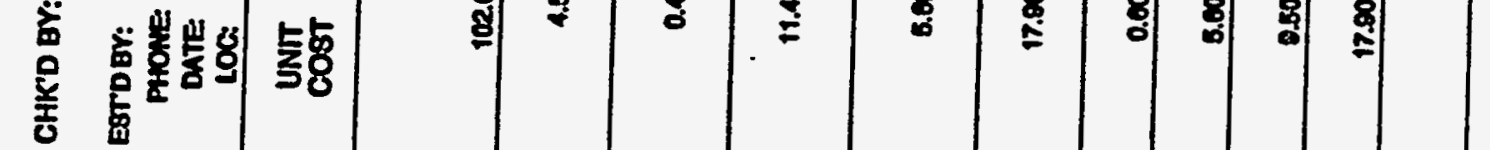

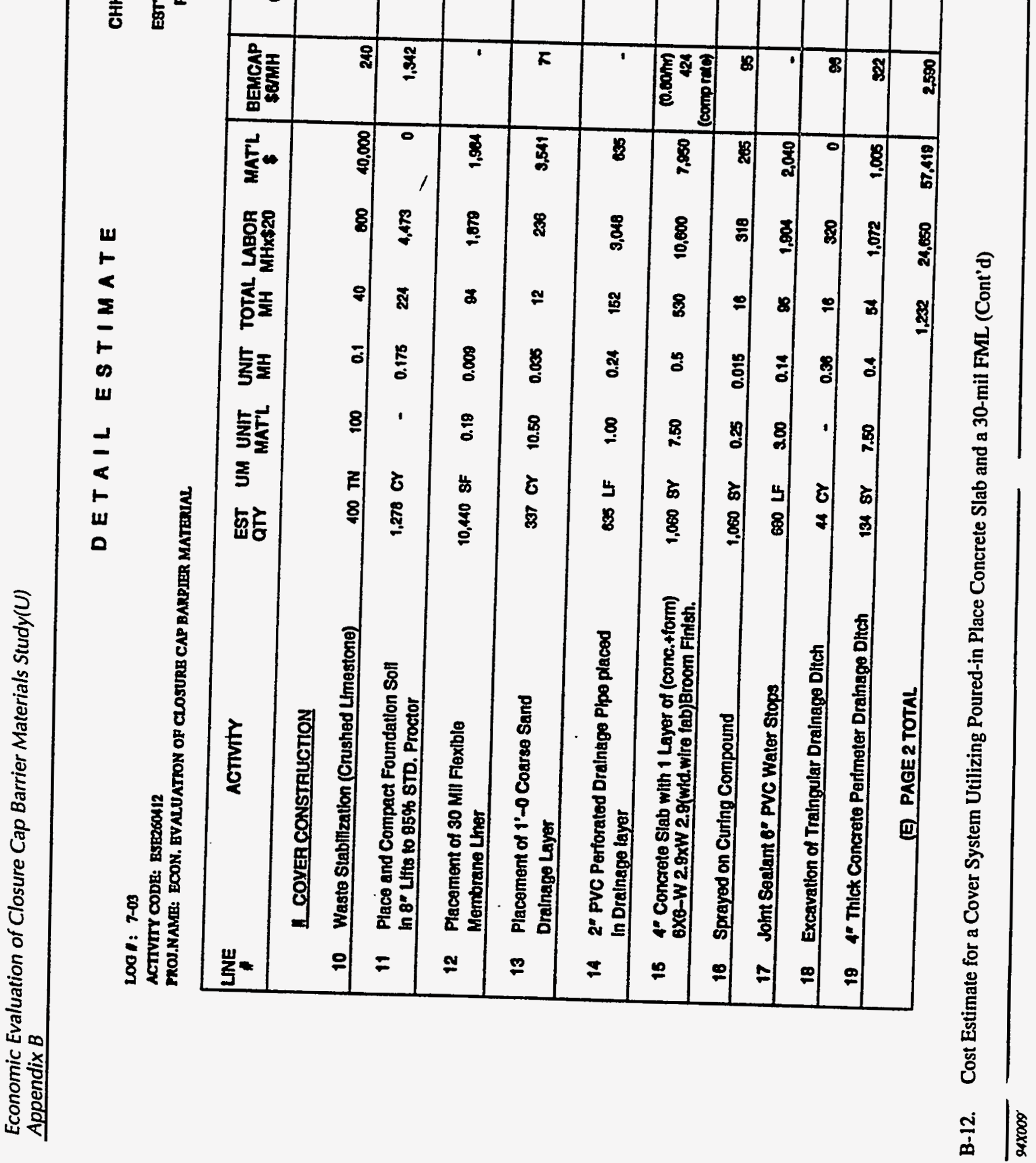




\section{B T A I L B S T I M A T B CHKDBY: S. MEAD}

L001: 7-03

ACTIVITY CODB: BSE2OOA12

PROJ.NAME: BCON. EYALUATTON OF CLOSURB CAP BARRIER MATERIAL.

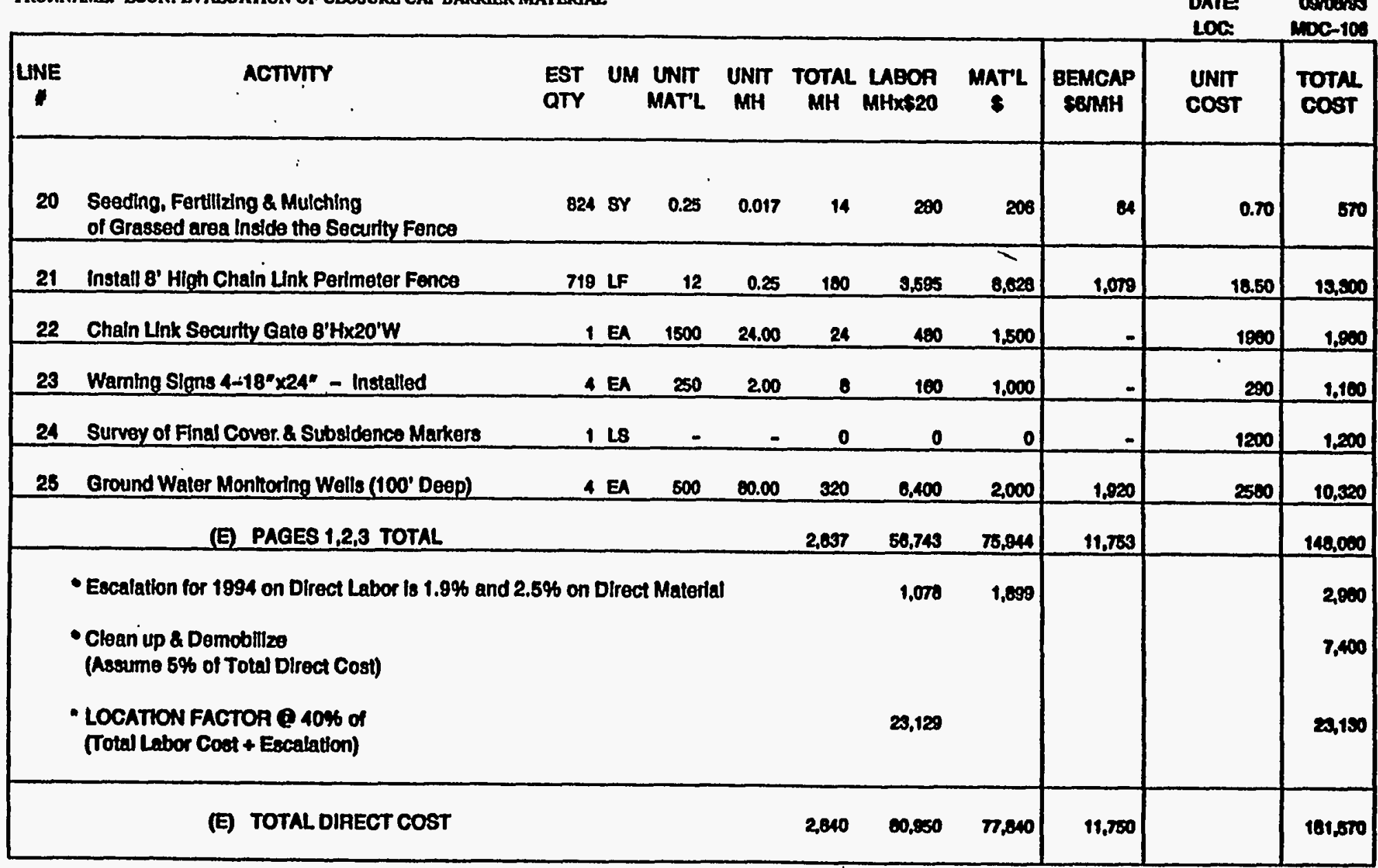

B-12. Cost Estimate for a Cover System Utilizing Poured-in Place Concrete Slab and a 30-mil FML (Cont'd) 
Lo01: 7-03

ACTVITY CODB: ESE20012

PROJ.NAME: ECON. EVALUATION OF CLOSURB CAP BARRIER MATERIAL.

D B T A I L B S T I M A T B

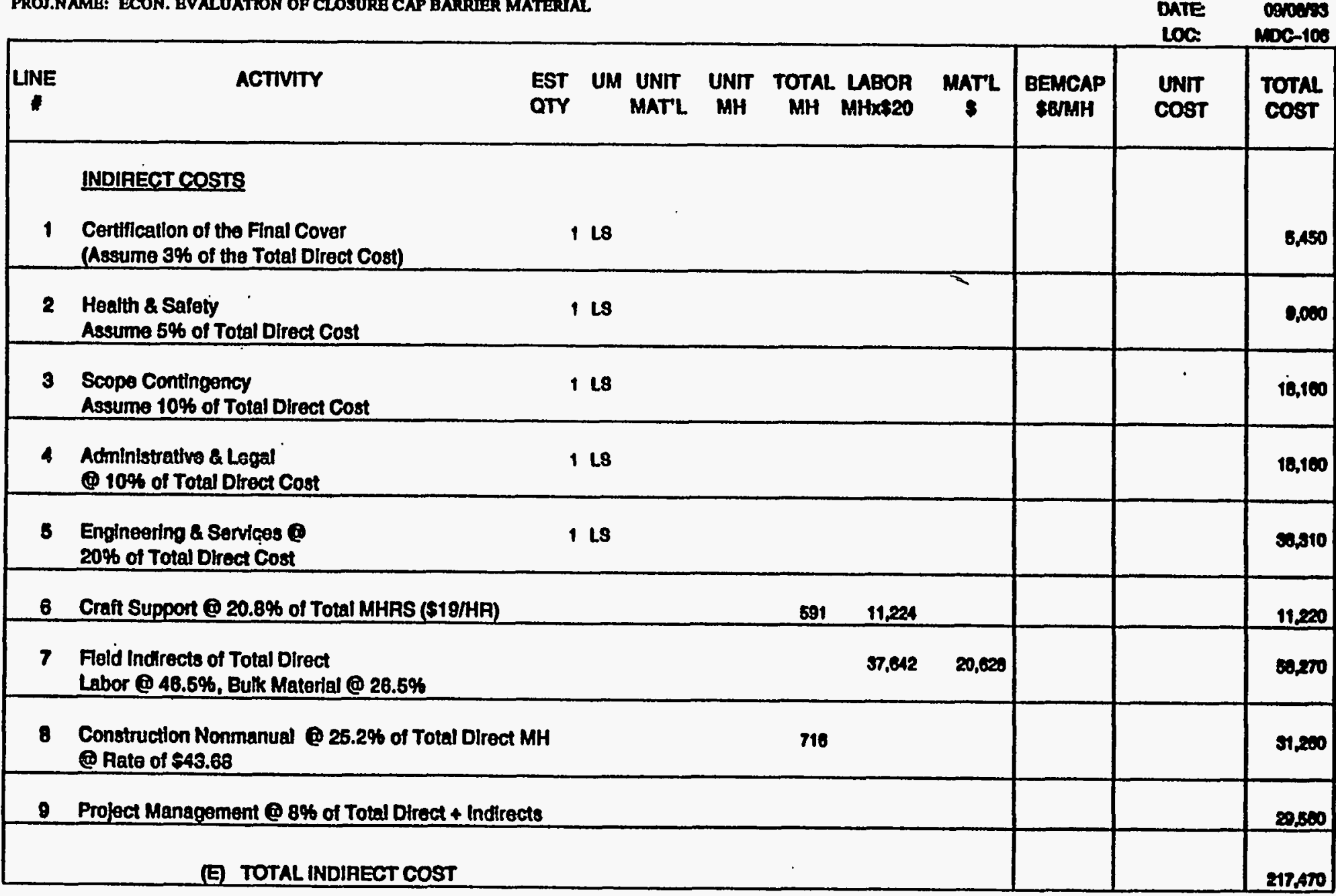

B-12. Cost Estimate for a Cover System Utilizing Poured-in Place Concrete Slab and a 30-mil FML (Cont'd) 
DETAIL B STIMATB

2001: $7-03$

ACTIVITY CODE: BSE26O412

PROJ.NAME: ECON. BVALUATION OP CLOSURB CAP BARRIRR MATERIAL

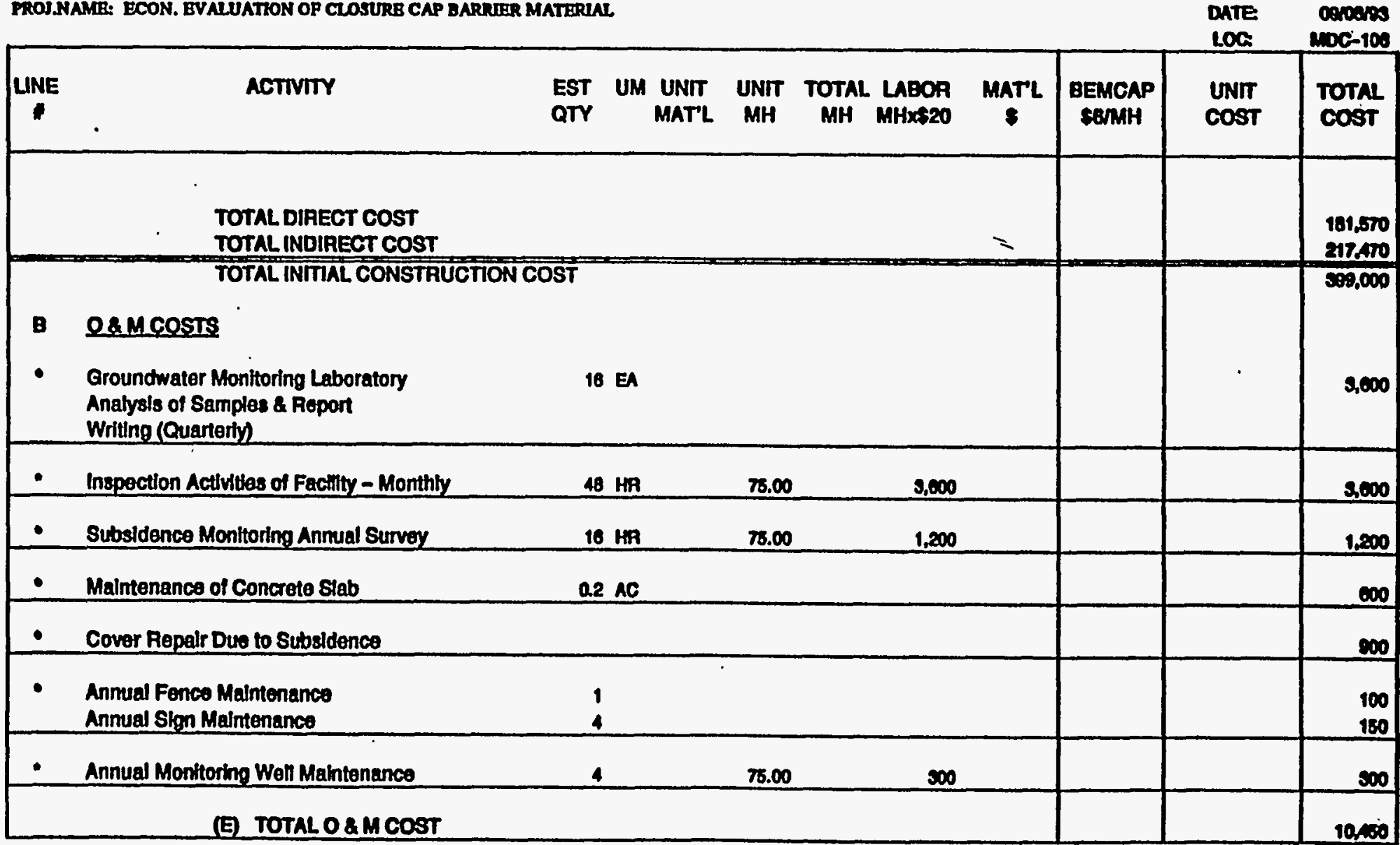

B-12. Cost Estimate for a Cover System Utilizing Poured-in Place Concrete Slab and a 30-mil FML (Cont'd) 
D B T A I L B S T I M A T B

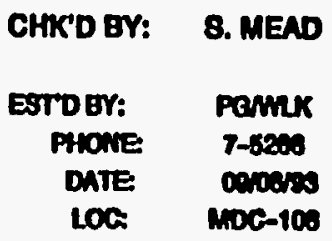

ACTIVTY CODB: BSB26012

PROJ.KAMR: BCON. EVALUATION OP CLOSURB CAP BARRIRR MATERINL

moc-100

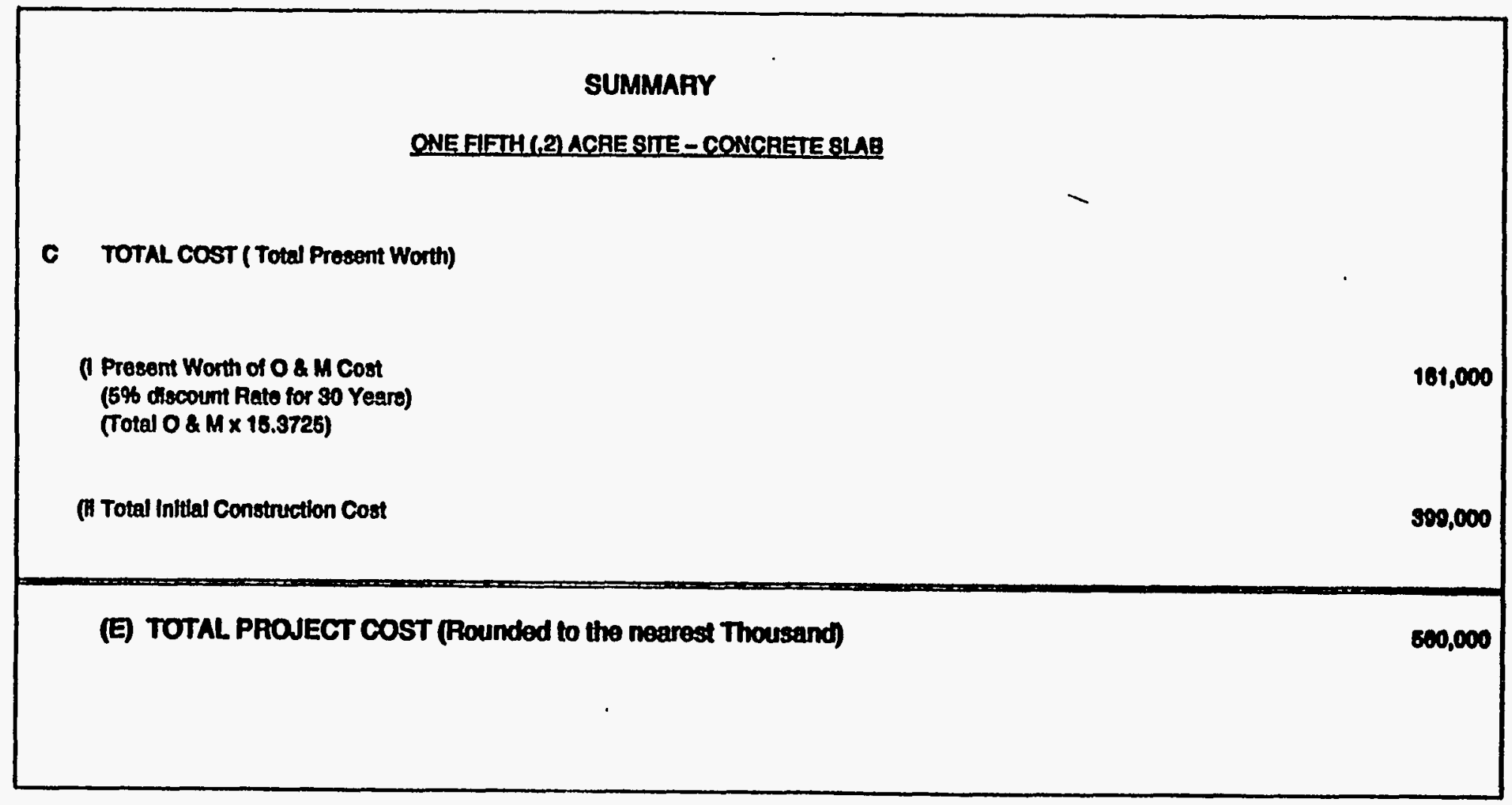

B-12. Cost Estimate for a Cover System Utilizing Poured-in Place Concrete Slab and a 30-mil FML (Cont'd) 


\section{Appendix C}

\section{Cost Estimates for 0.5 Acre Site}




\section{DETAIL ESTIMATE}

Lod : 7-03

ACTIVITY CODB: ESE260412

PROJ.NAME: BCON. EVALUATION OF CLOSURB CAP BARRIER MATERINL.

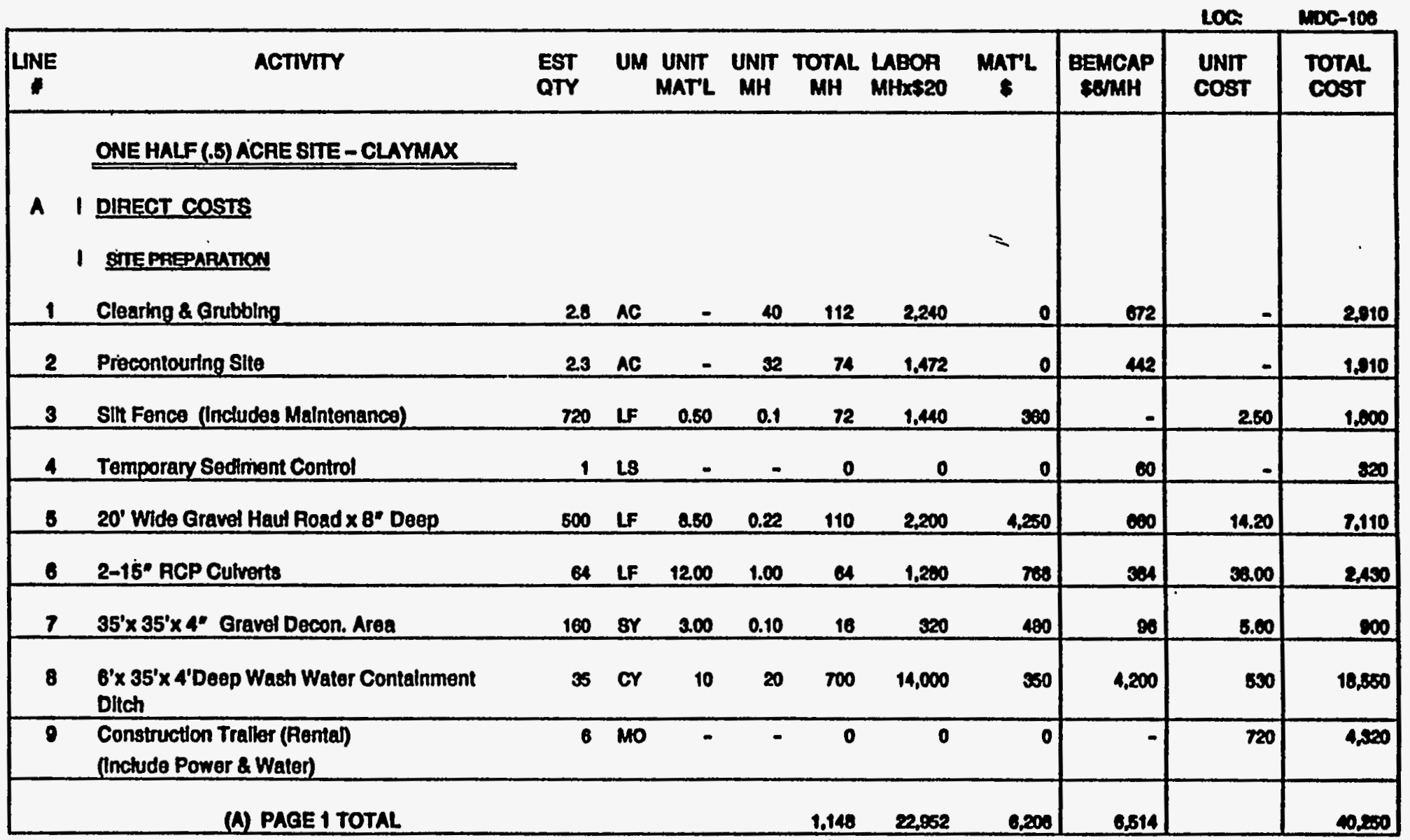

C-1. Cost Estimate for a Cover System Utilizing Claymax and a 30-mil FML 
DETAIL ESTIMATE CAKDEY QMEAD

L001: 7-03

ACTTVITY CODE: ESE260412

PROJ.NAME: ECON. EVALUATION OF CLOSURB CAP BARRIER MATERIAL

ESTO BY: Pamk

DATE

\begin{tabular}{|c|c|c|c|c|c|c|c|c|c|c|c|}
\hline UNE & ACTIVTY & $\begin{array}{l}\text { EST } \\
\text { QTY }\end{array}$ & UM & $\underset{\text { MAT'L }}{\text { UNIT }}$ & UNIT & $\underset{\mathbf{M H}}{\text { TOTAL }}$ & $\begin{array}{l}\text { LABOR } \\
\text { MHXS20 }\end{array}$ & MaTL & $\underset{\text { EEMMH }}{\text { BEMCAP }}$ & $\begin{array}{l}\text { UNm } \\
\text { COST }\end{array}$ & $\begin{array}{l}\text { Total } \\
\text { cost }\end{array}$ \\
\hline 10 & $\begin{array}{l}\text { D COVER CONSTRUCTION } \\
\text { Waste Stablization (Crushod Limestone) }\end{array}$ & 1.007 & $\mathbf{T N}$ & 100 & 0.1 & 101 & 2,014 & 100,700 & 604 & 10200 & \\
\hline 11 & $\begin{array}{l}\text { Place and Compact Foundation Soll } \\
\text { in 8" Litts to 95\% STD. Proctor }\end{array}$ & 4.035 & Cr & - & 0.163 & 008 & 13,318 & $=$ & 3,995 & 4.50 & 17,310 \\
\hline 12 & Placement of Claymax Geolextile Mat & 30,800 & SF & 0.50 & 0.01 & 300 & 0.180 & 15,400 & - & a.ro & 21,500 \\
\hline 13 & $\begin{array}{l}\text { Placement of } 30 \text { Mil Flexlblo } \\
\text { Membrane Liner }\end{array}$ & 30,800 & 8 & 0.18 & 0.000 & 246 & 4.928 & 5,544 & -1 & 0.30 & 10,470 \\
\hline 14 & $\begin{array}{l}\text { Placement of 1'-0 Coarse Sand } \\
\text { Drainage Layer }\end{array}$ & 1,082 & Cr & 10.50 & 0.035 & 37 & 743 & 11,181 & 223 & 11.40 & 12,120 \\
\hline 16 & $\begin{array}{l}\text { Placement of Geotextile (TYPAR) Filter on } \\
\text { 1'-0" Coarse Sand Oralnage Layer }\end{array}$ & 3,506 & sr & 0.10 & 0.006 & 21 & 421 & 691 & -1 & 0.30 & 1,050 \\
\hline 16 & Placement of 1'-6' Common FIn & 1,212 & or & 3.75 & 0.07 & $\infty$ & 1,009 & 4,545 & 500 & 8.00 & 0.750 \\
\hline 17 & Placement of 6" Topsoll & 455 & cy & 9.50 & 0.00 & 38 & 727 & 4,318 & 210 & 11.00 & 8.200 \\
\hline 18 & $\begin{array}{l}\text { Placement of Perimeter Exterior } \\
\text { Oralnage Layer } 34^{*} \times 3^{*} \text { DP (Crushed Stone) }\end{array}$ & 216 & SY & 1.50 & 0.22 & 40 & 850 & 324 & $2 \%$ & 7.20 & 1,600 \\
\hline 19 & Excavation of Trapezoldal Drainage Ditch & 221 & or & - & 0.185 & 41 & 818 & 0 & 245 & 4.00 & 1.000 \\
\hline & (A) PAGE 2 TOTAL & & & & & 1.500 & st.m2 & 142,619 & 0,000 & & 100,100 \\
\hline
\end{tabular}

C-1. Cost Estimate for a Cover System Utilizing Claymax@ and a 30-mil FML (Cont'd) 
D B T A L B S T IMATE

Lo01: $7-03$

ACTIVITY CODE: ESE260112

MROS.NAME: ECON. EVALUATION OP CLOSURB CAP BARRIER MATERIAL

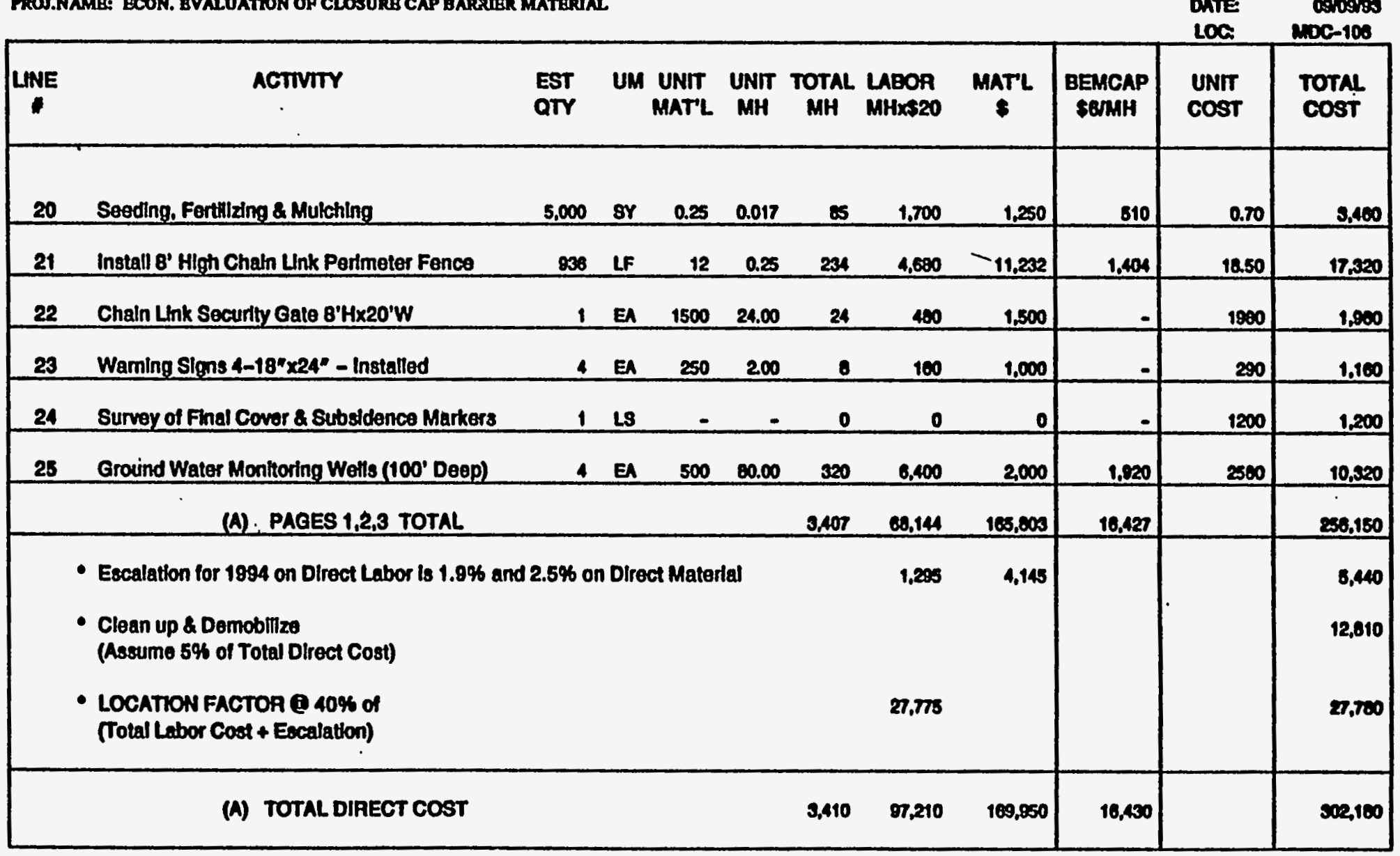

C-1. Cost Estimate for a Cover System Utilizing Claymax and a 30-mil FML (Cont'd) 
D B T A I L B S T I M T B CAKDOY: Q.MEAO

Lo01: $7-03$

ACTIVITY CODB: ESE260412

PROU.NAME: ECON. EVALUATTON OF CLOSURB CAP BARRIER MATERIAL.

MTO BY: Pamk

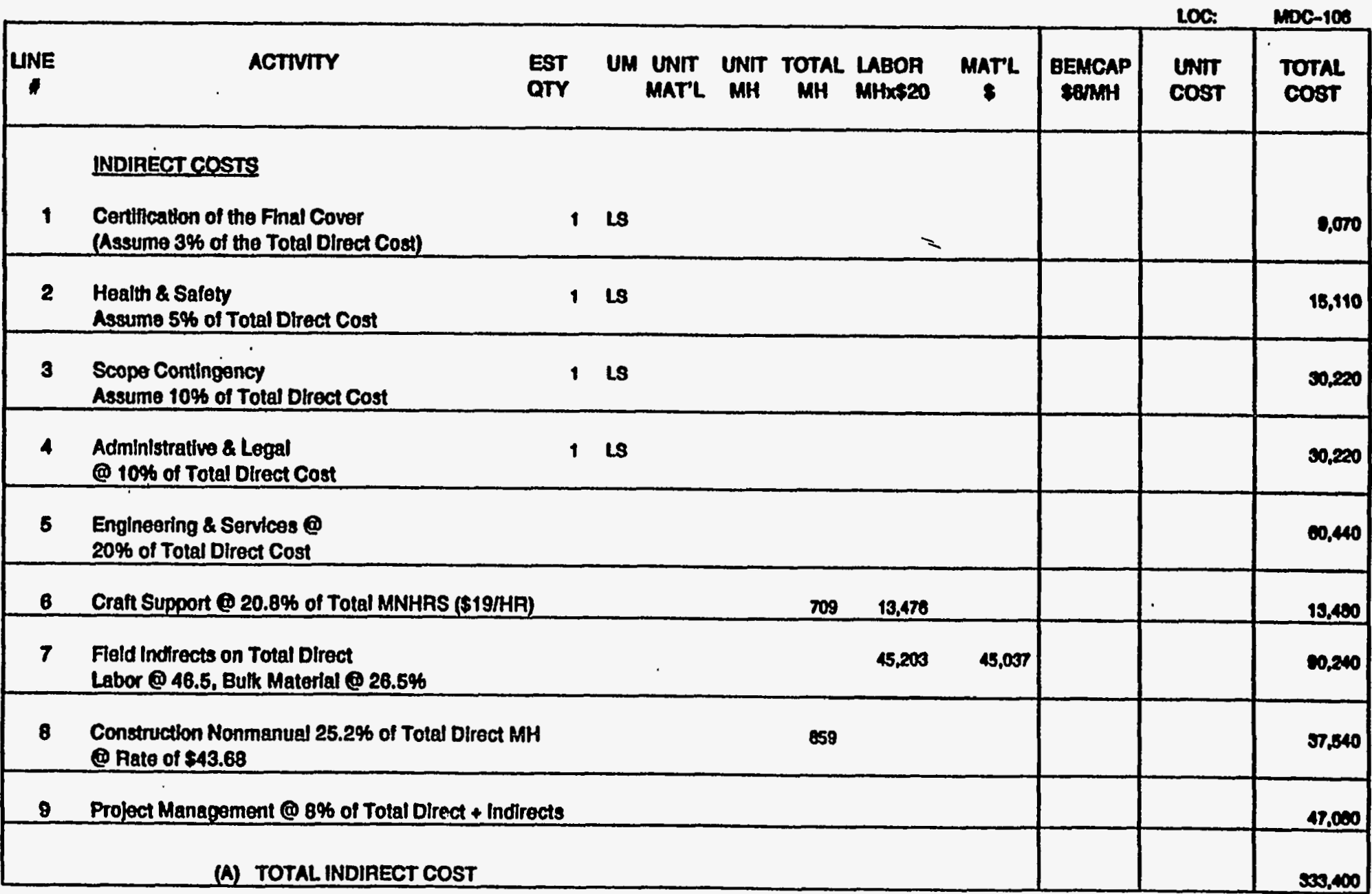

C-1. Cost Estimate for a Cover System Utilizing Claymax and a 30-mil FML (Cont'd) 


\section{LOO I: $7-03$}

ACTIVITY CODE: ESE260412

PROJ.AAMB: ECON. EVALUATION OR CLOSURB CAP BARRRER MATERIAL

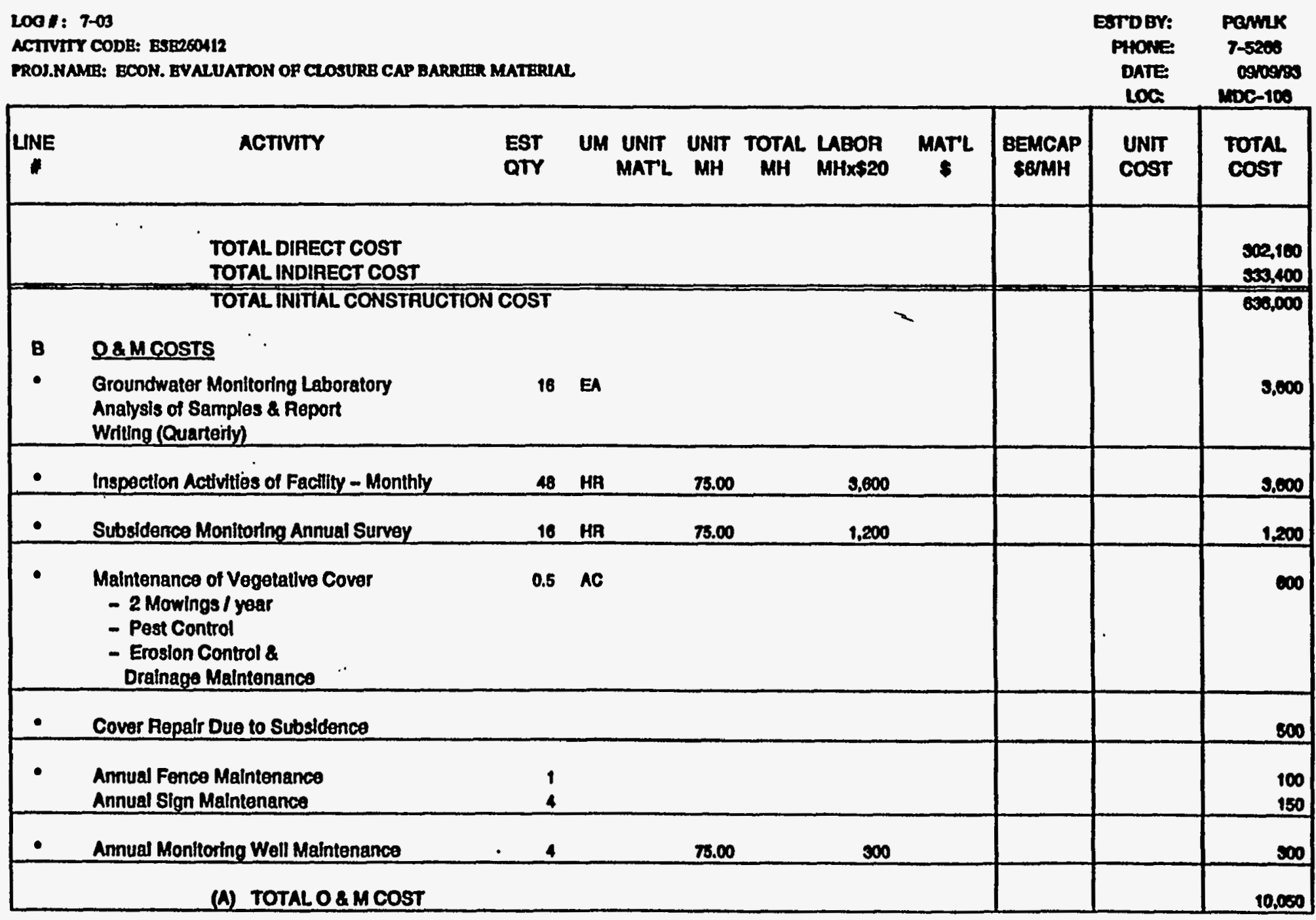

C-1. Cost Estimate for a Cover System Utilizing Claymax and a 30-mil FML (Cont'd) 


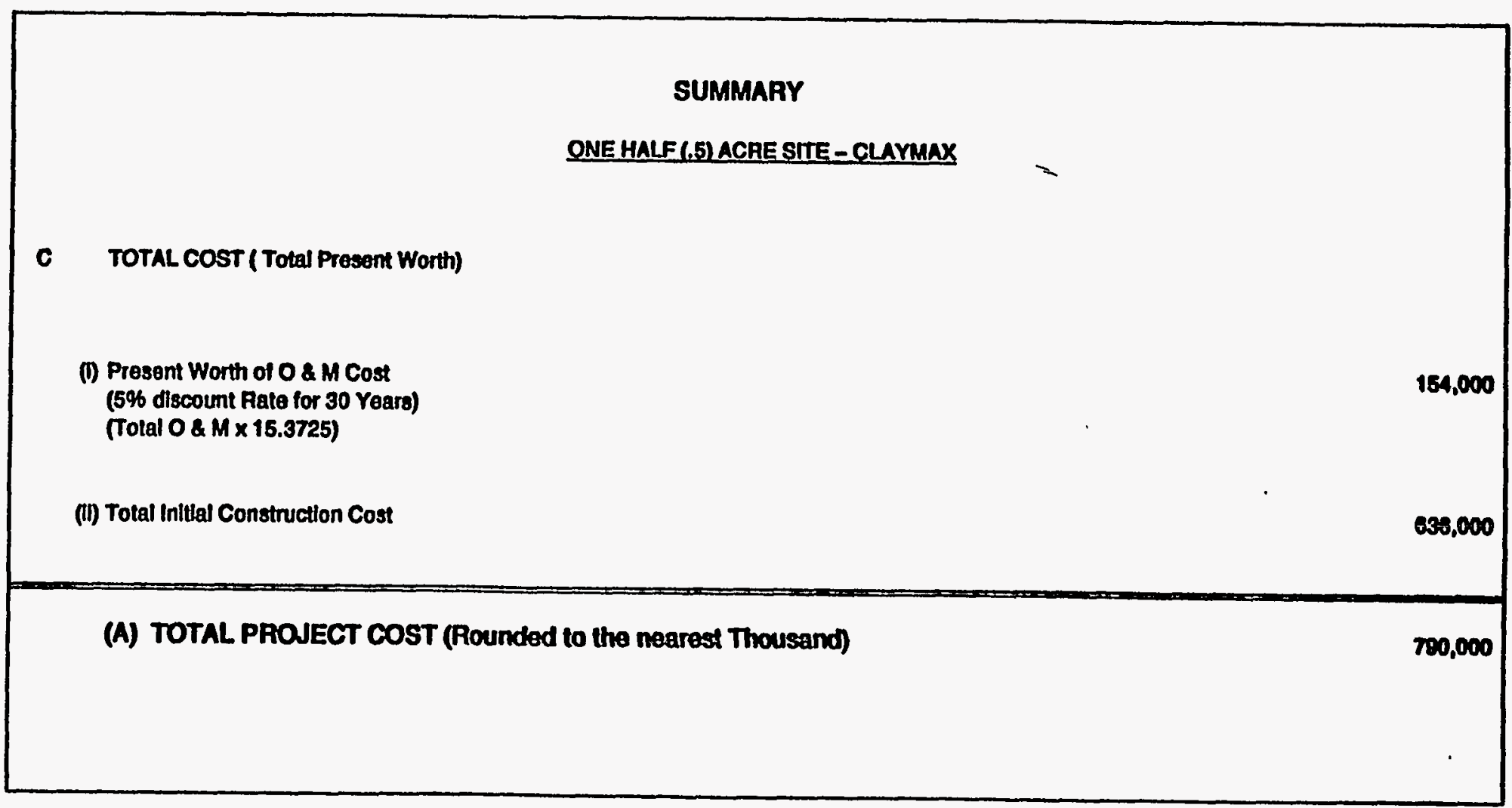

C-1. Cost Estimate for a Cover System Utilizing Claymax@ and a 30-mil FML (Cont'd) 


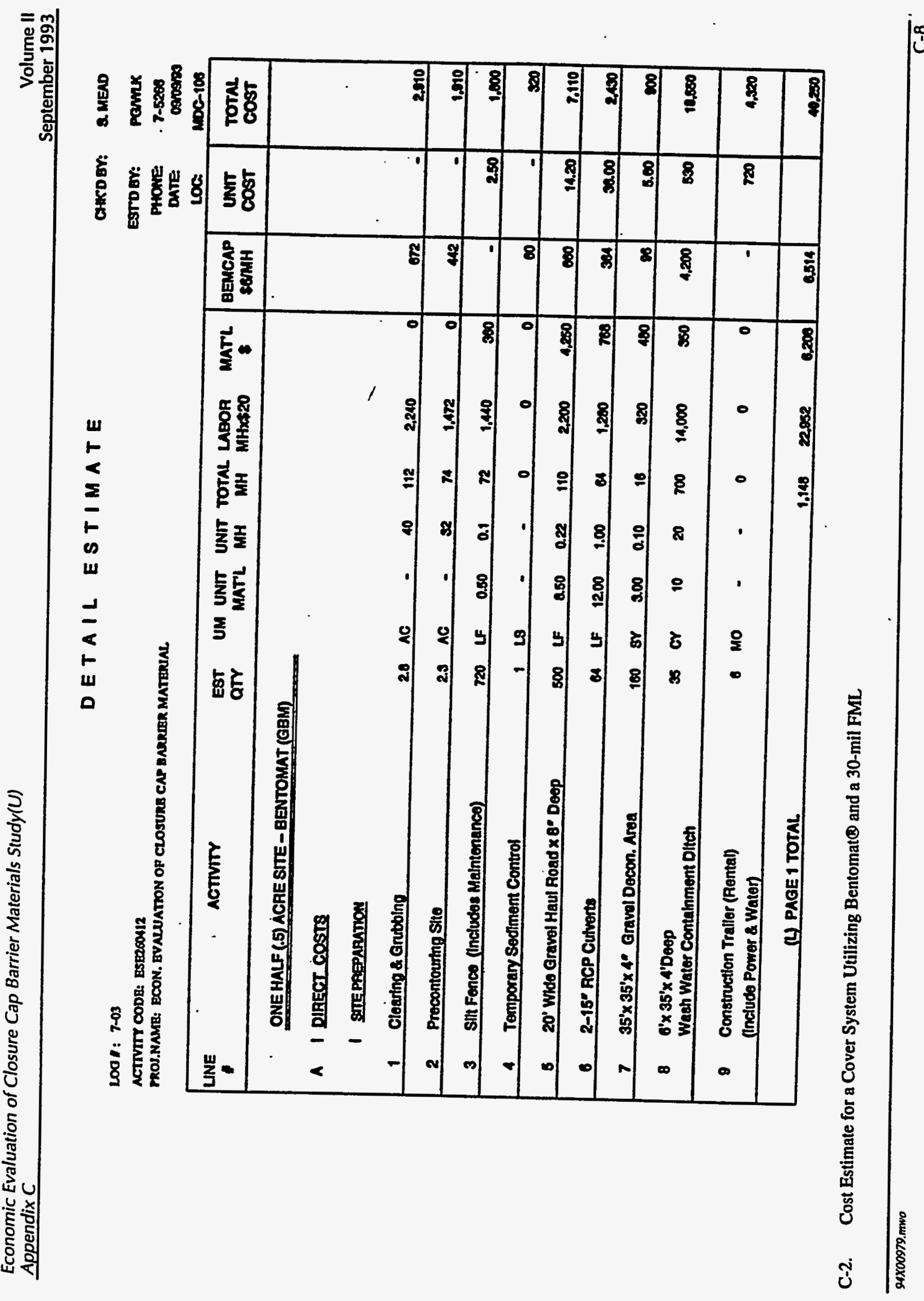


DETAIL ESTIMATE

CAKD BY: QMEAD

Lod : $7-03$

ACIIVITY CODB: BSE260112

PROJ.NAMR: BCON. BVALUATHOH OP CLOSURB CAP ENRURER MATERIAL

\begin{tabular}{|c|c|c|c|c|c|c|c|c|c|c|c|}
\hline UNE & ACTINTY & $\begin{array}{l}\text { EST } \\
\text { QTY }\end{array}$ & UM & $\begin{array}{l}\text { UNTT } \\
\text { MATLL }\end{array}$ & $\begin{array}{l}\text { UNIT } \\
\text { MH }\end{array}$ & $\underset{\text { MH }}{\text { TOTAL }}$ & $\begin{array}{l}\text { LABOA } \\
\text { MHLxS20 }\end{array}$ & $\underset{\$}{\operatorname{math}}$ & $\begin{array}{l}\text { BEHCAP } \\
\text { SBNMH }\end{array}$ & $\begin{array}{l}\text { UNT } \\
\text { COST }\end{array}$ & $\begin{array}{l}\text { Total } \\
\text { cost }\end{array}$ \\
\hline 10 & $\begin{array}{l}\text { CONER CONSTAUCTION } \\
\text { Waste Stabilization (Crushad Umastono) }\end{array}$ & 1,007 & $\mathbf{m}$ & 100 & 0.1 & 101 & 2,014 & 100,700 & $\infty$ & 10200 & $100,3 \times 0$ \\
\hline 11 & $\begin{array}{l}\text { Place and Compact Foundation Soll } \\
\text { in } 8^{\circ} \text { Litis to } 95 \% \text { 8TD. Proctor }\end{array}$ & 4,035 & or & - & 0.165 & 666 & 13,310 & - & 3,086 & 4.80 & 17,310 \\
\hline 12 & Placement of Bentonite Geotextine Mat & 30,600 & 8F & 0.50 & 0.01 & 300 & 6.160 & 15,400 & - & 0.70 & 21.500 \\
\hline 19 & $\begin{array}{l}\text { Placoment of } 30 \text { Mr Floxible } \\
\text { Membrane Lner }\end{array}$ & 30,600 & 8F & 0.18 & 0.000 & 248 & 4,920 & B.344 & - & 0.30 & 10,470 \\
\hline 14 & $\begin{array}{l}\text { Placement of t'-0 Coarsa Sand } \\
\text { Drainage Layer }\end{array}$ & 1,082 & CY & $\mathbf{8 0 . 5 0}$ & 0.035 & $n$ & 743 & 11,151 & 220 & 11.40 & 12,120 \\
\hline 15 & $\begin{array}{l}\text { Placement of Geotextle (TYPAF) Fiter on } \\
1^{\prime}-0^{\prime} \text { Coaree Sand Dralnage Layer }\end{array}$ & 3,508 & SY & 0.18 & 0.006 & 21 & 421 & 601 & - & 0.30 & 1,050 \\
\hline 16 & Placement of 1'-6' Common FIII & 1,212 & cr & 3.75 & 0.07 & $\infty$ & 1.097 & 4.545 & 800 & 8.00 & 6.750 \\
\hline 17 & Placemem of $6^{-}$Topsoll & 455 & or & 8.50 & 0.08 & $\boldsymbol{s}$ & 727 & 4,318 & 218 & 11.00 & 8200 \\
\hline 18 & $\begin{array}{l}\text { Placement of Pertmater Extertor } \\
\text { Drainage Layar } 314^{*} \times 3^{*} \text { DP (Crushod Stono) }\end{array}$ & 210 & sy & 1.50 & 0.22 & 40 & 850 & 324 & 200 & 7.20 & 1800 \\
\hline 19 & Excavation of Trapezoldal Dralnage Dich & 221 & Cr. & - & Q.165 & 41 & 818 & 0 & 245 & 1.00 & 1,000 \\
\hline & (L) PAGE 2 TOTAL & & & & & 1,500 & $31, \pi 2$ & 142,013 & s,or & & 100,400 \\
\hline
\end{tabular}

C-2. Cost Estimate for a Cover System Utilizing Bentomat@ and a 30-mil FML (Cont'd) 


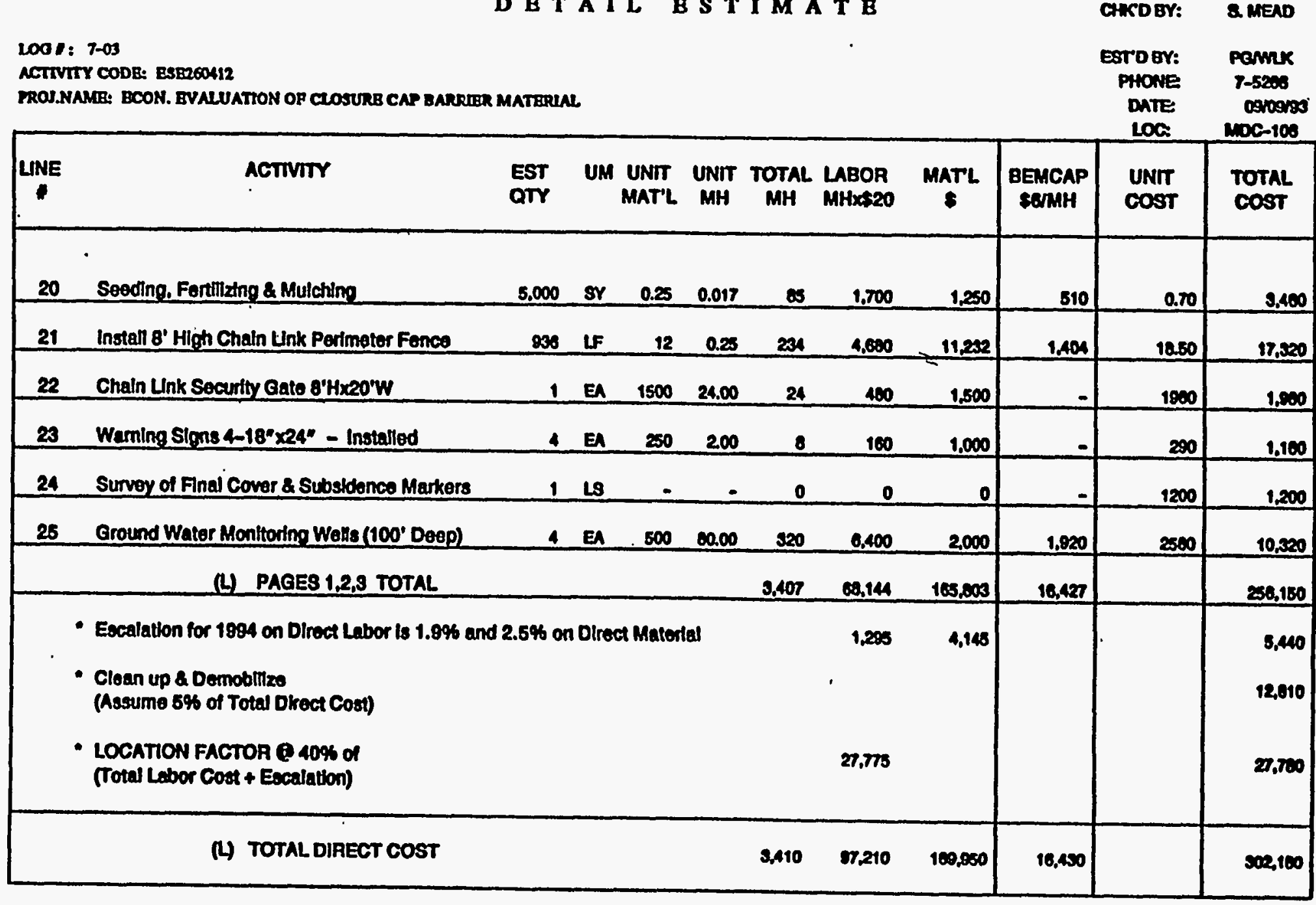




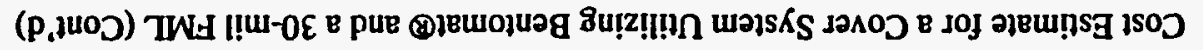

\begin{tabular}{|c|c|c|c|c|c|c|c|}
\hline 000000 & & & \multicolumn{5}{|c|}{ LSOS 109 GIONN 7Y1OL (7) } \\
\hline 00020 & & & \multicolumn{5}{|c|}{ 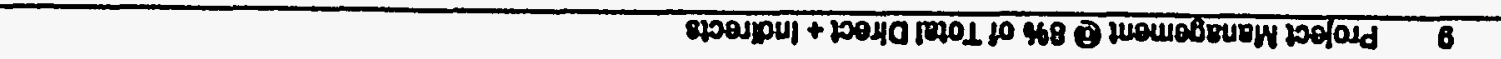 } \\
\hline $\cos 28$ & & & \multicolumn{3}{|c|}{690} & 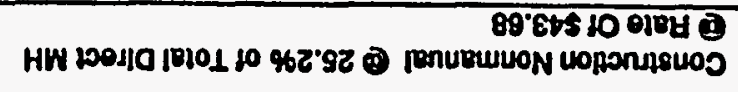 & 8 \\
\hline orecos & & & $400^{\circ} \mathrm{st}$ & $\cos \theta t$ & 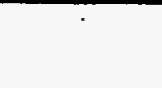 & 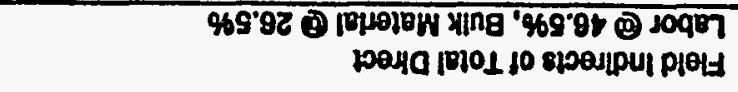 & 2 \\
\hline ariel & & & & QLP'Cl 602 & & 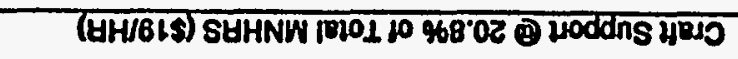 & 9 \\
\hline $0 \times 100$ & & & & & & 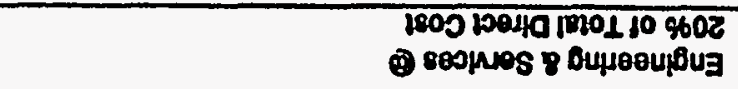 & 9 \\
\hline 00200 & & & & & & 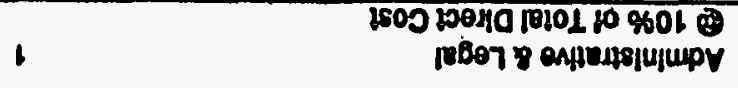 & $\bullet$ \\
\hline $\cos \theta$ & & & & & & 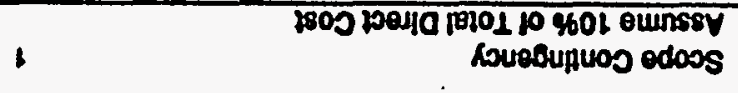 & 6 \\
\hline OL'st & & & & & & 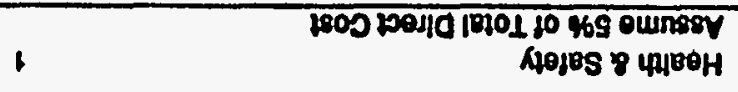 & $z$ \\
\hline $0<0 \%$ & & & & & & 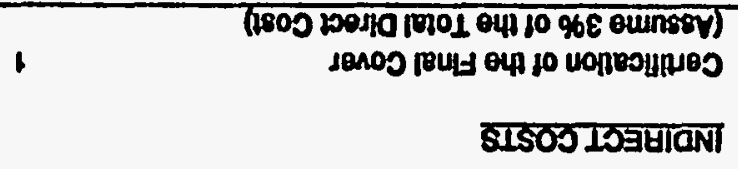 & $\downarrow$ \\
\hline $\begin{array}{l}1800 \\
\text { TV101 }\end{array}$ & $\begin{array}{l}1500 \\
\text { LIND }\end{array}$ & $\begin{array}{c}\text { HWwes } \\
\text { dVOWJa }\end{array}$ & T.Avm & $\begin{array}{l}\text { OZgXHW HW HW } \\
\text { HOQVI TVIOL LNN }\end{array}$ & $\begin{array}{l}\text { TaLVW } \\
\text { LINn wn }\end{array}$ & $\begin{array}{l}100 \\
159\end{array}$ & ann \\
\hline
\end{tabular}

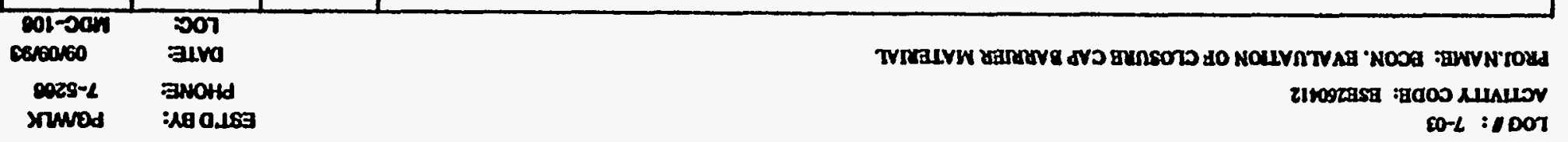

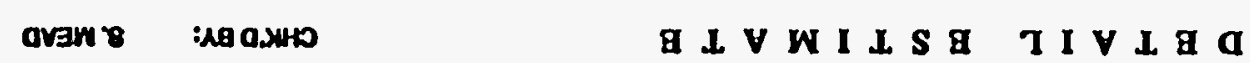


D B TAIL B S T IMATB

a MEAD

Loo1: $7-03$

ACTIVITY CODE: ESE260112

PROJ.NAMB: ECON. BVALUATION OF CLOSURB CAP BARRIER MATERIAL

carogr:

Permx

PHONE: 7-5200

DATE DONOSOS

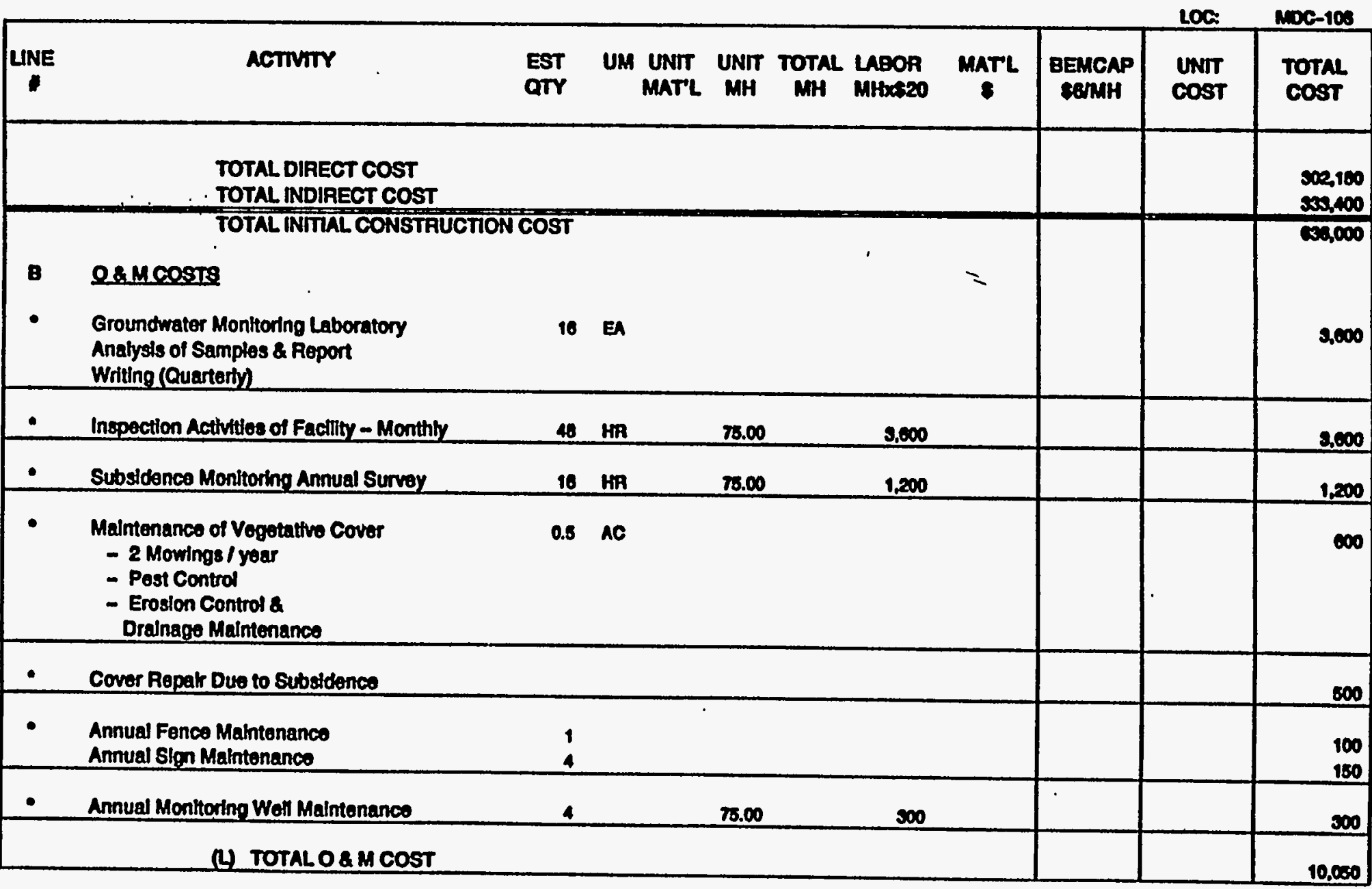

C-2. Cost Estimate for a Cover System Utilizing Bentomat@ and a 30-mil FML (Cont'd) 


\begin{tabular}{|c|c|}
\hline CAwco BV: & Q. MEND \\
\hline $\begin{array}{l}\text { ESTOBY: } \\
\text { PHONE } \\
\text { OATE } \\
\text { LOQ }\end{array}$ & $\begin{array}{c}\text { Pamuk } \\
7-5200 \\
0900000 \\
\text { noc- } 100\end{array}$ \\
\hline
\end{tabular}

2001: 7-03

ACTIVTYY CODB: ESE260112

MOO.NAME: BCON. EVALUATION OF CLOSURB CAP BARRIER MATERIAL.

toa

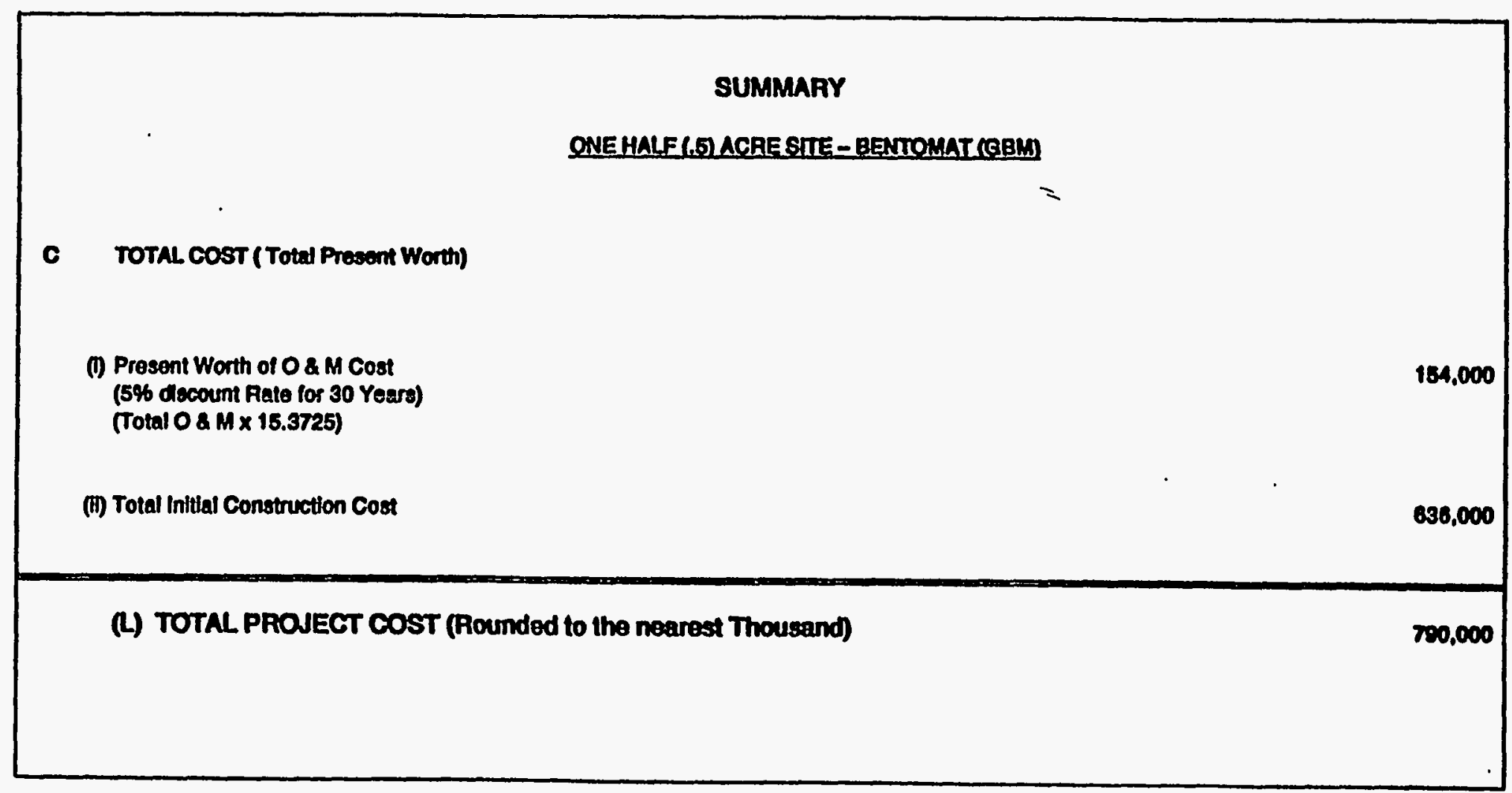

C-2. Cost Estimate for a Cover System Utilizing Bentomat $\circledast$ and a 30-mil FML (Cont'd) 
Loa: $7-03$

ACTIVTYY CODB: ESEZ60412

PROS.NAMIB: ECON. EVALUATION OP CLOSURB CAP EARRIER MATERIAL

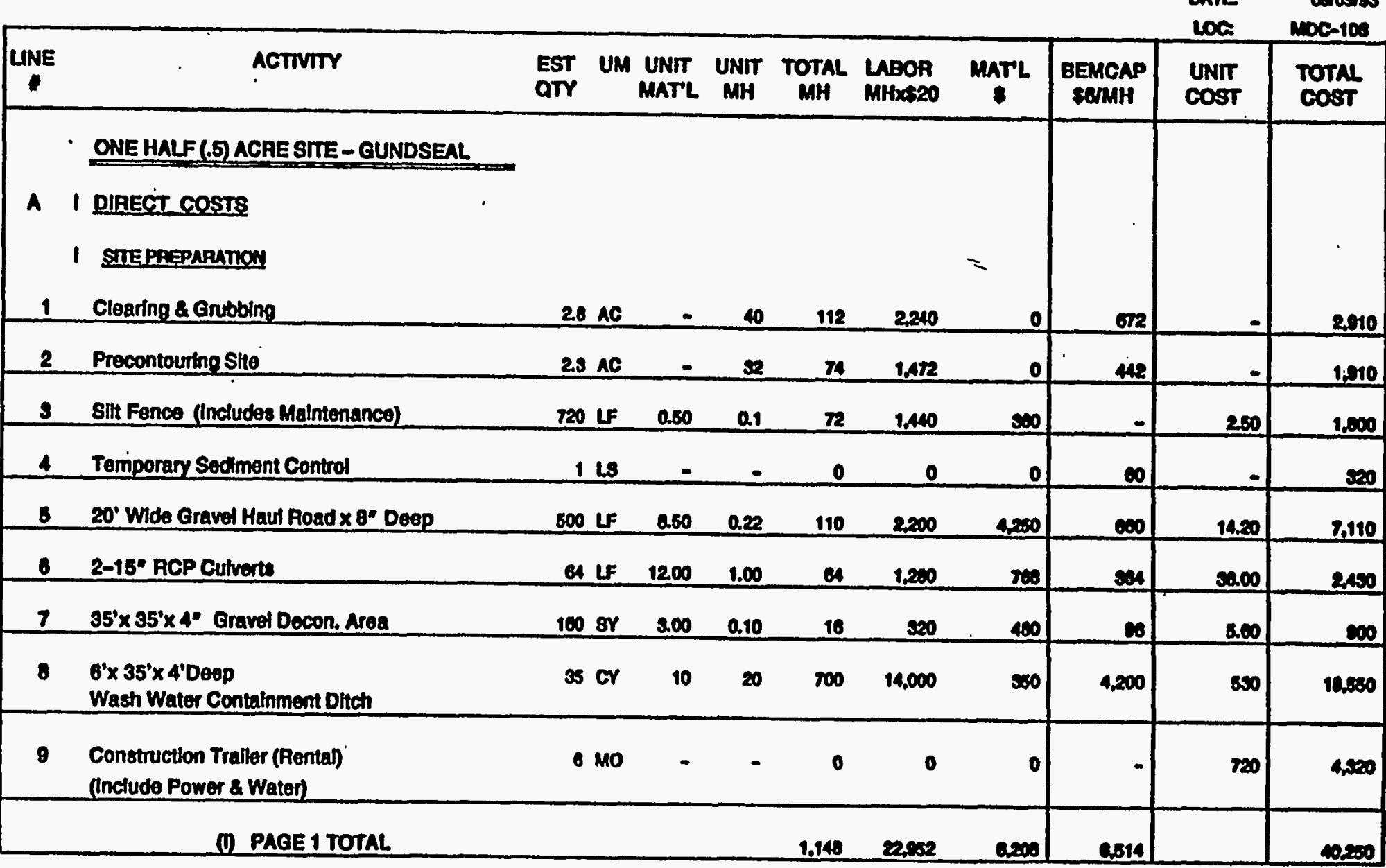

C-3. Cost Estimate for a Cover System Utilizing Gundseal(6 


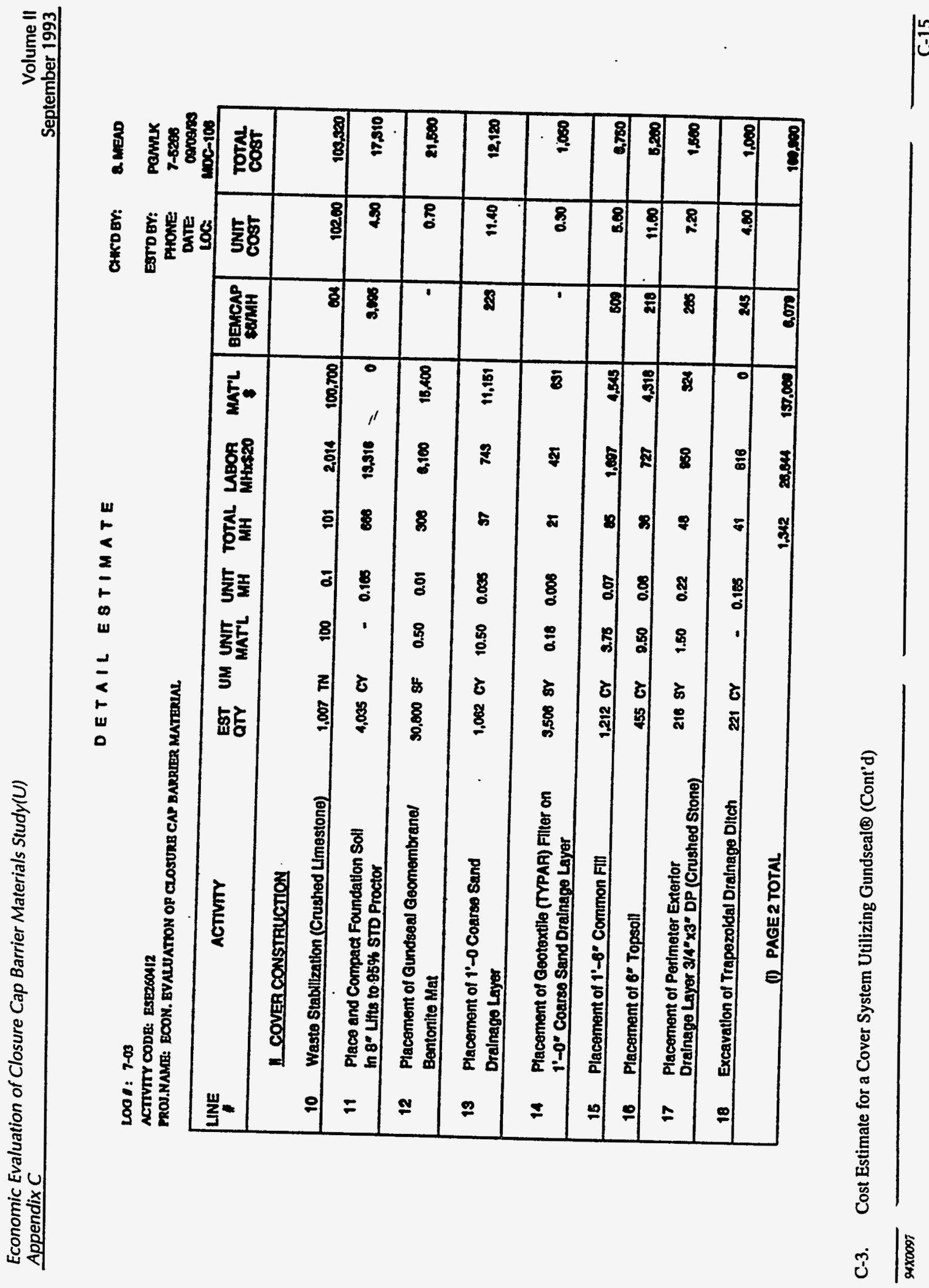




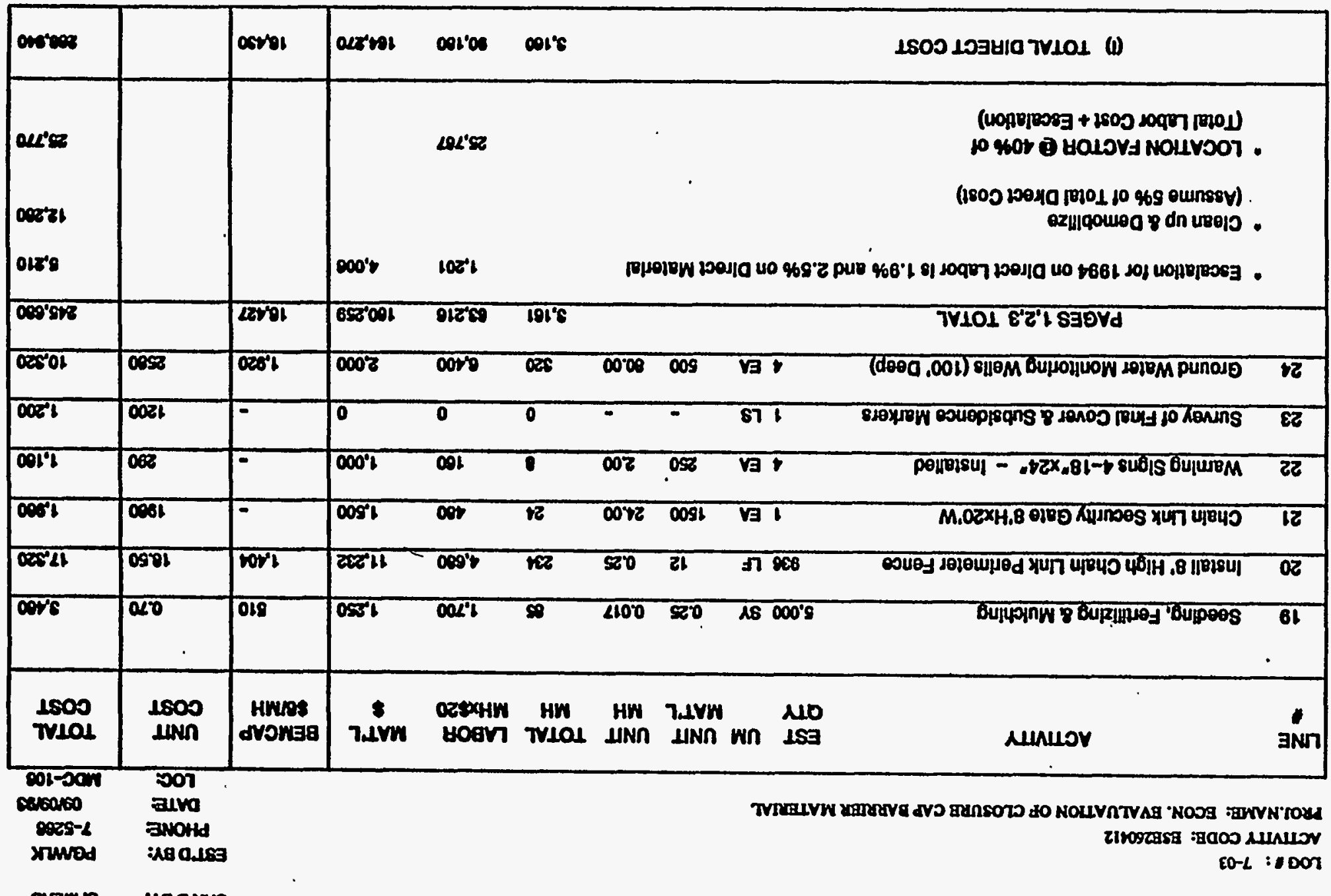

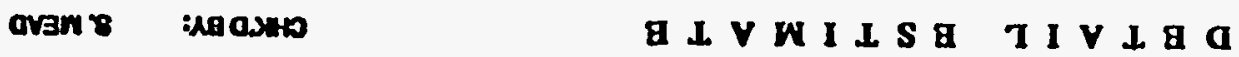


L001: 7-03

ACTIVTTY CODE: BSE260412

MOJ.NAMR: ECON. EVALUATION OF CLOSURB CAP BARRIER MATBRIAL.

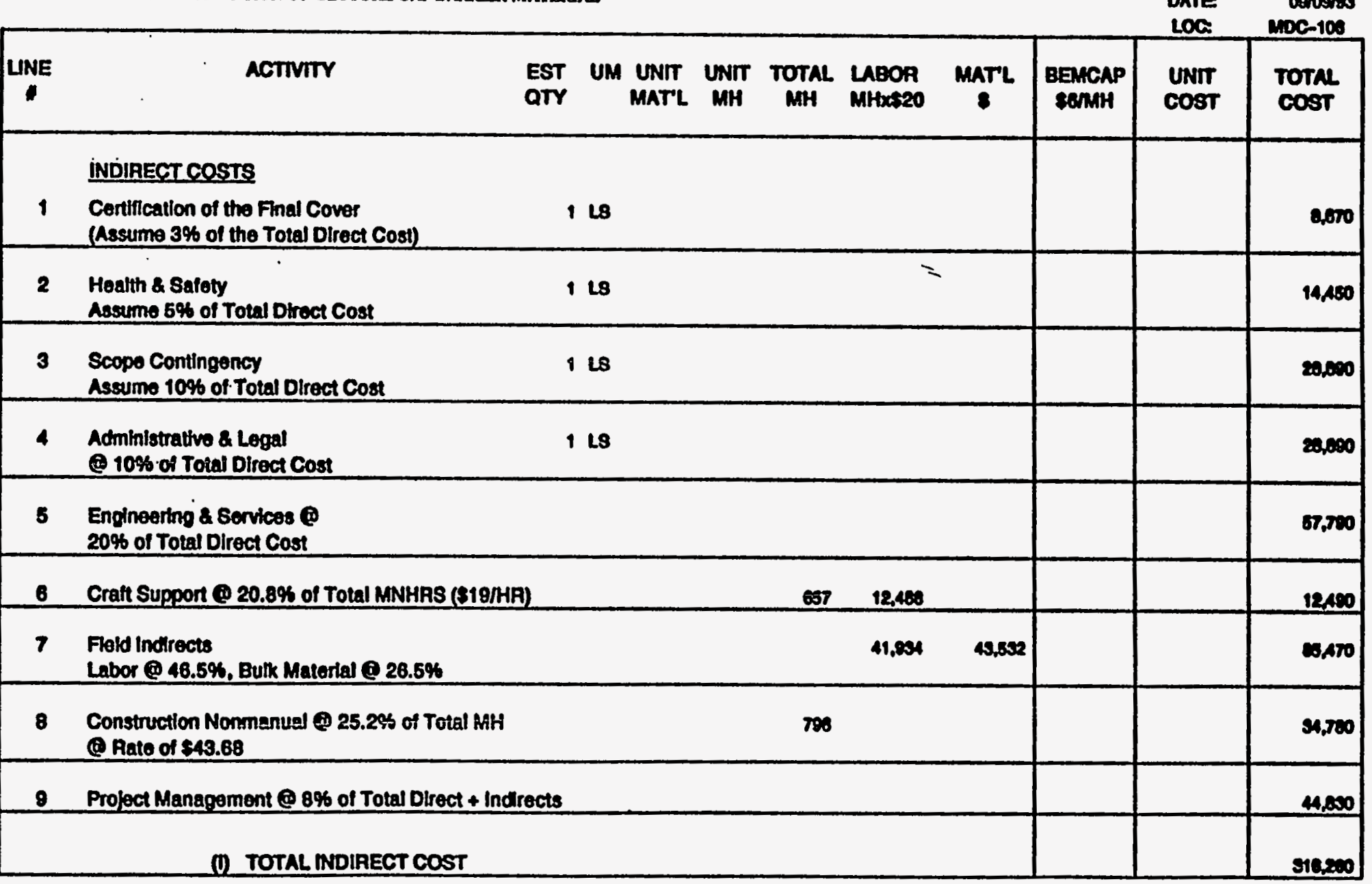

C-3. Cost Estimate for a Cover System Utilizing Gundseal@ (Cont'd) 
D B TAIL BSTIMATE

2001: $7-03$

ACTIVITY CODE: BSE260412

TROJ.NAMB: ECON. BVALUATION OF CLOSURB CAP BARRTSR MATERUAL

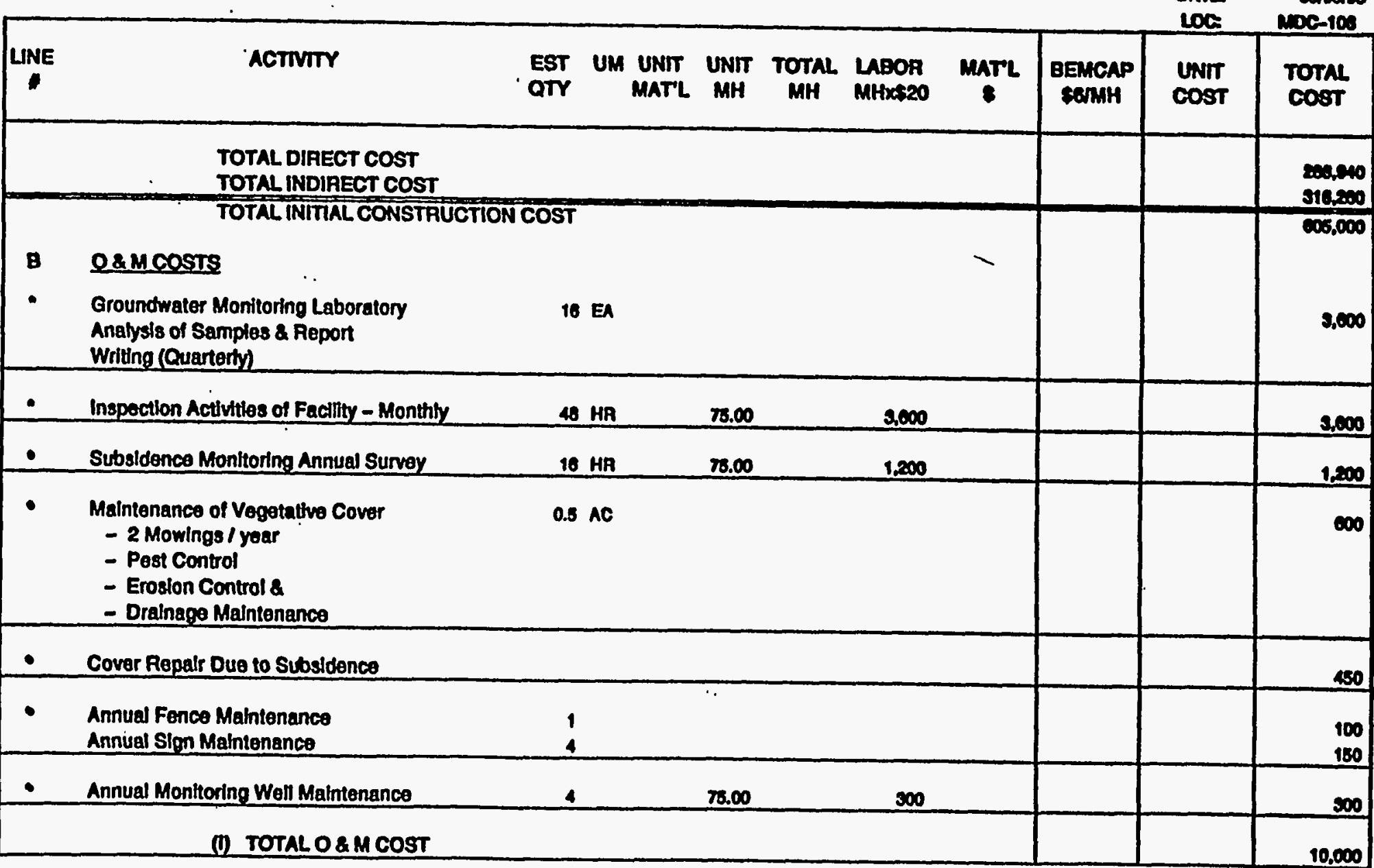

C-3. Cost Estimate for a Cover System Utilizing Gundsealo (Cont'd) 
L001: 7-03

ACTIVITY CODB: ESEPSON12

PROJ.NAME: ECON. EYALUATION OF CLOSURB CAP BARRIER MATERIAL
DBTAIL BSTIMATB

\begin{tabular}{|c|c|}
\hline CancD Br: & Q. MEND \\
\hline $\begin{array}{l}\text { ESTOQY: } \\
\text { PHONE } \\
\text { DATE } \\
\text { LOQ }\end{array}$ & 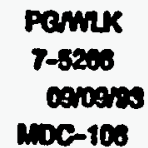 \\
\hline
\end{tabular}

\section{SUMMAPY}

\section{QNE HALE (.5) ACRE SIE-GUNDSEAL}

164,000

(i) Present Worth of 0 \& $M$ Cost

(5\% discount Pato for 30 Years)

(Total O\& M $\times$ 15.3725)

(ii) Totul Initial Construction Cost

608,000

(I) TOTAL PROJECT COST (Rounded to the nearest Thousend)

709,000

C-3. Cost Estimate for a Cover System Utilizing Gundseal@ (Cont'd) 
DETAIL ESTIMATE

200 I: $7-03$

ACTTVITY CODB: ESEZGOA12

MROI.NAME: ECON. BVALUATION OF CLOSURB CAP BARRIER MATBRIAL

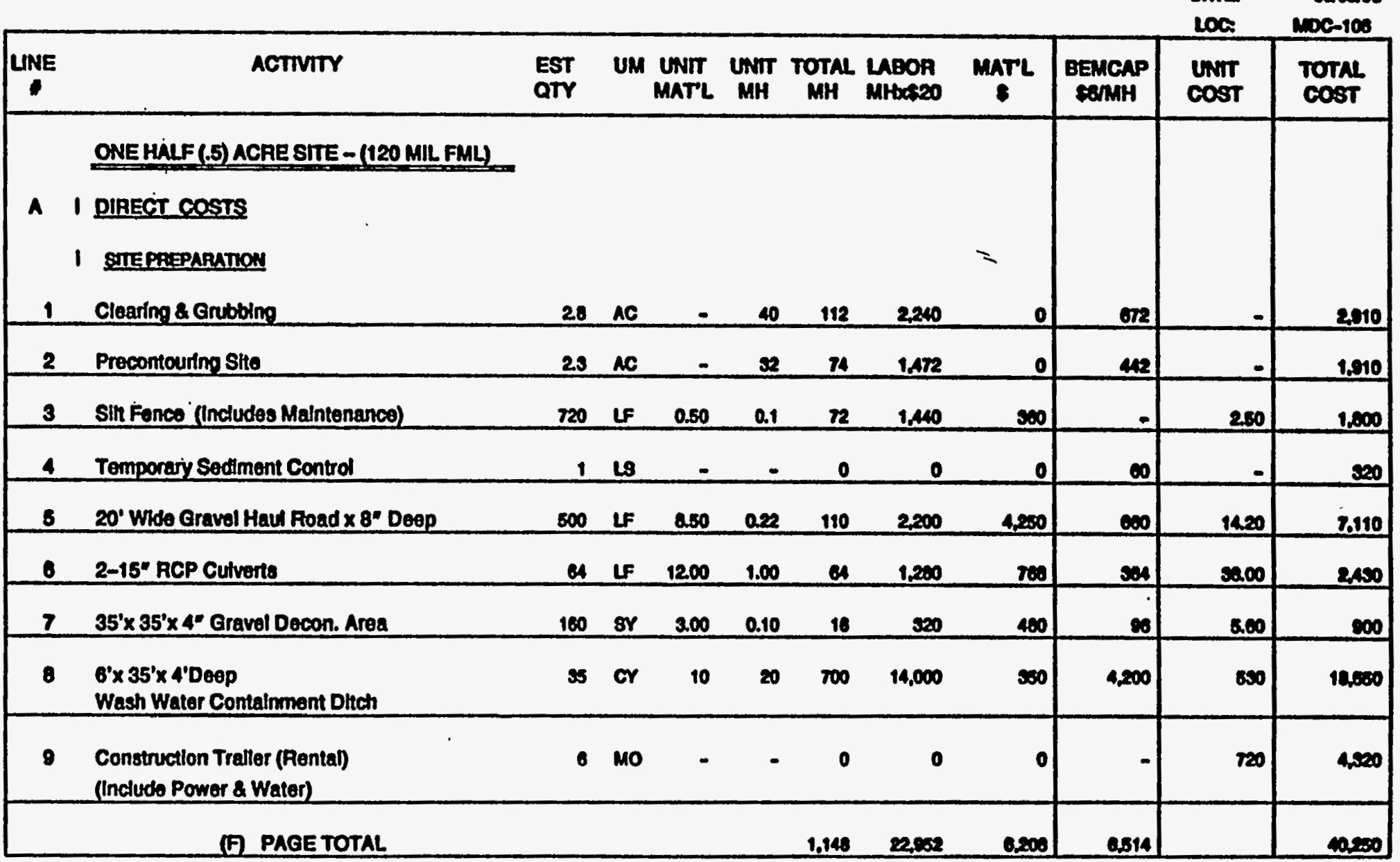

C-4. Cost Estimate for a Cover System Utilizing 120-mil High Density Polyethylene (HDPE) Liner 
DETAIL ESTIMATE

Loo I: $1-03$

ACTIVITY CODR: ESE26ON12

PROI.NAME: ECON. EVNLUATTON OP CLOSURE CAP BARRIER MATERIAR.

\begin{tabular}{|c|c|c|c|c|c|c|c|c|c|c|c|}
\hline & & & & & & & & & & LoG & 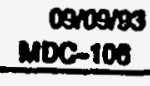 \\
\hline JNE & ACTIVITY & $\begin{array}{l}\text { EST } \\
\text { QTY }\end{array}$ & UM & $\begin{array}{c}\text { UNMT } \\
\text { MATLL }\end{array}$ & $\underset{M H}{\text { UNIT }}$ & $\begin{array}{l}\text { TOTAL } \\
\text { MH }\end{array}$ & $\begin{array}{l}\text { LAEOA } \\
\text { MHXOP2O }\end{array}$ & MATL & Bemcap & $\begin{array}{l}\text { UNm } \\
\text { cost }\end{array}$ & $\begin{array}{l}\text { TorAl } \\
\cos T\end{array}$ \\
\hline & COVER CONSTRUCTION & & & & & & & & & & \\
\hline 10 & Wasto Stabilization (Crushod Lmestono) & 1.007 & $\mathbf{T N}$ & 100 & 0.1 & 101 & 2.014 & 100,700 & $\infty$ & 10200 & 100.520 \\
\hline 11 & $\begin{array}{l}\text { Place and Compact Foundation Soll } \\
\text { m 8. Lits to 95\% STD. Proctor }\end{array}$ & 4,035 & or & - & 0.168 & 600 & $13,510=$ & o) & s,003 & 4.50 & 17.910 \\
\hline 12 & $\begin{array}{l}\text { Placement of } 120 \text { Min Flextble } \\
\text { Membrane Liner }\end{array}$ & 30,800 & sf & 0.50 & 0.01 & 508 & 6,100 & 18,400 & - & 0.70 & 21,500 \\
\hline 13 & $\begin{array}{l}\text { Piacoment of 1'-O Coarso Sand } \\
\text { Dralnage Layer }\end{array}$ & 1,062 & cr & 10.60 & 0.033 & 37 & 740 & 11,181 & 20 & 11.40 & 42,950 \\
\hline 14 & $\begin{array}{l}\text { Placoment of Geotextile (TYPAR) Filter on } \\
\text { 1'-0" Coarse Sand Drainage Layer }\end{array}$ & 3.500 & sy & 0.10 & 0.000 & 21 & 421 & 601 & - & 0.30 & 1.060 \\
\hline 16 & Placoment of Y'-8" Common Fil & $\$, 212$ & or & $2 \pi$ & $0.0 \%$ & $\omega$ & 1.007 & 4,845 & $\mathbf{\infty}$ & 8.60 & 0,700 \\
\hline 10 & Placement of 6" Topsoll & 455 & or & 0.50 & 0.00 & so & 227 & 4910 & 210 & 11.00 & 8,200 \\
\hline 17 & $\begin{array}{l}\text { Placement of Perimeter Exterlor } \\
\text { Oratheqe Layer } 34^{*} \times 3^{*} \text { Stong }\end{array}$ & 210 & or & 1.50 & 0.22 & 48 & 250 & s24 & 200 & 7.20 & 1,800 \\
\hline 18 & Excavation of Trapezoldal Drainage Ditch & 221 & cr & $=$ & 0.186 & 41 & 818 & of & 245 & 4.80 & 1.000 \\
\hline & (D) PAGE TOTAL & & & & & 1342 & 20,04 & 158.000 & con & & 16000 \\
\hline
\end{tabular}

C-4. Cost Estimate for a Cover System Utilizing 120-mil High Density Polyethylene (HDPE) Liner (Cont'd) 
D B T A I L B S TIMATB

L00 I: 7-03

ACTIVITY CODE: ESE2SON12

PROJ.NAME: BCON. BVALUATION OF CLOSURB CAP BAFRIER MATENUL

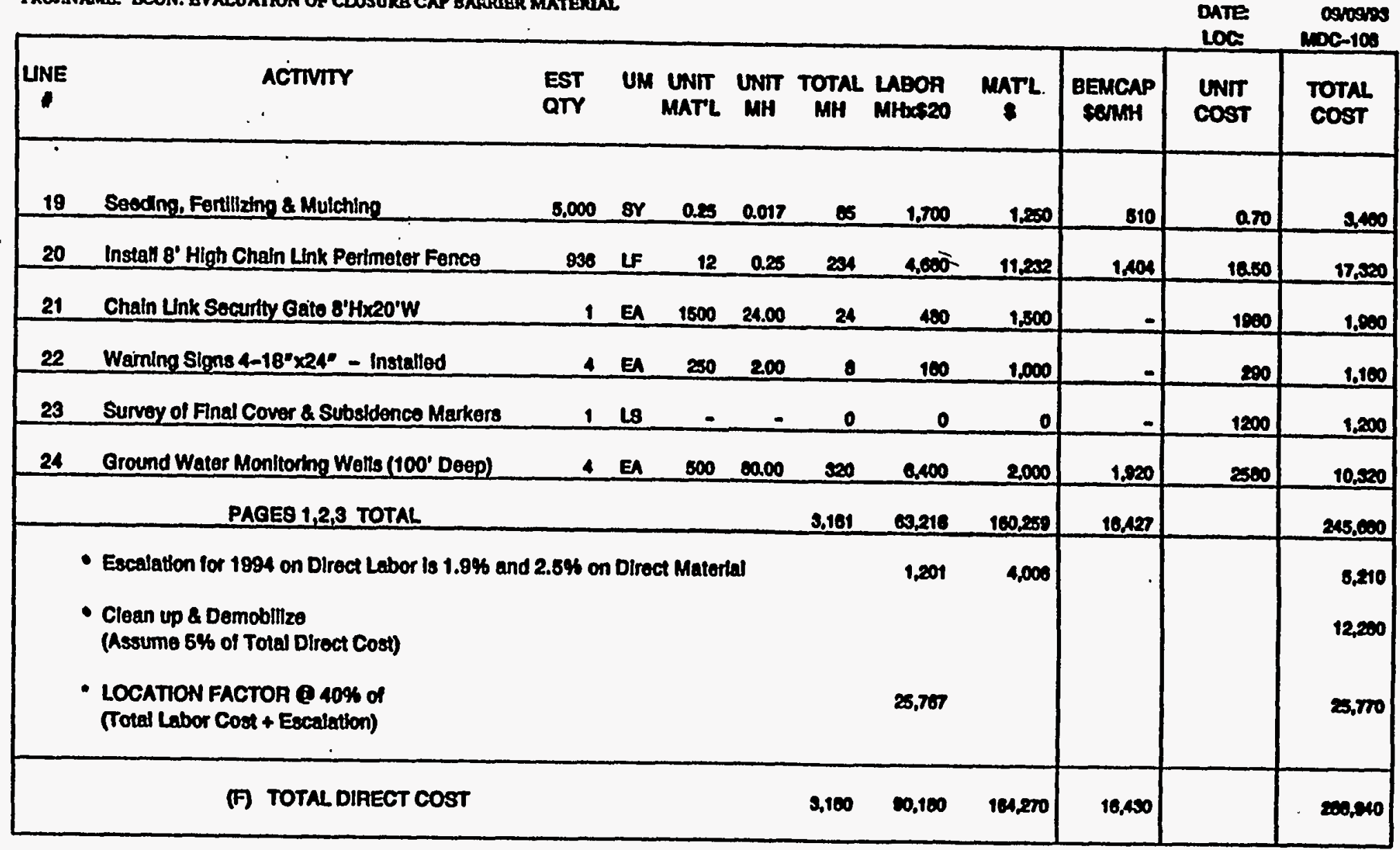

C-4. Cost Estimate for a Cover System Utilizing 120-mil High Density Polyethylene (HDPE) Liner (Cont'd) 
L001: 7-03

ACTIVITY CODE: ESE260412

MOJ.NAME: ECON. BVALUATION OF CLOSURB CAP BARRIER MATHRUAL

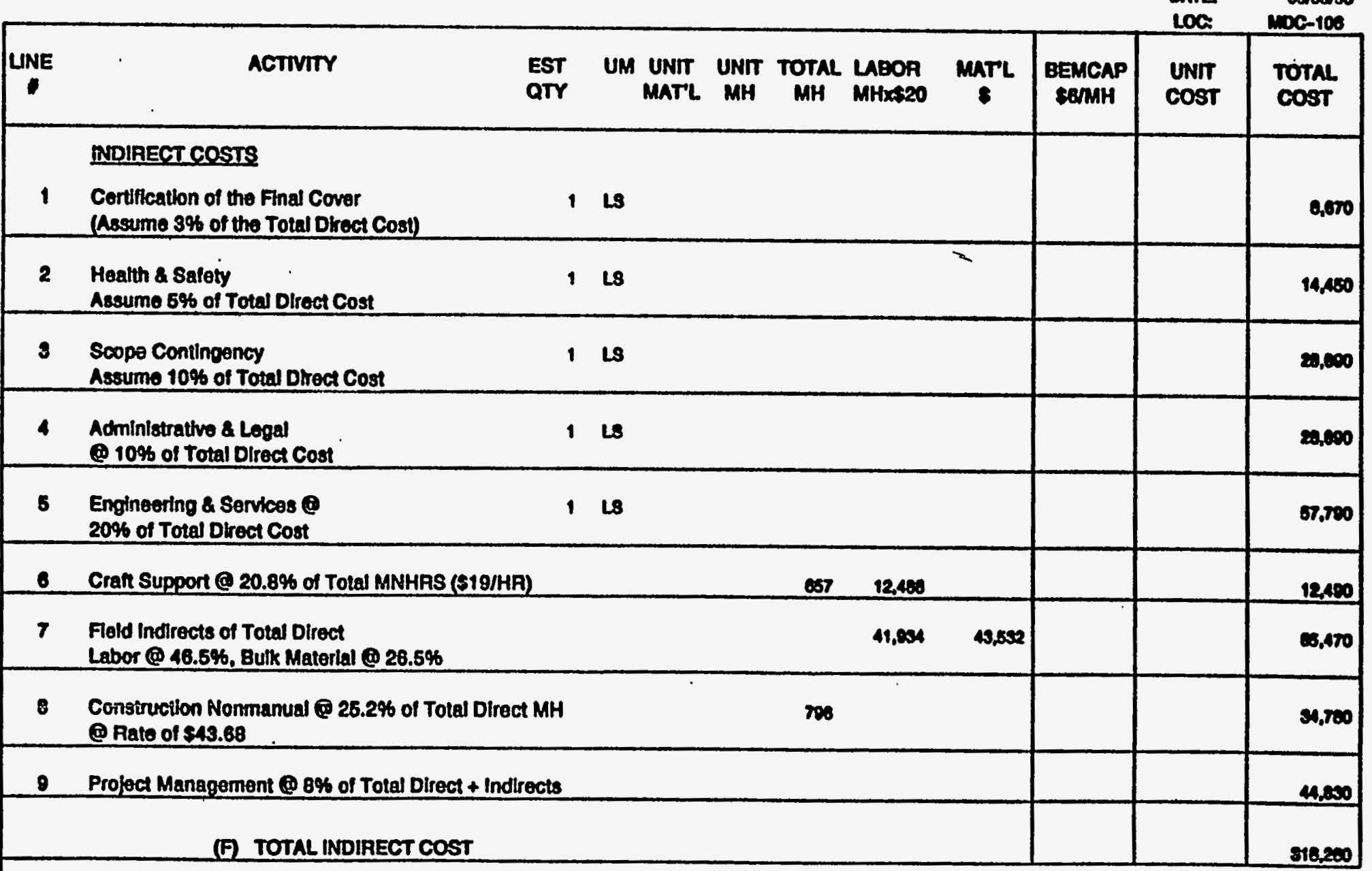

C-4. Cost Estimate for a Cover System Utilizing 120-mil High Density Polyethylene (HDPE) Liner (Cont'd) 
D B T A I L B TIMAT B

CAKD BY: Q.MEND

Lod : 7-03

ACTIVITY CODE: ESE260412

PROS.NAMB: BCON. EVALUATION OP CLOSURB CAP BARRIER MATERIAL

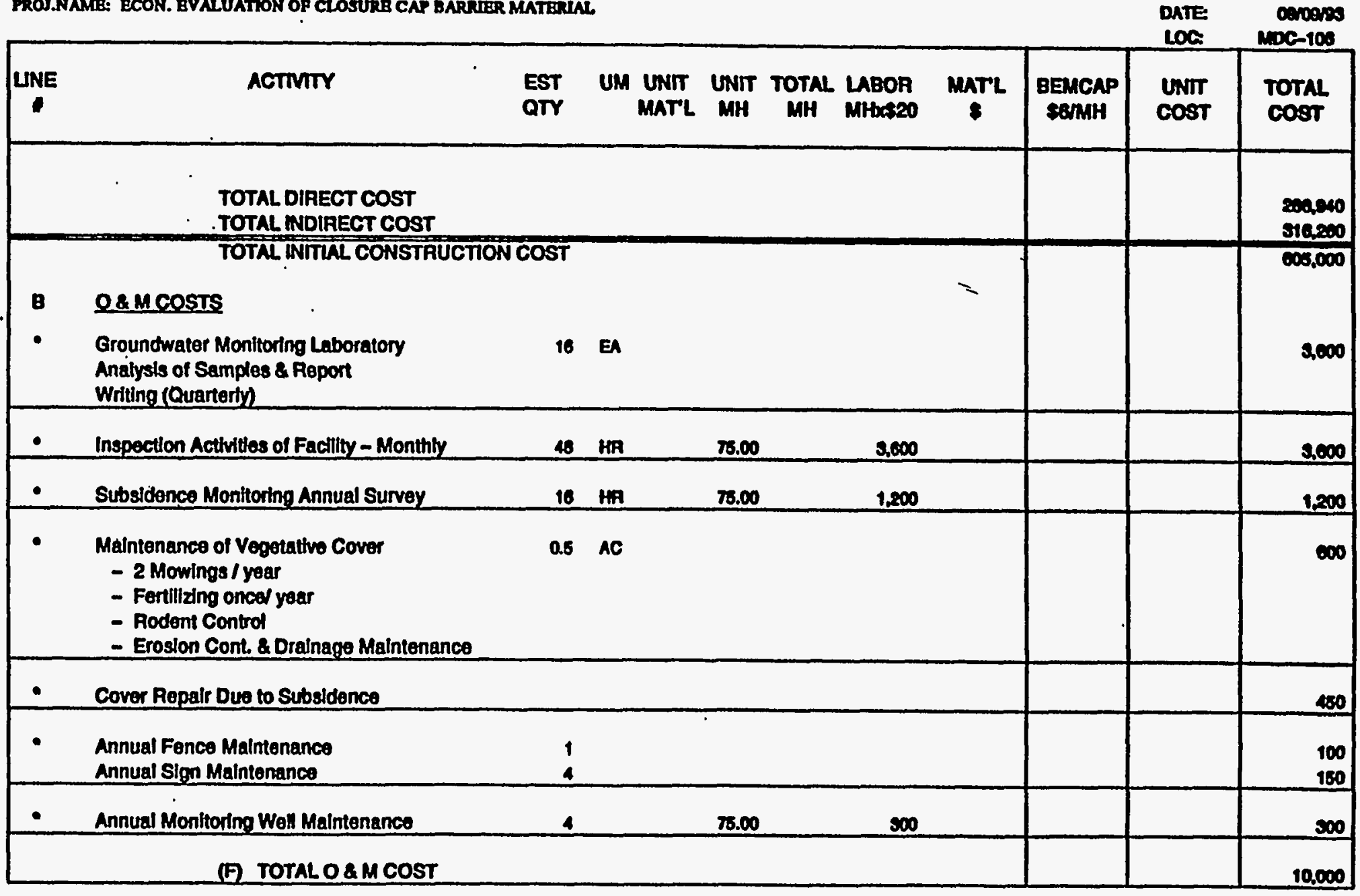

C-4. Cost Estimate for a Cover System Utilizing 120-mil High Density Polyethylene (HDPE) Liner (Cont'd) 
D B TAIL BSTIMATB

2001: $7-03$

ACTIVIT CODA: BSE200N12

FOU.NAME: BCON. EYALUATION OP CLOSUNB CAP DARRERR MATERIAL.
Grobr:

ESTD BV:

PHONE

DATE

Loc.
Q LEA

Panuk

7-6200

conoms moc-100

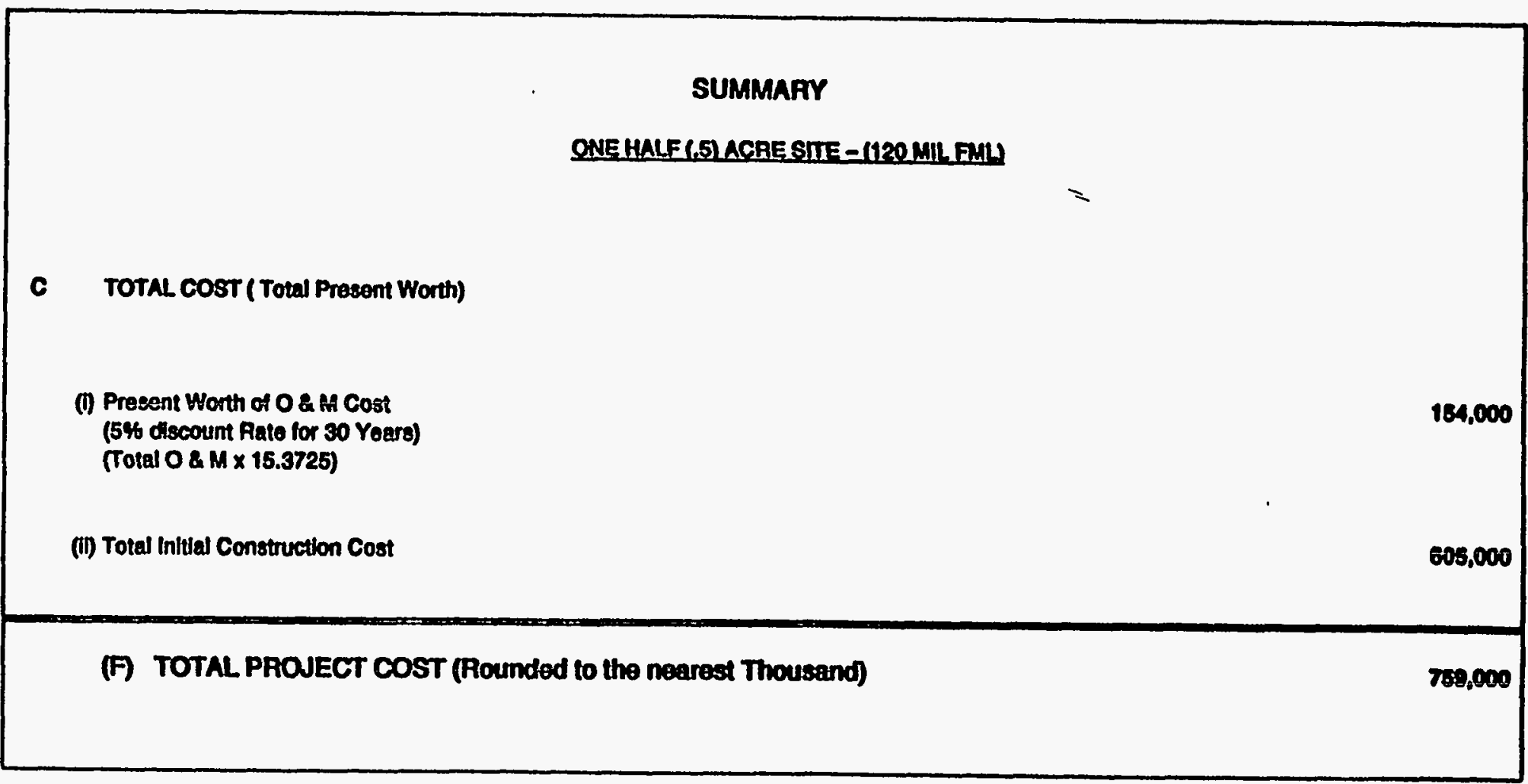

C-4. Cost Estimate for a Cover System Utilizing 120-mil High Density Polyethylene (HDPE) Liner (Cont'd) 
DETAIL ESTIMATE

L00 1: 7-03

ACTIVITY CODE: ESEZCON12

MOS.NAME: ECON, EVALUATION OP CLOSURB CAP BARRIER MATERUN

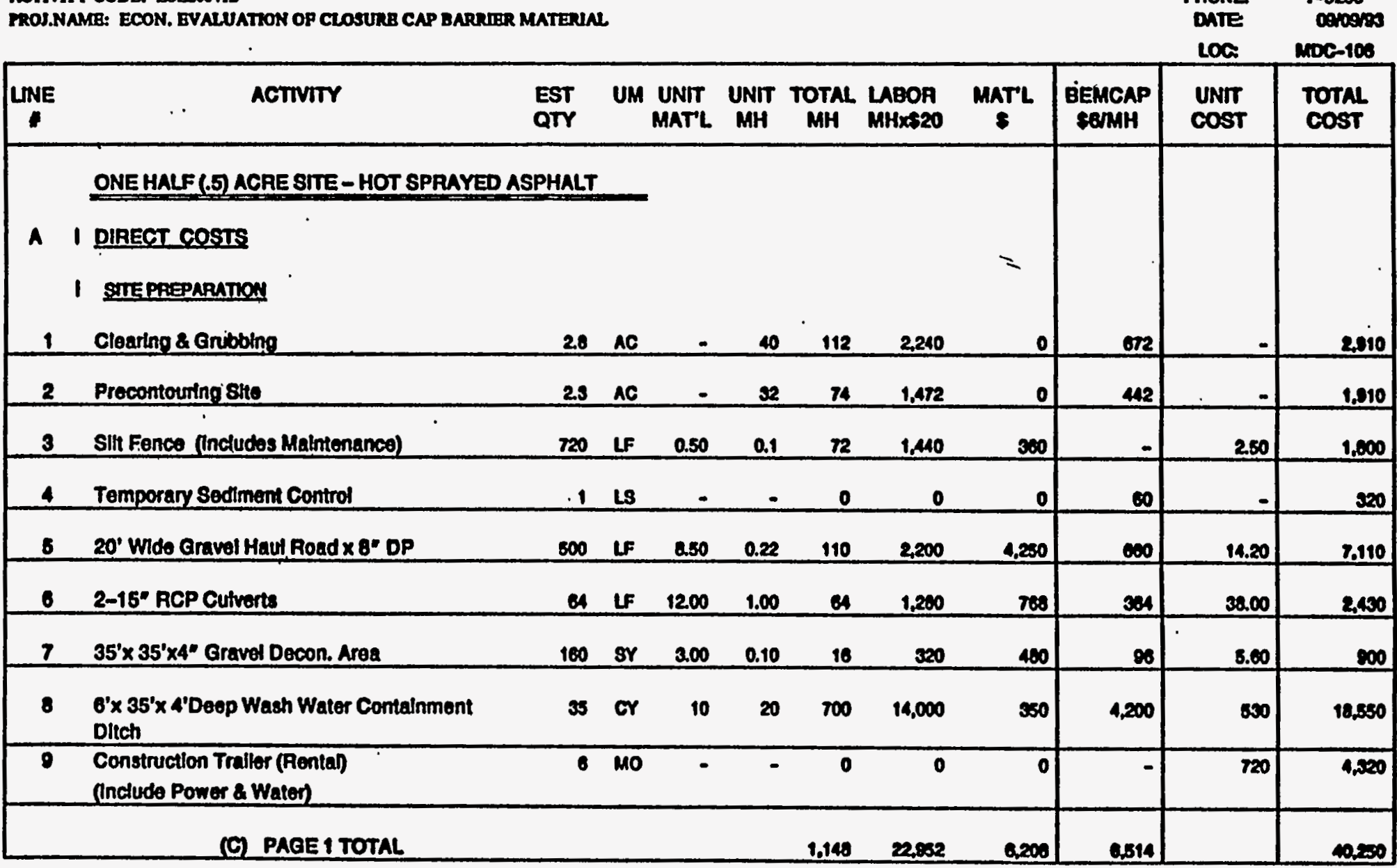

C-5. Cost Estimate for a Cover System Utilizing 1/4-inch Thick Hot Sprayed or Emulsified Asphalt 
Loo1: $7-03$

DETAILESTIMATE

MEAD

ACTTVTYY CODB: ESE2SON12

PROJ.NAME: BCON. EVALUATTON OF CLOSURB CAP BARRUER MATERIAL.

\begin{tabular}{|c|c|c|c|c|c|c|c|c|c|c|c|}
\hline & & & & & & & & & & Loa: & MOCG-106 \\
\hline LNEE & ACTIVITY & $\begin{array}{l}\text { EST } \\
\text { QTY }\end{array}$ & UM & $\begin{array}{l}\text { UNTT } \\
\text { MATL }\end{array}$ & UNIT & $\underset{\mathrm{MH}}{\text { TOTAL }}$ & $\begin{array}{l}\text { LABOR } \\
\text { MHXSEDO }\end{array}$ & $\underset{\$}{\operatorname{MATL}}$ & $\begin{array}{c}\text { BEMCAP } \\
\text { SGRMH }\end{array}$ & $\begin{array}{l}\text { UNIT } \\
\text { COST }\end{array}$ & $\begin{array}{l}\text { TOTAL } \\
\text { COST }\end{array}$ \\
\hline 10 & $\begin{array}{l}\text { 11 COVER CONSTAUCTION } \\
\text { Waste Stabilizalion (Crushed Umestone) }\end{array}$ & 1,007 & $T N$ & 100 & 0.1 & 101 & 2.014 & 100,700 & 604 & 102.00 & 100,320 \\
\hline 11 & $\begin{array}{l}\text { Place and Compact Foundation Soll } \\
\text { In } 8^{\circ} \text { Litts to } 85 \% \text { STD. Proctor }\end{array}$ & 4.035 & or & - & 0.165 & 668 & 13,316 & 0 & 3,995 & 4.00 & 17,310 \\
\hline 12 & $\begin{array}{l}\text { Placemient of } 30 \text { MH Floxible } \\
\text { Membrane Liner }\end{array}$ & 30,800 & $\mathbf{S F}$ & 0.18 & 0.000 & 246 & 4,928 & 5,544 & - & 0.30 & 10,470 \\
\hline 13 & $\begin{array}{l}\text { Applied Hot Asphath using } 3 \text { passes } \\
\text { at a rate of } 0.5 \text { gal / SY }\end{array}$ & $\begin{array}{l}2,711 \\
4,067 \\
\end{array}$ & $\begin{array}{l}\text { Sr } \\
\text { GAL }\end{array}$ & 2 & 0.18 & 407 & 0,133 & 8,422 & 2,440 & 5.90 & 15,900 \\
\hline 14 & $\begin{array}{l}\text { Placement of 1'-0 Coarse Sand } \\
\text { Dralnage Layer }\end{array}$ & 804 & or & 10.50 & 0.035 & 32 & $\infty 00$ & 0,492 & 190 & 11.40 & 10,310 \\
\hline 16 & $\begin{array}{l}\text { Placement of Geotextlie (TYPAP) Filter on } \\
1^{1}-0^{*} \text { Coarse Sand Dralnage Layer }\end{array}$ & 2,711 & 8Y & 0.18 & 0.006 & 16 & 325 & 468 & - & 0.30 & 810 \\
\hline 16 & Placement of $1^{\prime}-6^{\circ}$ Common FIII & 1.212 & or & 3.75 & 0.07 & 85 & 1.697 & 4,545 & 509 & 8.00 & 0.750 \\
\hline 17 & Placement of $\theta^{\prime \prime}$ Topsoil & 455 & or & 9.50 & 0.00 & $3 \mathbf{s}$ & 220 & 4,323 & 210 & $\$ 1.00$ & 8,270 \\
\hline 18 & $\begin{array}{l}\text { Placsment of Pertmeter Exierior } \\
\text { Dralnage Layer } 3 / 4^{\prime} \times 3^{\prime \prime} \text { Crushed Stone }\end{array}$ & 210 & 8Y & 1.50 & 0.22 & 48 & 950 & 324 & 200 & 7.20 & 1.500 \\
\hline 18 & Excavation of Trapezoldal Drahage Ditch & 221 & or & $=$ & 0.185 & 41 & 816 & 0 & 245 & 4.00 & 1.000 \\
\hline & (O) PAOE 2 TOTAL & & & & & $1.0 n$ & $\mathbf{5 0 , 5 4 0}$ & 130,005 & 8,400 & & 172000 \\
\hline
\end{tabular}

C-5. Cost Estimate for a Cover System Utilizing 1/4-inch Thick Hot Sprayed or Emulsified Asphalt (Cont'd) 
D B T A I B S T I MAT B

GACD BY: 2 MEAD

1001: 7-03

ACTIVITY CODB: BSE260N12

MOJ.NAME: BCON. BVALUATION OF CLOSURB CAP BARRIER MATERAAL.

ESTD BY: PaWnK

PHONE: 7-5206

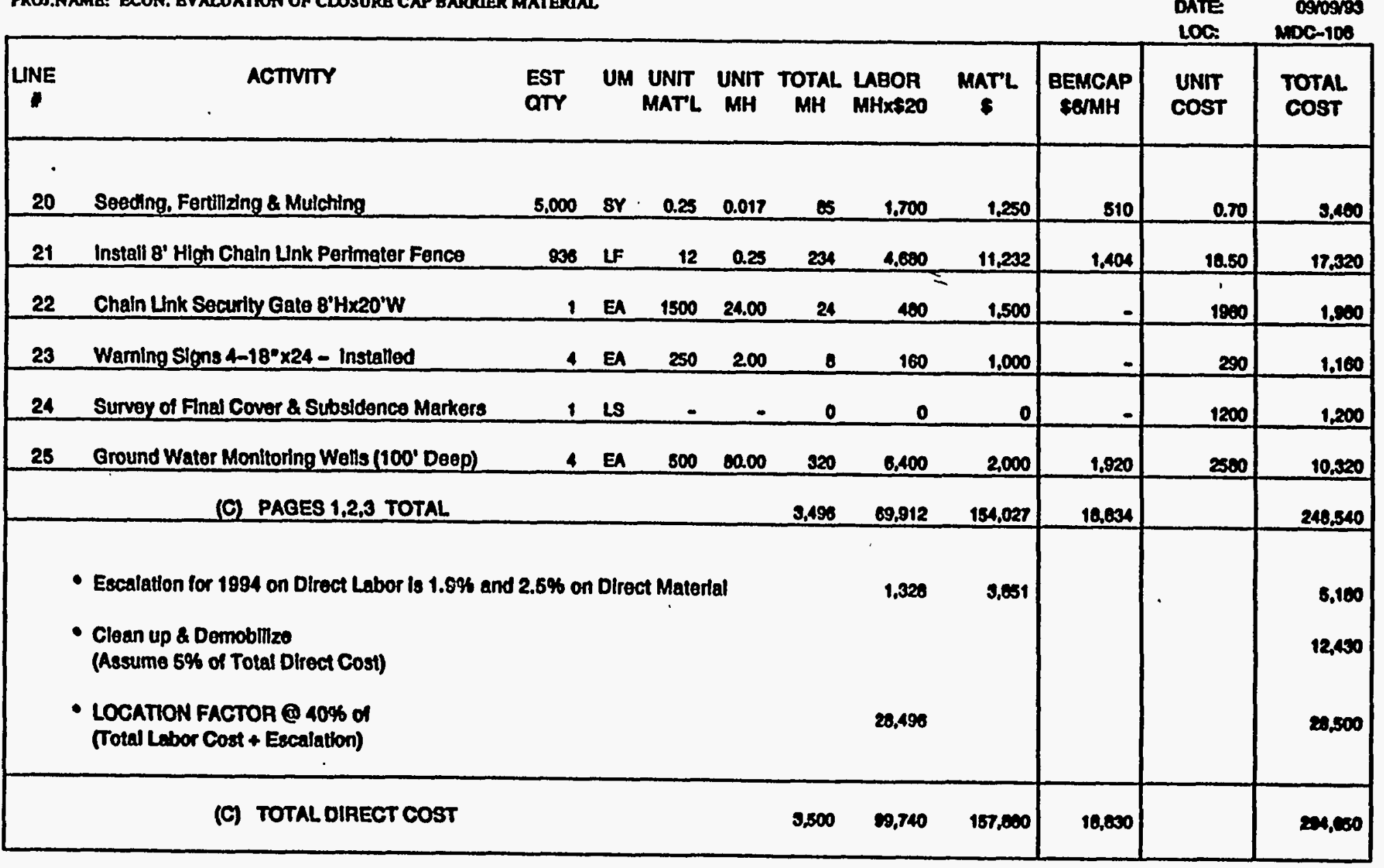

C-5. Cost Estimate for a Cover System Utilizing 1/4-inch Thick Hot Sprayed or Emulsified Asphalt (Cont'd) 
D B T A L B S TIMATB

Lod 1: 7-03

ACTTVITY CODE: BSE2SOA12

MOU.MAME: BCON. BYALUATION OF CLOSURB CAP BATRRER MATERSNL

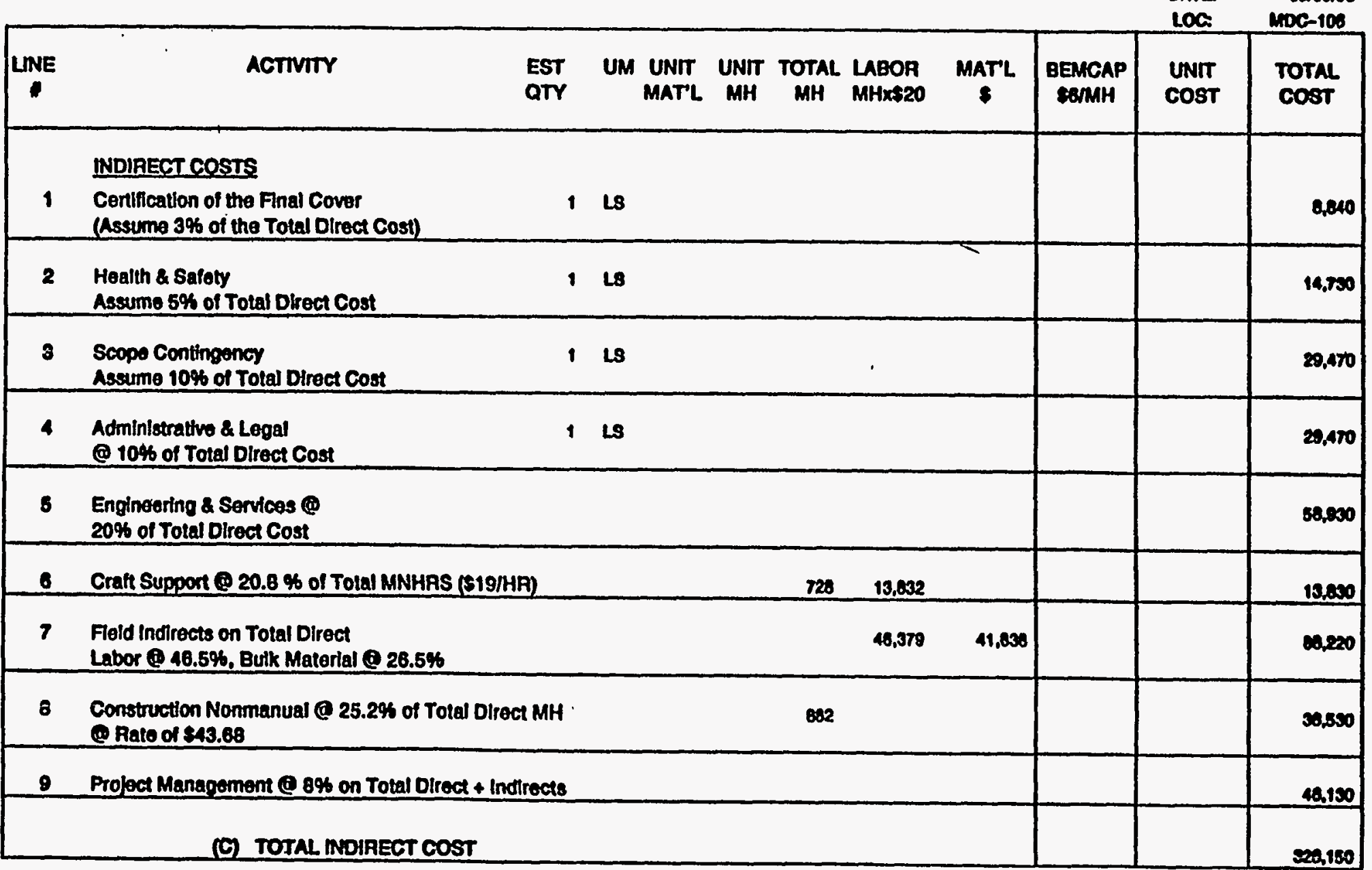

C-5. Cost Estimate for a Cover System Utilizing 1/4-inch Thick Hot Sprayed or Emulsified Asphalt (Cont'd) 
1001: $7-03$

ACTIVITY CODB: ESE260412

FOJ.NAME: ECON. BVALUATION OP CLOSURB CAP BARUIBR MATERIAL.

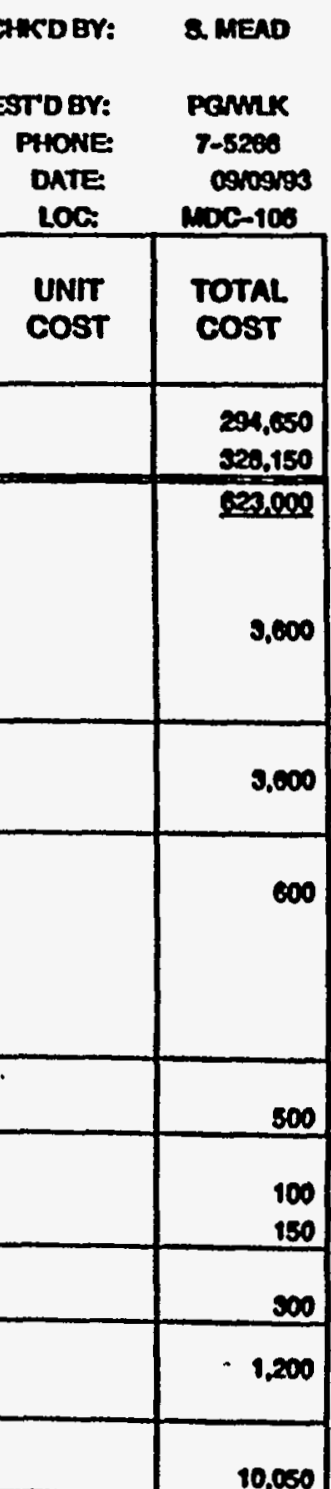

(C) TOTAL O \& M COST

16 EA

48 HR

75.00

3,600

$0.5 \quad A C$

- 2 Mowings / year

- Fortilizing Onceryr

- Pest Control

- Erosion Control \& Drain. Maint.

- Cover Repair Due to Subsidence

- Annual Fence Malntenanco Amual Sign Maintenanco

- Annual Monttoring Well Maintenance

- Subsidenco Monitoring (Annual Survoy)

EST UM UNIT UNIT TOTAL LABOR MAT'L MH MH MHXS20

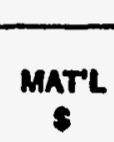

BEMCAP
SAMHH

C-5. Cost Estimate for a Cover System Utilizing 1/4-inch Thick Hot Sprayed or Emulsified Asphalt (Cont'd) 
D ETAIL BSTIMATE

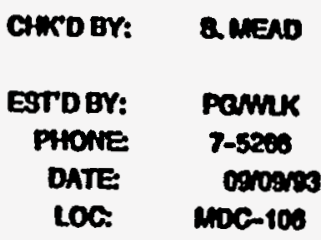

100 1: $7-00$

ACTVITY CODE: ESE200412

PROS.NAME: ECON. BVALUATTON OF CLOSURB CAP BARRRER MATERIAS.

LOC.

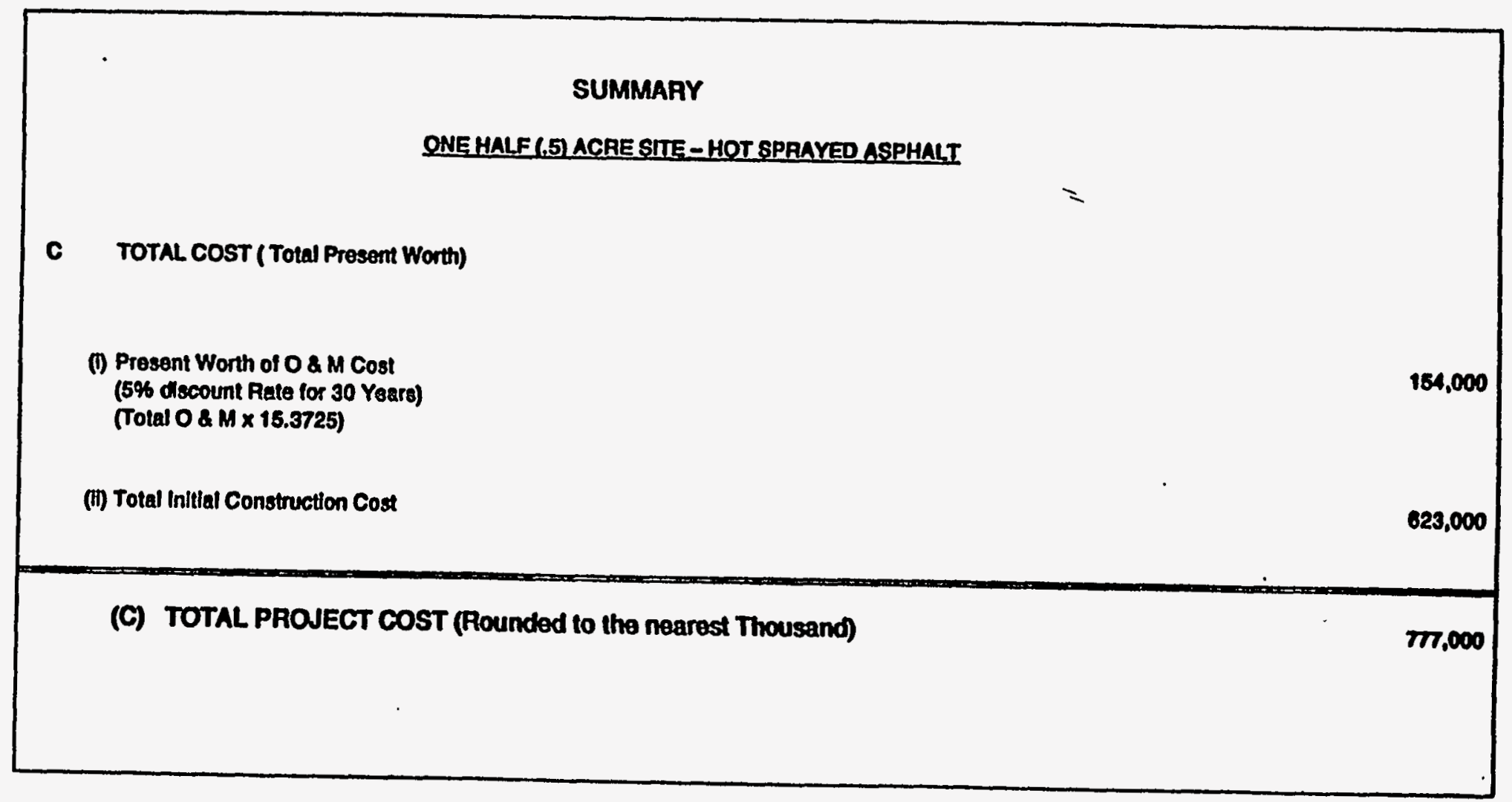

C-5. Cost Estimate for a Cover System Utilizing 1/4-inch Thick Hot Sprayed or Emulsified Asphalt (Cont'd) 
DETAIL ESTIMATE

LOG : : 7-03

ACTIVTY CODE: ESE260112

PROS.NAME: ECON. EVALUATION OF CLOSURE CAP BARAIER MATERIAL

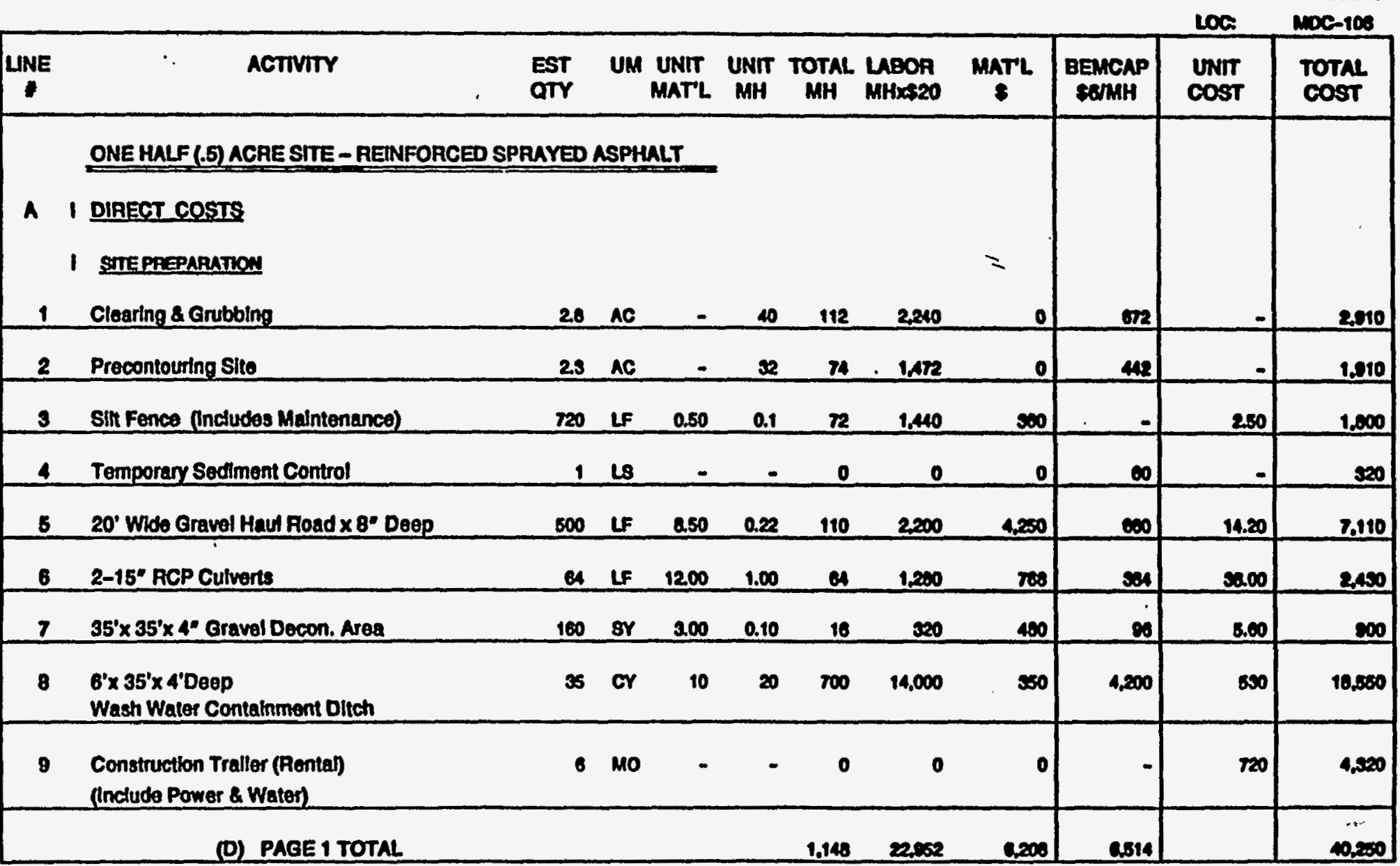

C-6. Cost Estimate for a Cover System Utilizing Reinforced Sprayed Asphalt 
DETAIL ESTIMATE

206 : : 7-03

ACTIVITY CODE: ESE260412

PROJ.NAME: ECON. EVALUATION OF CLOSURE CAP BARAIER MATERIAL

\begin{tabular}{|c|c|c|c|c|c|c|c|c|c|c|c|}
\hline UNE & ACTIVIY & $\begin{array}{l}\text { EST } \\
\text { QTY }\end{array}$ & um & $\begin{array}{l}\text { UNIT } \\
\text { MATL }\end{array}$ & $\underset{M H}{\text { UNT }}$ & TOTAL & $\begin{array}{l}\text { LABOR } \\
\text { MHx+20 }\end{array}$ & MATL & $\begin{array}{l}\text { BEMCAP } \\
\text { SBMMH }\end{array}$ & $\begin{array}{l}\text { UNT } \\
\text { Cost }\end{array}$ & $\begin{array}{l}\text { Toral } \\
\text { cosst }\end{array}$ \\
\hline 10 & $\begin{array}{l}\text { A COVER CONSTAUCTION } \\
\text { Waste Stablizallon (Crustrod Umestone) }\end{array}$ & 1,007 & $\mathrm{Im}$ & 100 & 0.1 & 101 & 2.014 & 100,700 & $\infty$ & 10200 & 103,520 \\
\hline 11 & $\begin{array}{l}\text { Place and Compact Foundation Soll } \\
\text { in 8. Lifts to 95\% STD. Proctor }\end{array}$ & 4.035 & or & - & 0.185 & 600 & 13,918 & 0 & 9,985 & 4.50 & 17.010 \\
\hline 12 & $\begin{array}{l}\text { Placement of Geotextlie Reinforcement } \\
\text { on Foundation Layer }\end{array}$ & 2,711 & gr & 0.30 & 0.01 & 27 & 842 & 810 & - & 0.50 & 1,000 \\
\hline 13 & $\begin{array}{l}\text { Placement of } 30 \text { Mill Flexible } \\
\text { Membrane Lher }\end{array}$ & 30,800 & $\mathbf{S F}$ & 0.18 & 0.000 & 248 & 4.8200 & 8,544 & - & $a .50$ & 10,470 \\
\hline 14 & $\begin{array}{l}\text { Applled Hot Asphalt using } 3 \text { passes at a rate } \\
\text { of } 0.5 \mathrm{gal} / \mathrm{sY} \text { for a total of } 1.5 \mathrm{~g} \text { USy }\end{array}$ & $\begin{array}{l}2,711 \\
4.007\end{array}$ & SY & 200 & 0.15 & 407 & 0.130 & BAser & 2,40 & 8.90 & 16,000 \\
\hline 16 & $\begin{array}{l}\text { Placement of } 4 \text { ' }-0 \text { Coarse Sand } \\
\text { Drainage Layer }\end{array}$ & 804 & or & 10.50 & 0.035 & 32 & 603 & 0,492 & 180 & 11.40 & 10,310 \\
\hline 16 & $\begin{array}{l}\text { Placement of Geotextile (TYPAR) Filter on } \\
1^{\prime}-0^{*} \text { Coarse Sand Dralnage Layer }\end{array}$ & 2.711 & SY & 0.10 & 0.000 & 10 & s2s & 460 & - & 0.50 & 910 \\
\hline 17 & - Placoment of 1'-6" Common FIII & 1,212 & cy & 3.75 & 0.07 & $\infty$ & $1.00 \pi$ & a.548 & 800 & 8.00 & 0,780 \\
\hline 18 & Placement of 6" Topson & 455 & or & 8.50 & 0.00 & $\boldsymbol{3 0}$ & 720 & 40200 & 210 & 11.00 & 8,200 \\
\hline 18 & $\begin{array}{l}\text { Placement of Perimeter Exterlor } \\
\text { Drainage Layer } 34^{\prime \prime} \times 3^{*} \text { DP (Crushed Stone) }\end{array}$ & 216 & 8Y & 1.50 & 0.22 & 40 & 150 & 324 & 200 & 7.20 & 1,560 \\
\hline 20 & Excavation of Trapezoldal Drainage Ditch & 221 & or & - & Q.1es & 41 & & 0 & 245 & 400 & 1.000 \\
\hline & (D) PAGE TOTAL & & & & & 1.704 & 34,002 & 131,051 & 8,400 & & 174210 \\
\hline
\end{tabular}

C-6. Cost Estimate for a Cover System Utilizing Reinforced Sprayed Asphalt (Cont'd) 
DETAIL ESTIMATE

AMCD OV: 2.MEAD

LOG : : 7-03

ACTIVITY CODE: ESE260412

PROI.NAME: ECON. EVALUATION OF CLOSURE CAP BARAIER MATERIAL

Troser: Pamk

PHone 7-6200

DATE
LOC

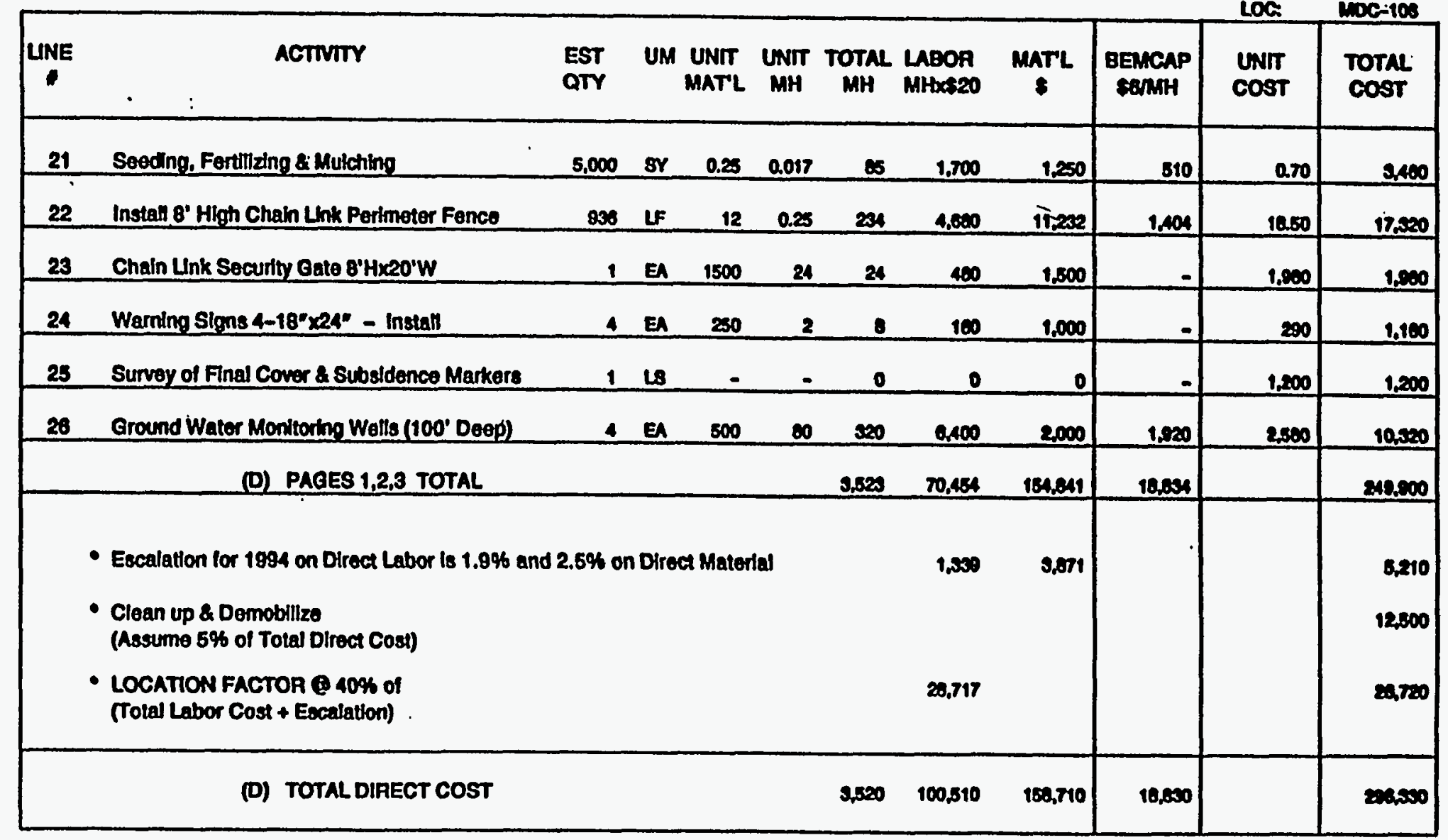

C-6. Cost Estimate for a Cover System Utilizing Reinforced Sprayed Asphalt (Cont'd) 
LOQ: : 7-03

ACTIVITY CODE: ESE260412

PROJ.NAME: ECON. EVALUATION OF CLOSUAE CAP BARAIER MATERIAL

CHCOBV: Q.MEAD

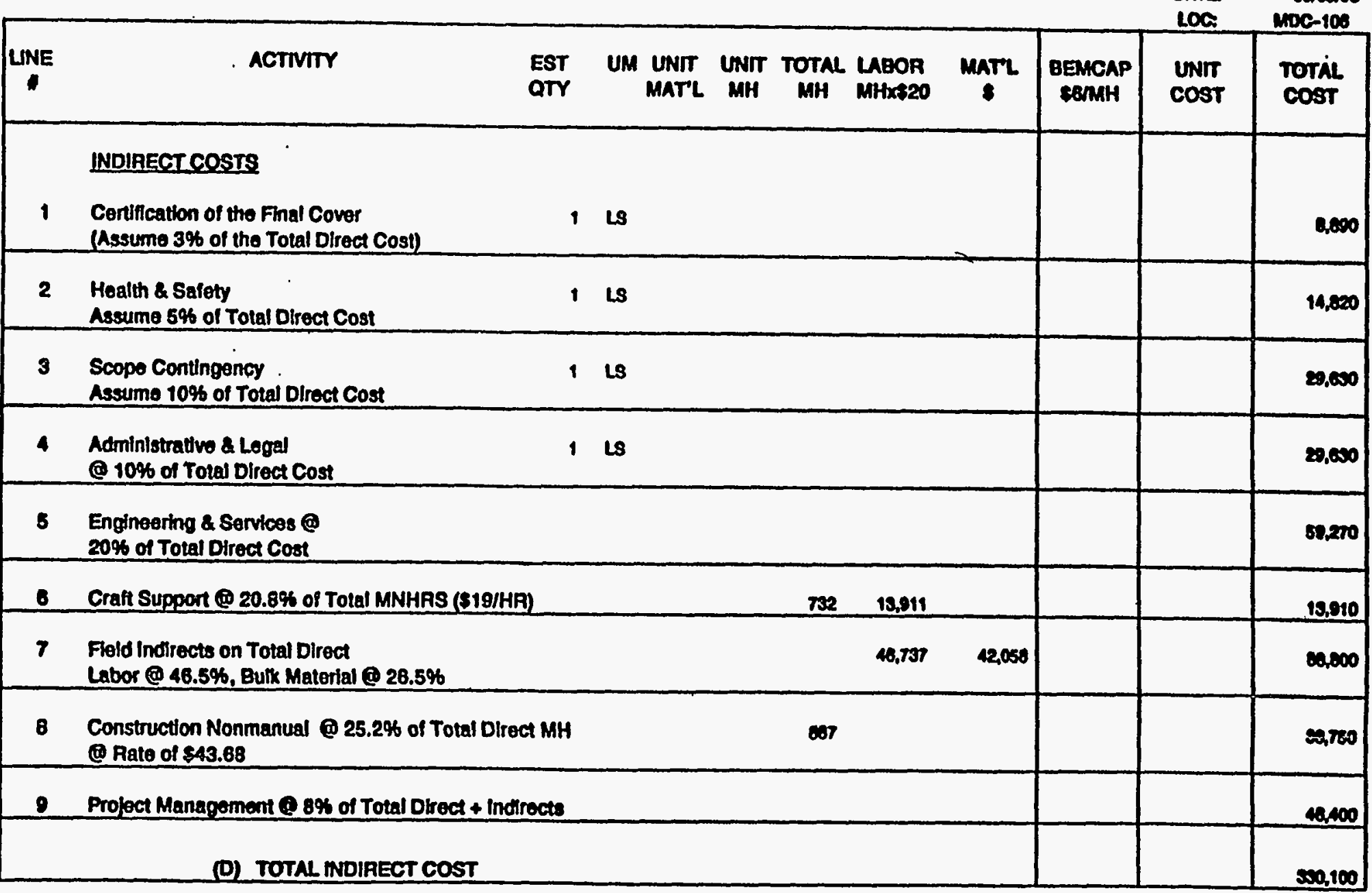

C-6. Cost Estimate for a Cover System Utilizing Reinforced Sprayed Asphalt (Cont'd) 
DETAIL ESTIMATE

LOG : 7-03

ACTIVIT CODE: ESE280412

PROJ.NAME: ECON. EVALUATION OF CLOSURE CAP BAARIER MATERIAL

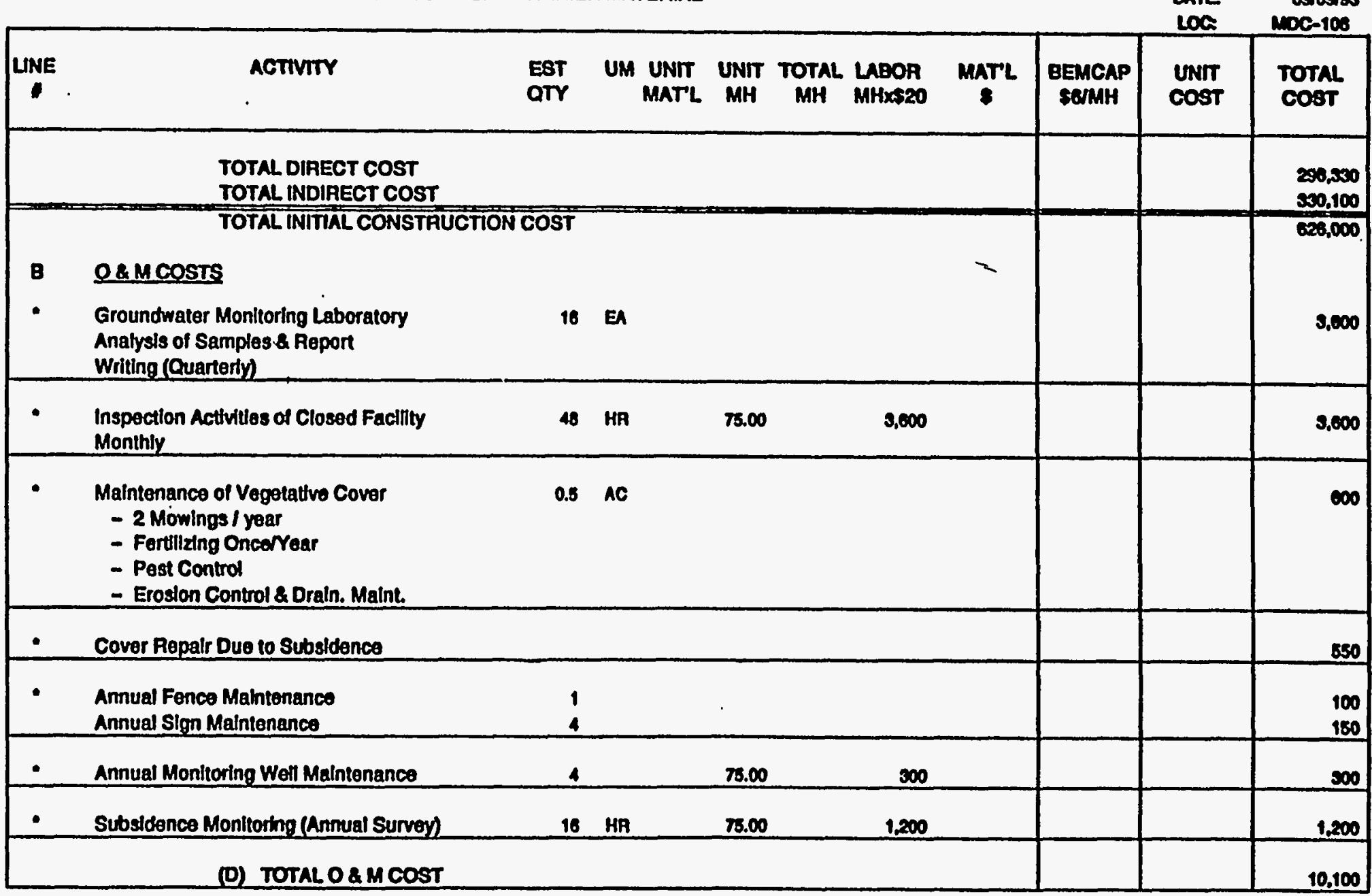

C-6. Cost Estimate for a Cover System Utilizing Reinforced Sprayed Asphalt (Cont'd) 
DETAIL ESTIMATE

LOQ : : 7-03

ACTIVITY CODE: ESE260412

PAOI.NAME: ECON. EVALUATION OF CLOSUAE CAP BARRIER MATERIAL

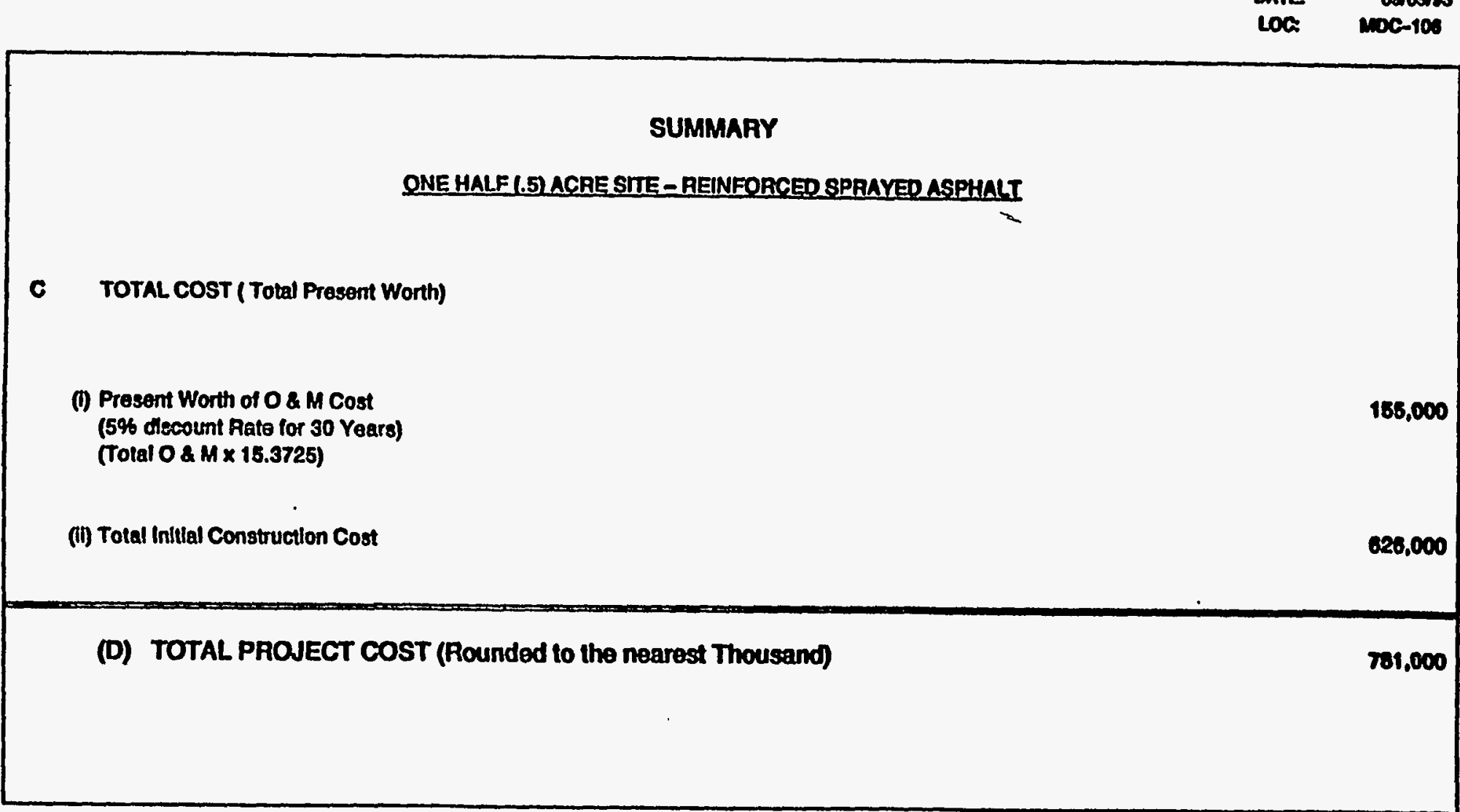

\begin{tabular}{|c|c|}
\hline Cruco er: & 2. MEAD \\
\hline $\begin{array}{l}\text { ESTOEY: } \\
\text { PHone } \\
\text { OATE } \\
\text { Loa }\end{array}$ & $\begin{array}{c}\text { Pomnк } \\
\text { r-5200 } \\
\text { comosiss } \\
\text { noc-10s }\end{array}$ \\
\hline
\end{tabular}

86,000

8,000

, 000

C-6. Cost Estimate for a Cover System Utilizing Reinforced Sprayed Asphalt (Cont'd) 
DETAIL ESTIMATE

CHWOEY: Q MEND

$100 \%: 2-03$

ACTIVITY CODE: ESE26ON12

PROJ.KAME: ECON. EVALUATION OP CLOSURB CAP BARRUER MATERLAL

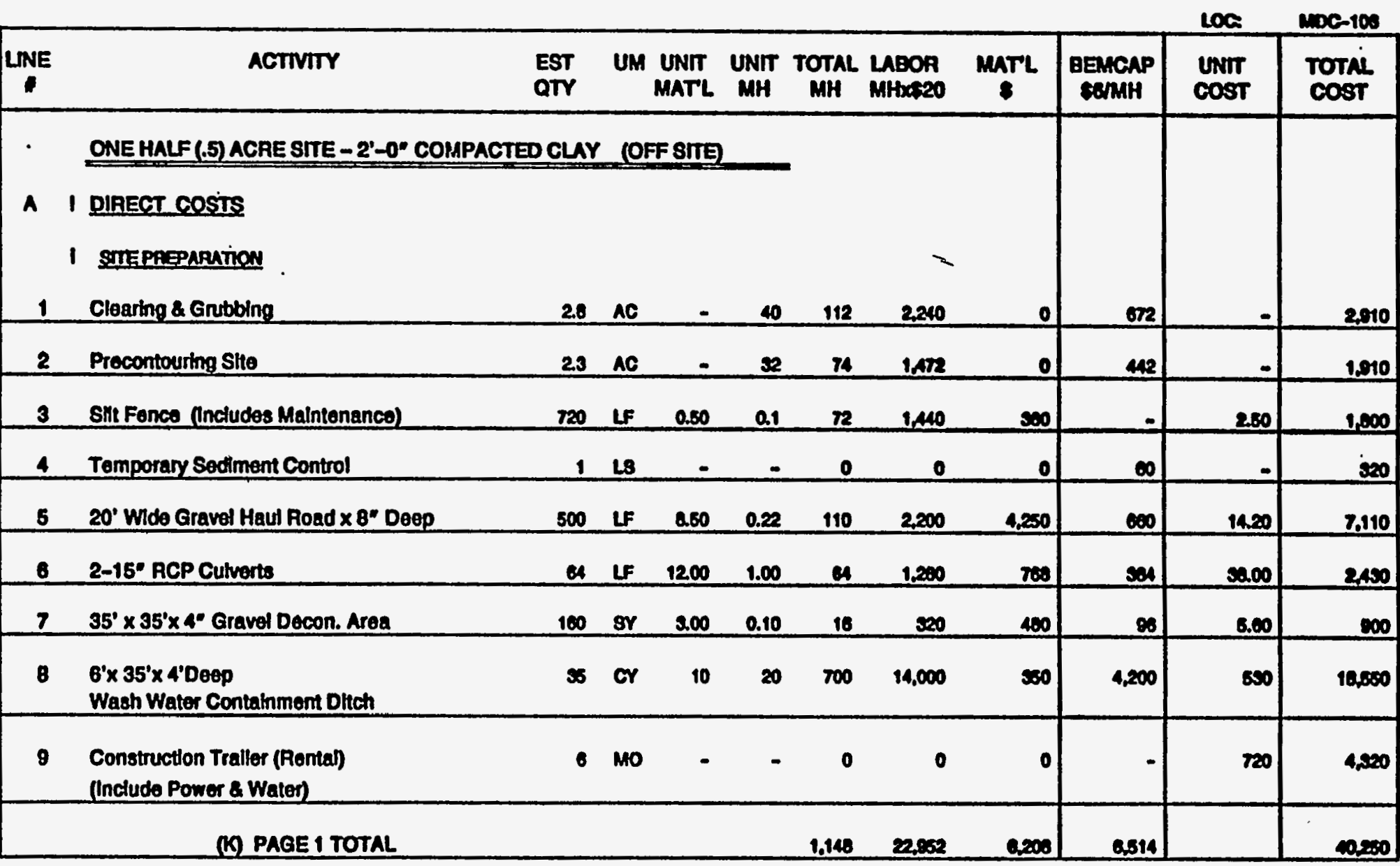

C-7. Cost Estimate for a Cover System Utilizing Compacted Kaolin (Ofisite) Clay and a 30-mil FML 


\section{DETAIL EBTIMATE}

2. MEND

Loo 1: $7-03$

ACTIVTTY CODE: BSE260112

PROJ.NAMGE: BCON. BVALUATTON OF CLOSURE CAP DARRURR MATERIAL

\begin{tabular}{|c|c|c|c|c|c|c|c|c|c|c|c|}
\hline & & & & & & & & & & 100 & inc-10s \\
\hline LINE & ACTIVITY & EST & UM & $\begin{array}{l}\text { UNTT } \\
\text { MAT'L }\end{array}$ & UNH $_{\text {MHT }}$ & TOTAL & $\begin{array}{l}\text { LABOR } \\
\text { MHDOEDO }\end{array}$ & $\underset{8}{\operatorname{MaTL}}$ & BEMCAP & $\begin{array}{l}\text { UNIT } \\
\text { Cost }\end{array}$ & $\begin{array}{l}\text { TOTAL } \\
\text { cost }\end{array}$ \\
\hline & LCOVER CONSTRUCTION & & & & & & & & & & \\
\hline 10 & Waste Stabllization (Crushed Limestone) & 1,007 & $\mathbf{T N}$ & 100 & 0.1 & 101 & 2,014 & 100,700 & $\mathbf{\infty}$ & 10200 & 100,590 \\
\hline 11 & $\begin{array}{l}\text { Prace and Compact Foundation Soll } \\
\text { in } 8^{\circ} \text { Lifts to } 95 \% \text { STD. Proctor }\end{array}$ & 4,035 & or & - & 0.165 & 606 & 13,516 & o & 2,098 & 4.50 & 17.910 \\
\hline 12 & $\begin{array}{l}\text { Compact 2'-0" Thick Clay Layer } \\
\text { in 8" Lits }\end{array}$ & 1,695 & or & 13.00 & 0.106 & 200 & 5,500 & 22,030 & 1,070 & 17.50 & 20,010 \\
\hline 13 & $\begin{array}{l}\text { Placement of } 30 \text { Mn F Fextblo } \\
\text { Membrane Lher }\end{array}$ & 26,100 & SF & 0.10 & 0.000 & 200 & 0.170 & 4,698 & - & 0.50 & 8,070 \\
\hline 14 & $\begin{array}{l}\text { Placement of 1'-0 Coarse Sand } \\
\text { Drainage Layer }\end{array}$ & 804 & or & 10.50 & 0.036 & 32 & $\infty 02$ & Q.48T & 100 & 11.40 & 10,510 \\
\hline 15 & $\begin{array}{l}\text { Placoment of Geotextlle (TYPAP) Fiter on } \\
y^{\prime}-0^{*} \text { Coarse Sand Drainage Layor }\end{array}$ & 2,785 & 8r & 0.10 & 0.006 & 17 & 334 & sot & - & a.so & 800 \\
\hline 10 & Placement of $1^{\prime}-6^{\prime}$ Common Fill & 1,212 & Or & 376 & 0.07 & $\infty$ & 1,097 & 4845 & 800 & 0.00 & 0.730 \\
\hline 17 & Placement of 6" Topsoll & 155 & or & 0.60 & 0.00 & $\boldsymbol{s}$ & 720 & 42020 & 210 & 11.00 & 8,270 \\
\hline 18 & $\begin{array}{l}\text { Fiacoment of Perimeter Exterfor } \\
\text { Dralnage Layer } 3 / 4^{*} \times 3^{\circ} \text { DP (Crushod Sione) }\end{array}$ & 216 & SY & 1.50 & 0.22 & 48 & 850 & 204 & 200 & 7.20 & 1,500 \\
\hline 19 & Excavation of Trapezoldal Dralnago Ditch & 221 & or & - & 0.100 & 41 & 016 & 0 & 245 & 400 & 1,000 \\
\hline & (1) PAGE 2 TOTAL & & & & & 1,515 & $\mathbf{5 0 , 2 0 7}$ & 1400012 & 7.724 & & 104.000 \\
\hline
\end{tabular}

C-7. Cost Estimate for a Cover System Utilizing Compacted Kaolin (Offsite) Clay and a 30-mil FML (Cont'd) 
D B T A L B S IMA T B CWKOBY OMEND

Lod I: 7-03

ACTIVTY CODB: BSEZ6OS12

PROJ.NAME: BCON. EVALUATION OP CLOSURB CAP BARRTER MATERIAL

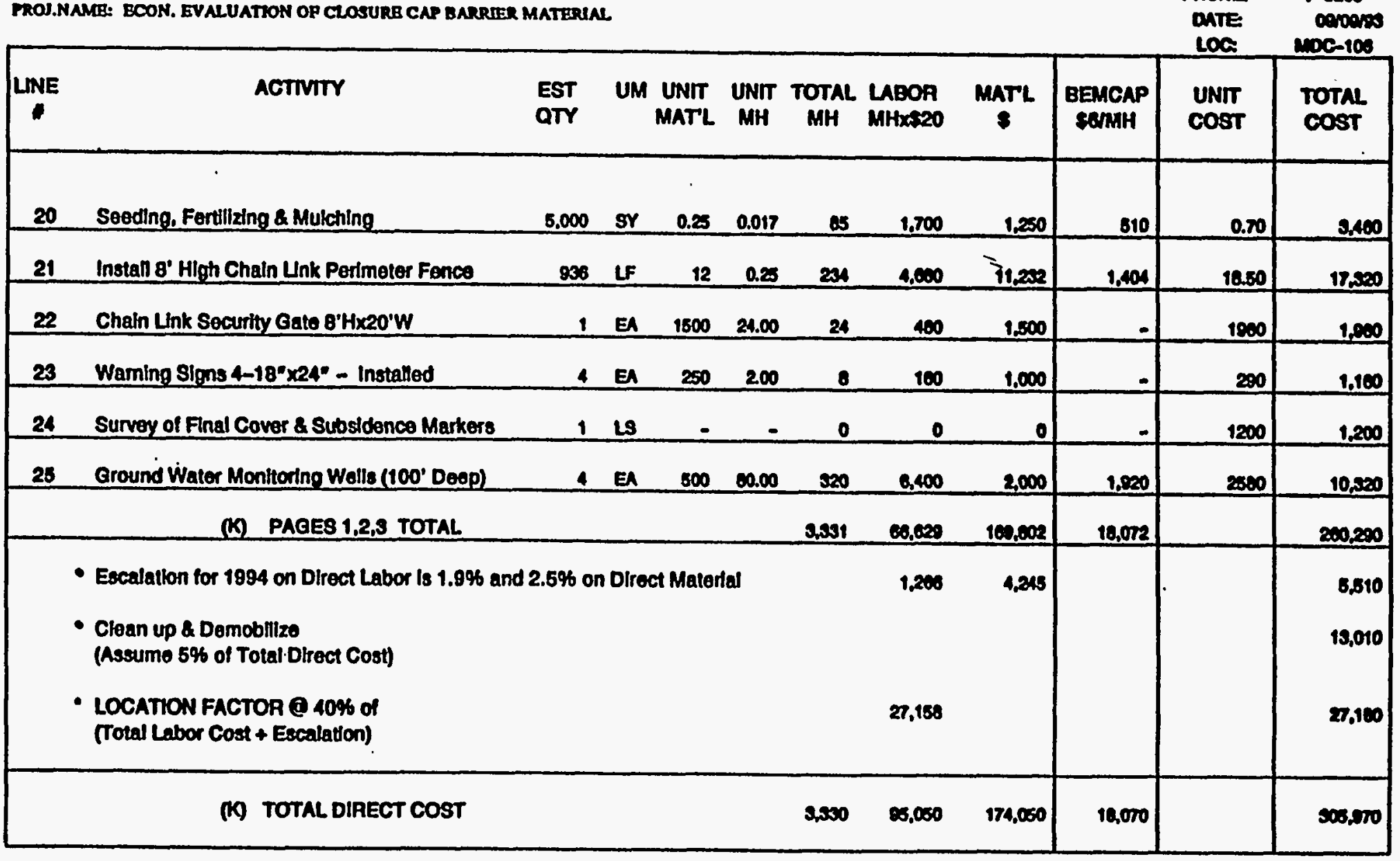

C-7. Cost Estimate for a Cover System Utilizing Compacted Kaolin (Offsite) Clay and a 30-mil FML (Cont'd) 
DETAIL BSTIMATE

Q. MEAD

200 I: 7-03

ACTIVITY CODE: BSE260412

RROJ.NAMG: BCON. BVALUATION OF CLOSURB CAP BAPRIER MATERLAL

CAwD BY:

ramix

SSTO BY

7-5006

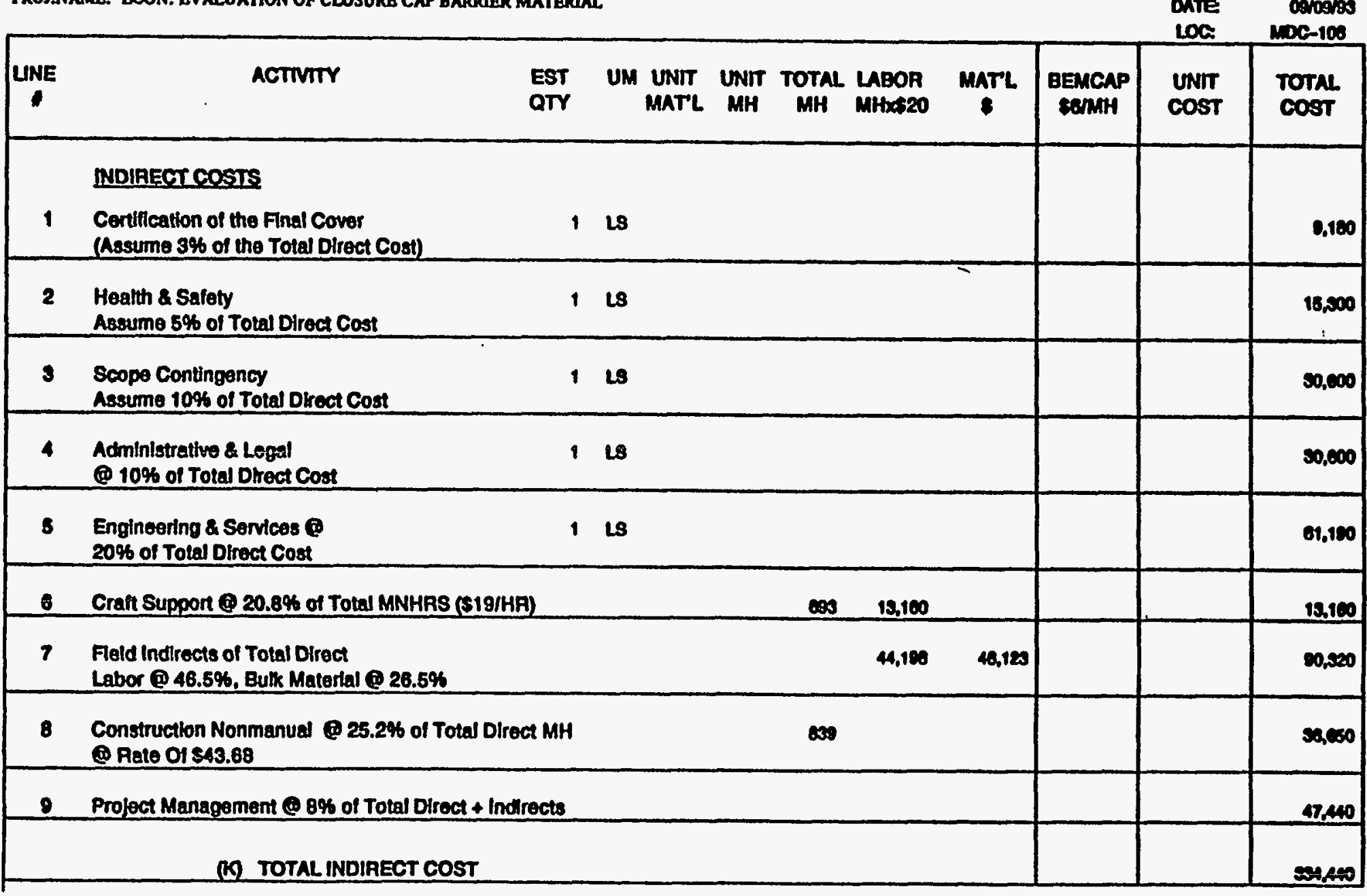

C-7. Cost Estimate for a Cover System Utilizing Compacted Kaolin (Offsite) Clay and a 30-mil FML (Cont'd) 
D B T A L B STIMATB

Q MEND

L00 I: 7-03

ACTIVTYY CODE: ESE26012

MOJ.NAME: ECON. EVALUATHOY OF CLOSURE CAP BARRIRR MATERIAL.

PATOEY: Pank
PHOME

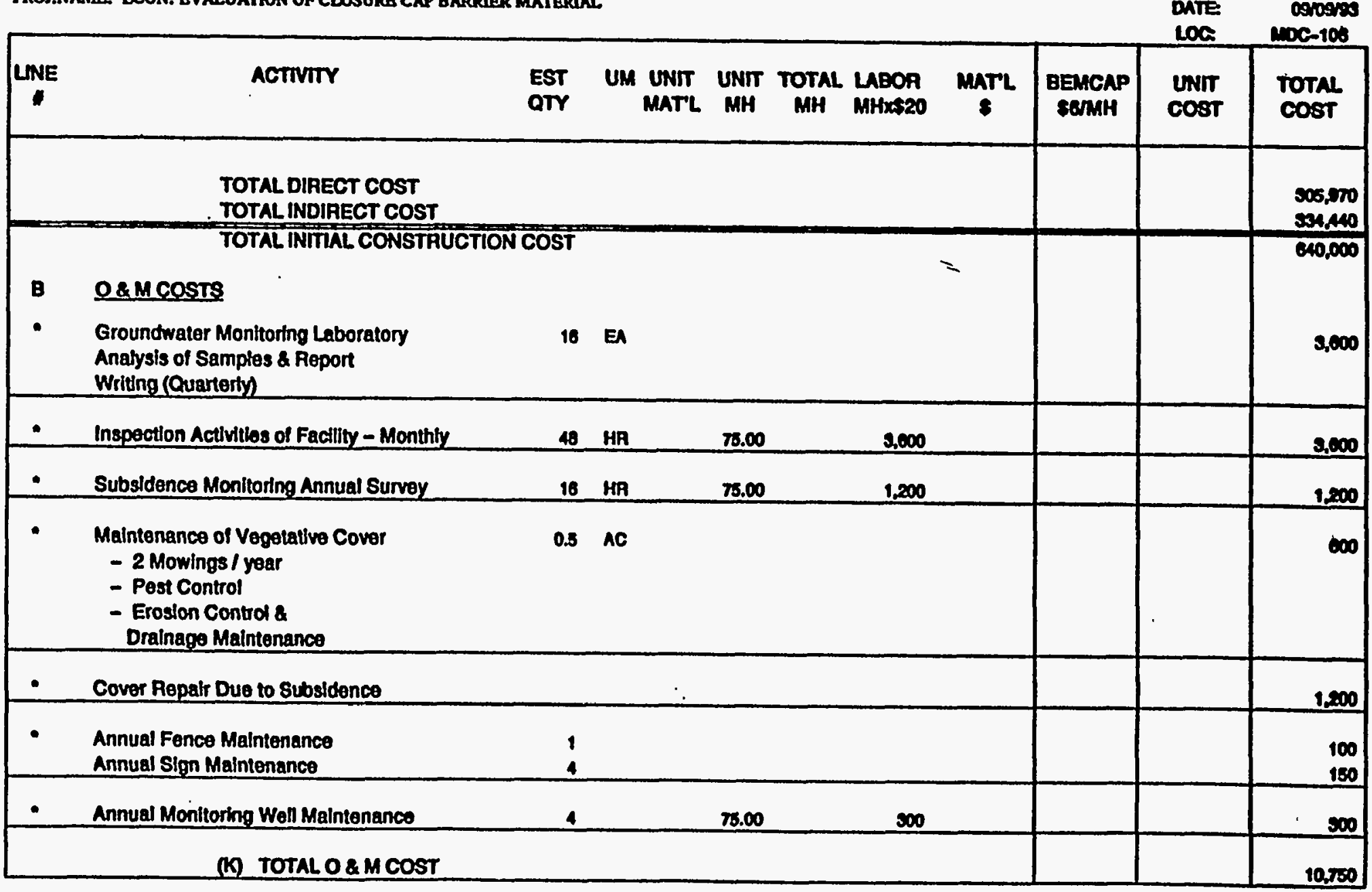

C-7. Cost Estimate for a Cover System Utilizing Compacted Kaolin (Offsite) Clay and a 30-mil FML (Cont'd) 
DETAILASTIMATB

\begin{tabular}{|c|c|}
\hline Atro or: & $8 . M=10$ \\
\hline $\begin{array}{l}\text { ESTD EY: } \\
\text { PHOMVE } \\
\text { OATE } \\
\text { LOa: }\end{array}$ & 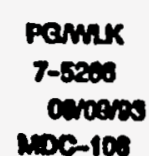 \\
\hline
\end{tabular}

\section{1: 2-03}

ACTTVITY CODE: BSE2OOM12

PROJ.NAMRE: BCON. BVALUATION OF CLOSURB CAP BARRIER MATERLAL

Low

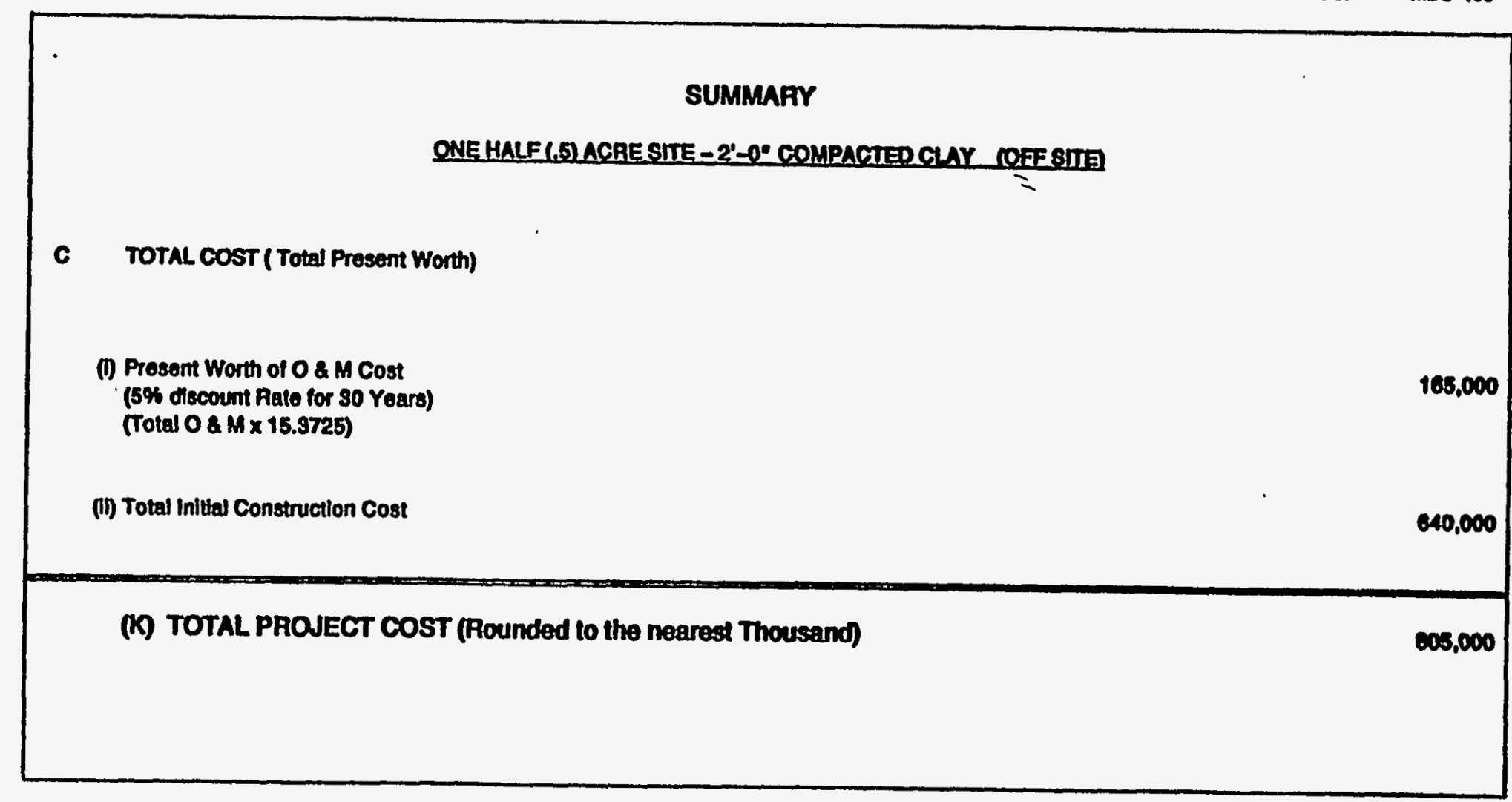

C-7. Cost Estimate for a Cover System Utilizing Compacted Kaolin (Offsite) Clay and a 30-mil FML (Cont'd) 


\section{DETAIL ESTIMATE}

CAED BY: S.MEND

L00 : $7-03$

ACTIVITY CODB: BSB260412

RROS.NAME: ECON. EVALUATION OF CLOSURB CAP BARRIER MATERIAL

esTogr:

PHowe

panke

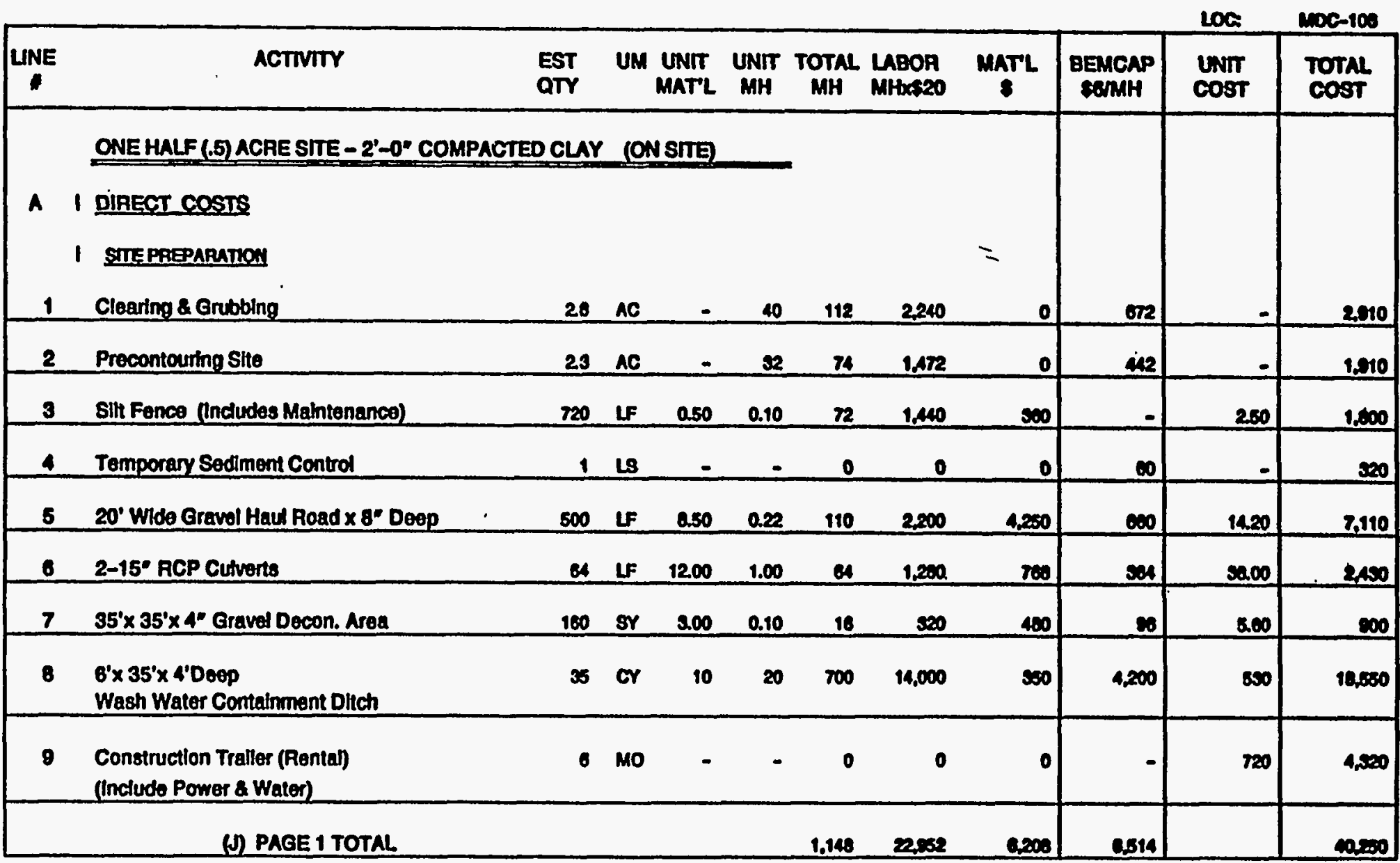

C-8. Cost Estimate a for Cover System Utilizing Compacted Onsite Sandy Clay and a 30-mil FML 
DETAIL ESTIMATE

Q.MEND

\section{0 : :-03}

ACTIVITY CODE: ESE260412

PROI.NAME: ECON. EVALUATTON OF CLOSURB CAP BARRIER MATERIAL.

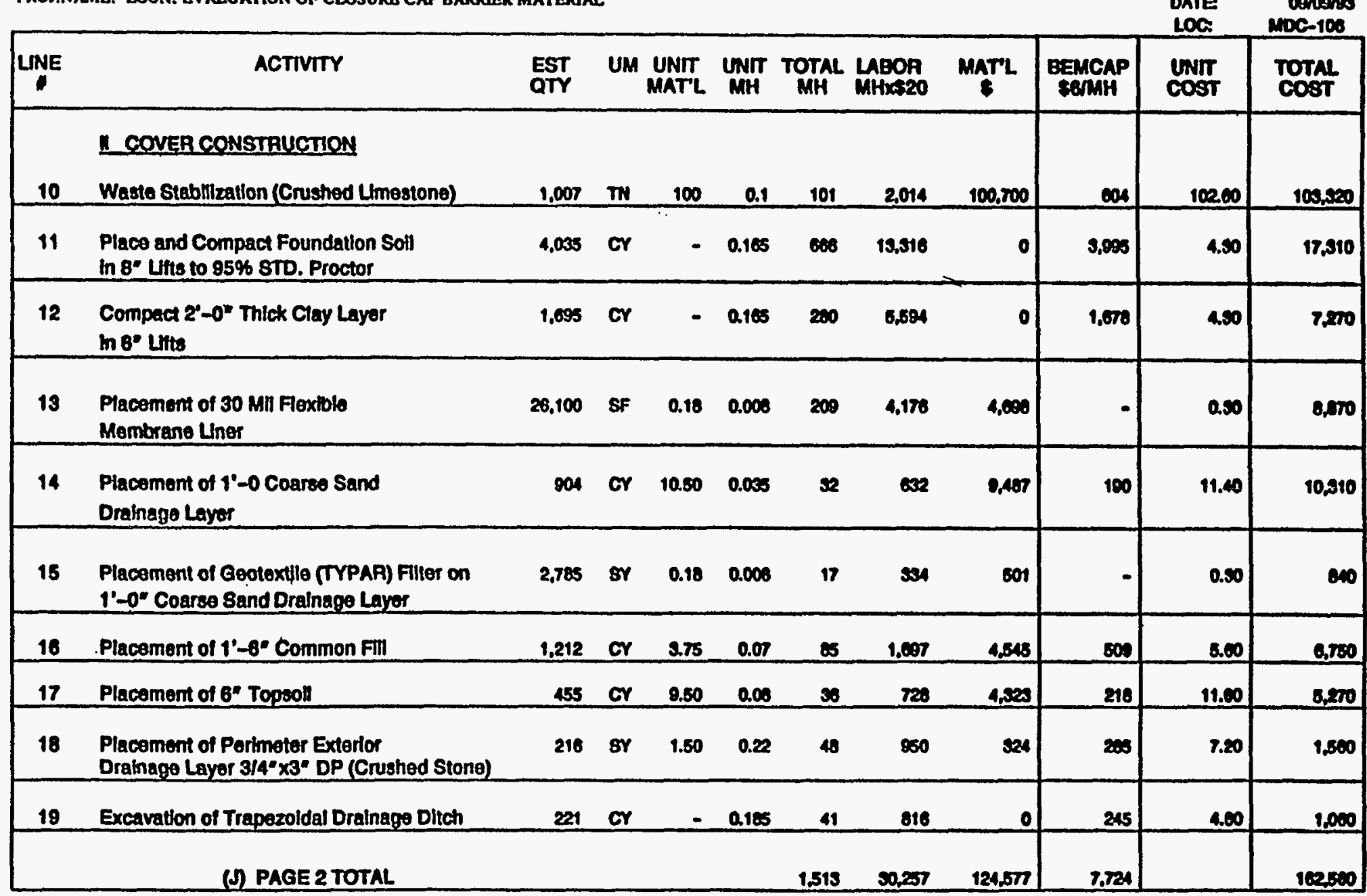

C-8. Cost Estimate a for Cover System Utilizing Compacted Onsite Sandy Clay and a 30-mil FML (Cont'd) 
D B T A L B T IMATE CHKOBY: RMEAO

Lo01: 7-03

ACTIVITY CODE: BSRESON12

PROS.NAME: BCON. BVALUATTON OF CLOSURB CAP BARIIER MATERLAL

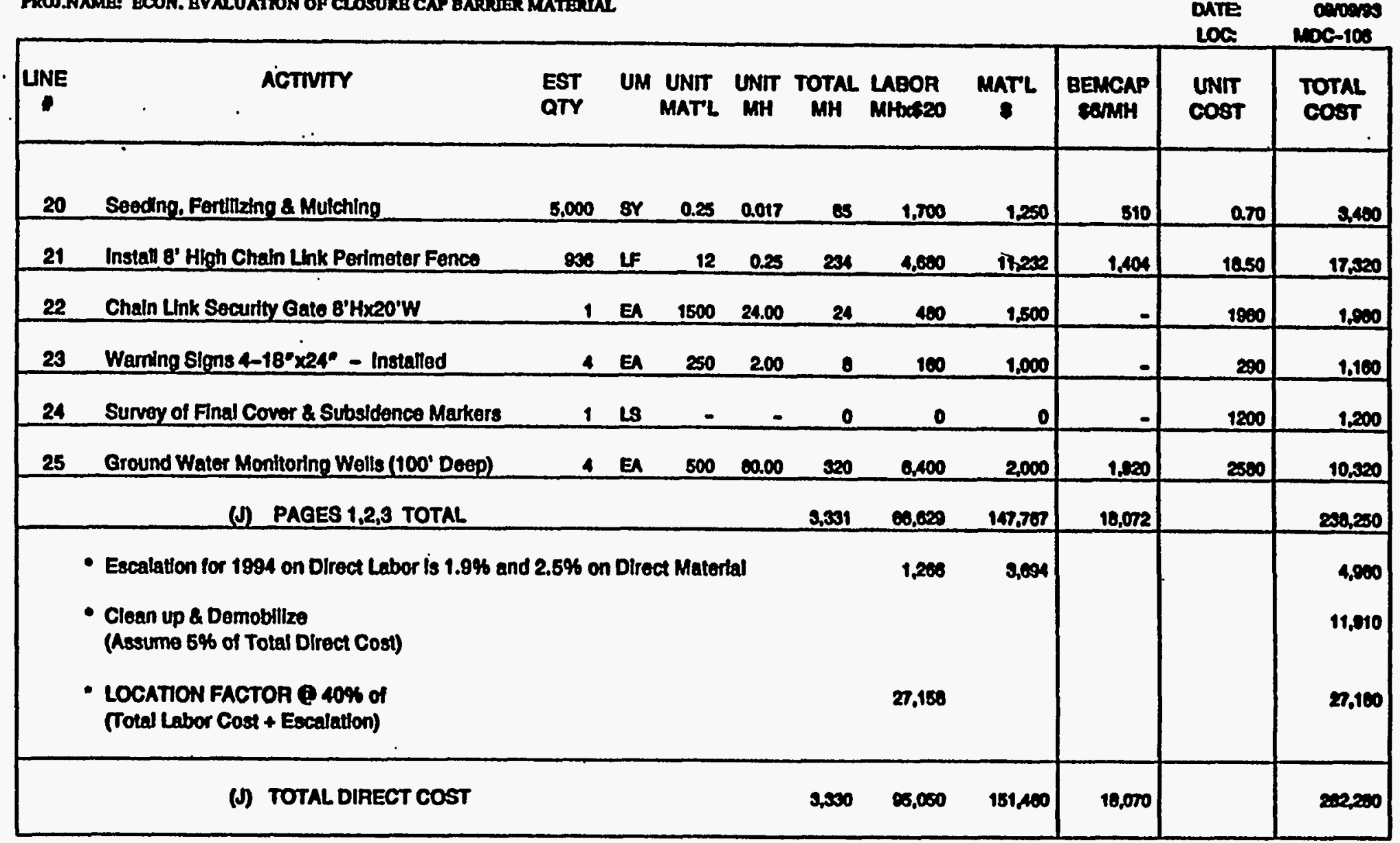

C-8. Cost Estimate a for Cover System Utilizing Compacted Onsite Sandy Clay and a 30-mil FML (Cont'd) 
Lod: 7-03

ACTTVITY CODB: ESR2CON12

FRO.MAME: ECON. EVALUATION OP CLOSURB CAP BARRIER MATBRUAL

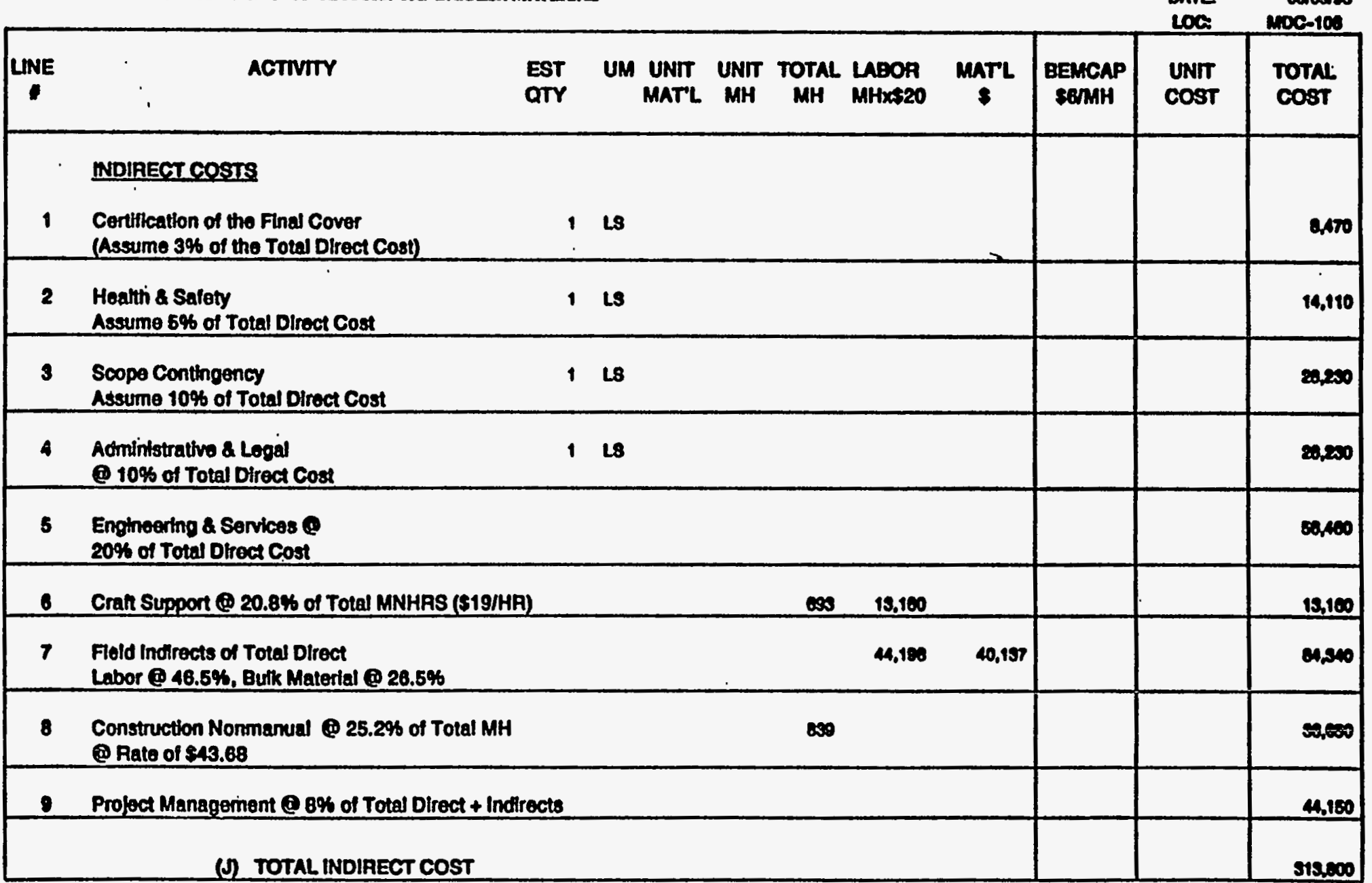

C-8. Cost Estimate a for Cover System Utilizing Compacted Onsite Sandy Clay and a 30-mil FML (Cont'd) 
Lod: : 7-03

ACTIVITY CODE: ESER6O412

PROJ.NAME: ECON. BVALUATON OF CLOSURE CAP BARRIER MATERUAL

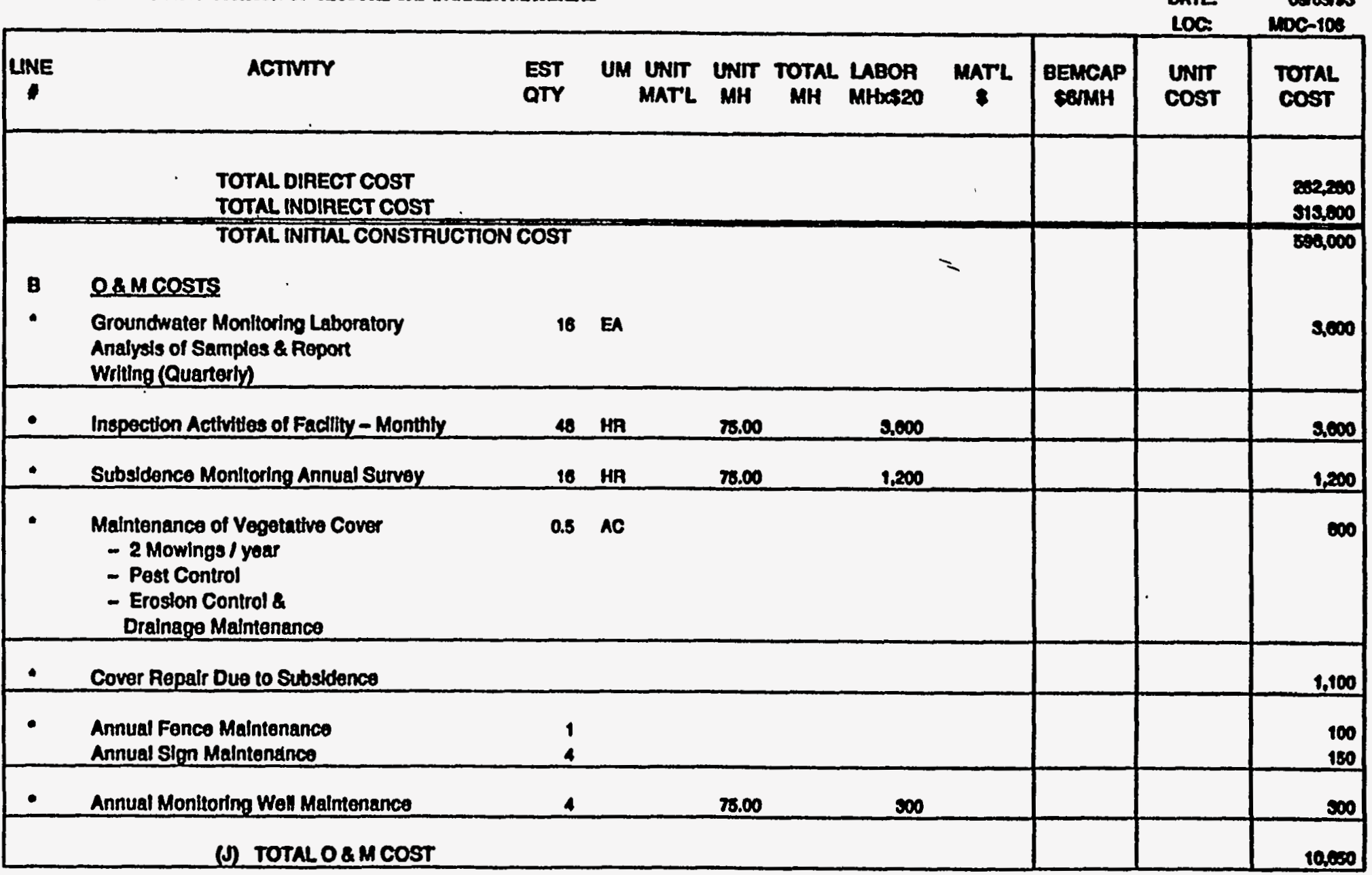

C-8. Cost Estimate a for Cover System Utilizing Compacted Onsite Sandy Clay and a 30-mil FML (Cont' d) 
D BTAIL BSTIMATE

\begin{tabular}{|c|c|}
\hline wD or: & a. $m E$ \\
\hline $\begin{array}{l}\text { EBTD BY: } \\
\text { PHONE } \\
\text { DATE } \\
\text { LOG }\end{array}$ & 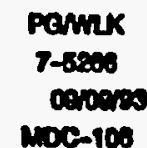 \\
\hline
\end{tabular}

1001: $7-03$

ACTIVITY CODB: ESEZCON12

PROJ.HAMM: BCON. EVALUATION OF CLOSURB CAP BARRIER MATERUAL.

Loc

Moc-100

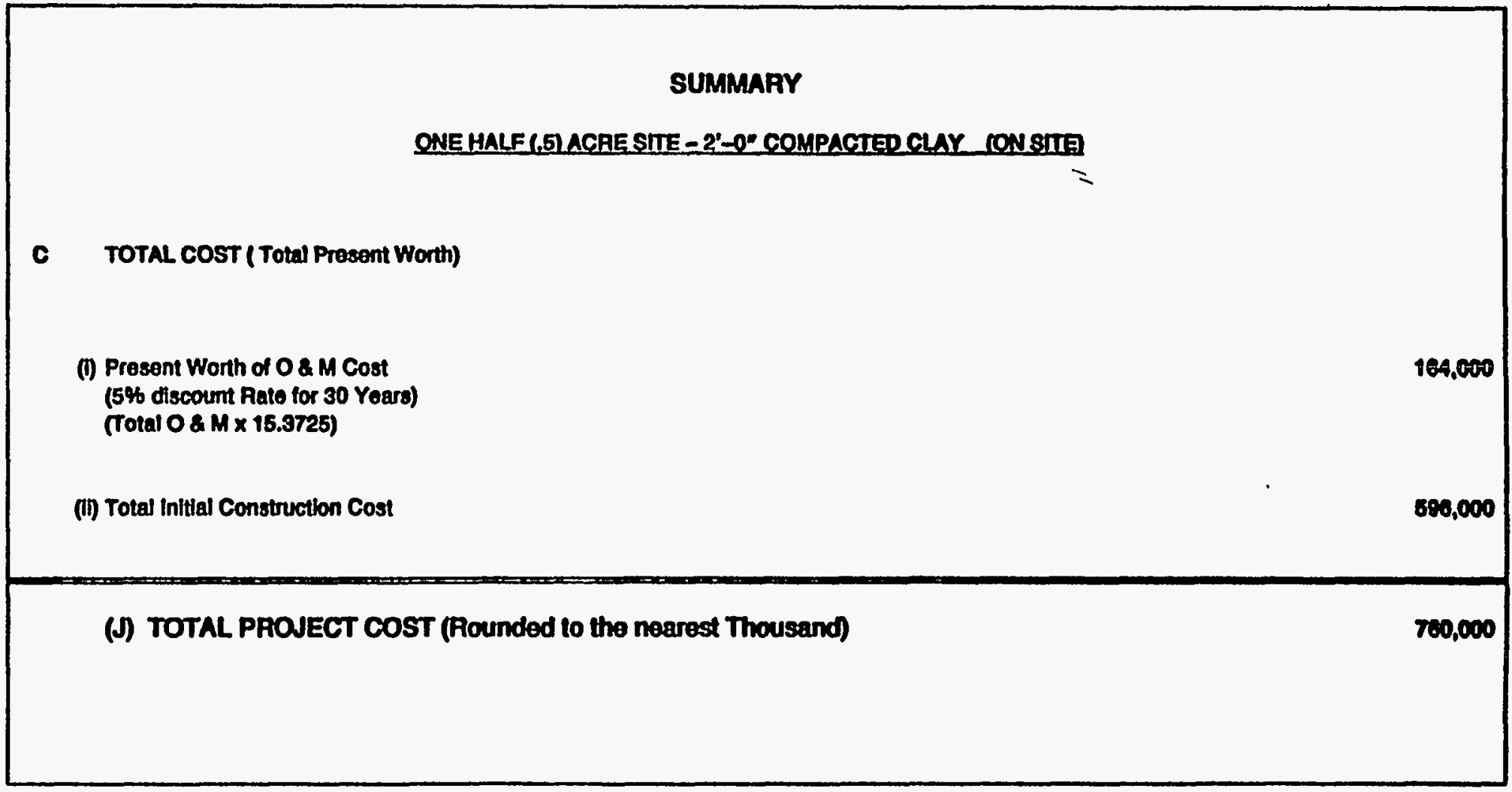

C-8. Cost Estimate a for Cover System Utilizing Compacted Onsite Sandy Clay and a 30-mil FML (Cont'd) 
DETAIL ESTIMATE

Loof: $7-03$

ACTVITY CODE: ESE260112

PROJ.NAME: ECON. EVALUATTON OF CLOSURB CAP BARRIER MATERTAL

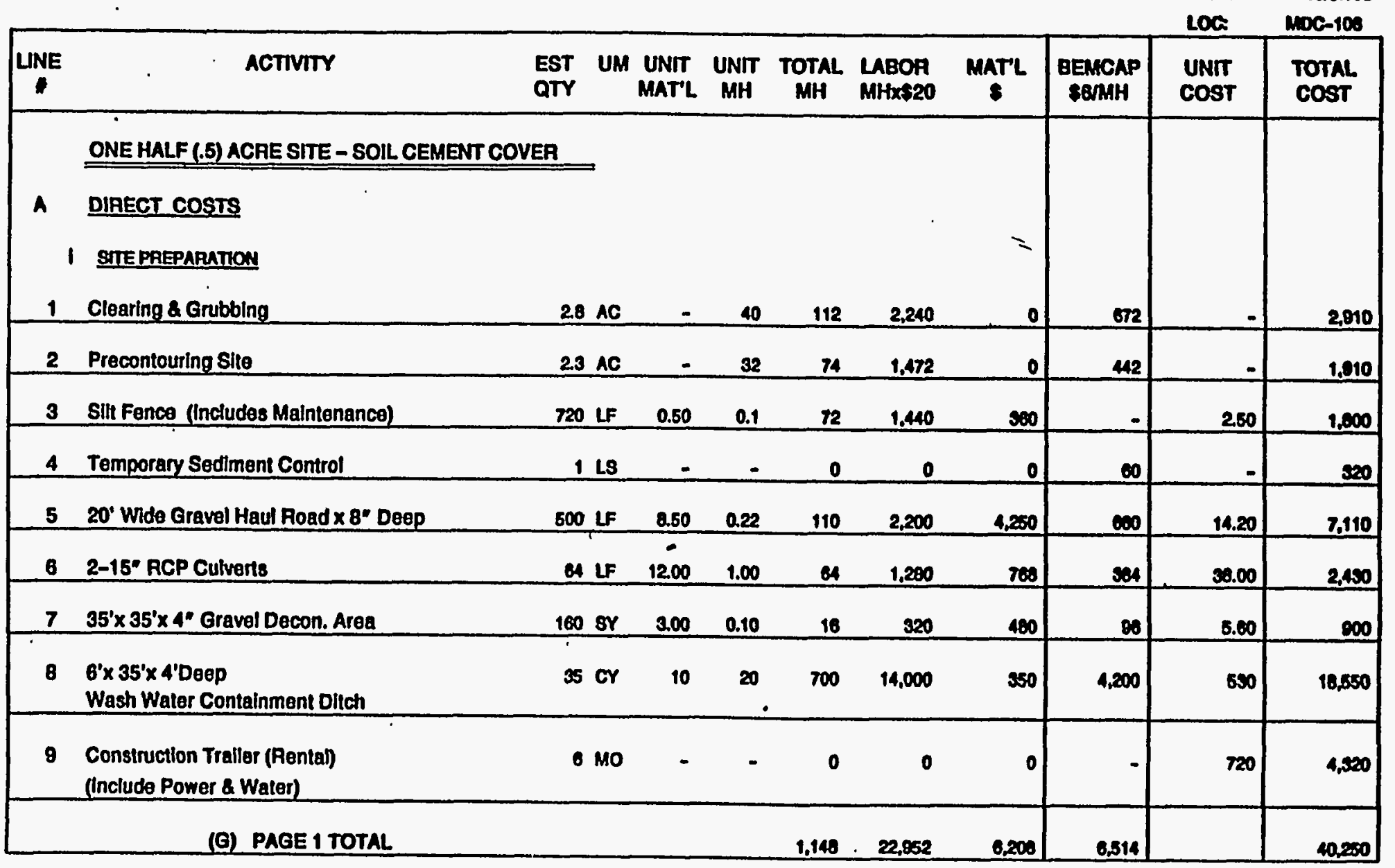

C-9. Cost Estimate for a Cover System Utilizing Soil Cement and 30-mil FML 
DETAIL ESTIMATE CAKDOY: 8.MEAD

Lo01: 7-03

ACTIVITY CODB: ESE260412

PROJ.NAME: ECON. BVALUATION OF CLOSURB CAP BARRIER MATERIAL

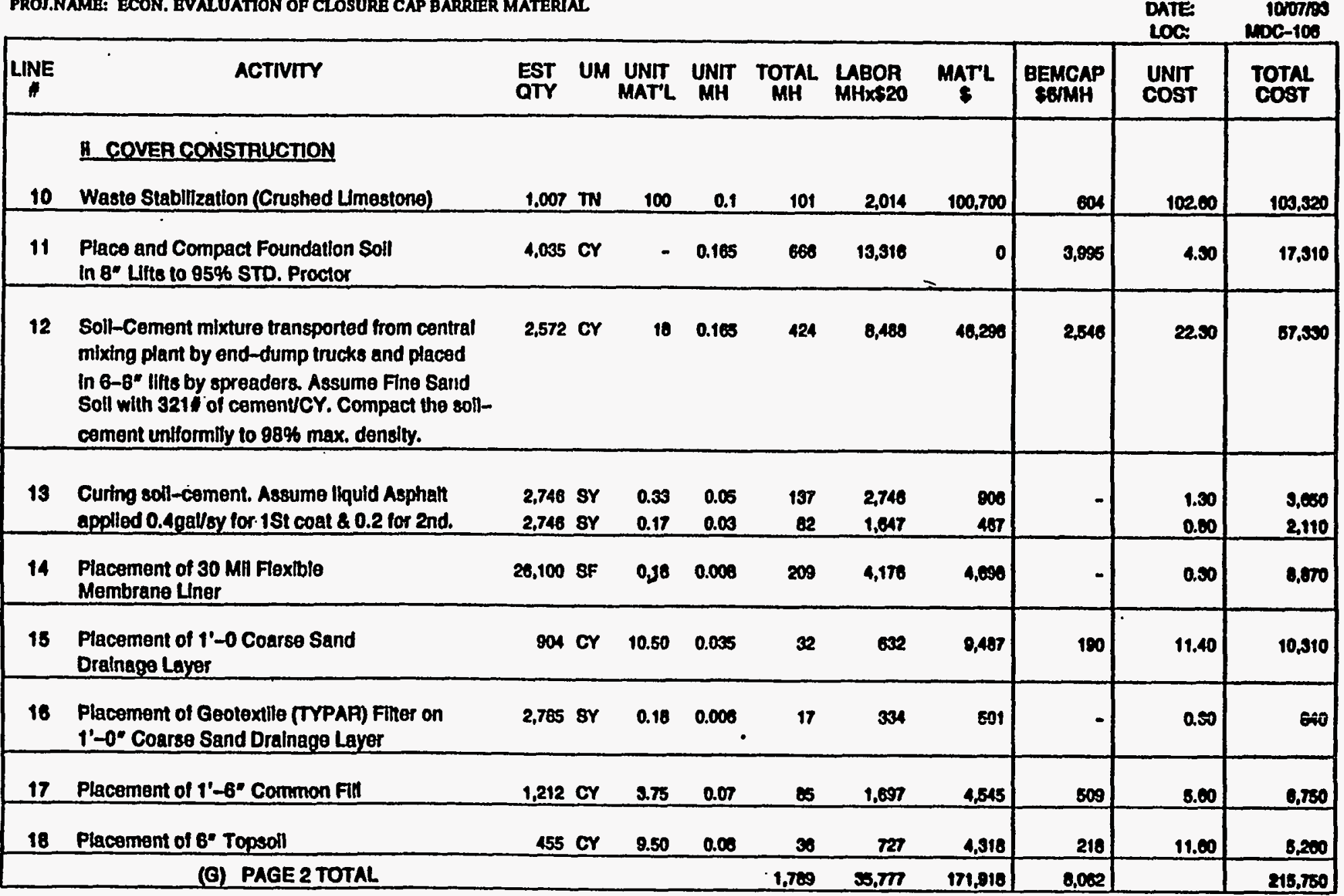

C-9. Cost Estimate for a Cover System Utilizing Soil Cement and 30-mil FML (Cont'd) 
D B TAIL E S T I M A T

CAWD BY: S.MEAD

LO0 I: 7-03

ACTIVTTY CODE: ESE26OH12

PROJ.NAMB: ECON. EVALUATION OF CLOSURE CAP BARRIER MATERIAI

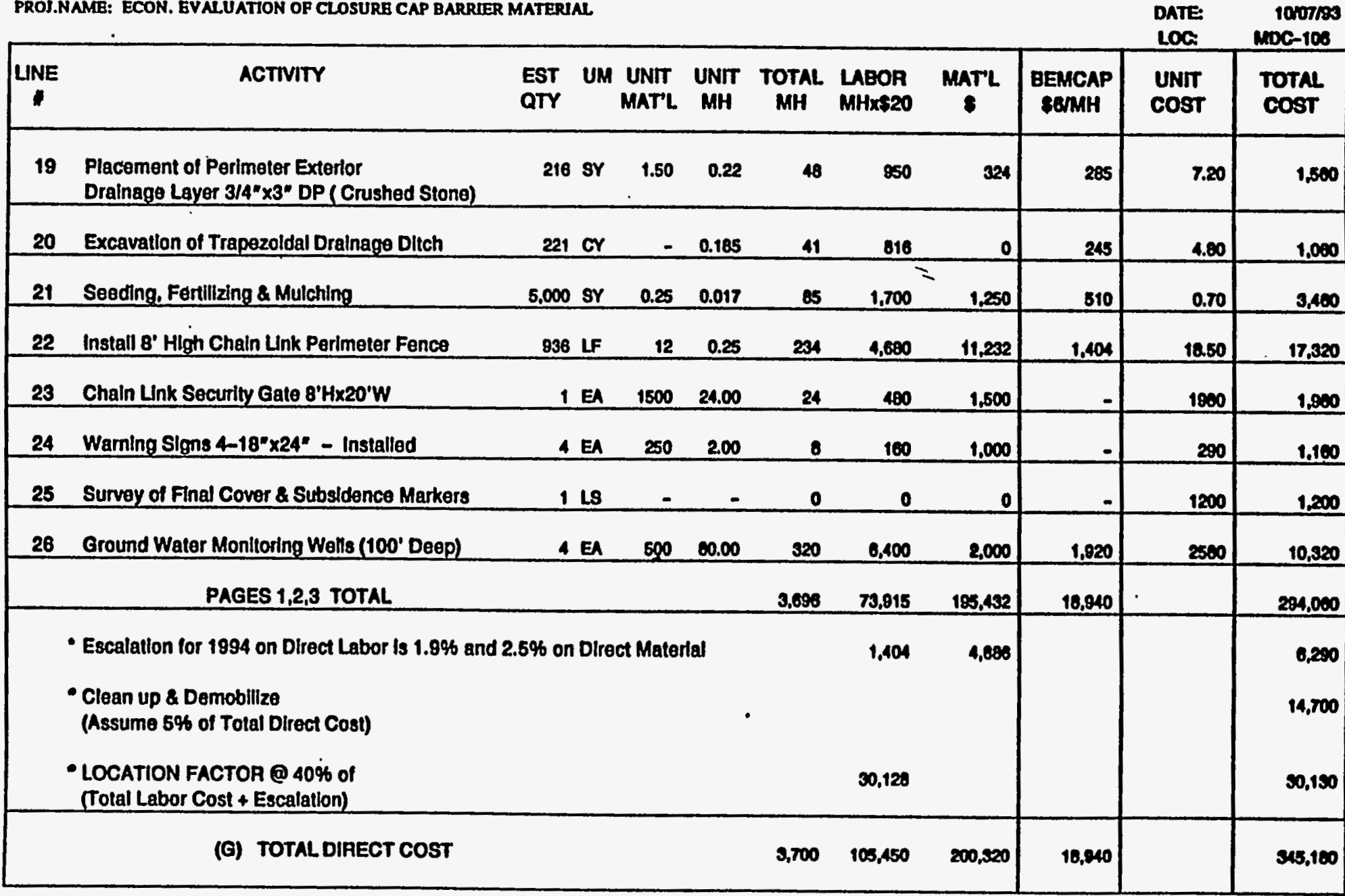

C-9. Cost Estimate for a Cover System Utilizing Soil Cement and 30-mil FML (Cont'd) 
D BTAIL B T I MAT B

L001: 7-03

ACTIVITY CODE: ESE260412

PROJ.NAME: ECON. EVALUATION OF CLOSURB CAP BARRIIR MATERIAL.

CAKDBY: Q.MEAD

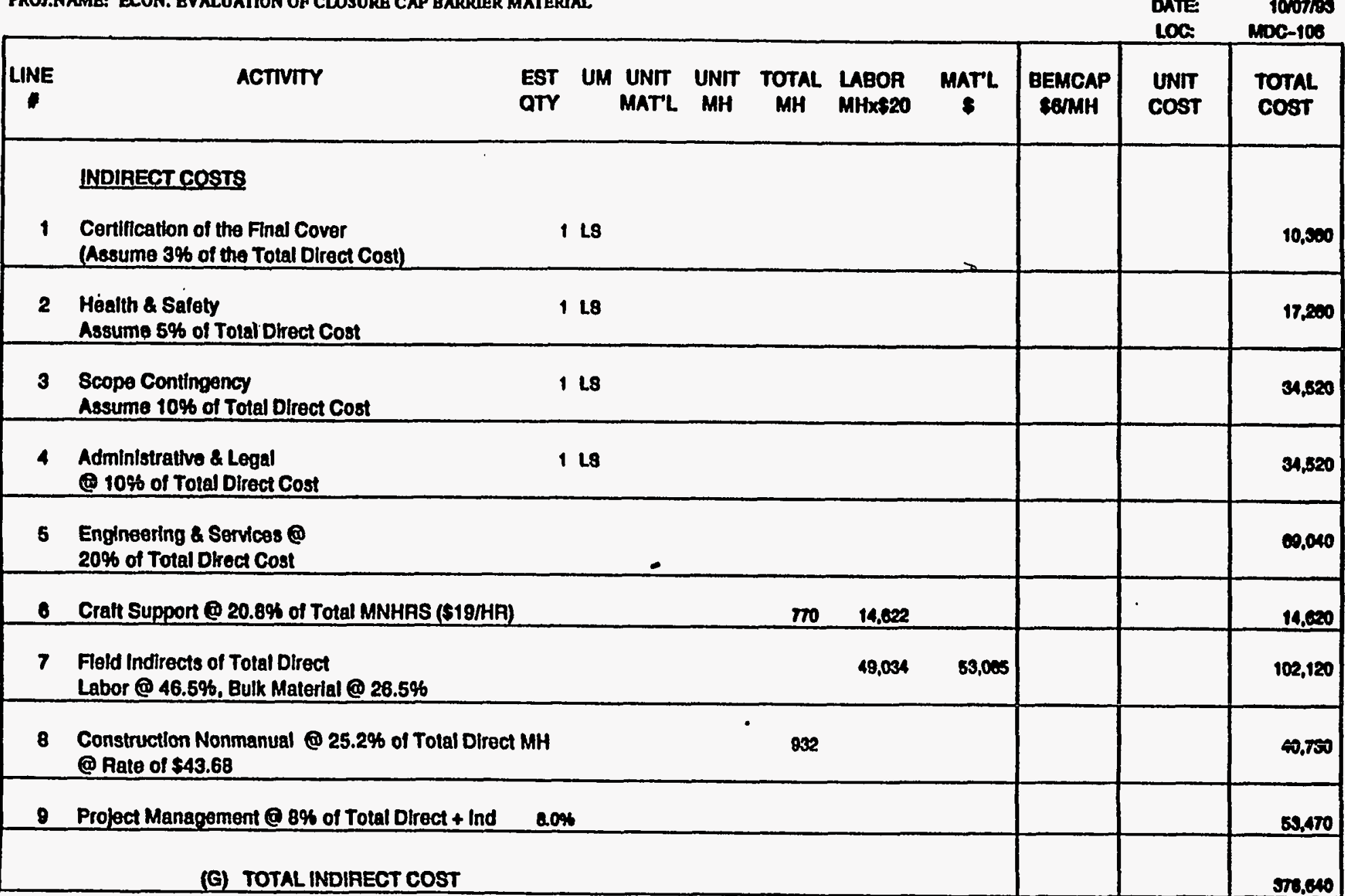

(G) TOTAL INDIAECT COST

C-9. Cost Estimate for a Cover System Utilizing Soil Cement and 30-mil FML (Cont'd) 
Economic Evaluation of Closure Cap Barrier Materials Study(U)

Volume II Appendix $C$

September 1993

I

D B T A L B S T I M T B

CHECD BY: S. MEND

Lo0 : : 7-03

ACTIVITY CODE: ESE2C0412

PROJ.NAME: ECON. EYALUATION OF CLOSURB CAP BARRER MATERIAL

ESTO 8Y: Pank

PHOME: 7-5200

DATE: 10NT/8S

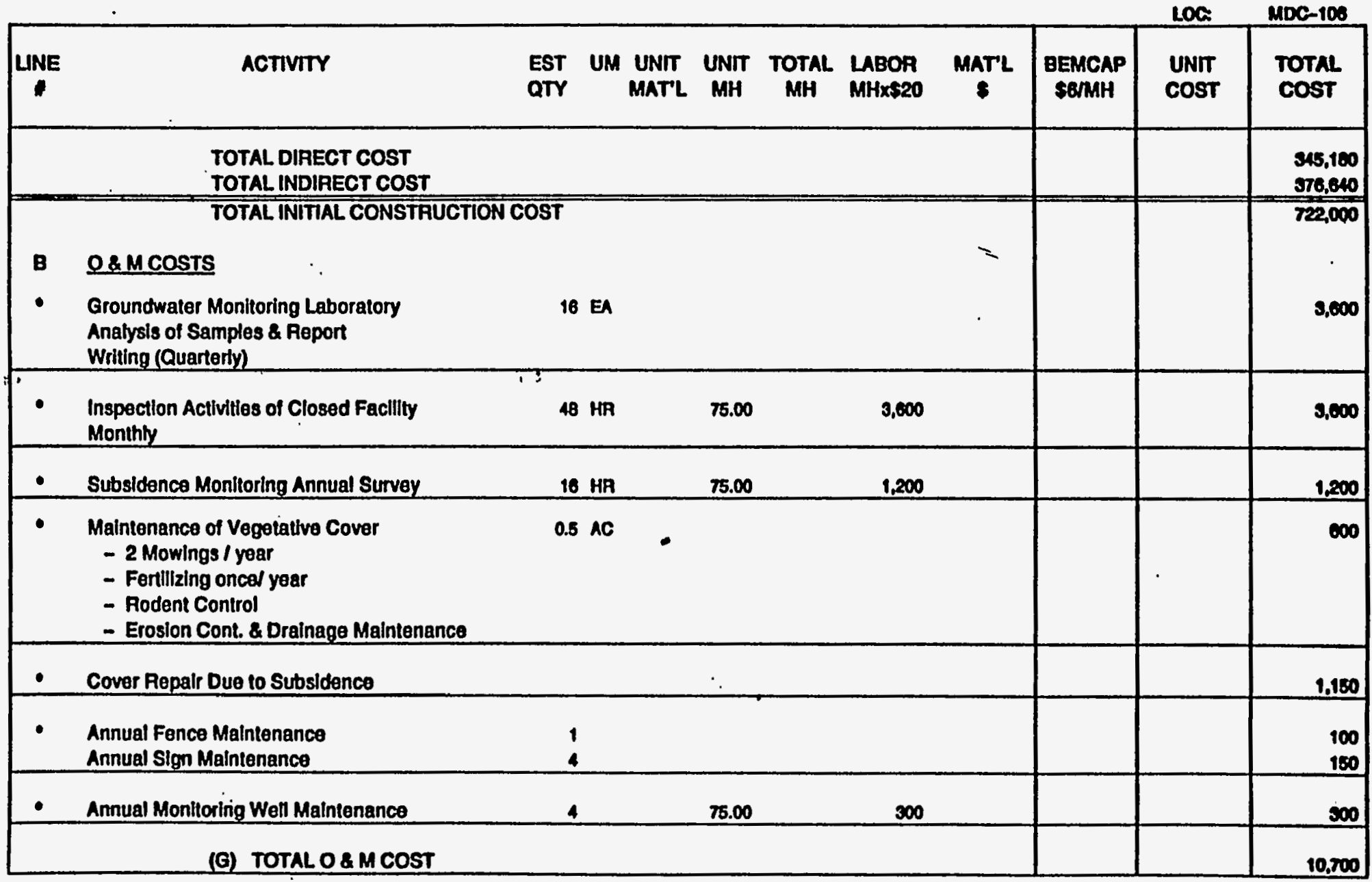

C-9. Cost Estimate for a Cover System Utilizing Soil Cement and 30-mil FML (Cont'd) 


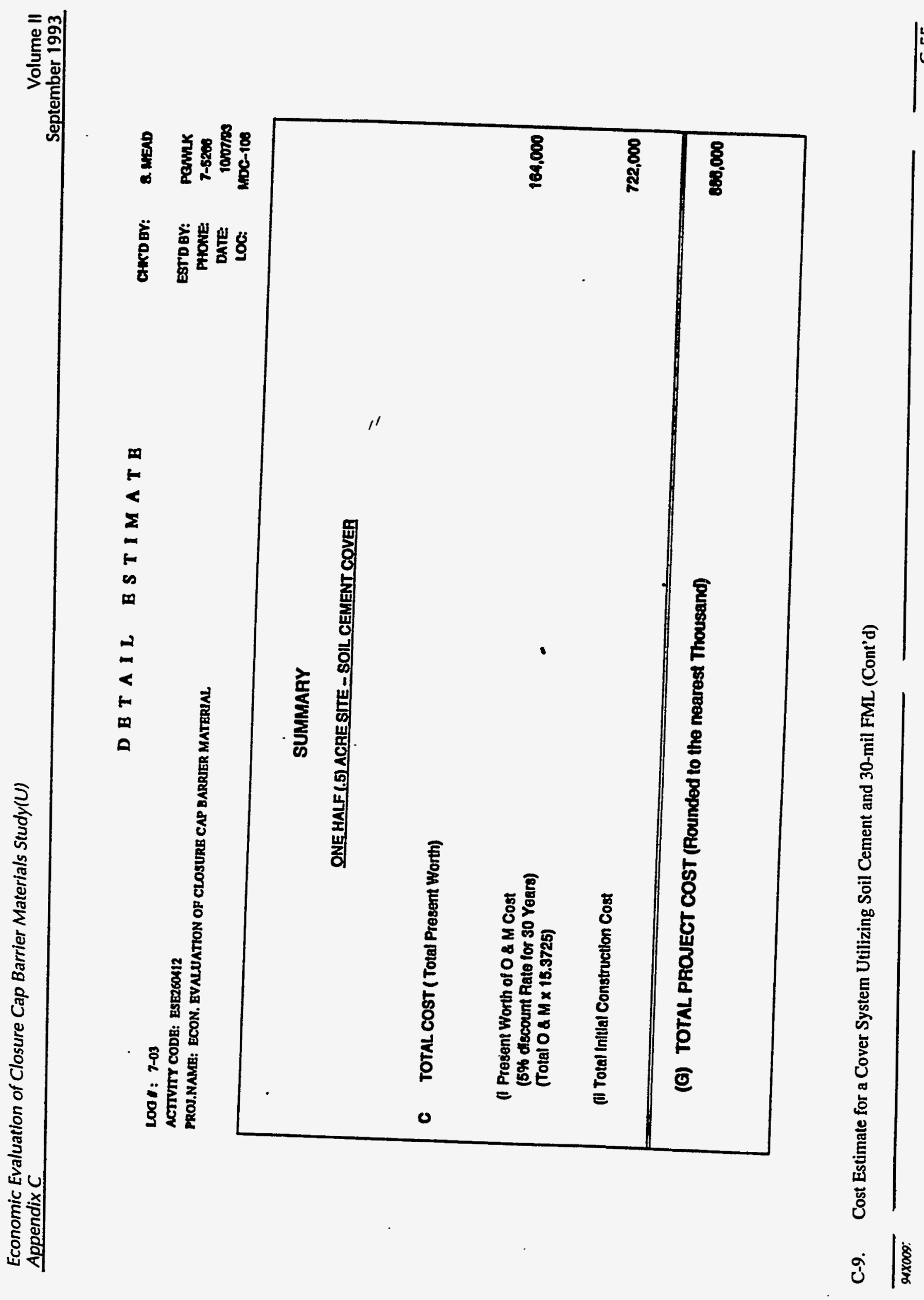


L00 I: 7-03

ACTIVITY CODE: ESE2 20412

PROJ.NAME: ECON. BVALUATION OF CLOSURB CAP BARRIER MATERIAL.

ESTOBY: PaAnK

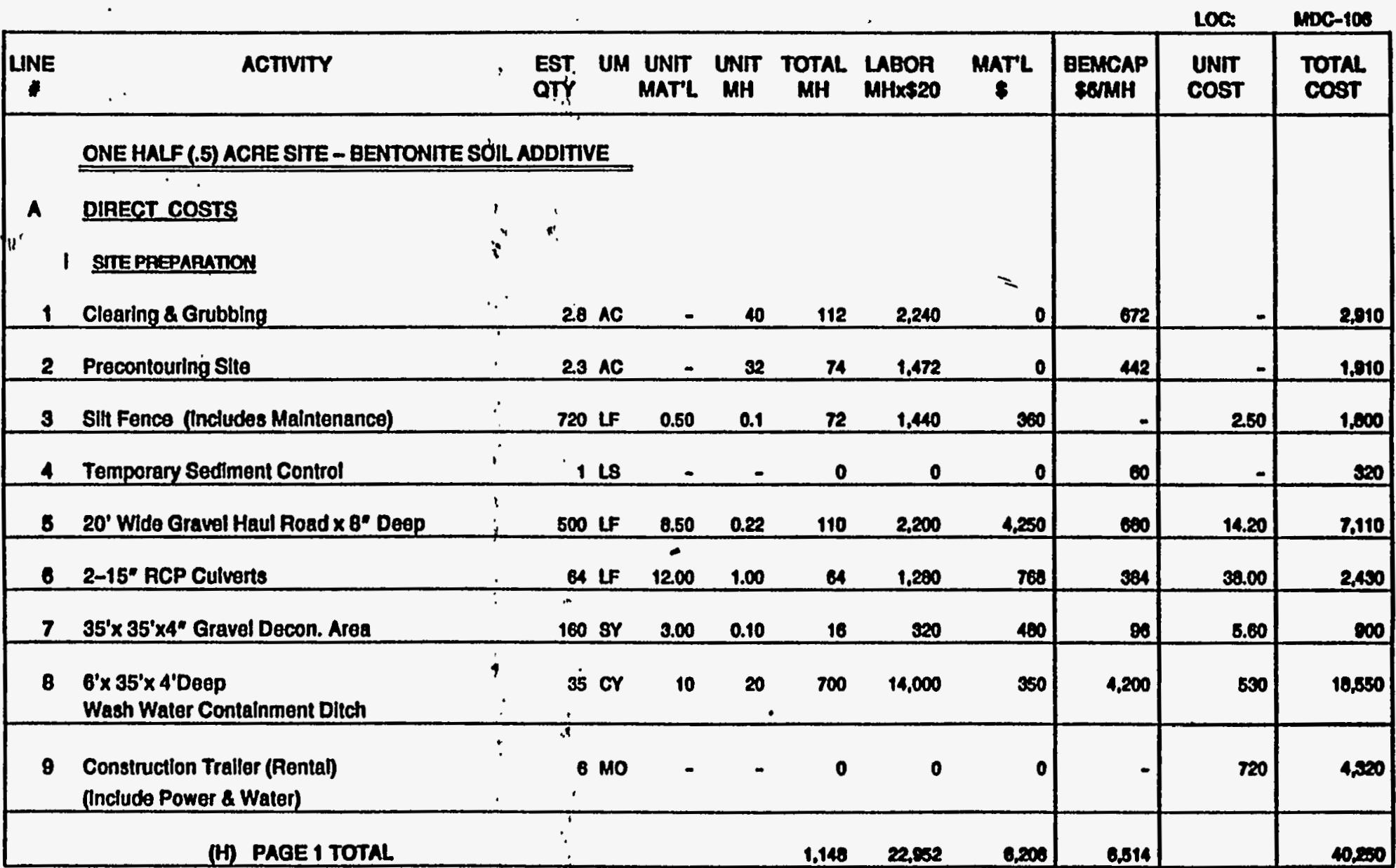

C-10. Cost Estimate for a Cover System Utilizing Bentonite Soil Aợditipgand a 30-mil FML • 


\section{DETAIL ESTIMATE}

Loof: $7-03$

ACTIVITY CODE: ESE26012

PROS.NAME: ECON. EYALUATION OF CLOSURE CAP BARRIER MATERIAL.

\begin{tabular}{|c|c|c|c|c|c|c|c|c|c|c|c|}
\hline LINE & ACTIVITY & $\begin{array}{l}\text { EST } \\
\text { QTY }\end{array}$ & UM & $\begin{array}{l}\text { UNIT } \\
\text { MAT'L }\end{array}$ & $\begin{array}{l}\text { UNIT } \\
\text { MH }\end{array}$ & TOTAL & $\begin{array}{l}\text { LABOA } \\
\text { MHX\$2O }\end{array}$ & $\underset{8}{\operatorname{MaTL}}$ & $\underset{\text { BEMCAP }}{\text { SEMHH }}$ & $\begin{array}{l}\text { UNIT } \\
\text { COST }\end{array}$ & $\begin{array}{l}\text { Total } \\
\text { COST }\end{array}$ \\
\hline 10 & $\begin{array}{l}1 \text { COVER CONSTRUCTION } \\
\text { Waste Stabilization (Crushed Limestone) }\end{array}$ & $.1,007$ & $\pi N$ & 100 & 0.1 & 101 & 2,014 & 100,700 & $\infty$ & 102.00 & 100,020 \\
\hline 11 & $\begin{array}{l}\text { Place and Compact Foundation Soll } \\
\text { in } 8^{\circ} \text { Lifts to } 95 \% \text { STO. Proctor }\end{array}$ & 4,035 & & - & 0.165 & 666 & 19,318 & $=$ & $\mathbf{9 , 9 8 5}$ & 4.30 & 17,910 \\
\hline 12 & $\begin{array}{l}\text { Bentonite Soll Admix Sodlum Bentonite wili } \\
\text { be added to a Silty Sand at an application } \\
\text { rate of } 2 \text { PSF per } 6 \text { Inch litt. Two lilts } \\
\text { required for a total barrler thickness of } \\
12^{\prime \prime} \text { compact, each lift to } 95 \% \text { std. } \\
\text { Proctor } 8 \text { Scarlly between lifts. } \\
\end{array}$ & $\begin{array}{l}25 \\
\text { So.Bent } \\
\\
\text { St.sa }\end{array}$ & $\begin{array}{l}\mathrm{TN} \\
\mathrm{Cr}\end{array}$ & 18.00 & 0.165 & 140 & 2,927 & $\begin{array}{l}3,665 \\
13,305\end{array}$ & 870 & 10.50 & $\begin{array}{r}8.100 \\
17,110\end{array}$ \\
\hline 13 & $\begin{array}{l}\text { Piacement of } 30 \text { Mil Flexible } \\
\text { Membrane Liner }\end{array}$ & 28,100 & SF & 0.10 & 0.000 & 209 & 4,170 & 4,0000 & - & 0.30 & 8,870 \\
\hline 14 & $\begin{array}{l}\text { Placement of 1'-0 Coarse Sand } \\
\text { Drainage Layer }\end{array}$ & 904 & or & 10.50 & 0.035 & 32 & 632 & 8,407 & 180 & 11.40 & 10,910 \\
\hline 15 & $\begin{array}{l}\text { Placement of Geotextile (TYPAR) Filter on } \\
\text { 1'-0" Coarse Sand Dralnage Layer }\end{array}$ & 2,785 & SY & 0.18 & 0.006 & 17 & 334 & 501 & - & 0.30 & 240 \\
\hline 16 & Placement of 1'-6" Common Fill & 1,212 & cr & 3.75 & 0.07 . & 85 & 1.697 & 4.545 & 800 & 8.00 & E, TEO \\
\hline 17 & Placement of 6" Topsoil & 455 & CY & 0.50 & 0.00 & 30 & 727 & 4,310 & 210 & 11,00 & 8,200 \\
\hline & (H) PAGE 2 TOTAL & s & & & & 1,300 & 27.5203 & 141,210 & 6.594 & & 174,500 \\
\hline
\end{tabular}

:

C-10. Cost Estimate for a Cover System Utilizing Bentonite Soil Additive and a 30-mil FML (Cont'd) 


\section{B T A I L B S T I M A T B}

Lo0 I: $7-03$

ACTIVITY CODE: ESE260112

PROJ.NAME: ECON. EVALUATTON OF CLOSURB CAP BARPJER MATERJAL

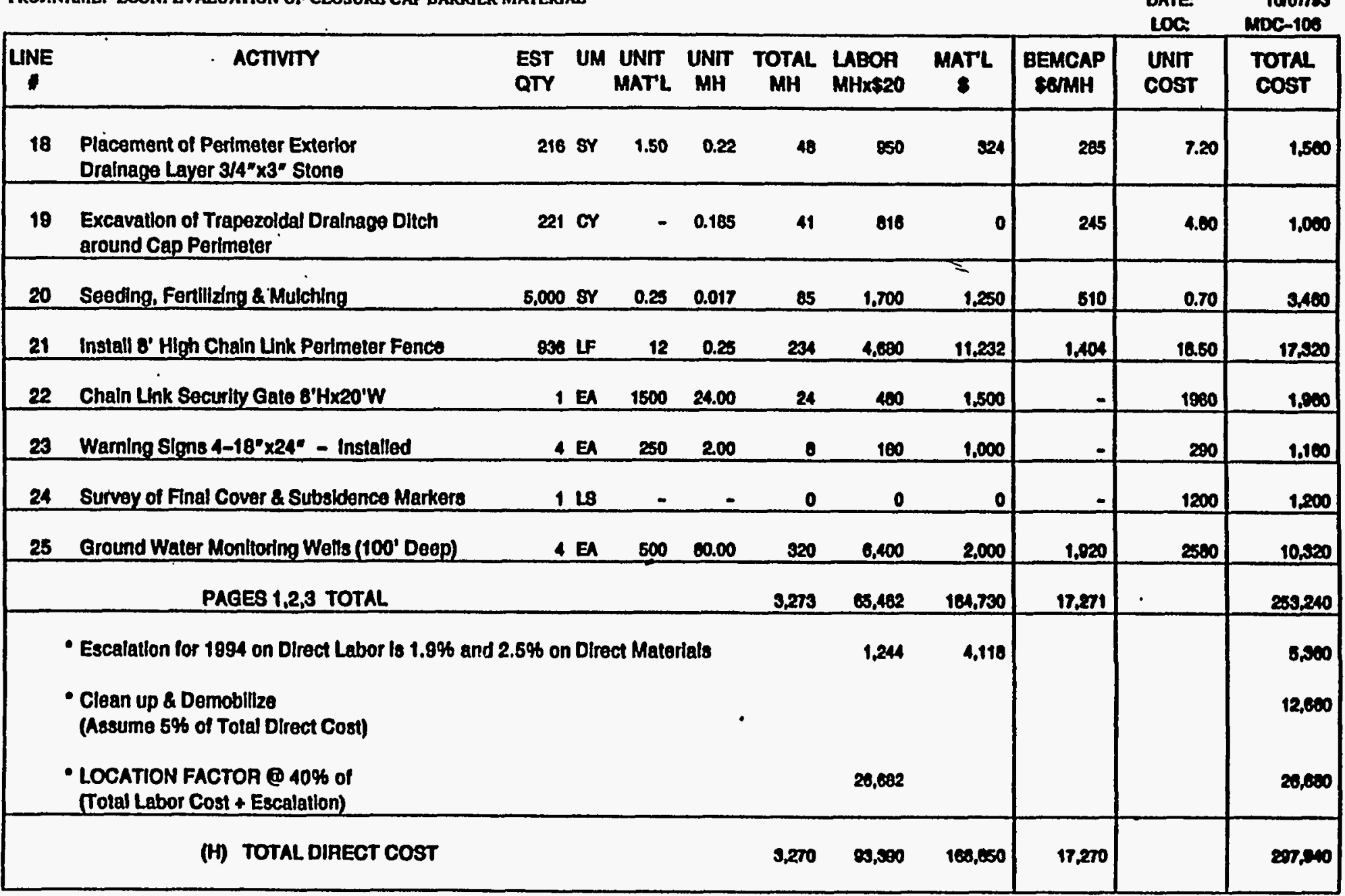

C-10. Cost Estimate for a Cover System Utilizing Bentonite Soil Additive and a 30-mil FML (Cont'd) 
DETA I L B S TIMAT B

8. MEAD

LOan: $7-03$

ACTIVITY CODE: ESE260412

PROJ.NAME: ECON. EYALUATTON OF CLOSURB CAP BARRIER MATERUAL

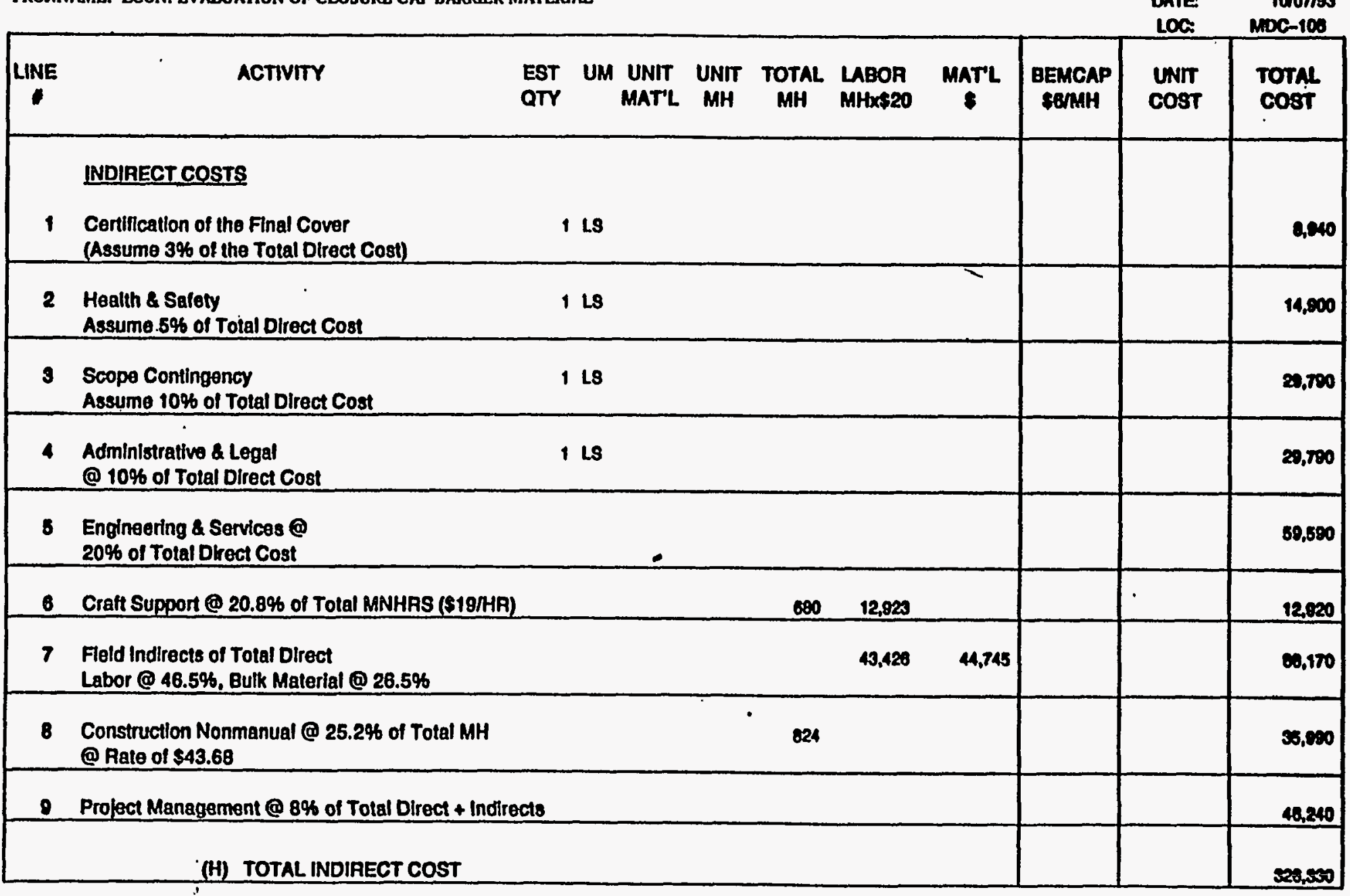

C-10. Cost Estimate for a Cover System Utilizing Bentonite Soil Additive and a 30-mil FML (Cont'd) 
D B T A L B S TIMAT B

CAKD BY: S. MEAD

L001: 7-03

ACTIVITY CODE: ESE260112

PROJ.NAMB: ECON. EVALUATTON OF CLOSURB CAP BARRIER MATERHAS

ESTDQY: PaWnK

PHONE: 7-5260

DATE: 1007/20

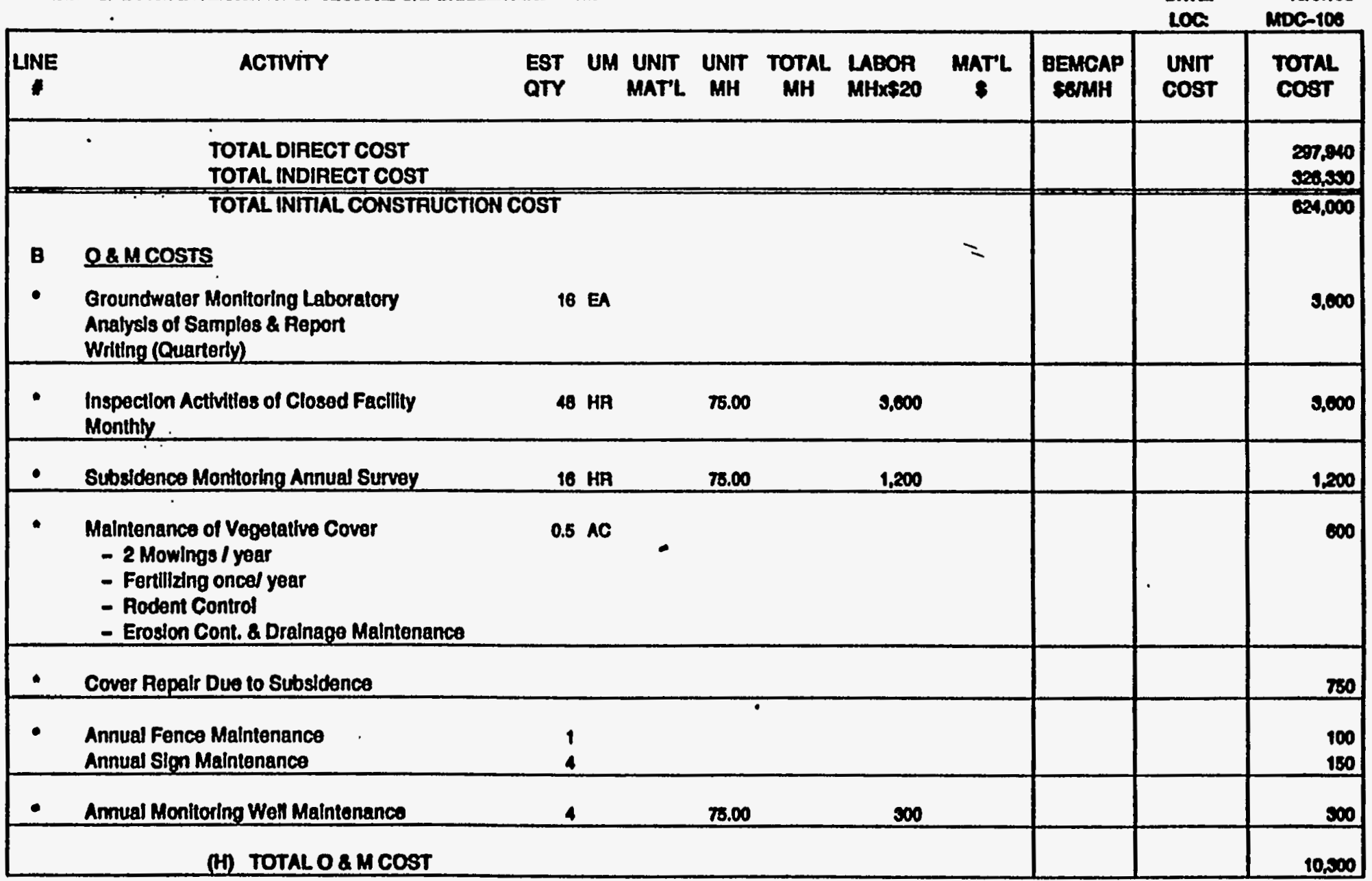

C-10. Cost Estimate for a Cover System Utilizing Bentonite Soil Additive and a 30-mil FML (Cont'd) 
FROJ.NAME: ECON. EVALUATTON OP CLOSURB CAP BARRIER MATERIAL.

MOC-100

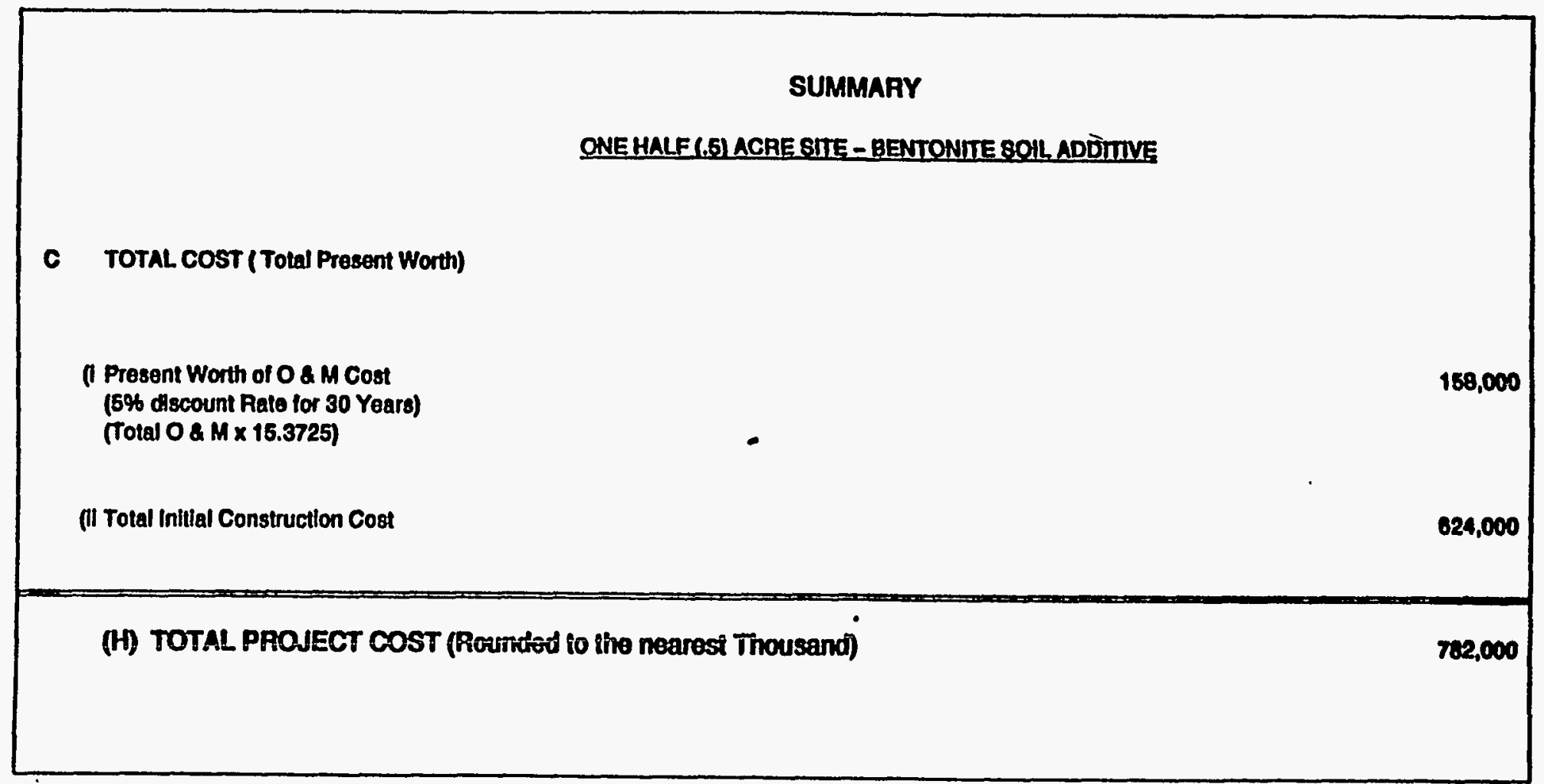

C-10. Cost Estimate for a Cover System Utilizing Bentonite Soil Additive and a 30-mil FML (Cont'd) 
DETAILESTIMATE

Lo0 $1: 2-03$

ACTIVITY CODB: ESERSOL12

PROJ.NAMB: BCON. BVALUATTON OF CLOSURES CAP BARRIER MATEDIAL.

\begin{tabular}{|c|c|c|c|c|c|c|c|c|c|c|}
\hline LINE & ACTIVITY & $\begin{array}{l}\text { EST } \\
\text { QTV }\end{array}$ & UM & $\begin{array}{l}\text { UNIT } \\
\text { MATLL }\end{array}$ & $\begin{array}{l}\text { UNIT } \\
\text { MH }\end{array}$ & $\begin{array}{l}\text { TOTAL LABOR } \\
\text { MH MHX\$20 }\end{array}$ & $\underset{\$}{\operatorname{MATL}}$ & $\begin{array}{l}\text { BEEMCAP } \\
\text { SBMHH }\end{array}$ & $\begin{array}{l}\text { UNIT } \\
\text { COST }\end{array}$ & $\begin{array}{l}\text { Total } \\
\text { cost }\end{array}$ \\
\hline$\wedge$ & $\begin{array}{l}\text { ONEHALF (.5) ACRE SITE - ASPHALTIC } \\
1 \text { DIAECT COSTS } \\
1 \text { SMIEPREPARATION } \\
\text { Clearing \& Grubbing }\end{array}$ & GRETE & AC & - & 40 & 2.240 & 0 & or2 & - & 2.010 \\
\hline 2 & Precontouring Slie & 2.3 & AC & $=$ & 32 & 1,472 & 0 & 42 & - & 1,010 \\
\hline 3 & Silt Fence (Inctudes Maintenanco) & 720 & LF & 0.50 & 0.1 & 1.440 & 300 & - & 250 & 1800 \\
\hline 4 & Tomporary Sodiment Control & 1 & $L 8$ & - & - & 0 & 0 & $\infty$ & - & 3200 \\
\hline 5 & $20^{\circ}$ Wide Graved Haut Road $\times 8^{\circ}$ Deep & 500 & $\underline{L F}$ & 8.50 & 0.22 & 2,200 & 4,250 & 600 & 14.20 & 2.110 \\
\hline 6 & 2-15. RCP Culverts & 6 & $\mathbf{L}$ & 1200 & 1.00 & 1,200 & res & 204 & 30.00 & 2450 \\
\hline 7 & $35^{\prime} \times 35^{\prime} \times 4^{\prime \prime}$ Gravel Docon. Area & 100 & or & 0.00 & 0.10 & 320 & 400 & $\infty$ & 8.00 & $\infty 00$ \\
\hline 8 & $\begin{array}{l}\text { O'x } 35^{\prime} \times 4^{\prime} \text { Deop Wash Water Containment } \\
\text { Ditch }\end{array}$ & 35 & Cr & 10 & 20 & $700 \quad 14,000$ & 350 & 4,200 & 530 & 125000 \\
\hline 9 & $\begin{array}{l}\text { Construction Traller (Ponta) } \\
\text { (include Power \& Water) }\end{array}$ & 6 & 140 & $\overline{-}$ & - & 0 & 0 & - & 720 & 4,5200 \\
\hline & (B) PAGE I TOTAL & & & & & 22052 & 2000 & 8.514 & & 19:0 \\
\hline
\end{tabular}

C-11. Cost Estimate for a Cover System Asphalt Concrete Hydraulic Barrier and a 30-mil FML 
DETAIL ESTIMATE

1001: 7-03

ACTIVTTY CODE: BSEQCOA12

PROJ.NAME: ECON. BVALUATHON OF CLOSURB CAP BARRIER MATERSAL

\begin{tabular}{|c|c|c|c|c|c|c|c|c|c|c|c|}
\hline LINE & ACTIVIT & $\begin{array}{l}\text { EST } \\
\text { QTY }\end{array}$ & UM & $\begin{array}{l}\text { UNIT } \\
\text { MAT'L }\end{array}$ & $\underset{M H}{\text { UNIT }}$ & $\underset{\text { MHAL }}{\text { TOTAL }}$ & $\begin{array}{l}\text { LABOR } \\
\text { MHXSE2O }\end{array}$ & $\underset{\&}{\operatorname{maTL}}$ & $\underset{\text { SEMMAP }}{\text { BEMCAP }}$ & $\begin{array}{l}\text { Unit } \\
\text { Cost }\end{array}$ & $\begin{array}{l}\text { TOTAL } \\
\text { cost }\end{array}$ \\
\hline 10 & $\begin{array}{l}\text { H_COVER CONSTRUCTION } \\
\text { Waste Stabilization (Crushed Limestone) }\end{array}$ & 1007 & $\mathrm{~m}$ & 100 & 0.1 & 101 & 2.014 & 100,700 & ene & 10200 & 100,520 \\
\hline 11 & $\begin{array}{l}\text { Place and Compact Foundation Soll } \\
\text { In 8" Lits to } 95 \% \text { STD. Proctor }\end{array}$ & 3195 & or & - & 0.165 & 527 & 10.54 & $=$ & 3,160 & 4.30 & 19,710 \\
\hline 12 & $\begin{array}{l}\text { Placement of } 30 \text { Mil Flextblo } \\
\text { Membrane Lner }\end{array}$ & 26.100 & SF & 0.18 & 0.000 & 209 & 0,170 & 4,090 & - & 0.30 & 8,870 \\
\hline 13 & $\begin{array}{l}\text { Placement of } f^{\prime}-0 \text { Coarse Sand } \\
\text { Drainage Layer }\end{array}$ & 843 & or & 10.50 & 0.035 & 30 & 690 & 8,052 & in & 11.40 & 0,620 \\
\hline 14 & $\begin{array}{l}\text { 2" PVC Perforated Drainage Pipe placed } \\
\text { In Drainage layer }\end{array}$ & 890 & LF & 1.0 & 0.24 & 225 & 4,704 & $\infty 00$ & - & 5.80 & 8,800 \\
\hline 15 & 2 1/2" Binder Course & 2050 & sY & 4.20 & 0.06 & 158 & 3.100 & 11,150 & S54 & 8.00 & 18,200 \\
\hline 16 & 4" Hyorautic Asphan Conc. & 2650 & SY & ars & 0.03 & $\infty$ & 1,590 & 17,0000 & $4 \pi$ & 7.80 & 10.550 \\
\hline 17 & Asphaln Mastlc Seal & 2650 & SY & 0.50 & 0.015 & 40 & 795 & 1,525 & - & 0.00 & 2.120 \\
\hline 18 & Sprayod Asphaltle Concrete Soalant & 2650 & 9Y & 0.78 & 0.02 & 63 & 1,000 & 1,000 & 310 & 1.30 & 20,0 \\
\hline 18 & Excavation of Tralngular Drainage Ditch & 111 & or & - & 0.3 & 33 & 606 & 0 & 200 & 7.00 & 870 \\
\hline 20 & 4" Thlck Asphalt Perlmeter Drainage Dltch & 395 & SY & 7.85 & 0.15 & 50 & 1,005 & 2,600 & 302 & 11.00 & ano \\
\hline & (B) PAGE 2 TOTAL & & & & & 1,510 & $\mathbf{5 0 , 5 2 4}$ & 150,100 & 6,105 & & 1 andine \\
\hline
\end{tabular}

C-11. Cost Estimate for a Cover System Asphalt Concrete Hydraulic Barrier and a 30-mil FML (Cont'd) 
D B T A I L B S T I MATE

Lo01: 7-00

ACTTVTY CODB: ESEZCON12

PKOJ.NAMG: ECON. BYNLUATTON OF CLOSURB CAP BARRIER MATERUAL.

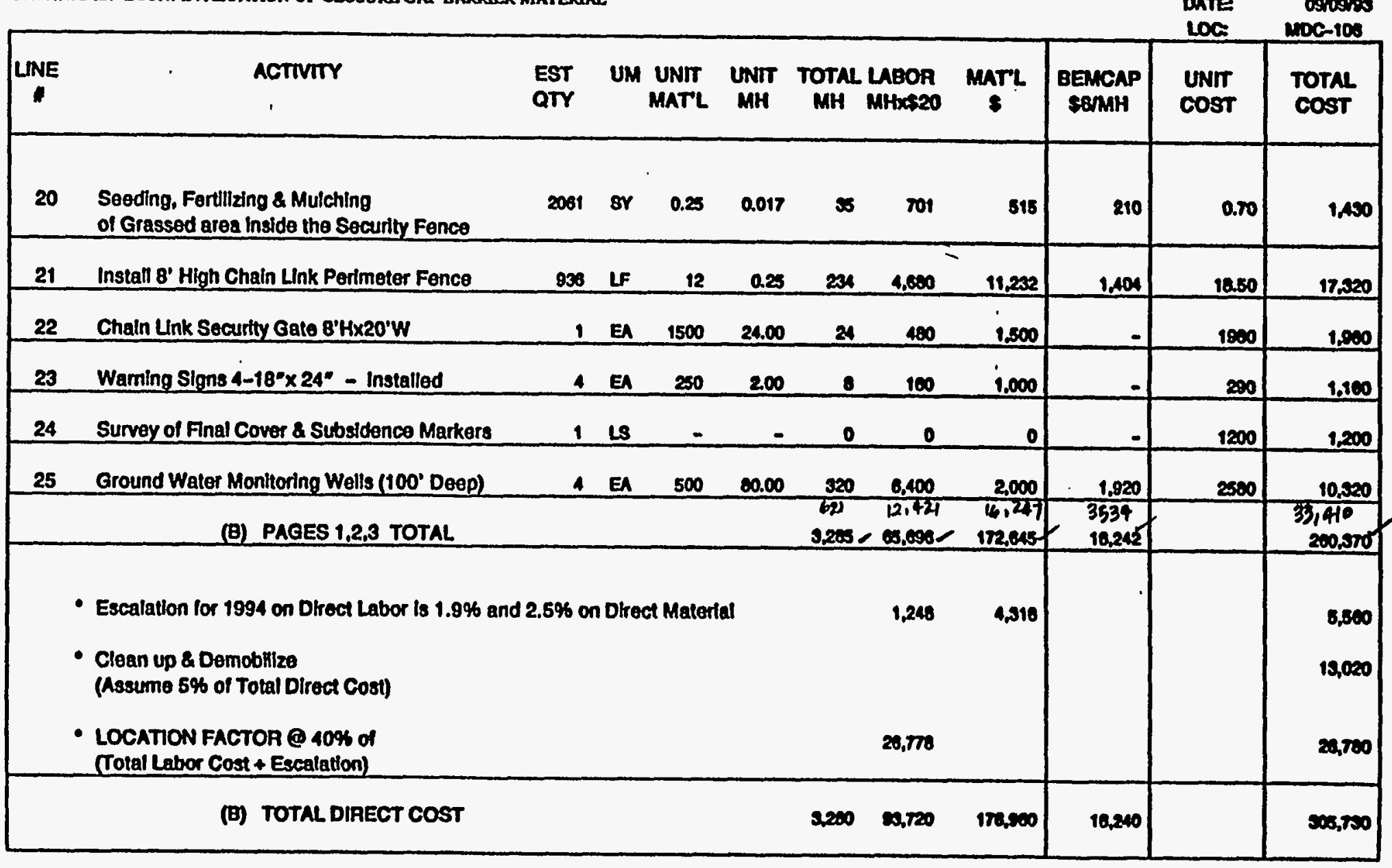

C-11. Cost Estimate for a Cover System Asphalt Concrete Hydraulic Barrier and a 30-mil FML (Cont'd) 
DBTAILESTIMATE

\begin{tabular}{|c|c|}
\hline $\begin{array}{l}\text { CHCD BY: } \\
\text { ESTD QY: } \\
\text { Prione: } \\
\text { OMTE: } \\
\text { LOC: }\end{array}$ & 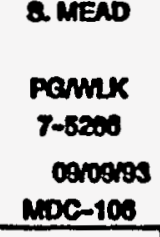 \\
\hline $\begin{array}{l}\text { UNIT } \\
\text { COST }\end{array}$ & $\begin{array}{l}\text { TOTAL } \\
\text { COST }\end{array}$ \\
\hline & 0,170 \\
\hline & 16,250 \\
\hline & 30,570 \\
\hline & 50,570 \\
\hline & 61.160 \\
\hline & \\
\hline & 90,470 \\
\hline & $\begin{array}{r}56,100 \\
210,280\end{array}$ \\
\hline & $47,2000^{2}$ \\
\hline & $300,840^{\circ}$ \\
\hline
\end{tabular}

\section{(B) TOTAL INDIAECT COST}

1 LS

1 Certilicallon of the Final Cover (Assume 3\% of the Total Dhect Cost)

2 Health \& Safoty

1 LS

3 Scope Contingency Assume $10 \%$ of Total Direct Cost

1 Ls

4 Aoministrative \& Logal $\quad 1$ is

5 Engineoring \& Sorvices (1) $20 \%$ of Total Direct Cost

6 Crall Support @ 20.8\% of Total MNHRS (\$19/HA)

7 Flold Indirects of Total Direct Labor @ 46.5\%. Bulk Matorial @i 26.5\%

43,500

8 Construction Nonmanual \& 25.2\% of Total Direct MH @ Rate of $\$ 43.68$

9 Project Managament @ 8\% of Total Direct + indirects

22 UNIT UNT TOTAL LABOA
MATL MH MH MHXS2O

MATLL

\begin{tabular}{c|c|c}
\hline BEMCAP & UN \\
samH & COST
\end{tabular}

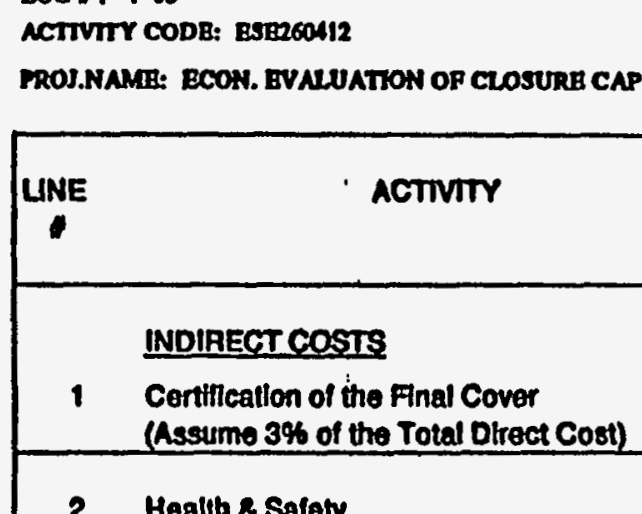

Ls

$\cdot$

C-11. Cost Estimate for a Cover System Asphalt Concrete Hydraulic Barrier and a 30-mil FML (Cont'd) 
D B T A I L B S TIMATB

GHSDBY: Q.MERD

1001: 7-03

ACTTVTY CODE: BSE2SOA12

PROJ.MAME: BCON. EVALUATION OP CLOSURE CAP BARRIER MATERIAL

ESTD BY:

PHowe

Pank

maver.

7-52006

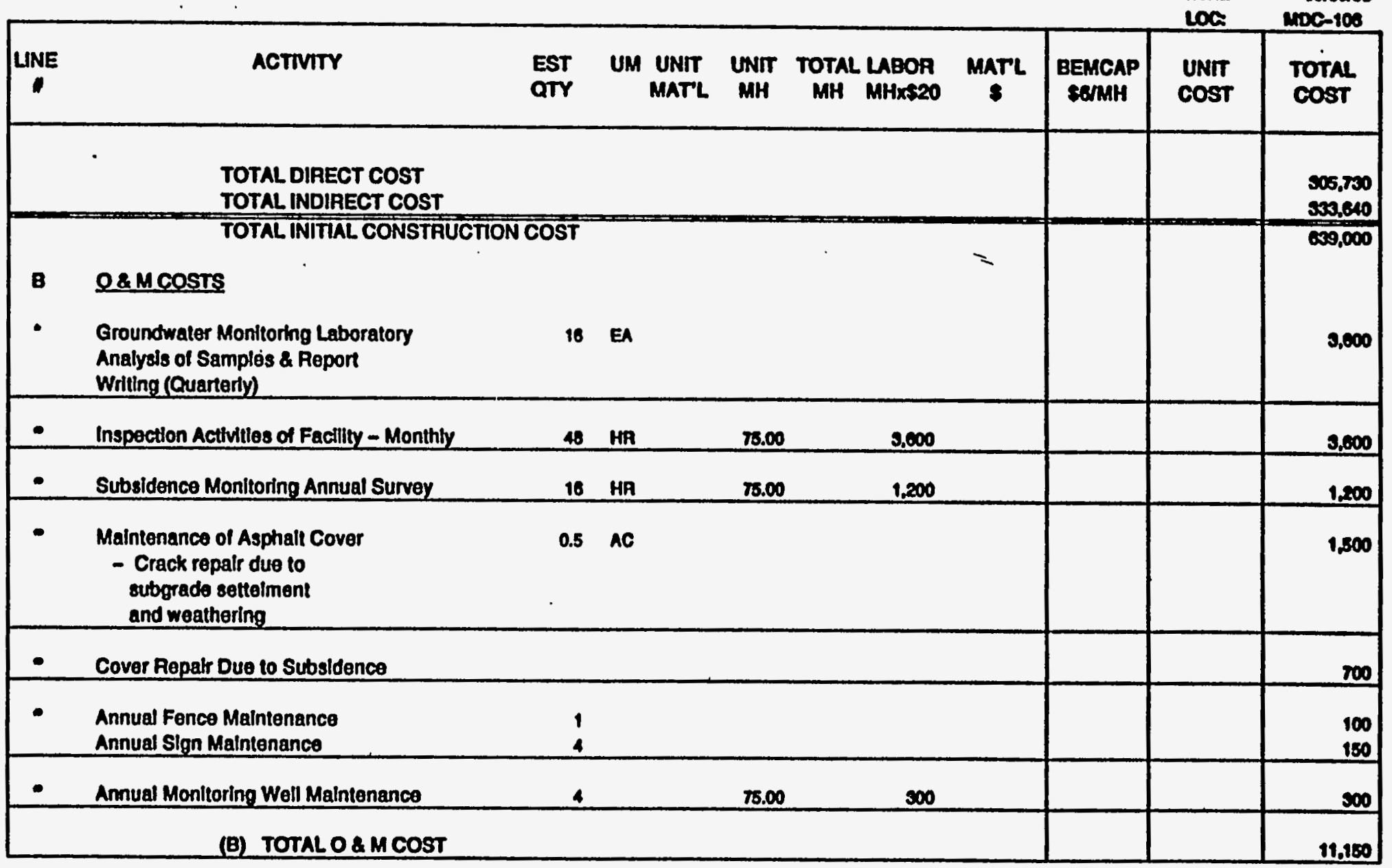

C-11. Cost Estimate for a Cover System Asphalt Concrete Hydraulic Barrier and a 30-mil FML (Cont'd) 


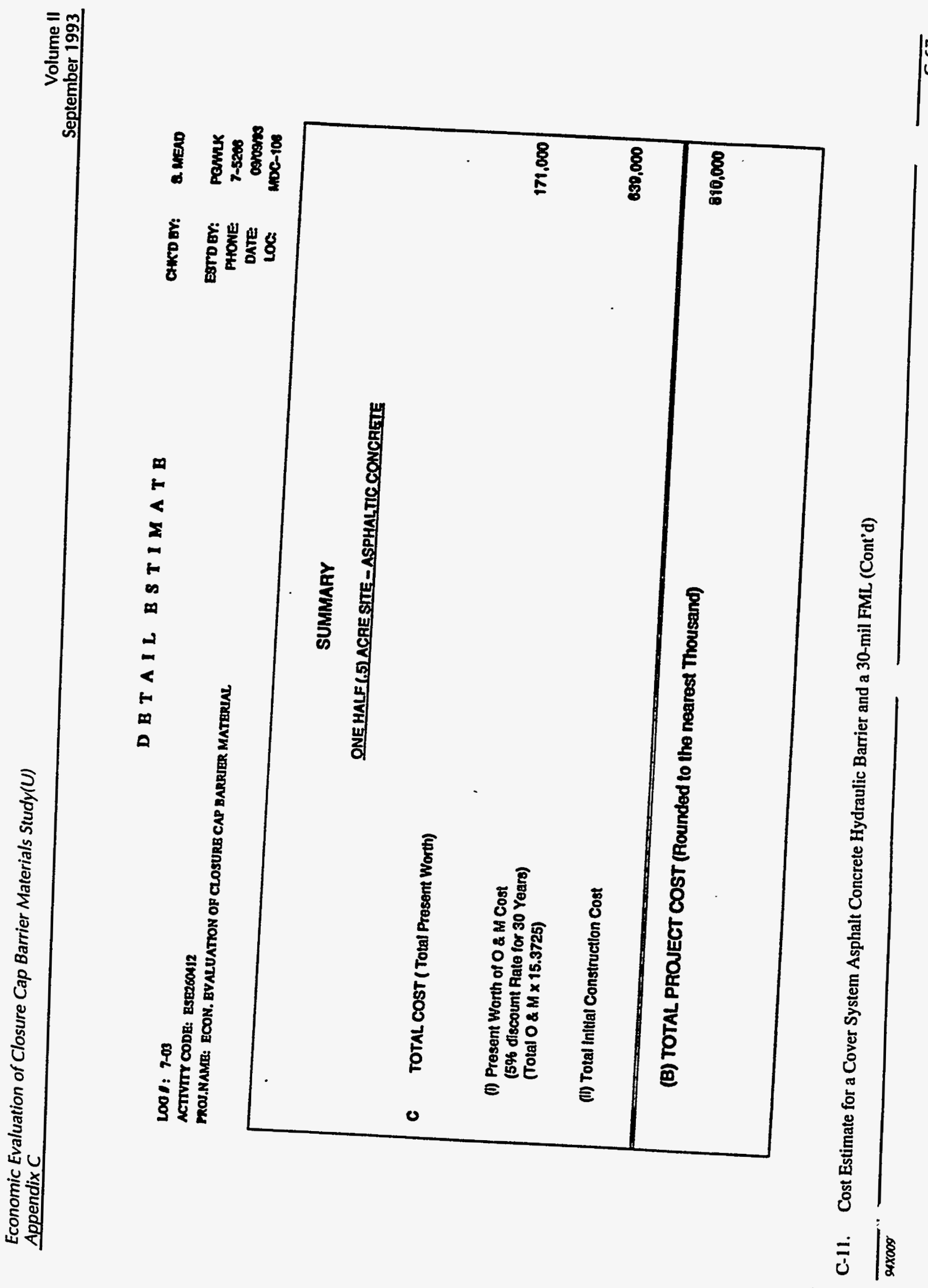




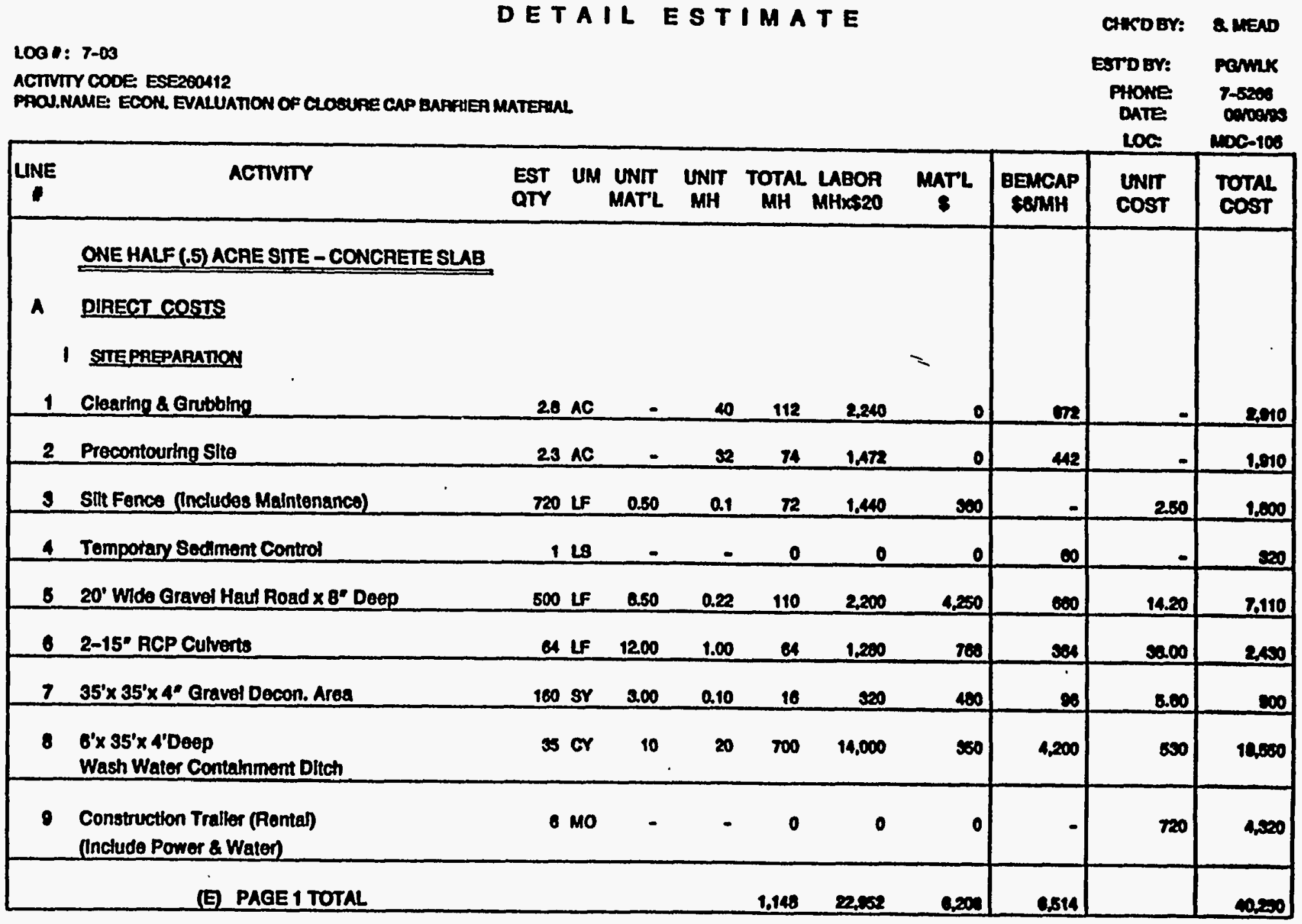

C-12. Cost Estimate for a Cover System Utilizing Poured-in Place Concrete Slab and a 30-mil FML 


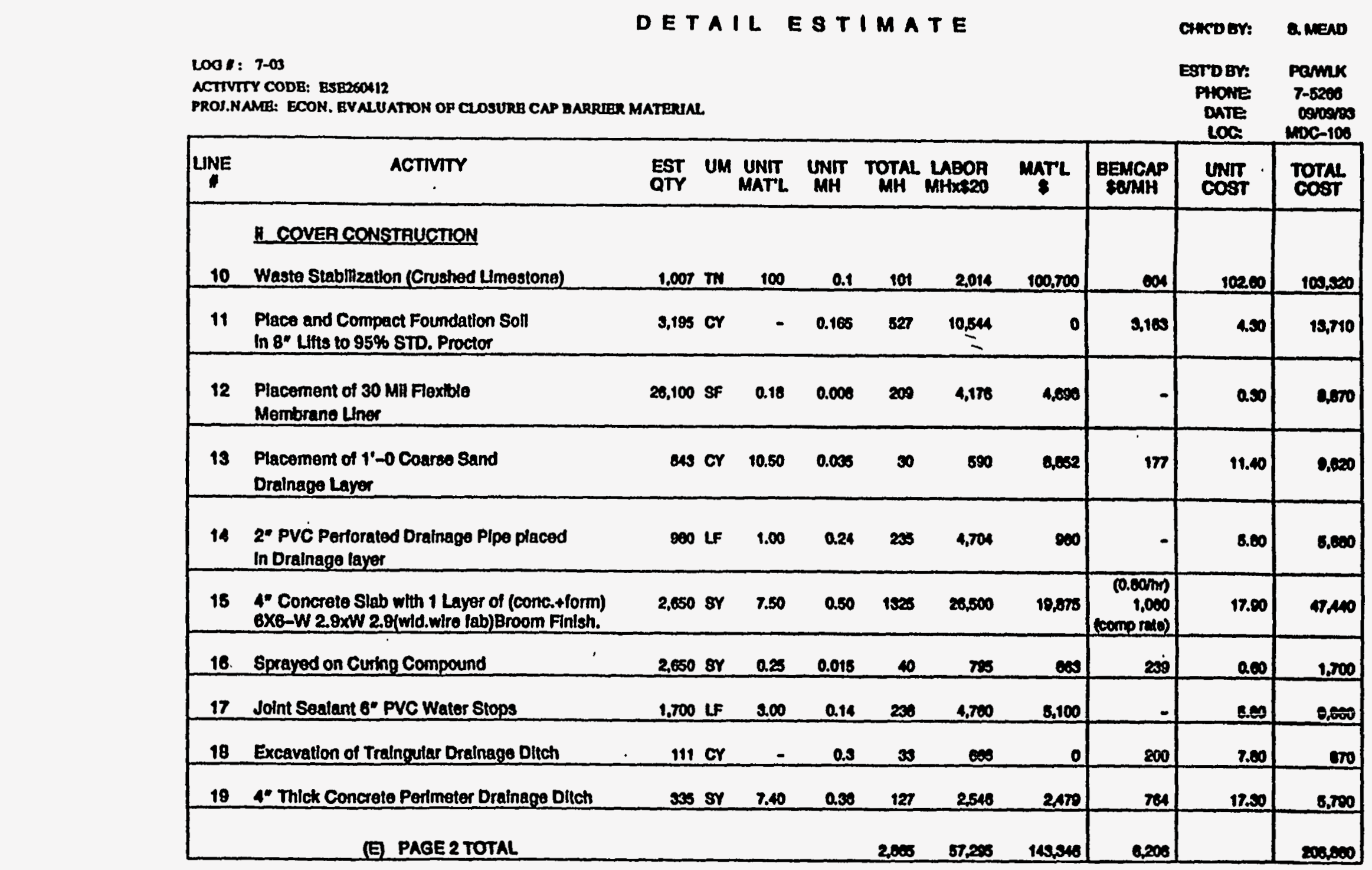

C-12. Cost Estimate for a Cover System Utilizing Poured-in Place Concrete Slab and a 30-mil FML (Cont'd) 
DETAIL BSTIMATB CAKOEY: RMEAO

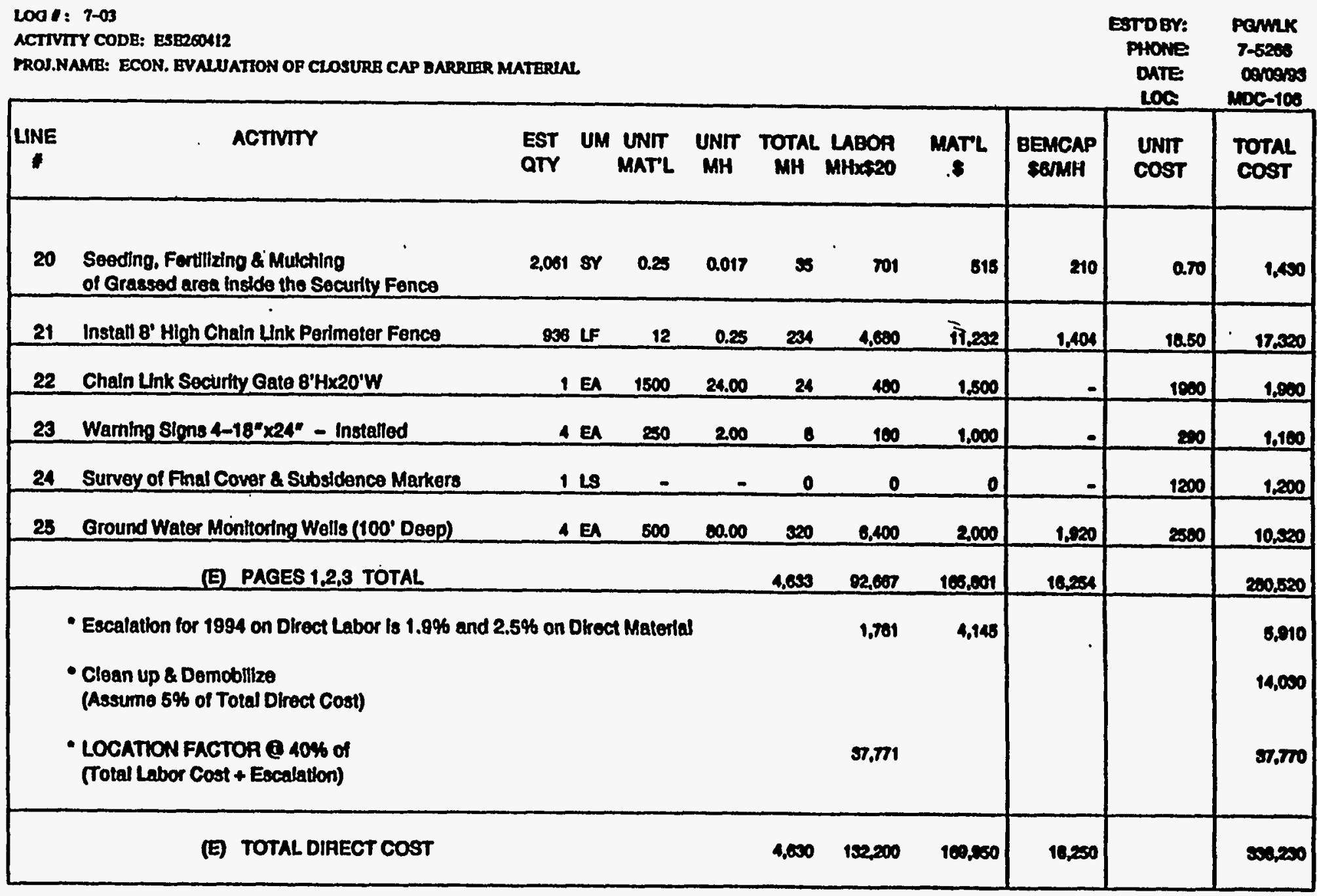

C-12. Cost Estimate for a Cover System Utilizing Poured-in Place Concrete Slab and a 30-mil FML (Cont'd) 
D B TAIL BSTIMATB

AWCD QV: QMEAD

L001: 7-03

ACTIVTTY CODB: ESE2SOA12

MOJ.NAME: BCON. EYALUATON OP CLOSURB CAP BARRTER MATERAAL

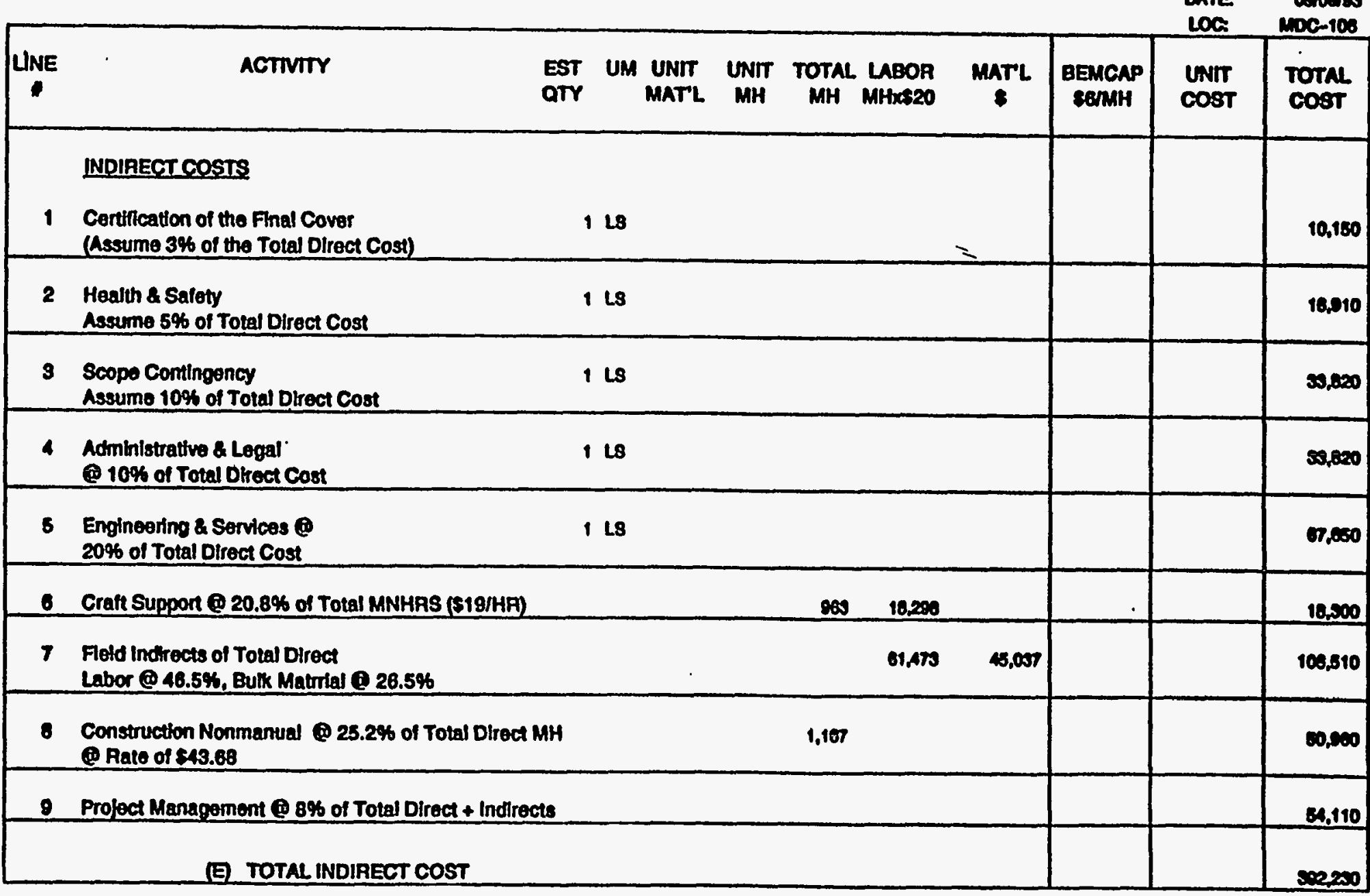

C-12. Cost Estimate for a Cover System Utilizing Poured-in Place Concrete Slab and a 30-mil FML (Cont'd) 
I

D B TAILA B TIMATB

$1001: 2-03$

ACTIVIYY CODE: ESB26OA12

MOS.NAME: BCON. BVALUATKON OF CLOSURE CAP BATRIER MATERULL

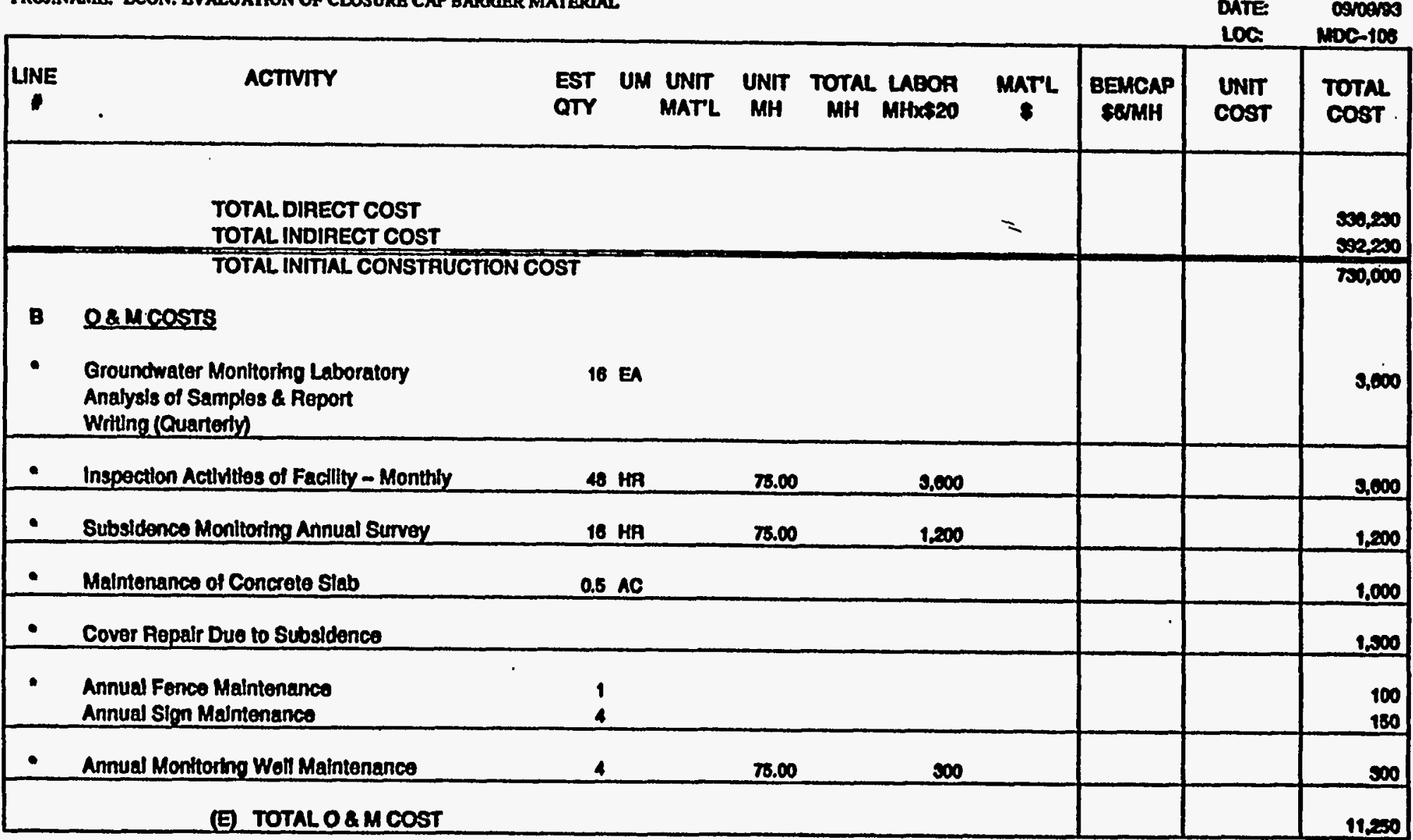

C-12. Cost Estimate for a Cover System Utilizing Poured-in Place Concrete Slab and a 30-mil FML (Cont'd) 


\begin{tabular}{|c|c|}
\hline AWCD BY: & Q. $M E \wedge 10$ \\
\hline 8170 & $\operatorname{Pen}$ \\
\hline $\begin{array}{l}\text { PHONE } \\
\text { DATE } \\
\text { LOC: }\end{array}$ & $\begin{array}{r}7-5200 \\
000010 \\
\text { moc-100 }\end{array}$ \\
\hline
\end{tabular}

100: : 7-03

ACTIVITY CODA: BSE250412

FOU.NAME: BCON. BVALUATHON OF CLOSURB CAP BARRIER MATRRUAL

100:

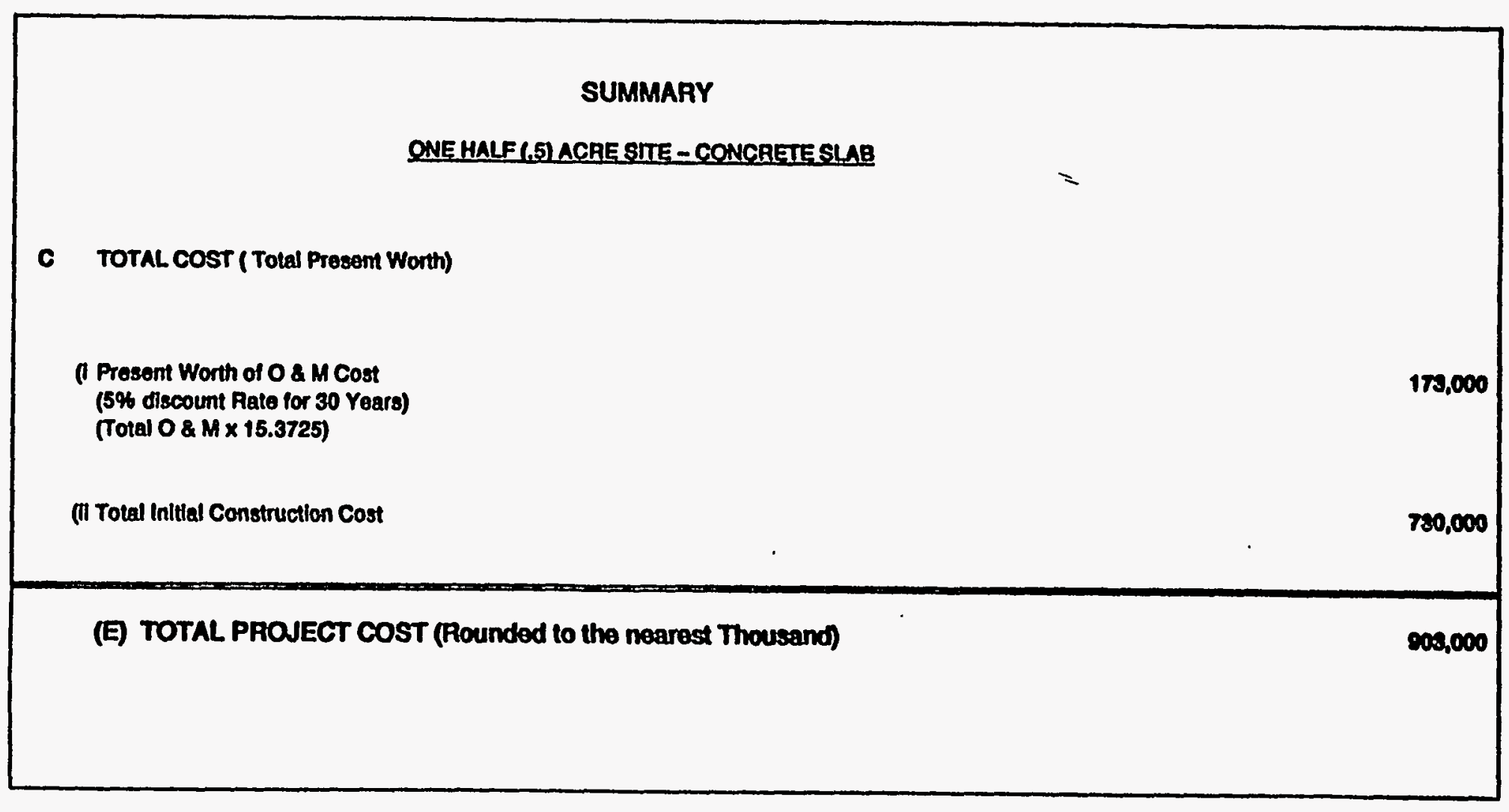

C-12. Cost Estimate for a Cover System Utilizing Poured-in Place Concrete Slab and a 30-mil FML (Cont'd) 


\section{Appendix D}

\section{Cost Estimates for 1-Acre Site}


DETAILESTIMATE

L001: 7-03

ACTIVITY CODE: ESERGON12

TROJ.NAME: ECON. EVALUATION OF CLOSURB CAP BARRIER MATERLAL

E

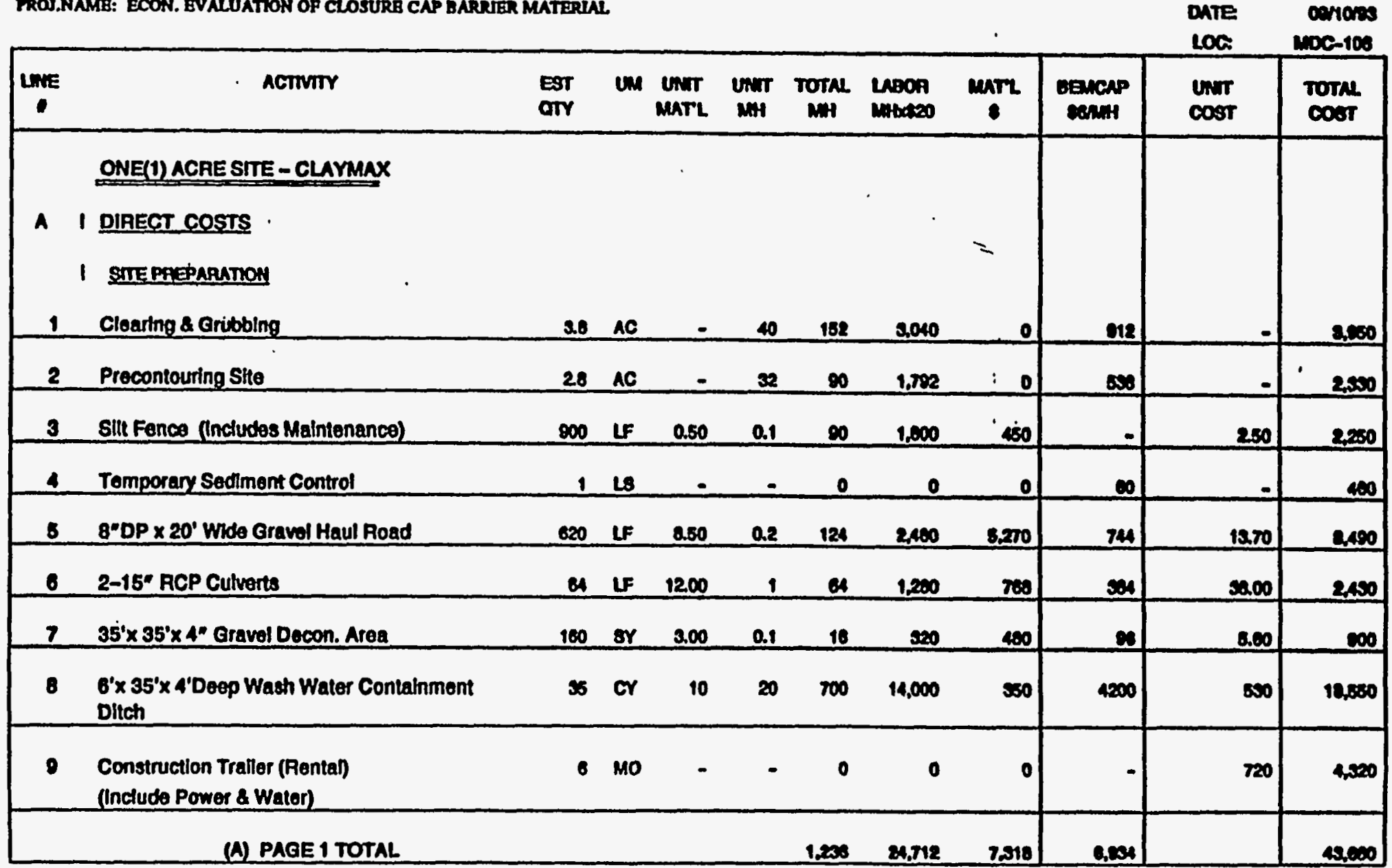

D-1. Cost Estimate for a Cover System Utilizing Claymax@ and a 30-mil FML 
DETAIL ESTIMATE

Le01: 7-03

ACTIVITY CODE: BSE260112

PROJ.NAME: ECOH. BVALUATION OP CLOSURB CAP EARRRER MATERLAL

CHKD BY: 2.MENO

\begin{tabular}{|c|c|c|c|c|c|c|c|c|c|c|c|}
\hline LINE & activirr & $\begin{array}{l}\text { EST } \\
\text { QTY }\end{array}$ & un & $\begin{array}{l}\text { UniT } \\
\text { MaTL }\end{array}$ & UNTI $_{\text {Mit }}$ & roThL & LABOA & $\operatorname{mart}$ & 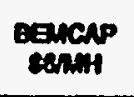 & $\operatorname{UnvosT}_{\text {Cost }}$ & $\begin{array}{l}\text { Totne } \\
\cos \end{array}$ \\
\hline & I COVER CONSTRUCTION & & & & & & & & & & \\
\hline 10 & Waste Stablization (Crushed Limestone) & 2,014 & $\mathrm{TN}$ & 100 & 0.1 & 201 & 4,020 & 201,400 & 12000 & 102.00 & 200,640 \\
\hline 11 & $\begin{array}{l}\text { Place and Compact Foundation Soll } \\
\text { in } 8^{\circ} \text { Lifts to } 95 \% \text { STD. Proctor }\end{array}$ & 8,070 & or & - & 0.100 & 1332 & 28,601 & -10 & 7000 & 4.50 & 3,020 \\
\hline 12 & Placoment of Claymax Geotextile Mat & 61,600 & SF & 0.45 & 0.01 & 018 & 12,320 & 87,720 & - & a.ro & $\mathbf{6 0 . 0 4 0}$ \\
\hline 13 & $\begin{array}{l}\text { Placement of } 30 \text { Mil Floxible } \\
\text { Membrane Liner }\end{array}$ & 61,600 & SF & 0.10 & 0.008 & 493 & 0.060 & 11,000 & - & 0.30 & $\mathbf{2 0 , 2 0 0}$ \\
\hline 14 & $\begin{array}{l}\text { Piacement of } 1^{\prime}-0 \text { Coarse Sand } \\
\text { Drainage Layer }\end{array}$ & 2.124 & or & 10.50 & 0.00 & 64 & 1.274 & 2espoe & 302 & 18.50 & 23,100 \\
\hline 16 & $\begin{array}{l}\text { Placement of Geotextile (TYPAR) Filter on } \\
\text { 1'-0. Coarse Sand Dralnage Layer }\end{array}$ & 7.011 & sr & 0.18 & 0.000 & 42 & OA1 & 1,202 & - & 0.50 & 2,100 \\
\hline 16 & Placement of 1'-6" Common Fill & 2,424 & or & 3.75 & 0.08 & 145 & 2.000 & 2.0000 & 870 & 8.50 & 120070 \\
\hline 17 & Placement of 6" Topsoll & 909 & or & 0.50 & 0.07 & 64 & 1,273 & 0,0000 & $\mathbf{s 0 2}$ & 11.30 & 10.200 \\
\hline 18 & $\begin{array}{l}\text { Placement of Perimeter Exterfior } \\
\text { Drainage Layer } 3 / 4^{\prime} \times 3^{*} \text { DP (Crushod Stone) }\end{array}$ & 297 & or & 1.50 & 0.22 & $\infty$ & 1,2007 & 40 & 302 & 7.20 & 2,140 \\
\hline 18 & Excavation of Trapezoldal Dralnage Ditch & 49 & or & - & 0.185 & 02 & 1.032 & 0 & 480 & 4.80 & 2120 \\
\hline & (A) PAGE 2 TOTAL & & & & & 3,104 & ceon & 201,000 & 11,70 & & 500,120 \\
\hline
\end{tabular}

D-1. Cost Estimate for a Cover System Utilizing Claymax \& and a 30-mil FML (Cont'd) 


\section{DETAIL BSTIMATB}

Lool: $2-03$

ACTIVITY CODE: ESE2SOA12

PROJ.NAME: ECON. EYALUATION OP CLOSURE CAP BARRIER MATERIAL.

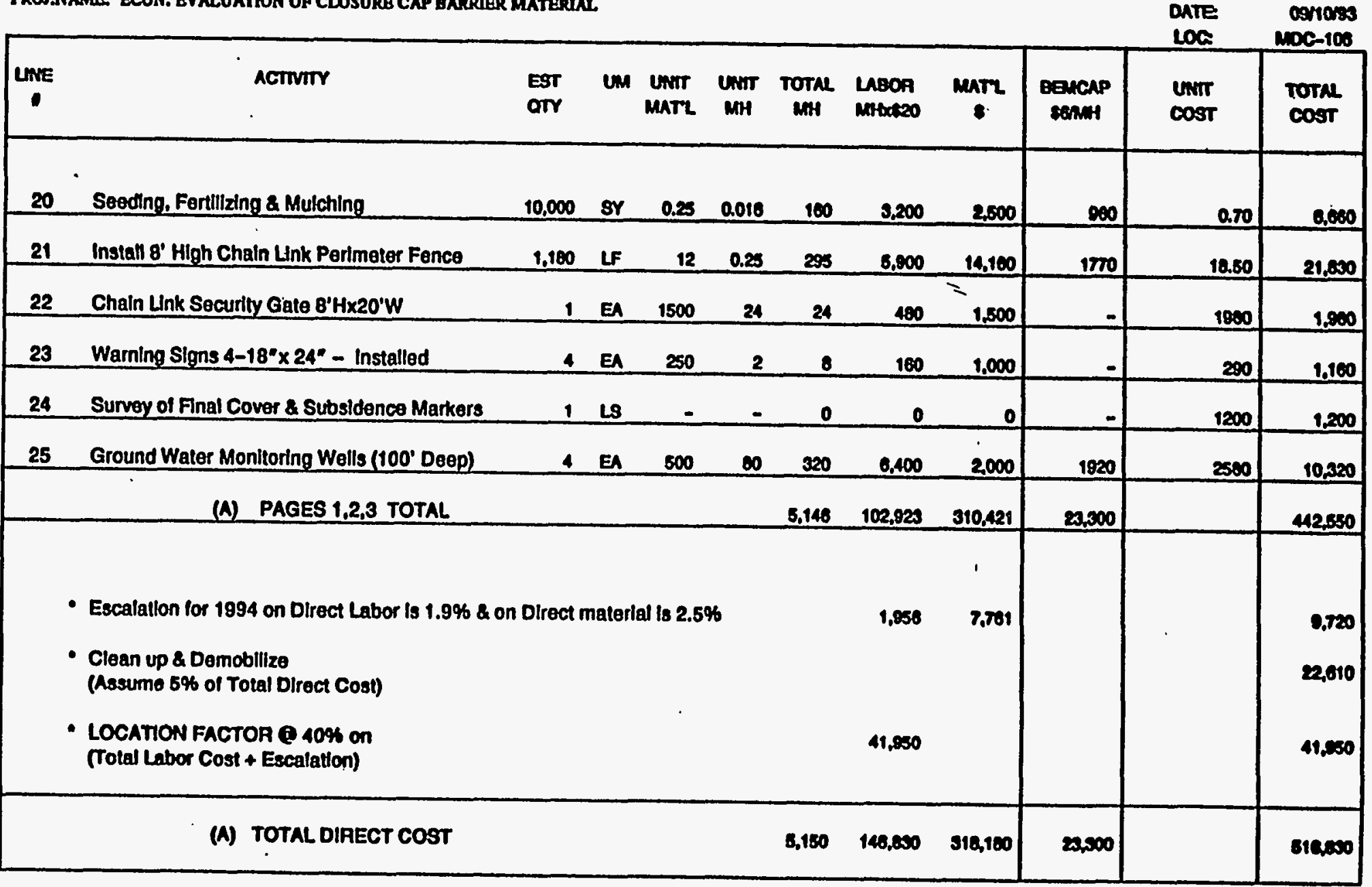

D-1. Cost Estimate for a Cover System Utilizing Claymax and a 30-mil FML (Cont'd) 
DETAIL BSTIMATB

Loo: $7-03$ ACTIVITY CODE: ESE260412

MOI.NAME: ECON. BVALUATION OF CLOSURB CAP BARRIER MATERIAL

PHONE PCWn

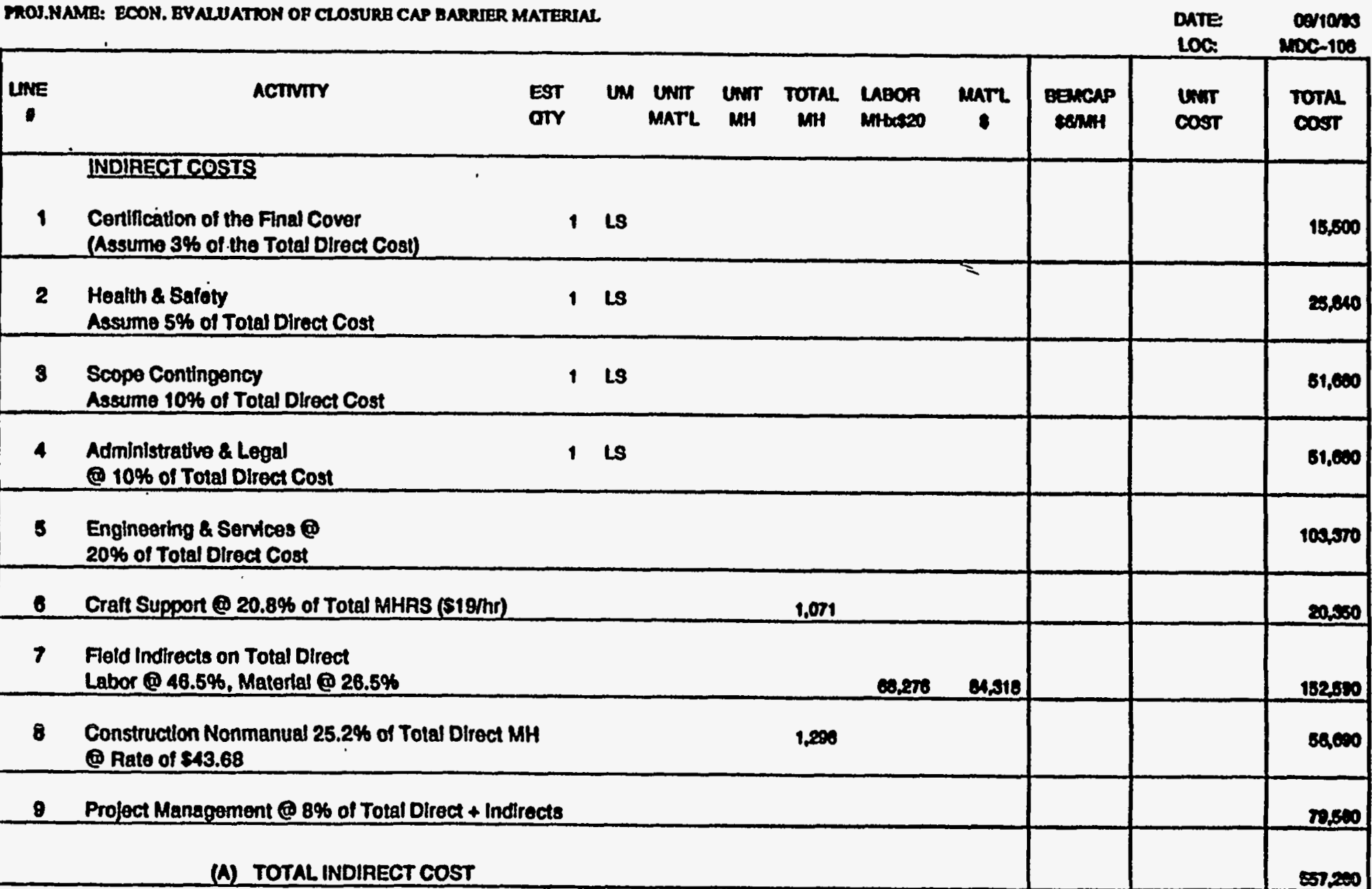

D-1. Cost Estimate for a Cover System Utilizing Claymax and a 30-mil FML (Cont'd) 
D B TAIL B S TIMATB

L00 1: 7-03

ACTIVTTY CODB: BSH260112

PROJ.NAME: ECON. EVALUATTON OF CLOSURB CAP BARRIER MATERIAL

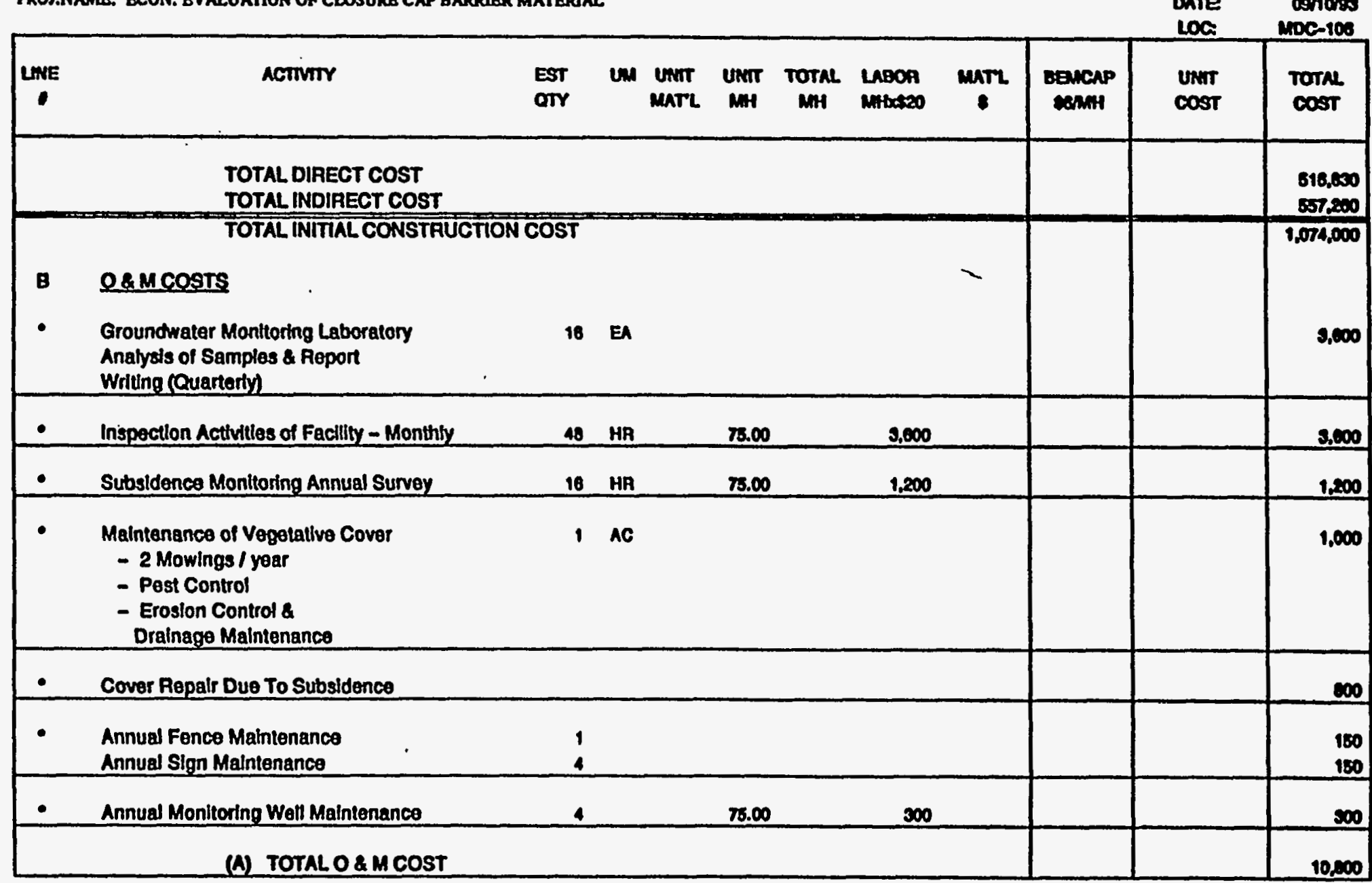

D-1. Cost Estimate for a Cover System Utilizing Claymax@ and a 30-mil FML (Cont'd) 
DETAIL BSTIMAT B

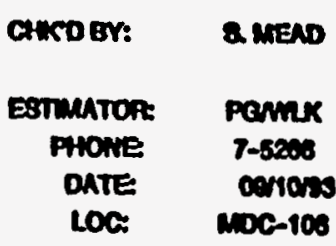

Lod: 7-03

ACTTVTY CODE: ESE26012

MROJ.NAME: ECON. BVALUATION OF CLOSURE CAP BARRIER MATERIAL

100

$\operatorname{moc}-108$

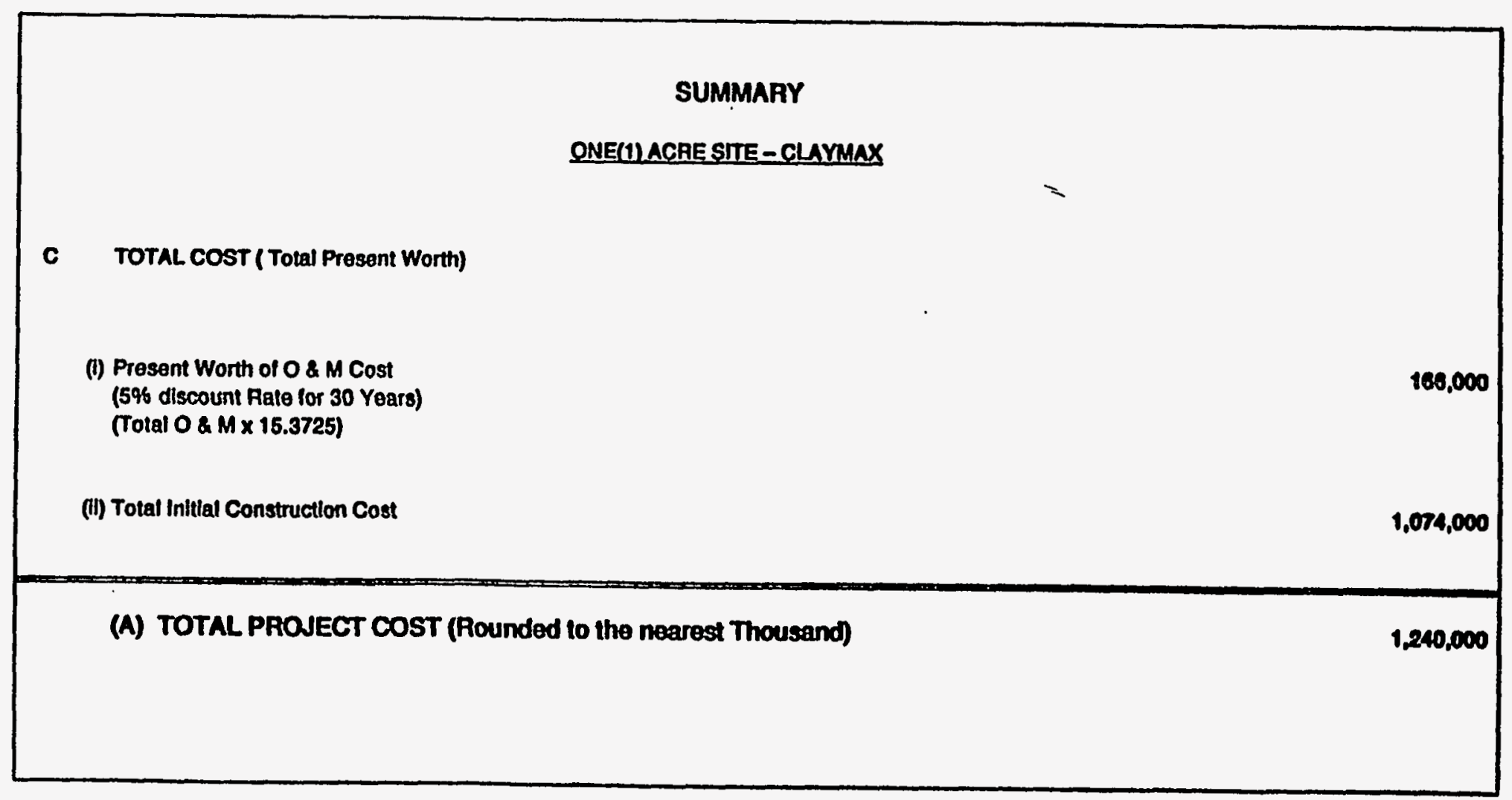

D-1. Cost Estimate for a Cover System Utilizing Claymax@ and a 30-mil FML (Cont'd) 
200: : 7-03

ACTIVIT CODB: BSE200112

PROJ.NAME: BCON. BVALUATION OP CLOSURB CAP BARRIER MATERLAL

\begin{tabular}{|c|c|c|c|c|}
\hline$E$ & & & $\begin{array}{l}\text { CHxD BY: } \\
\text { ESTmuTort } \\
\text { PHONE } \\
\text { OATE } \\
\text { LOA }\end{array}$ & $\begin{array}{l}\text { Q MEAD } \\
\text { PQMUK } \\
7-5200 \\
\text { OONOSO } \\
\text { MDC-100 }\end{array}$ \\
\hline $80 R$ & $\underset{8}{\operatorname{mir}}$ & $\begin{array}{l}\text { Bencap } \\
\text { saniny }\end{array}$ & $\begin{array}{l}\text { UnT } \\
\text { cost }\end{array}$ & $\begin{array}{l}\text { TOTAL } \\
\text { COST }\end{array}$ \\
\hline 3,040 & 0 & 012 & - & 3000 \\
\hline 1.782 & 0 & 50 & - & 20000 \\
\hline 1000 & 1400 & $=$ & 260 & $2 \pi 0$ \\
\hline 0 & 0 & $\infty$ & - & 400 \\
\hline 2400 & 8,270 & 744 & 18.70 & asco \\
\hline 1,200 & 700 & $\mathbf{s e n}$ & 80,00 & 2400 \\
\hline 200 & 400 & $\boldsymbol{c}$ & 6.00 & $\infty$ \\
\hline 14,000 & 500 & 4,200 & 550 & 18,500 \\
\hline 0 & . 0 & - & 720 & 1,000 \\
\hline 24.712 & 70318 & 0,934 & & 48,500 \\
\hline
\end{tabular}

D-2. Cost Estimate for a Cover System Utilizing Bentomat@ and a 30-mil FML 


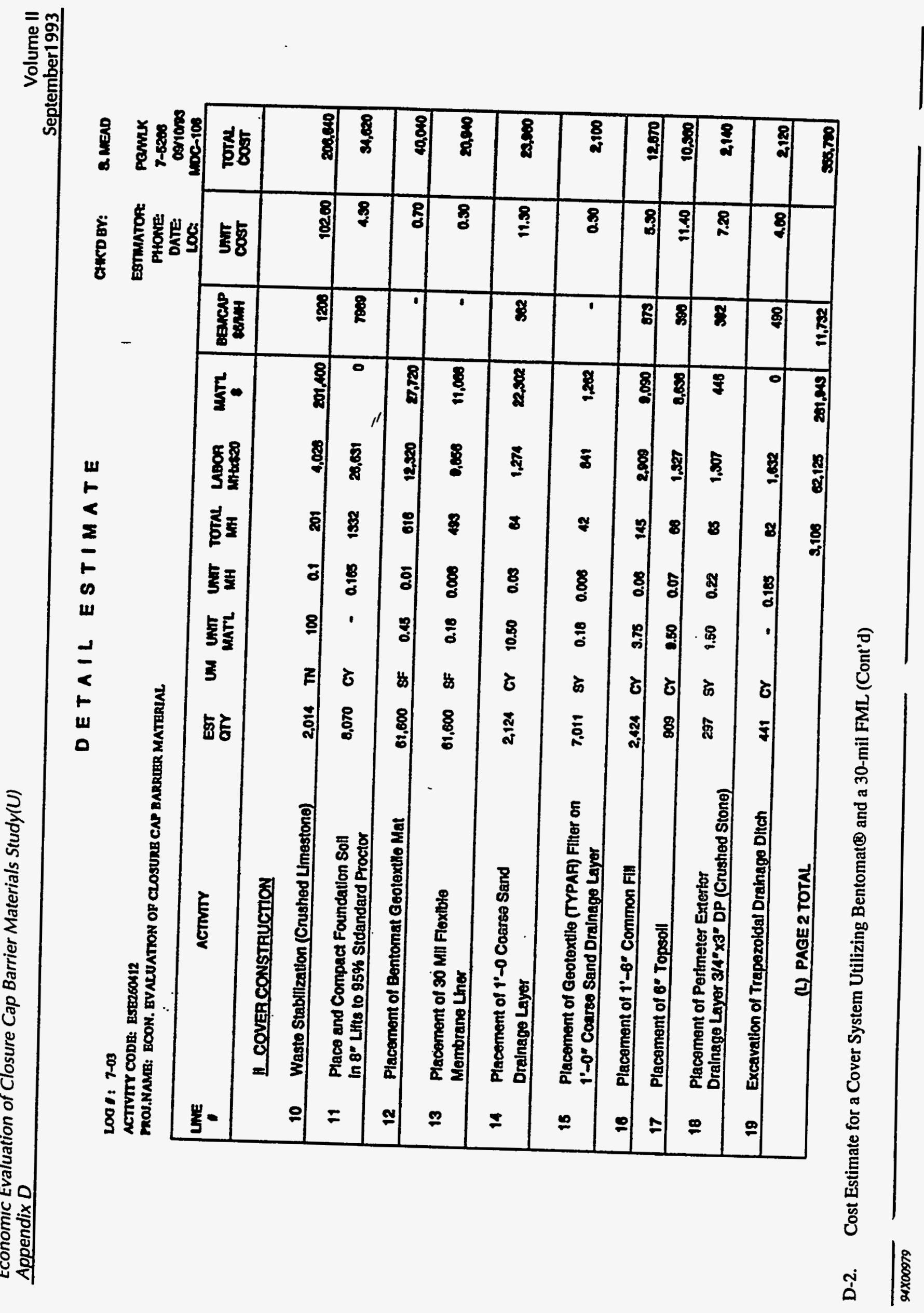




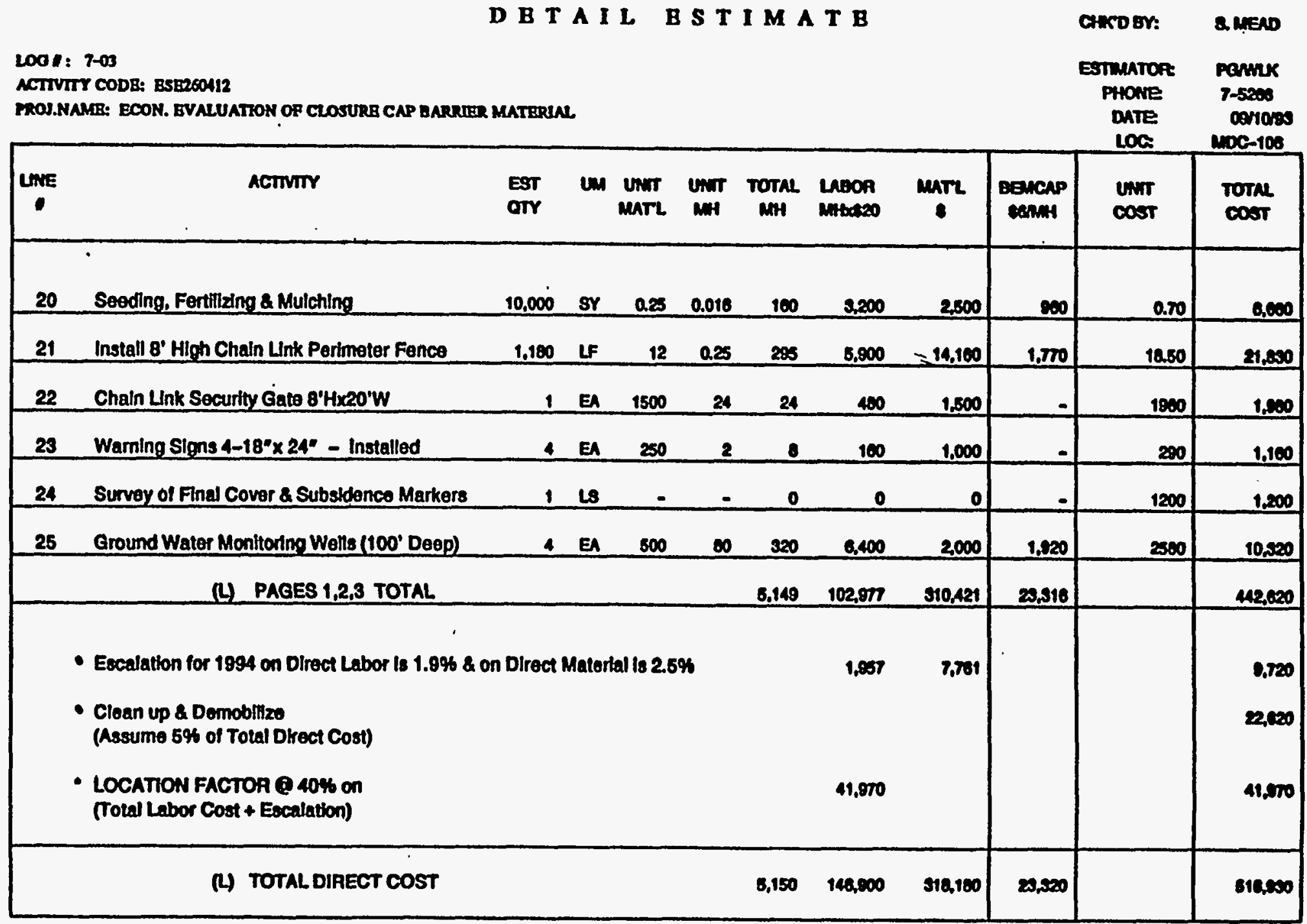

D-2. Cost Estimate for a Cover System Utilizing Bentomat and a 30-mil FML (Cont'd) 


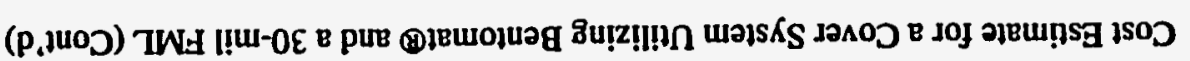

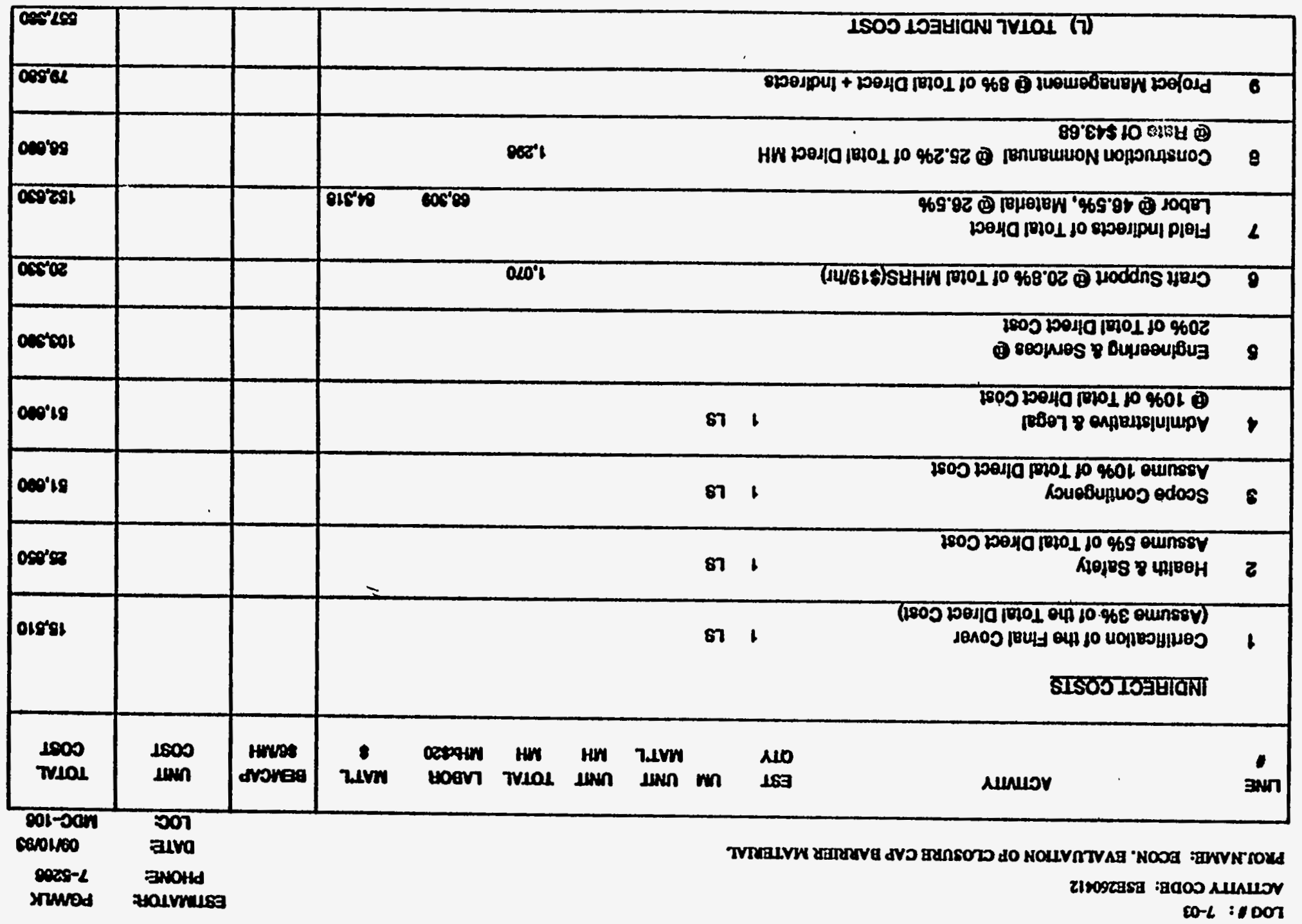

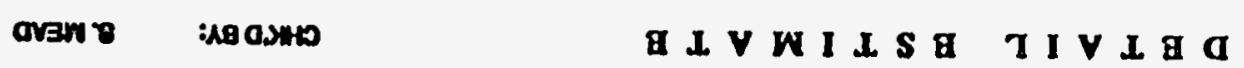


D B T A L B S T IMATB

Chrogr: 2.MENO

LO0 I: 7-03

ACTIVITY CODE: ESE260412

PROJ.NAME: ECON. EVALUATION OF CLOSURB CAP BARRTER MATERLAL

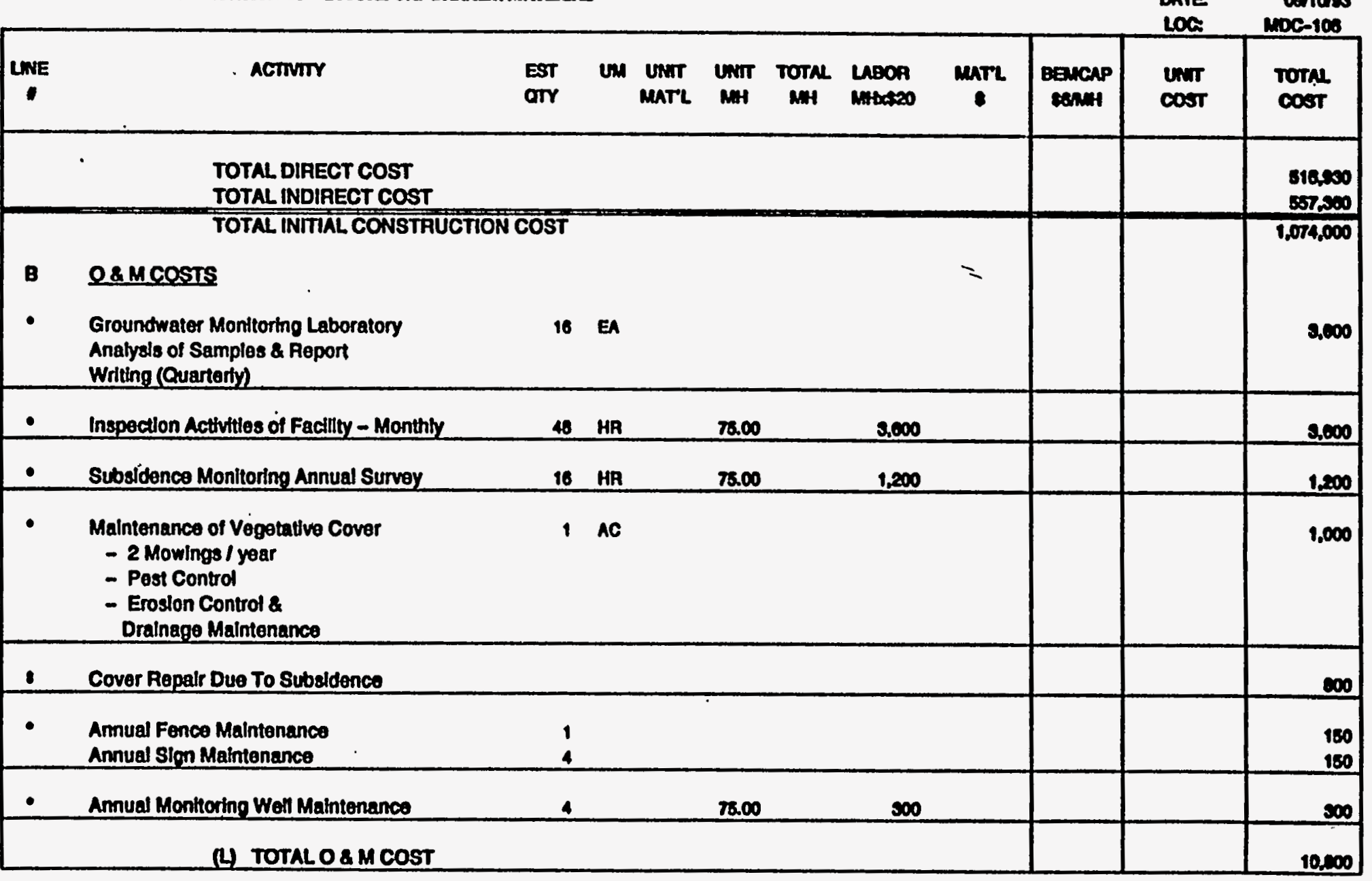

D-2. Cost Estimate for a Cover System Utilizing Bentomat@ and a 30-mil FML (Cont'd) 
D B T A I B S T I MAT B

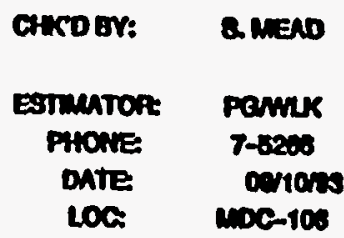

100 I: 7-03

ACTIVITY CODE: ESEZSOL12

PROJ.NAMGB: BCON. BVALUATION OP CLOSURB CAP BARRIER MATERLAL.

me-10.

\section{SUMMARY}

ONE(1) ACAE SITE-BENTOMAT (GBMD

C TOTAL COST ( Total Present Worth)

(1) Present Worth of $O$ \& $M$ Cost

(5\% dfscoumt Rate for 30 Years)

(Total $O \& M \times 15.3725$ )

(ii) Total Initial Construction Cost

(L) TOTAL PAOJECT COST (Rounded to the nearest Thousend)

D-2. Cost Estimate for a Cover System Utilizing Bentomat@ and a 30-mil FML (Cont'd) 
DETAIL ESTIMATE

Lod : $7-03$

ACTIVITY CODE: ESE2COH12

PROJ.NAME: BCON. EVALUATTON OF CLOSURE CAP BARRIER MATBRIAL

E

CHEOBV: ameno

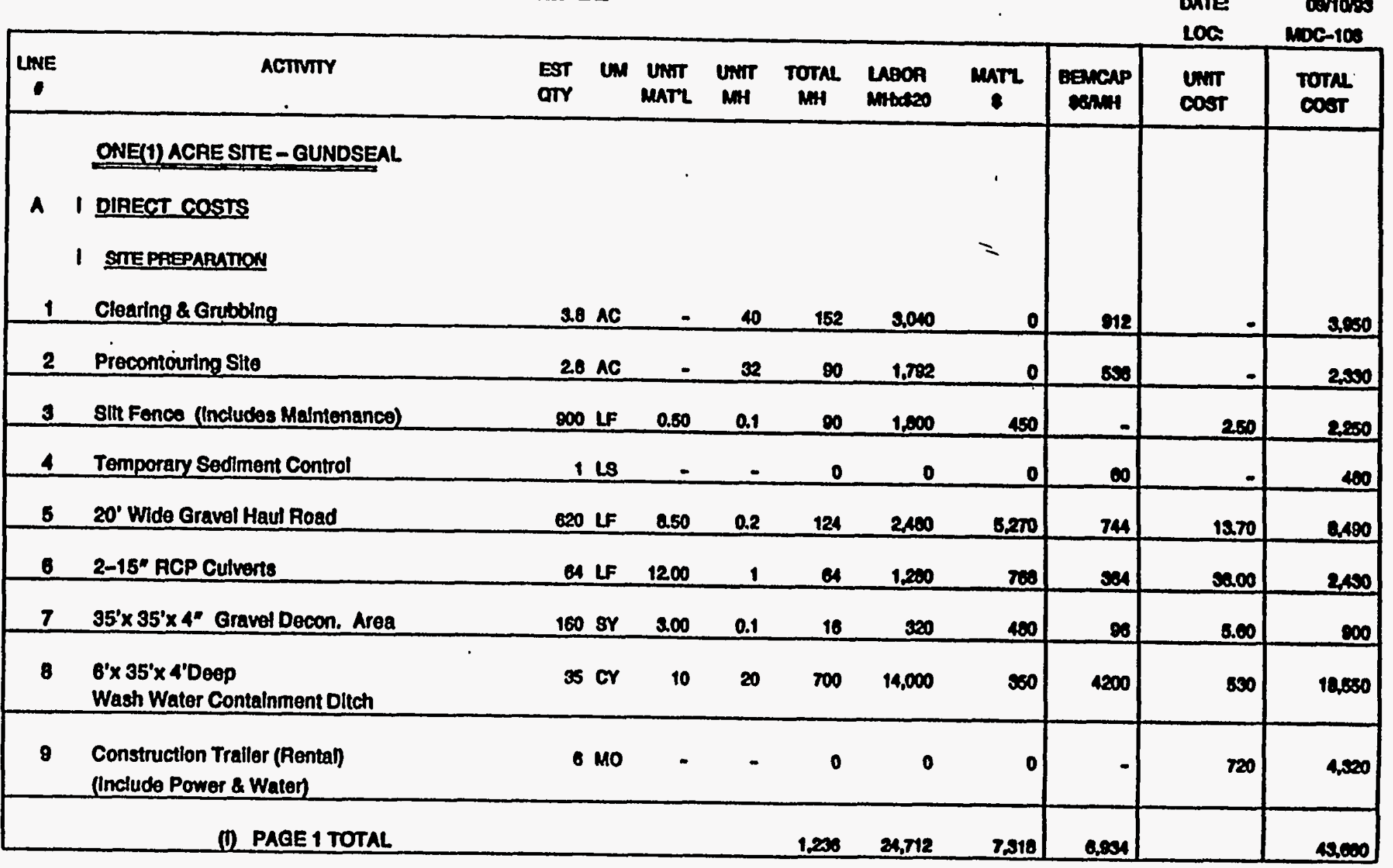

D-3. Cost Estimate for a Cover System Utilizing GundsealB 
DETAIL ESTIMATE CHNDEY: QMEAD

Loof: 7-03

ACTIVTY CODE: ESEOSOH12

PROJ.NAME: ECON. EVALUATTON OF CLOSURB CAP BARRUER MATERAAL.

\begin{tabular}{|c|c|c|c|c|c|c|c|c|c|c|c|}
\hline INE & ACTIVIV & EST & um & $\begin{array}{l}\text { UNMT } \\
\text { MATL }\end{array}$ & $\lim _{\min }$ & $\underset{M n}{\text { TOTAL }}$ & $\begin{array}{l}\text { LABOA } \\
\text { Mrtuteos }\end{array}$ & $\operatorname{marz}$ & $\operatorname{sencep}$ & $\begin{array}{l}\operatorname{lng} \pi \\
\cos \end{array}$ & $\begin{array}{l}\text { TotRl } \\
\cos \end{array}$ \\
\hline & LCOVER CONSTRUCTION & & & & & & & & & & \\
\hline 10 & Waste Stabillzation (Crushod Limestone) & 2.014 & $\mathbf{T}$ & 100 & 0.1 & 201 & 4,020 & 201,400 & 1200 & 10200 & 200,400 \\
\hline 11 & $\begin{array}{l}\text { Place and Compact Foundation Soil } \\
\ln 8^{*} \text { Litts to } 95 \% \text { Std. Proctor }\end{array}$ & 0,070 & or & - & 0.105 & 1332 & 200,601 & $=0$ & $m 00$ & 4.30 & 34,620 \\
\hline 12 & $\begin{array}{l}\text { Placement of Gundseal Geomembranel } \\
\text { Bentontte Mat }\end{array}$ & 61,600 & $8 F$ & 0.50 & 0.01 & 616 & 12,5000 & 50,000 & - & a.ro & 40,120 \\
\hline 13 & $\begin{array}{l}\text { Placement of 1'-0 Coarse Sand } \\
\text { Drainage Layer }\end{array}$ & 2,124 & or & 10.50 & 0.03 & 64 & 1,274 & 22,500 & 582 & 11.30 & 20,000 \\
\hline 14 & $\begin{array}{l}\text { Placement of Geotextile (TYPAR) Fitter on } \\
\text { 1'-0" Coareo Sand Drainage Layes }\end{array}$ & 7.011 & 8Y & 0.10 & 0.006 & 12 & 81 & 1,2002 & - & 0.50 & 2,100 \\
\hline 15 & Placoment of 1'-6" Common FIII & 2,424 & or & 3.75 & 0.08 & 145 & 2000 & 2000 & 89 & 8.00 & 12,070 \\
\hline 16 & Placement of $6^{\circ}$ Topsoll & 000 & or & 0.50 & 0.07 & of & 1200 & 0,000 & 302 & 11.00 & 10,200 \\
\hline 17 & $\begin{array}{l}\text { Placement of Perfimeter Exterlor } \\
\text { Drainaga Lajor } 3 / 4^{*} \times 3^{\circ} \text { DP (Crusined Sione) }\end{array}$ & 297 & 8Y & 1.50 & 0.22 & $\infty$ & 1,507 & 440 & 392 & 7.20 & 2,140 \\
\hline 18 & Excavation of Trapezoldal Drahage Ditch & 441 & or & - & 0.165 & 22 & 1.002 & 0 & 400 & 4.00 & 2.120 \\
\hline & (l) PAGE 2 TOTAL & & & & & 2.611 & $\$ 2215$ & 272036 & $11, r 18$ & & $\operatorname{sen} 2000$ \\
\hline
\end{tabular}

D-3. Cost Estimate for a Cover System Utilizing Gundseal@ (Cont'd) 
D B TAILE B TIMAT B

CHED BY: QMENO

2001: 7-03

ACTIVITY CODB: EsE200412

POI.MAME: BCON. EVALUATION OF CLOSURB CAP DARRIIR MATERIAL.

ESmantofe pamk

PPHONE 7-5200

DATE Derromos

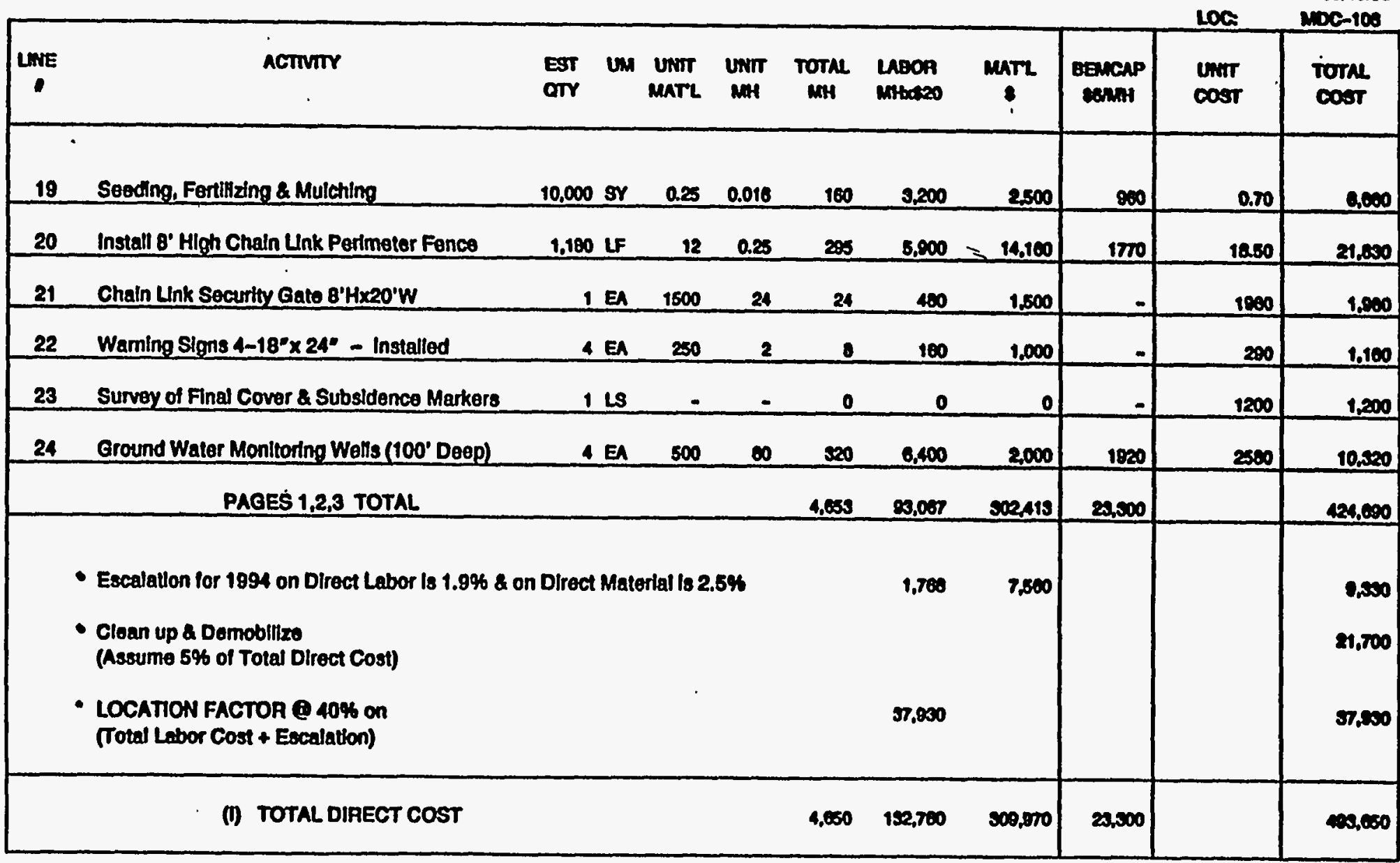

D-3. Cost Estimate for a Cover System Utilizing Gundseal@ (Cont'd) 
D B TAIL B STIMATE

Loo 1: 7-03

ACTIVTIY CODB: BSB260112

MOS.MAME: BCON. EYALUATION OF CLOSURB CAY DARRIER MATERIAL

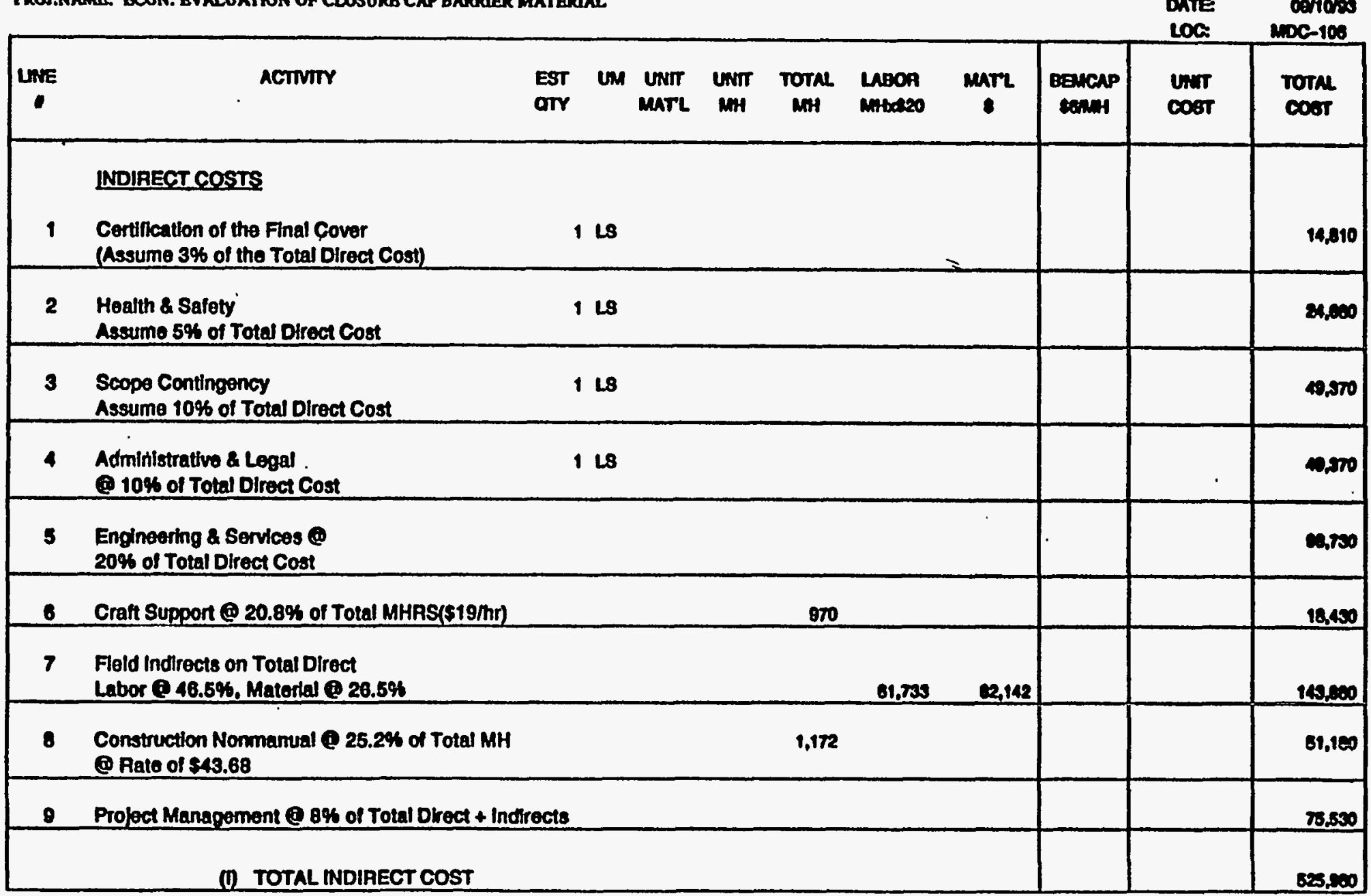

D-3. Cost Estimate for a Cover System Utilizing Gundseal@ (Cont'd) 
D B TAILE S TIMAT B

2008: 7-03

ACTIVTY CODB: ESE2OS12

PROJ.NAME: BCON. BVALUATION OP CLOSURB CAP BARRIBR MATBRLAL

CHKD BY: 2MEND

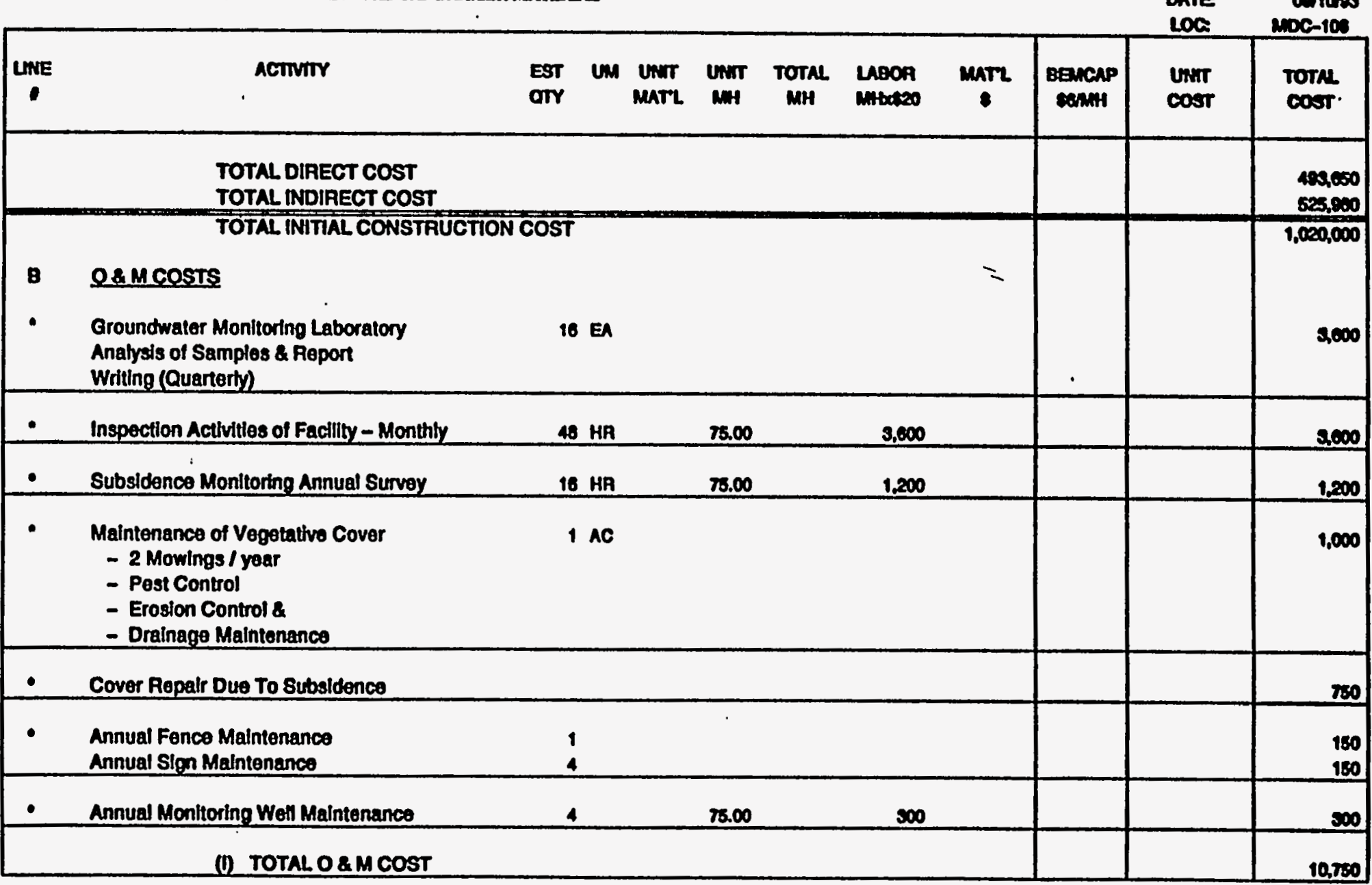

D-3. Cost Estimate for a Cover System Utilizing Gundseal@ (Cont'd) 
D B T A I L B S T IMAT B

\section{0 : 7-03}

ACTTVITY CODE: BSE26O112

PROJ.NAMR: ECON. BVALUATION OF CLOSURB CAP BARRIER MATERLAL.

\begin{tabular}{|c|c|}
\hline CHWCD BY: & Q MEND \\
\hline $\begin{array}{l}\text { Estumitor } \\
\text { PHone } \\
\text { DATE } \\
\text { LoG. }\end{array}$ & 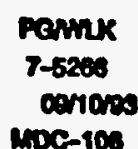 \\
\hline
\end{tabular}

\section{SUMMARY}

\section{ONE(1) ACRE SIIE-GUNDSEAL}

C TOTAL COST (Total Present Worth)

(D) Present Worth of $O \&$ Cost

(5\% discount Rate for 30 Years)

(Total O \& M $\times$ 15.3725)

165,000

(ii) Total Inltial Construction Cost

$1,020,000$

(i) TOTAL PAOSECT COST (Rounded to the nearest Thousand)

D-3. Cost Estimate for a Cover System Utilizing Gundseal® (Cont'd) 
DETAILESTIMATE

\section{LoOA: $7-03$}

ACTIVITY CODB: ESE2OON12

FROU.AANE: ECON. BYALUATION OP CLOSURB CAP BARRIBR MATERIAL

\begin{tabular}{|c|c|c|c|c|c|c|c|c|c|c|c|}
\hline LNE & ACTVIV & $\begin{array}{l}\text { EST } \\
\text { aTr }\end{array}$ & un & $\begin{array}{l}\text { UnNT } \\
\text { mart }\end{array}$ & UnT $_{\text {MH }}$ & TOTML & $\begin{array}{l}\text { LeOA } \\
\text { Mitureo }\end{array}$ & $\operatorname{marz}$ & $\begin{array}{l}\text { Bancap } \\
\text { senni }\end{array}$ & $\ln _{\cos \pi}$ & $\begin{array}{l}\text { TOTAL } \\
\text { Cost }\end{array}$ \\
\hline 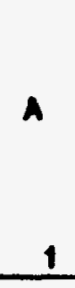 & $\begin{array}{l}\text { ONE(1) ACRE SIIE - (120 MIL FMI } \\
\text { I DIRECT COSTS } \\
1 \text { SUEPRAPARATON } \\
\text { Cloaring \& G Gubbing }\end{array}$ & 3.8 & AC & - & 40 & 152 & 5.040 & $=$ & 012 & - & 0.050 \\
\hline 2 & Precontouring Site & 28 & AC & - & 32 & $\infty$ & 1.702 & of & $\infty$ & - & 2050 \\
\hline 3 & Silt Fonce (inctudes Maintenance) & 900 & LF & 0.50 & 0.1 & $\infty$ & 1.000 & 450 & $=$ & 2050 & 22250 \\
\hline 4 & Temporary Sediment Control & 1 & LS & - & - & $\underline{0}$ & 0 & 0 & $\boldsymbol{\infty}$ & - & 400 \\
\hline 5 & 20' Wido Gravel Haul Road & 620 & $\underline{L}$ & 8.50 & 0.2 & 124 & 2,400 & 8,270 & $2 m$ & 19.70 & OASO \\
\hline$B$ & 2-15" RCP Cutvorts & a & LLF & 1200 & 1 & M & 1,200 & $r e 0$ & 30 & 3000 & 2450 \\
\hline 7 & $35^{\prime} \times 35^{\prime} \times 4^{\prime \prime}$ Gravel Decon. Area & 100 & 8Y & 3.00 & a.1 & 16 & 320 & 400 & $\boldsymbol{0}$ & 8.00 & $\infty 00$ \\
\hline 8 & $\begin{array}{l}\text { 6' } \times 35^{\prime} \times 4^{\prime} \text { Doep } \\
\text { Wash Water Containment Ditch }\end{array}$ & 35 & or & 10 & 20 & 700 & 14,000 & 350 & 4200 & 830 & 18550 \\
\hline 9 & $\begin{array}{l}\text { Construction Traller (Pental) } \\
\text { (include Power \& Water) }\end{array}$ & - & Mo & - & - & 0 & 0 & 0 & - & 120 & 4,5200 \\
\hline & (A) PAGE TOTAL & & & & & 1,230 & 24,712 & 7.018 & a.sen & & 45,000 \\
\hline
\end{tabular}

D-4. Cost Estimate for a Cover System Utilizing 120-mil High Density Polyethylene (HDPE) Liner 
DETAILESTIMATE

\section{0 : $7-03$}

ACTIVIT CODE: ESER60112

PROJ.NAME: ECON. BVALUATTON OP CLOSURB CAP DARXIER MATERIAL.

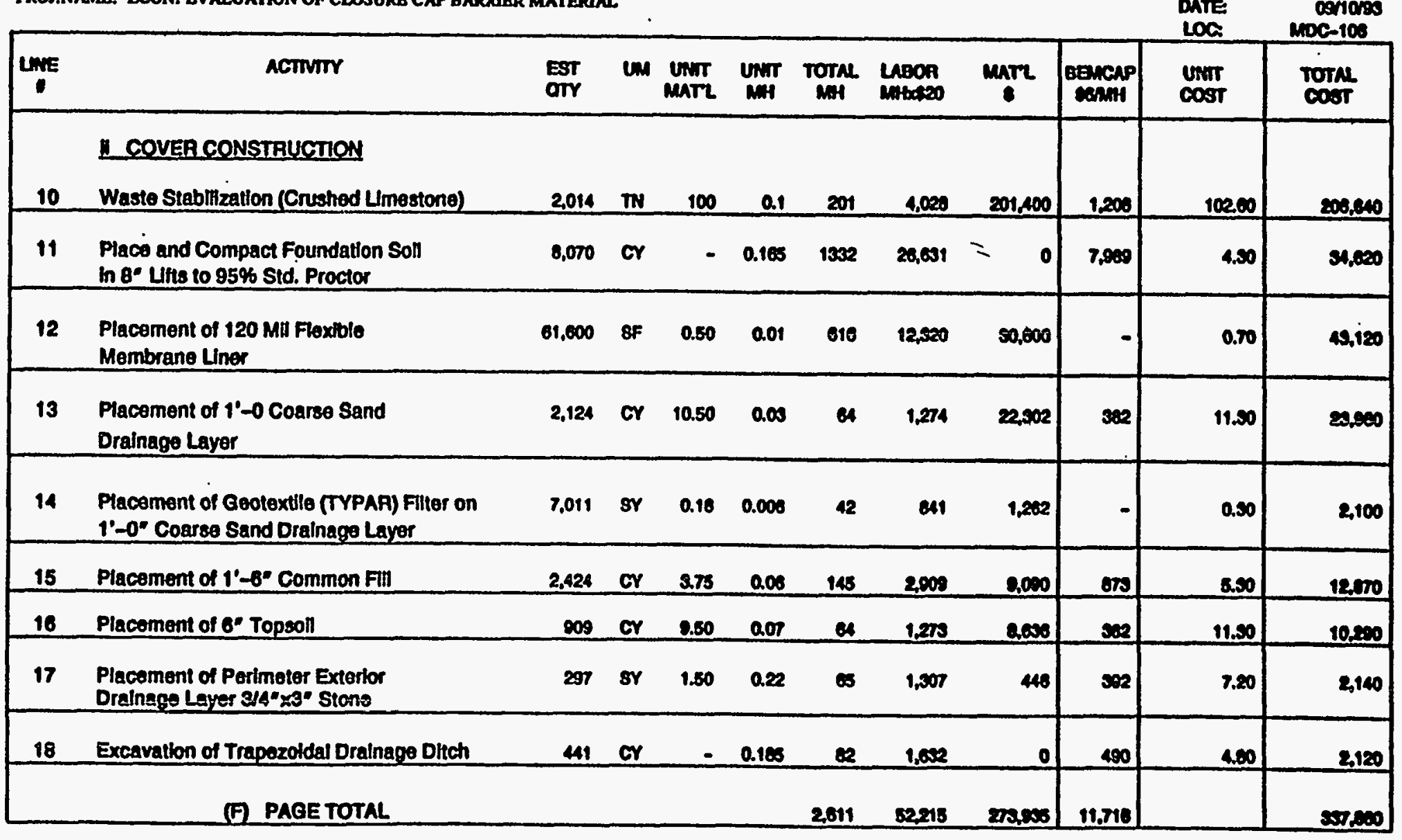

D-4. Cost Estimate for a Cover System Utilizing 120-mil High Density Polyethylene (HDPE) Liner (Cont'd) 
DBTAIL BSTIMATB

Caroor: 2.MENo

100 1: : 7-03

ACTIVITY CODE: BSB260412

PROJ.NAMB: BCON. BVALUATION OF CLOSURB CAP BARRIER MATERIAL

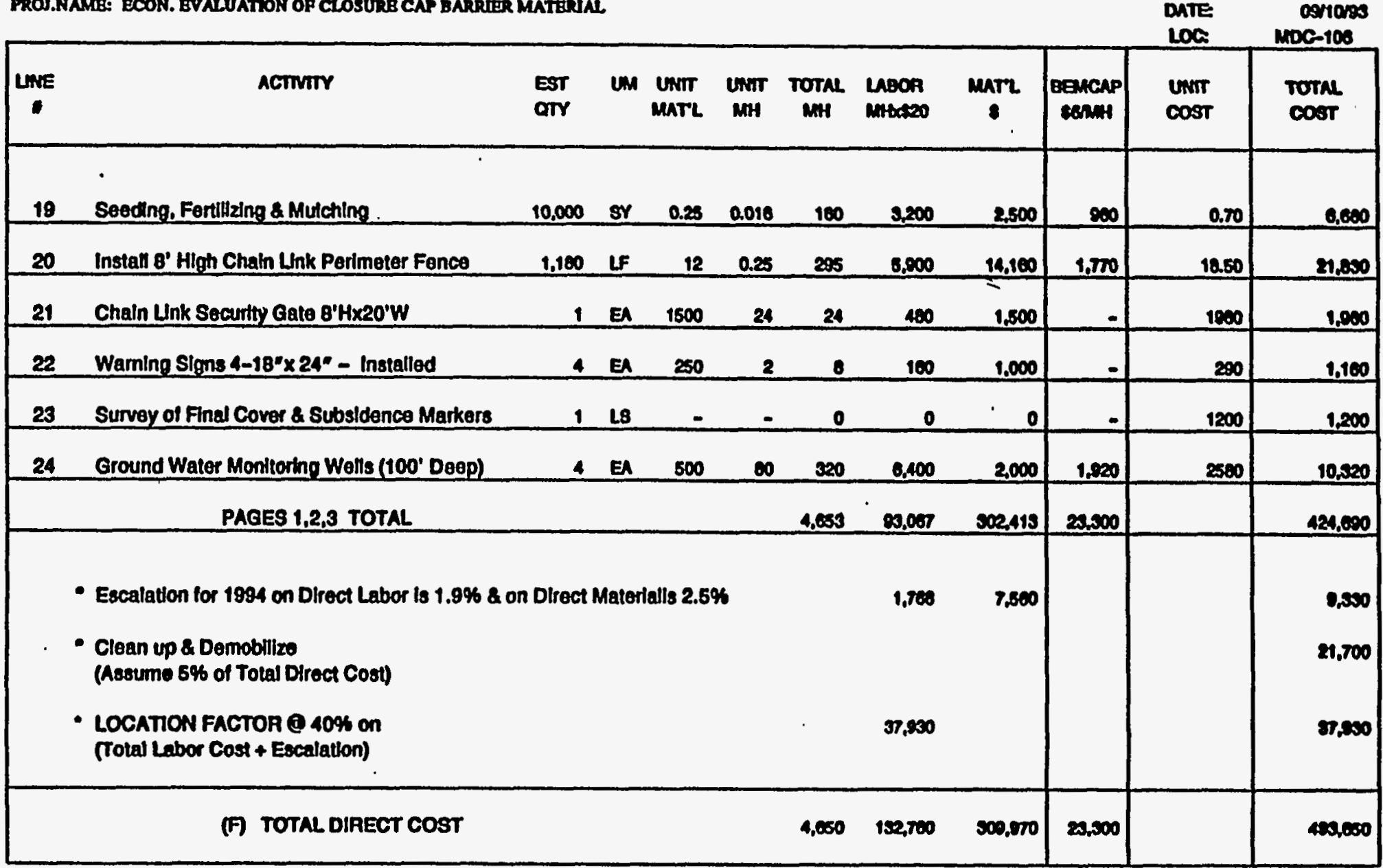

D-4. Cost Estimate for a Cover System Utilizing 120-mil High Density Polyethylene (HDPE) Liner (Cont'd) 
D B T A L B S T IMATB

L001: 7-03

ACTIVITY CODB: ESE2CON12

MOJ.NAME: BCON. EVALUATON OP CLOSURE CAP BARRRER MATERLAL

\begin{tabular}{|c|c|c|c|c|c|c|c|c|c|c|}
\hline LWE & ACIVITY & $\begin{array}{l}\text { EST } \\
\text { OTr }\end{array}$ & UM Unir & $\operatorname{Uim}_{\min }$ & TOTML & $\begin{array}{l}\text { LABOA } \\
\text { mitrated }\end{array}$ & $\operatorname{marL}$ & $\begin{array}{l}\text { egacap } \\
\text { senum }\end{array}$ & $\begin{array}{l}\operatorname{lng} \\
\cos t\end{array}$ & $\begin{array}{l}\text { Tothl } \\
\cos 21\end{array}$ \\
\hline & INDIRECT COSTS & & & & & & & & & \\
\hline 1 & $\begin{array}{l}\text { Certincation of the Final Cover } \\
\text { (Assume 3\% of the Total Direct Cost) }\end{array}$ & 1 & LS & & & & - & & & 14,010 \\
\hline 2 & $\begin{array}{l}\text { Heath \& Salety } \\
\text { Assume 5\% of Total Direct Cost }\end{array}$ & 1 & LS & & & & & & & 24,600 \\
\hline 3 & $\begin{array}{l}\text { Scope Contingency } \\
\text { Assume } 10 \% \text { of Total Direct Cost }\end{array}$ & 1 & 18 & & & & & & & 40,070 \\
\hline 4 & $\begin{array}{l}\text { Adminlstrativo \& Legal } \\
\text { (1) } 10 \% \text { of Total Direct Cost }\end{array}$ & 1 & LS & & & & & & & 40,370 \\
\hline $\mathbf{s}$ & $\begin{array}{l}\text { Engineering \& Sorvices } \\
20 \% \text { of Total Direct Cost }\end{array}$ & 1 & L8 & & & & & & & 60,750 \\
\hline 6 & Craft Suppon @ $20.8 \%$ of Total MHAS( $(\$ 19 / \mathrm{hr})$ & & & & 900 & & & & & 10,450 \\
\hline 7 & $\begin{array}{l}\text { Field Indirects of Total Direct } \\
\text { Labor Q 46.5. Material Q 28.5\% }\end{array}$ & & & & & 01,700 & 82,142 & & & 145,000 \\
\hline 8 & $\begin{array}{l}\text { Construction Nonmanual @ } 25.2 \% \text { of Total Direct } \\
\text { @ Rate of } \$ 43.88\end{array}$ & MH & & & 1,172 & . & & & & 61,900 \\
\hline 0 & Prolect Management @ 8\% of Tolal Dlroct + Indire & octs & & & & & & & & 7,550 \\
\hline & (F) TOTAL INDIRECT COST & & & & & & & & & 520,800 \\
\hline
\end{tabular}

D-4. Cost Estimate for a Cover System Utilizing 120-mil High Density Polyethylene (HDPE) Liner (Cont'd) 
D B T A I L B S T IMAT B

Loof: 7-03

ACTIVTY CODB: ESB260N12

PROJ.NAME: BCON. BVALUATION OF CLOSURB CAP BARRIER MATERLAL

CAKCD Br: 2 MeNo

Estmator: pamere

PHONE T-6200

oute corions

LOa $\quad$ moc-100

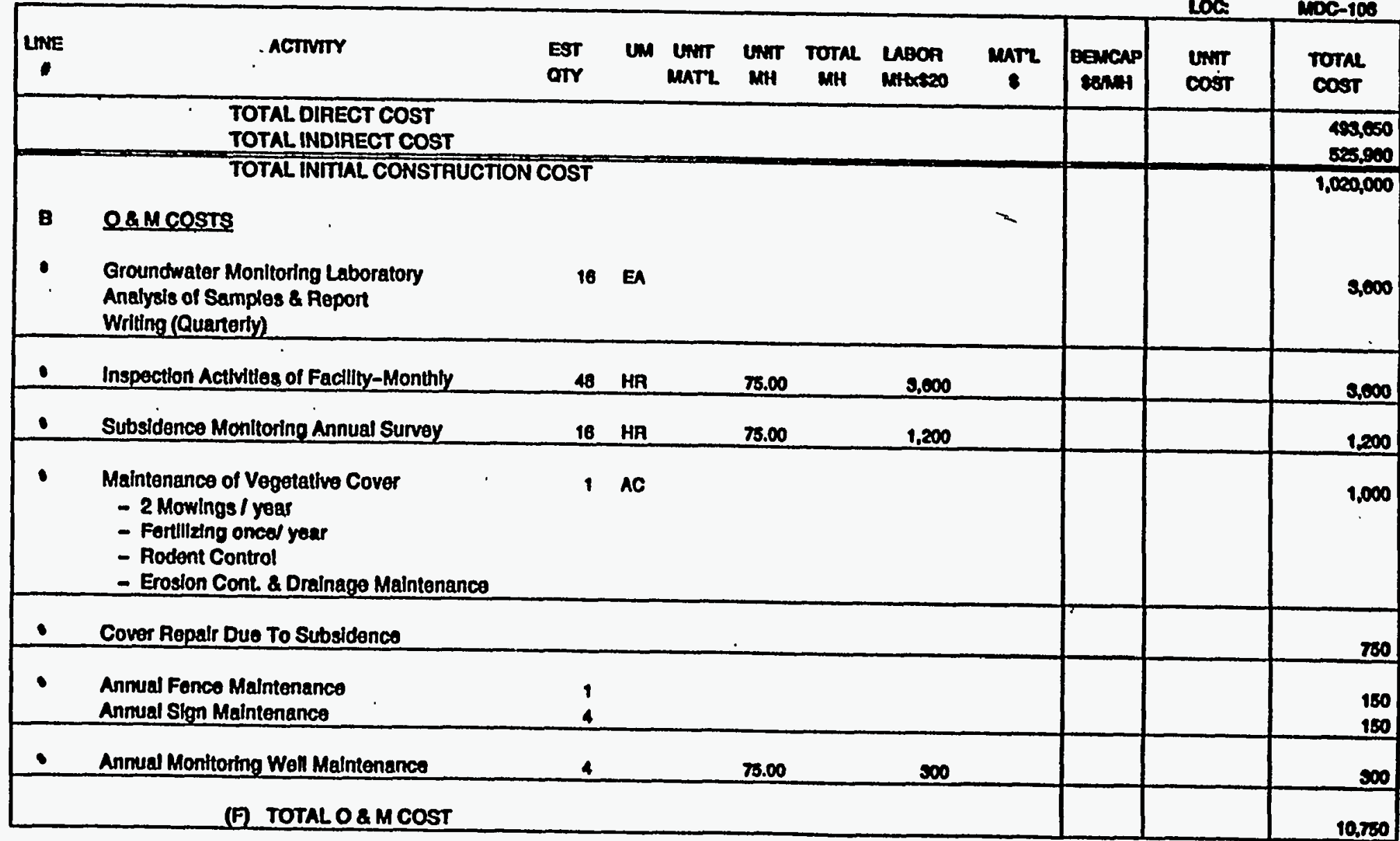

D-4. Cost Estimate for a Cover System Utilizing 120-mil High Density Polyethylene (HDPE) Liner (Cont'd) 


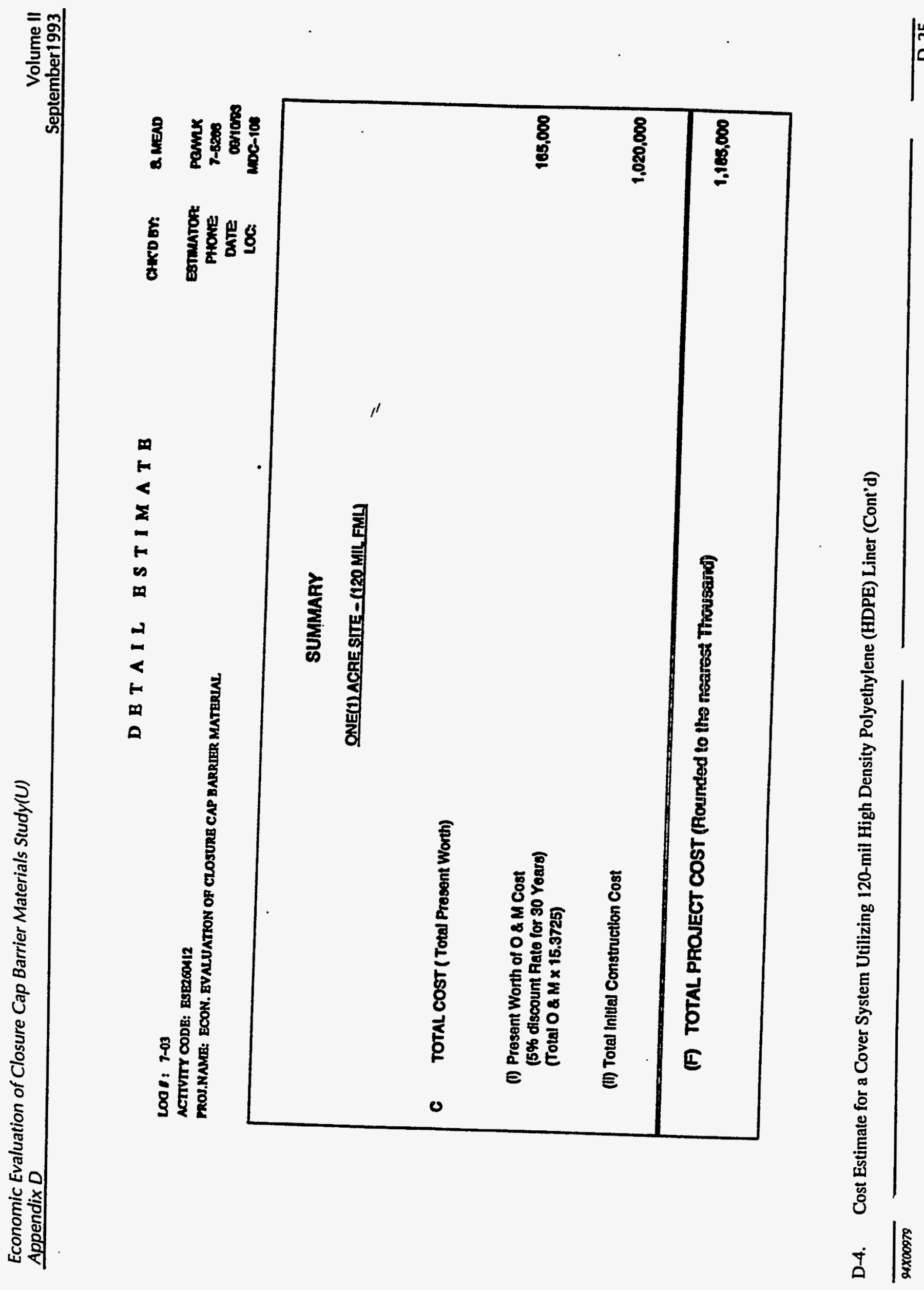


DETAIL ESTIMATE

1001: 7-03

ACTIVTY CODB: ESBESON12

PROJ.NAME: ECON. EYALUATTON CLOSURR CAP BARRIER MATERIAL

E CHCDOY: 2MEND

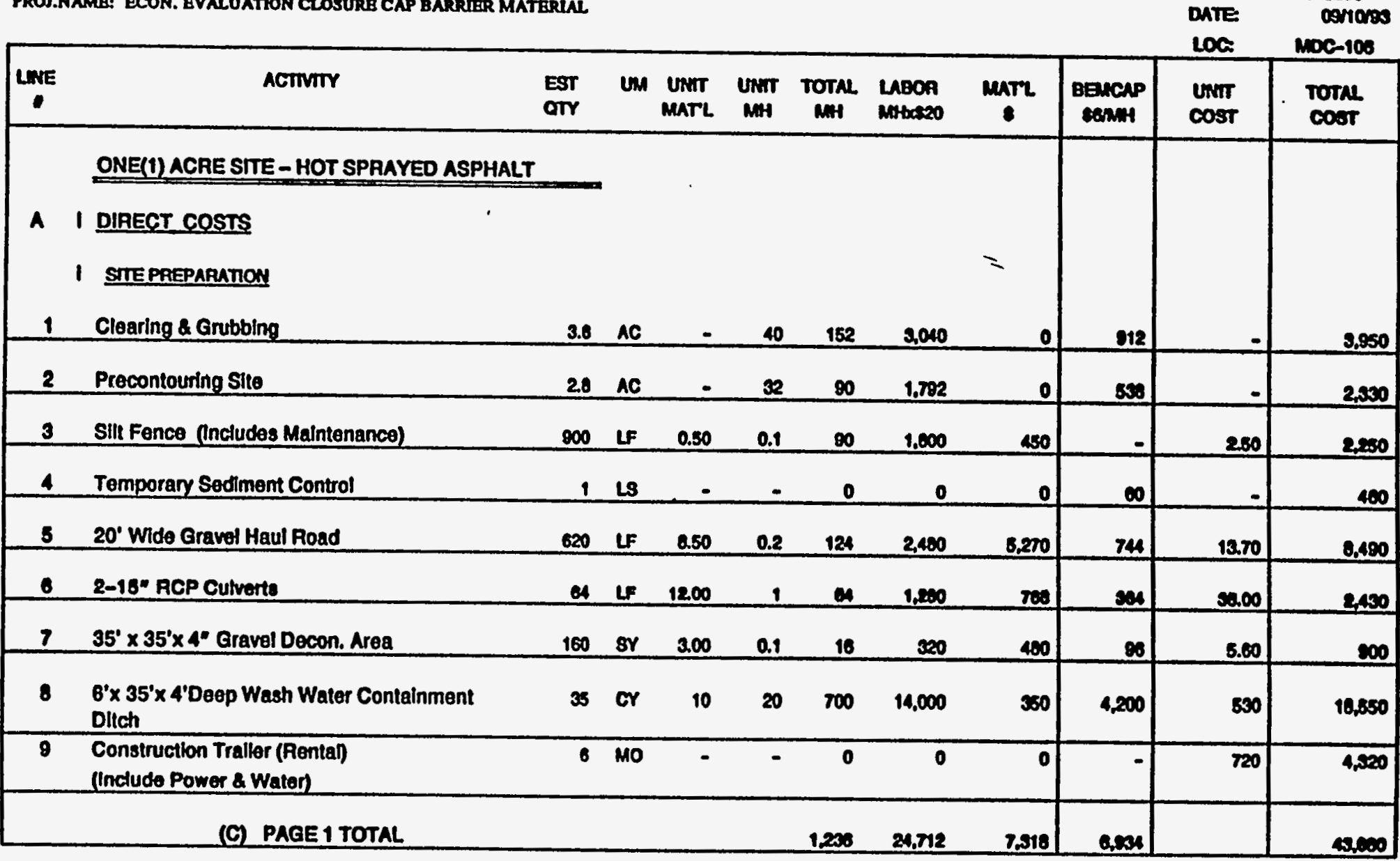

D-5. Cost Estimate for a Cover System Utilizing 1/4-inch Thick Hot Sprayed or Emulsified Asphalt 
DETAIL ESTIMATE

Lod 1: 7-03

ACTIVITY CODE: ESE2OON12

MOJ.NAMB: ECON. BYALUATTON CLOSURB CAP BARRRER MATERJAL

CHCOEV: 2MEAD

\begin{tabular}{|c|c|c|c|c|c|c|c|c|c|c|c|}
\hline UNE & ACTIVIV & $\begin{array}{l}\text { EST } \\
\text { OTY }\end{array}$ & UM & $\begin{array}{c}\text { UNMT } \\
\text { MATL }\end{array}$ & UMT & $\underset{M H}{\text { TOTAR }}$ & $\begin{array}{l}\text { LABOA } \\
\text { MHated }\end{array}$ & $\operatorname{maTL}^{\prime}$ & $\begin{array}{l}\text { Bemcap } \\
\operatorname{sennhy}\end{array}$ & $\begin{array}{l}\text { Unit } \\
\text { Cost }\end{array}$ & $\begin{array}{l}\text { Tothl } \\
\text { cosit }\end{array}$ \\
\hline 10 & $\begin{array}{l}\text { 11 COVER CONSTRUCTION } \\
\text { Waste Stabilization (Crushod LImestone) }\end{array}$ & 2.014 & $\mathrm{TN}$ & 100 & 0.1 & 201 & 4,020 & 201,400 & 1.200 & 102.00 & 200,640 \\
\hline 11 & $\begin{array}{l}\text { Place and Compact Foundation Soll } \\
\text { in 8" Lits to 95\% Std. Proctor }\end{array}$ & 8.070 & or & - & 0.168 & 1932 & 20,691 & 2 & 7,000 & 4.50 & $\mathbf{3 4 , 6 0 0}$ \\
\hline 12 & $\begin{array}{l}\text { Placoment of } 30 \mathrm{MnI} \text { Flexible } \\
\text { Membrane Lher }\end{array}$ & 61,600 & SF & 0.18 & 0.000 & 193 & 9.056 & 11,000 & - & 0.30 & 20,840 \\
\hline 13 & $\begin{array}{l}\text { Applied Hot Asphalt using } 3 \text { passes } \\
\text { at a rate of } 0.5 \text { gal / SY }\end{array}$ & $\begin{array}{l}5,422 \\
8,133 \\
\end{array}$ & $\begin{array}{l}\text { SY } \\
\text { QAL }\end{array}$ & 2 & 0.18 & 819 & 10,260 & 10,84 & 4,000 & 8.90 & 91,890 \\
\hline 14 & $\begin{array}{l}\text { Placement of 1'-0 Coarse Sand } \\
\text { Drainage Layor. }\end{array}$ & 1,807 & Cr & 10.50 & 0.00 & 54 & 1.004 & 18,974 & 325 & 11.30 & 20,500 \\
\hline 15 & $\begin{array}{l}\text { Placement of Geotextile (TYPAR) Fiter on } \\
1^{1}-0^{*} \text { Coarse Sand Dralnage Layer }\end{array}$ & 5,122 & SY & 0.16 & 0.006 & $\boldsymbol{s}$ & 851 & 970 & - & 0.30 & 1,000 \\
\hline 16 & Placement of $1^{\prime}-6^{\circ}$ Common Fill & 2,424 & cr & 3.75 & 0.00 & 145 & 2900 & 2,090 & o70 & 5.50 & 12.070 \\
\hline 17 & Placement of $B^{-}$Topsoll & 909 & cr & 8.50 & 0.08 & $\boldsymbol{a}$ & 1,279 & 0,030 & 302 & 11.30 & 10,200 \\
\hline 18 & $\begin{array}{l}\text { Placement of Perimeter Exterlor } \\
\text { Drainage Layer } 3 / 4^{*} \times 3^{*} \text { Stone }\end{array}$ & 297 & 9r & 1.50 & 0.22 & $\infty$ & 1,307 & 440 & 392 & 7.20 & 2,140 \\
\hline 19 & Excavatlon of Trapezoldal Drainage Ditch & 441 & or & $=$ & 0.185 & 82 & 1.032 & 0 & 490 & 4.60 & 2,120 \\
\hline & (C) PAGE 2 TOTAL & & & & & 2,202 & $\infty, 000$ & 201,452 & 10.550 & & $\sin , 020$ \\
\hline
\end{tabular}

D-5. Cost Estimate for a Cover System Utilizing 1/4-inch Thick Hot Sprayed or Emulsified Asphalt (Cont'd) 


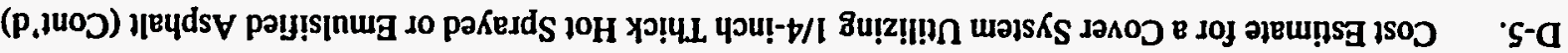

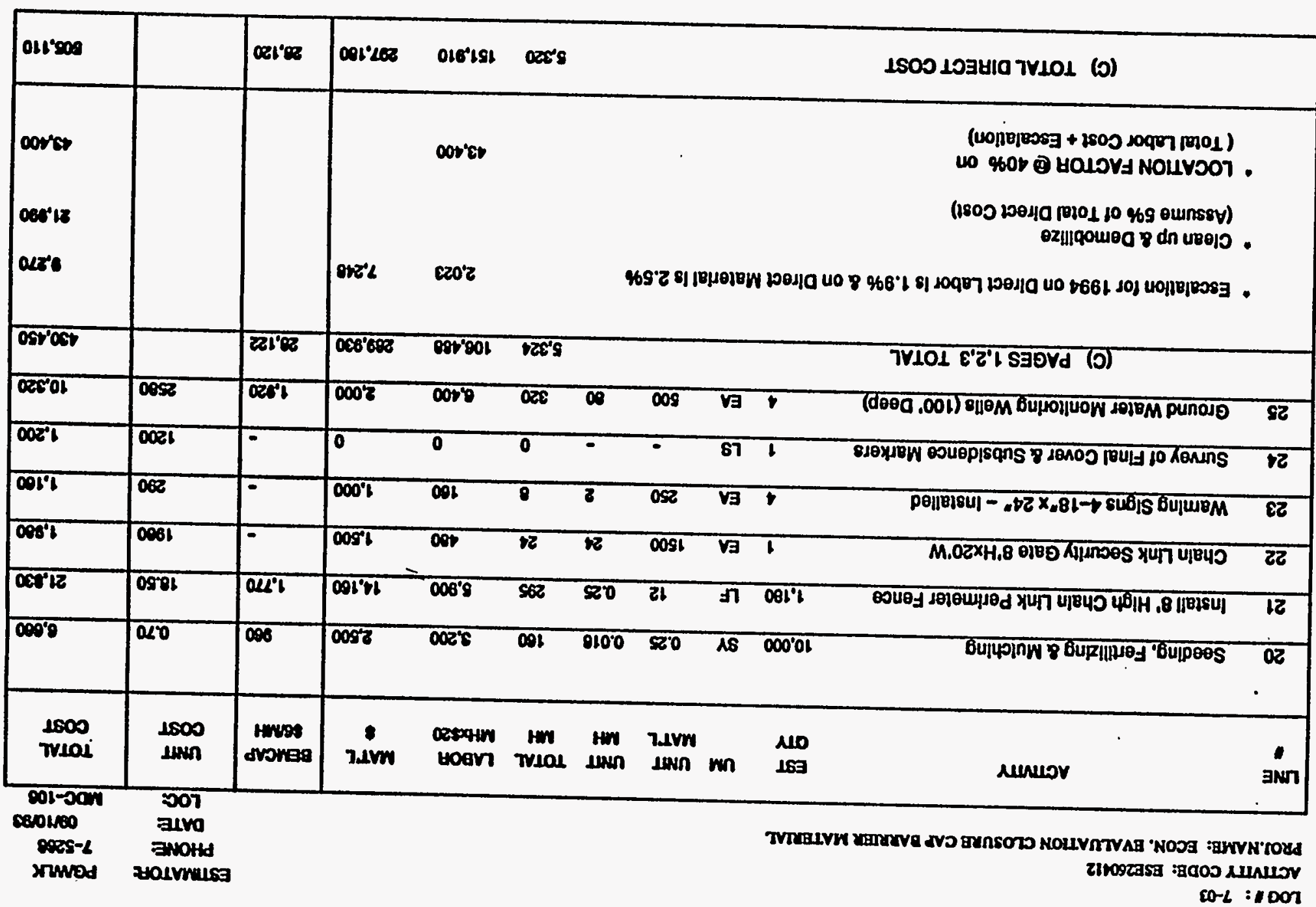

Gram 8 :

g $\perp \vee$ K I I S \& $\quad$ I I $\vee \perp$ B 
DETAIL BSTIMATB

CHCOQY: 2.MEAD

Loof: $7-03$

ACTIVTYY CODE: ESE26012

PROJ.NAMER: ECON. EYALUATION CLOSURB CAP BARRIER MATERJAL

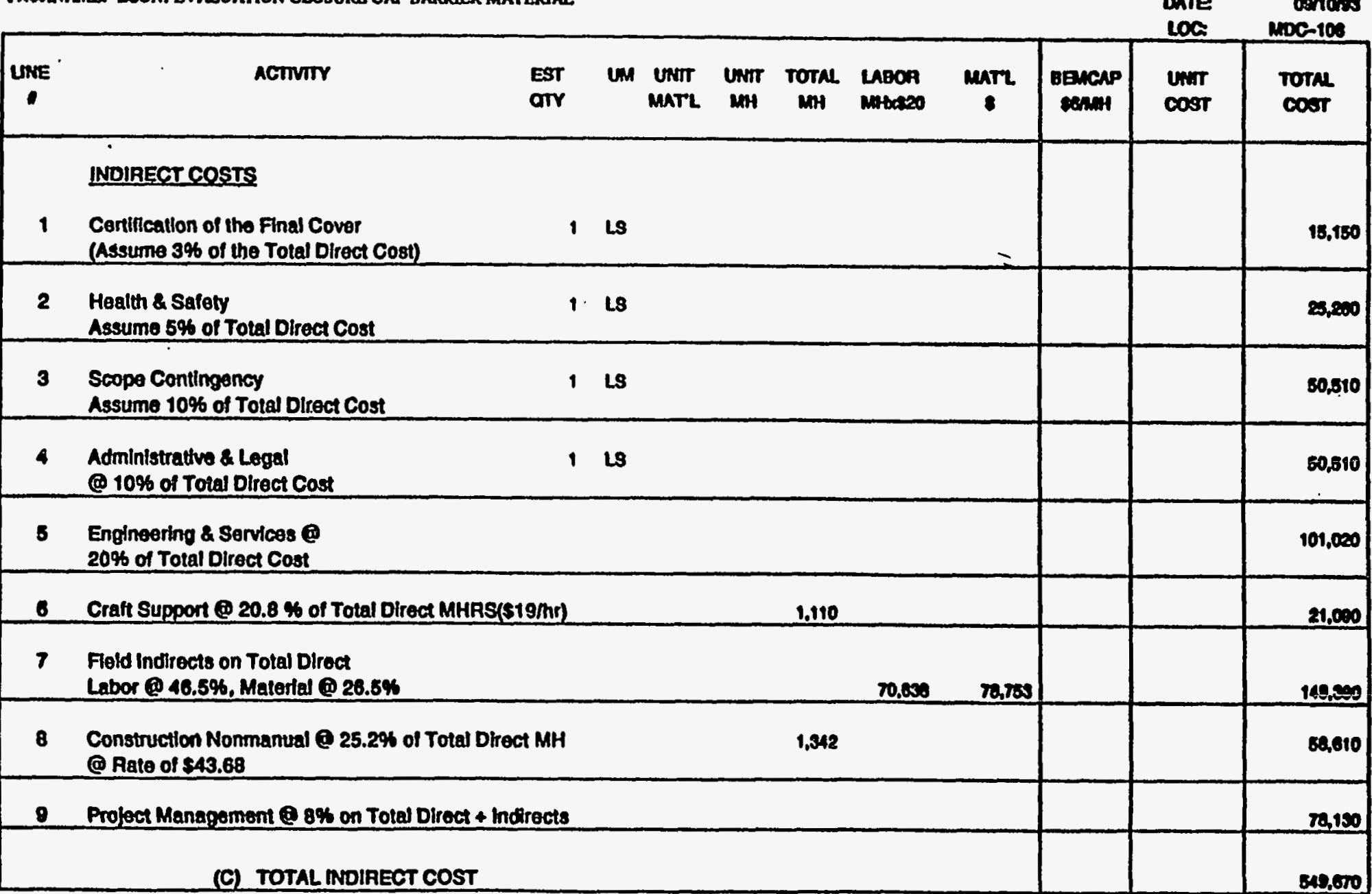

D-5. Cost Estimate for a Cover System Utilizing 1/4-inch Thick Hot Sprayed or Emulsified Asphalt (Cont'd) 


\section{DETAIL BSTIMAT B}

$1001: 7-03$

ACTTVITY CODE: ESE260412

PROI.NAMR: ECON. EVALUATION CLOSURB CAP BARRIER MATERIAL,

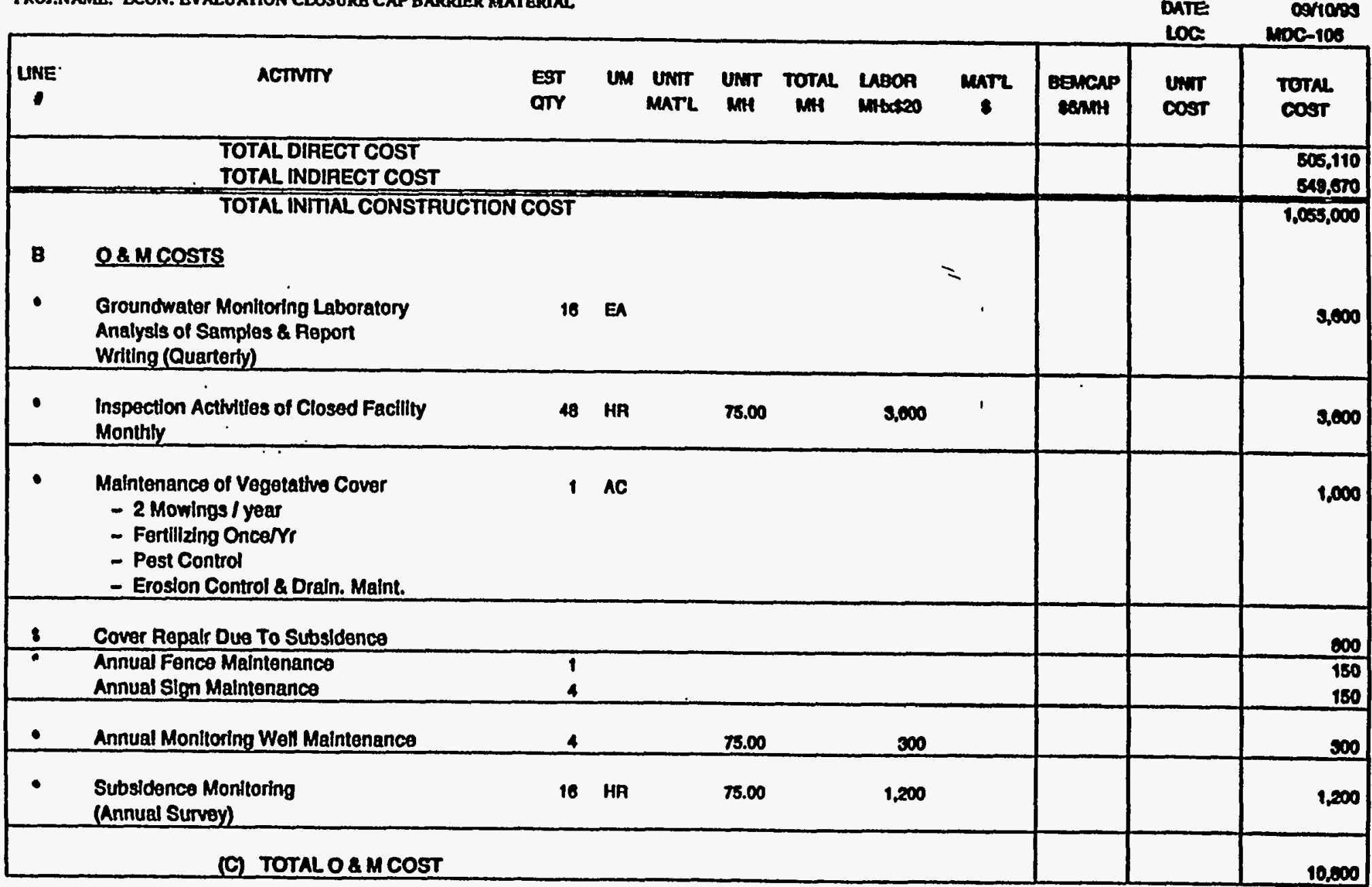

D-5. Cost Estimate for a Cover System Utilizing 1/4-inch Thick Hot Sprayed or Emulsified Asphalt (Cont'd) 
D B TAIL BSTIMATB

Loof: $7-03$

ACTIVTY CODE: ESE2CON12

PROJ.NAME: ECON. EYALUATKON CLOSURE CAP EARRIER MATERIAL

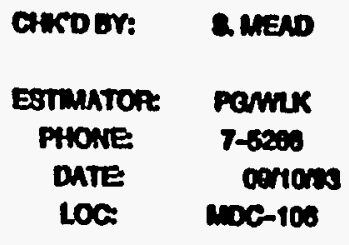

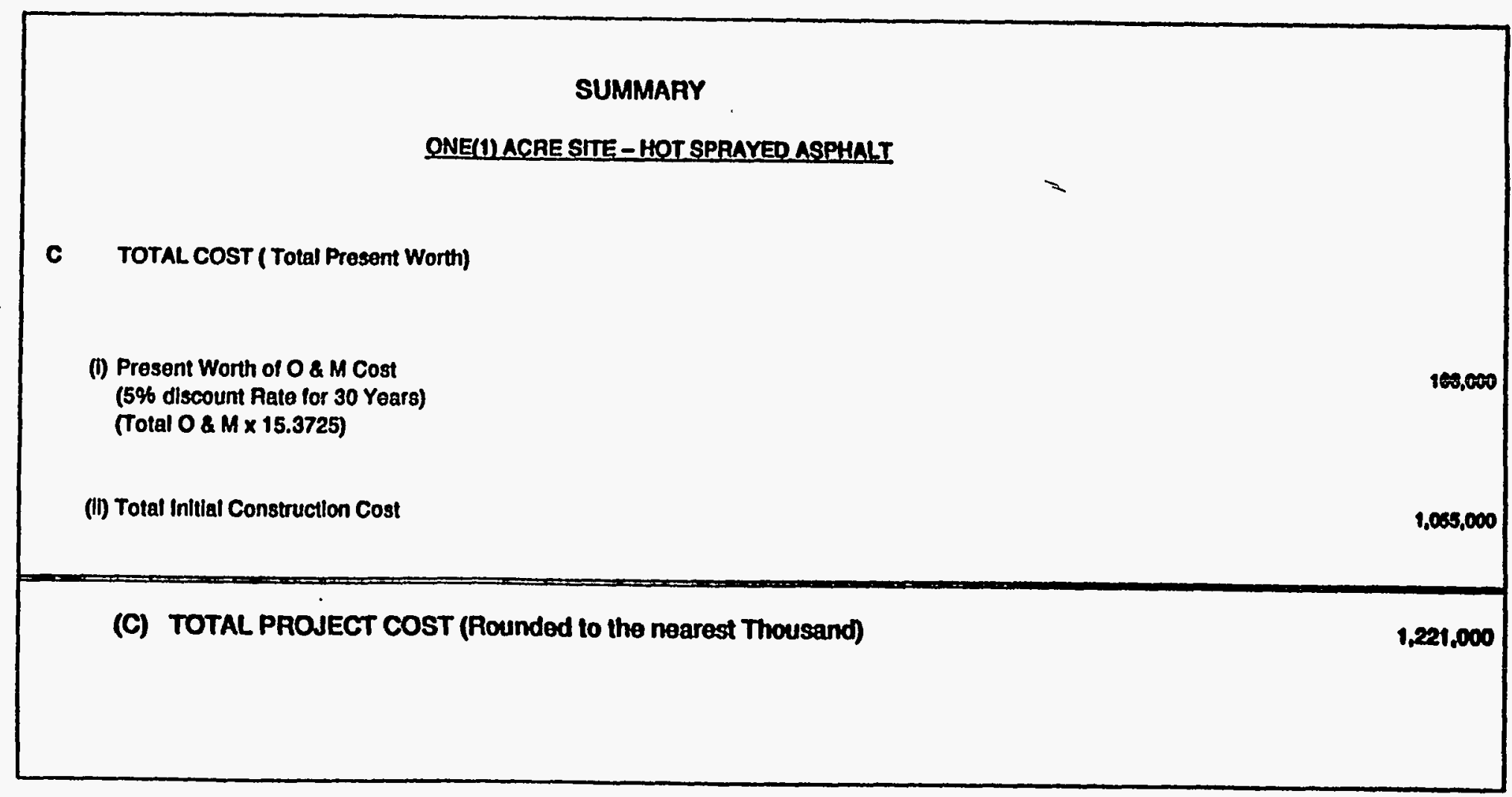

D-5. Cost Estimate for a Cover System Utilizing 1/4-inch Thick Hot Sprayed or Emulsified Asphalt (Cont'd) 


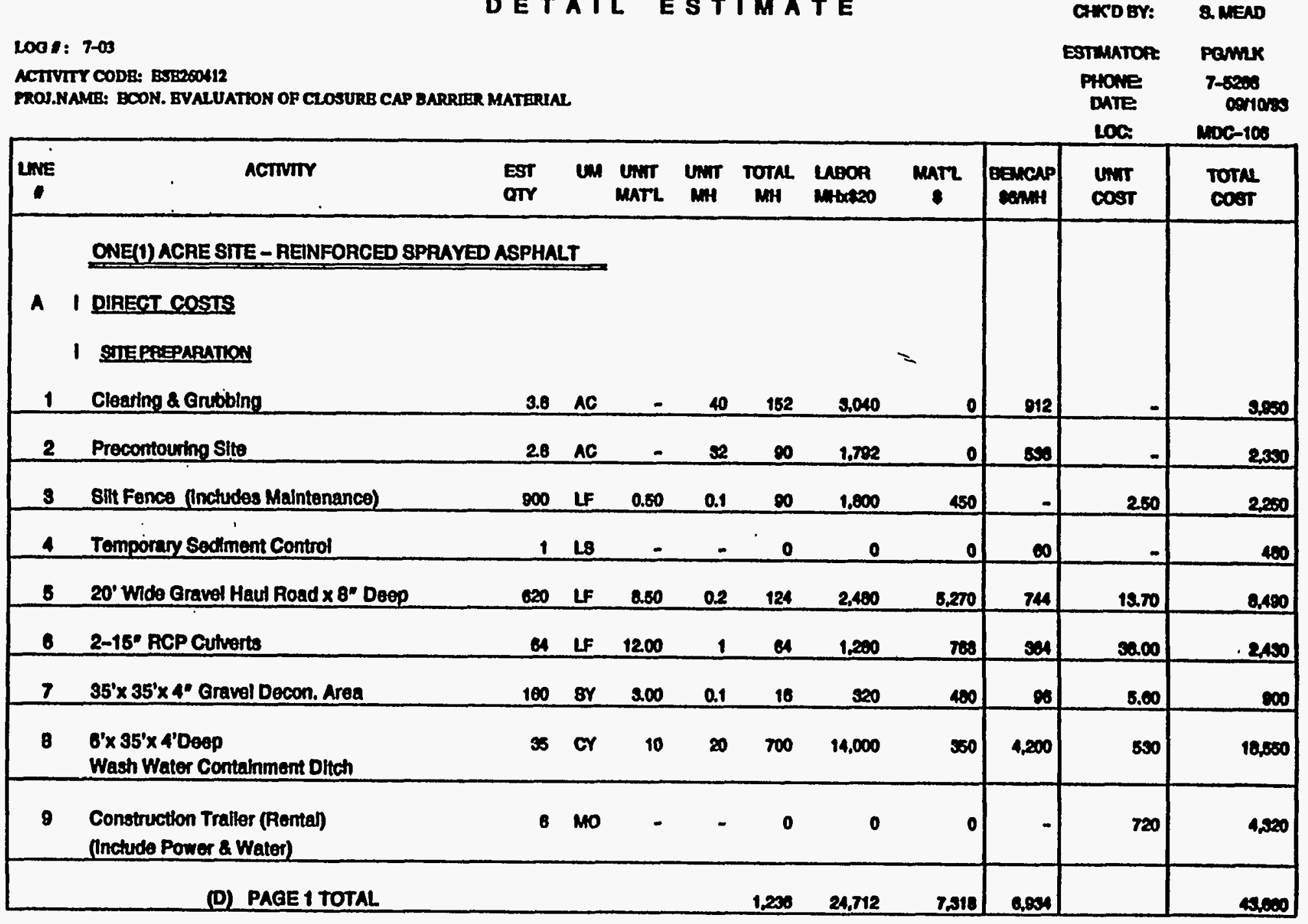

D-6. Cost Estimate for a Cover System Utilizing Reinforced Sprayed Asphalt 
CHCDEY: QMEND

LOO 1: 7-03

ACTIVTTY CODB: ESBE260412

PROU.NAME: ECON. BVALUATTON OF CLOSURB CAP BARRUER MATERIAL

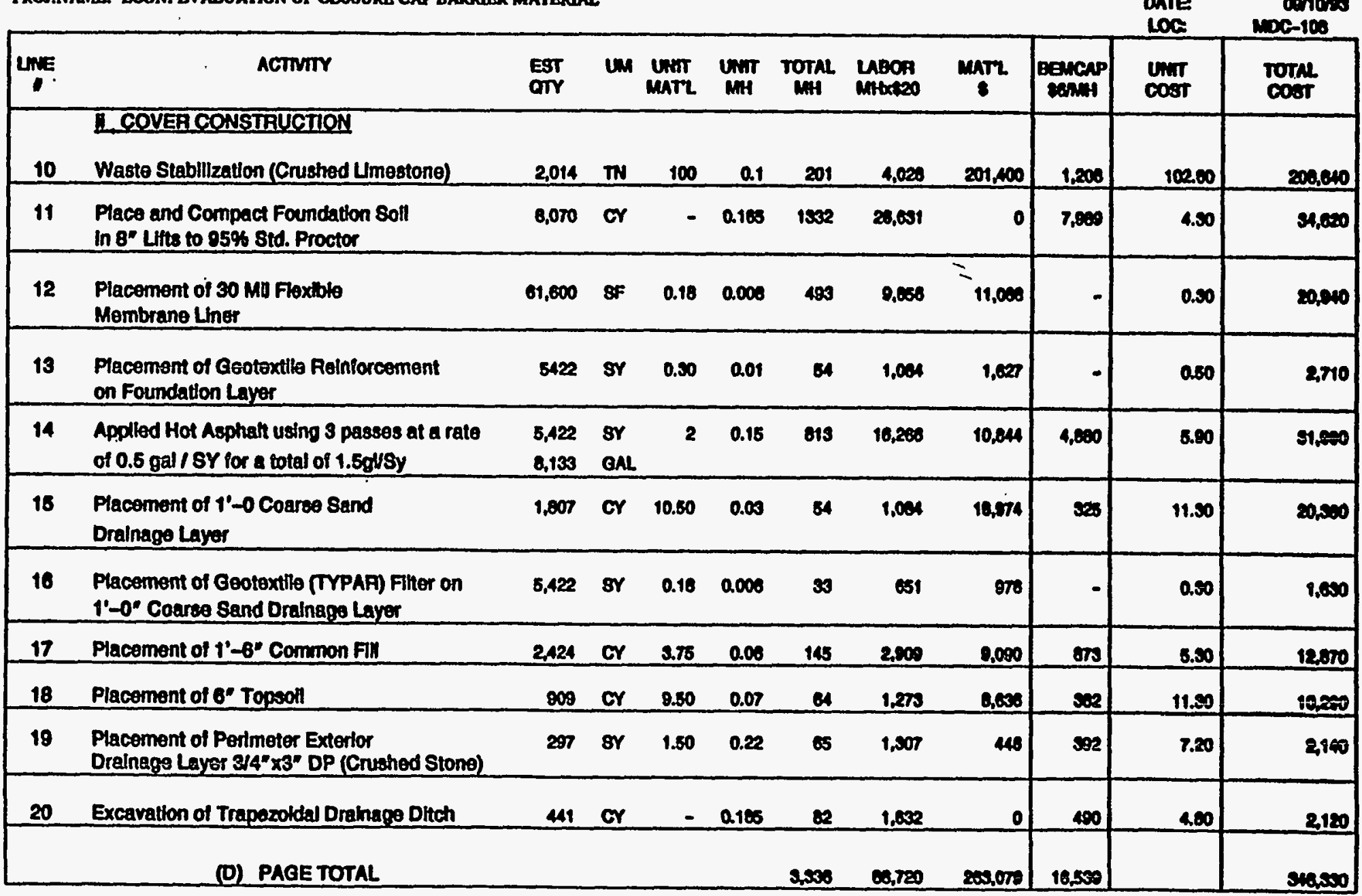

D-6. Cost Estimate for a Cover System Utilizing Reinforced Sprayed Asphalt (Cont'd) 


\section{DETAIL ESTIMATE}

L00 1: $7-03$

ACTIVITY CODB: BSEZOOA12

FROJ.NAMR: BCON. BVALUATION OF CLOSURB CAP DARRIER MATERLAL

\begin{tabular}{|c|c|c|}
\hline INATE & CHND or: & A. MEAD \\
\hline & $\begin{array}{l}\text { ESTIMATOR } \\
\text { PHOHE } \\
\text { DATE }\end{array}$ & $\begin{array}{l}\text { Panik } \\
7-5200 \\
\text { oenciose }\end{array}$ \\
\hline
\end{tabular}

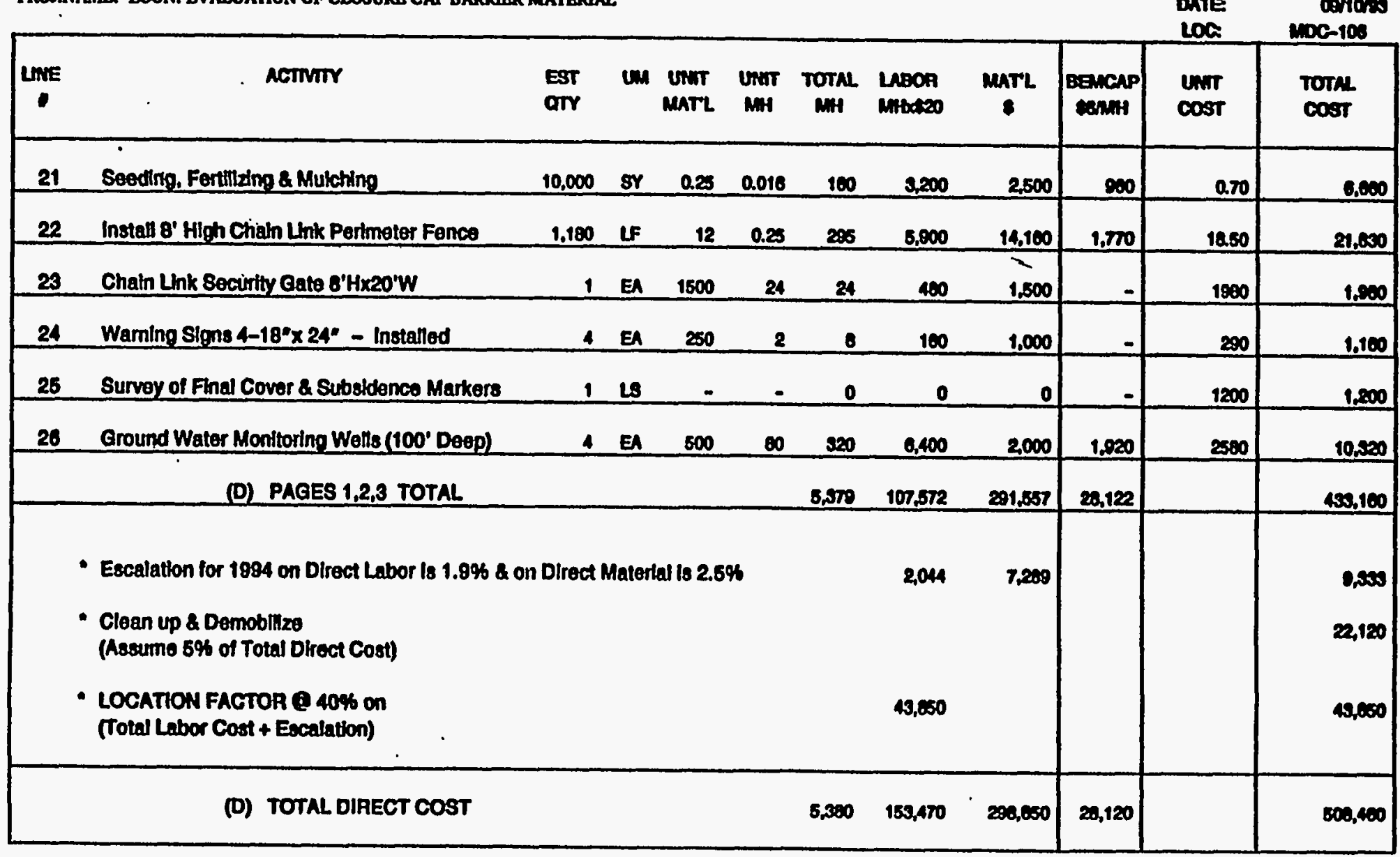

D-6. Cost Estimate for a Cover System Utilizing Reinforced Sprayed Asphalt (Cont'd) 
DETAIL ESTIMATE

\begin{tabular}{|c|c|}
\hline 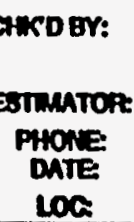 & 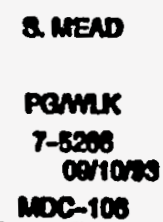 \\
\hline $\begin{array}{l}\text { Unirt } \\
\text { cost }\end{array}$ & $\begin{array}{l}\text { TotM } \\
\text { cosis }\end{array}$ \\
\hline & 16,2000 \\
\hline & 20,420 \\
\hline & 50,050 \\
\hline & 80.600 \\
\hline & 101,600 \\
\hline & 21,200 \\
\hline & 960,500 \\
\hline & 60,220 \\
\hline & ra,eos \\
\hline & $\cos 1010$ \\
\hline
\end{tabular}

(D) TOTAL INDIRECT COST

$1 \quad 18$

1 LS

Ls

LS

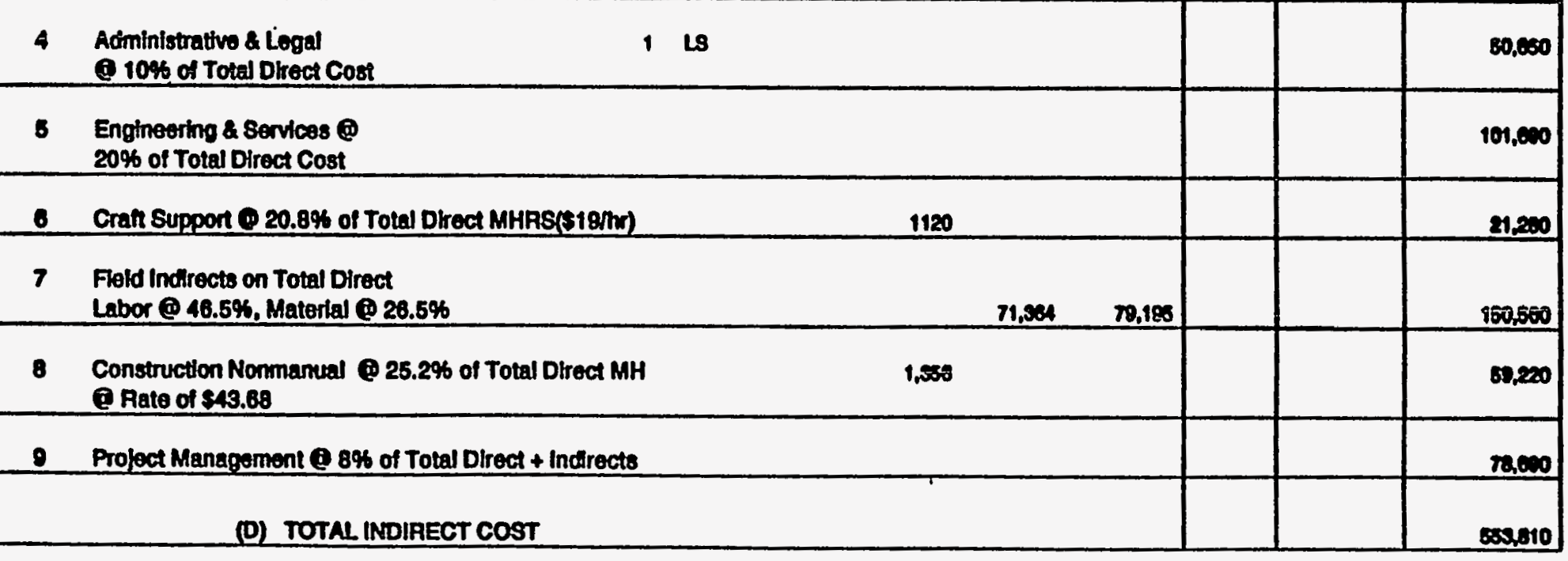

D-6. Cost Estimate for a Cover System Utilizing Reinforced Sprayed Asphalt (Cont'd) 
DETAIL ESTIMATE

\section{L001: 1-03}

ACTIVITY CODE: BSE260112

PROU.NAMB: ECON. EYALUATTON OF CLOSURB CAP BARRIER MATERUAL

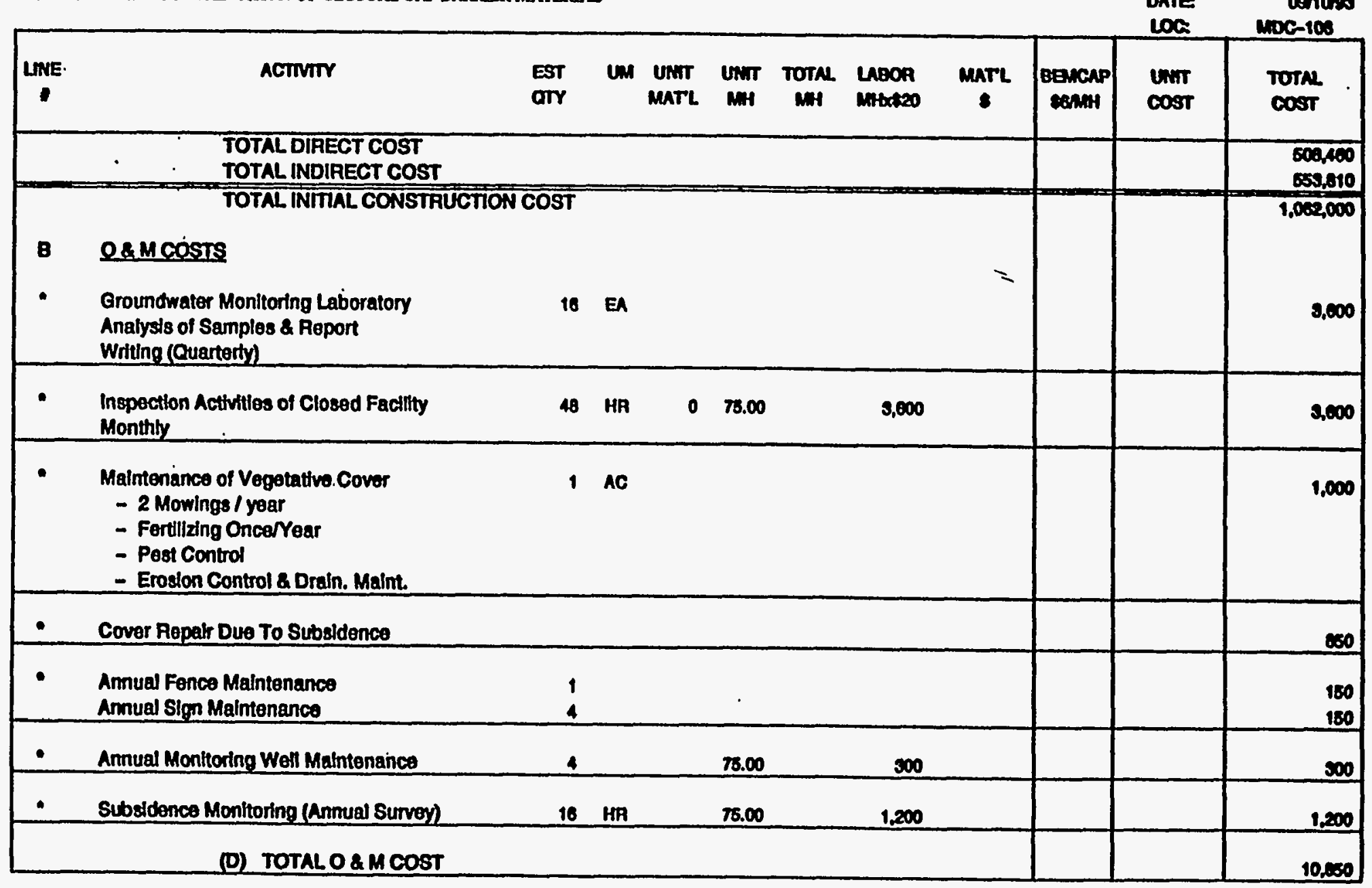

D-6. Cost Estimate for a Cover System Utilizing Reinforced Sprayed Asphalt (Cont'd) 
DETAIL ESTIMATE

Lod I: $7-03$

ACTIVITY CODE: ESEZSOL12

MOU.NAME: ECON. EVALUATTON OF CLOSURB CAP BARRRER MATEUANL.

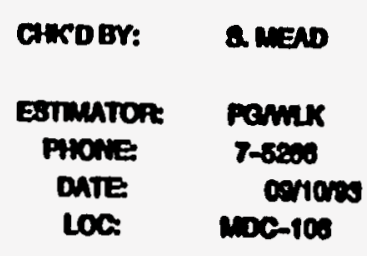

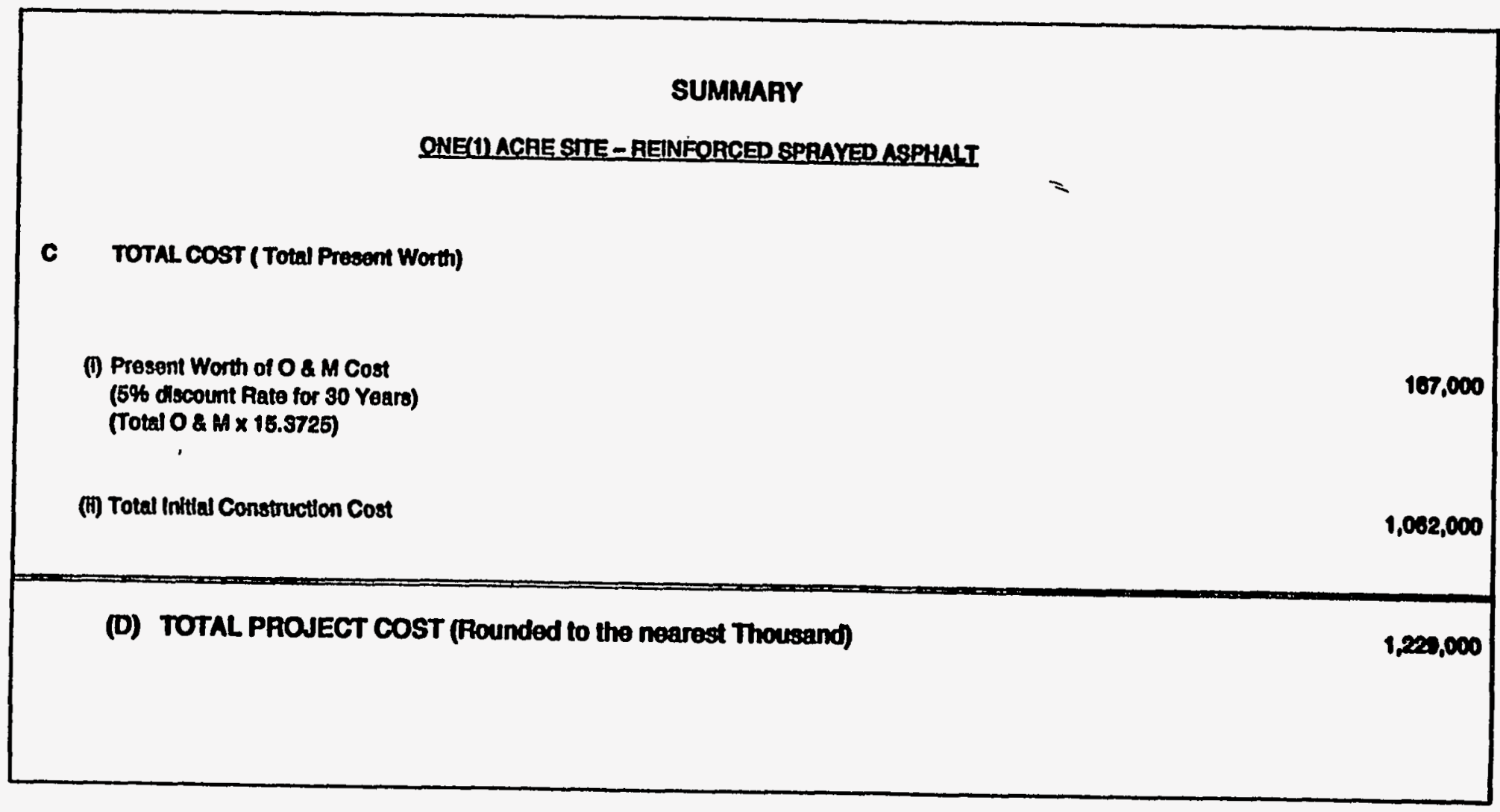

D-6. Cost Estimate for a Cover System Utilizing Reinforced Sprayed Asphalt (Cont'd) 
Loo : : 7-03

DETAIL ESTIMATE

ACTIVTY CODE: BSB200412

PROJ.NAME: BCON. BVALUATION OP CLOSURB CAP BARRIRR MATERIAL

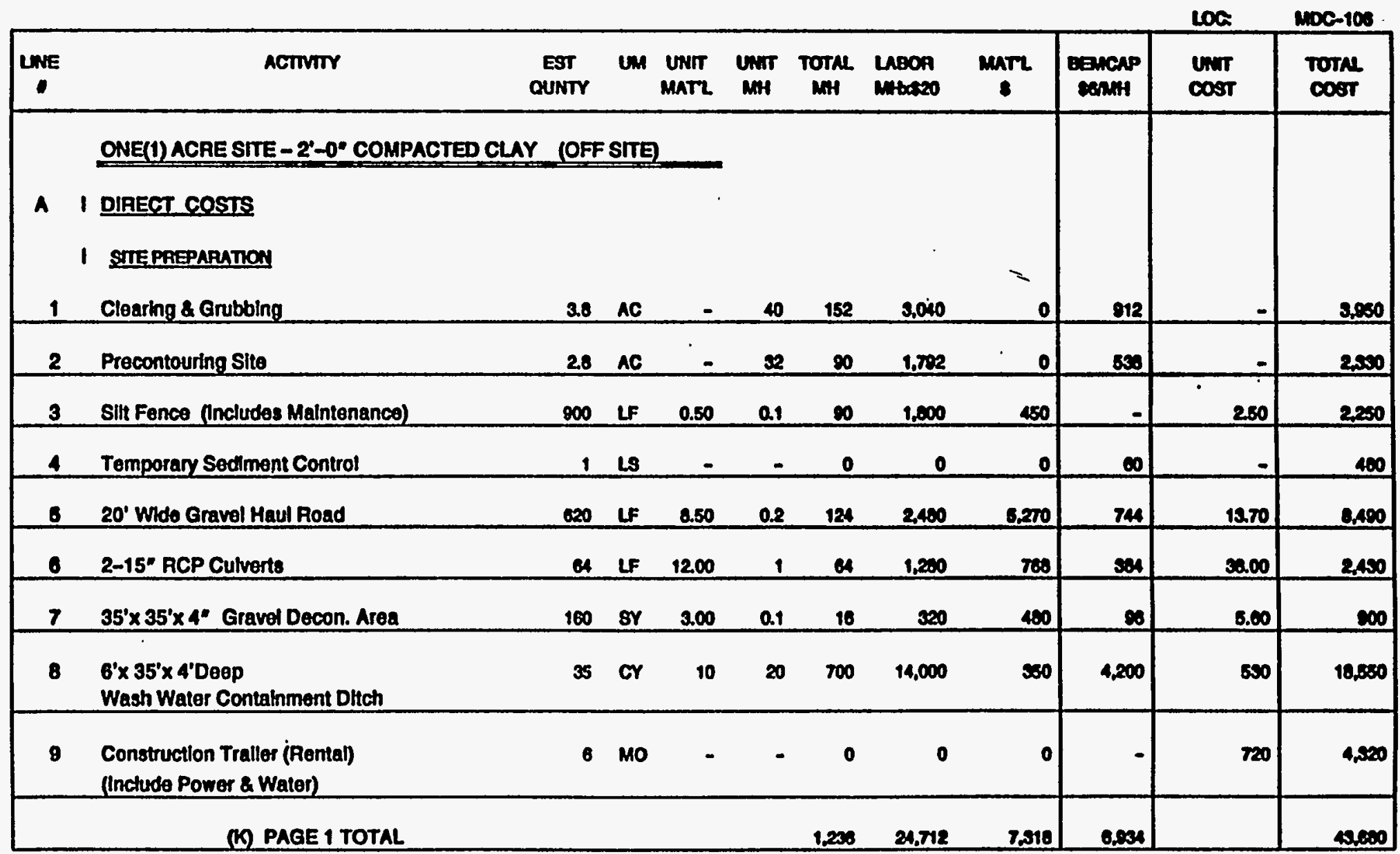

D-7. Cost Estimate for a Cover System Utilizing Compacted Kaolin (Offsite) Clay and a 30-mil FML 


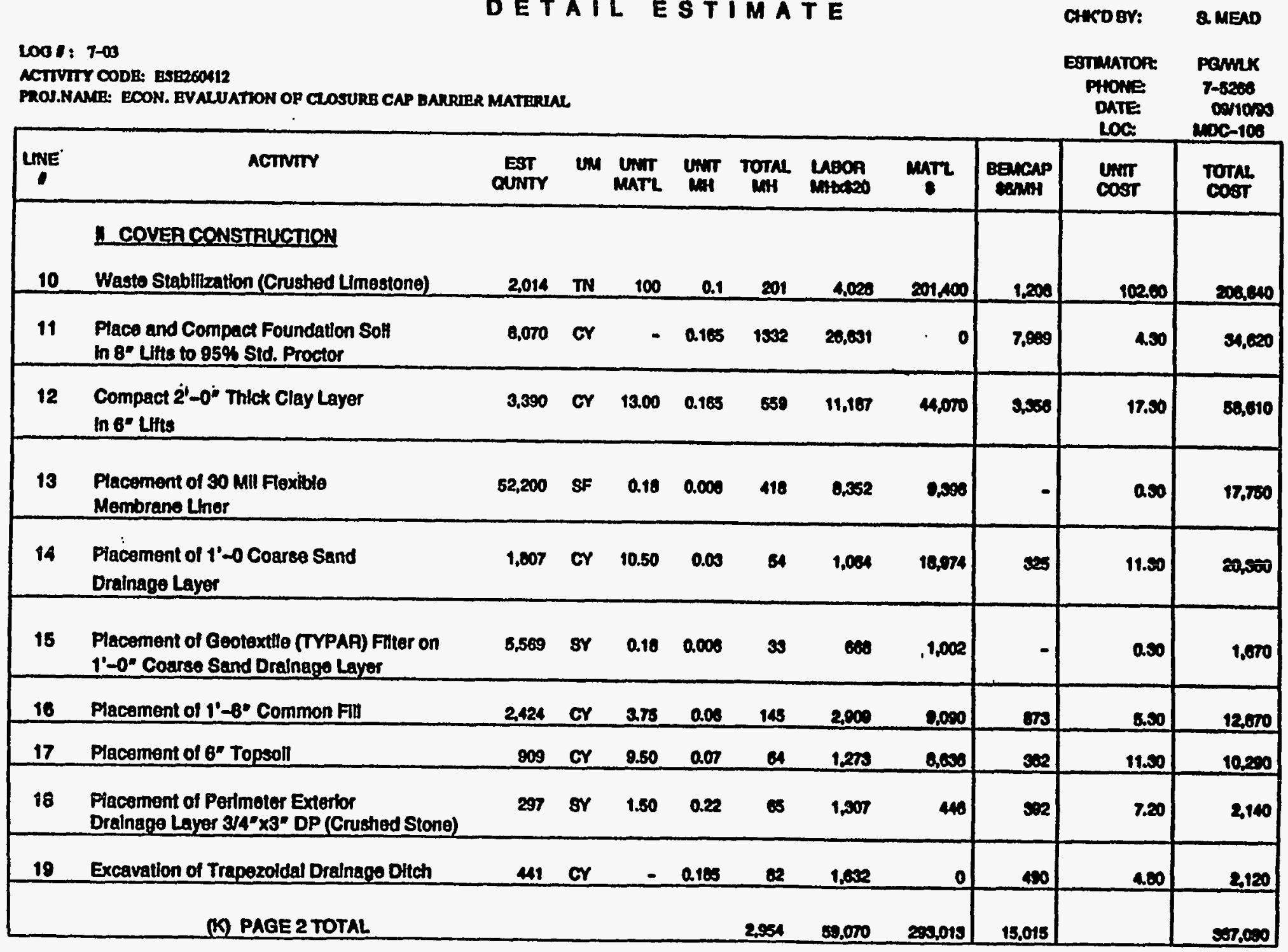

D-7. Cost Estimate for a Cover System Utilizing Compacted Kaolin (Offsite) Clay and a 30-mil FML (Cont'd) 


\section{DETAIL ESTIMATE}

Q.MEND

L001: 7-03

ACTIVTTY CODE: BSB2OOU12

PROJ.NAME: BCON. BVALUATION OR CLOSURB CAP BARRIER MATERIA

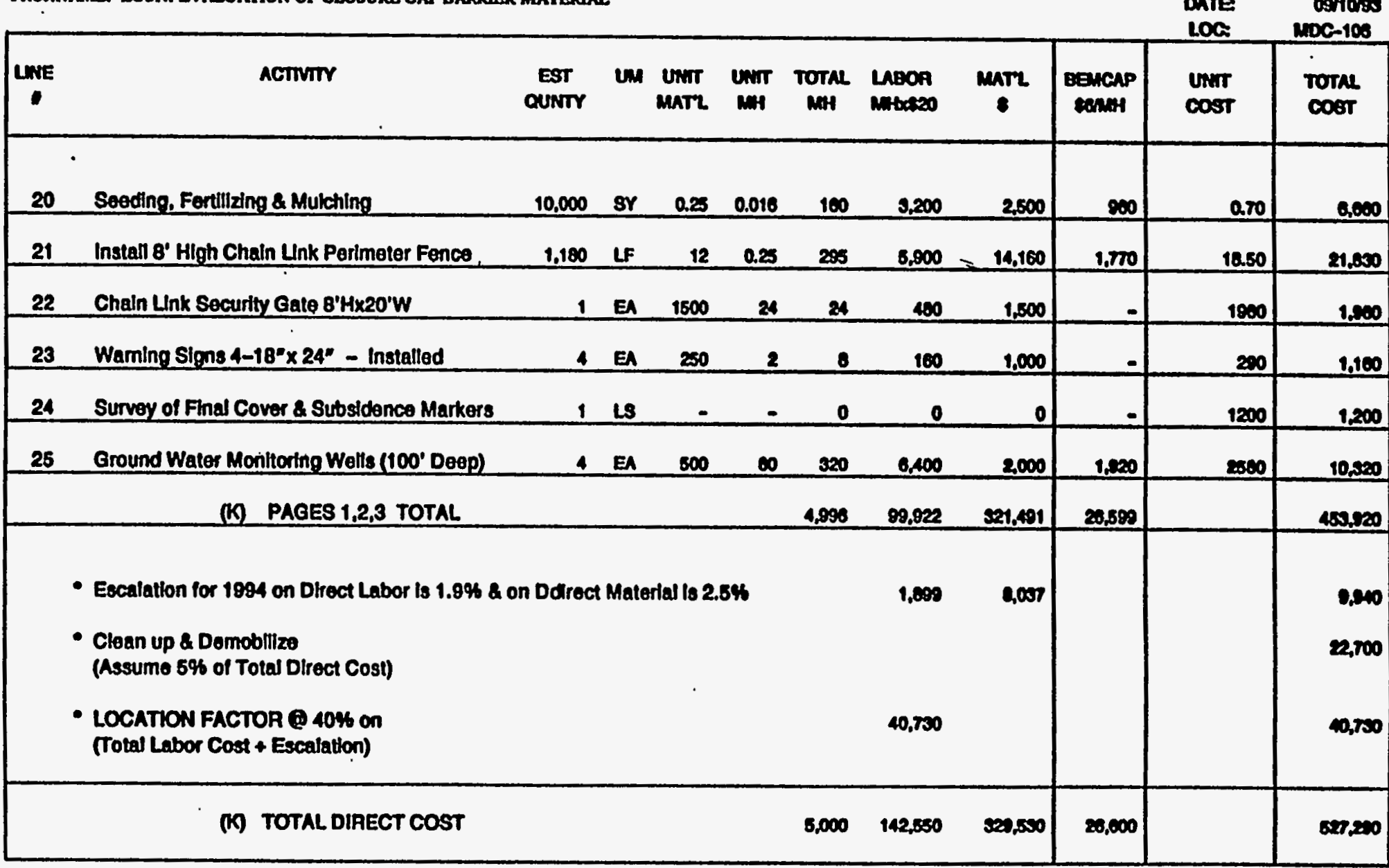

D-7. Cost Estimate for a Cover System Utilizing Compacted Kaolin (Offsite) Clay and a 30-mil FML (Cont'd) 


\section{DETAIL ESTIMATE}

Loos: $7-03$

ACTIVTTY CODE: BSEZCOA12

MOI.NAME: BCON. EVALUATION OP CLOSURB CAP DNRRIER MATERIAL

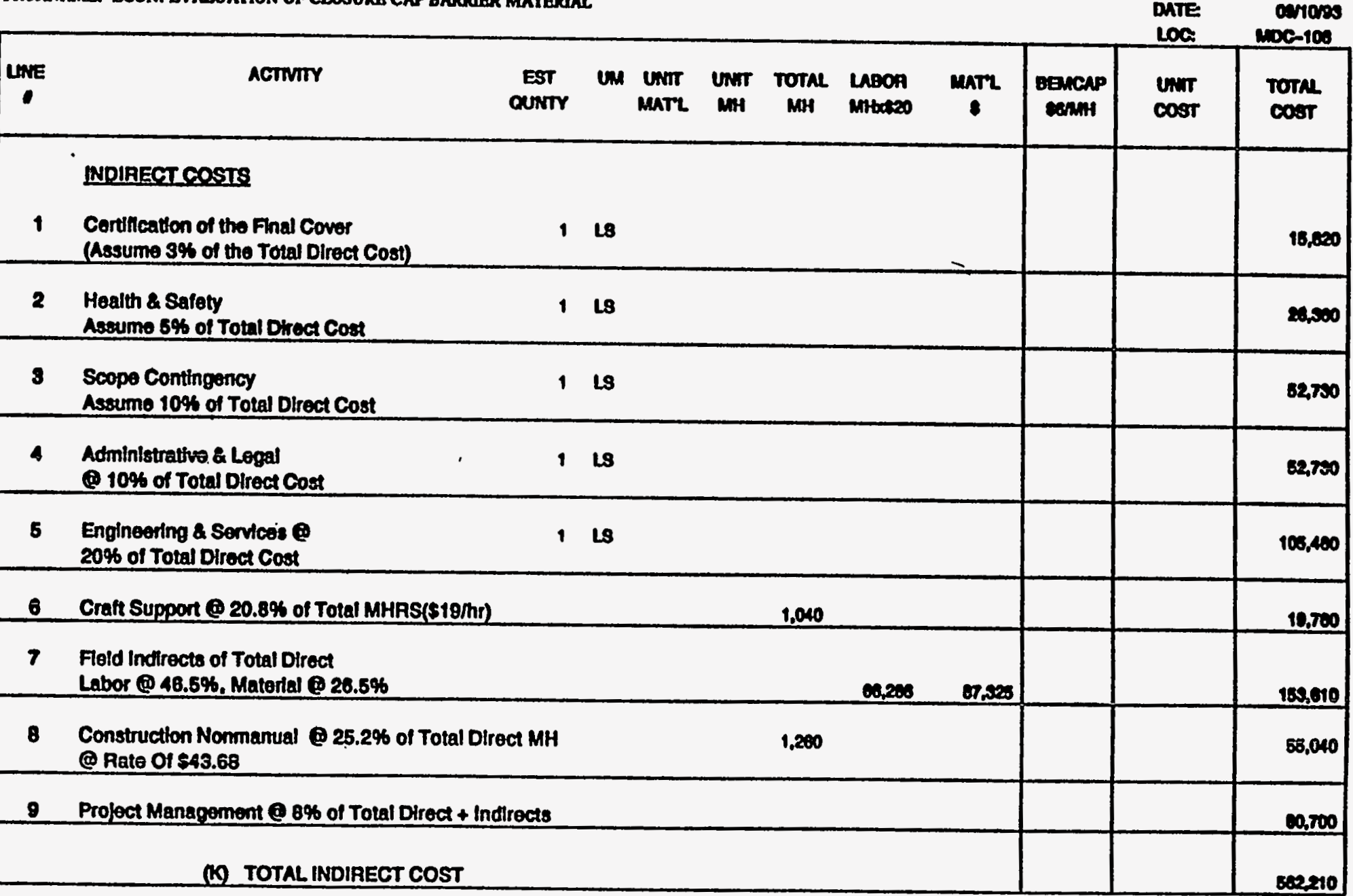

D-7. Cost Estimate for a Cover System Utilizing Compacted Kaolin (Offsite) Clay and a 30-mil FML (Cont'd) 
DETAIL ESTIMATE

Loa I: 7-03

ACTIVTTY CODE: RSE260412

PROY.NAMG: ECON. EVALUATHON OP CLOSURB CAP BARRIER MATERUAS

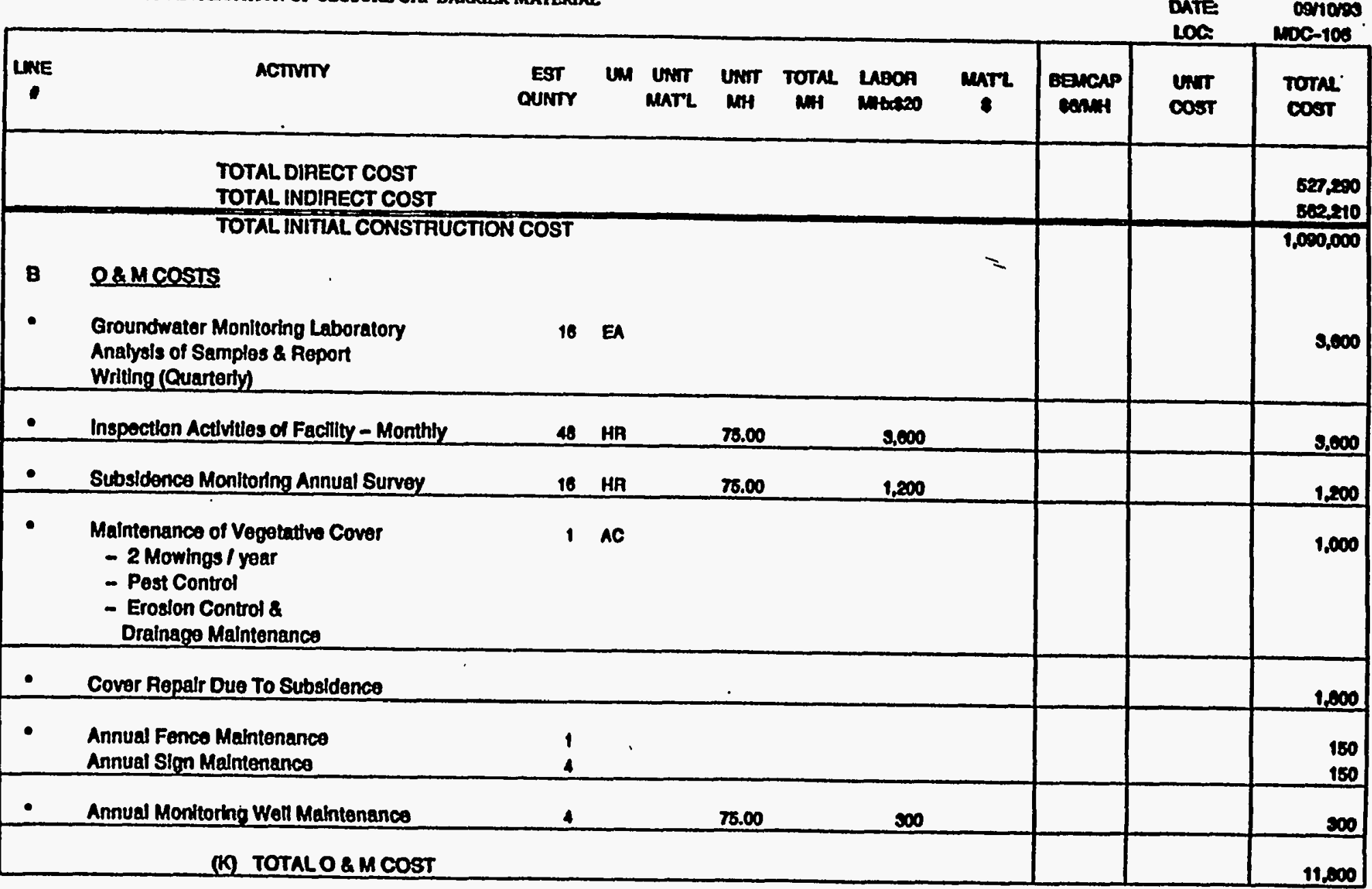

D-7. Cost Estimate for a Cover System Utilizing Compacted Kaolin (Offsite) Clay and a 30-mil FML (Cont'd) 


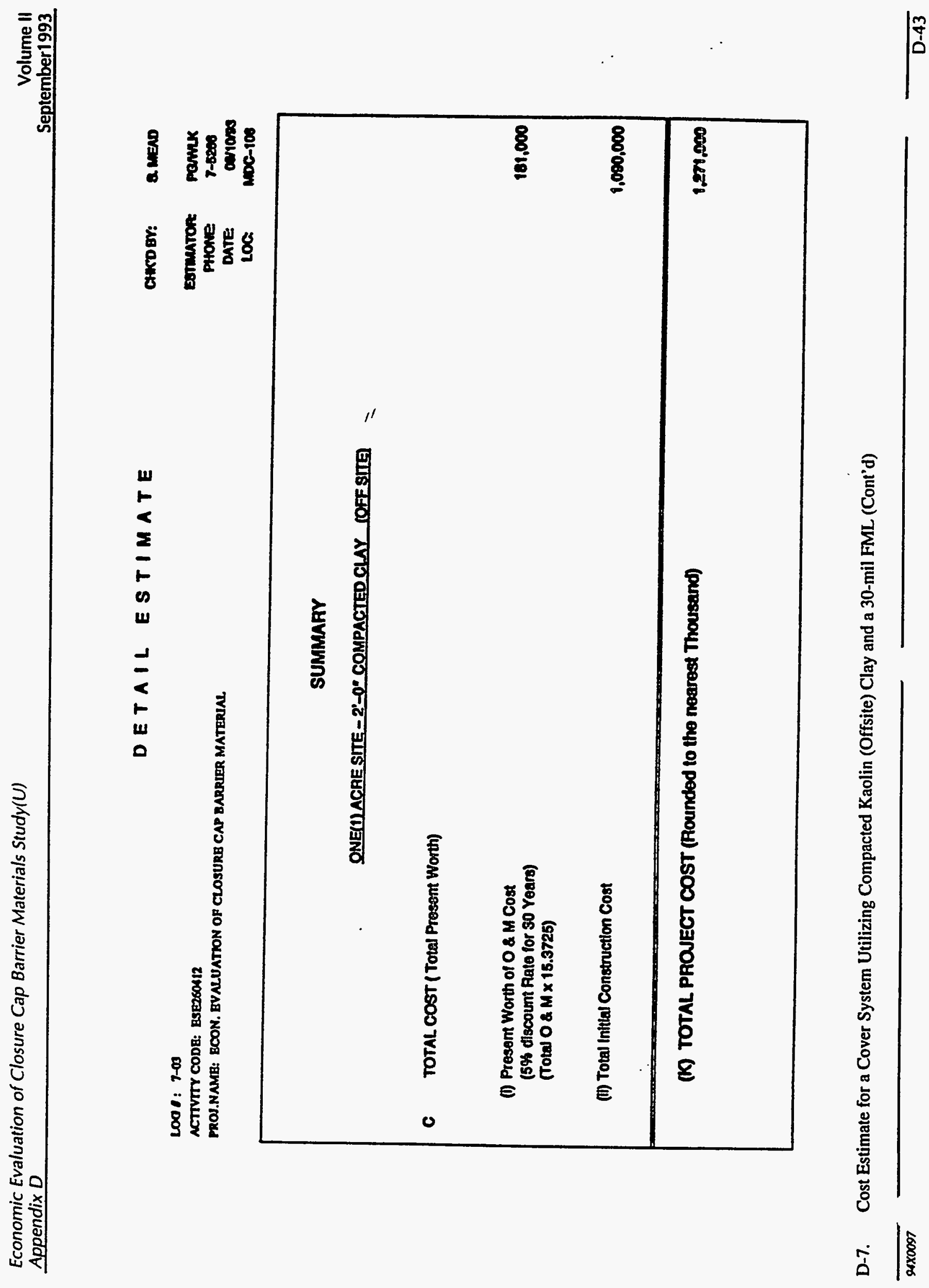




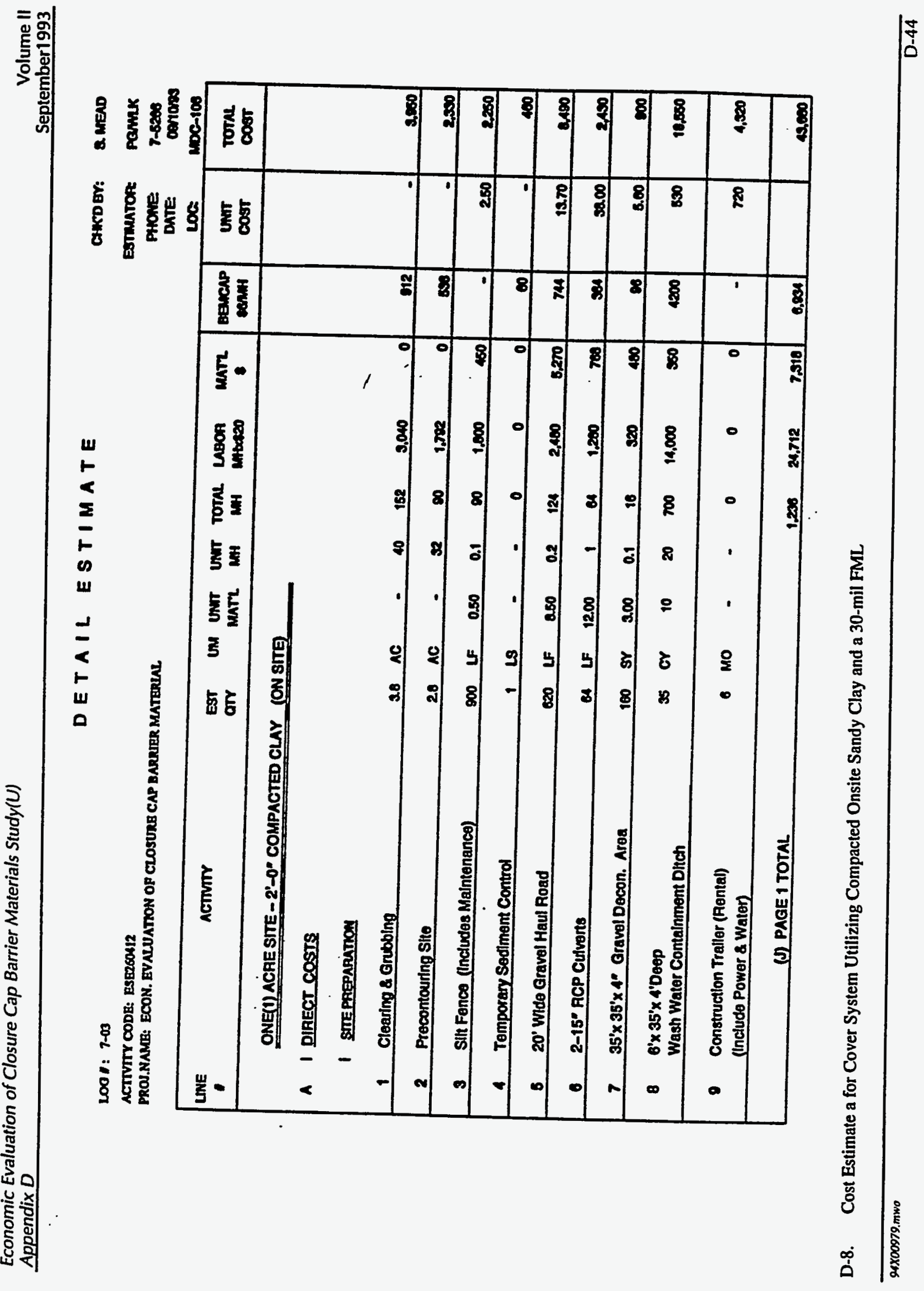


DETAIL ESTIMATE

2.MEND

1001: $7-03$

ACTIVTYY CODE: BsF260112

PROS.NAME: BCON. GVALUATHON OF CLOSURB CAP BARRIBR MATHRAAL

\begin{tabular}{|c|c|c|c|c|c|c|c|c|c|c|c|}
\hline UNE & activir & $\begin{array}{l}\text { EST } \\
\text { OTY }\end{array}$ & un & $\begin{array}{l}\text { UNTT } \\
\text { MATE }\end{array}$ & $\operatorname{Uim}_{\operatorname{mit}}$ & TOTML & $\begin{array}{l}\text { LABOA } \\
\text { Mttated }\end{array}$ & $\operatorname{manz}_{8}$ & Bexcap & $\lim _{\cos }$ & $\underset{\text { cost }}{\text { Tork }}$ \\
\hline & H COVER CONSTRUCTION & & & & & & & & & & \\
\hline 10 & Waste Stabilization (Crushed LImestone) & 2,014 & $\mathbf{T}$ & 100 & 0.1 & 201 & 4,020 & 201,000 & 1,200 & 10200 & 200800 \\
\hline 11 & $\begin{array}{l}\text { Piace and Compact Foundation Soll } \\
\text { in 8* Lits } 1095 \% \text { Sid. Proctor }\end{array}$ & 8,070 & or & - & 0.106 & 1932 & 20,001 & $=$ & 7,000 & 4.50 & 34,620 \\
\hline 12 & $\begin{array}{l}\text { Compact 2'-0" Thlck Clay Layer } \\
\text { In 6* Litte }\end{array}$ & 3,390 & or & - & 0.105 & 550 & 11,167 & o & 3,500 & 4.30 & 14.610 \\
\hline 13 & $\begin{array}{l}\text { Placement of } 30 \text { Mn Flexible } \\
\text { Membrane Uner }\end{array}$ & 52,200 & $8 F$ & 0.18 & 0.000 & 410 & 0,562 & 0,508 & - & 0.50 & 17.750 \\
\hline 14 & $\begin{array}{l}\text { Placement of 1'-0 Coarse Sand } \\
\text { Drainage Layor }\end{array}$ & 1,007 & or & $\mathbf{1 0 . 5 0}$ & 0.08 & sa & 1,004 & 10,074 & 5203 & 11.30 & 20,200 \\
\hline 13 & $\begin{array}{l}\text { Placement of Geotextlle (TYPAR) Filter on } \\
\text { 1-0" Coarse Sand Drainage Layer }\end{array}$ & 5,569 & $\mathbf{S Y}$ & 0.18 & 0.008 & $\boldsymbol{* 3}$ & $\infty$ & 1,002 & - & 0.50 & 1,070 \\
\hline 16 & Placoment of $1^{\prime}-6^{\prime \prime}$ Common Fill & 2,424 & or & 5.75 & 0.08 & 145 & 2,000 & 0.000 & $m$ & 8.50 & 12,070 \\
\hline 17 & Placement of $6^{\prime \prime}$ Topsoil & $\infty 08$ & or & 8.50 & 0.07 & 4 & 1.270 & 0.000 & 302 & 11.50 & 10200 \\
\hline 18 & $\begin{array}{l}\text { Placement of Permeter Exterior } \\
\text { Drainage Layer } 3 / 4^{\prime \prime} \times 3^{\circ} \text { DP (Crushod Stone) }\end{array}$ & 297 & sy & 1.50 & 0.22 & $\infty$ & 1,307 & Mo & 392 & 7.20 & 2140 \\
\hline 19 & Excavation of Trapezoidal Drainage Ditch & 41 & Cr & $=$ & 0.165 & 62 & 1.032 & o & 400 & 4.00 & 2,120 \\
\hline & (J) PAGE 2 TOTAL & & & & & 2.854 & 59.000 & 200.40 & 15.015 & & ses,oen \\
\hline
\end{tabular}

D-8. Cost Estimate a for Cover System Utilizing Compacted Onsite Sandy Clay and a 30-mil FML (Cont'd) 
DETAIL ESTIMATE

CHCD BV: S. MENO

L001: 7-03

ACTIYTY CODE: ESB20012

PROS.NANE: ECON. BVALUATION OF CLOSURB CNP BARRIER MATERUAL

ESTMATOR: Pamik

PHONe

$7-5206$

DATE Grions

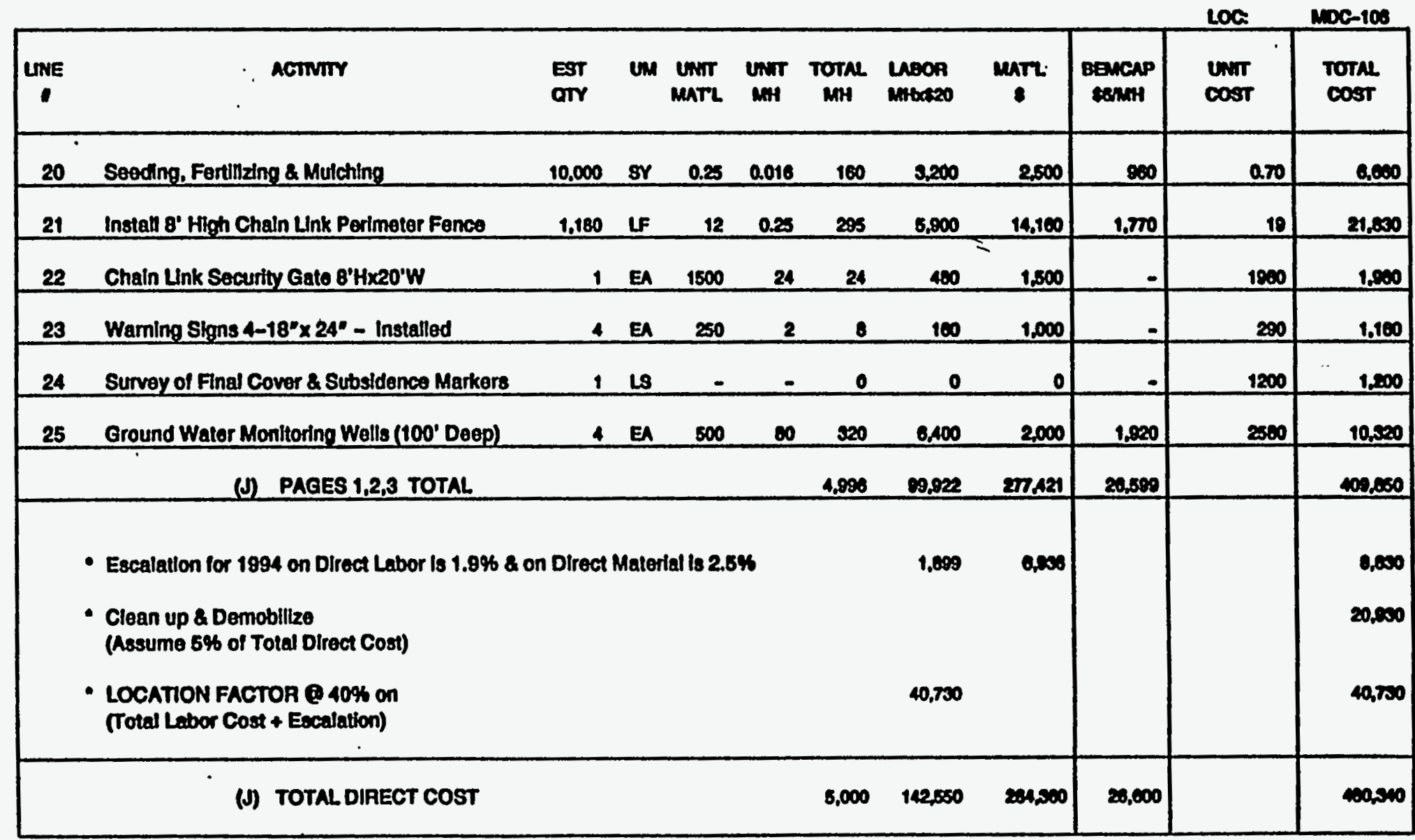

D-8. Cost Estimate a for Cover System Utilizing Compacted Onsite Sandy Clay and a 30-mil FML (Cont'd) 
DETAIL ESTIMATE CAKDOY: RMEAD

Loo 1: 7-03

ACTIVITY CODE: ESE2CON12

MOU.NAMR: BCON. BVALUATTON OF CLOSURE CAP DARRIER MATERIAL.

Esminaton: Pamk

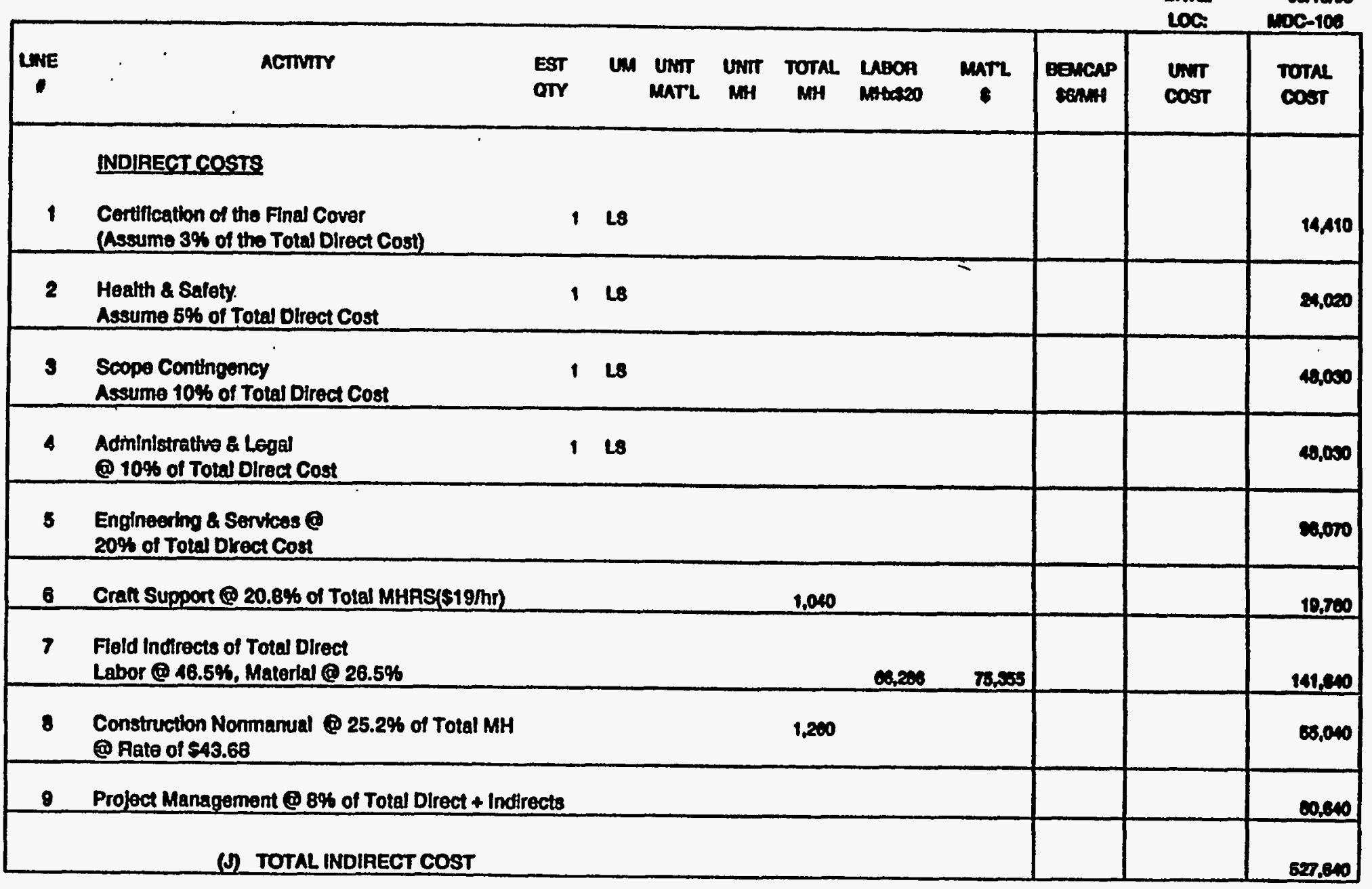

D-8. Cost Estimate a for Cover System Utilizing Compacted Onsite Sandy Clay and a 30-mil FML (Cont'd) 


\section{DETAIL ESTIMATE}

LoOI: 7-03

ACTIVITY CODE: ESEQOOH12

PROJ.NAMGB: BCON. BVALUATION OP CLOSURB CAP BARRIRR MATERINL

CHCDEV: QMEND

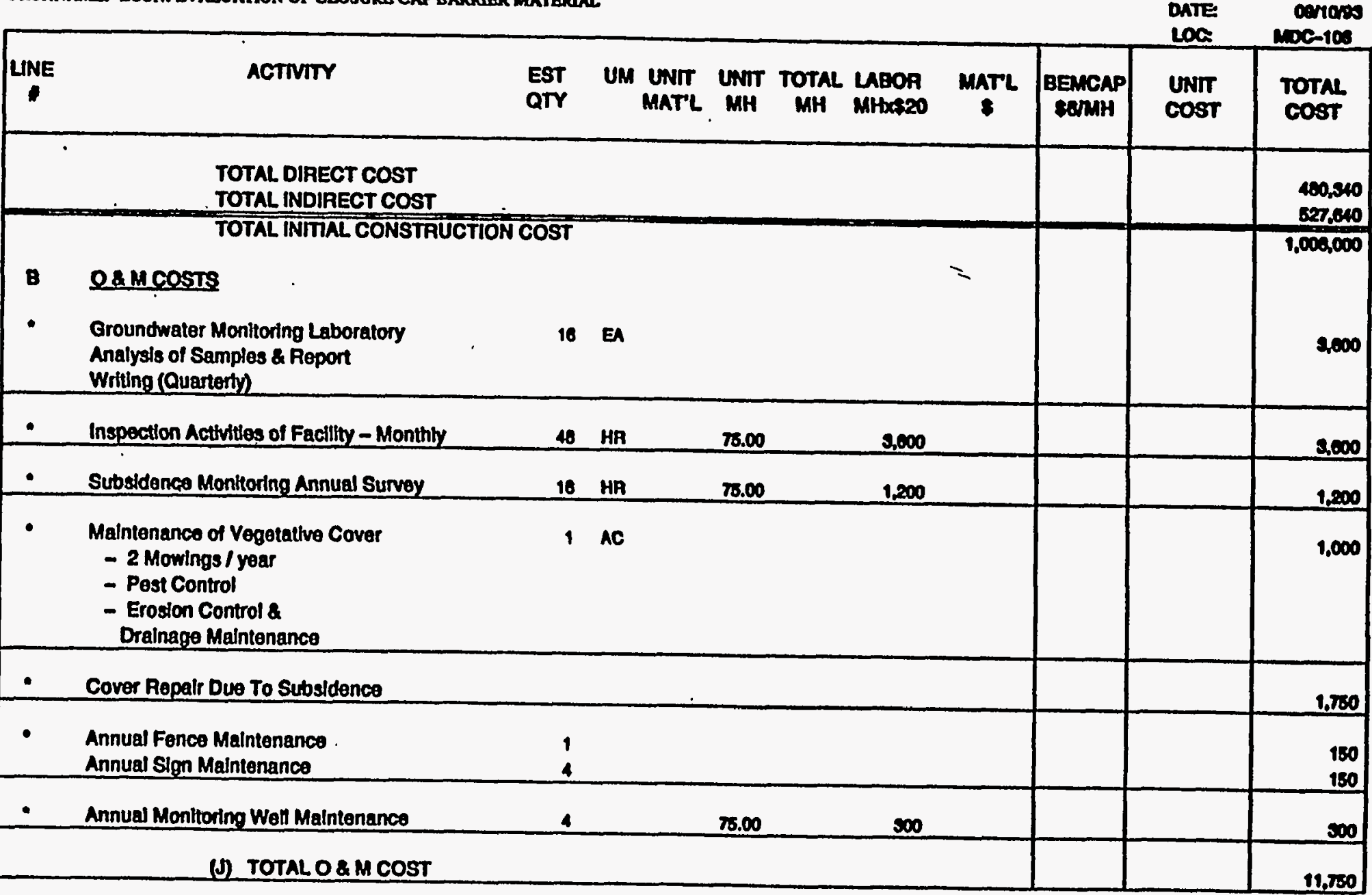

D-8. Cost Estimate a for Cover System Utilizing Compacted Onsite Sandy Clay and a 30-mil FML (Cont'd) 
DETAILESTIMATE

L001: 7-03

ACTIVITY CODB: ESE2CON12

PROJ.NAME: BCON. BVALUATION OP CLOSURB CAP DARRIER MATERIAL

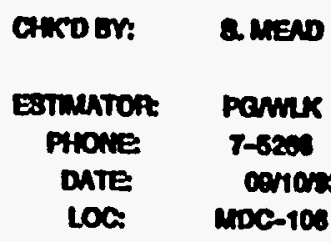

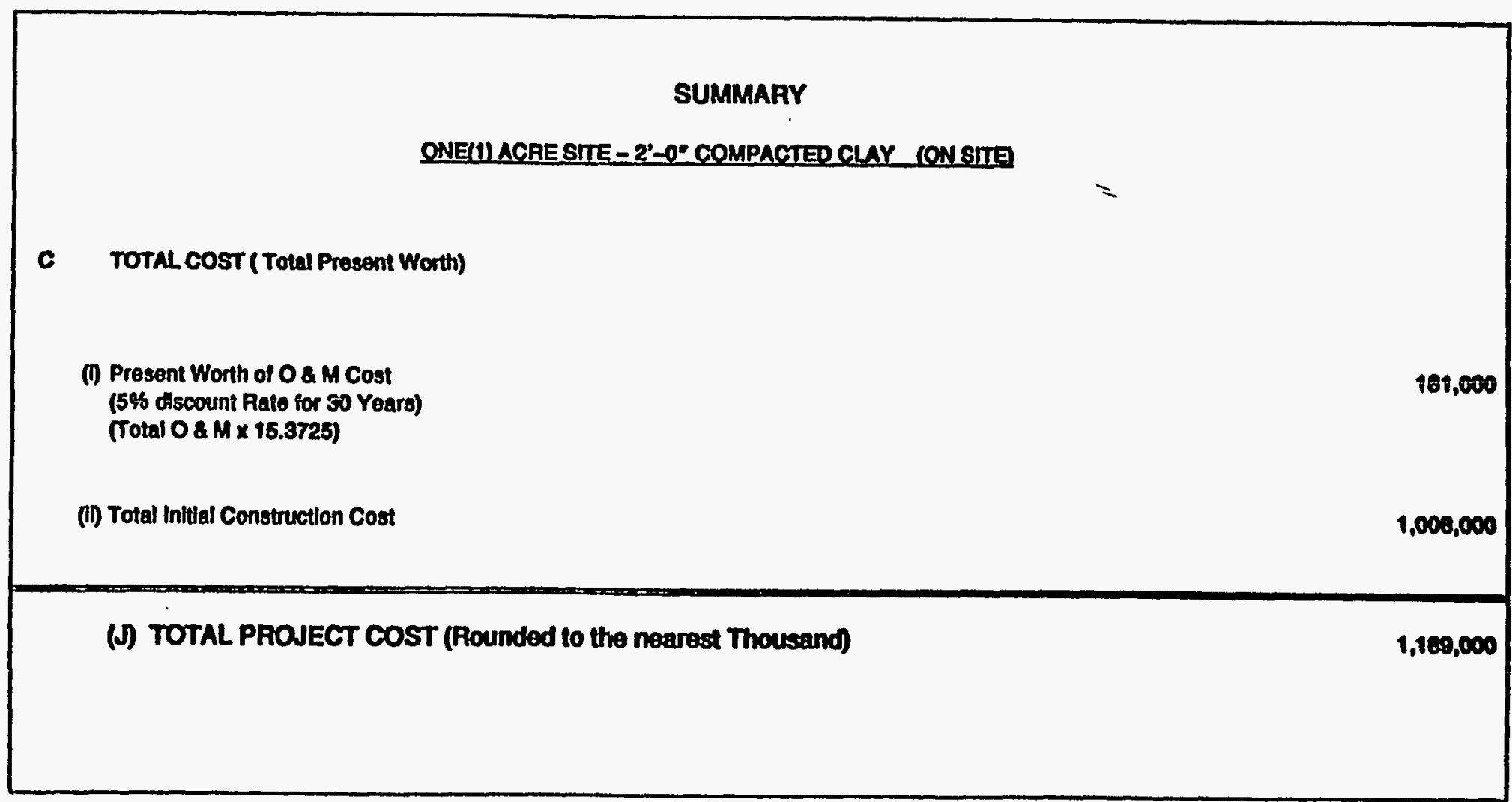

D-8. Cost Estimate a for Cover System Utilizing Compacted Onsite Sandy Clay and a 30-mil FML (Cont'd) 
DETAIL ESTIMATE CHKOBY: S.MEAD

100 : : 7-03

ACTIVTTY CODE: ESE260412

PROJ.NAME: BCON. EVALUATION OP CLOSURB CAP BARRIER MATERIAL

ESTMATOR: PaMnK

PHONE T-5200

\begin{tabular}{|c|c|c|c|c|c|c|c|c|c|c|}
\hline LNEE & ACTIVTY & $\begin{array}{l}\text { EST Un } \\
\text { OTY }\end{array}$ & $\begin{array}{l}\text { UNIT } \\
\text { MATL }\end{array}$ & $\begin{array}{l}\text { UNT } \\
\text { MH }\end{array}$ & $\underset{\text { TOTAL }}{\text { TOT }}$ & $\begin{array}{l}\text { LAOOR } \\
\text { MHXXPOD }\end{array}$ & mart & $\begin{array}{l}\text { Bencap } \\
\text { senmi }\end{array}$ & $\begin{array}{l}\text { Umit } \\
\text { cost }\end{array}$ & $\begin{array}{l}\text { TOTML } \\
\text { Cosst }\end{array}$ \\
\hline $\begin{array}{l}A \\
1 \\
\end{array}$ & $\begin{array}{l}\text { ONE(1) ACRE STIE - SOIL CEMEA } \\
\text { DIAECT COSTS } \\
\text { SIIE PREPARAMON } \\
\text { Clearing \& Grubbing }\end{array}$ & $3.8 \wedge \mathrm{C}$ & - & 40 & 152 & 3,040 & 0 & 912 & - & 2050 \\
\hline 2 & Procontouring Site & $2.8 \wedge C$ & - & 32 & 80 & 1,792 & 0 & 800 & - & 2,000 \\
\hline 3 & Sit Fence (includes Maintenance) & $900 \mathrm{LF}$ & 0.50 & 0.1 & $\boldsymbol{8 0}^{\circ}$ & 1,000 & 150 & - & 280 & 2,250 \\
\hline 4 & Temporary Sediment Control & $1 \mathrm{LS}$ & - & - & 0 & $\mathbf{0}$ & 0 & $\infty$ & - & 400 \\
\hline 6 & 20' Wide Graves Haul Road & $620 \mathrm{LF}$ & 8.50 & 0.2 & 124 & 2,400 & 8,270 & 794 & 13.70 & 0.480 \\
\hline 6 & 2-15" RCP Culverts & 64 LF & 12.00 & 1 & 64 & 1,280 & 700 & 304 & 30.00 & 2400 \\
\hline 7 & $35^{\prime} \times 35^{\prime} \times 4^{\prime \prime}$ Gravel Docon. Area & $160 \mathrm{SY}$ & 3.00 & 0.1 & 16 & 320 & 480 & 98 & 8.60 & $\infty$ \\
\hline 8 & $\begin{array}{l}6^{\prime} \times 35^{\circ} \times 4^{\prime} \text { Deep } \\
\text { Wash Water Containment Ditch }\end{array}$ & $35 \mathrm{Cr}$ & 10 & 20 & 700 & 14,000 & 350 & 4200 & 530 & 10,650 \\
\hline 8 & $\begin{array}{l}\text { Construction Traller (Renta) } \\
\text { (Include Power \& Water) }\end{array}$ & ( $\mathrm{MO}$ & - & - & o & 0 & 0 & - & 220 & 4,520 \\
\hline & (G) PAGE 1 TOTAL & & & & 1,230 & 24,712 & 7,518 & 0.924 & & 4,000 \\
\hline
\end{tabular}

D-9. Cost Estimate for a Cover System Utilizing Soil Cement and 30-mil FML 
DETAIL ESTIMATE

2001: $7-03$

ACTIVITY CODE: ESE260412

PROS.NAMR: ECON. BVALUATION OP CLOSURE CAP BARRIER MATERIAL

\begin{tabular}{|c|c|c|c|c|c|c|c|c|c|c|c|}
\hline \multicolumn{10}{|c|}{ 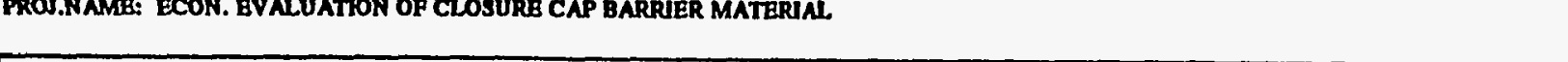 } & \multirow{2}{*}{$\begin{array}{l}\text { DAvE } \\
\text { LOG } \\
\lim \\
\cos \end{array}$} & \multirow{2}{*}{ 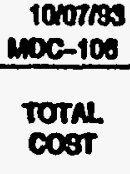 } \\
\hline LNE & ACTIVIT & $\begin{array}{l}\text { EST } \\
\text { aTr }\end{array}$ & un & $\begin{array}{l}\text { UNT } \\
\text { MATL. }\end{array}$ & UNT & ${ }_{M H}^{\text {TOTN }}$ & $\begin{array}{l}\text { LABOR } \\
\text { MHodeO }\end{array}$ & $\underset{8}{\operatorname{marL}}$ & $\underset{\operatorname{mencup}}{\operatorname{menc}}$ & & \\
\hline & D_COVER CONSTRIUCTION & & & & & & & & & & \\
\hline 10 & Waste Stabillzation (Crushed Uimestone) & 2.014 & $\mathbf{T N}$ & 100 & 0.1 & 201 & 4.020 & 201,400 & 1,200 & 10200 & 200,040 \\
\hline 11 & $\begin{array}{l}\text { Place and Compact Foundation Soll } \\
\text { in 8" Lifts to 95\% Std. Proctor }\end{array}$ & 8,070 & & - & 0.165 & 1332 & 26,631 & of & 7,009 & 4.50 & $\mathbf{3 4 , 6 2 0}$ \\
\hline 12 & $\begin{array}{l}\text { Soll-Cement mlxture transported from central } \\
\text { mixing plant by end-dump trucks and placed } \\
\text { in } 6-8 \text { " lifts by spreaders. Assume Fre Sand } \\
\text { Soll with } 321 \text { of coment/CY. Compact the soll- } \\
\text { coment unliormlly to } 88 \% \text { max. density. }\end{array}$ & 5,143 & & 18 & 0.165 & 849 & 16,972 & 92,574 & 3,092 & 22.30 & 114,640 \\
\hline 13 & $\begin{array}{l}\text { Curing soil-cement. Assume liquld Asphalt } \\
\text { appilled 0.4gallsy for ist coat } 20.2 \text { for } 2 \text { nd. }\end{array}$ & $\begin{array}{l}5,491 \\
5,491 \\
\end{array}$ & & $\begin{array}{l}0.33 \\
0.17 \\
\end{array}$ & $\begin{array}{l}0.05 \\
0.03\end{array}$ & $\begin{array}{l}275 \\
105 \\
\end{array}$ & $\begin{array}{l}5,491 \\
0,295 \\
\end{array}$ & $\begin{array}{r}1,012 \\
\text { 235 } \\
\end{array}$ & - & $\begin{array}{l}1.50 \\
0.00 \\
\end{array}$ & $\begin{array}{l}7,500 \\
4,200 \\
\end{array}$ \\
\hline 14 & $\begin{array}{l}\text { Placement of } 30 \text { ABII Flextble } \\
\text { Membrane Liner }\end{array}$ & 52,200 & & 0.16 & 0.000 & 410 & 0,352 & 9,390 & - & 0.30 & 17,750 \\
\hline 15 & $\begin{array}{l}\text { Placement of } 1^{\prime}-0 \text { Coarse Sand } \\
\text { Dralnage Layer }\end{array}$ & 1,807 & or & 10.50 & 0.03 & 54 & 1,004 & 18,974 & 325 & 11.30 & 20,000 \\
\hline 16 & $\begin{array}{l}\text { Placement of Cootextlie (TYPAR) Fiter on } \\
\text { 1'-0" Coarse Sand Drainage Layer }\end{array}$ & 5,569 & & 0.18 & 0.006 & $\mathbf{3 0}$ & 060 & 1,002 & - & 0.50 & 8,6000 \\
\hline 17 & Placement of 1'-6" Common FIII & 2.424 & or & 3.75 & 0.06 & 145 & 2,809 & 9,090 & 873 & 8.50 & 12,070 \\
\hline 18 & Placement of 6" Topsoll & 809 & or & 9.50 & 0.07 & 64 & 1,273 & 0.030 & 302 & 11.50 & 10,290 \\
\hline & (G) PAGE 2 TOTAL & & & & & 5,535 & 70,702 & s43,017 & 15,000 & & 450,590 \\
\hline
\end{tabular}

D-9. Cost Estimate for a Cover System Utilizing Soil Cement and 30-mil FML (Cont'd) 
DETAIL B S T I A A T

Lo0 I: 7-03

ACTIVITY CODE: ESE260112

PROI.NAME: ECON. GVALUATION OF CLOSURB CAP BARRIER MATERIAL

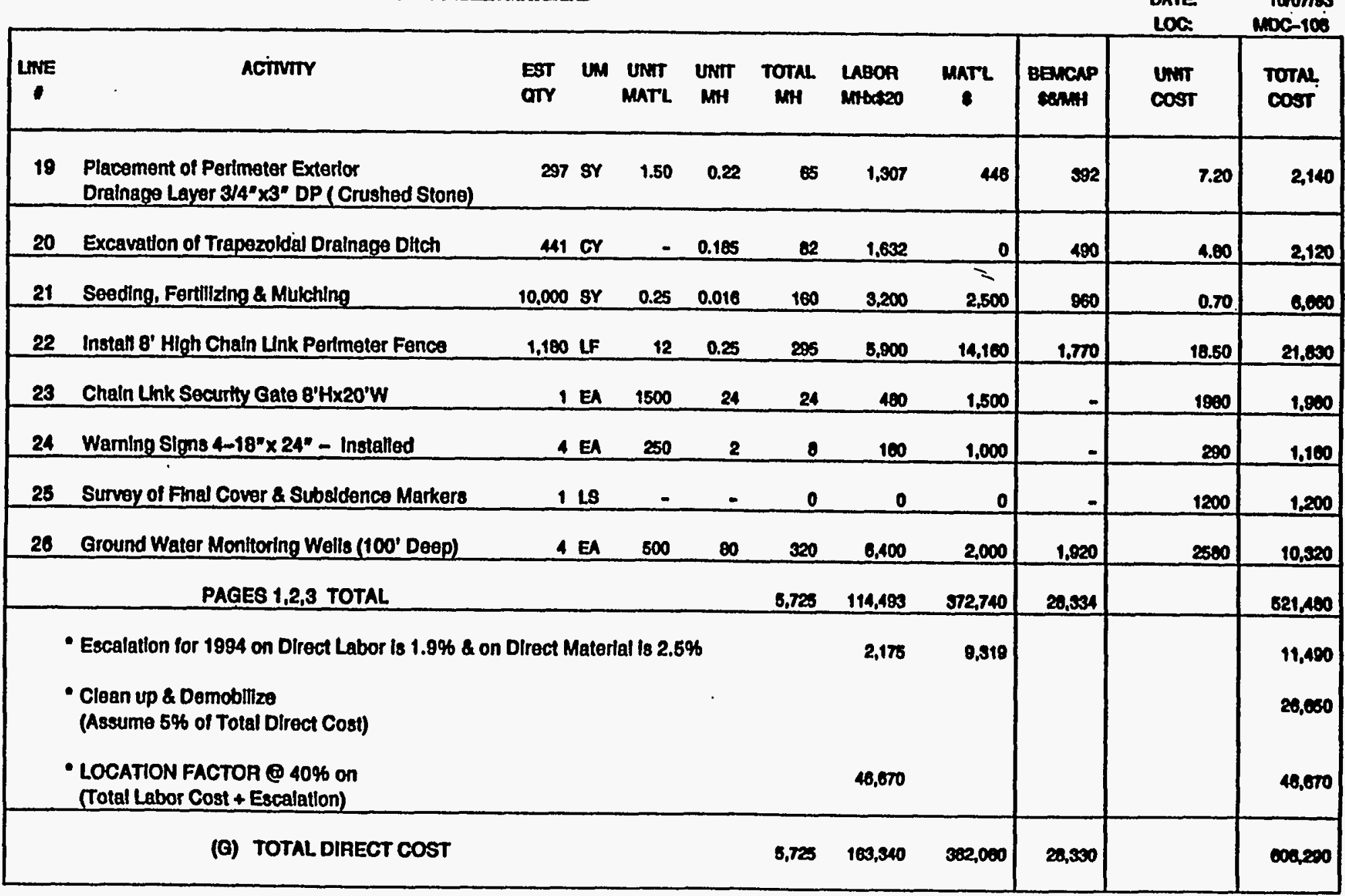

D-9. Cost Estimate for a Cover System Utilizing Soil Cement and 30-mil FML (Cont'd) 


$$
\text { Loof: 7-03 }
$$

PROJ.NAME: ECON. EVALUATION OP CLOSURB CAP BARRIRR MATERUAL

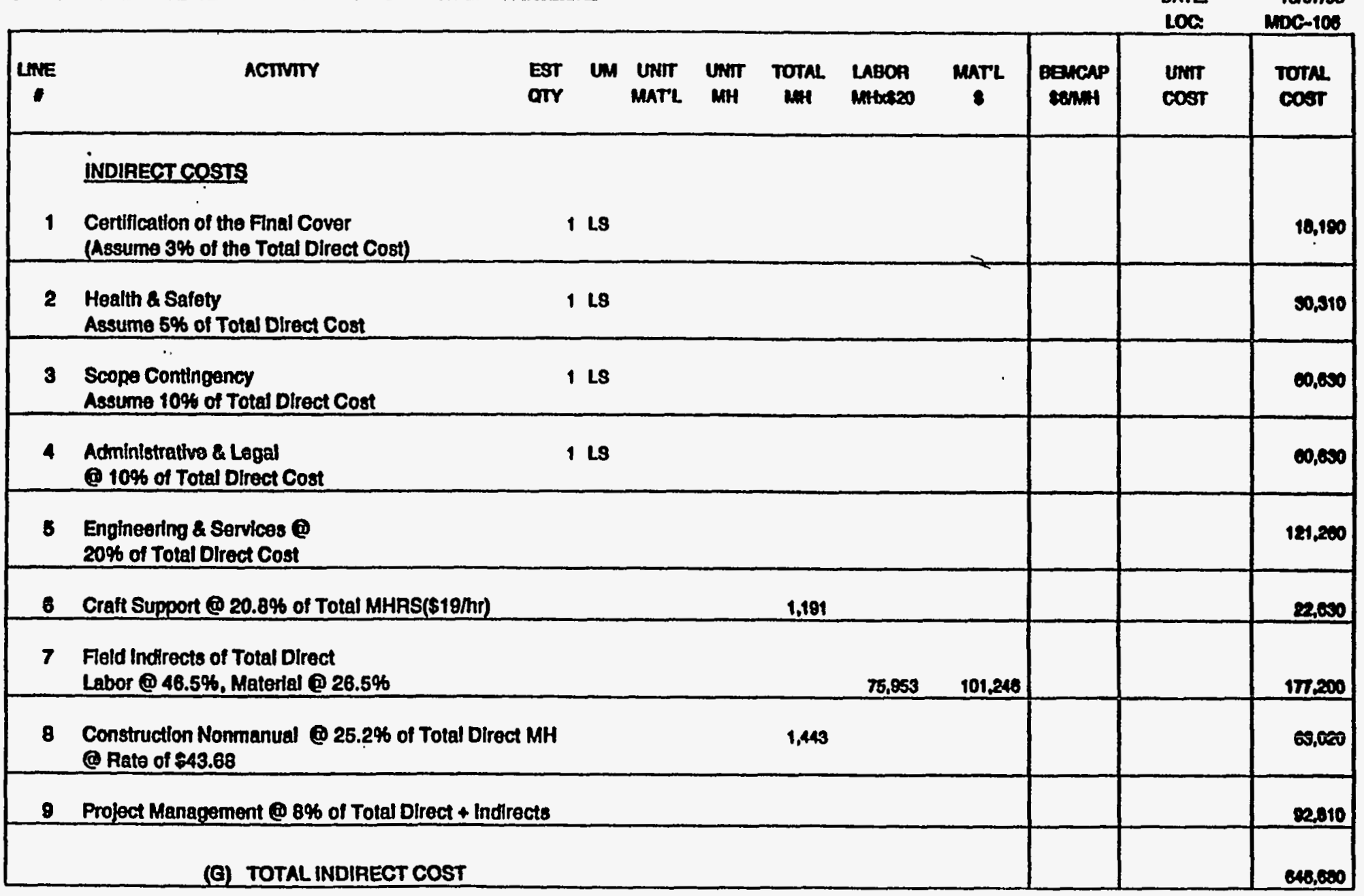

D-9. Cost Estimate for a Cover System Utilizing Soil Cement and 30-mil FML (Cont'd) 
D B T I L BSTIMAT B

Kro 8Y:

S. MEAD

LOO I: 7-03

ACTIVTYY CODE: ESE260112

PROJ.NAMB: ECON. BVALUATTON OF CLOSURB CAP BARRIER MATERIAL

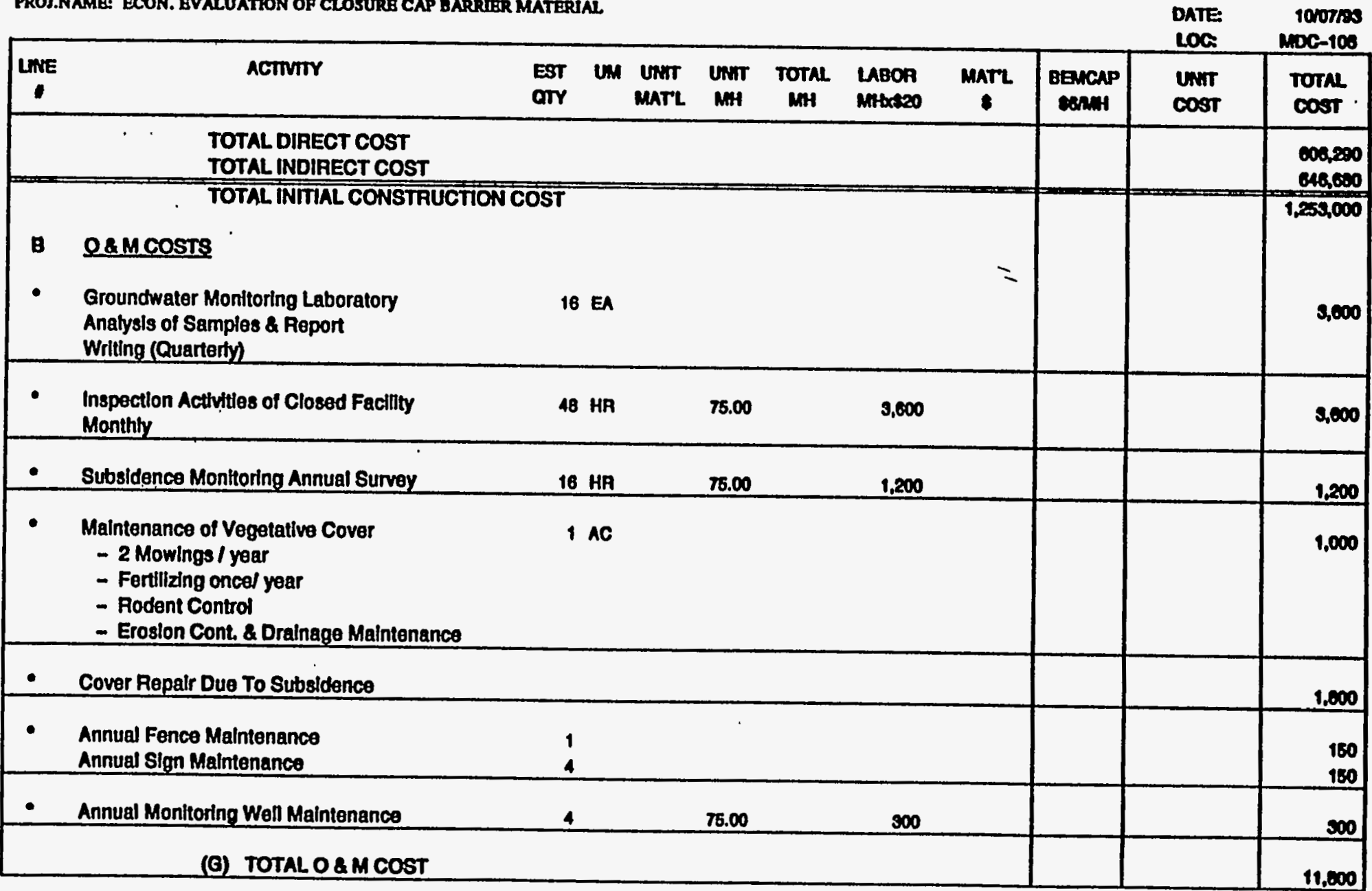

D-9. Cost Estimate for a Cover System Utilizing Soil Cement and 30-mil FML (Cont'd) 
Loo : : 7-03

ACTIVITY CODB: BSBZCOA12

LOC.

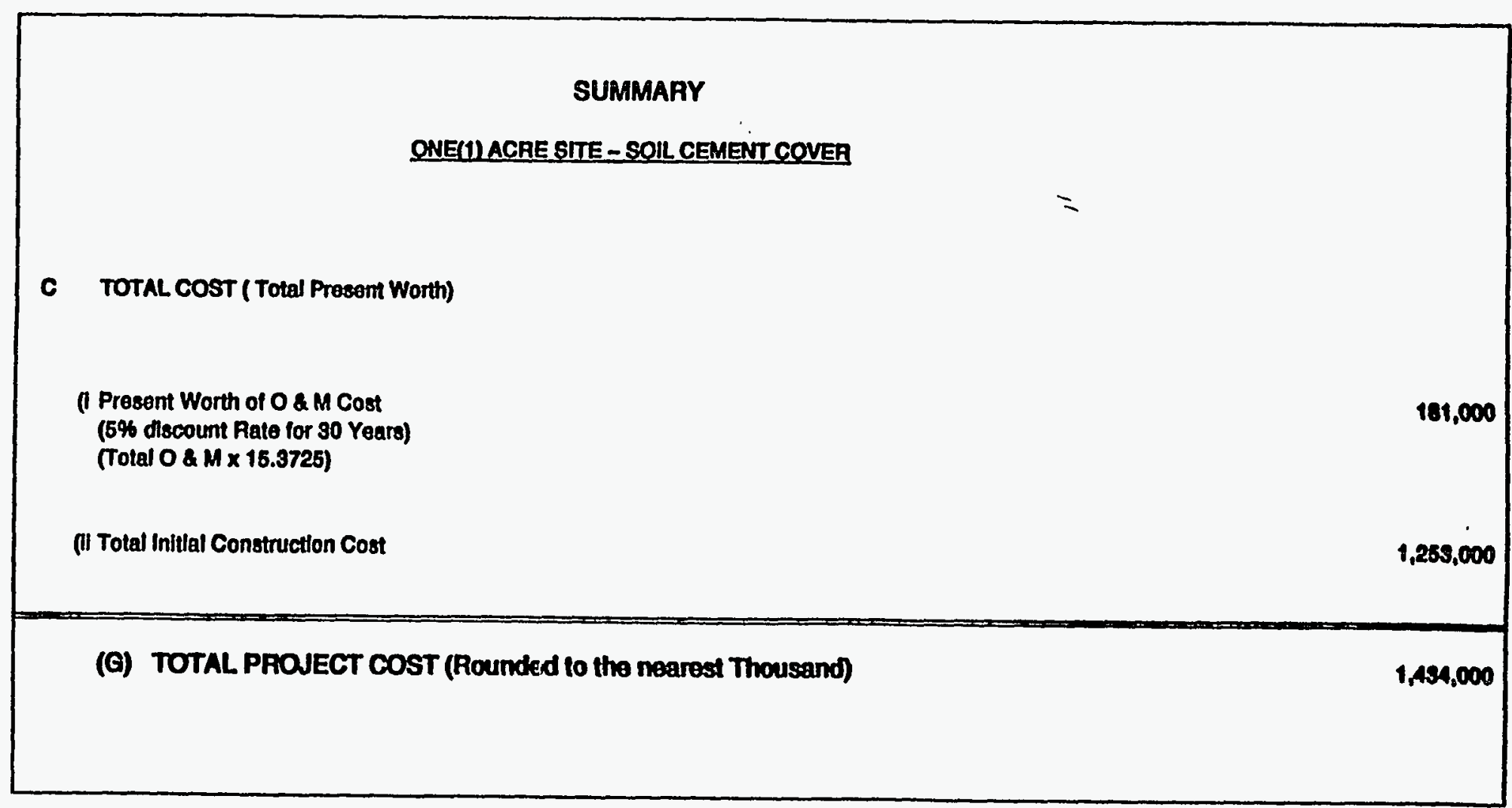

D-9. Cost Estimate for a Cover System Utilizing Soil Cement and 30-mil FML (Cont'd) 
DETAIL ESTIMATE

\section{Loof: 7-03}

ACTIVITY CODE: ESE260112

PROJ.NAME: ECON. EVALUATION OF CLOSURE CAP BARRIER MATERIAL

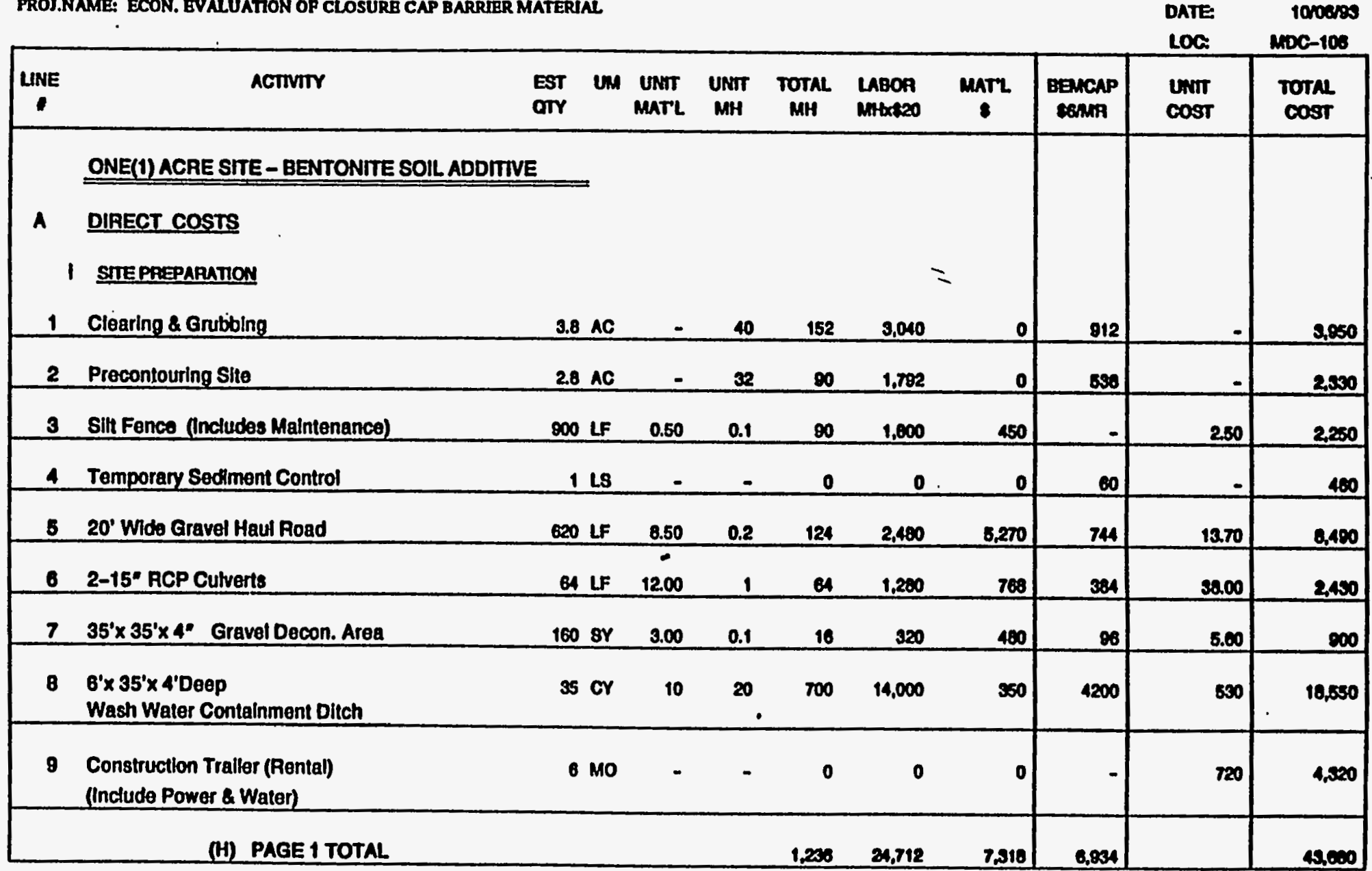

D-10. Cost Estimate for a Cover System Utilizing Bentonite Soil Additive and a 30-mil FML 
DETAIL ESTIMATE CHKDBY: 8.MEAD

LO0 I: $7-03$

ACTIVITY CODE: ESE260N12

PROJ.NAME: ECON. BVALUATION OF CLOSURE CAP BARRIER MATERIAL

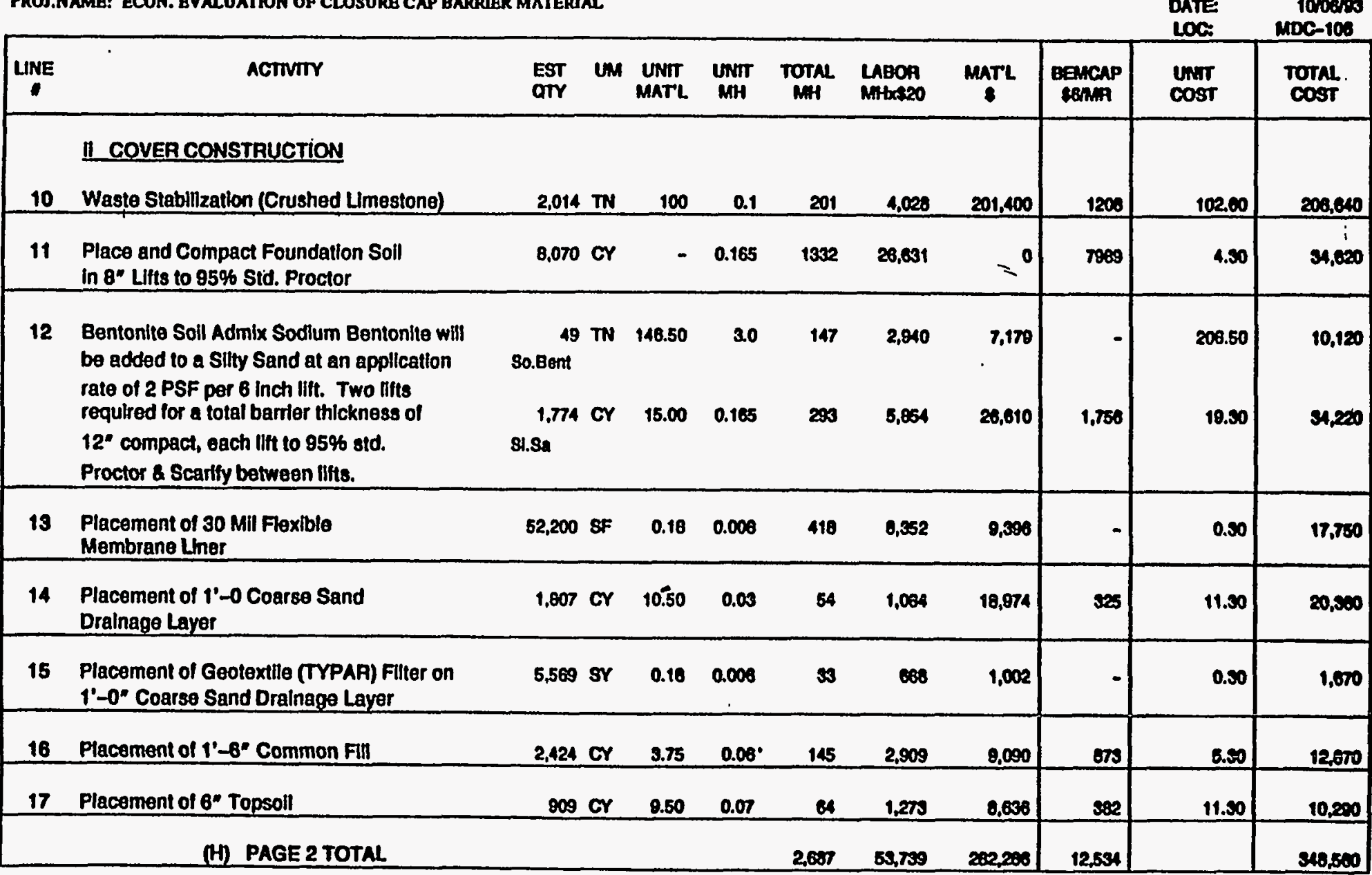

D-10. Cost Estimate for a Cover System Utilizing Bentonite Soil Additive and a 30-mil FML (Cont'd) 


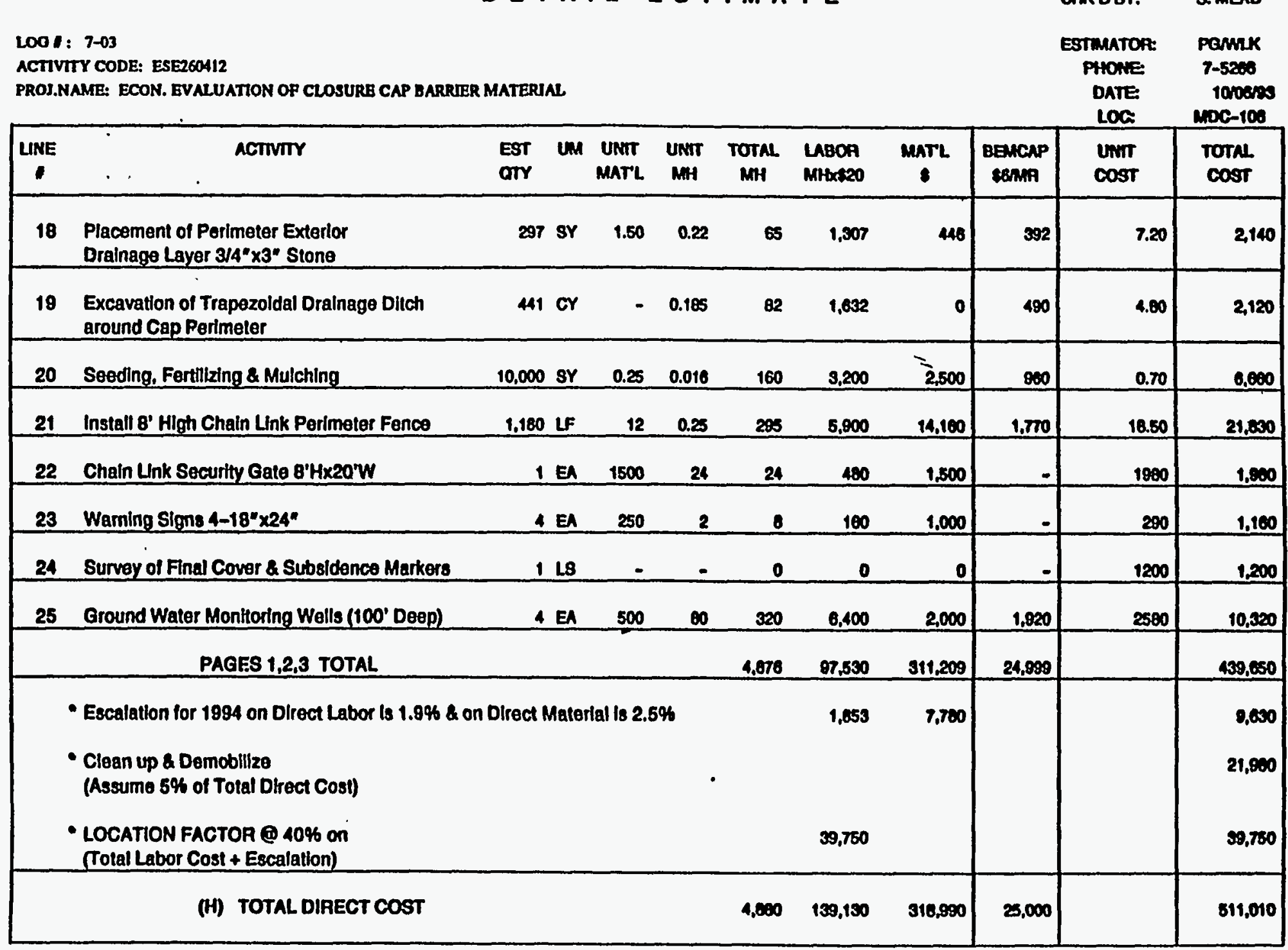

D-10. Cost Estimate for a Cover System Utilizing Bentonite Soil Additive and a 30-mil FML (Cont'd) 
DETAIL ESTIMATE

3. MEAD

LOO II: $7-03$

ACTIVTY CODE: ESE260412

PROJ.NAME: ECON. EYALUATION OF CLOSURE CAP BARRIER MATERIAL

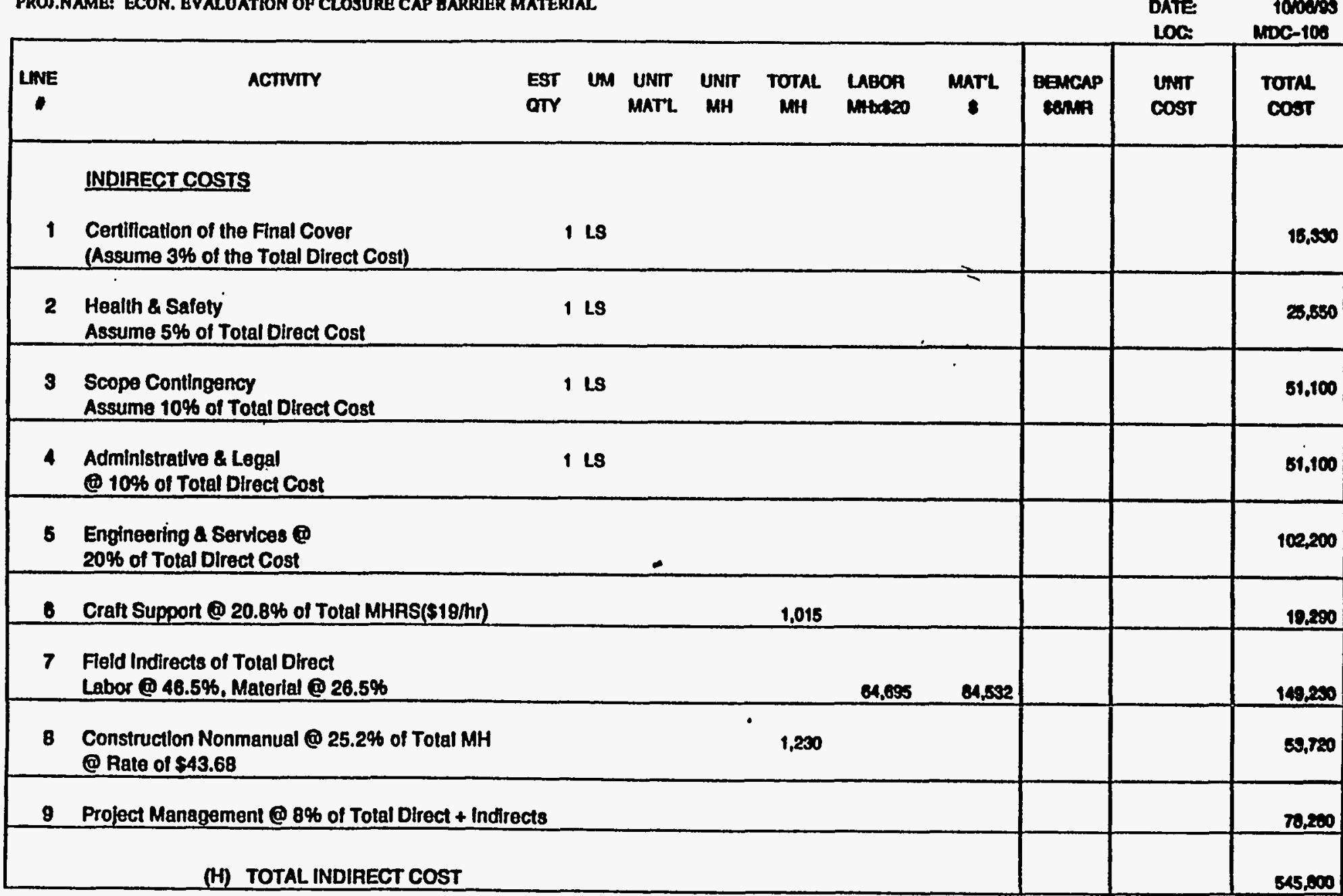

D-10. Cost Estimate for a Cover System Utilizing Bentonite Soil Additive and a 30-mil FML (Cont'd) 


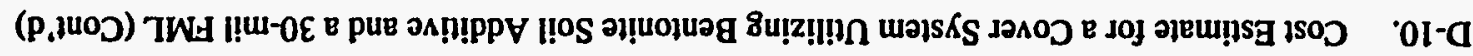

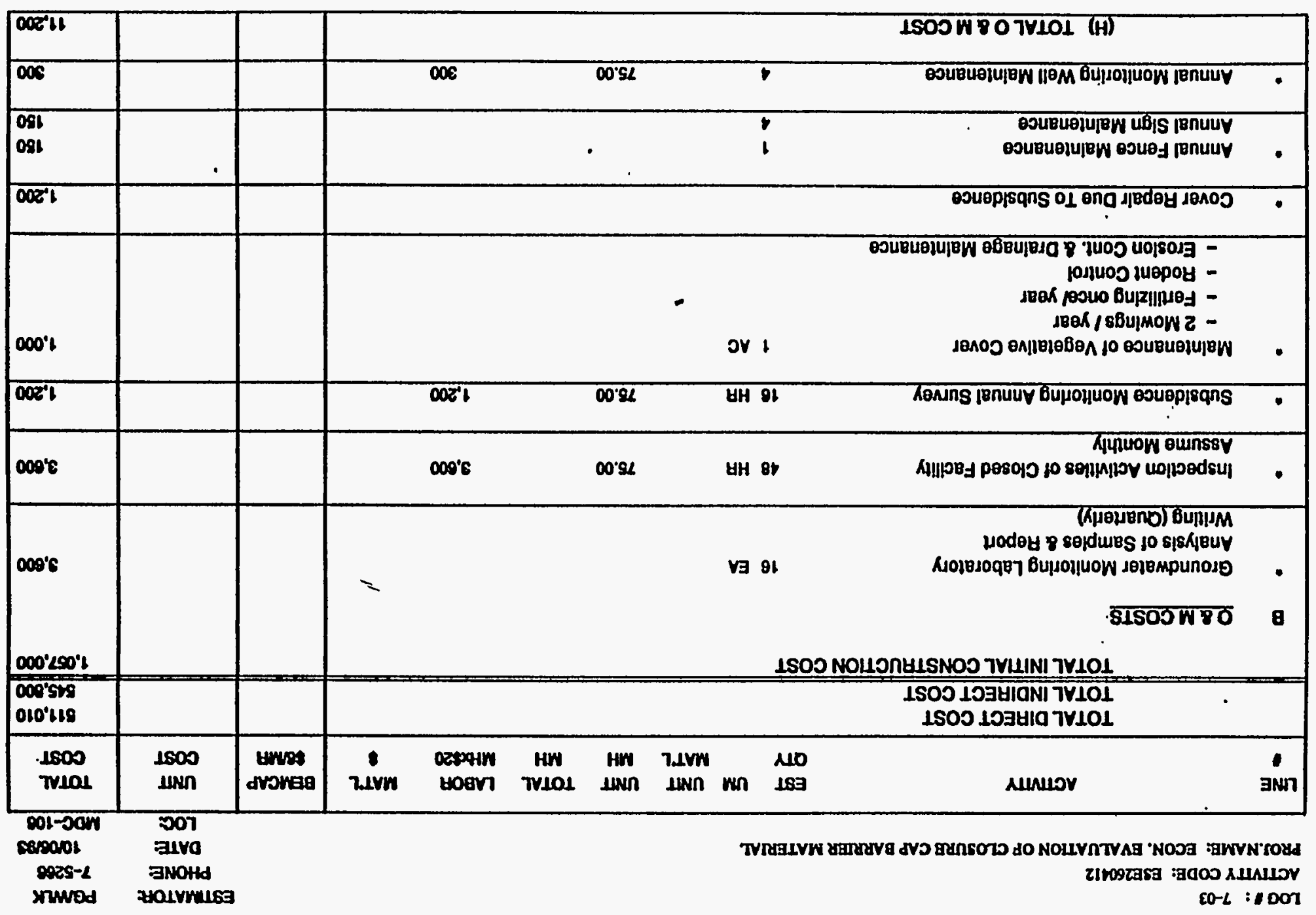
ovaw 8
:N90)
$\exists \perp \vee W 1 \perp s \exists 71 \forall \perp \exists a$ 
DETAIL ESTIMATE

\begin{tabular}{|c|c|}
\hline & a MEAD \\
\hline TMaAT & PannK \\
\hline $\begin{array}{l}\text { PHONE } \\
\text { DATE: } \\
\text { LOC: }\end{array}$ & MDC. \\
\hline
\end{tabular}

LO01: 7-03

ACTIVITY CODB: ESEZ6OM12

PROI.NAME: ECON. EVALUATION OF CLOSURE CAP BARRIER MATERIAL

LOC:

MOC-100

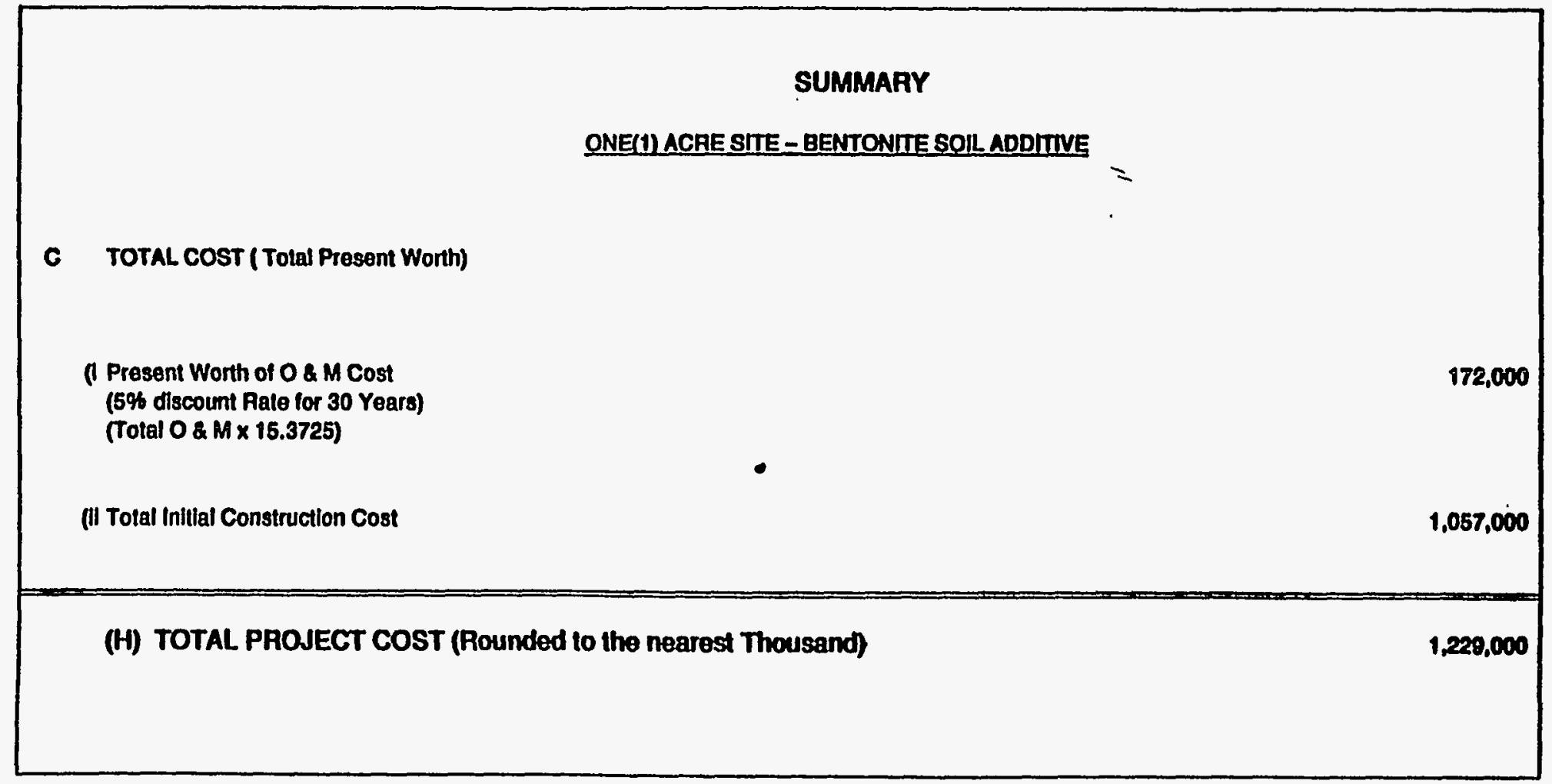

D-10. Cost Estimate for a Cover System Utilizing Bentonite Soil Additive and a 30-mil FML (Cont'd) 
DETAILESTIMATE

CHeo or: QMEAD

1001: 7-03

ACTIVTTY CODE: ESE260412

PROS.NAMB: ECON. EVALUATION OF CLOSURB CAP BARUIBR MATERIAL.

Esmantor:

PHone

pannk

Dare

100.

cosions

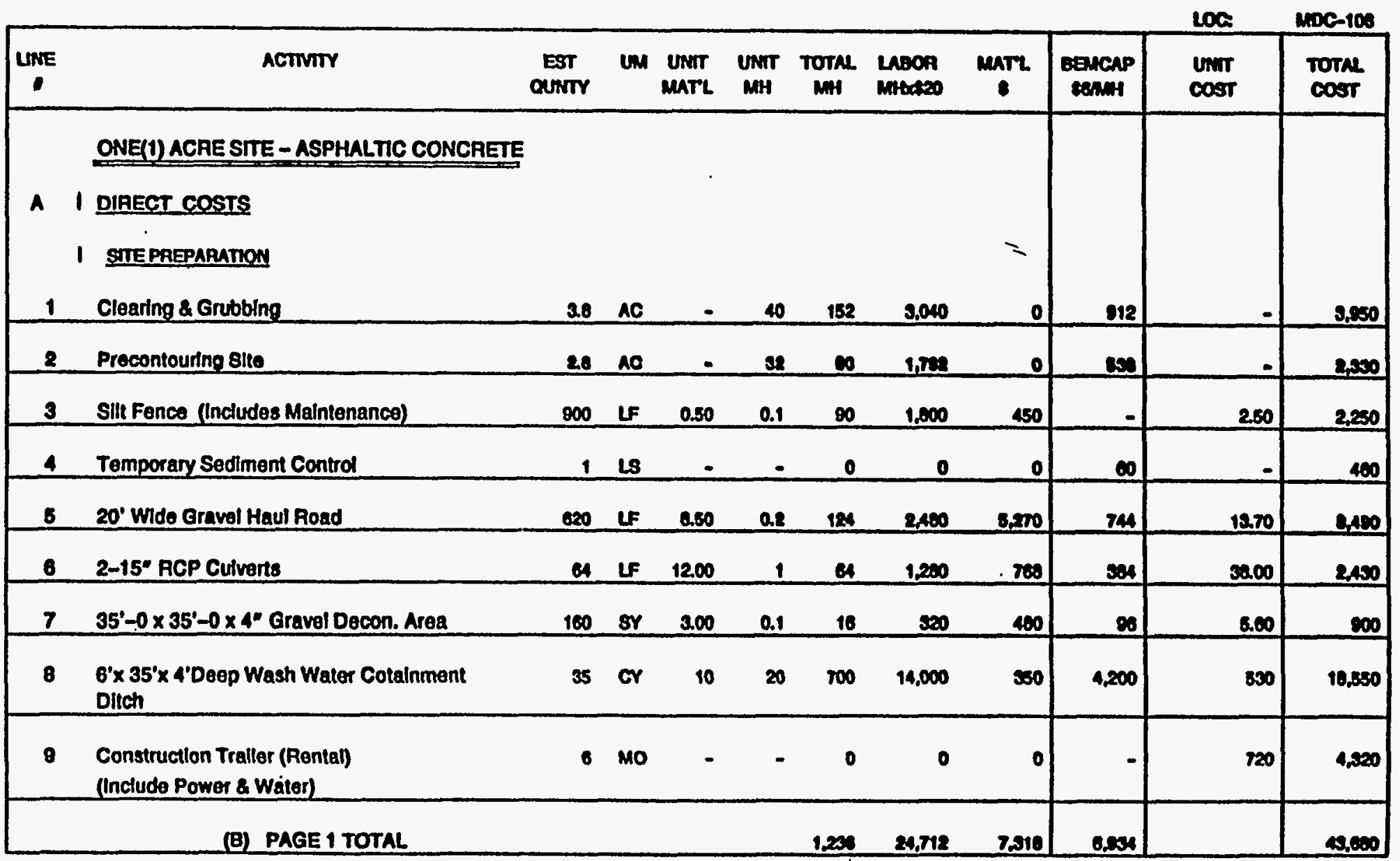

D-11. Cost Estimate for a Cover System Asphalt Concrete Hydraulic Barrier and a 30-mil FML 
DETAILESTIMATE

Loal: 7-03

ACTTVTTY CODE: Esp26012

MROJ.NAME: ECON. EVALUATION OF CLOSURB CAP BARRIER MATERUAL

\begin{tabular}{|c|c|c|c|c|c|c|c|c|c|c|c|}
\hline & & & & & & & & & & LOA: & $\log _{0 \rightarrow 100}$ \\
\hline LNE & netrutr & $\begin{array}{l}\text { EST } \\
\text { currr }\end{array}$ & Un & $\begin{array}{l}\text { UNTT } \\
\text { MATL }\end{array}$ & $\lim _{\min }$ & $\underset{\text { MH }}{\text { TOTAC }}$ & MBOA & $\operatorname{marl}^{2}$ & gexcenp & $\cos$ & $\underset{\cos T}{\operatorname{Torh}}$ \\
\hline & CONER CONSTRUCTION & & & & & & & & & & \\
\hline 10 & Wasre Stabllization (Crushod Limestone) & 2.014 & $T M$ & 100 & 0.1 & 201 & 1,020 & 201.400 & 1,200 & 102,00 & 2006040 \\
\hline 11 & $\begin{array}{l}\text { Place and Compact Foundation Soll } \\
\text { in } 8^{\circ} \text { Uits to } 95 \% \text { Sid. Proctor }\end{array}$ & 6,390 & or & - & 0.160 & 1054 & 21,007 & $=$. & 6,328 & 4.50 & 28,410 \\
\hline 12 & $\begin{array}{l}\text { Placement of } 30 \text { Min Flexible } \\
\text { Membrane Liner. }\end{array}$ & 52,200 & SF & 0.10 & 0.006 & 418 & 0,552 & 0,000 & - & 0.50 & 17,750 \\
\hline 13 & $\begin{array}{l}\text { Placement of 1'-0 Coarse Sand } \\
\text { Drainage Layer }\end{array}$ & 1,688 & or & 10.50 & 0.08 & 81 & 1.012 & 17,700 & 2000 & 11.50 & 10,000 \\
\hline 14 & $\begin{array}{l}\text { 2" PvC Perforatod Drainage Pipo placed } \\
\text { in Orainage layer }\end{array}$ & $1,380^{-}$ & LF & 1.6 & 0.20 & sot & 0,072 & 1,300 & - & 0.40 & 7,450 \\
\hline 18 & 21/2" Binder Course & 8,300 & SY & 4.20 & 0.06 & 910 & $\mathrm{es00}$ & 2,200 & 1,0000 & 5.00 & $\mathbf{s 0 , 5 0 0}$ \\
\hline 16 & 4" Hydraulic Asphatt Conc. & 5,300 & gr & e.tr & 0.06 & 159 & 3.180 & 30.310 & sest & 7.50 & 39,040 \\
\hline 17 & Asphait Mastlc Soal & 5,300 & gr & 0.50 & 0.016 & $\infty$ & 1,590 & 2050 & - & 0.00 & 4,240 \\
\hline 18 & Sprayed Asphaltic Concrete Sealant & 5,300 & SY & $0.75^{\prime}$ & $0.0 \dot{2}$ & 100 & 2,120 & 2,978 & $\Leftrightarrow$ & 8.50 & 0,00 \\
\hline 19 & Excavation of Traingular Drainage Ditch & 222 & or & - & $0 . \dot{s}^{\prime}$ & $\theta$ & 1,332 & 0 & 200 & 7.00 & 1,700 \\
\hline 20 & 4" Thlek Asphah Perlmeter Drainage Ditch & 000 & SY & 7.00 & 0.15 & 101 & 2.010 & 8,200 & $\infty$ & 11.70 & 1,070 \\
\hline & (B) PAGE 2 TOTAL & & & & & 2007 & 87,145 & 200,534 & 12500 & & 500.010 \\
\hline
\end{tabular}

D-11. Cost Estimate for a Cover System Asphalt Concrete Hydraulic Barrier and a 30-mil FML (Cont'd) 


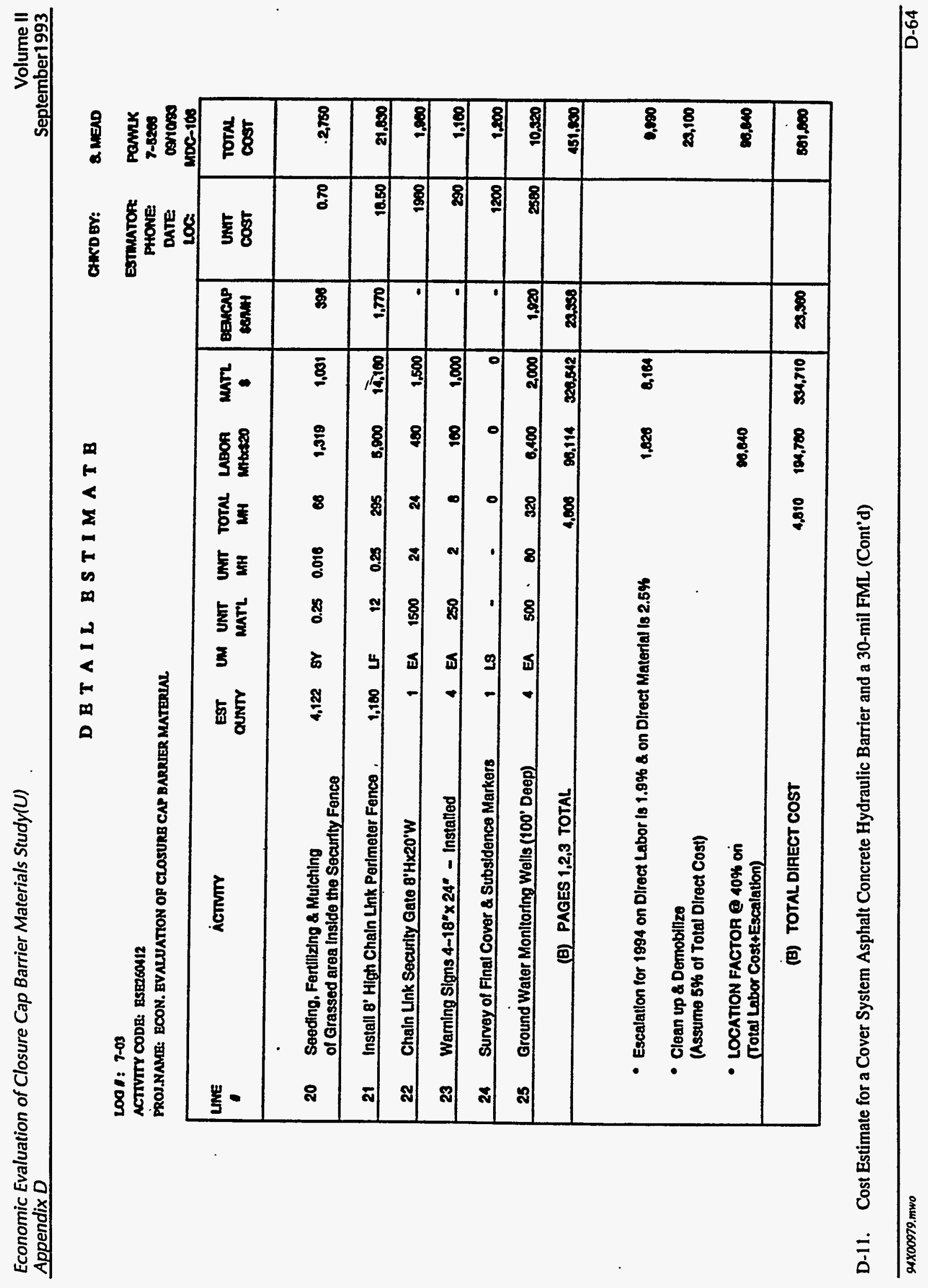




\section{I: 7-03}

ACTTVITY CODB: ESESSOA12

PROS.NAME: ECON. BVALUATTON OP CLOSURB CAP GARRIRR MATERIAL

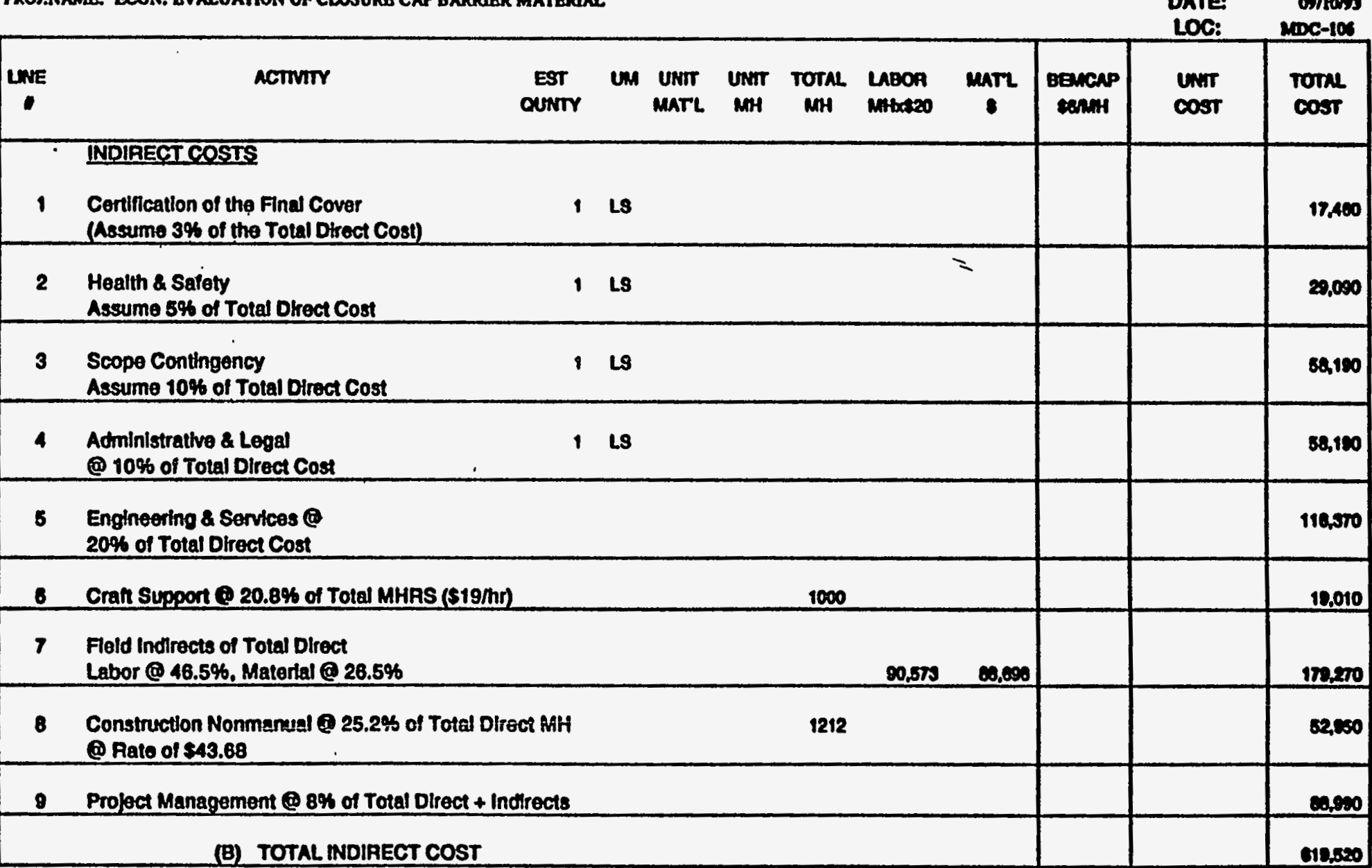

D-11. Cost Estimate for a Cover System Asphalt Concrete Hydraulic Barrier and a 30-mil FML (Cont'd) 
D B TAILESTIMATB

L001: 1-03

ACTIVITY CODE: ESE260412

MOJ.NAMR: ECON. BVALUATION OP CLOSURB CAP BARRIER MATENULL

QMEND

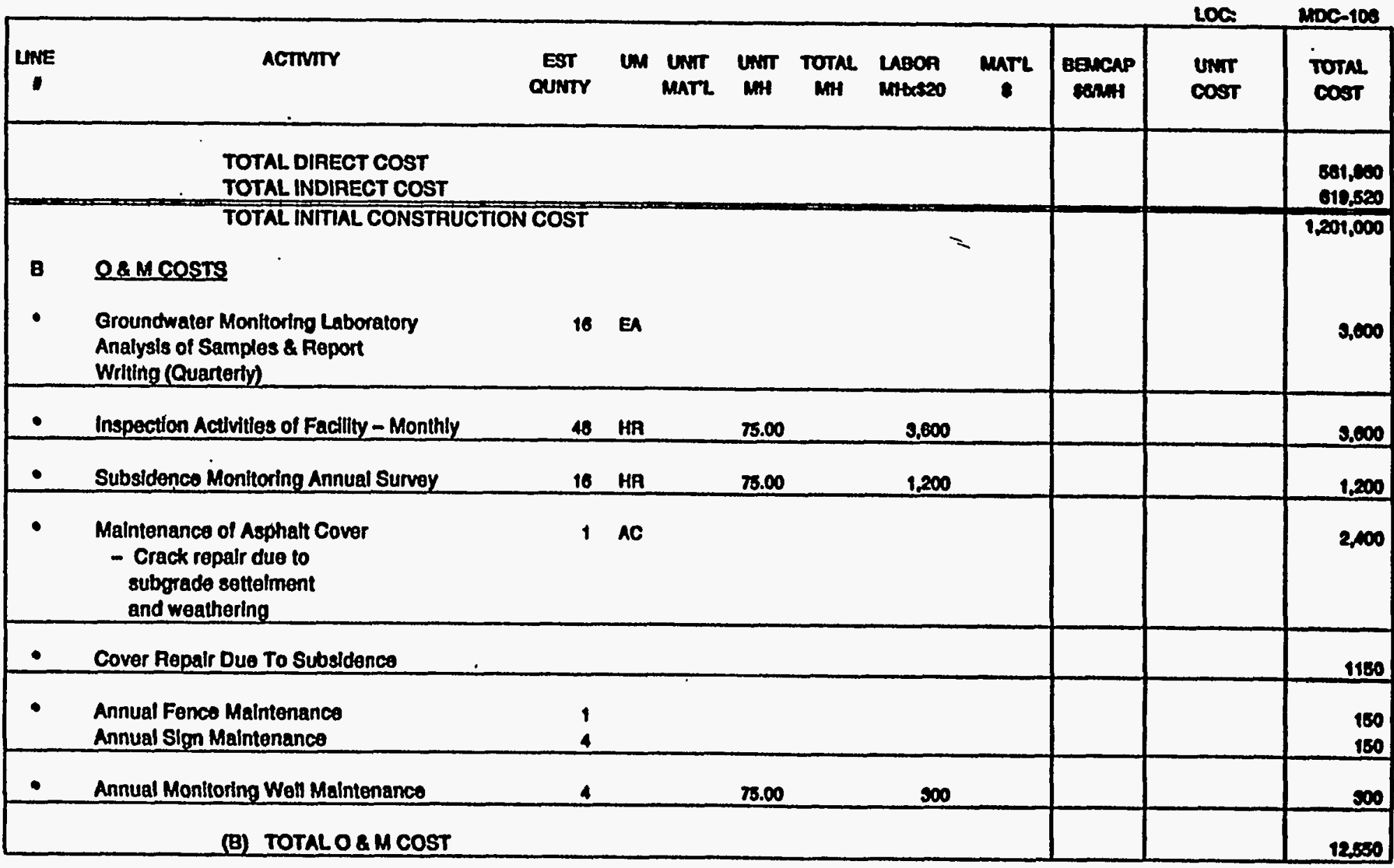

D-11. Cost Estimate for a Cover System Asphalt Concrete Hydraulic Barrier and a 30-mil FML (Cont'd) 
D B T A I L B S T I MAT B

\section{LO0 : : 7-03}

ACTIVTYY CODE: ESE260112

PROL.MAME: ECON. EYALUATION OF CLOSURB CAP BARRIER MATERIAL

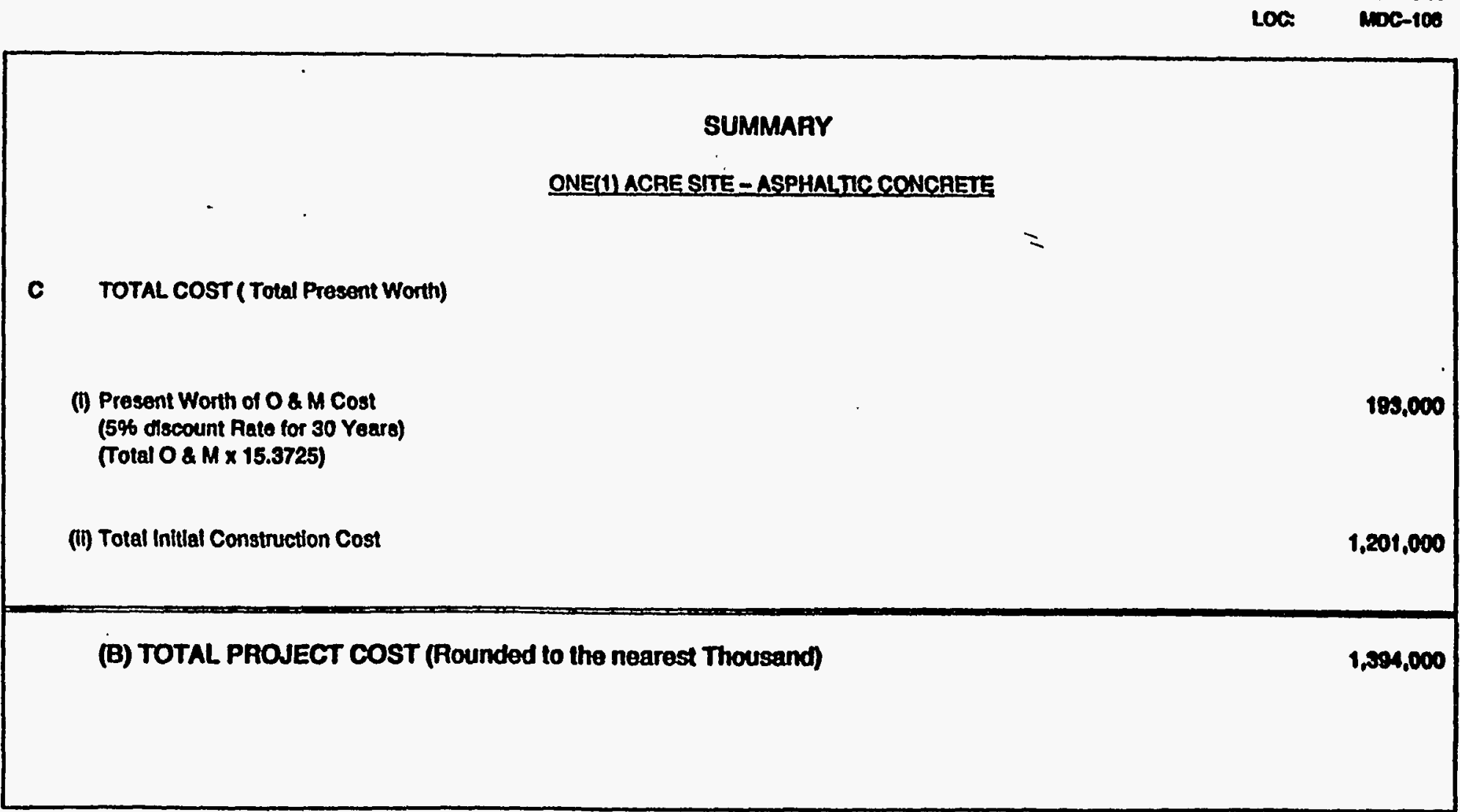

\begin{tabular}{|c|}
\hline CHWCD BY: \\
\hline $\begin{array}{l}\text { ESTMANTOR: } \\
\text { PHOAEE } \\
\text { DATE } \\
\text { LOC: }\end{array}$ \\
\hline
\end{tabular}

D-11. Cost Estimate for a Cover System Asphalt Concrete Hydraulic Barrier and a 30-mil FML (Cont'd)

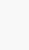




\section{DETAIL ESTIMATE}

\section{LO0 1: 7-03}

ACTIVIT CODE ESE200412

PPOU.NMME: ECON. EVALUATION OF CLOSUPE CAP BAPFUEA MATEEAR

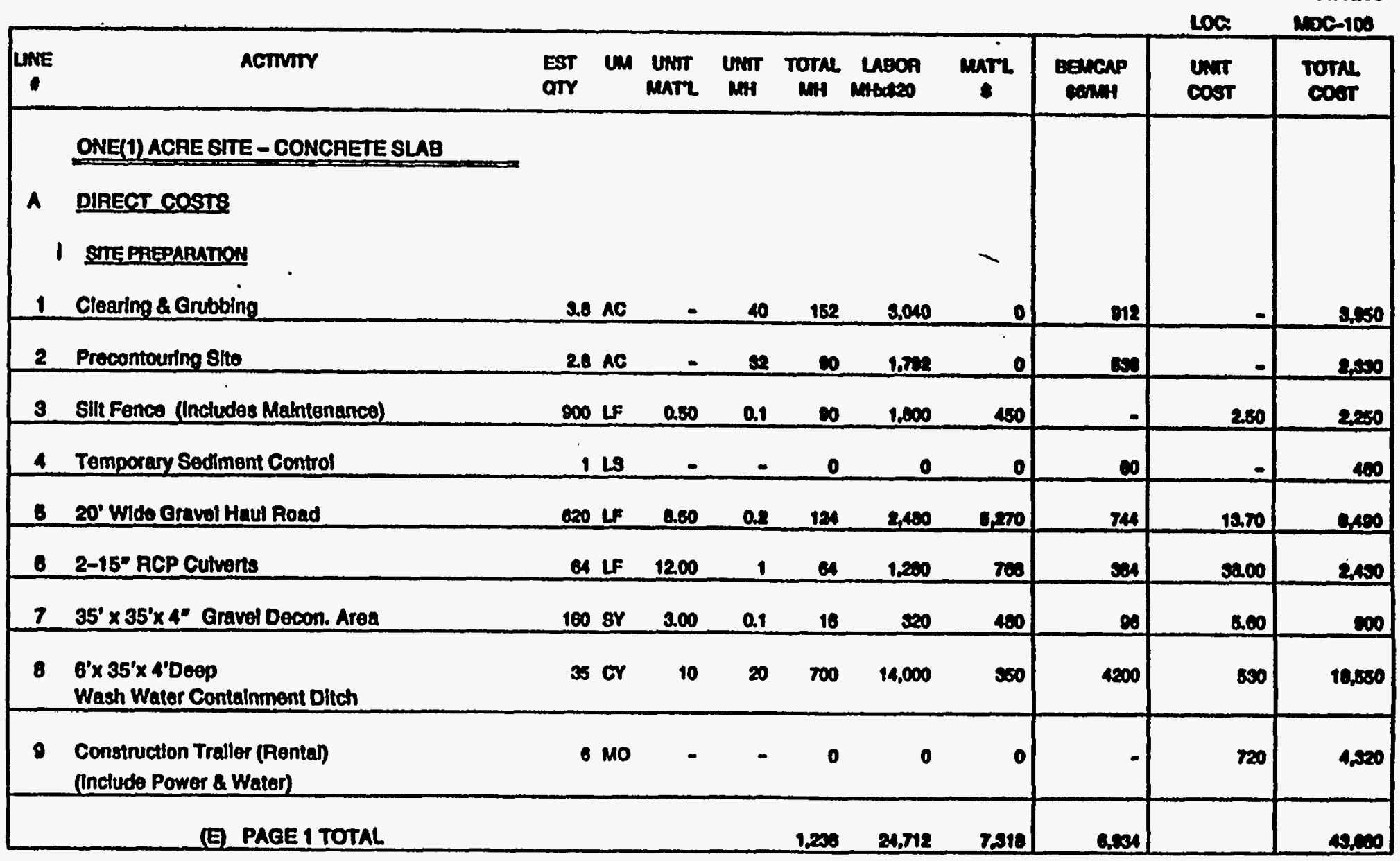

D-12. Cost Estimate for a Cover System Utilizing Poured-in Place Concrete Slab and a 30-mil FML 
DETAIL ESTIMATE CKCDOV: QMEAD

Lo0 : 7-03

ACTIVTY CODB: BSBESOA12

PROJ.NAME: BCON. BVALUATION OP CLOSURB CAP BARRUER MATERIAL.

\begin{tabular}{|c|c|c|c|c|c|c|c|c|c|c|c|}
\hline LNE & Actrvm & $\begin{array}{l}\text { EST } \\
\text { orr }\end{array}$ & um & $\begin{array}{l}\text { UnimT } \\
\text { MATL }\end{array}$ & $\lim _{\text {MNH }}$ & TOTAL & $\begin{array}{c}\text { LABCA } \\
\text { Mituaed }\end{array}$ & $\underset{?}{\operatorname{maTL}}$ & $\begin{array}{l}\text { Dencap } \\
\text { senmi }\end{array}$ & $\operatorname{lins}_{\cos }$ & $\begin{array}{l}\text { Total } \\
\text { Cost }\end{array}$ \\
\hline 10 & $\begin{array}{l}1 \text { COVER CONSTRUCTION } \\
\text { Waste Stablization (Crushod Lmestono) }\end{array}$ & 2.014 & TN & 100 & Q1 & 201 & 4,020 & 201.400 & 1200 & 102.00 & 200.810 \\
\hline 11 & $\begin{array}{l}\text { Place and Compact Foundation soli } \\
\text { In } 8^{\circ} \text { Lifte to } 95 \% \text { Std. Proctor }\end{array}$ & 6,390 & Cr & - & 0.183 & 1054 & 21.007 & $=$ & 6.520 & 4.00 & 21,110 \\
\hline 12 & $\begin{array}{l}\text { Placement of } 30 \text { Mn Flexiblo } \\
\text { Membrane Liner }\end{array}$ & 52,200 & SF & 0.10 & 0.006 & 418 & 8,252 & 2.500 & - & 0.50 & 17,760 \\
\hline 13 & $\begin{array}{l}\text { Placement of } 1^{\prime}-0 \text { Coarse Sand } \\
\text { Drainage Layer }\end{array}$ & 1.686 & Cr & 10.50 & 0.00 & 81 & 1.012 & 17,700 & $\operatorname{sos}$ & 11.30 & 10,020 \\
\hline 14 & $\begin{array}{l}\text { 2- PvC Perforaled Drainago Pipo placod } \\
\text { in Dralnage layer }\end{array}$ & 1,380 & $\mathbf{L F}$ & 1.00 & 0.22 & sod & 0,072 & 1,500 & - & . .40 & 7,150 \\
\hline 18 & $\begin{array}{l}\text { 4" Concrete Slab with } 1 \text { Layer of (conc.tform) } \\
6 \times 6-W 2.9 \times W 2.9 \text { (wld.wire fab) Broom Finish. }\end{array}$ & 5,300 & SY & 7.50 & 0.45 & 2503 & 97,700 & 59,760 & $\begin{array}{r}0.00 \mathrm{~min} \\
1,010 \\
\text { compresto }\end{array}$ & 18.50 & 8000 \\
\hline 16 & Sprayod on Curing Compound & 5,300 & SY & 0.20 & 0.016 & $\boldsymbol{\omega}$ & 1.590 & 1000 & $4 \pi$ & 0.00 & $\mathbf{3 , 1 8 0}$ \\
\hline 17 & Jolnt Sealant 6" PVC Water Stops & 3,400 & $\underline{L F}$ & 260. & Q1 & 340 & 8,000 & 2000 & - & $\mathbf{4 . 0 0}$ & 1000 \\
\hline 18 & Excavation of Traingular Drainage Ditch & 222 & Cr & - & 0.30 & $\boldsymbol{\theta r}$ & 1.052 & 0 & $\operatorname{sen}$ & 8.60 & 1.750 \\
\hline 19 & 4" Thick Concrete Perimeter Drainage Dltch & 670 & sr & 7.40 & a.so & 255 & 8,092 & 4058 & 1.820 & 17.50 & 11.500 \\
\hline & (E) PAGE 2 TOTAL & & & & & 8.153 & 100,000 & 204,147 & 12,152 & & 20250 \\
\hline
\end{tabular}

D-12. Cost Estimate for a Cover System Utilizing Poured-in Place Concrete Slab and a 30-mil FML (Cont'd) 
D B T A I L BSTIMATB

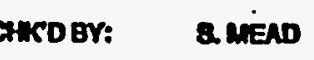

1001: $7-03$

ACTIVIT CODE: BSE2SOL12

FROJ.NANG: BCON. EVALUATION OF CLOSURB CAP BARRIER MATERIAL.

Esmuator: Pamk

PHONE $7-52006$

OATE Derroses

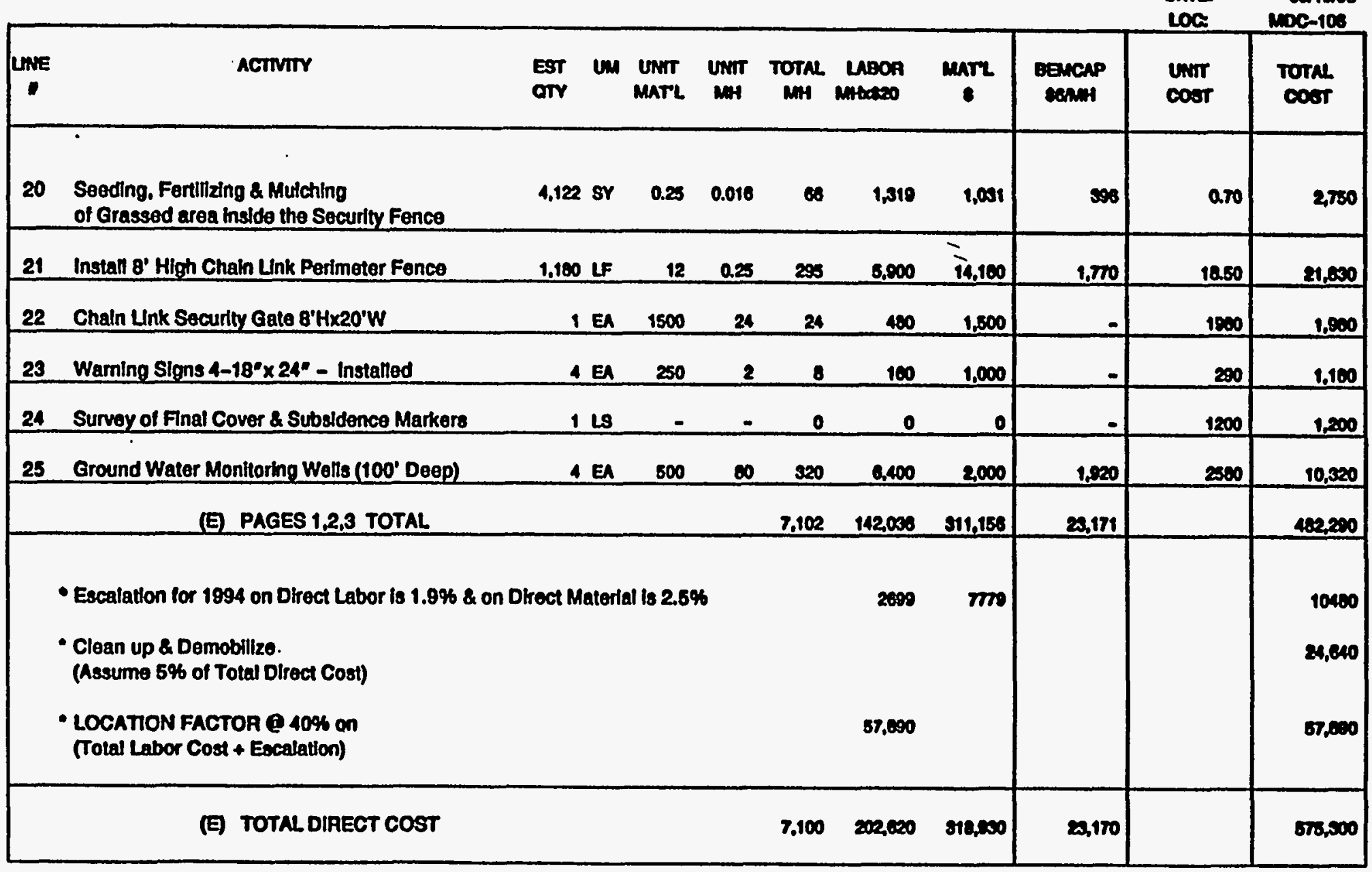

D-12. Cost Estimate for a Cover System Utilizing Poured-in Place Concrete Slab and a 30-mil FML (Cont'd) 
DBTAIL BSTIMATB

Loof: 2-03

ACTIVTY CODB: ESE260012

MOI.MAME: BCON. BYALUATION OF CLOSURB CAP BARRIER MATERLAL.

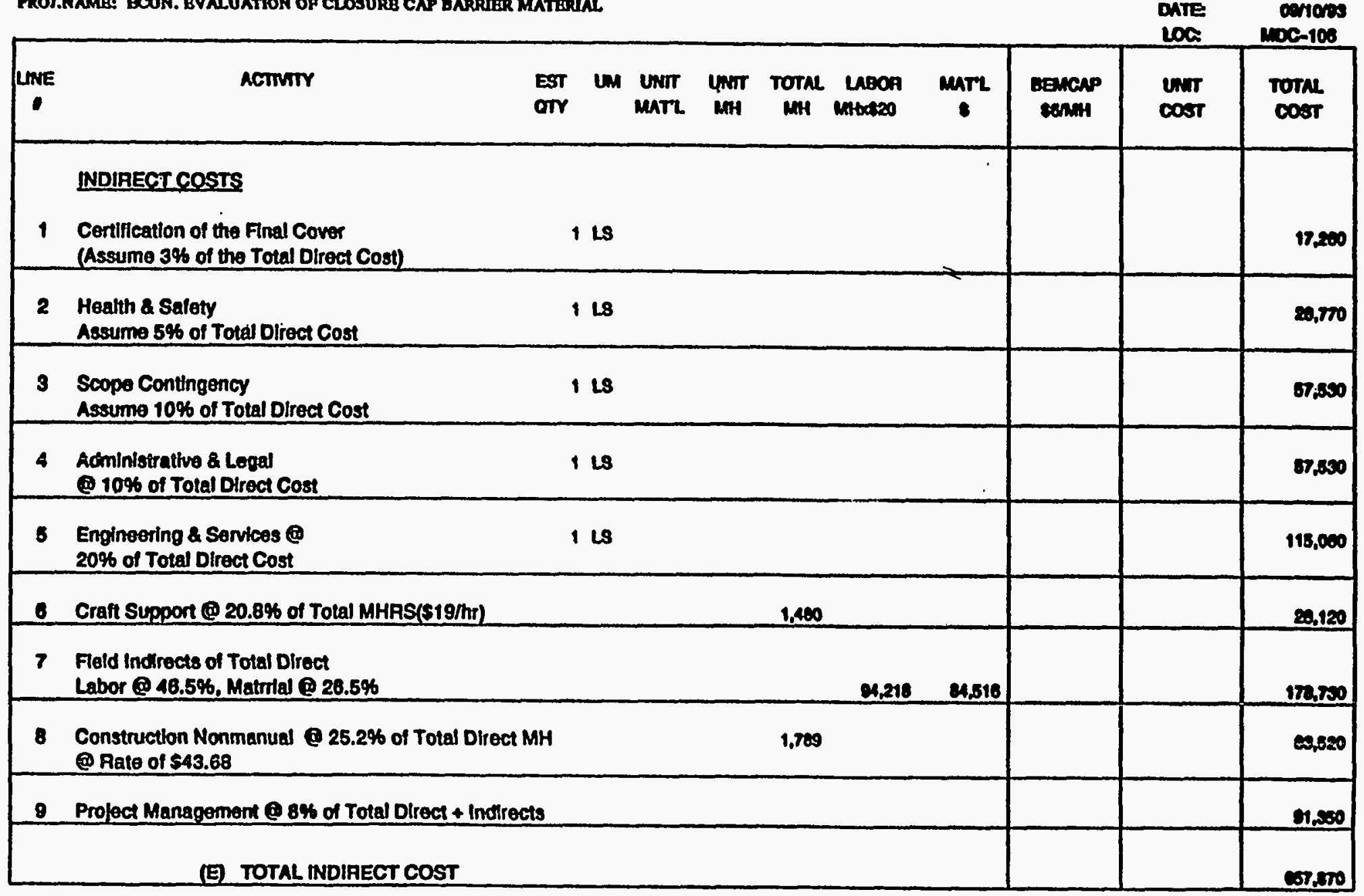

D-12. Cost Estimate for a Cover System Utilizing Poured-in Place Concrete Slab and a 30-mil FML (Cont'd) 
D B TAILE S T MATB

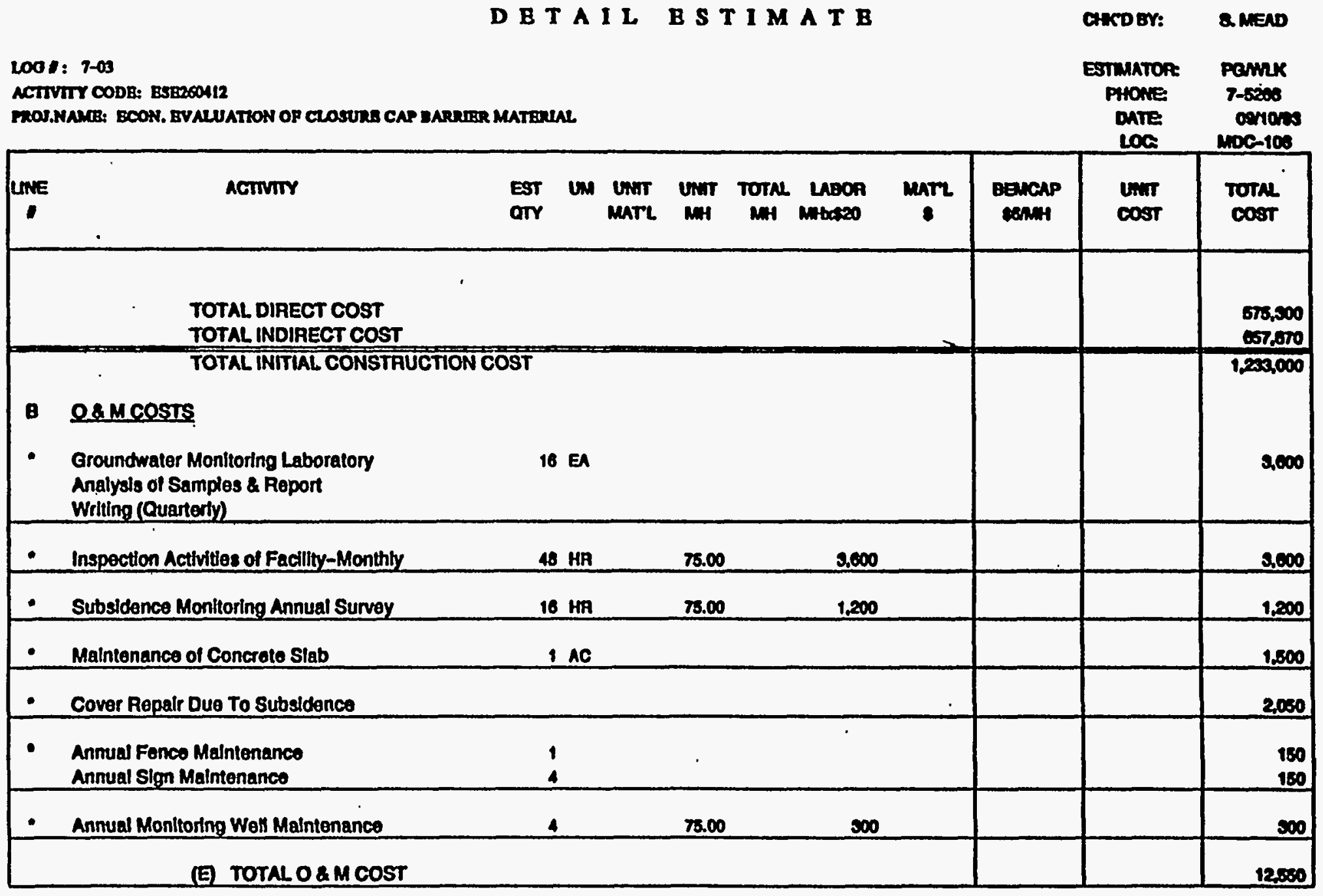

2001: 7-03

ACTIVITY CODE: BSE260412

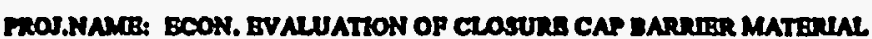

D-12. Cost Estimate for a Cover System Utilizing Poured-in Place Concrete Slab and a 30-mil FML (Cont'd) 
D B T A I L B S T I A T B

\begin{tabular}{|c|c|}
\hline & a mead \\
\hline $\begin{array}{l}\text { STmantor: } \\
\text { PHONE } \\
\text { DATE }\end{array}$ & $P$ \\
\hline
\end{tabular}

\section{Lod : 7.03}

ACTVITY CODA: BSB260412

PROJ.MAME: BCON. EVALUATION OP CLOSURB CAP BARRIER MATERTAL.

10a

$\operatorname{mos}$

\section{SUAMMARY}

\section{ONE(1) ACRE SITE - CONCAETE SLAB}

C TOTAL COST ( Total Present Worth)

(1) Present Worth of 0 \& M Cost

(5\% discount Rato for 30 Years)

(Total O\& M X 15.3725)

(1) Total Initial Construction Cost

(E) TOTAL PROJECT COST (Rounded to the nearest Thousand) 


\section{Appendix E}

\section{Cost Estimates for 2-Acre Site}


DETAILESTIMATE

L000: 7-03

ACTIVITY CODB: BSE2SOU12

FROS.NAMR: BCON. EVALUATION OF CLOSURB CAP BARRURR MATERUAL.

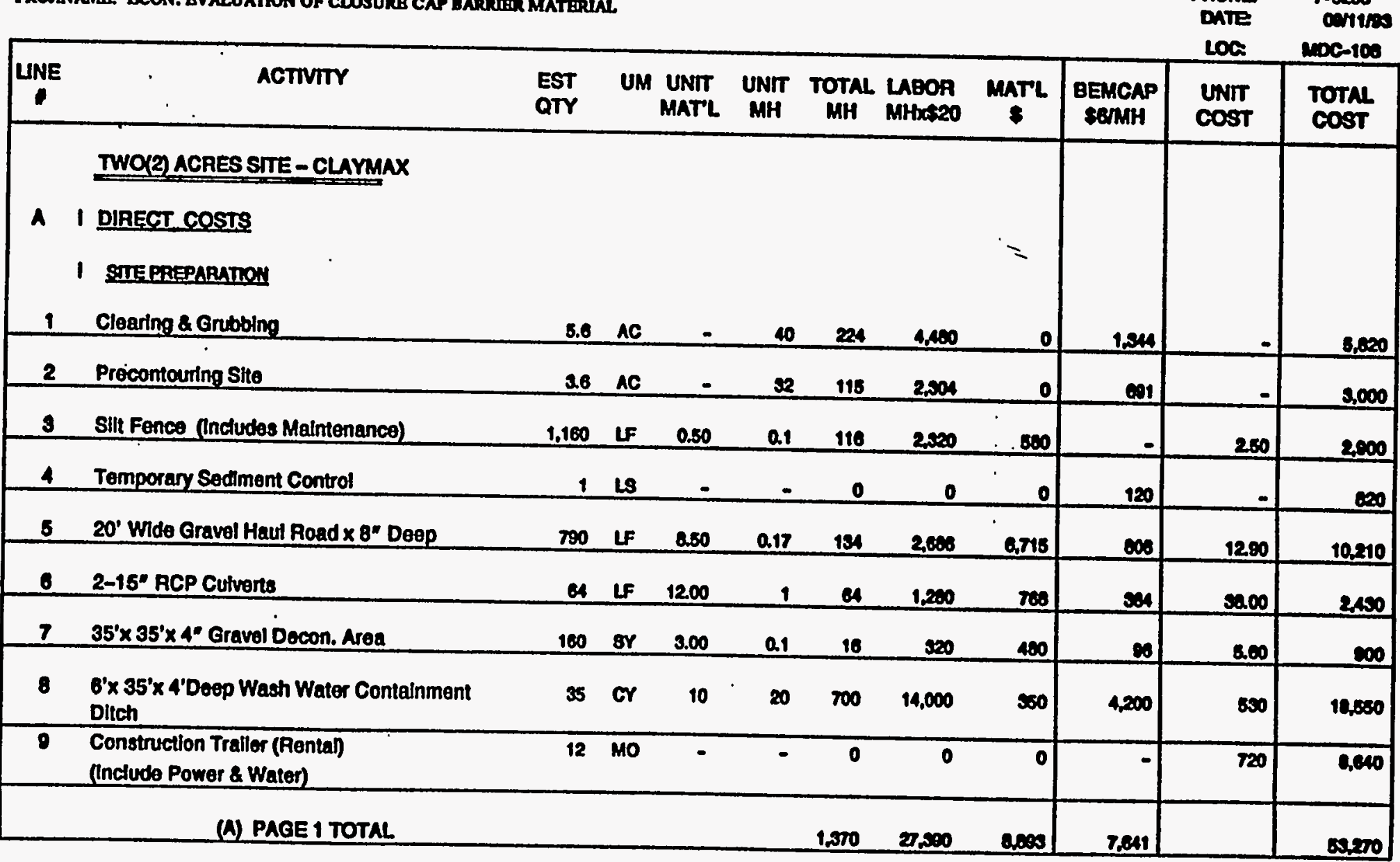

E-1. Cost Estimate for a Cover System Utilizing Claymax $\circledast$ and a 30-mil FML 
DETAIL ESTIMATE

Loo I: $7-03$

ACTIVTTY CODE: BSTESOA12

PROJ.NAME: BCON. BVALUATTON OP CLOSURB CAP BARRIIR MATERIAL

Chrogr: $2 . M E N O$

\begin{tabular}{|c|c|c|c|c|c|c|c|c|c|c|c|}
\hline LNE & ACTIVIr & $\begin{array}{l}\text { EST } \\
\text { QTYY }\end{array}$ & UM & $\begin{array}{l}\text { UNIT } \\
\text { MATL }\end{array}$ & $\underset{\text { MHI }}{\text { UNH }}$ & TOTAL & $\begin{array}{l}\text { LABOR } \\
\text { MHXA20 }\end{array}$ & $\underset{8}{\operatorname{MaTL}}$ & $\begin{array}{l}\text { BEMCAP } \\
\text { SOMHH }\end{array}$ & $\begin{array}{l}\text { UNIT } \\
\text { COST }\end{array}$ & $\begin{array}{l}\text { Toral } \\
\text { cost }\end{array}$ \\
\hline 10 & $\begin{array}{l}\text { H_ COVER CONSTRUCTION } \\
\text { Waste Stabillzation (Crushod LImestone) }\end{array}$ & 4.028 & $\mathbf{T}$ & 100 & 0.1 & 400 & 8,056 & 400,000 & 2,418 & 102.00 & 410220 \\
\hline 11 & $\begin{array}{l}\text { Place and Compact Foundation Soll } \\
\text { In 8. Uits to 95\% STD. Proctor }\end{array}$ & 16,140 & or & - & 0.155 & 2502 & 50,084 & $=$ & 18,010 & 4.00 & 6,040 \\
\hline 12 & Placement of Claymax Geotextilo Mat & 123,200 & SF & 0.45 & 0.01 & 1232 & 24,040 & $\$ 0,440$ & - & 0.70 & 60,000 \\
\hline 13 & $\begin{array}{l}\text { Placement of } 30 \text { Mil Flexible } \\
\text { Membrane Liner }\end{array}$ & 123,200 & SF & 0.17 & 0.0075 & 924 & 18,460 & 20,84 & - & 0.30 & 50,480 \\
\hline 14 & $\begin{array}{l}\text { Placement of 1'-0 Coares Sand } \\
\text { Drainage Layer }\end{array}$ & 4,240 & or & 10.50 & 0.00 & 127 & 2840 & 40,000 & 700 & 11.50 & 47,920 \\
\hline 15 & $\begin{array}{l}\text { Placement of Geotextile (TYPAR) Filter on } \\
1^{1}-0^{\circ} \text { Coarse Sand Drainage Layer }\end{array}$ & 14,022 & Sr & 0.17 & 0.006 & 70 & 1,402 & 2,504 & - & 0.50 & 3,200 \\
\hline 16 & Placement of $1^{\prime}-6^{\prime \prime}$ Common Fill & 4.848 & or & 3.75 & 0.06 & 291 & 8.010 & 18,100 & 1,746 & 8.50 & 25,740 \\
\hline 17 & Placement of $6^{\prime}$ Topsoll & 1.020 & or & 9.50 & 0.06 & 109 & 2.184 & 17,280 & $=08$ & 11.10 & 20,1200 \\
\hline 18 & $\begin{array}{l}\text { Placement of Perlmeter Exterlor } \\
\text { Drahnape Layer } 34^{*} \times 3^{*} \text { DP (Crushod Stone) }\end{array}$ & A12 & sr & 1.50 & 0.2 & 82 & 1.640 & 618 & 494 & 0.70 & 2,700 \\
\hline 19 & Excavation of Trapezoldal Drainage Ditch & 682 & or & - & 0.176 & 164 & $\mathbf{2 0 0 0}$ & - & 20 & 4.50 & 9.010 \\
\hline & (A) PAGE 2 TOTAL & & & & & 8.005 & 117.000 & 502200 & 22,018 & & 702,100 \\
\hline
\end{tabular}

E-1. Cost Estimate for a Cover System Utilizing Claymax@ and a 30-mil FML (Cont'd) 
D B TAILE T T I A T B

\section{CHCOBY: Q.MENo}

$2001: 2-03$

ACTIVITY CODB: BSEREOA12

PROS.NAMR: BCON. BVALUATION OF CLOSURB CAP BARRIER MATERIAL

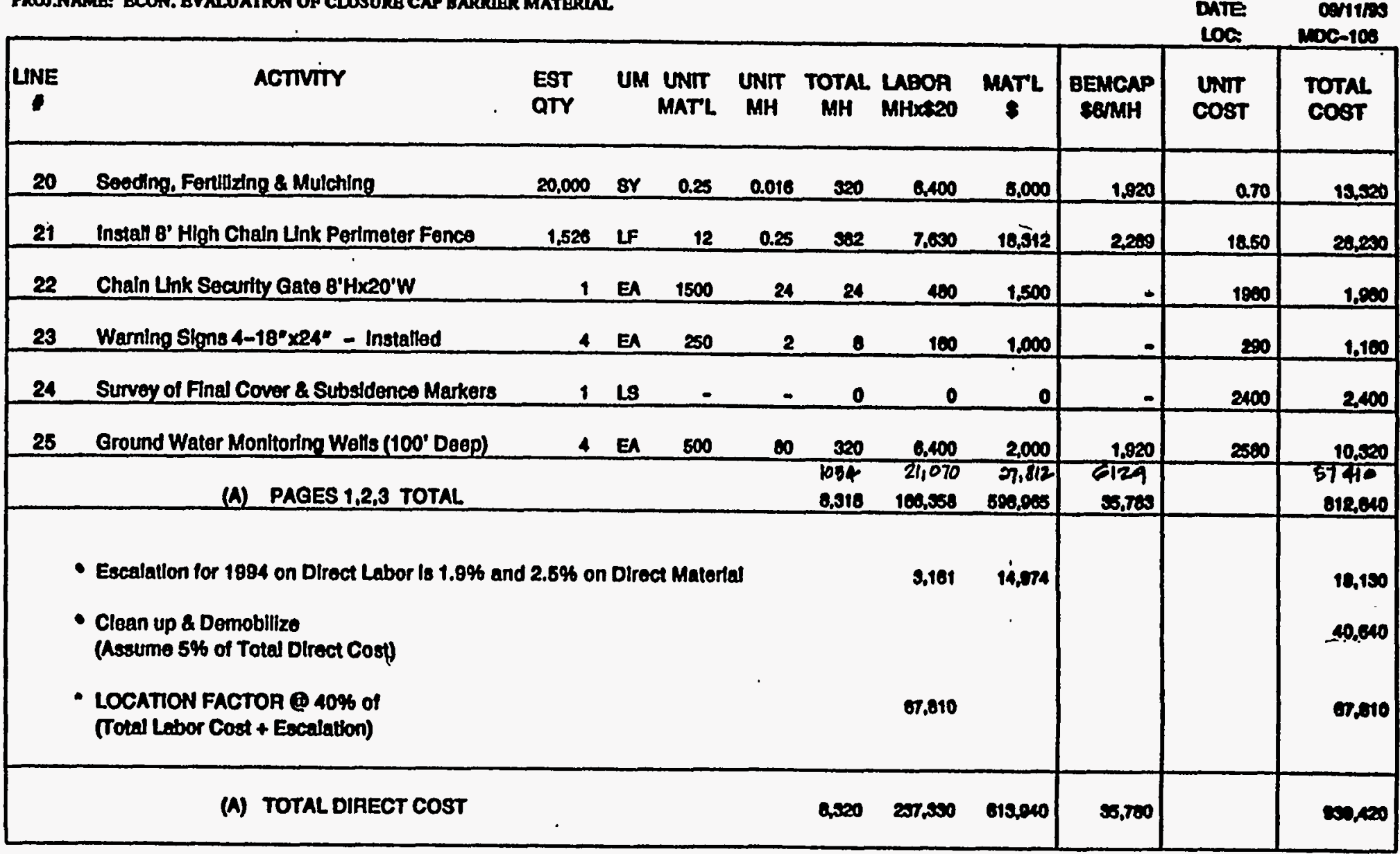

E-1. Cost Estimate for a Cover System Utilizing Claymax@ and a 30-mil FML (Cont'd) 


\section{B T A I L B S T I M A T B}

$1001: 7-03$

ACTIVITY CODE: BSE260412

MOJ.MAMR: ECON. EVALUATTON OF CLOSURE CAP BARRRRR MATBRIAL

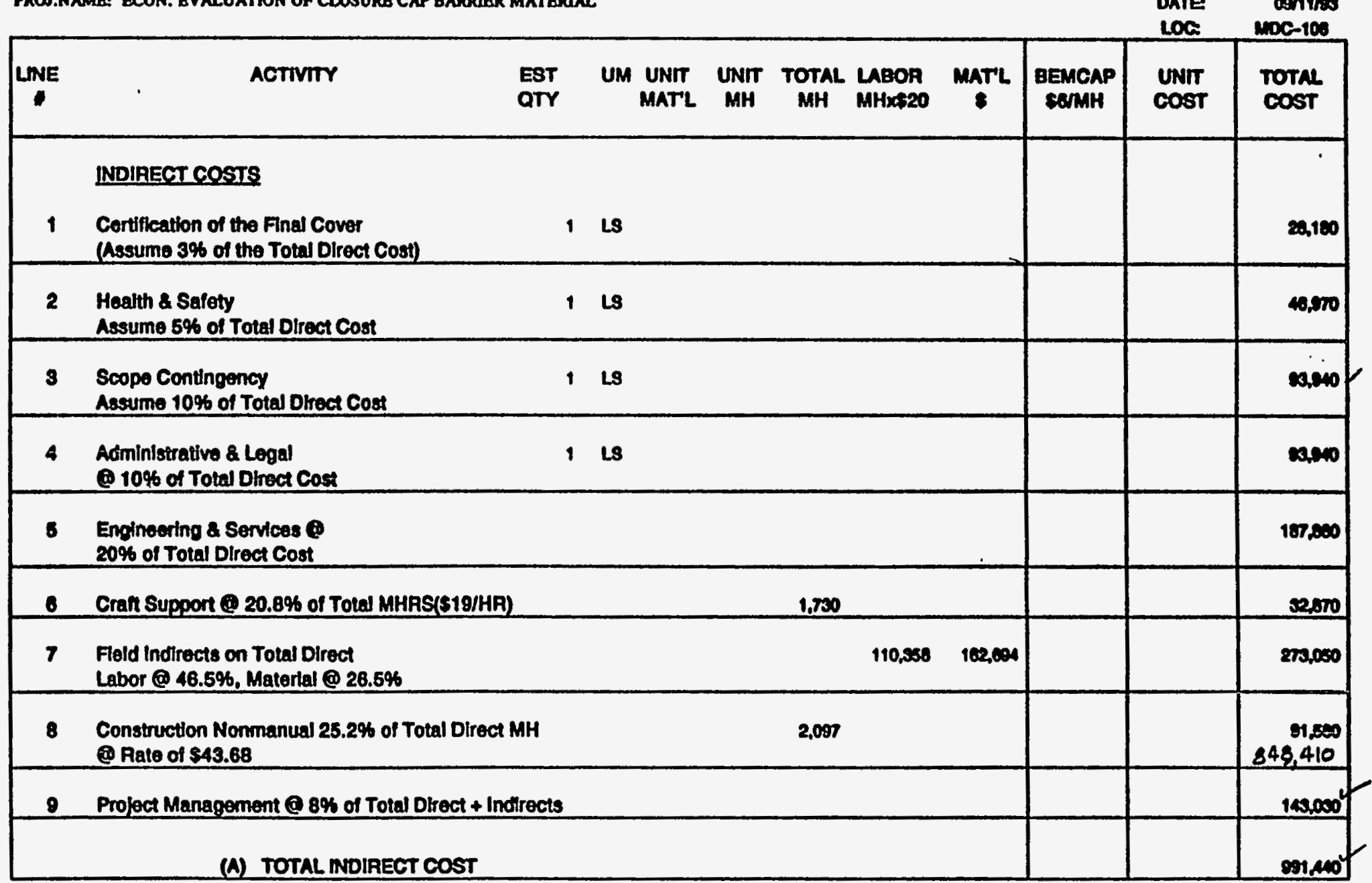

E-1. Cost Estimate for a Cover System Utilizing Claymax and a 30-mil FML (Cont'd) 


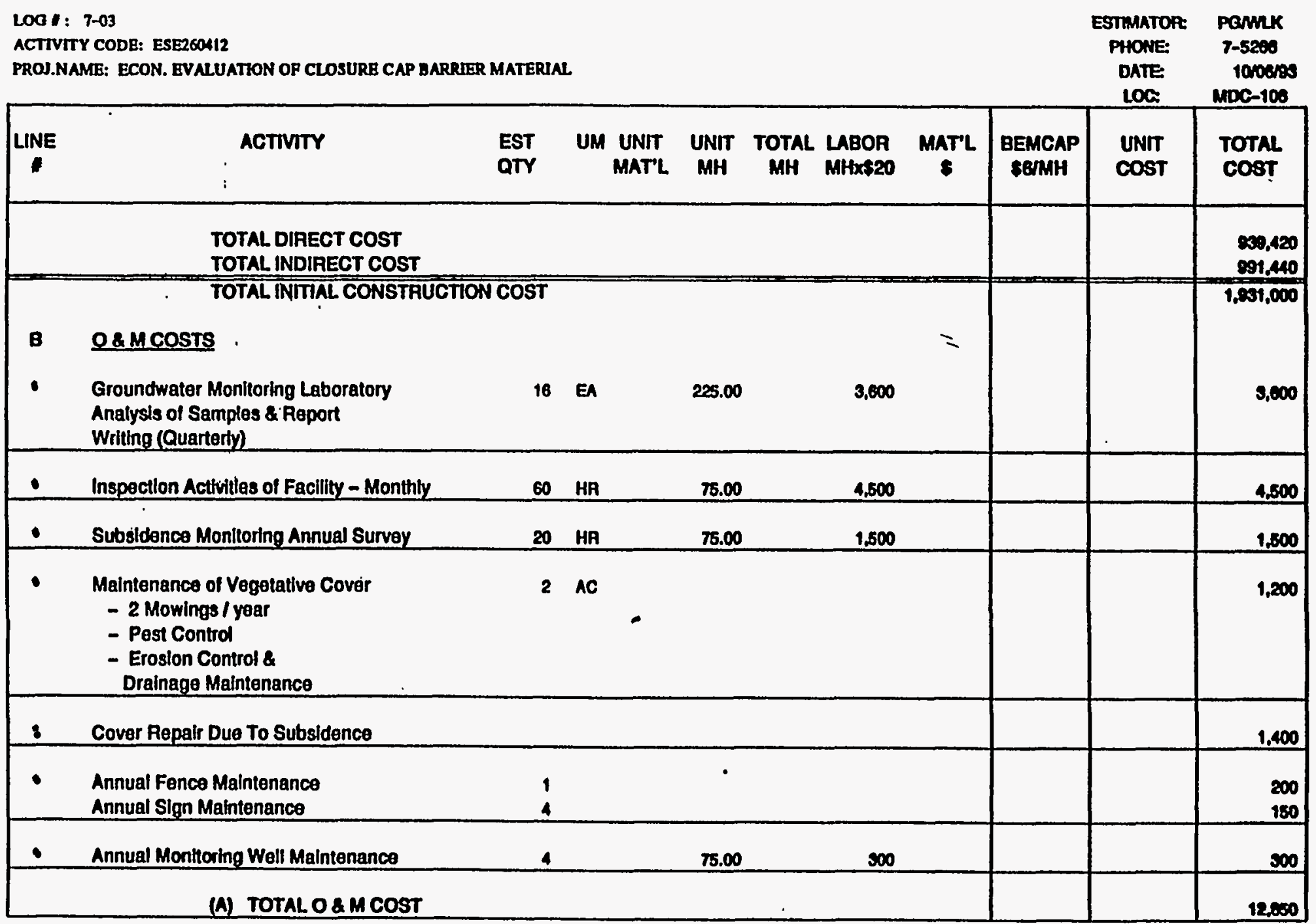

E-1. Cost Estimate for a Cover System Utilizing Claymax and a 30-mil FML (Cont'd) 
1001: 7-03

ACTIVITY CODB: BSE260412

MROY.MAMR: BCON. BVALUATION OP CLOSURB CAP BARRIER MATBRLAL noc-10s

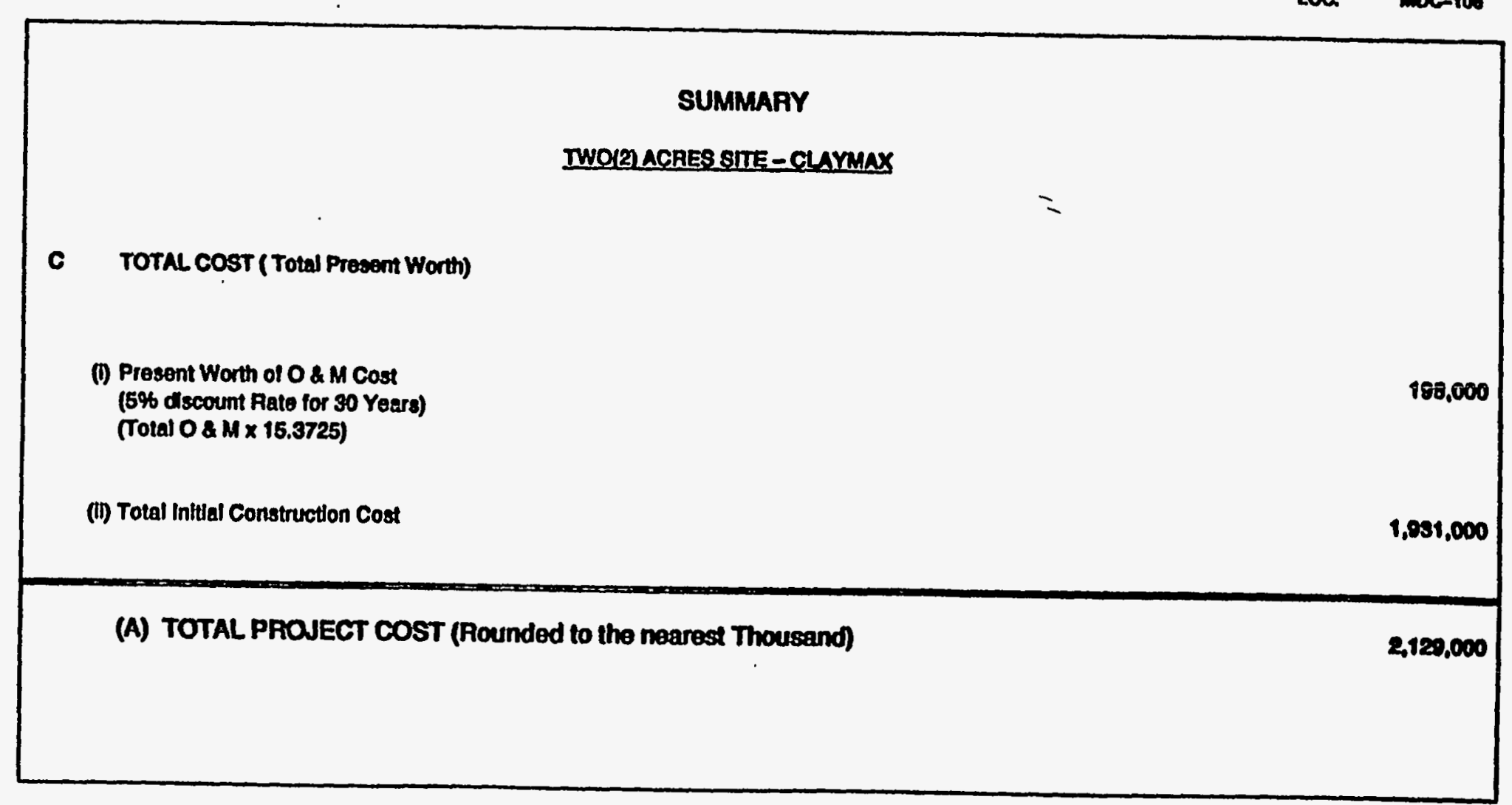

E-1. Cost Estimate for a Cover System Utilizing Claymax@ and a 30-mil FML (Cont'd) 


\section{DETAIL ESTIMATE}

2001: 7-03

ACTIVITY CODE: BSE2SON12

PROJ.NAME: ECON. BVALUATION OF CLOSURB CAP BARRIER MATERIAS

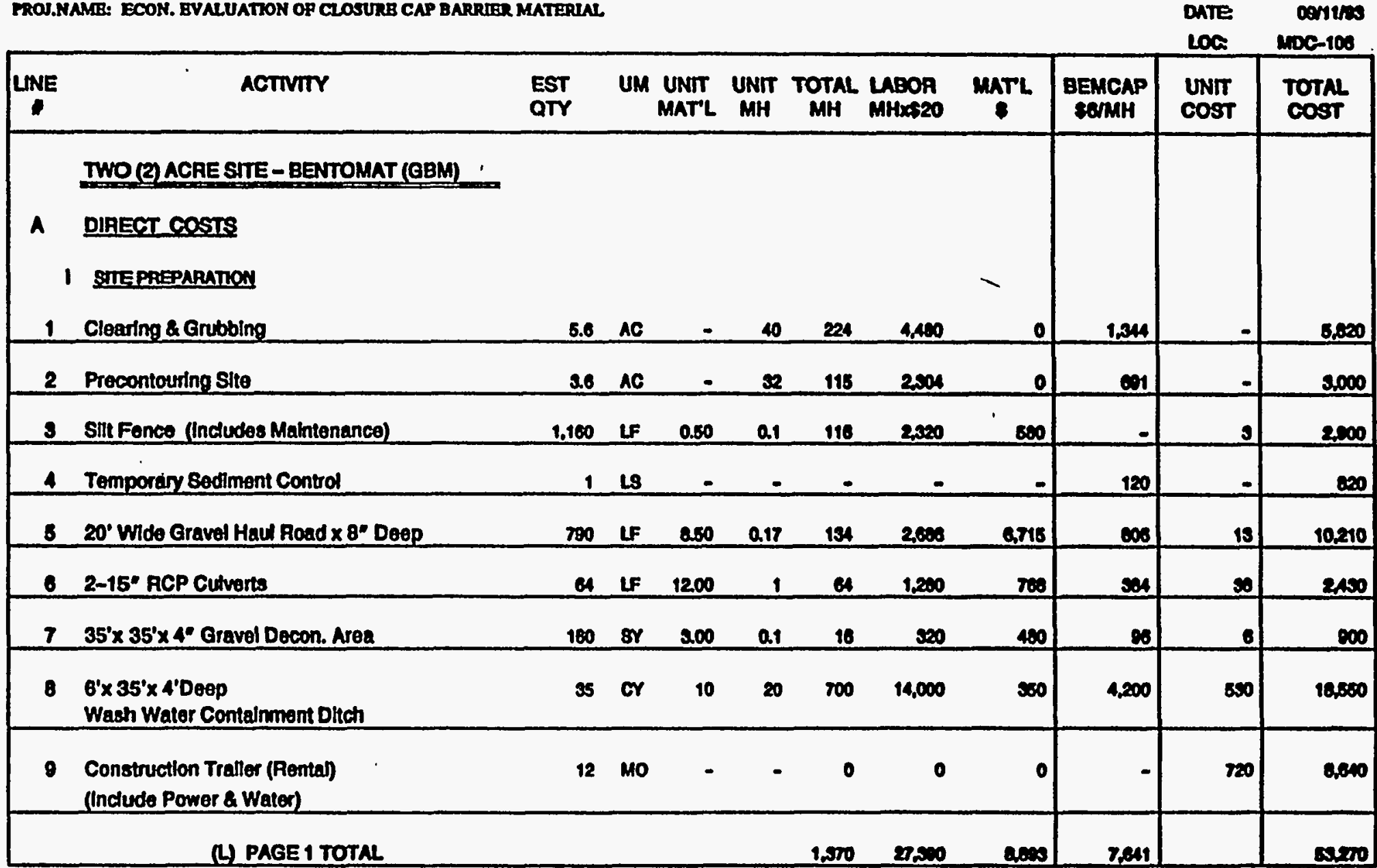

E-2. Cost Estimate for a Cover System Utilizing Bentomat@ and a 30-mil FML 


\section{DETAIL ESTIMATE}

AND BY: MEND

$2001,7-03$

ACTVITY CODE: ESE260N12

PROI.NAMR: ECON. BVALUATOON OP CLOSURB CAP BARRIER MATERUAL

\begin{tabular}{|c|c|c|c|c|c|c|c|c|c|c|c|}
\hline UNE & ACTIVITY & $\begin{array}{l}\text { EST } \\
\text { QTY }\end{array}$ & & $\underset{\text { MNTL }}{\text { UNT }}$ & UNIT & TOTAL & $\begin{array}{l}\text { LABOR } \\
\text { MHXat20 }\end{array}$ & $\underset{s}{\operatorname{MarL}}$ & $\begin{array}{l}\text { BEMCAP } \\
\text { SGMMH }\end{array}$ & $\begin{array}{l}\text { UnNIT } \\
\text { COST }\end{array}$ & $\begin{array}{l}\text { Total } \\
\text { cost }\end{array}$ \\
\hline 10 & $\begin{array}{l}\text { LCONER CONSTRUCTION } \\
\text { Waste Stabilization (Crushod Limestone) }\end{array}$ & 4.028 & $\pi$ & 100 & $a_{1}$ & 403 & 0,050 & 400,000 & 2,417 & 100 & 413,270 \\
\hline 11 & $\begin{array}{l}\text { Place and Compact Foundatton Soll } \\
\text { h } 8^{\circ} \text { Litis to } 95 \% \text { Stctandard Proctor }\end{array}$ & 16,140 & or & - & 0.158 & 2,502 & 80,084 & 0 & 18,010 & 4 & 60,040 \\
\hline 12 & Placement of Bentonite Geotextile Mat & 123,200 & 8f & 0.45 & 0.01 & 1,202 & 24,640 & 00,100 & - & 1 & $\mathbf{\infty 0 , 0 0 0}$ \\
\hline 13 & $\begin{array}{l}\text { Placomant of } 50 \text { un Fexible } \\
\text { Mombrane Uner }\end{array}$ & 123,200 & 8F & 0.17 & 0.0078 & 284 & 18,400 & 20,040 & - & 0 & 50,400 \\
\hline 14 & $\begin{array}{l}\text { Piacement of 1'-0 Coarse Sand } \\
\text { Dralnege Layor }\end{array}$ & 4,240 & or & 10.50 & 0.08 & 127 & 2,840 & 40,004 & $7 \infty$ & 11 & 47,500 \\
\hline 16 & $\begin{array}{l}\text { Placememt of Gootextho (TYPAR) Filter on } \\
\text { 1'-0" Coarse Sand Dralnage Layer }\end{array}$ & 14,022 & 8r & 0.17 & 0.006 & $\pi$ & 1.402 & 2,304 & - & 0 & $2, \pi \infty 0$ \\
\hline 16 & Placoment of $1^{\prime}-6^{\prime}$ Common Fil & 4,048 & Cr & 2.75 & 0.06 & 201 & 8,818 & 10,100 & 1.745 & 8 & 20,740 \\
\hline 17 & Placoment of $\theta^{\prime}$ Topsoll & 1.020 & or & 0.50 & 0.06 & 100 & 2,184 & 17,2900 & $\infty$ & 11 & 20.150 \\
\hline 18 & $\begin{array}{l}\text { Placement of Perimater Exterior } \\
\text { Drainage Layer } 3 / 4 " \times 3^{*} \text { OP (Crushod Stone) }\end{array}$ & $\$ 12$ & 8Y & 1.50 & 0.2 & 82 & 1,048 & 818 & $\mathbf{9 4}$ & 7 & 2,200 \\
\hline 19 & Excavation of Trapezoldal Dralnage Ditch & 682 & or & - & 0.175 & 164 & 3.007 & 0 & 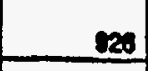 & 8 & 4.010 \\
\hline & (L) PAGE 2 TOTAL & & & & & 8,000 & 11rowe & 8202200 & 22,015 & & 700,100 \\
\hline
\end{tabular}

E-2. Cost Estimate for a Cover System Utilizing Bentomat $\$$ and a 30-mil FML (Cont'd) 
Loo 1: 7-03

ACTIVTY CODE: BSE260412

MROS.NAME: ECON. BYALUATTON OP CLOSURB CAP BARRIER MATERLAL

estanator pormL

DATE

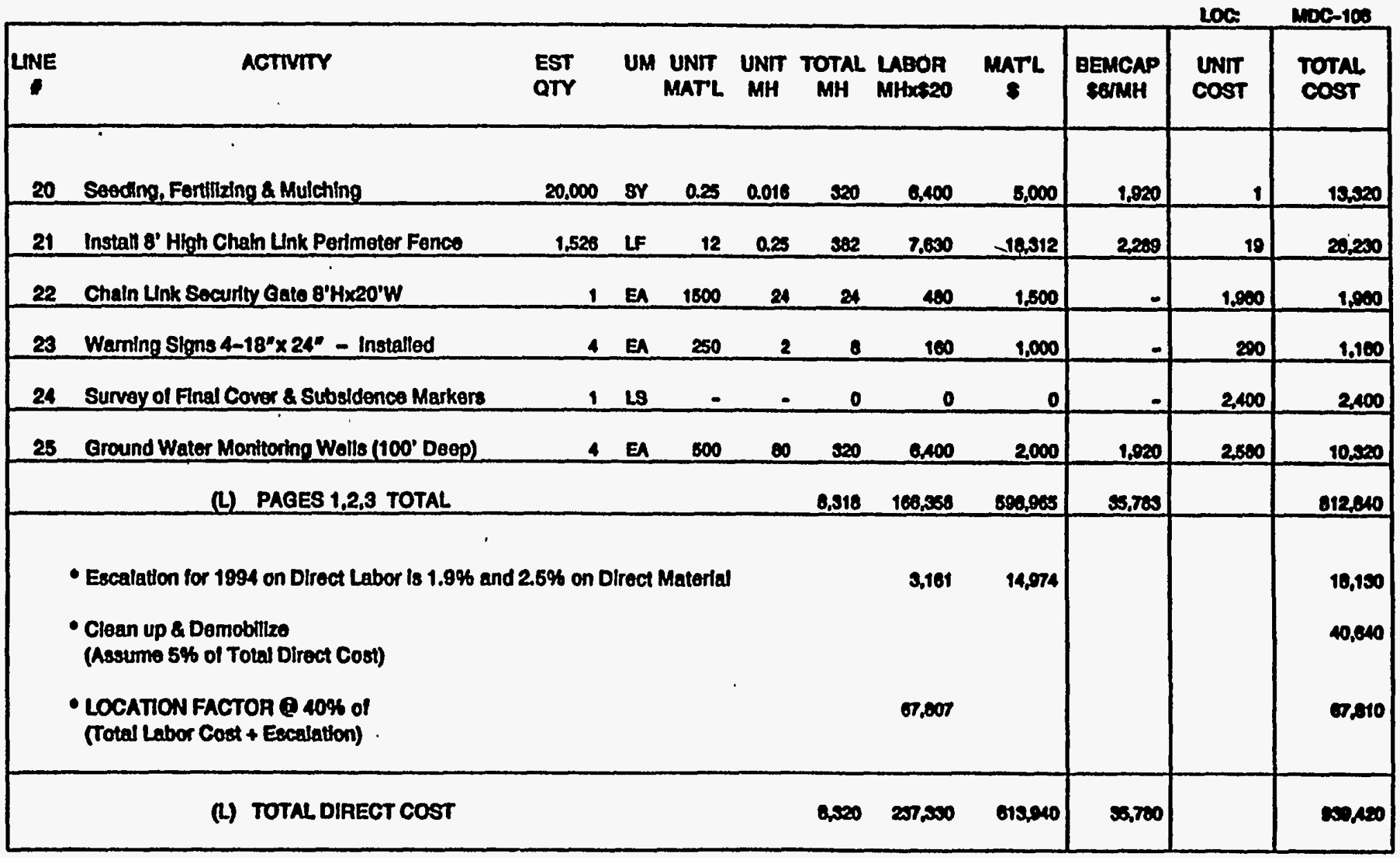

E-2. Cost Estimate for a Cover System Utilizing Bentomat $₫$ and a 30-mil FML (Cont'd) 
DETAIL BSTIMAT B

1001: 2-03

ACTIVITY CODB: ESE260412

MOS.NAMAR: BCON. BVALUATTON OF CLOSURB CAP BARRTRR MATERIALL

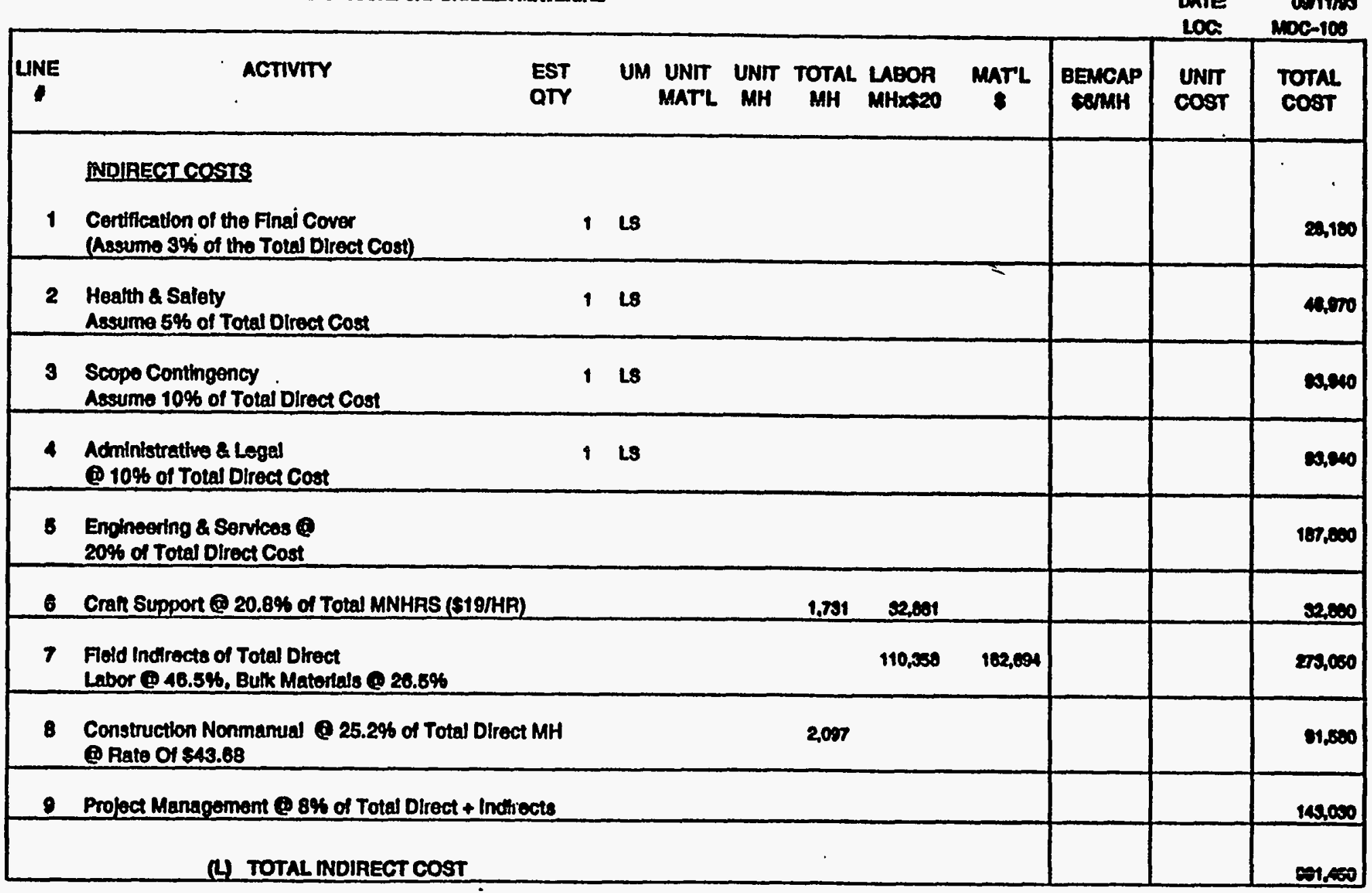

E-2. Cost Estimate for a Cover System Utilizing Bentomat@ and a 30-mil FML (Cont'd) 
D B T A I L B S T I M A T B

Loos: $1-03$

ACTVITY CODE: BSE26012

PROS.NAME: BCON. EVALUATION OP CLOSURB CAP BARRIER MAATERUAL

CHEDBY: QMEAD

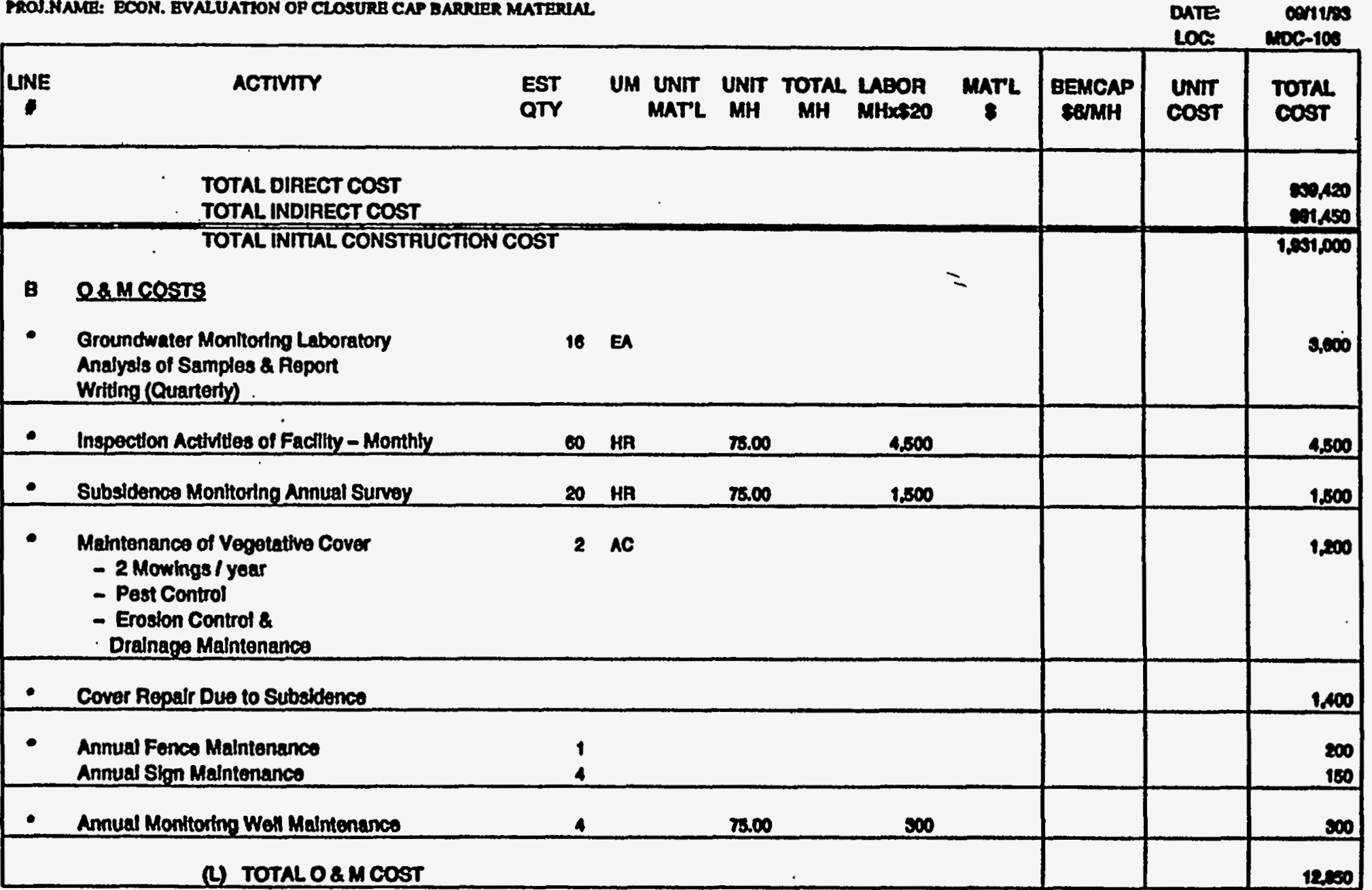

E-2. Cost Estimate for a Cover System Utilizing Bentomat and a 30-mil FML (Cont'd) 
D B TA I L B S T I A T B

\begin{tabular}{|c|c|}
\hline Carco or: & a MEAD \\
\hline $\begin{array}{l}\text { Estminton } \\
\text { PHone } \\
\text { onte } \\
\text { Loc: }\end{array}$ & $\begin{array}{c}\text { Pannk } \\
7-62006 \\
0011190 \\
\text { moc-100 }\end{array}$ \\
\hline
\end{tabular}

2001: 7-03

ACTVITY CODB: BSB260412

FOU.MAME: BCON. EVALUATION OP CLOSURB CAP DARRIER MATERULL.

Loc.

$\operatorname{moc}-100$

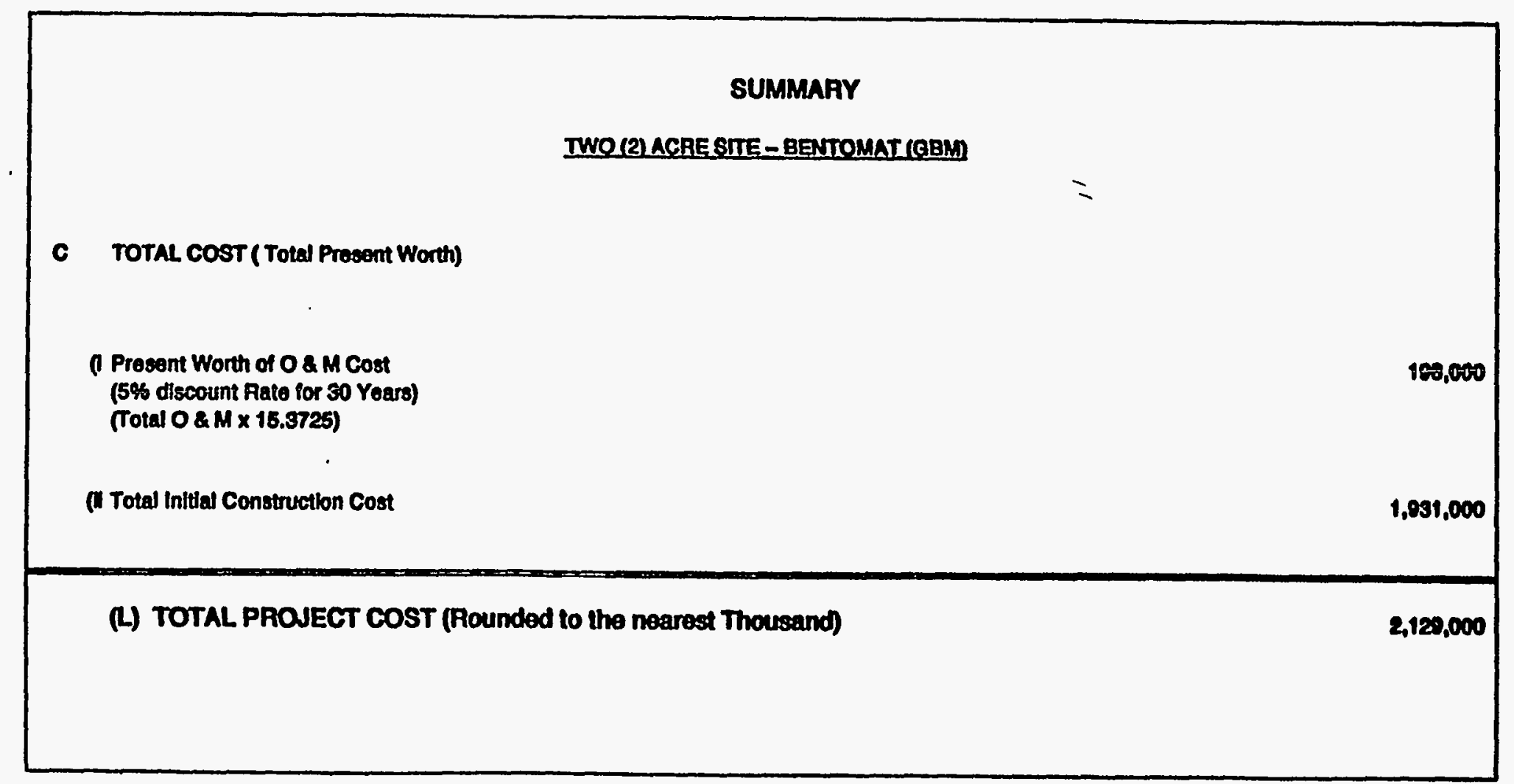

E-2. Cost Estimate for a Cover System Utilizing Bentomat@ and a 30-mil FML (Cont'd) 
D B TAILE B T IMATE

L001: 7-03

ACTIVITY CODE: ESE2CON12

FROU.NAME: ECON. EVALUATION OF CLOSURB CAP BARRRBR MATERIAL

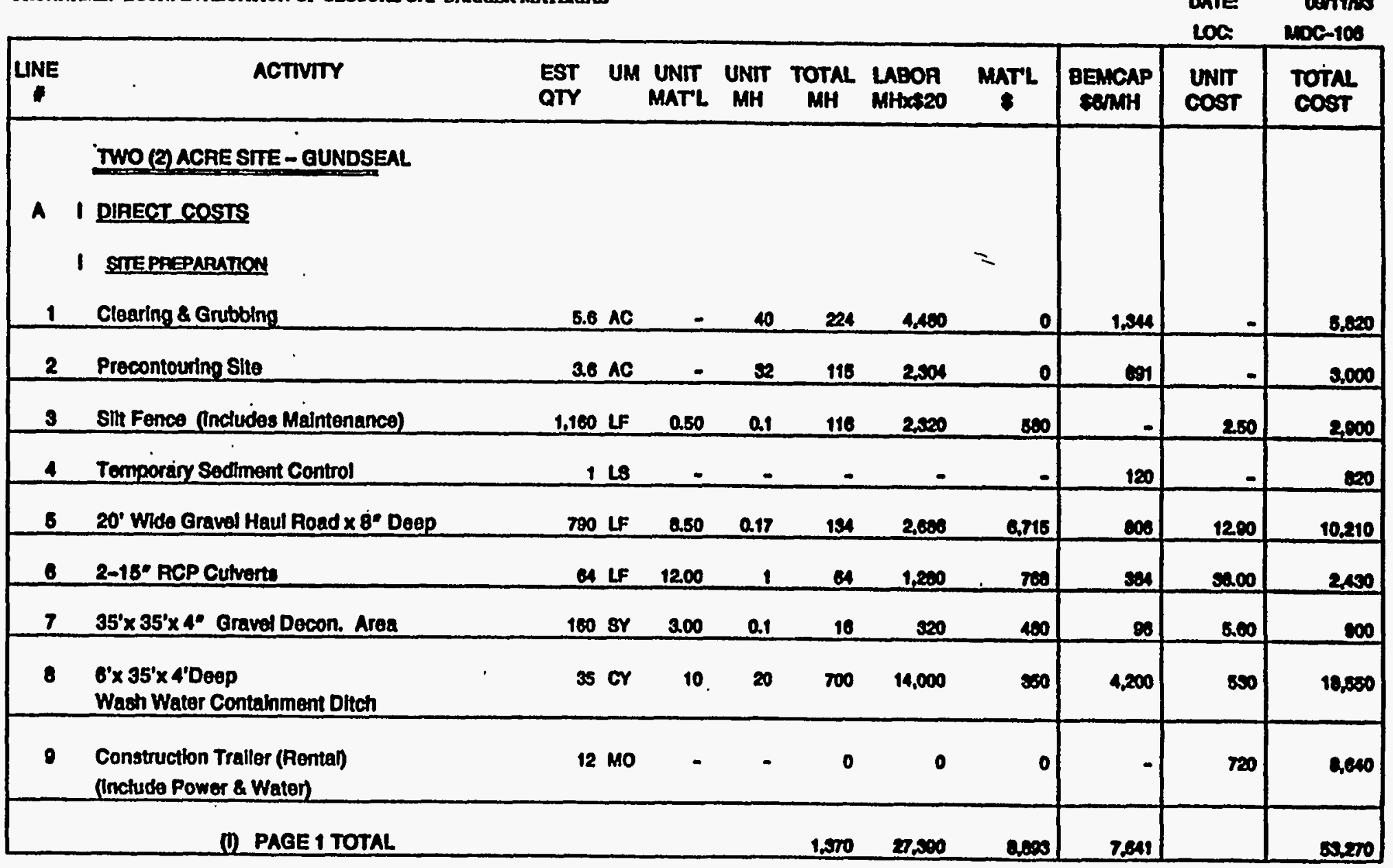

E-3. Cost Estimate for a Cover System Utilizing Gundseal(8) 
DETAIL BSTIMATB

CHCD 8Y: S.MEND

\section{Lo01: 7-03}

ACTIVITY CODE: BSE200412

PROJ.NAME: ECON. EVALUATTON OP CLOSURB CAP BANRURR MATRRIAL

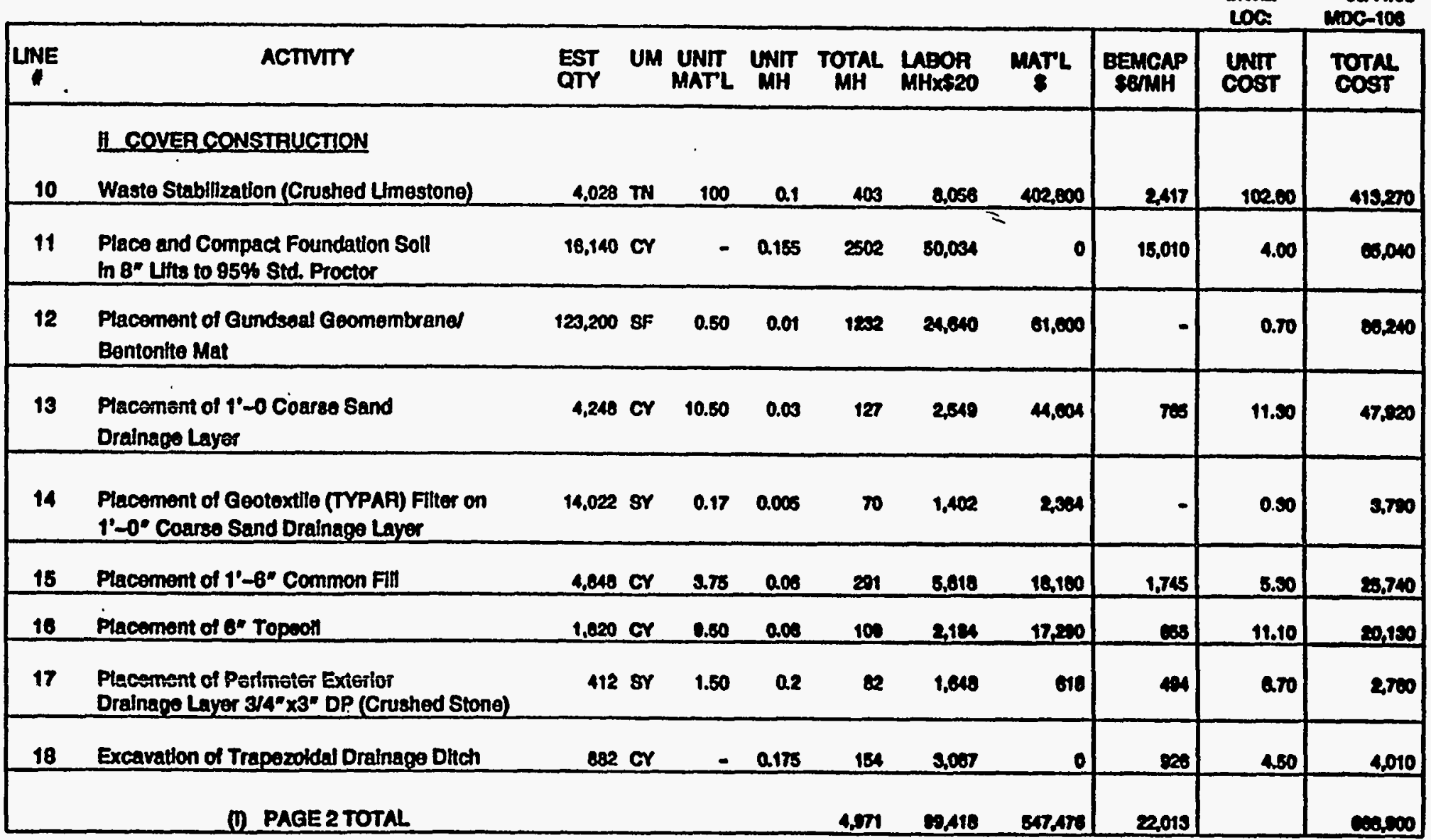

E-3. Cost Estimate for a Cover System Utilizing Gundseal@ (Cont'd) 


\section{B T A I L B S T I M A T B}

2001: 7-03

ACTIVITY CODB: BSE260412

FWOU.MAMER BCON. EUALUATION OP CLOSURB CAP BNRRIER MATERLAL

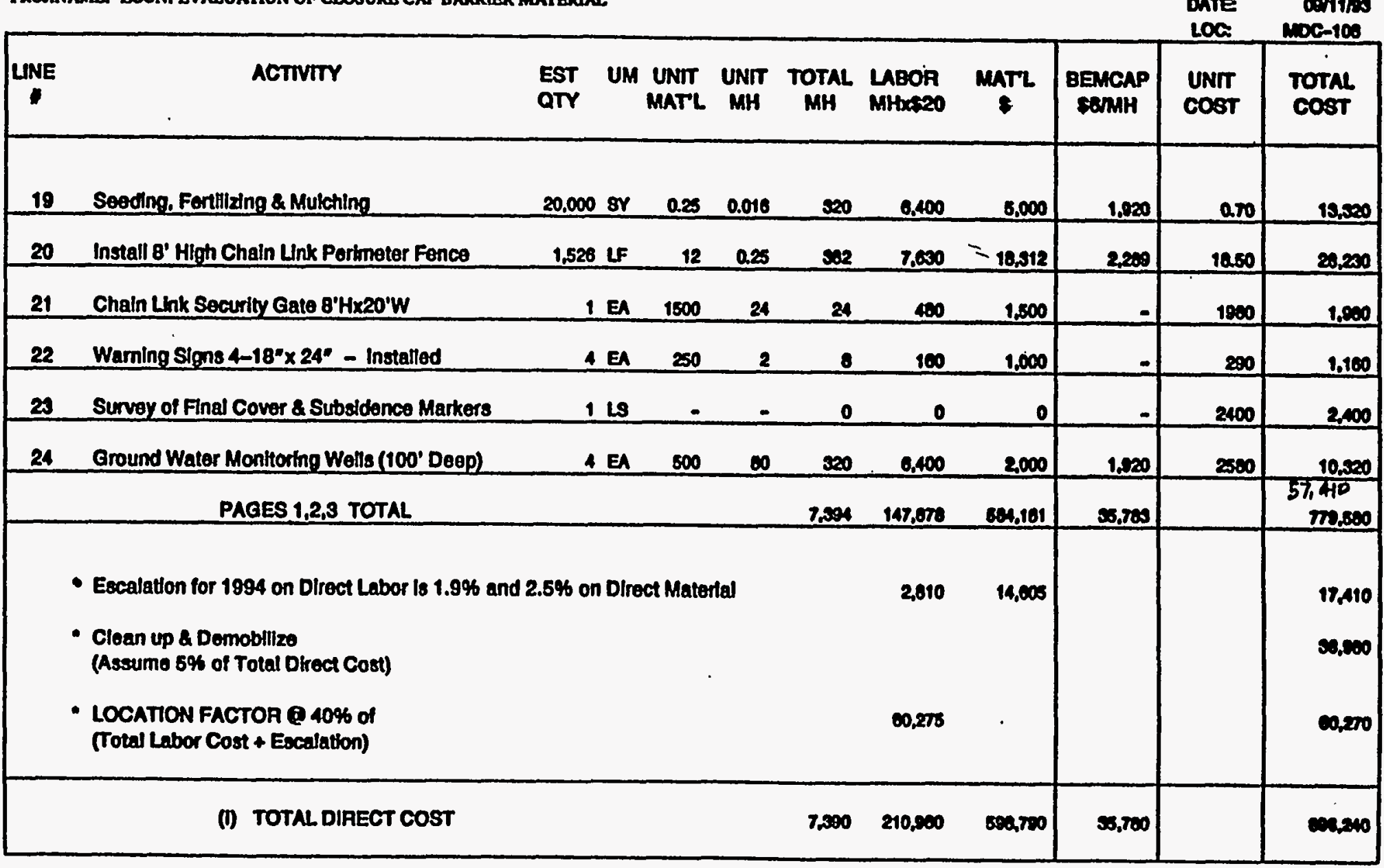

E-3. Cost Estimate for a Cover System Utilizing Gundseal@ (Cont'd) 
D B TAIL BSTIMATB

Loo $0: 7-03$

ACTTVTY CODB: BS1E26012

TROS.NAME: BCON. EVALUATION OF CLOSURRB CAP BARRIER MATERUAL

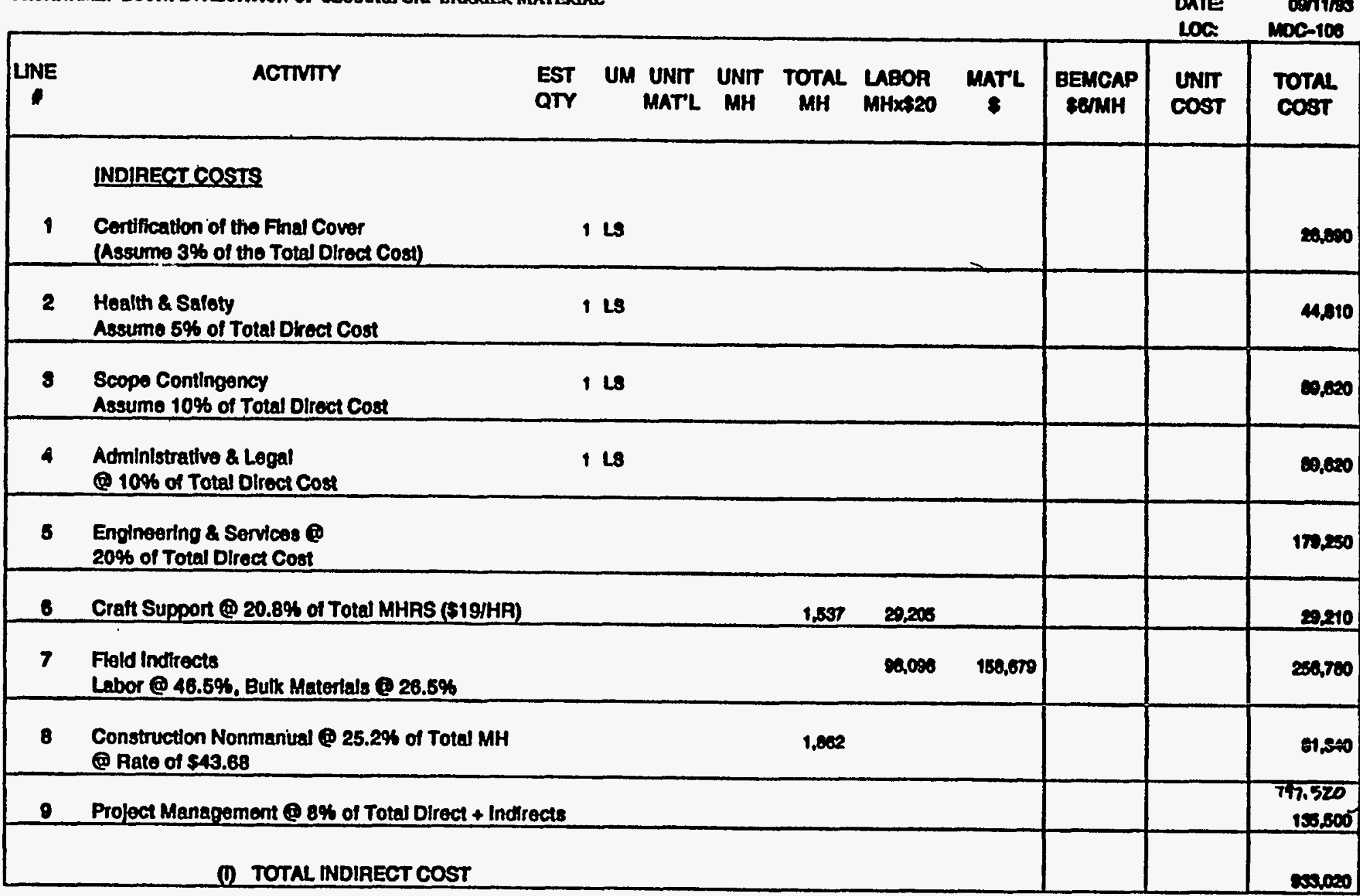

E-3. Cost Estimate for a Cover System Utilizing Gundseal $\$$ (Cont'd) 
CHED BY: 2 MEND

\section{1: 7-03}

ACTIVITY CODB: BSE200412

PLOY.NAME: BCON. BVALUATION OP CLOSURB CAP BARUIBR MATERIAL

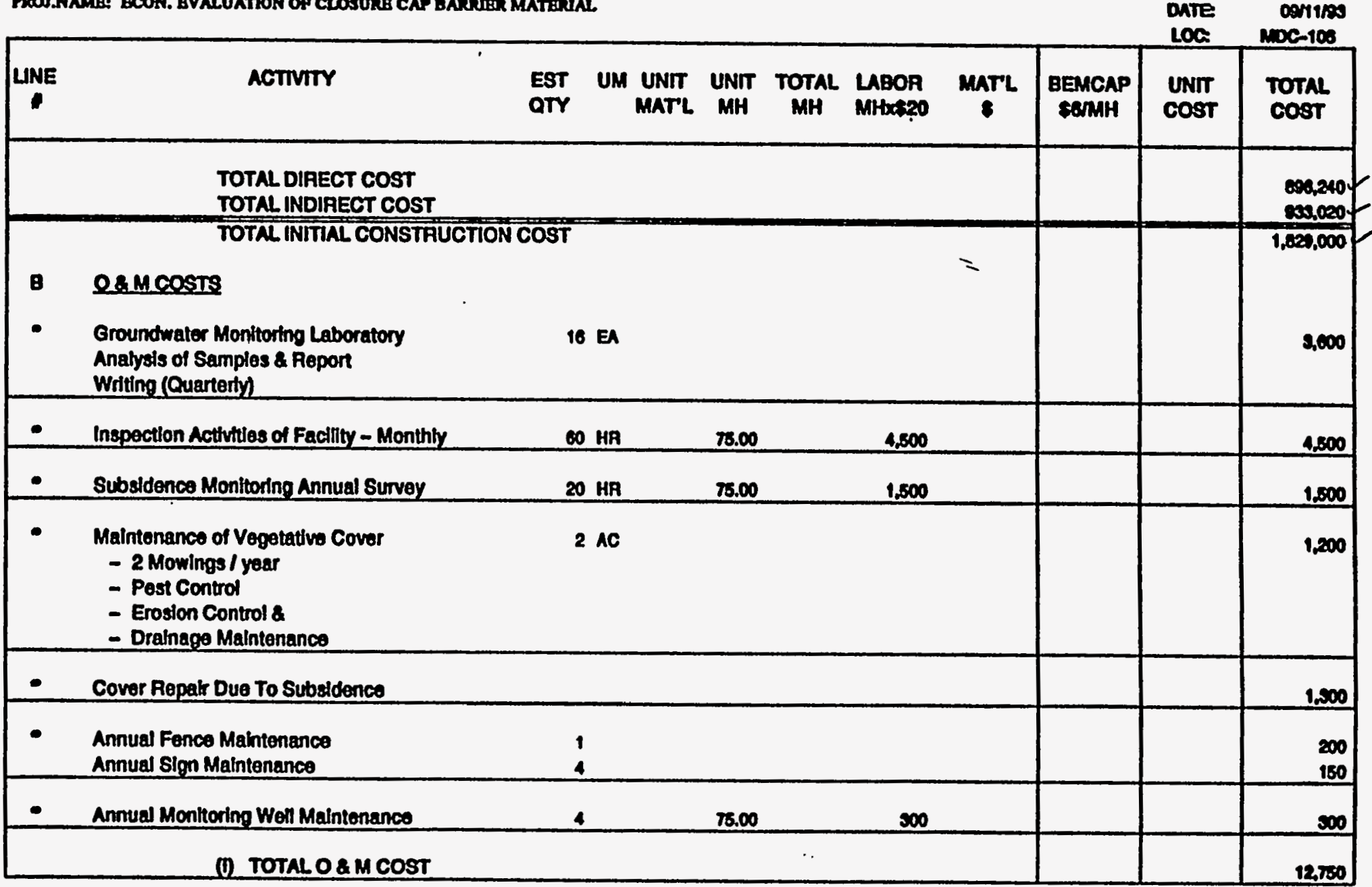

E-3. Cost Estimate for a Cover System Utilizing Gundseal@ (Cont'd) 


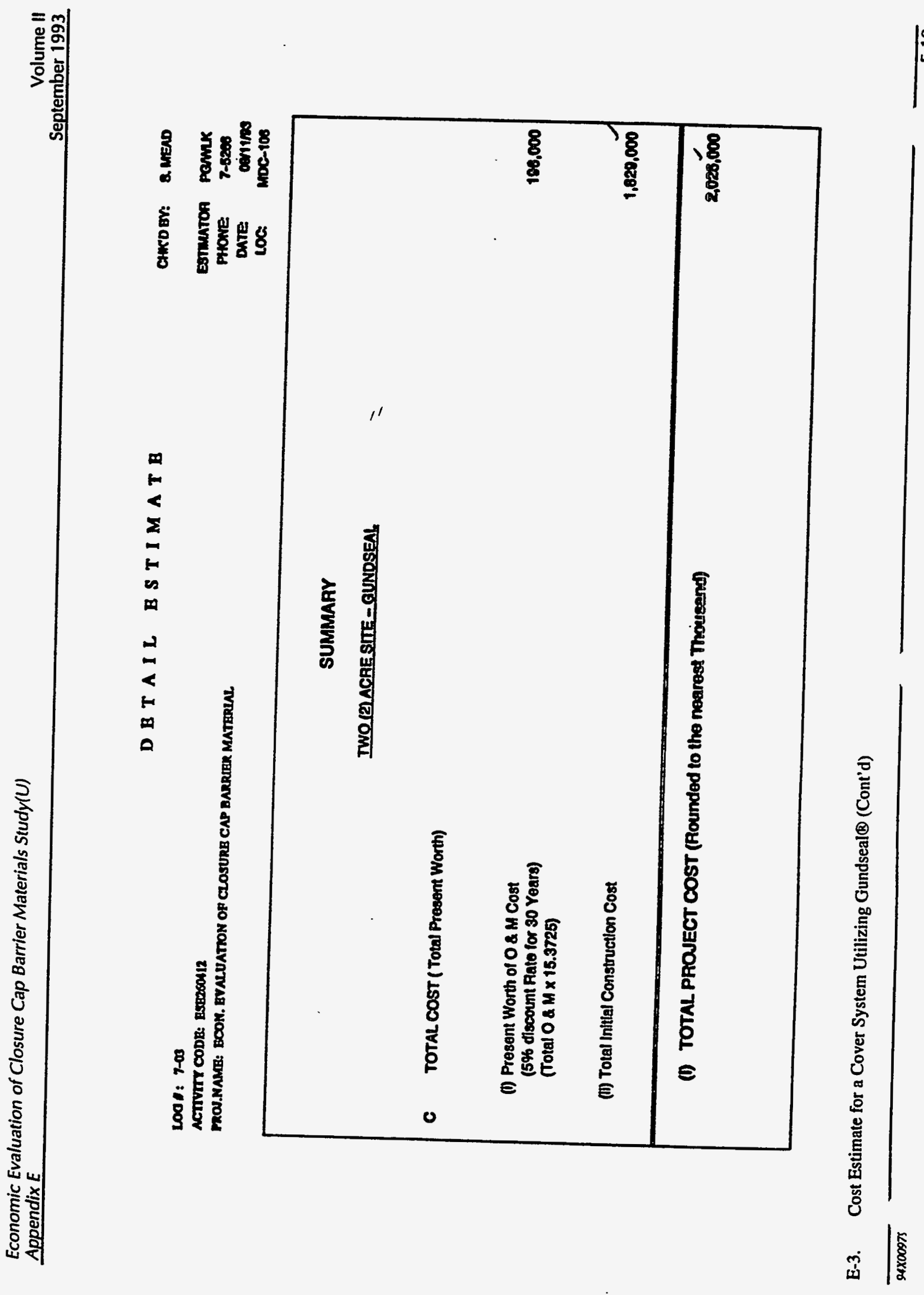




\section{DETAIL ESTIMATE}

$1001: 7-03$

ACTIVITY CODE: BSE260112

MOJ.NAME: BCON. BVALUATTON OP CLOSURB CAP BARRRER MATBRIAS

\begin{tabular}{|c|c|c|c|c|c|c|c|c|c|c|c|}
\hline LNE & ACTIVITY & $\begin{array}{l}\text { EST } \\
\text { QTY }\end{array}$ & UM & $\begin{array}{l}\text { UNIT } \\
\text { MATL }\end{array}$ & $\begin{array}{c}\text { UNIT } \\
\text { MH }\end{array}$ & $\underset{\text { MH }}{\text { TOTAL }}$ & $\begin{array}{l}\text { LABOR } \\
\text { MHxat20 }\end{array}$ & $\underset{*}{\operatorname{MaTL}}$ & $\begin{array}{l}\text { BEMCAP } \\
\text { SOMHH }\end{array}$ & $\begin{array}{l}\text { UNIT } \\
\text { Cost }\end{array}$ & $\begin{array}{l}\text { Total } \\
\text { Cost }\end{array}$ \\
\hline $\begin{array}{l}A \\
1 \\
\end{array}$ & $\begin{array}{l}\text { TWO ACAES (2) ACRE SITE - (120 MII } \\
\text { I DIRECT COSTS } \\
1 \text { SIEPPRPARAMON } \\
\text { Cloarting \& G Gubbing }\end{array}$ & 5.6 & AC & - & 40 & 234 & 1400 & $=$ & $\tan$ & - & 8,020 \\
\hline 2 & Precontourng Sile & 3.6 & AC & - & 32 & 115 & 2,504 & 0 & ert & $=$ & 8,000 \\
\hline 3 & Silt Fence (Inctudes Malntenanco) & 1.100 & LF & 0.50 & 0.1 & 118 & 2,2000 & 800 & - & 250 & 2000 \\
\hline 4 & Tomporary Sediment Control & 1 & Le & - & - & - & $=$ & - & 120 & - & 820 \\
\hline 5 & $20^{\circ}$ Whot Gravel Haut Road $\times \theta^{\circ}$ Deep & 780 & LF & $\mathbf{0 . 5 0}$ & 0.17 & 134 & 2608 & 0,718 & $\infty$ & 1200 & 10,210 \\
\hline B & 2-15* RCP Culverts & 64 & LFF & 1200 & 1 & 64 & 1,200 & 700 & $\operatorname{sen}$ & 30.00 & 2400 \\
\hline 7 & $35^{\prime} \times 35^{\prime} \times 4^{\prime}$ Gravel Decon. Area & 160 & BY & 3.00 & 0.1 & 16 & 520 & 400 & $\boldsymbol{\omega}$ & 0.00 & 000 \\
\hline 8 & $\begin{array}{l}\text { 6'x } 35^{\prime} \times 4^{\prime} \text { Deep } \\
\text { Wash Water Contahment Ditch }\end{array}$ & 35 & Cr & 10 & 20 & 700 & 14,000 & $5 s 0$ & $\$ 200$ & 850 & 185000 \\
\hline 9 & $\begin{array}{l}\text { Construction Tralier (Rental) } \\
\text { (Include Power \& Waler) }\end{array}$ & 12 & MO & - & - & 0 & 0 & 0 & - & 720 & 2010 \\
\hline & (F) PAGE TOTAL & & & & & 1,370 & 21,500 & 0,000 & 7.041 & & 50,210 \\
\hline
\end{tabular}

E-4. Cost Estimate for a Cover System Utilizing 120-mil High Density Polyethylene (HDPE) Liner 
DETAIL ESTIMATE

CHco or: a meno

LOa I: 1-03

ACTVITY CODB: ESE260412

PROJ.NAME: ECON. BVALUATION OP CLOSURB CAP BARUIER MATERIAL

EgTmuTon Pamix

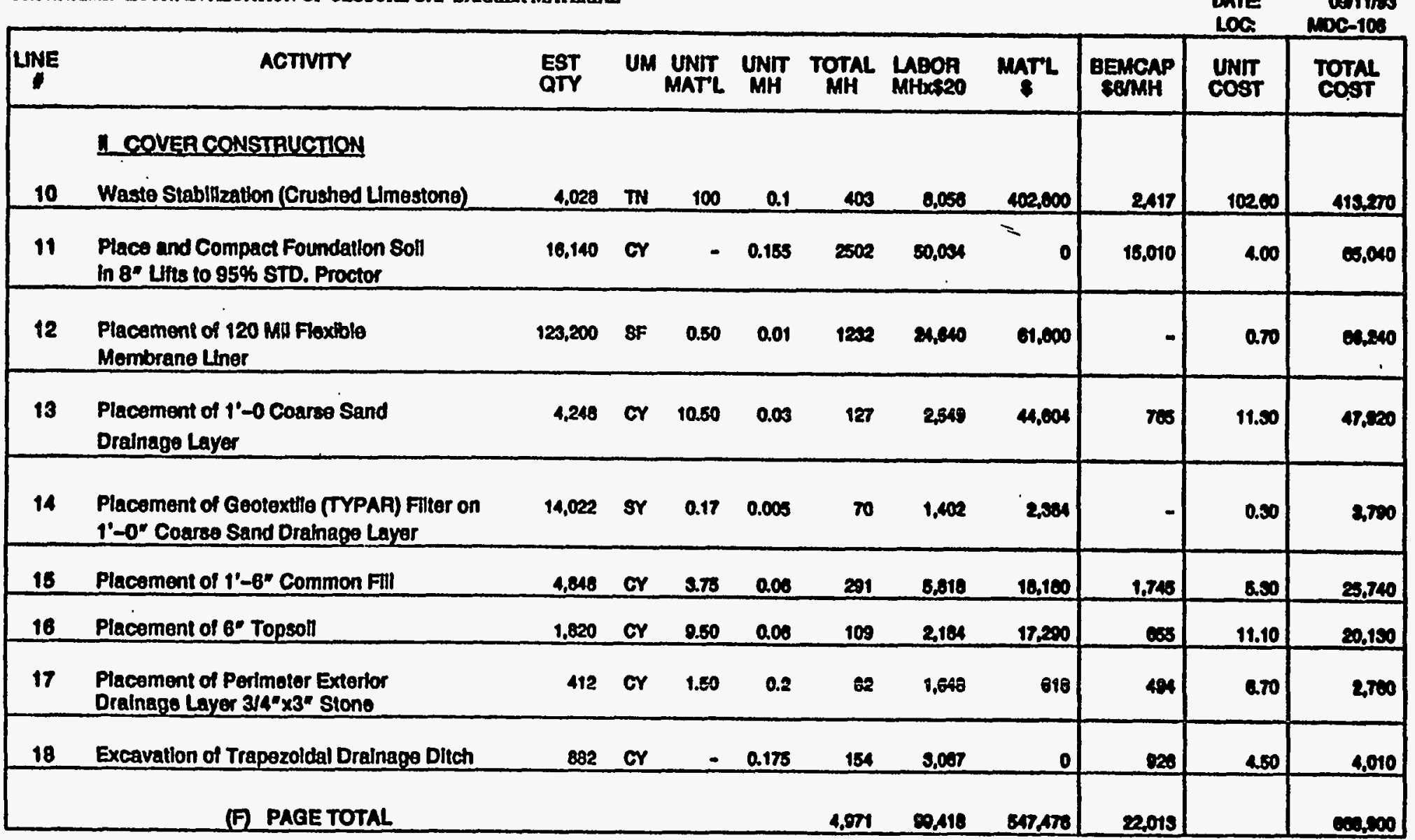

E-4. Cost Estimate for a Cover System Utilizing 120-mil High Density Polyethylene (HDPE) Liner (Cont'd) 
D B TAILE S T IMA B

CAxo BV: 2 MEND

Loo : : 7-03

ACTIVTY CODE: ESE2SO412

MOS.MAME: ECON. BVALUATHON OR CLOSURE CAP BARRER MATERIAL

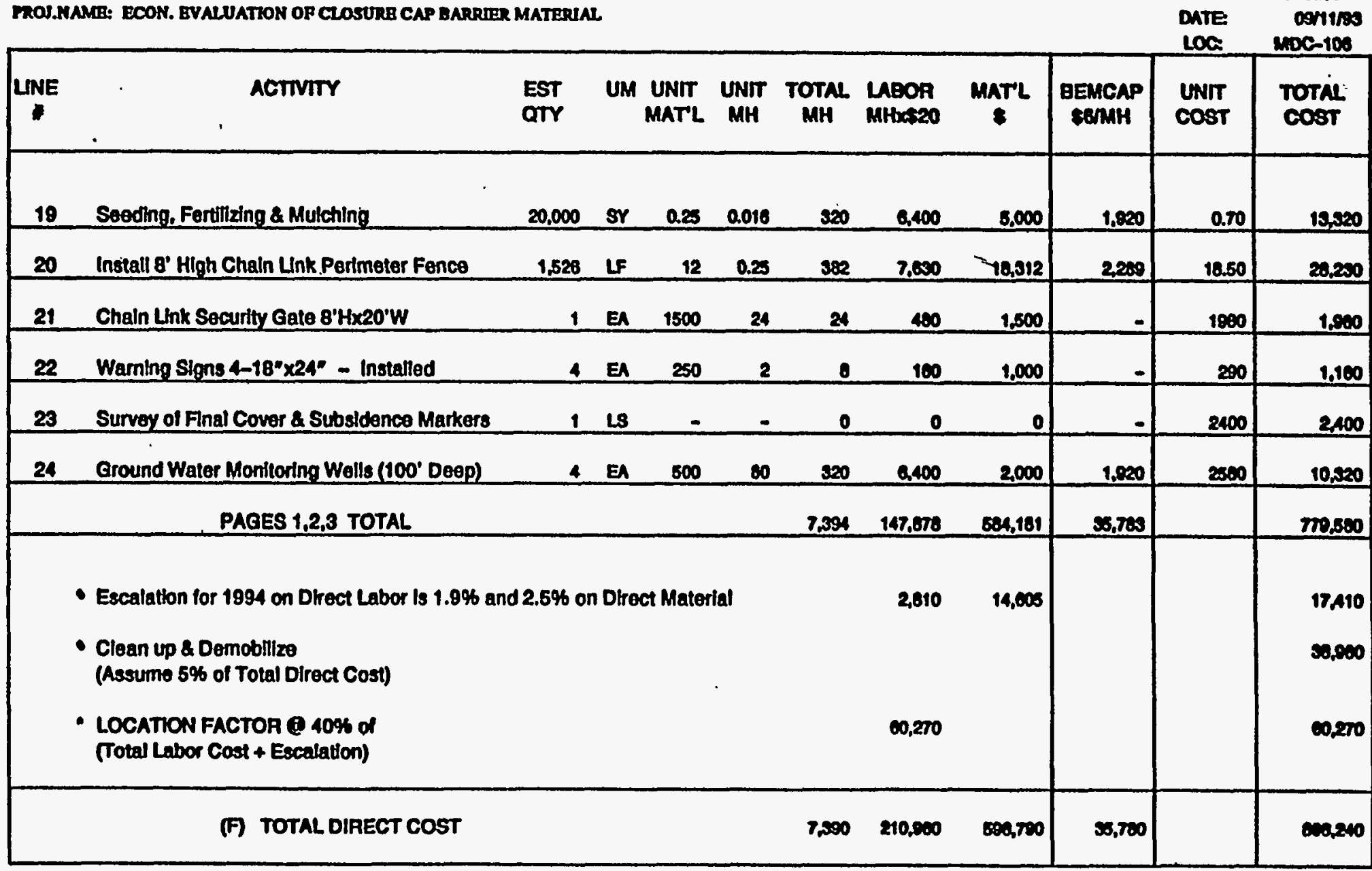

E-4. Cost Estimate for a Cover System Utilizing 120-mil High Density Polyethylene (HDPE) Liner (Cont'd) 
1001: $1-03$

ACTIVITY CODB: BSB260012

FROJ.NAMB: ECON. BVALUATTON OP CLOSURB CAP BARRIER MATERUAL

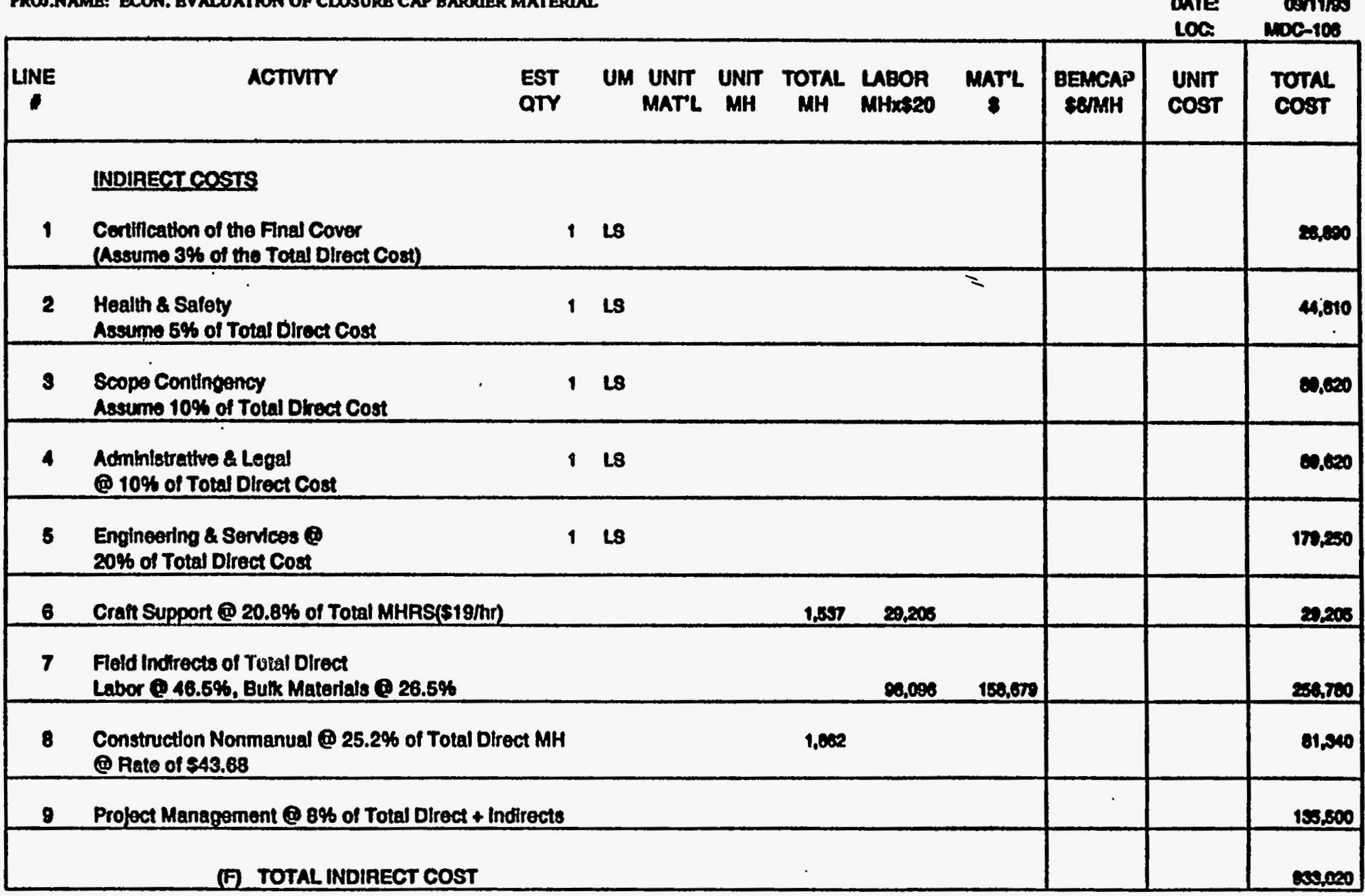

E-4. Cost Estimate for a Cover System Utilizing 120-mil High Density Polyethylene (HDPE) Liner (Cont'd) 
1001: 7-03

ACTIVTY CODB: BSB260412

PROJ.NAMBE: BCON. BVALUATTON OF CLOSURB CAP BARRRER MATERUAL.

Cuso Br: Q MEAD

ESTMATOR Pannk

PHowe 7-5206

DATE OON1190

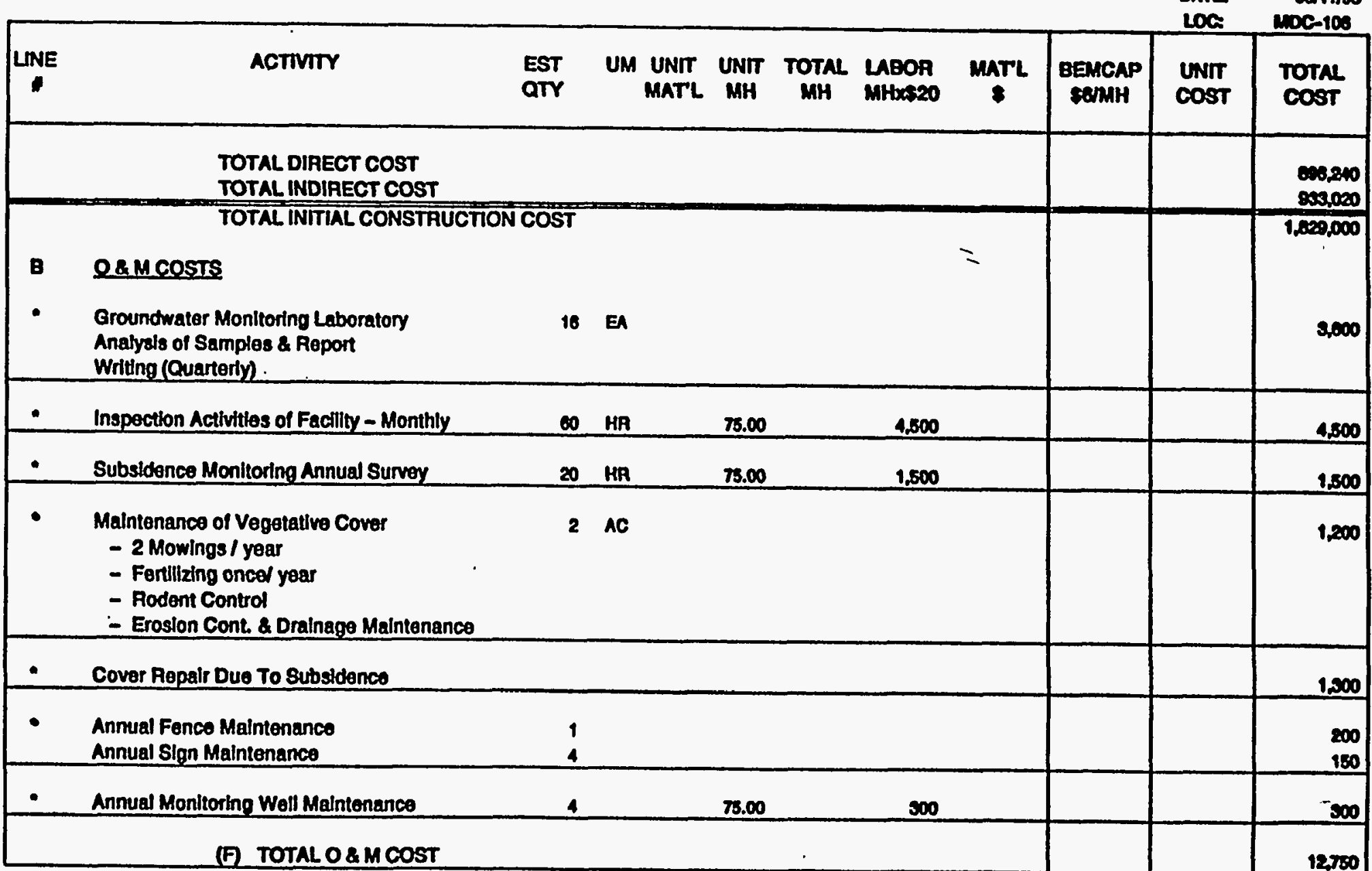

E-4. Cost Estimate for a Cover System Utilizing 120-mil High Density Polyethylene (HDPE) Liner (Cont'd) 
D B TAIL BSTIMATB

\section{1: 7-03}

ACTIVTY CODE: ESE26012

PROJ.NAMER: BCON. BVALUATHON OP CLOSURB CAP BARRIER MATERIAL.

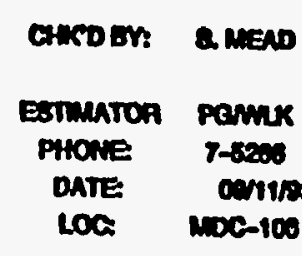

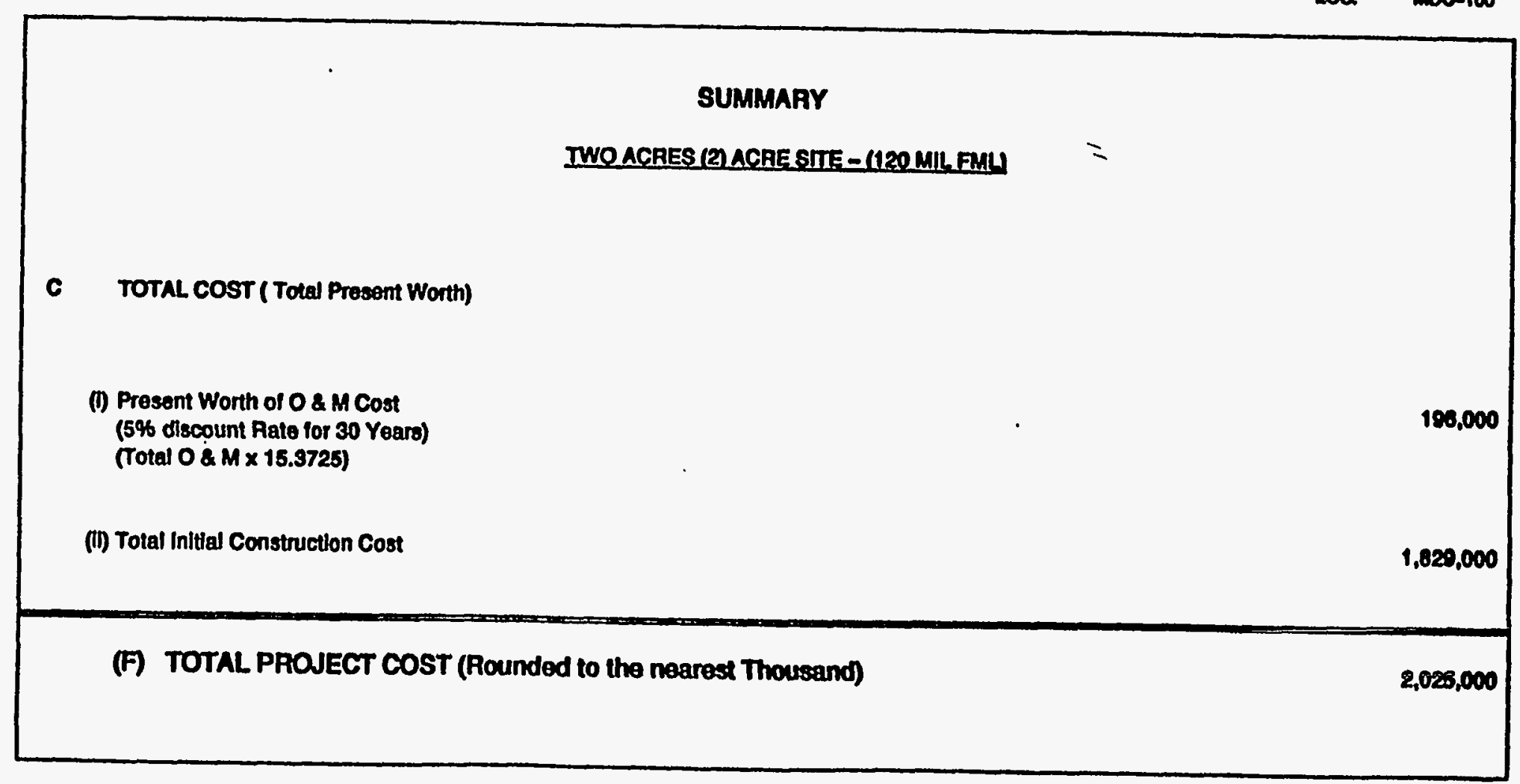

E-4. Cost Estimate for a Cover System Utilizing 120-mil High Density Polyethylene (HDPE) Liner (Cont'd) 
DETAIL ESTIMATE

Lo0 I: $7-03$

ACTIVITY CODB: ESEQSOH12

PROJ.NAME: ECON. BVALUATON OF CLOSURB CAP BARRRRR MATERIAL

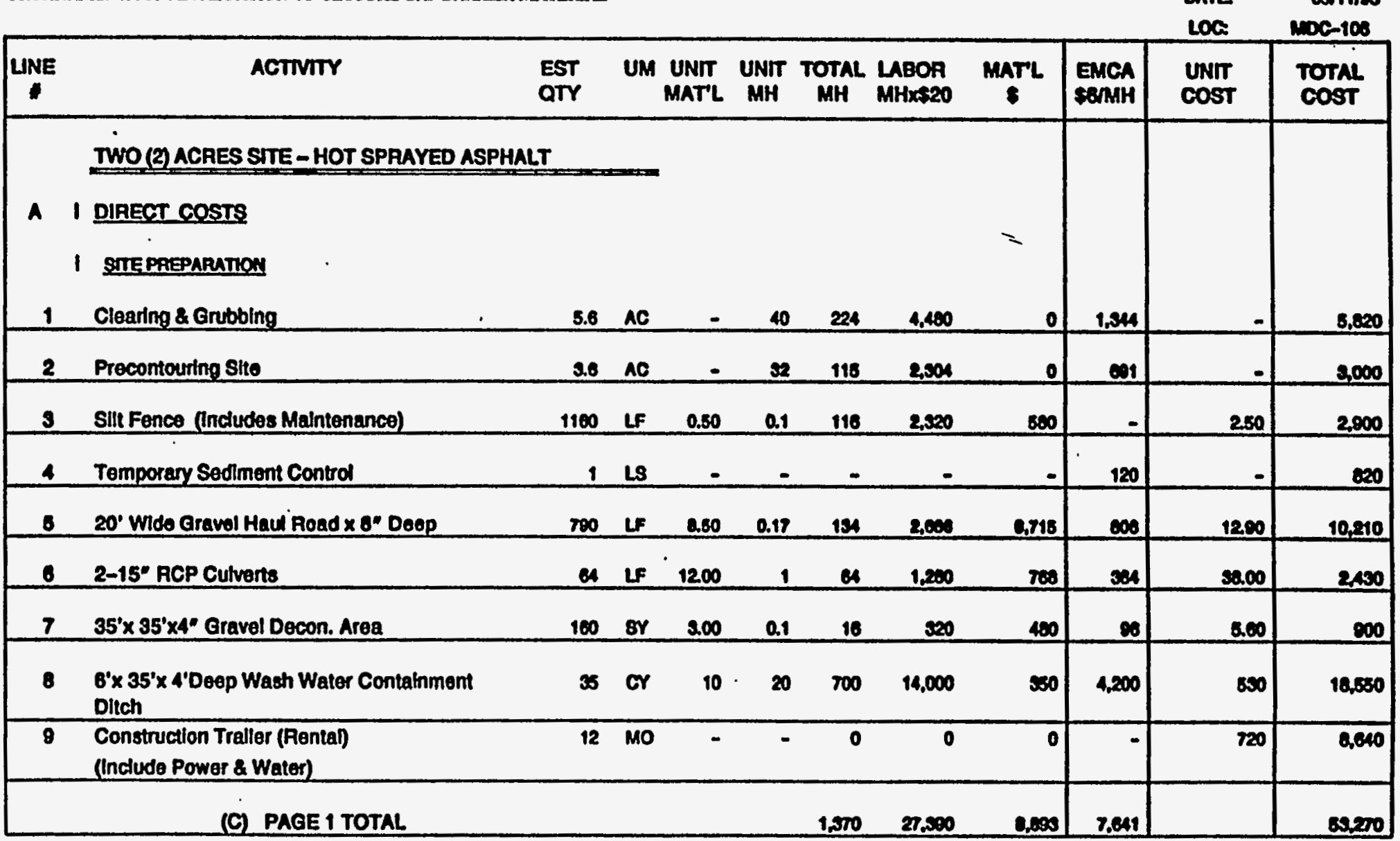

E-5. Cost Estimate for a Cover System Utilizing 1/4-inch Thick Hot Sprayed or Emulsified Asphalt 
D B T A L B S T IMATB CHWOBV: O.MEND

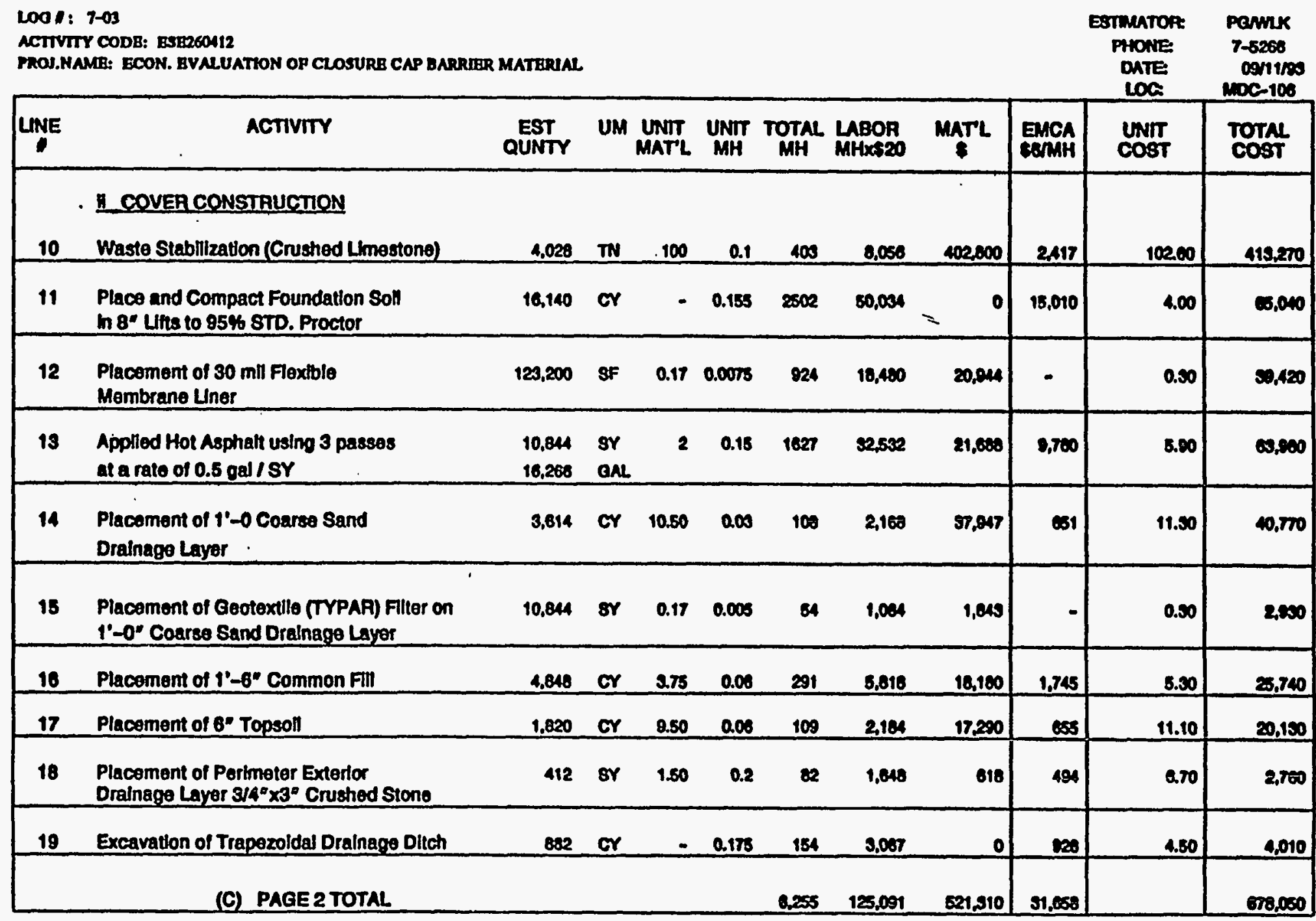

E-5. Cost Estimate for a Cover System Utilizing 1/4-inch Thick Hot Sprayed or Emulsified Asphalt (Cont'd) 


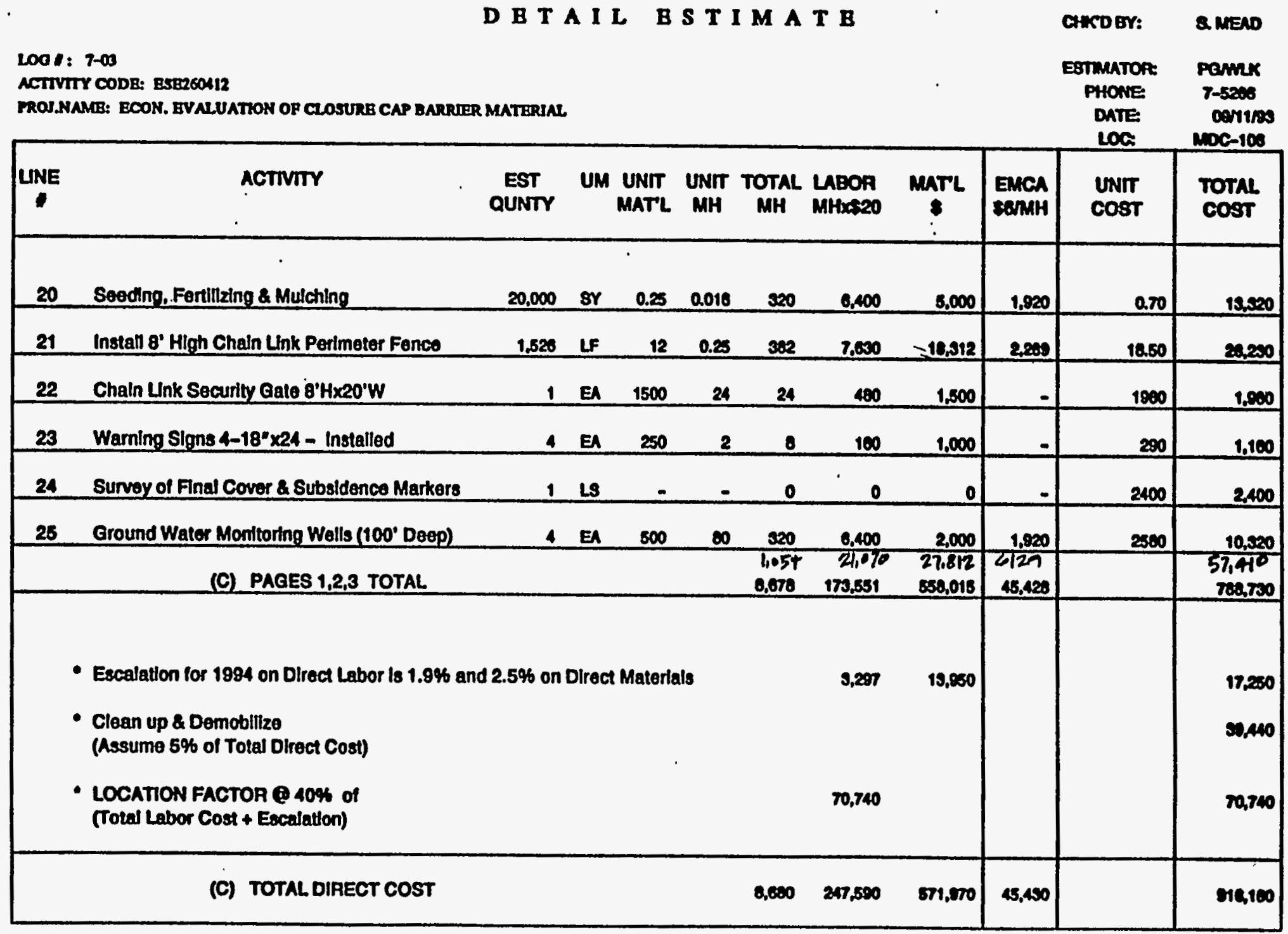

E-5. Cost Estimate for a Cover System Utilizing 1/4-inch Thick Hot Sprayed or Emulsified Asphalt (Cont'd) 
200 : 7-03

ACTIVTTY CODB: BSE260412

PROJ.NAME: ECON. BVALUATHON OF CLOSURB CAP BARRIRK MATBRIAL

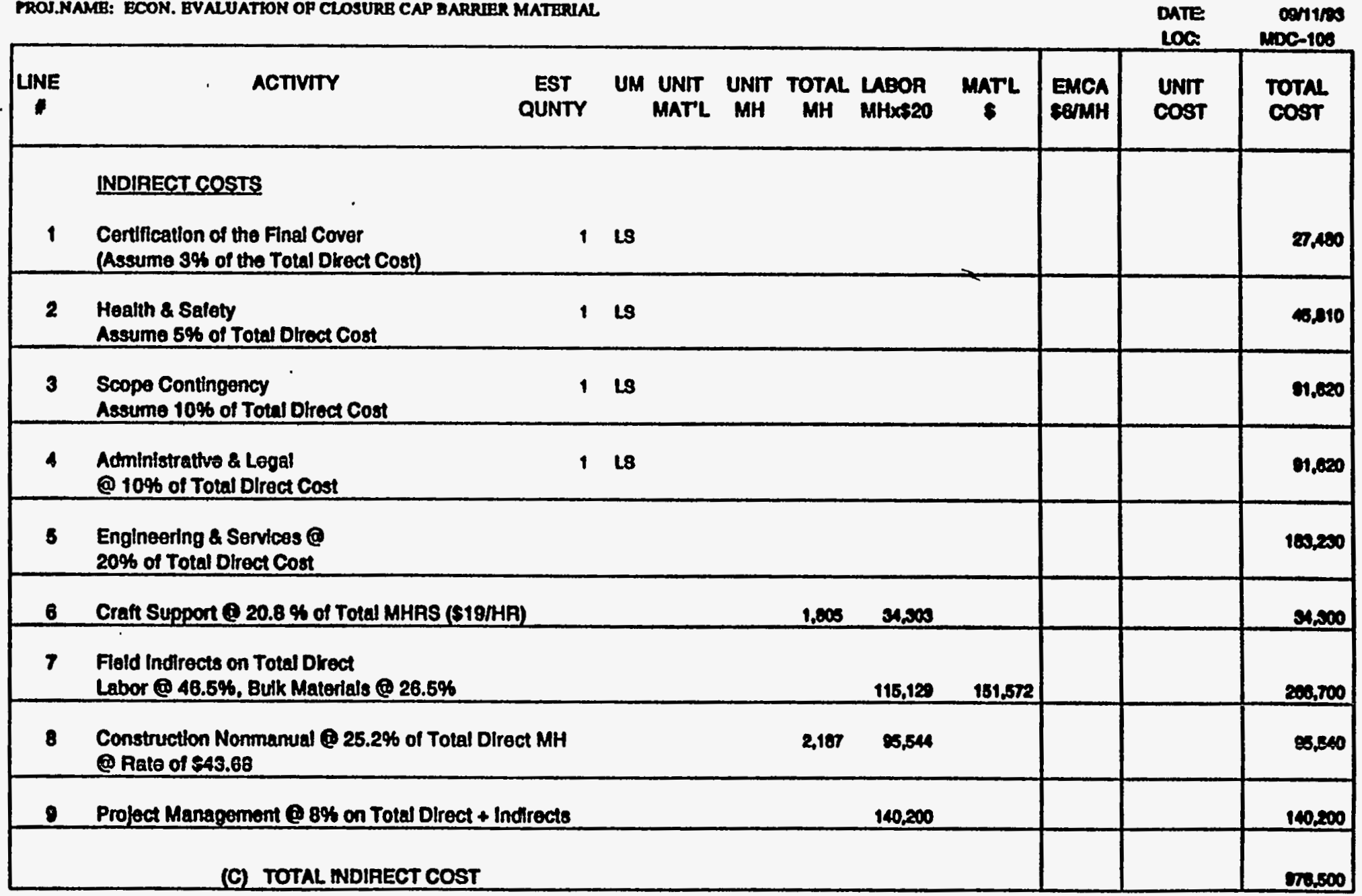

E-5. Cost Estimate for a Cover System Utilizing 1/4-inch Thick Hot Sprayed or Emulsified Asphalt (Cont'd) 
LOO I: 7-03

ACTIVITY CODE: ESEQSOH12

PROU.NAME: ECON. BVNLUATION OF CLOSURE CAP BARRTRR MATERUNL.

Pamink

PHONE 7-5200

DATE

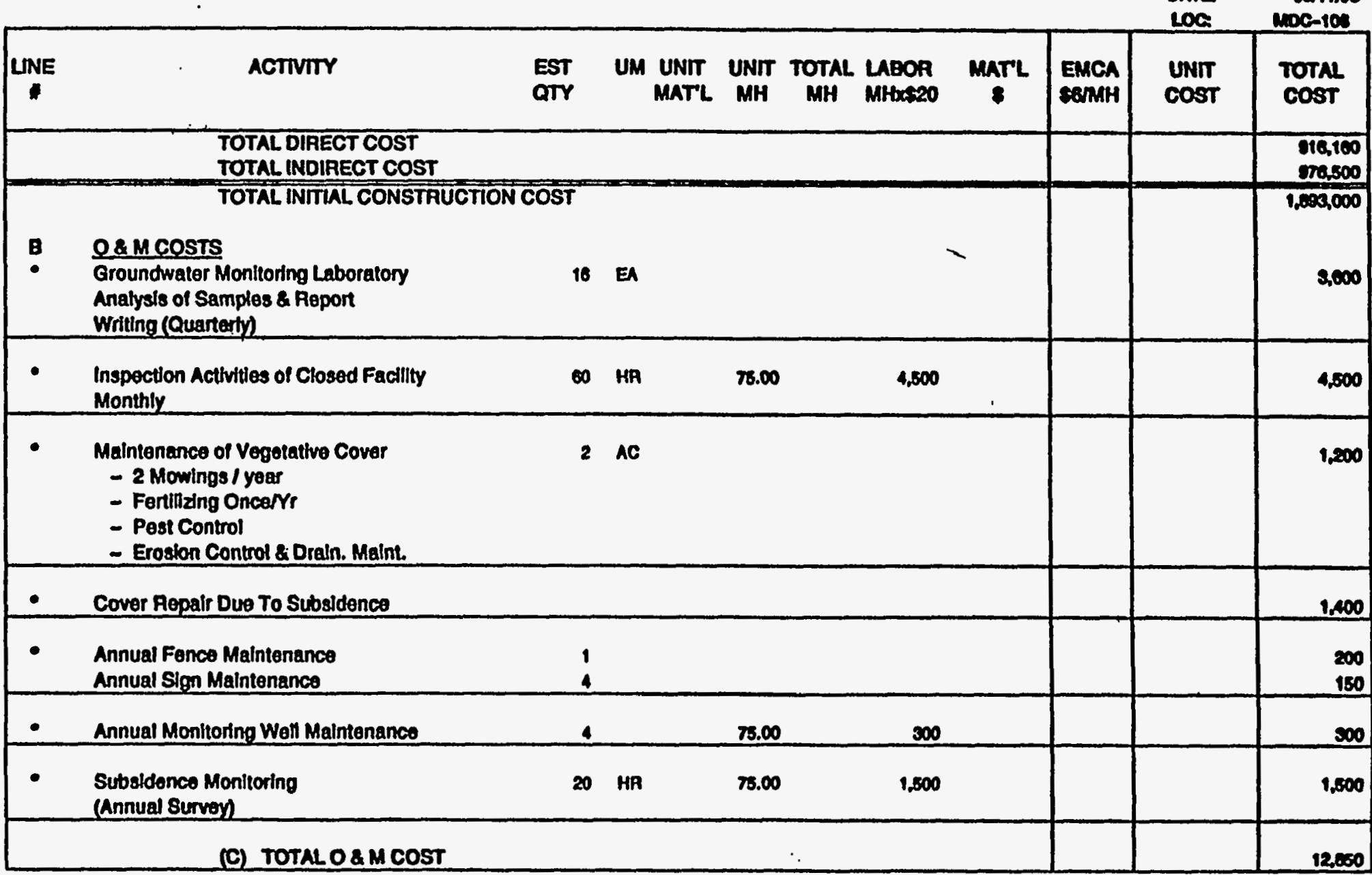

E-5. Cost Estimate for a Cover System Utilizing 1/4-inch Thick Hot Sprayed or Emulsified Asphalt (Cont'd) 
D B T A I L B S TIMATB

1001: $7-03$

ACTIVITY CODB: BSEECON12

MROJ.NAMB: BCON. BVALUATION OP CLOSURE CAP BARRIER MATERUAL

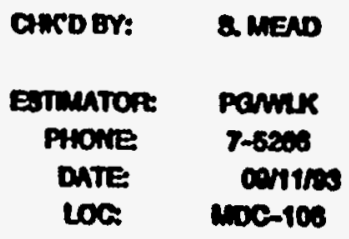

SUMMARY

TWQ(2) ACAES SITE - HOT SPAAYED ASPHALT

C TOTAL COST (Total Present Worth)

(i) Present Worth of 0 \& $M$ Cost

(5\% discount Rate for 30 Years)

(Total O\& M X 15.3725)

(ii) Total Inlilat Conatruction Cost

(C) TOTAL PROJECT COST (Rounded to the nearest Thousand)

E-5. Cost Estimate for a Cover System Utilizing 1/4-inch Thick Hot Sprayed or Emulsified Asphalt (Cont'd) 
DETAILESTIMATE

Lo0 1: $7-00$

ACTIVTY CODE: ESE2SOL12

PFOU.MUME ECON. EVALUATTON OF CLOSUPE CAP BARRAER MATERAML

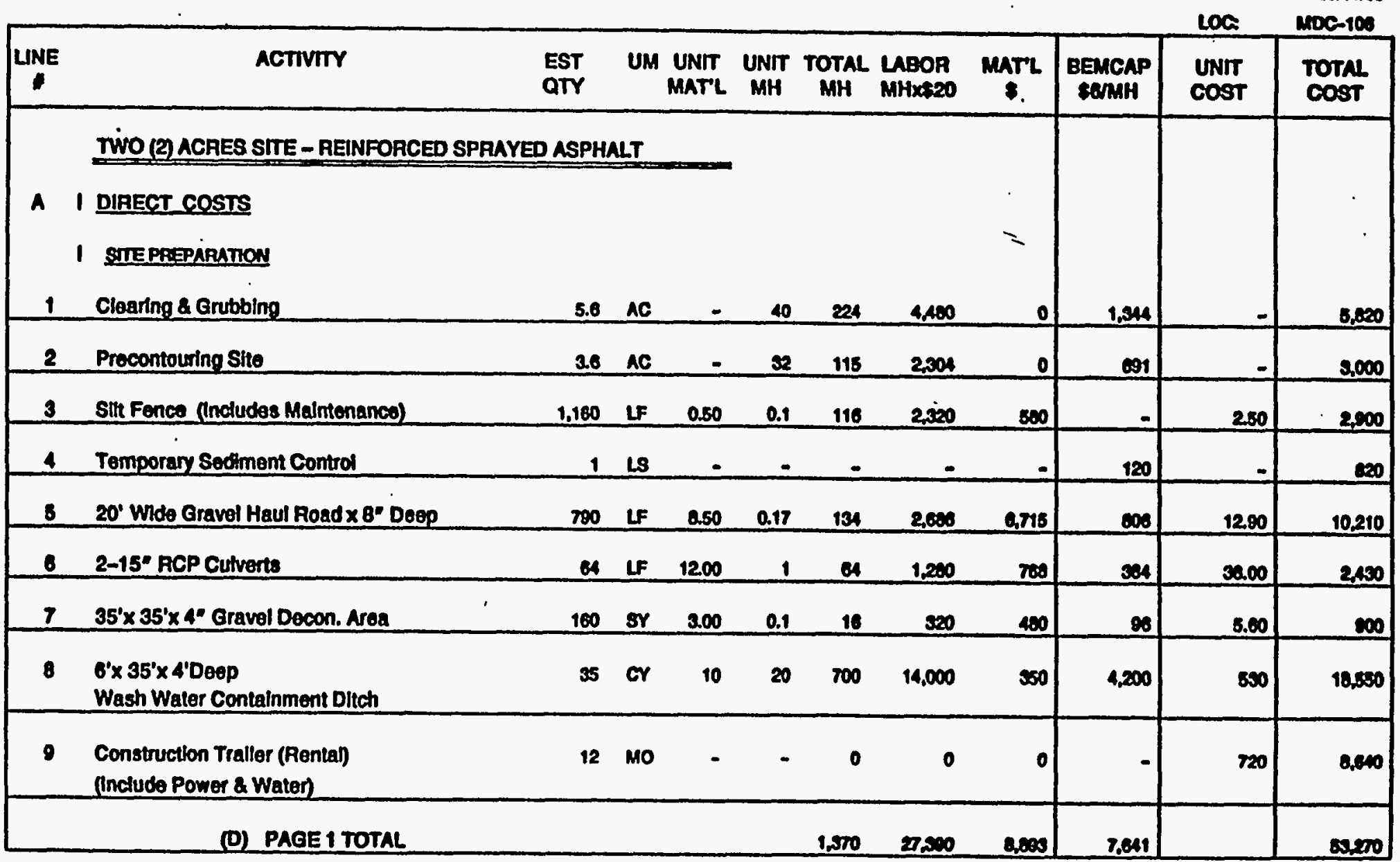

E-6. Cost Estimate for a Cover System Utilizing Reinforced Sprayed Asphalt 
DETAILESTIMATE

Lo0 1: $7-03$

ACTVITY CODE: ESE260412

PFOU.MUME: ECON. EVALUATION OF CLOSURE CAP BARRIER MATERUL.

CHCo or: 2.MElo

Estmatoft pamik

PGOMAME ECON. EVALUATION OF GLOURE CAP BARAIERMATERAL

\begin{tabular}{|c|c|c|c|c|c|c|c|c|c|c|c|}
\hline UNE & ACTIVITY & $\begin{array}{l}\text { EST } \\
\text { QTY }\end{array}$ & UM & $\begin{array}{l}\text { UNIT } \\
\text { MAT'L }\end{array}$ & $\underset{M H}{\text { UNIT }}$ & TOTAL & $\begin{array}{l}\text { LABOR } \\
\text { MHXAZOZ }\end{array}$ & $\underset{*}{\operatorname{MaTL}}$ & BEMCAP & $\begin{array}{l}\text { UNIT } \\
\text { COST }\end{array}$ & $\begin{array}{l}\text { TOTAL } \\
\text { COST }\end{array}$ \\
\hline & II COVER CONSTRUCTION & & & & & & & & & & \\
\hline 10 & Waste Stabilization (Crushed LImestone) & 4,020 & $T M$ & 100 & a.1 & 400 & 0.006 & 402000 & 2,417 & 10200 & A19,250 \\
\hline 11 & $\begin{array}{l}\text { Place and Compact Foundation Soll } \\
\text { in } 8^{\circ} \text { Lilts to } 95 \% \text { STO. Proctor }\end{array}$ & 18,140 & or & - & 0.155 & 2502 & 50,084 & 0 & 18,010 & 4.00 & 0.040 \\
\hline 12 & $\begin{array}{l}\text { Placement of Gootextlie Reinforcement } \\
\text { on Foundatlon Layer }\end{array}$ & 10,844 & gr & 0.30 & 0.01 & 100 & 2,100 & $\mathbf{5 2 5 0}$ & - & 0.60 & B,400 \\
\hline 13 & Placement of 30 mill Flexible Membrane Liner & 123,200 & $8 F$ & 0.17 & 0.0075 & 224 & 10,400 & 20,041 & - & 0.30 & 50,420 \\
\hline 14 & $\begin{array}{l}\text { Appllod Hot Asphat using } 9 \text { passes at a rate } \\
\text { of } 0.5 \text { gal / SY for a fotzl of } 1.5 \mathrm{~g} / \mathrm{Sy}\end{array}$ & $\begin{array}{r}10,844 \\
16,2606 \\
\end{array}$ & $\begin{array}{l}\text { or } \\
\text { onL }\end{array}$ & 2 & 0.15 & 1627 & 32,532 & 21,600 & 0,700 & 8.00 & 04,900 \\
\hline 18 & $\begin{array}{l}\text { Placement of } 1^{-}-0 \text { Co:arse Sand } \\
\text { Drainage Layer }\end{array}$ & 3,614 & or & 10.50 & 0.03 & 100 & 2,160 & 37,90 & $\infty \mathbf{1}$ & 11.50 & 40,770 \\
\hline 10 & $\begin{array}{l}\text { Placement of Geotextile (TYPAR) Fitter on } \\
\text { 1'-0" Coarse Sand Drainege Layer }\end{array}$ & 10.84 & SY & 0.17 & 0.006 & 84 & 1,004 & 1,840 & - & 0.30 & 2,000 \\
\hline 17 & Placement of $1^{\prime}-6^{\prime}$ Common Fill & 4.840 & or & $\mathbf{3 . 7 5}$ & 0.00 & 201 & 8,010 & 10,100 & 1.746 & 8.50 & 200,740 \\
\hline 18 & Plecement of $\sigma^{*}$ Topsolt & 1.020 & Cr & 2.50 & 0.06 & 109 & 2,184 & 17.200 & $\infty$ & 11.10 & 20,180 \\
\hline 19 & $\begin{array}{l}\text { Placement of Perlmeter Exterfor } \\
\text { Drainage Layer } 3 / 4^{-} \times 3^{\circ} \text { DP (Crushed Stone) }\end{array}$ & 412 & or & 1.50 & 0.2 & 82 & 1,640 & 618 & 194 & 0.70 & $2, \pi 00$ \\
\hline 20 & Excavation of Trapezoldal Drainage Ditch & 202 & Cr & - & a.175 & 154 & 2,007 & 0 & 928 & 4.50 & 4,010 \\
\hline & (D) PAGE TOTAL & & & & & asess & 127,200 & 824,504 & $\mathbf{3 1 , 0 6 8}$ & & 00,470 \\
\hline
\end{tabular}

E-6. Cost Estimate for a Cover System Utilizing Reinforced Sprayed Asphalt (Cont'd) 
DETAIL ESTIMATE

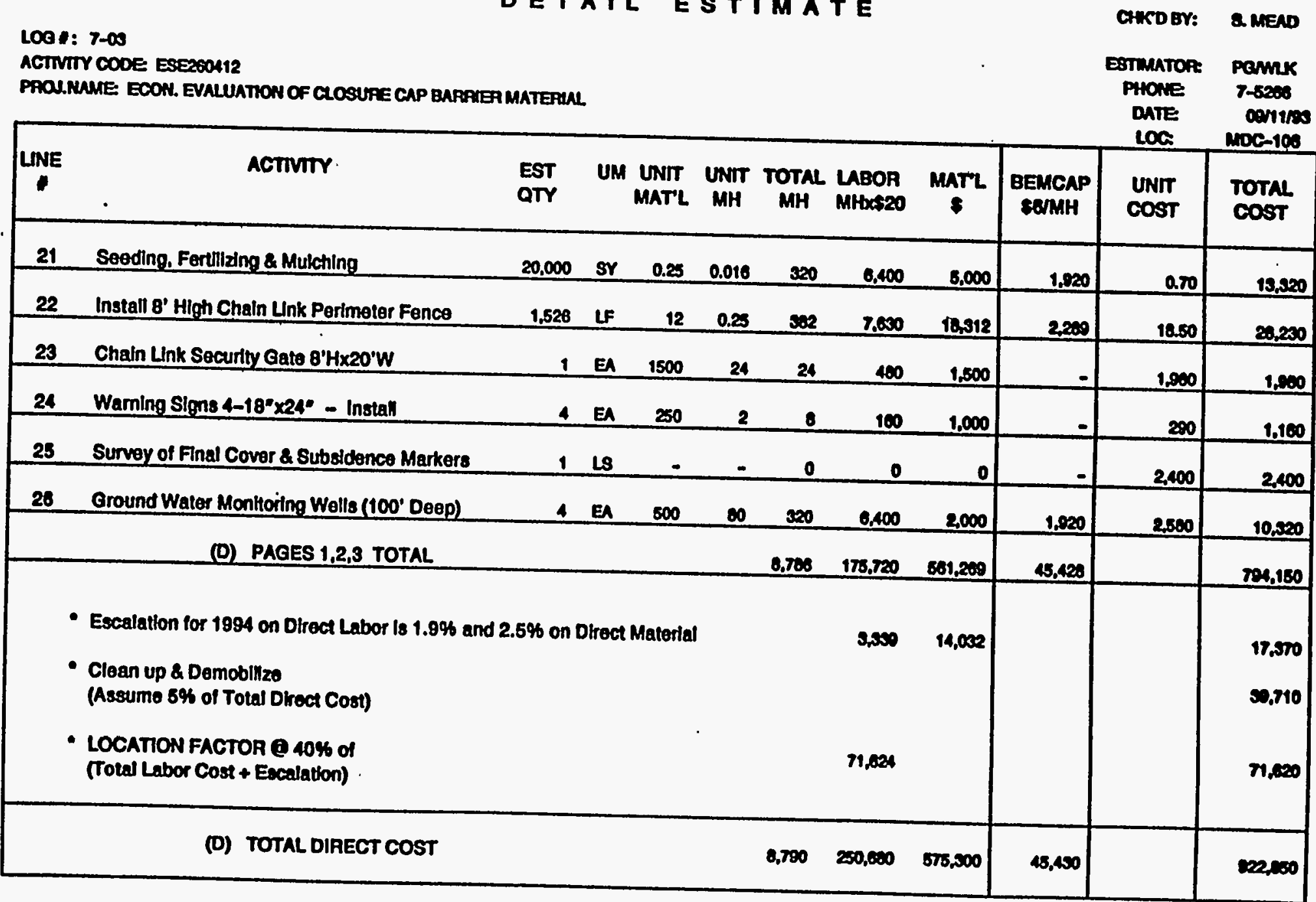

E-6. Cost Estimate for a Cover System Utilizing Reinforced Sprayed Asphalt (Cont'd) 


\section{DETAIL ESTIMATE}

200: $7-03$

ACTIVTY CODE: ESERSON12

PAOU.NANE ECON. EVALUATKON OF CLOSURE CAP BARARERMATERELL.

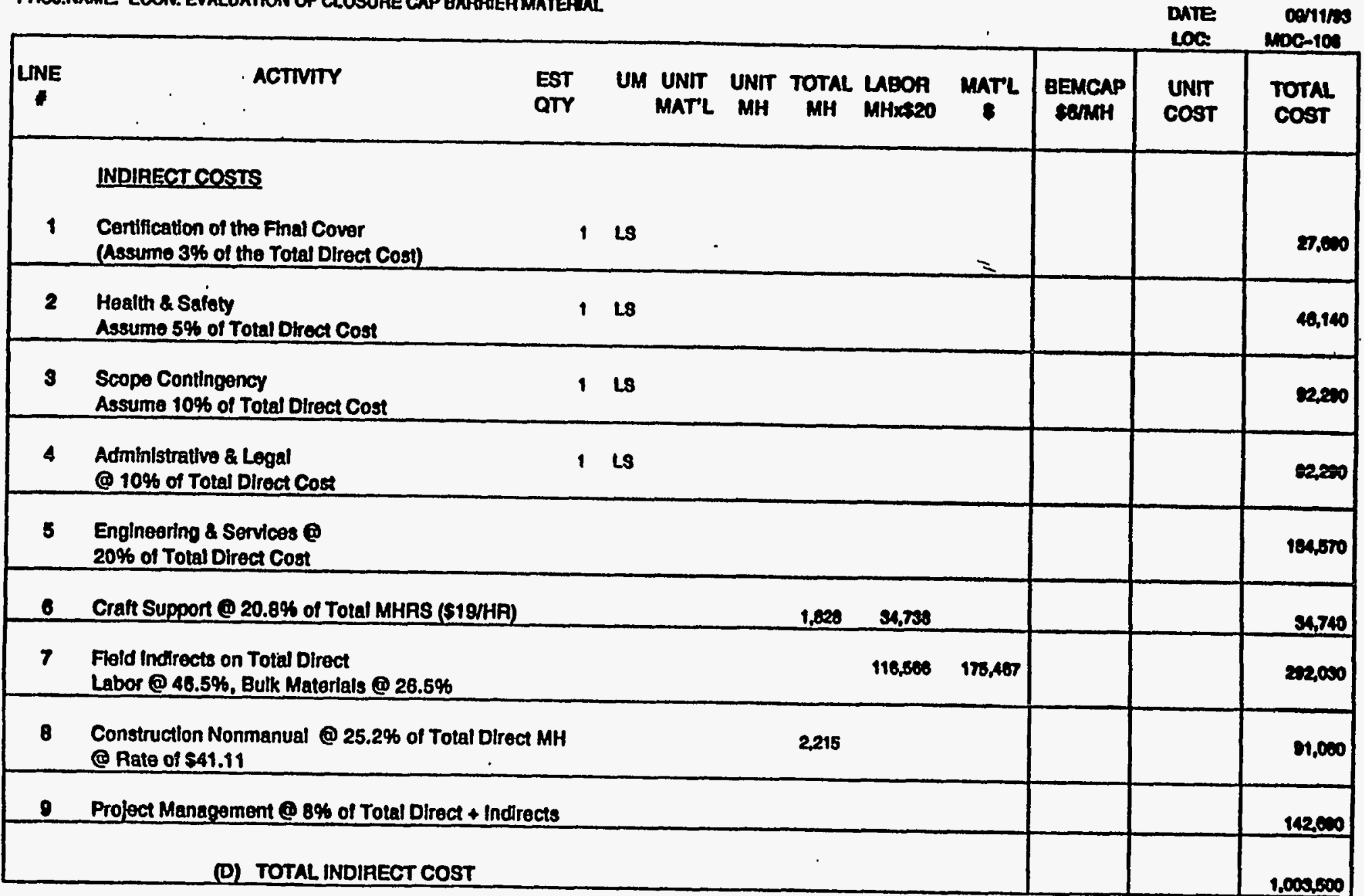

E-6. Cost Estimate for a Cover System Utilizing Reinforced Sprayed Asphalt (Cont'd) 


\section{DETAIL ESTIMATE}

CWCD BY: S. MESD

LO0 I: $7-00$

ACTIVTY CODE: ESE260112

PFOU.MAME: ECON. EVALUATON OF CLOSUPE CAP BAPRUER MATEFANL

Estuantore pamk

PHONE:

DATE

$7-5200$

Loa

$\operatorname{cosing}$

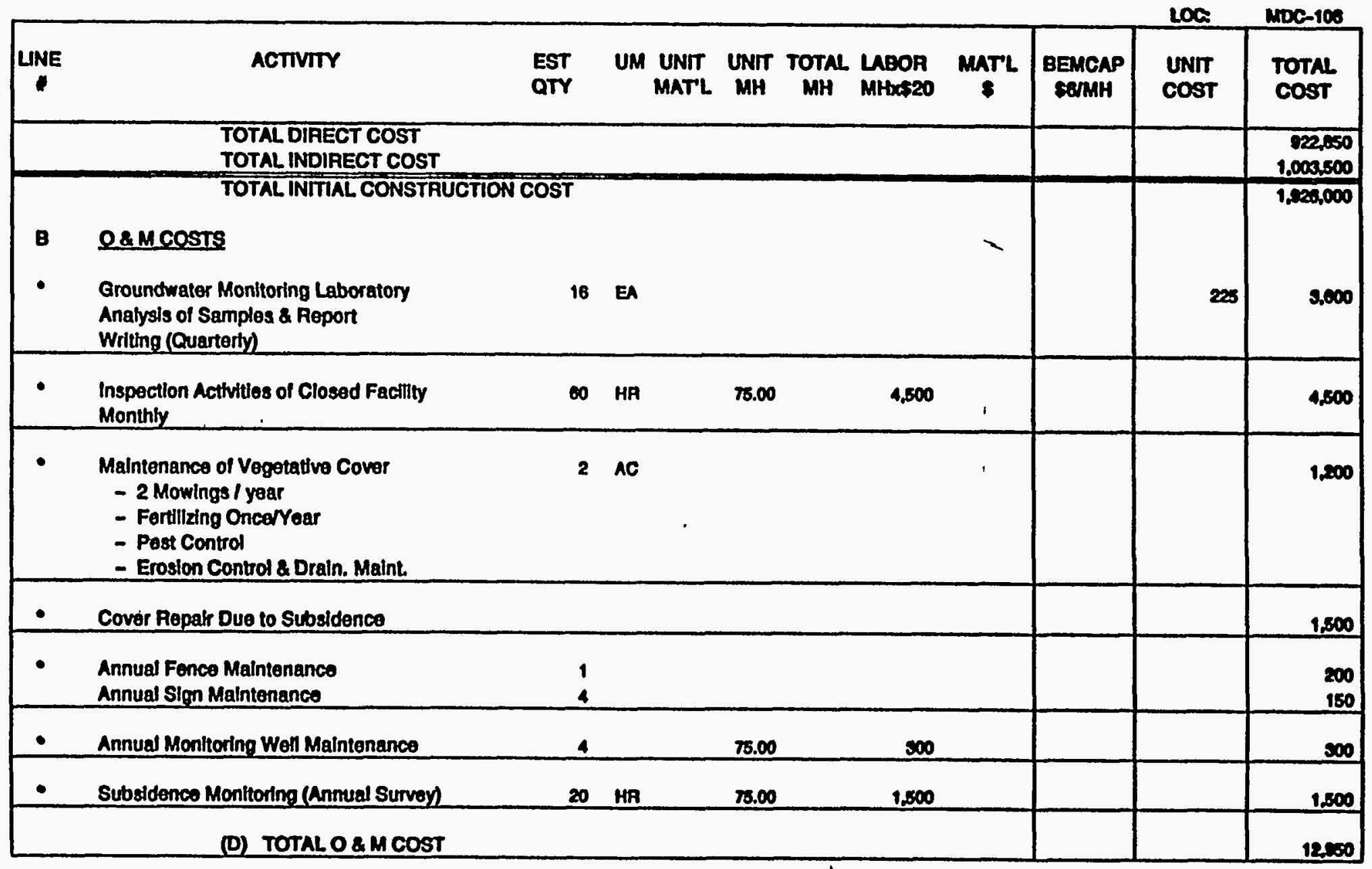

E-6. Cost Estimate for a Cover System Utilizing Reinforced Sprayed Asphalt (Cont'd) 
DETAIL ESTIMATE

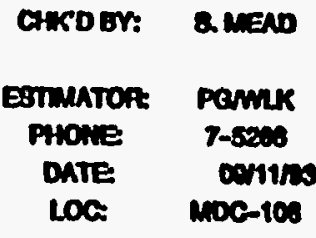

L001: 7-03

ACTIVTY COOE ESE200412

PPOU.RUME ECON. EVALUATION OF CLOSURE CAP BAPRUEAMATERUL

Loa. noc-100

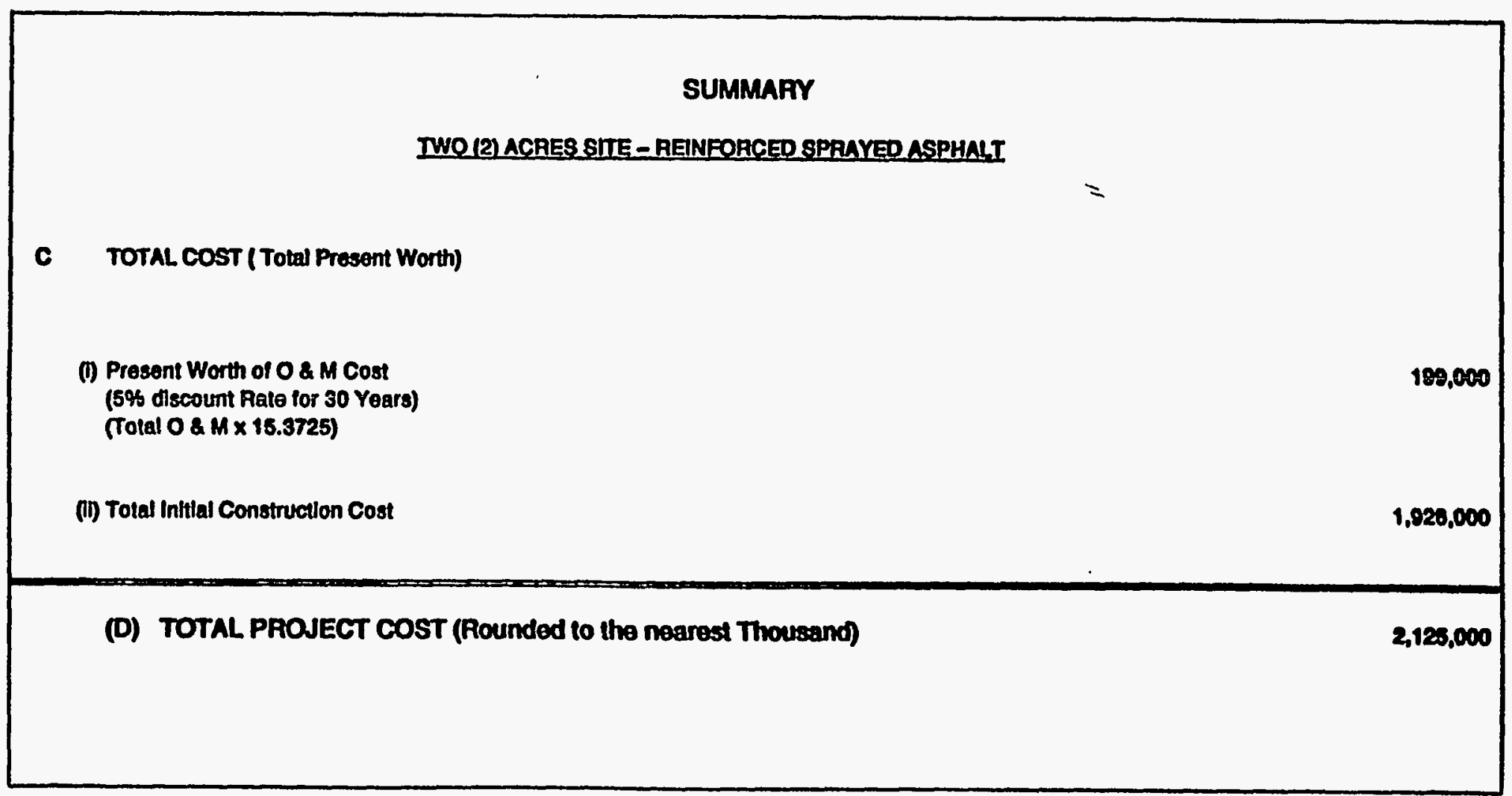

E-6. Cost Estimate for a Cover System Utilizing Reinforced Sprayed Asphalt (Cont'd) 


\section{DETAILESTIMATB}

\section{Lod: $7-\infty$}

ACTIVIYY CODE: BSE260412

PROY.NAME: BCON. EVALUATON OP CLOSURB CAP BARRRER MATERIAL

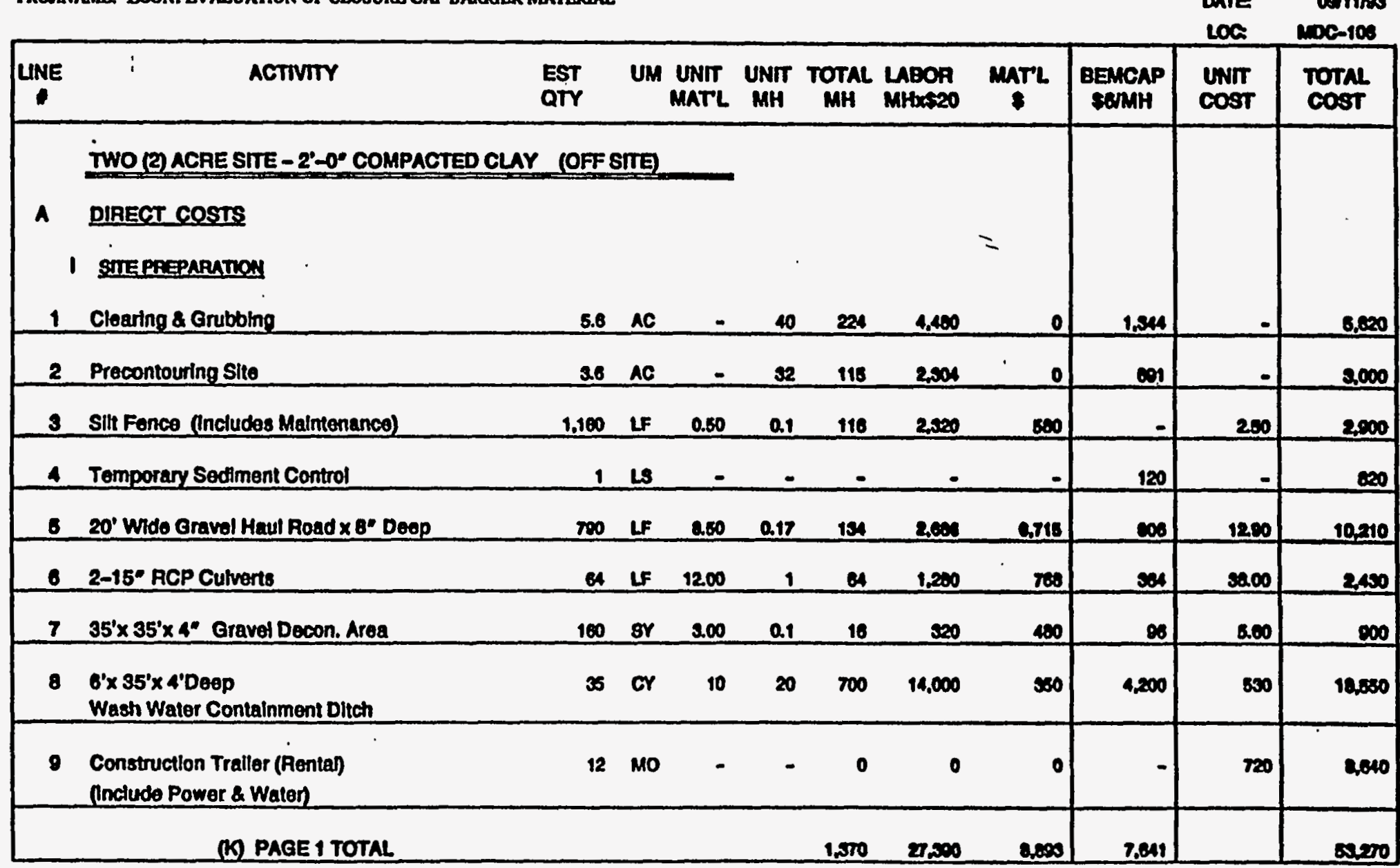

E-7. Cost Estimate for a Cover System Utilizing Compacted Kaolin (Offsite) Clay and a 30-mil FML 
DETAIL BSTIMATE

KCDEY: 2. MEAD

$100 / 17-03$

ACTIVTY CODA: BSB2SON12

PROU.NAMB: BCON. BVALUATHON OF CLOSURB CAP BARRURR MATERUAL

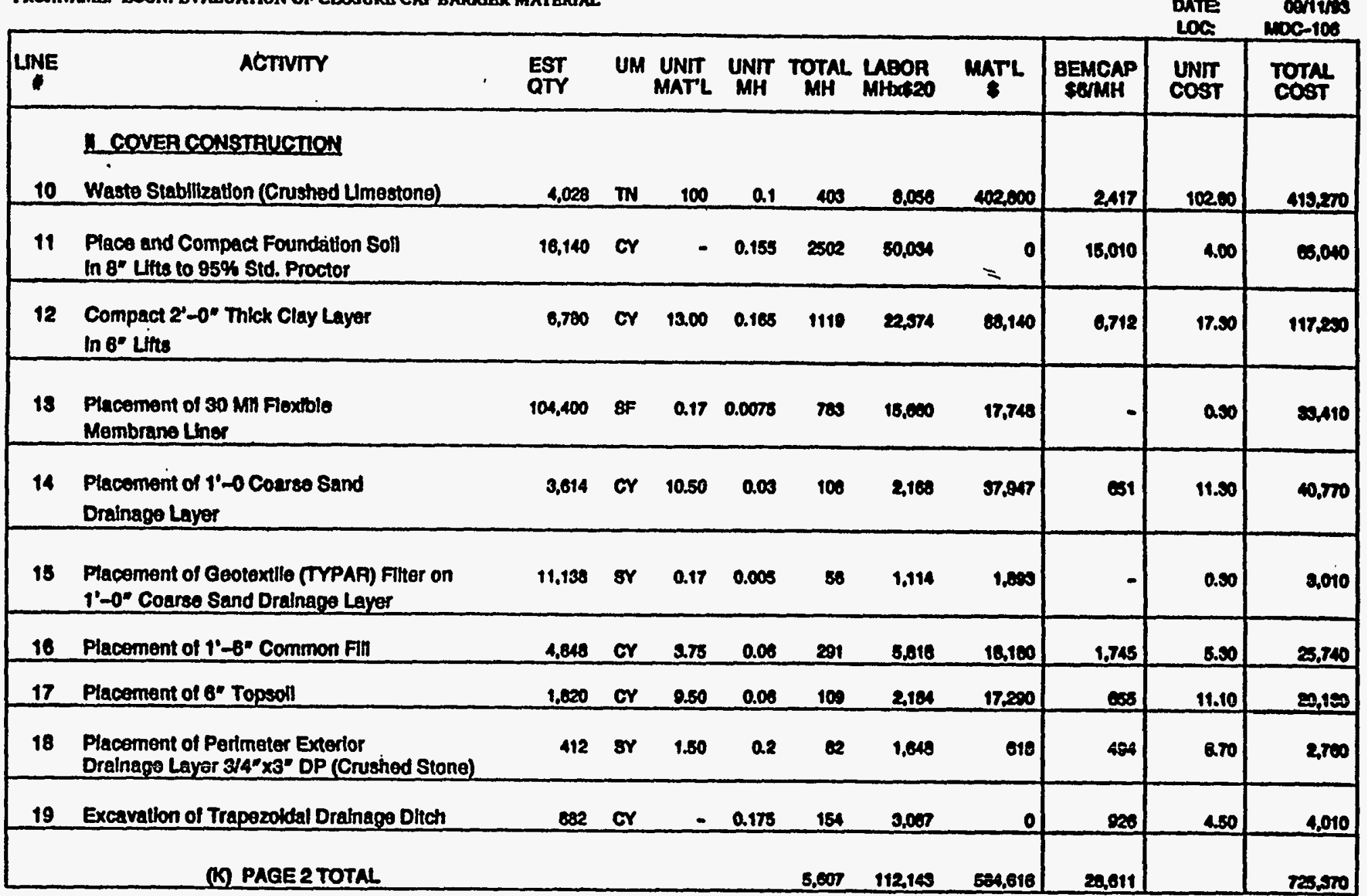

E-7. Cost Estimate for a Cover System Utilizing Compacted Kaolin (Offsite) Clay and a 30-mil FML (Cont'd) 
D B TAIL BSTIMATB

\section{Lo0 I: $7-03$ \\ ACTIVTY CODE: BSE26012}

MEOS.NAME: ECON. BYALUATION OF CLOSURB CAP BARRIER MATERLAL,

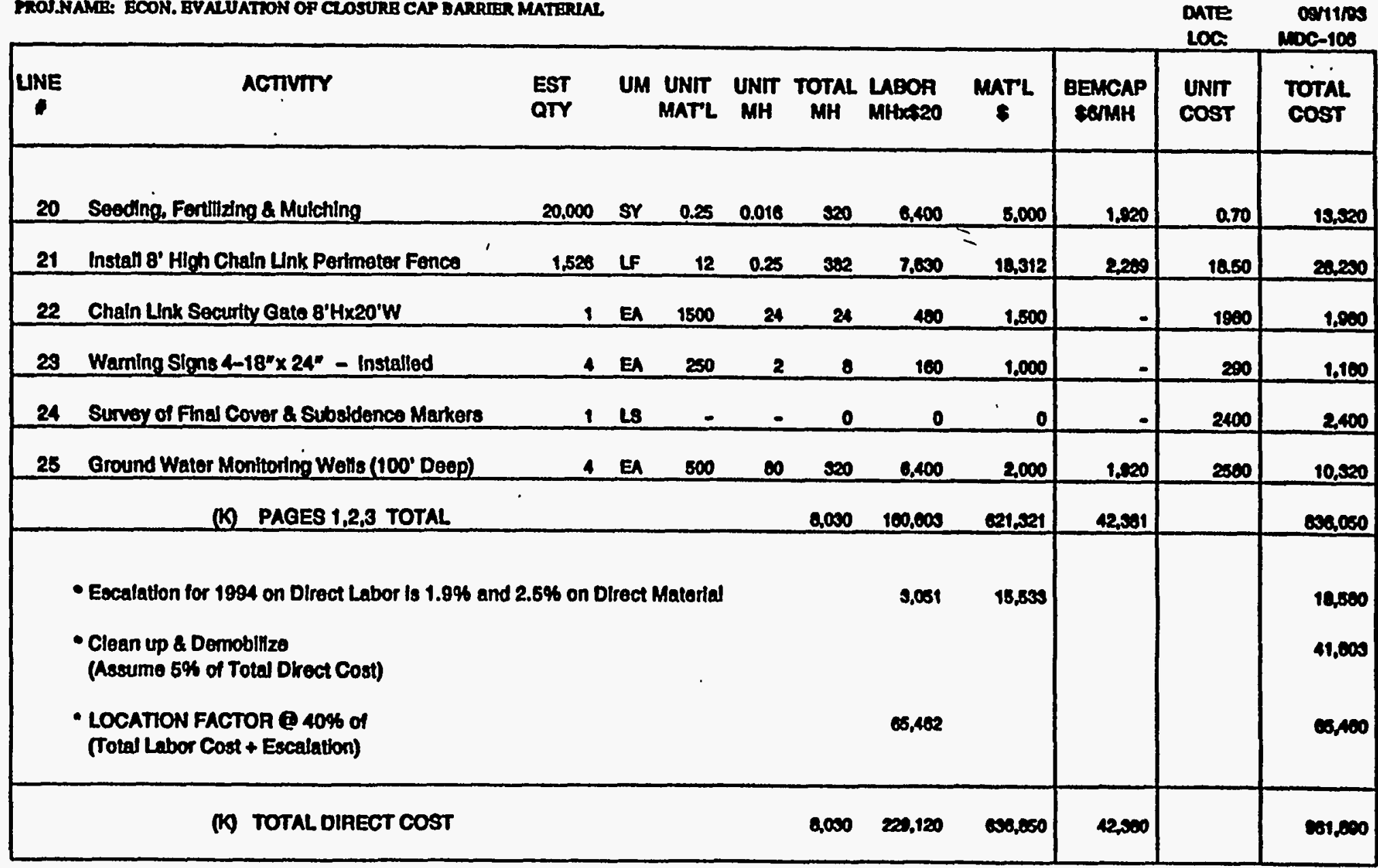

E-7. Cost Estimate for a Cover System Utilizing Compacted Kaolin (Offsite) Clay and a 30-mil FML (Cont'd) 
CHCO EV: Q.MEAD

$1001: 7-03$

ACTIVITY CODB: BSE260112

MROU.NAMG: BCON. BVALUATION OP CLOSURE CAP BARUIBR MATERIAL.

egrmator parme

PHONE

$7-52000$

DATE DOTros

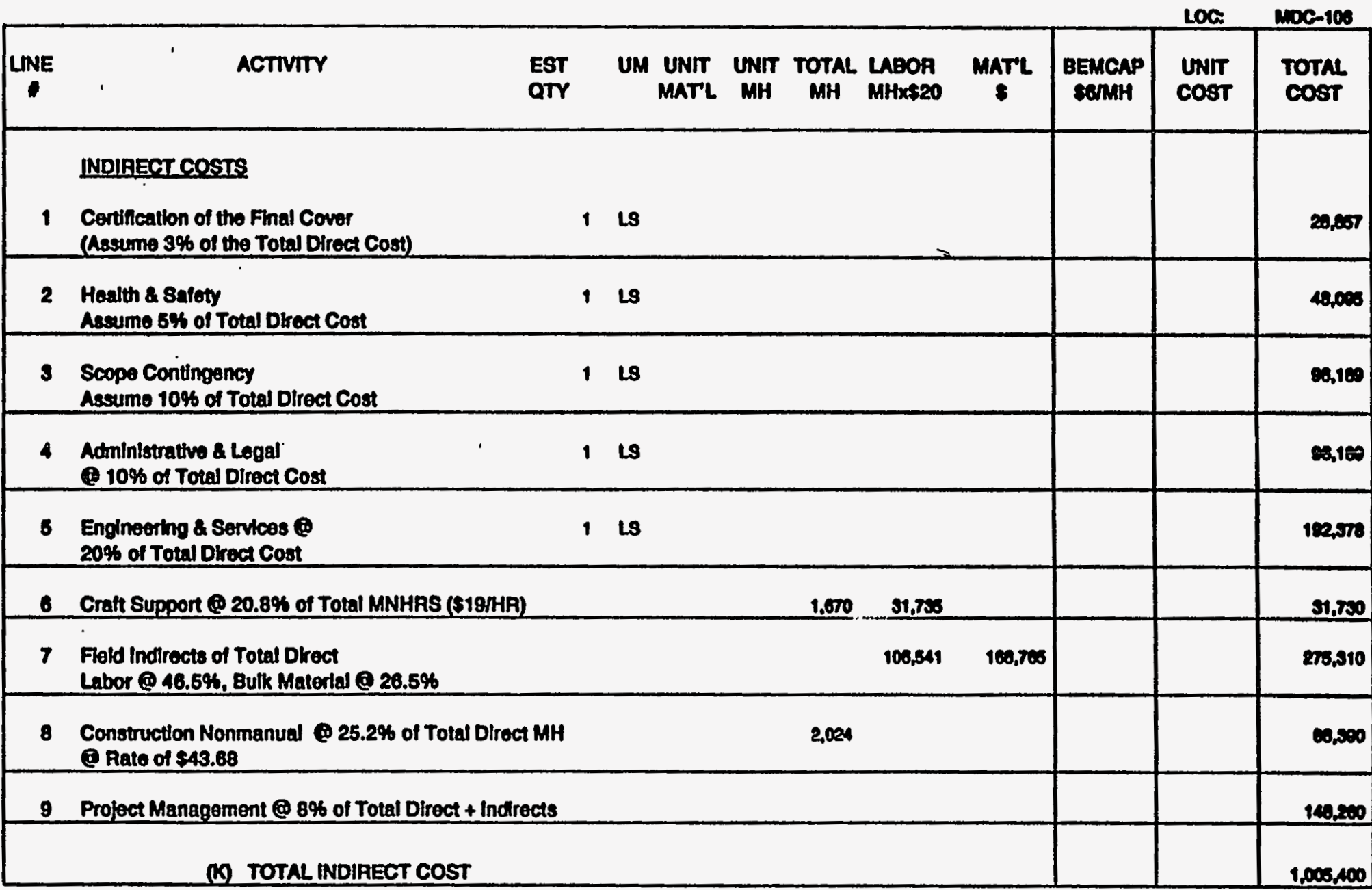

E-7. Cost Estimate for a Cover System Utilizing Compacted Kaolin (Offsite) Clay and a 30-mil FML (Cont'd) 


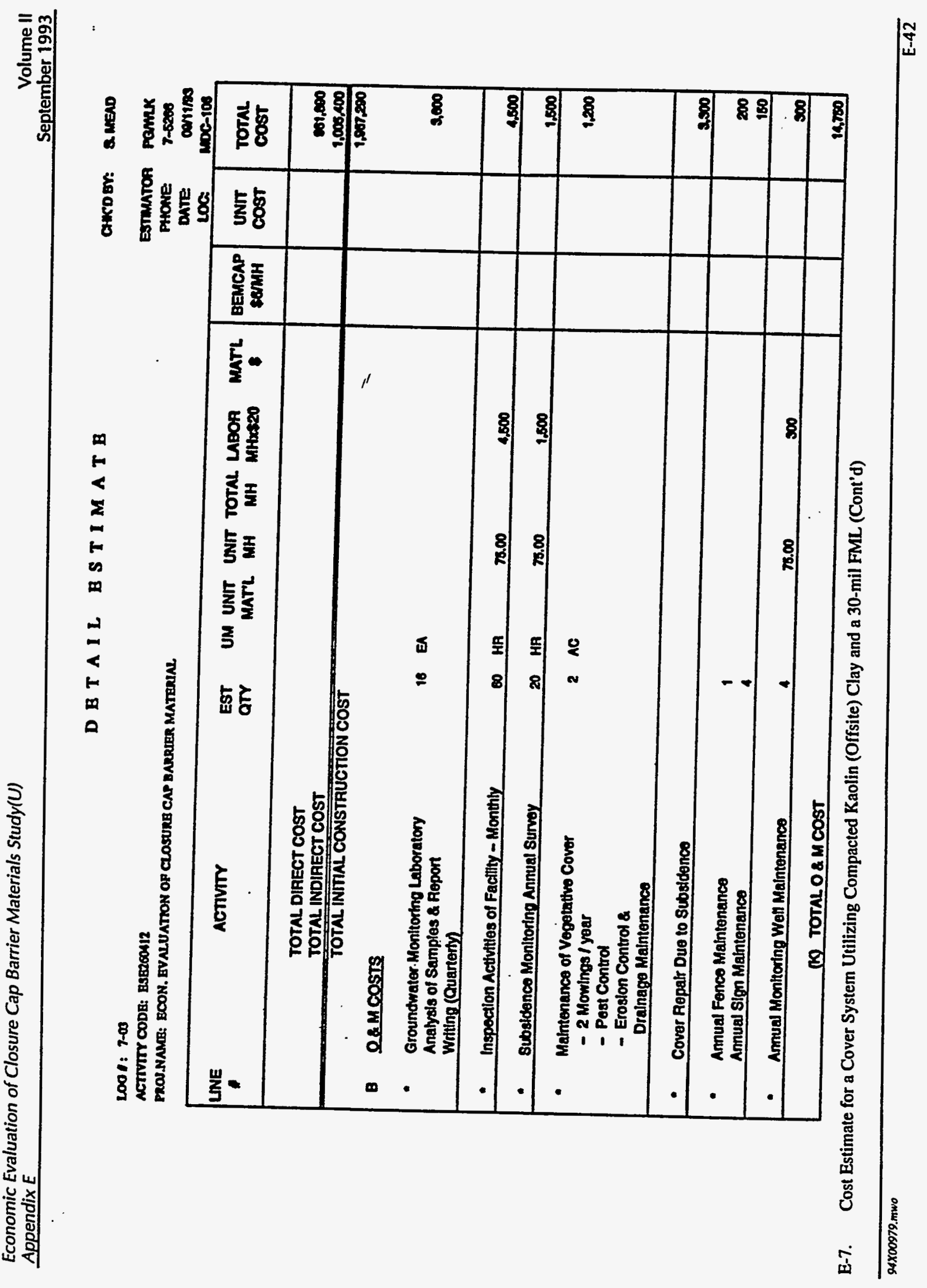


D B T A I L B S T I M T B

Loof: 7-03

ACTIVITY CODB: BST200412

PROJ.NAMR: BCON. BVALUATTON OR CLOSURE CAP BARRIER MATERUAL.

\section{CAKCD BY: 2 MENO \\ ESTmaton Pamk \\ PHone \\ $7-6200$ \\ $2 \operatorname{cones}$ \\ LOC. MDC-100}

\section{SUMMARY}

TWO (2) ACRE SITE - 2'-0. COMPACTED CLAY IOFF EITE

TOTAL COST ( Total Present Worth)

(i) Present Worth of 0 \& $M$ Cost

(5\% discount Rate for 30 Years)

(Total O \& $M \times 18.3725)$

(il Total Initial Construction Cost

$1,967,200$

(M) TOTAL PROJECT COST (Rounded to the nearest Thouseand)

$2,194,000$

E-7. Cost Estimate for a Cover System Utilizing Compacted Kaolin (Offsite) Clay and a 30-mil FML (Cont'd) 
D B TAIL B TIMATB

Lo0 1: $7-03$

ACTIVTY CODE: BSEZSON12

FROS.NAME: ECON. EVALUATONO OP CLOSURB CAP BAERIER MAATERLAL.

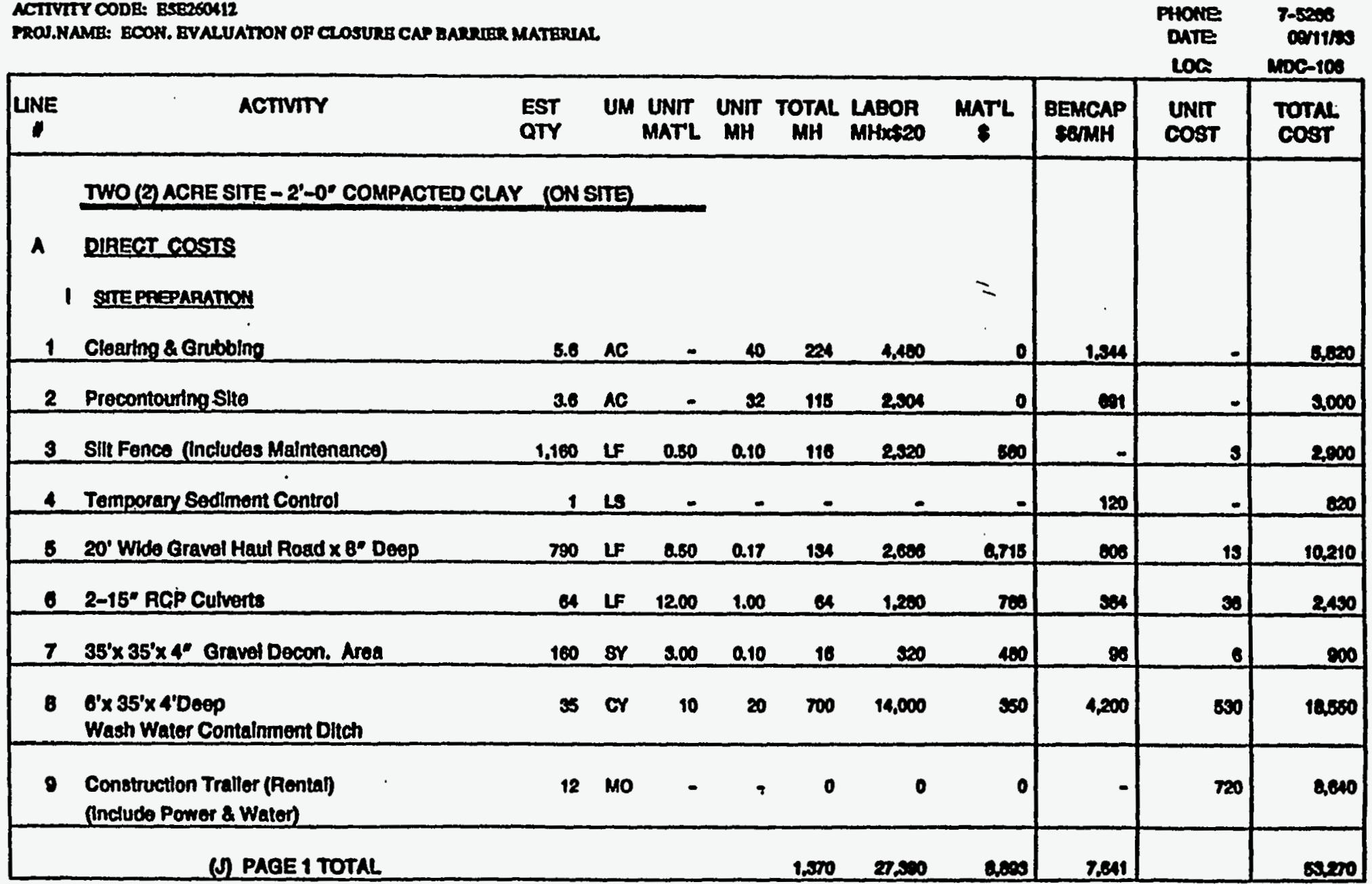

E-8. Cost Estimate a for Cover System Utilizing Compacted Onsite Sandy Clay and a 30-mil FML 
$100: 7-03$

NCTIVITY CODE: BSBESOA12

MROJ.NAME: BCON. EVALUATION OP CLOSURB CAP BARRIER MATERLAL

\begin{tabular}{|c|c|c|c|c|c|c|c|c|c|c|c|}
\hline JNE & ACTIVTY & $\begin{array}{l}\text { EST } \\
\text { QTY }\end{array}$ & UM & $\begin{array}{l}\text { UNTT } \\
\text { MATL }\end{array}$ & $\begin{array}{l}\text { UNIT } \\
\text { MH }\end{array}$ & $\underset{\text { MH }}{\text { TOTAL }}$ & $\begin{array}{l}\text { LABOR } \\
\text { MHAT20 }\end{array}$ & $\underset{8}{\operatorname{MarL}}$ & $\begin{array}{l}\text { BEMCAP } \\
\text { SAMMH }\end{array}$ & $\begin{array}{l}\text { UNT } \\
\text { COST }\end{array}$ & $\begin{array}{l}\text { Total } \\
\cos \end{array}$ \\
\hline & LQOVER CONSTAUCTION & & & & & & & & & & \\
\hline 10 & Waste Stabilizatlon (Crushed Limestone) & 4,028 & $\mathbf{m}$ & 100 & 0.1 & 403 & 8,000 & 102000 & 2417 & 100 & 480,270 \\
\hline 11 & $\begin{array}{l}\text { Place and Compact Foundation Soll } \\
\text { in } 8^{\circ} \text { Lifts to } 85 \% \text { Std. Proctor }\end{array}$ & 16,140 & Cr & - & 0.150 & 2002 & 80,084 & $=$ & 18,010 & 4 & 0.040 \\
\hline 12 & $\begin{array}{l}\text { Compact 2'-0" Thick Clay Layer } \\
\text { In 6० Ufts }\end{array}$ & 6,780 & CY & - & 0.106 & 1110 & 22,074 & $\mathbf{0}$ & 6,712 & 4 & 20,000 \\
\hline 13 & $\begin{array}{l}\text { Placoment of } 30 \text { Min Flexiblo } \\
\text { Membrane Lner }\end{array}$ & 104,400 & SF & 0.17 & 0.0076 & 703 & 15,600 & 17,748 & - & $\mathbf{0}$ & 38,410 \\
\hline 14 & $\begin{array}{l}\text { Placement of } t^{\prime}-0 \text { Coarse Sand } \\
\text { Drainage Layer }\end{array}$ & 3,614 & or & 10.50 & 0.00 & 100 & 2,160 & 57.⿻17 & $\mathbf{\infty 1}$ & 11 & 40,770 \\
\hline 16 & $\begin{array}{l}\text { Placement of Ceotextlle (TYPAR) Filter on } \\
\text { I'-0* Course Sand Drainage Layer }^{-0}\end{array}$ & 11.138 & 8r & $\mathbf{a . 1 7}$ & 0.006 & 86 & 1,114 & 1,000 & - & $\mathbf{0}$ & 3,010 \\
\hline 16 & Placememt of $1^{\prime}-6^{\prime \prime}$ Common Fin & 4,840 & CY & 273 & Q.00 & 291 & 8.010 & 19.100 & 1,745 & 8 & 20.740 \\
\hline 17 & Placement of 6" Topsoll & 1.020 & cr & $\mathbf{9 . 5 0}$ & $\mathbf{0 . 0 0}$ & 109 & 2,184 & 17200 & $\mathbf{6 0 8}$ & 11 & 20,150 \\
\hline 18 & $\begin{array}{l}\text { Placement of Perlmeter Exterlor } \\
\text { Drainage Layer } 34^{*} \times 3^{*} \text { DP (Crustrad Stone) }\end{array}$ & 412 & sr & 1.50 & 0.2 & 02 & 1,648 & 68 & 484 & $\boldsymbol{7}$ & $2 \pi 00$ \\
\hline 19 & Excavation of Trapezoldal Drainage Ditch & 882 & or & - & a.175 & 154 & 2,007 & $\mathbf{0}$ & $\operatorname{ces}$ & 8 & 4.010 \\
\hline & (J) PAGE 2 TOTAL & & & & & 5.007 & 112,149 & sines & 20.011 & & $\cos , \infty 0$ \\
\hline
\end{tabular}

E-8. Cost Estimate a for Cover System Utilizing Compacted Onsite Sandy Clay and a 30-mil FML (Cont'd) 
D B T A I L B T I M T B

\section{LOC 1: 7-03}

ACTIVITY CODB: BSE260112

PROJ.NAMR: BCON. BYALUATION OR CLOSURB CAP BARRIER MATERUAL

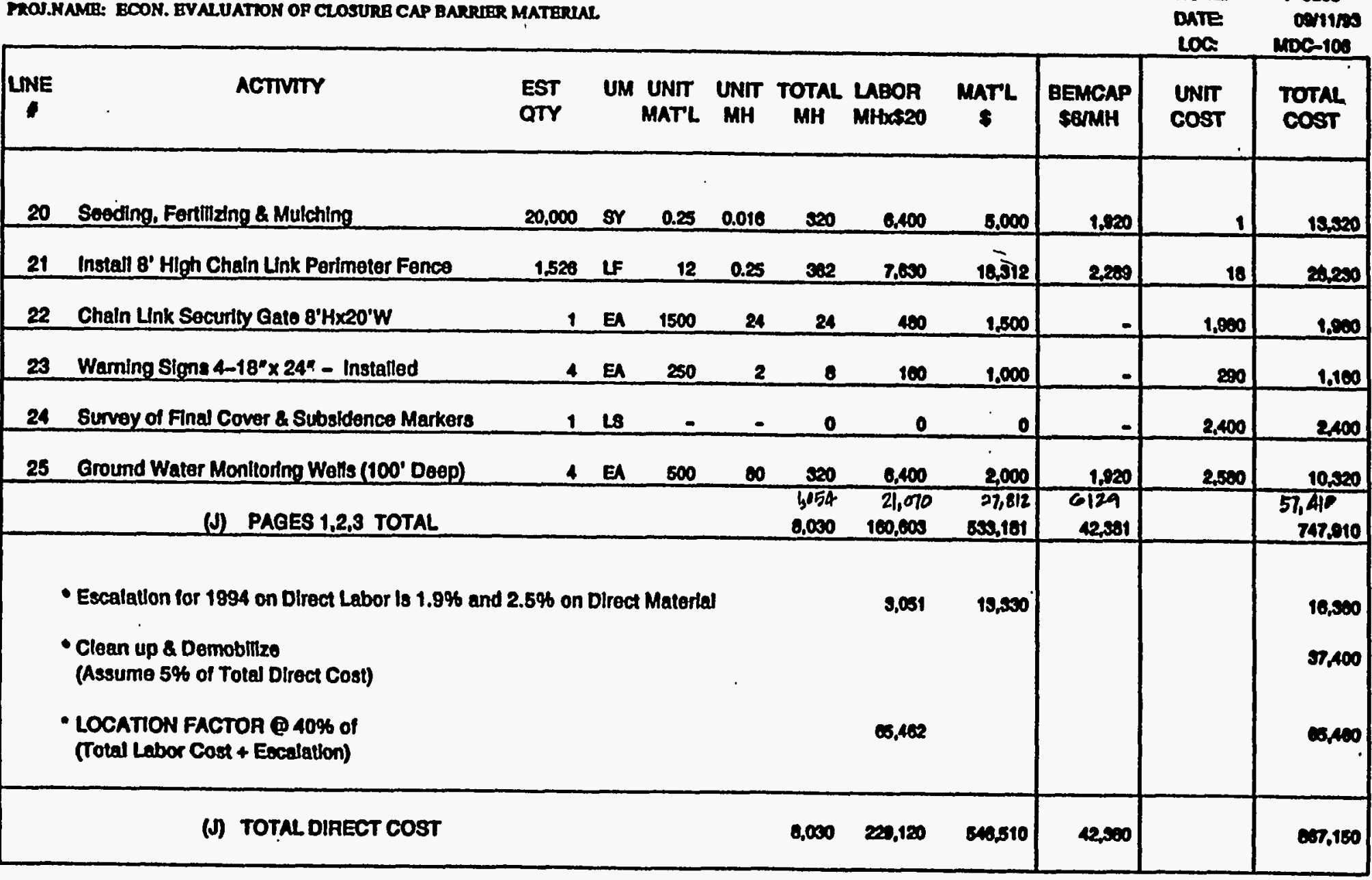

E-8. Cost Estimate a for Cover System Utilizing Compacted Onsite Sandy Clay and a 30-mil FML (Cont'd) 
D B T A IL BSTIMATB CHEOV: QMEAO

$10018: 7-00$ ACTIVITY CODB: ESBZ6012

MROS.NAME: ECON. BYALUTION OF CLOSURB CAP BARRIER MATERLAL.

\begin{tabular}{|c|c|c|c|c|c|c|c|c|c|}
\hline LNE & ACTIVITY & UM UNIT & $\begin{array}{l}\text { UNIT } \\
\text { MH }\end{array}$ & $\begin{array}{c}\text { TOTAL } \\
\text { MH }\end{array}$ & $\begin{array}{l}\text { LABOA } \\
\text { MHX*20 }\end{array}$ & $\underset{8}{\operatorname{MATL}}$ & $\begin{array}{l}\text { BEMCAP } \\
\text { SONHH }\end{array}$ & $\begin{array}{l}\text { UNIT } \\
\text { COST }\end{array}$ & $\begin{array}{l}\text { Total } \\
\text { cost }\end{array}$ \\
\hline & NNDIAECT COSTS & & & & & & & & \\
\hline 1 & $\begin{array}{l}\text { Certification of the Final Cover } \\
\text { (Asserme } 3 \% \text { of the Total Direct Cost) }\end{array}$ & LS & & & & $=$ & & & 8010 \\
\hline 2 & $\begin{array}{l}\text { Heaith \& 8afity } \\
\text { Aosume } 5 \% \text { of Total Orect Cost }\end{array}$ & is & & & & & & & 40.500 \\
\hline $\mathbf{3}$ & $\begin{array}{l}\text { Scope Contingancy } \\
\text { Assume } 10 \% \text { of Total Direct Cost }\end{array}$ & 18 & & & & & . & & $\infty 6,720$ \\
\hline 4 & $\begin{array}{l}\text { Administrative \& Logal } \\
\text { (10) 10\% of Total Oiroct Cost }\end{array}$ & Lo & & & & & & & 00,720 \\
\hline 3 & $\begin{array}{l}\text { Enginoering \& Services } 0 \\
20 \% \text { of Total Dlrect Cost }\end{array}$ & & & & & & & & 170,430 \\
\hline 6 & Craft Suppon Q 20.8\% of Total MNHRS (\$19/HR) & & & 1.070 & 31,703 & & & & 51,700 \\
\hline 7 & $\begin{array}{l}\text { Frald Indirects of Total Direct } \\
\text { Labor Q 46.5\%, Bulk Malerials @ 26.5\% }\end{array}$ & & & & 100,64t & 14,000 & & & 201,570 \\
\hline 8 & $\begin{array}{l}\text { Construction Nonmanual @ } 25.2 \% \text { of Total MH } \\
\text { e Rato of } \$ 49.68\end{array}$ & & & 2,024 & & & & & 00000 \\
\hline 9 & Propect Managament @ 8\% of Total Direct + Indirects & & & & & & & & 152,500 \\
\hline & (v) TOTAL NNDIRECT COST & & & & & & & & 200,120 \\
\hline
\end{tabular}

E-8. Cost Estimate a for Cover System Utilizing Compacted Onsite Sandy Clay and a 30-mil FML (Cont'd) 
2001: 7-03

ACTIVITY CODB: BSE2SON12

PROJ.NAME: ECON. BVALUATON OF CLOSURB CAP BARRRER MATERUNL

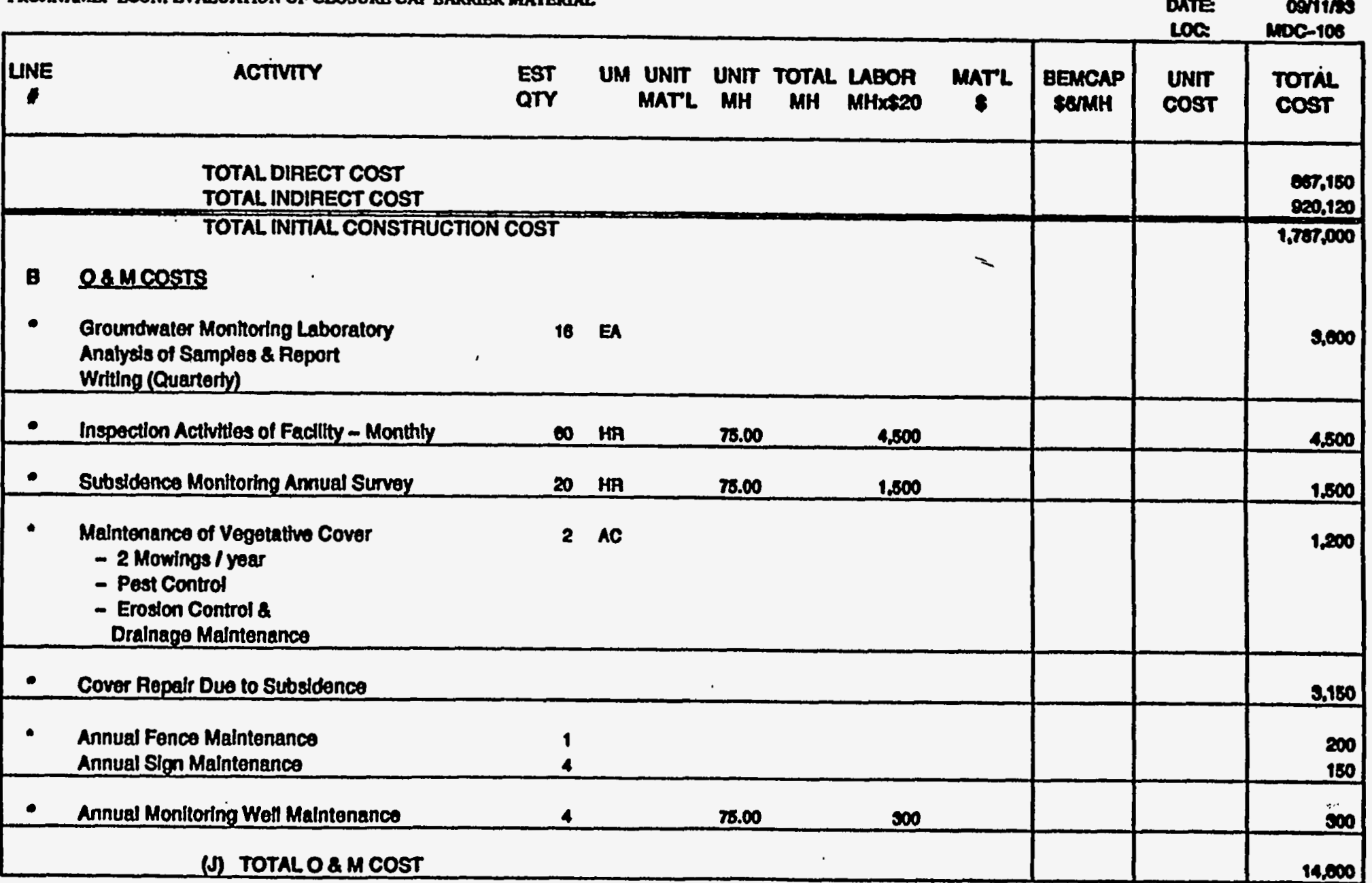

E-8. Cost Estimate a for Cover System Utilizing Compacted Onsite Sandy Clay and a 30-mil FML (Cont'd) 
D B TAIL B T IMATB

\begin{tabular}{|c|c|}
\hline CAES Or: & Q. MEAD \\
\hline $\begin{array}{l}\text { DSTmantore } \\
\text { PHONE } \\
\text { DATE } \\
\text { LOG }\end{array}$ & 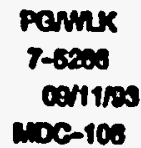 \\
\hline
\end{tabular}

L001: 7-03

ACTIVITY CODE: BSE2COA12

PROJ.NAMT: BCON. EVALUATTON OF CLOSURB CAP BARRURR MATERIAL.

Loc

\section{SUMMARY \\ TWO (2) ACAE SITE - 3'-0' COMPACTED CLAY ION SIIE}

C TOTAL COST ( Total Present Worth)

(1) Present Worth of $O$ a $M$ Cosi

(5\% discount Rate for 30 Yeare)

(Total 0 \& M $\times$ 16.3725)

(i) Totel Intrial Construction Cost

$1,787,000$

(v) TOTAL PROJECT COST (Rounded to the nearest Thousand)

E-8. Cost Estimate a for Cover System Utilizing Compacted Onsite Sandy Clay and a 30-mil FML (Cont'd) 
DETAIL ESTIMATE eptember 1993

\section{LOO : : 7-03}

ACTTVITY CODB: ESE260412

PROJ.NAME: ECON. EVALUATION OF CLOSURE CAP BARRIER MATERIAL.

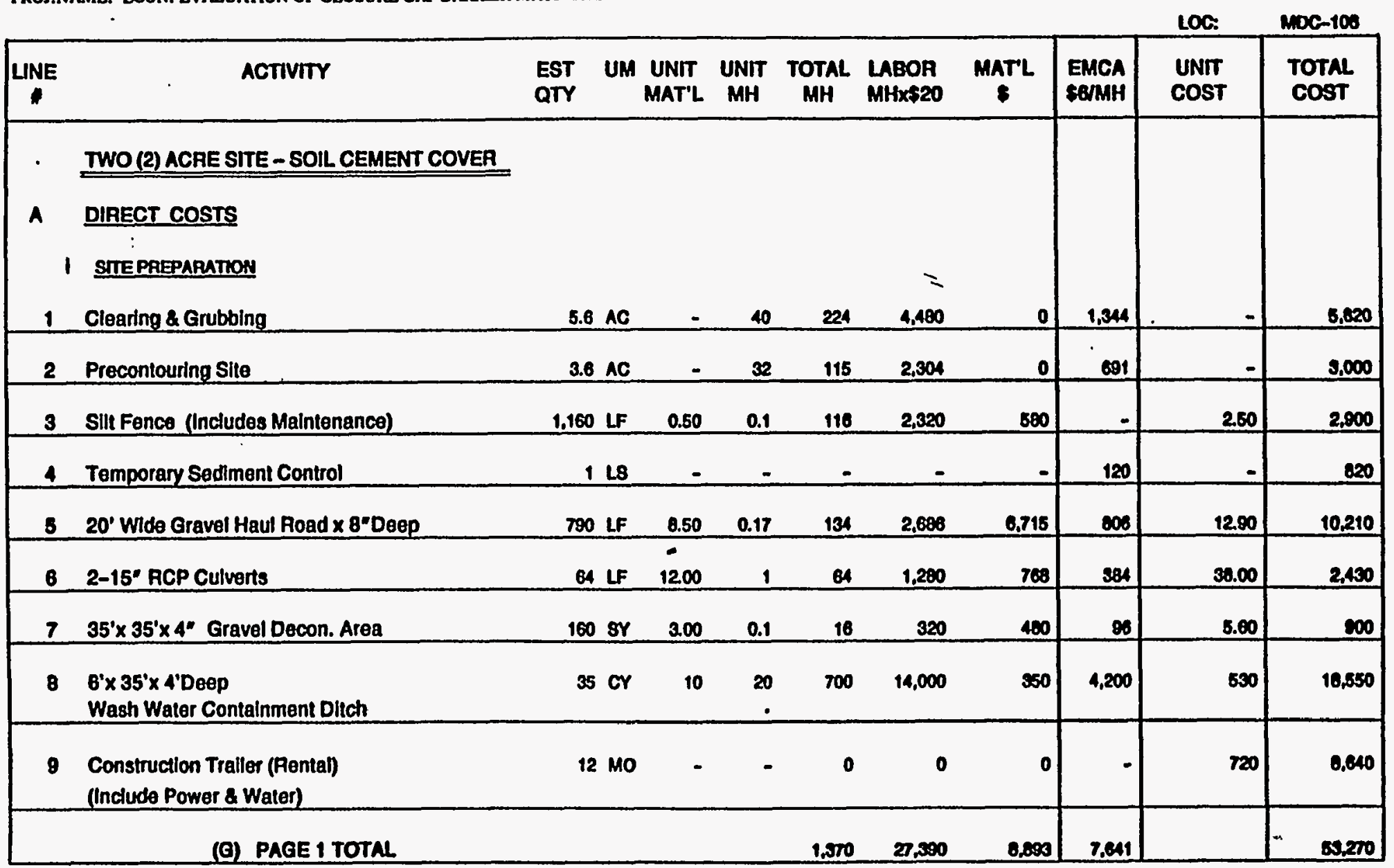

E-9. Cost Estimate for a Cover System Utilizing Soil Cement and 30-mil FML 
LOOA: 7-03

ACTIVITY CODE: ESE260412

PROI.NAME: ECON. EVALUATION OF CLOSURE CAP BARRIER MATERIAL

ESTMATOR: PQWMK

PHONE: 7-5266

\begin{tabular}{|c|c|c|c|c|c|c|c|c|c|c|c|}
\hline LINE & ACTIVITY & $\begin{array}{l}\text { EST } \\
\text { QTY }\end{array}$ & UM & $\begin{array}{l}\text { UNIT } \\
\text { MAT'L }\end{array}$ & $\underset{M H}{\text { UNIT }}$ & $\underset{M H}{\text { TOTAL }}$ & $\begin{array}{l}\text { LABOR } \\
\text { MHX\$20 }\end{array}$ & $\operatorname{MATL}$ & $\underset{S G M H A}{\operatorname{EMCA}}$ & $\begin{array}{l}\text { UNIT } \\
\text { Cost }\end{array}$ & $\begin{array}{l}\text { Total } \\
\text { cost }\end{array}$ \\
\hline & H COVER CONSTRUCTION & & & & & & & & & & \\
\hline 10 & Waste Stabillzation (Crushed Limestone) & 4,028 & TN & 100 & 0.1 & 403 & 0,058 & 402,600 & 2,417 & 102.60 & 415,270 \\
\hline 11 & $\begin{array}{l}\text { Place and Compact Foundation Soll } \\
\text { in 8" Lilts to } 95 \% \text { Sid. Proctor }\end{array}$ & 16,140 & cr & - & 0.155 & 2502 & 50,034 & 0 & 18,010 & 4.00 & 65,040 \\
\hline 12 & $\begin{array}{l}\text { Soll-Cement mixture transported from central } \\
\text { mixing plant by end-dump trucks and placed } \\
\text { in 6-8" lifts by spreaders. Assume Fine Sand } \\
\text { Soll with } 321 \text { of of cementfCY. Compact the soll- } \\
\text { cement uniformily to } 98 \% \text { max. density. }\end{array}$ & 10,286 & cr & 18 & 0.165 & 1697 & 33,944 & 185,140 & 10,183 & 22.30 & 229,270 \\
\hline 13 & $\begin{array}{l}\text { Curing soll-cement. Assume llquild Asphalt } \\
\text { appolled 0.4gallsy for } 15 t \text { coat } 80.2 \text { for 2nd. }\end{array}$ & $\begin{array}{l}10,982 \\
10,982 \\
\end{array}$ & $\begin{array}{l}\text { sr } \\
\text { sr }\end{array}$ & $\begin{array}{l}0.33 \\
0.17 \\
\end{array}$ & $\begin{array}{l}0.05 \\
0.03 \\
\end{array}$ & $\begin{array}{l}549 \\
329 \\
\end{array}$ & $\begin{array}{r}10,862 \\
6,589 \\
\end{array}$ & $\begin{array}{l}3,024 \\
1,067 \\
\end{array}$ & - & $\begin{array}{l}1.50 \\
0.80 \\
\end{array}$ & $\begin{array}{r}14,610 \\
0,400 \\
\end{array}$ \\
\hline 14 & $\begin{array}{l}\text { Placement of } 30 \text { Mil Flexible } \\
\text { Membrane Uner }\end{array}$ & 104,400 & $\mathbf{S F}$ & 0.17 & 0.0075 & 783 & 15,660 & 17,740 & - & 0.30 & $\mathbf{s 0 , 4 1 0}$ \\
\hline 15 & $\begin{array}{l}\text { Placement of 1'-0 Coarse Sand } \\
\text { Drainage Layer }\end{array}$ & 3,614 & or & 10.50 & 0.03 & 108 & 2,100 & 37,947 & 651 & 11.30 & $40, \mathrm{~m}$ \\
\hline 16 & $\begin{array}{l}\text { Placement of Geotextile (TYPAR) Filter on } \\
1^{1}-0^{\prime \prime} \text { Coarse Sand Drainage Layer }\end{array}$ & 11,138 & SY & 0.17 & 0.005 & 56 & 1,114 & 1,893 & - & 0.30 & 3,010 \\
\hline 17 & Placement of $1^{\prime}-6^{x}$ Common Fill & 4,848 & or & 3.75 & 0.06 & 291 & 5,810 & 10,100 & 1.745 & 8.30 & 20,74 \\
\hline 18 & Placement of 6" Topsoil & 1,820 & or & 9.5 & 0.06 & 109 & 2,184 & 17,200 & 655 & 11.10 & 20,130 \\
\hline & (G) PAGE 2 TOTAL & & & & & 6,827 & 139,549 & 600,497 & 30,661 & & 053,71 \\
\hline
\end{tabular}

E-9. Cost Estimate for a Cover System Utilizing Soil Cement and 30-mil FML (Cont'd) 
ACTIVTTY CODE: ESE260112

PROJ.NAME: ECON. BVALUATTON OF CLOSURB CAP BARRTER MATERIAL

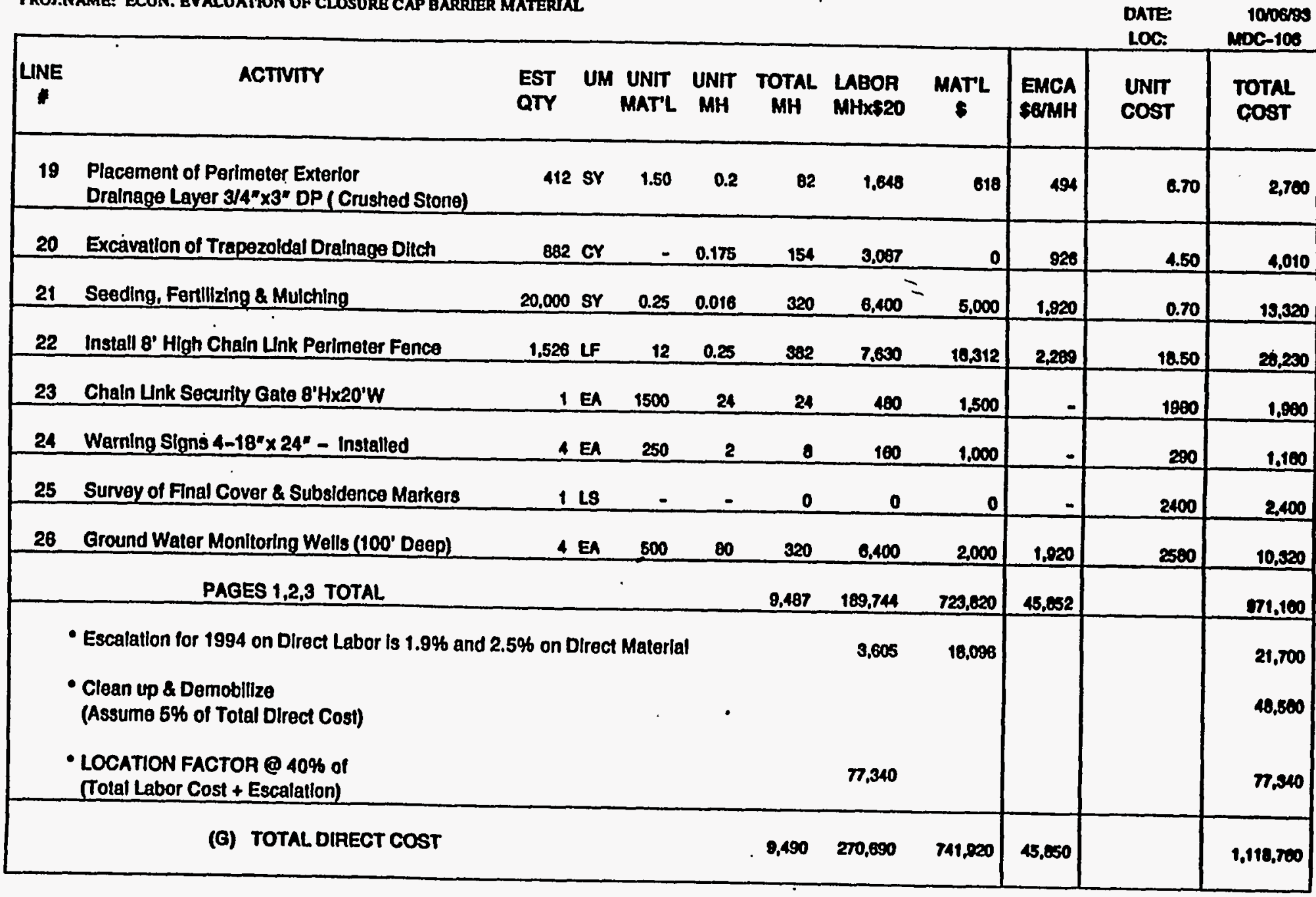

E-9. Cost Estimate for a Cover System Utilizing Soil Cement and 30-mil FML (Cont'd) 
D B T A I L B S T I M A B

LOO 1: 7-03

ACTIVITY CODE: ESE260412

PROJ.NAME: ECON. EVALUATION OP CLOSURE CAP BARRIER MATERIAL

\begin{tabular}{|c|c|c|c|c|c|c|c|c|c|c|}
\hline LINE & ACTIVITY & $\begin{array}{l}\text { EST } \\
\text { QTY }\end{array}$ & $\begin{array}{l}\text { UM UNIT } \\
\text { MAT'L }\end{array}$ & $\begin{array}{l}\text { UNIT } \\
\text { MH }\end{array}$ & $\begin{array}{c}\text { TOTAL } \\
\text { MH }\end{array}$ & $\begin{array}{l}\text { LABOR } \\
\text { MHX } \$ 20\end{array}$ & $\underset{\$}{\text { MATLL }}$ & $\begin{array}{l}\text { EMCA } \\
\text { SAMMH }\end{array}$ & $\begin{array}{l}\text { UNIT } \\
\text { COST }\end{array}$ & $\begin{array}{l}\text { TOTAL } \\
\text { Cost }\end{array}$ \\
\hline & INDIRECT COSTS & & & & & & & & & \\
\hline 1 & $\begin{array}{l}\text { Certification of the Final Cover } \\
\text { (Assume 3\% of the Total Direct Cost) }\end{array}$ & & 28 & & & & $=$ & & & 33,600 \\
\hline 2 & $\begin{array}{l}\text { Health \& Salety } \\
\text { Assume } 5 \% \text { of Total Direct Cost }\end{array}$ & & L.9 & & & & & & & 85,840 \\
\hline 3 & $\begin{array}{l}\text { Scope Contingency } \\
\text { Assume 10\% of Total Direct Cost }\end{array}$ & & L8 & & & & & & & 111,000 \\
\hline 4 & $\begin{array}{l}\text { Administrative \& Legal } \\
\text { @ 10\% of Total Direct Cost }\end{array}$ & & LS & & & & & & & 111,860 \\
\hline $\mathbf{5}$ & $\begin{array}{l}\text { Engineering \& Services @ } \\
20 \% \text { of Total Dlrect Cost }\end{array}$ & & - & & & & & & & 223,750 \\
\hline 6 & Craft Support @ $20.8 \%$ of Total MHRS ( $\$ 19 / \mathrm{HR}$ ) & & & & 1,974 & & & & & 37.504 \\
\hline 7 & $\begin{array}{l}\text { Field Indirects of Tolal Direct } \\
\text { Labor @ 46.5\%, Bulk Material @ 26.5\% }\end{array}$ & & & & & 125,871 & 196,609 & & & 322,400 \\
\hline 8 & $\begin{array}{l}\text { Construction Nonmanual @ } 25.2 \% \text { of Total Direct } \\
\text { @ Rate of } \$ 43.68\end{array}$ & & & & 2,399 & & & & & 104,400 \\
\hline 9 & Profect Management @ 8\% of Tolal Direct + Ind & 8.09 & & & & & & & & 100.620 \\
\hline & (G) TOTAL INDIRECT COST & & & & & & & & & $1,171,070$ \\
\hline
\end{tabular}

E-9. Cost Estimate for a Cover System Utilizing Soil Cement and 30-mil FML (Cont'd) 
D B T A L B S T I A T B

C.MEDY:

LOa A: $7-03$

ACTIVITY CODE: ESE260112

PROS.NAME: ECON. BVALUATION OF CLOSURB CAP BARRRER MATERIAL

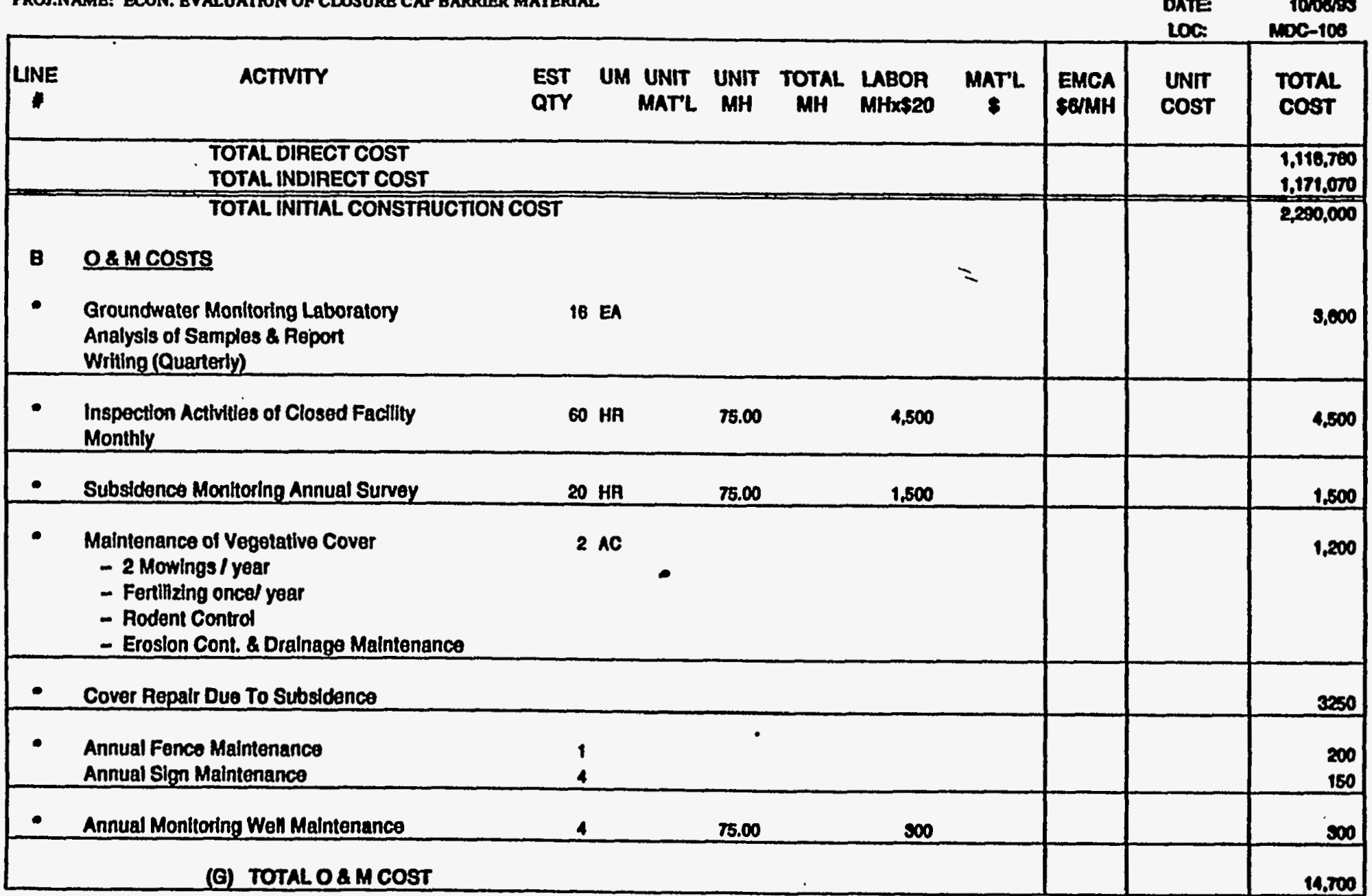

E-9. Cost Estimate for a Cover System Utilizing Soil Cement and 30-mil FML (Cont'd) 
DETAIL BSTIMATE

\begin{tabular}{|c|c|}
\hline WCD BY: & 8. MEAD \\
\hline ETmaATo & Pam \\
\hline $\begin{array}{l}\text { PHOME: } \\
\text { DATE: } \\
\text { LOC: }\end{array}$ & 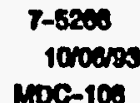 \\
\hline
\end{tabular}

L001: 7-03

ACTIVITY CODE: ESEZGON12

PROI.NAME: ECON. EYALUATHON OF CLOSURB CAP BARRIER MATERIAL

nac-10.

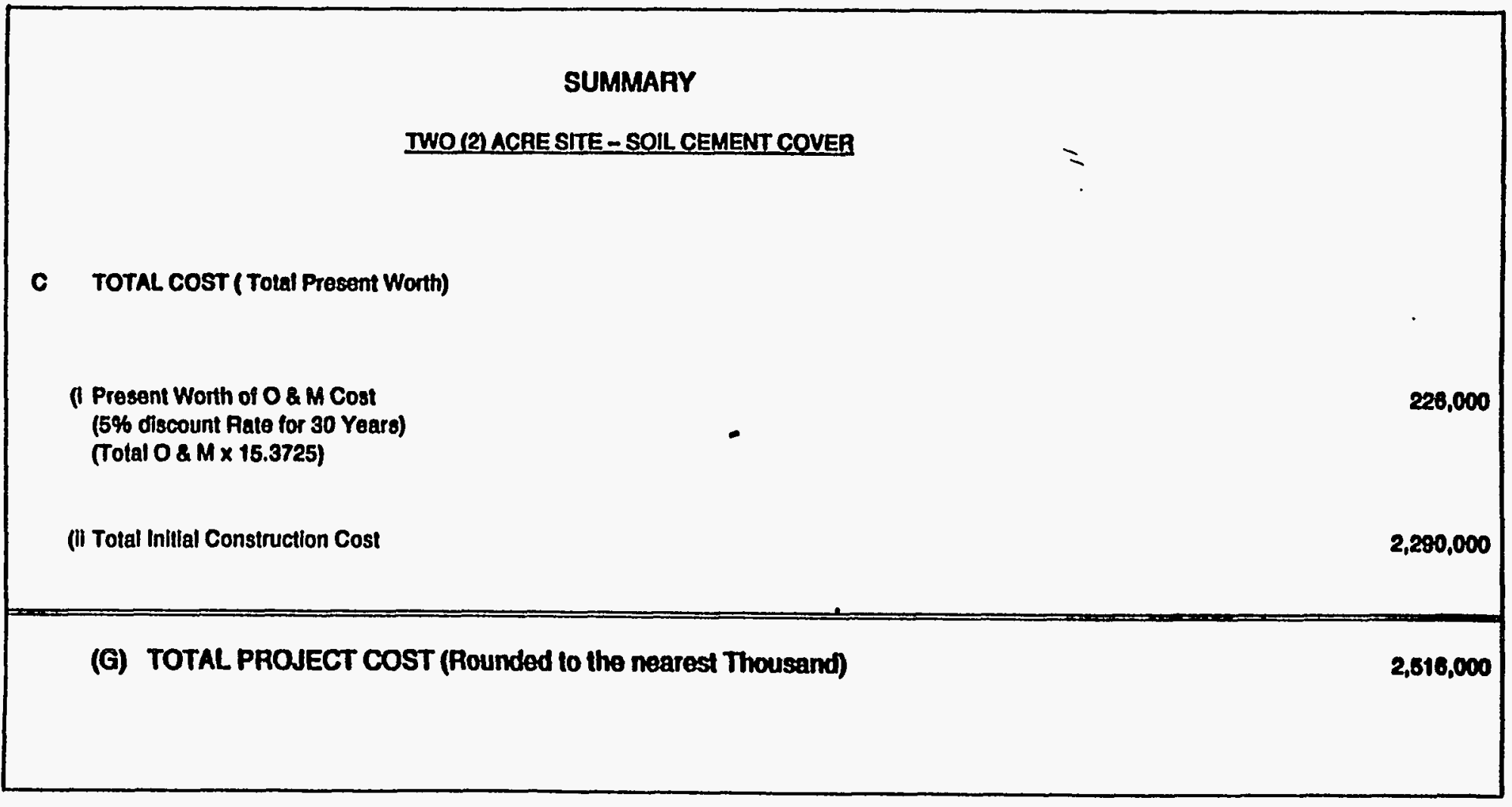

E-9. Cost Estimate for a Cover System Utilizing Soil Cement and 30-mil FML (Cont'd) 


\section{Lo0 I: $7-03$}

ACTIVTTY CODE: ESE260412

PROJ.NAME: ECON. EVALUATION OF CLOSURE CAP BARRTER MATERIAL

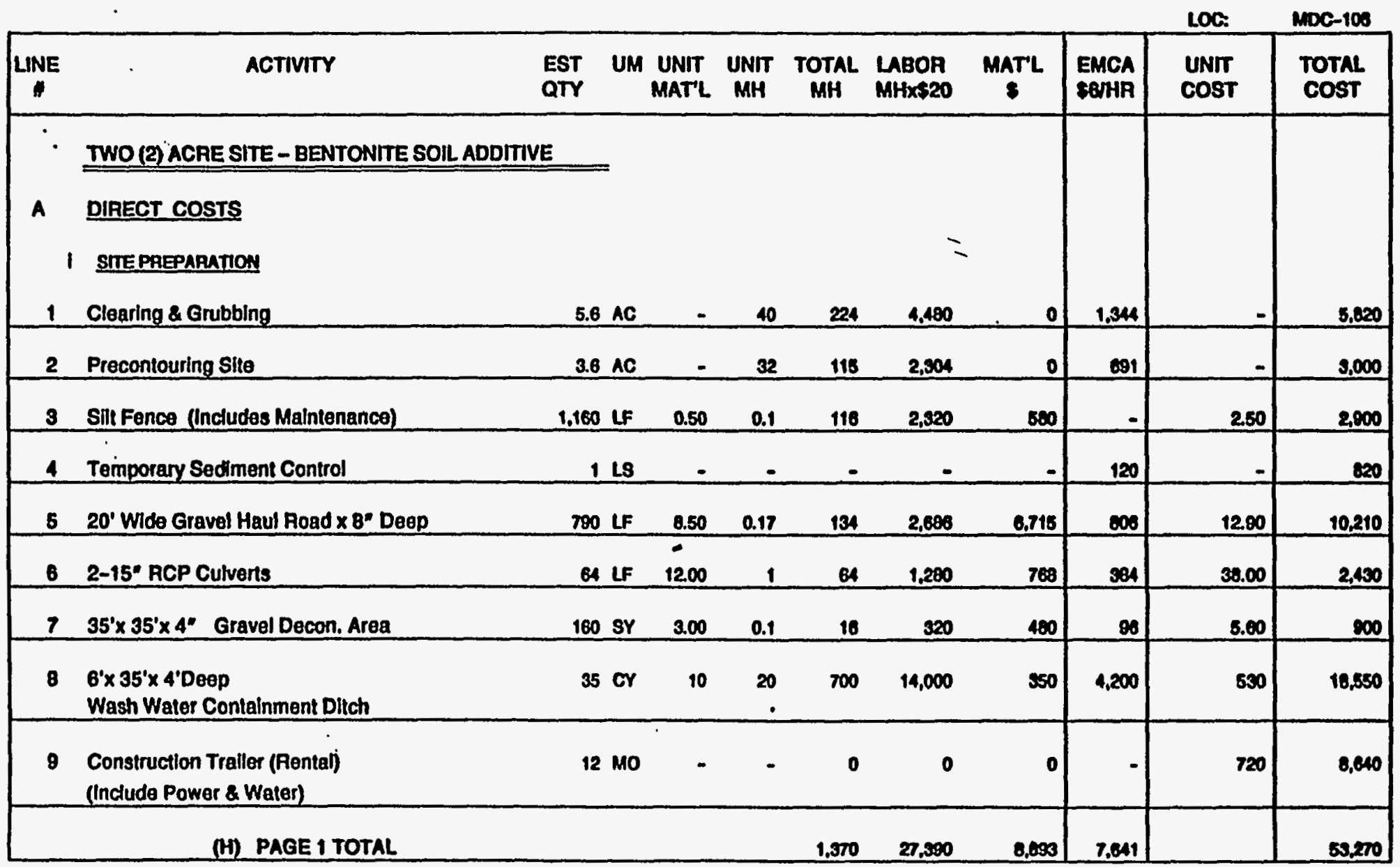

E-10. Cost Estimate for a Cover System Utilizing Bentonite Soil Additive and a 30-mil FML 
D ETAIL BSTIMATB

CAKDOBY: Q.MEAD

L.001: 7-03

ACTIVITY CODE: ESE260412

PROJ.NAME: ECON. EVALUATTON OF CLOSURE CAP BARPIER MATERIAL

ESTMATOF: PAMMK

PHONE 7-5260

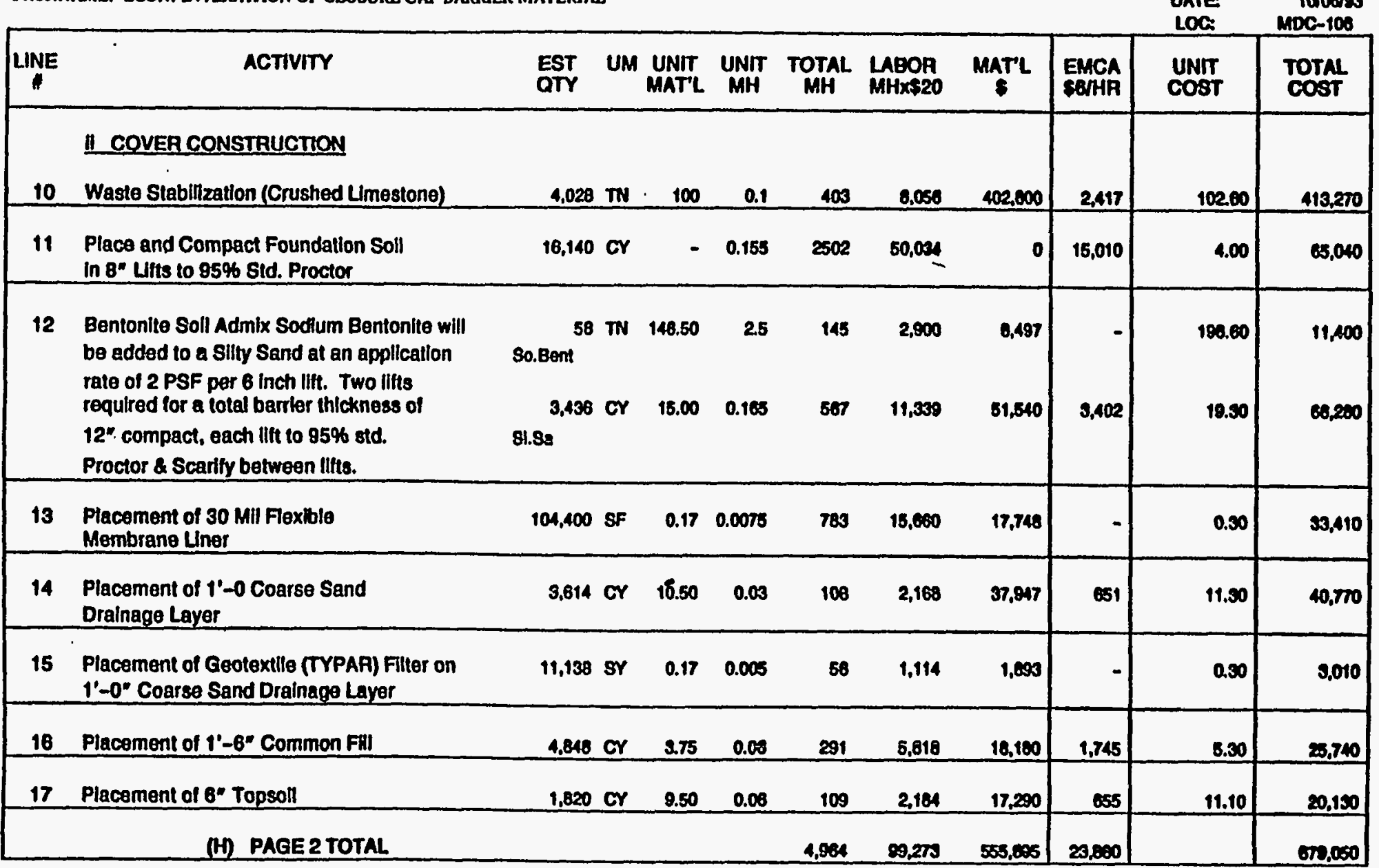

E-10. Cost Estimate for a Cover System Utilizing Bentonite Soil Additive and a 30-mil FML (Cont'd) 
D E T A I L E S T I M A T B

CHKDQY: S.MEAD

LOO A: 7-03

ACTIVITY CODE: ESE260112

PROJ.NAME: ECON. EVALUATION OR CLOSURE CAP BARRIER MATERJAL

ESTMATOR: POMnK

DATE: 100699

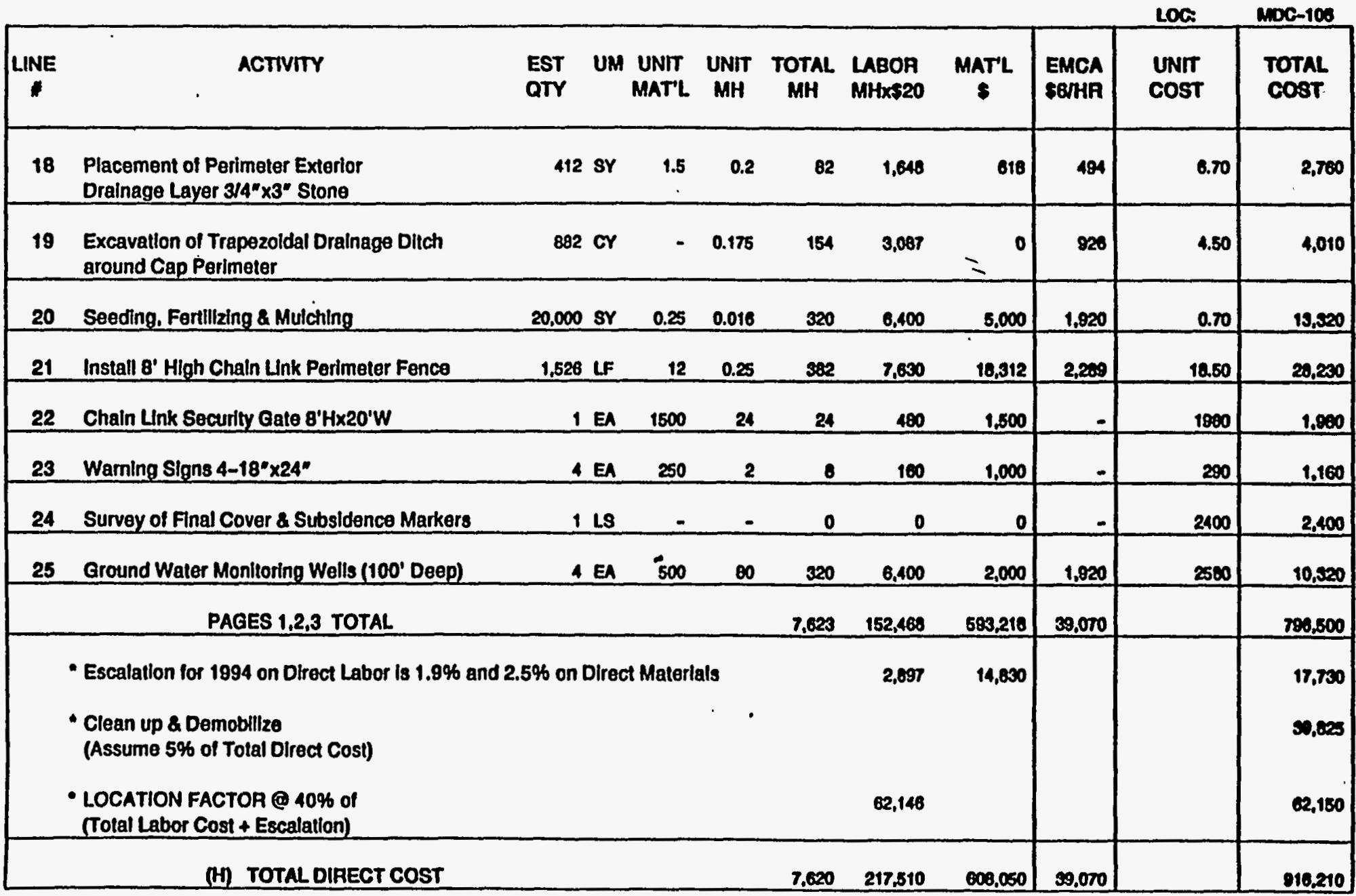

E-10. Cost Estimate for a Cover System Utilizing Bentonite Soil Additive and a 30-mil FML (Conl'd) 
D E T A L E S T I A T B

GHCD BY: Q.MEAD

LOO 11: 7-03

ACTIVITY CODE: ESE260112

ESTMATOR: PQMAK

PROJ.NAMB: ECON. EVALUATION OF CLOSURB CAP BARRIER MATERIAL

PHOWE: 7-5260

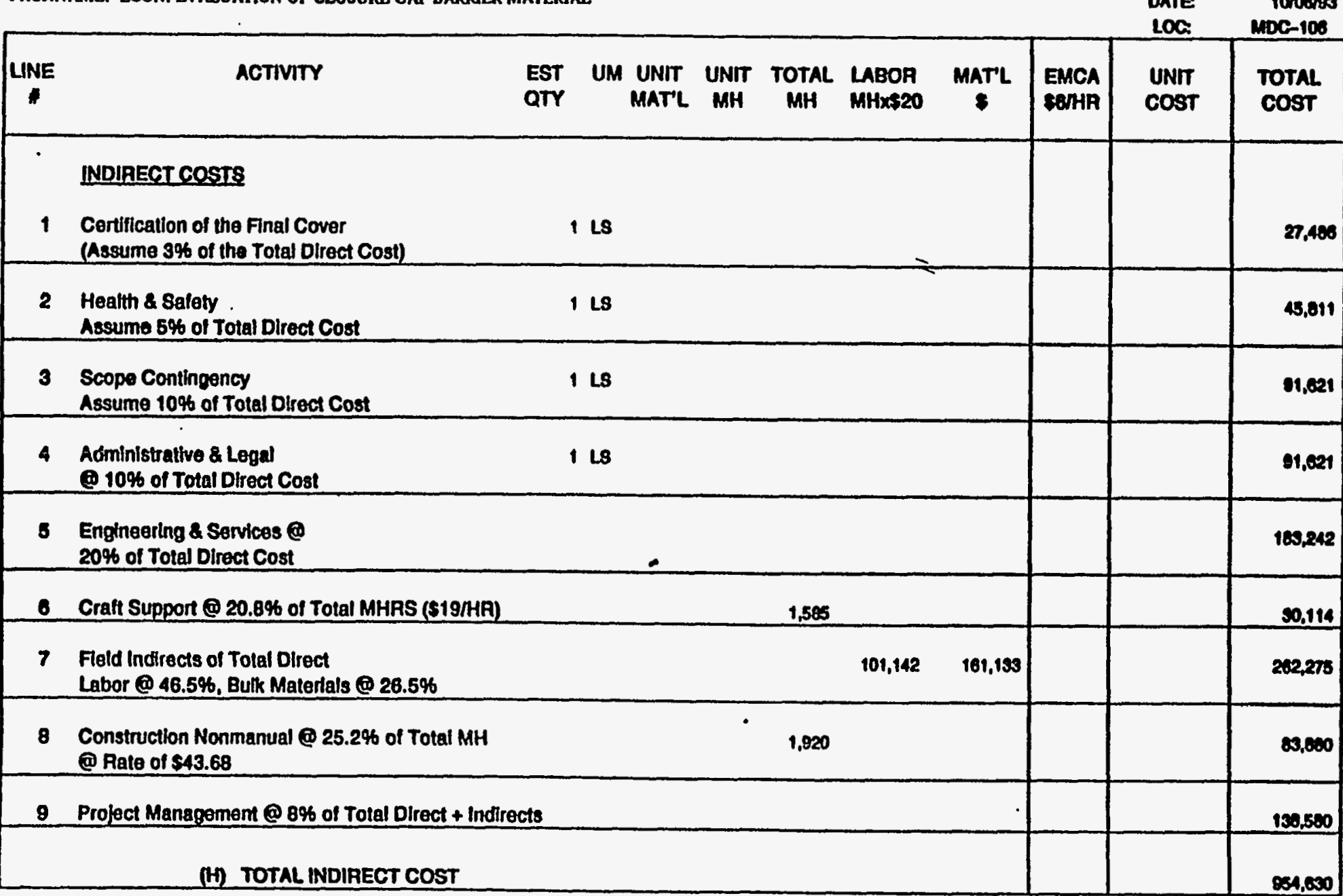

E-10. Cost Estimate for a Cover System Utilizing Bentonite Soil Additive and a 30-mil FML (Cont'd) 
D BTAIL B S T I A T B

9. MEAD

LOOI: :-03

ACTIVITY CODE: ESE2601/2

PROJ.NAME: ECON. EVALUATION OF CLOSURE CAP BARRIER MATERINL

Amso Br:

ESTMATOR:

PHONE:

PHONE

Pank

$7-5800$

10C:

$1000 / 23$

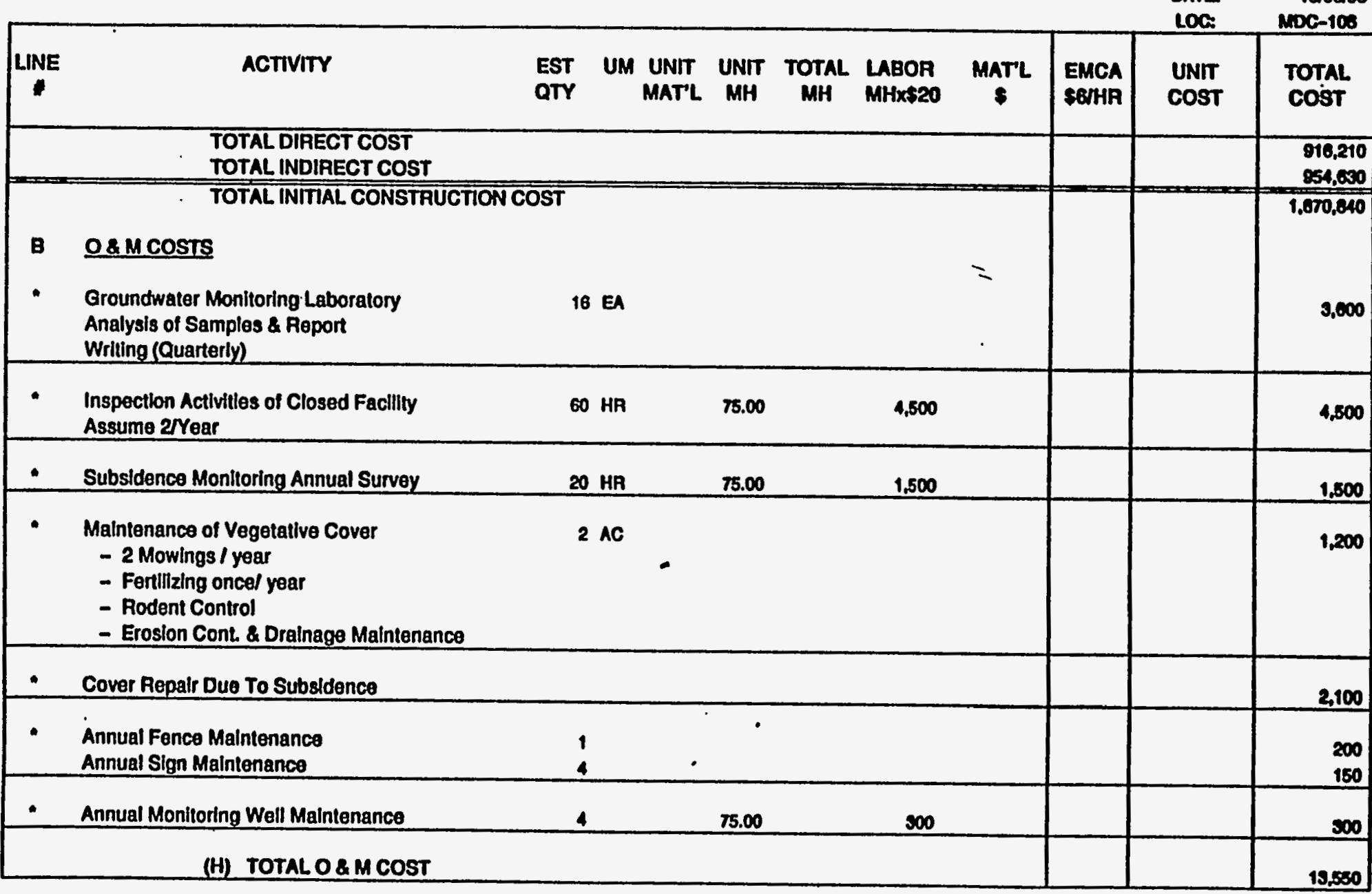

E-10. Cost Estimate for a Cover System Utilizing Bentonite Soil Additive and a 30-mil FML (Cont'd) 


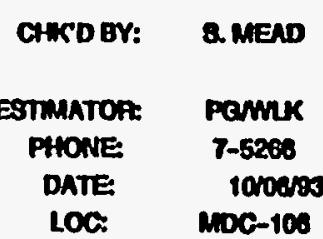

ACTIVITY CODE: ESE26012

PROI.MAME: ECON. BVALUATON OP CLOSURE CAP BARPUER MATERIAL.

Moc-100

\section{SUMMARY}

TWO (2) ACAE SITE - BENTONITE SOIL ADDITIVE

C TOTAL cost ( Total Present Worth)

(1) Present Worth of $O \& M$ Cost

(5\% discount Rate for 30 Yeare)

208,000

(Total O\& $\mathrm{B} \times$ 15.3725)

(il Total Initial Construction Cost

(H) TOTAL PROJECT COST (Rounded to the nearest Thousand)

$2,079,000$

E-10. Cost Estimate for a Cover System Utilizing Bentonite Soil Additive and a 30-mil FML (Cont'd) 
DETAILESTIMATE

L001: 7-03

ACTIVITY CODE: ESE260412

PROU.NAMB: ECON, BVALUATTON OR CLOSURB CAP BARRRER MATERIAL

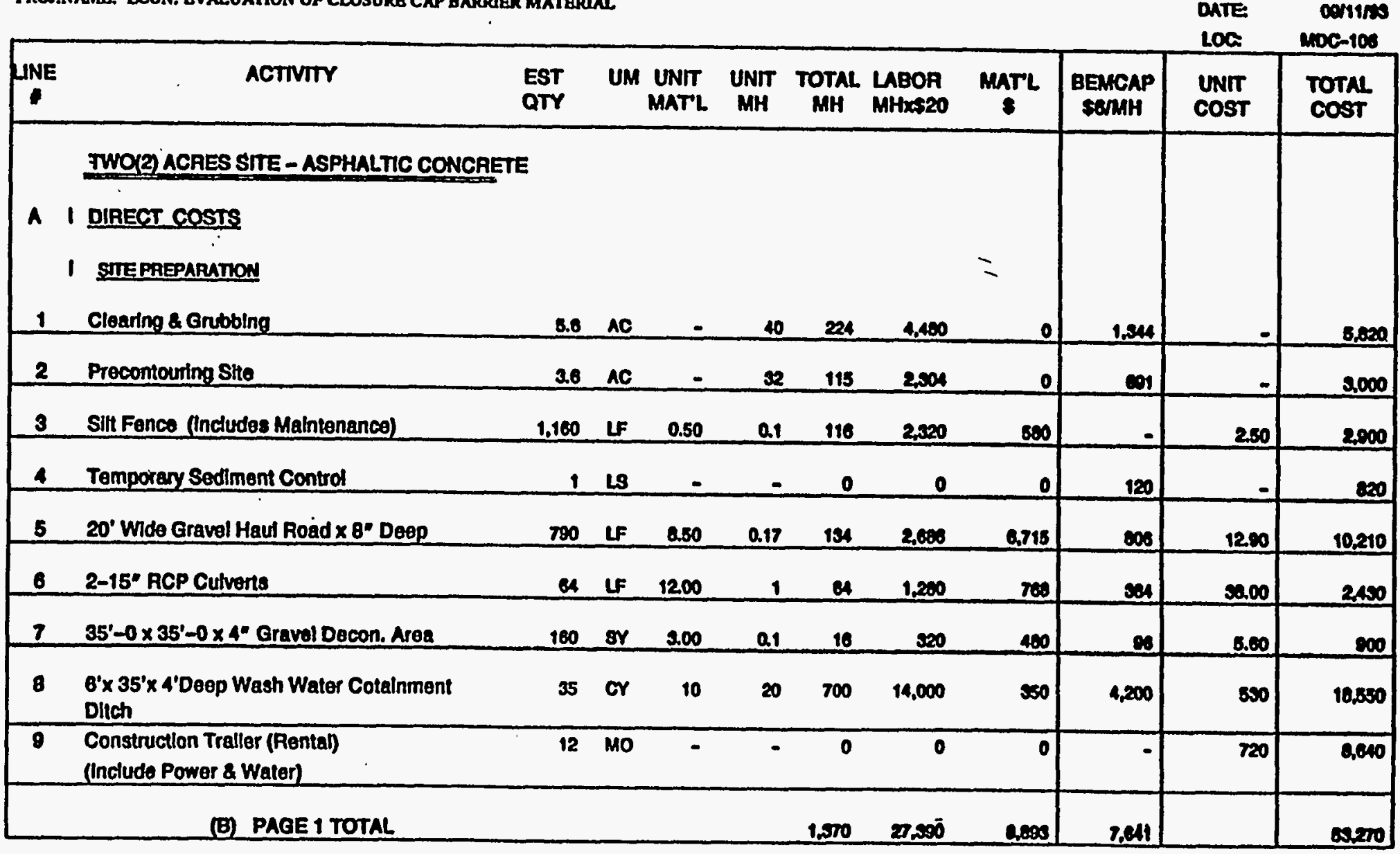

E-11. Cost Estimate for a Cover System Asphalt Concrete Hydraulic Barrier and a 30-mil FML 
DETAIL ESTIMATE

200 : 1-03

ACTIVTY CODB: BSE260412

PROSNAMB: 'ECON. BVALUATYON OP CLOSURB CAP IARUIER MATERIAL.

QHEOEY: MEN

ESTMATOF: PanuK

PHONE: T-6200

DATE: OOI1M

LOa MOC-100

\begin{tabular}{|c|c|c|c|c|c|c|c|c|c|c|c|}
\hline JNE & ACTIMT & $\begin{array}{l}\text { Est } \\
\text { aTr }\end{array}$ & UM & $\begin{array}{l}\text { UNIT } \\
\text { MAT'L }\end{array}$ & UNI & $\begin{array}{c}\text { TOTAL } \\
\text { MH }\end{array}$ & $\begin{array}{l}\text { LABOR } \\
\text { MHbeteo }\end{array}$ & MATL & $\begin{array}{l}\text { Bgucap } \\
\text { PanMH }\end{array}$ & $\begin{array}{l}\text { UNIT } \\
\text { COST }\end{array}$ & Totel \\
\hline 10 & $\begin{array}{l}\text { DCOVEA CONSTRUCTION } \\
\text { Waste Stabilization (Crushed Limestono) }\end{array}$ & 4,020 & TN & 100 & 0.1 & 403 & 0,050 & 102,000 & 2417 & 10200 & 41220 \\
\hline 11 & $\begin{array}{l}\text { Place and Compact Foundation 80: } \\
\text { In 8* Lifts } 10 \text { 95\% Std. Proctor }\end{array}$ & 12,780 & Cr & - & 0.155 & 1801 & 29,618 & $\mathbf{0}$ & 11,000 & 4.00 & 81.000 \\
\hline 12 & $\begin{array}{l}\text { Piacement of } 30 \text { Mil Flexble } \\
\text { Membrane Liner }\end{array}$ & 104,400 & SF & 0.17 & 0.0075 & 700 & 18,600 & 17,748 & - & 0.50 & 30.410 \\
\hline 13 & $\begin{array}{l}\text { Placement of 1'-0 Coarse Sand } \\
\text { Dralnage Layer }\end{array}$ & 3,372 & Cr & 10.50 & 0.08 & 101 & 2,000 & 25,400 & 607 & 11.50 & sespento \\
\hline 14 & $\begin{array}{l}\text { 2* PVC Porforated Drainage Pipo placed } \\
\text { in Orainage layer }\end{array}$ & 1,960 & LF & 1.0 & 0.22 & 431 & 2024 & 1,800 & - & 6.40 & 10,500 \\
\hline 16 & 2 1/2" Binder Course & 10,600 & $\mathbf{S r}$ & 4.2 & 0.06 & $\infty 6$ & 12,720 & 4680 & 3,018 & 2.80 & 01,000 \\
\hline 10 & 4" Hydraulle Asphalt Conc. & 10,600 & SY & a.70 & 0.00 & 310 & 6,000 & $n, 000$ & 1,000 & 7.50 & 7,280 \\
\hline 17 & Asphalt Mastlc Soal & 10,600 & 8Y & 0.50 & 0.015 & 169 & 3,160 & 8.000 & - & 0.00 & 2000 \\
\hline 18 & Sprayod Aophaltic Concrete Sealant & 10,000 & Br & 0.75 & 0.02 & 212 & 8,240 & 7.50 & 8,872 & 1.00 & 12000 \\
\hline 19 & Excavation of Traingular Drainage Ditch & 44 & Cr & $=$ & 0.2 & 8 & 1,70 & 0 & 803 & 8.20 & 2010 \\
\hline 20 & 4" Thick Asphatt Perimoter Drainage Dltch & 1,340 & SY & 7.85 & 0.15 & 201 & 4,020 & 10,510 & 1,200 & 11.00 & 18,750 \\
\hline & (B) PAGE2 TOTAL & & & & & B.,314 & $100.2 n$ & $\sin .223$ & 29,64 & & 722.150 \\
\hline
\end{tabular}

E-11. Cost Estimate for a Cover System Asphalt Concrete Hydraulic Barrier and a 30-mil FML (Cont'd) 
D B T A I L B S T I M A T B

LOO II: 7-03
ACTIVITY CODB: BSE20O412

FROJ.NAME: BCON. BVALUATTON OF CLOSURB CAP BNIRIER MATERINL

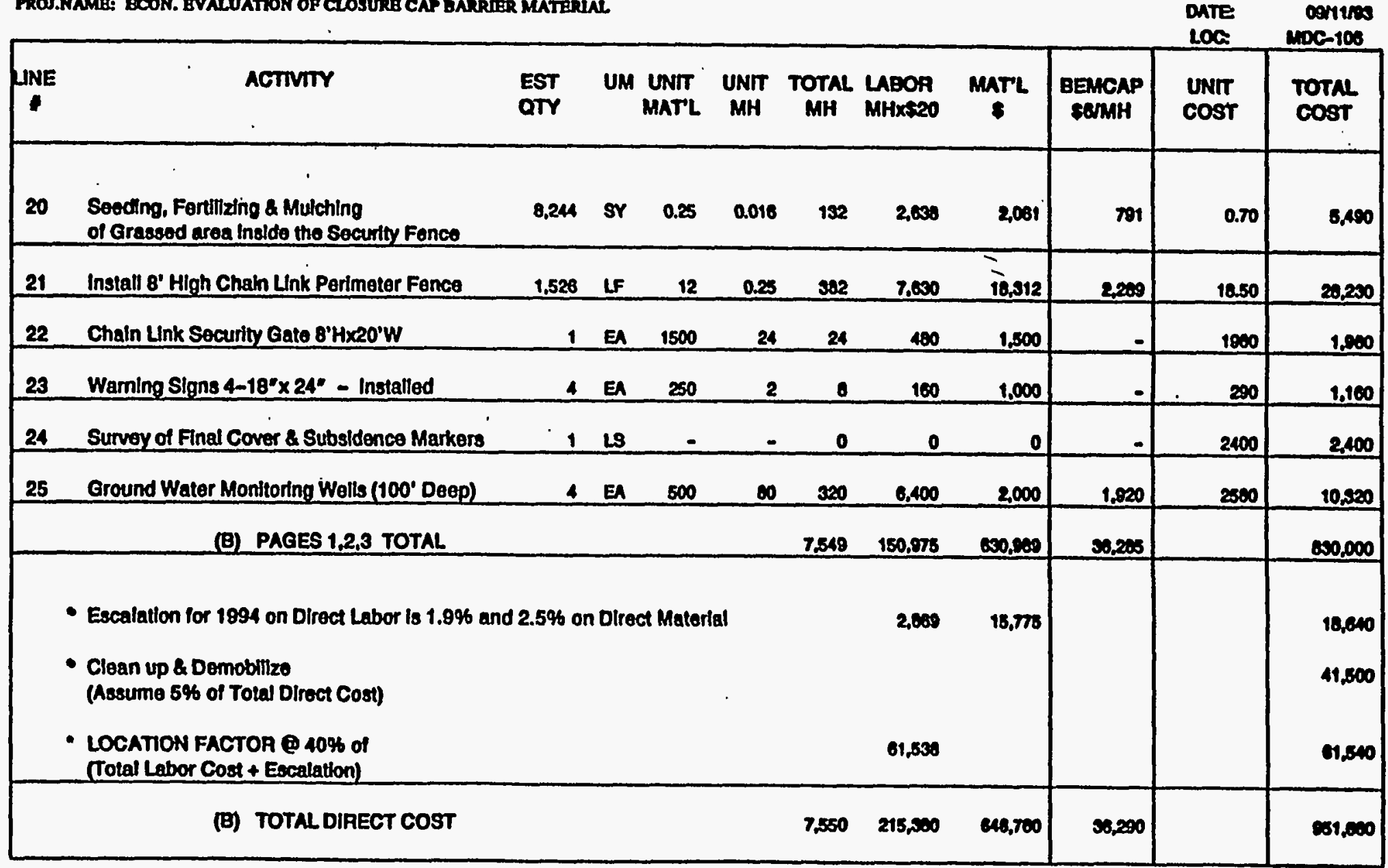

E-11. Cost Estimate for a Cover System Asphalt Concrete Hydraulic Barrier and a 30-mil FML (Cont'd) 
Economic Evaluation of Closure Cap Barrier Materials Study(U)

Volume II Appendix E

D B T A I L E B T I M A T B September 1993

Loa : $7-03$

ACTIVITY CODE: BSER60412

TROJ.NAME: BCON. EVALUATION OP CLOSURB CAP BARRIER MATERLAL.

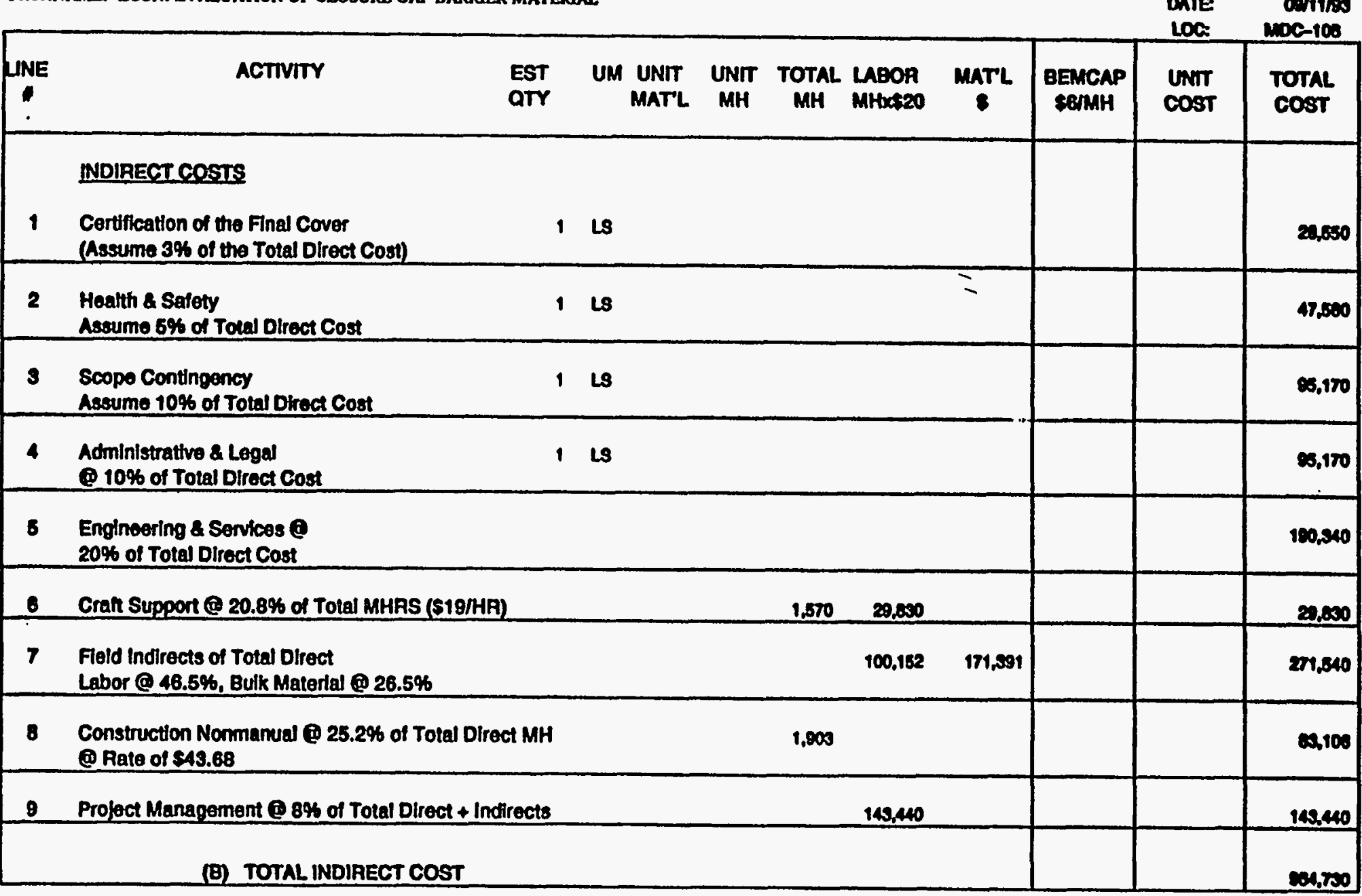

E-11. Cost Estimate for a Cover System Asphalt Concrete Hydraulic Barrier and a 30-mil FML (Cont'd) 
D B T A I B S T IMAT B

Carogr: 2MEND

2001: 7-03

ACTIVTY CODE: BSELSON12

FROJ.NAME: ECON. BVALUATKON OF CLOSURE CAP BARRRER MATERUAL

\begin{tabular}{|c|c|c|c|c|}
\hline & & & $\begin{array}{l}\text { CHCogr: } \\
\text { Estmutort } \\
\text { PHone: } \\
\text { DATE: } \\
\text { loa }\end{array}$ & 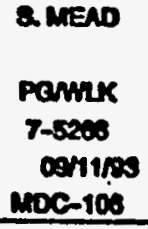 \\
\hline $\begin{array}{l}\text { ABOA } \\
A H x+20\end{array}$ & $\underset{8}{\text { MATL }}$ & $\begin{array}{l}\text { BEMCAP } \\
\text { SEMMH }\end{array}$ & $\begin{array}{l}\text { UNIT } \\
\text { COST }\end{array}$ & $\begin{array}{l}\text { TOTAL } \\
\text { COST }\end{array}$ \\
\hline & & & & $\begin{array}{l}061,000 \\
\operatorname{ses}, 700\end{array}$ \\
\hline & $=$ & & & $\begin{array}{r}1,086,000 \\
9,000\end{array}$ \\
\hline 4,500 & & & & 4,500 \\
\hline 1,500 & & & & 1,500 \\
\hline & & & & 9,000 \\
\hline & & & & 2000 \\
\hline & & & & $\begin{array}{l}200 \\
150 \\
\end{array}$ \\
\hline 500 & & & & 500 \\
\hline & & & & 18,250 \\
\hline
\end{tabular}

E-11. Cost Estimate for a Cover System Asphalt Concrete Hydraulic Barrier and a 30-mil FML (Cont'd) 
D B TAIL B S TIMATB

\begin{tabular}{|c|c|}
\hline CAKD DV: & \\
\hline $\begin{array}{l}\text { ESTmantort } \\
\text { PHONE: } \\
\text { DATE } \\
\text { LOC }\end{array}$ & 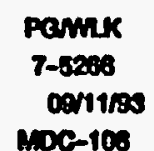 \\
\hline
\end{tabular}

Loo : 7-03

ACTIVITY CODE: ESE260412

MOU.NAME: ECON. EVALUATION OF CLOSURB CAP BARRIER MATERLAL

Loa Mor-108

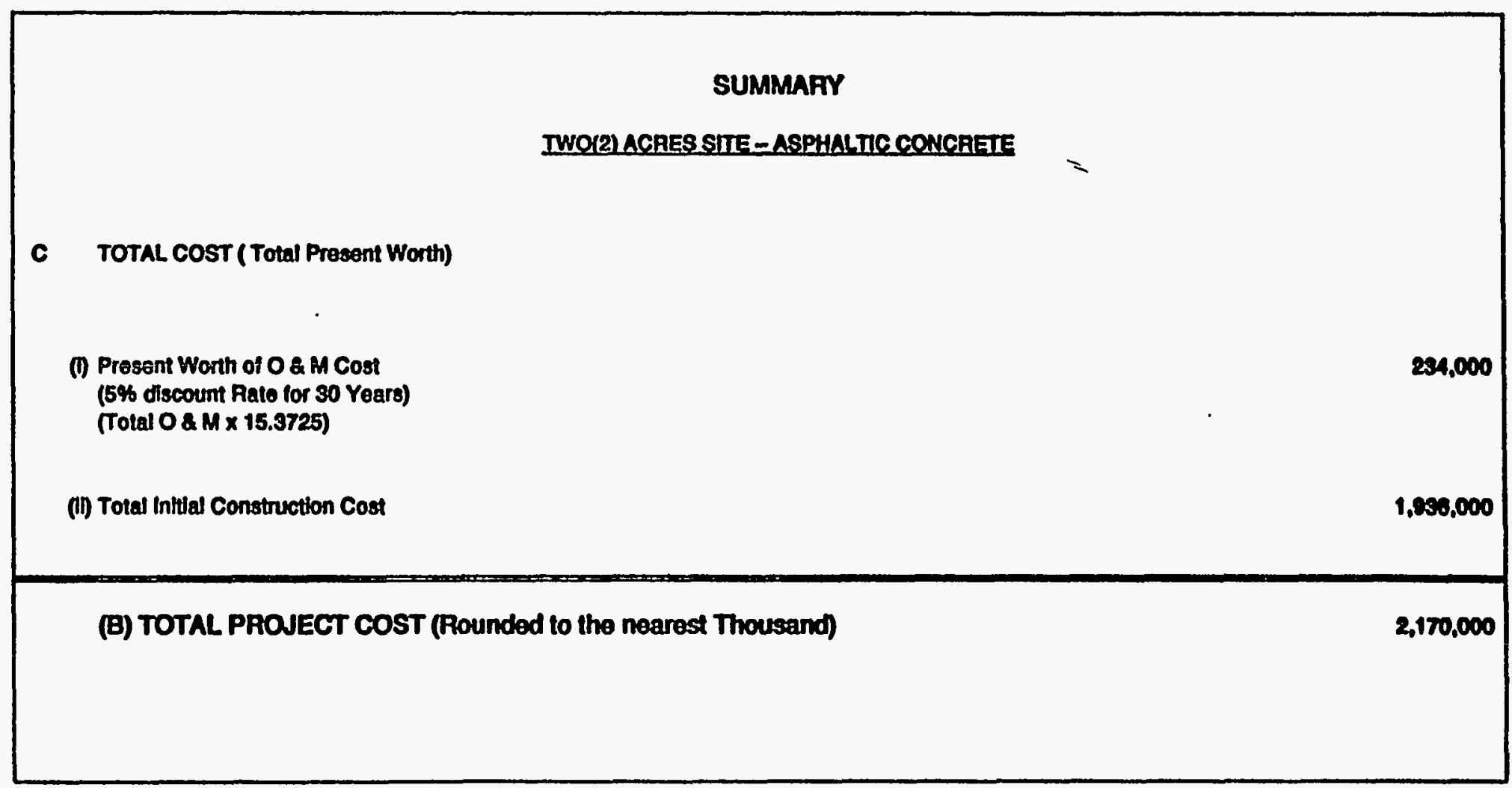

E-11. Cost Estimate for a Cover System Asphalt Concrete Hydraulic Barrier and a 30-mil FML (Cont'd) 


\section{LOQ : : $7-\infty$}

DETAIL ESTIMATE

ACTIVTY CODE ESE260412

PFOO.NAME: ECON. EVALUATHON OF CLOSURE CAP BARAREAMATERAL

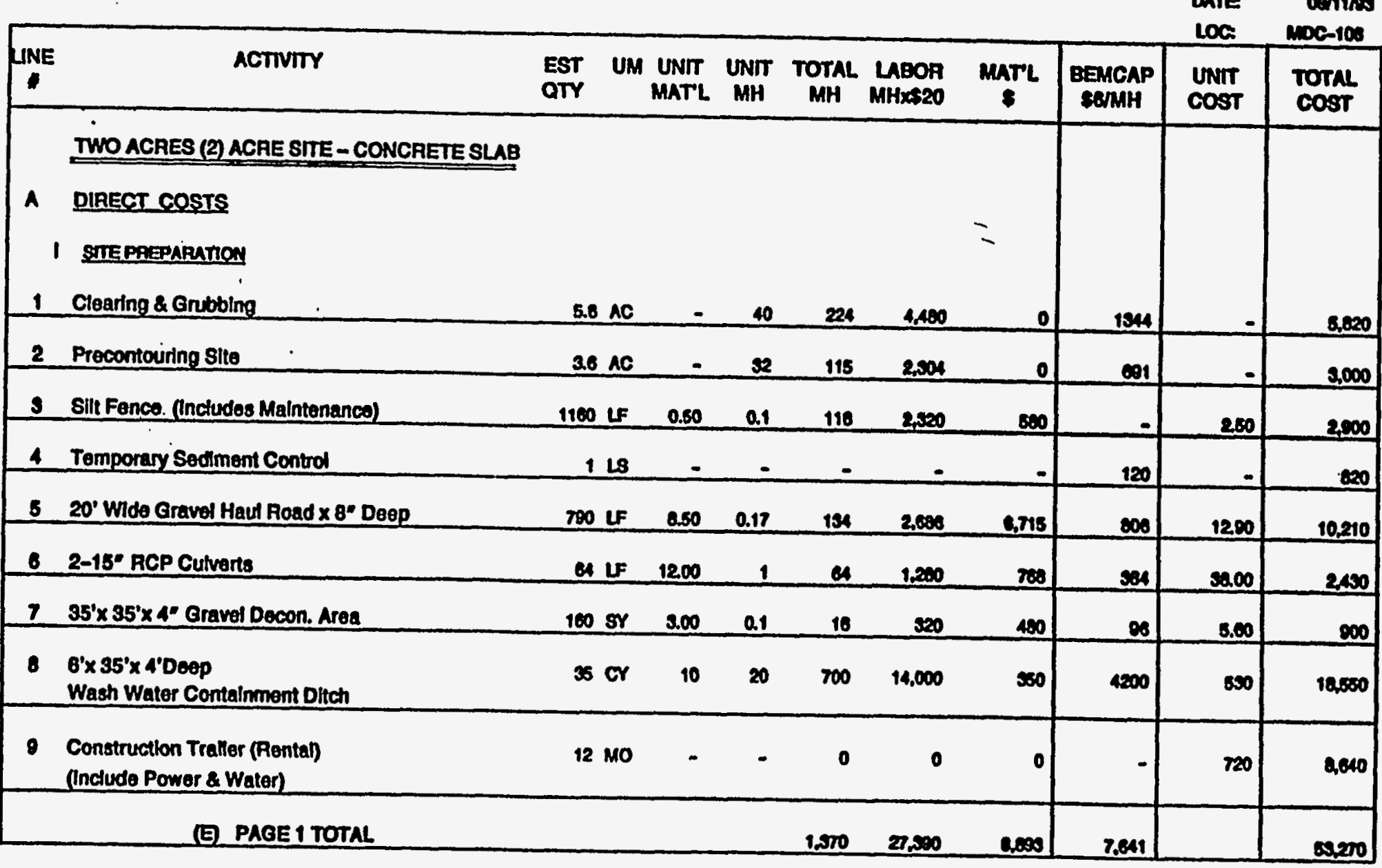

E-12. Cost Estimate for a Cover System Utilizing Poured-in Place Concrete Slab and a 30-mil FML 


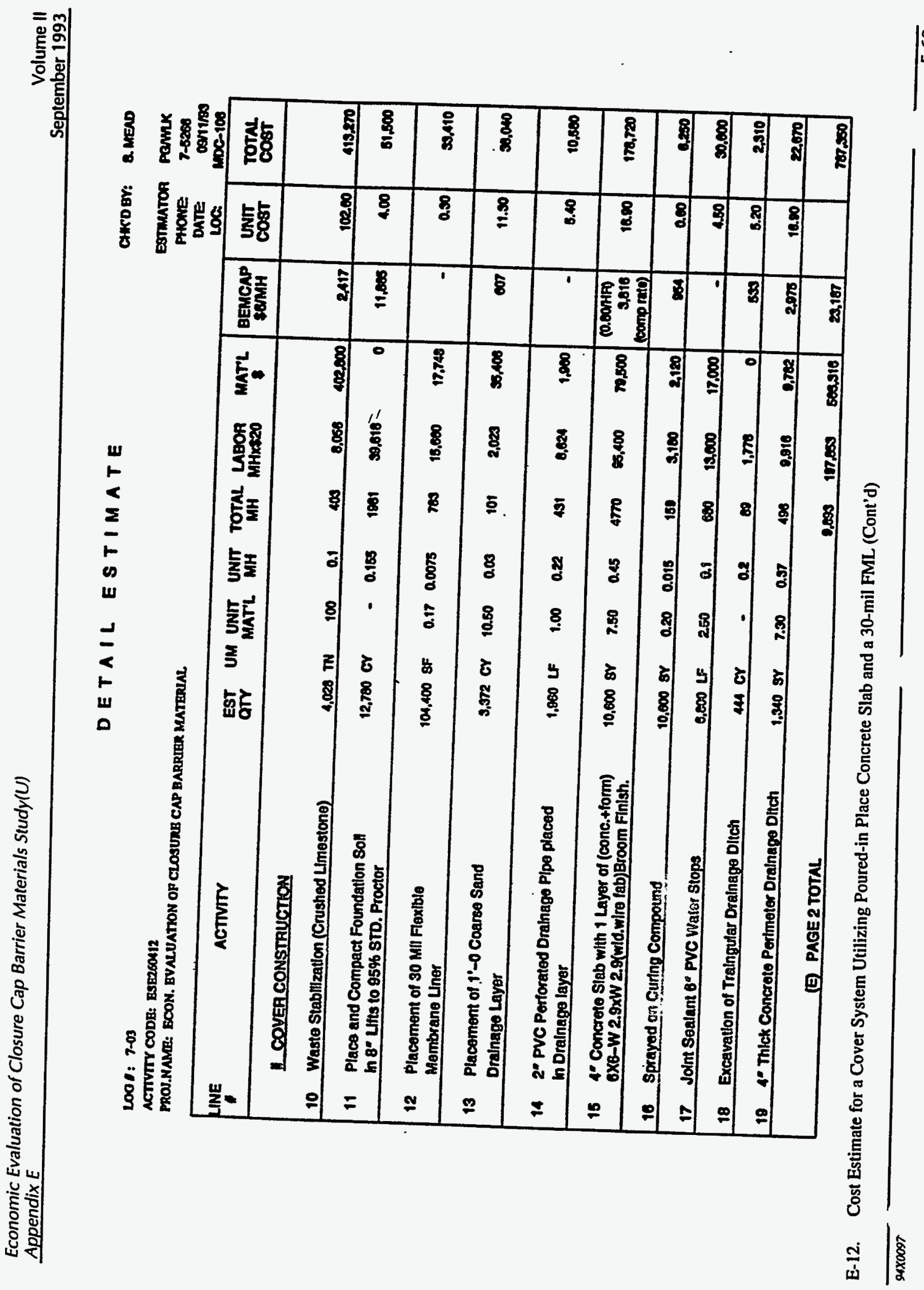


D B TA I L B S TIMATB

1001: 7-03

ACTIVTTY CODE: ESERSOH12

TROU.NAME: BCON. EVALUATION OP CLOSURB CAP BARRIER MATERLAL

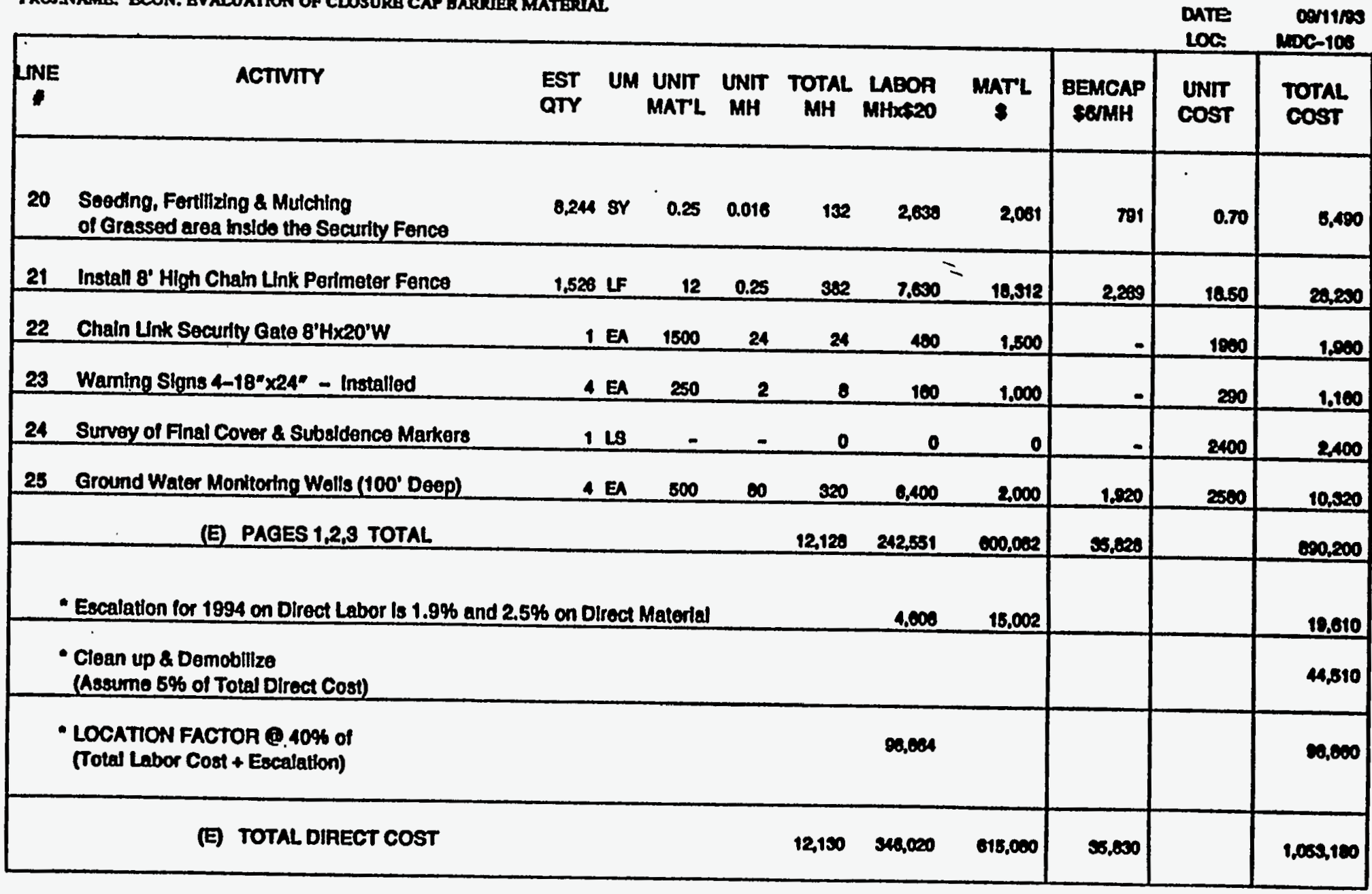

E-12. Cost Estimate for a Cover System Utilizing Poured-in Place Concrete Slab and a 30-mil FML (Cont'd) 
D BTAIL BSTIMATB CHEDEY: QMEAO

Loof: $7-03$

ACTTVTY CODE: BSE200112

MOJ.MAME: BCON. EVALUATTON OP CLOSURR CAP QARRRRR MATERUAL,

Estmator PanmK

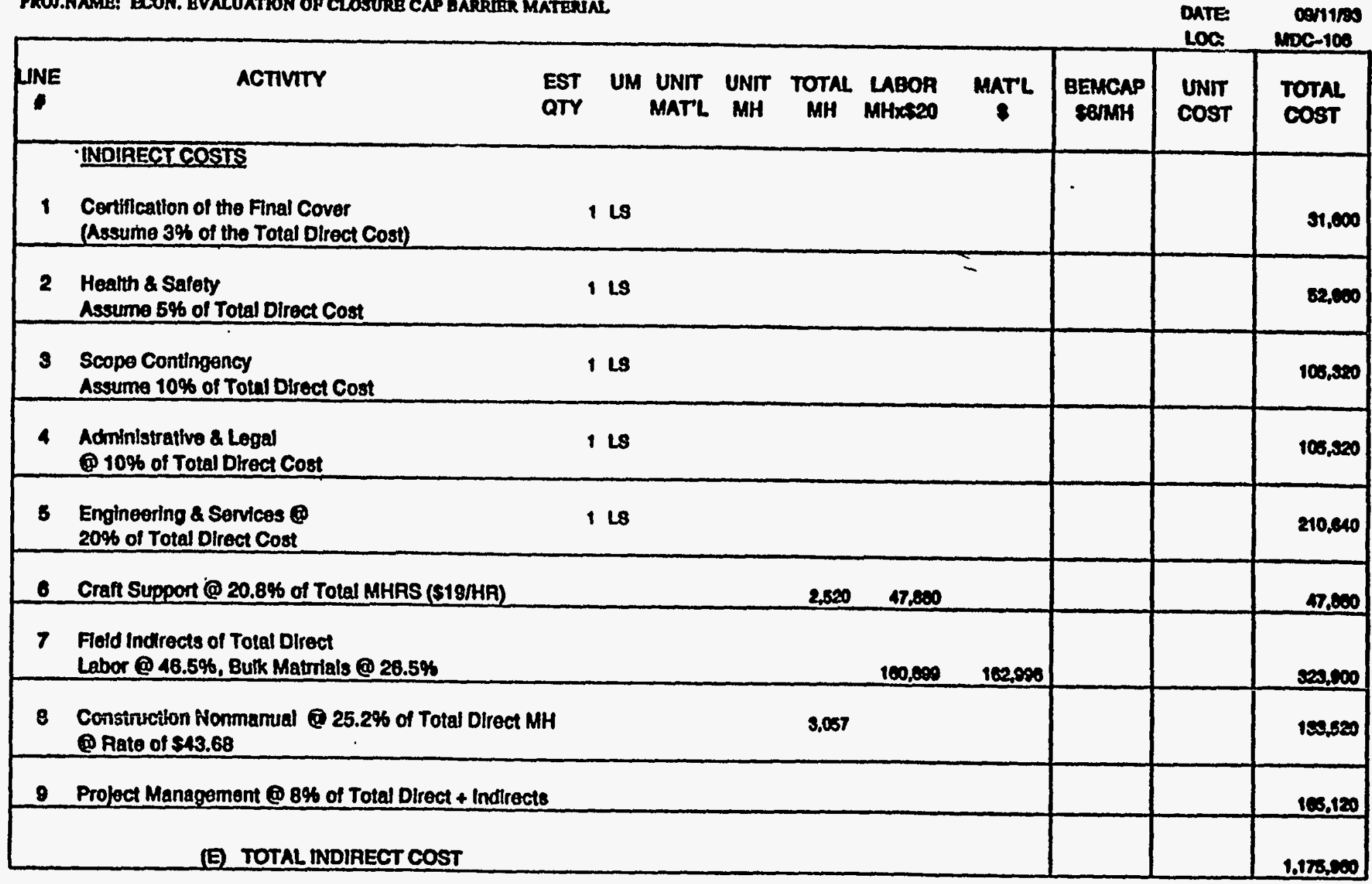

E-12. Cost Estimate for a Cover System Utilizing Poured-in Place Concrete Slab and a 30-mil FML (Cont'd) 
D B T A I L B TIMATB

\section{1: 7-03}

ACTVITY CODE: BSHESON12

MOJ.NAME: BCON. EVALUATION OR CLOSURE CAP BARRIIRR MATERURL.

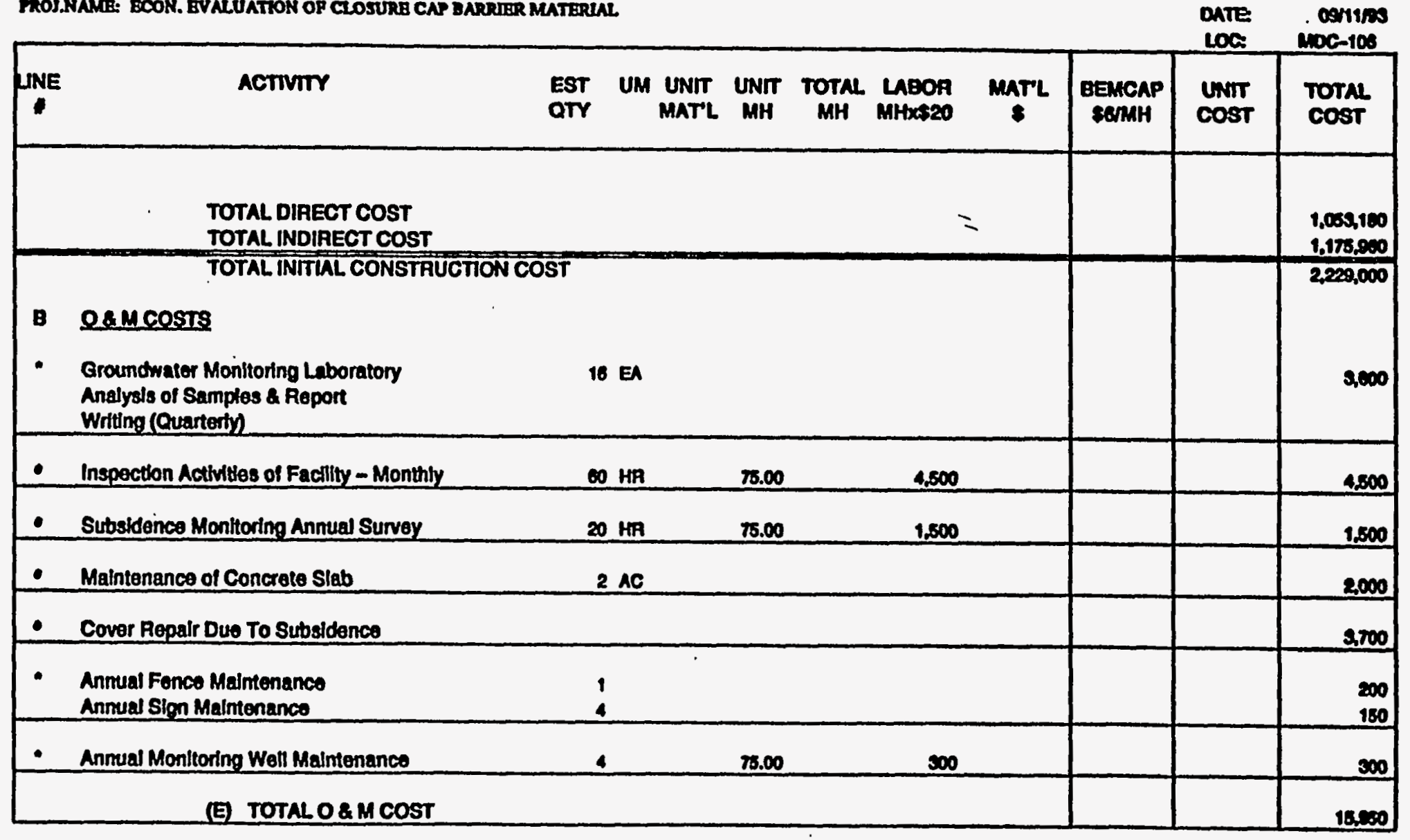

E-12. Cost Estimate for a Cover System Utilizing Poured-in Place Concrete Slab and a 30-mil FML (Cont'd) 
D BTAIL BSTIMATB

CHKOBY: Q MENO

Lo01: $7-03$

ACTIVTY CODE: RSE260112

MOO.NAME: BCON. EVALUATKON OP CLOSURB CAP BARRIER MATERUAS

Eomanton ramux

PHONE $7-6200$

DATE DON100

100. $100-100$

\section{SUMMARY}

TWOACAES (2) ACRE SITE-CONCARIE SQ AB

$=$

C TOTAL COST ( Totel Prosent Worth)

(i) Prosent Worth of 0 \& $M$ Cost

( $5 \%$ efiscount Rate for 30 Yoars)

(Total O\& $M \times$ 15.3725)

(il Total Initial Construction Cost

$2,229,000$

(E) TOTAL PROJECT COST (Rounded to the nearest Thousand)

$2,474,000$

E-12. Cost Estimate for a Cover System Utilizing Poured-in Place Concrete Slab and a 30-mil FML (Cont'd) 


\section{Appendix F}

\section{Cost Estimates for 5-Acre Site}




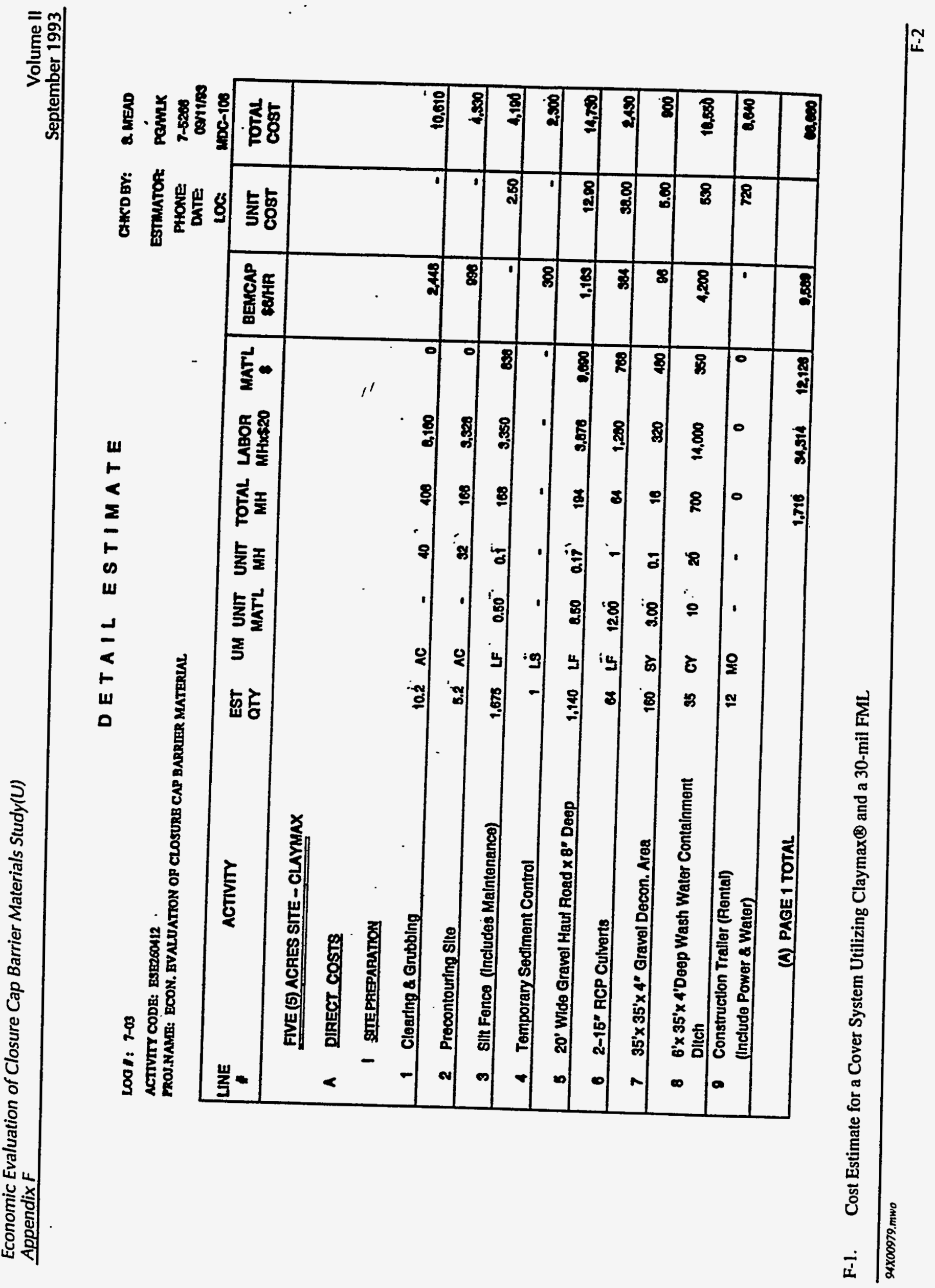


DETAIL ESTIMATE

CHCD BY: Q.MEND

Lod : 7-03

ACTIVITY CODB: BSE2CON12

TROJ.NAMB: ECON. BVALUATION OP CLOSURB CAP BARRIRR MATERIAL.

mimaroft pamk

PHOME: 7-5800

\begin{tabular}{|c|c|c|c|c|c|c|c|c|c|c|c|}
\hline LNNE & ACTrVIr & $\begin{array}{l}\text { EST } \\
\text { QTY }\end{array}$ & UM & $\begin{array}{l}\text { UNTT } \\
\text { MATLL }\end{array}$ & $\begin{array}{l}\text { UNIT } \\
\text { MH }\end{array}$ & TOTAL & $\begin{array}{l}\text { LABOA } \\
\text { MHxateO }\end{array}$ & $\underset{8 .}{\operatorname{MaTL}}$ & $\begin{array}{c}\text { BEMCAP } \\
\text { SAHA }\end{array}$ & $\begin{array}{l}\text { UNIT } \\
\text { cost }\end{array}$ & Toth \\
\hline 10 & $\begin{array}{l}\text { I. COVER CONSTRUCTION } \\
\text { Wasto Stabilization (Crushod Limestono) }\end{array}$ & 10,070 & $\mathrm{TN}$ & 100 & 0.1 & 1.007 & 20,140 & $1,007,000$ & 6,042 & 10200 & $1.053,100$ \\
\hline 11 & $\begin{array}{l}\text { Place and Compact Foundation Soll } \\
\text { in 8" Lifts to } 95 \% \text { STD. Proctor }\end{array}$ & 10,350 & or & - & 0.185 & 6,254 & 125,005 & $=$ & 37,620 & 4.00 & 162,610 \\
\hline 12 & Placement of Claymax Qeotextile Mat & 308,000 & SF & 0.45 & $0.0 \dot{0}$ & 3,080 & 61,000 & 100,000 & - & 0.70 & 200,200 \\
\hline 13 & $\begin{array}{l}\text { Placement of } 30 \text { Mil Flexiblo } \\
\text { Membrane Liner }\end{array}$ & 308,000 & SF & $0.17^{\circ}$ & 0.0075 & 2,310 & 46,200 & 82,500 & - & 0.00 & 60800 \\
\hline 14 & $\begin{array}{l}\text { Placement of ' } 1 \text { ' }-0 \text { Coarse Sand } \\
\text { Drainage Layer }\end{array}$ & 10,620 & Cr & $10.80^{\circ}$ & 0.00 & 810 & 6,572 & 111,810 & 1,012 & 11.50 & $110, \pi 0$ \\
\hline 16 & $\begin{array}{l}\text { Placement of Geotextile (TYPAR) Filter on } \\
\text { 1'-0" Coarse Sand Drainage Layer }\end{array}$ & $35.055^{\circ}$ & sy & 0.15 & 0.005 & 175 & 5,500 & 8,859 & - & 0.50 & 2,400 \\
\hline 10 & Piacement of $1^{\prime}-6^{\prime \prime}$ Common FIII & 12,120 & or & 3.75 & $0.00^{\circ}$ & 72 & 19,844 & 46,450 & 4.0009 & 8.00 & caseo \\
\hline 17 & Placemen of 6" Topsoll & 4,545: & or & 0.60 & a.od & 273 & 8.454 & 43,170 & 1.000 & 11.10 & 80270 \\
\hline 18 & $\begin{array}{l}\text { Placement of Perimeter Exterior } \\
\text { Dralnage Layer } 3 / 4^{\circ} \times 3^{*} \text { DP (Crushed Stono) }\end{array}$ & 641 & Sir & 1.60 & 0.2 & 120 & 2584 & $\operatorname{sos}$ & 700 & 8.70 & 4800 \\
\hline 19 & Excavation of Trapezoidal Drainage Ditch & 2,205 & or & - & Q.175. & 380 & 7.710 & 0 & 2016 & 400 & 10,000 \\
\hline & (A) PAGE 2 TOTAL & & & & & 14,008 & 289,102 & $1,105,010$ & 84500 & & $1,702, \pi 00$ \\
\hline
\end{tabular}

F-1. Cost Estimate for a Cover System Utilizing Claymax $\$$ and a 30-mil FML (Cont'd) 
D B TA I L B S TIMATB

CHCD BY: Q MEAD

L001: 7-03

ACTIVIY CODE: ESE2COA12

PROJ.NAME: ECON. EYALUATTON OF CLOSURB CAP BARRIER MATERUAL

Estmator: Parmik

PHONE 7-6200

DATE OOMTRO

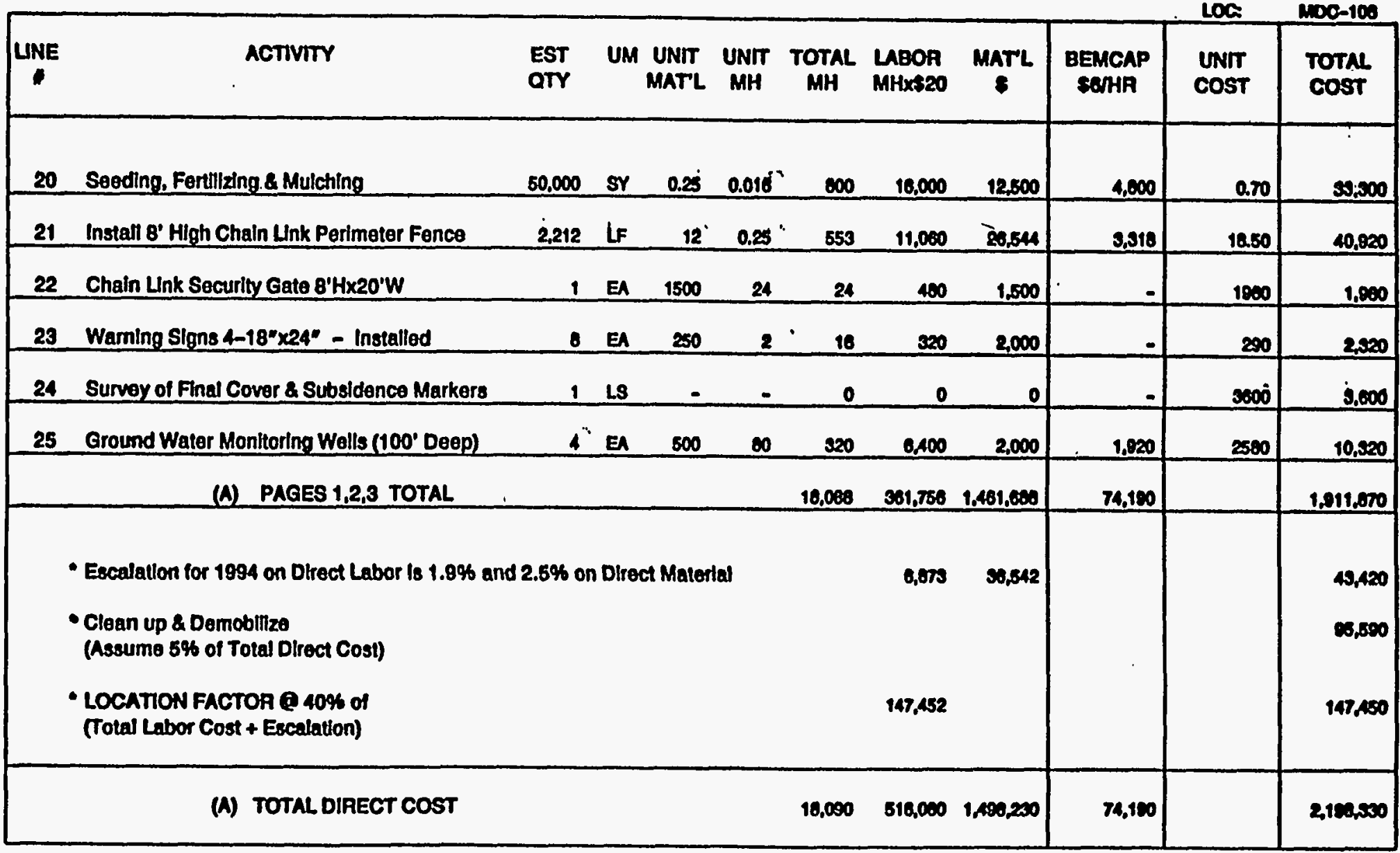

F-1. Cost Estimate for a Cover System Utilizing Claymax $®$ and a 30-mil FML (Cont'd) 
D B TAIL B S T I A T B

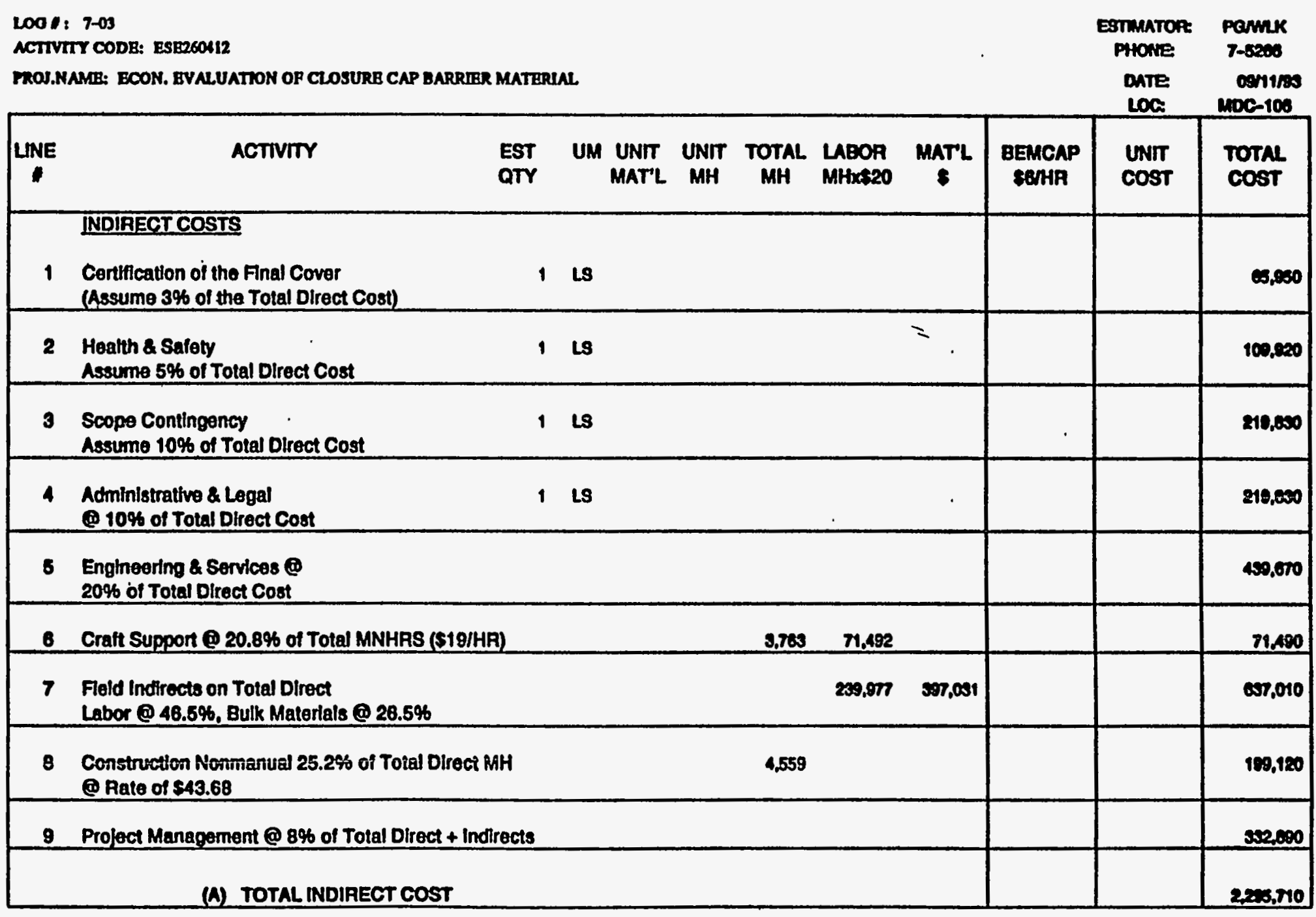

F-1. Cost Estimate for a Cover System Utilizing Claymax and a 30-mil FML (Cont'd) 
D B T A I L B S T I M A T B

CHKD BY: S.MEAD

2001: $7-03$

ACTTVITY CODB: ESE26012

PROJ.NAME: BCON. EVALUATION OF CLOSURB CAP BARRIER MATERIAL.

Ptore: Pamk

DATE CANTrs

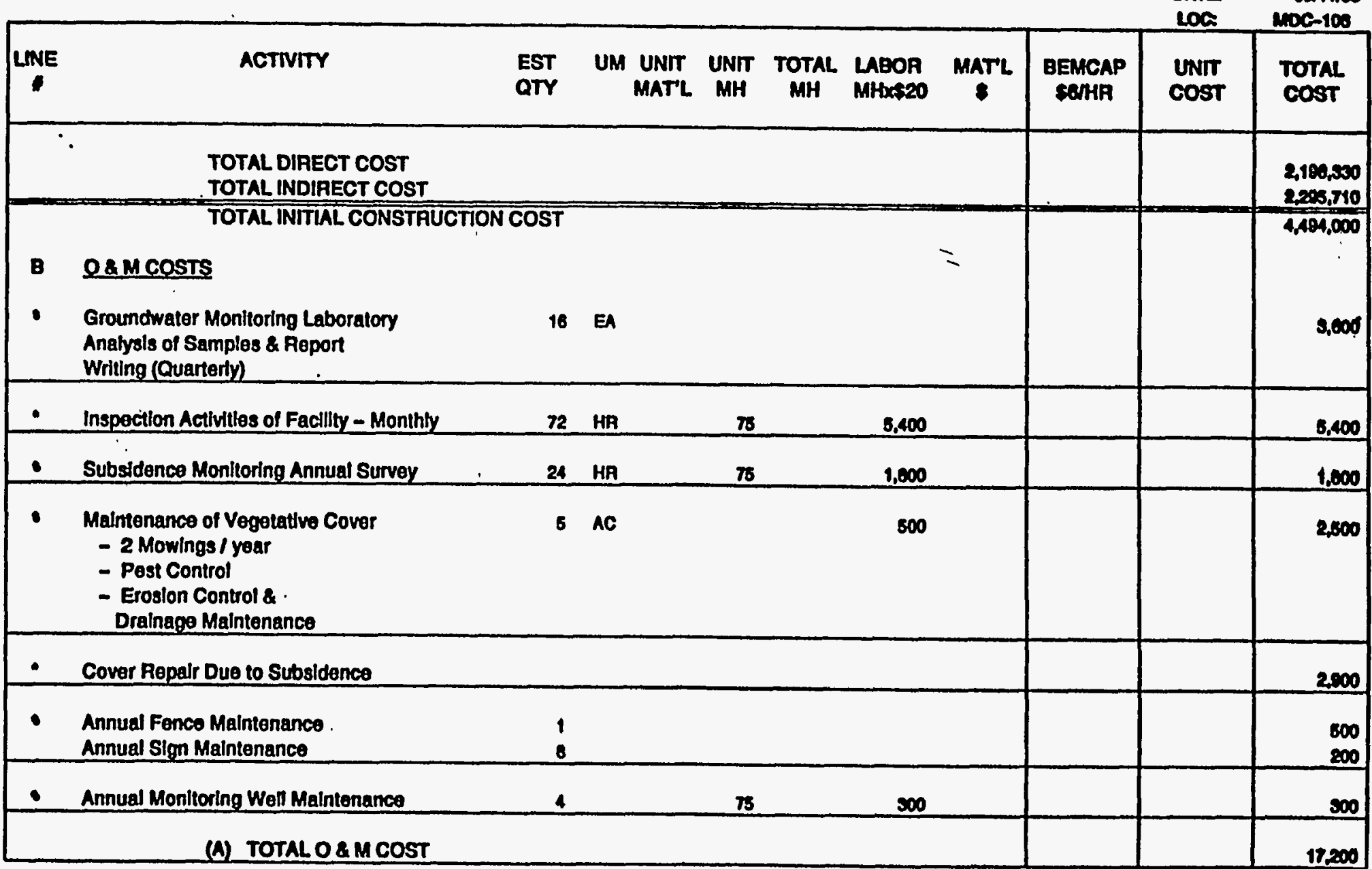

F-1. Cost Estimate for a Cover System Utilizing Claymax and a 30-mil FML (Cont'd) 
D B T A I B S T IMATB

1001: 7-03

ACTIVTYY CODB: ESE2SOH12

FROJ.NAME: BCON. BVALUATION OP CLOSURB CAP BARRIER MATERUAL.
CHero or: 2. MESTO

ESTMAToR: Pamuk

PHONE 7-5200

DATE DEN1/20

LOC. MDC-10s

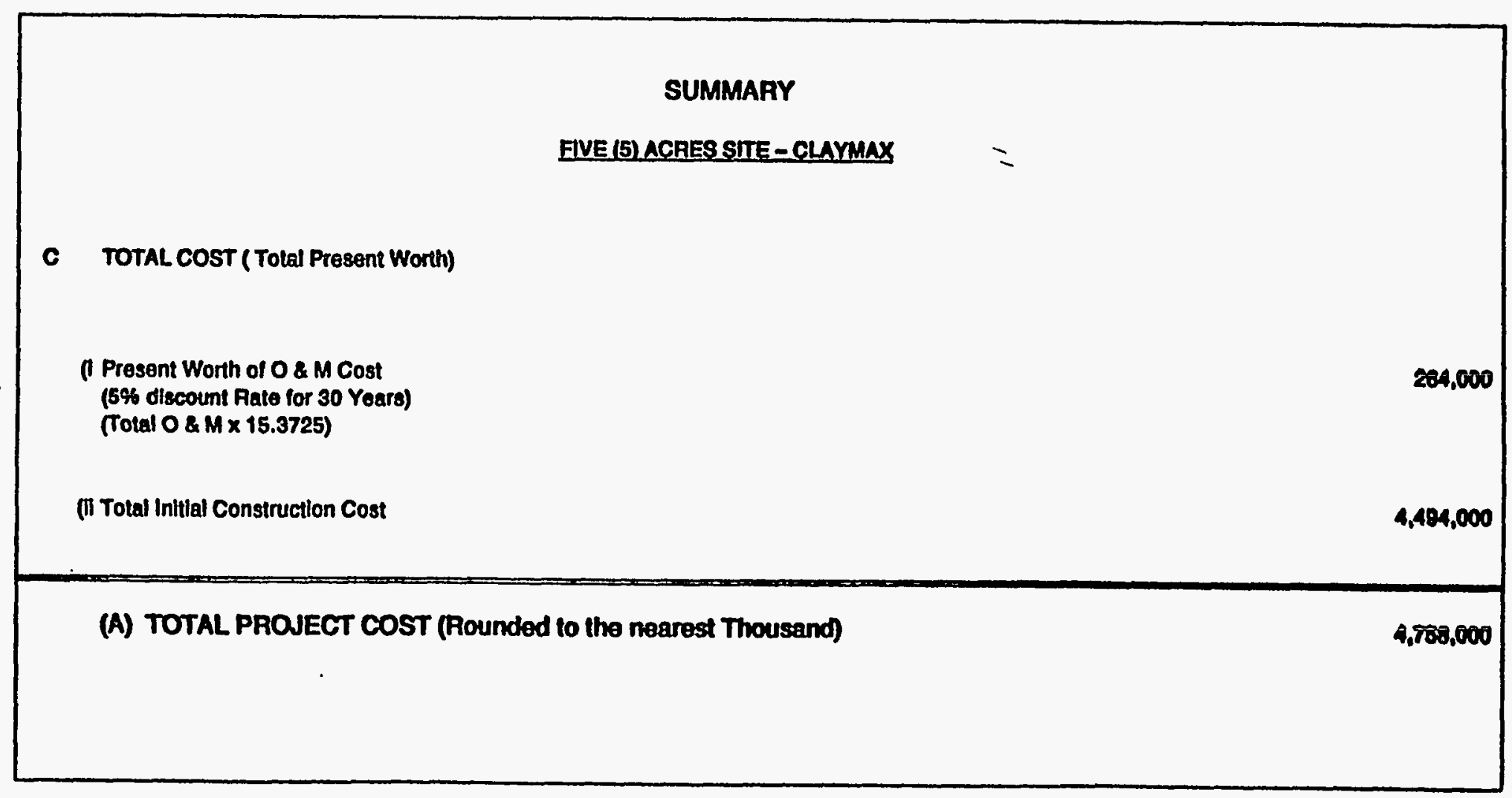

F-1. Cost Estimate for a Cover System Utilizing Claymax and a 30-mil FML (Cont'd) 
DETAIL ESTIMATE

CACDBY: Q MEAD

L00 / : 7-03

ACTIVITY CODE: ESE260112

PROJ.NAME: ECON. EVALUATION OP CLOSURB CAP BARRIER MATERIAL

\section{Estmantor: Pawn}

PHONE:

$7-5206$

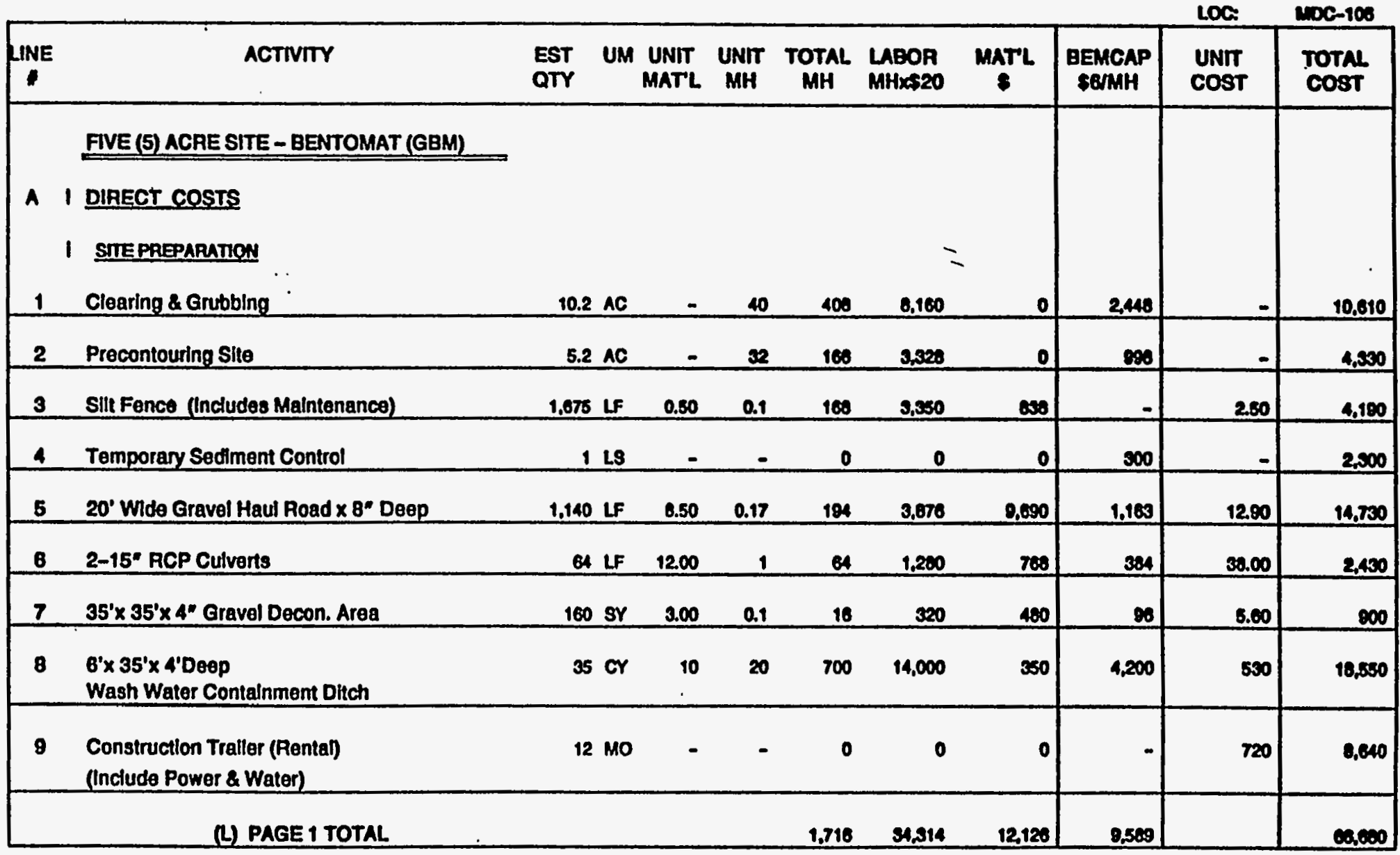

F-2. Cost Estimate for a Cover System Utilizing Bentomat $@$ and a 30-mil FML 


\section{DETAIL ESTIMATE}

200 : 7-03

ACTTVITY CODE: ESE260112

PROI.NAME: ECON. EVALUATION OR CLOSURA CAP BARRTER MATERIAL.

CHCOBY: Q MEAD

\begin{tabular}{|c|c|c|c|c|c|c|c|c|c|c|c|}
\hline LNE & ACTIVITY & $\begin{array}{l}\text { EST } \\
\text { QTY }\end{array}$ & UM & $\begin{array}{l}\text { UNIT } \\
\text { MAT'L }\end{array}$ & $\begin{array}{l}\text { UNIT } \\
\text { MH }\end{array}$ & TOTAL & $\begin{array}{l}\text { LABOR } \\
\text { MHXas0 }\end{array}$ & $\underset{\$}{\text { MATL }}$ & $\begin{array}{l}\text { BEMCAP } \\
\text { SGMH }\end{array}$ & $\begin{array}{l}\text { UNT } \\
\text { COST }\end{array}$ & $\begin{array}{l}\text { Total } \\
\text { cost }\end{array}$ \\
\hline 10 & $\begin{array}{l}\text { D COVEA CONSTRUCTION } \\
\text { Waste Stabllization (Crushed LImestone) }\end{array}$ & 10,070 & $\mathrm{TN}$ & 100 & 0.1 & 1007 & 20,140 & $1,007,000$ & 8.042 & 102.00 & $1,038,160$ \\
\hline 11 & $\begin{array}{l}\text { Place and Compact Foundation Soll } \\
\text { In } 8^{\circ} \text { Lilts to } 95 \% \text { Stdandard Proctor }\end{array}$ & 40,350 & CY & - & 0.155 & 6254 & 125,085 & 0 & 37.528 & 4.00 & 162,610 \\
\hline 12 & Placememt of Bentonite Geotextle Mat & 308,000 & SF & 0.45 & 0.01 & 3000 & 81,000 & 150,000 & - & 0.70 & 200,200 \\
\hline 13 & $\begin{array}{l}\text { Placement of } 30 \text { Mil Flexible } \\
\text { Membrane Liner }\end{array}$ & 308,000 & SF & 0.17 & 0.0076 & 2310 & 46,200 & 62,300 & - & $\mathbf{0 . 0 0}$ & 80,800 \\
\hline 14 & $\begin{array}{l}\text { Placement of } 1 \text { ' }-0 \text { Coarse Sand } \\
\text { Dralnage Layer }\end{array}$ & 10.620 & or & 10.50 & 0.03 & ง19 & 6.372 & 111,810 & 1,012 & 11.00 & 119,700 \\
\hline 15 & $\begin{array}{l}\text { Placememt of Geotextlle (TYPAR) Filter on } \\
1^{\circ}-0^{\circ} \text { Coarse Sand Dralnage Layer }\end{array}$ & 35,055 & sY & 0.17 & 0.005 & 175 & 3,506 & 8,950 & - & 0.50 & 0,400 \\
\hline 16 & Piacement of $1^{\prime}-6^{\prime \prime}$ Common Fill & 12,120 & CY & 3.75 & 0.06 & 727 & 1434 & 45.450 & 40003 & 8.50 & 04,500 \\
\hline 17 & Placement of 6* Topsoil & 4,545 & CY & 9.50 & 0.00 & 273 & B,454 & 49,170 & 1.036 & 11.10 & 80.270 \\
\hline 98 & $\begin{array}{l}\text { Placement of Pertmater Extertor } \\
\text { Orainage Layer } 3 / 4^{*} \times 3^{*} \text { DP (Crushed Stone) }\end{array}$ & 641 & sY & 1.50 & 0.2 & 126 & 2,564 & $\operatorname{san}$ & $\pi$ & $\mathbf{2 \pi 0}$ & 4,200 \\
\hline 19 & Excavation of Trapezoldal Orainage Ditch & 2,205 & OY & - & 0.175 & 306 & 7.710 & 0 & 2,518 & 4.60 & 10.050 \\
\hline & (ㄴ) PAGE 2 TOTAL & & & & & 14,669 & 290,162 & $1,405,018$ & SA,503 & & $1,752,750$ \\
\hline
\end{tabular}

F-2. Cost Estimate for a Cover System Utilizing Bentomat@ and a 30-mil FML (Cont'd) 
D E T A I E S TIMATB

GKCDEY: S MEND

Loa A: 2-03

NCTIVTY CODE: ESE26ON12

PROJ.NAME: ECON. EVALUATION OF CLOSURB CAP BARRIER MATERIAL

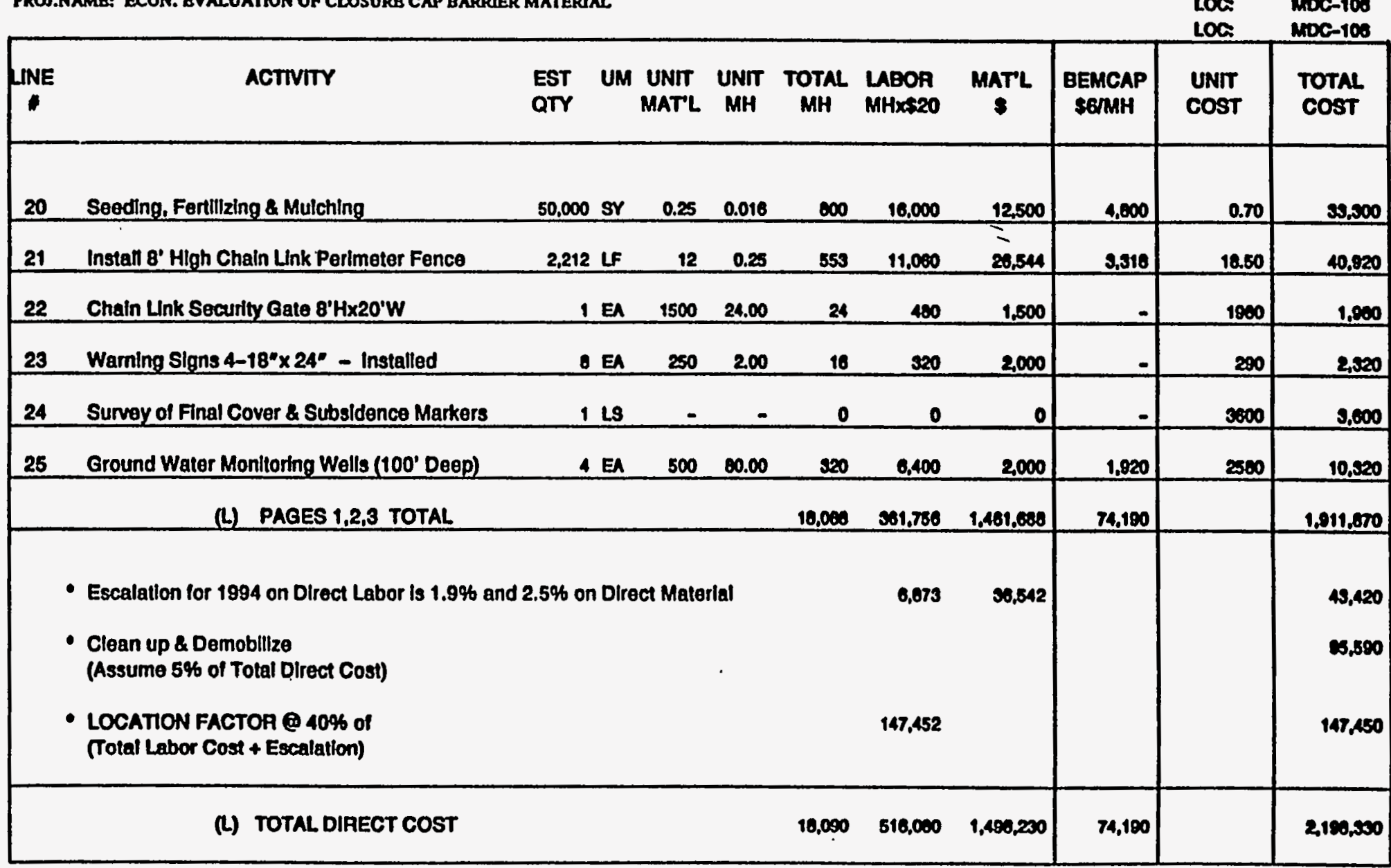

F-2. Cost Estimate for a Cover System Utilizing Bentomat and a 30-mil FML (Cont'd) 
D E T A I L B S T I M A T B

Lo0 1: 7-03

ACTIVITY CODB: ESE260412

PROI.NAME: ECON. BVALUATTON OP CLOSURE CAP BARRIER MATERJAS

CHCOBY: Q MEAD

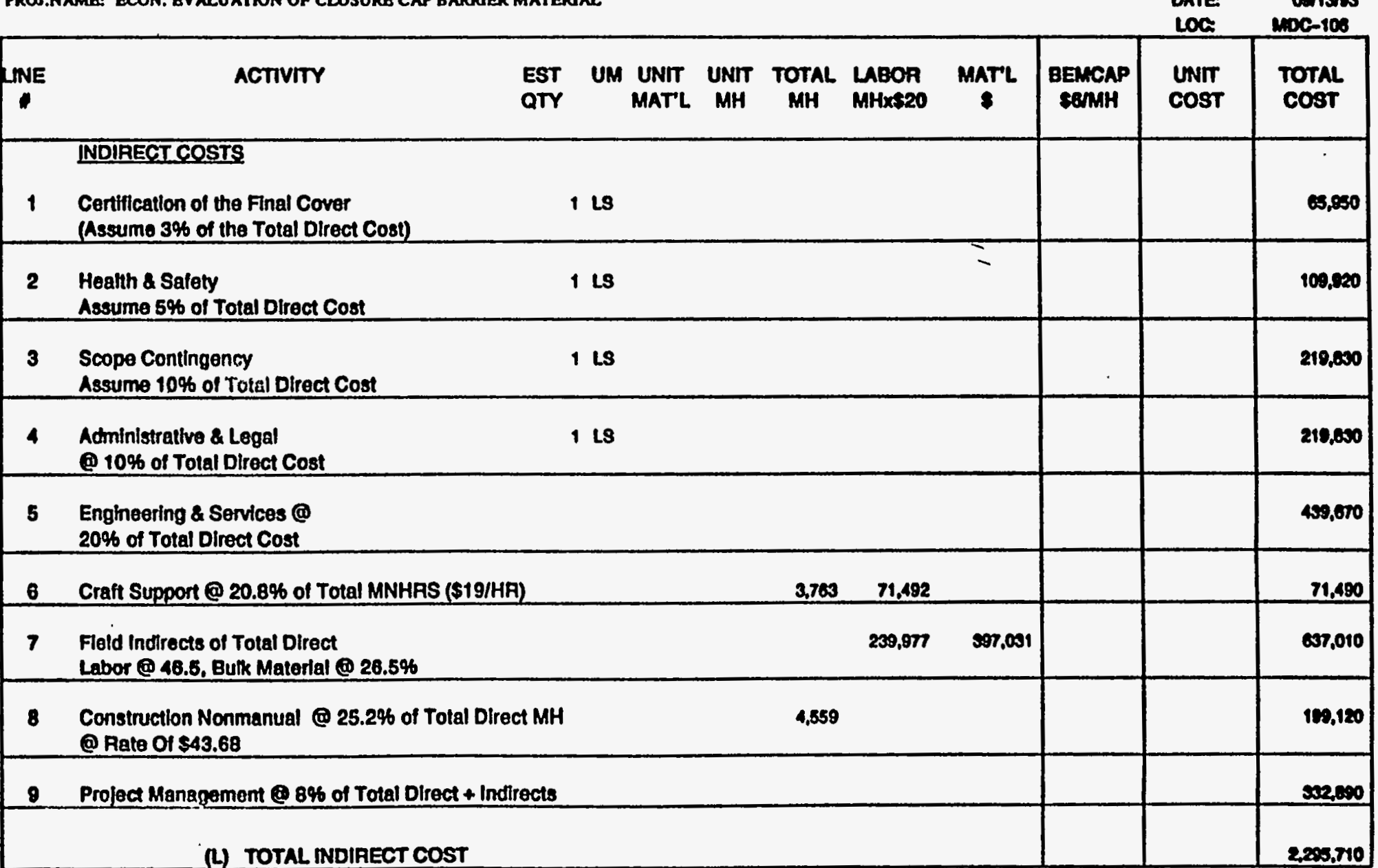

F-2. Cost Estimate for a Cover System Utilizing Bentomat@ and a 30-mil FML (Cont'd) 


\section{$1001: 7-03$}

ACTIVTY CODE: ESE260412

PROJ.NANE: ECON. BVALUATION OF CLOSURB CAP BARRTER MATERIAL.

CTMCOQY: S.MEAD

ESTMATOR: PaMnk

PHONE: 7-5268

DATE: $\quad 09 / 13 \% 9$

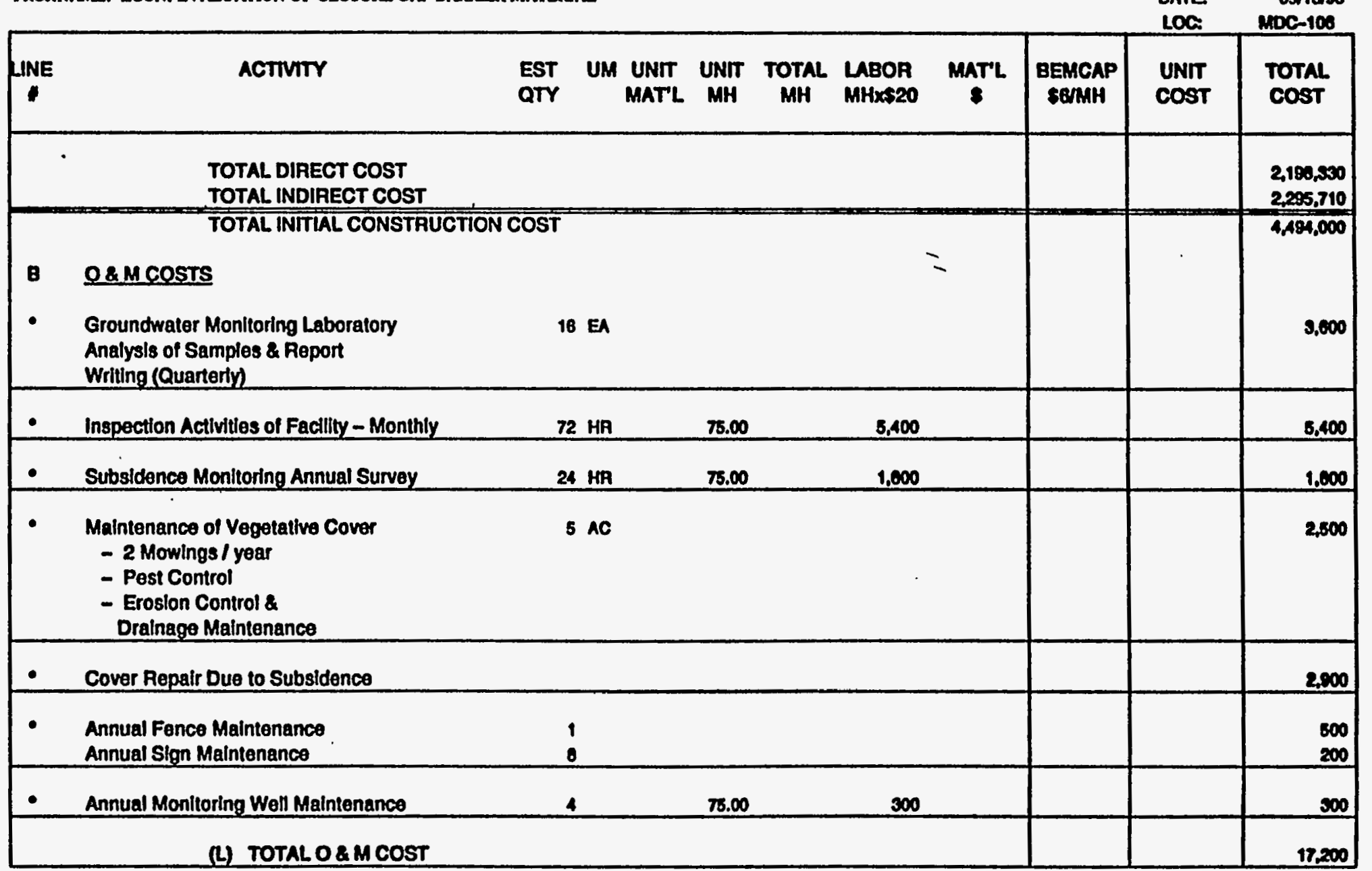

F-2. Cost Estimate for a Cover System Utilizing Bentomat@ and a 30-mil FML (Cont'd) 
D BTAIL BSTIMAT B

\begin{tabular}{|c|c|}
\hline 7 & R MEAD \\
\hline $\begin{array}{l}\text { ESTMATOR } \\
\text { PHONE: } \\
\text { DATE: } \\
\text { LOC: }\end{array}$ & 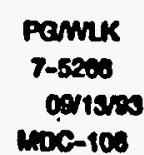 \\
\hline
\end{tabular}

Lo0 I: $7-03$

ACTIVITY CODE: ESE260412

PROJ.NAME: ECON. EVALUATHON OF CLOSURB CAP BARRIER MATERIAL

LOC.

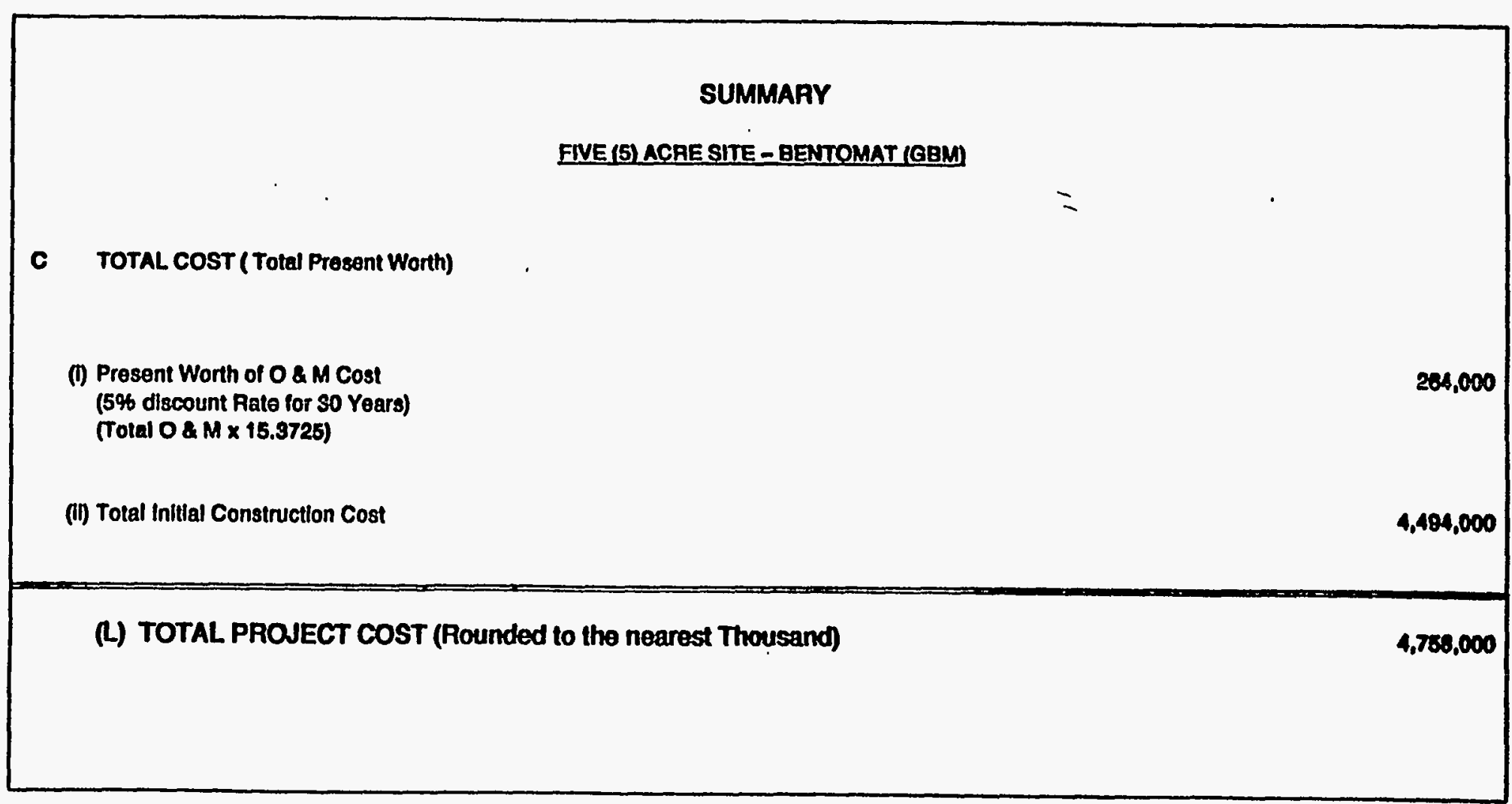

F-2. Cost Estimate for a Cover System Utilizing Bentomat@ and a 30-mil FML (Cont'd) 


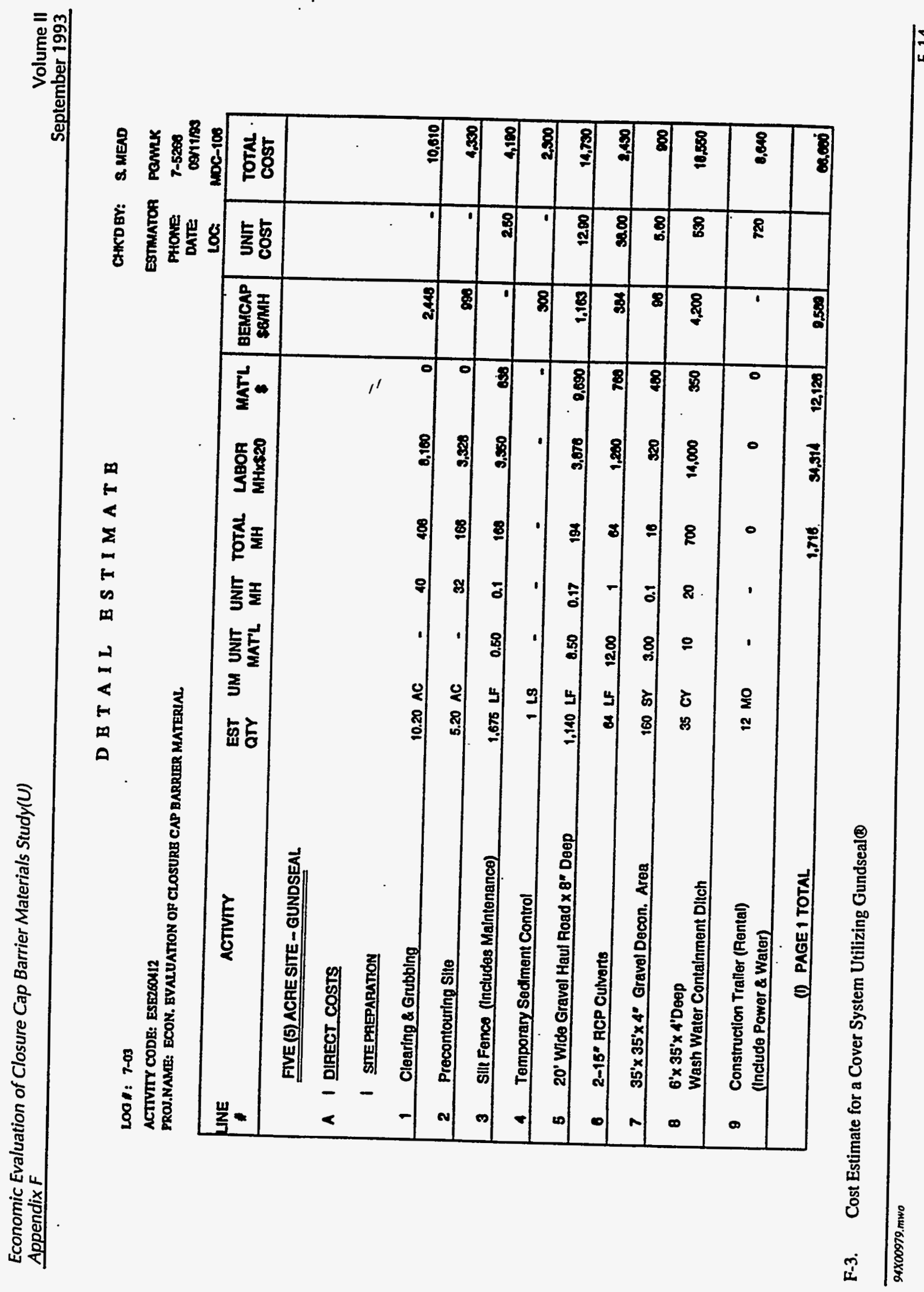




\section{DETAIL RSTIMATE}

Lod: $7-03$

ACTIVITY CODE: ESE260112

PROS.NAMR: ECON. EVALUATION OP CLOSURB CAP BARRIER MATERIAL.

\begin{tabular}{|c|c|c|c|c|c|c|c|c|c|c|c|}
\hline & & & & & & & & & & LOC: & $\operatorname{moc}-100$ \\
\hline SNE & Activir & $\begin{array}{l}\text { EST } \\
\text { QTY }\end{array}$ & UM & $\underset{\text { MATL }}{\text { UNT }}$ & $\underset{M H}{\text { UNIT }}$ & TOTAL & $\begin{array}{l}\text { LABOR } \\
\text { MHXS2O }\end{array}$ & $\underset{\$}{\operatorname{maTL}}$ & $\underset{\text { BEMCAP }}{\text { BQMH }}$ & $\begin{array}{l}\text { UNIT } \\
\text { COST }\end{array}$ & $\begin{array}{l}\text { TOTAL } \\
\text { COST }\end{array}$ \\
\hline 10 & $\begin{array}{l}\text { H_COVER CONSTRUCTION } \\
\text { Waste Stabllization (Crushed LImestone) }\end{array}$ & 10,070 & $\mathrm{TM}$ & 100 & 0.1 & 1.007 & 20.140 & $1.007,000$ & 0.042 & 10200 & 1.033 .160 \\
\hline 11 & $\begin{array}{l}\text { Place and Compact Foundation Soll } \\
\text { in 8. Lifts to } 95 \% \text { Std. Proctor }\end{array}$ & 10,350 & or & - & 0.155 & 6,254 & 125,005 & $=0$ & 37,520 & 4.00 & 162,610 \\
\hline 12 & $\begin{array}{l}\text { Placement of Gundseal Geomembranel } \\
\text { Benionite Mat }\end{array}$ & 308,000 & SF & 0.50 & 0.01 & 3,000 & 61,600 & 154,000 & - & 0.70 & 215,600 \\
\hline 13 & $\begin{array}{l}\text { Placement of 1'-0 Coarse Sand } \\
\text { Drainage Layer }\end{array}$ & 10,620 & or & 10.50 & 0.05 & 319 & 0,372 & 111,510 & 1,912 & 11.50 & 110,700 \\
\hline 14 & $\begin{array}{l}\text { Placement of Geotextlle (TYPAP) Filter on } \\
1^{1}-0^{*} \text { Coarse Sand Drainage Layer }\end{array}$ & 35,055 & or & 0.17 & 0.005 & 175 & 3,506 & 8,059 & - & 0.30 & $8,400^{7}$ \\
\hline 15 & Placement of $1^{\prime}-6^{*}$ Common Fill & 12,120 & Cr & 2.75 & 0.00 & 727 & 14.544 & 45,450 & 4.360 & 8.50 & Q4,500 \\
\hline 16 & Placement of 6" Topsoil & 4.545 & or & 9.50 & 0.06 & 293 & 8,454 & 4.,170 & 1.030 & 11.10 & 60,200 \\
\hline 17 & $\begin{array}{l}\text { Placement of Perimeter Exterlor } \\
\text { Drainage Layer } 3 / 4^{\prime \prime} \times 3^{\prime} \text { DP (Crushed Stone) }\end{array}$ & on1 & sY & 1.50 & 0.2 & 128 & 2,564 & 862 & $\pi$ & 6.50 & 4,200 \\
\hline 18 & Excavation of Trapazoldal Drainage Ditch & 2,205 & or & - & 0.175 & 306 & 7.720 & 0 & 2,310 & 4.60 & 10,040 \\
\hline & (i) PAGE 2 TOTAL & & & & & 12,349 & 260,800 & $1,300,050$ & su,sest & & $1,000,000$ \\
\hline
\end{tabular}

F-3. Cost Estimate for a Cover System Utilizing Gundseal@ (Cont'd) 
DETAIL ESTIMATE

GHSDBY: 2 MEAD

L00 R: 7-03

ACTIVTTY CODE: ESE260112

FROJ.MAME: ECON. EVALUATTON OP CLOSURB CAP BARRIER MATERIAL

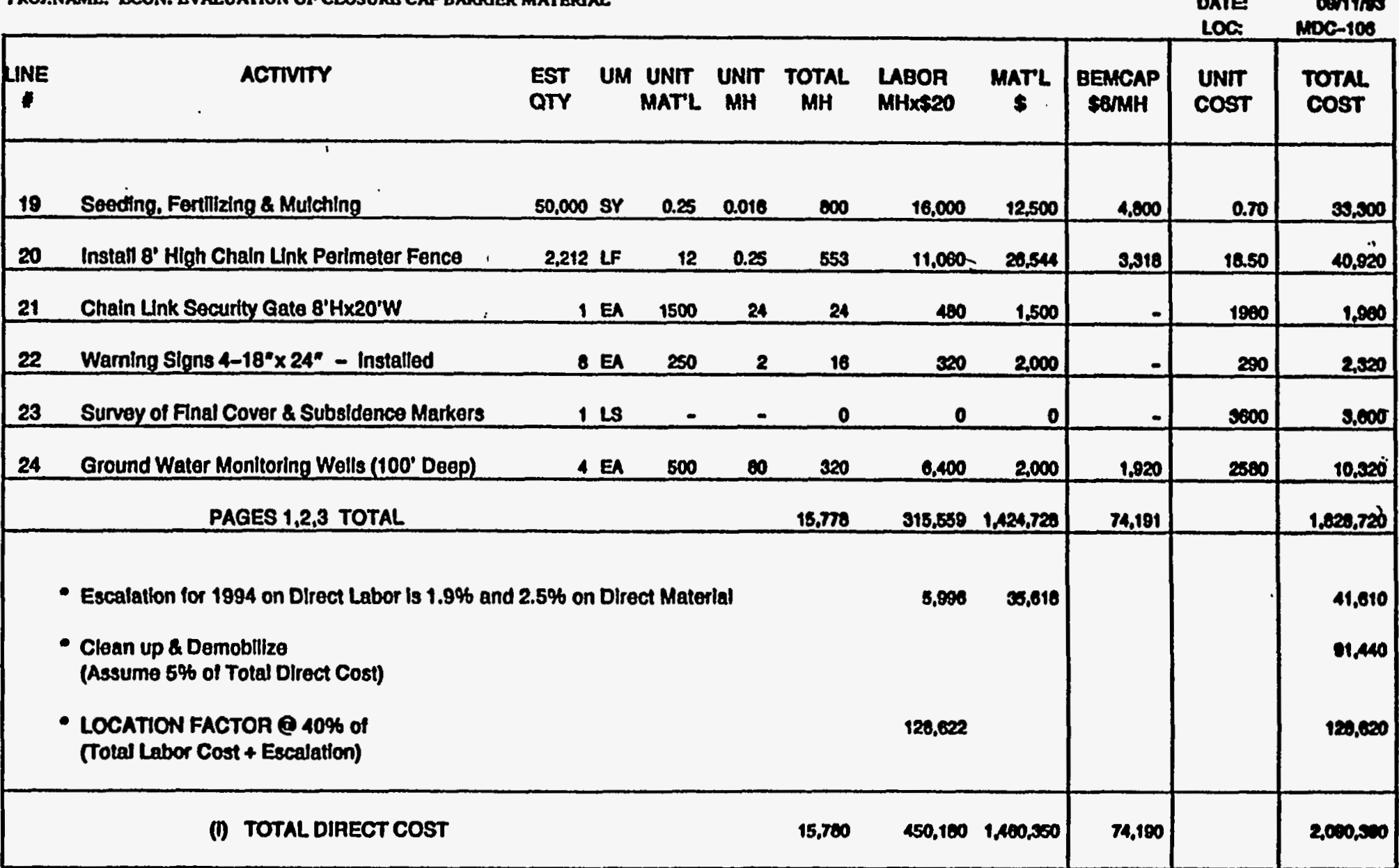

F-3. Cost Estimate for a Cover System Utilizing Gundseal (Cont'd) 
LO01: 7-03

ACTIVTTY CODB: BSE26012

MROJ.NAME: ECON. EVALUATTON OF CLOSURE CAP BARRIER MATERIAL,

CATSOBY: 8.MEAD

Estimaton parnik

PHOWE T-5200

DATE OST1100

LOC. $\quad$ MOC-108

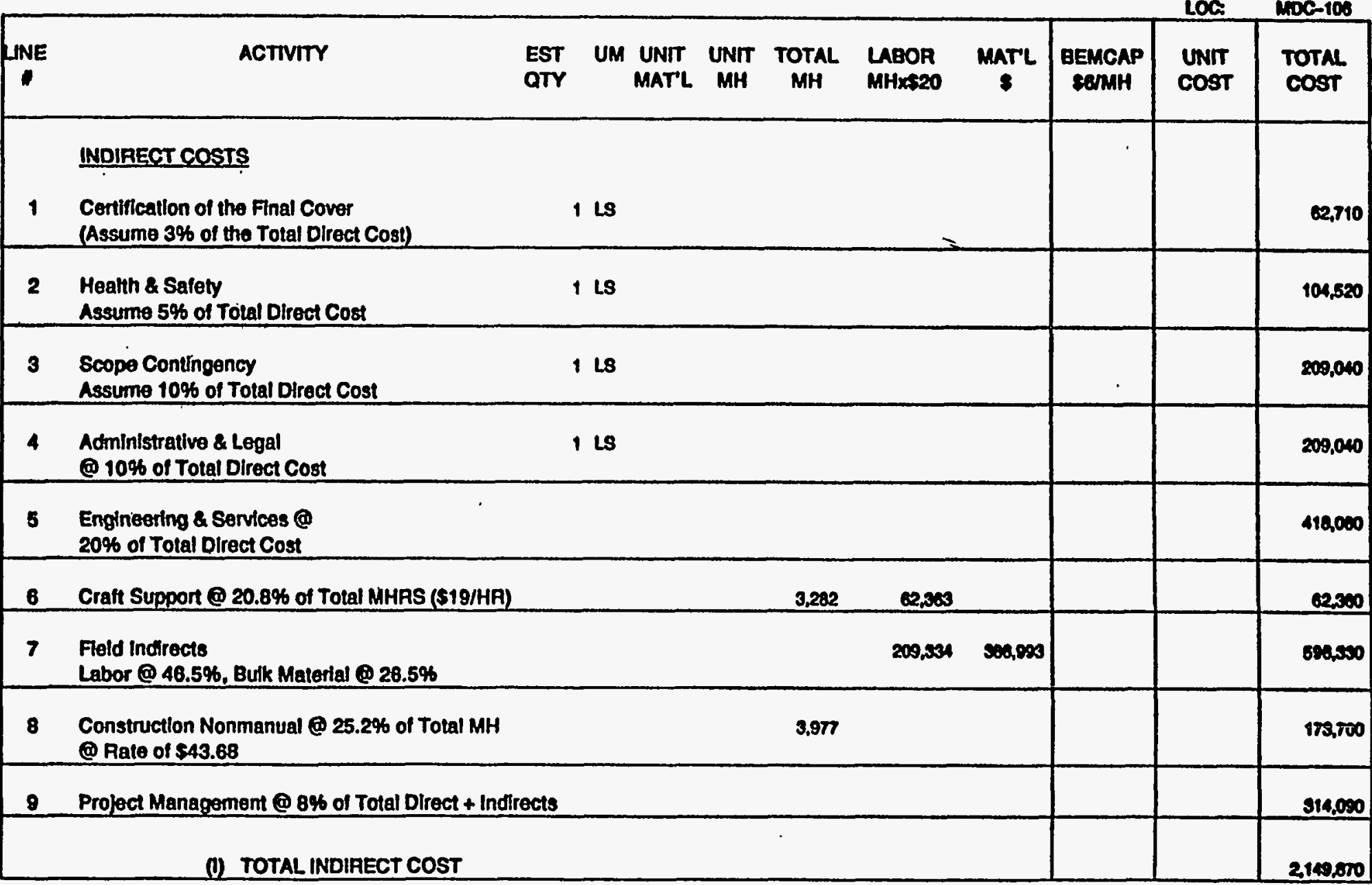

\section{TOTALINDIAECT COST}

F-3. Cost Estimate for a Cover System Utilizing Gundseal@ (Cont'd) 
Loof: $7-03$

ACTIVITY CODE: ESE260412

PROJ.NAME: ECON. EVALUATTON OF CLOSURE CAP BARRIER MATERIAL

\begin{tabular}{|c|c|c|c|c|c|c|c|}
\hline INE & ACTIVITY & $\begin{array}{l}\text { EST UM UNT } \\
\text { QTY } \\
\text { MAT'L }\end{array}$ & $\begin{array}{l}\text { UNIT TOTAL } \\
\text { MH MH }\end{array}$ & $\begin{array}{l}\text { LABOA } \\
\text { MHxis20 }\end{array}$ & $\begin{array}{l}\text { BEMCAP } \\
\text { SENMH }\end{array}$ & $\begin{array}{l}\text { UNIT } \\
\text { COST }\end{array}$ & $\begin{array}{l}\text { TOTAL } \\
\text { COST }\end{array}$ \\
\hline & $\begin{array}{l}\text { TOTAL DIRECT COST } \\
\text { TOTAL INDIRECT COST }\end{array}$ & & & & & & $\begin{array}{l}2,090,0000^{\circ} \\
2,149,070\end{array}$ \\
\hline $\mathbf{B}$ & $\begin{array}{l}\text { TOTAL INITIAL CONSTRU } \\
\text { O\&M COSTS } \\
\text { Groundwater Monitoring Laboratory } \\
\text { Analysis of Samples \& Report } \\
\text { Writing (Quarterly) }\end{array}$ & 16 EA & & $=$ & & & $\begin{array}{r}4,240,000 \\
9,000\end{array}$ \\
\hline$\bullet$ & Inspection Activities of Facility - Monthly & $72 \mathrm{HA}$ & 75.00 & 5,400 & & & 8,400 \\
\hline$\bullet$ & Subsidence Monitoring Annual Survoy & $24 \mathrm{HA}$ & 73.00 & 1,000 & & & $1.0000^{\circ}$ \\
\hline & $\begin{array}{l}\text { Malntenance of Vegetative Cover } \\
\text { - } 2 \text { Mowings / year } \\
\text { - Pest Control } \\
\text { - Erosion Control \& } \\
\text { - Drainage Maintenance } \\
\end{array}$ & $B A C$ & & & & & 2.0000 \\
\hline & Cover Repair Due to Subsidence & & & & & & 2,000 \\
\hline & $\begin{array}{l}\text { Annual Fence Maintenance. } \\
\text { Annual Sign Maintenance }\end{array}$ & $\begin{array}{l}1 \\
4 \\
\end{array}$ & & $\mathbf{0}$ & & & $\begin{array}{l}500 \\
200\end{array}$ \\
\hline$\bullet$ & Annual Monitoring Well Maintenance & 4 & 75.00 & 500 & & & 300 \\
\hline & (1) TOTALO \& M COST & & & & & & 10.50 \\
\hline
\end{tabular}

F-3. Cost Estimate for a Cover System Utilizing Gundseal@ (Cont'd) 
D B T A I E B T I M A T B

CHCo Br: S Meno

$2001: 7-03$

ACTIVITY CODE: ESE260412

PROJ.NAME: ECON. EVALUATION OF CLOSURB CAP BARRIER MATERIN

menton pamk

PFIOME 7-5260

DATE: OSN11/90

LOC: MDC-100

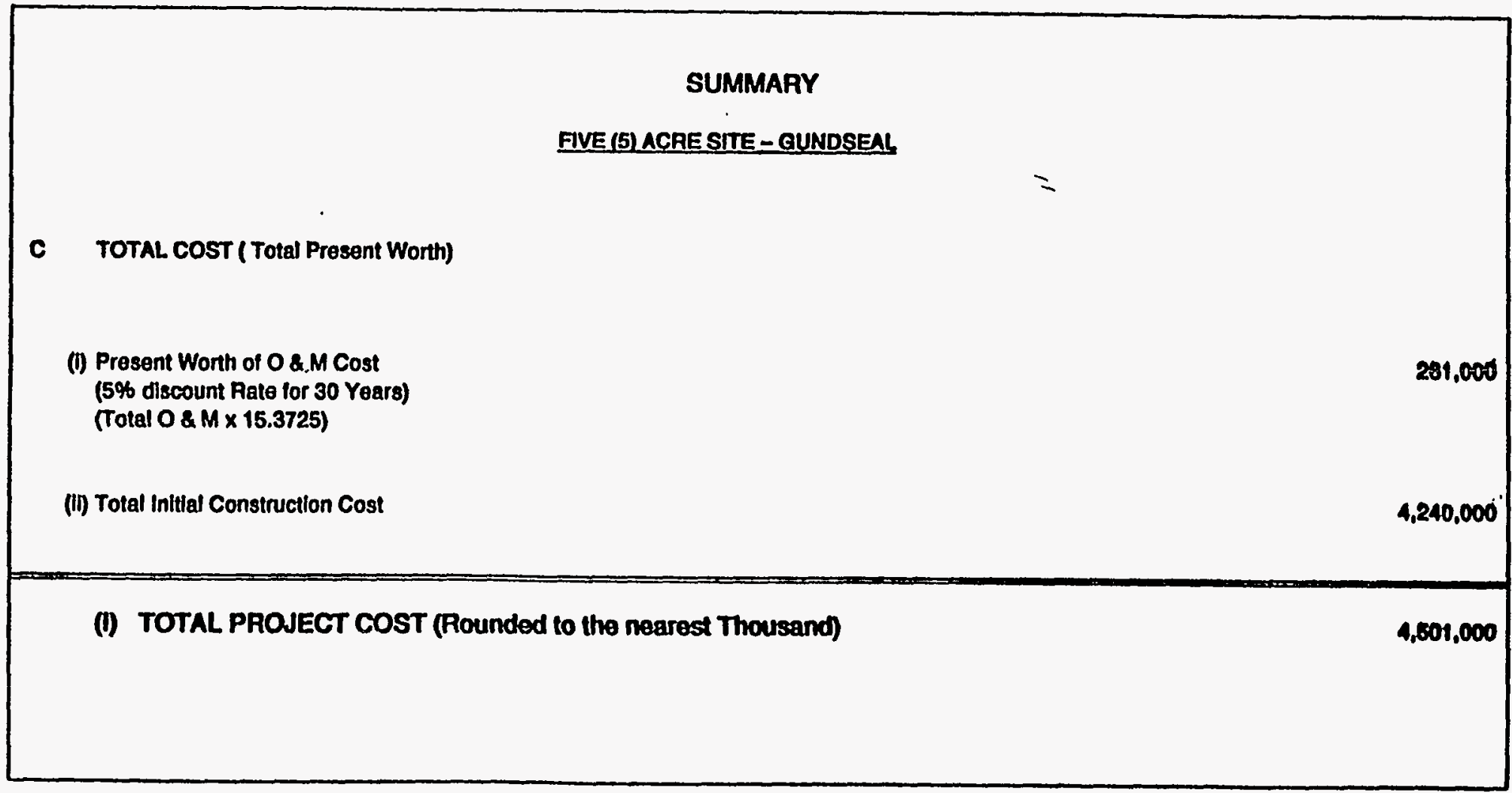

F-3. Cost Estimate for a Cover System Utilizing Gundseal@ (Cont'd) 


\section{DETAIL ESTIMATE}

\section{Loo 1: 7-03}

ACTIVITY CODE: ESE260112

PROJ.NAME: ECON. EVALUATTON OF CLOSURB CAP BARRRER MATERIAL.

\begin{tabular}{|c|c|c|c|c|c|c|c|c|c|c|c|}
\hline LNE & ACTIVITY & $\begin{array}{l}\text { EST } \\
\text { QTY }\end{array}$ & UM & $\begin{array}{l}\text { UNIT } \\
\text { MATL }\end{array}$ & $\begin{array}{l}\text { UNTT } \\
\text { MH }\end{array}$ & $\underset{M H}{\text { TOTAL }}$ & $\begin{array}{l}\text { LABOR } \\
\text { MHX } \$ 20\end{array}$ & $\underset{\&}{\text { MATL }}$ & $\begin{array}{l}\text { BEMCAP } \\
\text { SERHR }\end{array}$ & $\begin{array}{l}\text { UNIT } \\
\text { COST }\end{array}$ & $\begin{array}{l}\text { TOTAL } \\
\text { COST }\end{array}$ \\
\hline $\begin{array}{l}1 \\
1\end{array}$ & $\begin{array}{l}\text { FIVE ACRES (5) ACAE SITE - (120 MII } \\
1 \text { DIRECT COSTS } \\
1 \text { SIIE PREPARATION } \\
\text { Cloaring \& GRubbing }\end{array}$ & 10.2 & AC & - & 40 & 400 & 0.100 & $=$ & 2.48 & $=$ & 10,610 \\
\hline & Precontouring Sile & 5.2 & AC & - & 32 & 166 & 3,320 & 0 & 990 & - & 4,500 \\
\hline 3 & Sill Fence (inctudes Malntenance) & 1.675 & LF & 0.50 & 0.1 & 160 & 3,350 & 830 & - & 250 & 4.180 \\
\hline 4 & Temporary Sodiment Control & 1 & Ls & - & - & - & - & $=$ & $\$ 00$ & - & 2,200 \\
\hline 5 & 20' Wide Graval Haul Road $\times$ 8" Deep & 1.140 & LF & 0.50 & 0.17 & 194 & 3,070 & 2,090 & 1,160 & 12.90 & 14,700 \\
\hline 6 & 2-15. RCP Culverts & 64 & LF & 12.00 & 1 & 64 & 1,200 & 788 & sen & 30.00 & 2450 \\
\hline 7 & $35^{\prime} \times 35^{\prime} \times 4^{\prime \prime}$ Gravel Docon. Area & 160 & 8Y & 3.00 & 0.1 & 16 & 320 & 400 & $\theta$ & 6.00 & 200 \\
\hline 8 & $\begin{array}{l}6^{\prime} \times 35^{\prime} \times 4 \text { 'Deep } \\
\text { Wash Water Contalnment Ditch }\end{array}$ & ${ }^{35}$ & or & 10 & 20 & 700 & 14,000 & 350 & 4,200 & 830 & 10,560 \\
\hline $\boldsymbol{\theta}$ & $\begin{array}{l}\text { Construction Traller (Rental) } \\
\text { (Include Power \& Water) }\end{array}$ & 12 & мо & - & - & 0 & 0 & 0 & - & 720 & 8,820 \\
\hline & (F) PAGE TOTAL & & & & & 1.716 & sesta & 12,120 & 0,500 & & 0,000 \\
\hline
\end{tabular}

F-4. Cost Estimate for a Cover System Utilizing 120-mil High Density Polyethylene (HDPE) Liner 
DETAIL ESTIMATE

1001: $7-03$

ACTIVITY CODE: ESE260412

PROJ.NAME. BCON BVALUATION OP CLOSURB CAP BAREIRR MATERUAL

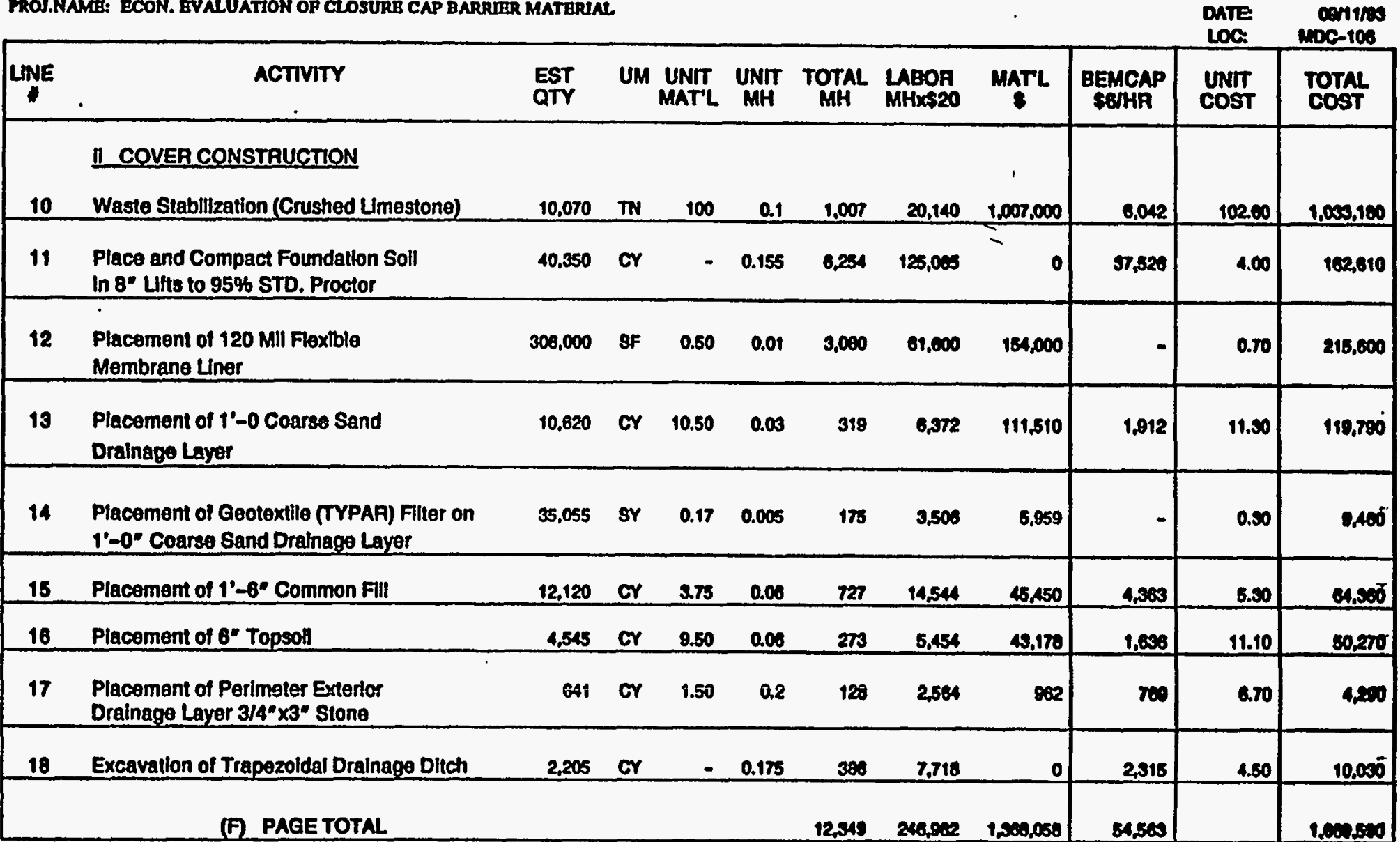

F-4. Cost Estimate for a Cover System Utilizing 120-mil High Density Polyethylene (HDPE) Liner (Cont'd) 


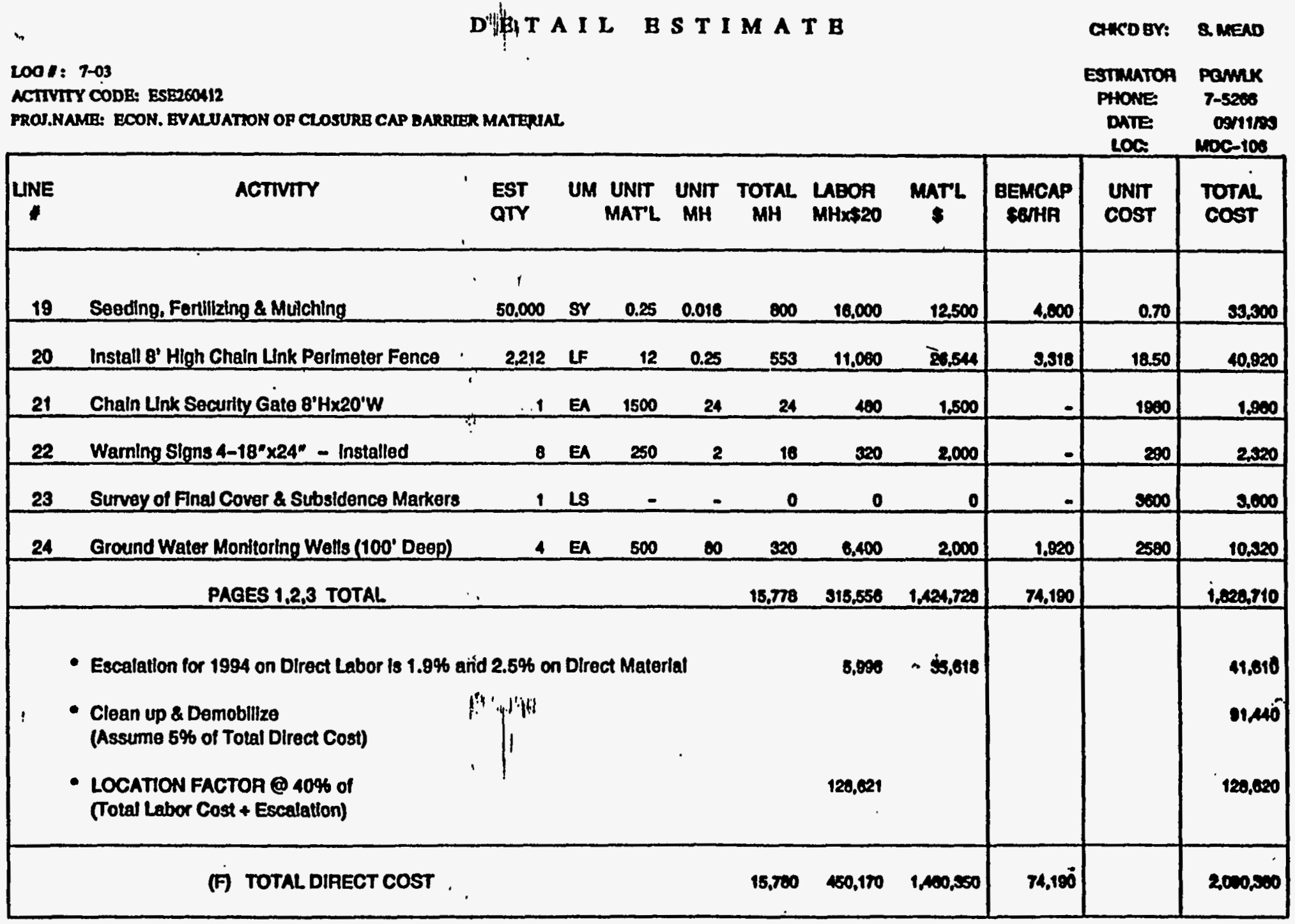

F-4. Cost Estimate for a Cover System Utilizing 120-mil High Density Polyethylene (HDPE) Liner (Cont'd) 
CHCD BY: Q MEAD

L001: 7-03

ACTIVTTY CODE: ESE260012

PROJ.NAME: ECON. BVALUATION OF CLOSURB CAP BARRIER MATERIAL

esmator pamk

PHowe 7-5286

DATE DON1MS

\begin{tabular}{|c|c|c|c|c|c|c|c|c|c|}
\hline LUNE & ACTIVITY & UM UNIT & UNIT & $\underset{M H}{\text { TOTAL }}$ & $\begin{array}{l}\text { LABOR } \\
\text { MHX } \$ 20\end{array}$ & $\underset{\$}{\operatorname{MATL}}$ & $\begin{array}{l}\text { BEMCAP } \\
\text { SOMHR }\end{array}$ & $\begin{array}{l}\text { UNIT } \\
\text { COST }\end{array}$ & $\begin{array}{l}\text { TOTAL } \\
\text { COST }\end{array}$ \\
\hline & INDIRECT COSTS & & & & & & & & \\
\hline 1 & $\begin{array}{l}\text { Certification of the Final Cover } \\
\text { (Assume 3\% of the Total Direct Cost) }\end{array}$ & LS & & & & & & & 02,710 \\
\hline 2 & $\begin{array}{l}\text { Health \& Safety } \\
\text { Assume 5\% of Total Direct Cost }\end{array}$ & LS & & & & & & & 104,520 \\
\hline 3 & $\begin{array}{l}\text { Scope Coningency } \\
\text { Assume } 10 \% \text { of Total Direct Cost }\end{array}$ & 48 & & & & & & & 200,040 \\
\hline 4 & $\begin{array}{l}\text { Administrative \& Legal } \\
\text { @ } 10 \% \text { of Total Olroct Cost }\end{array}$ & Ls & & & & & & & 200,000 \\
\hline 6 & $\begin{array}{l}\text { Engineering \& Services @ } \\
20 \% \text { of Total Direct Cost }\end{array}$ & Ls & & & & & & & 416,000 \\
\hline 8 & Craft Support @ $20.8 \%$ of Total MNHAS (\$19/HA) & & & 0,282 & 02,300 & & & & 02,300 \\
\hline 7 & $\begin{array}{l}\text { Fleld Indirects of Total Direct } \\
\text { Labor @ 46.6\%,8ulk Material @ 26.5\% }\end{array}$ & & & & 209,329 & $\$ 80,993$ & & & 896,320 \\
\hline 8 & $\begin{array}{l}\text { Construction Nonmanual @ } 25.2 \% \text { of Total Direct MH } \\
\text { @ Rate of \$43.68 }\end{array}$ & & & s.9n & & & & & 170,700 \\
\hline 8 & Project Management @ 8\% of Total Direct + Inflirects & & & & & & & & 914,000 \\
\hline & (F) TOTAL INDIRECT COST & & & & & & & & $2,149,000$ \\
\hline
\end{tabular}

F-4. Cost Estimate for a Cover System Utilizing 120-mil High Density Polyethylene (HDPE) Liner (Cont'd) 
D ETAIL E S T I M A T B

CHCOBY: QMEAD

2001: 7-03

ACTIVTY CODE: ESEZ6OL12

PROI.NAME: ECON. EYALUATHON OF CLOSURB CAP BARRTER MATERUAL

ESTMATOA PaWLK

PHONE 7-5206

DATE OSMIITS

ton

MOCG-100

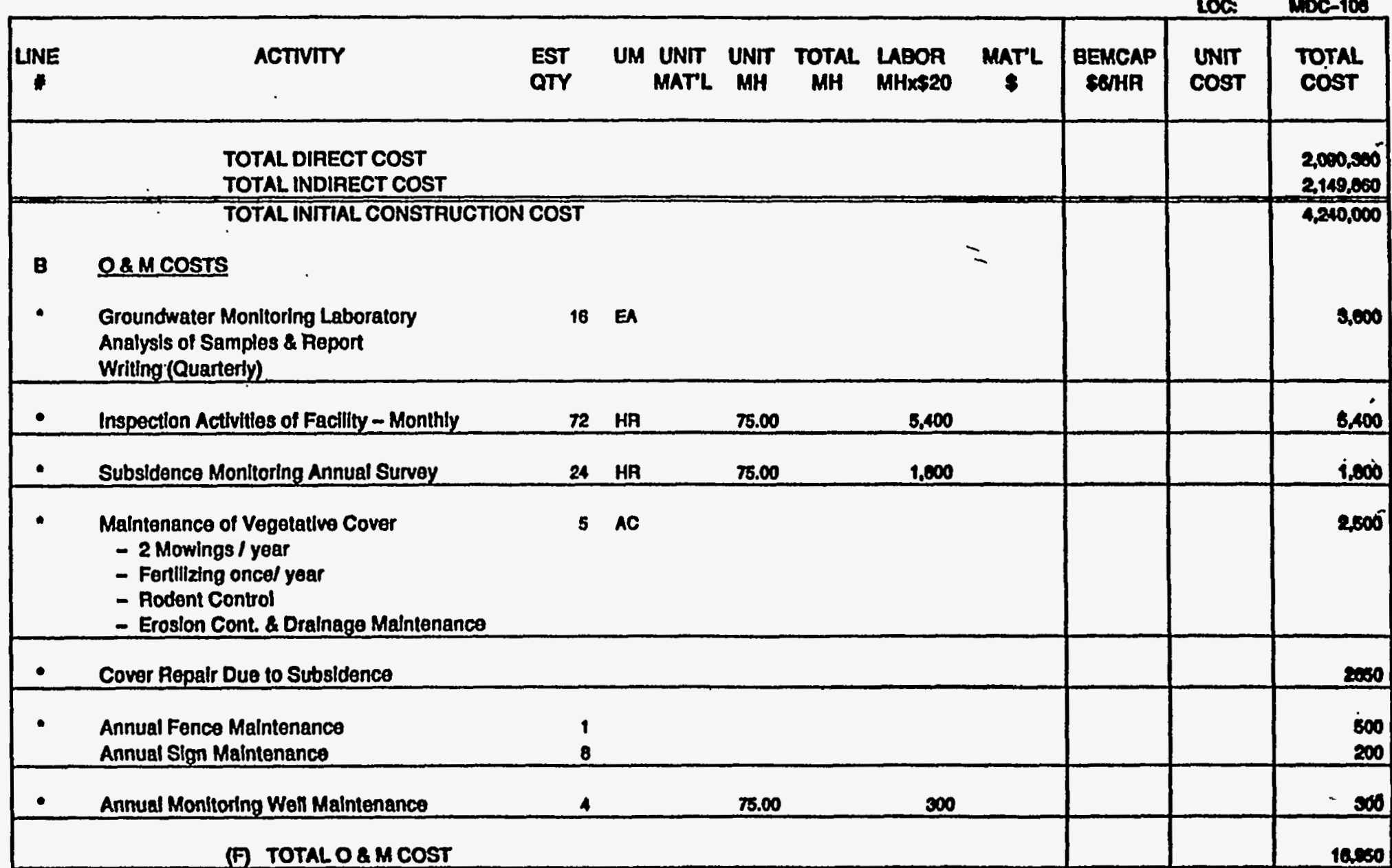

F-4. Cost Estimate for a Cover System Utilizing 120-mil High Density Polyethylene (HDPE) Liner (Cont'd) 


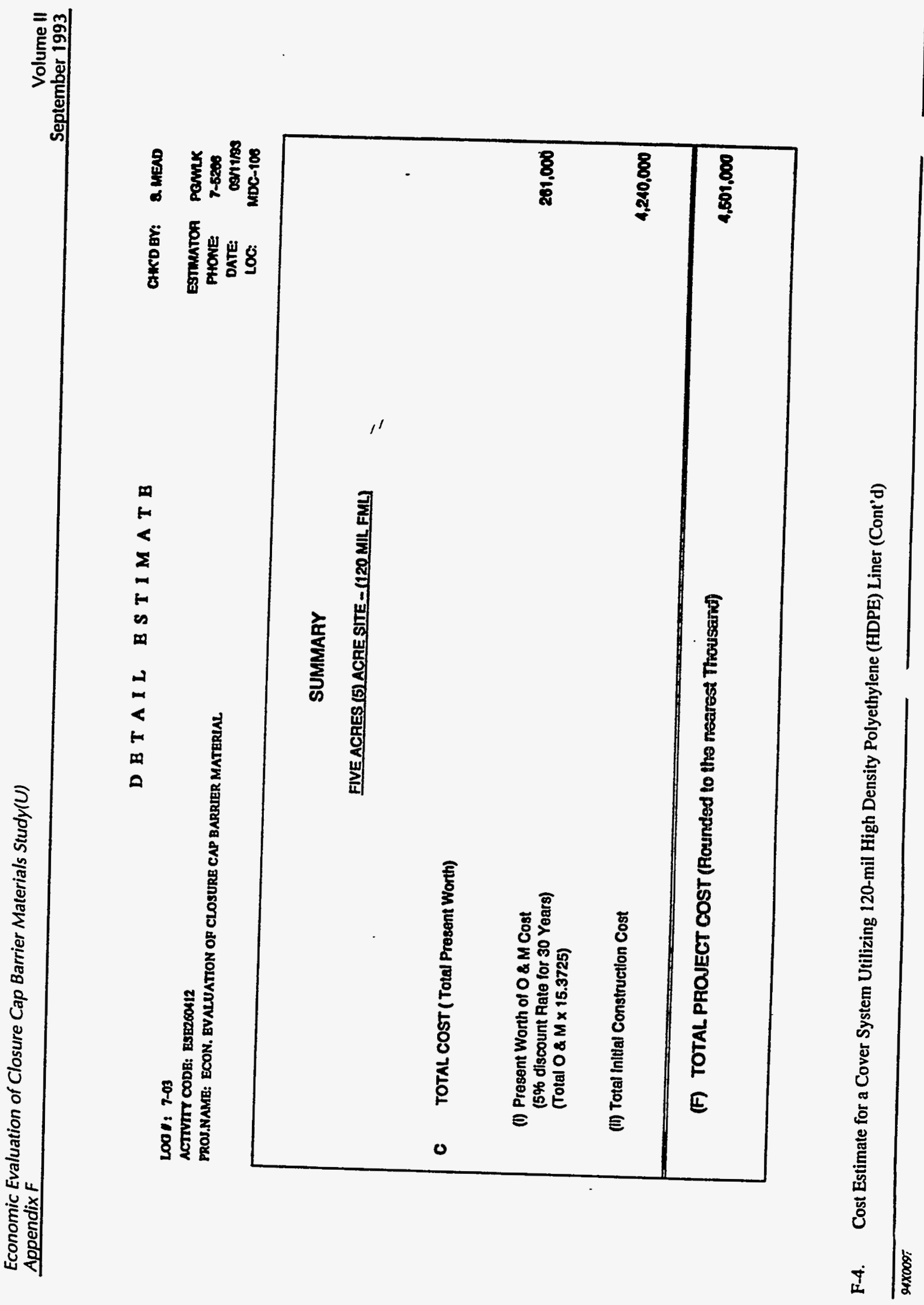


DETAIL ESTIMATE

Lo01: 7-03

ACTIVITY CO:DE: BSE2SOU12

PROJ.NAME: ECON. EVALUATION OF CLOSURB CAP BARRIRR MATERIAL

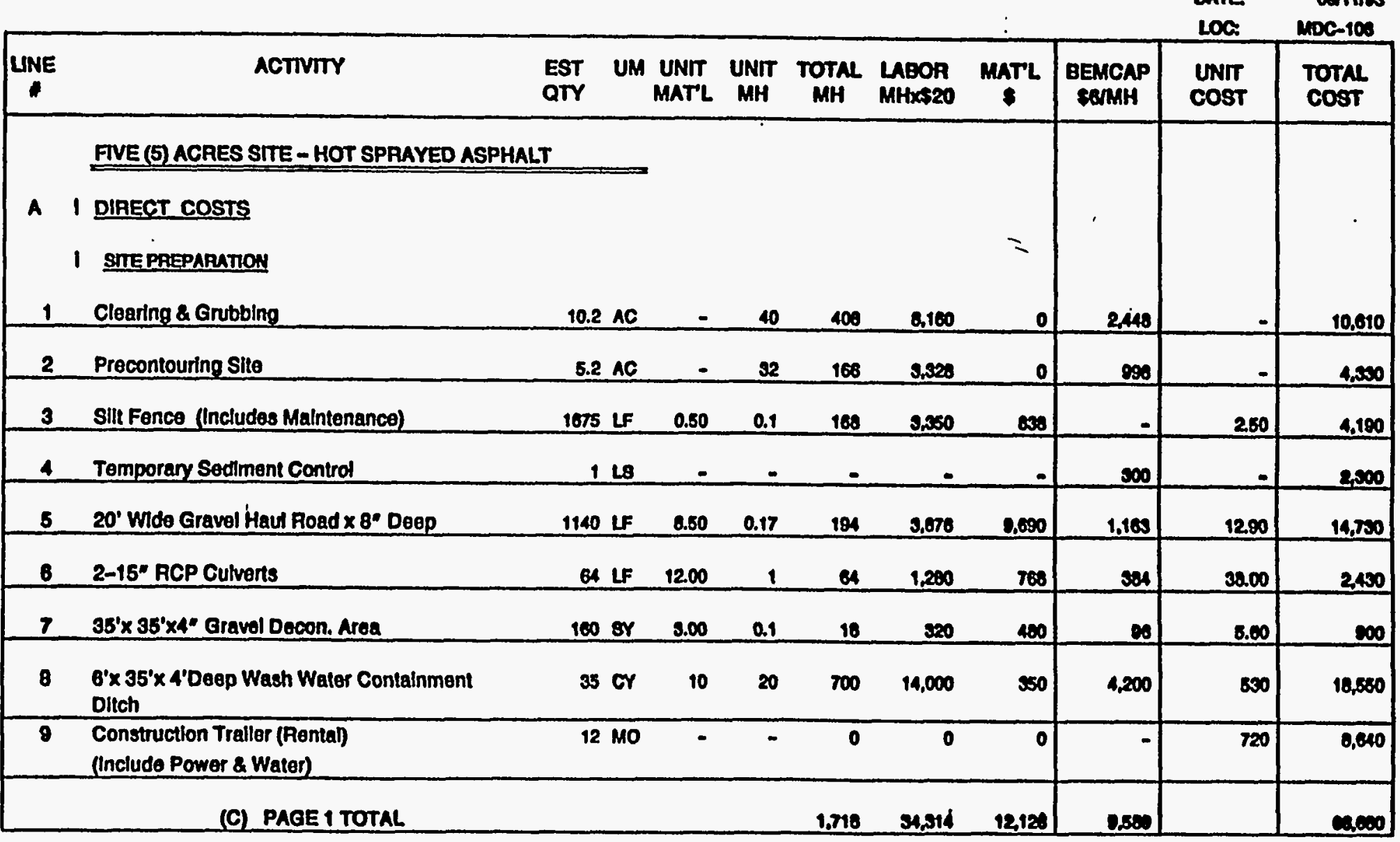

F-5. Cost Estimate for a Cover System Utilizing 1/4-inch Thick Hot Sprayed or Emulsified Asphalt 
D B T A L BSTIMAT B

Loo A: 7-03

NCTVITY CODE: ESE260112

PROJ.NAME: ECON. BVALUATTON OF CLOSURR CAP BARRUER MATERLAL.

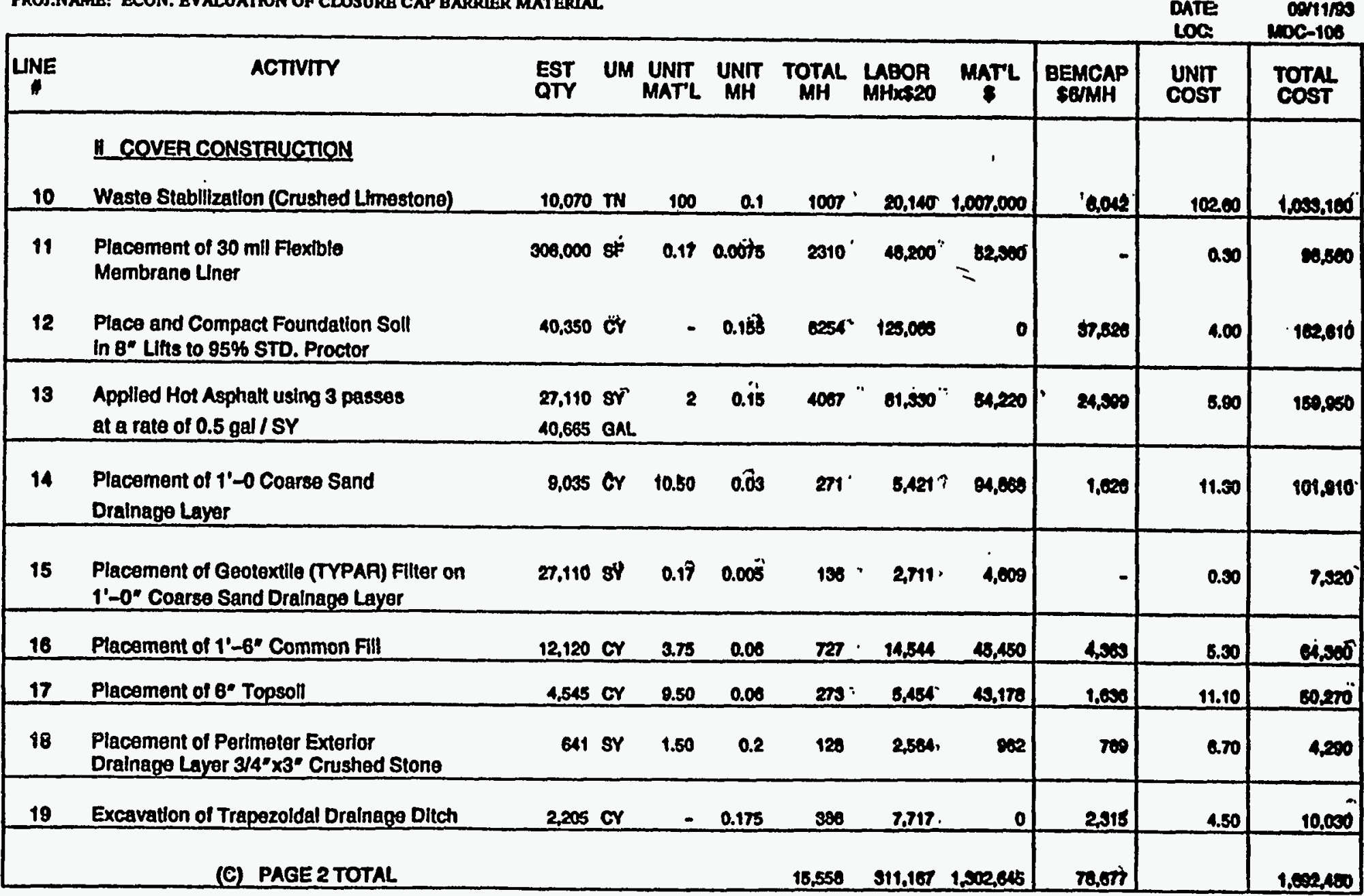

F-5. Cost Estimate for a Cover System Utilizing 1/4-inch Thick Hot Sprayed or Emulsified Asphalt (Cont'd) 
D B TAIL B S TIMAT B

CWRD QY: QMEAD

2001: 7-03

ACTIVITY CODE: ESE260112

MOJ.NAMG: BCON. BYALUATTON OF CLOSURB CAP BARRRRR MATERIAL.

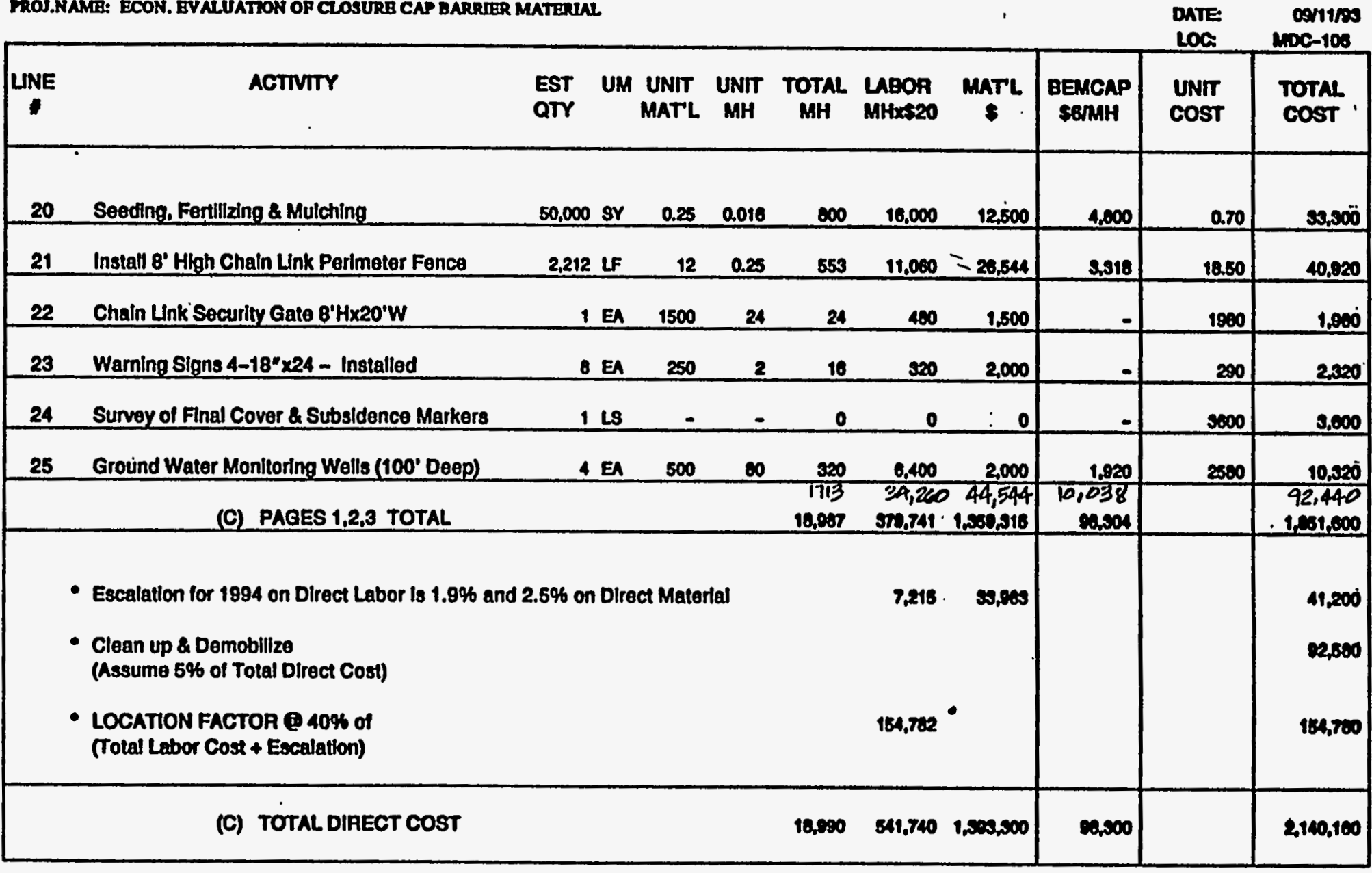

F-5. Cost Estimate for a Cover System Utilizing 1/4-inch Thick Hot Sprayed or Emulsified Asphalt (Cont'd) 
D B TAIL E S TIMATA

Q. MEAD

\section{8: 7-03}

NCTIVITY CODB: ESE260412

PROJ.NAME: ECON. EVALUATION OR CLOSURB CAP BANRTER MATERIAL.

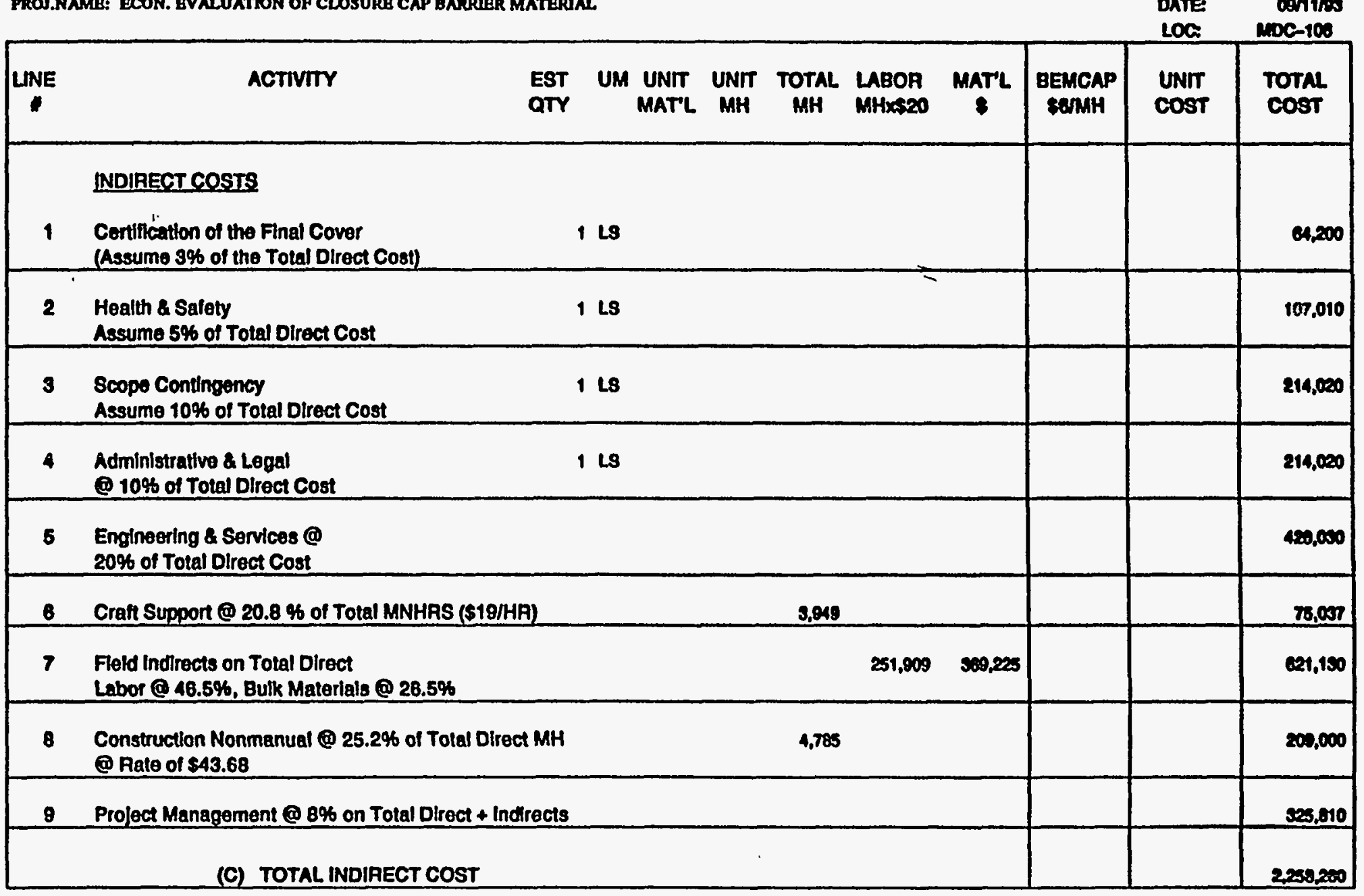

F-5. Cost Estimate for a Cover System Utilizing 1/4-inch Thick Hot Sprayed or Emulsified Asphalt (Cont'd) 
D B TAIL B S TIMATE

CKDDOY: QMEND

200 I: 7-03

ACTIVTY CODE: BSE260112

PROS.NAMG: ECON. BVALUATION OP CLOSURB CAP BARRIER MATERIAL.

EsTmatort PaWr

PHONE: 7-5200

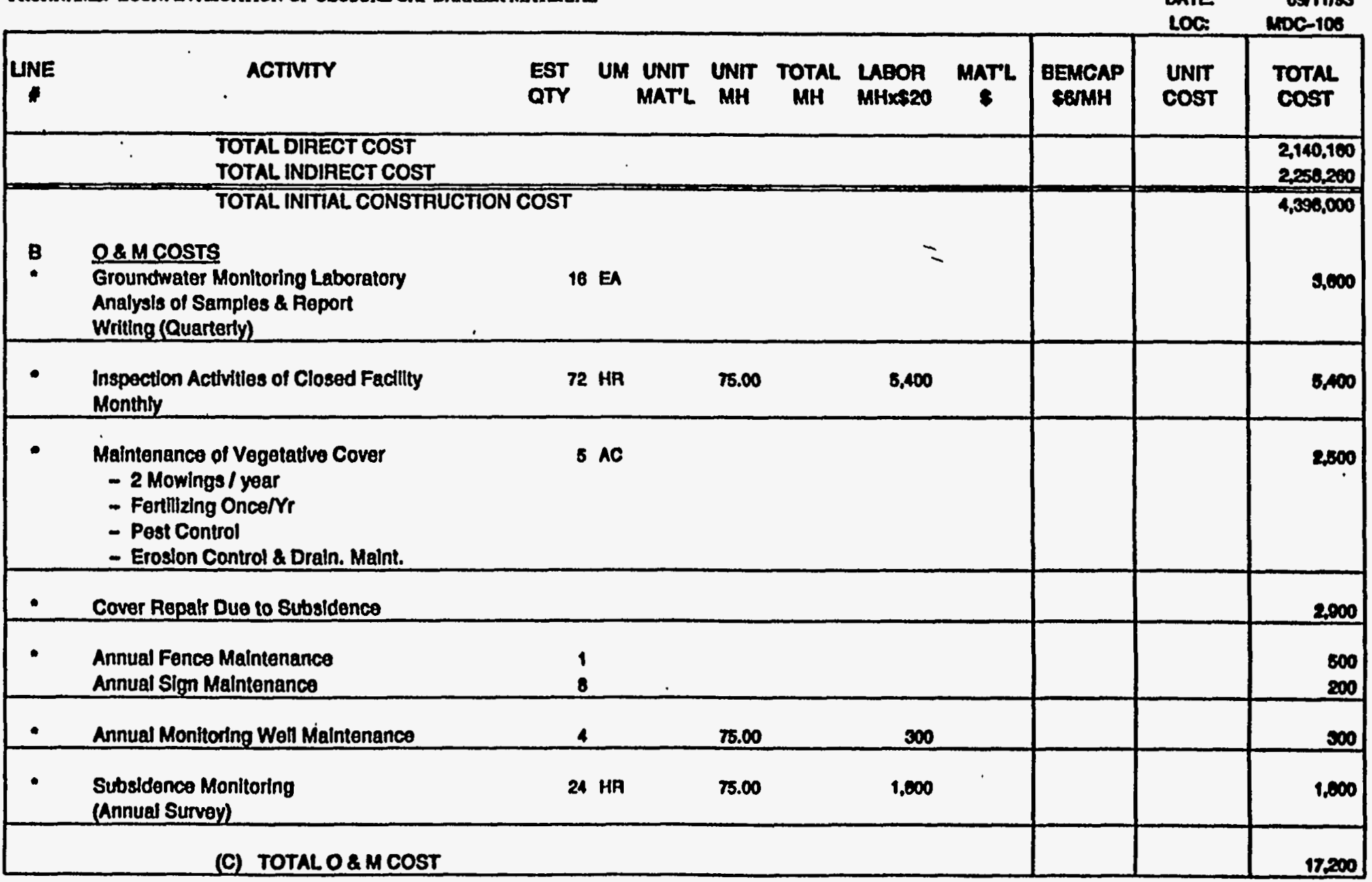

F-5. Cost Estimate for a Cover System Utilizing 1/4-inch Thick Hot Sprayed or Emulsified Asphalt (Cont'd) 
$1000,7-03$

ACTIVITY CODE: BSE260412

PROJ.NAME: ECON. EVALUATTON OP CLOSURB CAP BARRIER MATERIAL

100:

ance-10.0.

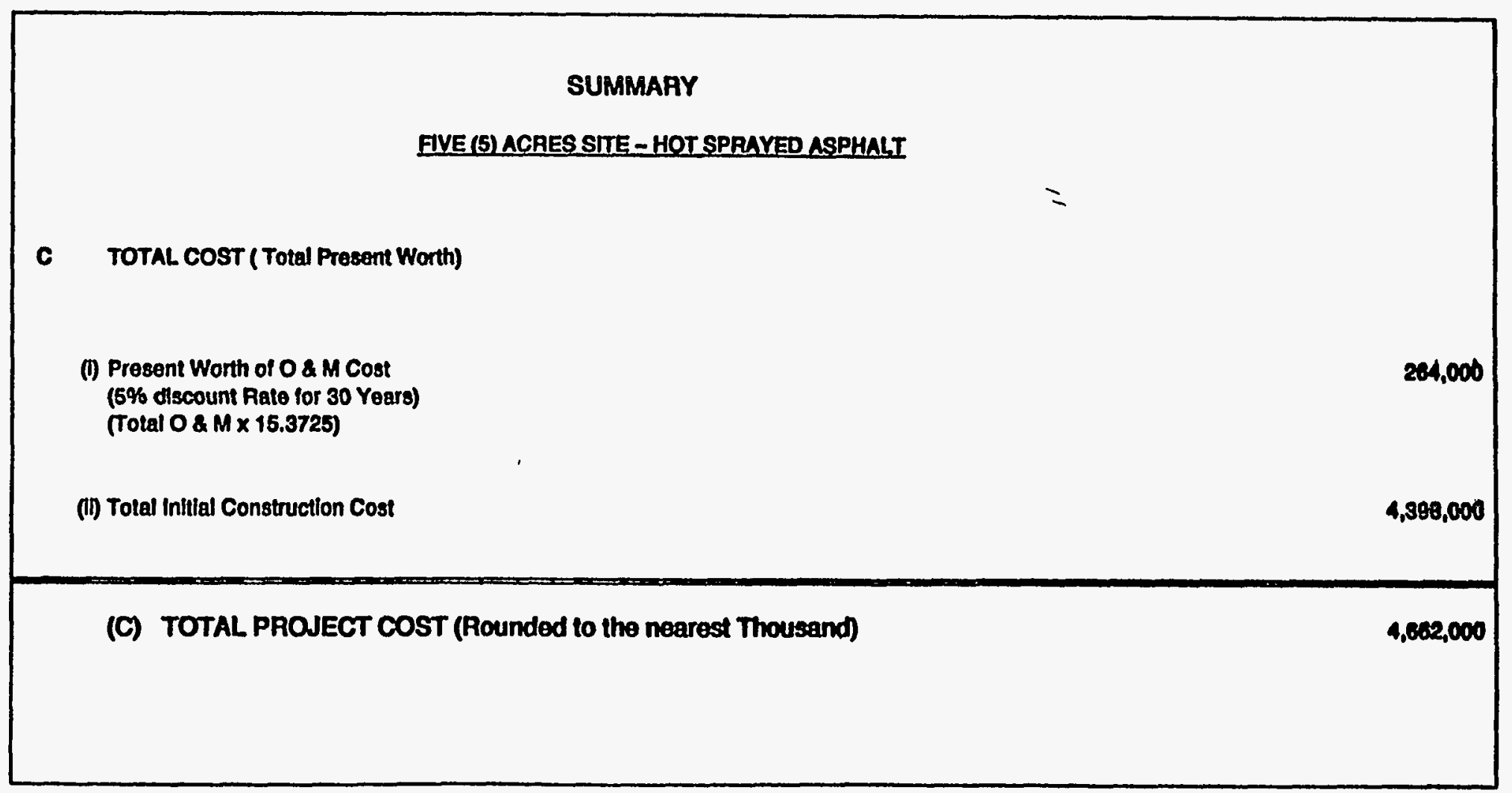

F-5. Cost Estimate for a Cover System Utilizing 1/4-inch Thick Hot Sprayed or Emulsified Asphalt (Cont'd) 


\section{DETAIL ESTIMATE}

LOQ $1: 7-00$

ACTIVTY CODE ESEPGO112

PAOU.NAME: ECON. EVALUATION OF CLOSUAE CAP BAPFIER MATERUAL

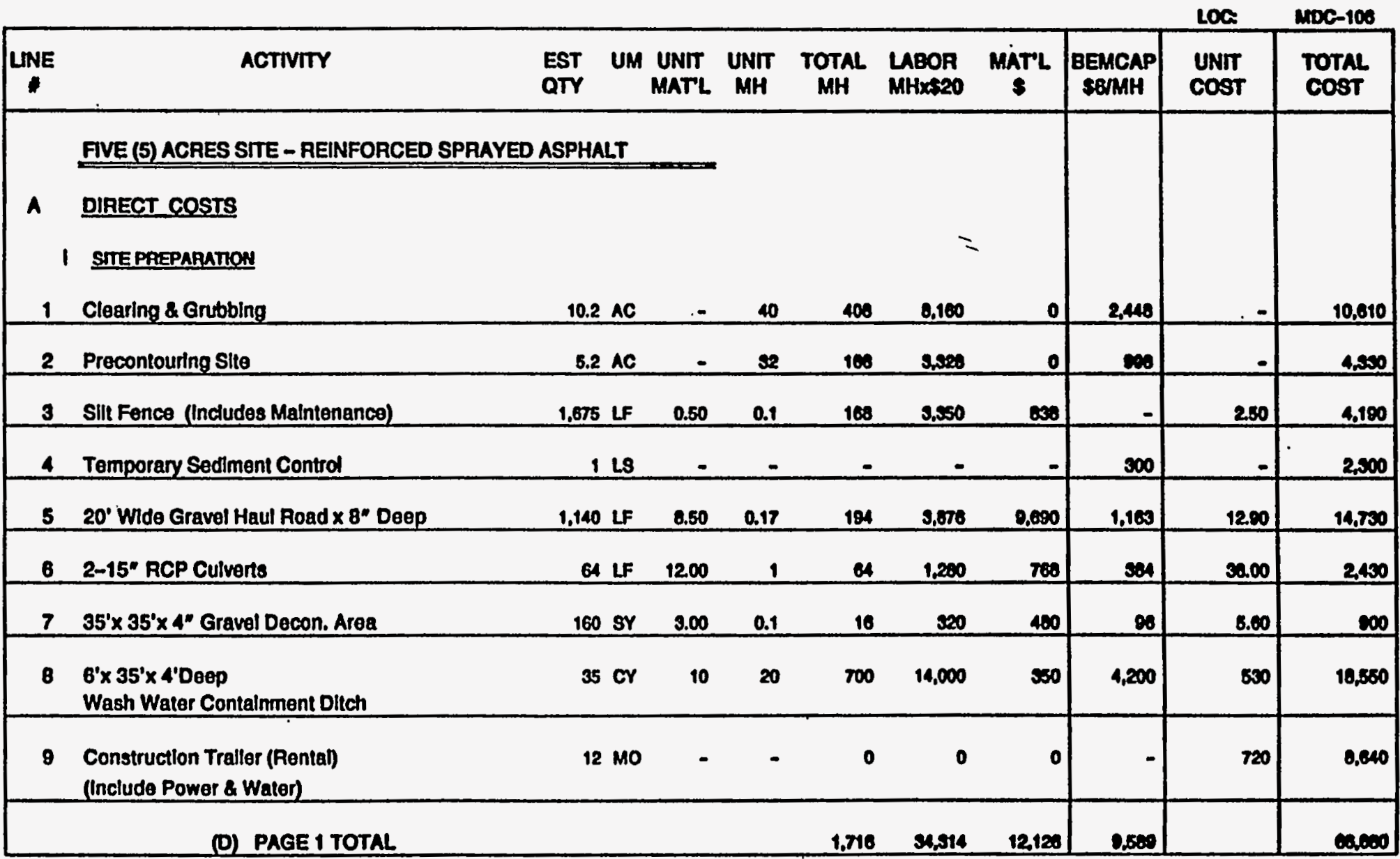

F-6. Cost Estimate for a Cover System Utilizing Reinforced Sprayed Asphalt 
DETAIL ESTIMATE

CAKD BY: 8.MEAD

1001: 7-25

PAOS.NO: KBGQ21100

PFOU.MAME ECON. EVALUATION CLOSURE CAP BARRAEA MATERARL

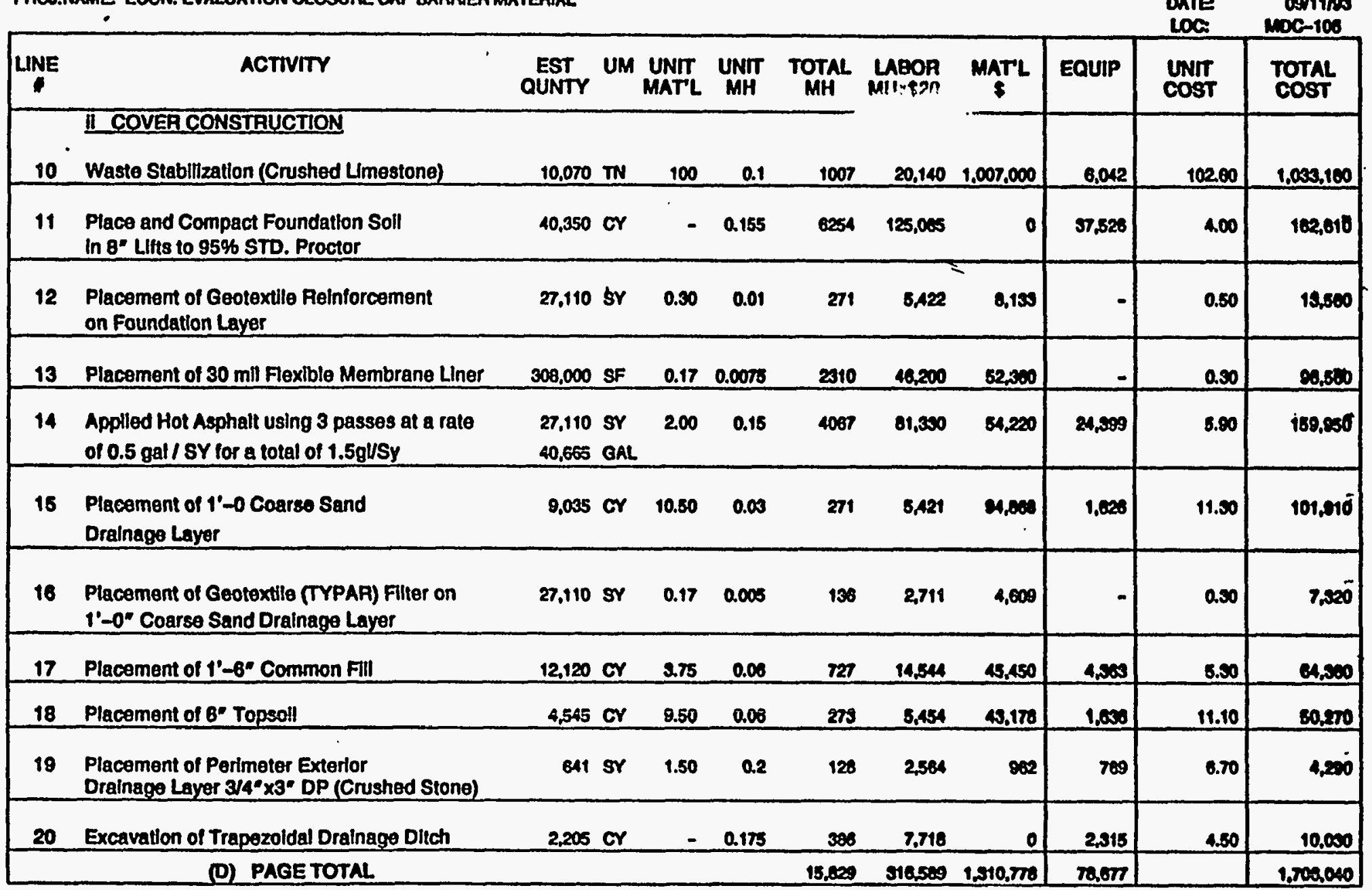

F-6. Cost Estimate for a Cover System Utilizing Reinforced Sprayed Asphalt (Cont'd) 
DETAIL ESTIMATE

CHECD BY: S.MEND

LOQ $7-25$

PPOI.NO: K86821100

PROJ.NMUE ECON. EVALUATION CLOSURE CAP BAPRAER MATERARL

ETmantor: Pamik

PHONE T-5260

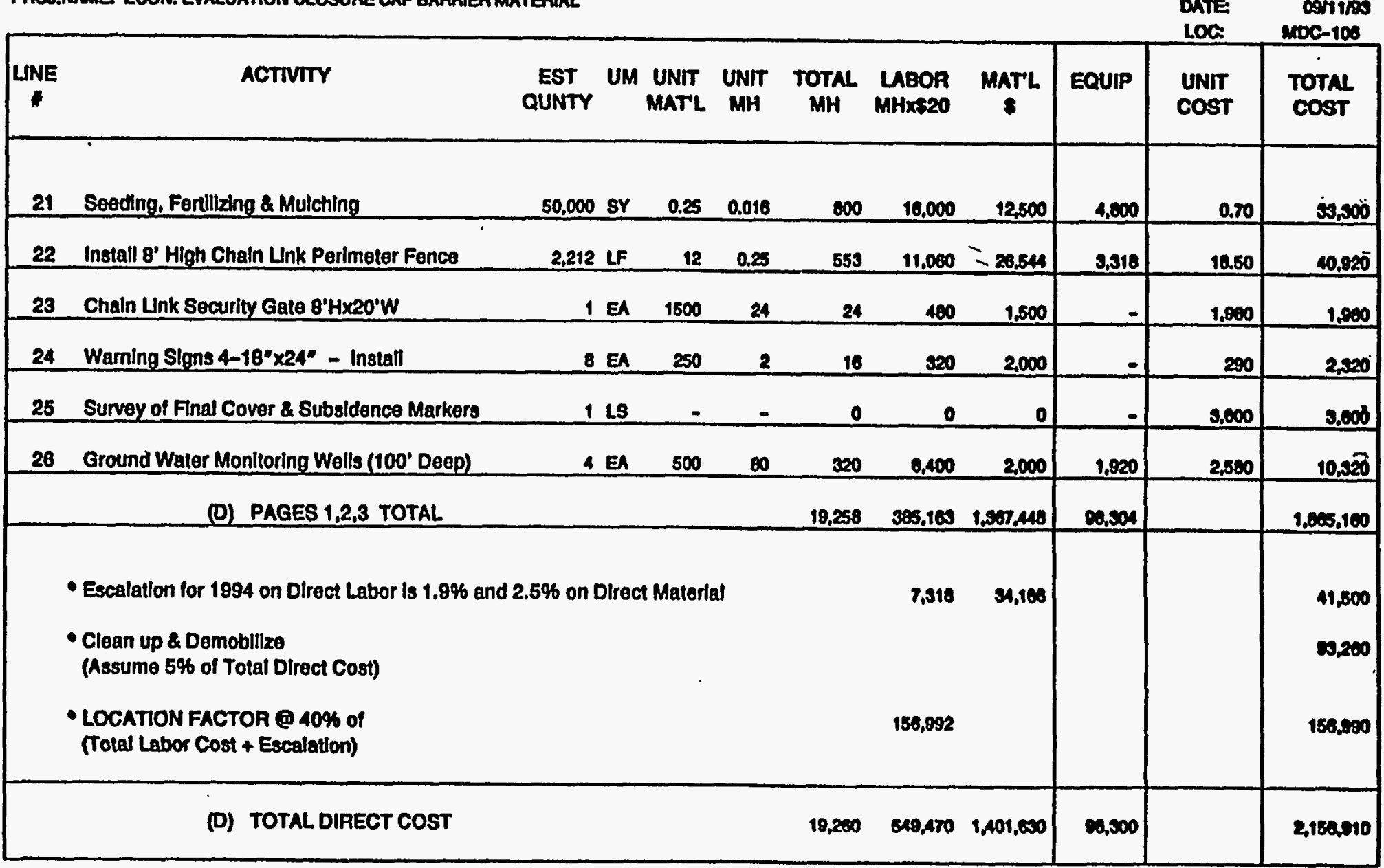

F-6. Cost Estimate for a Cover System Utilizing Reinforced Sprayed Asphalt (Cont'd) 
CHKD BY: QMEAD

LOO I: $7-25$

PAOS.NO: K86021100

PAOI.NAME: ECON. EVALUATION GLOSURE CAP BAARIER MATERIAL.

Esmmator: parnux

PHONE

$7-5268$

DATE DOM1100

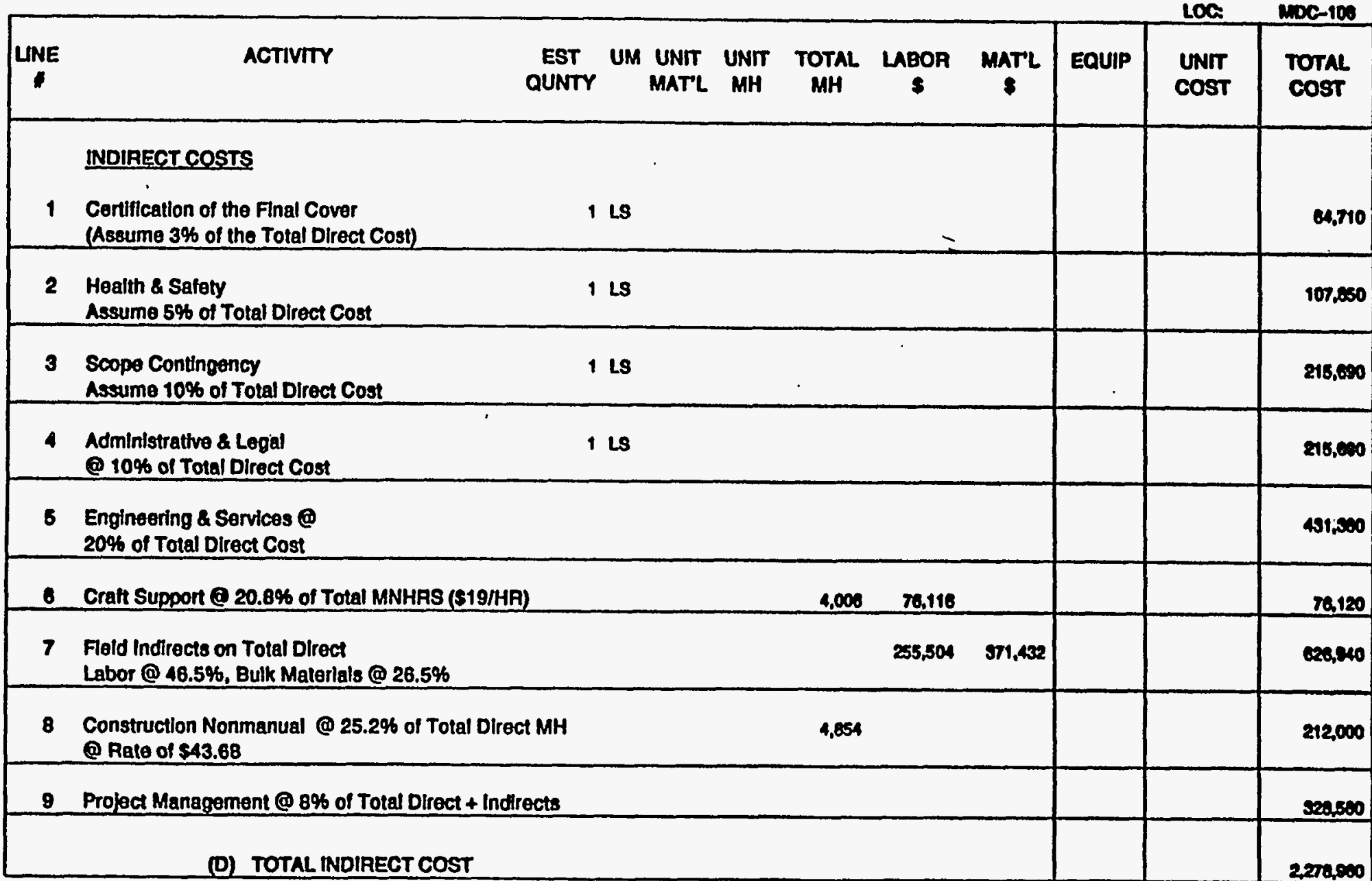

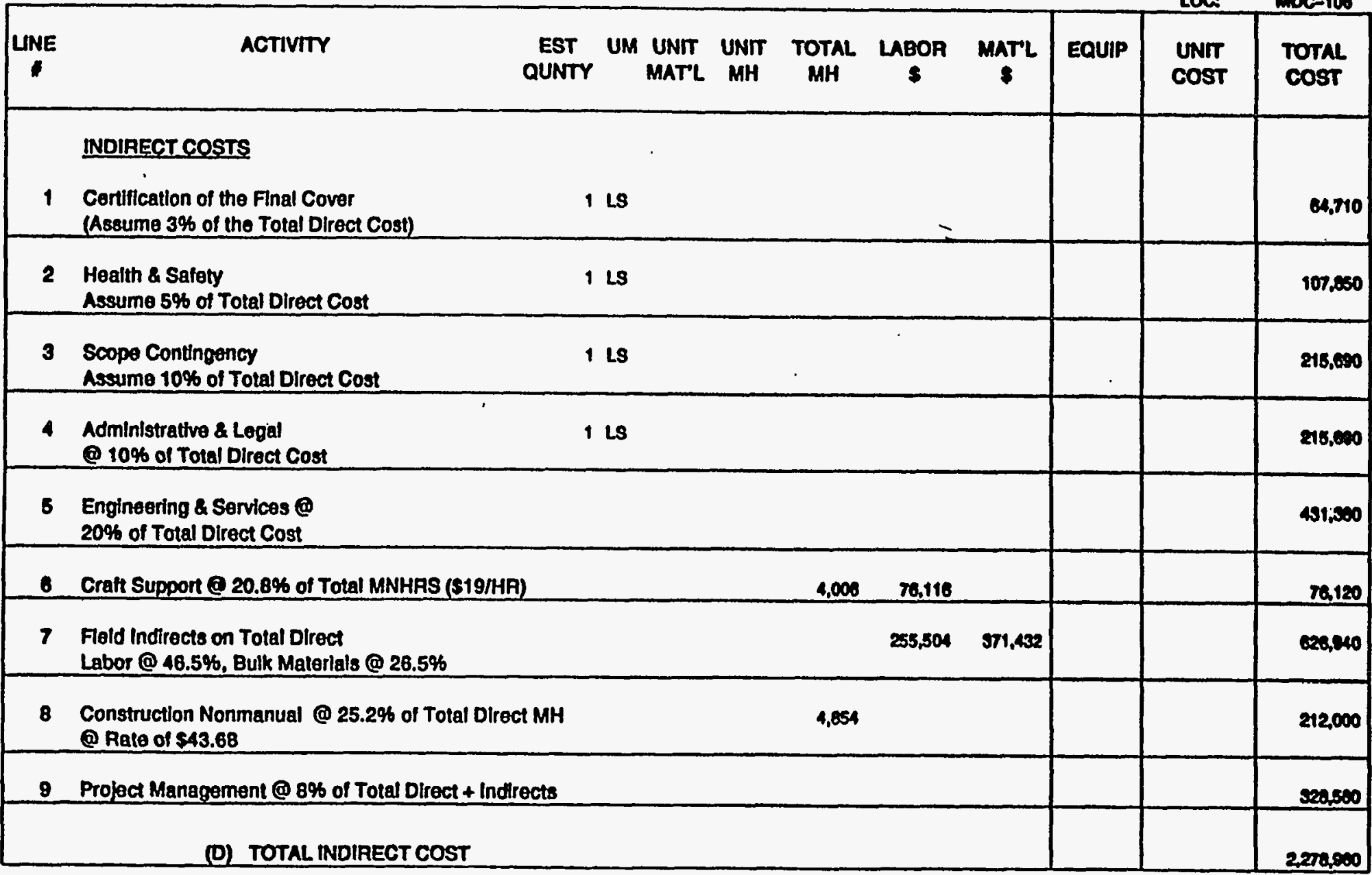

F-6. Cost Estimate for a Cover System Utilizing Reinforced Sprayed Asphalt (Cont'd) 


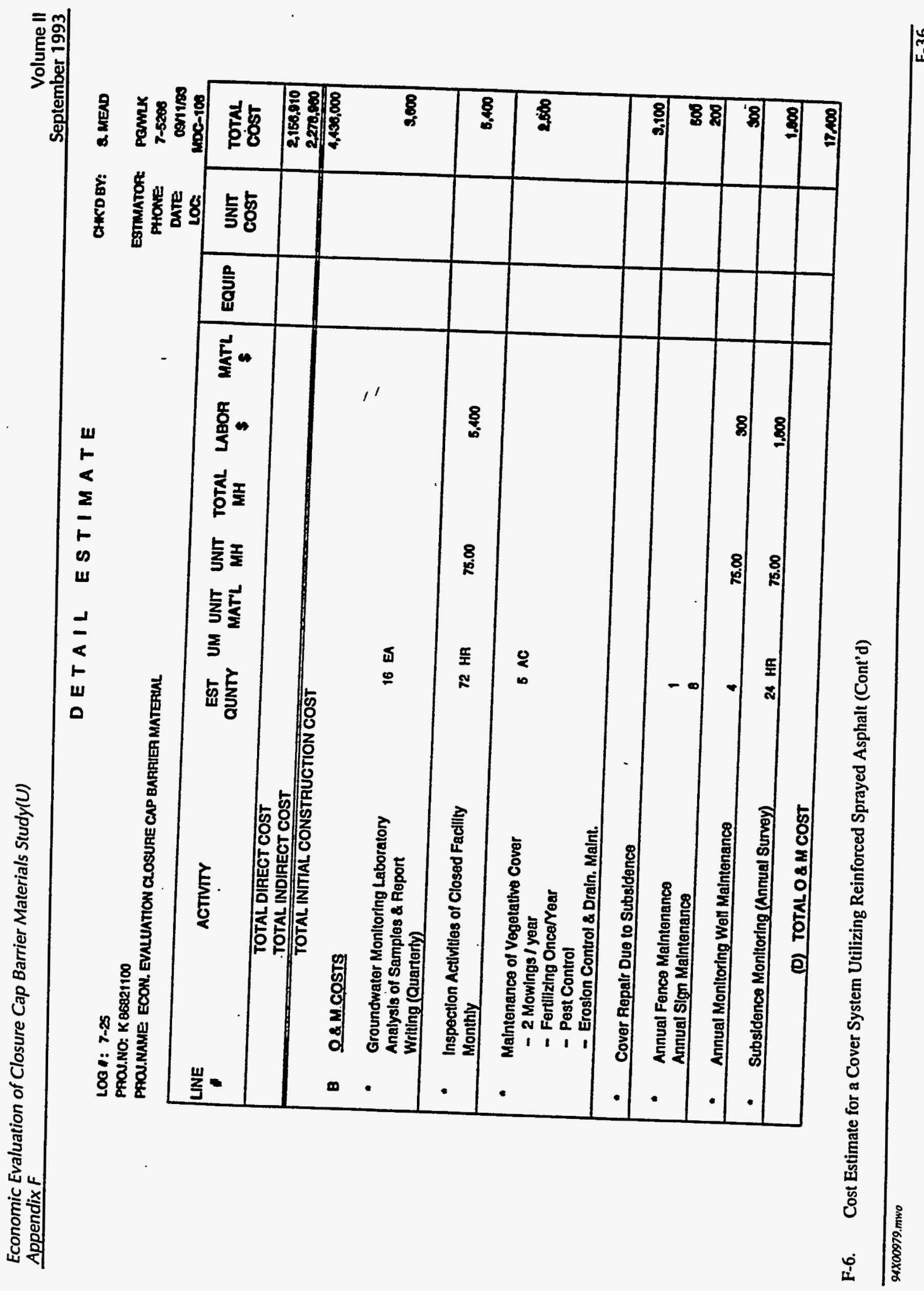


DETAIL ESTIMATE

CAKOBQY: Q.MEAD

LOQ I: $7-25$

PAOU.NO: K86821100

PAOU.NAME: ECON. EVALUATION CLOSURE CAP BARAIER MATERIAL

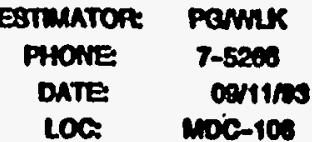

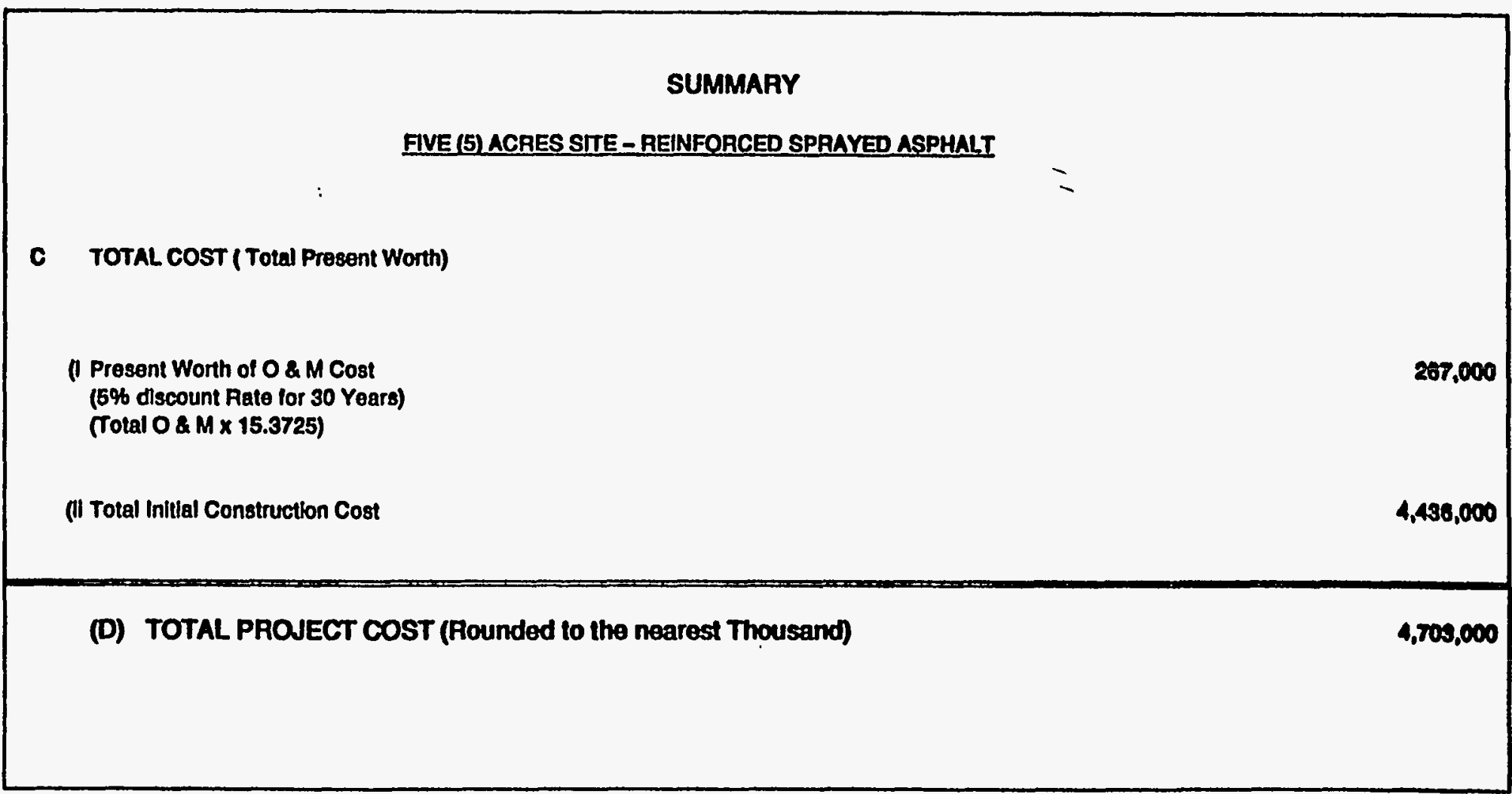

F-6. Cost Estimate for a Cover System Utilizing Reinforced Sprayed Asphalt (Cont'd) 
Lo01: 7-03

ACTIVITY CODE: ESE260412

PROJ.NAMB: ECON. EVALUATION OF CLOSURE CAP BARRIER MATERIAL

CHCD BY: Q MEND

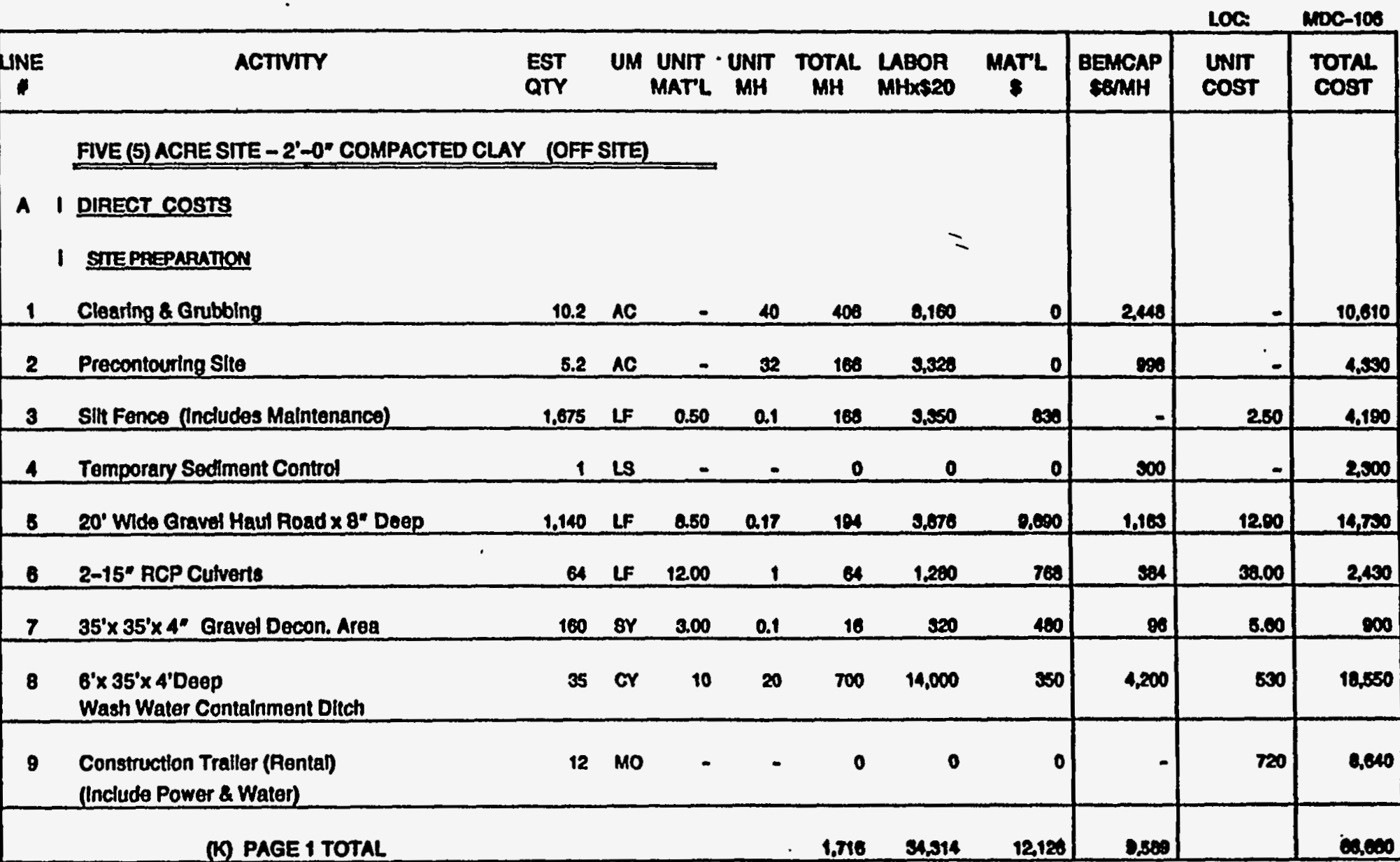

F-7. Cost Estimate for a Cover System Utilizing Compacted Kaolin (Offsite) Clay and a 30-mil FML 
DETAIL BSTIMATB

Q. MEAD

Loo e: $7-03$

ACTIVITY CODE: ESE260112

PROJ.NAME: ECON. BVALUATION OF CLOSURB CAP BARRIER MATERIAL

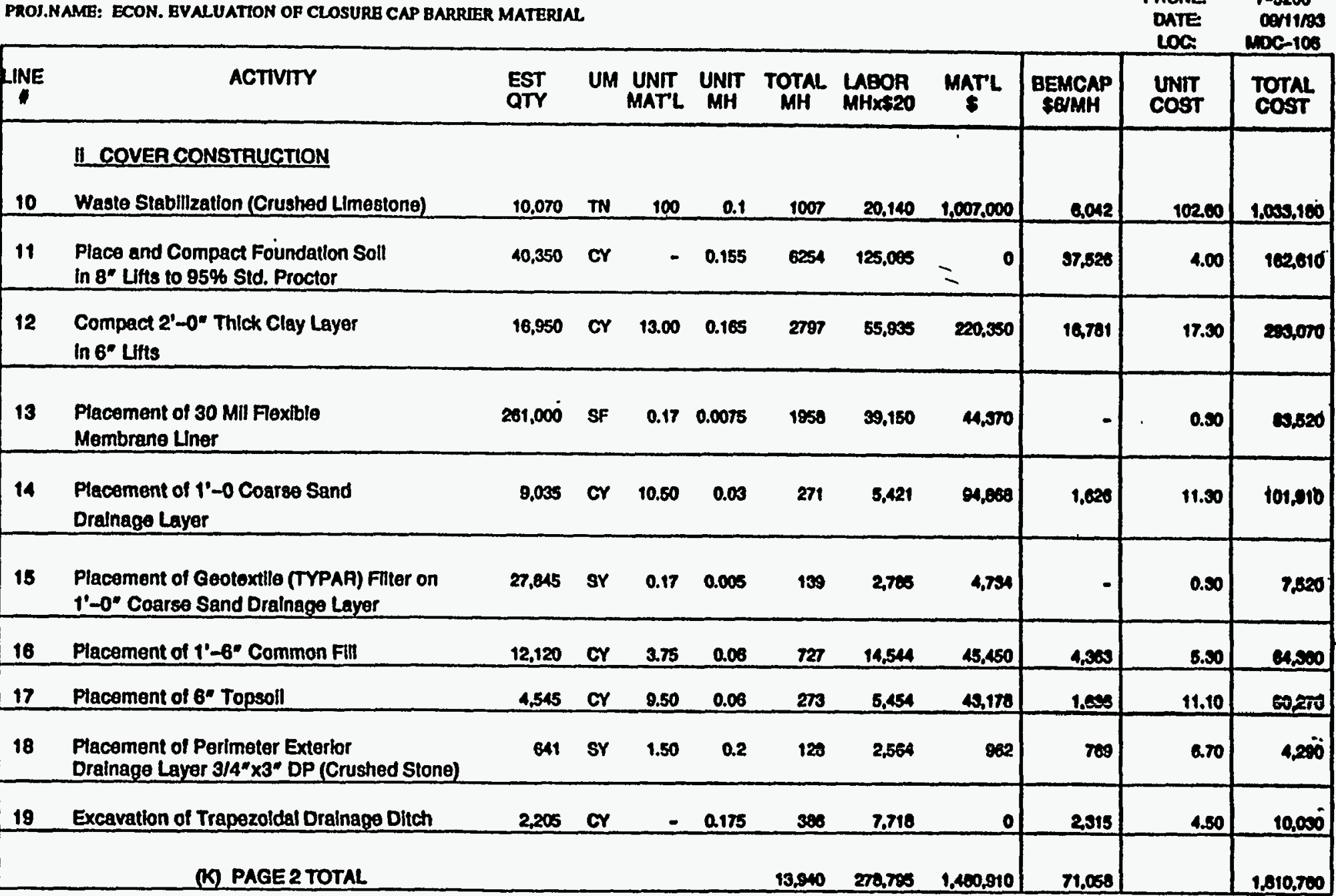

F-7. Cost Estimate for a Cover System Utilizing Compacted Kaolin (Offsite) Clay and a 30-mil FML (Cont'd) 
D B TA I L B S T IMATA

CHCD BV: Q.MEND

Loo : $7-03$

ACTIVITY CODE: ESE260\$12

PROJ.NAME: ECON. EVALUATION OF CLOSURE CAP BARRIER MATERIAL

Estuntor: PankK

PHONE $7-5268$

DATE

\begin{tabular}{|c|c|c|c|c|c|c|c|c|c|c|c|}
\hline UNE & ACTIVITY & $\begin{array}{l}\text { EST } \\
\text { QTY }\end{array}$ & UM & $\begin{array}{l}\text { UNIT } \\
\text { MATL }\end{array}$ & $\begin{array}{l}\text { UNIT } \\
\text { MH }\end{array}$ & $\begin{array}{c}\text { TOTAL } \\
\text { MH }\end{array}$ & $\begin{array}{l}\text { LABOR } \\
\text { MHX } \$ 20\end{array}$ & $\underset{\$}{\text { MAT'L }}$ & $\begin{array}{l}\text { BEMCAP } \\
\text { \$GMMH }\end{array}$ & $\begin{array}{l}\text { UNIT } \\
\text { COST }\end{array}$ & $\begin{array}{l}\text { TOTAL } \\
\text { COST }\end{array}$ \\
\hline 20 & Seeding, Fertilizing \& Mulching & 50.000 & sy & 0.25 & 0.016 & 000 & 16.000 & 12.500 & 4,000 & 0.70 & 30,300 \\
\hline 21 & Install 8' High Chain LInk Perlmeter Fence & 2,212 & LF & 12 & 0.25 & 553 & 11.060 & 20,544 & 3.318 & 18.50 & 40,920 \\
\hline 22 & Chain LInk Security Gate 8'Hx20'W & 1 & EA & 1500 & 24 & 24 & 400 & 1,500 & - & 1800 & 1.000 \\
\hline 23 & Warning Signs 4-18" $\times 24^{\prime \prime}-$ Installed & - & EA & 250 & 2 & 10 & 320 & 2.000 & $=$ & 290 & 2,020 \\
\hline 24 & Survey of Final Cover \& Subsidence Markers & 1 & LS & - & - & $\mathbf{0}$ & o & 0 & - & 3000 & 0.000 \\
\hline 25 & Ground Water Monitoring Wells (100' Deep) & 4 & EA & 500 & $\infty$ & 320 & 0,400 & 2,000 & 1,020 & 2580 & 10,520 \\
\hline & (M) PAGES 1,2,3 TOTAL & & & & & 17.360 & $\mathbf{3 4 7 , 3 0 9}$ & $1,517,500$ & $\mathbf{0 0 , 6 0 5}$ & & $1,009,000$ \\
\hline & $\begin{array}{l}\text { Escalation for } 1994 \text { on Direct Labor is } 1.9 \% 8 \\
\text { Clean up \& Demobilize } \\
\text { (Assume 5\% of Total Drect Cost) }\end{array}$ & $2.5 \%$ on & Direc & Materis & & & 0,600 & $\mathbf{5 7 . 0 3 9}$ & & & $\begin{array}{l}4,540 \\
00,490\end{array}$ \\
\hline & $\begin{array}{l}\text { LOCATION FACTOR C } 40 \% \text { of } \\
\text { (Total Labor Cost + Escalation) }\end{array}$ & & & & & & 141,580 & & & & 141,5000 \\
\hline & (M) TOTAL DIRECT COST & & & & & 17,370 & 485,500 & $1,565,520$ & $\infty 0,600$ & & 22504,500 \\
\hline
\end{tabular}

F-7. Cost Estimate for a Cover System Utilizing Compacted Kaolin (Offsite) Clay and a 30-mil FML (Cont'd) 
DETAIL B S T I A A B

CHCD BY: S.MEAD

Lool: $7-03$

ACTIVITY CODB: BSE260112

PROJ.NAME: ECON. EVALUATKON OF CLOSURE CAP BARRIER MATERAAL.

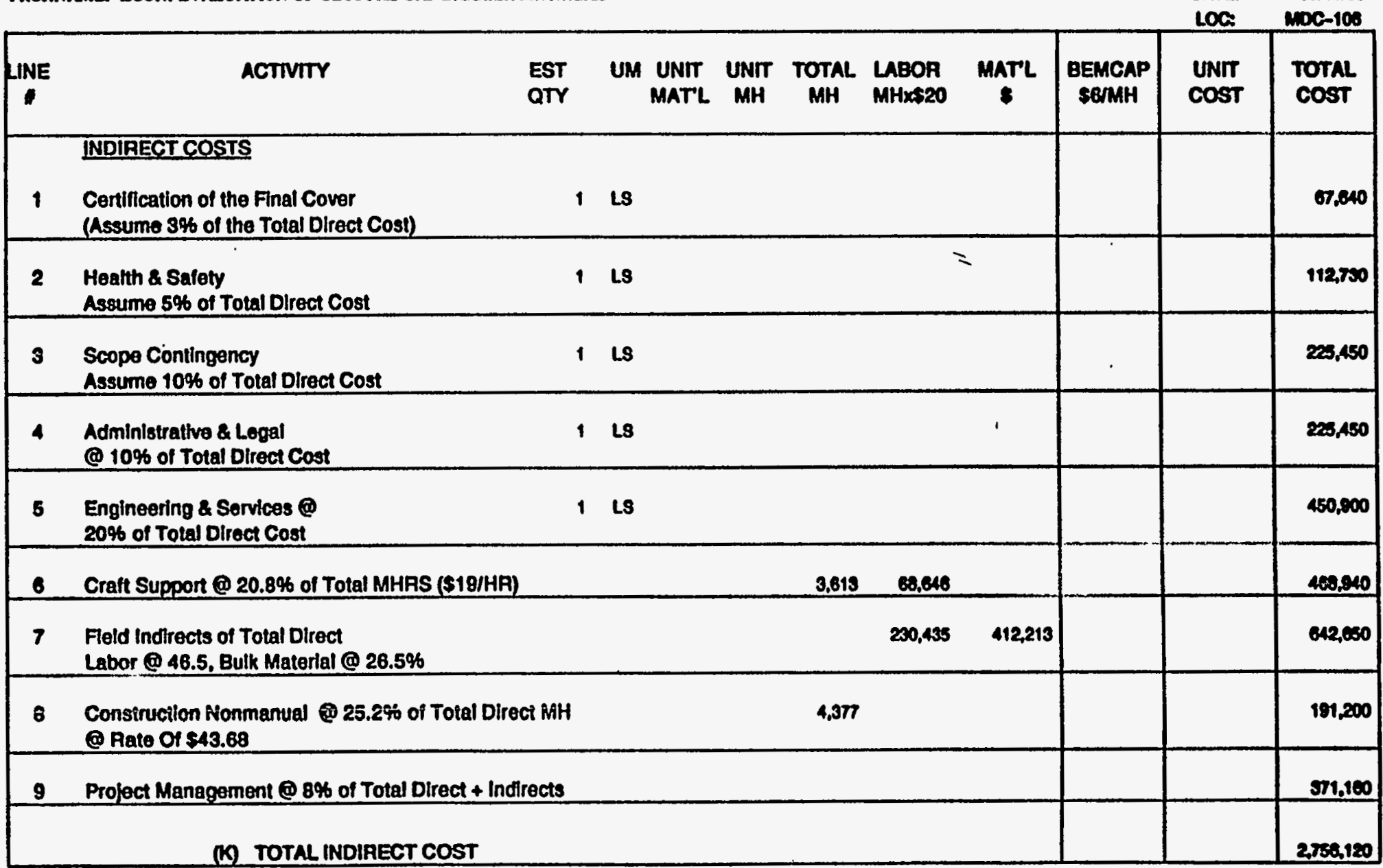

F-7. Cost Estimate for a Cover System Utilizing Compacted Kaolin (Offsite) Clay and a 30-mil FML (Cont'd) 
DETAIL E S T I MAT B

Q MERD

Lo01: $7-03$

ACTIVITY CODE: ESE260412

PROJ.NAME: ECON. EVALUATION OF CLOSURB CAP BARRIER MATERIAL

\begin{tabular}{|c|c|c|c|c|c|c|c|c|c|c|}
\hline JNE & ACTIVITY & $\begin{array}{l}\text { EST } \\
\text { QTY }\end{array}$ & $\begin{array}{l}\text { UM UNIT } \\
\text { MATL }\end{array}$ & $\begin{array}{l}\text { UNIT } \\
\text { MH }\end{array}$ & $\underset{\text { MH }}{\text { TOTAL }}$ & $\begin{array}{l}\text { LABOA } \\
\text { MHX\$20 }\end{array}$ & $\underset{*}{\text { MATLL }}$ & $\begin{array}{l}\text { BEMCAP } \\
\text { SBMMH }\end{array}$ & $\begin{array}{l}\text { UNIT } \\
\text { COST }\end{array}$ & $\begin{array}{l}\text { TOTAL } \\
\text { COST }\end{array}$ \\
\hline & $\begin{array}{l}\text { TOTAL DIRECT COST } \\
\text { TOTAL INDIRECT COST }\end{array}$ & & & & & & & & & $\begin{array}{l}2,254,600 \\
2,756,120\end{array}$ \\
\hline • & $\begin{array}{l}\text { TOTAL INITIAL CONSTAU } \\
\text { O\&M cosTs } \\
\text { Groundwater Monitoring Laboratory } \\
\text { Analysis of Samples \& Report } \\
\text { Writing (Quarterly) }\end{array}$ & $\begin{array}{r}\operatorname{COST} \\
16\end{array}$ & EA & & & & $=$ & & & $5,010,620$ \\
\hline- & Inspectlon Activitles of Facillty - Monthly & 72 & HA & 75.00 & & 8,400 & & & & 8,400 \\
\hline- & Subsidence Monitoring Annual Survey & 24 & HR & 75.00 & & 1.000 & & & & 1,000 \\
\hline & $\begin{array}{l}\text { Malntenance of Vegatative Cover } \\
\text { - } 2 \text { Mowings / year } \\
\text { - Pest Control } \\
\text { - Eroslon Control \& } \\
\text { Drainage Maintenance } \\
\end{array}$ & 5 & AC & & & & & & & 2,000 \\
\hline$\bullet$ & Cover Repalr Due to Subsidence & & & & & & & & & 8.0050 \\
\hline & $\begin{array}{l}\text { Annual Fonce Malntenance } \\
\text { Annual Slgn Malmtenance }\end{array}$ & $\begin{array}{l}1 \\
8 \\
\end{array}$ & & & & & & & & $\begin{array}{l}800 \\
200 \\
\end{array}$ \\
\hline - & Annual Moniloring Weil Maintenance & 4 & & 75.00 & & 5000 & & & & 300 \\
\hline & (K) TOTAL O\&MCOST & & & & & & & & & 20,00 \\
\hline
\end{tabular}

F-7. Cost Estimate for a Cover System Utilizing Compacted Kaolin (Offsite) Clay and a 30-mil FML (Cont'd) 
MROJ.NAME: ECON. EVALUATKON OP CLOSURE CAP BARRIER MATERAAL.

loc

MOC-108

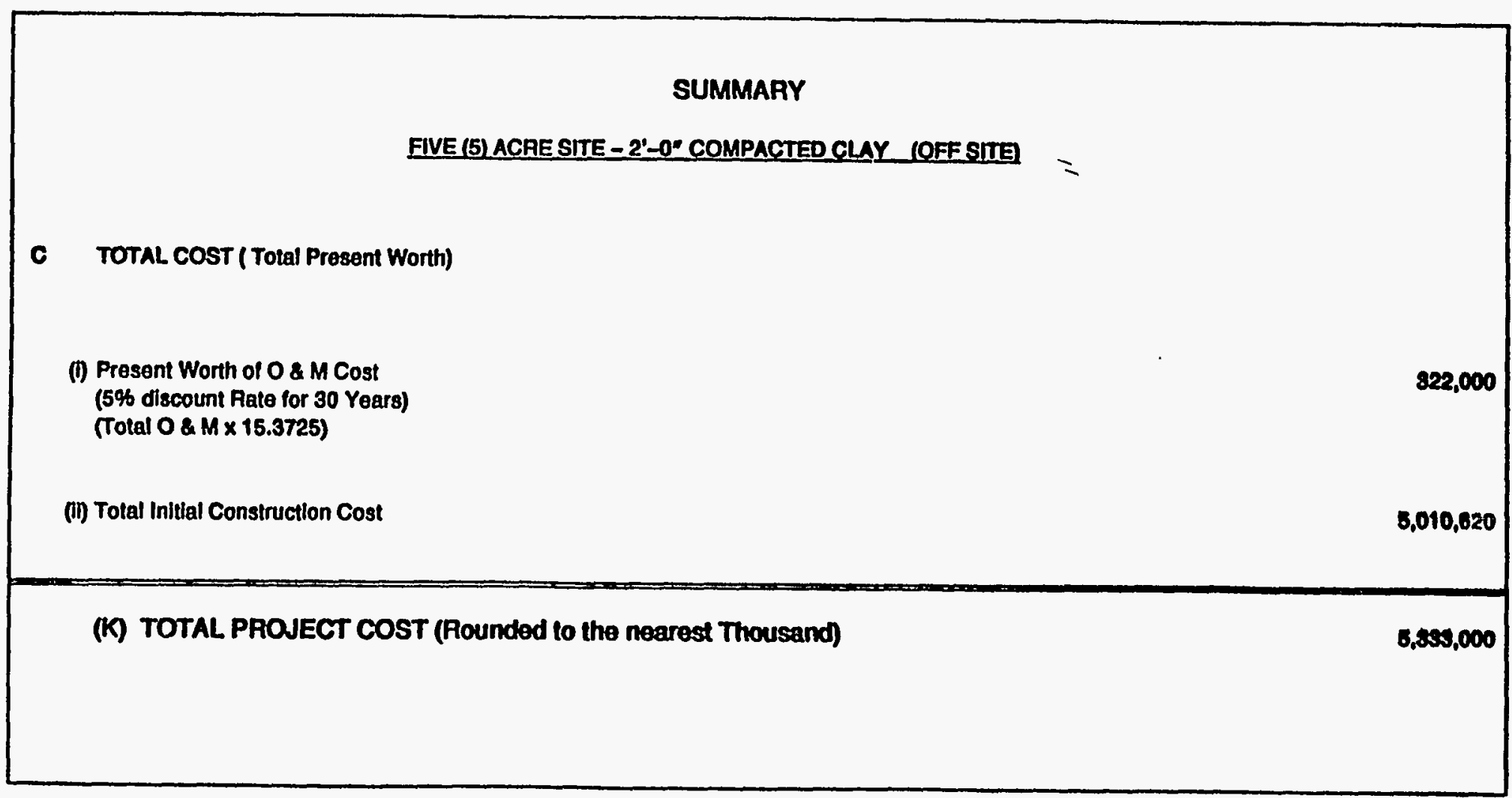

F-7. Cost Estimate for a Cover System Utilizing Compacted Kaolin (Offsite) Clay and a 30-mil FML (Cont'd) 
D E T A I L B T I M A T B

CWKD BY: Q MEAO

Loof: $7-03$

ACTIVITY CODE: ESE2GON12

PROI.NAME: BCON. EVALUATION OF CLOSURB CAP BARRIER MATERUAL

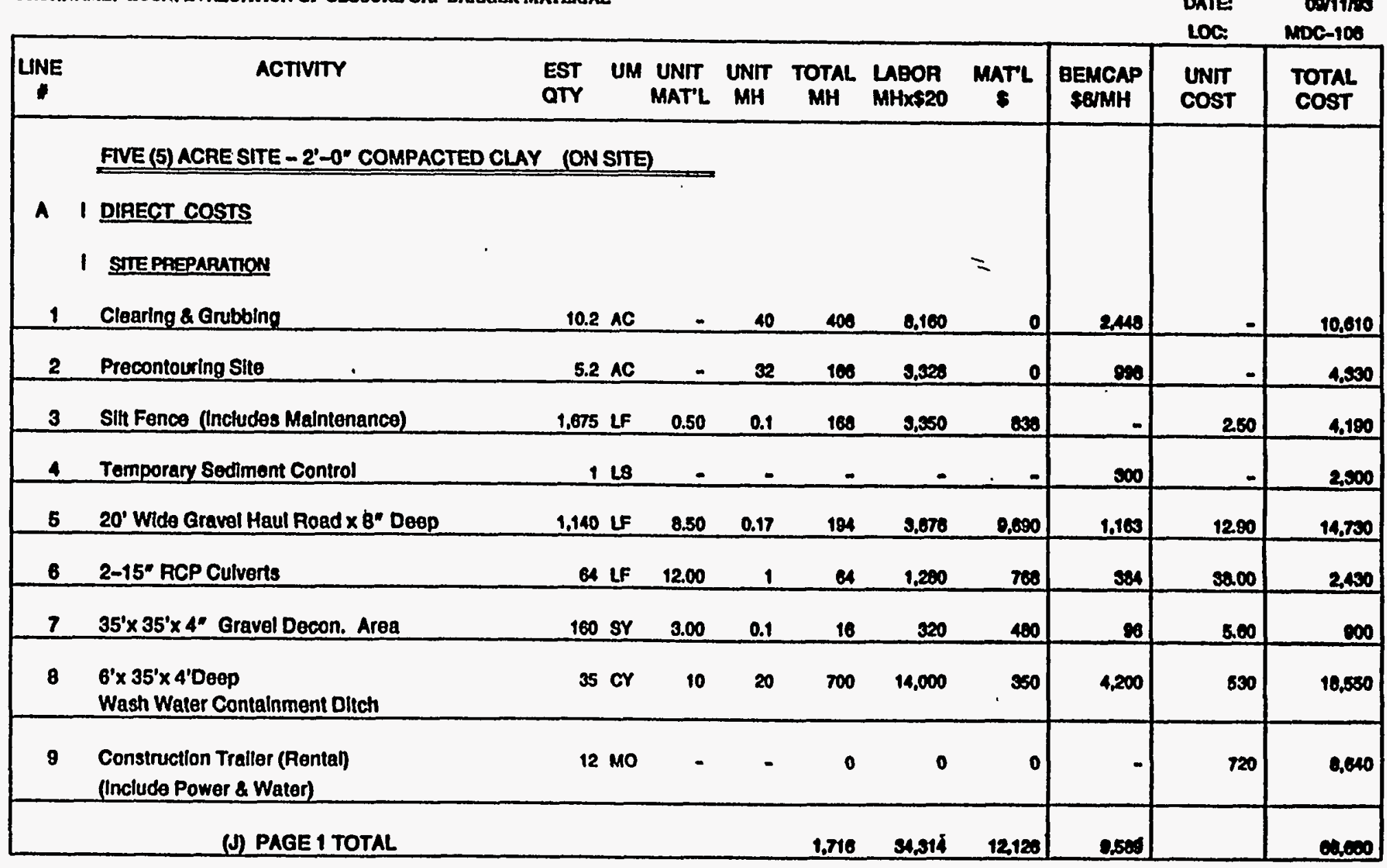

F-8. Cost Estimate a for Cover System Utilizing Compacted Onsite Sandy Clay and a 30-mil FML 
D B T A L B S T I MAT B

CAMDOY: Q.MEAD

Loo : :-03

ACTIVTYY CODE: ESE260412

PROJ.NAME: ECON. EYALUATION OP CLOSURB CAP BARRIER MATERIAL

\begin{tabular}{|c|c|c|c|c|c|c|c|c|c|c|c|}
\hline LUNE & ACTIVITY & $\begin{array}{l}\text { EST } \\
\text { QTY }\end{array}$ & UM & $\begin{array}{l}\text { UNIT } \\
\text { MATL }\end{array}$ & $\underset{M H}{\text { UNIT }}$ & TOTAL & $\begin{array}{l}\text { LAB:OR } \\
\text { MHX } \$ 20\end{array}$ & $\underset{\$}{\operatorname{MaTL}}$ & $\begin{array}{l}\text { BEMCAP } \\
\text { SGMMH }\end{array}$ & $\begin{array}{l}\text { UNIT } \\
\text { COST }\end{array}$ & $\underset{\text { COTAL }}{\text { TOST }}$ \\
\hline & II COVER CONSTRUCTION & & & & & & & & & & \\
\hline 10 & Waste Stabilization (Crushed Limestone) & 10,070 & $\mathbf{T N}$ & 100 & 0.1 & 1007 & 20,140 & $1,007,000$ & 0.042 & 102,00 & $1,000,100$ \\
\hline 11 & $\begin{array}{l}\text { Place and Compact Foundation Soll } \\
\text { In } 8^{\circ} \text { Lifts to } 95 \% \text { Std. Proctor }\end{array}$ & 40,350 & or & - & 0.155 & 6254 & 1205.005 & o) & 57.520 & 1.00 & 162,010 \\
\hline 12 & $\begin{array}{l}\text { Compact 2'-0" Thick Clay Layer } \\
\text { In 6" Llits }\end{array}$ & 16,950 & or & - & 0.165 & 2797 & 55,935 & o & 18,701 & 4.50 & 72,720 \\
\hline 13 & $\begin{array}{l}\text { Placement of } 30 \text { Mil Flexible } \\
\text { Membrane Liner }\end{array}$ & 261,000 & $\dot{\mathbf{S F}}$ & $0.17^{\circ}$ & 0.0076 & 1958 & 39,150 & 44,970 & - & 0.50 & 03,520 \\
\hline 14 & $\begin{array}{l}\text { Placement of 1'-0 Coarse Sand } \\
\text { Drainage Layar }\end{array}$ & 9,035 & or & 10.50 & 0.03 & 271 & 3,421 & 24,060 & 1,020 & 11.30 & 101,910 \\
\hline 18 & $\begin{array}{l}\text { Piacement of Geotextlle (TYPAP) Filter on } \\
1^{1}-0^{*} \text { Coarse Sand Dralnage Layer }\end{array}$ & 27,845 & $\boldsymbol{s i r}$ & 0.17 & 0.005 & 139 & 2,785 & 4,734 & - & 0.30 & 7,520 \\
\hline 16 & Placement of $1^{\prime}-6^{\prime \prime}$ Common Fill & 12,120 & or & 3.75 & 0.06 & 727 & 14,544 & 45,450 & 4,363 & 5.30 & 0,300 \\
\hline 17 & Placement of 6" Topsoil & 4,545 & or & 9.50 & 0.06 & 273 & 3,454 & 4s,170 & 1.006 & 11.10 & $80.270^{\circ}$ \\
\hline 18 & $\begin{array}{l}\text { Placement of Perimeter Exterlor } \\
\text { Drainage Layer } 3 / 4^{n} \times 3^{*} \text { DP (Crushed Stone) }\end{array}$ & G11 & sy & 1.50 & 0.2 & 120 & 2,564 & 802 & $r \infty$ & 0.70 & 4,200 \\
\hline 19 & Excavation of Trapezoldal Drainage Ditch & 2,205 & or & - & 0.175 & 306 & 7.710 & o. & 2,315 & 4.50 & 10.030 \\
\hline & (J) PAGE 2 TOTAL & & & & & 18,940 & $270, \pi 5$ & $1,240,500$ & $n .050$ & & 1,500A10 \\
\hline
\end{tabular}

F-8. Cost Estimate a for Cover System Utilizing Compacted Onsite Sandy Clay and a 30-mil FML (Cont'd) 
DETAIL B S T I M A T B

Lool: $7-03$

ACTIVITY CODB: BSE260412

PROJ.NAME: ECON. EVALUATION OP CLOSURB CAP BARRIER MATERIAL

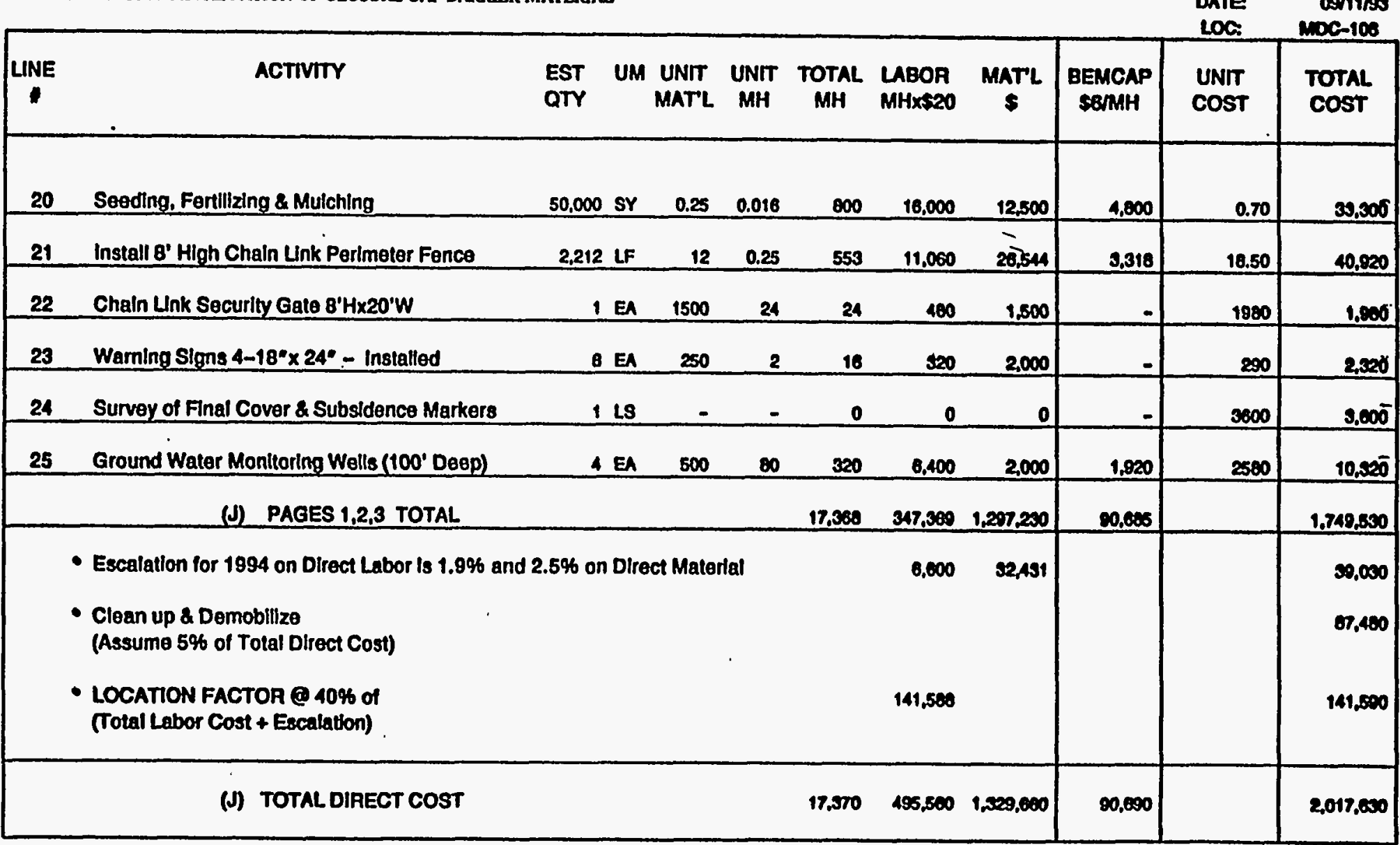

F-8. Cost Estimate a for Cover System Utilizing Compacted Onsite Sandy Clay and a 30-mil FML (Cont'd) 


\section{DETAIL ESTIMAT B}

Lo01: $7-03$

ACTTVTTY CODE: ESE260412

PROJ.NAME: ECON. EVALUATHON OF CLOSURE CAP BARRTER MATERIAL

CHKD BY: QMENO

Estmatofe Pamk

PHONE: 7-5260

DATE DOM1MS

LOC. $\quad M D C-100$

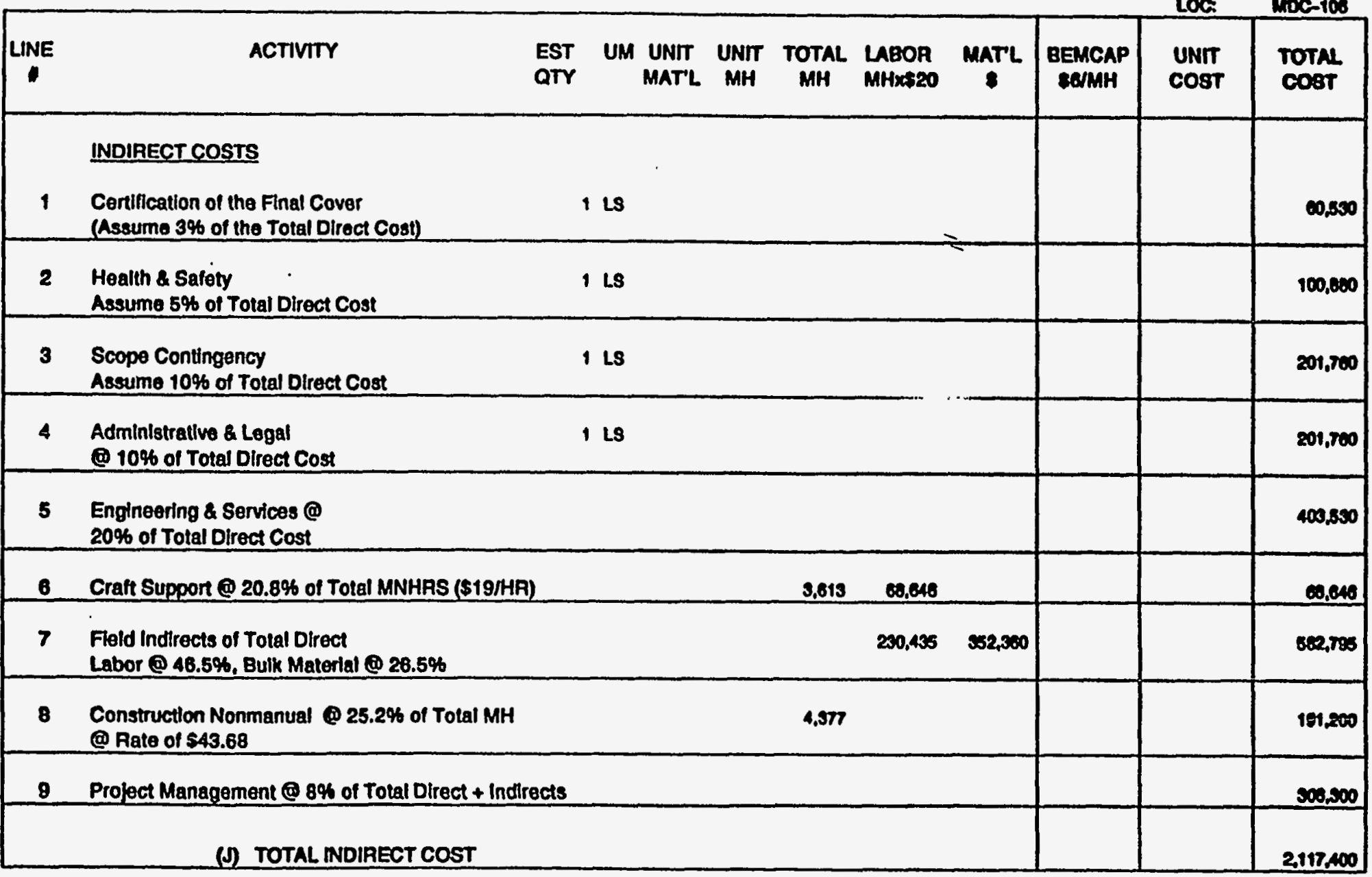

F-8. Cost Estimate a for Cover System Utilizing Compacted Onsite Sandy Clay and a 30-mil FML (Cont'd) 
DETAIL B S T I A T B

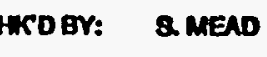

Lo0 I: 7-03

ACTIVTTY CODE: ESE260412

PROJ.NAME: ECON. EVALUATION OP CLOSURB CAP BARRIER MATERIAL

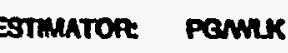

PHONE T-5260

DATE DOMISS

LOG. MOC-108

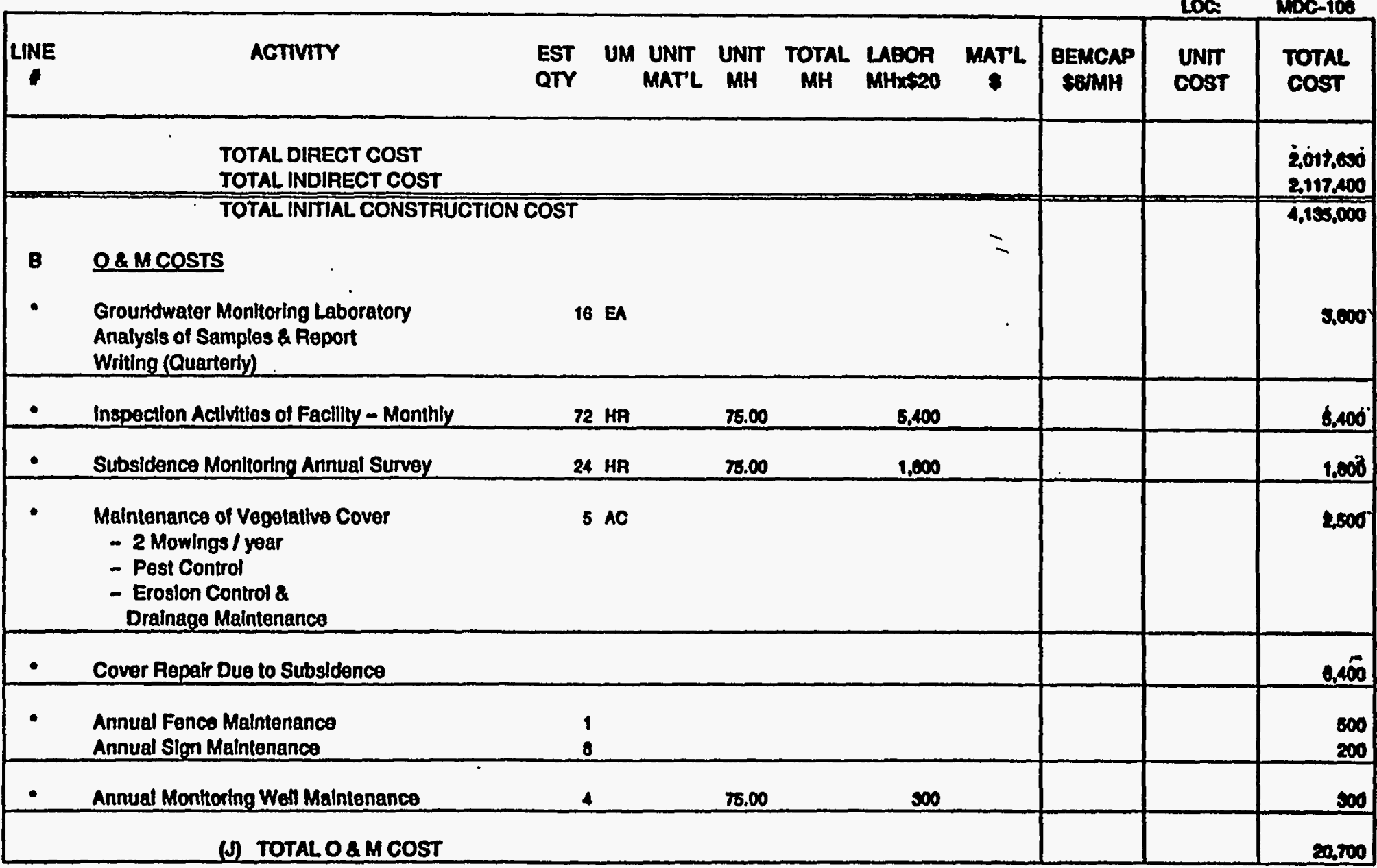

F-8. Cost Estimate a for Cover System Utilizing Compacted Onsite Sandy Clay and a 30-mil FML (Cont'd) 


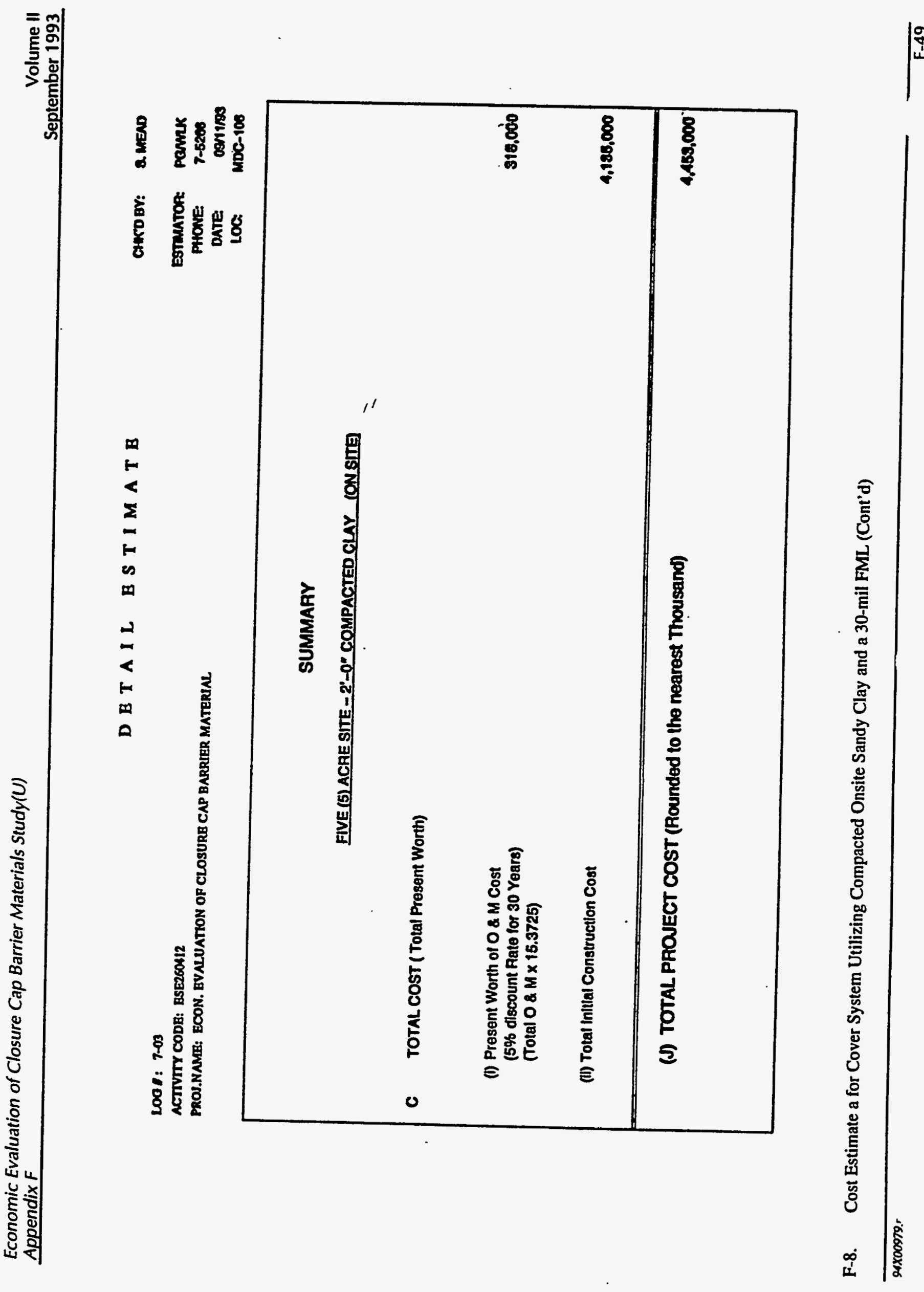


D E T A I L E S T I M A T

CHKD BY: S.MEAD

Lo0 1: $7-03$

ACTIVITY CODE: ESE260412

PROS.NAME: ECON. EVALUATION OF CLOSURE CAP BARRIER MATERIAL

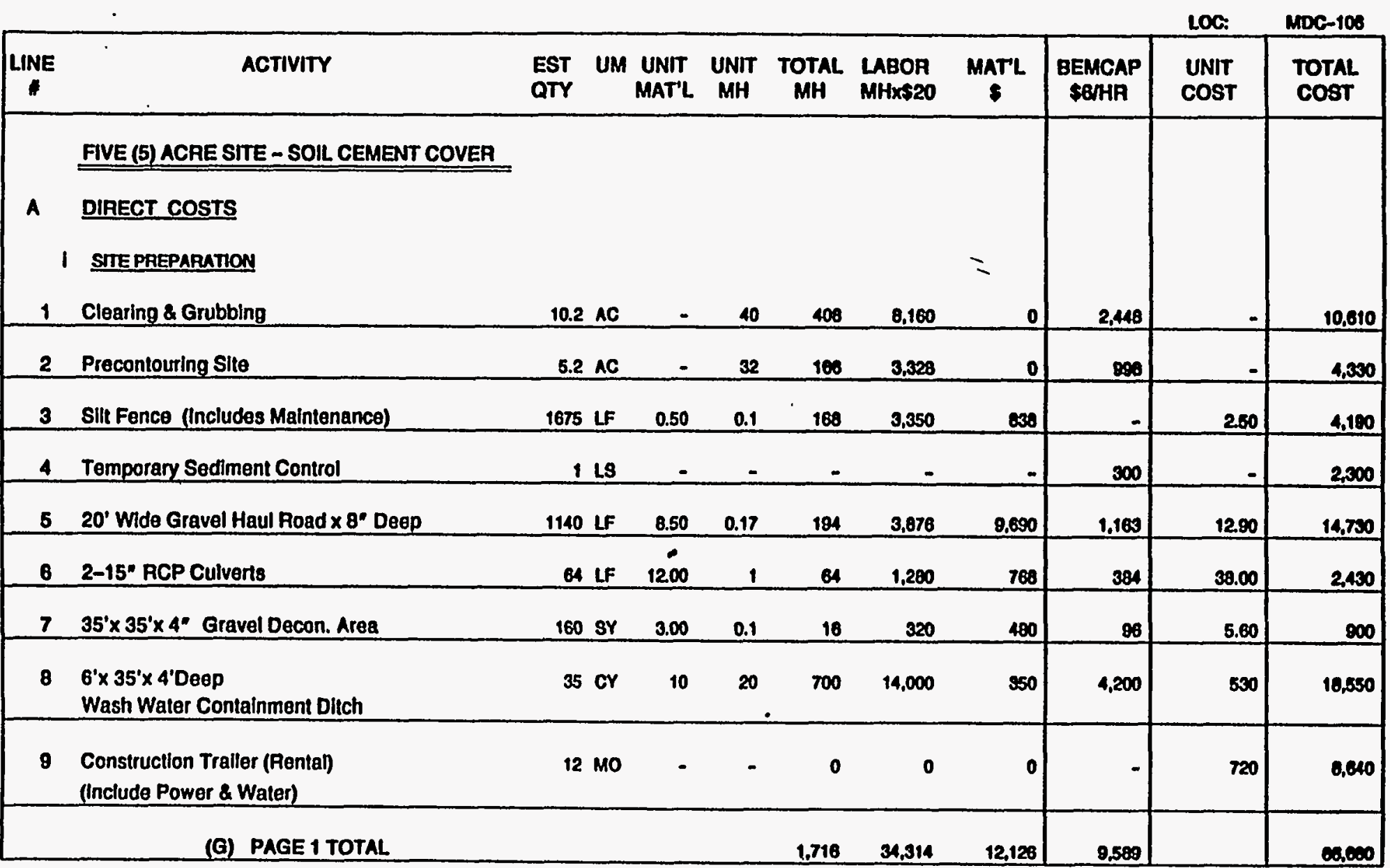

F-9. Cost Estimate for a Cover System Utilizing Soil Cement and 30-mil FML 
DETAIL ESTIMAT B

CHKD BY: 8. MEND

LoOI: 7-03

ACTIVTTY CODE: ESE2GON12

PROJ.NAME: ECON. EVALUATION OF CLOSURE CAP BARRIER MATERIAL

\begin{tabular}{|c|c|c|c|c|c|c|c|c|c|c|c|}
\hline & & & & & & & & & & LOC: & MDC-100. \\
\hline LINE & ACTIVIT & $\begin{array}{l}\text { EST } \\
\text { QTY }\end{array}$ & UM & $\begin{array}{l}\text { UNIT } \\
\text { MATL }\end{array}$ & $\underset{\text { UH }}{\text { UNIT }}$ & $\begin{array}{l}\text { TOTAL } \\
\text { MH }\end{array}$ & $\begin{array}{l}\text { LABOR } \\
\text { MHXSE2O }\end{array}$ & $\underset{\$}{\operatorname{MaTL}}$ & $\begin{array}{l}\text { BEMGAP } \\
\text { SQ/HR }\end{array}$ & $\begin{array}{l}\text { UNIT } \\
\text { COST }\end{array}$ & $\begin{array}{l}\text { Total } \\
\text { cost }\end{array}$ \\
\hline & I COVER CONSTRUCTION & & & & & & & & & & \\
\hline 10 & Waste Stabllization (Crushed Limestone) & 10,070 & $T N$ & 100 & 0.1 & 1007 & 20,140 & $1,007,000$ & 0,042 & 102.60 & $1,030,180$ \\
\hline 11 & $\begin{array}{l}\text { Place and Compact Foundation Soll } \\
\text { in } 8^{\prime \prime} \text { Lilts } 1095 \% \text { Sid. Proctor }\end{array}$ & 40,350 & or & - & 0.155 & 6254 & 125,085 & 0 & $\mathbf{9 7 , 5 2 0}$ & 4.00 & 162.610 \\
\hline 12 & $\begin{array}{l}\text { Soll-Cement mixture transported from central } \\
\text { mixing plant by end-dump trucks and placed } \\
\text { In 6-8" Iffts by spreaders. Assume Fine Sand } \\
\text { Soll with } 3211 \text { of cement/CY. Compact the soll- } \\
\text { cement unlformlly to } 98 \% \text { max. density. }\end{array}$ & 25,715 & or & 18 & 0.165 & 4243 & 84,860 & 462,670 & 25,458 & 22.30 & 575,190 \\
\hline 13 & $\begin{array}{l}\text { Curing soil-cement. Assume liquid Asphalt } \\
\text { applied } 0.4 \text { gallsy for } 1 \text { St coal } \& 0.2 \text { for } 2 \text { nd. }\end{array}$ & $\begin{array}{l}27.455 \\
27.455\end{array}$ & $\begin{array}{l}\text { SY } \\
\text { SY }\end{array}$ & $\begin{array}{l}0.33 \\
0.17 \\
\end{array}$ & $\begin{array}{l}0.05 \\
0.03\end{array}$ & $\begin{array}{r}1373 \\
824 \\
\end{array}$ & $\begin{array}{l}27,455 \\
16,479\end{array}$ & $\begin{array}{l}9,060 \\
4,687\end{array}$ & - & $\begin{array}{l}1.30 \\
0.60\end{array}$ & $\begin{array}{l}36,520 \\
21.140\end{array}$ \\
\hline 14 & $\begin{array}{l}\text { Placement of } 30 \text { Mil Flexible } \\
\text { Membrane Liner }\end{array}$ & 261,000 & $\mathbf{S F}$ & 0.97 & 0.0075 & 1858 & $\mathbf{3 9 , 1 5 0}$ & 4,370 & - & 0.30 & 00,620 \\
\hline 15 & $\begin{array}{l}\text { Placement of 1'-0 Coarse Sand } \\
\text { Drainäge Layer }\end{array}$ & 9,035 & or & 10.50 & 0.03 & 271 & 8,421 & 92,668 & 1.626 & 11.30 & 101,910 \\
\hline 16 & $\begin{array}{l}\text { Placement of Geolextile (TYPAR) Filter on } \\
Y^{\prime}-0^{*} \text { Coarse Sand Drainage Layer }\end{array}$ & 27,845 & sq & 0.17 & 0.005 . & 139 & 2,765 & 4,734 & - & 0.50 & 1,820 \\
\hline 17 & Placement of 1'-6" Common Fin & 12,120 & or & 2.75 & 0.06 & 727 & 14,544 & 45,450 & 4,363 & 5.30 & 60,000 \\
\hline 18 & Placement of 6" Topsoll & 4,545 & or & 9.50 & 0.06 & 273 & 5.454 & 43,170 & 1.636 & 11.10 & 50,270 \\
\hline & (G) PAGE 2 TOTAL & & & & & 17.000 & $\mathbf{5 4 1 , 3 6 0}$ & $1,716,106$ & 78,651 & & $2,134,200$ \\
\hline
\end{tabular}

F-9. Cost Estimate for a Cover System Utilizing Soil Cement and 30-mil FML (Cont'd) 
DETAIL B S T MAT B

Loof: $7-03$

ACTIVITY CODE: ESE260412

PROJ.NAME: ECON. EVALUATION OF CLOSURE CAP BARRJER MATERIAL

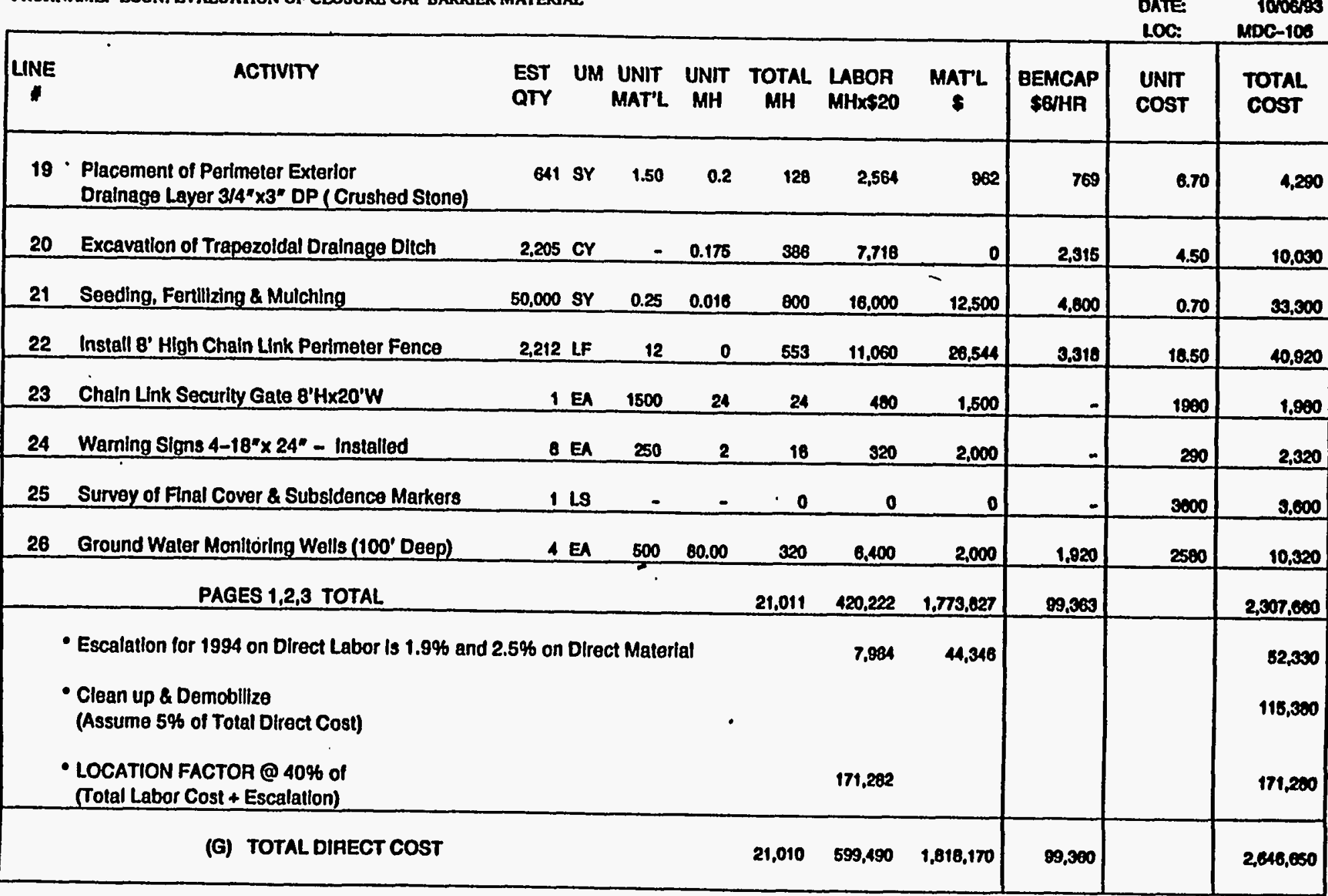

F-9. Cost Estimate for a Cover System Utilizing Soil Cement and 30-mil FML (Cont'd) 


$$
\text { Lod : 7-03 }
$$

ACTIVITY CODE: ESE260412 .

PROJ.NAME: ECON. BUALUATION OF CLOSURE CAP BARRIER MATERIAL.

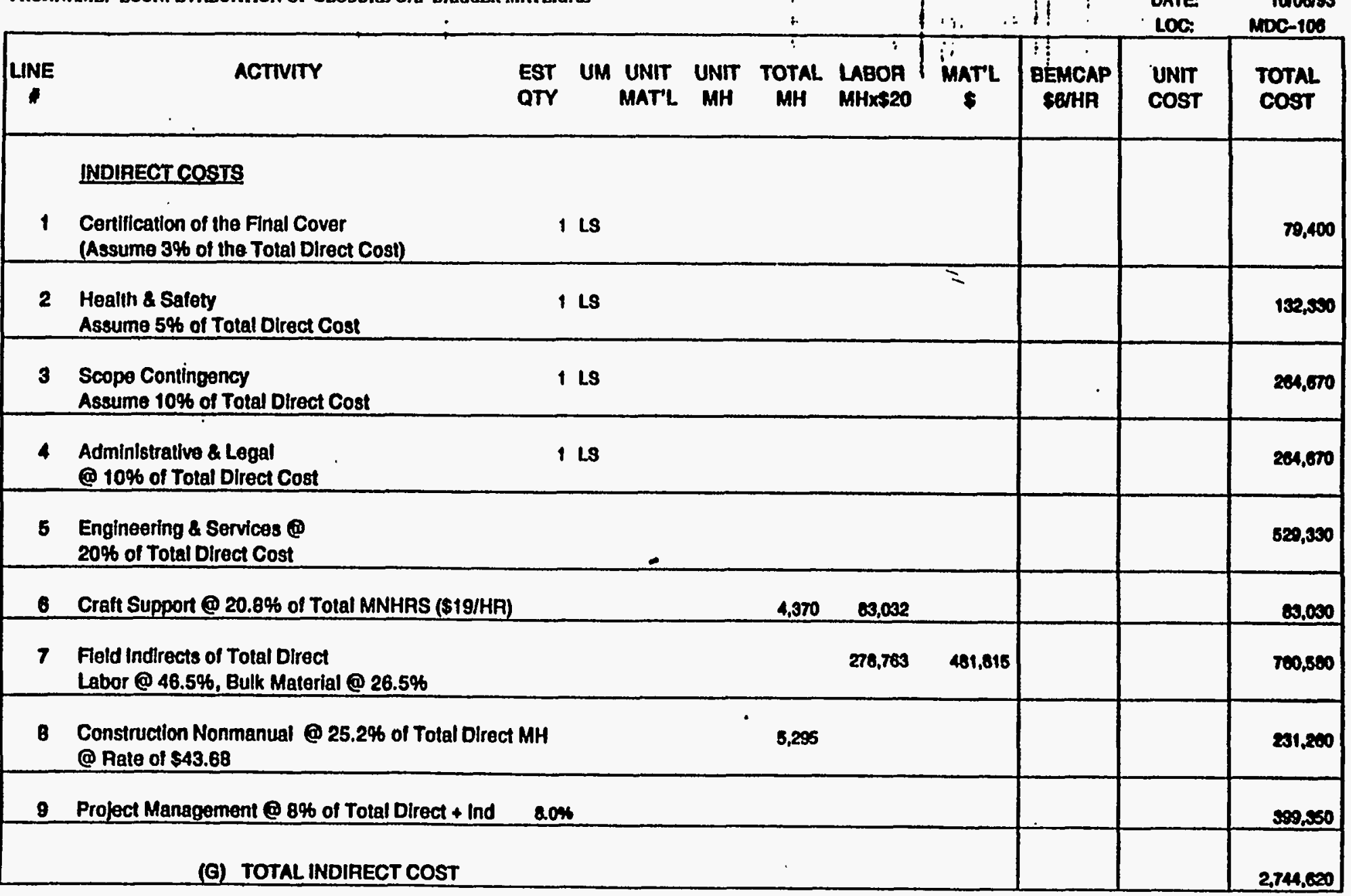

F-9. Cost Estimate for a Cover System Utilizing Soil Cement and 30-mil FML (Cont'd) 
D B T A I L E S T I M A T E

CHKDQYY: S.MEAD

LOO A : 7-03

ACTIVITY CODE: ESE260112

PROJ.NAME: ECON. EVALUATION OF CLOSURB CAP BARRIER MATERAAL

ESTMATOR: PGAMK

PHONE: 7-5260

DATE 1006/25

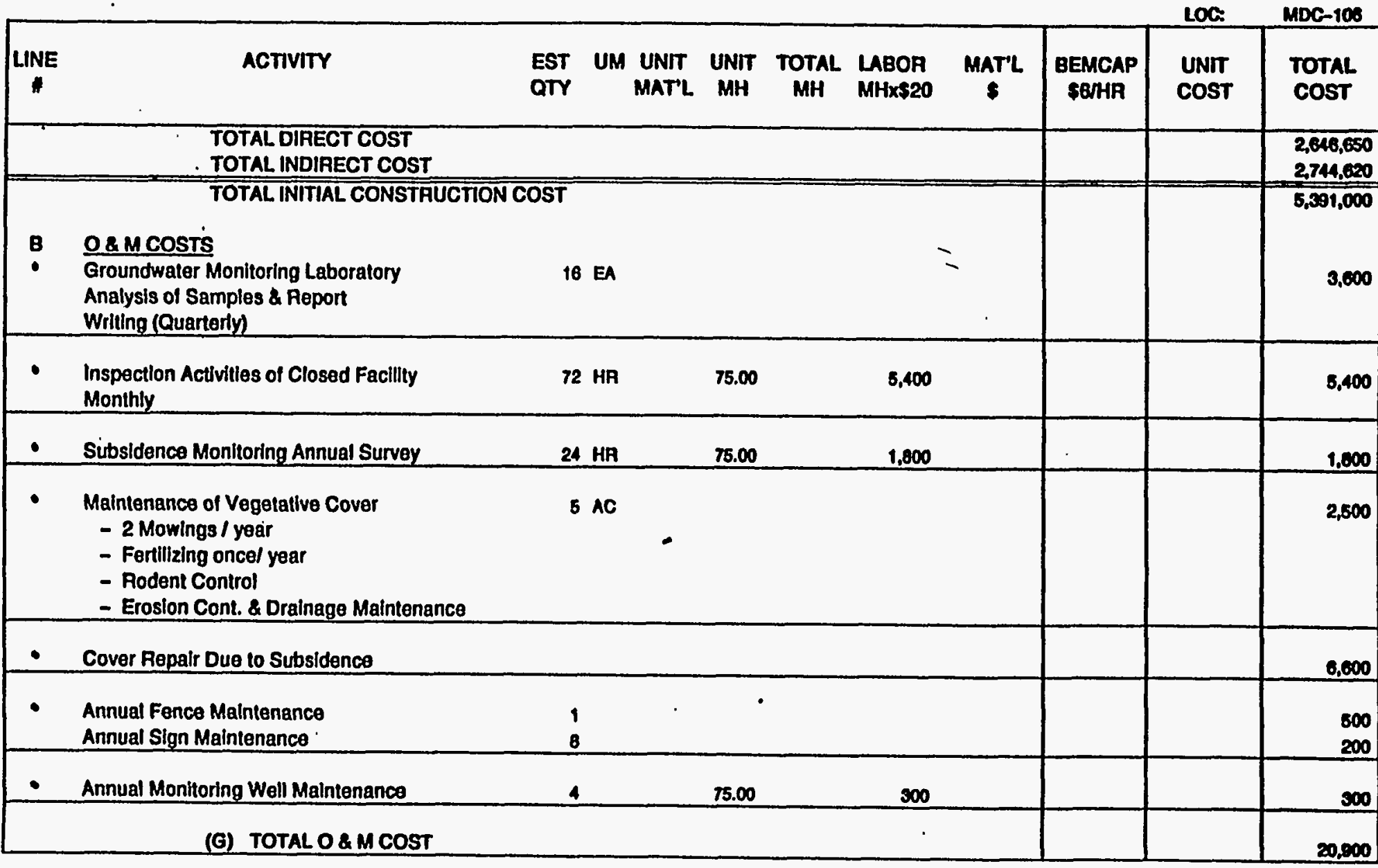

F-9. Cost Estimate for a Cover System Utilizing Soil Cement and 30-mil FML (Cont'd) 


\section{CAKD BY: 8.MEAD \\ Estmatort PaMk \\ PHONE: T-6260 \\ DATE TONers \\ LOC: MDC-100}

\section{SUMMAFY}

EIVE (5) ACAE SITE - SOIL CEMENT COVER

C TOTAL COST (Total Present Worth)

(i Present Worth of 0 \& $M$ Cost

(5\% ofscount Rate for 30 Years)

(Total O \& M X 15.3725)

$-$

321,000

(ii Total Initial Construction Cost

$8,391,000$

(G) TOTAL PROJECT COST (Rounded to the nearest Thousand)

$5,712,000$

F-9. Cost Estimate for a Cover System Utilizing Soil Cement and 30-mil FML (Cont'd) 
DETAIL ESTIMATE

CHEBV: Q MEAD

200 1: 7-03

ACTIVTTY CODE: ESE2GOA12

PROJ.MAME: ECON. EYALUATKON OF CLOSURB CAP BARRER MATERIAL

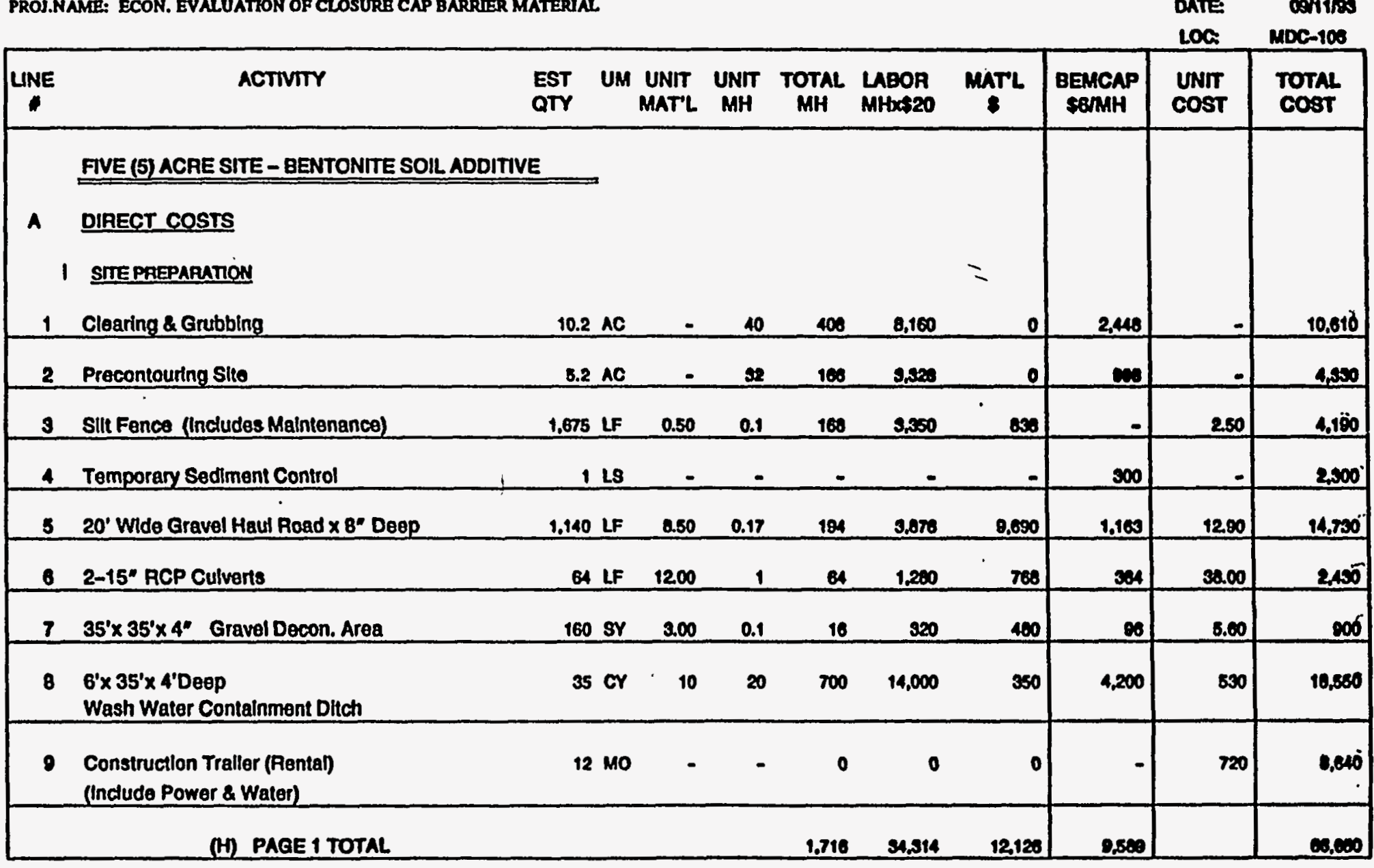

F-10. Cost Estimate for a Cover System Utilizing Bentonite Soil Additive and a 30-mil FML 
DETAIL ESTIMATE

Loo: $7-\infty$

ACTIVTYY CODE: ESE260112

PROJ.NAME: ECON. EVALUATION OF CLOSURE CAP BARRIER MATERIAL

\begin{tabular}{|c|c|c|c|c|c|c|c|c|c|c|c|}
\hline UNE & ACTIVITY & $\begin{array}{l}\text { EST } \\
\text { aTr }\end{array}$ & UM & $\underset{\text { MAT'L }}{\text { UNT }}$ & $\begin{array}{l}\text { UNIT } \\
\text { MH }\end{array}$ & TOTAL & $\begin{array}{l}\text { LABOR } \\
\text { MHXA20 }\end{array}$ & $\underset{\&}{\operatorname{MaTL}}$ & $\underset{\text { SEMCAP }}{\text { BEMCAP }}$ & $\begin{array}{l}\text { UNIT } \\
\text { cost }\end{array}$ & $\begin{array}{l}\text { Total } \\
\text { cost }\end{array}$ \\
\hline 10 & $\begin{array}{l}\text { in COVER CONSTRUCTION } \\
\text { Waste Stabillzatlon (Crushed Limestone) }\end{array}$ & 10,070 & $T N$ & 100 & 0.1 & 1007 & 20,140 & $1,007,000$ & 0,042 & 102.00 & $1,033,180$ \\
\hline 11 & $\begin{array}{l}\text { Place and Compact Foundation Soll } \\
\text { In } 8^{*} \text { Lifis to } 95 \% \text { Sid. Proctor }\end{array}$ & 10,350 & CY & - & 0.155 & 6254 & 125,005 & $=$ & 57,020 & 4.00 & 102,610 \\
\hline 12 & $\begin{array}{l}\text { Bentonite Soll Admix Sodium Bentonite will } \\
\text { be added to a Silty Sand at an application } \\
\text { rate of } 2 \text { PSF per } 6 \text { inch litt. Two lifts } \\
\text { required for a total bartier thlckness of } \\
12^{\prime \prime} \text { compact, each lift to } 95 \% \text { std. } \\
\text { Proctor \& Scarliy betwoen lifts. }\end{array}$ & Si.3a & cr & 146.50 & 0.165 & $19 \pi$ & 11,450 & $\begin{array}{r}30,549 \\
125,180\end{array}$ & 8,203 & 180.50 & 100,090 \\
\hline 13 & $\begin{array}{l}\text { Placement of } 30 \text { Mil Flexible } \\
\text { Membrane Liner }\end{array}$ & 261,000 & SF & 0.17 & 0.0075 & 1958 & 59,150 & 44,570 & - & 0.50 & 00,820 \\
\hline 14 & $\begin{array}{l}\text { Placement of 1'-0 Coarse Sand } \\
\text { Dralnage Layer }\end{array}$ & 9,035 & CY & 10.50 & 0.03 & 271 & 8,421 & 84,000 & 1,020 & 11.30 & 101,910 \\
\hline 15 & $\begin{array}{l}\text { Placement of Geotextile (TYPAR) Filter on } \\
1^{\prime}-0^{*} \text { Coarse Sand Drainage Layer }\end{array}$ & 27,845 & sr & 0.17 & 0.005 & 139 & 2,785 & 4,754 & - & 0.30 & 7340 \\
\hline 16 & Piacement of $1^{\prime}-6^{\circ}$ Common Fill & 12,120 & Cr & $\mathbf{3 . 7 5}$ & 0.06 & 727 & 14,644 & 45,450 & 4,300 & 8.30 & CAs.0 \\
\hline 17 & Placement of 6- Topsoll & 4,545 & Cr & 9.50 & 0.00 & 273 & E,454 & 48,170 & 1,000 & 11.10 & 30220 \\
\hline & (H) PAGE2 TOTAL & & & & & 12550 & 231,070 & $1,530,007$ & 60,450 & & 1,700500 \\
\hline
\end{tabular}

F-10. Cost Estimate for a Cover System Utilizing Bentonite Soil Additive and a 30-mil FML (Cont'd) 
DETAIL ESTIMATE

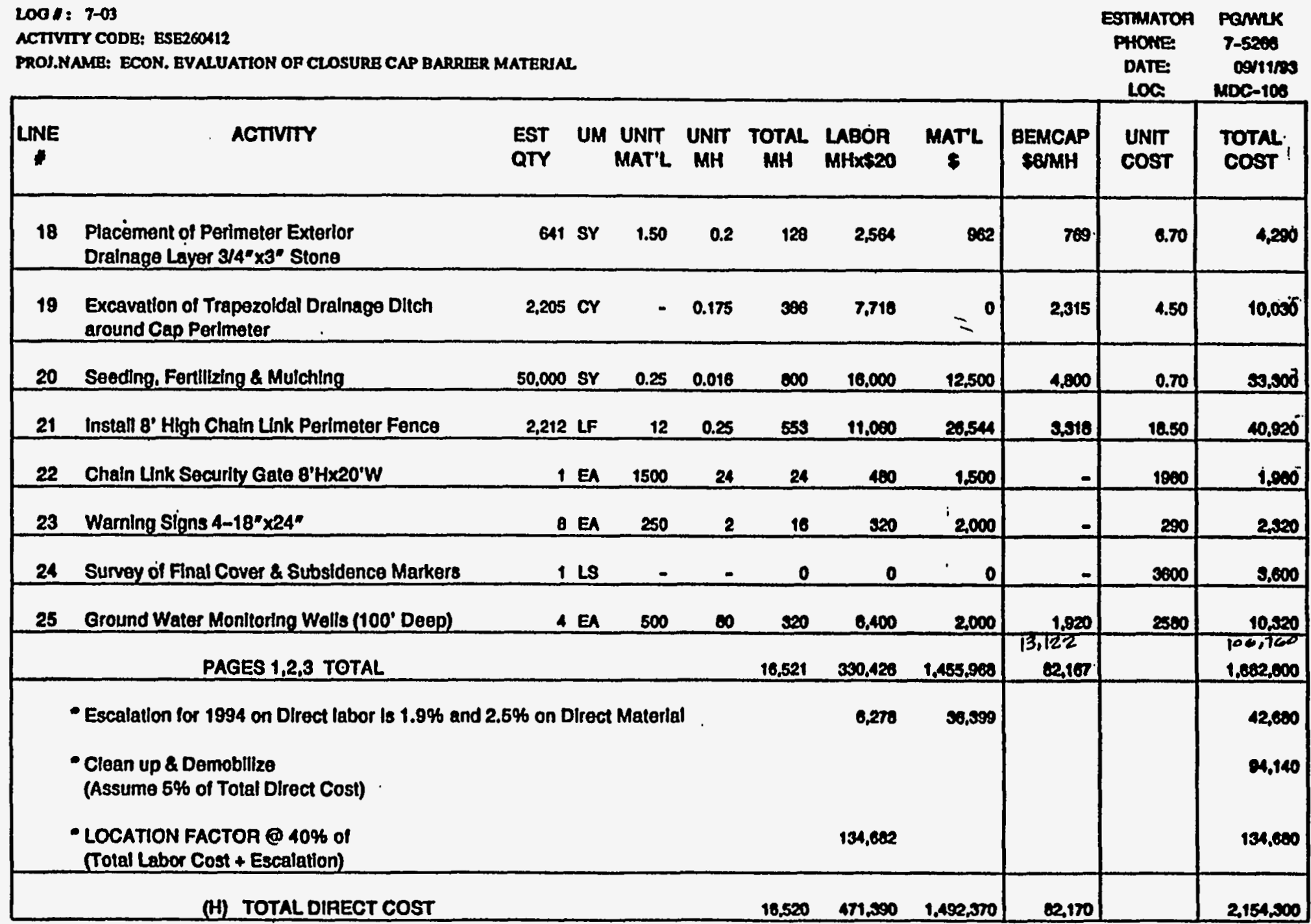

F-10. Cost Estimate for a Cover System Utilizing Bentonite Soil Additive and a 30-mil FML (Cont'd) 


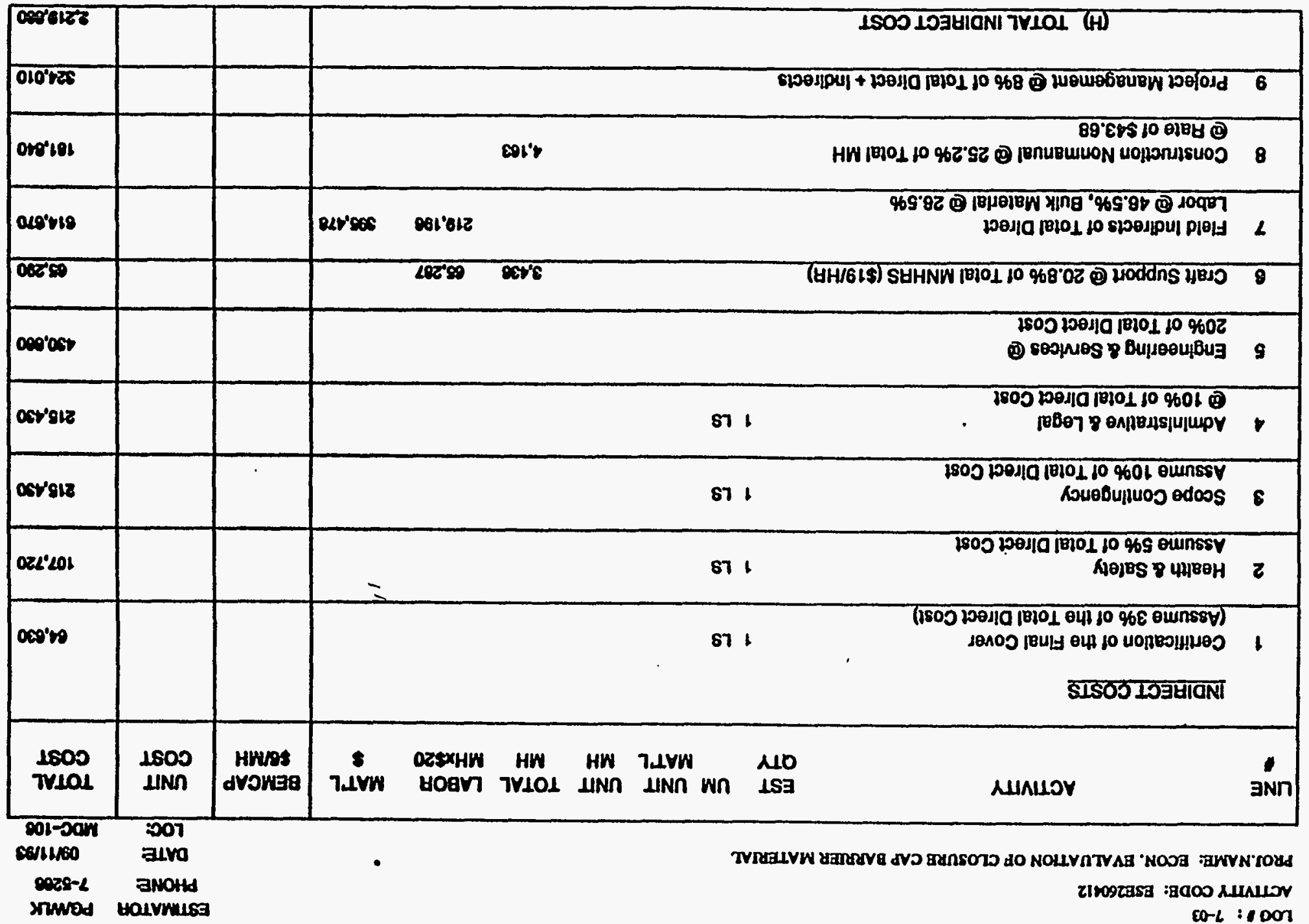

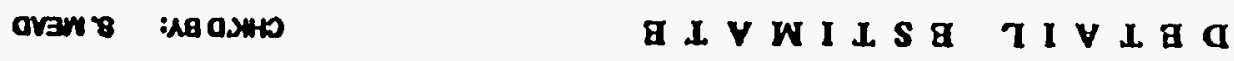


DETAILASTIMATE

CHKD BY: S.MEAD

Loof: $7-03$

ACTIVTTY CODE: ESE26012

PROJ.NAME: ECON. EVALUATION OF CLOSURB CAP BARRIER MATERIAL

estmator pamix

PHONE T-5268

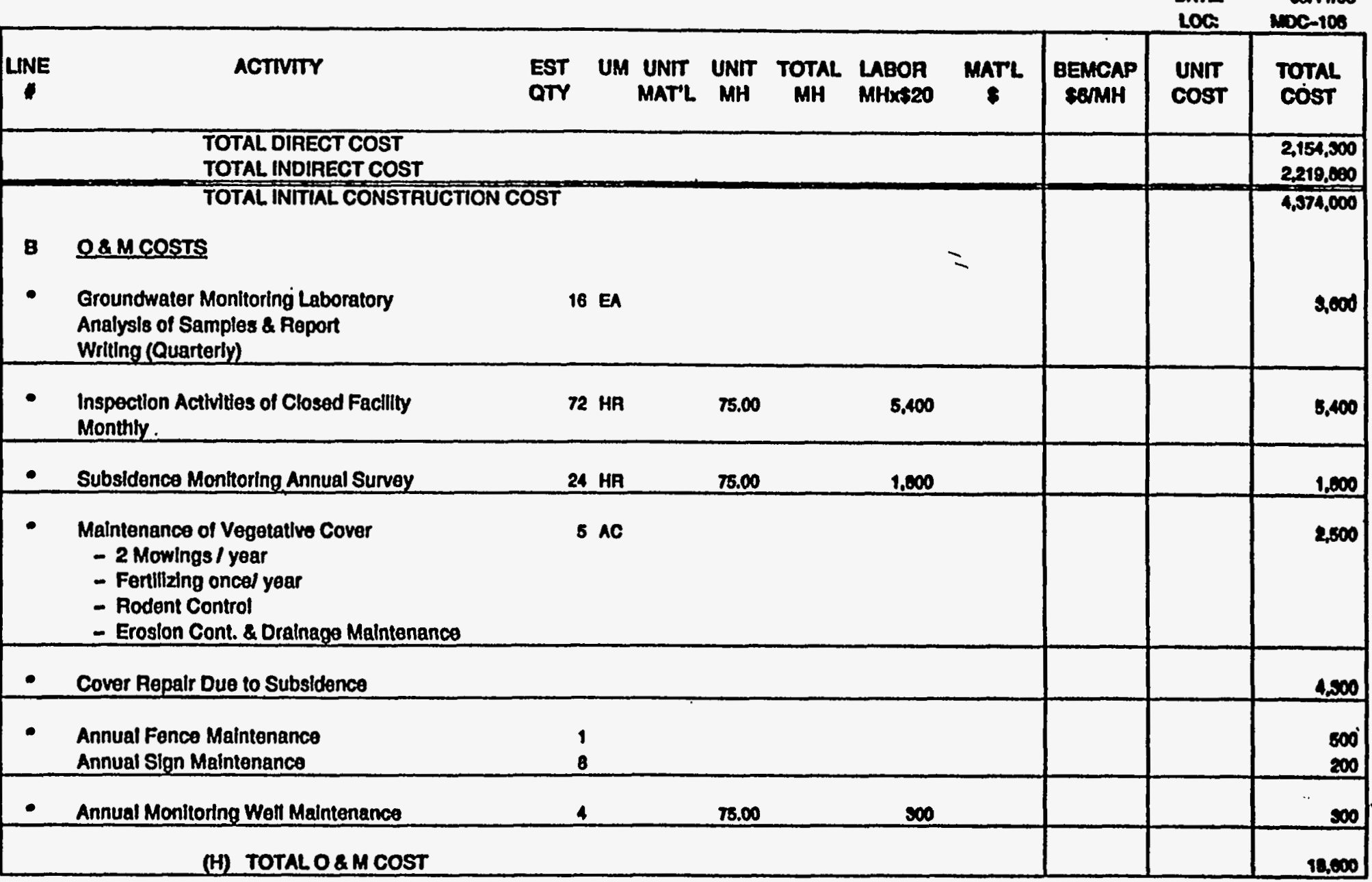

F-10. Cost Estimate for a Cover System Utilizing Bentonite Soil Additive and a 30-mil FML (Cont'd) 
D E T A I L B S TIMATB

\begin{tabular}{|c|c|}
\hline CAKCD BY: & 2. MEAD \\
\hline $\begin{array}{l}\text { EOMMATOA } \\
\text { PHONE } \\
\text { DATE: } \\
\text { LOC: }\end{array}$ & 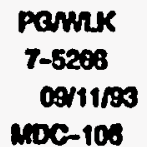 \\
\hline
\end{tabular}

Lo0 : : 7-03

NCTIVITY CODE: BSE260412

PROS.NAME: ECON. EVALUATION OP CLOSURE CAP BARPIER MATERINS.

LOC: MOC-100

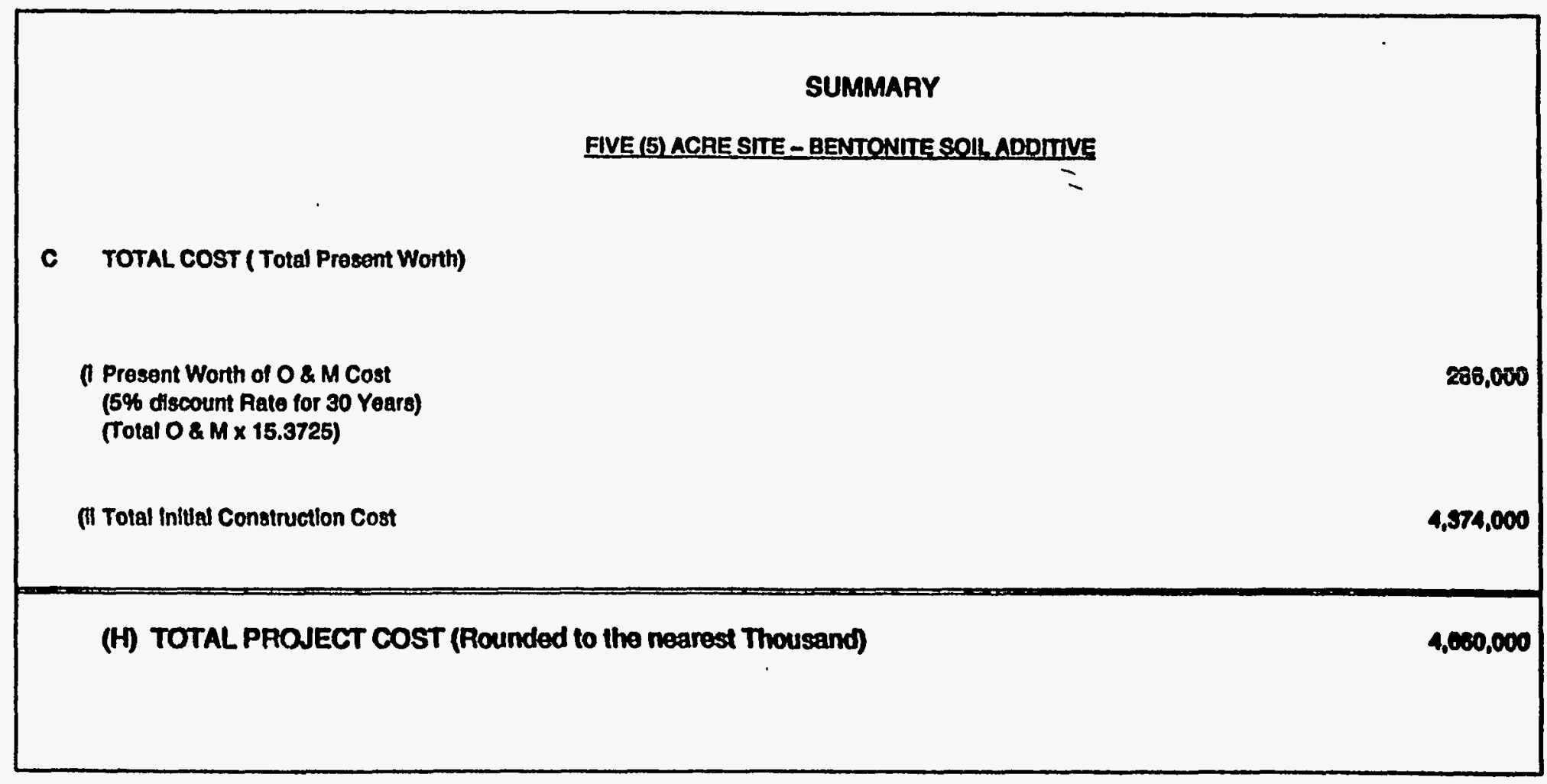

F-10. Cost Estimate for a Cover System Utilizing Bentonite Soil Additive and a 30-mil FML (Cont'd) 
DETAIL ESTIMATE CHCOEY QMEAD

$2001: 7-03$

ACTIVITY CODE: ESEZGOL12

PROJ.NAME: ECON. BVALUATION OP CLOSURB CAP BARRIER MATERIAL

Estmator panmK

PHONE T-5200

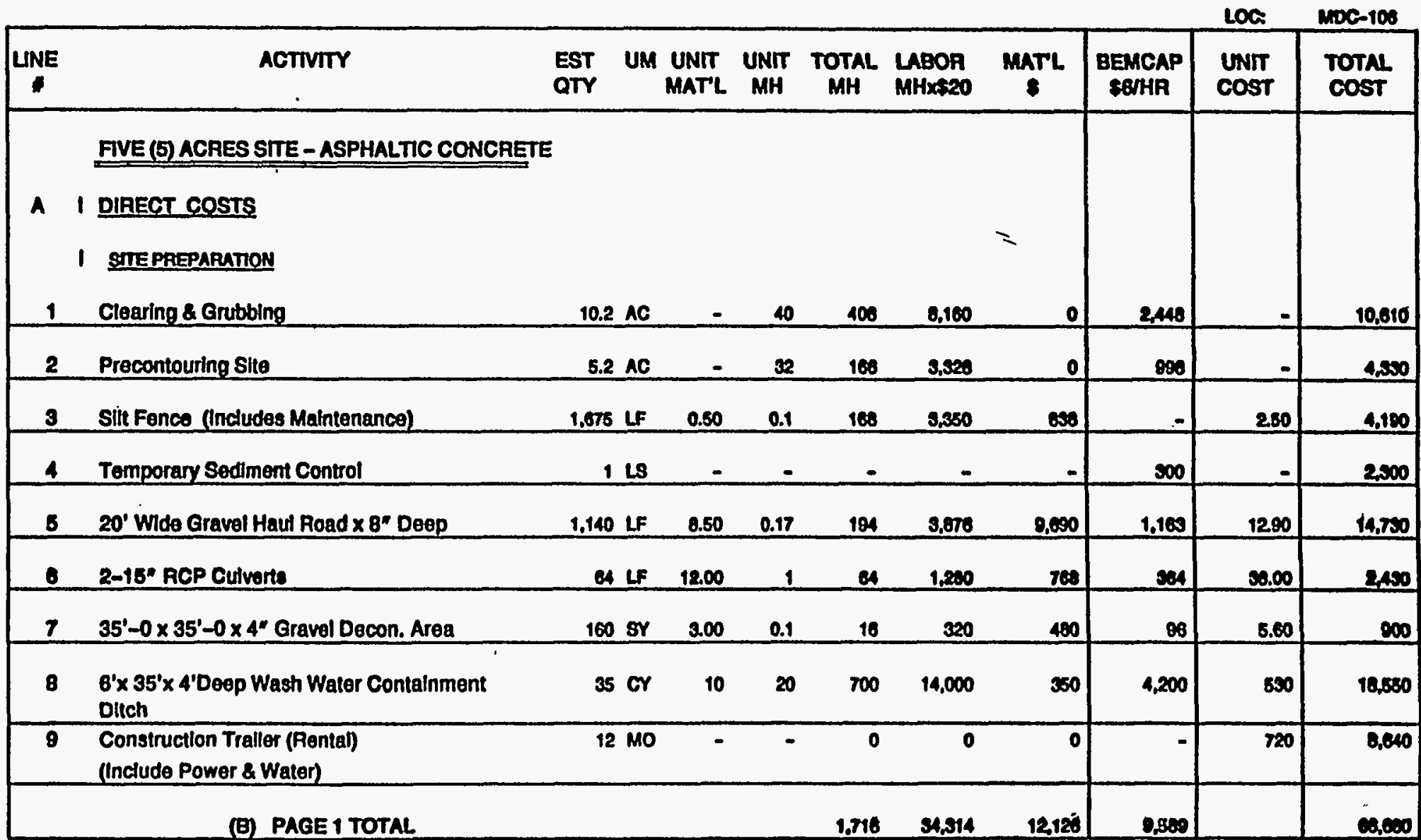

F-11. Cost Estimate for a Cover System Asphalt Concrete Hydraulic Barrier and a 30-mil FML 
DETAILESTIMATE

CHKOBV: Q MEAD

Lod: $7-03$

ACTIVITY CODE: BSE260412

MOU.NAMB: BCON. BVALUATHON OF CLOSURB CAP BARRIRR MATBUIAL,

Estmator pankK

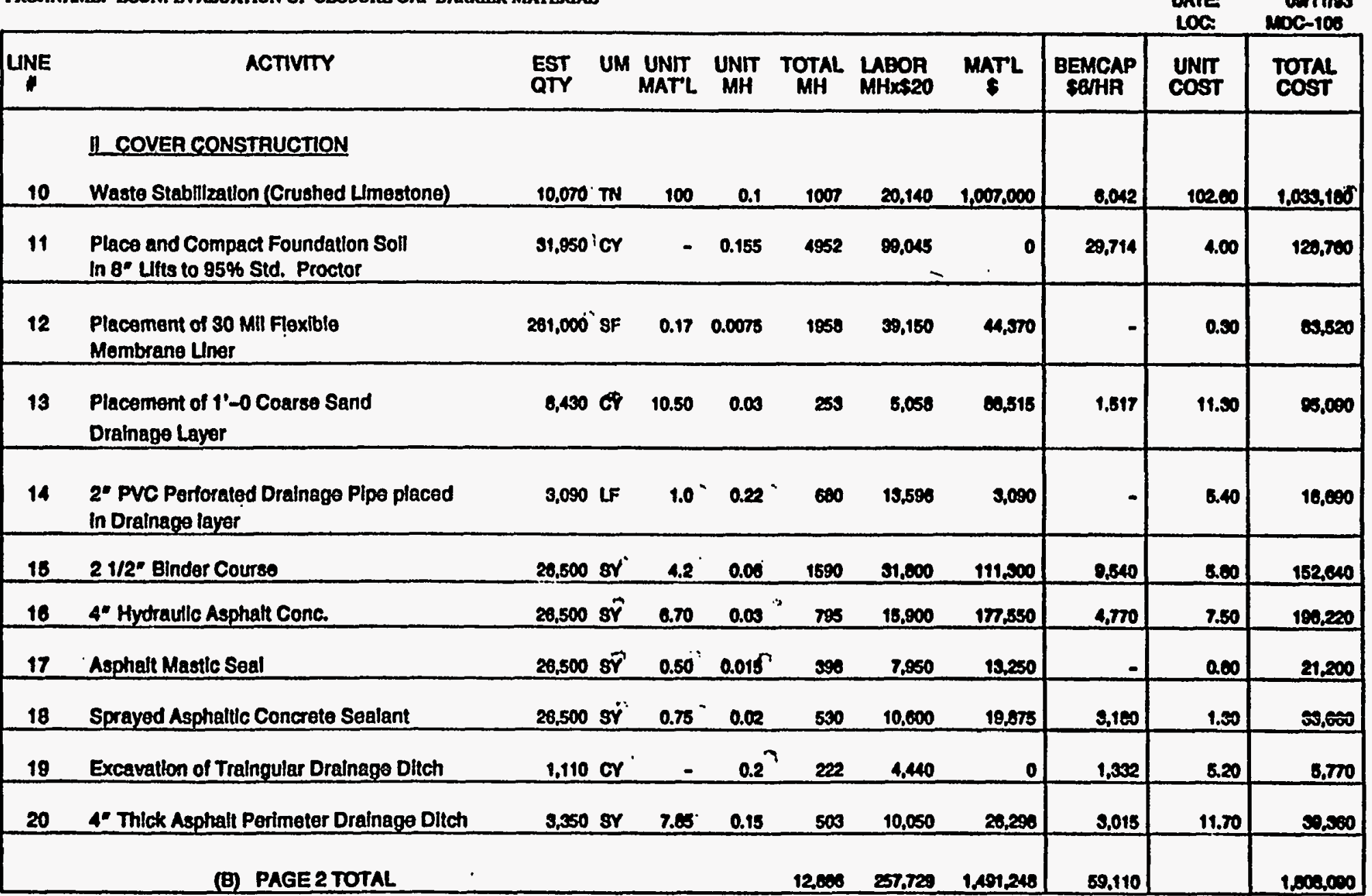

F-11. Cost Estimate for a Cover System Asphalt Concrete Hydraulic Barrier and a 30-mil FML (Cont'd) 
D B TAIL B S T I MATE

Lod: 7-03

ACTIVITY CODE: ESE2SOH12

MROI.NAMER ECON. EVALUATION OR CLOSURB CAP BARRUER MATERUL

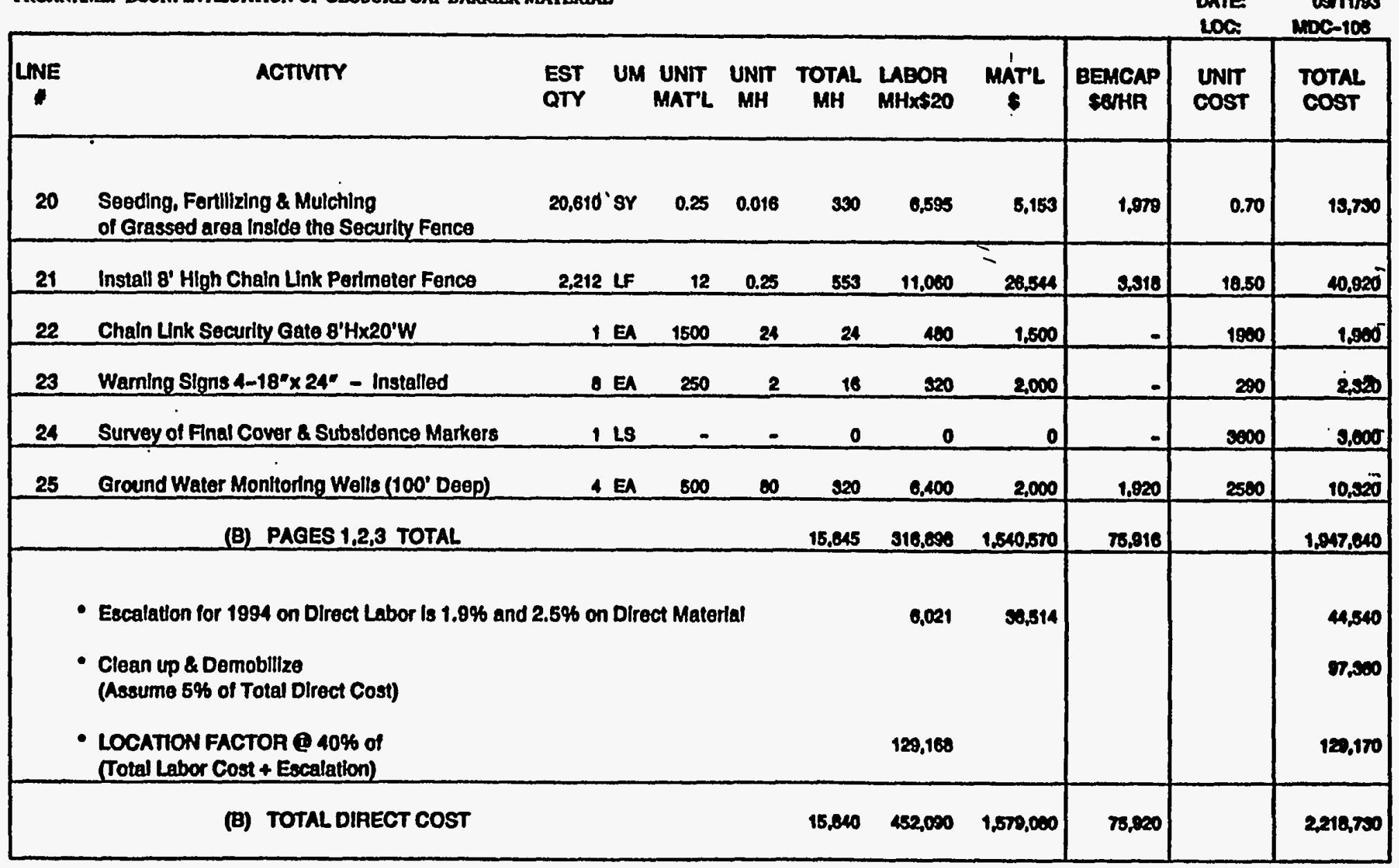

F-11. Cost Estimate for a Cover System Asphalt Concrete Hydraulic Barrier and a 30-mil FML (Cont'd) 
$1001: 7-03$

ACTIVITY CODB: ESE2CON12

MROJ.NAME: BCON. EVALUATTON OP CLOSURB CAP BARRIER MATERIAL

ESTMATOA PankK

PHONE: $7-5200$

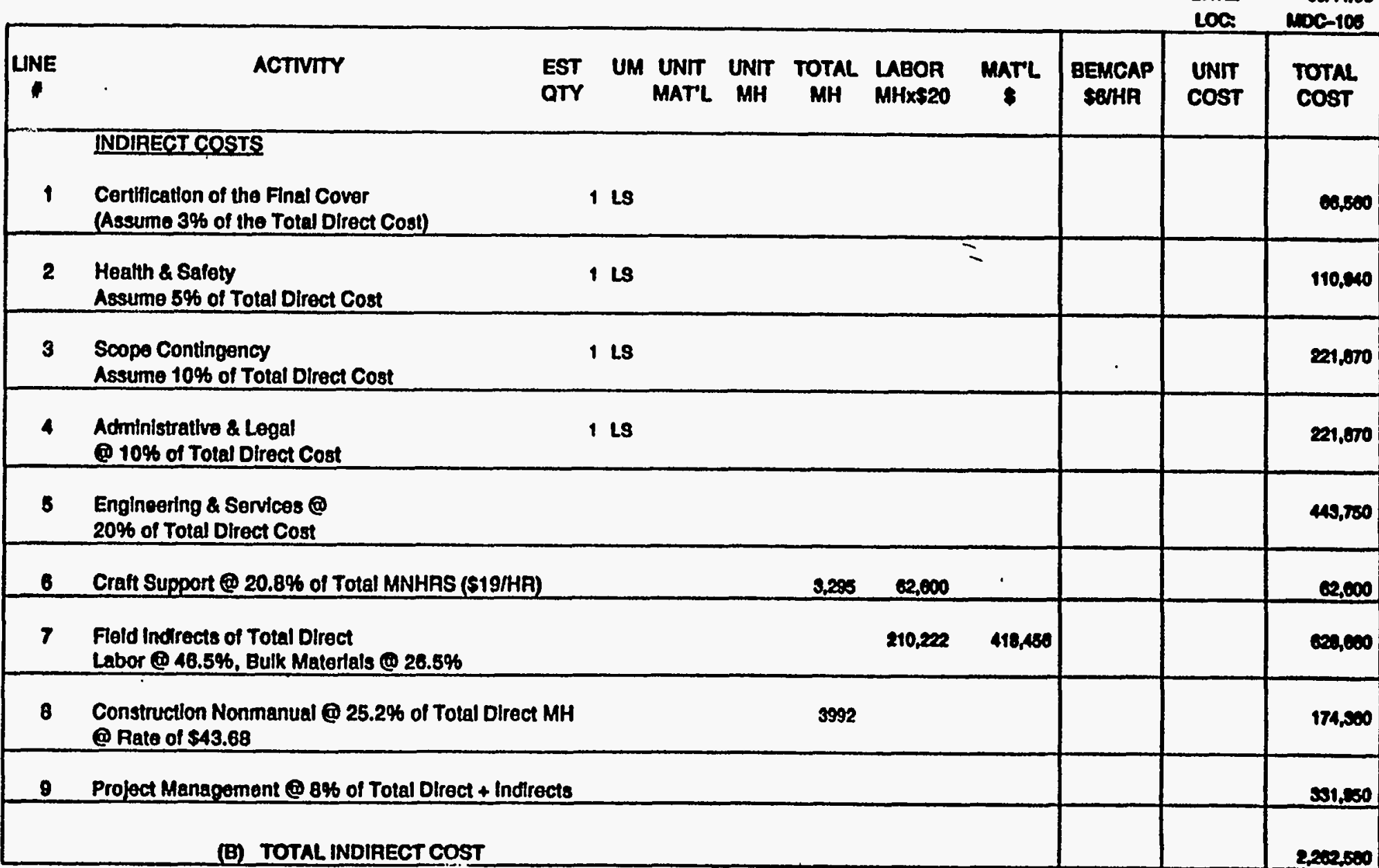

F-11. Cost Estimate for a Cover System Asphalt Concrete Hydraulic Barrier and a 30-mil FML (Cont'd) 
DETAIL BSTIMAT B

\section{8: 7-03}

ACTIVITY CODE: ESE260412

PROI.NAMB: ECON. EVALUATION OP CLOSURB CAP BARRTER MATERIAL

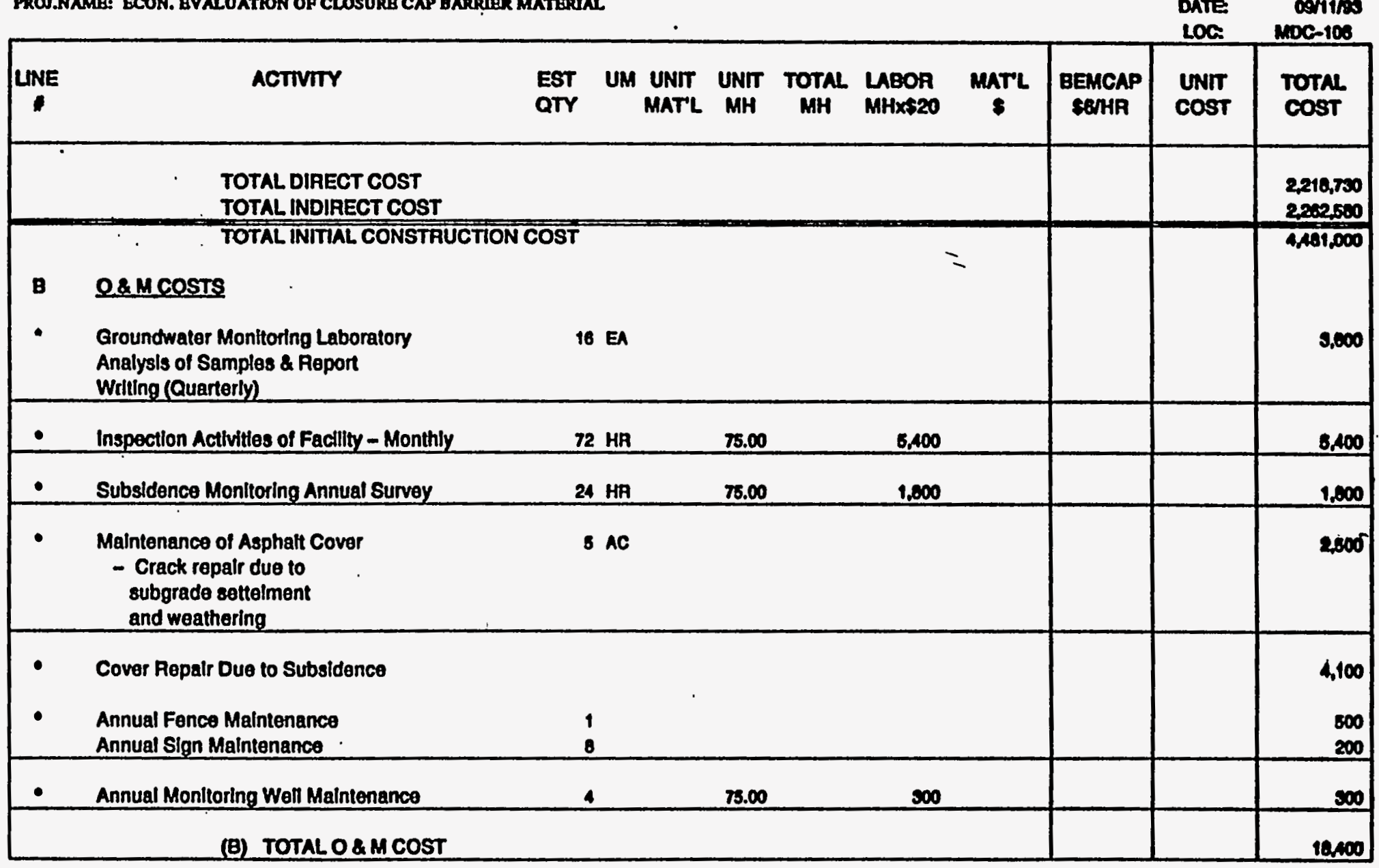

F-11. Cost Estimate for a Cover System Asphalt Concrete Hydraulic Barrier and a 30-mil FML (Cont'd) 
D B T A I L B S T IMATB

\section{Lool: $7-03$}

ACTIVIT CODE: BSE260112

MOJ.NAME: ECON. BVALUATION OP CLOSURE CAP BARRTER MATERIAL
CHKD BY: Q.MEND

Estmutor pamk

PHONE:

DATE

LOC:
$7-6200$

con1ms usc-10s

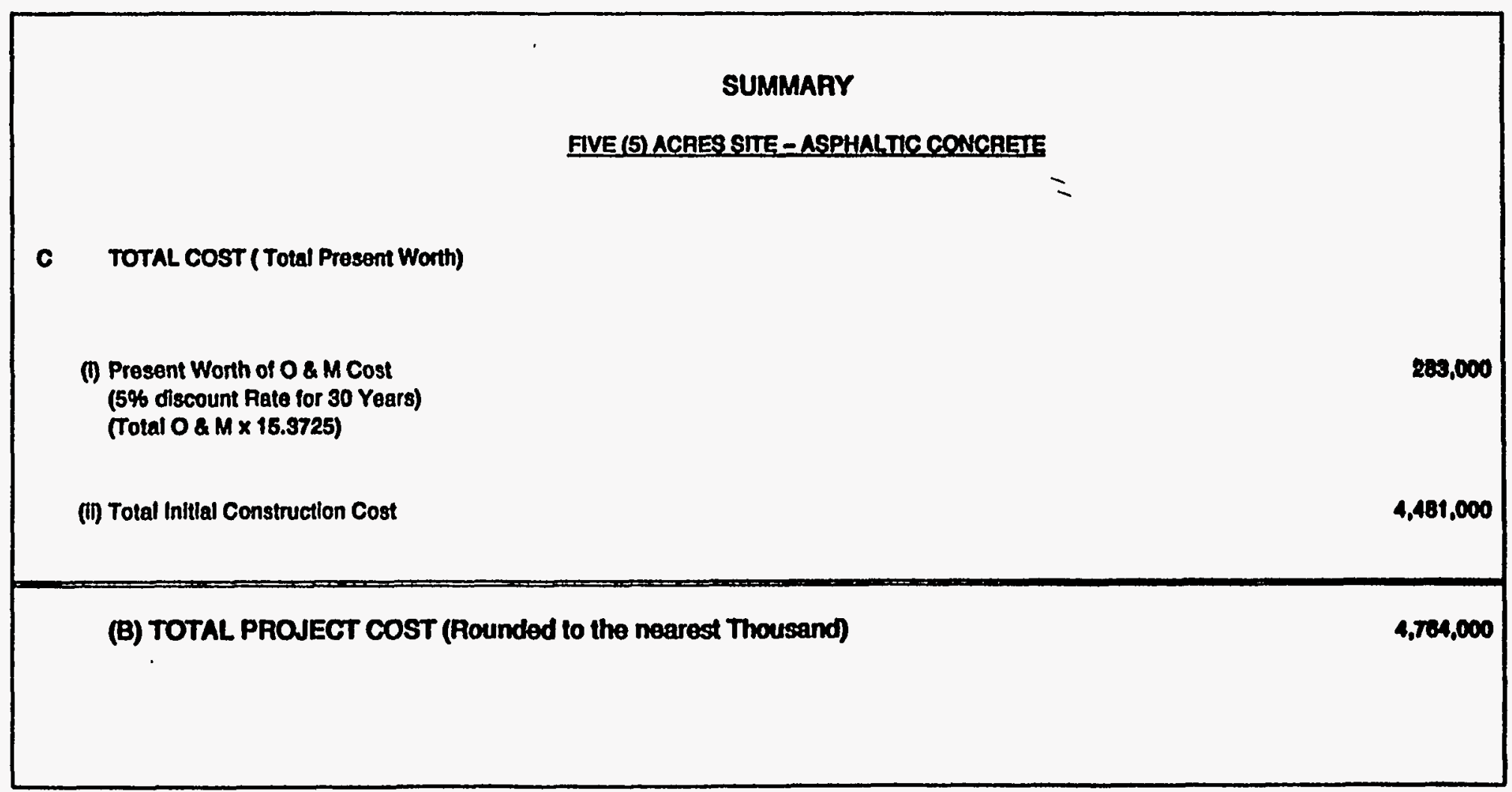

F-11. Cost Estimate for a Cover System Asphalt Concrete Hydraulic Barrier and a 30-mil FML (Cont'd) 
DETAIL ESTIMATE

Lo0 $1:$ : $7-03$

ACTIVTTY CODE ESE260412

PROU.NAME: ECON. EVALUATION OF CLOSURE CAP BAFAUEA MATERALL

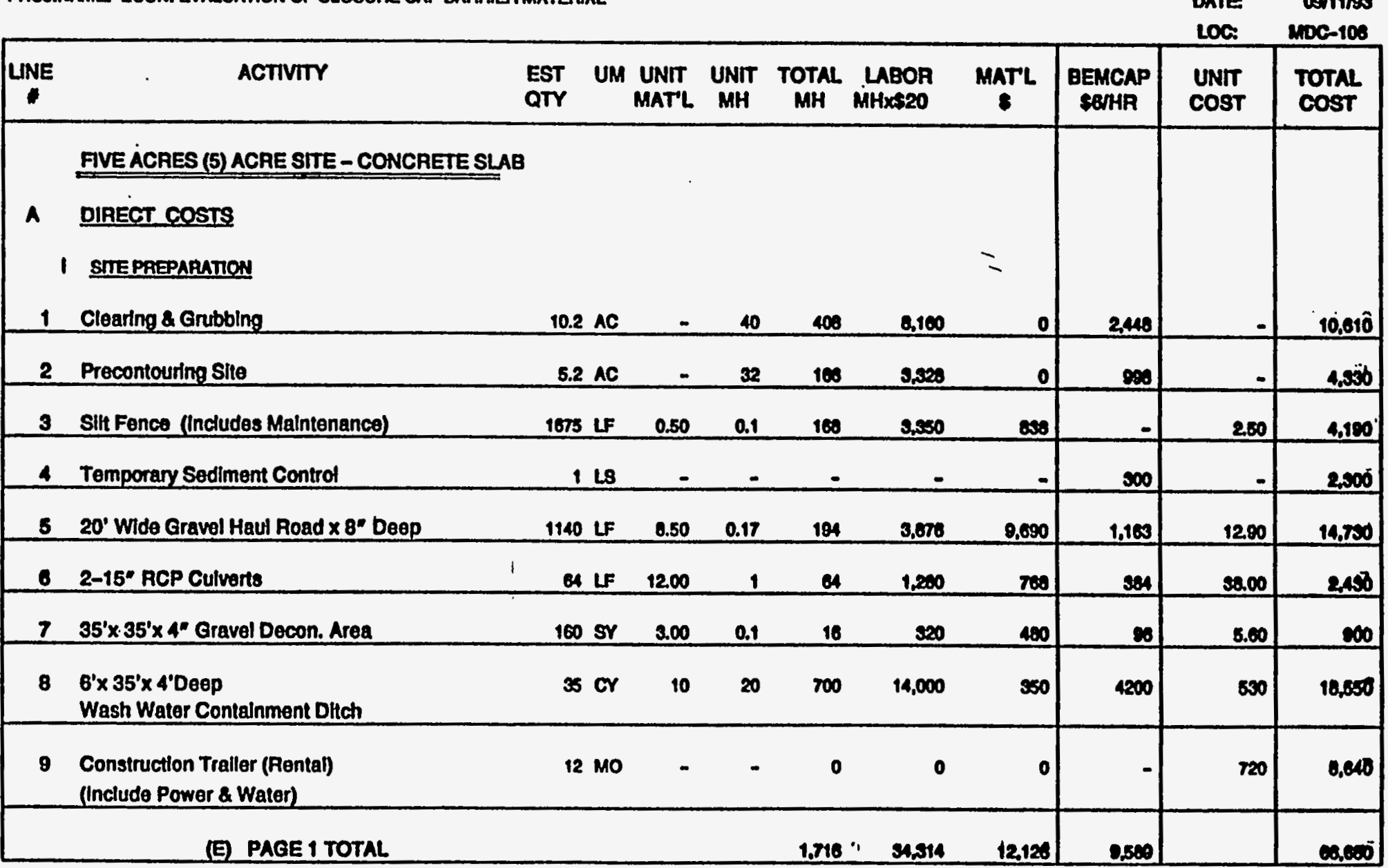

F-12. Cost Estimate for a Cover System Utilizing Poured-in Place Concrete Slab and a 30-mil FML 


\section{DETAIL ESTIMATE}

LoO1: 7-25

PROT.NO: $\mathrm{K} 8682821100$

PROJ.NAME: ECON. EVALUATFON CLOSURB CAP BARRIRR MATERIAR

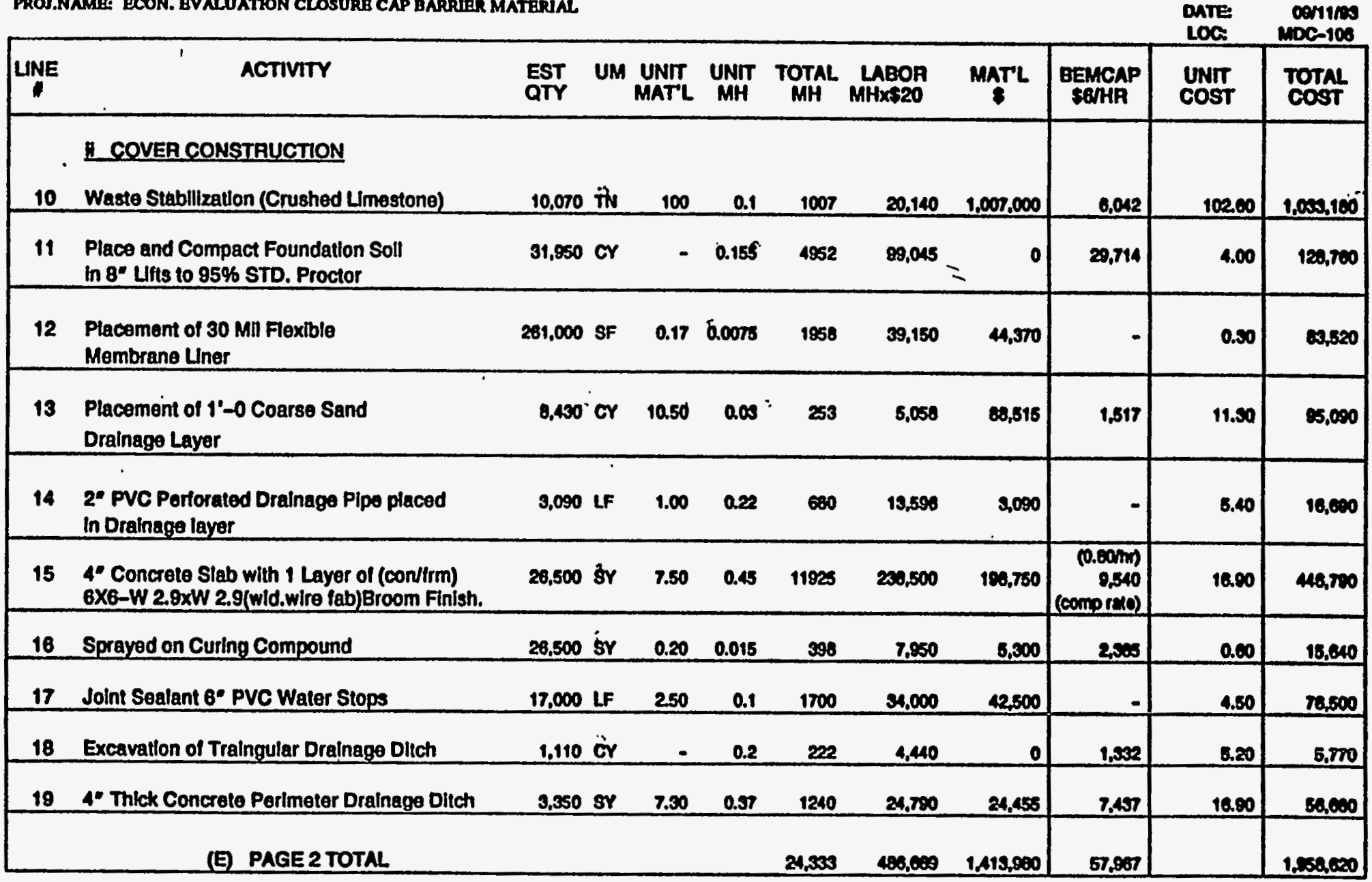

F-12. Cost Estimate for a Cover System Utilizing Poured-in Place Concrete Slab and a 30-mil FML (Cont'd) 
D B TAIL B S TIMATE

LOA : $7-25$

PROJ.NO: K 86821100

PROJ.NAMB: ECON. BVALUATION CLOSURB CAP BARRUER MATERIAL.

CHCO BY: 8.MEAD

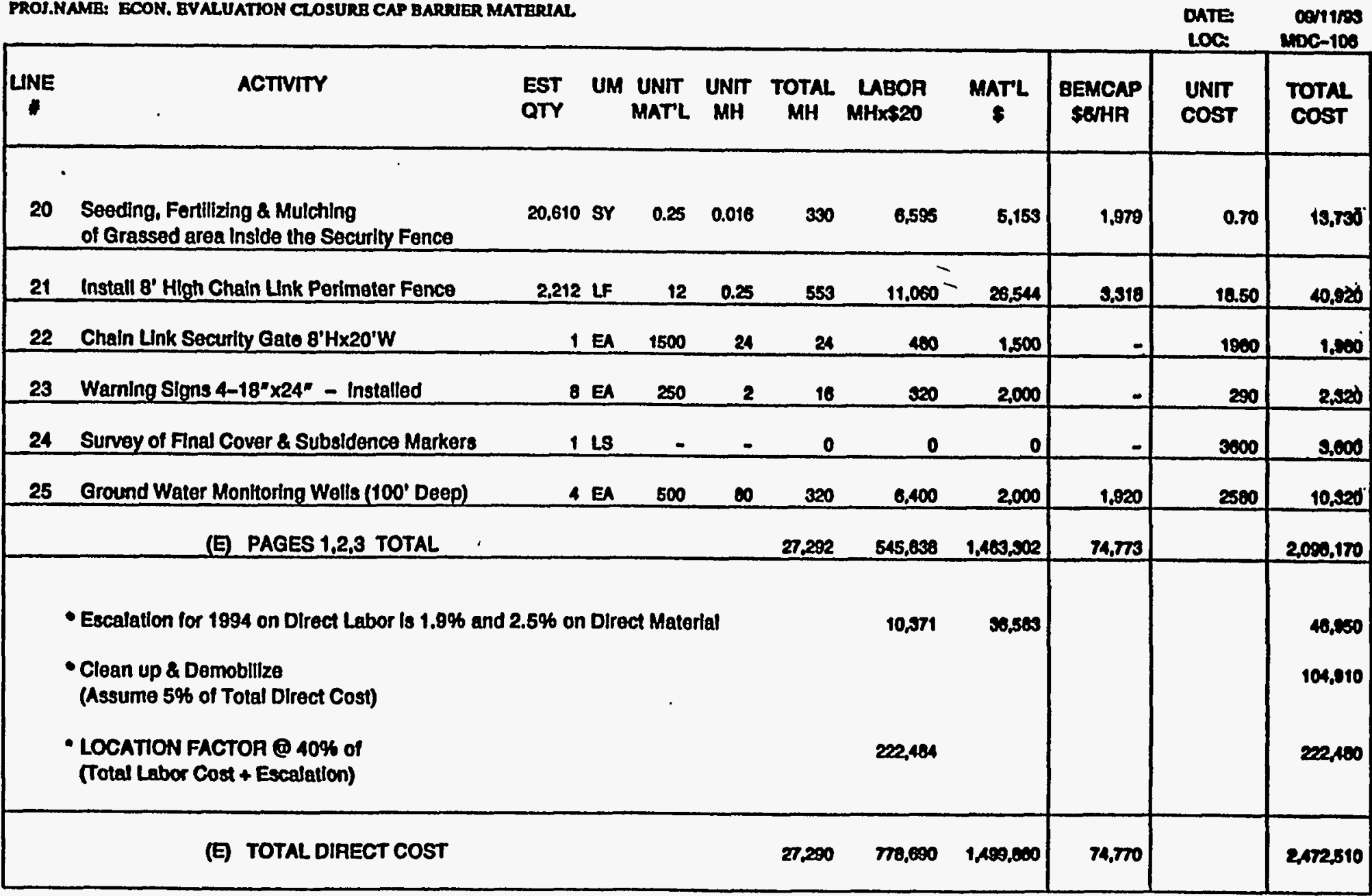

F-12. Cost Estimate for a Cover System Utilizing Poured-in Place Concrete Slab and a 30-mil FML (Cont'd) 


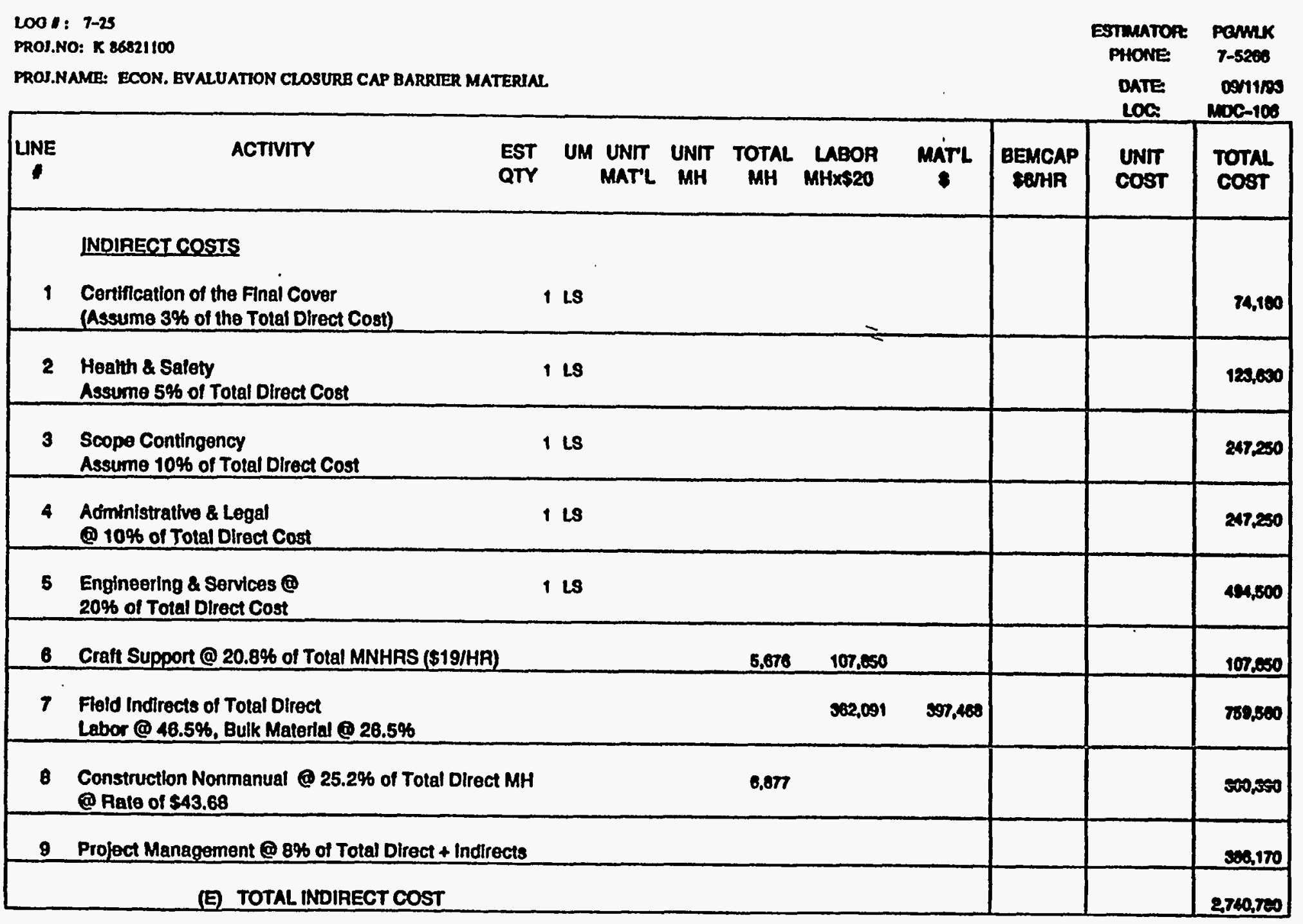

F-12. Cost Estimate for a Cover System Utilizing Poured-in Place Concrete Slab and a 30-mil FML (Cont'd) 


\section{L001: $7-25$}

PROS.NO: K 86521100

PROJ.NANB: ECON. EVALUATTON CLOSURB CAP BARRIER MATERIAL.

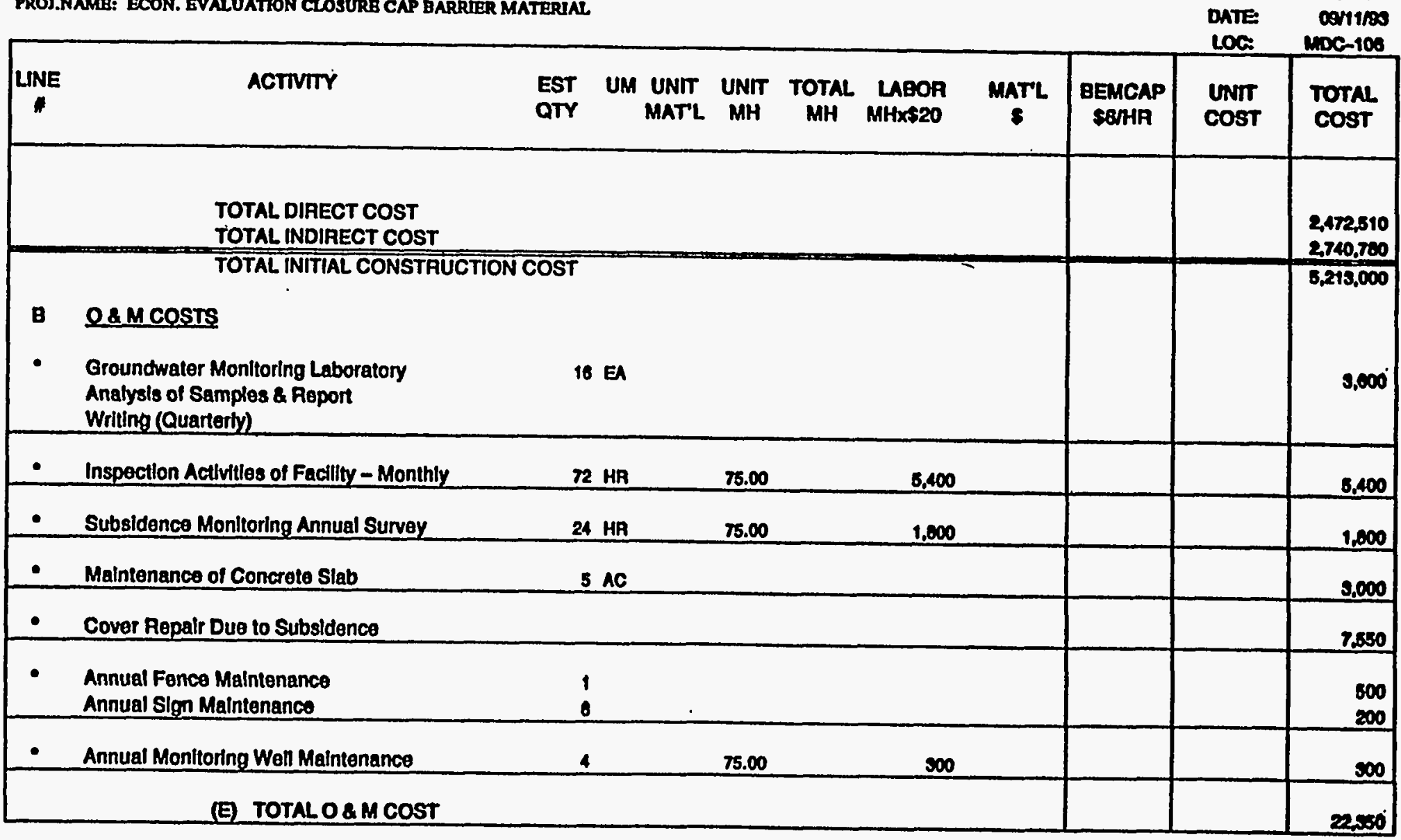

F-12. Cost Estimate for a Cover System Utilizing Poured-in Place Concrete Slab and a 30-mil FML (Cont'd) 
D B T A I L B S T I MATB

100 0: 7-25

PROJ.NO: $\mathrm{K} 86821100$

PROJ.NAME: ECON. EVALUATLON CLOSURB CAP BARRIBR MATERJAL

SUMMARY

EIVE ACAES (5) ACRE SITE - CONCAETE SLAB

C TOTAL COST (Total Present Worth)

(1 Present Worth of O \& $M$ Cost

(5\% discount Rate for 30 Years)

(Total O \& M X 15.3725)

(il Total Inittal Construction Cost

$6,218,000$

(E) TOTAL PROJECT COST (Rounded to the nearest Thousand)

\section{CANOBY: Q.MEND \\ Estmuntort pamix \\ PHONE 7-6200 \\ 0e/11/m \\ 100. Moc-100}

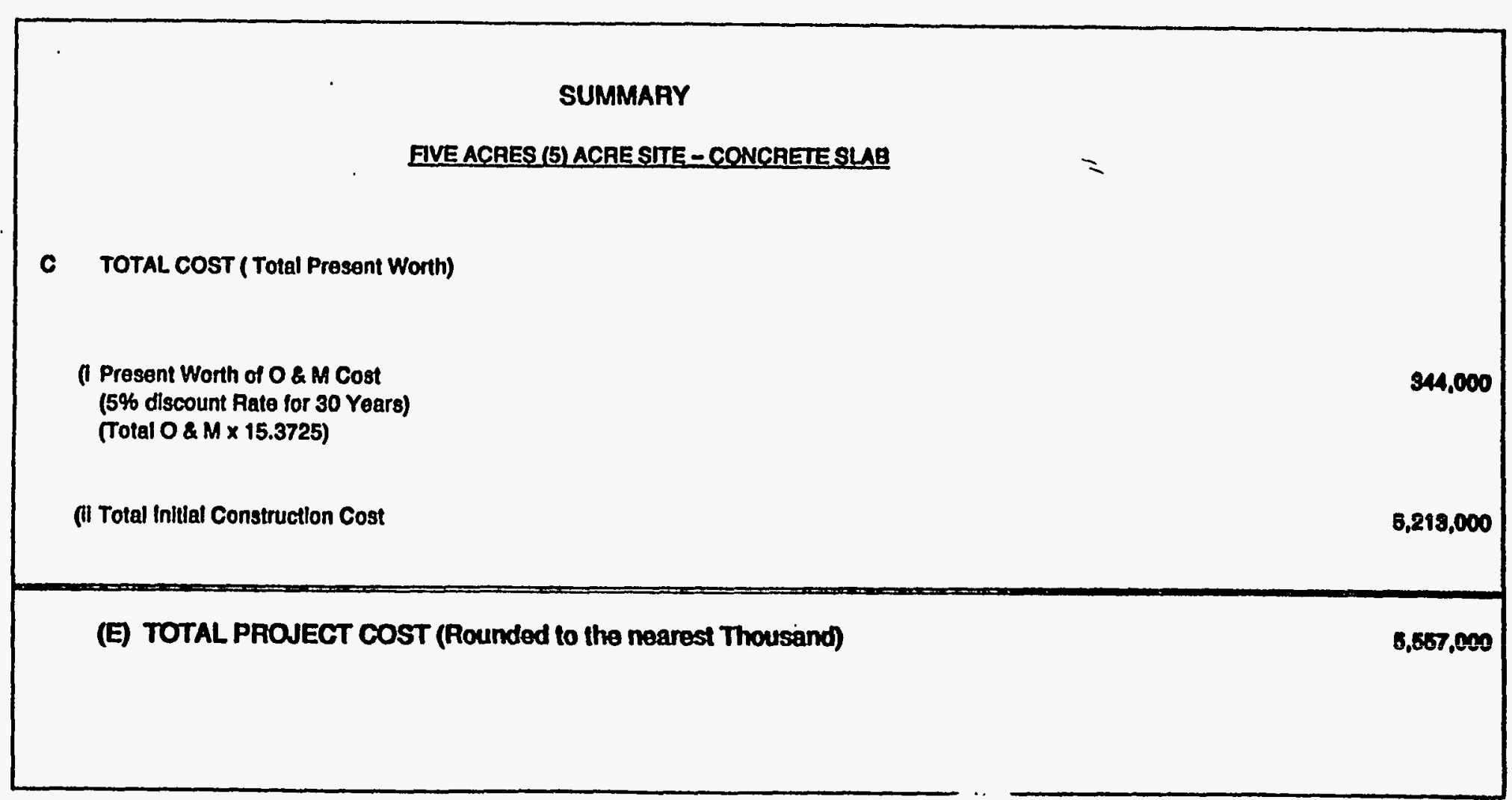

F-12. Cost Estimate for a Cover System Utilizing Poured-in Place Concrete Slab and a 30-mil FML (Cont'd) 

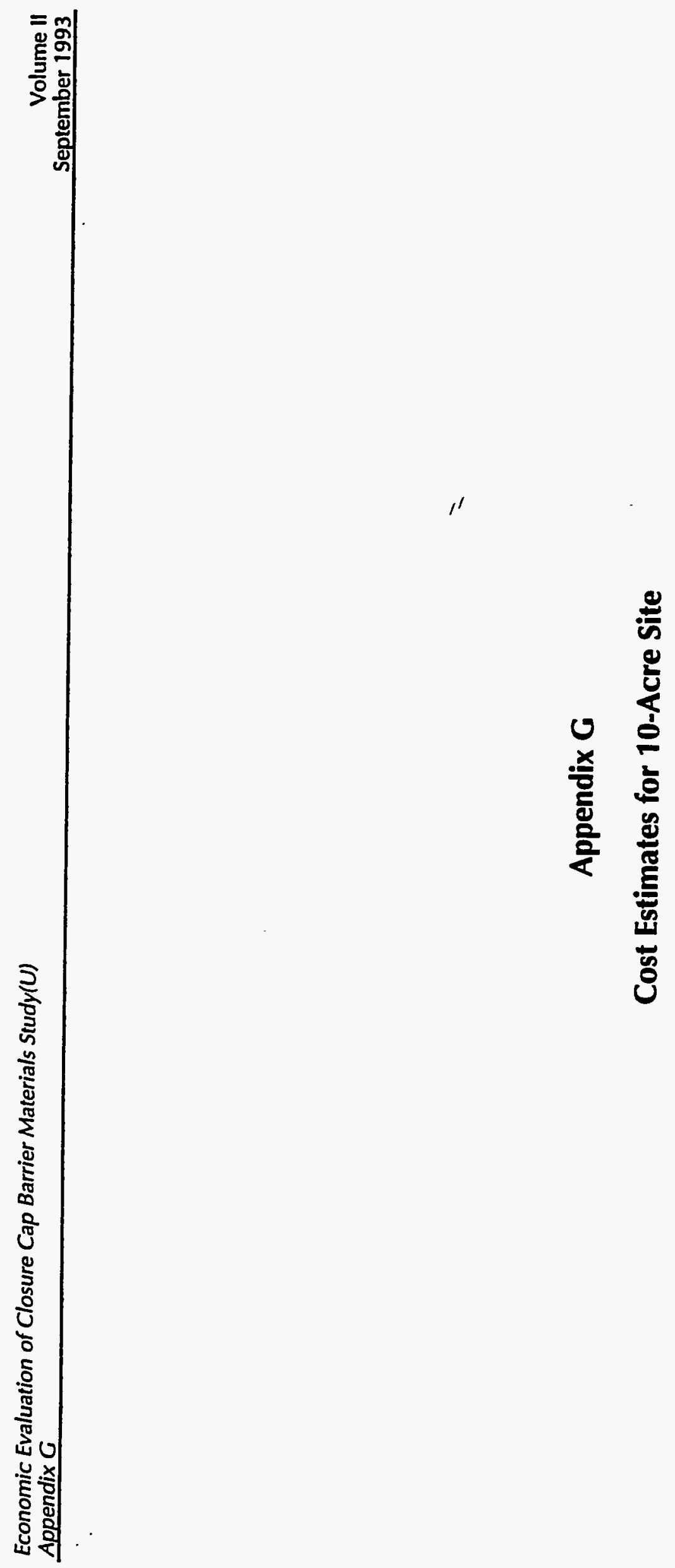
DETAIL ESTIMATE

\section{1: 7-03}

ACTIVITY CODE: ESEDCOA12

PROI.NAME: ECON. BVALUATION OF CLOSURE CAP DARRIER MATEPIAL

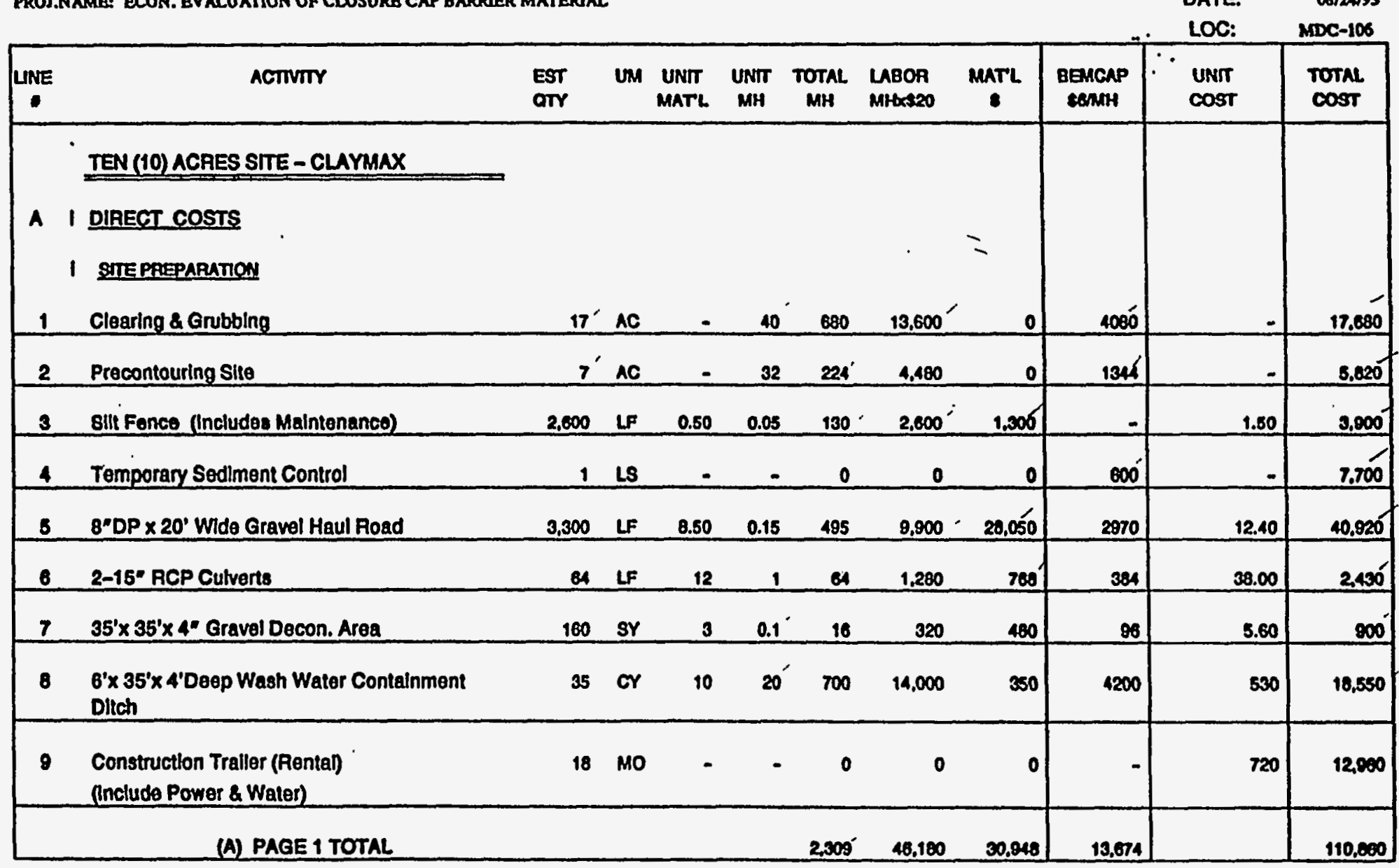

G-1. Cost Estimate for a Cover System Utilizing Claymax@ and a 30-mil FML 
DETAIL ESTIMATE

\section{LoOf: $7-03$}

ACTIVITY CODE: R9E260112

PROI.NAMB: ECON. BVALUATION OR CLOSURB CAP BARRRER MATERIAL

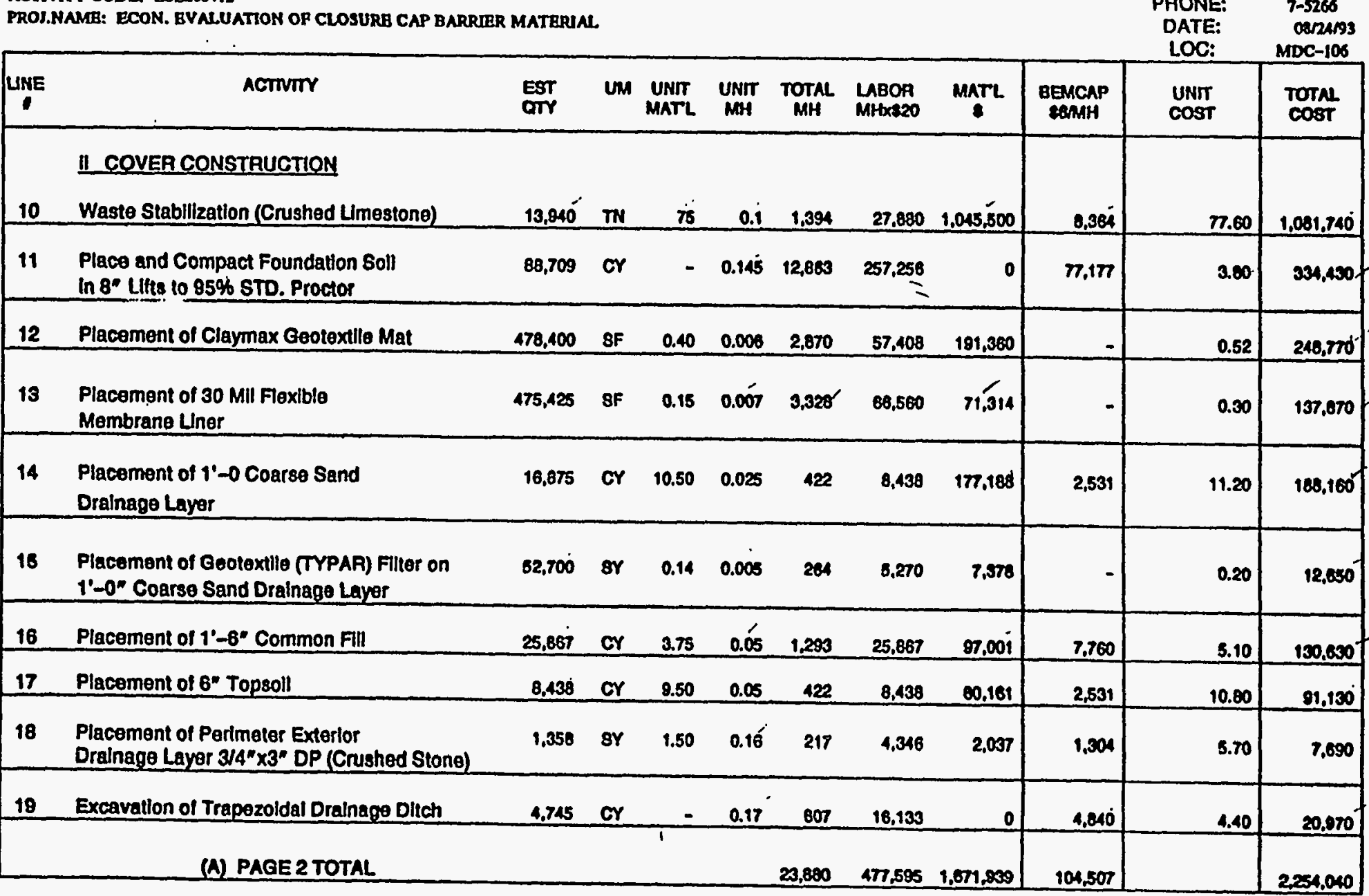

G-1. Cost Estimate for a Cover System Utilizing Claymax® and a 30-mil FML (Cont'd) 
DETAIL BSTIMATE

\section{1: $7-03$}

ACTTVITY CODB: ESE260412

PROS.NAMB: ECON. EVALUATTON OF CLOSURB CAP BARRRER MATERIAL

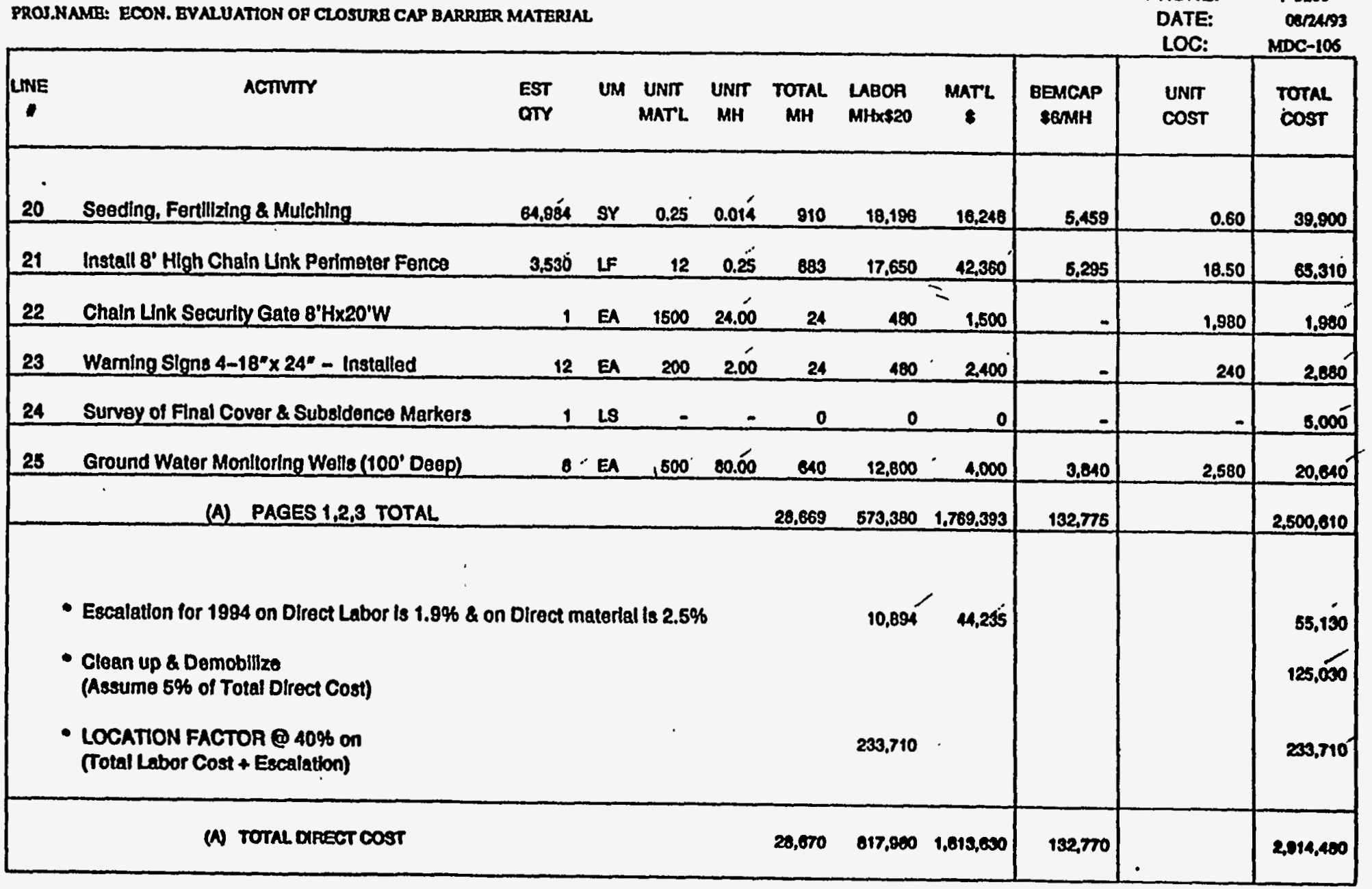

G-1. Cost Estimate for a Cover System Utilizing Claymax ${ }^{\circledR}$ and a 30-mil FML (Cont'd) 


\section{DETAIL B S T IMATB}

LOA : $: 7-03$

ACTTVITY CODB: ESE260412

PROS.NAME: ECON. EVALUATION OF CLOSURB CAP BARRIER MATERIAL

\begin{tabular}{|c|c|c|c|c|c|c|c|c|c|c|c|}
\hline LNNE & ACTVITY & $\begin{array}{l}\text { EST } \\
\text { arr }\end{array}$ & UM & $\begin{array}{l}\text { UNT } \\
\text { MATL }\end{array}$ & $\begin{array}{l}\text { UNIT } \\
\text { MH }\end{array}$ & $\begin{array}{l}\text { TOTAL } \\
\text { MH }\end{array}$ & $\begin{array}{l}\text { LABOR } \\
\text { MHXXs20 }\end{array}$ & $\underset{8}{\text { MATL }}$ & $\begin{array}{l}\text { BENCAP } \\
\text { SGMMH }\end{array}$ & $\begin{array}{l}\text { Unit } \\
\text { COST }\end{array}$ & $\begin{array}{l}\text { TOTAL } \\
\text { COST }\end{array}$ \\
\hline 1 & $\begin{array}{l}\text { INDIRECT COSTS } \\
\text { Centilication of tho Final Cover } \\
\text { (Assume 2.5\% of the Total Direct Cost) }\end{array}$ & 1 & LS & & & & & & & & 72,660 \\
\hline 2 & $\begin{array}{l}\text { Heath \& Safoty } \\
\text { Assume } 4 \% \text { of Total Direct Cost }\end{array}$ & 1 & LS & & & & - & & & & 116,580 \\
\hline 3 & $\begin{array}{l}\text { Scopo Contingency } \\
\text { Assume } 10 \text { se of Tolal Olrect Cost }\end{array}$ & 1 & LS & & & & & & & $\dot{-}$ & 291,450 \\
\hline 4 & $\begin{array}{l}\text { Administralke \& Logal } \\
\text { id a\% of Total Direct Cost }\end{array}$ & 1 & L8 & & & & & & & & 233,160 \\
\hline 8 & $\begin{array}{l}\text { Engineering \& Services } \\
20 \% \text { of Total Drect cost }\end{array}$ & & & & & & & & . & & 882,900 \\
\hline 6 & Crat support $020.8 \%$ of Tolal MHAS (819m) & & & & & 5,903 & & & & & 113,300 \\
\hline 7 & $\begin{array}{l}\text { Fiold Indirects on Total Direct } \\
\text { Lebor Q 46.5\%, Material Q 26.5\% }\end{array}$ & & & & $\because$ : & & 380,361 & 480,612 & & & 860,070 \\
\hline 8 & $\begin{array}{l}\text { Construction Nonmenued } 25.2 \% \text { of Total Direci AiH } \\
\text { Q Rato of \$43.68 }\end{array}$ & & & & & 7,225 & & & & & 318,580 \\
\hline $\boldsymbol{9}$ & Project Management o $8 \%$ of Total Diroct + Indirocts & & & & & & & & & & 40,100 \\
\hline & (A) TOTAL INDTAECT COST & & & & & & & & & & $3,028,900$ \\
\hline
\end{tabular}

G-1. Cost Estimate for a Cover System Utilizing Claymax@ and a 30-mil FML (Cont'd) 
DETAIL ESTIMATE

CHK'D BY: S.MEAD

Lo01: 7-03

ACTTVTY CODE: ESEZ60112

PROJ.NAMB: ECON. BY ALUATOON OP CLOSURB CAP BARRIRR MATERHAL

ESTIMATOR: POAMX

PHONE: T-5266

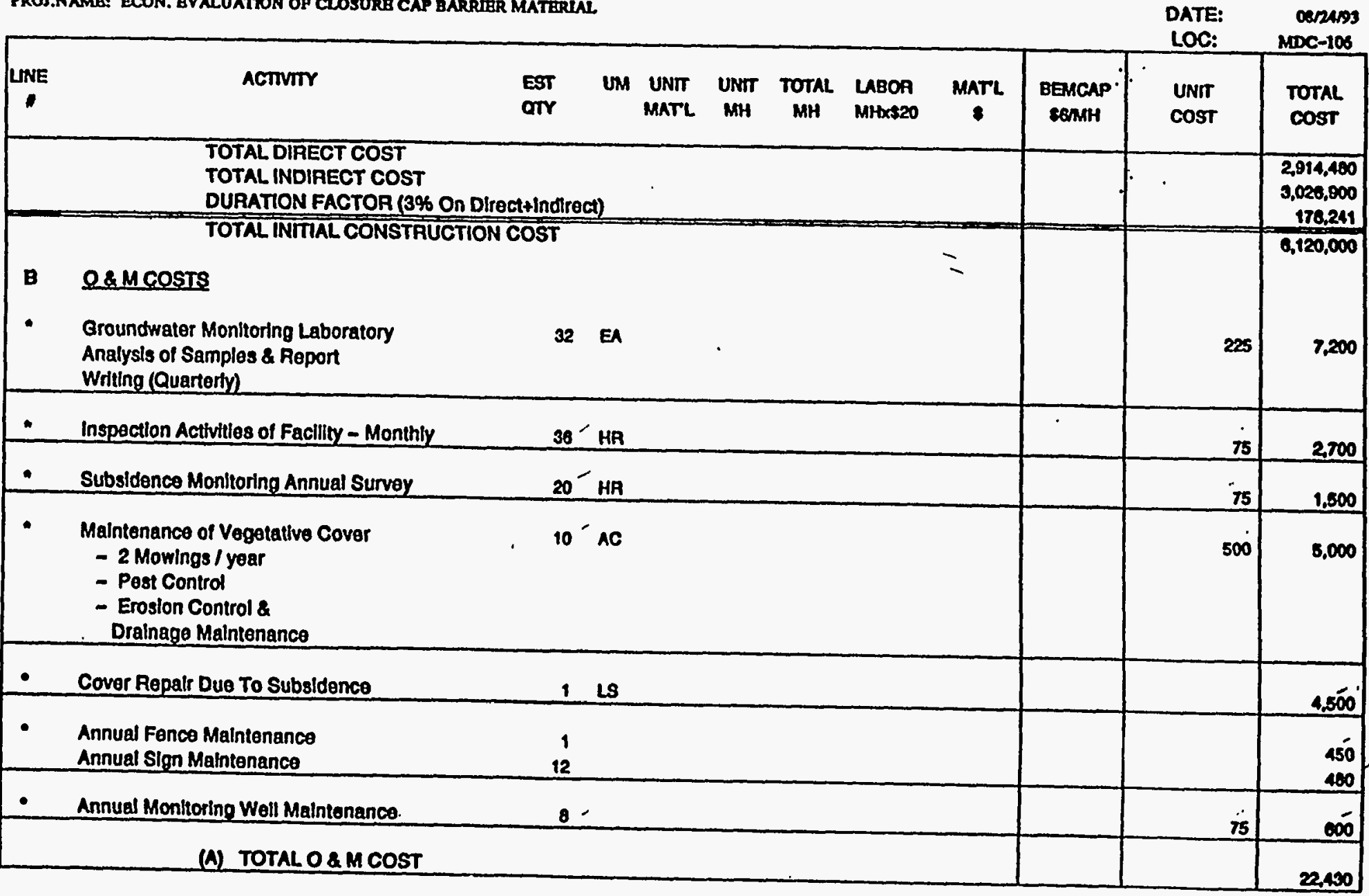

G-1. Cost Estimate for a Cover System Utilizing Claymax@ and a 30-mil FML (Cont'd) 
D B TAIL BSTIMATE

CHK'D BY: S.MEAD

Loof: $7-03$

ACTTVITY CODE: ESE260112

PROI.MAME: ECON. BYALUATION OP CLOSURB CAP BARRERR MATERIAL.

ESTIMATOR:

PHONE: 7-5265

DATE: 00242493

LOC: MDC-106

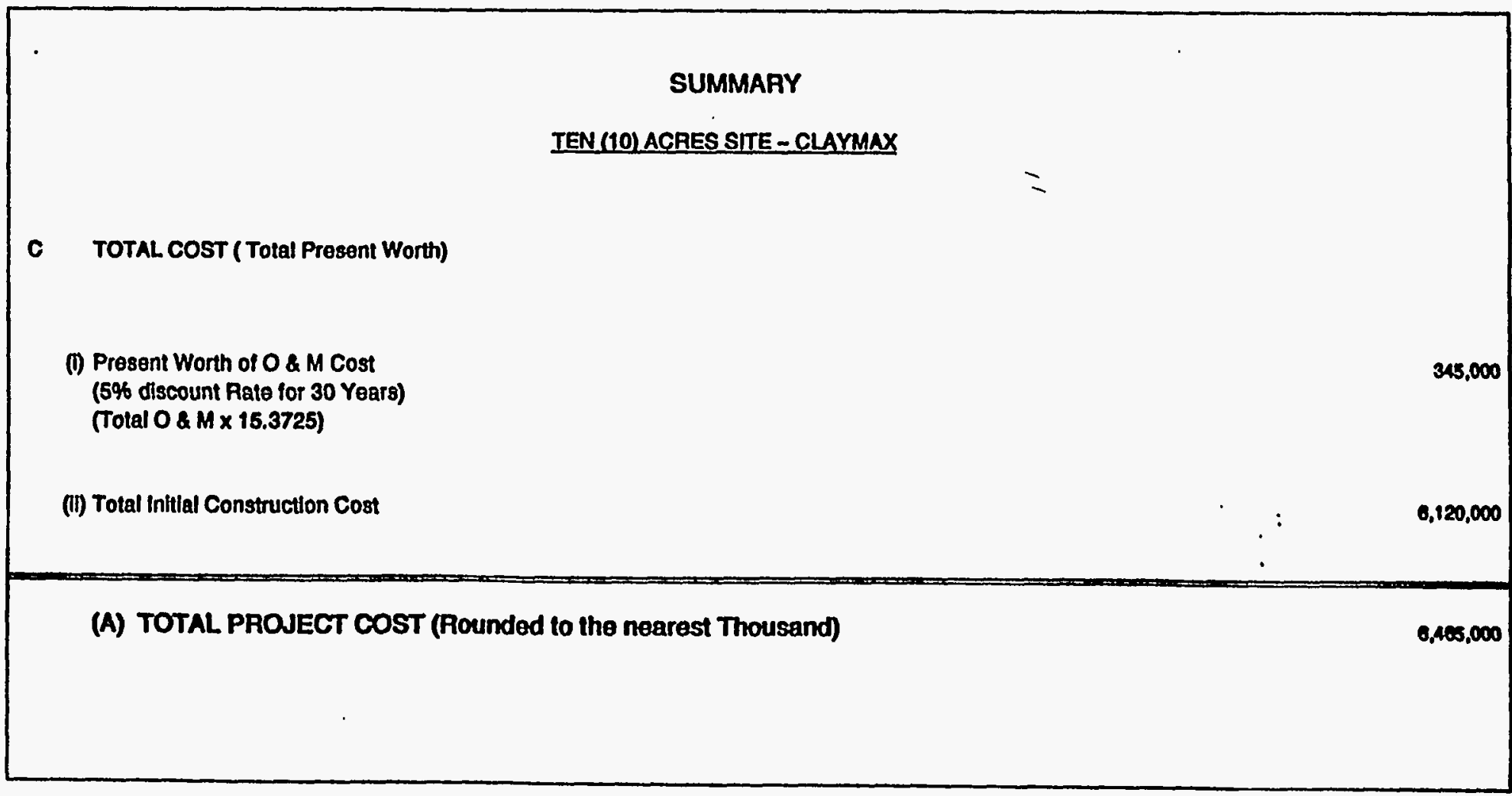

G-1. Cost Estimate for a Cover System Utilizing Claymax $\otimes^{\circledR}$ and a 30-mil FML (Cont'd) 
Economic Evaluation of Closure Cap Barrier Materials Study(U)

Volume II Appendix $\mathrm{C}$

DETAIL ESTIMATE

LOO I: : 7-03

ACTIVITY CODE: ESE260412

PROJ.NAME: ECON. EVALUATON OF CLOSURE CAP BARRIER MATERUAL

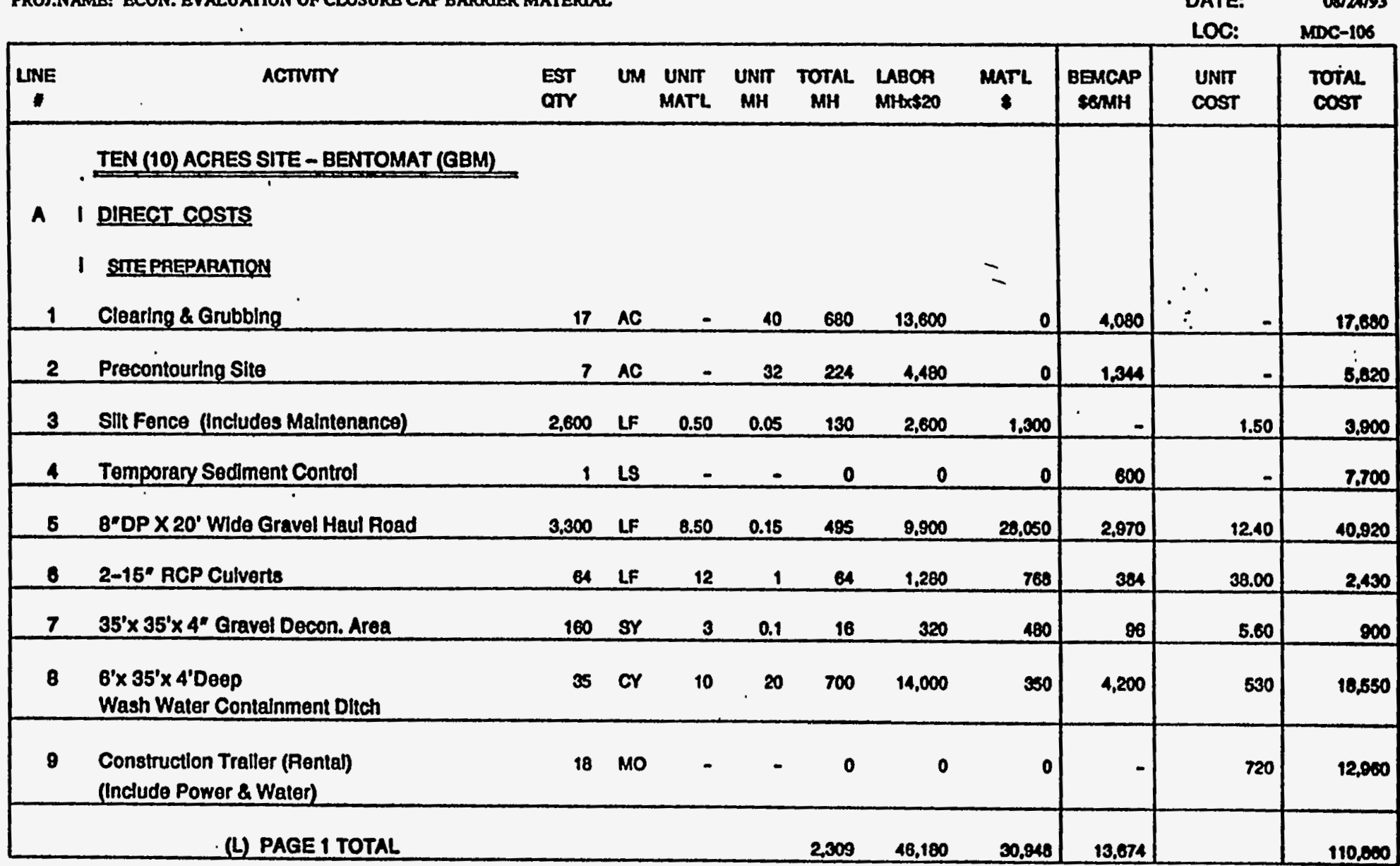

G-2. Cost Estimate for a Cover System Utilizing Bentomat and a 30-mil FML 
DETAIL ESTIMATE

LOO : : 7-03

ACTIVITY CODB: BSEZ6OA12

PROINAMB: ECON, EVALUATION OF CLOSURB CAP BARRIER MATERIAL

\begin{tabular}{|c|c|c|c|c|c|c|c|c|c|c|c|}
\hline UNE & ACTVITY & $\begin{array}{l}\text { EST } \\
\text { GTY }\end{array}$ & UM & $\begin{array}{l}\text { UNT } \\
\text { MATL }\end{array}$ & $\begin{array}{l}\text { UNIT } \\
\text { MH }\end{array}$ & $\underset{\text { MH }}{\text { TOTAL }}$ & $\begin{array}{l}\text { LABOR } \\
\text { MHAxtzO }\end{array}$ & $\underset{8}{M A T L}$ & $\begin{array}{l}\text { BEACAP } \\
\text { SEMMH }\end{array}$ & $\begin{array}{l}\text { UNIT } \\
\text { COST }\end{array}$ & $\begin{array}{l}\text { TOTAL } \\
\text { Cost }\end{array}$ \\
\hline & I COVER CONSTRUCTION & & & & & & & & & & \\
\hline 10 & Waste Stabilization (Crushed Limestone) & 13,940 & TN & .76 & 0.1 & 1,394 & 27,880 & $1,045,500$ & 8,364 & 77.60 & $1,001,740$ \\
\hline 11 & $\begin{array}{l}\text { Place and Compact Foundation Soll } \\
\text { in } 8^{\circ} \text { Ufts to } 95 \% \text { Stdandard Proctor }\end{array}$ & 88,709 & CY & - & 0.145 & 12,863 & 257,256 & $=$ & 77.177 & 3.60 & 334,430 \\
\hline 12 & Placement of Bentomat Geotextllo Mat & 478,400 & SF & 0.40 & 0.006 & 2,070 & 57,408 & 191,360 & - & 0.52 & 248,770 \\
\hline 13 & $\begin{array}{l}\text { Placement of } 30 \text { Mil Flexible } \\
\text { Membrane Uner }\end{array}$ & 475,425 & SF & 0.15 & 0.007 & 3,320 & 66,560 & 71,314 & - & 0.30 & 137,070 \\
\hline 14 & $\begin{array}{l}\text { Placement of 1'-0 Coarse Sand } \\
\text { Drainage Layer }\end{array}$ & 18,875 & OY & 10.50 & 0.025 & 422 & 8,438 & 177.188 & 2,531 & 11.20 & 180,160 \\
\hline 15 & $\begin{array}{l}\text { Placement of Geotextile (TYPAR) Filter on } \\
1^{\prime}-0^{\circ} \text { Coarse Sand Dralnage Layer }\end{array}$ & 52,700 & SY & 0.14 & 0.005 & 264 & 5,270 & 7,378 & - & 0.20 & 12,650 \\
\hline 16 & Placement of 1'-6* Common Fill & 25,867 & CY & 3.75 & 0.05 & 1,293 & 25,867 & 97.001 & 7,760 & 5.10 & 130.030 \\
\hline 17 & Placement of 6* Topsoll & 8,438 & CY & 8.50 & 0.05 & 422 & 8,438 & 80.161 & 2,531 & 10.80 & 81.130 \\
\hline 18 & $\begin{array}{l}\text { Placement of Perlmeter Exterlor } \\
\text { Dralnuge Layar } 3 / 4^{*} \times 3^{*} \text { DP (Crushed Stone) }\end{array}$ & 1,358 & sr & 1.50 & 0.18 & 217 & 4,346 & 2,037 & 1,304 & 5.70 & 7.690 \\
\hline 19 & Excavation of Trapezoidal Drainage Ditch & 4,745 & cr & - & 0.17 & 807 & 16,133 & 0 & 4,840 & 4.40 & 20.970 \\
\hline & (ㄴ) PAGE 2 TOTAL & & & & & 23,680 & 477.595 & $1,071,909$ & 104,507 & & $2,204,040$ \\
\hline
\end{tabular}

G-2. Cost Estimate for a Cover System Utilizing Bentomat@ and a 30-mil FML (Cont'd) 
D B TAIL BSTIMAT B

LOO : $:$ 7-03

ACTIVITY CODE: BSE26OL12

PROJ.NAME: ECON. EVALUATION OF CLOSURB CAP BARRIER MATERUAL
CHK'D BY: S. MEAD

ESTIMATOR: POANK

PHONE: 7-5266

DATE:

\begin{tabular}{|c|c|c|c|c|c|c|c|c|c|c|c|}
\hline UNE & ACTIVI & $\begin{array}{l}\text { Est } \\
\text { ary }\end{array}$ & UM & $\begin{array}{l}\text { UNIT } \\
\text { MATL }\end{array}$ & $\begin{array}{l}\text { UNIT } \\
\text { MH }\end{array}$ & $\begin{array}{l}\text { TOTAL } \\
\text { MH }\end{array}$ & $\begin{array}{l}\text { LABOA } \\
\text { MHXX\$2O }\end{array}$ & $\underset{*}{\text { MATL }}$ & $\begin{array}{l}\text { BEMCAP } \\
\text { SEMMH }\end{array}$ & $\begin{array}{l}\text { UNIT } \\
\text { COST }\end{array}$ & $\begin{array}{l}\text { Total } \\
\text { cost }\end{array}$ \\
\hline 20 & Seeding, Fortillzing \& Mulching & 64,984 & sY & 0.25 & 0.014 & 910 & 18.196 & 16,248 & 5,459 & 0.60 & 39,800 \\
\hline 21 & Install 8' High Chaln Link Perimater Fence & 3.530 & LF & 12 & 0.25 & 683 & 17,650 & 12,360 & 5,295 & 18.50 & 63,310 \\
\hline 22 & Chain Lnk Security Gate 8'Hx20'W & 1 & EA & 1500 & 24.00 & 24 & 180 & 1,500 & - & 1.980 & 1.980 \\
\hline 23 & Waming Signs 4-18" $\times 24^{\prime \prime}-$ Installed & 12 & EA & 200 & 2.00 & 24 & 480 & 2,400 & - & 240 & 2,880 \\
\hline 24 & Survey of Final Cover \& Subsidence Markers & 1 & LS & - & - & 0 & 0 & 0 & - & 5,000 & 8,000 \\
\hline 25 & Ground Water Monitoring Wells (100' Deep) & 8 & EA & 500 & 80.00 & 640 & 12,600 & 4.000 & 3,240 & 2,580 & 20,640 \\
\hline & (L) PAGES 1,2,3 TOTAL & & & & & 28,669 & 573,380 & $1,769,393$ & 132,775 & & $2,500,010$ \\
\hline & $\begin{array}{l}\text { Escalation for } 1994 \text { on Direct Labor is } 1.946 \text { \& } \\
\text { Clean up \& Demobillze } \\
\text { (Assume 5\% of Total Dlrect Cost) } \\
\text { LOCATION FACTOR (1 } 40 \% \text { on } \\
\text { (Total Labor Cost + Escalation) }\end{array}$ & Direct N & laterle & al is 2.5 & & & 10,894 & 44,235 & & & $\begin{array}{r}65,130 \\
123,030 \\
223,710\end{array}$ \\
\hline & (L) TOTAL DIAECT COST & & & & & 20,670 & 817,900 & $1,013,630$ & 132,770 & & $2,914,460$ \\
\hline
\end{tabular}

G-2. Cost Estimate for a Cover System Utilizing Bentomat@ and a 30-mil FML (Cont'd) 
DBTAIL BSTIMATE

$200 \div: 7-03$

ACTTVITY CODB: ESE260412

PROJ.NAME: ECON. BVALUATION OP CLOSURE CAP BARRJPR MATERIAL
CHK'D BY: S. MEAD

ESTIMATOR: PONWK PHONE: 7-5266

DATE: OORA93

LOC: $\quad$ MDC-100

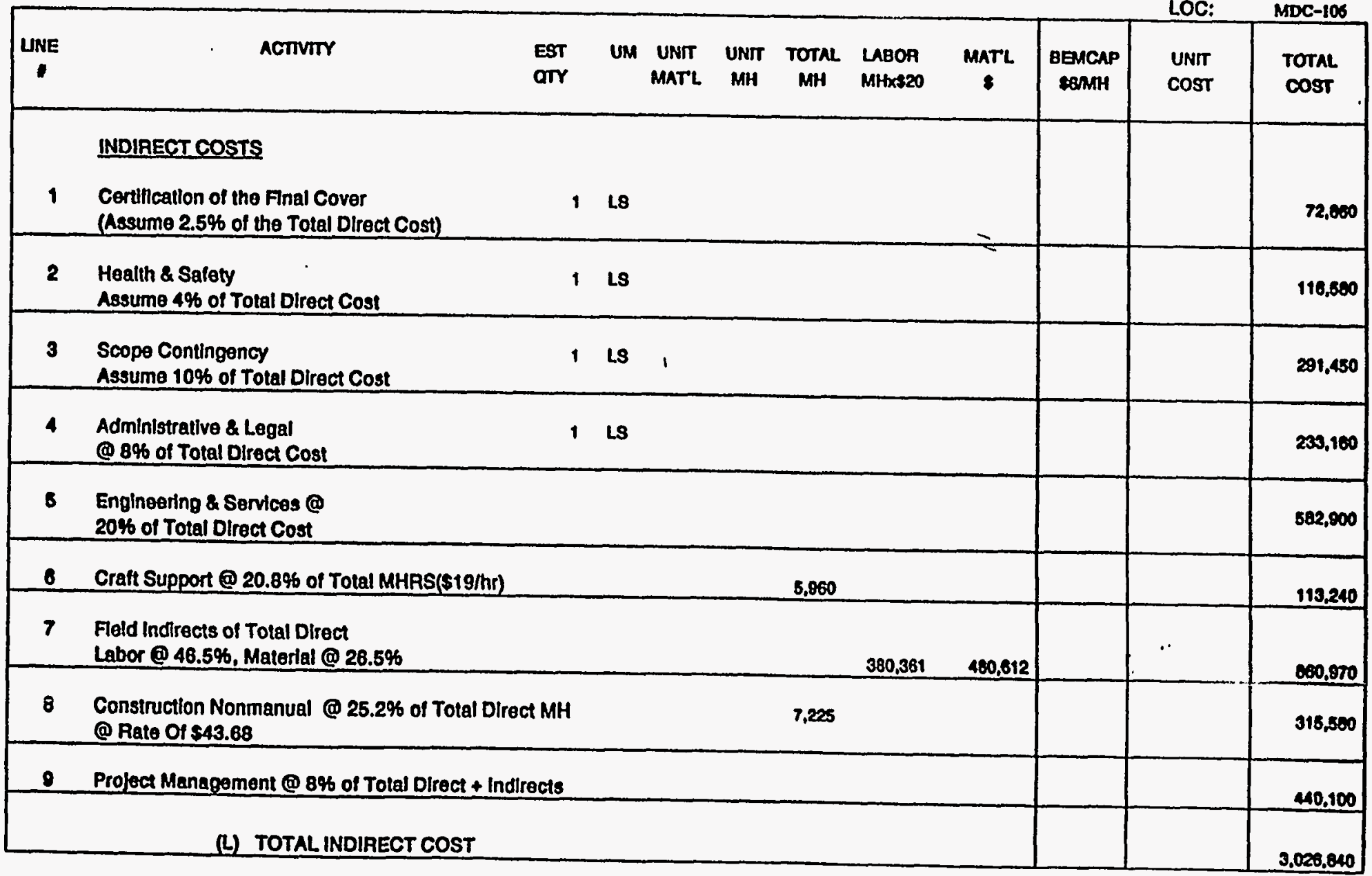

G-2. Cost Estimate for a Cover System Utilizing Bentomat@8 and a 30-mil FML (Cont'd) 
D E T A I L E S T I MAT B

Loof: 7-03

ACTIVITY CODB: BSE260112

PROJ.NAMB: ECON. BVALUATION OF CLOSURB CAP BARRTER MATERIAL

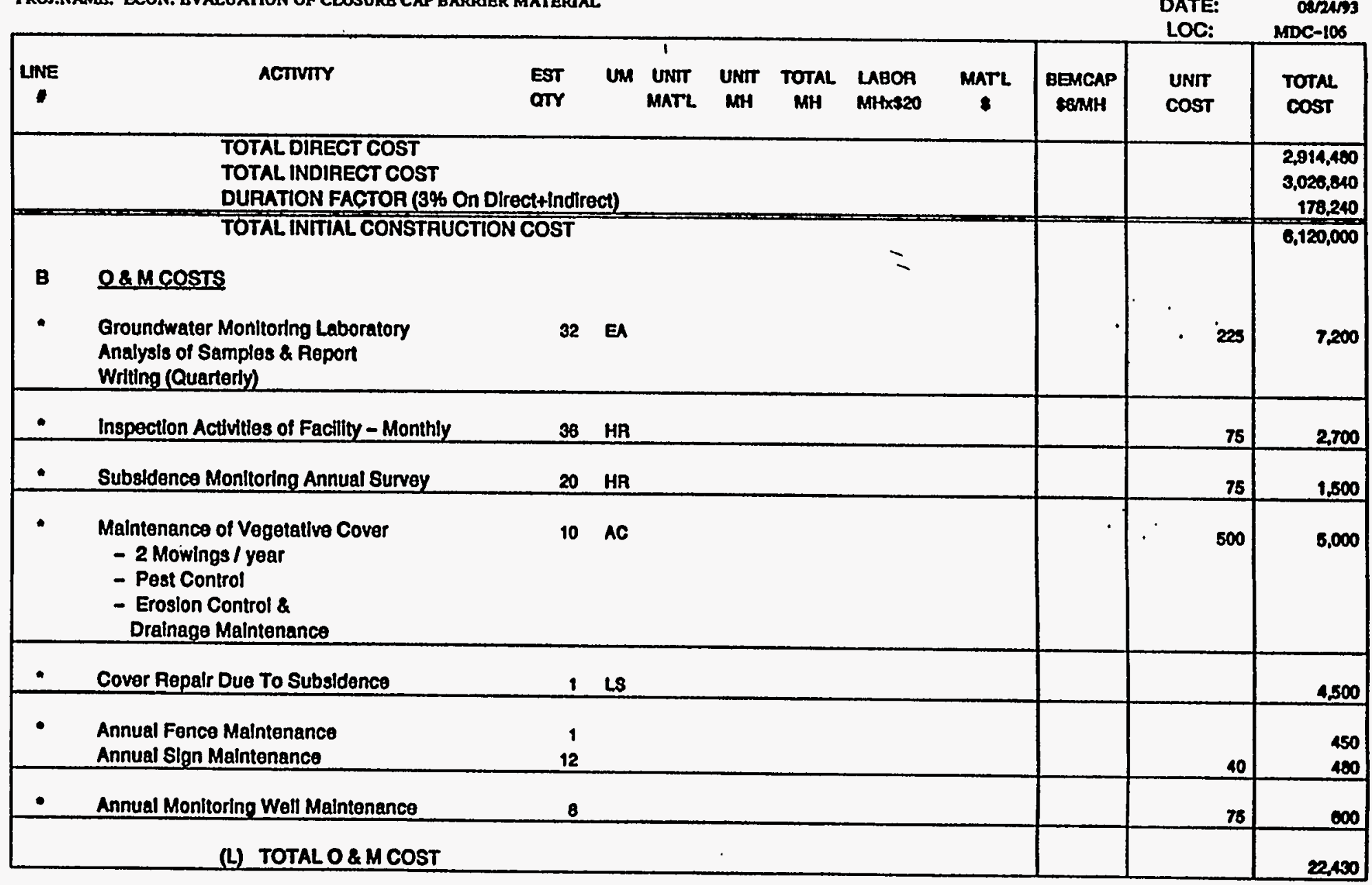

G-2. Cost Estimate for a Cover System Utilizing Bentomat $\$$ and a 30-mil FML (Cont'd) 
D B T A I L B T I M A T

100 \&: 7-03

ACTIVITY CODB: ESE260412

PROJ.NAME: ECON. EVALUATION OF CLOSURB CAP DARRIER MATERIAL
' CHKK'D BY: S. MEAD

ESTIMATOR: POWHX

PHONE: 7-5266

DATE:

LOC: MmC-10B

\section{SUMMARY}

IEN (10) ACRES SITE - BENTOMAT (GBM)

$=\quad \cdot \cdot$

C TOTAL COST (Total Present Worth)

(i) Present Worth of $O \& M$ Cost

(5\% discount Rate for 30 Years)

(Total O\&M $\times$ 15.3725)

(ii) Total Inittal Construction Cost

$0,120,000$

(L) TOTAL PROJECT COST (Rounded to the nearest Thousand)

G-2. Cost Estimate for a Cover System Utilizing Bentomat@ and a 30-mil FML (Cont'd) 
DETAIL ESTIMATE

Loo : 7-03

ACTIVITY CODB: ESEQ6ON12

PROJ.NAMB: ECON. BVALUATION OF CLOSURB CAP BARRIER MATERIAL

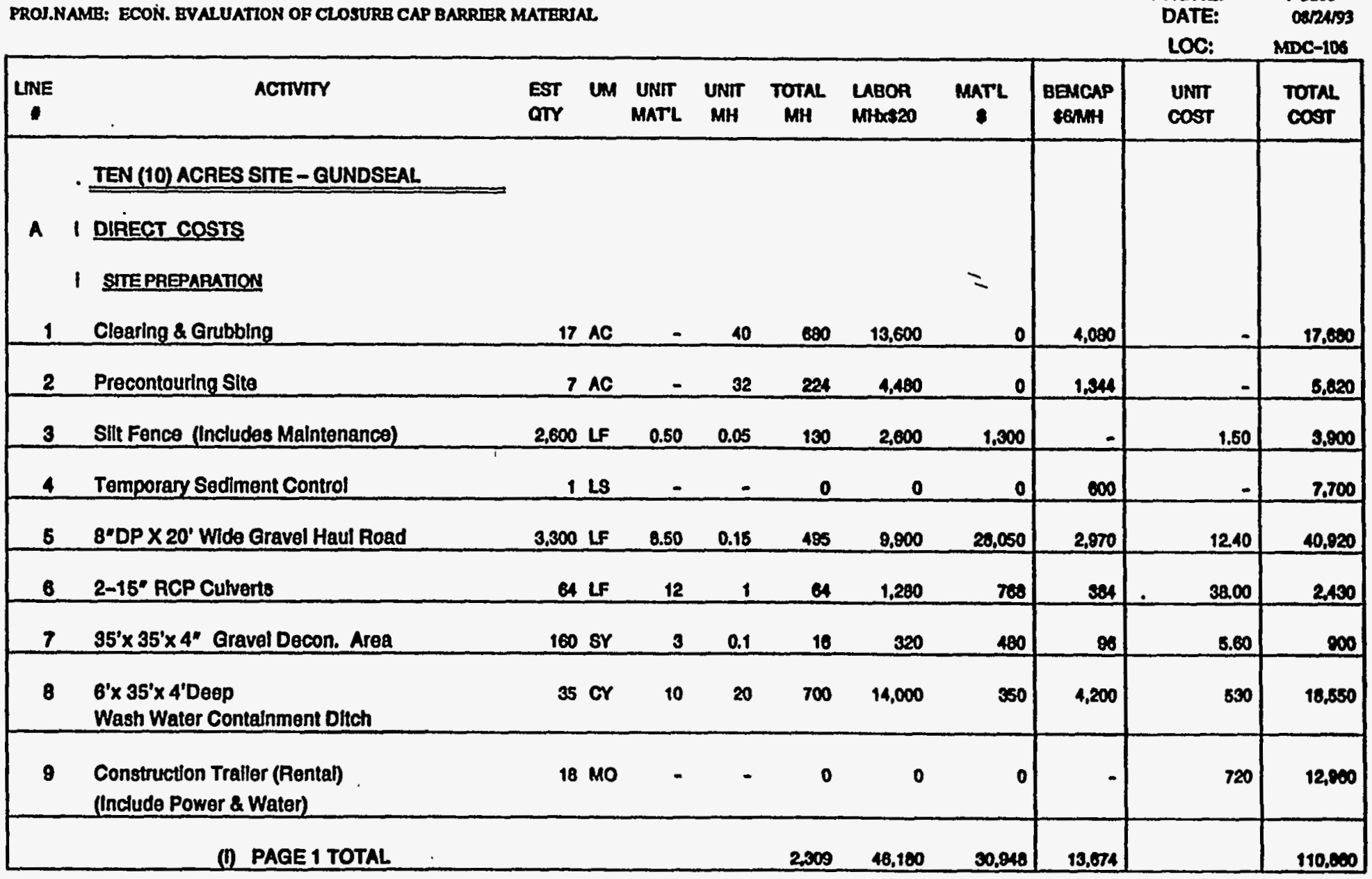

G-3. Cost Estimate for a Cover System Utilizing Gundseal@ 
DETAIL ESTIMATE

CHK'D BY: S. MEAD

Lo01: 7-03

ACTIVTTY CODB: ESE260112

PROJ.NAMR: ECON. EVALUATION OF CLOSURB CAP BARRIIR MATERIAL

\begin{tabular}{|c|c|c|c|c|c|c|c|c|c|c|c|}
\hline UNE & ACTIVTYY & $\begin{array}{l}\text { EST } \\
\text { aTr }\end{array}$ & Um & MaTT & UNHT & TOTAL & $\begin{array}{l}\text { LABOA } \\
\text { MHtaseO }\end{array}$ & MATL & $\begin{array}{l}\text { BEMCAP } \\
\text { BGAMH }\end{array}$ & $\begin{array}{l}\text { UNIT } \\
\text { COST }\end{array}$ & $\begin{array}{l}\text { Torre } \\
\operatorname{cost}\end{array}$ \\
\hline & A COVER CONSTRUCTION & & & & & & & & & & \\
\hline 10 & Waste Stabillzation (Crushed Limestone) & 13,940 & $\mathrm{TN}$ & 75 & 0.1 & 1.394 & 27,880 & $1,045,500$ & 8,364 & $\mathbf{7 7 . 6 0}$ & $1,001,740$ \\
\hline 11 & $\begin{array}{l}\text { Place and Compact Foundation Soll } \\
\text { In } 8^{*} \text { Lifts to } 95 \% \text { Std. Proctor }\end{array}$ & 88,709 & or & - & 0.145 & 12,803 & 257,258 & $=$ & 77,177 & 3.80 & 334,450 \\
\hline 12 & $\begin{array}{l}\text { Placement of Gundseal Geomembrane/ } \\
\text { Bentonite Mat }\end{array}$ & 478,400 & SF & 0.40 & 0.006 & 2,870 & 57,400 & 191,360 & - & 0.52 & 248,70 \\
\hline 13 & $\begin{array}{l}\text { Placement of 1'-0 Coarse Sand } \\
\text { Drainage Layer }\end{array}$ & 16,875 & or & 10.5 & 0.025 & 422 & 8,438 & $1 \pi, 180$ & 2,531 & 11.20 & 160,100 \\
\hline 14 & $\begin{array}{l}\text { Placement of Geotextlle (TYPAR) Fiter on } \\
\text { I'-0" Coarse Sand Drainage Layer }\end{array}$ & 62,700 & sr & 0.14 & 0.005 & 264 & 5,270 & 7,370 & - & 0.20 & 12,050 \\
\hline 15 & Placement of 1'-6" Common Fill & 25,867 & or & 3.75 & 0.05 & 1,293 & 25,867 & 97,001 & 7.760 & 5.10 & 130,600 \\
\hline 16 & Placement of 6" Topsoil & 8.438 & or & 9.50 & 0.05 & 422 & 0,438 & 80.181 & 2,531 & 10.80 & 91,130 \\
\hline 17 & $\begin{array}{l}\text { Placement of Perimeter Extertor } \\
\text { Dralnage Layer } 3 / 4^{*} \times 3^{*} \text { DP (Crushed Stone) }\end{array}$ & 1.358 & sy & 1.50 & 0.16 & 217 & 4,346 & 2,037 & 1,304 & 5.70 & 7,000 \\
\hline 18 & Excavation of Trapezoidal Drainage Ditch & 4,745 & CY & $=$ & 0.17 & 607 & 16,133 & o & 4.840 & 4.40 & 20,970 \\
\hline & (1) PAGE 2 TOTAL & & & & & 20.552 & 411.035 & $1,000,0625$ & 104,507 & & $2,116,170$ \\
\hline
\end{tabular}

G-3. Cost Estimate for a Cover System Utilizing Gundseal@ (Cont'd) 
D B TAIL ESTIMATE

Loof: 7-03

ACTIVTTY CODE: ESE2SOA12

PROJ.NAMB: ECON. BVALUATION OF CLOSURB CAP BARRIER MATERIAL.

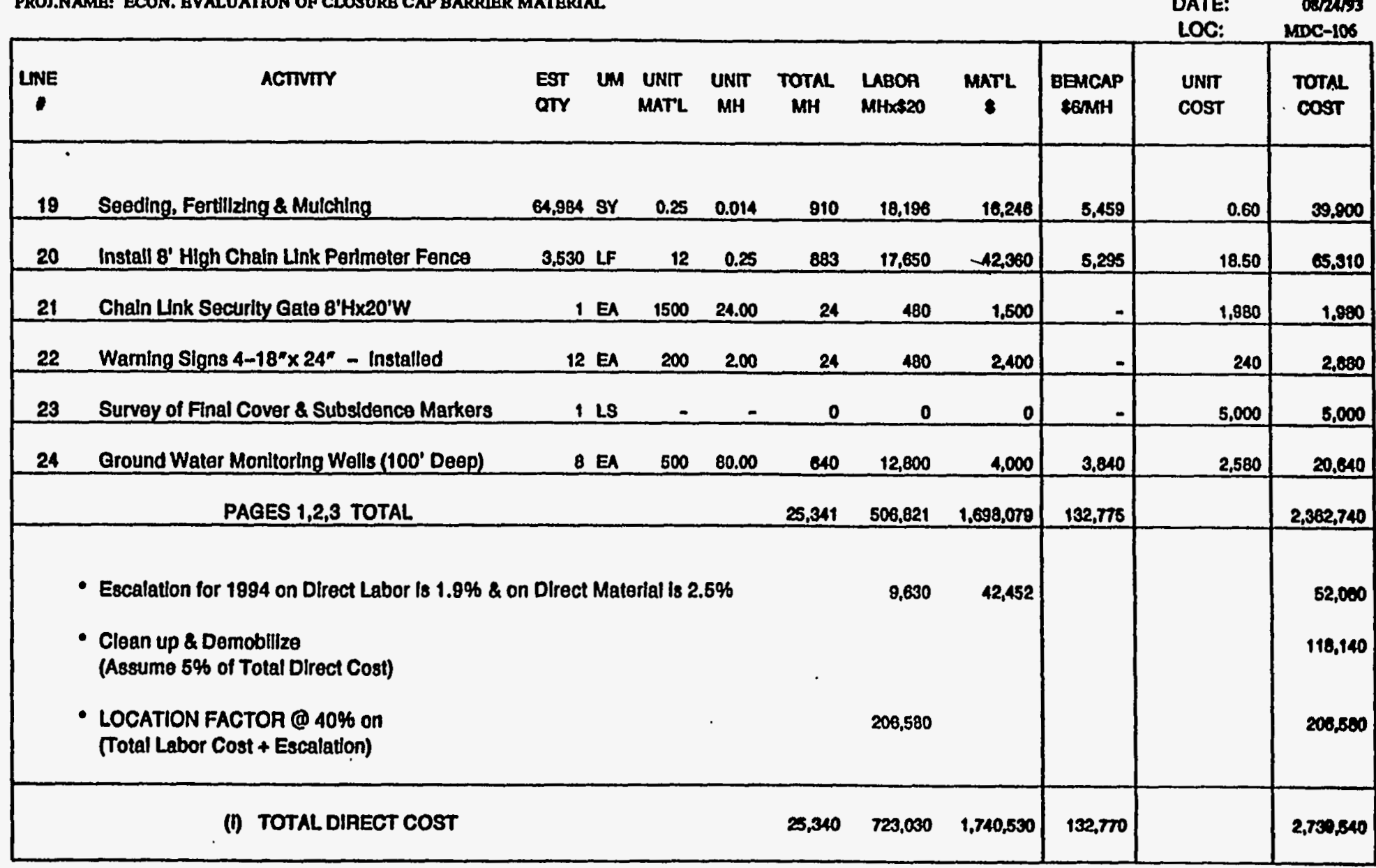

G-3. Cost Estimate for a Cover System Utilizing Gundseal@ (Cont'd) 
200 : : 7-03

D B T A I L

ACTIVITY CODE: BSE260412

PROJ.NAME: ECON. BVALUATION OF CLOSURB CAP BARRIER MATERIAL

CHK'D BY: 8. MEAD

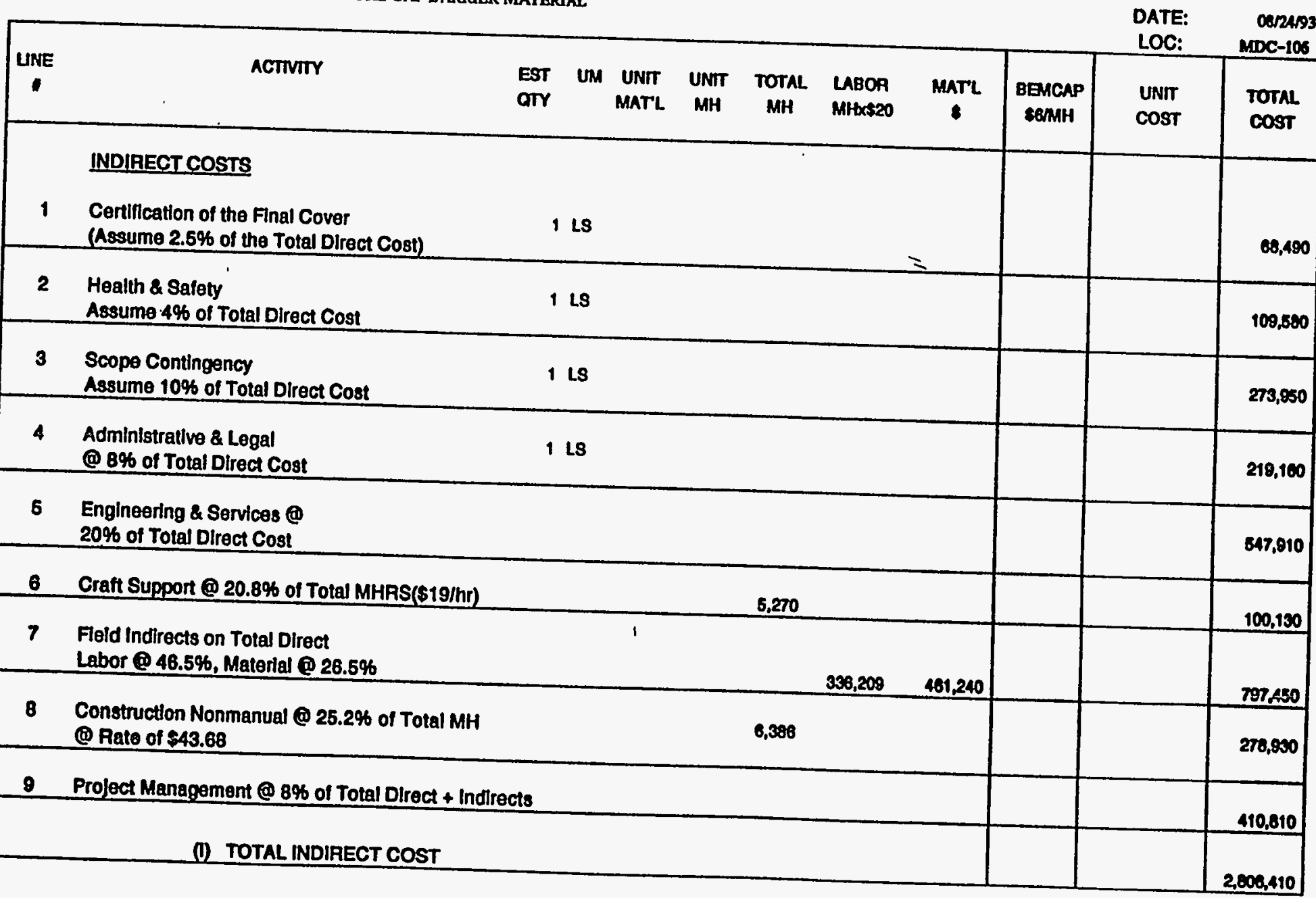

G-3. Cost Estimate for a Cover System Utilizing Gundseal@ (Cont'd) 
D B T A I L E S T I MAT B

CHK'D BY: S. MEAD

Lo0 I: $7-03$

ACTIVTTY CODE: ESE260412

PROJ.NAME: ECON. EVALUATION OF CLOSURE CAP BARRIER MATERSAL

ESTIMATOR: ParknK

PHONE: 7-5206

DATE: Densus

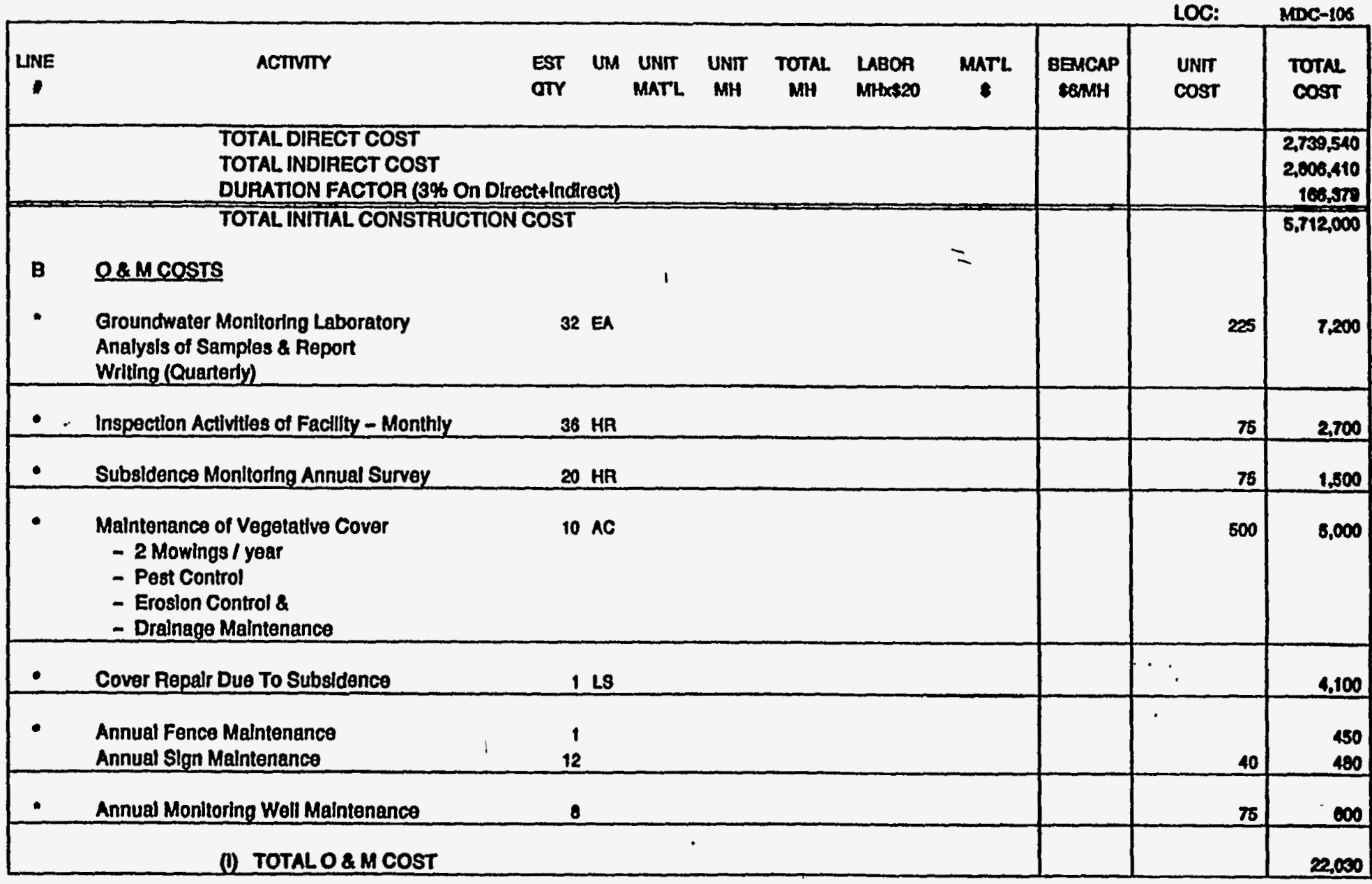

G-3. Cost Estimate for a Cover System Utilizing Gundseal (Cont'd) 
D B T A I L B S T I M A T B

$1001: 7-03$

ACTIVITY CODB: ESE260412

PROI.NAME: ECON. EVALUATION OP CLOSURB CAP BARRIER MATERIAL
CHK'D BY: S. MEAD

ESTIMATOR: POWR:

PHONE: 7-5266

DATE: OA/24r93

MDC-106

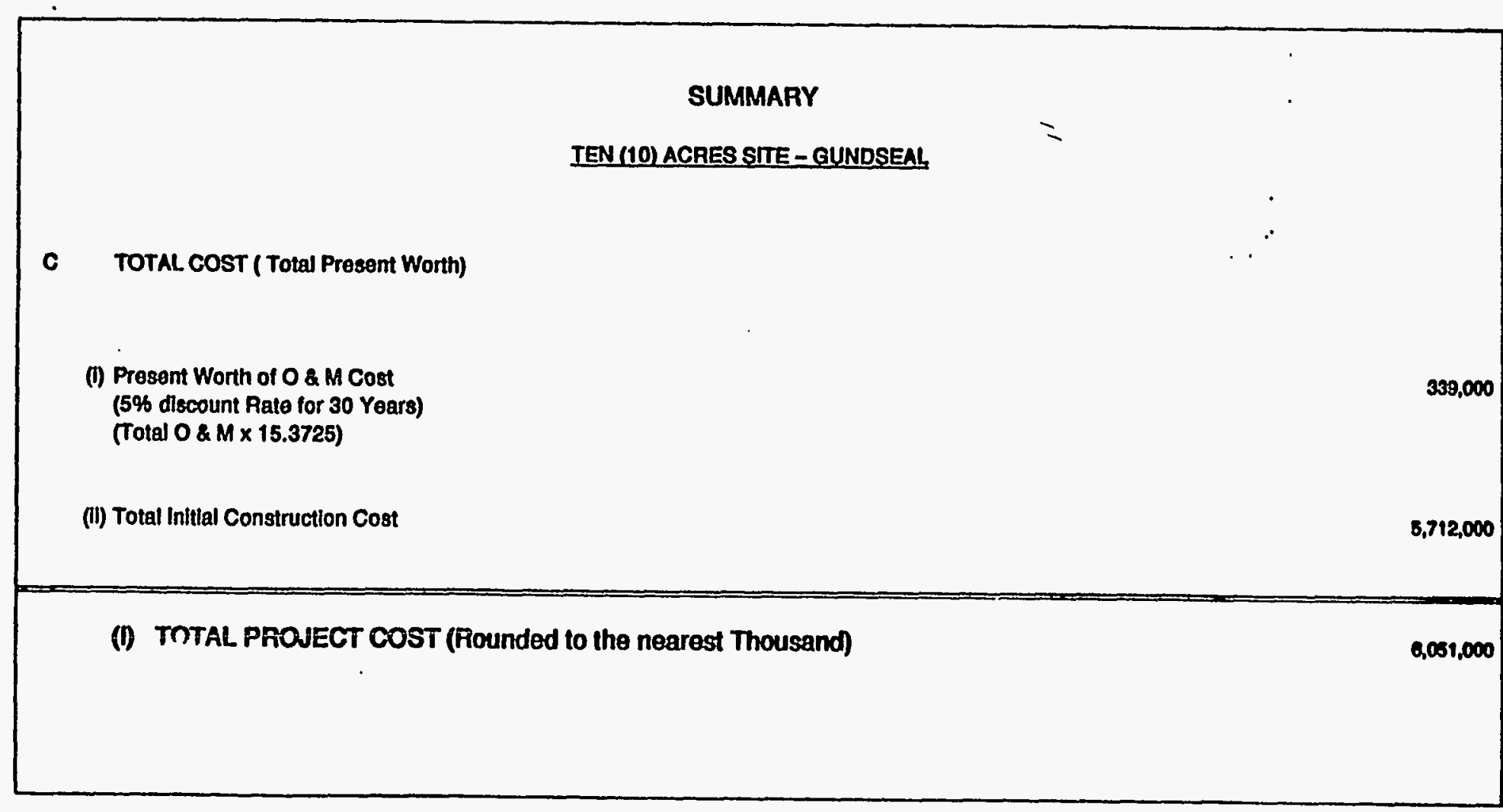

G-3. Cost Estimate for a Cover System Utilizing Gundseal@ (Cont'd) 
DETAIL ESTIMATE

L001: $7-03$

ACTIVTTY CODB: BSE260412

PROJ.NAMB: ECON. BYALUATION OF CLOSURB CAP BARRIBR MATERYAL

CHK'D BY: S. MEAD

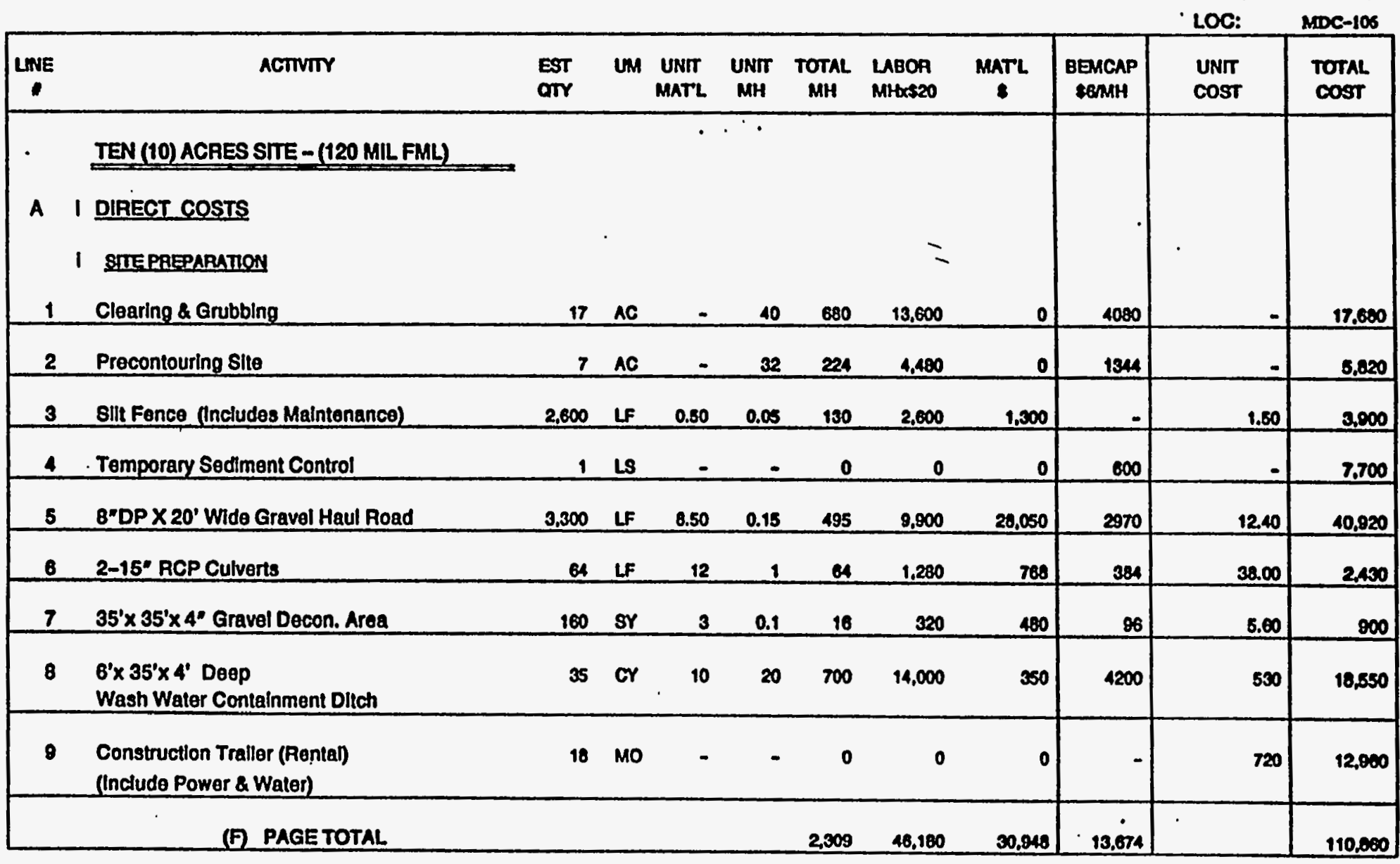

G-4. Cost Estimate for a Cover System Utilizing 120-mil High Density Polyethylene (HDPE) Liner 
DETAIL ESTIMATE

CHK'D BY: S. MEAD

Lo01: 7-03

ACTIVTTY CODE: ESE26O412

PROJ.NAME: ECON. EVALUATTON OF CLOSURE CAP BARRIER MATERIAL

ESTIMATOR: POARLX

\begin{tabular}{|c|c|c|c|c|c|c|c|c|c|c|c|}
\hline & & & & & & & & & & LOC: & MDC-106 \\
\hline UNE & ACTivir & $\begin{array}{l}\text { EST } \\
\text { GTY }\end{array}$ & UM & $\begin{array}{l}\text { UNTT } \\
\text { MATL }\end{array}$ & ${ }_{M H}^{U N T}$ & $\underset{M H}{\text { TOTAL }}$ & $\begin{array}{l}\text { LABOA } \\
\text { MHX+20 }\end{array}$ & $\underset{:}{\operatorname{MATL}}$ & $\begin{array}{l}\text { BEMCAP } \\
\text { SEMMH }\end{array}$ & $\begin{array}{l}\text { UNTT } \\
\text { COST }\end{array}$ & $\begin{array}{l}\text { Total } \\
\cos t\end{array}$ \\
\hline & 11 COVER CONSTRUCTION & & & & & & & & & & \\
\hline 10 & Waste Stabllization (Crushed Limestone) & 13,240 & $\pi \mathbf{N}$ & 75 & 0.1 & 1,394 & 27,880 & $1.045,500$ & 0.364 & 77.60 & 1.001 .740 \\
\hline 11 & $\begin{array}{l}\text { Place and Compact Foundation Soll } \\
\text { In } 8^{\circ} \text { Lifts } 1095 \% \text { Std. Proctor }\end{array}$ & 88,709 & or & - & 0.145 & 12,883 & 257,256 & $=$ & n7.17n & 3.80 & 334,430 \\
\hline 12 & $\begin{array}{l}\text { Placement of } 120 \text { Mil Flexible } \\
\text { Membrane Liner }\end{array}$ & 478,400 & SF & 0.40 & 0.008 & 3,827 & 76,544 & 191,360 & - & 0.60 & 287,900 \\
\hline 13 & $\begin{array}{l}\text { Placement of 1'-0 Coarse Sand } \\
\text { Dralnage Layer }\end{array}$ & 16,875 & or & 10.50 & 0.025 & 122 & 8.438 & $\mathbf{1 7 , 1 8 8}$ & 2,531 & 11.20 & 188.160 \\
\hline 14 & $\begin{array}{l}\text { Placement of Geotextile (TYPAR) Filter on } \\
1^{1}-0^{\prime} \text { Coarse Sand Drainage Layer }\end{array}$ & 52,700 & sr & 0.14 & 0.005 & 234 & 5,270 & 7,378 & - & $\therefore$ & 12,650 \\
\hline 18 & Placement of $1^{\prime}-6^{*}$ Common Fil & 25,867 & or & 3.75 & 0.05 & 1,293 & 25.867 & 97.001 & 7,780 & 8.10 & 130,630 \\
\hline 16 & Placement of 6" Topsoil & 0,438 & or & 9.50 & 0.05 & 422 & 8.438 & $\$ 0,161$ & 2.531 & 10.00 & 91,130 \\
\hline 17 & $\begin{array}{l}\text { Placoment of Perlmeter Exterlor } \\
\text { Dralnage Layer } 2 / 44^{\prime \prime} \times 3^{\prime \prime} \text { Stono }\end{array}$ & 1,358 & sy & 1.50 & 0.16 & 217 & 4,346 & 2,037 & 1,304 & 3.70 & 7,000 \\
\hline 18 & Excavation of Trapezoldal Drainage Dilich & 4.745 & or & $=$ & 0.17 & 807 & 18.133 & 0 & 4,640 & 1.40 & 20,970 \\
\hline & (F) PAGE TOTAL & & & & & 21,509 & 430.171 & $1,000,625$ & 104,507 & & $2.155,300$ \\
\hline
\end{tabular}

G-4. Cost Estimate for a Cover System Utilizing 120-mil High Density Polyethylene (HDPE) Liner (Cont'd) 
D B T A I L E S T I M A T B

CHK'D BY: S. MEAD

2001: 7-03

ACTVITY CODE: ESE260412

PROJ.NAME: ECON. EVALUATION OF CLOSURB CAP BARRIER MATERIAL

ESTIMATOR: POARK

DATE: 0.21193

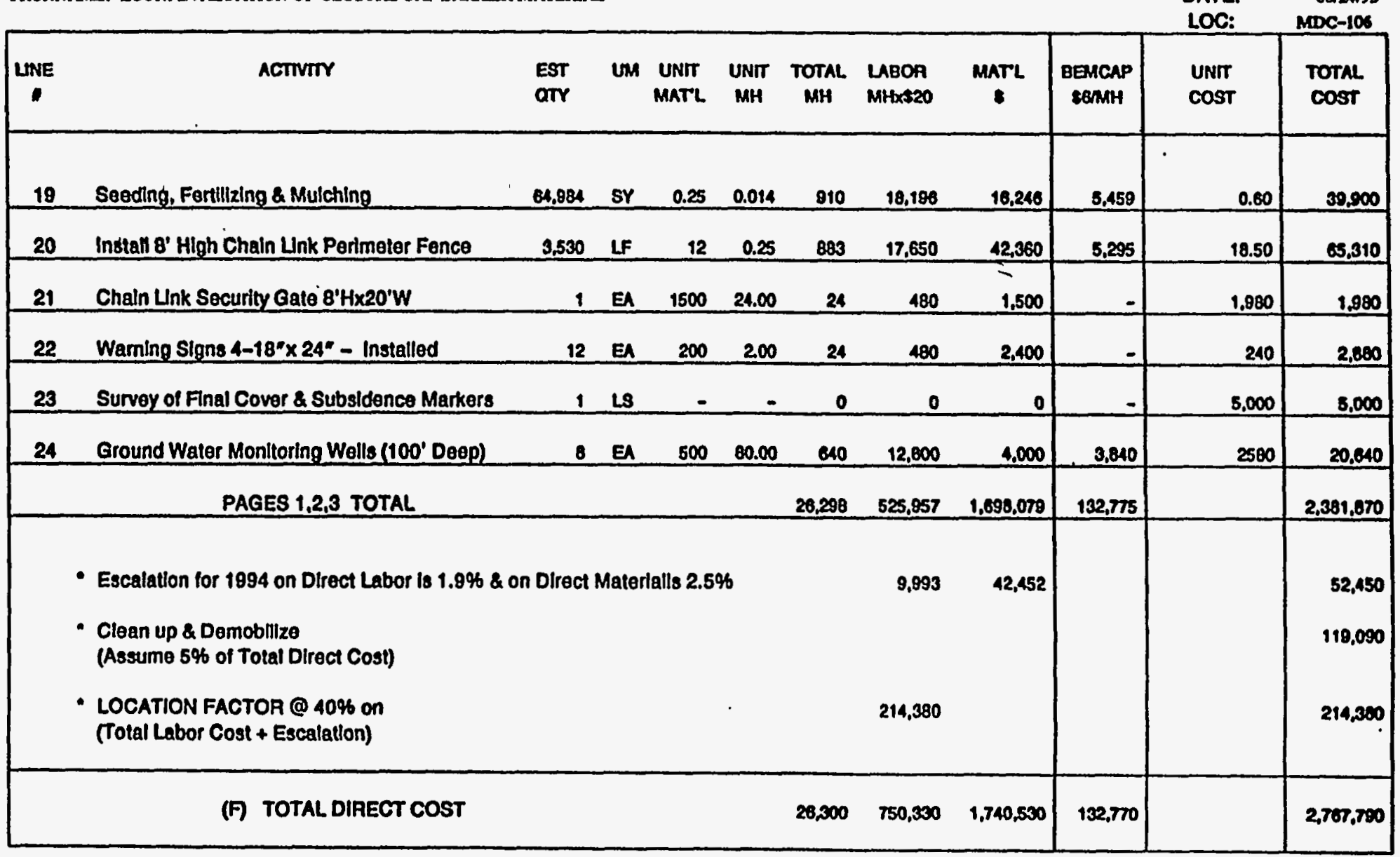

G-4. Cost Estimate for a Cover System Utilizing 120-mil High Density Polyethylene (HDPE) Liner (Cont'd) 
D BTAIL BSTIMAT B

CHK'D BY: S. MEAD

2001: $7-03$

ACTIVITY CODB: ESE260412

PROJ.NAMR: BCON. EVALUATION OF CLOSURB CAP BARRIER MATERINL

ESTIMATOR: POWWX

PHONE: 7-5266

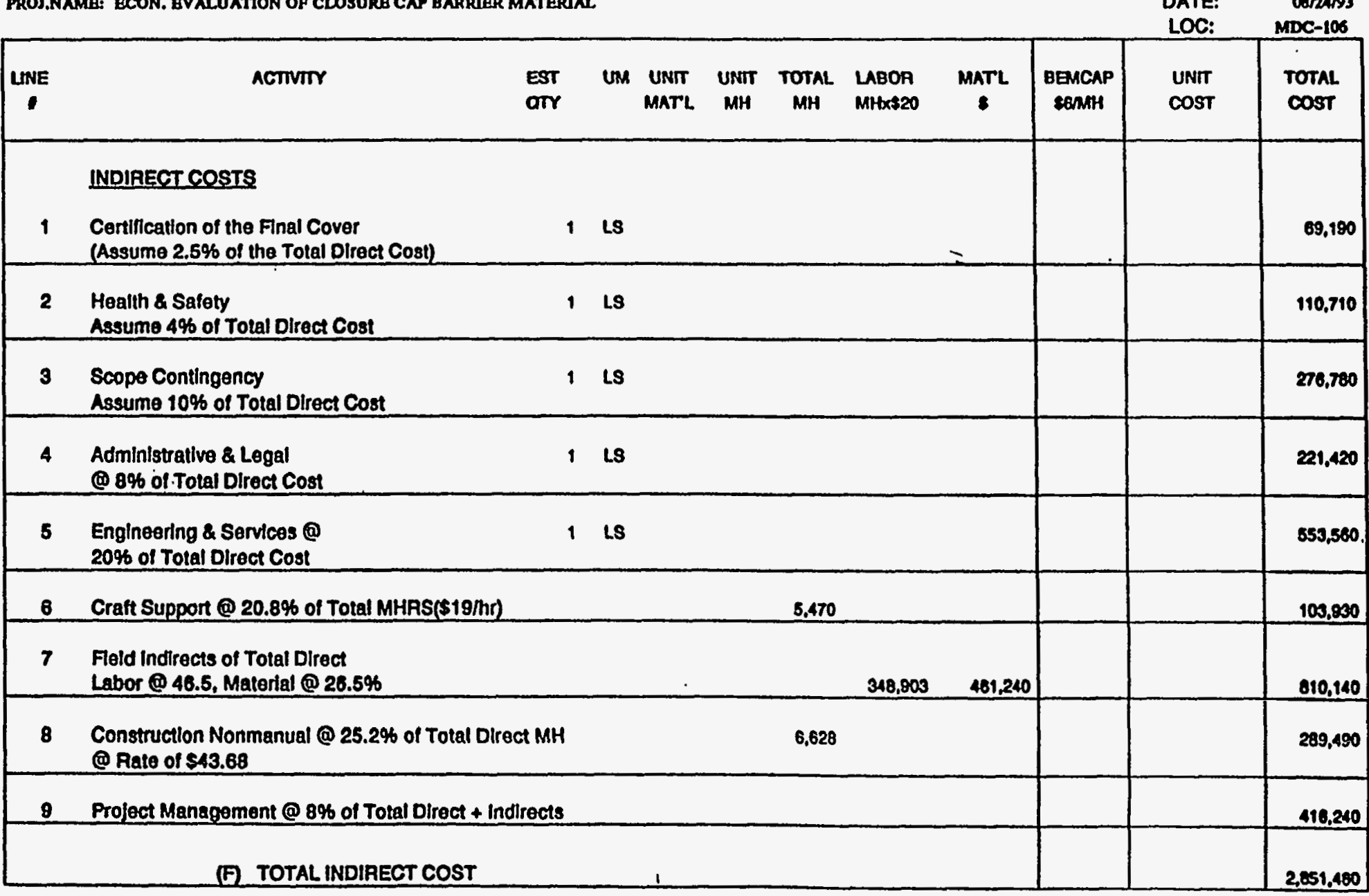

G-4. Cost Estimate for a Cover System Utilizing 120-mil High Density Polyethylene (HDPE) Liner (Cont'd) 
D B T A L ESTIMATB

LOOI: $7-03$

ACTIVITY CODE: ESE260112

PROS.NAME: ECON. EVALUATTON OP CLOSURB CAP BARRTER MATERAAL

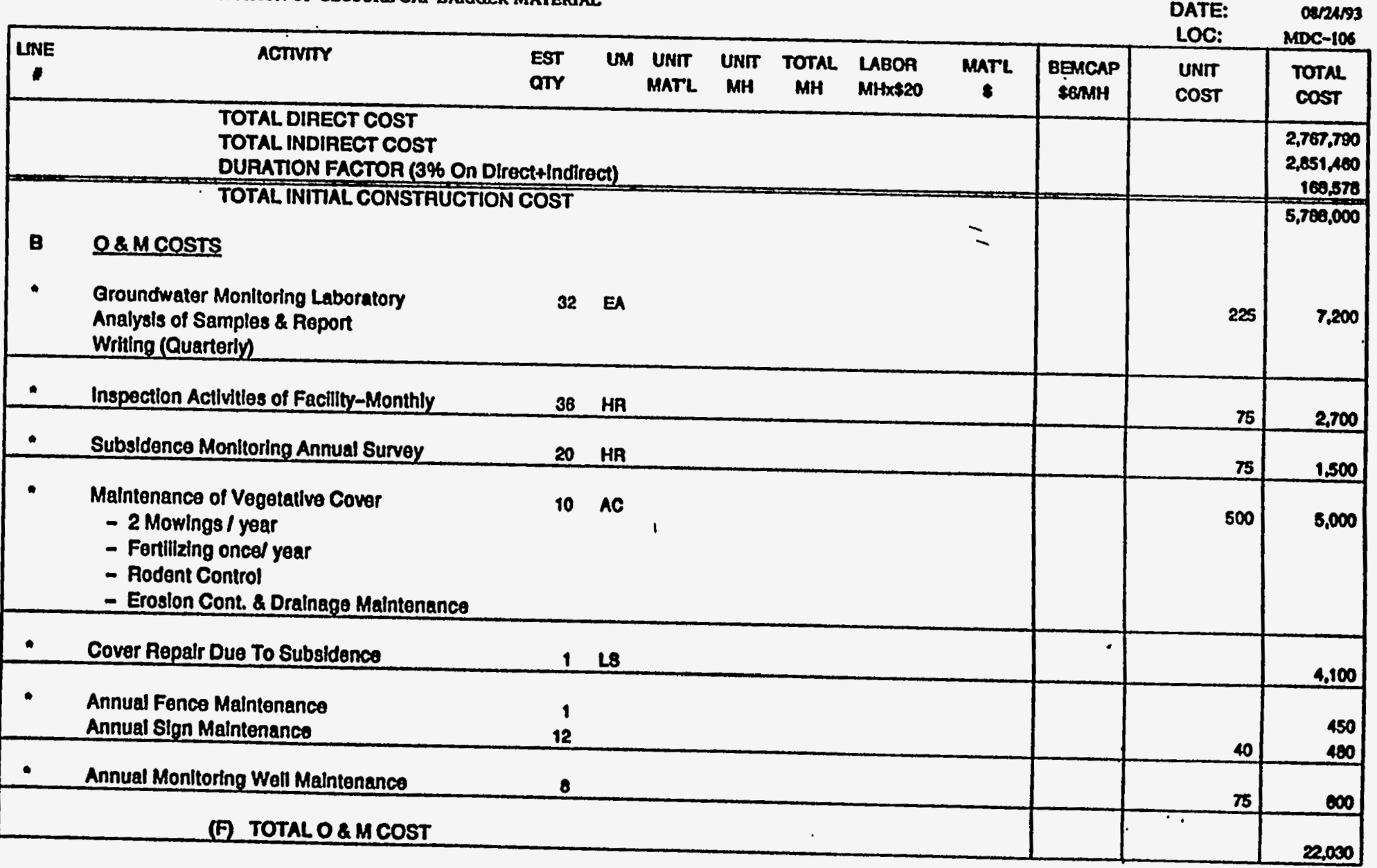

G-4. Cost Estimate for a Cover System Utilizing 120-mil High Density Polyethylene (HDPE) Liner (Cont'd) 
D B T A I L E S T I M A T B

CHK'D BY: S. MEAD

\section{L00 : $7-03$}

ACTIVITY CODR: ESEZ6O412

PROJ.NAME: ECON. BYALUATION OF CLOSURB CAP BARRIER MATERAAL

$\begin{array}{cc}\text { ESTIMATOR: } & \text { PONLK } \\ \text { PHONE: } & 7-5266 \\ \text { DATE: } & \text { OEN193 } \\ \text { LOC: } & \text { MDC-106 }\end{array}$

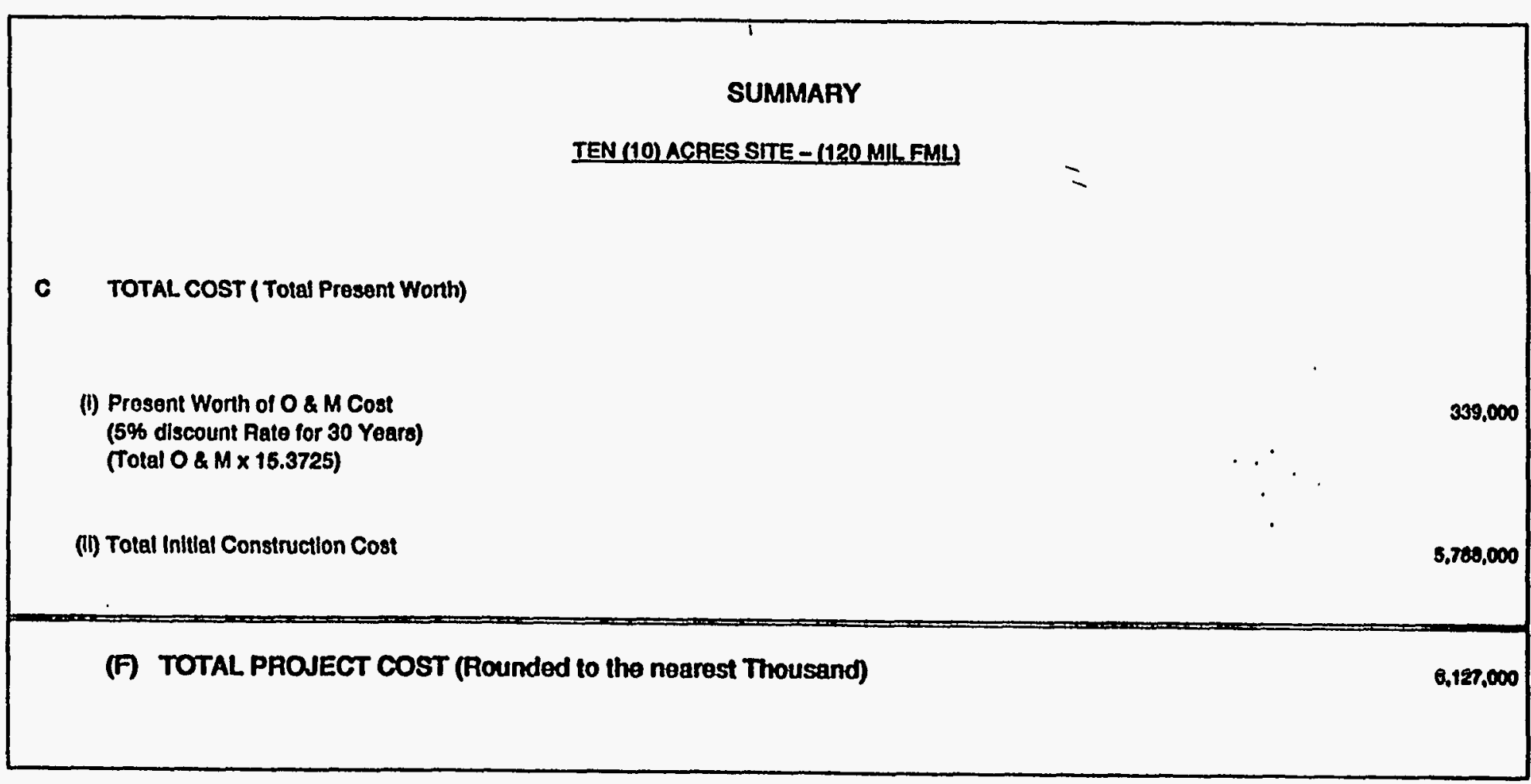

G-4. Cost Estimate for a Cover System Utilizing 120-mil High Density Polyethylene (HDPE) Liner (Cont'd) 
DETAIL ESTIMATE

CHK'D BY: S. MEAD

LOOI: 7-03

ACTTVITY CODE: ESE260A12

PROJ.NAME: BCON. EVALUATTON CLOSURB CAP BARRIER MATERIAL

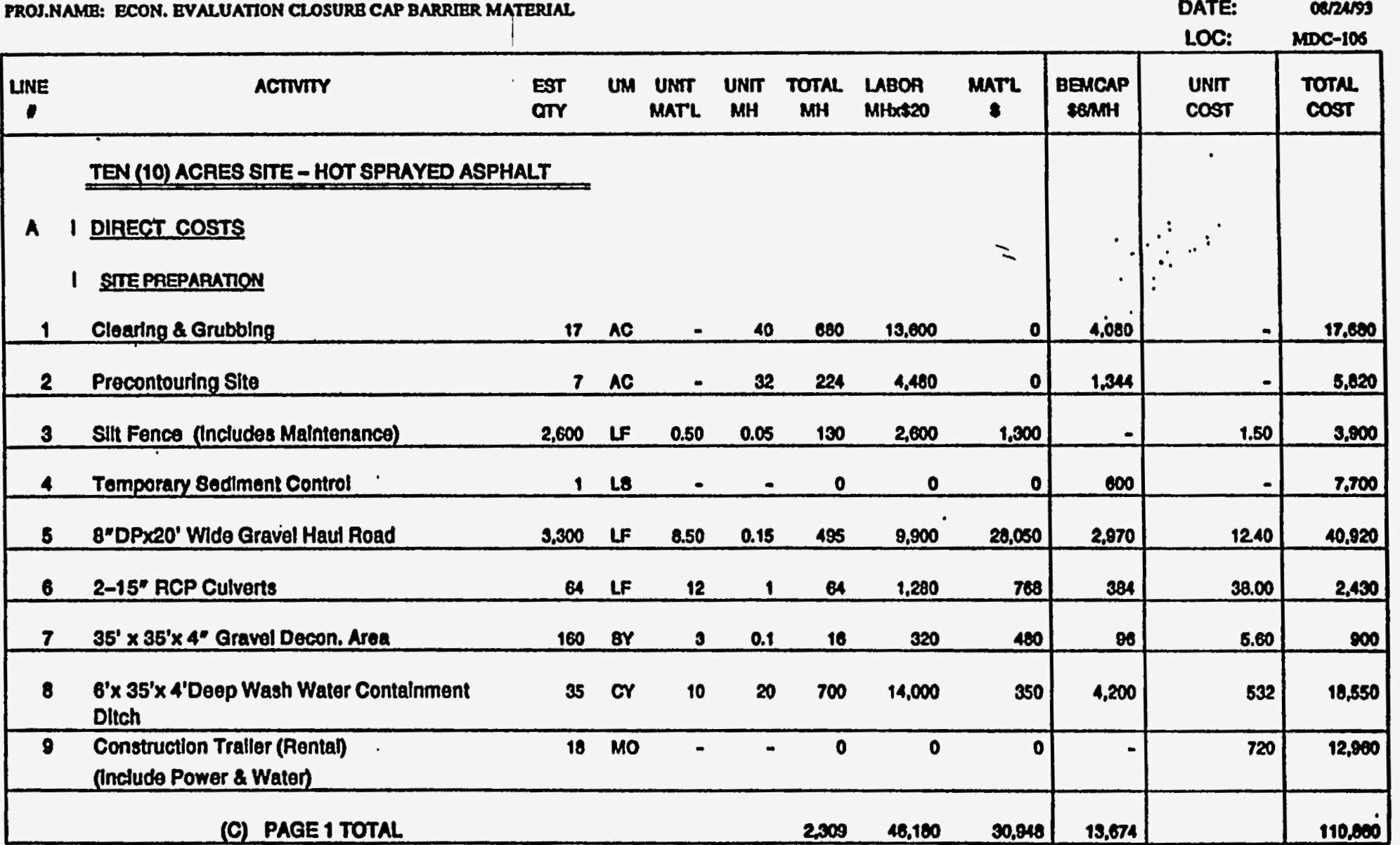

G-5. Cost Estimate for a Cover System Utilizing 1/4-inch Thick Hot Sprayed or Emulsified Asphalt 


$$
\text { LOOA: 7-03 }
$$

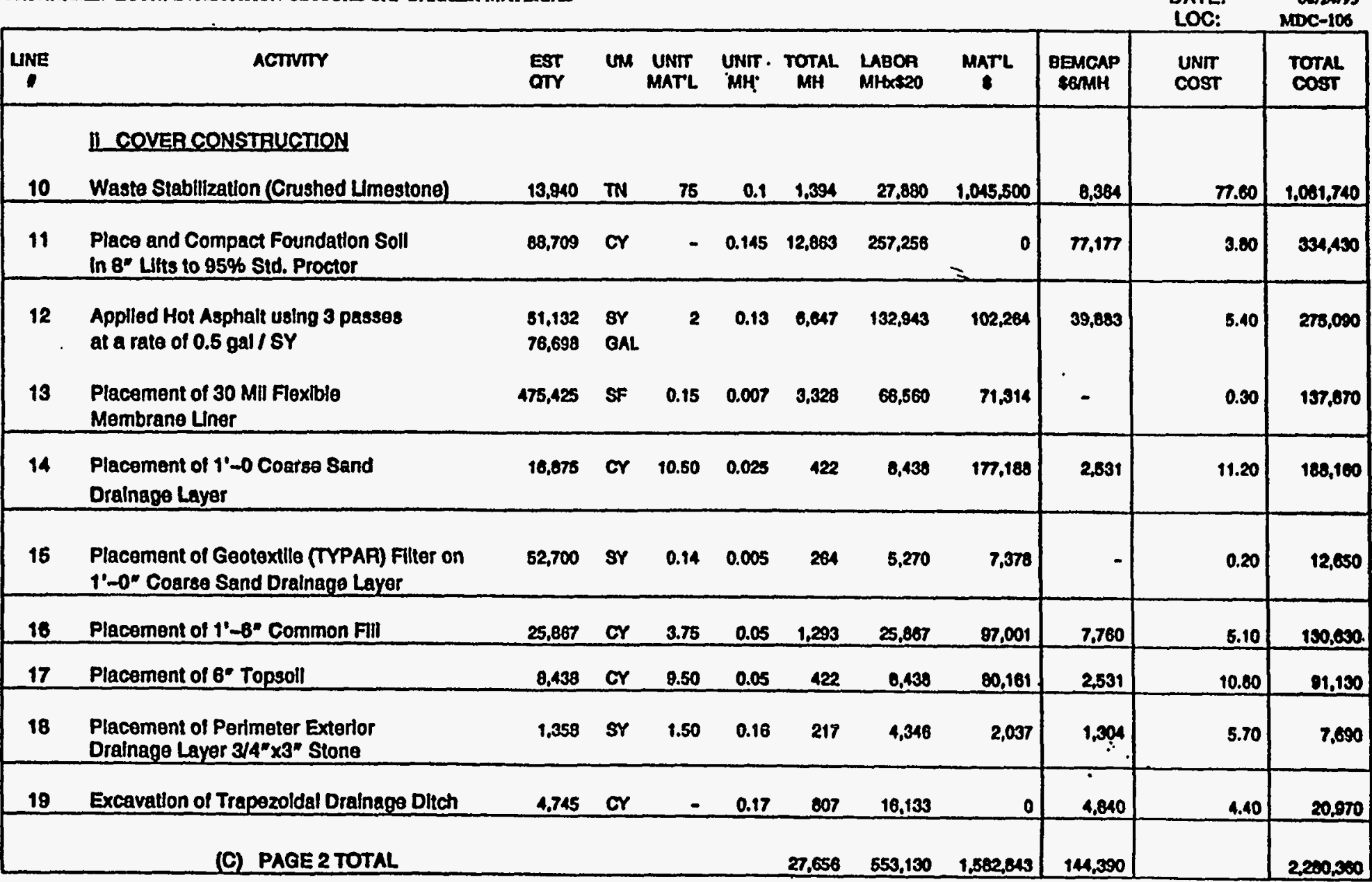

G-5. Cost Estimate for a Cover System Utilizing 1/4-inch Thick Hot Sprayed or Emulsified Asphalt (Cont'd) 
D BTA I L B S T I A T B

Loof: $7-03$

ACTIVITY CODB: ESE260112

PROJ.NAMB: ECON, BVALUATION CLOSURB CAP BARRTER MATERIAL

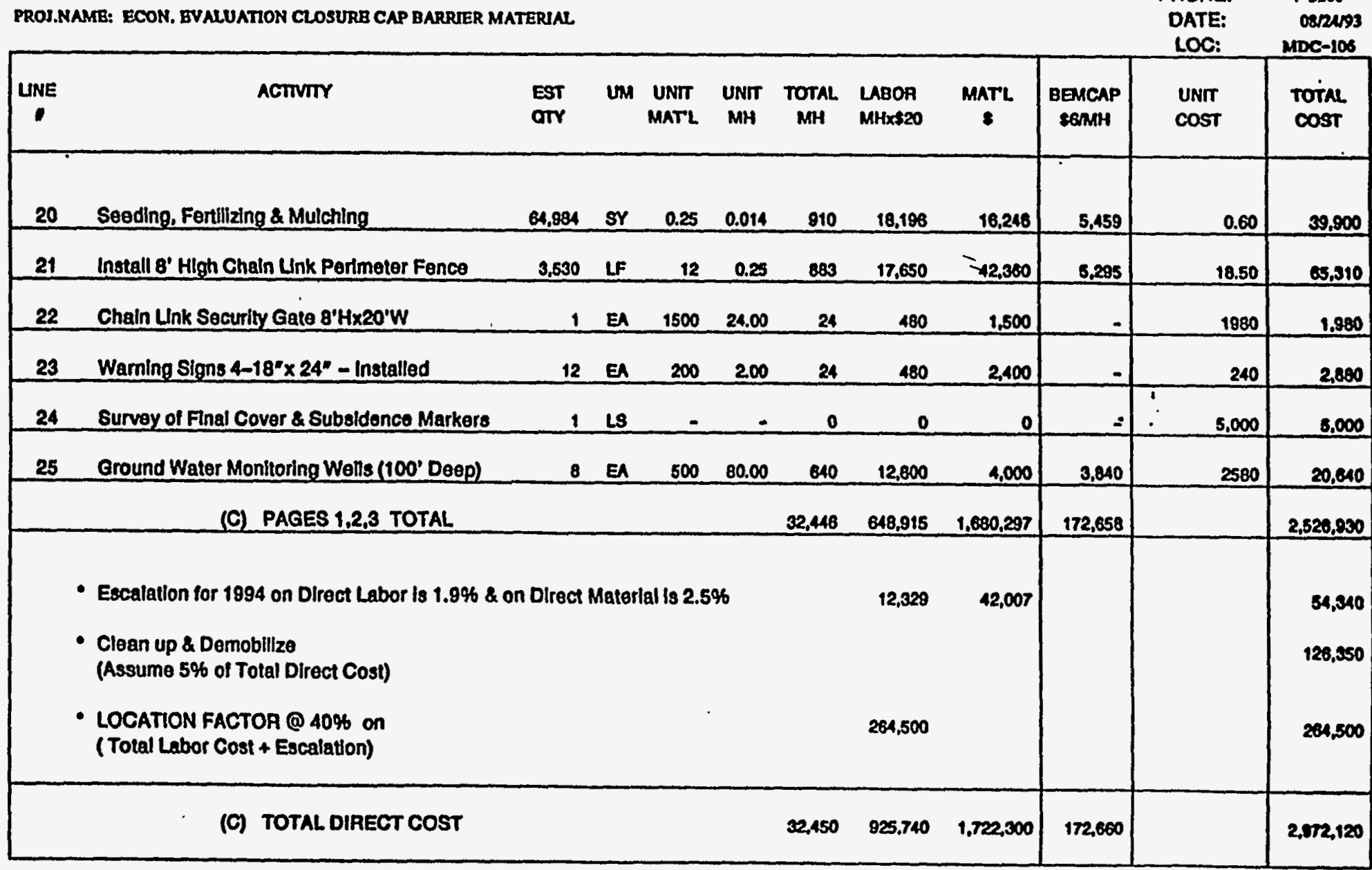

G-5. Cost Estimate for a Cover System Utilizing 1/4-inch Thick Hot Sprayed or Emulsified Asphalt (Cont'd) 
100 1: 7-03

ACTIVTTY CODE: ESE260112

ESTIMATOR: POAMRK

PROJ.NAMR: BCON. BVALUATTON CLOSURE CAP BARRIER MATERIAL

DATE: $0: 2903$

\begin{tabular}{|c|c|c|c|c|c|c|c|c|c|}
\hline UNE & ACTIVTIY & UM UNT & $\begin{array}{l}\text { UNIT } \\
\text { MH }\end{array}$ & $\begin{array}{c}\text { TOTAL } \\
\text { MH }\end{array}$ & $\begin{array}{l}\text { LABOA } \\
\text { MHtas:20 }\end{array}$ & MaTL. & $\begin{array}{l}\text { BEMCAP } \\
\text { SGMH }\end{array}$ & $\begin{array}{l}\text { UNrT } \\
\text { cost }\end{array}$ & $\begin{array}{l}\text { TOTAL } \\
\text { COST }\end{array}$ \\
\hline & INDIRECT COSTS & & & & & & & & \\
\hline 1 & $\begin{array}{l}\text { Certification of the Final Cover } \\
\text { (Assume } 2.5 \% \text { of the Total Direct Cosi) }\end{array}$ & LS & & & & & & & 74,300 \\
\hline 2 & $\begin{array}{l}\text { Health \& Salety } \\
\text { Assume } 4 \% \text { of Total Dlrect Cost }\end{array}$ & LS & & & & & & & 110,600 \\
\hline 3 & $\begin{array}{l}\text { Scope Conthingency } \\
\text { Assume } 10 \% \text { of Total Direct Cost }\end{array}$ & LS & & & & & & & 297,210 \\
\hline 4 & $\begin{array}{l}\text { Administrallve \& Legal } \\
@ 8 \% \text { of Total Direct Cost }\end{array}$ & LS & & & ' & & & & 237.770 \\
\hline 5 & $\begin{array}{l}\text { Engineering \& Services @ } \\
20 \% \text { of Total Direct Cost }\end{array}$ & & & & & & & & 890,420 \\
\hline $\mathbf{B}$ & Craft Support @ $20.8 \%$ of Tolal Dlrect MHRS $(\$ 19 / \mathrm{hr})$ & & & 0,750 & & & & & 128,250 \\
\hline 7 & $\begin{array}{l}\text { Field indirects on Total Direct } \\
\text { Labor @ 46.5\%, Material @ 26.5\% }\end{array}$ & & & & 430,469 & 456,410 & & & 868.060 \\
\hline 8 & $\begin{array}{l}\text { Construction Nonmanual @ } 25.2 \% \text { of Total Direct MH } \\
\text { @ Rate of } \$ 43.68\end{array}$ & & & 0.178 & & & & & 357.140 \\
\hline 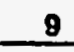 & Project Management @ 8\% on Total Direct + Indirects & & & & & & & & 153,360 \\
\hline & (C) TOTAL INDIAECT COST & & & & & & & & $\mathbf{5 . 1 4 0 , 2 1 0}$ \\
\hline
\end{tabular}

G-5. Cost Estimate for a Cover System Utilizing 1/4-inch Thick Hot Sprayed or Emulsified Asphalt (Cont'd) 
D BTAIL BSTIMATB

CHK'D BY: S. MEAD

Lo01: $7-03$

ACTIVITY CODE: ESEZ60412

PROJ.NAME: ECON. EVALUATION CLOSURB CAP BARRIER MATERIAL

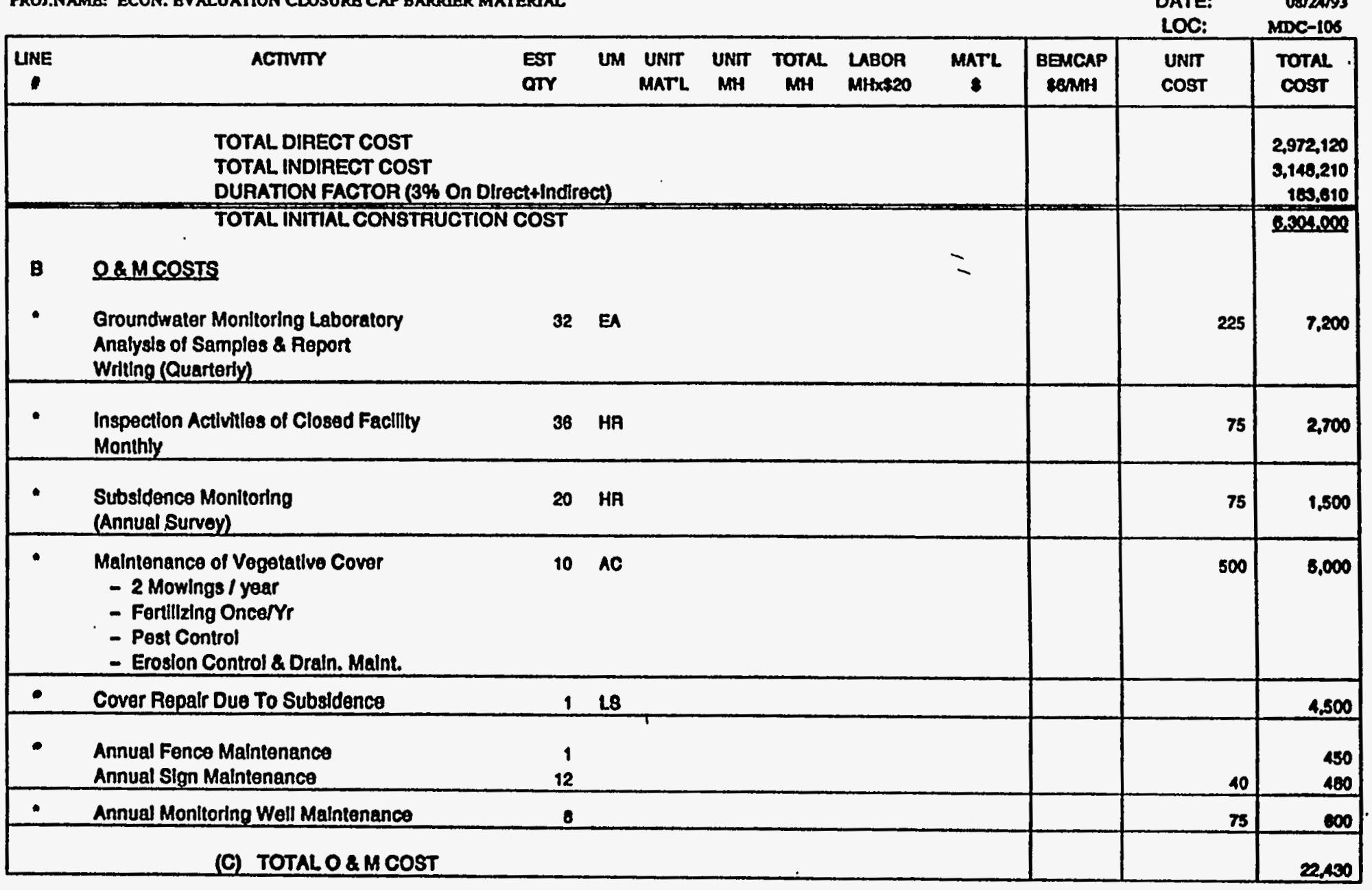

G-5. Cost Estimate for a Cover System Utilizing 1/4-inch Thick Hot Sprayed or Emulsified Asphalt (Cont'd) 
D B T A I L B S I M A B

CHK'D BY: 8. MEAD

Loo 1: 2-03

ACTIVITY CODE: ESE260412

PROJ.NAME: ECON. BVALUATION CLOSURA CAP DARRIER MATERIAL.

$\begin{array}{lc}\text { ESTIMATOR: } & \text { ra/mx } \\ \text { PHONE: } & 7-5266 \\ \text { DATE: } & 0212493 \\ \text { LOC: } & \text { MDC-106 }\end{array}$

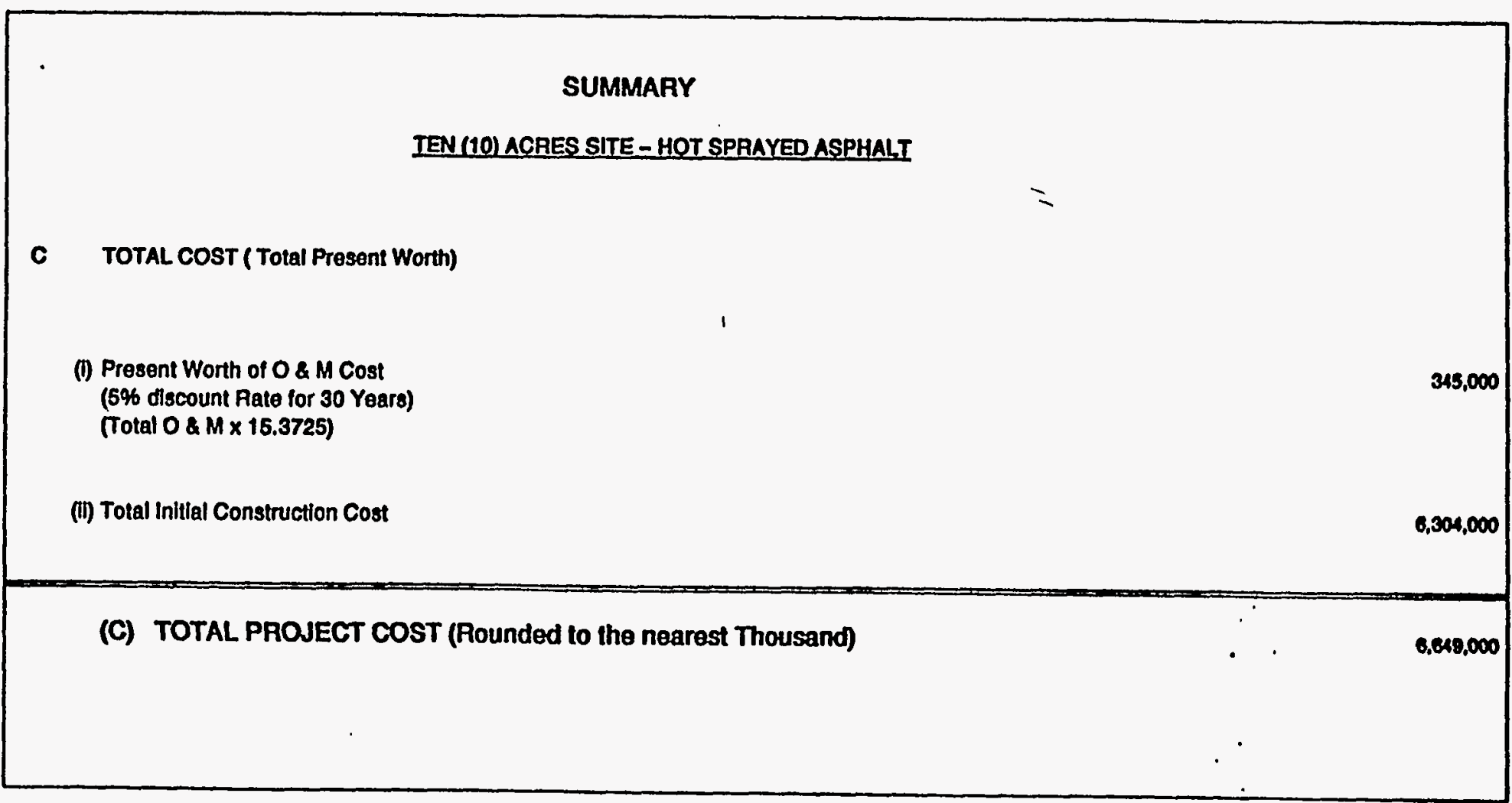

G-5. Cost Estimate for a Cover System Utilizing 1/4-inch Thick Hot Sprayed or Emulsified Asphalt (Cont'd) 
DETAIL ESTIMATE

LOG : 7-03

ACTIVITY CODE: ESE260412

PRON.NAME: ECON. EVALUATION OF CLOSURE CAP BARRIER MATĘRIAL

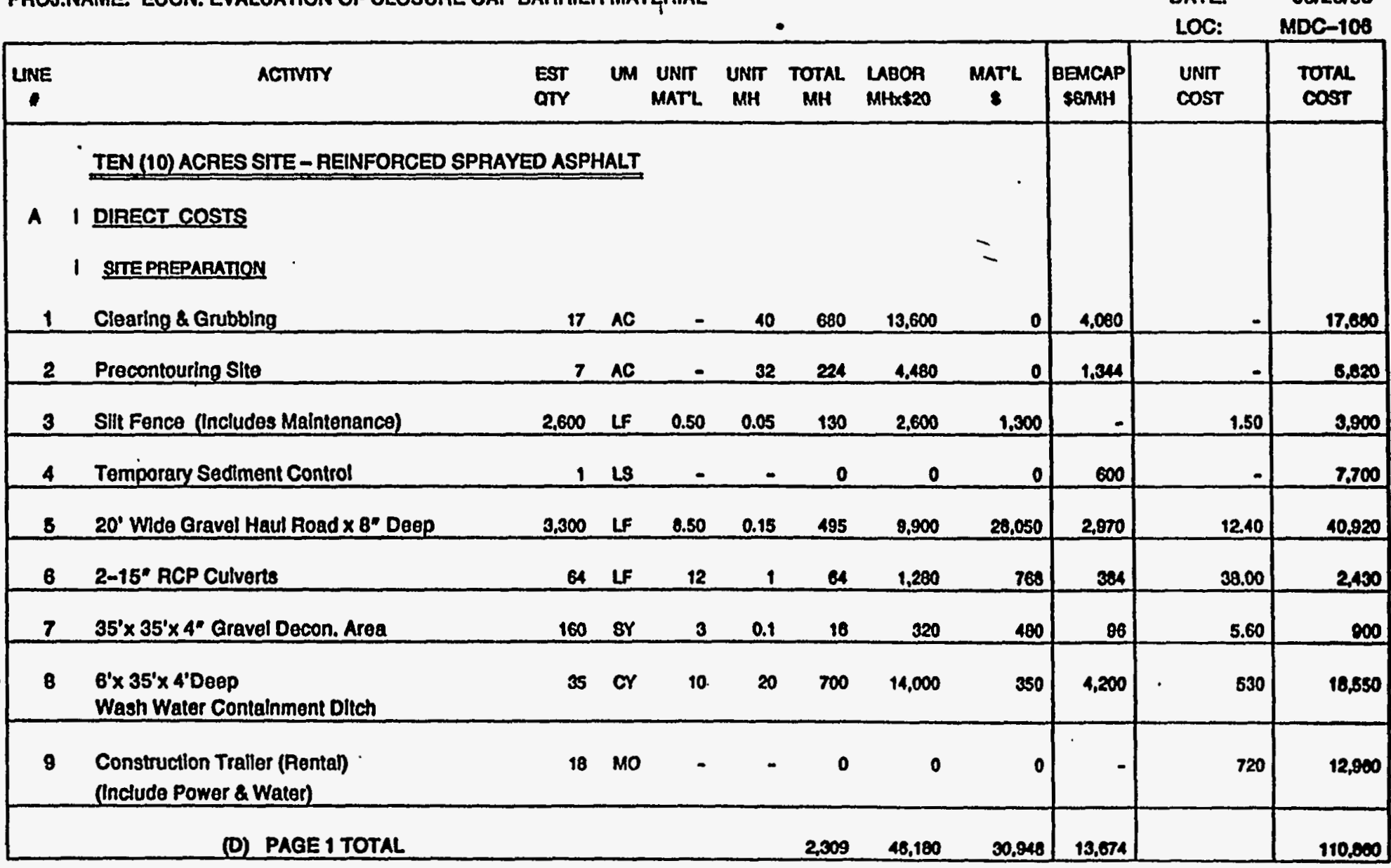

\section{G-6. Cost Estimate for a Cover System Utilizing Reinforced Sprayed Asphalt}


DETAIL ESTIMATE

- CंH'D BY: S. MEAD

LOG A : 7-03

ACTIVITY CODE: ESE260412

PROS.NAME: ECON. EVALUATION OF CLOSURE CAP BARAIER MATERIAL

\begin{tabular}{|c|c|c|c|c|c|c|c|c|c|c|c|}
\hline UNE & ACTIVTr & $\begin{array}{l}\text { EST } \\
\text { QTY }\end{array}$ & UM & $\begin{array}{l}\text { UNTT } \\
\text { MATL }\end{array}$ & $\begin{array}{l}\text { UNTI } \\
\text { MH }\end{array}$ & $\underset{M H}{\text { TOTAL }}$ & $\begin{array}{l}\text { LABOR } \\
\text { MHX } \$ 20\end{array}$ & MATL & $\begin{array}{l}\text { BEMCAP } \\
\text { SOMMH }\end{array}$ & $\begin{array}{l}\text { UNT } \\
\text { COST }\end{array}$ & $\begin{array}{l}\text { TOTAL } \\
\text { COST }\end{array}$ \\
\hline & II COVER CONSTRUCTION & & & & & & & & & & \\
\hline 10 & Waste Stabilization (Crushed Limestone) & 13,940 & TN & 75 & 0.1 & 1.394 & 27.880 & $1,045,500$ & 8,364 & 77.60 & $1.081,740$ \\
\hline 11 & $\begin{array}{l}\text { Place and Compact Foundallon Soll } \\
\text { in } 8^{*} \text { Lifts to } 95 \% \text { Sid. Proctor }\end{array}$ & 88,709 & or & - & 0.143 & 12,803 & 257,256 & $\mathbf{0}$ & $n, 1 n$ & 3.80 & $\mathbf{3 3 4 , 4 3 0}$ \\
\hline 12 & $\begin{array}{l}\text { Placement of } 30 \text { Mill Flexible } \\
\text { Membrane Lner }\end{array}$ & 475,425 & SF & 0.15 & 0.007 & 3,328 & 66,560 & 71,314 & - & 0.30 & 137,870 \\
\hline 13 & $\begin{array}{l}\text { Placement of Geotextlle Reinforcement } \\
\text { on Foundation Layer }\end{array}$ & 53,200 & sy & 0.20 & 0.005 & 286 & 5,320 & 13,832 & $\therefore$ & 0.40 & 19,150 \\
\hline 14 & $\begin{array}{l}\text { Applled Hot Asphalt using } 3 \text { passes at a rale } \\
\text { of } 0.5 \text { gal / SY for a total of } 1.5 \mathrm{~g} / \mathrm{Sy}\end{array}$ & $\begin{array}{l}51,132 \\
76,698\end{array}$ & $\begin{array}{l}\text { SY } \\
\text { GAL }\end{array}$ & 2.00 & 0.13 & 8,647 & 132,943 & 102,264 & 39,883 & 5.40 & 273,090 \\
\hline 15 & $\begin{array}{l}\text { Placement of } 1 '-0 \text { Coarse Sand } \\
\text { Dralnage Layer }\end{array}$ & 16,875 & or & 10.50 & 0.025 & 122 & 8,438 & 177,160 & 2,531 & 11.20 & 188.160 \\
\hline 18 & $\begin{array}{l}\text { Placement of Geotextile (TYPAR) Filter on } \\
1^{\prime}-0^{\prime} \text { Coarse Sand Dralnage Layer }\end{array}$ & 52,700 & SY & 0.14 & 0.005 & 284 & 5,270 & 7,378 & - & 0.20 & 12,050 \\
\hline 17 & Placement of $1^{\prime}-6^{\prime}$ Common Fil & 25,867 & or & 3.75 & 0.05 & 1,293 & 25.867 & 97,001 & 7.760 & 5.10 & 130.600 \\
\hline 18 & Placement of 6" Topsoll & 0.438 & or & 8.50 & 0.05 & 422 & 8,438 & 80,161 & 2.531 & 10.80 & 91,130 \\
\hline 18 & $\begin{array}{l}\text { Placement of Perlmeter Exterlor } \\
\text { Drainage Layer } 3 / 4^{\prime \prime} \times 3^{*} \text { DP (Crushed Stone) }\end{array}$ & 1,358 & SY & 1.50 & 0.16 & 217 & 4,346 & 2,037 & 1,304 & 5.70 & 7,090 \\
\hline 20 & Excavation of Trapezoldal Drainage Ditch & 4.745 & cr & - & 0.17 & 807 & 16,133 & 0 & 4.840 & 4.40 & 20.070 \\
\hline & (D) PAGE TOTAL & & & & & 27,922 & 550,450 & $1,596,675$ & 144,390 & & $2,299,510$ \\
\hline
\end{tabular}

G-6. Cost Estimate for a Cover System Utilizing Reinforced Sprayed Asphalt (Cont'd) 
DETAIL ESTIMATE

CHK'D BY: S. MEAD

LOG : : 7-03

ACTIVITY CODE: ESE260412

PROS.NAME: ECON. EVALUATION OF CLOSURE CAP BARRIER MATERIAL

ESTIMATOR: PONMLK

PHONE: 7-5266

DATE: $\quad 08 / 26 / 93$

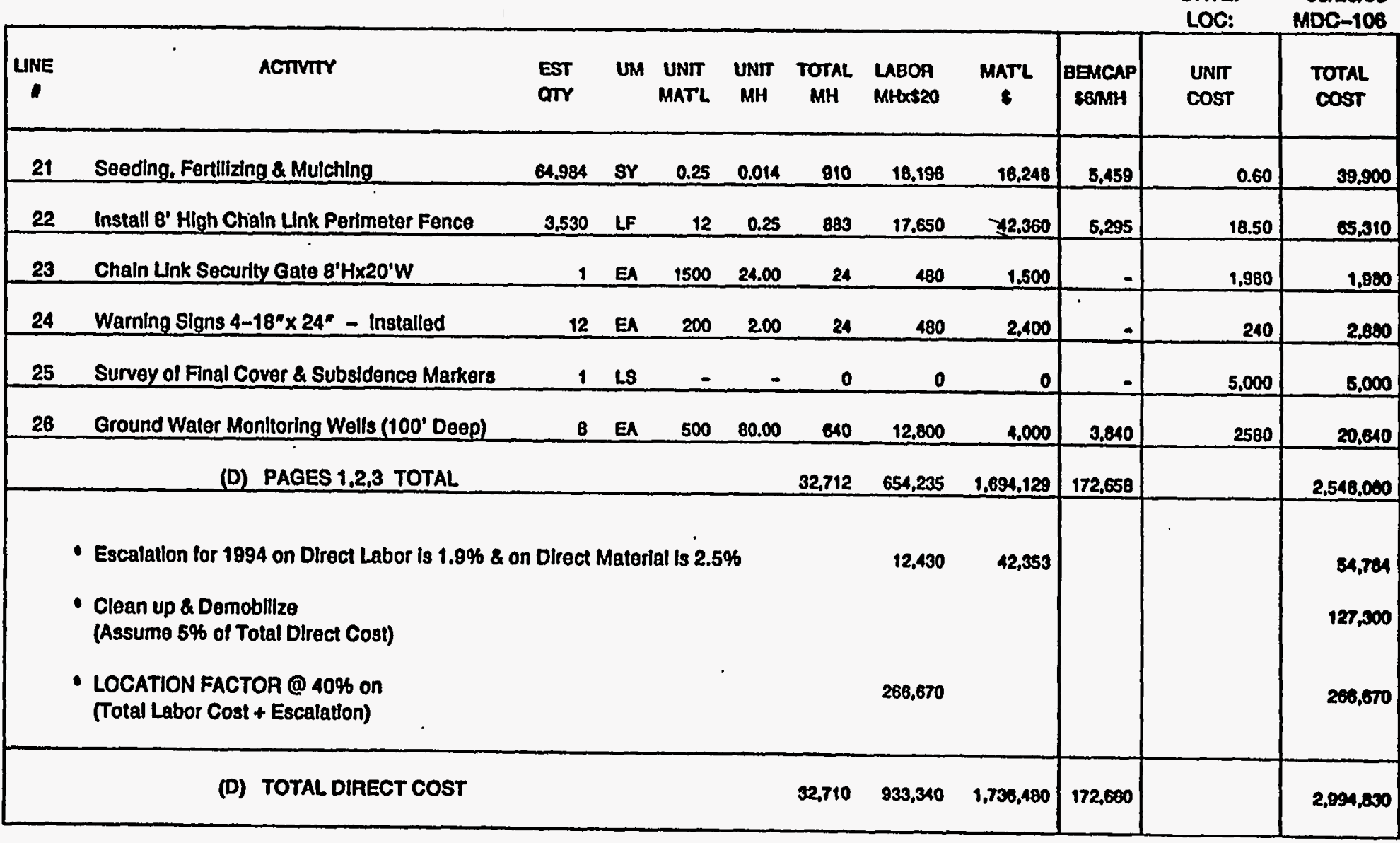

G-6. Cost Estimate for a Cover System Utilizing Reinforced Sprayed Asphalt (Cont'd) 
DETAIL ESTIMATE CHK'DBY: S. MEAD

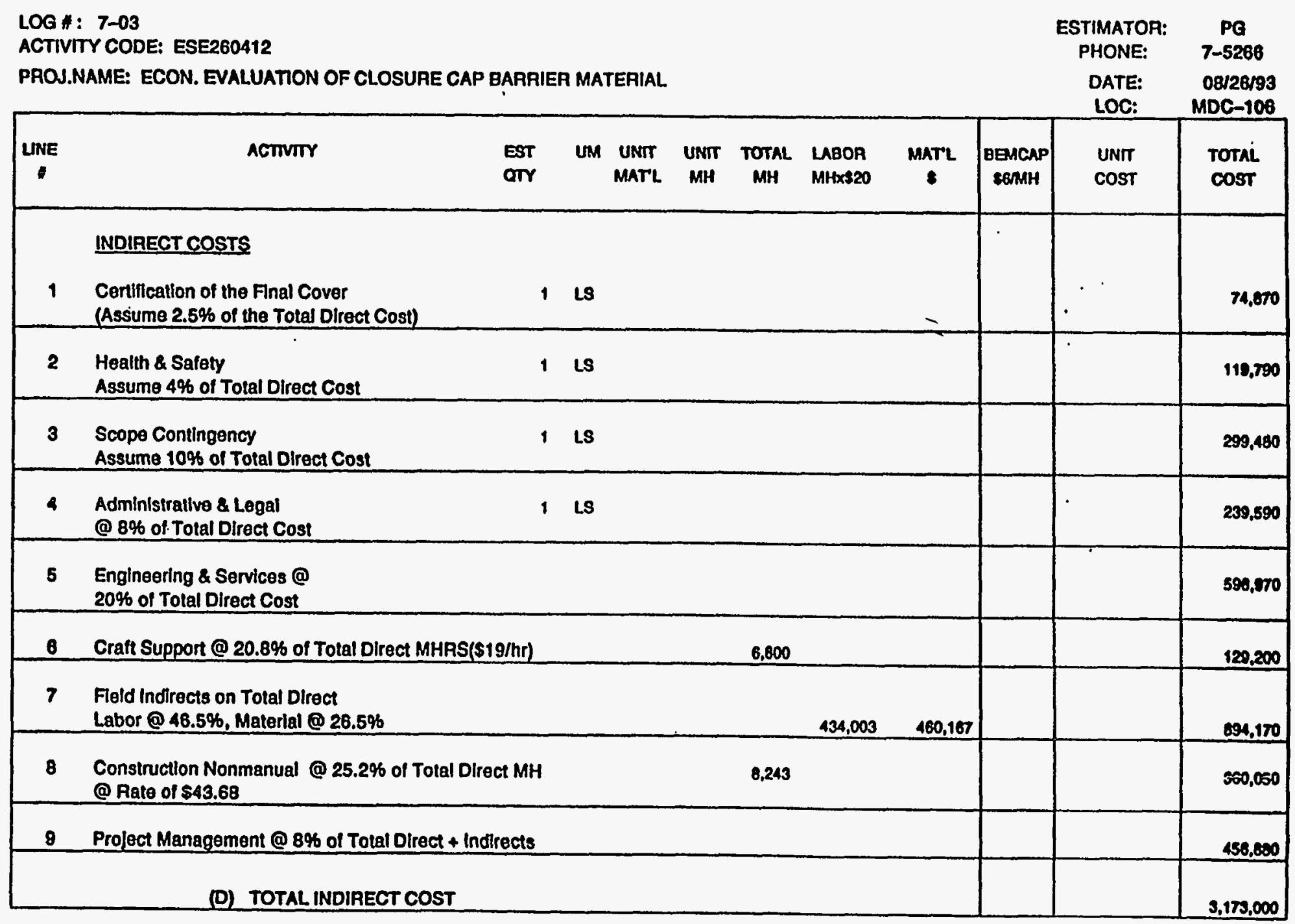

G-6. Cost Estimate for a Cover System Utilizing Reinforced Sprayed Asphalt (Cont'd) 
DETAIL ESTIMATE CHK'DBY: S.MEAD.

LOG A : 7-03

ACTIVITY CODE: ESE260412

PROJ.NAME: ECON. EVALUATION OF CLOSURE CAP BARRIER MATERIAL

ESTIMATOR: $\quad$ PG

DATE: 08/26/93

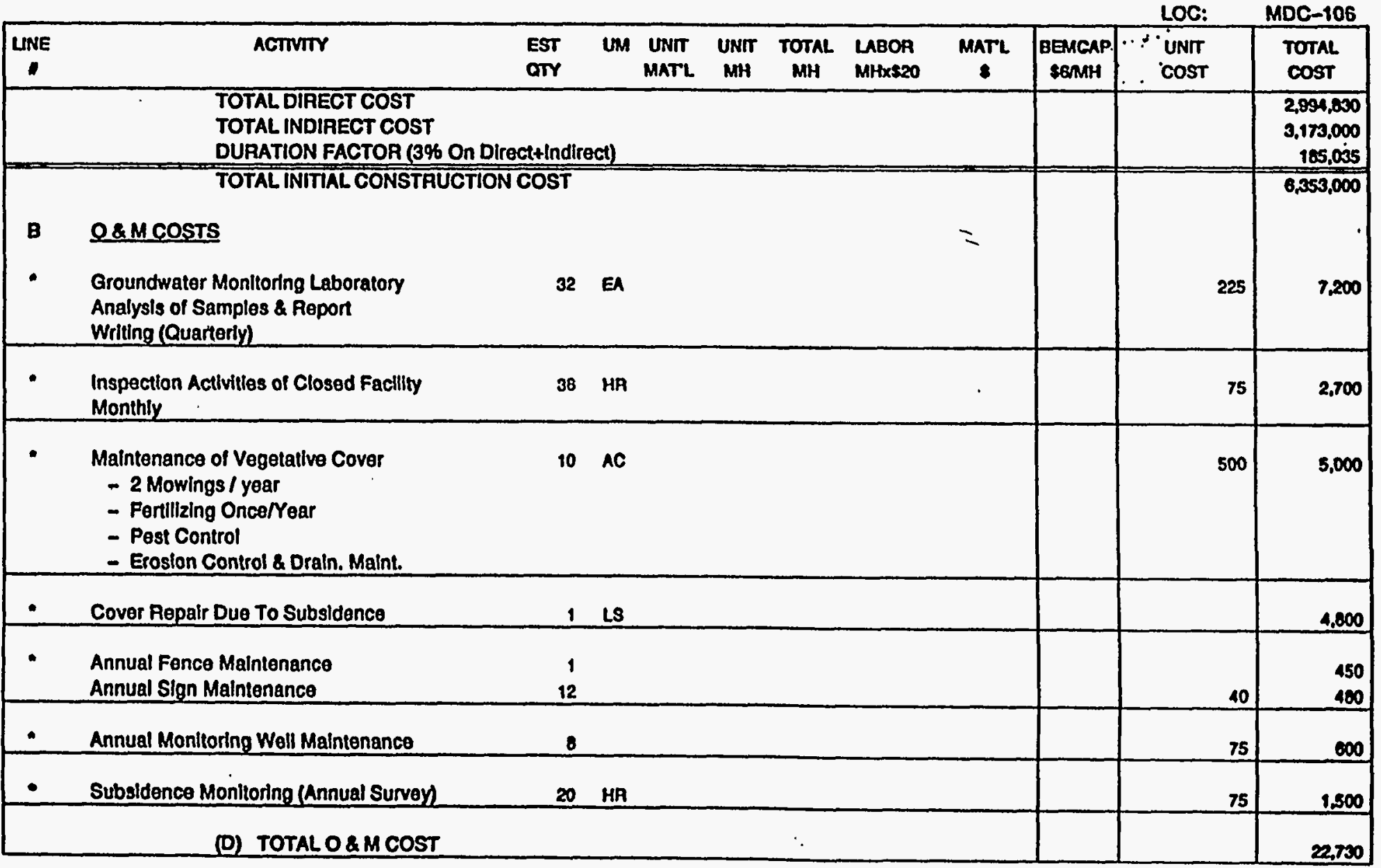

G-6. Cost Estimate for a Cover System Utilizing Reinforced Sprayed Asphalt (Cont'd) 
DETAIL ESTIMATE

$\begin{array}{cc}\text { CHK'O BY: } & \text { S. MEAD } \\ \text { ESTIMATOR: } & \text { PG } \\ \text { PHONE: } & 7-5286 \\ \text { DATE: } & 08 / 26 / 93 \\ \text { LOC: } & \text { MDC-106. }\end{array}$

LOG : : 7-03

ACTIVITY CODE: ESE260412

PROJ.NAME: ECON. EVALUATION OF CLOSURE CAP BARRIER MATERIAL.

LOC: MDC-106

\begin{tabular}{l} 
TEN (10) ACRES SITE - REINFORCED SPRAYED ASPHALT \\
SUMMARY \\
C TOTAL COST ( Total Present Worth) \\
(i) Present Worth of O \& M Cost \\
(5\% discount Rate for 30 Years) \\
(Total O \& $M \times 15.3725)$ \\
(ii) Total Inttlai Construction Cost \\
(D) TOTAL PROJECT COST (Rounded to the nearest Thousand) \\
\hline
\end{tabular}

G-6. Cost Estimate for a Cover System Utilizing Reinforced Sprayed Asphalt (Cont'd) 
DETAIL ESTIMATE

LOO I: :-03

ACTIVITY CODE: ESE260412

PROJ.NAMB: ECON. BVALUATION OF CLOSURB CAP BARRIER MATERUAL.
CHK'D BY: S. MEAD

ESTIMATOR: POWRK

PHONE: 7-5256

DATE:

LOC: Mncolos

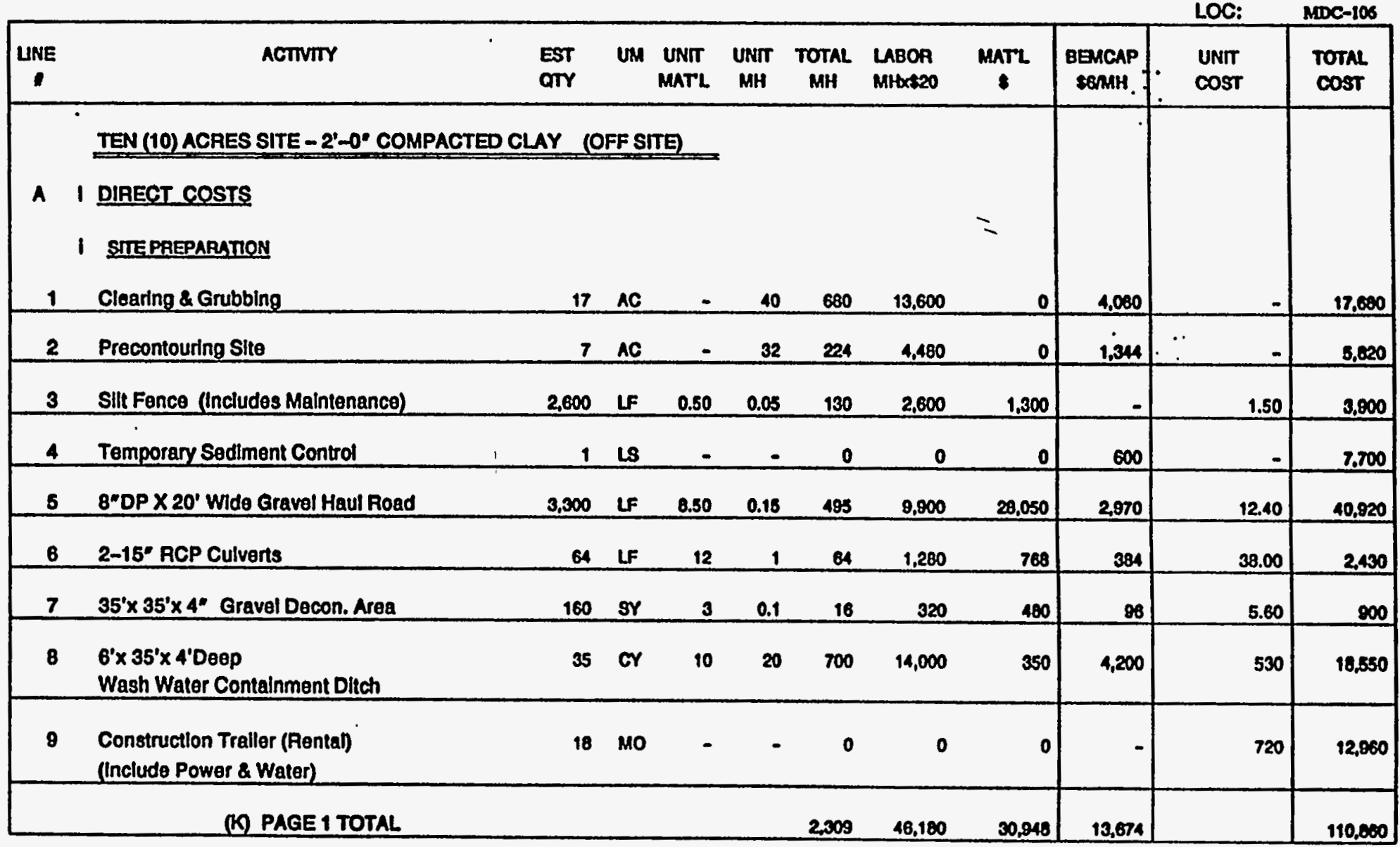

G-7. Cost Estimate for a Cover System Utilizing Compacted Kaolin (Offsite) Clay and a 30-mil FML 
DETAIL ESTIMATE

Loo : : 7-03

ACTTVITY CODE: ESE260412

PROJ.NAME: ECON. EVALUATION OP CLOSURB CAP BARRIER MATERIAL
CHK'D BY: S. MEAD

ESTIMATOR: PONRK

PHONE: 7-5265

DATE: $\quad 0824193$

LOC: MDC-10s

\begin{tabular}{|c|c|c|c|c|c|c|c|c|c|c|c|}
\hline LNE & ACTIVITY & $\begin{array}{l}\text { EST } \\
\text { arr }\end{array}$ & UM & $\begin{array}{l}\text { UNTI } \\
\text { MATL }\end{array}$ & $\underset{M H}{\text { UNT }}$ & $\begin{array}{c}\text { TOTAL } \\
\text { MH }\end{array}$ & $\begin{array}{l}\text { LABOR } \\
\text { MHXE20 }\end{array}$ & $\underset{8}{\operatorname{MaTL}}$ & $\begin{array}{l}\text { BENCAP } \\
\text { SEMIH }\end{array}$ & $\begin{array}{l}\text { UNT } \\
\text { COST }\end{array}$ & $\begin{array}{l}\text { TOTAL } \\
\text { COST }\end{array}$ \\
\hline & ¿ COVER CONSTRUCTION & & & & & & & & & & \\
\hline 10 & Waste Stabllization (Crushed Limestone) & 13,840 & TN & 75 & 0.1 & 1,394 & 27.680 & $1,045,500$ & 8,364 & 77.60 & $1.081,740$ \\
\hline 11 & $\begin{array}{l}\text { Place and Compact Foundation Soll } \\
\text { in } 8^{n} \text { Lifts to } 95 \% \text { Std. Proctor }\end{array}$ & 68.709 & CY & - & 0.145 & 12,863 & 257,256 & 0 & 77,177 & 3.80 & 334,430 \\
\hline 12 & $\begin{array}{l}\text { Compact 2'-0" Thlck Clay Layer } \\
\text { In } 8^{a} \text { Lifts }\end{array}$ & 32,660 & Cr & 13.00 & 0.165 & $\mathbf{5 . 3 8 9}$ & 107.778 & 424,580 & $\mathbf{3 2 , 3 3 3}$ & 17.30 & 564,690 \\
\hline 13 & $\begin{array}{l}\text { Placement of } 30 \text { Mil Flexlble } \\
\text { Membrane Lner }\end{array}$ & 475.425 & SF & 0.15 & 0.007 & 3,320 & 66,560 & 71,314 & - & 0.30 & 137.070 \\
\hline 14 & $\begin{array}{l}\text { Placement of 1'-0 Coarse Sand } \\
\text { Dralnage Layer }\end{array}$ & 16,875 & or & 10.50 & 0.025 & 422 & 8,438 & 177,180 & 2,531 & 11.20 & 100,160 \\
\hline 15 & $\begin{array}{l}\text { Placement of Geotextile (TYPAP) Filter on } \\
1^{\prime}-0^{\circ} \text { Coarse Sand Drainage Layer }\end{array}$ & 62,200 & 8Y & 0.14 & 0.005 & 281 & 6,220 & 7,308 & - & 0.20 & 12,330 \\
\hline 16 & Placement of $1^{\prime}-6^{\circ}$ Common Fill & 25,667 & CY & 3.75 & 0.05 & 1,293 & 25,867 & 97,001 & 7.760 & 5.10 & 130,630 \\
\hline 17 & Placement of $6^{*}$ Topsoil & 8.438 & CY & 8.50 & 0.05 & 422 & 8,438 & 80,161 & 2.531 & 10.80 & 91,130 \\
\hline 18 & $\begin{array}{l}\text { Placement of Perimeter Exterlor } \\
\text { Drainage Layer } 3 / 4^{*} \times 9^{\prime \prime} \text { DP (Crushed Stone) }\end{array}$ & 1.358 & sy & 1.50 & 0.16 & 217 & 4,346 & 2,037 & 1,304 & 3.70 & 7,690 \\
\hline 19 & Excavation of Trapezoldal Drainage Ditch & 4.745 & CY & $=$ & 0.17 & 007 & 16,133 & 음 & 4,840 & 4.40 & 20,970 \\
\hline & (K) PAGE 2 TOTAL & & & 1 & & 26,390 & 527,915 & $1,005,069$ & 130,841 & & $2,560,040$ \\
\hline
\end{tabular}

G-7. Cost Estimate for a Cover System Utilizing Compacted Kaolin (Offsite) Clay and a 30-mil FML (Cont'd) 


\section{L00 I: :-03}

ACTTVITY CODE: ESE2SOS12

PROJ.NAME: ECON. EVALUATION OF CLOSURB CAP BARRIER MATERSAL

ESTIMATOR: PO/WLR

PHONE: 7-5208

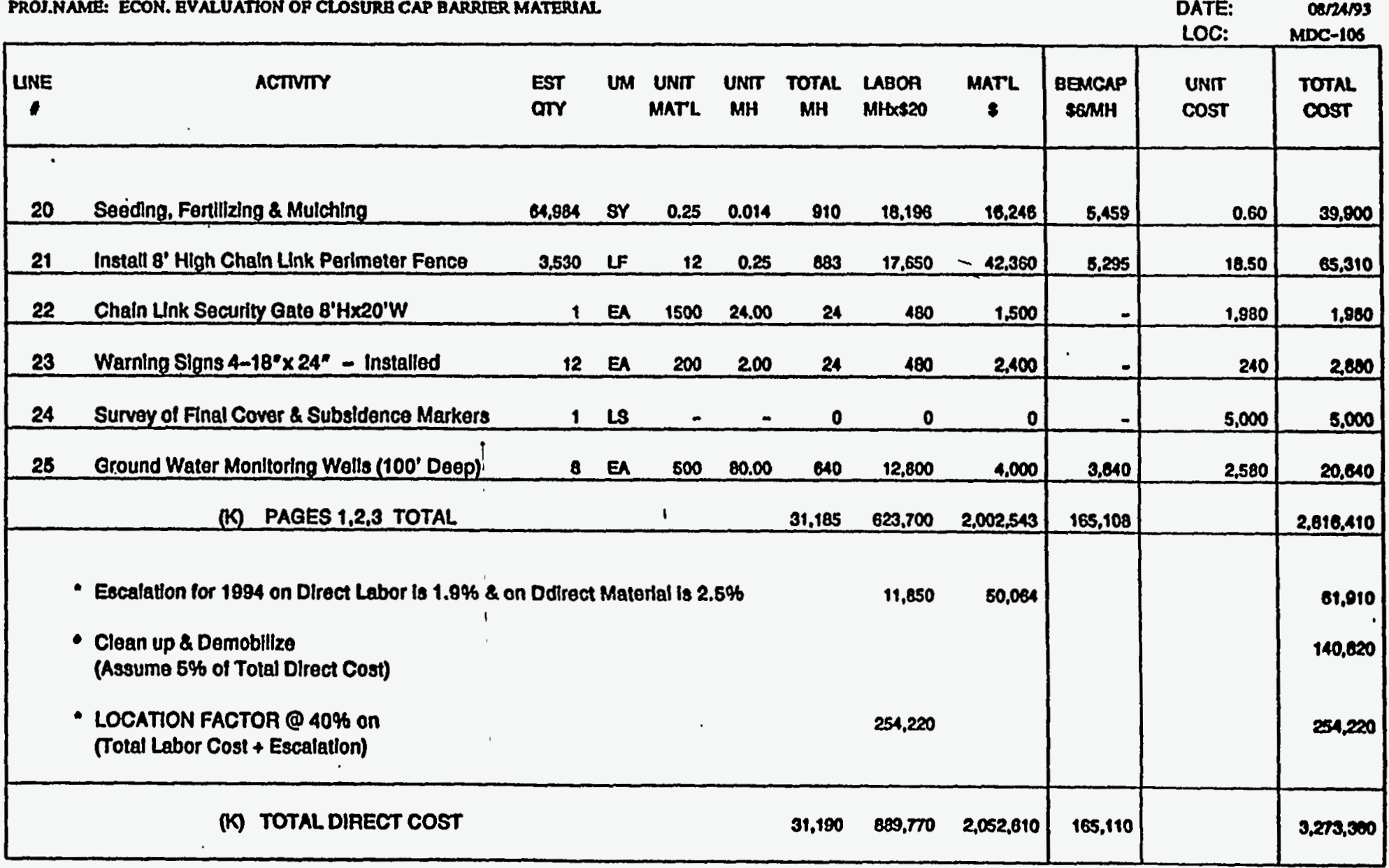

G-7. Cost Estimate for a Cover System Utilizing Compacted Kaolin (Offsite) Clay and a 30-mil FML (Cont'd) 
CHK'O BY: S. MEAD

Loall: $7-03$

ACTIVITY CODE: ESE260412

PROJ.NAME: ECON. EVALUATION OF CLOSURB CAP BARRIER MATERIAL.

ESTIMATOR: PONHR

PHONE: 7-5266

DATE: $\quad 0224193$

LOC: MAC-106

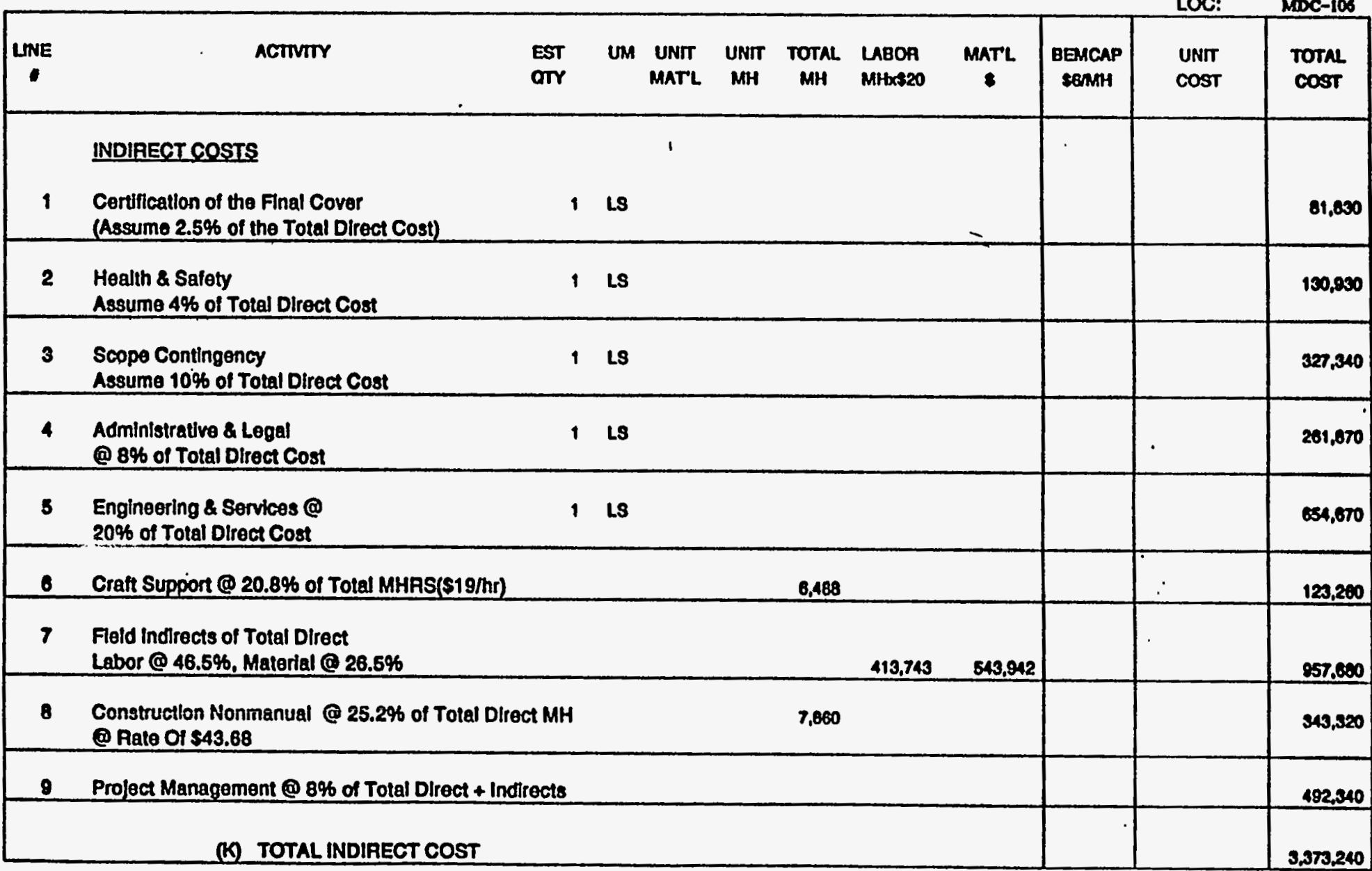

G-7. Cost Estimate for a Cover System Utilizing Compacted Kaolin (Offsite) Clay and a 30-mil FML (Cont'd) 
DETAIL ESTIMATE

CHK'D BY: S. MEAD

Loo \&: 7-03

ACTIVITY CODE: ESE260412

PROS.NAME: ECON. BVALUATION OP CLOSURB CAP BARRIER MATERIAL

STIMATOR: PONMX

PHONE: 7-5265

DATE: OSRAOS

LOC: MDC-106

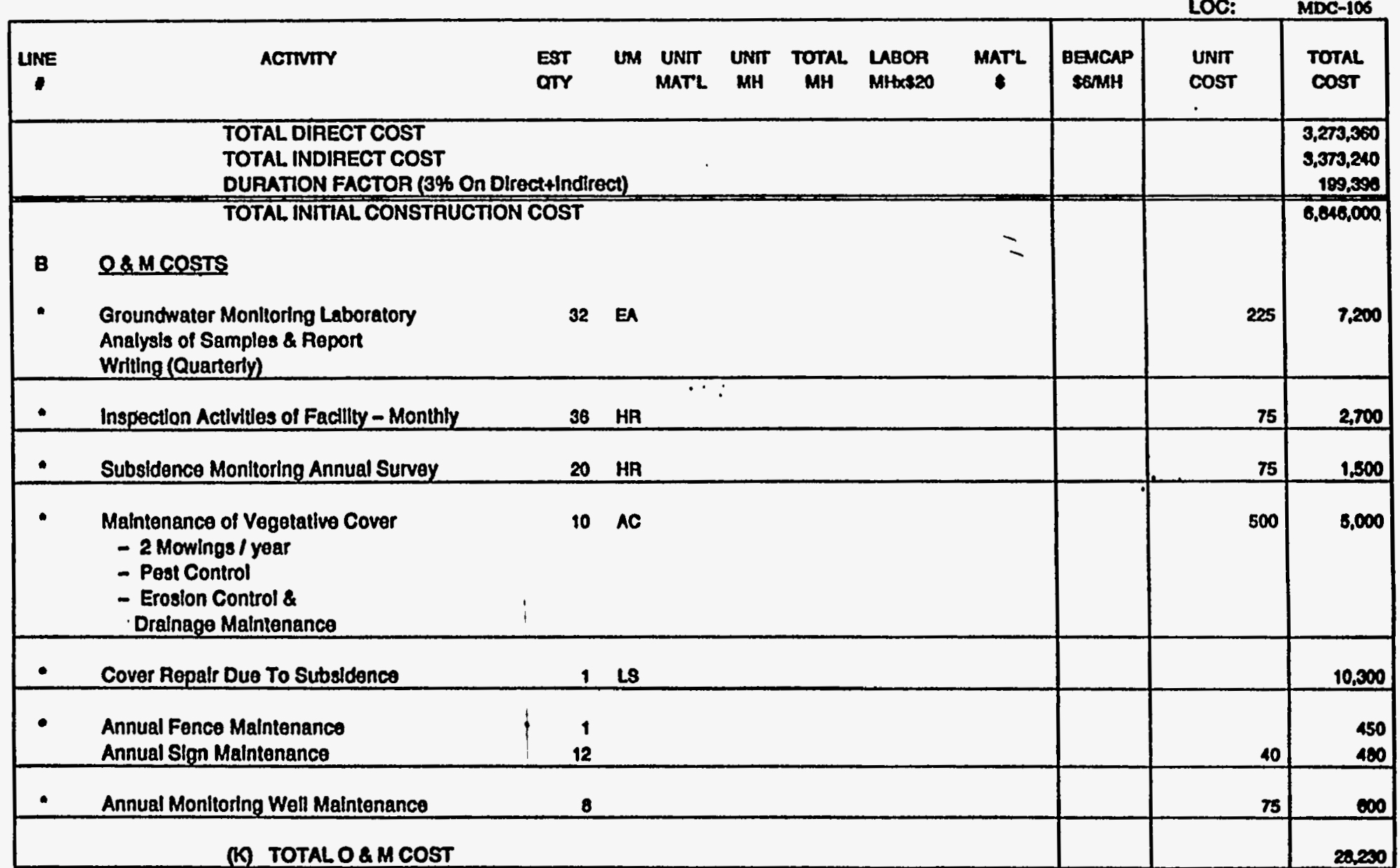

G-7. Cost Estimate for a Cover System Utilizing Compacted Kaolin (Offsite) Clay and a 30-mil FML (Cont'd) 
DETAIL ESTIMATE

\section{I: 7-03}

ACTIVITY CODE: BSE260412

PROJ.NAME: ECON. BVALUATION OP CLOSURB CAP BARRIER MATERUAL
CHK'D BY: S. MEAD

ESTIMATOR: PONMK

PHONE: 7-S268

DATE: DanAS3

LOC: $\quad$ MDC-106

\section{SUMMARY \\ IEN (10) ACRES SITE - 2'-0" COMPACTED CLAY IOFF SITE}

C TOTAL COST (Total Present Worth)

(i) Present Worth of 0 \& M Cost

(5\% discount Rate for 30 Yeare)

434,000

(Total O \& M $\times$ 15.3725)

(II) Total Initial Construction Cost

$8,846,000$

(K) TOTAL PROJECT COST (Rounded to the nearest Thousand)

$7,200,000$

G-7. Cost Estimate for a Cover System Utilizing Compacted Kaolin (Offsite) Clay and a 30-mil FML (Cont'd) 


\section{DETAIL ESTIMATE}

Lo0 If: 7-03

ACTTVTYY CODE: ESE260412

PROJ.NAME: ECON. BVALUATION OF CLOSURE CAP BARRIER MATERIAS

CHK'D BY: S. MEAD

ESTIMATOR: POIMRX

PHONE: 7-5265

DATE: 0012493

LOC: MDC-106

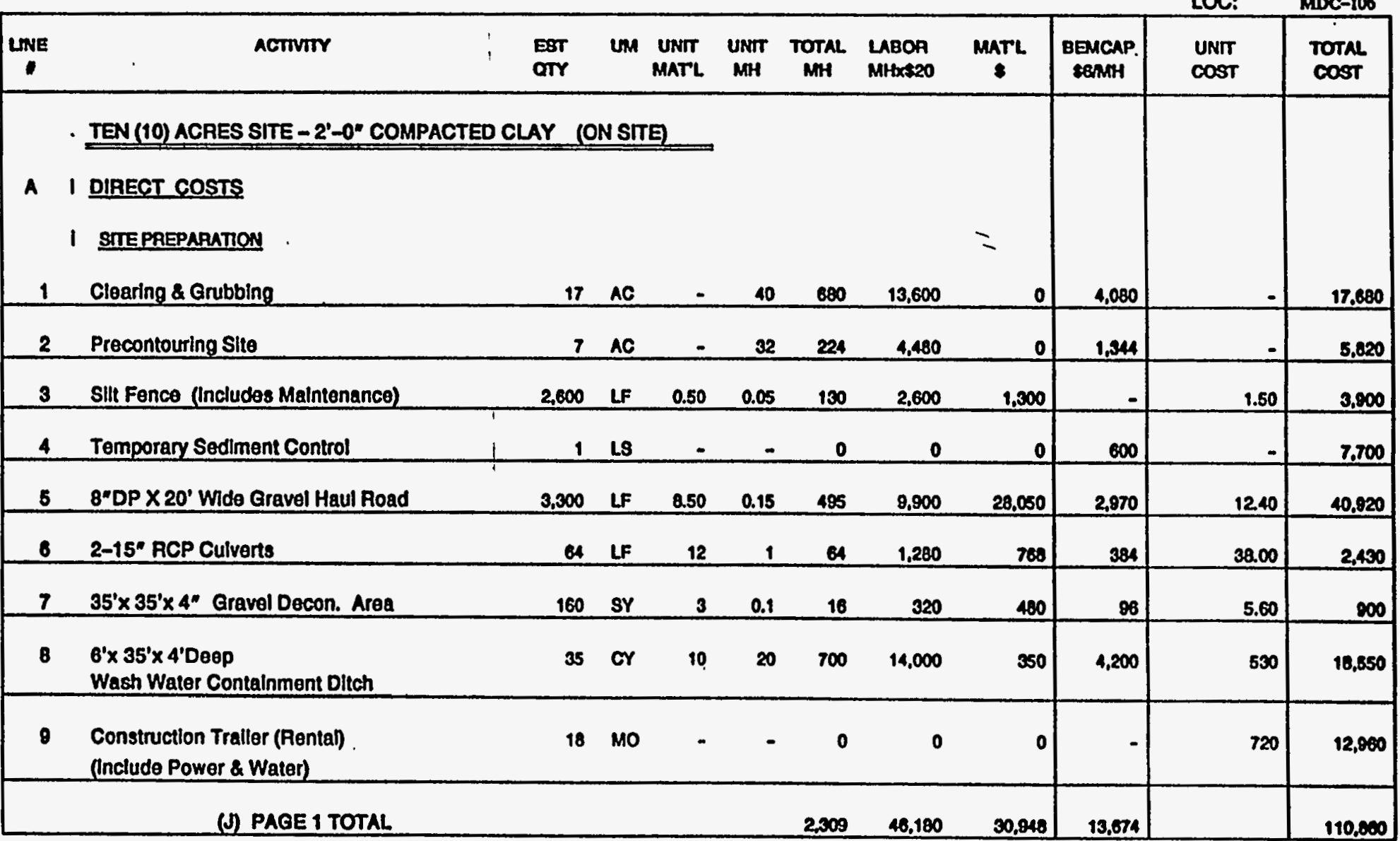

G-8. Cost Estimate a for Cover System Utilizing Compacted Onsite Sandy Clay and a 30-mil FML 
DETAIL ESTIMATE

CHK'D BY: S. MEAD

Lo0 1: 7-03

ACTIVITY CODB: ESE260412

PROI.NAME: ECON. BVALUATTON OP CLOSURB CAP BARRIER MATERUAL

\begin{tabular}{|c|c|c|c|c|c|c|c|c|c|c|c|}
\hline UNE & ACTIVITY & $\begin{array}{l}\text { EST } \\
\text { OTY }\end{array}$ & UM & $\begin{array}{l}\text { UNTT } \\
\text { MATL }\end{array}$ & $\begin{array}{l}\text { UNIT } \\
\text { MH }\end{array}$ & TOTAL & $\underset{M H \times \$ 20}{\text { UBOA }}$ & maTL & $\underset{\text { EEMCAP }}{\text { SEMH }}$ & $\begin{array}{l}\text { UNrT } \\
\text { COST }\end{array}$ & $\begin{array}{l}\text { TOTAL } \\
\text { Cost }\end{array}$ \\
\hline & 11 COVER CONSTRUCTION & & & & & & & & & & \\
\hline 10 & Waste Stablization (Crushed Umestone) & 13,940 & $\mathrm{~m}$ & 75 & 0.1 & 1,394 & 27,880 & $1,045,500$ & 0,364 & 77.60 & $1.061,740$ \\
\hline 11 & $\begin{array}{l}\text { Place and Compact Foundation Soll } \\
\text { In } 8^{*} \text { Litis to } 95 \% \text { Std. Proctor }\end{array}$ & 88,709 & or & - & 0.145 & 12,063 & 257.256 & 0 & $n, 177$ & 3.80 & 334,430 \\
\hline 12 & $\begin{array}{l}\text { Compact 2'-0" Thick Clay Layer } \\
\text { In 8" Lits }\end{array}$ & 32,660 & cr & - & 0.165 & 5,389 & 107.778 & 0 & 32,333 & 4.30 & 140,110 \\
\hline 13 & $\begin{array}{l}\text { Placement of } 30 \text { Mil Flexlble } \\
\text { Membrane Liner }\end{array}$ & 475,425 & SF & 0.15 & 0.007 & 3,328 & 66,560 & 71,314 & - & 0.30 & 137,070 \\
\hline 14 & $\begin{array}{l}\text { Placement of 1'-0 Coarse Sand } \\
\text { Drainaga Layer }\end{array}$ & 16,875 & or & 10.50 & 0.025 & 422 & 8,438 & 177.168 & 2,531 & 11.20 & 188,160 \\
\hline 15 & $\begin{array}{l}\text { Placement of Geotextile (TYPAP) Filter on } \\
\text { 1'-0" Coarse Sand Drainago Layer }\end{array}$ & 52,700 & sy & 0.14 & 0.005 & 264 & 5,270 & 7,378 & - & 0.20 & 12,650 \\
\hline 16 & Placement of $1^{\prime}-6^{\prime \prime}$ Common Fill & 25,887 & or & 3.75 & 0.05 & 1.293 & 25,867 & 97,001 & 7.760 & 8.10 & 150,600 \\
\hline 17 & Placement of 6" Topsoll & 8,438 & or & 9.50 & 0.05 & 422 & 8,438 & 80,161 & 2,531 & 10.80 & 91,130 \\
\hline 18 & $\begin{array}{l}\text { Placement of Perimeter Exterlor } \\
\text { Drainage Layer } 34^{\prime \prime} \times 3^{*} \text { DP (Crushed Stone) }\end{array}$ & 1,358 & sr & 1.50 & 0.18 & 217 & 4,348 & 2,037 & 1,304 & 5.70 & 7,690 \\
\hline 19 & Excavalion of Trapozoldal Drainage Dilch & 4,745 & or & - & 0.17 & 807 & 16,133 & 0 & 4,040 & 4.40 & 20,070 \\
\hline & (J) PAGE 2 TOTAL & & & & & 20,398 & 527,065 & $1,100,579$ & 190,841 & & $2,145,300$ \\
\hline
\end{tabular}

G-8. Cost Estimate a for Cover System Utilizing Compacted Onsite Sandy Clay and a 30-mil FML (Cont'd) 
DETAIL ESTIMATE

CHK'D BY: S. MEAD

L001: $7-03$

ACTIVTTY CODB: ESE26OA12

PROJ.NAME: ECON. EVALUATION OF CLOSURB CAP BARRIER MATERIAL

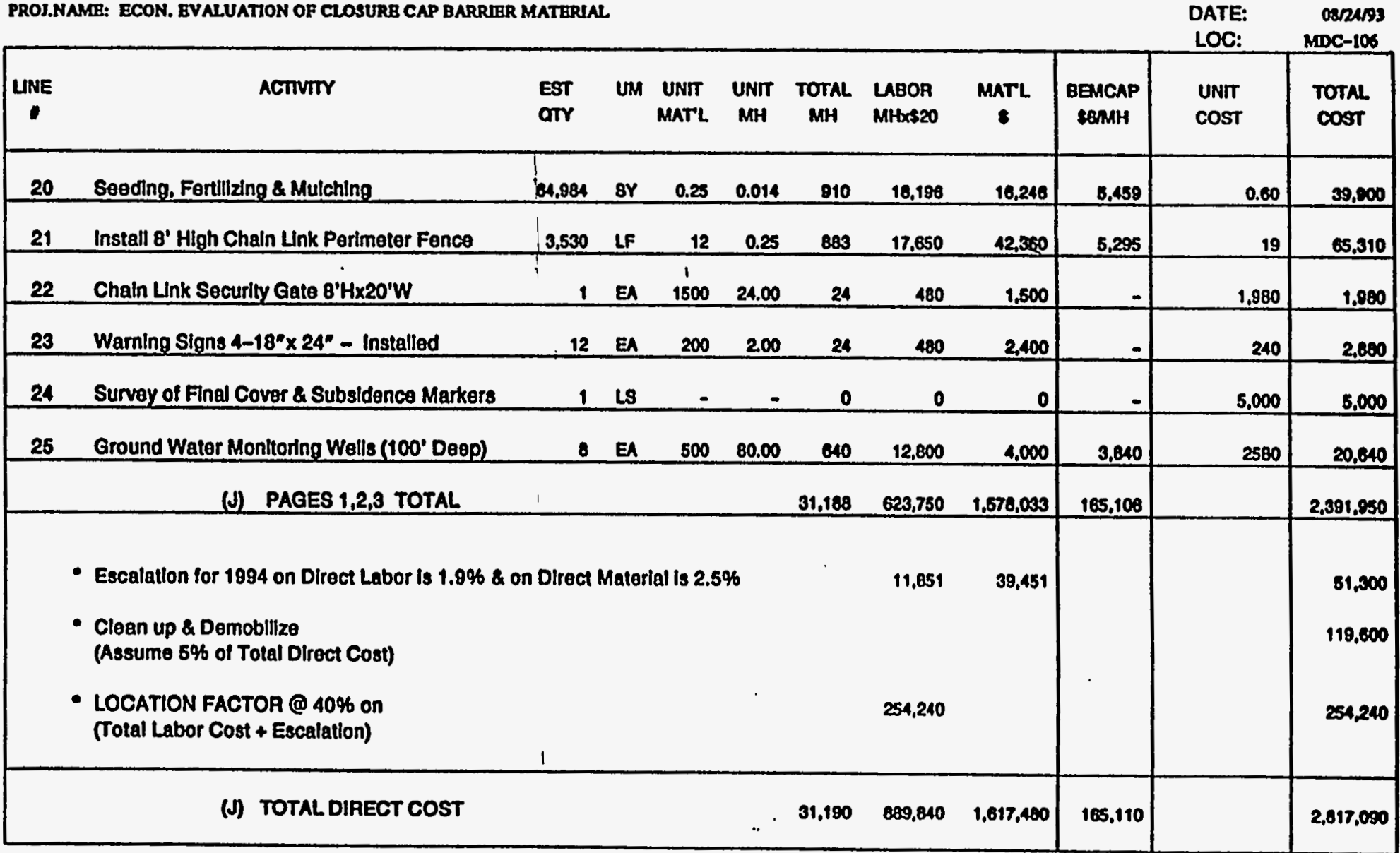

G-8. Cost Estimate a for Cover System Utilizing Compacted Onsite Sandy Clay and a 30-mil FML (Cont'd) 
DETAIL ESTIMATE

1001: 7-03

ACTIVITY CODE: ESE200412

PROI.NAMB: BCON. EVALUATION OF CLOSURE CAP BARRTER MATERIAL.

\begin{tabular}{|c|c|c|c|c|c|c|c|c|c|c|c|}
\hline UNE & ACTIVTr & $\begin{array}{l}\text { EST } \\
\text { OTY }\end{array}$ & un & $\begin{array}{l}\text { UNRT } \\
\text { MATL }\end{array}$ & $\begin{array}{l}\text { UNT } \\
M H\end{array}$ & $\begin{array}{l}\text { TOTAL } \\
M H\end{array}$ & $\begin{array}{l}\text { LABOA } \\
\text { MHtrS2O }\end{array}$ & $\stackrel{\operatorname{MaTL}}{*}$ & $\begin{array}{l}\text { BEMCAP } \\
\text { SEMMH }\end{array}$ & $\begin{array}{l}\text { UNT } \\
\text { COST }\end{array}$ & $\begin{array}{l}\text { TOTAL } \\
\text { Cost }\end{array}$ \\
\hline 1 & $\begin{array}{l}\text { NNDIBECT COSTS } \\
\text { Cortification of the Final Cover } \\
\text { (Assume 2.5\% of the Total Direct Cost) }\end{array}$ & 1 & LS & & & & & & & . & 70,430 \\
\hline 2 & $\begin{array}{l}\text { Health } \& \text { Safety } \\
\text { Aosume } 4 \% \text { of Total Direct Cost }\end{array}$ & 1 & 18 & & & & & & & & 112,600 \\
\hline 3 & $\begin{array}{l}\text { Scope Contingency } \\
\text { Assume } 10 \% \text { of Tolal Disect Cost }\end{array}$ & 1 & LS & & & & & & & & 201.710 \\
\hline 4 & $\begin{array}{l}\text { Administrativo \& Legal } \\
\text { @ 8\% of Total Direct Cost }\end{array}$ & 1 & LS & & . & & & & . & & 225,370 \\
\hline 5 & $\begin{array}{l}\text { Engineering \& Services @ } \\
20 \% \text { of Total Direct Cost }\end{array}$ & & & & & & & & & & 583,420 \\
\hline 6 & Craft Support @ 20.8\% of Total MHRS(S19/hr) & & & & & 6,490 & & & & & 123,310 \\
\hline 7 & $\begin{array}{l}\text { Feld Indlirects of Total Direct } \\
\text { Labor @ 46.5\%, Materlal @ 26.5\% }\end{array}$ & & & & & & 413,776 & 428,632 & & & O21,410 \\
\hline 8 & $\begin{array}{l}\text { Construction Nonmanual @ } 25.2 \% \text { of Total MH } \\
\text { @ Rate of } \$ 43.68\end{array}$ & & & & & 7,860 & & & & & 343,320 \\
\hline 8 & Project Management @ 8\% of Total Direct + Ind & ects & & & & & & & & & 467,000 \\
\hline & (J) TOTAL INDIRECT COST & & & & & & & & & & $3,050,450$ \\
\hline
\end{tabular}

CHK'DBY: S. MEAD

ESTIMATOR: POIMRK

PHONE: 7-5265

DATE: DoRAM3

LOC: $\quad$ MDC-100

.450

12.600

9.710

5,370

3,420

23,310

190

3,320

7,000

$.000,450$

G-8. Cost Estimate a for Cover System Utilizing Compacted Onsite Sandy Clay and a 30-mil FML (Cont'd) 
DETAIL ESTIMATE

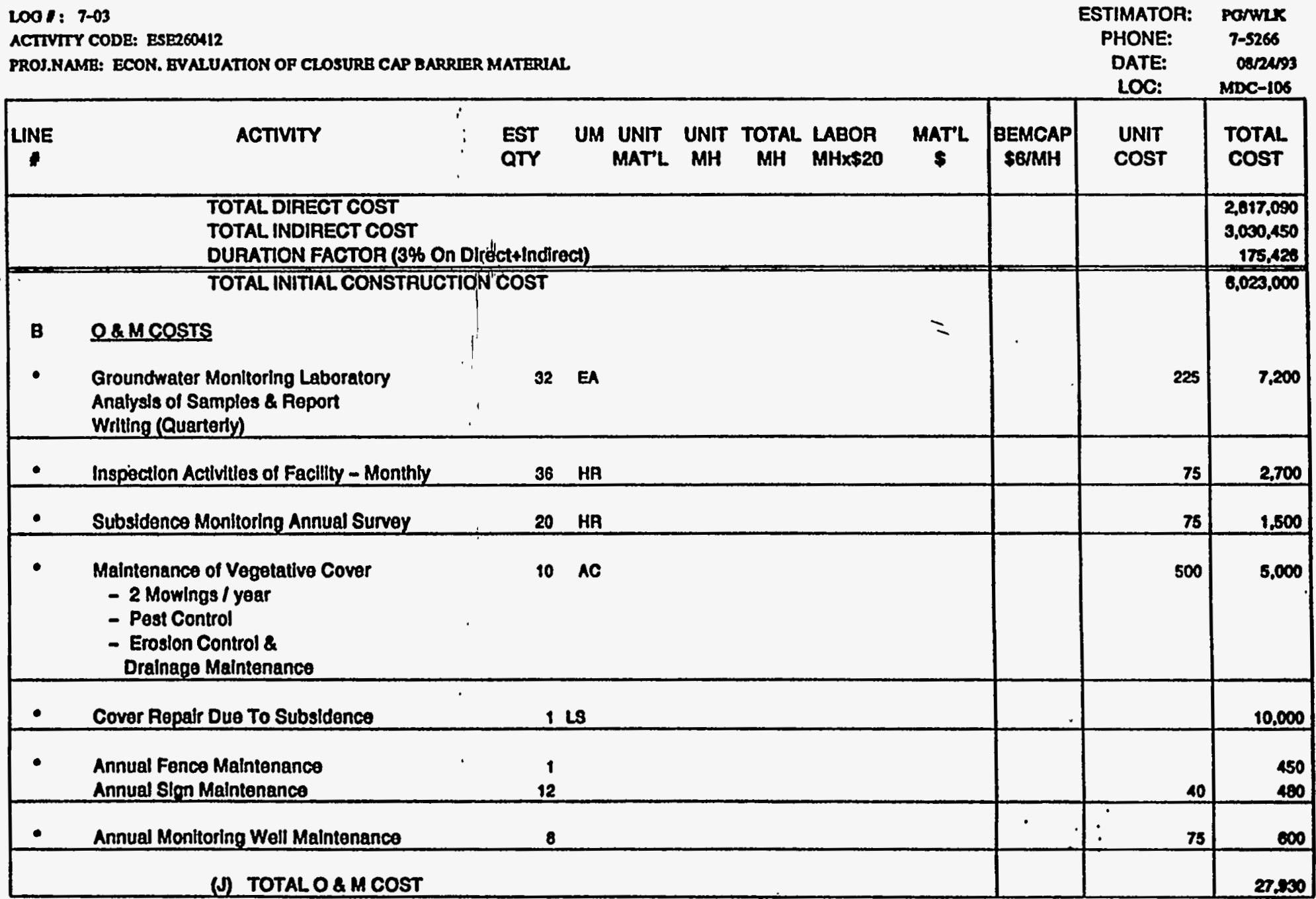

G-8. Cost Estimate a for Cover System Utilizing Compacted Onsitespandy Clay and a 30-mil FML (Cont'd) 
DETAIL ESTIMATE

Loo : : 7-03

ACTIVITY CODE: ESE260412

PROJ.NAME: ECON. EVALUATION OP CLOSURB CAP BARRIER MATERJAL
CHK'D BY: S. MEAD

ESTIMATOA: Mamx

PHONE: 7.5266

DATE:

LOC: MDC-108

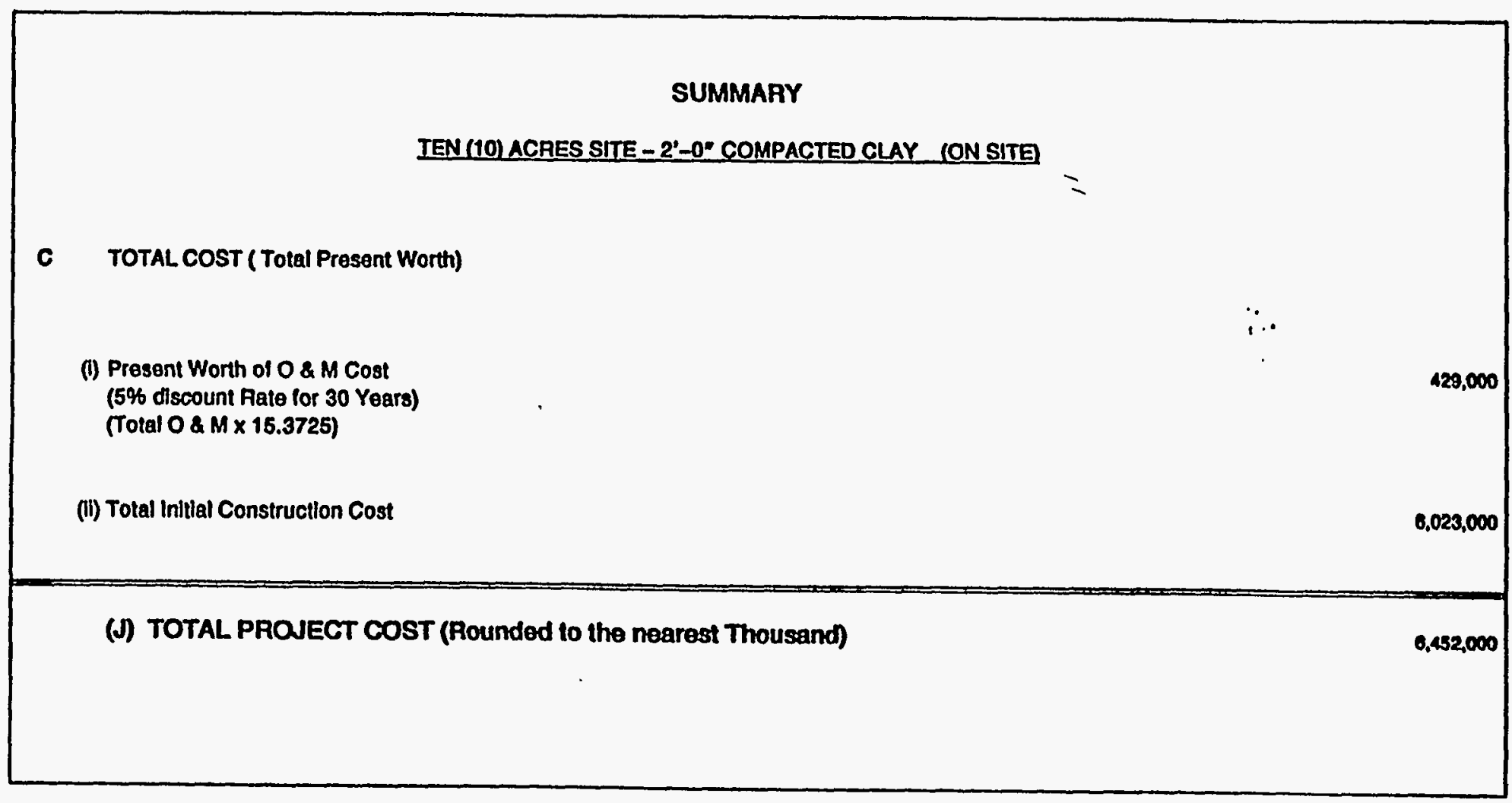

G-8. Cost Estimate a for Cover System Utilizing Compacted Onsite Sandy Clay and a 30-mil FML (Cont'd) 


\section{DETAIL ESTIMATE}

LO01: $7-03$

ACTTVITY CODE: ESE260412

PROJ.NAME: ECON. EVALUATION OF CLOSURE CAP BARRTER MATERIAL.

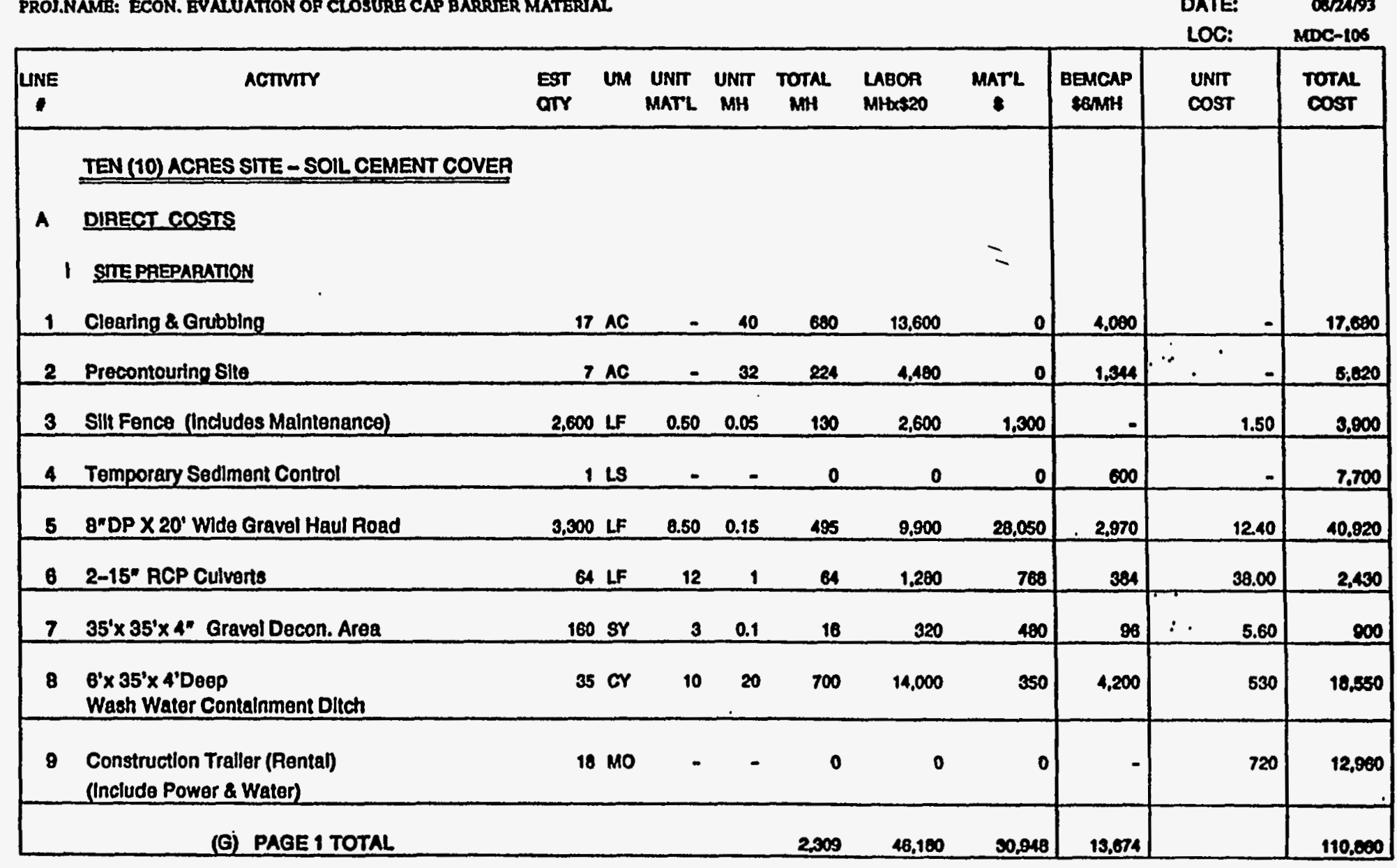

G-9. Cost Estimate for a Cover System Utilizing Soil Cement and 30-mil FML 
CHK'D BY: S. MEAD

LOO : :-03

ACTIVITY CODE: ESE26O412

MROJ.NAME: ECON. BVALUATTON OF CLOSURE CAP BARRIER MATERIAL

\begin{tabular}{|c|c|c|c|c|c|c|c|c|c|c|c|}
\hline UNE & ACTIVTY & $\begin{array}{l}\text { EST } \\
\text { arY }\end{array}$ & UM & $\begin{array}{l}\text { UNT } \\
\text { MATL }\end{array}$ & $\underset{\text { MH }}{\text { UNT }}$ & $\underset{M H}{\text { TOTAL. }}$ & $\begin{array}{l}\text { LABOR } \\
\text { MHX } \$ 20\end{array}$ & MATL & $\begin{array}{l}\text { BEMCAP } \\
\text { SGMHH }\end{array}$ & $\cdot$ UNIT & $\begin{array}{l}\text { Total } \\
\text { cost }\end{array}$ \\
\hline & \# COVER CONSTRUCTION & & & & & & & & & & \\
\hline 10 & Waste Stabilization (Crushed Umestone) & 13,940 & $\pi N$ & 75 & 0.1 & 1,394 & 27,880 & $1,045,500$ & 8,364 & 77.60 & $1.081,740$ \\
\hline 11 & $\begin{array}{l}\text { Place and Compact Foundation Soll } \\
\text { in } 8^{\circ} \text { Lifis to } 95 \% \text { Std. Proctor }\end{array}$ & 88,709 & Cr & - & 0.145 & 12,863 & 257,256 & $=$ & 77.17 & 3.80 & 334,430 \\
\hline 12 & $\begin{array}{l}\text { Soll-Cement mixture transported from central } \\
\text { mixing plant by end-dump trucks and placed } \\
\text { in } 6-8 \text { " lifts by spreaders. Assume Fine Sand } \\
\text { Soll with } 321 \text { of cement/CY. Compact the soll- } \\
\text { cement uniformily to } 98 \% \text { max. density. }\end{array}$ & 49,285 & or & 18 & 0.12 & 5,914 & 118,284 & 887,130 & 35,485 & 21.10 & $1,040,900$ \\
\hline 13 & $\begin{array}{l}\text { Curing soll-cement. Assume liquid Asphalt } \\
\text { applled } 0.4 \text { gallsy for } 1 \text { St coat } 20.2 \text { for } 2 \text { nd. }\end{array}$ & $\begin{array}{l}50,388 \\
50,386 \\
\end{array}$ & $\begin{array}{l}\text { SY } \\
\text { Sr }\end{array}$ & $\begin{array}{l}0.33 \\
0.17 \\
\end{array}$ & $\begin{array}{l}0.03 \\
0.02 \\
\end{array}$ & $\begin{array}{l}1.512 \\
1.008 \\
\end{array}$ & $\begin{array}{l}30,232 \\
20,154 \\
\end{array}$ & $\begin{array}{r}16,627 \\
8,566 \\
\end{array}$ & - & $\begin{array}{l}0.90 \\
0.60 \\
\end{array}$ & $\begin{array}{l}16,860 \\
22,720 \\
\end{array}$ \\
\hline 14 & $\begin{array}{l}\text { Placement of } 30 \text { Mill Flexible } \\
\text { Membrane Liner }\end{array}$ & 475,425 & SF & 0.15 & 0.007 & 3,328 & 66,560 & 71,314 & - & 0.30 & 137.070 \\
\hline 15 & $\begin{array}{l}\text { Placement of } 1 \text { '-0 Coarse Sand } \\
\text { Dralnage Layer }\end{array}$ & 16,875 & or & 10.5 & 0.025 & 422 & 8,438 & 177,188 & 2,531 & 11.20 & 180,160 \\
\hline 16 & $\begin{array}{l}\text { Placement of Geotextile (TYPAF) Filter on } \\
1^{\prime}-0^{*} \text { Coarse Sand Dralnage Layer }\end{array}$ & 62,700 & 8Y & 0.14 & 0.005 & 284 & 6,270 & 7,378 & - & 0.20 & 12,650 \\
\hline 17 & Placement of $1^{\prime}-6^{\prime \prime}$ Common Fill & 25,867 & or & 3.75 & 0.05 & 1,293 & 25,667 & 87.001 & 7,760 & 5.10 & 130.630 \\
\hline 18 & Placement of $6^{\prime}$ Topsoll & 0,438 & Cr & 9.5 & 0.05 & 422 & 8,438 & 80.161 & 2,531 & 10.60 & 91,130 \\
\hline & (G) PAGE 2 TOTAL & & & & & 20,701 & 534,073 & $2,213,702$ & 123,557 & & $3,093,090$ \\
\hline
\end{tabular}

G-9. Cost Estimate for a Cover System Utilizing Soil Cement and 30-mil FML (Cont'd) 
LOO : $7-03$

ACTIVITY CODE: ESE260412

PROJ.NAME: ECON. EVALUATION OP CLOSURB CAP BARRIER MATERIAL.

ESTIMATOR: POWRX

PHONE: 7-5266

DATE:

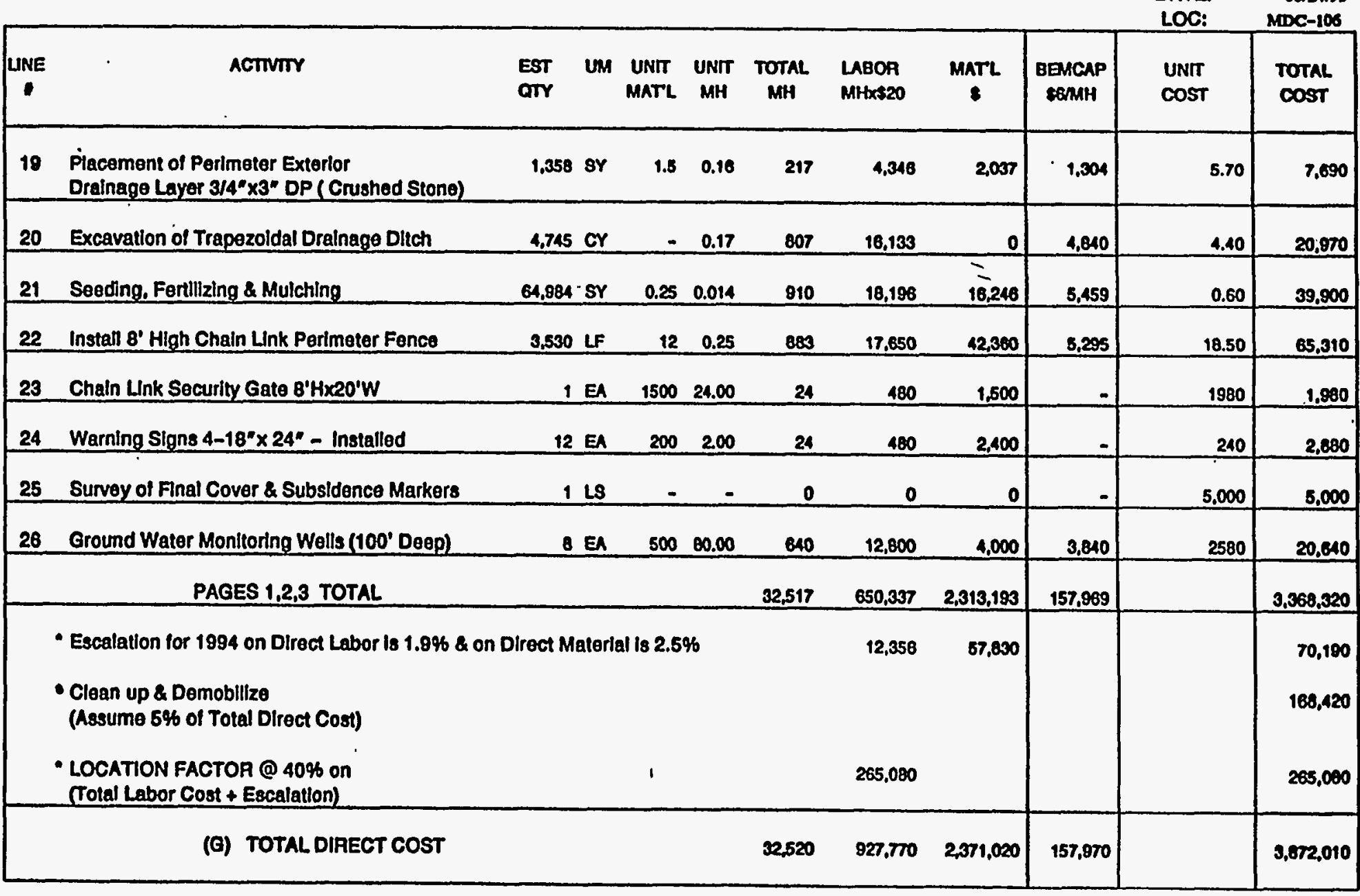

G-9. Cost Estimate for a Cover System Utilizing Soil Cement and 30-mil FML (Cont'd) 
D E T A I L E S T I M A T B CHK'D BY: S. MEAD

Lo0 : :-03 ACTIVTY CODE: ESE260112

PROJ.KAMB: RCON. BYALUATTON OF CLOSURB CAP BARRRER MATERALL

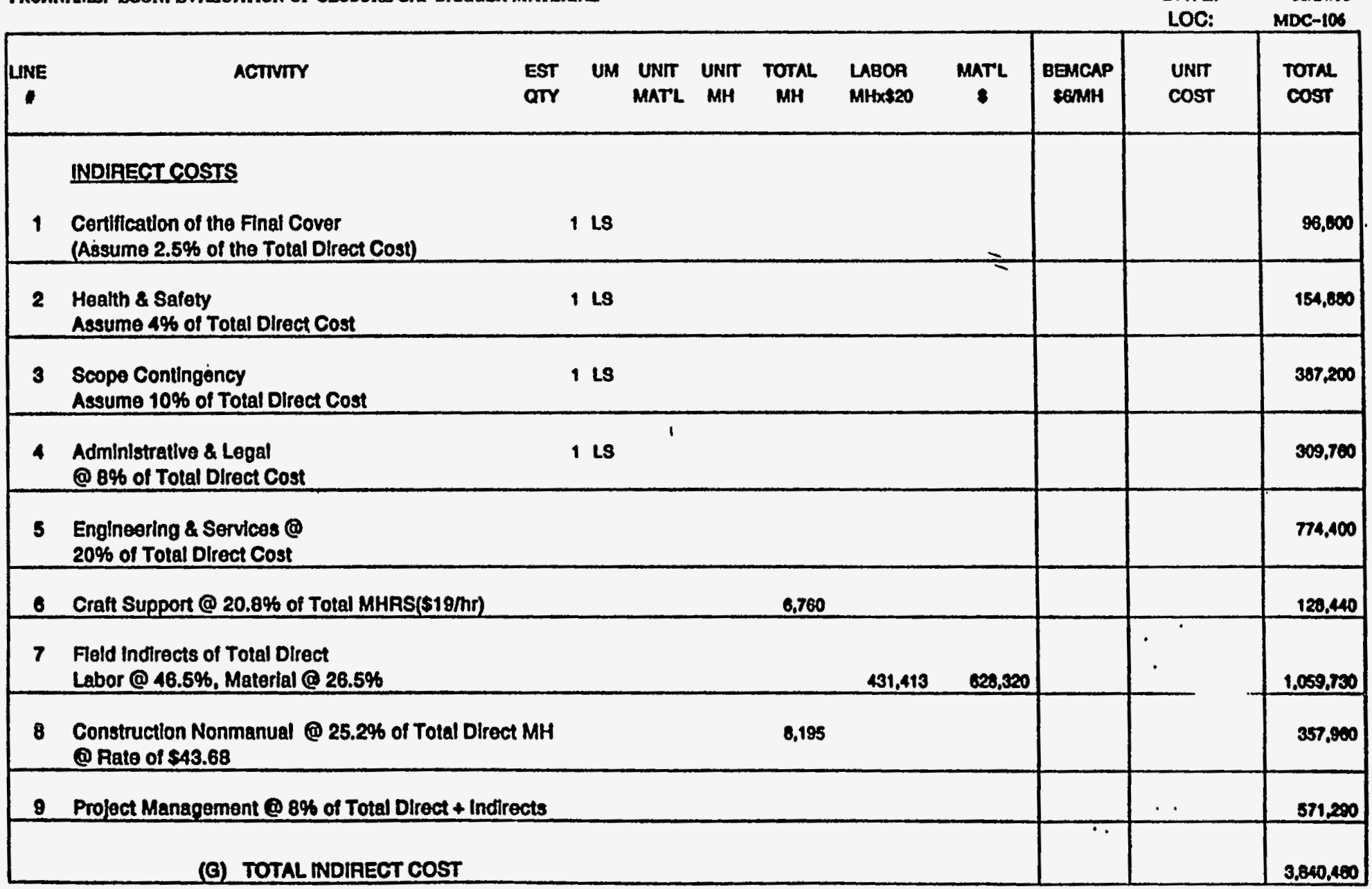

G-9. Cost Estimate for a Cover System Utilizing Soil Cement and 30-mil FML (Cont'd) 
L00\%: 7-03

ACTIVITY CODE: BSE20O412

PROI.NAME: ECON. EVALUATTON OP CLOSURB CAP BARRIER MATERLAL

ESTIMATOR: POWWK
PHONE:

DATE: Conars

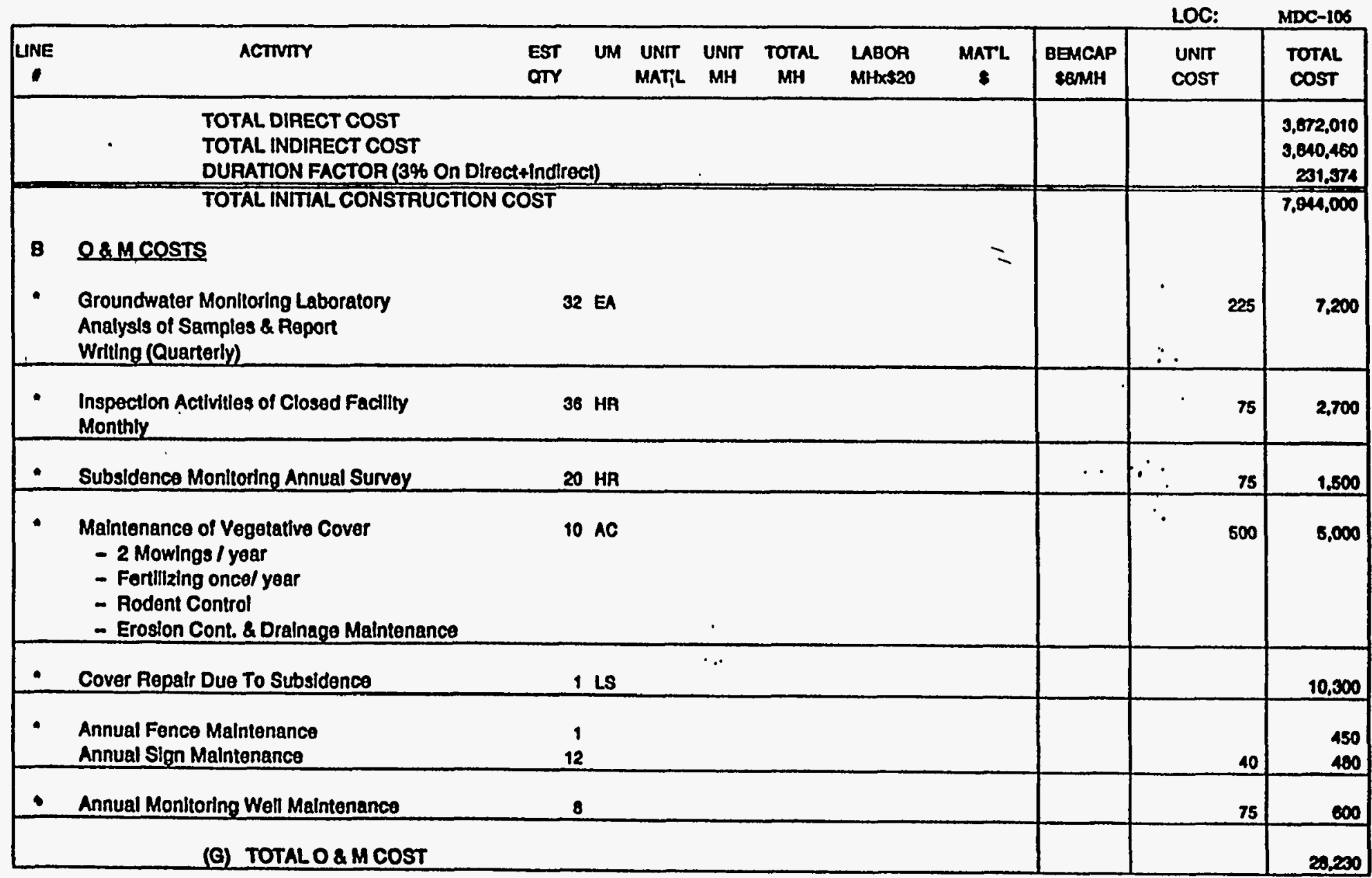

G-9. Cost Estimate for a Cover System Utilizing Soil Cement and 30-mil FML (Cont'd) 


\section{Lod : $7-03$}

ACTIVITY CODB: ESB260112

PROJ.NAMR: ECON. EVALUATION OF CLOSURE CAP BARRIER MATERIAL

CHK'D BY: S. MEAO

Estimator:

PHONE:

DATE:

Porme

LOC: MDC-100

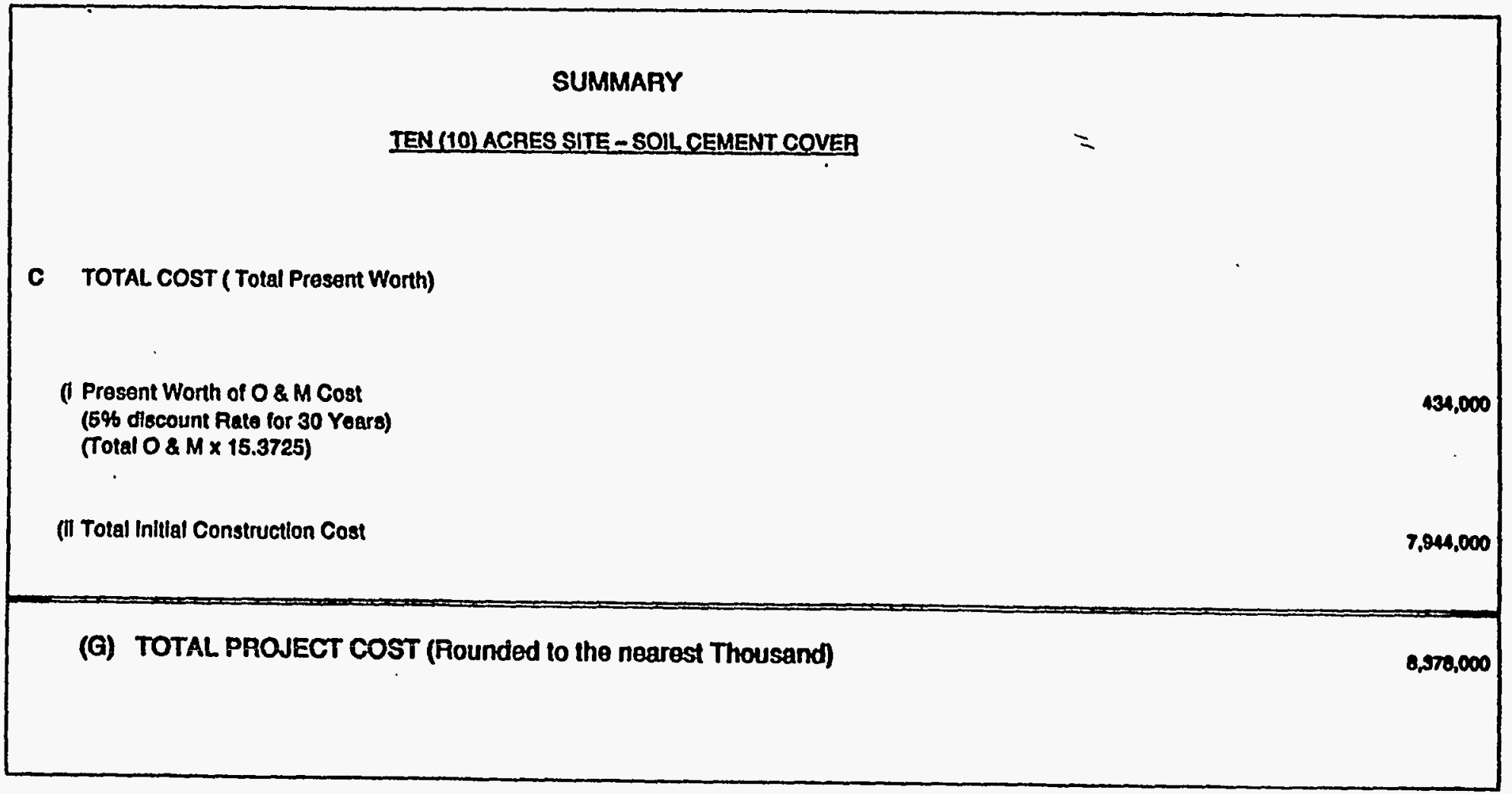

G-9. Cost Estimate for a Cover System Utilizing Soil Cement and 30-mil FML (Cont'd) 
DETAIL ESTIMATE

Lo01: $7-03$

ACTIVITY CODE: ESE260412

PROJ.NAMB: ECON. EVALUATTON OF CLOSURB CAP BARRIER MATERIAL.

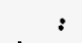

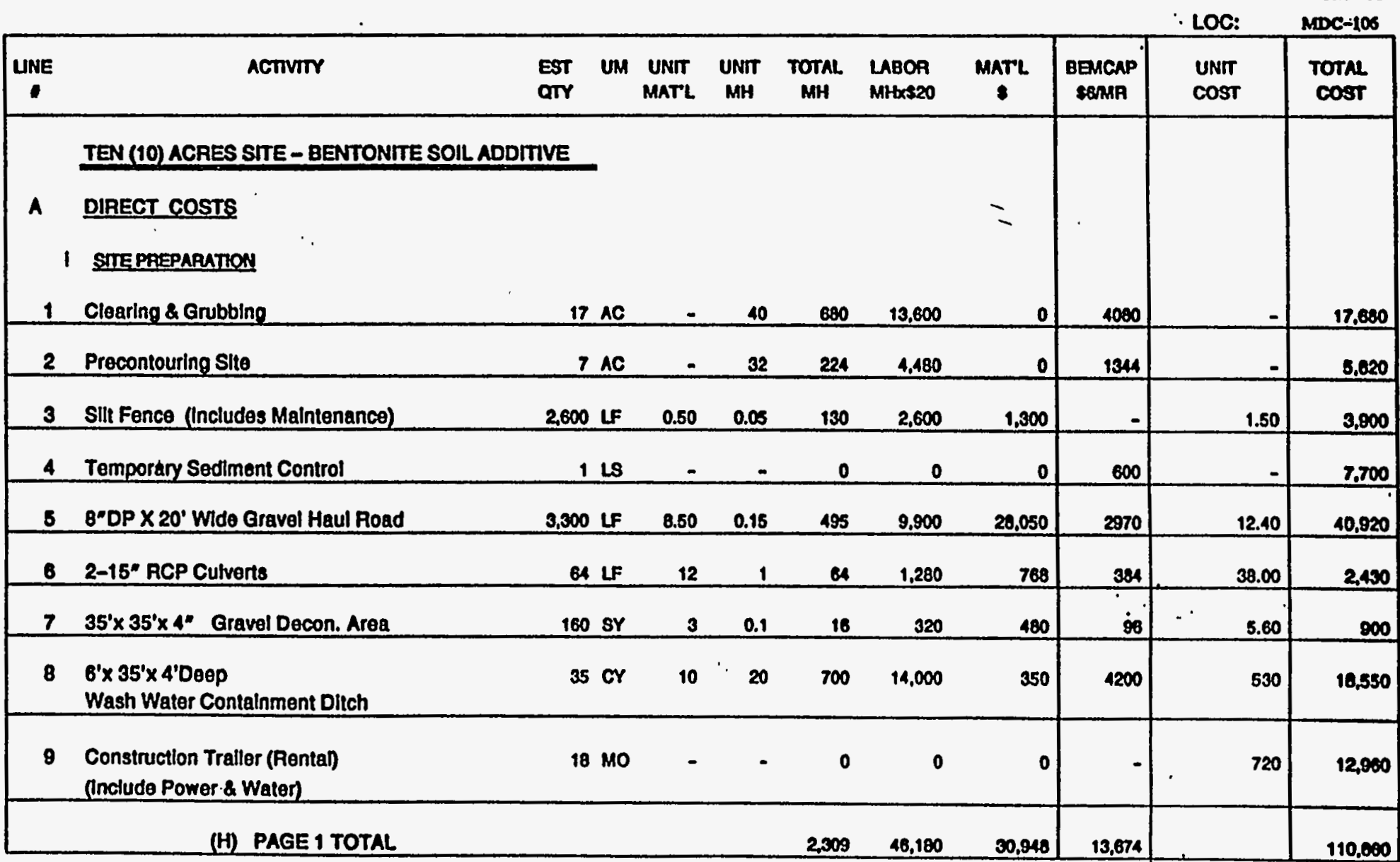

G-10. Cost Estimate for a Cover System Utilizing Bentonite Soil Additive and a 30-mil FML 
DETAIL ESTIMATE

CHK'D BY: S. MEAD

Lo01: $7-03$

ACTTVTY CODE: ESE2 60412

PROI.NAME: ECON. BVALUATION OF CLOSURB CAP BARRIRR MATERIAL

ESTIMATOR: PONHX

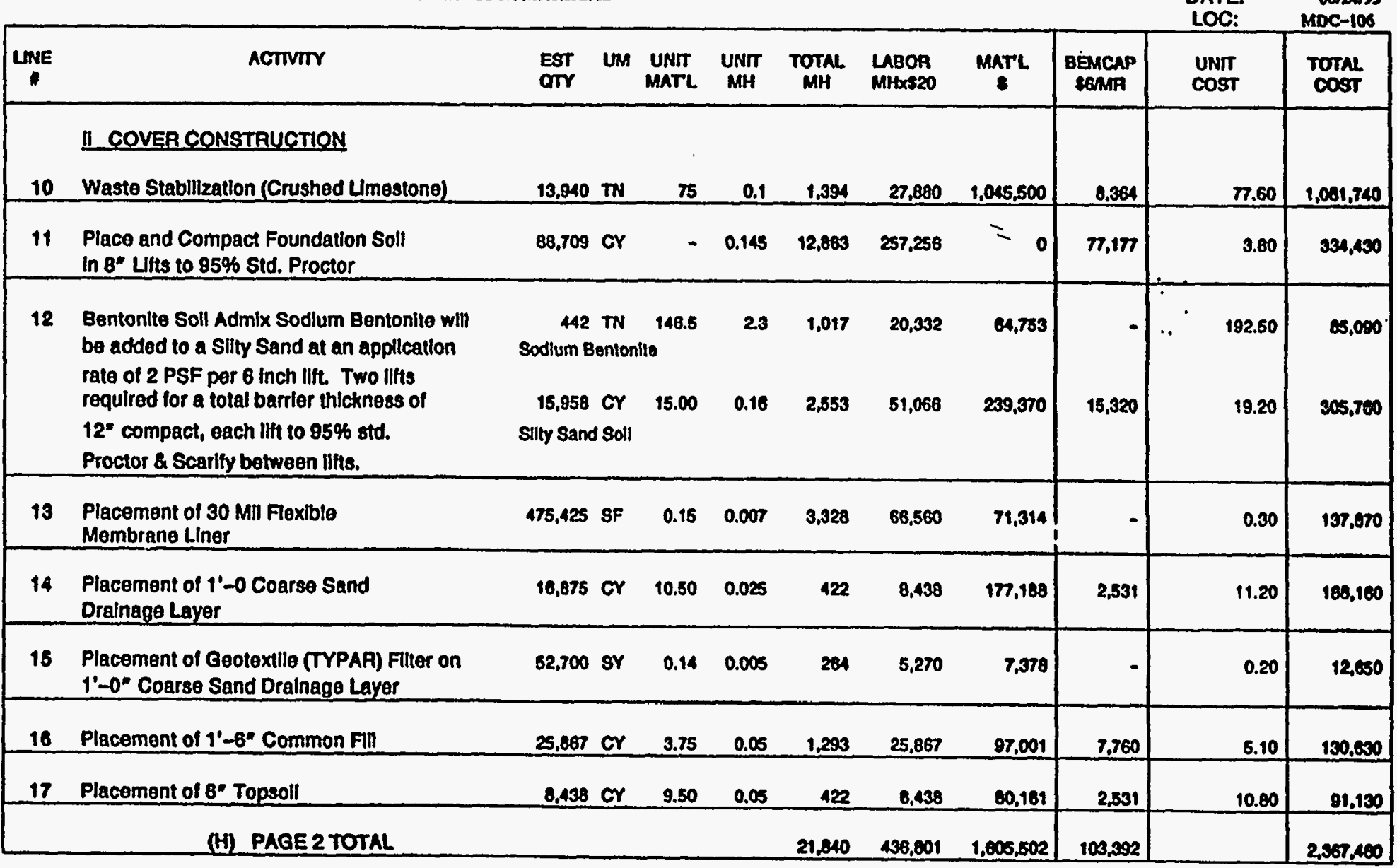

G-10. Cost Estimate for a Cover System Utilizing Bentonite Soil Additive and a 30-mil FML (Cont'd) 


\section{DETAIL ESTIMATE}

LOC1: $7-03$

ACTIVITY CODE: ESE260412

PROJ.NAMB: ECON. BVALUATION OF CLOSURB CAP BARRIER MATHRIAL.

CHK'D BY: S. MEAD

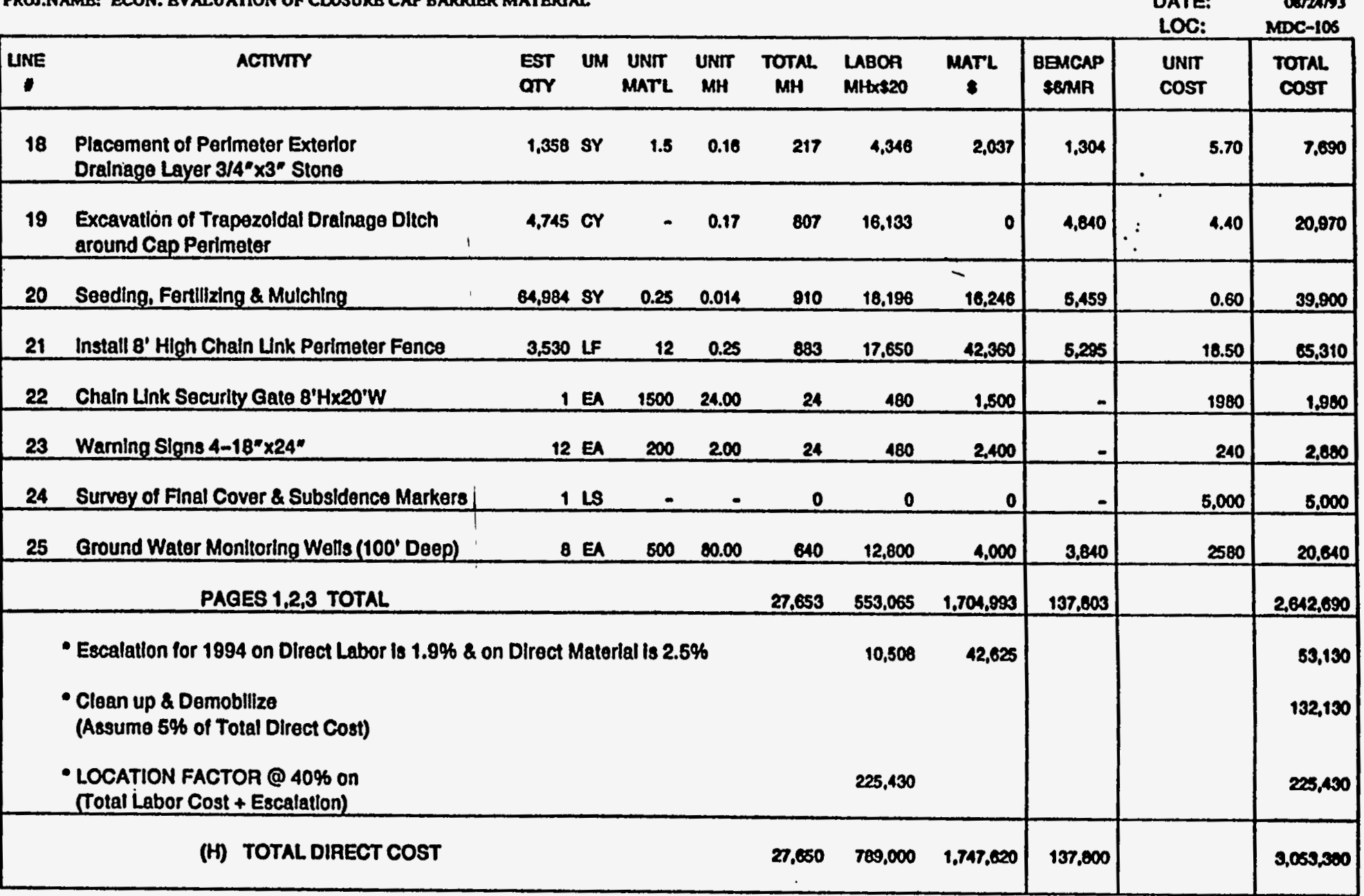

G-10. Cost Estimate for a Cover System Utilizing Bentonite Soil Additive and a 30-mil FML (Cont'd) 
DETAIL ESTIMATE

L00 1: 7-03

ACTIVITY CODE: ESE260112

PROJ.NAME: ECON. EYALUATION OF CLOSURR CAP BARRIER MATERIAL.

\begin{tabular}{|c|c|c|c|c|c|c|c|c|c|c|c|}
\hline LINE & ACTIVTIY & $\begin{array}{l}\text { EST } \\
\text { arr }\end{array}$ & UM & $\begin{array}{l}\text { UNT } \\
\text { MATL }\end{array}$ & $\begin{array}{l}\text { UNT } \\
\text { MH }\end{array}$ & $\begin{array}{l}\text { TOTAL } \\
\text { MH }\end{array}$ & $\begin{array}{l}\text { LABOR } \\
\text { MHXe20 }\end{array}$ & $\stackrel{\operatorname{maTL}}{*}$ & $\begin{array}{l}\text { BEMCAP } \\
\text { EGMR }\end{array}$ & $\begin{array}{l}\text { UNrT } \\
\text { Cost }\end{array}$ & $\begin{array}{l}\text { TOTAL } \\
\text { COST }\end{array}$ \\
\hline & INDIRECT COSTS & & & & & & & & & & \\
\hline 1 & $\begin{array}{l}\text { Certification of the Final Cover } \\
\text { (Assume 2.6\% of the Total Direct Cost) }\end{array}$ & & LS & & & & & & & & 76,330 \\
\hline 2 & $\begin{array}{l}\text { Health \& Safety } \\
\text { Assume } 490 \text { of Total Direct Cost }\end{array}$ & & LS & & & & & & & & 122,140 \\
\hline 3 & $\begin{array}{l}\text { Scope Contingency } \\
\text { Assume 10\% of Total Direct Cost }\end{array}$ & & LS & & & & & & & & 305.310 \\
\hline 4 & $\begin{array}{l}\text { Administrative \& Legal } \\
\text { @ B\% of Total Direct Cost }\end{array}$ & & LS & & & & & & & & 244,270 \\
\hline 8 & $\begin{array}{l}\text { Engineering \& Services @ } \\
20 \% \text { of Total Direct Cost }\end{array}$ & & & & & & & & & & 610,680 \\
\hline 6 & Craft Support @ 20.8\% of Total MHRS(\$19/hr) & & & & & 3,750 & & & & & 109,250 \\
\hline 7 & $\begin{array}{l}\text { Field Indirects of Total Direct } \\
\text { Labor @ 46.5\%, Material @ 26.5\% }\end{array}$ & & & 1 & & & 366,885 & 463,110 & & & 800,000 \\
\hline 8 & $\begin{array}{l}\text { Construction Nonmanual @ } 25.2 \% \text { of Total MH } \\
\text { Q Rale of } \$ 43.68\end{array}$ & & & & & 8,980 & & & & & 200,300 \\
\hline 9 & Projoct Managament @ 8\% of Total Direct + ind & acts & & & & & & & & & 452,460 \\
\hline & (H) TOTAL INDIRECT COST & & & & & & & & & & $3,054,020$ \\
\hline
\end{tabular}

CHK'D BY: S. MEAD

ESTIMATOR: POANLX

PHONE: 7-5266

DATE: C-106

that

G-10. Cost Estimate for a Cover System Utilizing Bentonite Soil Additive and a 30-mil FML (Cont'd) 


\section{DETAIL ESTIMATE}

CHK'D BY: S. MEAD

2001: $2-03$

ACTIVITY CODB: ESE2SOS12

PROJ.NAME: ECON. EVALUATTON OF CLOSURE CAP BARRIER MATERIAL

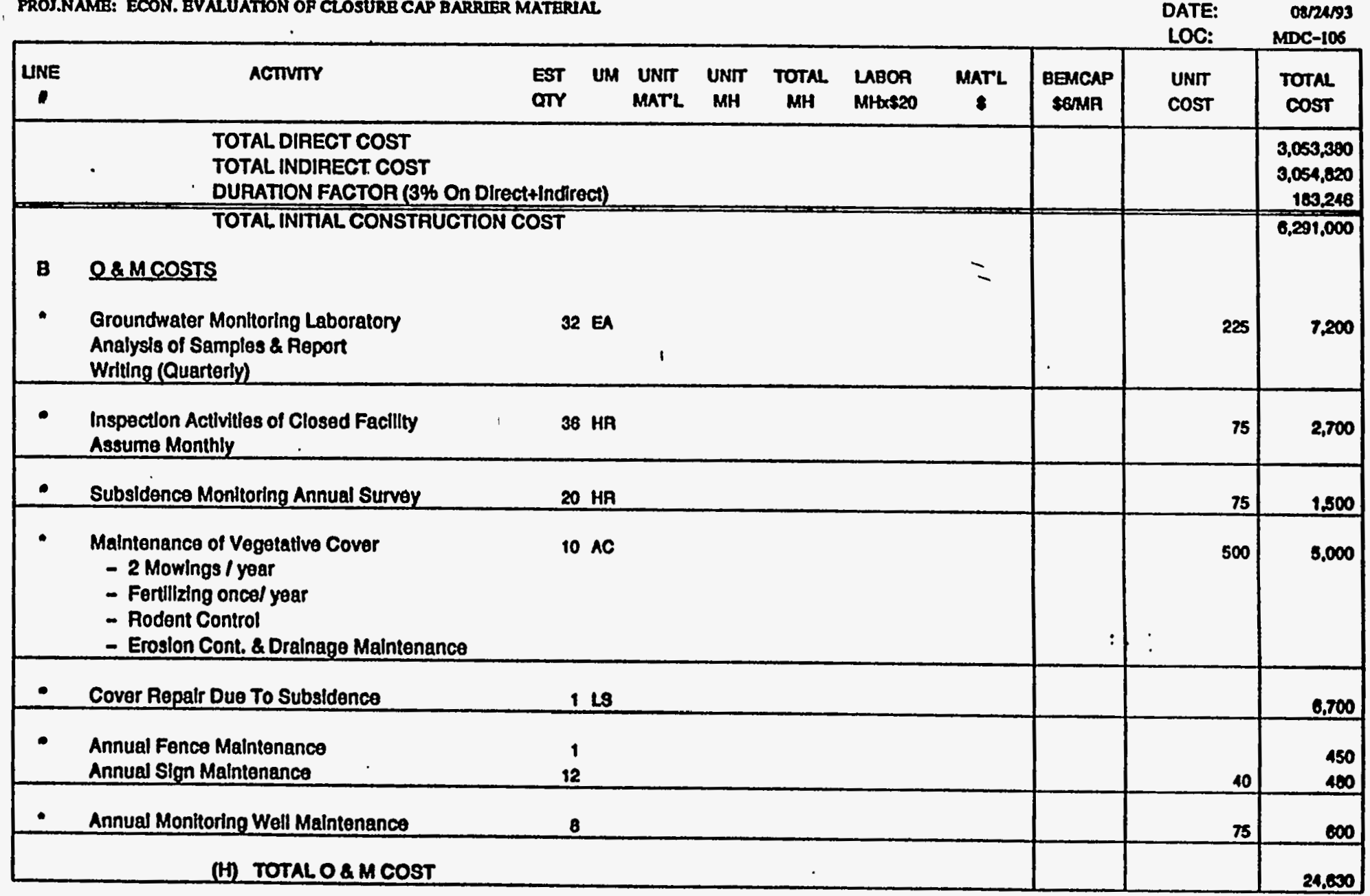

G-10. Cost Estimate for a Cover System Utilizing Bentonite Soil Additive and a 30-mil FML (Cont'd) 
DETAIL ESTIMATE

\section{Loo 1: 7-03}

ACTIVITY CODE: ESE2QS1:12

PROJ.NAMB: ECON. BVALLUATON OF CLOSURB CAP BARRIER MATERIAL
CHK'D BY: S. MEAD

ESTIMATOR: raWWX

PHONE: 7-5266

DATE: CORAM3

LOC: MDC-105

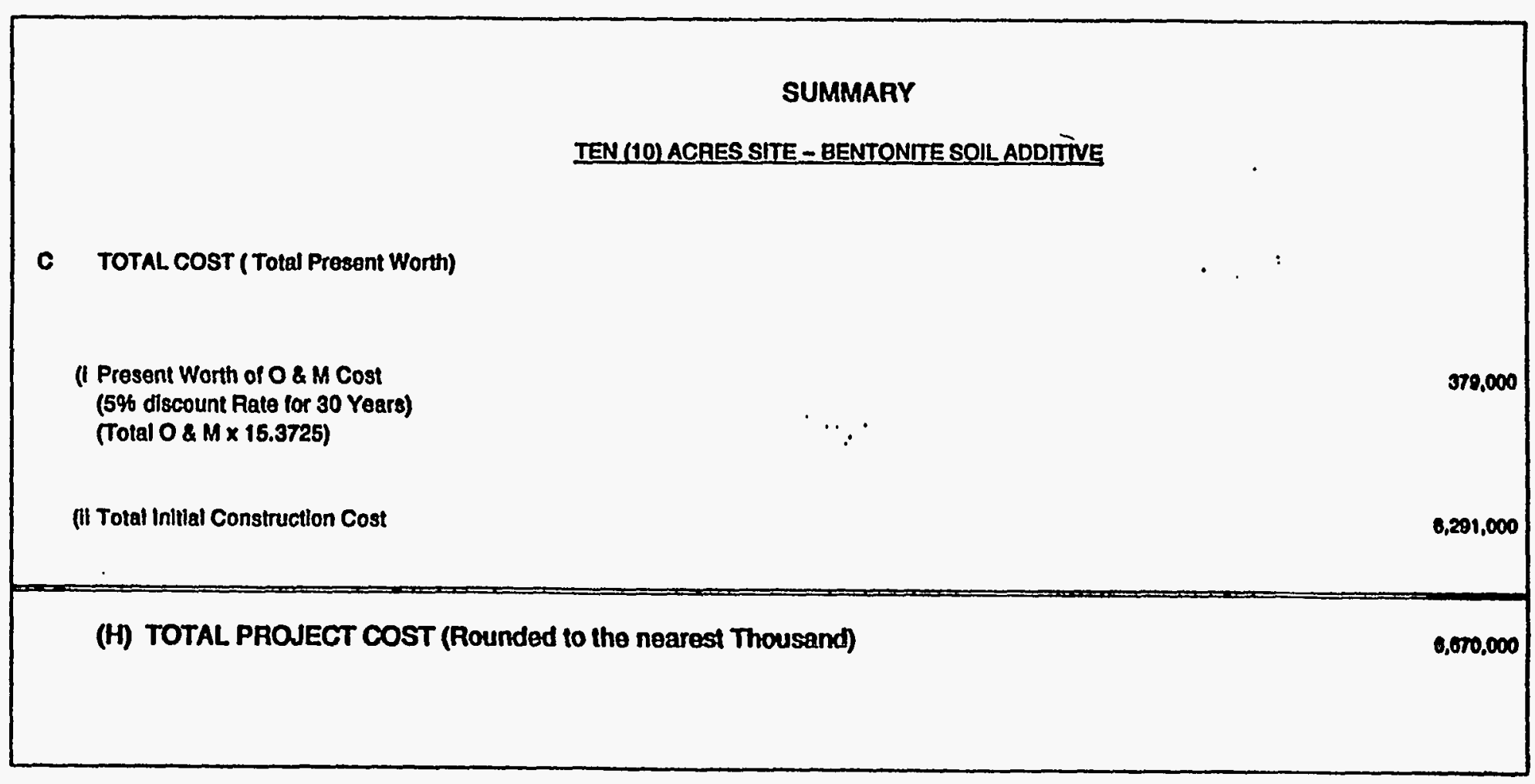

G-10. Cost Estimate for a Cover System Utilizing Bentonite Soil Additive and a 30-mil FML (Cont'd) 
DETAIL ESTIMATE

CHK'D BY: S. MEAD

Loo $x: 7-03$

ACTIVITY CODE: ESE260112

PROJ.NAME: BCON. EVALUATTON OF CLOSURB CAP BARRIER MATERIAL

ESTIMATOR: POAMKX

PHONE: 7-S265

DATE: Der25s3

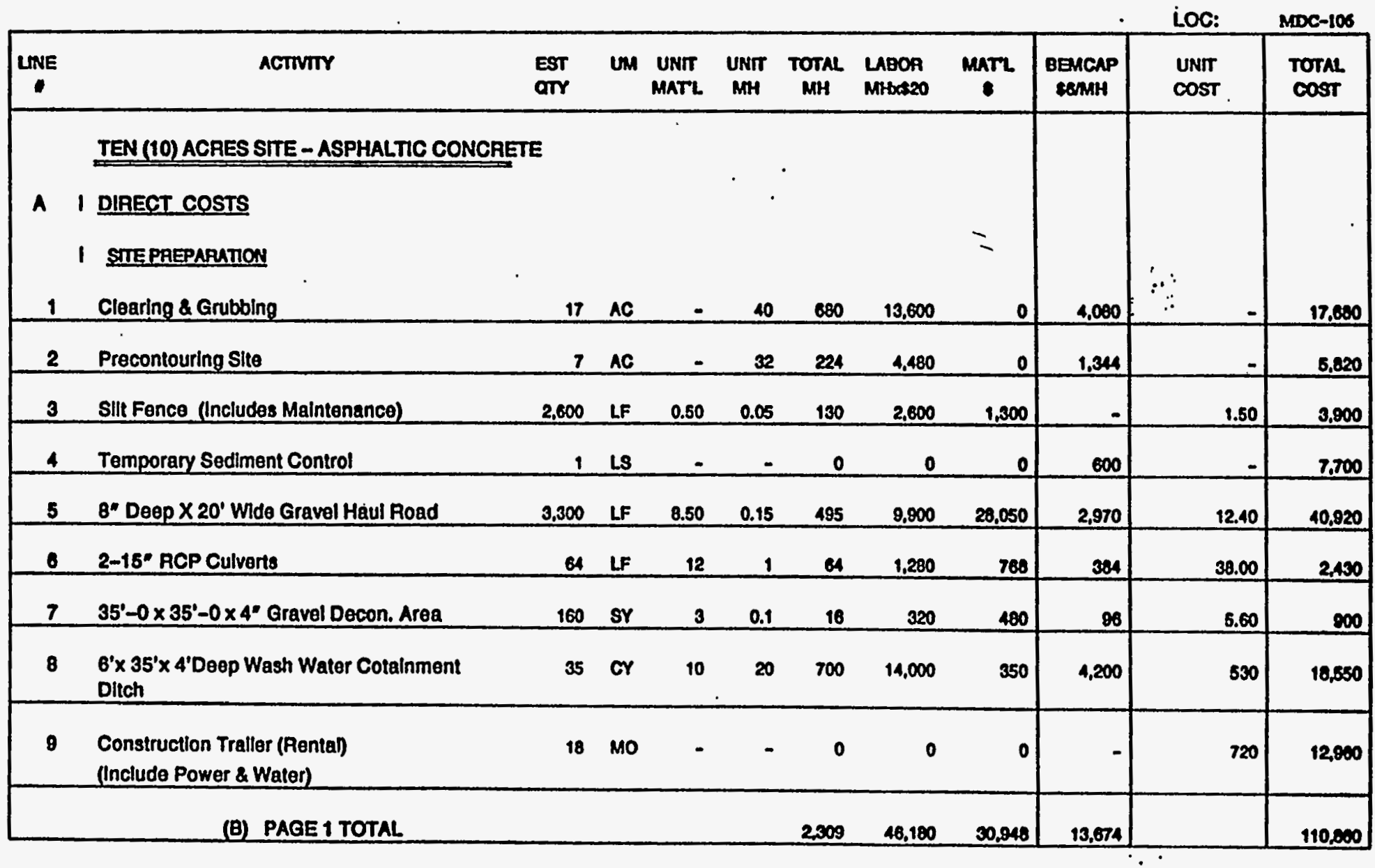

G-11. Cost Estimate for a Cover System Asphalt Concrete Hydraulic Barrier and a 30-mil FML 
DETAIL ESTIMATE

$2001: 7-03$

ACTTVTY CODB: 8SB2SOA12

PROJ.NAMB: BCON. BYALUATION OF CLOSURB CAP BARRIRR MATRPIAL.

\begin{tabular}{|c|c|c|c|c|c|c|c|c|c|c|c|}
\hline UINE & ACTIVIY & $\begin{array}{l}\text { Est } \\
\text { OTY }\end{array}$ & un & $\begin{array}{c}\text { UNTT } \\
\text { MATL }\end{array}$ & $\underset{M H}{\text { UNIT }}$ & TOTAL & $\begin{array}{l}\text { LABOR } \\
M H+\times+20\end{array}$ & $\stackrel{\text { MATL }}{:}$ & $\begin{array}{c}\text { BEMGAP } \\
\text { SGMMH }\end{array}$ & $\begin{array}{l}\text { UNT } \\
\text { Cost }\end{array}$ & $\begin{array}{l}\text { TotAl } \\
\text { cost }\end{array}$ \\
\hline 10 & $\begin{array}{l}1 \text { COVER CONSTRUCTION } \\
\text { Waste Stabilization (Crushed Umestone) }\end{array}$ & 13,940 & $T N$ & 75 & 0.1 & 1394 & 27,800 & $1,045,500$ & 8,364 & 77.60 & $1.001,740$ \\
\hline 11 & $\begin{array}{l}\text { Place and Compact Foundation Soll } \\
\text { In } 8^{\circ} \text { Lifts to } 95 \% \text { Std. Proctor }\end{array}$ & 73,907 & or & - & 0.145 & 10,717 & 214,330 & $=$ & 84,299 & 3.80 & 278,650 \\
\hline 12 & $\begin{array}{l}\text { Placement of } 30 \text { Mill Flexible } \\
\text { Membrane Liner }\end{array}$ & 421,078 & SF & 0.15 & 0.007 & 2.948 & 58,851 & 03,161 & - & 0.30 & 122,110 \\
\hline 13 & $\begin{array}{l}\text { Placement of 1'-0 Coarse Sand } \\
\text { Drainage Layer }\end{array}$ & 15,202 & or & 10.50 & 0.025 & 380 & 7.601 & 159,621 & 2,280 & 11.10 & 169,500 \\
\hline 14 & $\begin{array}{l}\text { 2" PVC Perforated Dralnage Plpe placed } \\
\text { in Drainage layer }\end{array}$ & 5,016 & LF & 1.0 & 0.2 & 1,003 & 20,064 & 8,016 & - & 5.00 & 23,080 \\
\hline 18 & 2 1/2" Binder Course & 45,840 & sY & 4.15 & 0.055 & 2,510 & 50,204 & 189,408 & 15,061 &.$\cdot \cdot$ & 254,070 \\
\hline 16 & 4" Hydraulle Asphalt Conc. & 45,640 & sY & 8.60 & 0.03 & 1,369 & 27,384 & 301,224 & 8,215 & 7.40 & 338,020 \\
\hline 17 & Asphalt Mastic Soal & 45.640 & SY & 0.50 & 0.015 & 685 & 13,692 & 22,020 & $=$ & 0.80 & 36,510 \\
\hline 18 & Sprayed Asphaltic Concrete Sealant & 45,640 & sr & 0.75 & 0.015 & 605 & 13,692 & 34,230 & 4,108 & 1.10 & 52,050 \\
\hline 19 & Excavatlon of Traingular Drainage Dilch & 2.592 & or & $=$ & 0.17 & 41 & 8,813 & $\underline{0}$ & 2.644 & 1.40 & 11,460 \\
\hline 20 & 10 Thick Asphalt Perlmeler Drainage Ditch & 5,854 & sY & 7.6 & 0.144 & 843 & 18,860 & 44.490 & 5,058 & 11.30 & 60,410 \\
\hline & (B) PAGE 2 TOTAL & & & & & 22,974 & $\mathbf{4 5 9 , 4 7 0}$ & $1,865,469$ & 110,029 & & $2,134,800$ \\
\hline
\end{tabular}

G-11. Cost Estimate for a Cover System Asphalt Concrete Hydraulic Barrier and a 30-mil FML (Cont'd) 
DBTAIL B S TIMATE

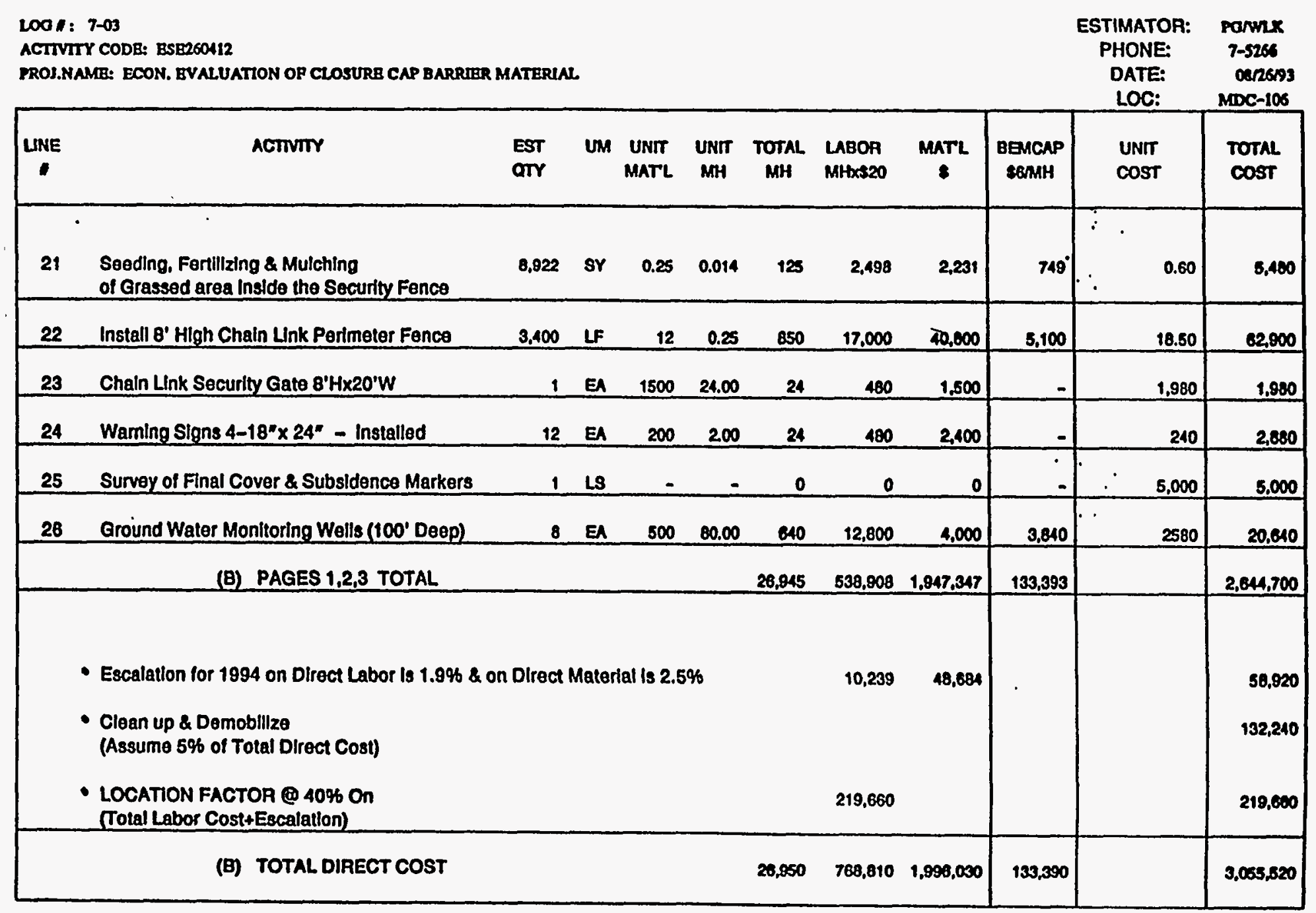

G-11. Cost Estimate for a Cover System Asphalt Concrete Hydraulic Barrier and a 30-mil FML (Cont'd) 
D B T A I L B S T I M A B

CHK'D BY: S. MEAD

Lo0 1: 7-03

ACTIVITY CODE: BSE260412

PROJ.NAMBE: ECON. EVALUATTON OR CLOSURB CAP BARRIER MATERINL

ESTIMATOR: PONMKK

PHONE: 7-5266

DATE: 1000609

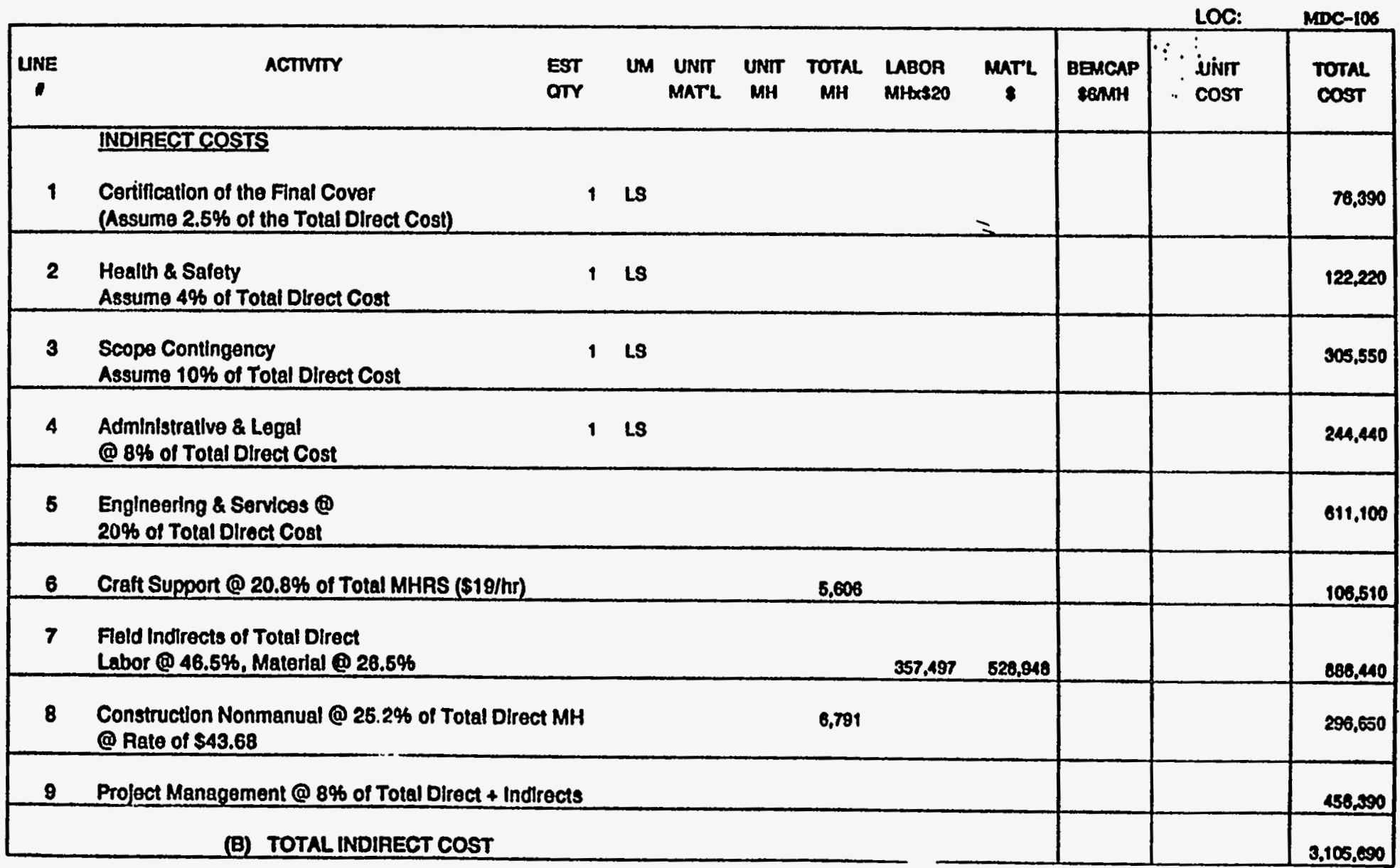

G-11. Cost Estimate for a Cover System Asphalt Concrete Hydraulic Barrier and a 30-mil FML (Cont'd) 
DETAIL ESTIMATE

CHKDBY: S.MEAD

L00 I: $7-03$

ACTIVTTY CODE: ESE26012

PROJ.NAME: ECON. EVALUATION OF CLOSURB CAP BARRIER MATERIAL

STIMATOR: PaMnK

PHONE: 7-5260

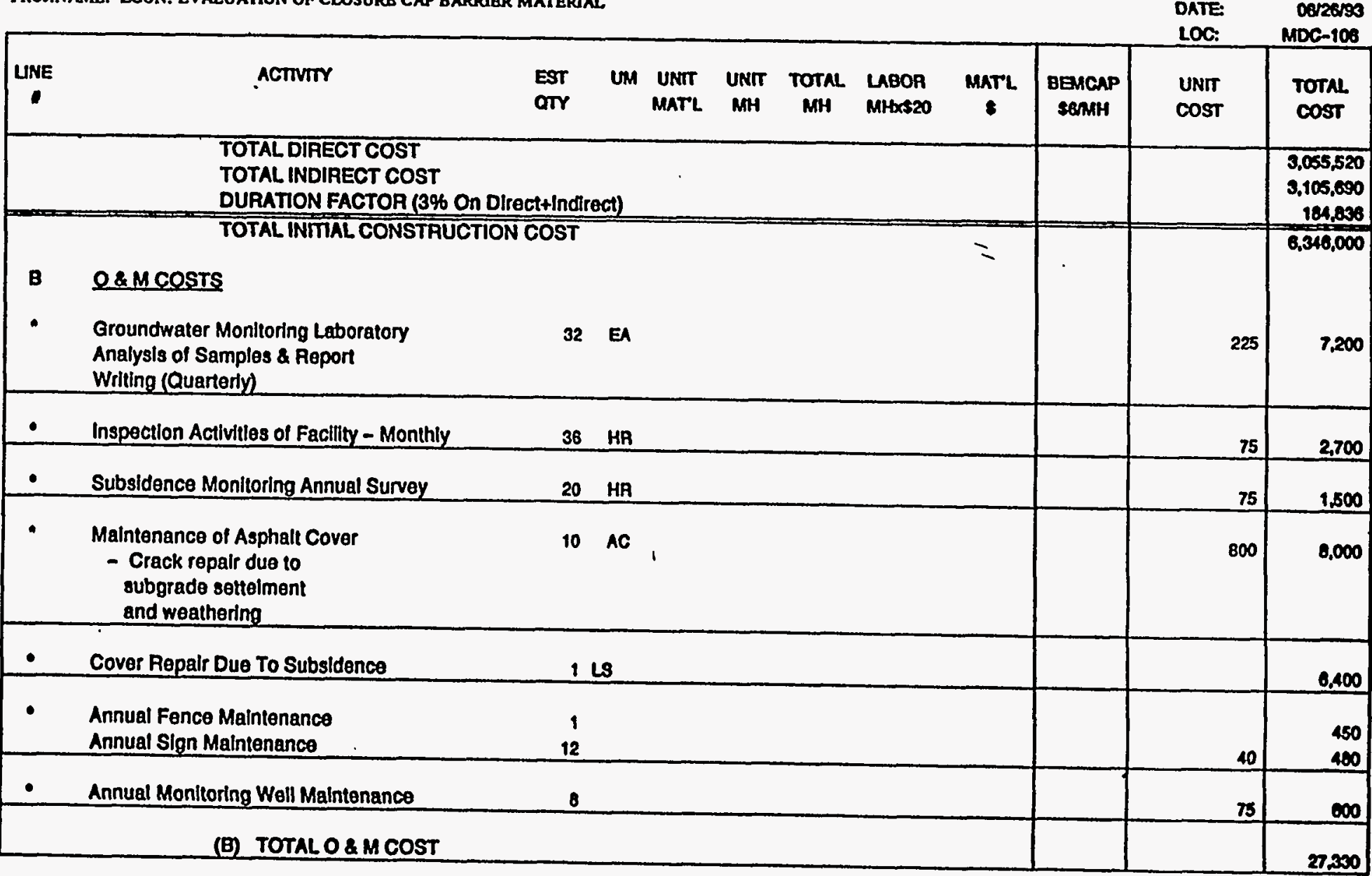

G-11. Cost Estimate for a Cover System Asphalt Concrete Hydraulic Barrier and a 30-mil FML (Cont'd) 
D B T A I L B S T I M A T B

\section{Loof: 7-03}

ACTVITY CODE: ESE260012

PROJ.NAMM: ECON. EVALUATTON OR CLOSURB CAP УARRIER MATERIAL
CHK'D BY: S. MEAD

ESTIMATOR: POWWK

PHONE: 7-5266

DATE: Darass

LOC: MDC-106

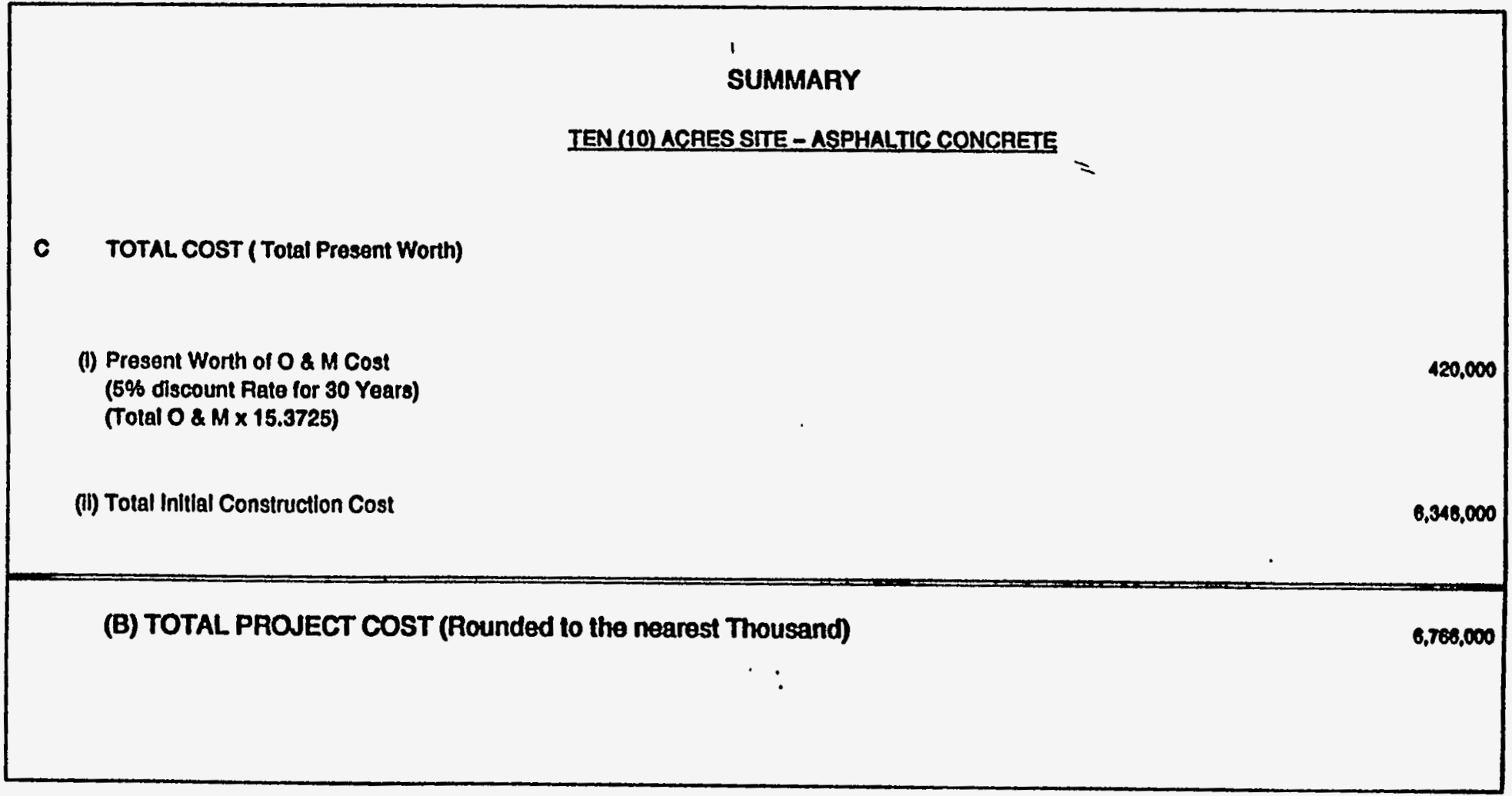

G-11. Cost Estimate for a Cover System Asphalt Concrete Hydraulic Barrier and a 30-mil FML (Cont'd) 
DETAIL ESTIMATE

CHK'D BY: S. MEAD

L.0. I: $7-0.03$

ACTUVTY CODE: ESE200412

PFOU.NAME: ECON. EVALUATION OF CLOSURE CAP QARRIER MATEFAAL

ESTIMATOR: $\operatorname{man}$

PHONE: 7-5266

DATE: $\quad 0026593$

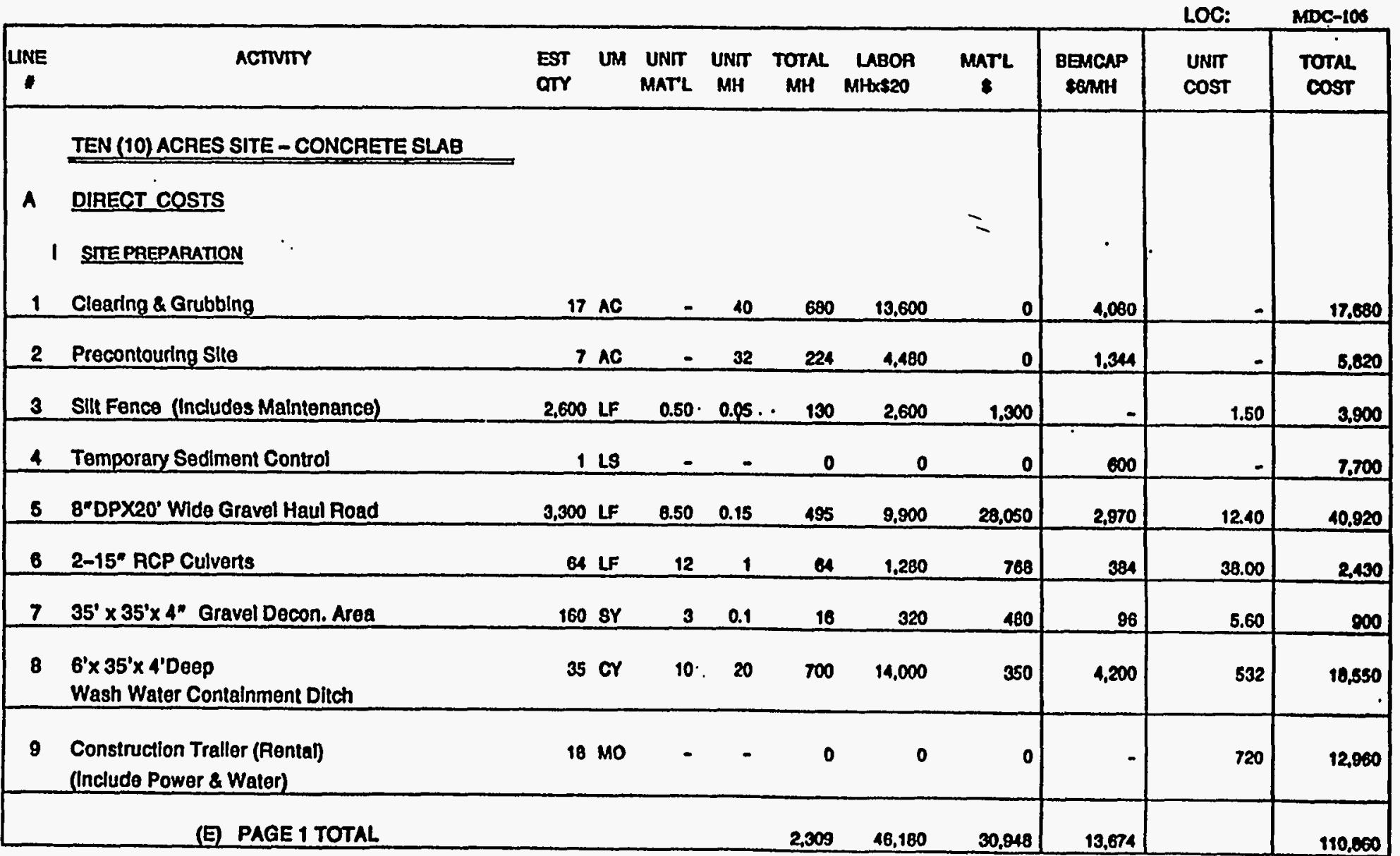

G-12. Cost Estimate for a Cover System Utilizing Poured-in Place Concrete Slab and a 30-mil FML 
DETAIL ESTIMATE CHK'DBY: KULP

L001: 7-03

ACTTVTTY CODE: ESE260412

PROI.NAME: ECON. EVALUATTON OF CLOSURB CAP BARRIER MATERYAL

PO

PHONE: 7-5266

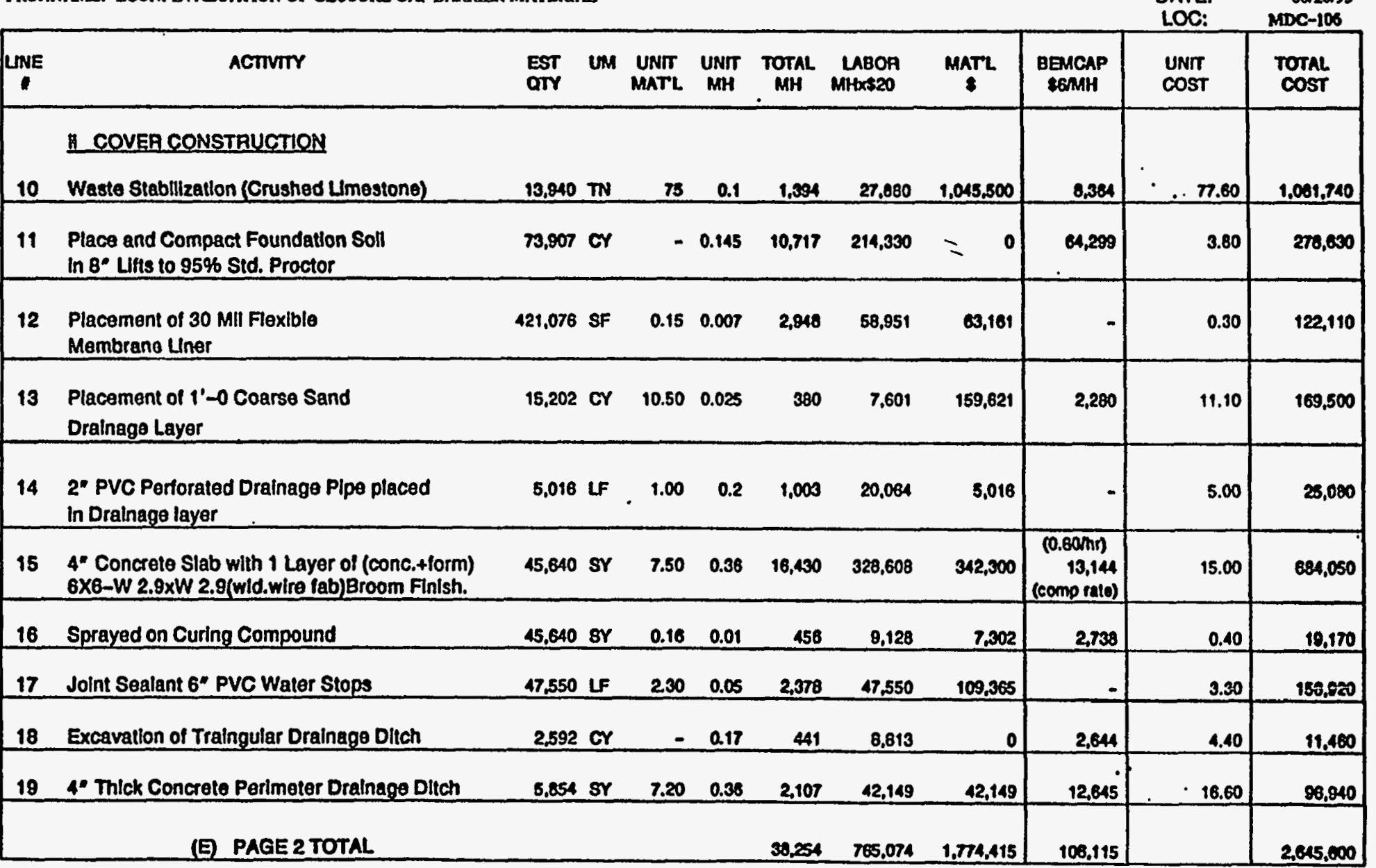

G-12. Cost Estimate for a Cover System Utilizing Poured-in Place Concrete Slab and a 30-mil FML (Cont'd) 


\section{ETAIL B S T I MAT E}

CHK'D BY: $\quad$ KULP

Lo0: : 7-03

ACTIVITY CODE: ESE260412

PROI.NAME: ECON. EVALUATION OF CLOSURB CAP BARRIER MATERIAL. DATE: DSOS

ESTIMATOR: PO

PHONE: 7-5266

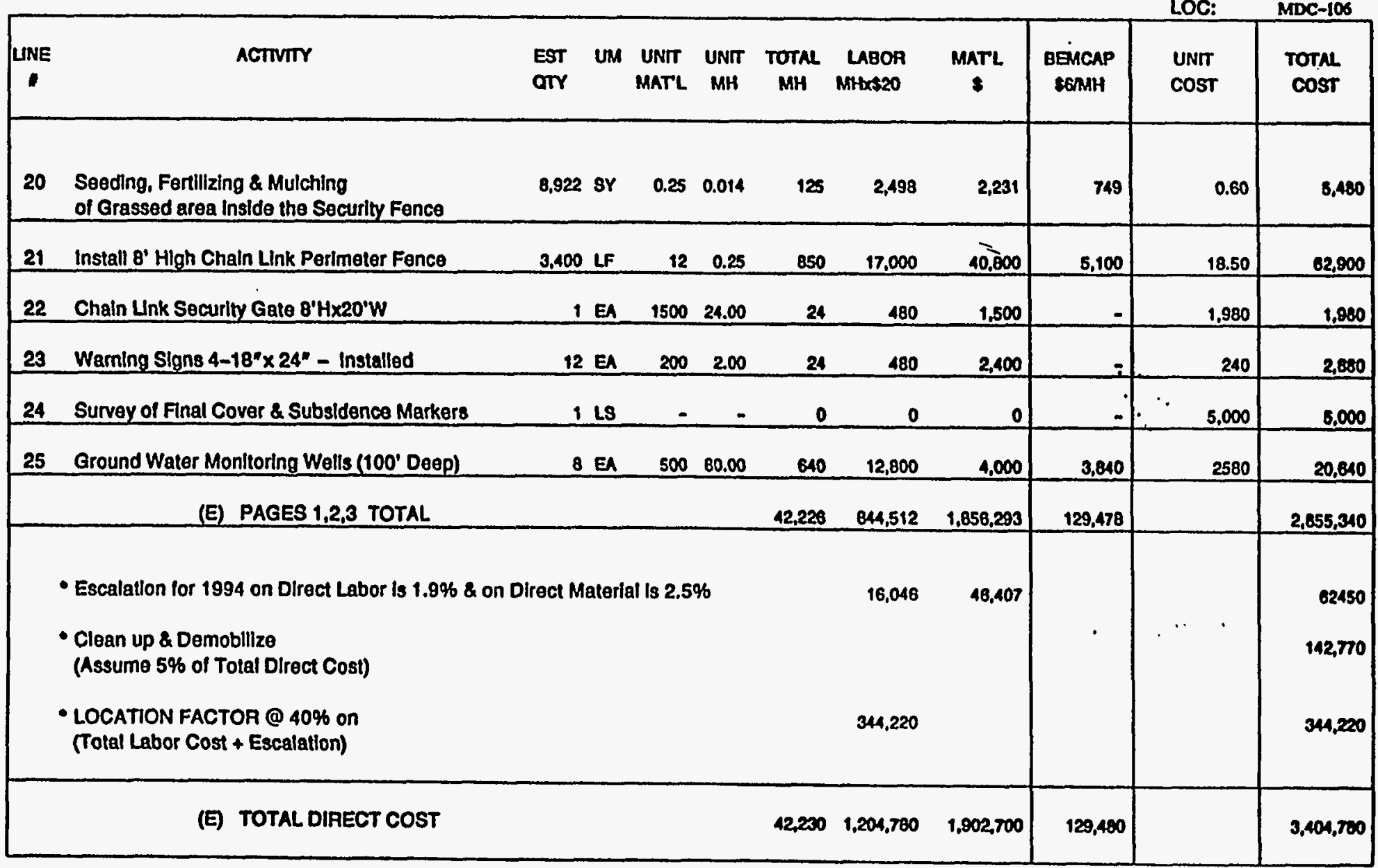

G-12. Cost Estimate for a Cover System Utilizing Poured-in Place Concrete Slab and a 30-mil FML (Cont'd) 
Lo01: 7-03

ACTIVTYY CODE: ESE2600112

PROS.NAME: ECON. EVALUATTON OR CLOSURE CAP BAPRIER MATERRAL

DATE: $\quad 0026193$

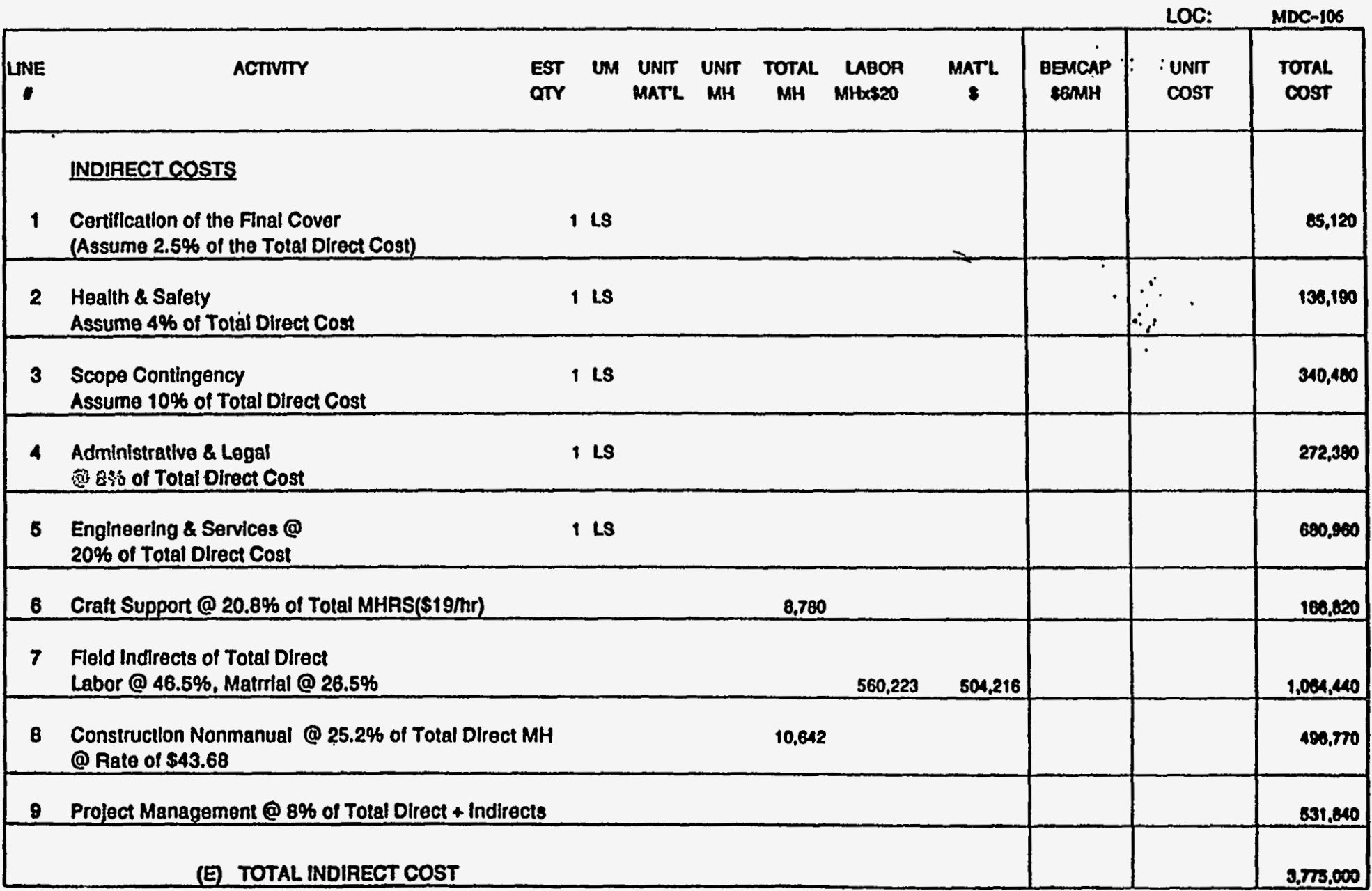

G-12. Cost Estimate for a Cover System Utilizing Poured-in Place Concrete Slab and a 30-mil FML (Cont'd) 
DETAIL ESTIMATE

Loof: $7-03$

ACTIVITY CODB: ESE260412

PROJ.NAMB: ECON. EVALUATTON OF CLOSURB CAP BARRRER MATERIAL.

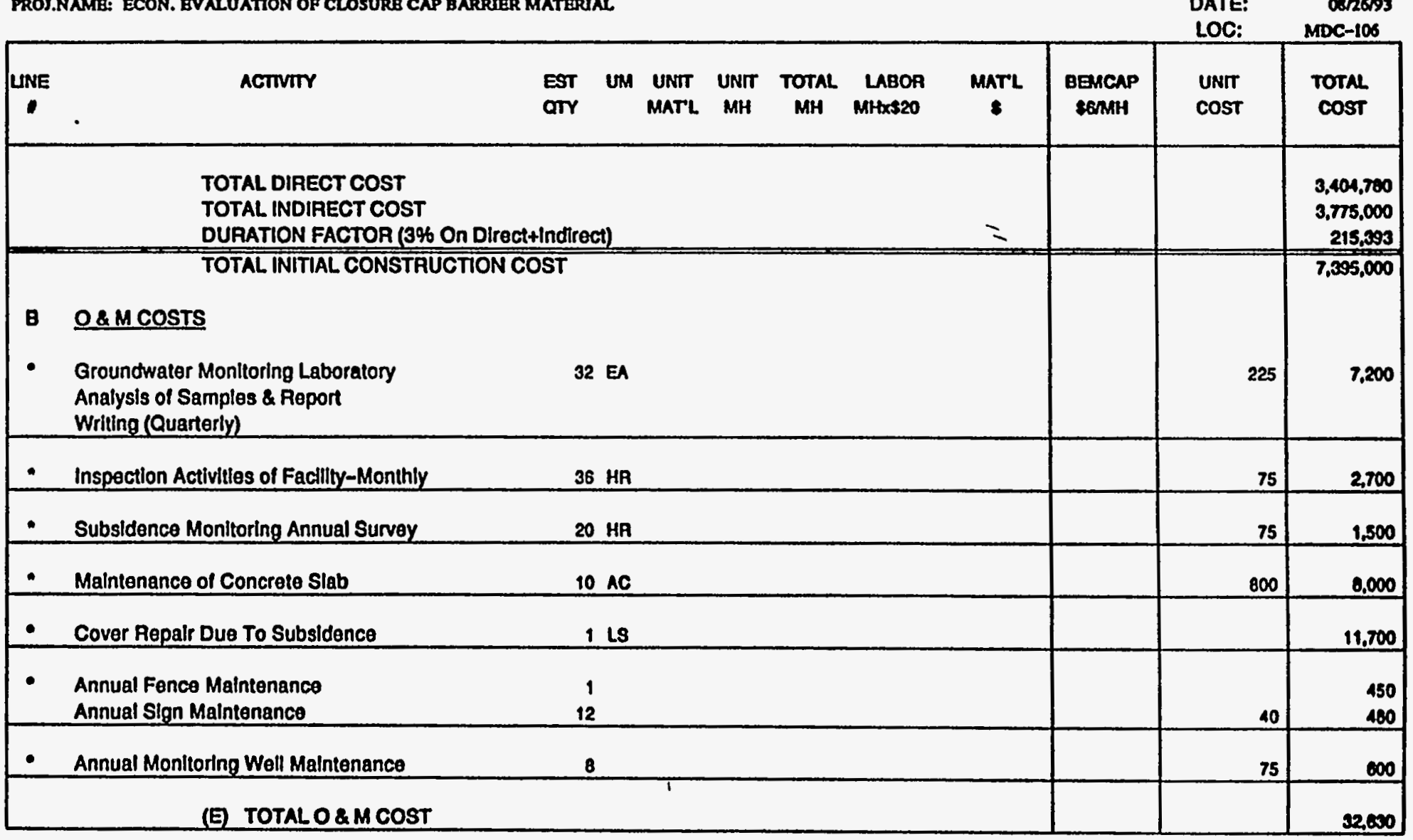

G-12. Cost Estimate for a Cover System Utilizing Poured-in Place Concrete Slab and a 30-mil FML (Cont'd) 
DETAIL ESTIMAT B

$\begin{array}{cc}\text { CHK'O BY: } & \text { KULP } \\ \text { ESTIMATOR: } & \text { PO } \\ \text { PHONE: } & 7-5266 \\ \text { DATE: } & \text { TOR26193 } \\ \text { LOC: } & \text { MDC-106 }\end{array}$

Loo : : 7-03

ACTIVITY CODE: ESE260+12

PROI.NAME: ECON. EVALUATION OF CLOSURB CAP BARRIER MATERIAL,

LOC: MDC-105

\begin{tabular}{|l|c|}
\hline & SUMMARY \\
TEN (10) ACRES SITE - CONCRETE SLAB \\
C TOTAL COST ( Total Present Worth) \\
(I Present Worth of O \& M Cost \\
(5\% discount Rate for 30 Years) \\
(Total O \& M x 15.3725) \\
(II Total Inllial Constructlon Cost \\
(E) TOTAL PROJECT COST (Rounded to the nearest Thousand) \\
\hline
\end{tabular}

G-12. Cost Estimate for a Cover System Utilizing Poured-in Place Concrete Slab and a 30-mil FML (Cont'd) 


\section{Appendix H}

\section{Cost Estimates for 20-Acre Site}




\section{DETAIL ESTIMATE}

Loo A: 7-03

ACTIVITY CODB: BSE2SOH12

MOU.NAME: BCON. BYALUATTON OP CLOSURE CAP BARRIER MATERIAN

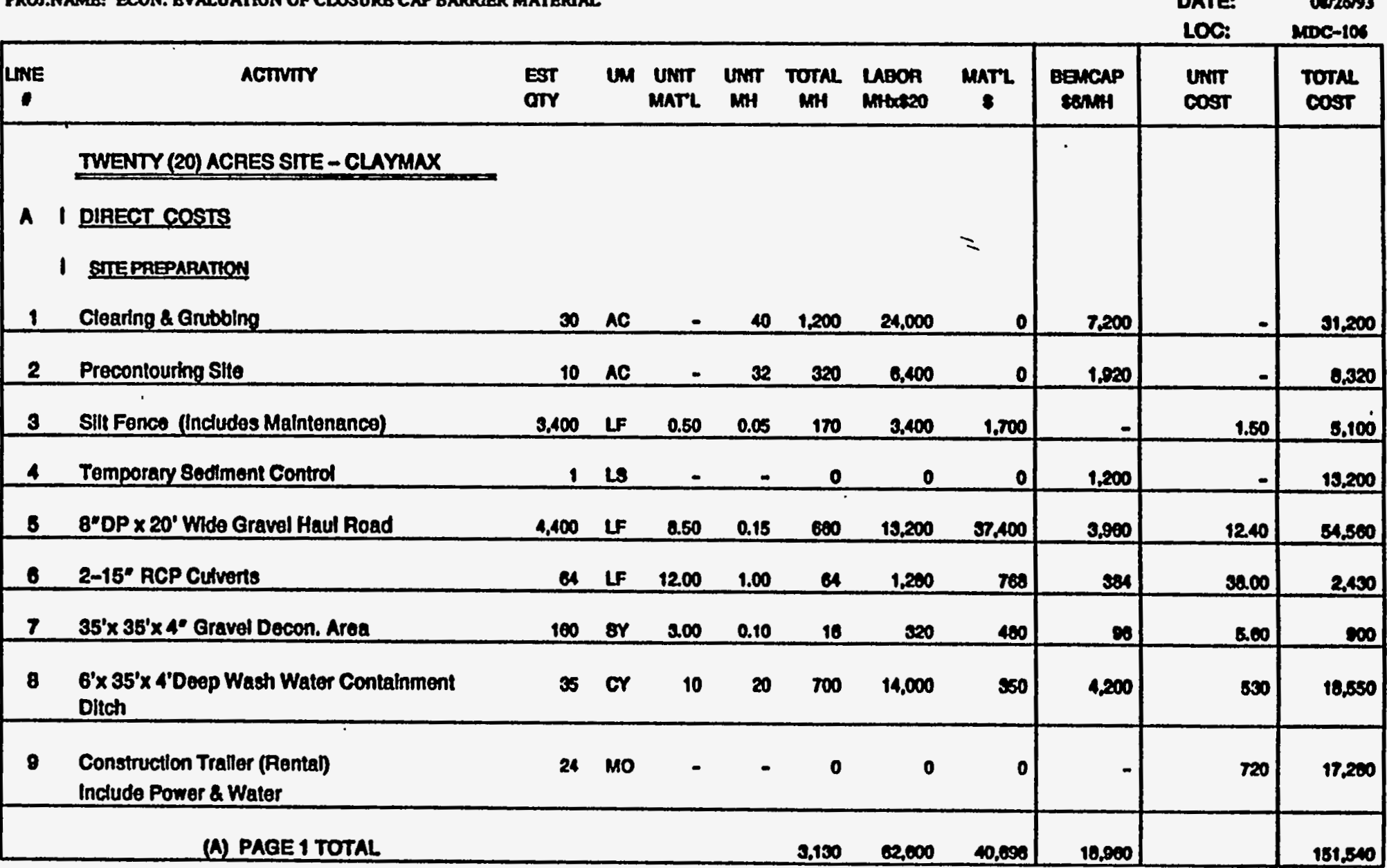

H-1. Cost Estimate for a Cover System Utilizing Claymax and a 30-mil FML 
DETAIL ESTIMATE

2001: 7-03

ACTIVITY CODE: ESE260412

MROJ.NAME: ECON. EVALUATON OP CLOSURB CAP BARKRER MATERAAL

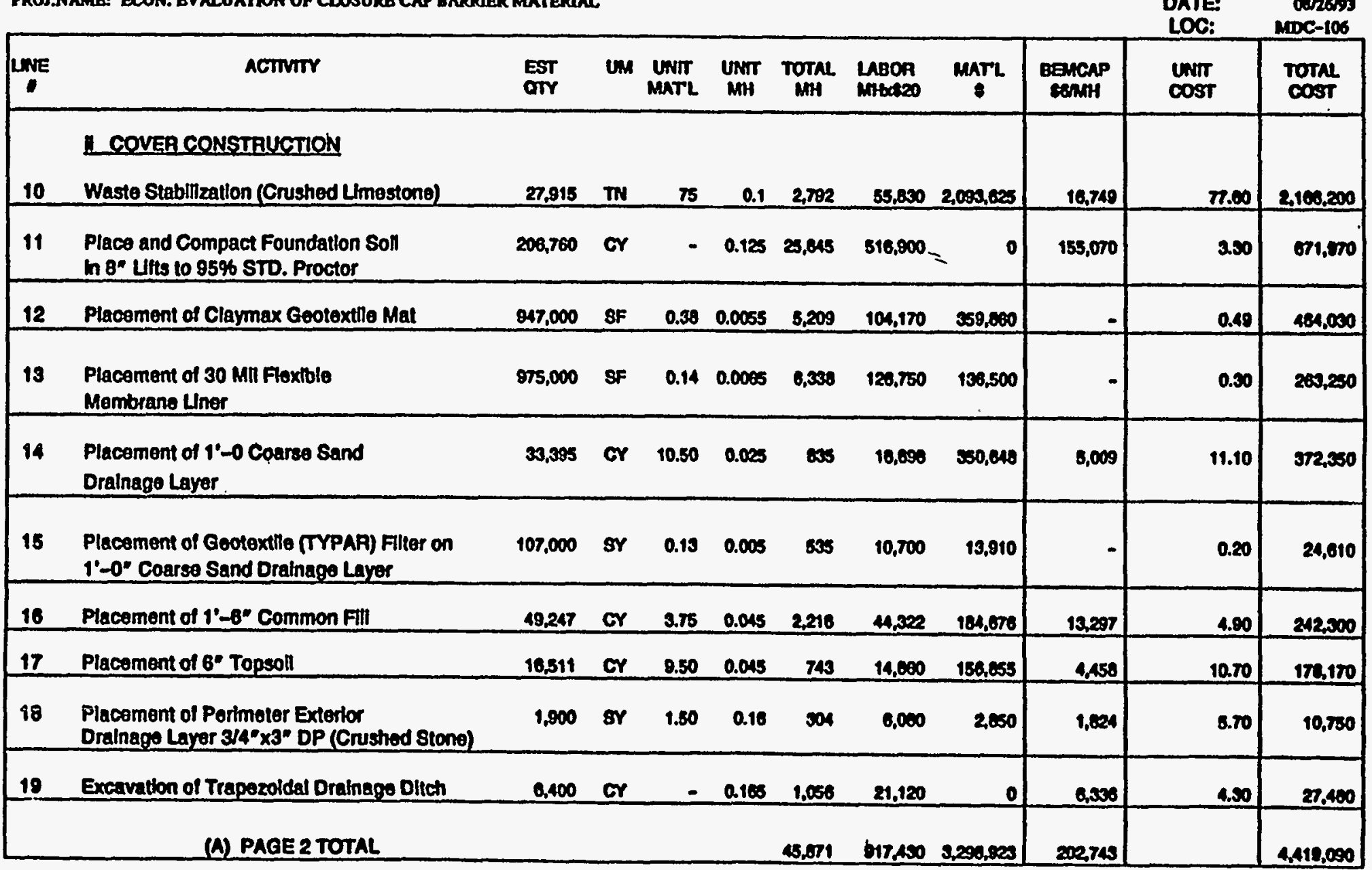

H-1. Cost Estimate for a Cover System Utilizing Claymax @ and a 30-mil FML (Cont'd) 
CHK'D BY: S. MEAD

Lo01: $7-03$

ACTIVITY CODB: ESE26012

PROU.KAME: BCON. BVALUATION OP CLOSURB CAP BARRIER MATERIAL.

ESTIMATOA: NamR

PHONE:

7-5266

DATE:

ourcess

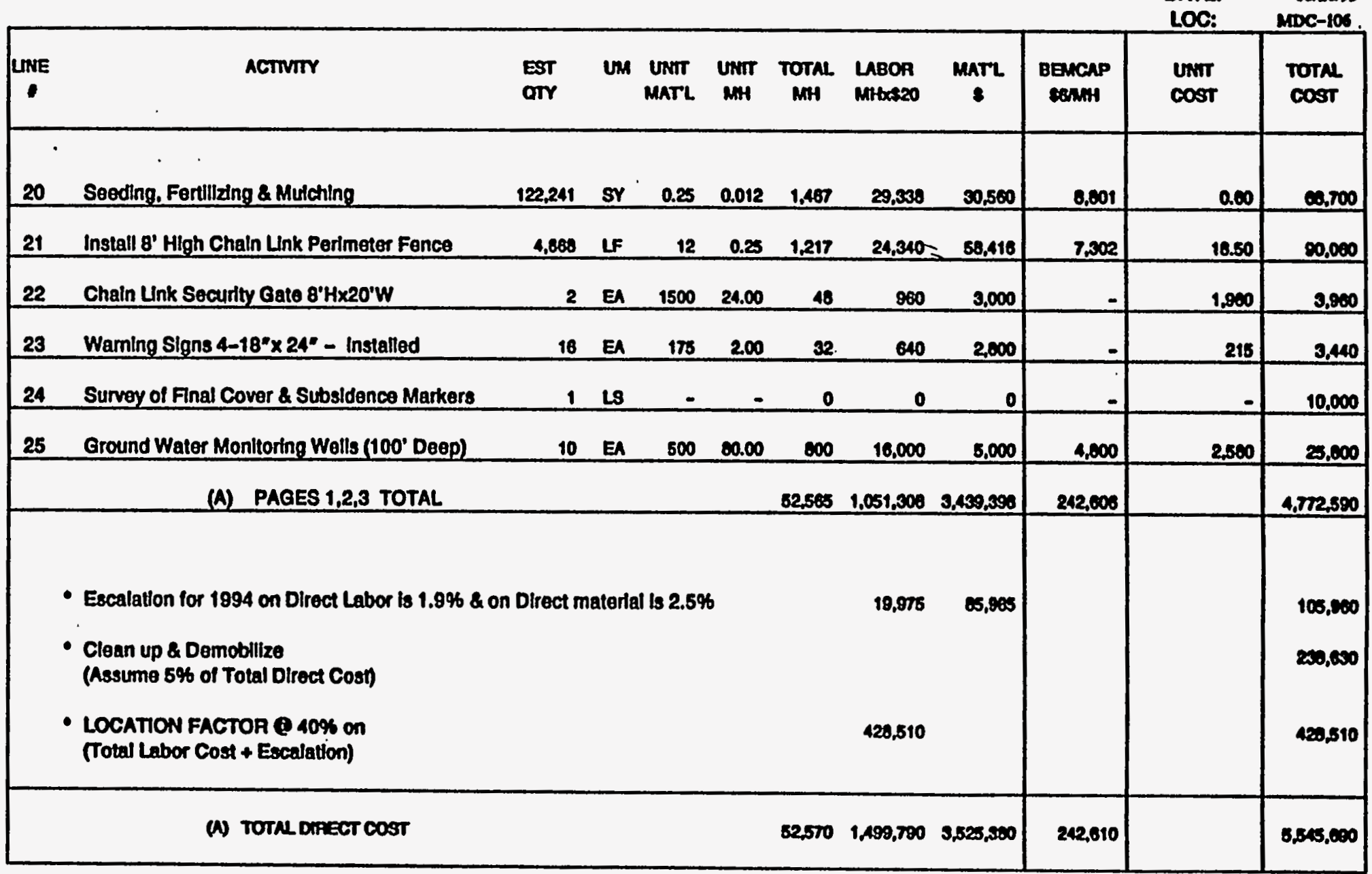

H-1. Cost Estimate for a Cover System Utilizing Claymax and a 30-mil FML (Cont'd) 
D B TAIL BSTIMATB

$1001: 7-03$

ACTIVITY CODE: ESE260412

MOJ.NAME: ECY' EVALUATTON OP CLOSURB CAP BANRRER MATERSAL

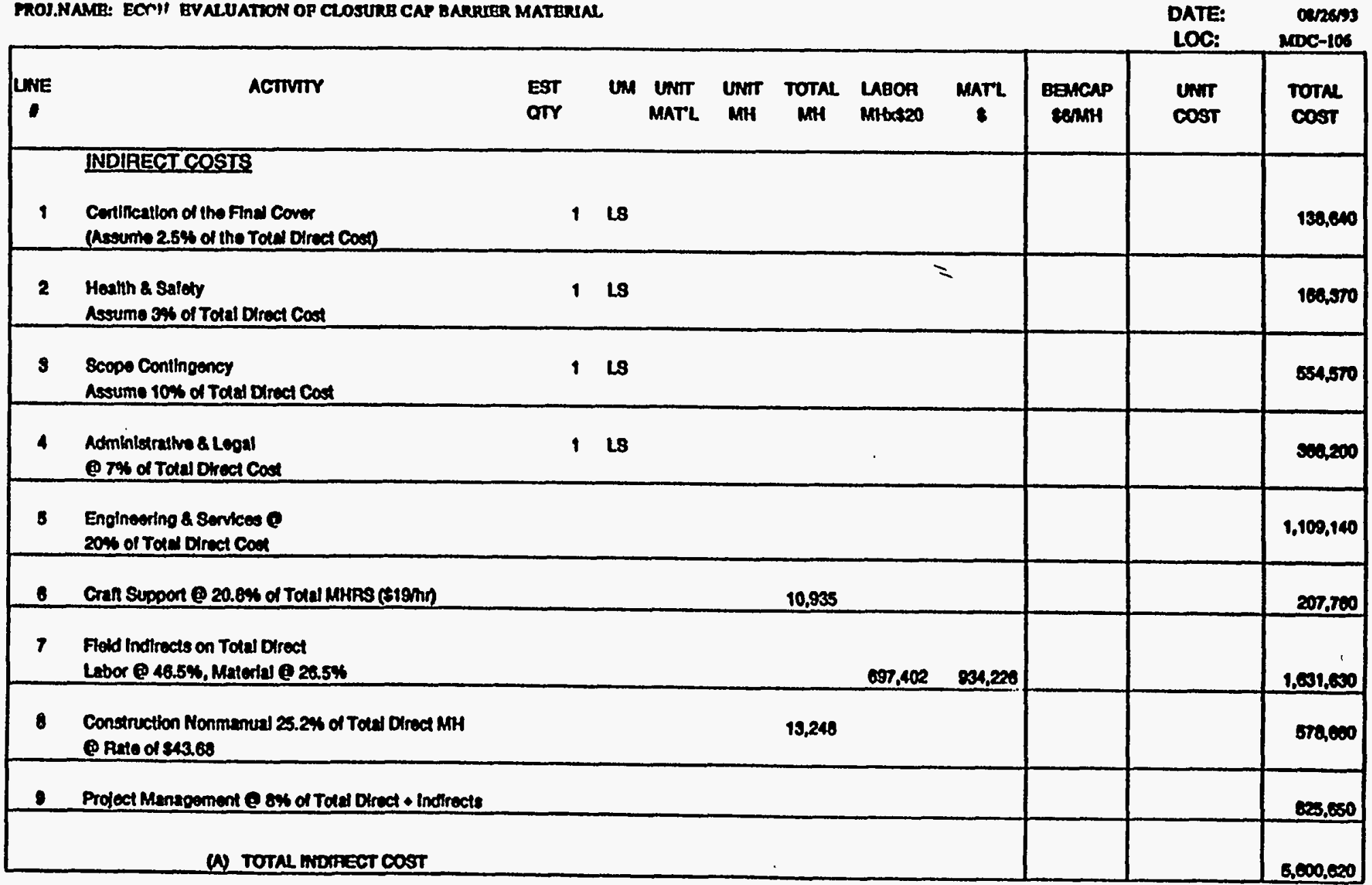

CHKD BY: 8. MEAD

ESTIMATOR: Namx PHONE: 7-5266

DATE: ORZMS - -106

H-1. Cost Estimate for a Cover System Utilizing Claymax and a 30-mil FML (Cont'd) 
Lo01: 7-03

ACTTVTY CODB: ESEZOON12

TROJ.NAME: ECON. BVALUATON OF CLOSURE CAP BARRIER MATERIAL

ESTIMATOA: raws

PHONE:

7-5266

DATE: Densos

\begin{tabular}{|c|c|c|c|c|c|c|c|c|c|c|c|}
\hline LINE & ACTIVIY & $\begin{array}{l}\text { EST } \\
\text { OTY }\end{array}$ & Um & $\begin{array}{l}\text { UNTT } \\
\text { MATL }\end{array}$ & $\begin{array}{l}\text { UNT } \\
\text { MH }\end{array}$ & $\begin{array}{c}\text { TOTAL } \\
\text { MH }\end{array}$ & $\begin{array}{l}\text { LABOR } \\
\text { MHQAPO }\end{array}$ & $\underset{*}{\text { MATL }}$ & $\begin{array}{c}\text { BEMCAP } \\
\text { EgAMH }\end{array}$ & $\begin{array}{l}\text { UNT } \\
\text { Cost }\end{array}$ & $\begin{array}{l}\text { Total } \\
\text { cost }\end{array}$ \\
\hline & $\begin{array}{l}\text { TOTAL DIRECT COST } \\
\text { TOTAL INDIRECT COST } \\
\text { DURATION FACTOR }(3 \%\end{array}$ & ot+lndire & & & & & & & & & $\begin{array}{r}5,645,690 \\
6,000,020 \\
334,309 \\
\end{array}$ \\
\hline B & $\begin{array}{l}\text { TOTAL INITIAL CONSTRU } \\
\text { Q\&MCOSTS } \\
\text { Groundwater Monitoring Laboratory } \\
\text { Analysis of Samples \& Report } \\
\text { Writing (Quarterty) }\end{array}$ & 40 & EA & & & & . & $=$ & & 225 & $\begin{array}{r}11,401,000 \\
8,000\end{array}$ \\
\hline$\bullet$ & Inspection Activities of Facilly - Monthly & 72 & HR & & & & & & & 76 & 8,400 \\
\hline- & Subsidence Monltoring Annual Survoy & 20 & HR & & & & & & & $\pi$ & 1.500 \\
\hline$\bullet$ & $\begin{array}{l}\text { Malntenance of Vegetative Cover } \\
\text { - } 2 \text { Mowings / year } \\
\text { - Pest Control } \\
\text { - Eroslon Control \& } \\
\text { Drainage Maintenance }\end{array}$ & 20 & AC & & & & & & & 500 & 10,000 \\
\hline$\bullet$ & Cover Repalr Due To Subsidence & 1 & LS & $\therefore$ & & & & & & & 0.400 \\
\hline$\bullet$ & $\begin{array}{l}\text { Annual Fenco Malntenance } \\
\text { Annual Stgn Maintenancs }\end{array}$ & $\begin{array}{r}1 \\
16 \\
\end{array}$ & & & & & . & & & 40 & $\begin{array}{l}800 \\
640 \\
\end{array}$ \\
\hline - & Anmual Monitorine Well Maintenance & 10 & & & & & & & & $\pi$ & 900 \\
\hline & (A) TOTAL O \& M COST & & & & & & & & & & $3 \times 280$ \\
\hline
\end{tabular}

H-1. Cost Estimate for a Cover System Utilizing Claymax and a 30-mil FML (Cont'd) 
CHKD BY: 8. MEAD

100 I: $7-03$

ACTIVITY CODB: BSE2SOH12

PROJ.NAMGE: ECON. EVALUATTON OF CLOSURB CAP BARRIBR MATERIAL

ESTIMATOR:

PHONE:

DATE

LOC:

rowex

7-52es

coszests

MDC-106

\section{SUMMARY}

TWENTY (20) AGRES SITE-CLAYMAX

527,000

(i) Present Worth of 0 \& $M$ Cost

(5\% discount Hato for 30 Years)

(Total $O \& M \times$ 15.3725)

(ii) Total Initiad Construction Cost

$11,401,000$

(A) TOTAL PROJECT COST (Rounded to the nearest Thousand)

$12,000,000$

H-1. Cost Estimate for a Cover System Utilizing Claymax and a 30-mil FML (Cont'd) 


\section{DETAIL ESTIMATE}

1001: 7-03

ACTIVTYY CODE: ESE2OOA12

PROJ NAME: ECON. EVALUATION OR CLOSURB CAP BARRIER MATERIAL

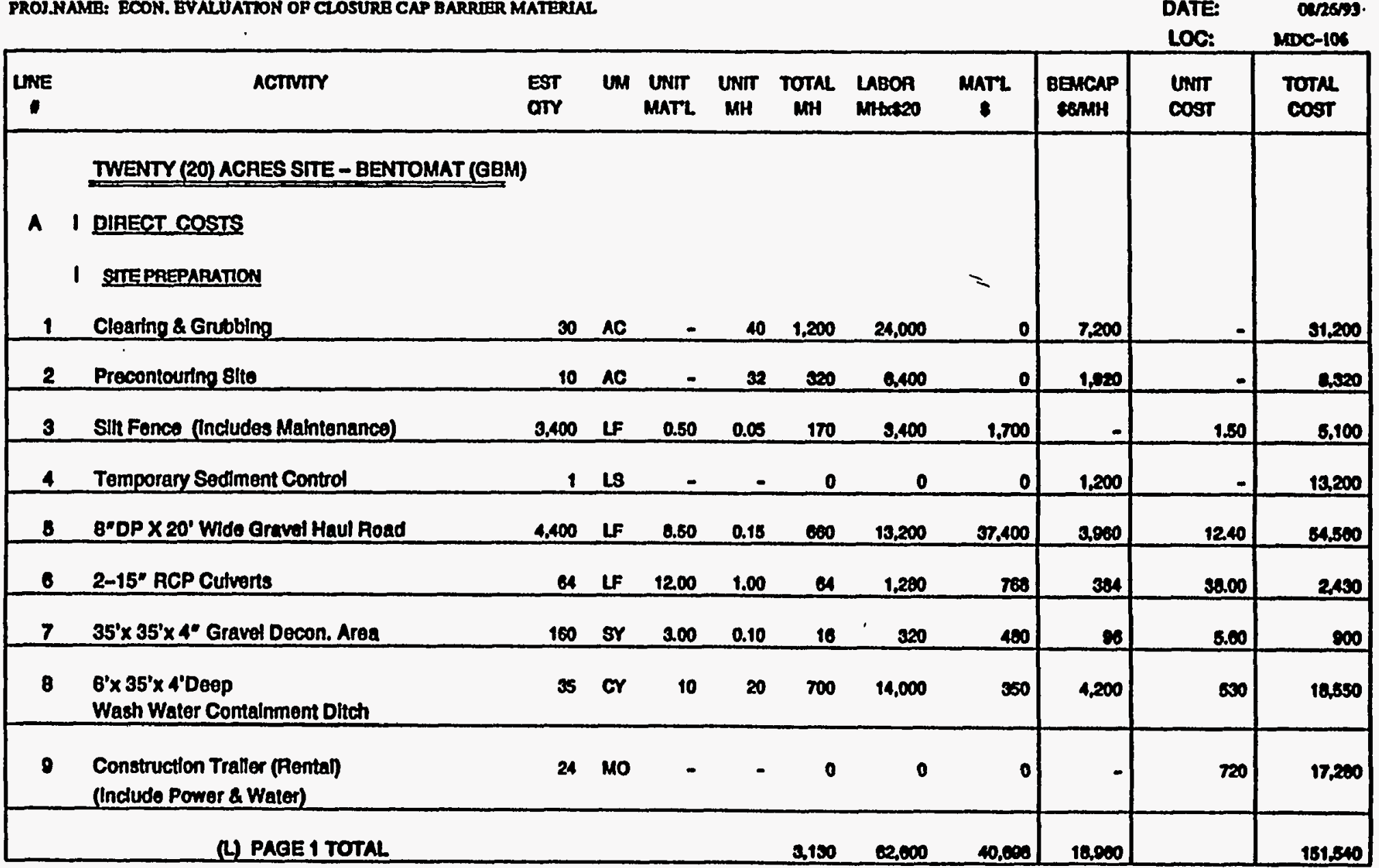

H-2. Cost Estimate for a Cover System Utilizing Bentomat@ and a 30-mil FML 


\section{DETAIL ESTIMATE CHKDBY: 8.MEAO}

Lod : $7-03$

ACTIVITY CODB: ESEQCOA12

PROJ.NAME: BCON. BVALUATION OP CLOSURB CAP DARRIER MATERUAL.

\begin{tabular}{|c|c|c|c|c|c|c|c|c|c|c|c|}
\hline $\operatorname{me}$ & ACTIVIY & $\begin{array}{l}\text { EST } \\
\text { arr }\end{array}$ & UM & $\begin{array}{l}\text { UNT } \\
\text { MATL }\end{array}$ & $\operatorname{Umm}_{\operatorname{Min}}$ & $\begin{array}{l}\text { Torme } \\
\text { Mat }\end{array}$ & $\begin{array}{l}\text { LABOA } \\
\text { Amtxi20 }\end{array}$ & MATL & $\begin{array}{l}\text { Bexcap } \\
\text { senmi }\end{array}$ & $\begin{array}{l}\text { Unit } \\
\text { cost }\end{array}$ & $\begin{array}{l}\text { Total } \\
\text { cost }\end{array}$ \\
\hline 10 & $\begin{array}{l}\text { COQVER CONSTRUCTION } \\
\text { Waste Stablization (Crushed Lmestone) }\end{array}$ & 27,915 & $\pi$ & $\pi 5$ & 0.1 & 2,792 & 55,830 & $2,093,020$ & 16,749 & 7.60 & $2,160,200$ \\
\hline 11 & $\begin{array}{l}\text { Piace and Compact Foundation Soll } \\
\text { In 8* Ufts to } 95 \% \text { Stdandard Proctor }\end{array}$ & 200.760 & Cr & - & 0.125 & 25,045 & 816,900 & $=$ & 155,070 & 3.30 & 671.070 \\
\hline 12 & Piacement of Bentomat Geotextlle Mas & 947,000 & SF & 0.38 & 0.0055 & 8,209 & 104,170 & 359,060 & - & 0.49 & 404,030 \\
\hline 13 & $\begin{array}{l}\text { Placement of } 30 \text { Mil Flexlble } \\
\text { Membrane Uner }\end{array}$ & 975.000 & $8 F$ & 0.14 & 0.0065 & 6,330 & 128,750 & 130,600 & - & 0.30 & 203,250 \\
\hline 14 & $\begin{array}{l}\text { Piacement of } 1^{\prime}-0 \text { Coarse Sand } \\
\text { Dralnage Layor }\end{array}$ & 33.395 & CY & 10.50 & 0.025 & 830 & 16,690 & 50,440 & 8,000 & 11.10 & 372,350 \\
\hline 18 & $\begin{array}{l}\text { Placement of Geotextlle (TYPAR) Fitter on } \\
1^{\prime}-0^{\circ} \text { Coarse Sand Dralnage Layor }\end{array}$ & 107,000 & 8Y & 0.13 & 0.005 & 835 & 10,700 & 15,910 & - & 0.20 & 24.610 \\
\hline 10 & Placoment of $1^{\prime}-6^{\prime \prime}$ Common FII & 49,247 & Cr & 3.75 & 0.045 & 2,216 & 44,322 & 184,078 & 19,207 & 4.80 & 242,000 \\
\hline 17 & Placement of $6^{-}$Topsoll & 16,511 & Or & 8.50 & 0.045 & 743 & 14,000 & 150,655 & 4,458 & 10.70 & 170,170 \\
\hline 18 & $\begin{array}{l}\text { Placememt of Pertmeter Exterior } \\
\text { Drainage Layer } 3 / 4^{*} \times 3^{*} \text { DP (Crushed Stone) }\end{array}$ & 1,900 & $8 Y$ & 1.50 & a.18 & 504 & 0,000 & 2,650 & 1.024 & $\mathbf{5 . 7 0}$ & $10, \pi 50$ \\
\hline 19 & Excavation of Trapezoidal Drainage Ditch & 6,400 & CY & - & 0.165 & 1.056 & 21.120 & 0 & 6.230 & 4.50 & 21.400 \\
\hline & (4) PAQE 2 TOTAL & & & & & $45,07 \pi$ & 917,450 & $3,290,020$ & 202,743 & & $4,412.050$ \\
\hline
\end{tabular}

H-2. Cost Estimate for a Cover System Utilizing Bentomat@ and a 30-mil FML (Cont'd) 


\section{B T A L B S TIMAT B}

CHK'D BY: 8. MEAD

2001: 7-03

ACTIVITY CODB: ESB2SOL12

PROJ.NAMB: ECON. BVALUATION OR CLOSURB CAP BARRIER MATERIAL.

\section{ESTMMATOR: ramx}

PHONE: 7-S265

DATE: OARSOS3

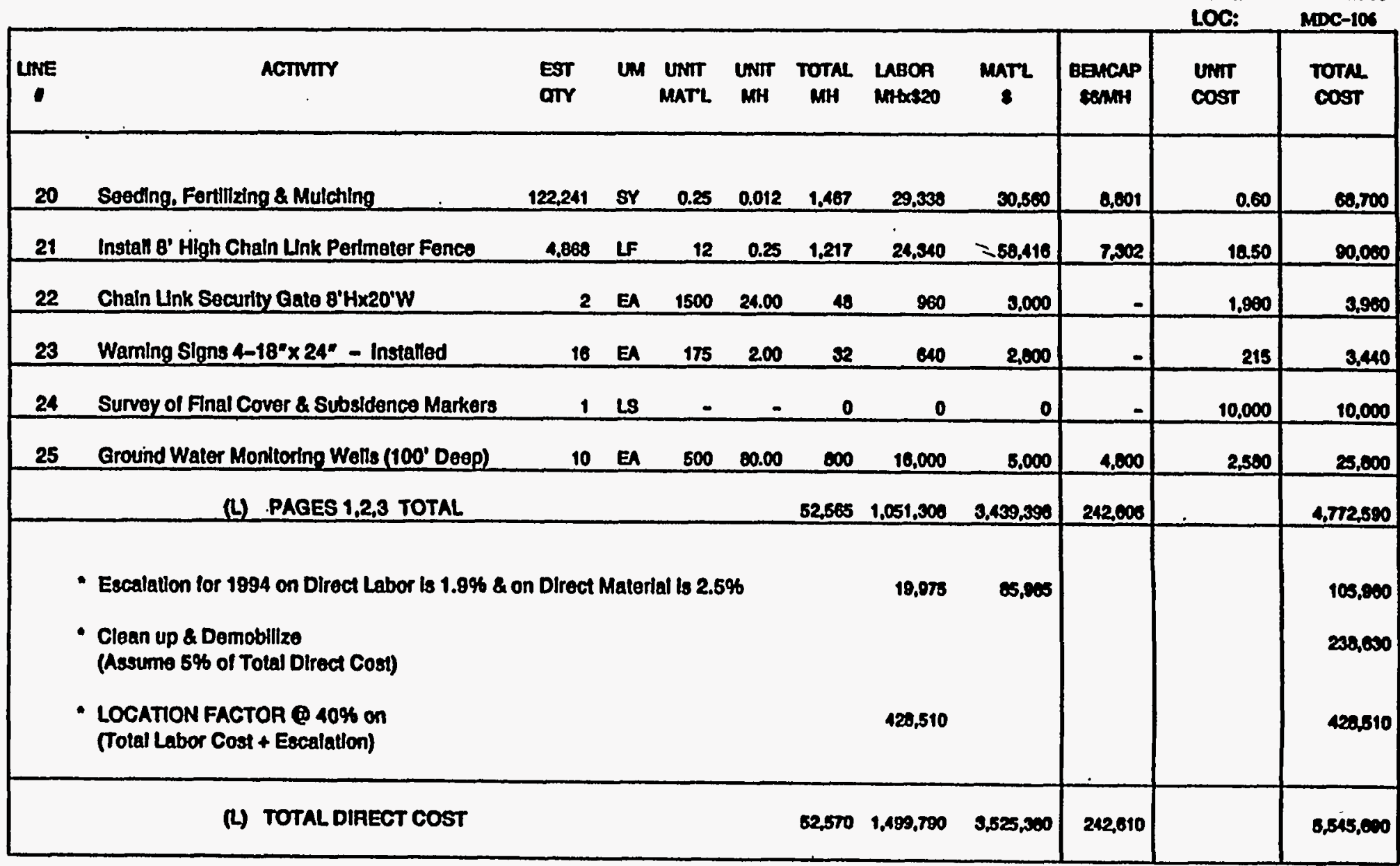

H-2. Cost Estimate for a Cover System Utilizing Bentomat and a 30-mil FML (Cont'd) 
DETAIL B S TIMATB

CHK'O BY: 8. MEAD

Lo01: $7-03$

ACTIVITY CODE: ESE2OON12

PROI.NAME: BCON. EVALUATION OF CLOSURB CAP BARRIER MATERIAL.

ESTIMATOA: murns

PHONE: 7-5256

DATE: OAZSOS

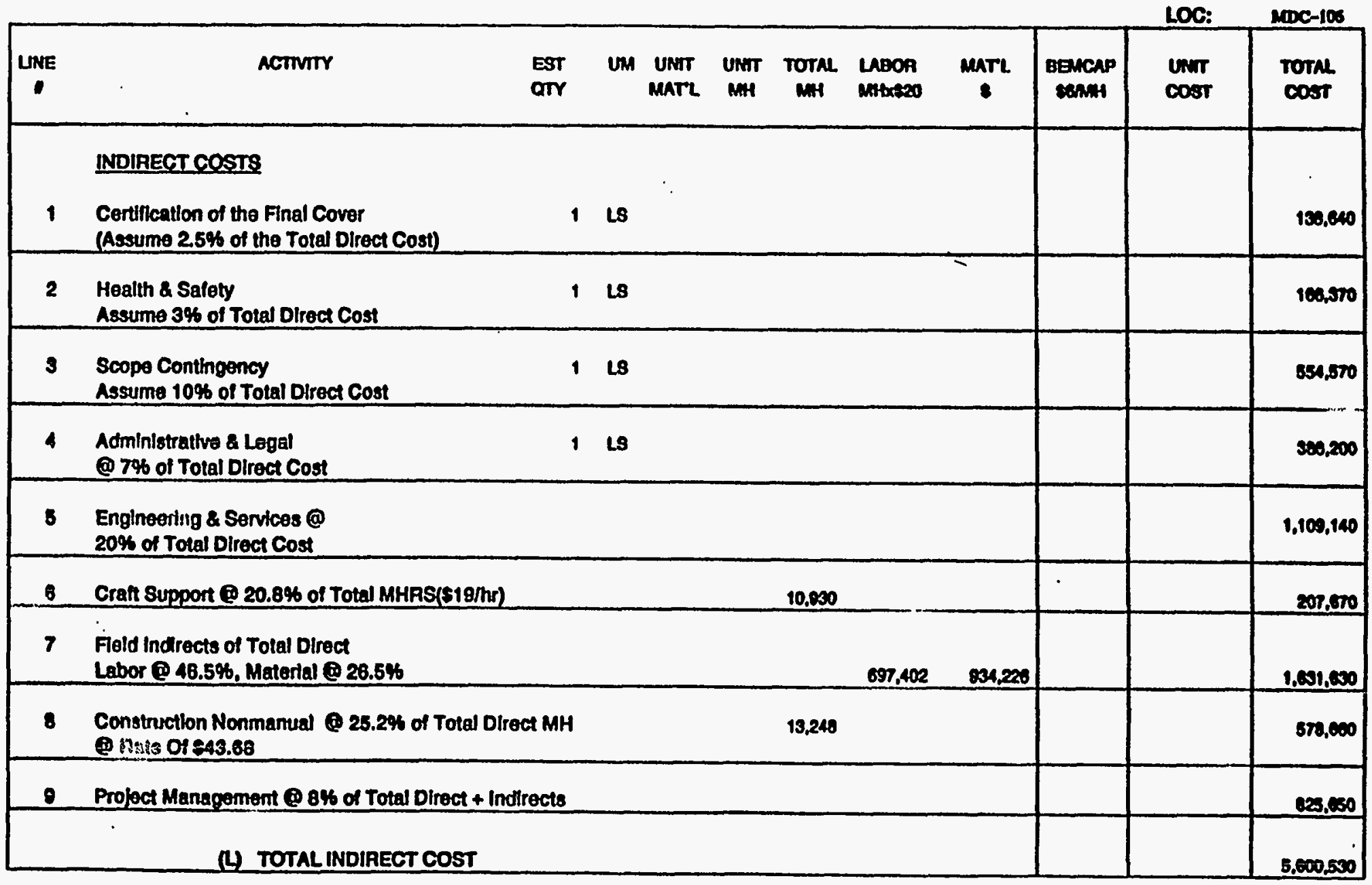

H-2. Cost Estimate for a Cover System Utilizing Bentomat@ and a 30-mil FML (Cont'd) 
D B T A I L B T IMAT B

CHKD BY: S. MEAD

Lo0 1: 7-03

ACTIVITY CODE: ESE260412

PROJ.NAME: ECON. EVALUATION OP CLOSURB CAP BARRIER MATERIAL

ESTIMATOR: rownx

PHONE: 7-5268

DATE: CarGos

\begin{tabular}{|c|c|c|c|c|c|c|c|c|c|c|c|}
\hline UNE & ACTIVIY & $\begin{array}{l}\text { EST } \\
\text { OTY }\end{array}$ & end & $\begin{array}{l}\text { UNIT } \\
\text { MATL }\end{array}$ & $\begin{array}{l}\text { UNIT } \\
\text { MH }\end{array}$ & TOTAL & $\begin{array}{l}\text { LABOA } \\
\text { MAPX }\end{array}$ & MATL & $\begin{array}{l}\text { DEMCAP } \\
\text { senNH }\end{array}$ & $\begin{array}{l}\text { Untr } \\
\text { cost }\end{array}$ & $\begin{array}{l}\text { Totn } \\
\text { cost . }\end{array}$ \\
\hline & $\begin{array}{l}\text { TOTAL DIRECT COST } \\
\text { TOTAL INDIRECT COST } \\
\text { DURATION FACTOA (3\% }\end{array}$ & ctindir & & & & & & & & & $\begin{array}{r}6,515,000 \\
6,000,550 \\
504,507\end{array}$ \\
\hline B & $\begin{array}{l}\text { TOTAL INITIAL CONSTRU } \\
\text { O.8M costs } \\
\text { Groundwater Monitoring Laboratory } \\
\text { Analysis of Samples \& Peport } \\
\text { Writing (Quarterly) }\end{array}$ & $\begin{array}{r}\text { COST } \\
40\end{array}$ & EA & & & & & $=$ & & 225 & $11,481,000$ \\
\hline$\bullet$ & Inapoction Activitles of Facilly - Monthly & $\pi$ & MA & & & & & & & 75 & 8,400 \\
\hline$\bullet$ & Subsidence Monltoring Annual Survoy & 20 & HA & & & & & & & 75 & 1.500 \\
\hline$\bullet$ & $\begin{array}{l}\text { Maintenance of Vegetative Cover } \\
\text { - } 2 \text { Mowings / yoar } \\
\text { - Pest Controt } \\
\text { - Erosion Controt \& } \\
\text { Drainage Maintenance }\end{array}$ & 20 & AC & & & & & & & 800 & 10,000 \\
\hline$\bullet$ & Cover Repalr Due To Subsidence & 1 & L8 & & & & & & & & 8.400 \\
\hline$\bullet$ & $\begin{array}{l}\text { Annual Fence Maimtenanco } \\
\text { Annual Sign Malntenance }\end{array}$ & $\begin{array}{r}1 \\
18 \\
\end{array}$ & & & & & & & & 40 & $\begin{array}{l}000 \\
640 \\
\end{array}$ \\
\hline$\bullet$ & Annual Monitoring Well Maintenance & 10 & & & & & & & & 75 & 750 \\
\hline & (L) TOTAL OEM COST & & & & & & & & & & sures \\
\hline
\end{tabular}

H-2. Cost Estimate for a Cover System Utilizing Bentomat and a 30-mil FML (Cont'd) 


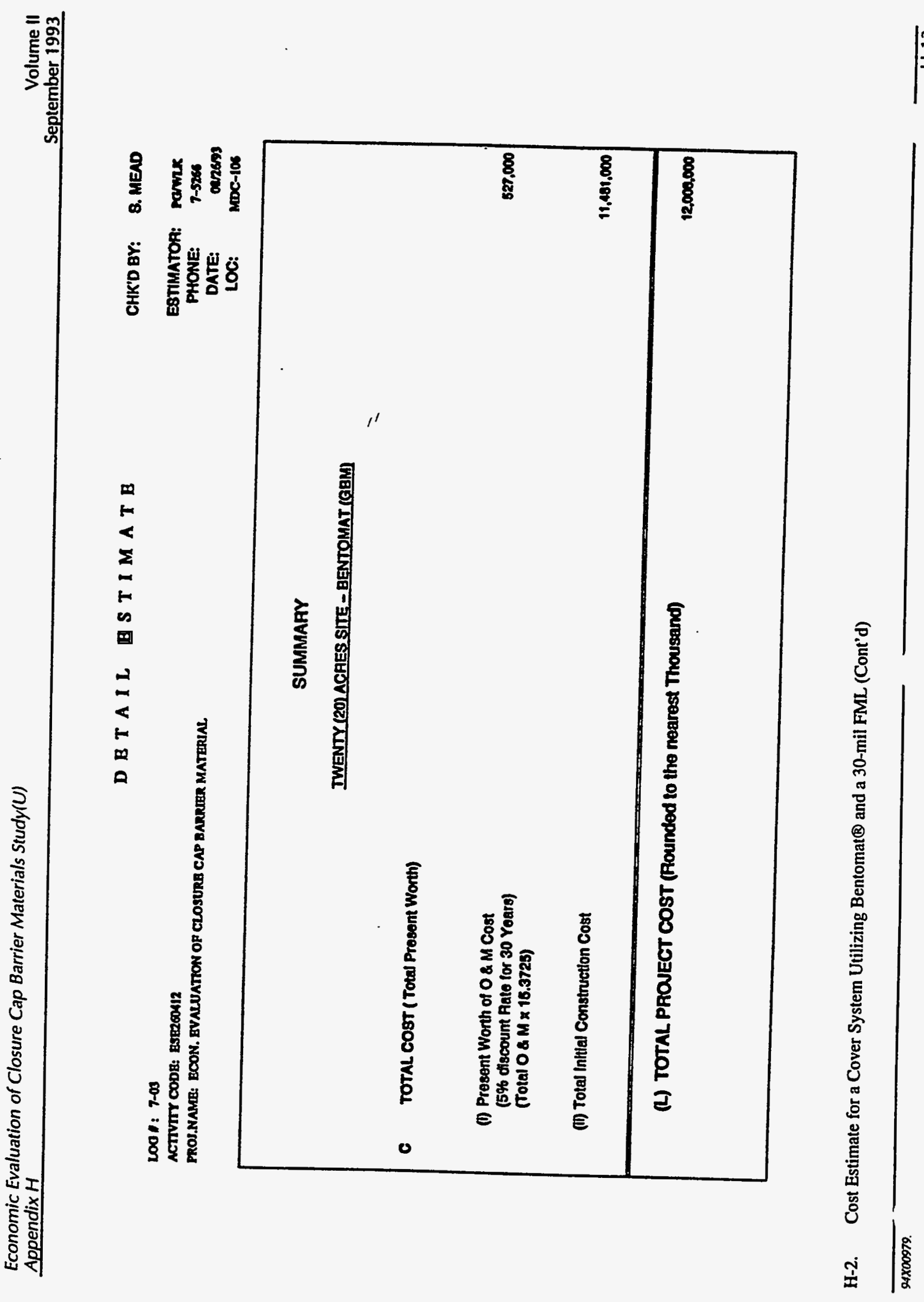

$\mid \frac{m}{1}$ 
Lo : : 7-03

DETAIL ESTIMATE

CHK'D BY: S. MEND

ACTIVITY CODB: ESEZ 600112

PROJ.NAME: ECON. EYALUATION OP CLOSURB CAP BARRIER MATERUAL.

ESTIMATOR: RaIMK

PHONE: 2-S266

DATE: Oarzses

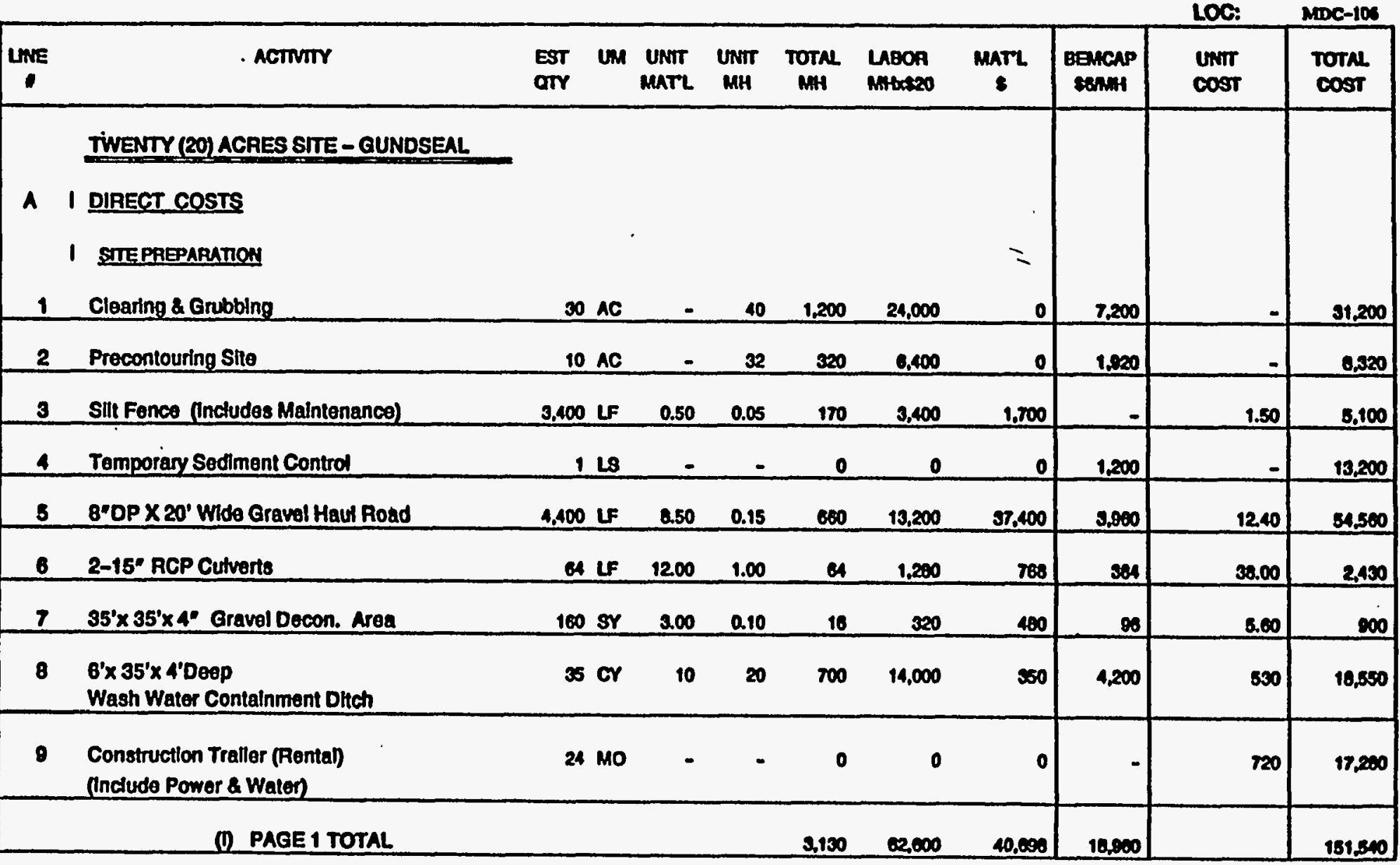

H-3. Cost Estimate for a Cover System Utilizing Gundseal@ 
DETAIL ESTIMATE

CHK'D BY: S. MEAD

2001: 7-03

ACTIVIT CODE: BSEZOOA12

FOL.KAME: ECON. BVALUATION OR CLOSURB CAP BARRIER MATERIAL

STIMATOR: NaWr:

PHONE: 7-5266

DATE: DORGOS

\begin{tabular}{|c|c|c|c|c|c|c|c|c|c|c|c|}
\hline LNE & NCTIVIY & $\begin{array}{l}\text { EST } \\
\text { OTY }\end{array}$ & un & $\begin{array}{l}\text { UNTT } \\
\text { MATLL }\end{array}$ & Umin & $\underset{M H}{\text { TOTAL }}$ & $\begin{array}{l}\text { LABOA } \\
\text { MAtret20 }\end{array}$ & mate & $\begin{array}{l}\text { Bencap } \\
\text { renny }\end{array}$ & $\begin{array}{l}\text { Unm } \\
\text { cost }\end{array}$ & $\begin{array}{l}\text { TorAl } \\
\text { cost }\end{array}$ \\
\hline 10 & $\begin{array}{l}\text { LCOVER CONSTRUCTION } \\
\text { Waste Siabiltration (Crushed Lmestone) }\end{array}$ & 27,815 & $\mathrm{~m}$ & 75 & 0.1 & 2,792 & 55,830 & $2,003,025$ & 16,740 & $\pi .00$ & $2,160,200$ \\
\hline 11 & $\begin{array}{l}\text { Place and Compact Foundation Soll } \\
\text { in } 8^{\circ} \text { Ulfts to } 95 \% \text { Std. Proctor } \\
\end{array}$ & 200.760 & CY & - & 0.125 & 25,245 & 516,900 & $=$ & 155,070 & 3.30 & 671.070 \\
\hline 12 & $\begin{array}{l}\text { Placement of Gundseal Geomembranel } \\
\text { Bentonite Mat }\end{array}$ & 947,000 & BF & 0.38 & 0.0055 & 8,209 & 104,170 & 339,800 & - & 0.60 & 404,050 \\
\hline 13 & $\begin{array}{l}\text { Placement of } 1^{\prime}-0 \text { Coarse Sand } \\
\text { Drainage Layer }\end{array}$ & 30,395 & Cr & 10.50 & 0.025 & 835 & 18,698 & 350,840 & 8,009 & $\$ 1.10$ & 372,500 \\
\hline 14 & $\begin{array}{l}\text { Placement of Geotextile (TYPAR) Filter on } \\
\text { 1'-0" Coarse Sand Drainage Layer }\end{array}$ & 107,000 & SY & 0.13 & 0.005 & 535 & 10.700 & 13.910 & - & 0.20 & 24,610 \\
\hline 15 & Placement of $1^{\prime}-6^{\circ}$ Common Fm & 48,247 & or & 3.75 & 0.045 & 2,216 & 44,322 & 164.670 & 19,297 & 4.90 & 242,300 \\
\hline 16 & Placement of $6^{\prime \prime}$ Topsoll & 18,511 & CY & 8.50 & 0.045 & 743 & 14,600 & 156,055 & 4,458 & 10.70 & 170.170 \\
\hline 17 & $\begin{array}{l}\text { Placement of Perlmeter Exterfor } \\
\text { Dralnage Layer } 34^{*} 3^{*} \text { DP (Crushod Stone) }\end{array}$ & 1,900 & SY & 1.50 & 0.16 & 304 & 6,000 & 2,850 & 1,024 & 8.70 & $10, \pi 50$ \\
\hline 18 & Excavation of Trapezoldal Drainage Ditch & 6,400 & Cr & - & 0.165 & 1.056 & 21,120 & 0 & 6,330 & 4.30 & 27.400 \\
\hline & (1) PAGE 2 TOTAL & & & & & 30,534 & 790,600 & $2,102,420$ & 202,743 & & $0.153,840$ \\
\hline
\end{tabular}

H-3. Cost Estimate for a Cover System Utilizing Gundseal@ (Cont'd) 
D B T A I L BSTIMAT B

CHKD BY: S. MEAD

Loo A: 7-03

ACTIVITY CODB: ESE260112

PROJ.NAME: ECON. BYALUATKON OP CLOSURB CAP BARRIER MATERIAL

ESTIMATOR: ronmK

PHONE: 7-5206

DATE: Darsess

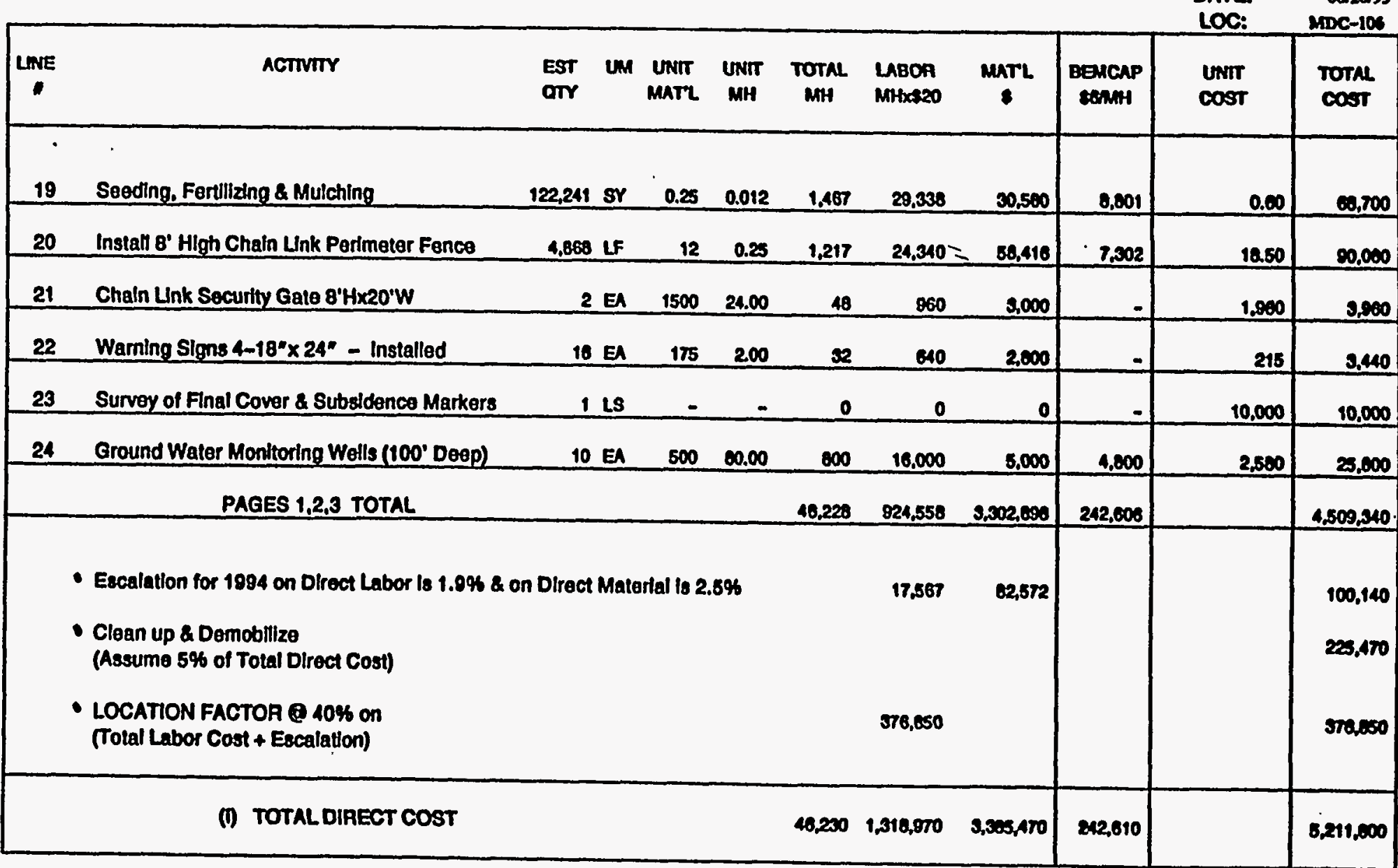

H-3. Cost Estimate for a Cover System Utilizing Gundseal@ (Cont'd) 
D B T A I L B S T I A T B

Lo0 I: 7-03

ACTIVITY CODB: ESEZ6O412

PROJ.NAMR: ECON. EVALUATION OP CLOSURB CAP DARRIER MATERIAL.

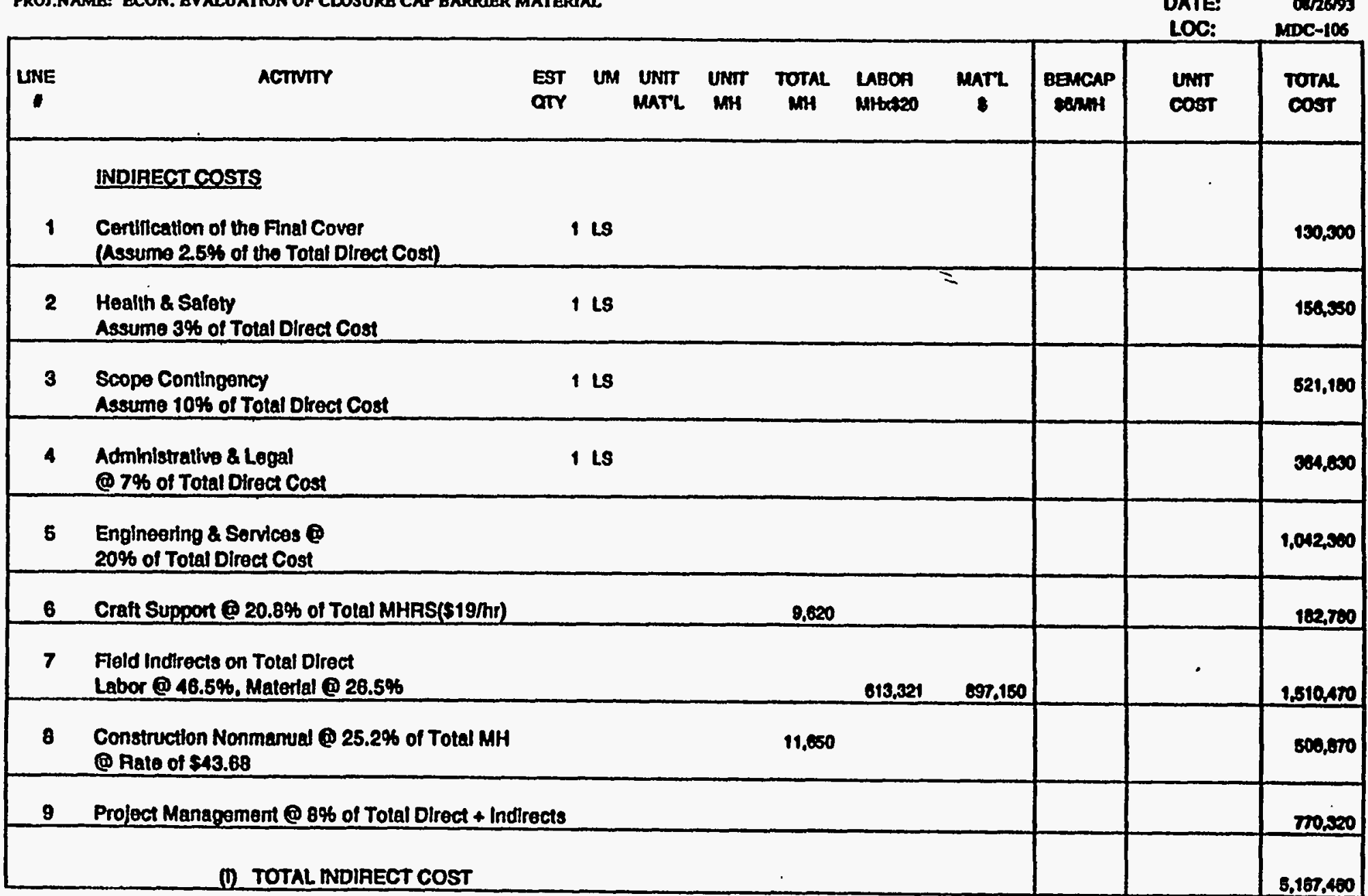

H-3. Cost Estimate for a Cover System Utilizing Gundseal@ (Cont'd) 
DETAIL BSTIMATE

Loo : $7-03$

ACTIVTY CODB: ESB26O412

PROJ.NAMR: BCON. BVALUATION OF CLOSURE CAP BARRIER MATERIAL

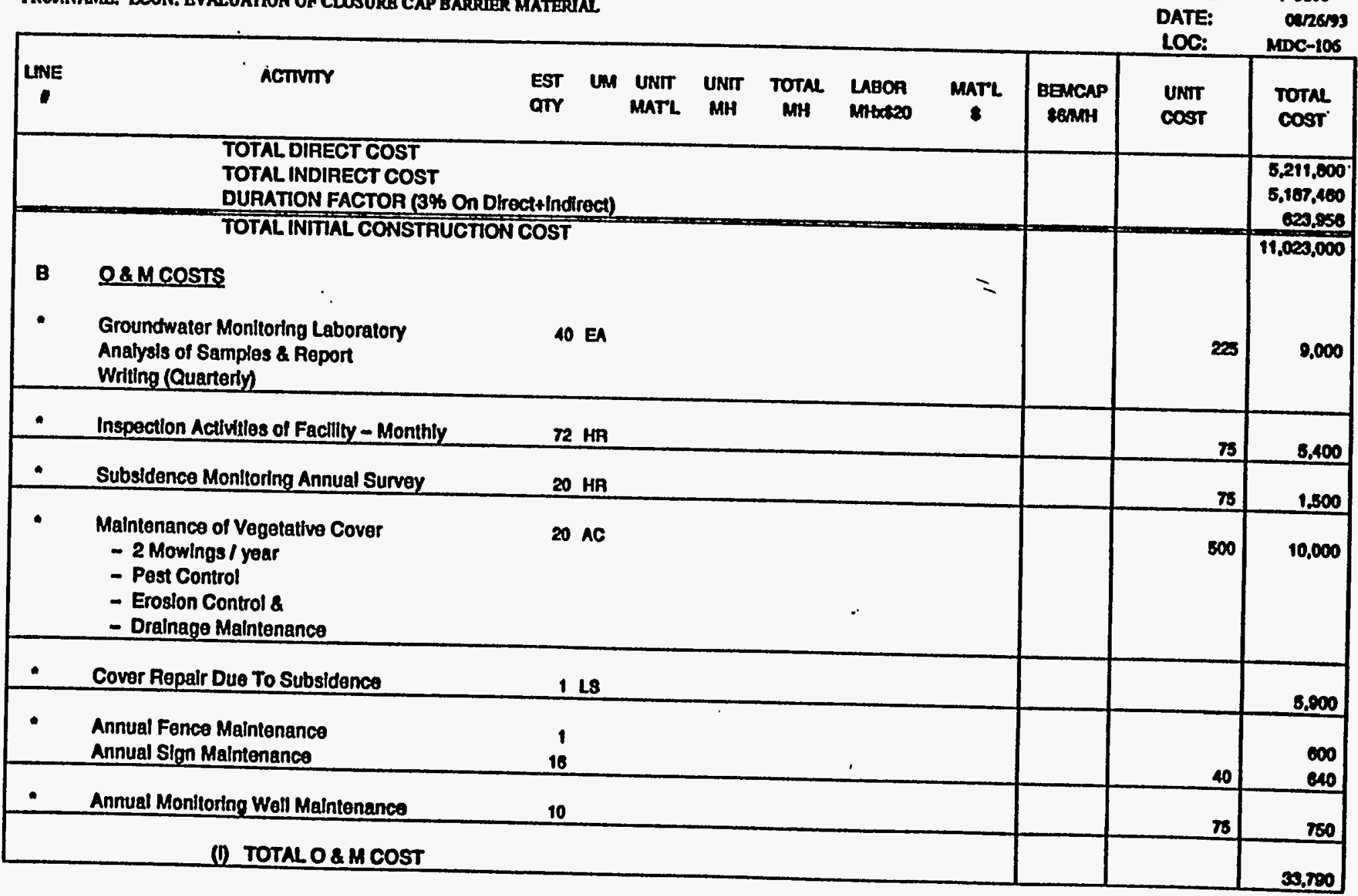

H-3. Cost Estimate for a Cover System Utilizing Gundseal@ (Cont'd) 
D B TAIL B S TIMAT B

$1002: 7-03$

ACTIVIT CODE: ESB2OOA12

PROS.NAMB: BCON. BVALUATKON OF CLOSURB CAP BARRER MATERUAL

$\begin{array}{lc}\text { CHKD BY: } & \text { S. MEAD } \\ \text { ESTIMATOR: } & \text { NOMHX } \\ \text { PHONE: } & 7-5266 \\ \text { DATE: } & \text { OA2GN } \\ \text { LOC: } & \text { MDC-106 }\end{array}$

\section{SUMMARY}

TWENTY (20) ACAES SITE - GUNDSEAL

C TOTAL COST (Total Present Worth)

(1) Present Worth of $O$ \& M Cost

(5\% ollscount Rate for 30 Years)

819,000

(Total O \& $M \times 15.3725$ )

(ii) Total Initial Construction Cost

$11,023,000$

(i) TOTAL PROJECT COST (Rounded to the nearest Thousand)

11512000

H-3. Cost Estimate for a Cover System Utilizing Gundseal[ (Cont'd) 


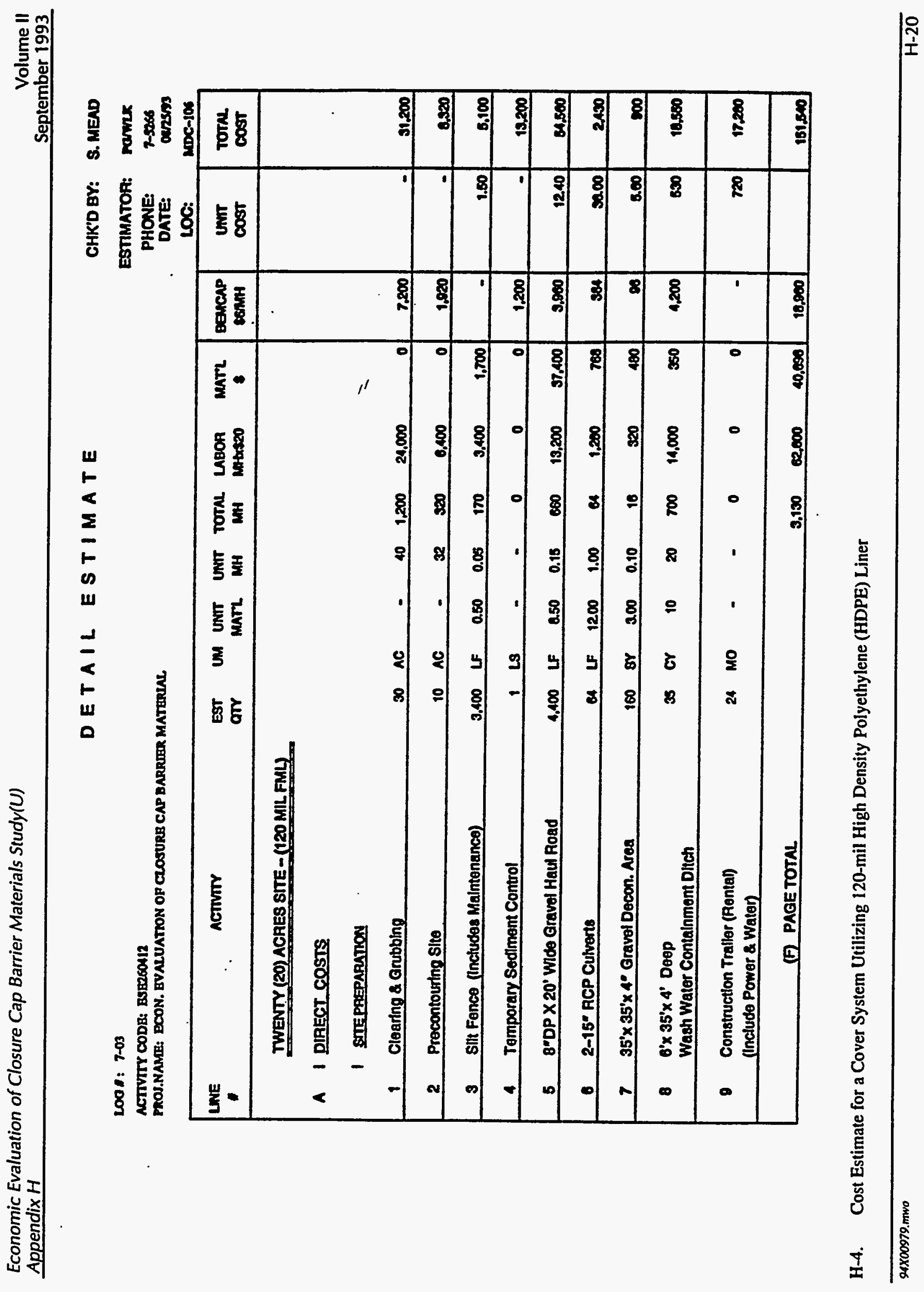


DETAIL ESTIMATE

CHK'D BY: 8. MEAD

1001: 7-03

ACTIVITY CODB: ESE260412

PROJ.NAMB: ECON. EVALUATTON OF CLOSURB CAP DARRURR MATRRIAL.

ESTIMATOR: NormK

PHONE: 7-5266

\begin{tabular}{|c|c|c|c|c|c|c|c|c|c|c|c|}
\hline & & & & & & & & & & LOC: & $20 C-106$ \\
\hline LWE & ACTVIIY & $\begin{array}{l}\text { Est } \\
\text { arr }\end{array}$ & un & $\begin{array}{l}\text { UNmT } \\
\text { MATL }\end{array}$ & UNHTH $_{M H}$ & TOTMe & $\begin{array}{l}\text { LABOA } \\
\text { MPbat2O }\end{array}$ & $\operatorname{maTL}$ & $\begin{array}{l}\text { Bencap } \\
\text { senmin }\end{array}$ & $\begin{array}{l}\operatorname{Unm} \\
\cos T\end{array}$ & $\begin{array}{l}\text { Totn } \\
\text { cost }\end{array}$ \\
\hline & I COVEA CONSTRUCTION & & & & & & & & & & \\
\hline 10 & Waste Stabillzation (Crushed Umestone) & 27,915 & $\pi$ & 73 & 0.1 & 2,792 & 55.630 & $2,095,023$ & 16,749 & 77.60 & $2,160,200$ \\
\hline 11 & $\begin{array}{l}\text { Place and Compact Foundation Soll } \\
\text { in } 8^{\circ} \text { Uits to } 95 \% \text { sto. Proctor }\end{array}$ & 206,700 & or & - & 0.125 & 25,645 & 516,000 & 2 & 165,070 & 3.30 & 6ri.mo \\
\hline 12 & $\begin{array}{l}\text { Placement of } 120 \text { MII Flextble } \\
\text { Membrane Lmer }\end{array}$ & 947,000 & SF & 0.39 & 0.007 & 0,023 & 132,560 & 309,230 & - & 0.50 & 801,910 \\
\hline 13 & $\begin{array}{l}\text { Placement of } 1^{\prime}-0 \text { Coarse Sand } \\
\text { Dralnage Layer }\end{array}$ & $\mathbf{3 3 , 3 9 5}$ & or & 10.50 & 0.025 & 605 & 16,689 & 350,640 & 6,000 & 11.10 & 572,360 \\
\hline 14 & $\begin{array}{l}\text { Placement of Geotextite (TYPAR) Filler on } \\
1^{1}-0^{\prime} \text { Coarse Sand Dralnage Layer }\end{array}$ & 107,000 & sr & 0.13 & 0.005 & 535 & 10,700 & 10,910 & - & 0.20 & 24,810 \\
\hline 16 & Placement of $1^{\prime}-6^{\circ}$ Common Fil & 49.247 & cr & 0.75 & 0.045 & 2,216 & 4,322 & 184,078 & 10,297 & 4.90 & 242,300 \\
\hline 16 & Placement of $6^{*}$ Topsoil & 16,511 & or & 9.50 & 0.045 & 740 & 14,000 & 160,055 & 4,458 & 10.70 & 178,170 \\
\hline 17 & $\begin{array}{l}\text { Placement of Perlmeter Exterfor } \\
\text { Drainage Layer } 314^{*} \times 3^{*} \text { "Stone }\end{array}$ & 1,900 & sy & 1.50 & 0.16 & 304 & 0,000 & 2,650 & 1.024 & 5.70 & 10,750 \\
\hline 18 & Excavation of Trapezotdal Drainage Ditch & 0,400 & or & - & 0.165 & 1,058 & 21.120 & 0 & 0,336 & 4.00 & $27 \times 00$ \\
\hline & (F) PAQE TOTAL & & & & & 40,254 & 819.000 & $0,171,090$ & 202,740 & & $4,108,720$ \\
\hline
\end{tabular}

H-4. Cost Estimate for a Cover System Utilizing 120-mil High Density Polyethylene (HDPE) Liner (Cont'd) 


\section{B T A I B S T I MAT B}

CHKD BY: S. MEAD

Lool: 7-03

ACTIVTTY CODB: ESE260012

PROJ.NAMTE: ECON. BVALUATION OF CLOSURB CAP BARRIER MATERIAL

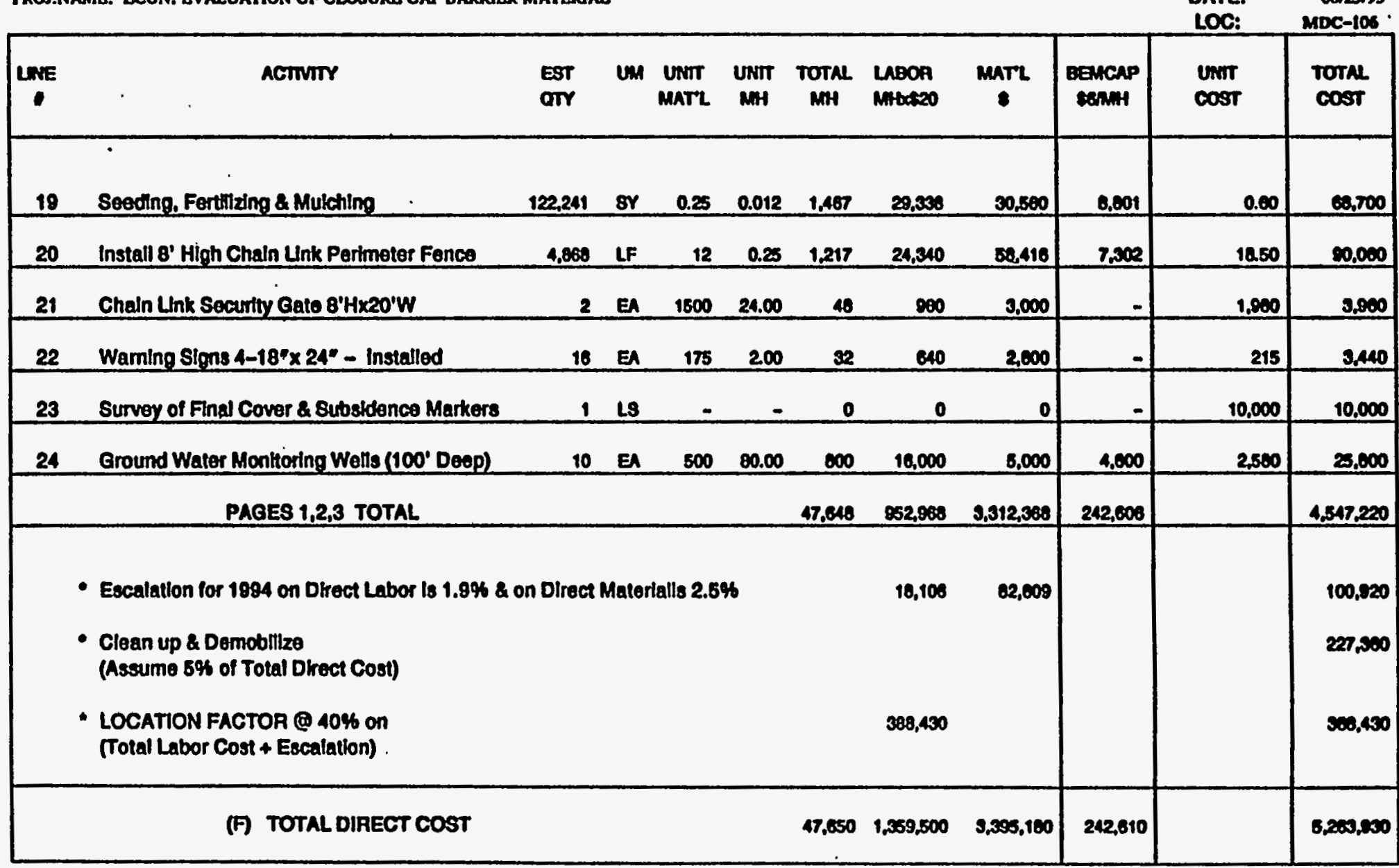

H-4. Cost Estimate for a Cover System Utilizing 120-mil High Density Polyethylene (HDPE) Liner (Cont'd) 
D B TAIL BSTIMATE

CHK'D BY: 8. MEAD

200 1: $7-03$

ACTIVTY CODB: ESEZOOS12

PROJ.NAME: ECON. EVALUATTON OF CLOSURB CAP BARRIBR MATERIAL

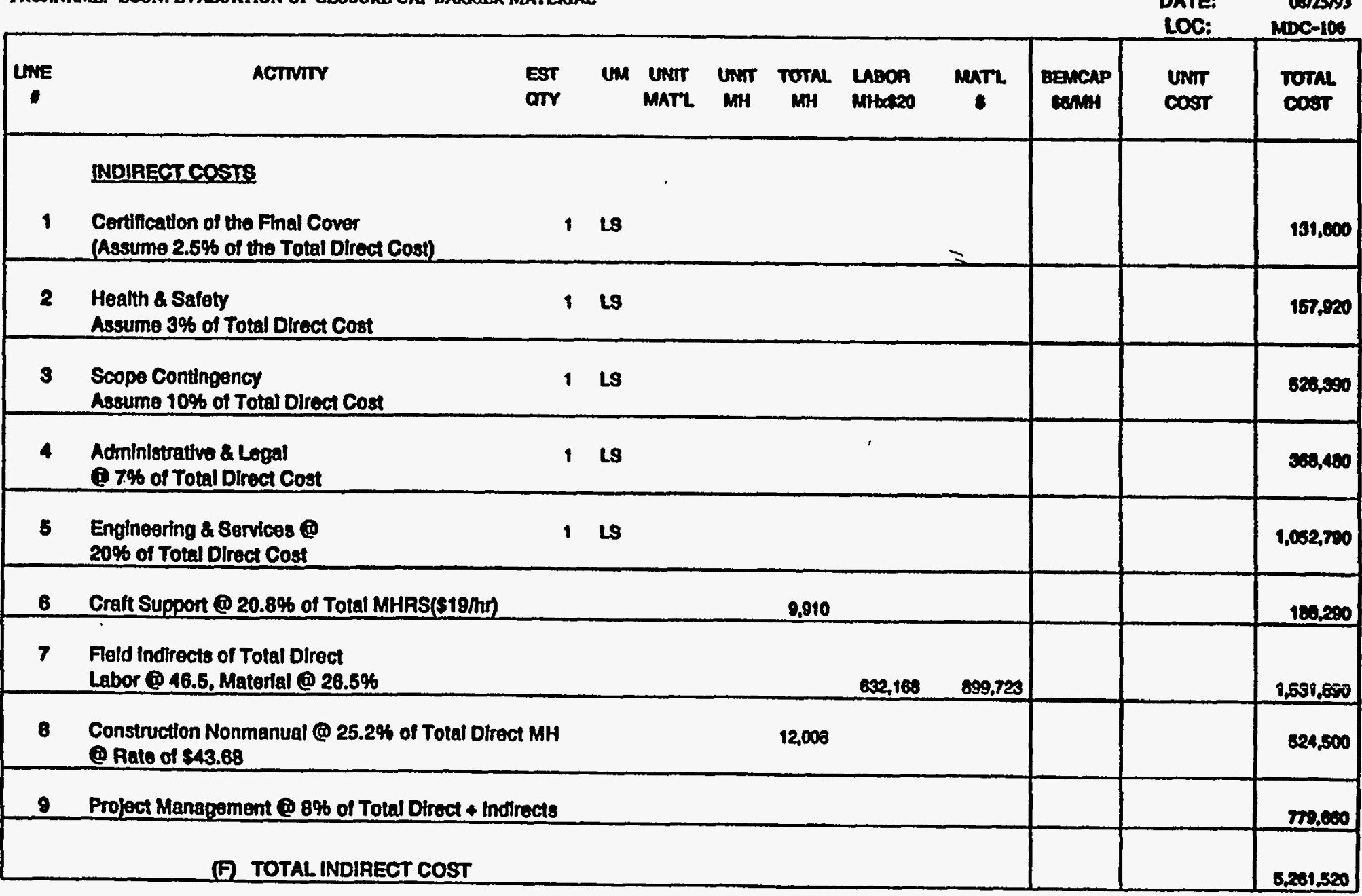

H-4. Cost Estimate for a Cover System Utilizing 120-mil High Density Polyethylene (HDPE) Liner (Cont'd) 


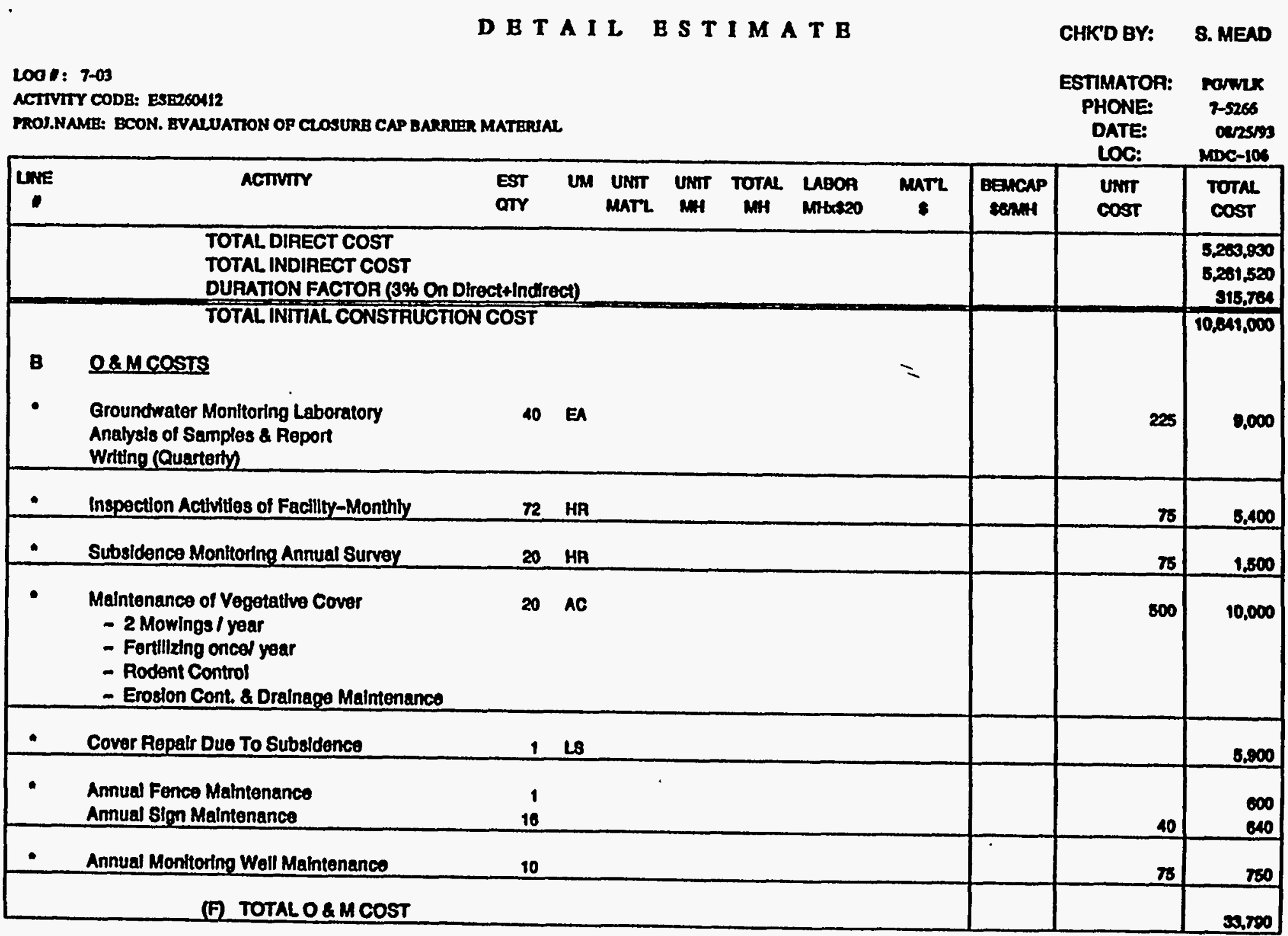

H-4. Cost Estimate for a Cover System Utilizing 120-mil High Density Polyethylene (HDPE) Liner (Cont'd) 
D BTAIL BSTIMATB

\begin{tabular}{|c|c|}
\hline HKCD BY: & 8. MEAD \\
\hline $\begin{array}{l}\text { E8TIMATOR: } \\
\text { PHONE: } \\
\text { DATE: } \\
\text { LOC: }\end{array}$ & 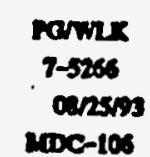 \\
\hline
\end{tabular}

Lon: 7-03

NCTIVTY CODE: BSE260N12

TROS.NAME: BCON. EVALUATION OF CLOSURB CAP BARRURR MATERIAL

LOC:

SUMMAFY

TWENTY (20) ACRES SITE-(120 MIL FML)

C TOTAL COST (Total Present Worth)

(i) Present Worth of 0 \& M Cost

(5\% discount Pato for 30 Yeare)

(Total O\& M X 18.3725)

(ii) Total Inttal Construction Cost

$10,811,000$

(F) TOTAL PROJECT COST (Poundad to the mearest Thousand)

$11,500,000$

H-4. Cost Estimate for a Cover System Utilizing 120-mil High Density Polyethylene (HDPE) Liner (Cont'd) 
DETAIL ESTIMATE

L001: $7-03$

ACTIVITY CODB: BSE260412

MOO.NAME: ECON. BVALUATION CLOSURB CAP BARRTER MATERLAL

\begin{tabular}{|c|c|c|c|c|c|c|c|c|c|c|c|}
\hline Lwe & ACTrum & $\begin{array}{l}\text { EST } \\
\text { arr }\end{array}$ & un & $\begin{array}{l}\text { UNTT } \\
\text { MATLL }\end{array}$ & $\begin{array}{l}\text { UNT } \\
\text { Int }\end{array}$ & TOTML & $\begin{array}{l}\text { LABOA } \\
\text { entexteo }\end{array}$ & $\stackrel{\operatorname{maTh}}{*}$ & $\begin{array}{l}\operatorname{sencap} \\
\operatorname{sen} 4\end{array}$ & $\begin{array}{l}\operatorname{lnmin} \\
\cos t\end{array}$ & $\begin{array}{l}\text { Total } \\
\text { cosst }\end{array}$ \\
\hline $\begin{array}{l}A \\
1 \\
\end{array}$ & $\begin{array}{l}\text { TWENTY (20) ACRES STIE-HOT SPRAY } \\
1 \text { DIRECT COSTS } \\
1 \text { SMEPREPARATION } \\
\text { Clearing \& Grubbing }\end{array}$ & SPHALT & $A C$ & $=$ & 40 & 1,200 & 24,000 & $=$ & 7,200 & $=$ & 31,200 \\
\hline 2 & Precontouring Sile & 10 & AC & - & 32 & 320 & 6,400 & 0 & 1,0020 & - & 0.320 \\
\hline 3 & Sill Fence (includes Malntenance) & 3,400 & LF & 0.50 & 0.05 & 170 & 3,400 & 1,700 & - & 1.60 & 8.100 \\
\hline 4 & Temporary Sodiment Control & 1 & LS & - & - & 0 & 0 & 0 & 1,200 & - & 13,200 \\
\hline 8 & 8"DPX20' Whde Gravel Haul Road & 4,400 & LF & 2.50 & 0.15 & 000 & 13,200 & 37.400 & 2.000 & 1240 & 84,500 \\
\hline 6 & 2-15" RCP Cutverts & 64 & LF & 12.00 & 1.00 & 64 & 1,260 & 700 & 304 & 30,00 & 2,430 \\
\hline 7 & $35^{\prime} \times 35^{\prime} \times 4^{\prime \prime}$ Gravel Docon. Area & 160 & sY & 3.00 & 0.10 & 10 & 320 & 400 & 80 & 8.60 & $\infty 00$ \\
\hline 8 & $\begin{array}{l}6^{\prime} \times 35^{\circ} \times 4^{\prime} \text { Deep Wash Water Containment } \\
\text { Ditch }\end{array}$ & 35 & or & 10 & 20 & 700 & 14,000 & 350 & 4,200 & 532 & 10,060 \\
\hline 8 & $\begin{array}{l}\text { Construction Traller (Rental) } \\
\text { (Include Power \& Water) }\end{array}$ & 24 & MO & - & - & 0 & 0 & 0 & - & 720 & 17,200 \\
\hline & (C) PAGE 1 TOTAL & & & & & 2,130 & 02,000 & $\$ 0.000$ & 18,900 & & 181,510 \\
\hline
\end{tabular}

H-5. Cost Estimate for a Cover System Utilizing 1/4-inch Thick Hot Sprayed or Emulsified Asphalt 
DETAIL ESTIMATE

Lod: $7-0$ s

ACTIVITY CODA: BSE260412

MOS.NAME: BCON. BVALUATTON CLOSURB CAP DARRIER MATBRIAL

\begin{tabular}{|c|c|c|c|c|c|c|c|c|c|c|c|}
\hline UNE & ACTIMT & $\begin{array}{l}\text { EST } \\
\text { OTY }\end{array}$ & um & $\underset{\text { MATIT }}{\text { UNT }}$ & $\underset{\text { UMH }}{\text { Uim }}$ & $\underset{M H}{\text { TOTAL }}$ & $\begin{array}{l}\text { LABOA } \\
\text { MHAdA2O }\end{array}$ & MATL & $\begin{array}{l}\text { BEwCAP } \\
\text { EunH }\end{array}$ & $\begin{array}{l}\text { und } \\
\text { cost }\end{array}$ & $\begin{array}{l}\text { TOTAL } \\
\text { Cost. }\end{array}$ \\
\hline & I CQVER CONSTIRUCTION & & & & & & & & & & \\
\hline 10 & Waste Stabillzation (Crushed Umestone) & 27,915 & TN & 75 & Q.1 & 2,792 & 55,6030 & $2,093,605$ & 10,749 & $\pi .00$ & $2,160,200$ \\
\hline 11 & $\begin{array}{l}\text { Piace and Compact Foundation 8oll } \\
\text { in } 8^{\circ} \text { Lits to } 85 \% \text { Std. Proctor }\end{array}$ & 206,700 & or & - & 0.123 & 20,245 & 816,900 & o & 185,070 & 2.00 & 071,010 \\
\hline 12 & $\begin{array}{l}\text { Applled Hot Asphatt using } 3 \text { passes } \\
\text { at a rate of } 0.5 \text { gal / SY }\end{array}$ & $\begin{array}{l}105,2222 \\
157,233 \\
\end{array}$ & $\begin{array}{l}\text { SY } \\
\text { GAL }\end{array}$ & 2 & 0.13 & 13.679 & 273.57 & 210,44 & 82,073 & $\mathbf{5 . 4 0}$ & 500,000 \\
\hline 18 & $\begin{array}{l}\text { Placement of } 30 \text { MH Flexible } \\
\text { Membrane Lmer }\end{array}$ & 975.000 & BF & 0.14 & 0.0005 & 6,350 & 128,760 & 150,600 & - & 0.00 & 200,200 \\
\hline 14 & $\begin{array}{l}\text { Piacsmont of 1'-0 Coarea Sand } \\
\text { Dralnage Layer }\end{array}$ & 33,395 & Cr & 10.50 & 0.025 & 2035 & 10,698 & 250,648 & 8,000 & 11.10 & 372500 \\
\hline 15 & $\begin{array}{l}\text { Placement of Geotextlle (TYPAP) Fiter on } \\
t^{\prime}-0^{\circ} \text { Coarse Sand Drainage Layer }\end{array}$ & 107,000 & $\mathbf{8 r}$ & 0.18 & 0.005 & 635 & 10,700 & 19,210 & - & 0.20 & 24,010 \\
\hline 16 & Piacement of $1^{\prime}-6^{\prime \prime}$ Common Fill & 48,247 & CY & $\mathbf{3 . 7 5}$ & 0.045 & 2.216 & 44,322 & 184,078 & 13,297 & 4.00 & 242000 \\
\hline 17 & Placement of 6" Topson & 16,511 & or & 8.50 & Q.015 & 743 & 14,060 & 150,055 & 4,450 & 10.70 & 170,170 \\
\hline 18 & $\begin{array}{l}\text { Plecement of Parlmeter Exteriot } \\
\text { Dralnage Layer } 34^{*} \times 3^{*} \text { Stone }\end{array}$ & 1,800 & 8Y & 1.50 & 0.16 & 304 & 6.060 & 2,050 & 1,024 & 8.70 & 10,700 \\
\hline 18 & Excavation of Trapezoldal Dralnage Dltch & 6,400 & or & - & Q.165 & 9.058 & 21.120 & 0 & 6,330 & 4.00 & 27,400 \\
\hline & (C) PAGE 2 TOTAL & & & & & 54,342 & $1,000,037$ & $3,149,501$ & 294,818 & & $1,621,160$ \\
\hline
\end{tabular}

H-5. Cost Estimate for a Cover System Utilizing 1/4-inch Thick Hot Sprayed or Emulsified Asphalt (Cont'd) 
D B T A I L S T I MAT B

CHK'D BY: 8. MEAD

L00 $1: 7-03$

ACTTVTYY CODE: ESE260412

PROJ.NAME: ECON. BVALUATION CLOSURB CAP BARRIER MATERIAI

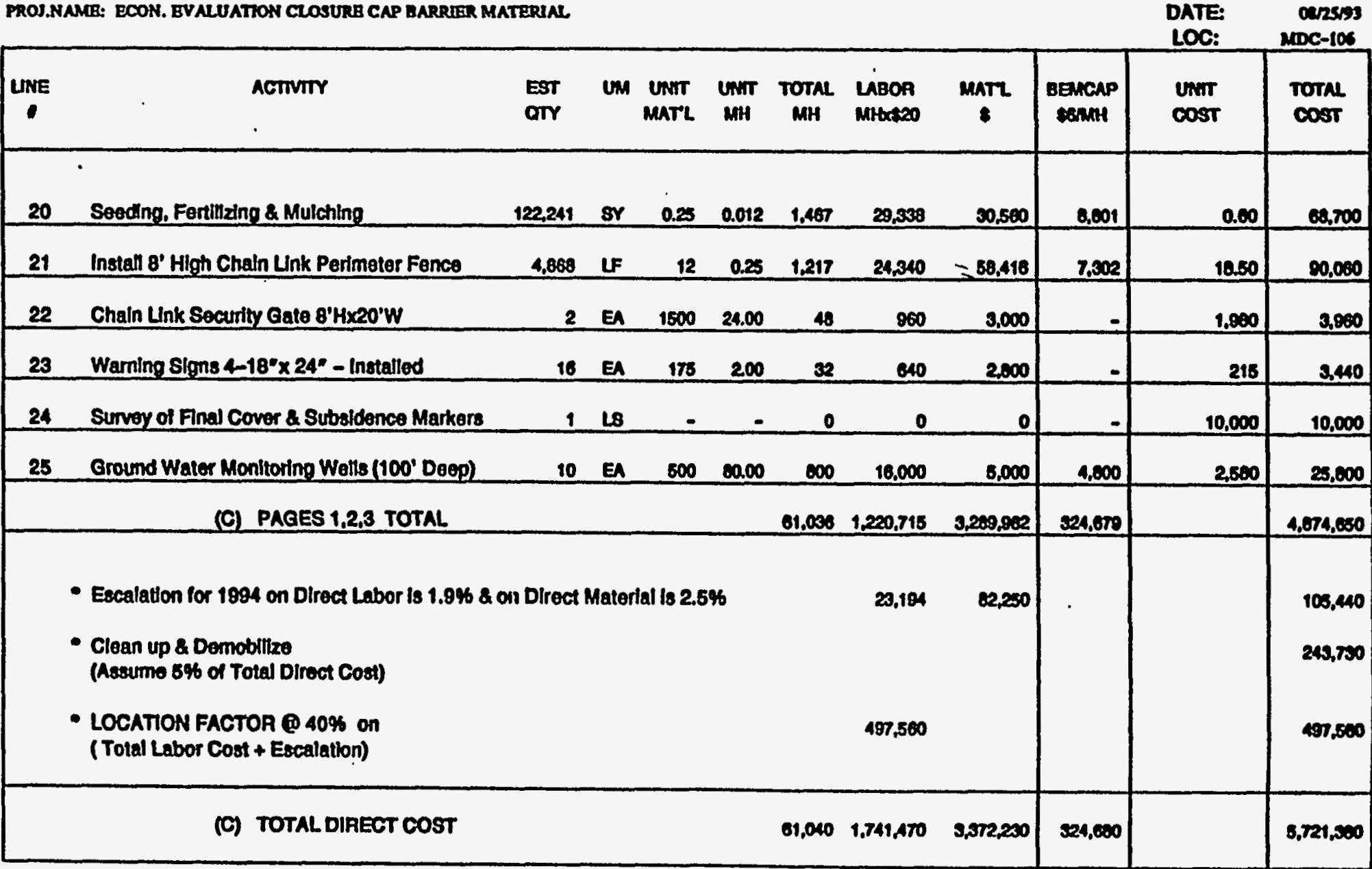

H-5. Cost Estimate for a Cover System Utilizing 1/4-inch Thick Hot Sprayed or Emulsified Asphalt (Cont'd) 
LOON: 7-03

ACTIVITY CODE: ESE260112

PROJ.NAME: BCON. EVALUATION CLOSURB CAP BARRIER MATERIAI

ESTIMATOR: NOMIX

PHONE: T-S26S

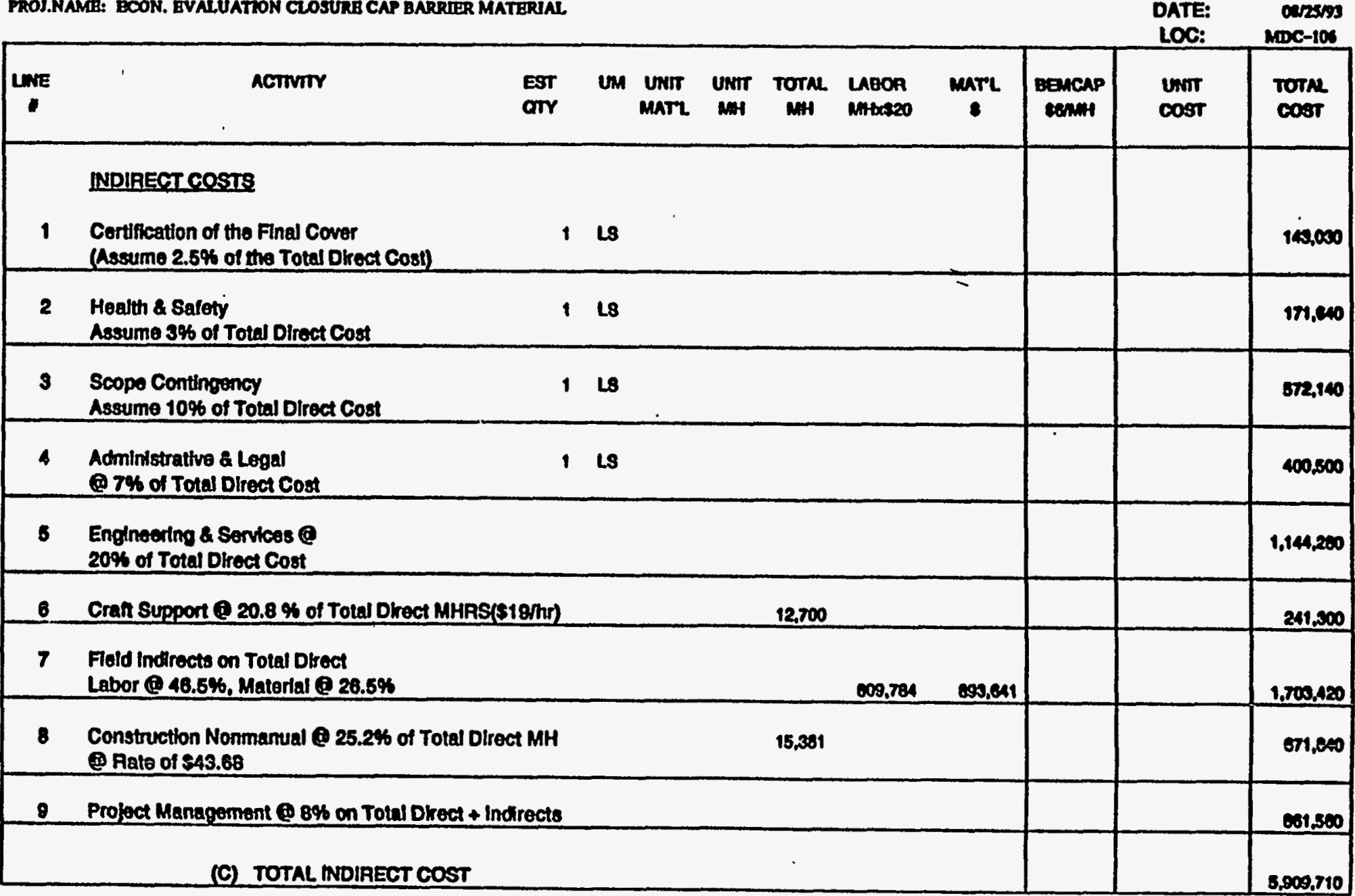

H-5. Cost Estimate for a Cover System Utilizing 1/4-inch Thick Hot Sprayed or Emulsified Asphalt (Cont'd) 
L00 : $7-03$

ACTIVITY CODE: ESE260412

ROSNAME: BCON. BVALUATION CLOSURE CAP BARUIR MATERLAL

ESTIMATOR: TOWR

PHONE: 7-5266

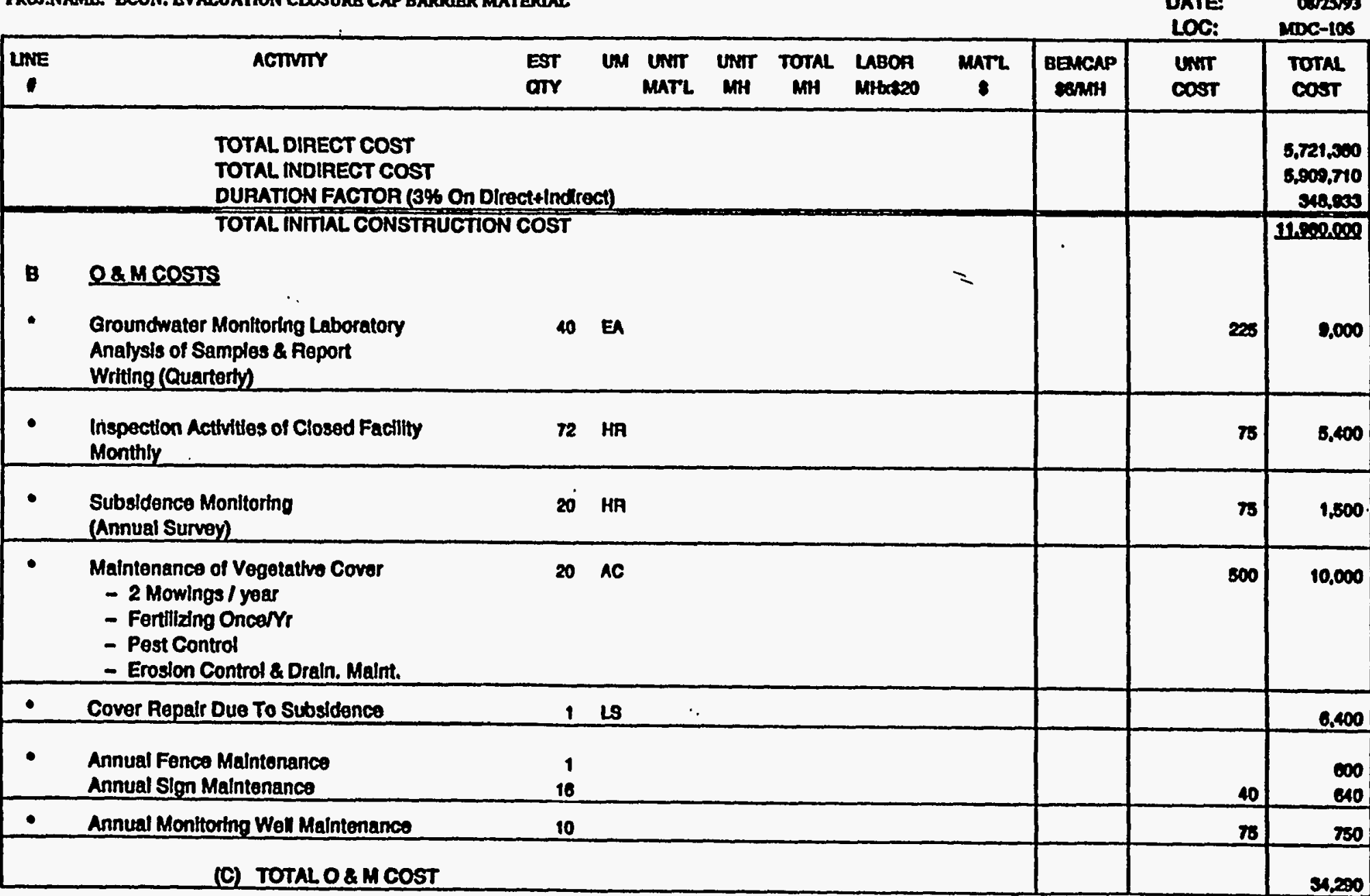

H-5. Cost Estimate for a Cover System Utilizing 1/4-inch Thick Hot Sprayed or Emulsified Asphalt (Cont'd) 


\begin{tabular}{|c|c|}
\hline CHK'D BY: & 8. MEAD \\
\hline $\begin{array}{l}\text { ESTIMATOA: } \\
\text { PHONE: } \\
\text { DATE: } \\
\text { LOC: }\end{array}$ & $\begin{array}{c}\text { rownx } \\
7-5266 \\
\text { ourssiss } \\
\text { Made-106 }\end{array}$ \\
\hline
\end{tabular}

\section{1: 7-03}

ACTIVITY CODR: BSECSOA12

PROJ.NAME: ECON. EVALUATTON CLOSUIRR CAP BARRIER MATEREAL.

LOC:

Mote-100

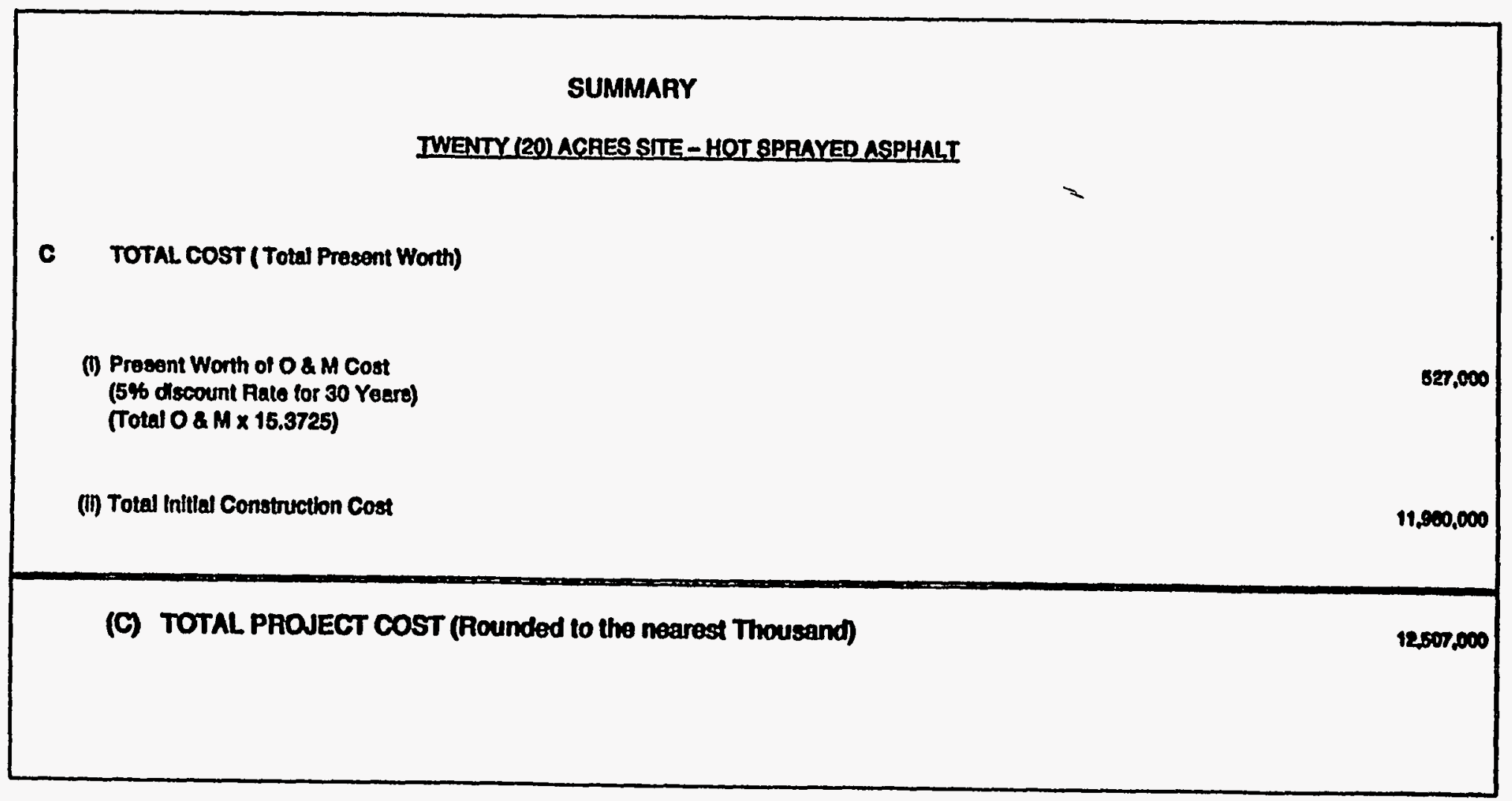

H-5. Cost Estimate for a Cover System Utilizing 1/4-inch Thick Hot Sprayed or Emulsified Asphalt (Cont'd) 


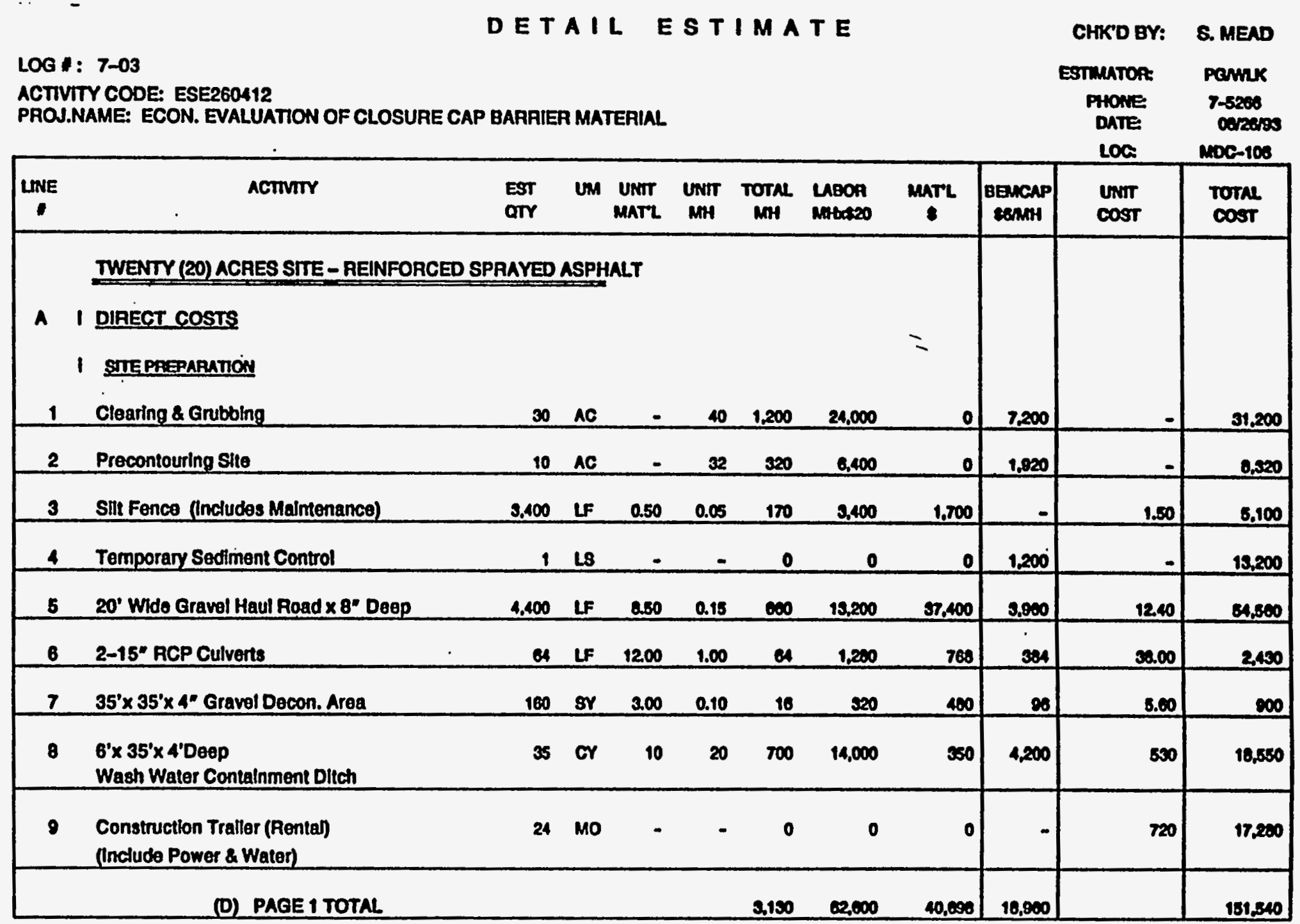

H-6. Cost Estimate for a Cover System Utilizing Reinforced Sprayed Asphalt 
LOG If: :-03

ACTIVITY CODE: ESE260412

PROS.NAME: ECON. EVALUATION OF CLOSURE CAP BARRIER MATERIAL D DATE DOR2\%

EsTmator: PaMnK

PHONE 7-5260

\begin{tabular}{|c|c|c|c|c|c|c|c|c|c|c|c|}
\hline LUNE & ACTIVIY & EST & $u$ & $\begin{array}{l}\text { UNTI } \\
\text { MATL }\end{array}$ & UMrT & $\underset{\text { MH }}{\text { TOTAL }}$ & $\begin{array}{l}\text { LaBon } \\
\text { imtectoon }\end{array}$ & $\operatorname{manz}$ & Bencap & $\operatorname{lnm}_{\cos \pi}$ & $\begin{array}{l}\text { Total } \\
\cos \end{array}$ \\
\hline & I COVER CONSTRUCTION & & & & & & & & & & \\
\hline 10 & Waste Stabilization (Crushed Limestone) & 27,915 & TN & $\pi$ & 0.1 & 2.792 & 55,030 & $2,093,625$ & 16,748 & $\mathbf{n . \infty}$ & $2,106,200$ \\
\hline 11 & $\begin{array}{l}\text { Place and Compact Foundation Soil } \\
\text { in } 8^{*} \text { Litis to } 95 \% \text { Sid. Proctor }\end{array}$ & 206.760 & CY & - & 0.125 & 25,045 & 516,900 & o & 155,070 & 3.30 & 671,970 \\
\hline 12 & $\begin{array}{l}\text { Placement of } 30 \text { Mil Floxible } \\
\text { Membrane Liner }\end{array}$ & 975,000 & SF & 0.14 & 0.0065 & 6,330 & 126,750 & 130,500 & - & 0.50 & 2003,250 \\
\hline 13 & $\begin{array}{l}\text { Placement of Geotextile Retniorcement } \\
\text { on Foundation Layer }\end{array}$ & 105,222 & SY & 0.28 & 0.003 & 520 & 10,522 & 27,358 & - & 0.40 & $\mathbf{5 7 , 8 0 0}$ \\
\hline 14 & $\begin{array}{l}\text { Applled Hot Asphalt using } 3 \text { passes at a rate } \\
\text { of } 0.5 \text { gal / sY for a total of } 1.5 \mathrm{~g} \text { USy }\end{array}$ & $\begin{array}{l}105,2222 \\
157,633 \\
\end{array}$ & $\begin{array}{l}\text { SY } \\
\text { OAL }\end{array}$ & 2.00 & 0.13 & 13,679 & 273.57 & 210,44 & $\$ 2,073$ & 8.40 & 600,090 \\
\hline 18 & $\begin{array}{l}\text { Piacement of } 1 \text { ' }-0 \text { Coarse Sand } \\
\text { Orainage Layer }\end{array}$ & 33,395 & or & 10.50 & 0.025 & 035 & 10.650 & 350,848 & 3.000 & 11.10 & 372,530 \\
\hline 18 & $\begin{array}{l}\text { Placement of Geotextlle (TYPAR) Filter on } \\
1^{1}-0^{*} \text { Coarse Sand Drainage Layer }\end{array}$ & 107.000 & sy & 0.13 & 0.005 & 535 & 10,700 & 10,910 & - & 0.20 & 24,610 \\
\hline 17 & Piacement of $1^{\prime}-6^{*}$ Common Fin & 49,247 & or & 3.75 & 0.045 & 2.216 & 44.352 & 184.076 & 19.297 & 4.80 & 242,300 \\
\hline 18 & Placement of 6" Topsoil & 16,511 & or & 9.50 & 0.045 & 743 & 14,000 & 150,055 & 4.,138 & 10.70 & 170.17 \\
\hline 89 & $\begin{array}{l}\text { Piacement of Pertmeter Exterior } \\
\text { Drainage Layer } 3 / 4^{*} \times 3^{*} \text { DP (Crushed Stone) }\end{array}$ & 1,900 & 8Y & 1.50 & 0.16 & son & 0,000 & 2.050 & 1,624 & 8.70 & $10, \pi$ \\
\hline 20 & Excavation of Trapezotdal Drainage Ditch & 6,400 & or & - & a.165 & 1.056 & 21,120 & o. & 8.030 & 4,30 & 27,400 \\
\hline & (D) PAGE TOTAL & & & & & 54,800 & $1,097,309$ & $3,170,000$ & 204.016 & & 1000,0 \\
\hline
\end{tabular}

H-6. Cost Estimate for a Cover System Utilizing Reinforced Sprayed Asphalt (Cont'd) 
DETAIL ESTIMATE CHKDBY: 8.MEAD

LOG I: :-03

ACTIVITY CODE: ESE260412

PROJ.NAME: ECON. EVALUATION OF CLOSUAE CAP BARRIER MATERIAL

ESTMATOR: Pamk

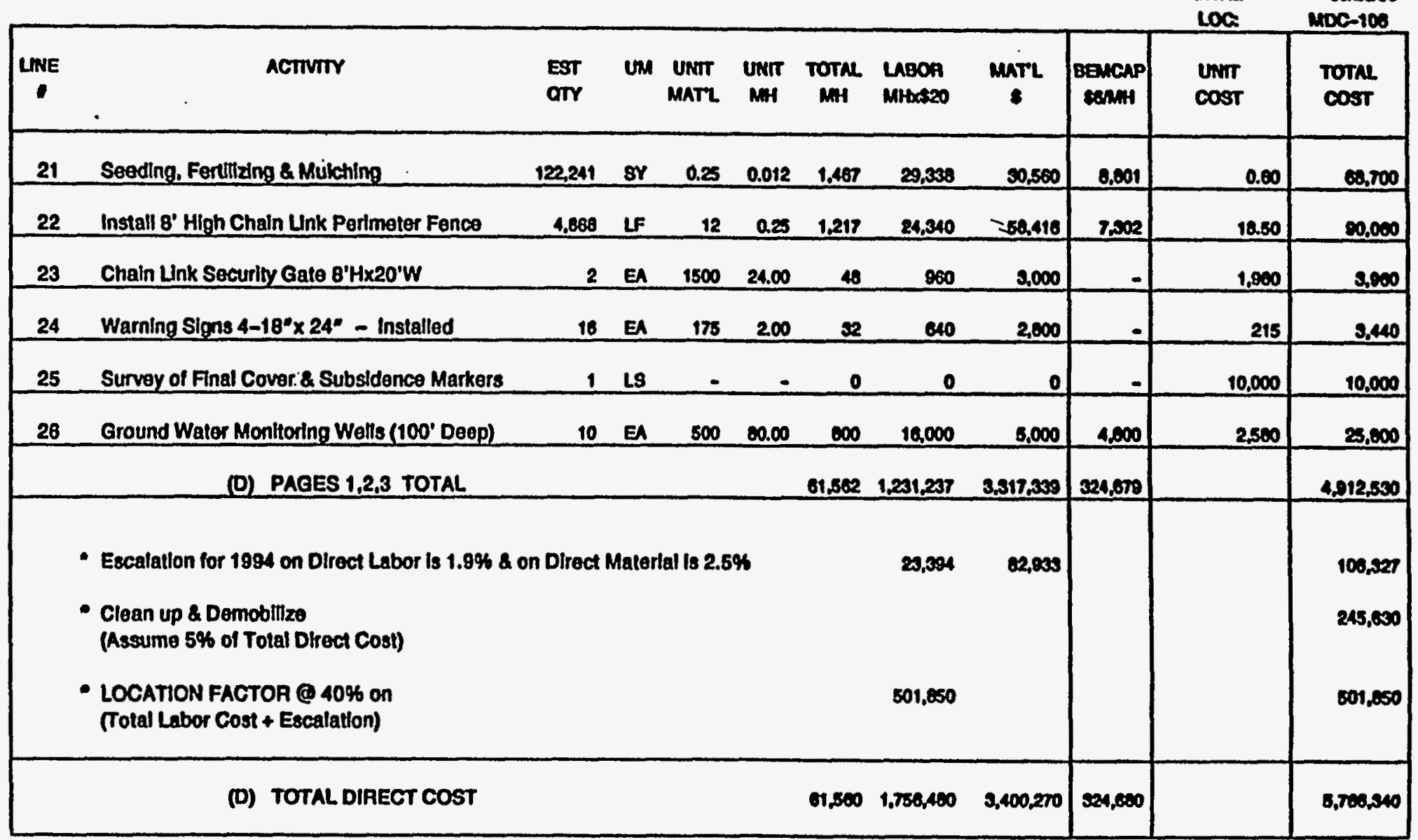

H-6. Cost Estimate for a Cover System Utilizing Reinforced Sprayed Asphalt (Cont'd) 
DETAIL ESTIMATE

LOG : : 7-03

ACTIVITY CODE: ESE260412

PROI.NAME: ECON. EVALUATION OF CLOSURE CAP BARAIEA MATERIAL

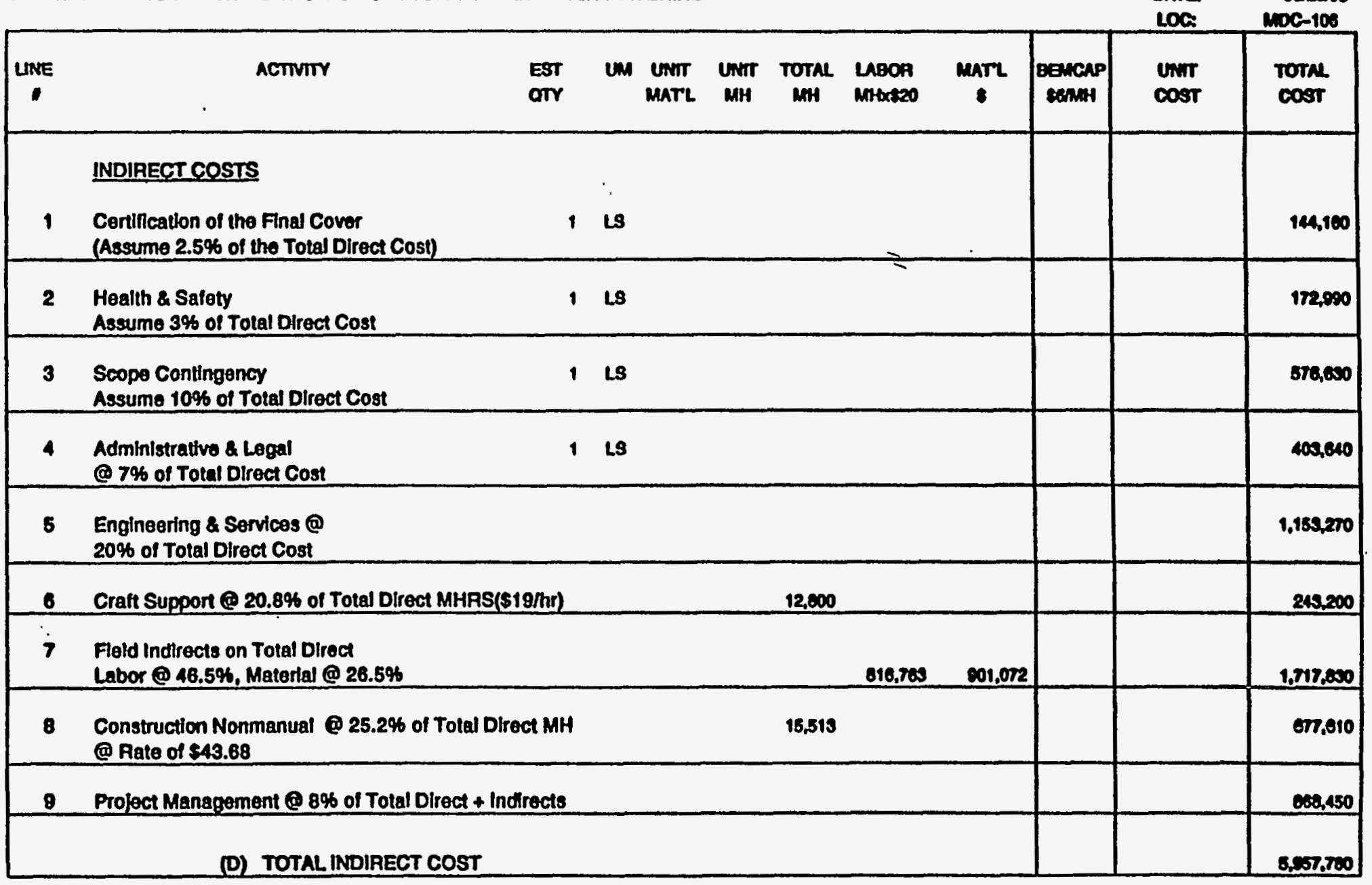

H-6. Cost Estimate for a Cover System Utilizing Reinforced Sprayed Asphalt (Cont'd) 
DETAIL ESTIMATE

8. MEAD

LOG : : 7-03

ACTIVTTY CODE: ESE260412

PROI.NAME: ECON. EVALUATION OF CLOSURE CAP BARAIER MATERIAL

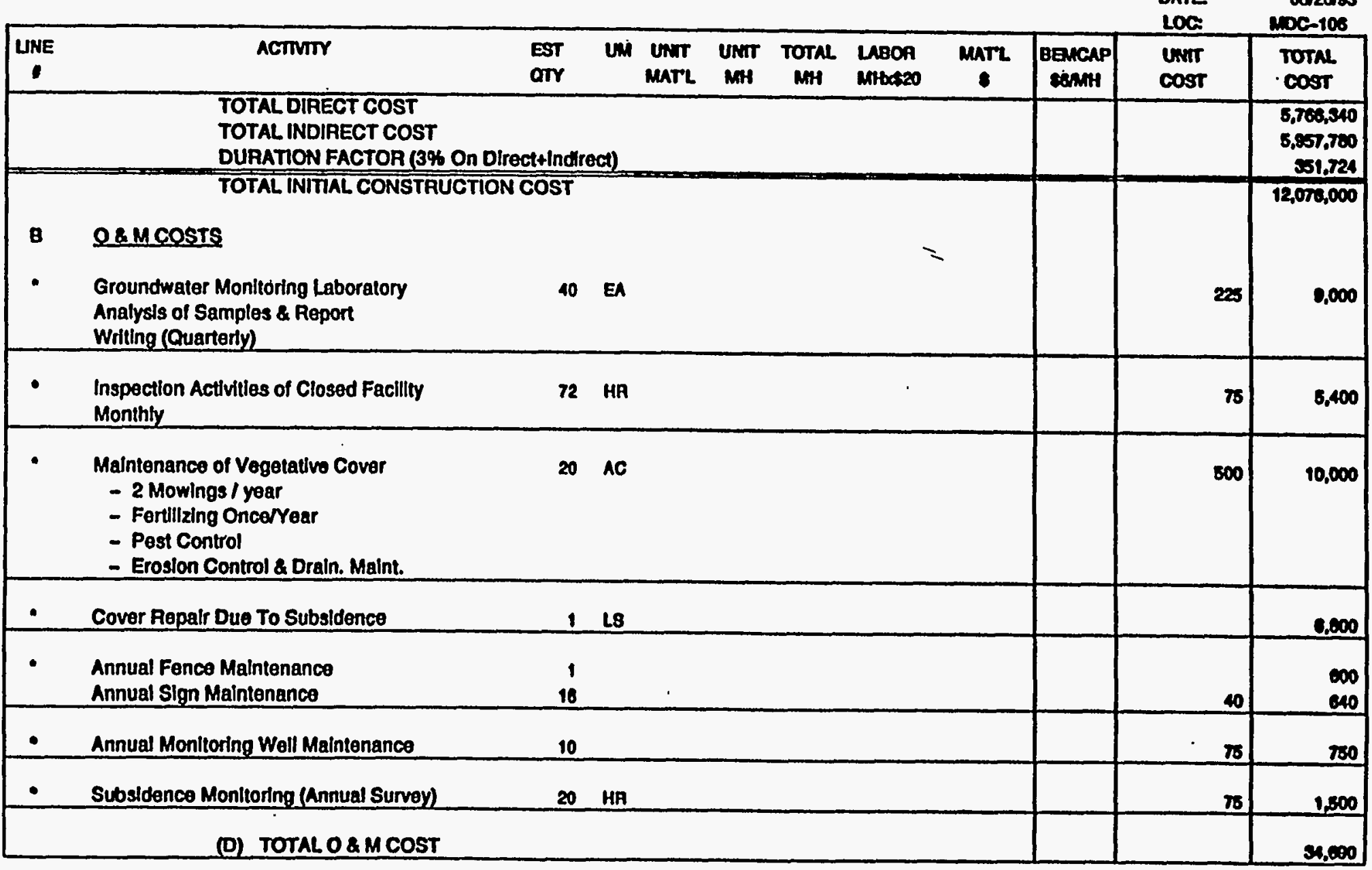

H-6. Cost Estimate for a Cover System Utilizing Reinforced Sprayed Asphalt (Cont'd) 
DETAIL ESTIMATE

LOG I: : $7-03$

ACTIVITY COOE: ESE260412

PROS.NAME: ECON. EVALUATION OF GLOSURE CAP BAAAIER MATERIAL

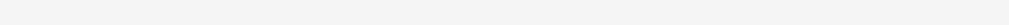

\begin{tabular}{|c|c|}
\hline CHK'D BY: & 8. MEAD \\
\hline $\begin{array}{l}\text { ESTmator: } \\
\text { PHONE: } \\
\text { DATE } \\
\text { LOC. }\end{array}$ & $\begin{array}{c}\text { Pomik } \\
7-3200 \\
\text { circess } \\
\text { moc-10s }\end{array}$ \\
\hline
\end{tabular}

\section{SUMMARY}

TWENTY (20) ACRES SITE- REINFORGED SPRAYED ASPHALI =

C TOTAL COST ( Total Present Worth)

(1) Present Worth of $O \& M$ Cost

(5\% discount Rate for 30 Years)

600,050

(Total O\& $M \times 15.3725$ )

(II) Total Initial Construction Cost

$12,078,000$

(D) TOTAL PROJECT COST (Rounded to the nearest Thousand)

H-6. Cost Estimate for a Cover System Utilizing Reinforced Sprayed Asphalt (Cont'd) 


\section{DETAIL ESTIMATE}

CHKD BY: 8. MEAD

Loof: 7-03

ESTIMATOF: rannx

ACTIVITY CODB: ESE260112

PROJ.NAME: ECON. BVALUATTON OP CLOSURB CAP BARRIER MATERIAL

PHONE: T-5265

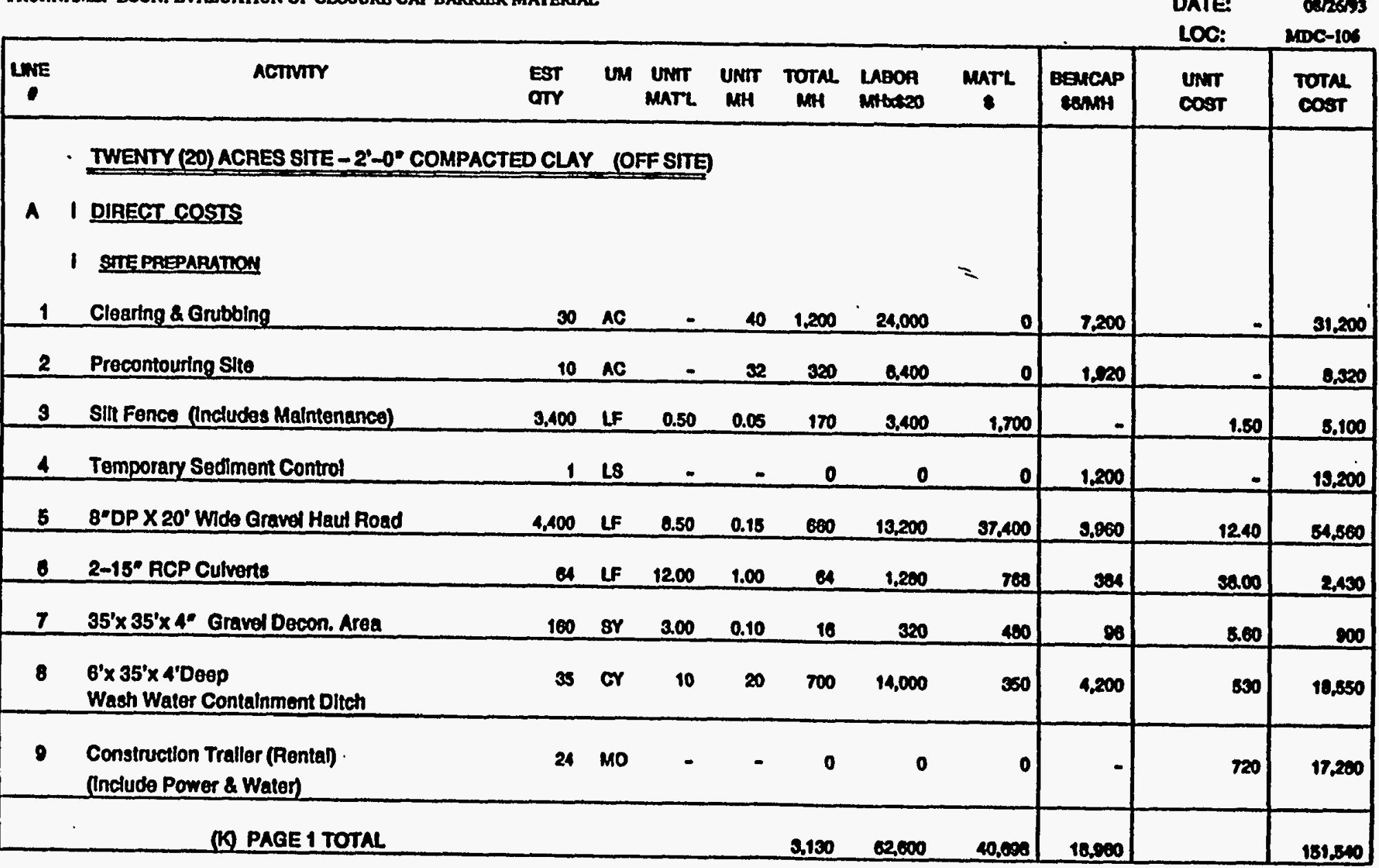

H-7. Cost Estimate for a Cover System Utilizing Compacted Kaolin (Offsite) Clay and a 30-mil FML 
DETAILESTIMATE

CHKD BY: S. MEAD

Loof: 7-03

ACTIVITY CODB: BSB2CON12

PROJ.NAMB: BCON. BVALUATION OP CLOSURB CAP BARRIER MATBRALL

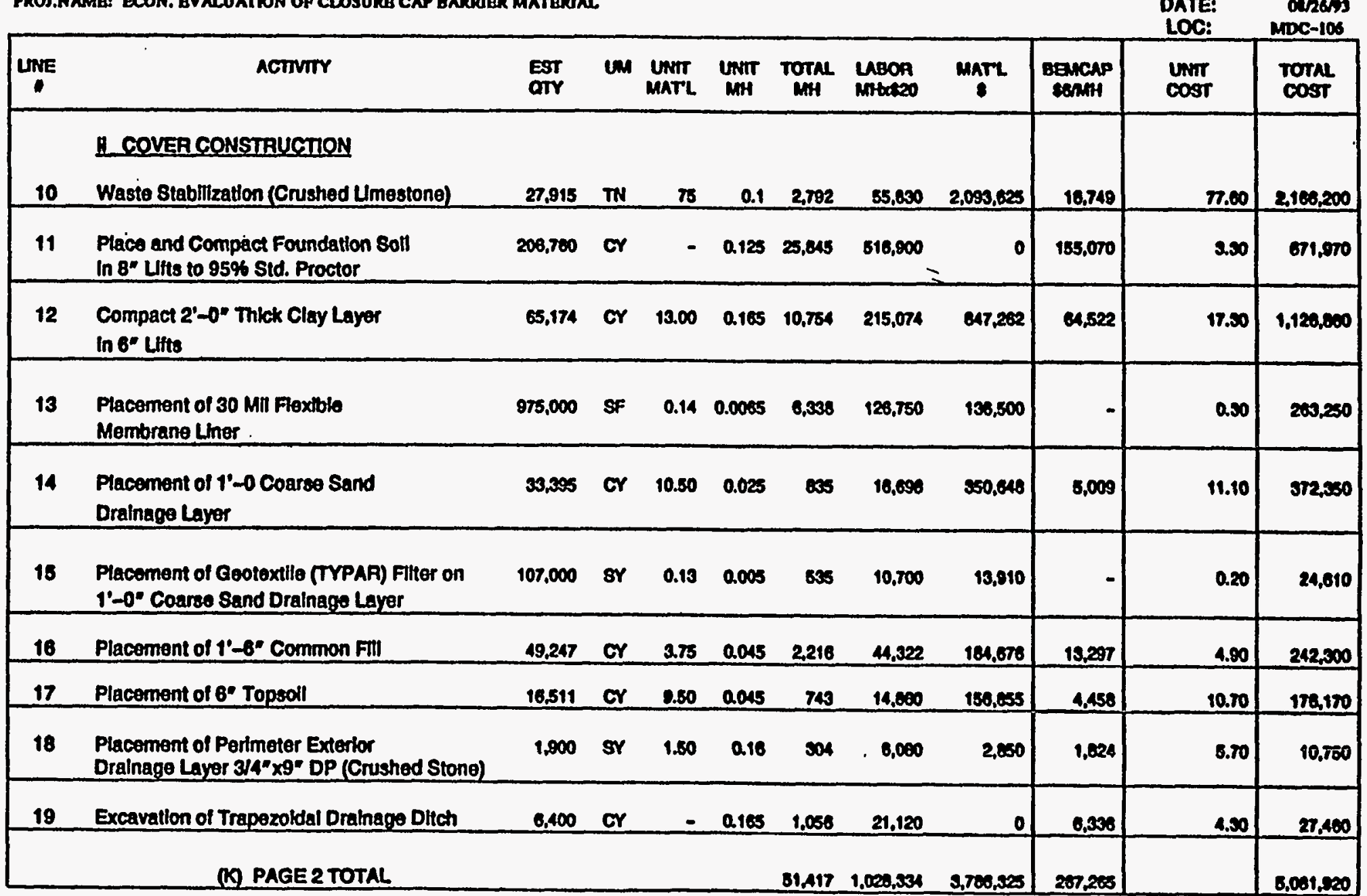

H-7. Cost Estimate for a Cover System Utilizing Compacted Kaolin (Offsite) Clay and a 30-mil FML (Cont'd) 
CHKO BY: S. MEAD

200 : :-03

ACTIVTYY CODE: ESE200412

PROJ.NAME: ECON. EVALUATION OP CLOSURB CAP BARRUER MATERIAL

ESTIMATOA: TOMWT

PHONE: 7-5265

DATE:

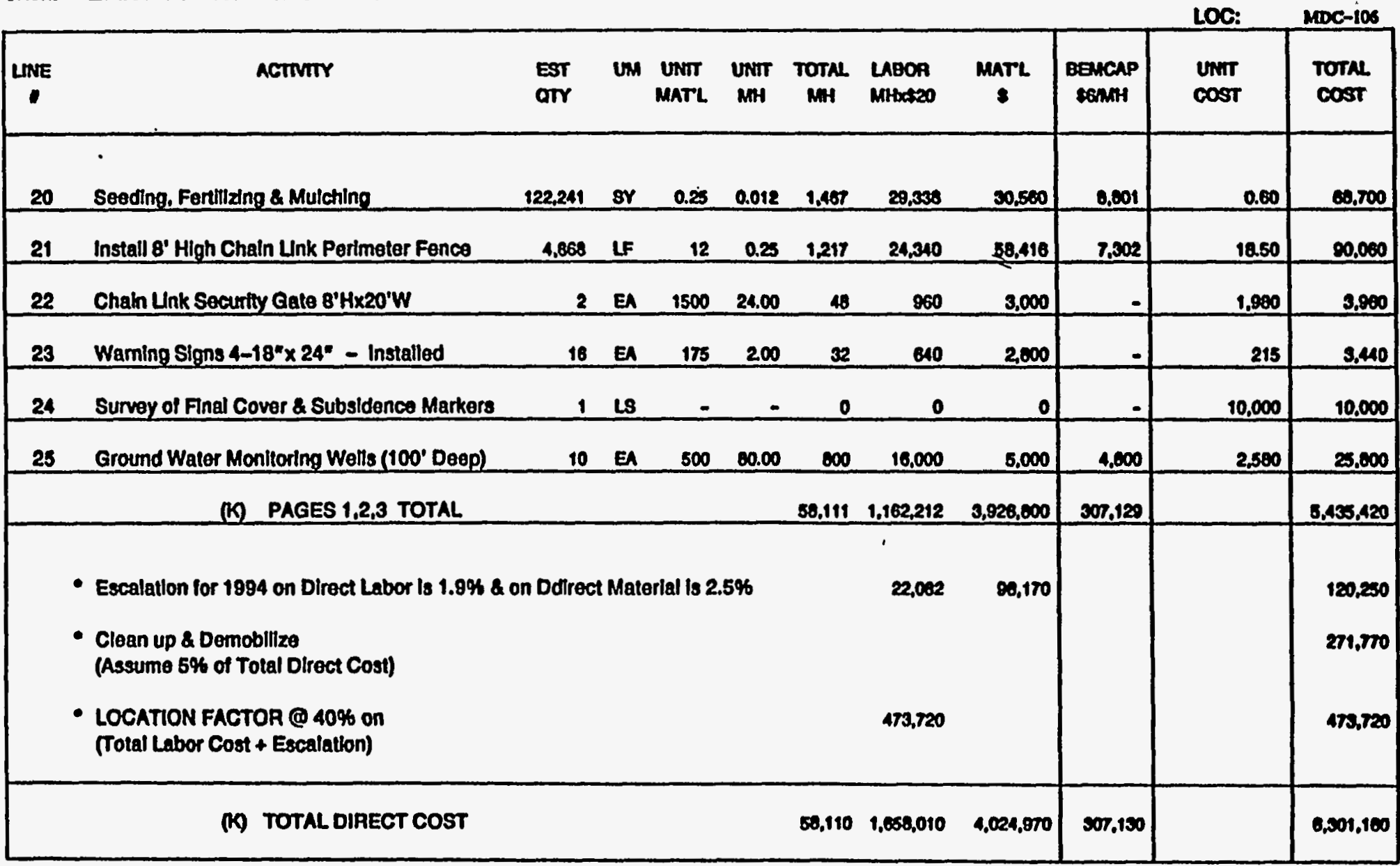

H-7. Cost Estimate for a Cover System Utilizing Compacted Kaolin (Offsite) Clay and a 30-mil FML (Cont'd) 
DETAIL ESTIMATE

CHKD BY: S. MEAD

Lod: $7-03$

ACTTVTYY CODE: ESB260412

ESTIMATOR: TOWL

PROJ.NAMB: ECON. BVALUATOK OP CLOSURB CAP BARRER MATERAL.

PHONE:

$7-5256$

DATE:

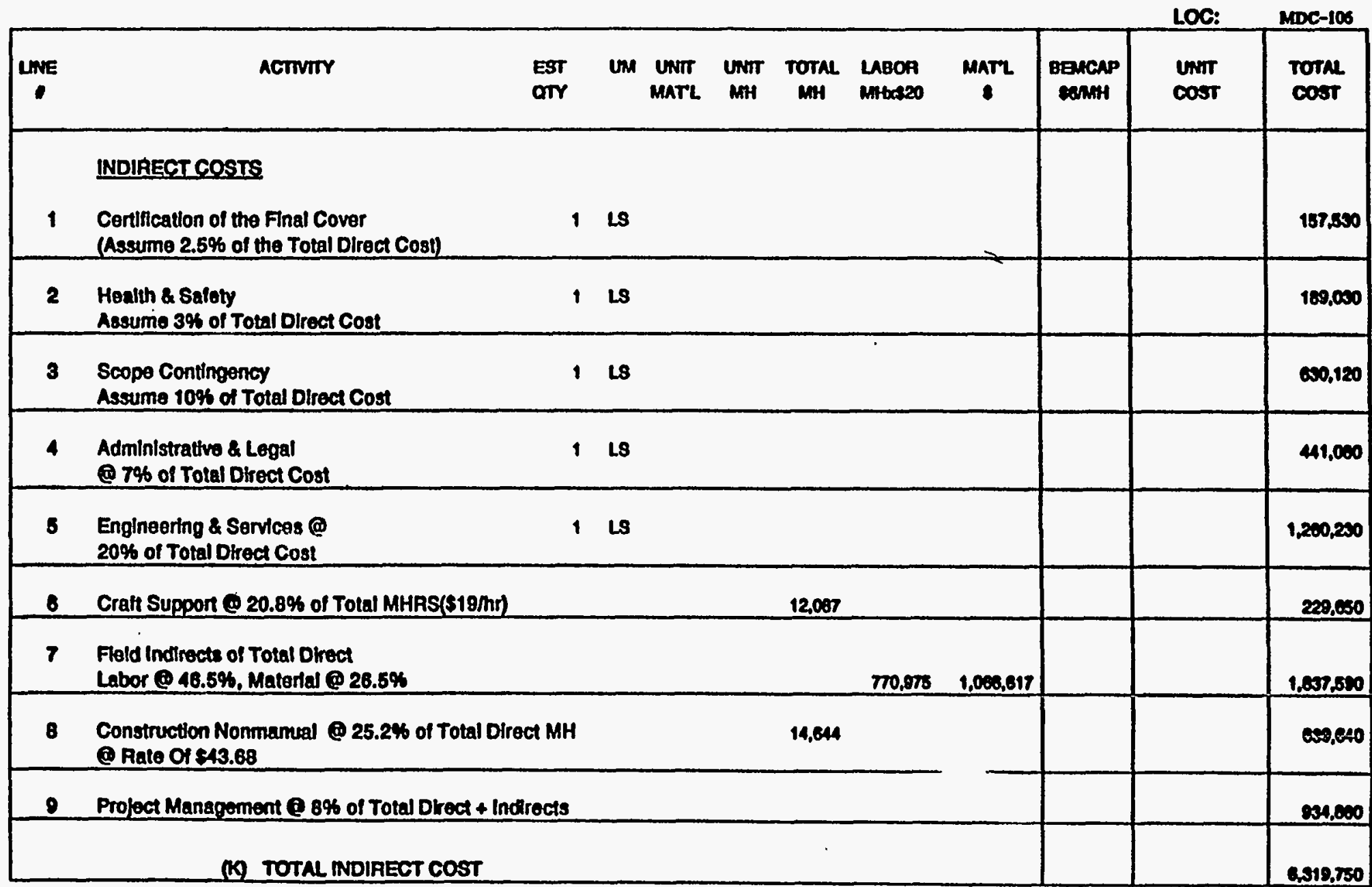

H-7. Cost Estimate for a Cover System Utilizing Compacted Kaolin (Offsite) Clay and a 30-mil FML (Cont'd) 
DETAIL ESTIMATE

CHKD BY: S. MEAD

\section{I: $7-03$}

ACTIVTTY CODE: BSE260412

PROS.NAME: ECON. BVALUATHON OP CLOSURB CAP DARUIER MATERIAL

PHONE: Nank

DATE:

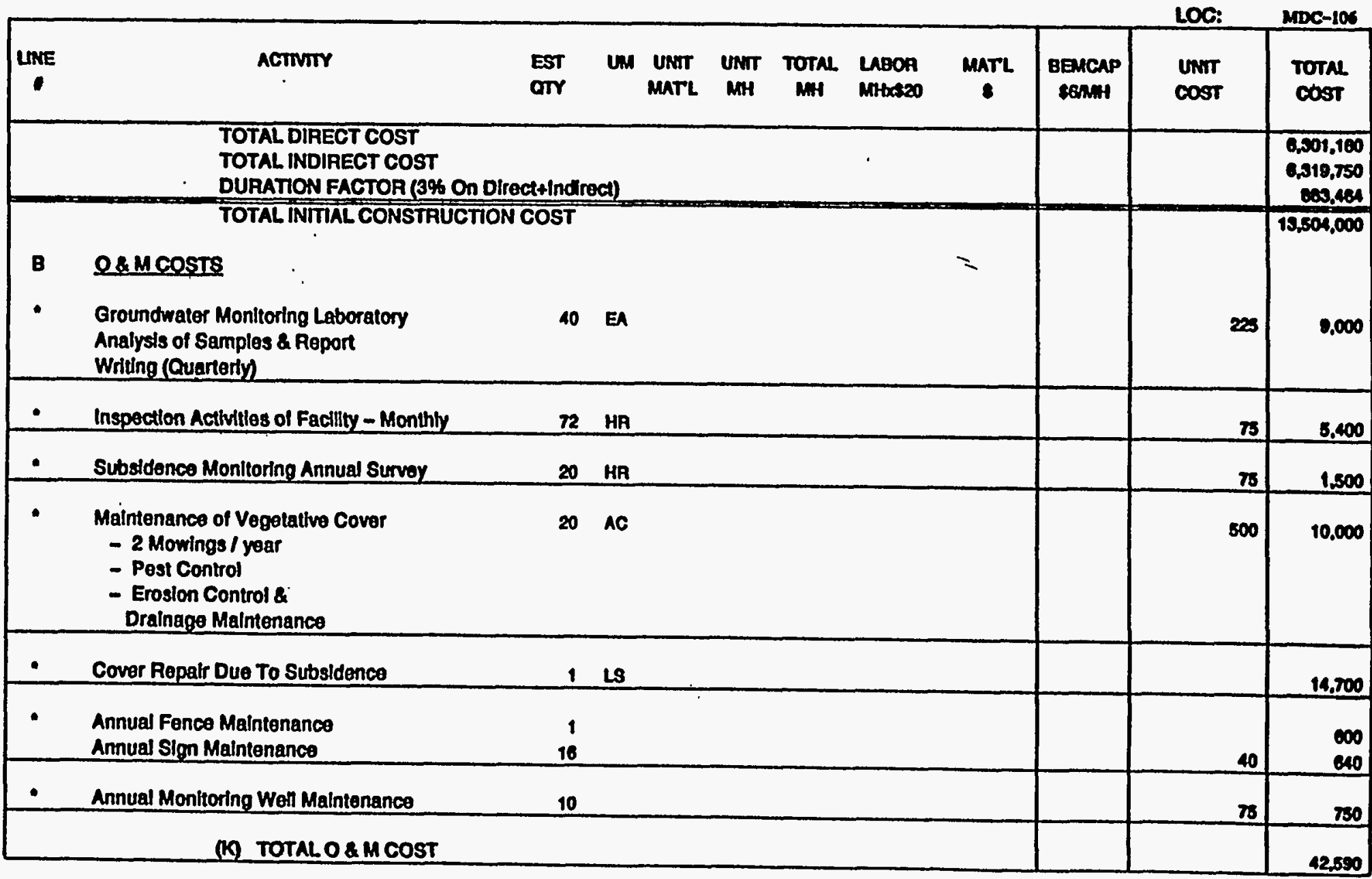

H-7. Cost Estimate for a Cover System Utilizing Compacted Kaolin (Offsite) Clay and a 30-mil FML (Cont'd) 
DETAIL ESTIMATE

200 1: 7-03

ACTTVITY CODEs BSR260412

MOJ.NAMR: BCON. BVALUATTON OF CLOSURB CAP BARRTER MATERJAL.
CHKD BY: 8. MEAD

ESTIMATOA: ramx

PHONE: 7-S206

DATE: Densess

LOC: MDC-106

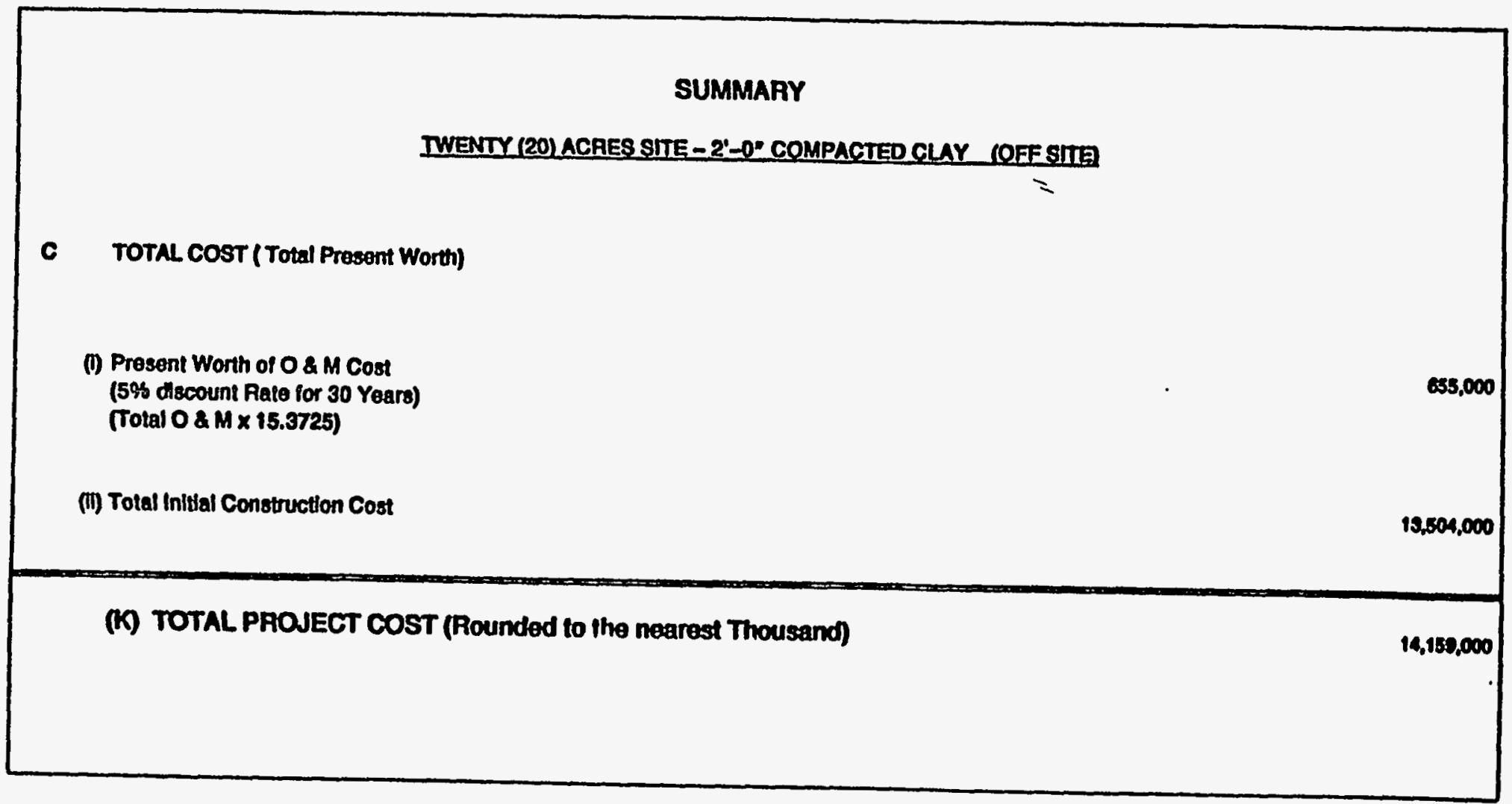

H-7. Cost Estimate for a Cover System Utilizing Compacted Kaolin (Offsite) Clay and a 30-mil FML (Cont'd) 
DETAIL ESTIMATE

Lo0 $1:$ 7-03

ACTIVITY CODB: ESE2OON12

PROJ.NAME: ECON. EVALUATTON OF CLOSURE CAP BARRIER MATERIAL.
CHKD BY: S.MEAD

ESTIMATOA: ramix

PHONE: 7-5268

DATE: OARSSBs

MDC-105

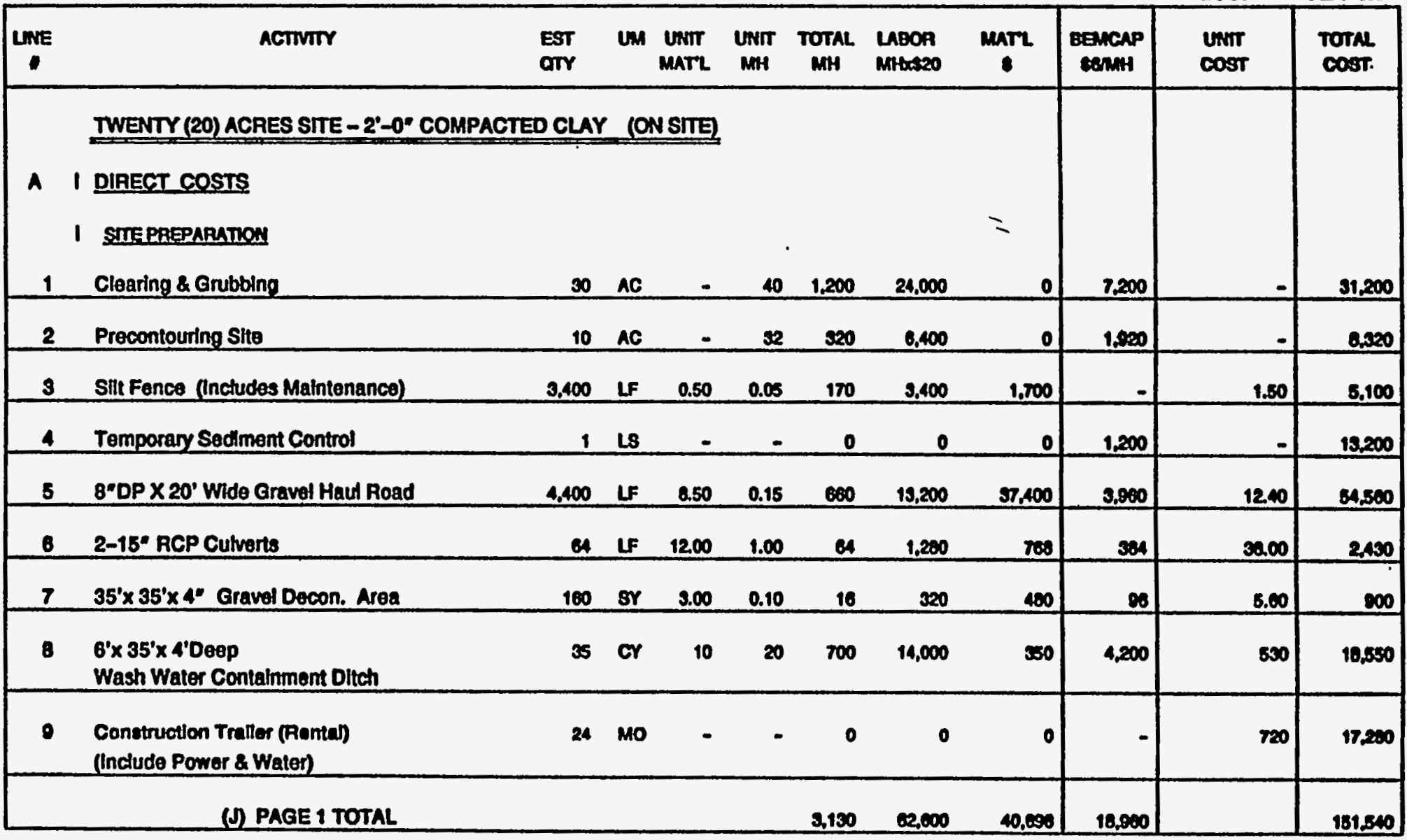

H-8. Cost Estimate a for Cover System Utilizing Compacted Onsite Sandy Clay and a 30-mil FML 
Loo : $7-03$

ACTIVITY CODE: ESE260412

PROJ.NAME: ECON. EVALUATION OF CLOSURB CAP BARRIRR MATERIAL.

DETAIL ESTIMATE

CHK'D BY: S.MEAD

ESTIMRTOR: ROMU:

PHONE: 7-5260

DATE:

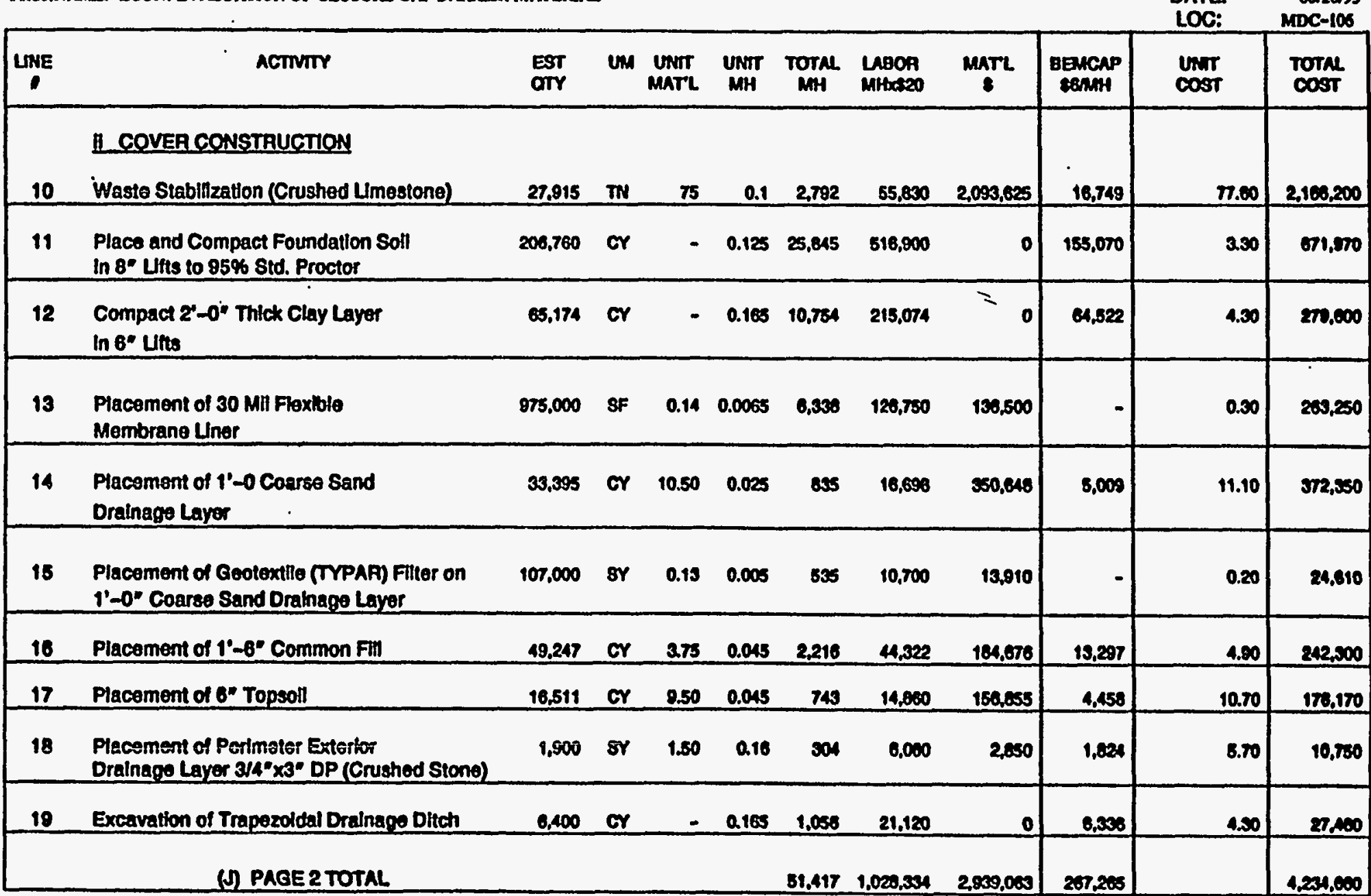

H-8. Cost Estimate a for Cover System Utilizing Compacted Onsite Sandy Clay and a 30-mil FML (Cont'd) 


\section{DETAIL ESTIMATE}

CHKD BY: S.MEAD

Loaf: 7-03

ACTIVIT CODE: BSE260412

PROJ.NAME: ECON, EVALUATION OP CLOSURB CAP BARRIER MATERLAL.

ESTIMATOR: ramix

DATE:

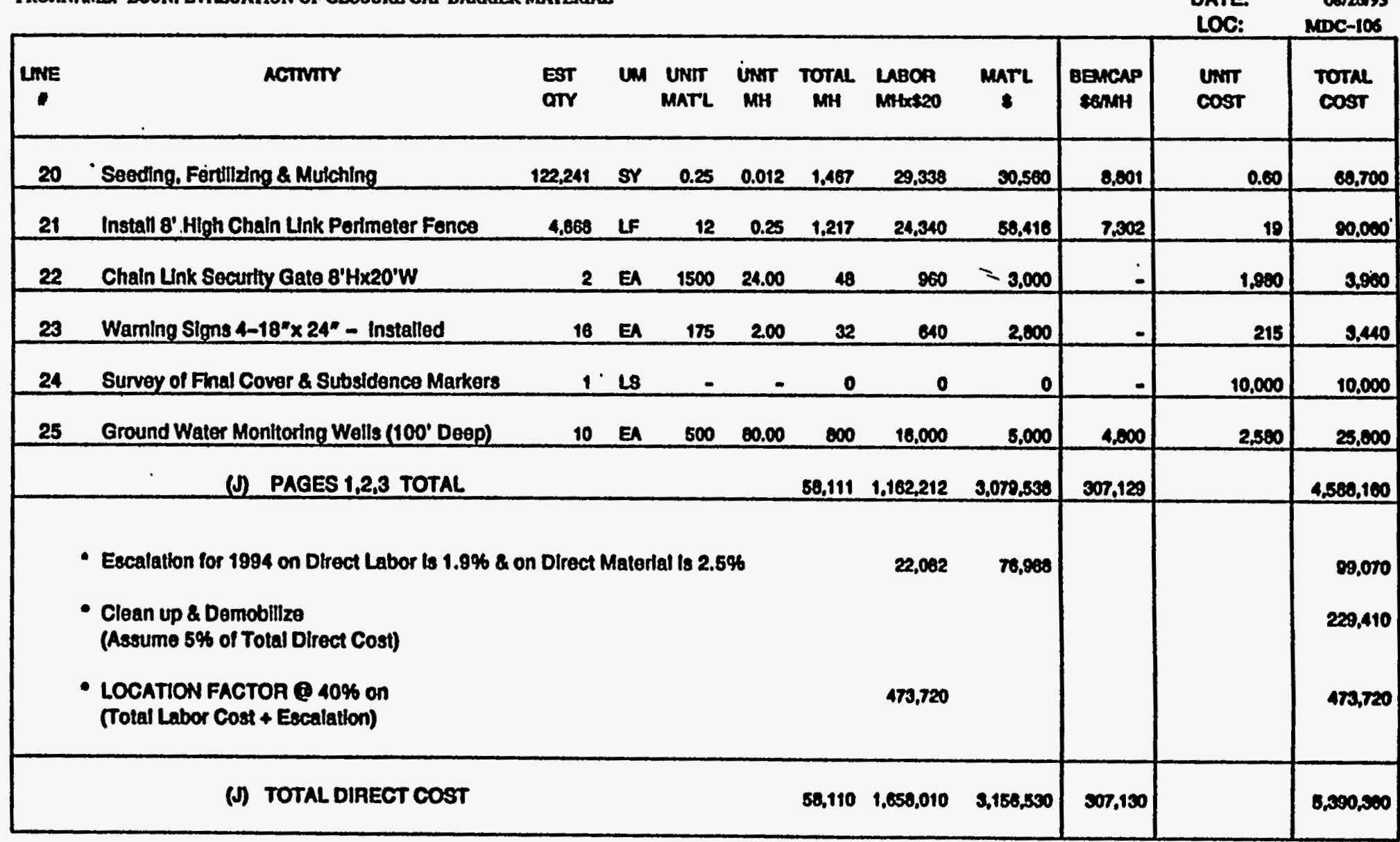

H-8. Cost Estimate a for Cover System Utilizing Compacted Onsite Sandy Clay and a 30-mil FML (Cont'd) 
DETAIL ESTIMATE

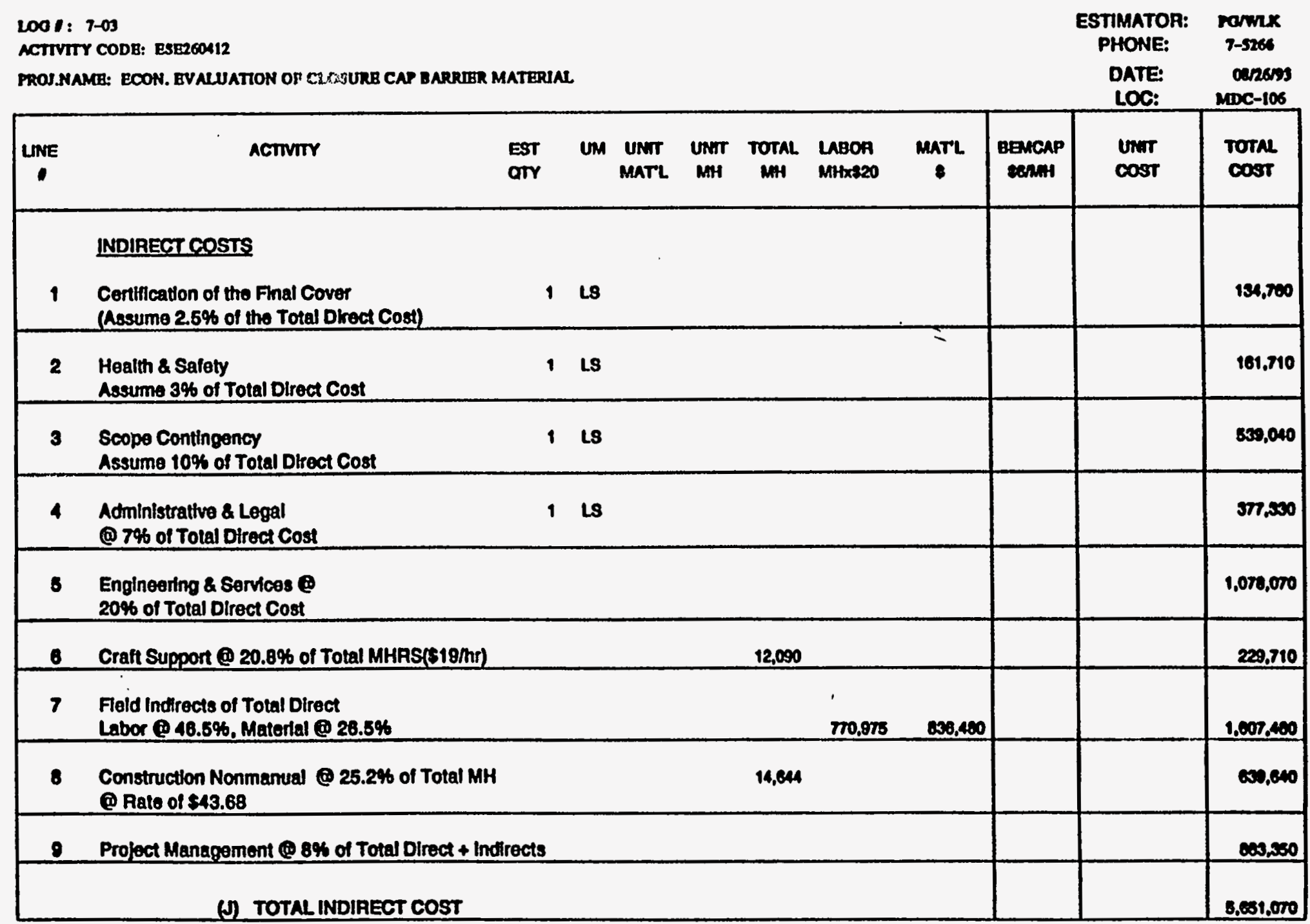

H-8. Cost Estimate a for Cover System Utilizing Compacted Onsite Sandy Clay and a 30-mil FML (Cont'd) 


\section{DETAIL ESTIMATE}

CHKD BY: S.MEAD

\section{$200,7-03$}

ACTIVIT CODE: ESE260412

PROJ.NANB: ECON. EVALUATHON OP CLOSURB CAP BARRURR MATERIAL

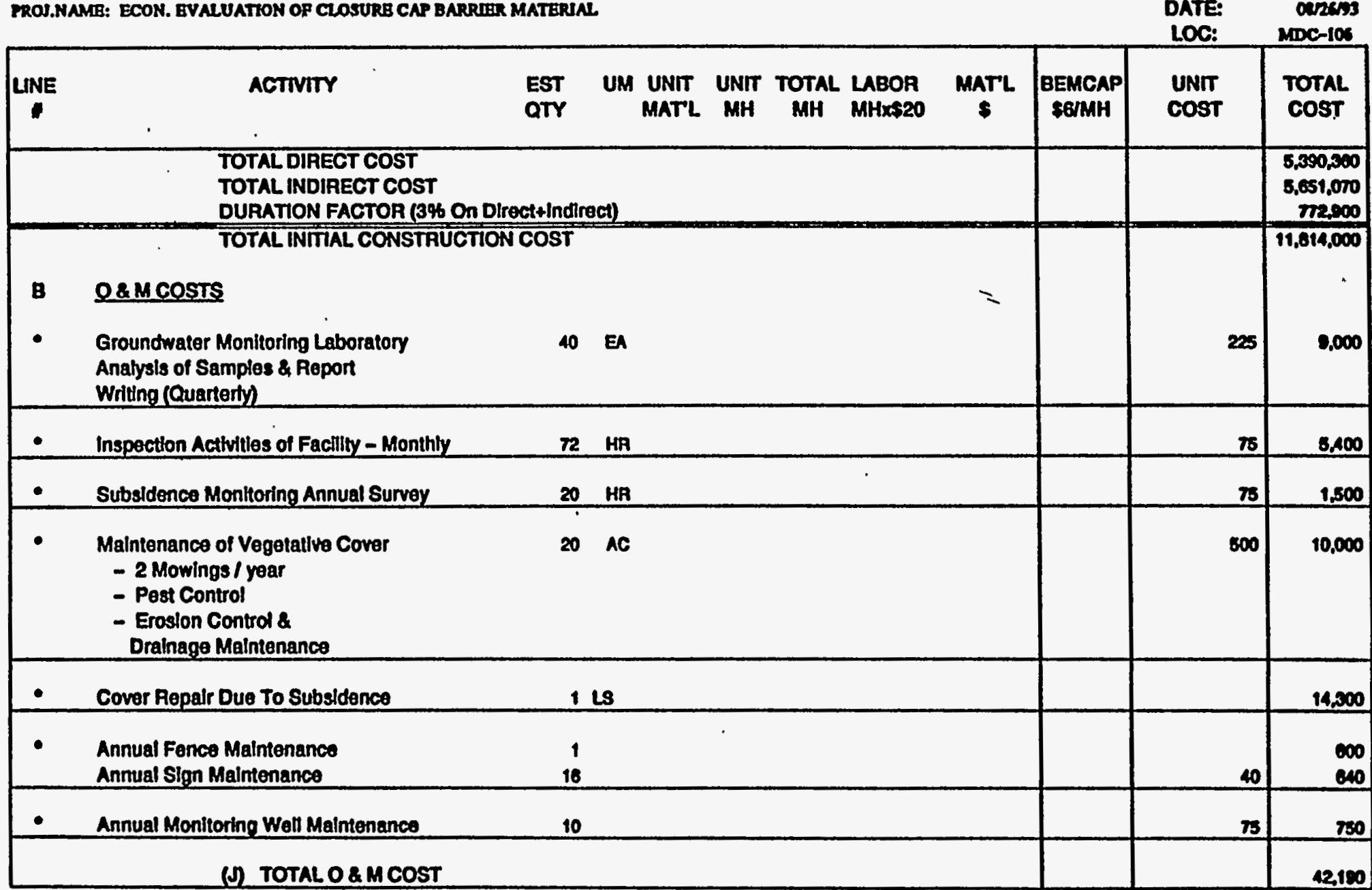

H-8. Cost Estimate a for Cover System Utilizing Compacted Onsite Sandy Clay and a 30-mil FML (Cont'd) 
$1001: 7-03$

ACTIVITY CODE: ESE2COA12

PROJ.NAME: ECON. BVALUATION OP CLOSUIRB CAP BARRIER MATERIAL

10C:

$10 \mathrm{DC}-10$

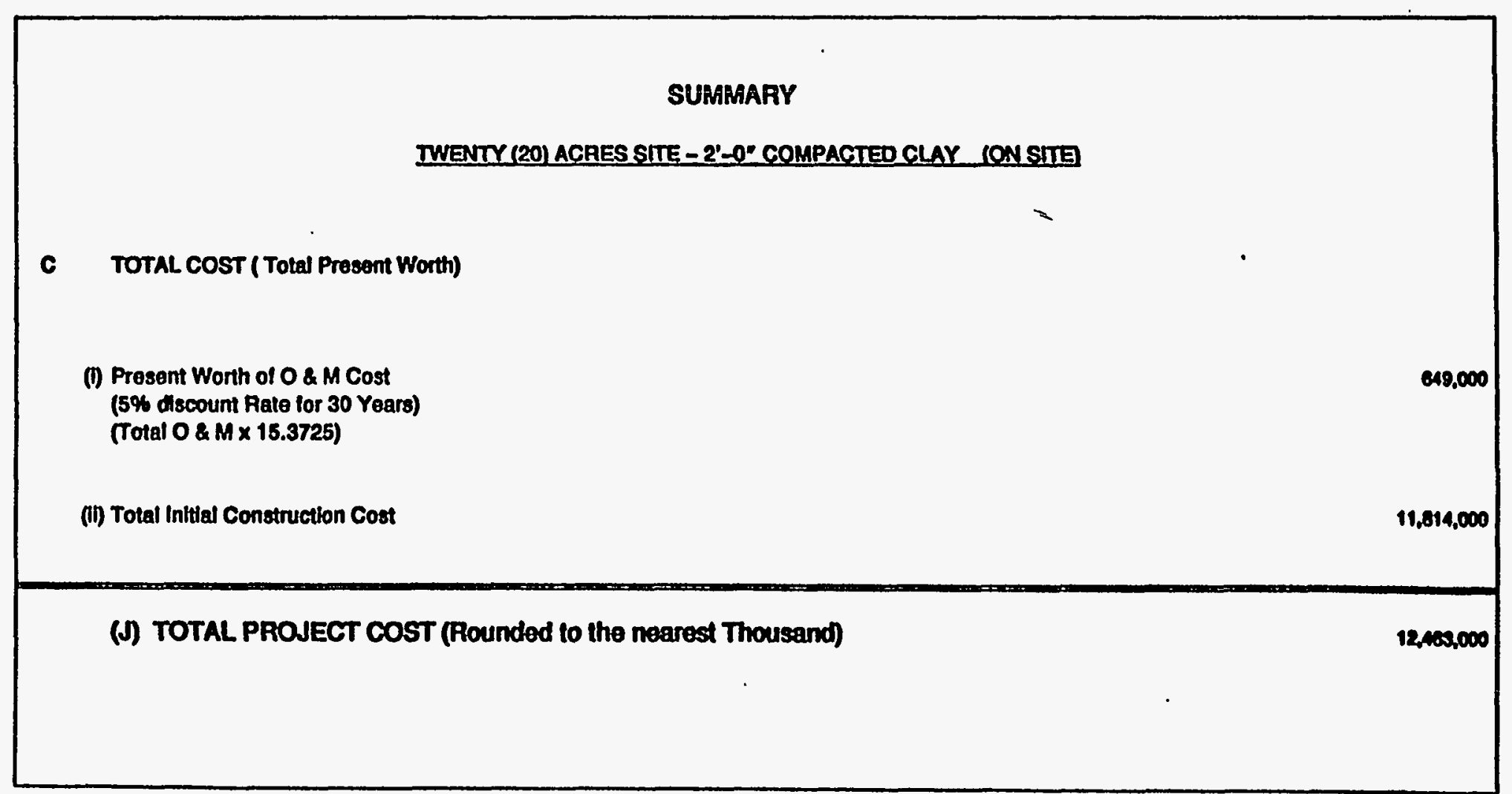

H-8. Cost Estimate a for Cover System Utilizing Compacted Onsite Sandy Clay and a 30-mil FML (Cont'd) 


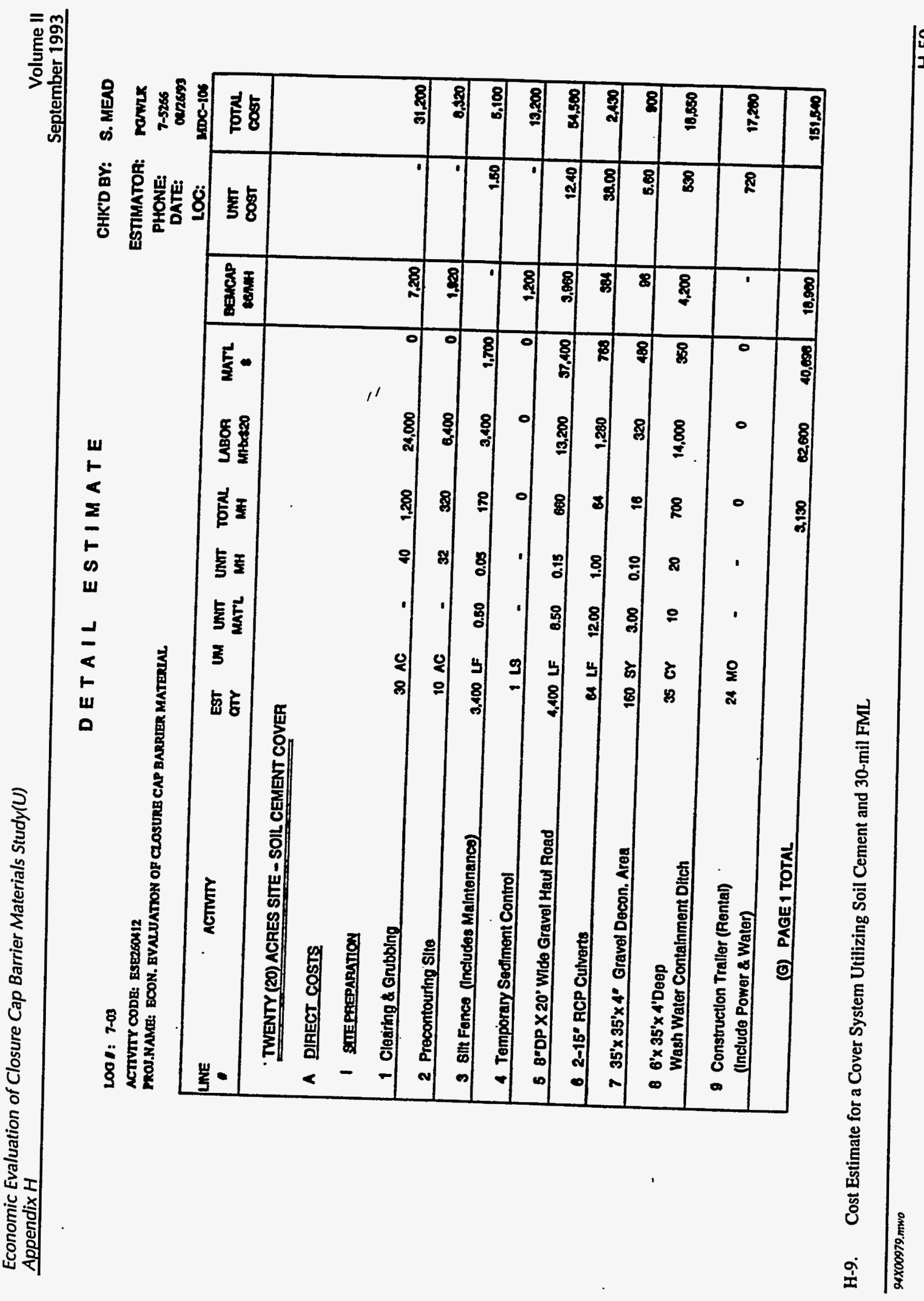




\section{DETAIL ESTIMATE}

CHK'D BY: S. MEAD

1001: 7-03

ACTVITY CODE: ESE260412

MROJ.MAME: ECON. BVALUATION OP CLOSURB CAP DARRIER MATERIAL

\begin{tabular}{|c|c|c|c|c|c|c|c|c|c|c|}
\hline & & & & & & & & & LOG: & MDC-105 \\
\hline ACTVITY & $\begin{array}{l}\text { EST } \\
\text { OTY }\end{array}$ & UM & $\begin{array}{l}\text { UNIT } \\
\text { MATL }\end{array}$ & ${ }_{M H H}^{\text {UNT }}$ & Trotal & $\begin{array}{l}\text { LABOA } \\
\text { MAtrezeO }\end{array}$ & $\operatorname{marL}$ & $\begin{array}{c}\text { gencap } \\
\text { senmH }\end{array}$ & $\begin{array}{l}\text { UNT } \\
\text { COST }\end{array}$ & $\begin{array}{l}\text { Total } \\
\text { Cost }\end{array}$ \\
\hline $\begin{array}{l}1 \text { COVER CONSTRUCTION } \\
10 \text { Wasto Stabilization (Crushod Umestono) }\end{array}$ & 27.915 & $\pi N$ & 73 & 0.1 & 2,792 & 35,030 & $2,093,025$ & 16,749 & $n . \infty$ & $2,100,200$ \\
\hline $\begin{array}{l}11 \text { Place and Compact Foundation Soll } \\
\text { in } 8^{\circ} \text { Uits to } 95 \% \text { Std. Proctor }\end{array}$ & 208,760 & or & - & 0.125 & 25,045 & 516,900 & 0 & 155,070 & 3.50 & 071.070 \\
\hline $\begin{array}{l}12 \text { Soll-Cemenl mixture transported from central } \\
\text { mixing plant by end-dump trucks and placed } \\
\text { In o-8" Ifts by spreadere. Assume Fine Sand } \\
\text { Soll with } 321 \text { of comentcY. Compact the soil- } \\
\text { cement uniformily to } 98 \% \text { max. densily. }\end{array}$ & 98,179 & or & 18 & 0.12 & 11,781 & 235,630 & $1,701,202$ & 70,600 & 21.10 & 2,073510 \\
\hline $\begin{array}{l}13 \text { Curing soll-cement. Assume liquid Asphatt } \\
\text { applied } 0.4 \text { gallsy for } 15 t \text { coat } \& 0.2 \text { for } 2 \text { nd. }\end{array}$ & $\begin{array}{l}99,725 \\
89.725 \\
\end{array}$ & $\begin{array}{l}\text { SY } \\
\text { sr }\end{array}$ & $\begin{array}{l}0.33 \\
0.17 \\
\end{array}$ & $\begin{array}{l}0.03 \\
0.02\end{array}$ & $\begin{array}{l}2,992 \\
1,895 \\
\end{array}$ & $\begin{array}{r}69,635 \\
39,690 \\
\end{array}$ & $\begin{array}{l}32,000 \\
16,250 \\
\end{array}$ & - & $\begin{array}{l}0.00 \\
0.600 \\
\end{array}$ & $\begin{array}{r}82,740 \\
80,040 \\
\end{array}$ \\
\hline $\begin{array}{l}14 \text { Placement of } 30 \text { Mill Floxible } \\
\text { Membrana Liner }\end{array}$ & 973,000 & $\mathbf{S F}$ & 0.14 & 0.0005 & 6,330 & 120,750 & 130,500 & - & 0.30 & 200,250 \\
\hline $\begin{array}{l}\text { 15 Placement of 1'-0 Coarse Sand } \\
\text { Drainage Layer }\end{array}$ & 39,393 & Cr & 10.50 & 0.025 & 635 & 16,690 & 350,640 & 8,000 & 11.10 & 972,330 \\
\hline $\begin{array}{l}16 \text { Placement of Geotextile (TYPAR) Filter on } \\
1^{\prime}-0^{*} \text { Coarse Sand Drainspe Loyer }\end{array}$ & 107,000 & sY & 0.13 & 0.005 & 536 & 10,700 & 19.910 & - & 0.20 & 24,810 \\
\hline 17 Plecement of $1^{\prime}-6^{\circ}$ Common Fil & 49,247 & Cr & 3.75 & 0.045 & 2,216 & 44,322 & 184,078 & 13,297 & 4.00 & 242,300 \\
\hline 18 Placement of $6^{\prime \prime}$ Topsoll & 16,511 & or & 9.50 & 0.045 & 743 & 14.000 & 150,055 & 4,450 & 10.70 & 170,170 \\
\hline (G) PAGE 2 TOTAL & & & & & SS,112 & $1,002,232$ & $4,411,707$ & 247.517 & & $0,100,010$ \\
\hline
\end{tabular}

H-9. Cost Estimate for a Cover System Utilizing Soil Cement and 30-mil FML (Cont'd) 
D B T A I L B S T M A T B

\section{Lool: $7-03$}

ACTIVITY CODB: ESE2SOA12

PROJ.NAMB: ECON. EYALUATION OF CLOSURB CAP BARRIER MATERIAL.

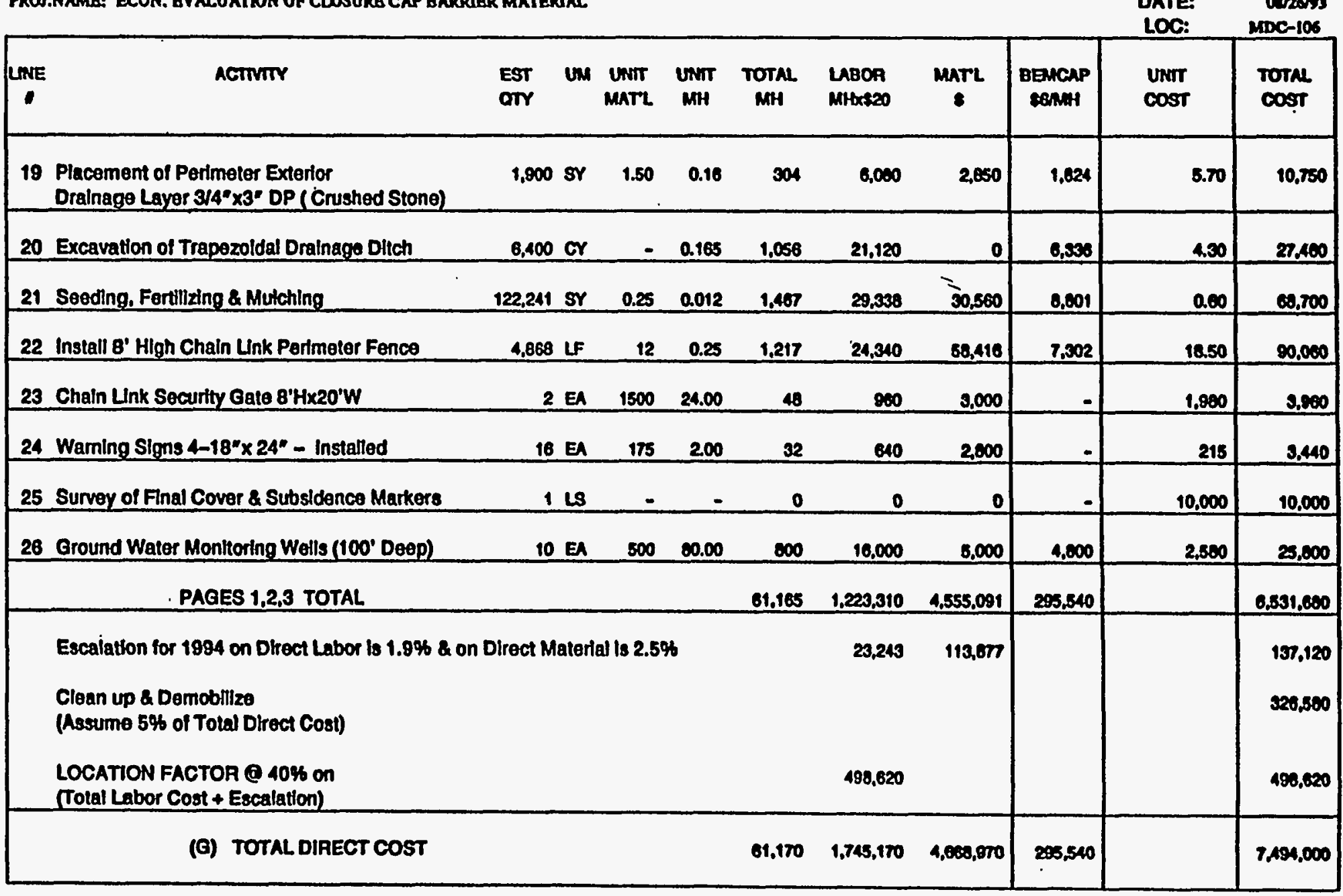

H-9. Cost Estimate for a Cover System Utilizing Soil Cement and 30-mil FML (Cont'd) 
Lo01: 7-03

ACTIVITY CODE: EsB260412

MROJ.MAMB: ECON. EVALUATION OP CLOSURB CAP BARRIER MATERULL

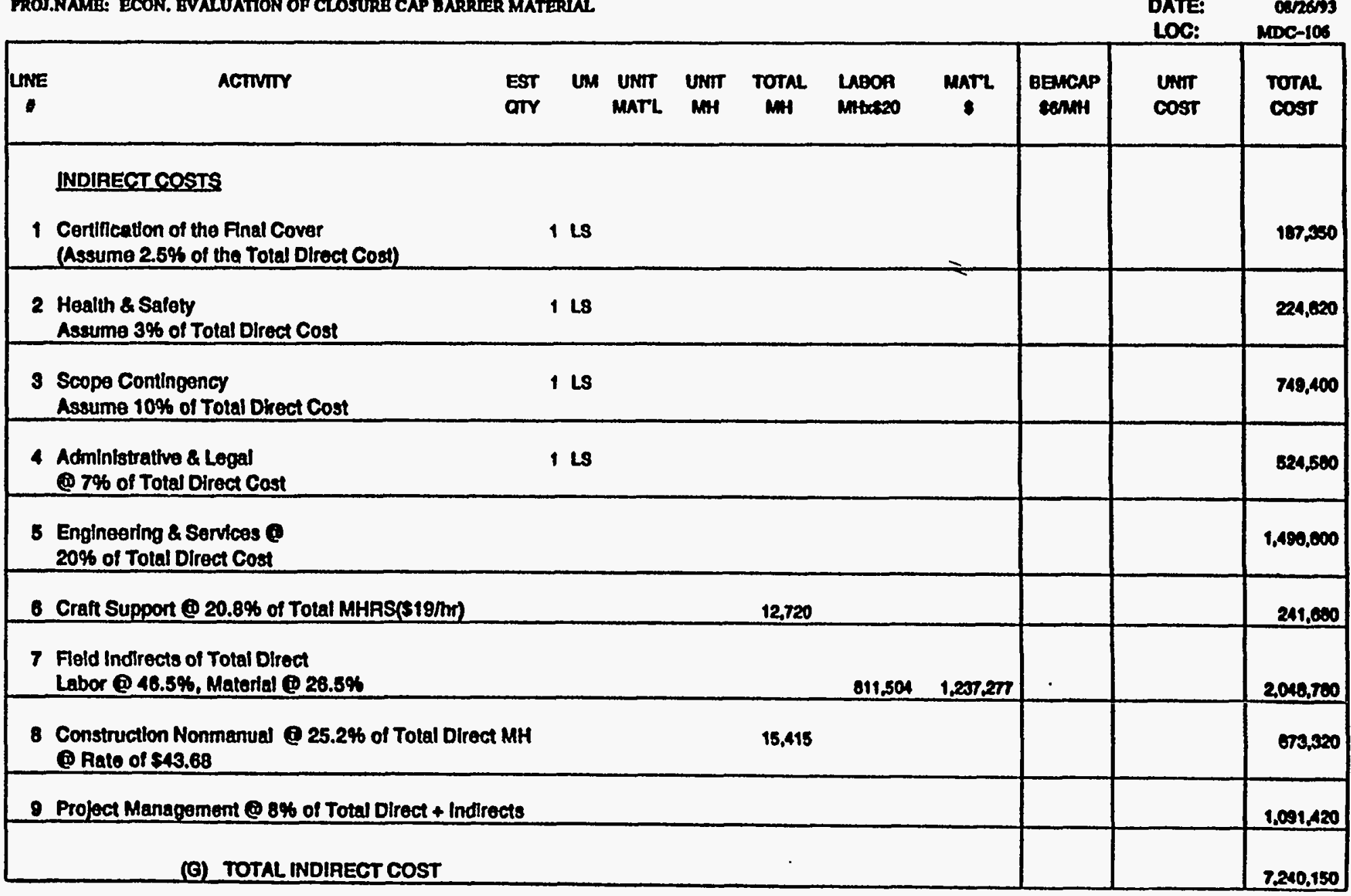

H-9. Cost Estimate for a Cover System Utilizing Soil Cement and 30-mil FML (Cont'd) 


\section{DBTAIL BSTIMATA}

Lo0 I: 7-03

ACTIVITY CODE: ESE260412

PROJ.NAMB: BCON. BVALUATON OP CLOSURE CAP BATRUER MATERIAL

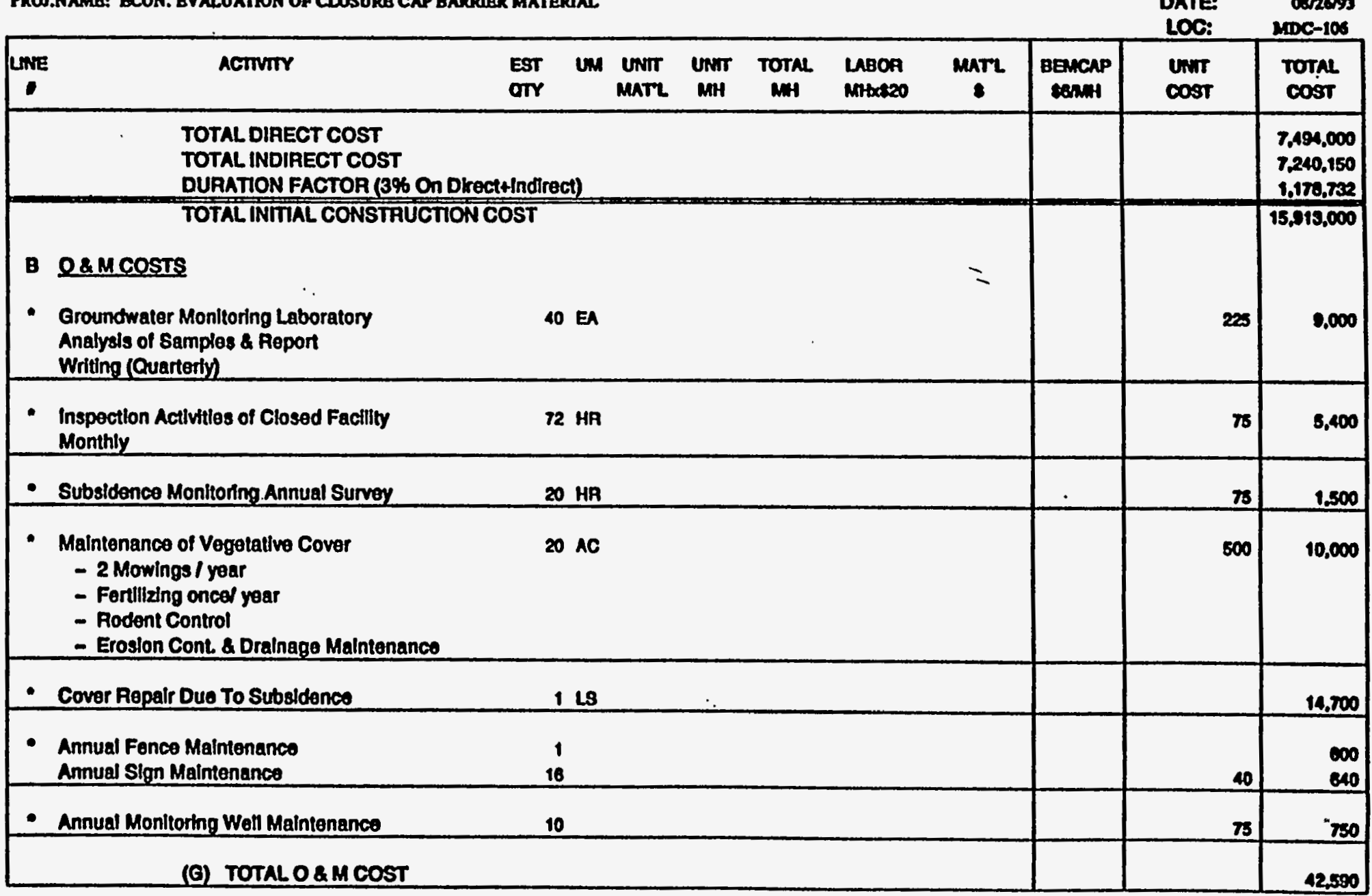

H-9. Cost Estimate for a Cover System Utilizing Soil Cement and 30-mil FML (Cont'd) 
D B TAIL BSTIMATE

\begin{tabular}{|c|c|}
\hline$C$ & 8. ME \\
\hline R: & \\
\hline $\begin{array}{l}\text { PHON } \\
\text { DAT } \\
\text { LOC }\end{array}$ & \\
\hline
\end{tabular}

Loof: 2-03

ACTTVITY CODE: ESE2OOA12

PROJ.NAME: ECON. BVALUATTON OP CLOSURE CAP BARRTER MATERIAS.

LOC:

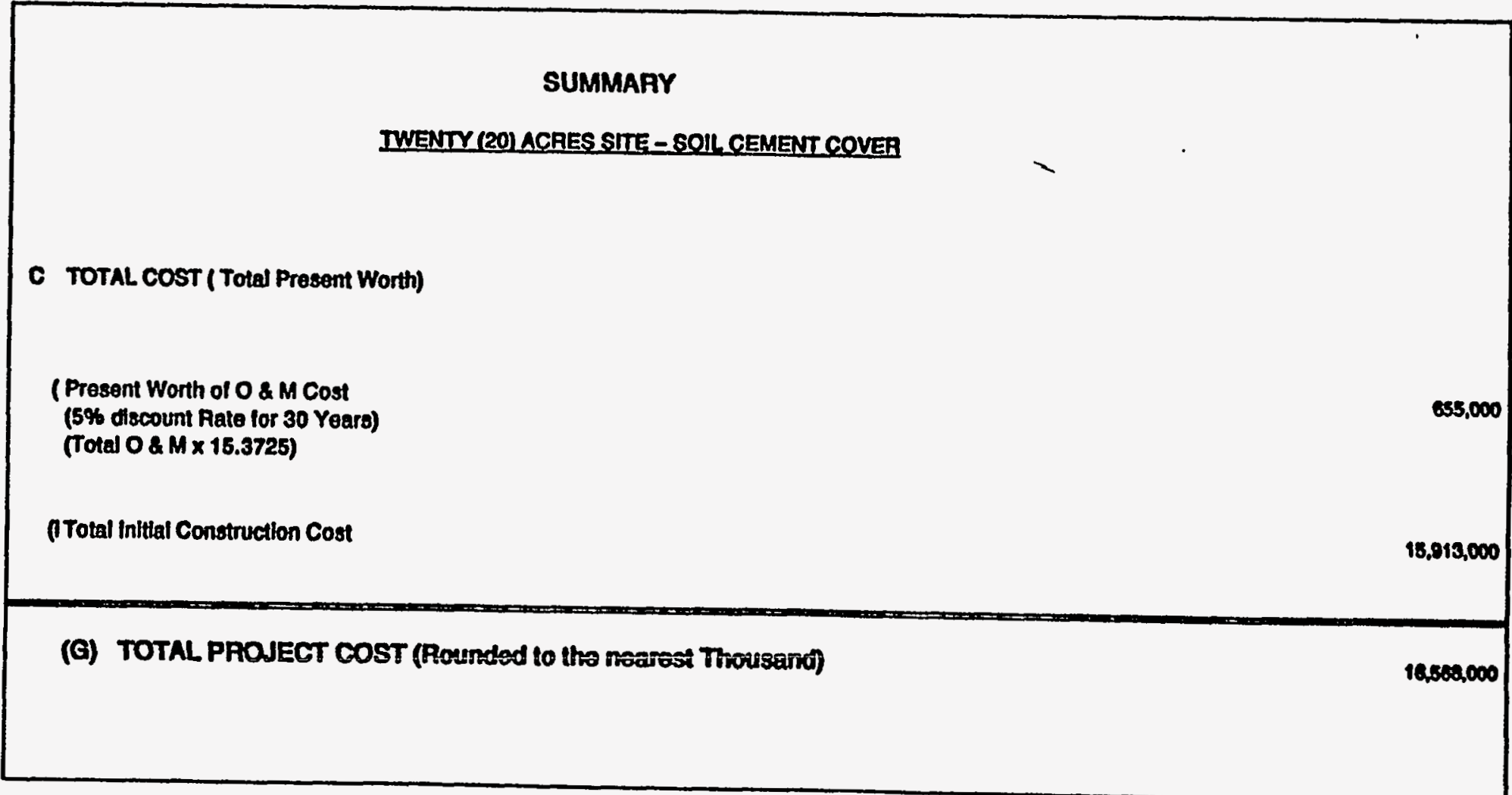

H-9. Cost Estimate for a Cover System Utilizing Soil Cement and 30-mil FML (Cont'd) 


\section{DETAIL ESTIMATE}

Lod: $7-03$

ACTIVTYY CODB: BSE2SON12

FROJ.NAME: ECON. EVALUATTON OF CLOSURB CAP BARRRER MATERSAL

i'

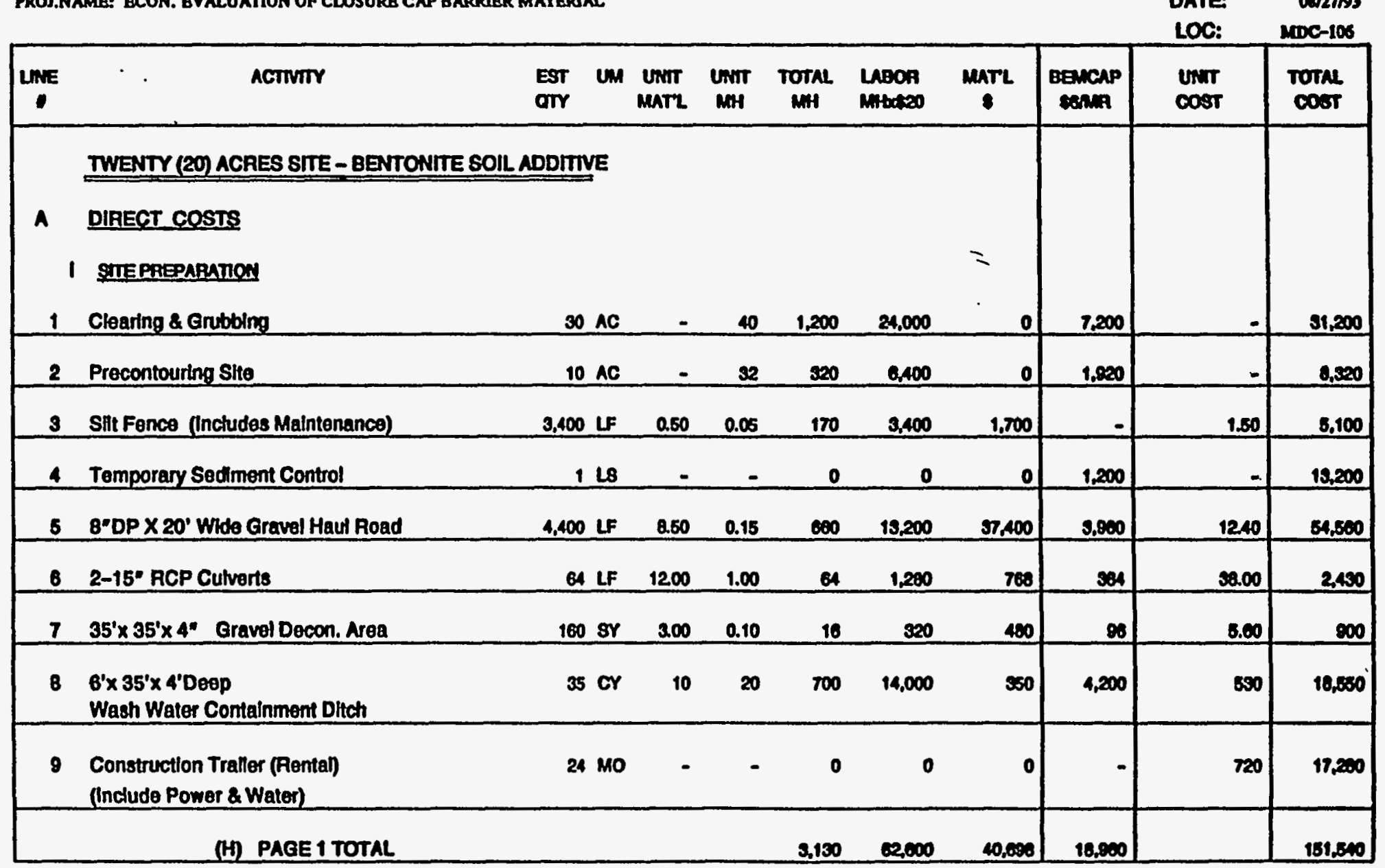

H-10. Cost Estimate for a Cover System Utilizing Bentonite Soil Additive and a 30-mil FML 
DETAIL ESTIMATE

L001: 7-03

ACTIVTYY CODB: ESE26OA12

PROJ.NAME: BCON. EYALUATION OF CLOSURB CAP BARRRRR MATERIAL

\begin{tabular}{|c|c|c|c|c|c|c|c|c|c|c|c|}
\hline UME & ACTVITY & $\begin{array}{l}\text { Est } \\
\text { aTr }\end{array}$ & & $\begin{array}{l}\text { MNTr } \\
\text { MATL }\end{array}$ & $\bigcup_{M H 1}^{\min }$ & $\underset{\text { MOT }}{\text { Totre }}$ & $\begin{array}{l}\text { LABOA } \\
\text { inteaszo }\end{array}$ & $\operatorname{marL}_{8}$ & $\begin{array}{l}\text { Bencap } \\
\operatorname{senNR}\end{array}$ & $\begin{array}{l}\operatorname{untr}_{\cos } \\
\cos t\end{array}$ & $\begin{array}{l}\text { TotAl } \\
\text { cosit }\end{array}$ \\
\hline 10 & $\begin{array}{l}\text { COVER CONSTRUCTION } \\
\text { Waste Stablization (Crushod Lmestone) }\end{array}$ & 27,915 & & 78 & 0.1 & 2.792 & 55,630 & $2,093,625$ & 16,749 & 7.00 & $2,160,200$ \\
\hline 11 & $\begin{array}{l}\text { Place and Compact Foundation Soll } \\
\text { In 8. Lins to } 95 \% \text { std. Proctor }\end{array}$ & 206,760 & or & - & 0.125 & 25,845 & 516,0000 & $=$ & 185,070 & 2.30 & 071,970 \\
\hline 12 & 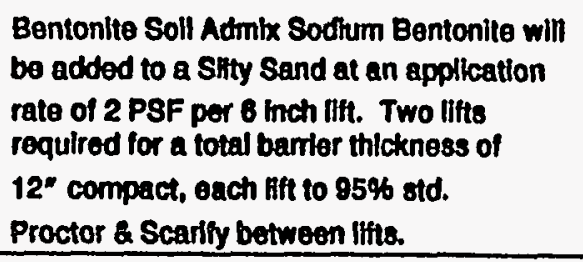 & $\begin{array}{r}880 \\
\text { Sodium B } \\
\text { s1,907 } \\
\text { 811ty Sand }\end{array}$ & $\operatorname{TN}_{\text {centonk }}$ & 146.50 & 0.155 & 4,946 & 80,012 & 470,005 & 29.674 & 19.00 & 0007.100 \\
\hline 13 & $\begin{array}{l}\text { Placement of } 30 \text { Mh Flextble } \\
\text { Membrane Liner }\end{array}$ & 975,000 & SF & 0.14 & 0.0068 & 6,390 & 128,750 & 130,600 & - & 0.50 & 200,250 \\
\hline 14 & $\begin{array}{l}\text { Placement of 1'-0 Coarse Sand } \\
\text { Drainage Layer }\end{array}$ & 33,395 & or & 10.50 & 0.025 & 635 & 18,690 & 350,648 & 5,009 & 11.10 & 972,350 \\
\hline 15 & $\begin{array}{l}\text { Placement of Geotextile (TYPAP) Fitter on } \\
1^{\prime}-0^{\prime \prime} \text { Coarse Sand Dralnage Layer }\end{array}$ & 107,000 & SY & 0.13 & 0.005 & 535 & 10,700 & 13,910 & - & 0.20 & 24,610 \\
\hline 16 & Placement of $1^{\prime}-6^{\prime \prime}$ Common FIII & 49,247 & or & 3.75 & 0.045 & 2,216 & 4,322 & 184,678 & 15,297 & 4.00 & 212,300 \\
\hline 17 & Placement of $\theta^{\circ}$ Topsoll & 16,511 & cr & 0.50 & 0.045 & 743 & 14,000 & 156,065 & 4,458 & 10.70 & 170,170 \\
\hline & (H) PAGE 2 TOTAL & & & & & 43,181 & 800.600 & $3,202,200$ & 200,502 & & 4,000000 \\
\hline
\end{tabular}

H-10. Cost Estimate for a Cover System Utilizing Bentonite Soil Additive and a 30-mil FML (Cont'd) 
Economic Evaluation of Closure Cap Barrier Materials Study(U)

Volume II Appendix $H$ September 1993

L001: 7-03

ACTIVITY CODE: BSE260012

PROJ.NAME: ECON. BVALUATTON OF CLOSURB CAP BARRIER MATERUAL

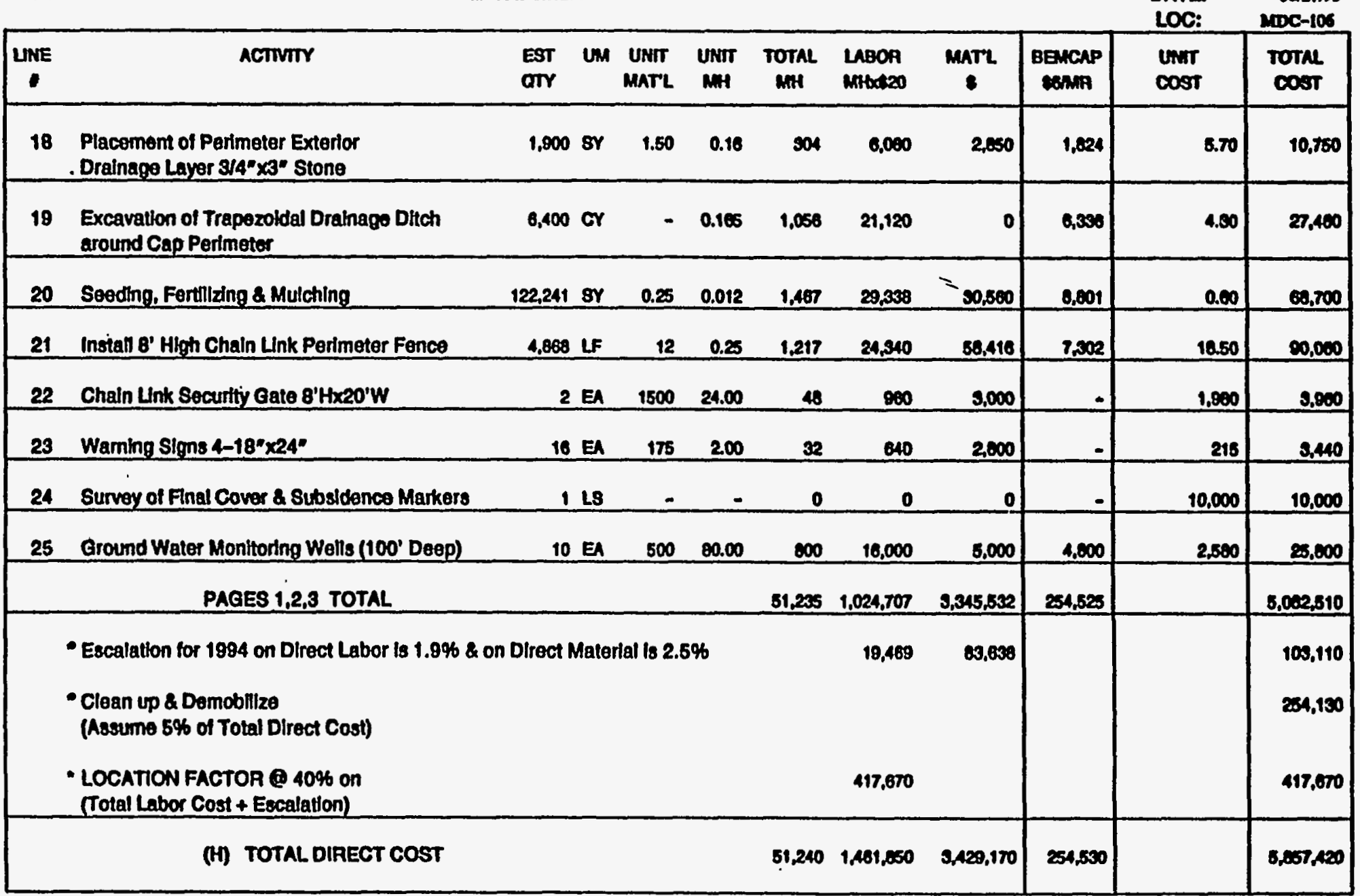

H-10. Cost Estimate for a Cover System Utilizing Bentonite Soil Additive and a 30-mil FML (Cont'd) 
L00 I: 7-03

ACTTVITY CODE: ESE260412

PROJ.NAME: ECON. EVALUATTON OF CLOSURB CAP BARRIER MATERIAL.

DETAIL ESTIMATE

CHK'D BY: 8. MEAD

STIMATOA: nomx

PHONE: 7-5266

DATE: OAMAS

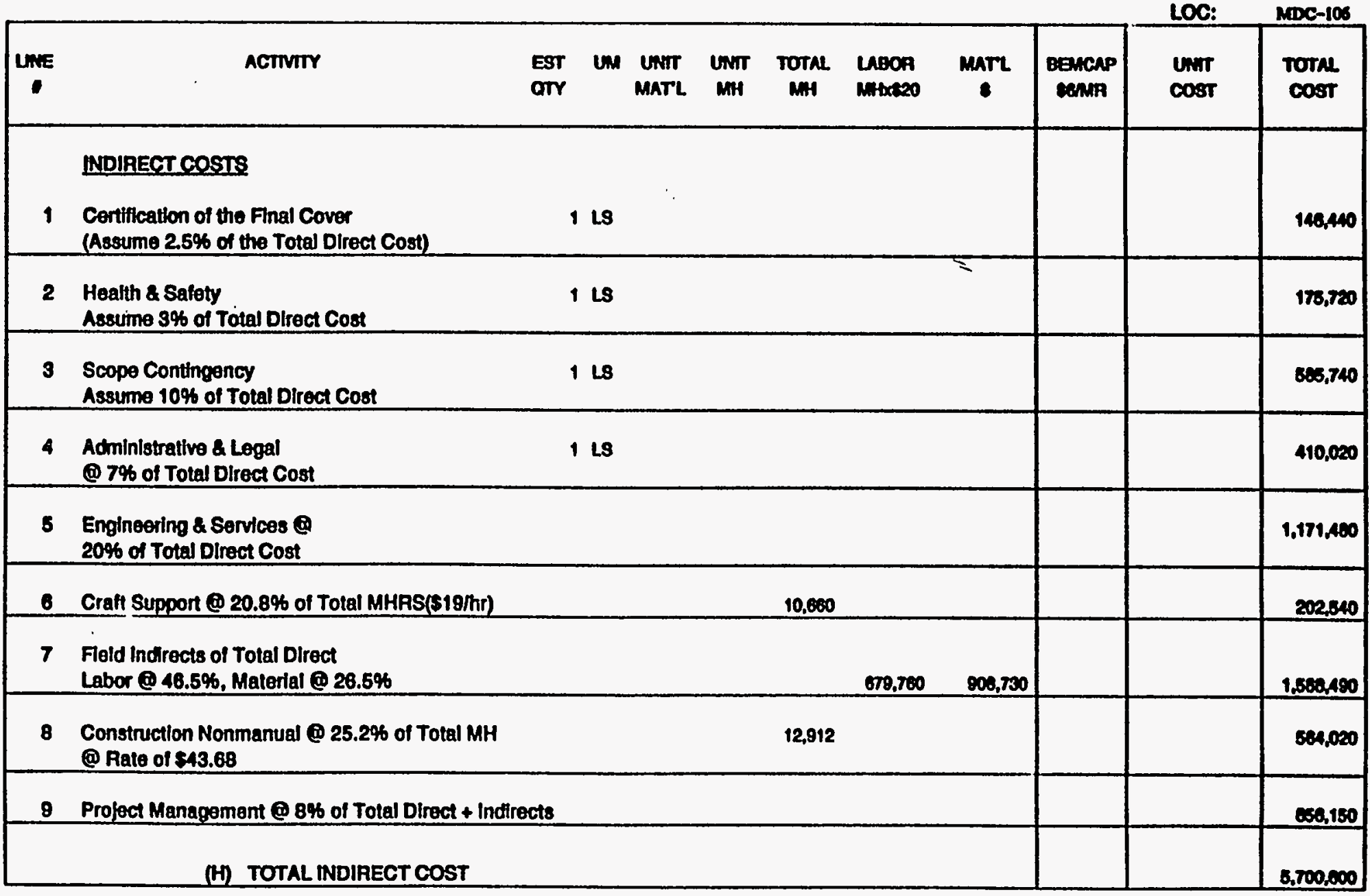

H-10. Cost Estimate for a Cover System Utilizing Bentonite Soil Additive and a 30-mil FML (Cont'd) 
DETAIL ESTIMATE

8. MEAD

Loof: $7-03$

ACTIVTTY CODE: ESE2SOS12

MROJ.NAMB: ECON. EVALUATION OF CLOSURB CAP BARRIER MATERINL

\begin{tabular}{|c|c|c|c|c|c|c|}
\hline & & & & & $\begin{array}{c}\text { ESTIMATOR: } \\
\text { PHONE: } \\
\text { DATE: } \\
\text { LOC: } \\
\end{array}$ & 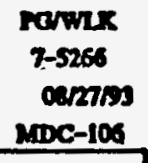 \\
\hline \multirow[t]{9}{*}{$\begin{array}{l}\text { UniT } \\
\text { MH }\end{array}$} & $\begin{array}{c}\text { TOTAL. } \\
\text { HHA }\end{array}$ & $\begin{array}{l}\text { LABOR } \\
\text { MHSACOEO }\end{array}$ & $\stackrel{m a r t}{*}$ & Bencap & $\begin{array}{l}\text { Unist } \\
\text { cost }\end{array}$ & $\begin{array}{l}\text { TOTAL } \\
\text { COST }\end{array}$ \\
\hline & & & & & & $\begin{array}{r}5,057,480 \\
5,700,000 \\
446,741\end{array}$ \\
\hline & & & $=$ & & 225 & $\begin{array}{c}11,005,000 \\
\ldots \\
2,000\end{array}$ \\
\hline & & & & & 78 & 8,400 \\
\hline & & & & & $\pi$ & 1,600 \\
\hline & & & & & 800 & 10,000 \\
\hline & & & & & & 0.000 \\
\hline & & & & & 40 & $\begin{array}{l}000 \\
400 \\
\end{array}$ \\
\hline & & & & & $\pi$ & $\pi 00$ \\
\hline & & & & & & 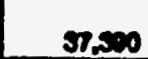 \\
\hline
\end{tabular}

H-10. Cost Estimate for a Cover System Utilizing Bentonite Soil Additive and a 30-mil FML (Cont'd) 
DETAIL ESTIMATE

CHK'D BY: 8. MEAD

Lo01: 7-03

ACTIVTT CODB: BSE2SOA12

PROJ.NAME: BCON. EVALUATION OP CLOSURB CAP BARRIER MATERAAL.

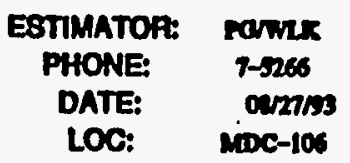

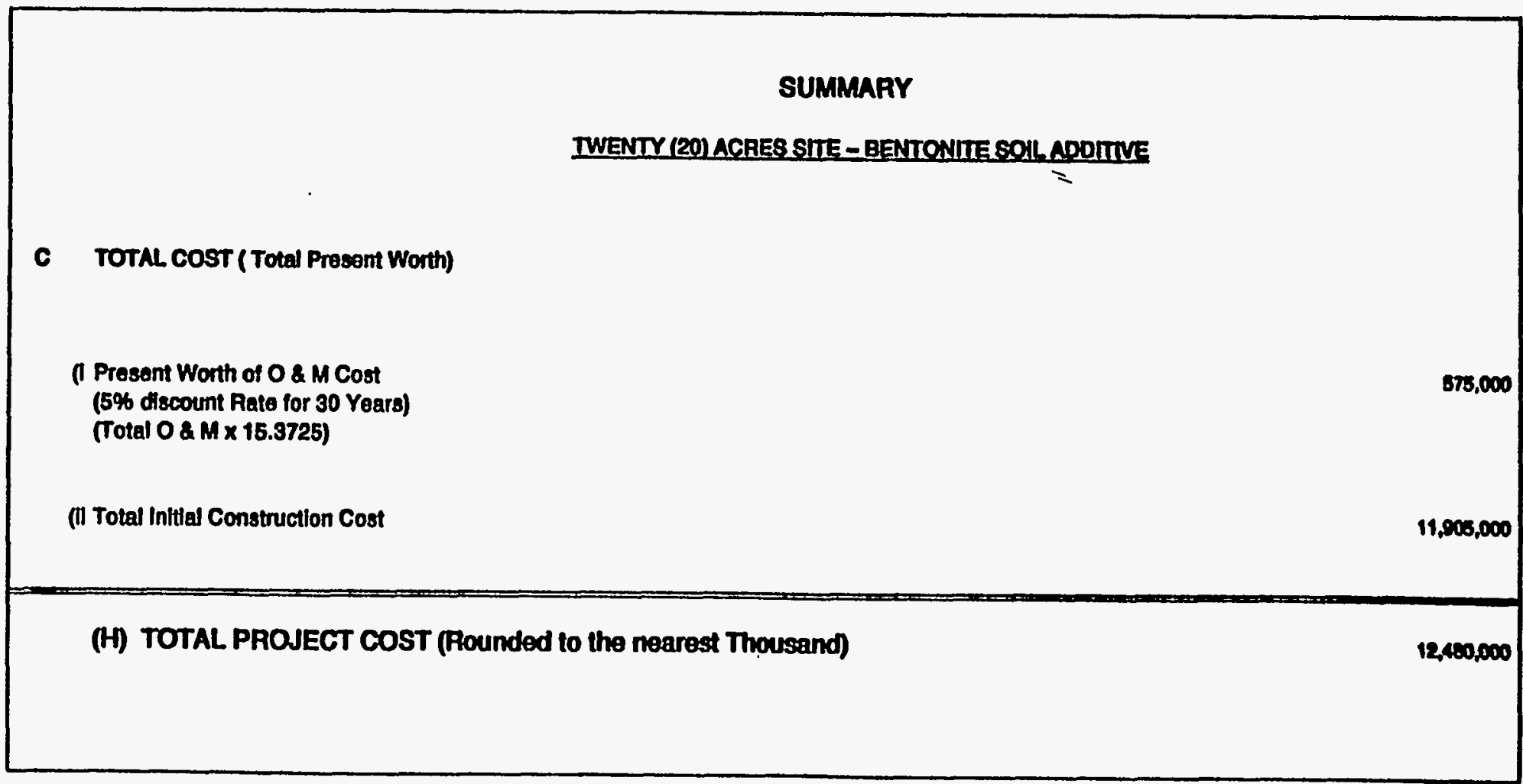

H-10. Cost Estimate for a Cover System Utilizing Bentonite Soil Additive and a 30-mil FML (Cont'd) 
DETAILESTIMATE

LO0 I: 7-03

ACTIVITY CODE: ESE260112

PROJ.NAMB: BCON. BVALUATTON OF CLOSURB CAP BARRUBR MATERIAL

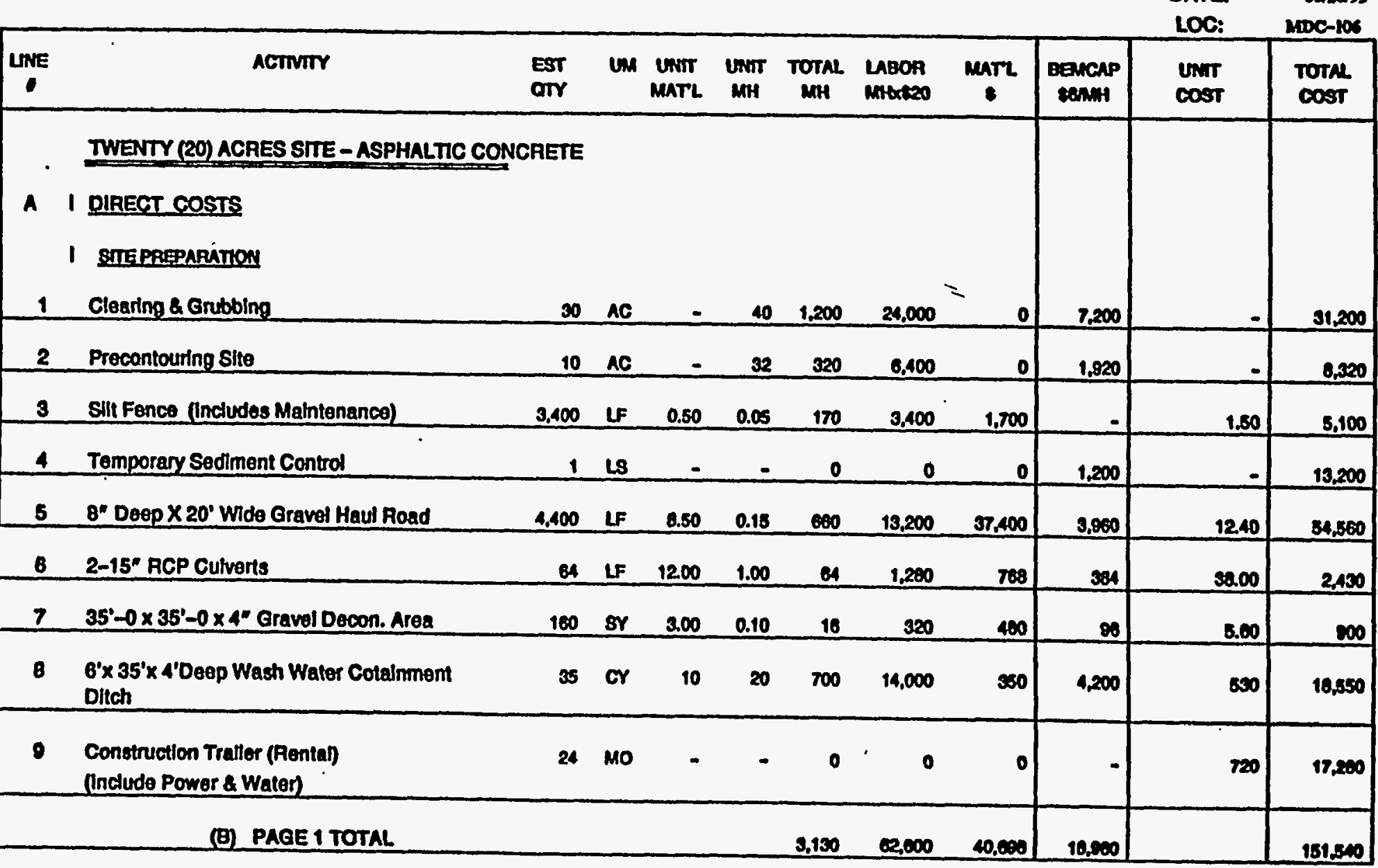

H-11. Cost Estimate for a Cover System Asphalt Concrete Hydraulic Barrier and a 30-mil FML 
DETAIL ESTIMATE

L001: 7-03

ACTIVITY CODE: ESE200012

PROJ.NAME: BCON. EVALUATHON OF CLOSURB CAP DARRIER MATERUAL

\begin{tabular}{|c|c|c|c|c|c|c|c|c|c|c|c|}
\hline & & & & & & & & & & LOC: & $200-106$ \\
\hline LWE & ACTIVTY & $\begin{array}{l}\text { EST } \\
\text { QTY }\end{array}$ & un & $\begin{array}{l}\text { UNTR } \\
\text { MATL }\end{array}$ & $\underset{M H H}{\text { UNT }}$ & TOTAL $_{\text {MH }}$ & $\begin{array}{l}\text { LABOA } \\
\text { MHtext20 }\end{array}$ & $\operatorname{maTL}$ & $\begin{array}{l}\text { Bencap } \\
\text { SeAMH }\end{array}$ & $\begin{array}{l}\ln (\pi) \\
\operatorname{cost}\end{array}$ & $\begin{array}{l}\text { Torm } \\
\text { cost }\end{array}$ \\
\hline & LCOVER CONSTRUCTION & & & & & & & & & & \\
\hline 10 & Waste Stabilization (Crushod Umestono) & 27,915 & $\mathbf{T N}$ & 75 & 0.1 & 2,792 & 55,030 & $2,093,625$ & 10,749 & $\mathbf{n . 0 0}$ & $2,100,200$ \\
\hline 11 & $\begin{array}{l}\text { Place and Compact Foundation Soll } \\
\text { in } 8^{\circ} \text { Uits to } 85 \% \text { Std. Proctor }\end{array}$ & 168,245 & or & - & 0.125 & 21,031 & 480,610 & 0 & 128,184 & s.so & 346,000 \\
\hline 12 & $\begin{array}{l}\text { Placement of } 30 \text { Mil Fexible } \\
\text { Membrane Uner }\end{array}$ & 900,562 & SF & 0.14 & 0.0065 & 5,854 & $\$ 17,076$ & 128,081 & - & 0.30 & 243,100 \\
\hline 13 & $\begin{array}{l}\text { Placement of } 1 \text { '-0 Coarse Sand } \\
\text { Drainage Layer }\end{array}$ & 33,076 & or & 10.50 & 0.025 & 827 & 16,530 & $\mathbf{s 4 7 , 2 9 0}$ & 4,801 & 11.20 & 308,000 \\
\hline 14 & $\begin{array}{l}\text { 2" PVC Perforated Drainage Pipe placed } \\
\text { in Drainage layer }\end{array}$ & 7.916 & LF & 1.00 & 0.16 & 1,267 & 25,331 & 7,018 & - & 4.20 & 33,250 \\
\hline 15 & $21 / 2^{\prime}$ Binder Course & 98,441 & sr & 4.15 & 0.055 & 5,414 & 100,205 & 406,530 & 32,408 & 5.60 & 810.300 \\
\hline 16 & 4" Hydraulie Asphalt Cone. & 90,441 & sr & $\mathbf{6 0 0}$ & 0.03 & 2,053 & 59,065 & 649.711 & 17.719 & 7.40 & 7280,480 \\
\hline 17 & Asphait Mastlc Soal & 90,449 & 8Y & 0.50 & 0.015 & $1,4 n$ & 29,532 & 49.221 & - & 0.80 & 70,750 \\
\hline 18 & Sprayed Asphatite Concreto Sealam & 80,441 & 8r & 0.73 & 0.015 & $1, A \pi$ & 20,532 & 70.231 & 0,000 & 1.10 & 112,200 \\
\hline 19 & Excavation of Trainguiar Drainage Ditch & 3,070 & or & - & 0.105 & 607 & 12.137 & 0 & 3,641 & 4.30 & 18,700 \\
\hline 20 & 4" Thlek Asphialt Pertmeter Drainage Ditch & 8,268 & or & 7.00 & 0.144 & 1,191 & 23,012 & 02,637 & 7.144 & 11.50 & $\omega 0, \pi 00$ \\
\hline & (B) PAGE 2 TOTAL & & & & & 40808 & 097,751 & $3.018,049$ & 217.74 & & 4,534,540 \\
\hline
\end{tabular}

H-11. Cost Estimate for a Cover System Asphalt Concrete Hydraulic Barrier and a 30-mil FML (Cont'd) 


$$
\text { L001: } 7-03
$$

ACTIVITY CODB: BSE200412

MOS.NAME: ECON. BVALUATON OP CLOSURB CAP BARRRER MATERUAL

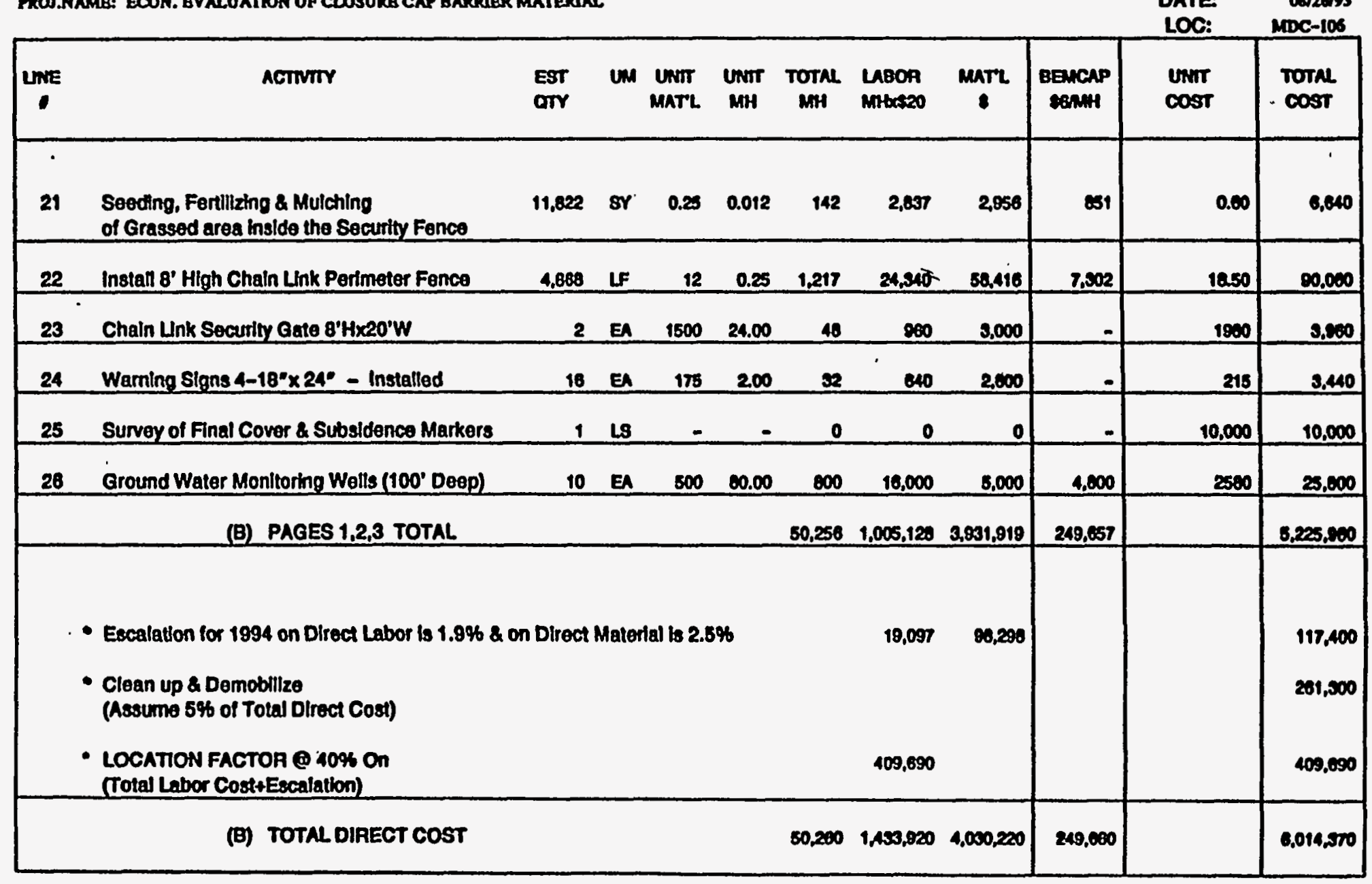

H-11. Cost Estimate for a Cover System Asphalt Concrete Hydraulic Barrier and a 30-mil FML (Cont'd) 
D B T A I L B S T IMAT B

CHK'D BY: 8. MEAD

LOa I: 7-03

ACTIVITY CODB: ESE26012

PROJ.NAME: BCON. BVALUATION OP CLOSURB CAP BARRIER MATERINL

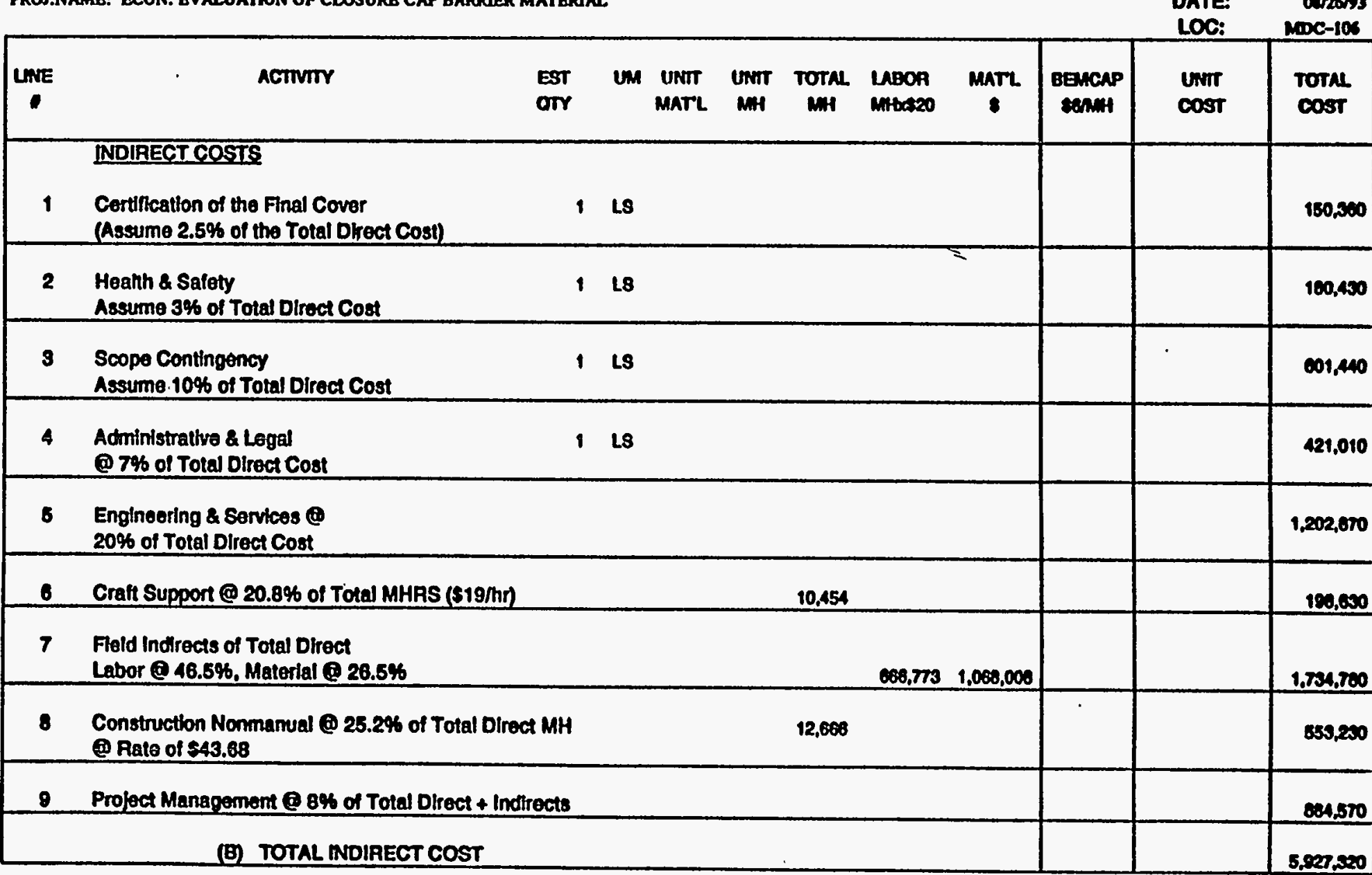

H-11. Cost Estimate for a Cover System Asphalt Concrete Hydraulic Barrier and a 30-mil FML (Cont'd) 


\section{DETAIL ESTIMATE}

CACD BY: 2. MEND

Lo01: $7-03$

ACTVITY CODE: ESB2SOH12

PROS.NAME: ECON. EVALUATION OF CLOSURB CAP BARRIER MATERINL.

ESTMATOR: PaAnK

PAONE 7-5266

DATE Dararso

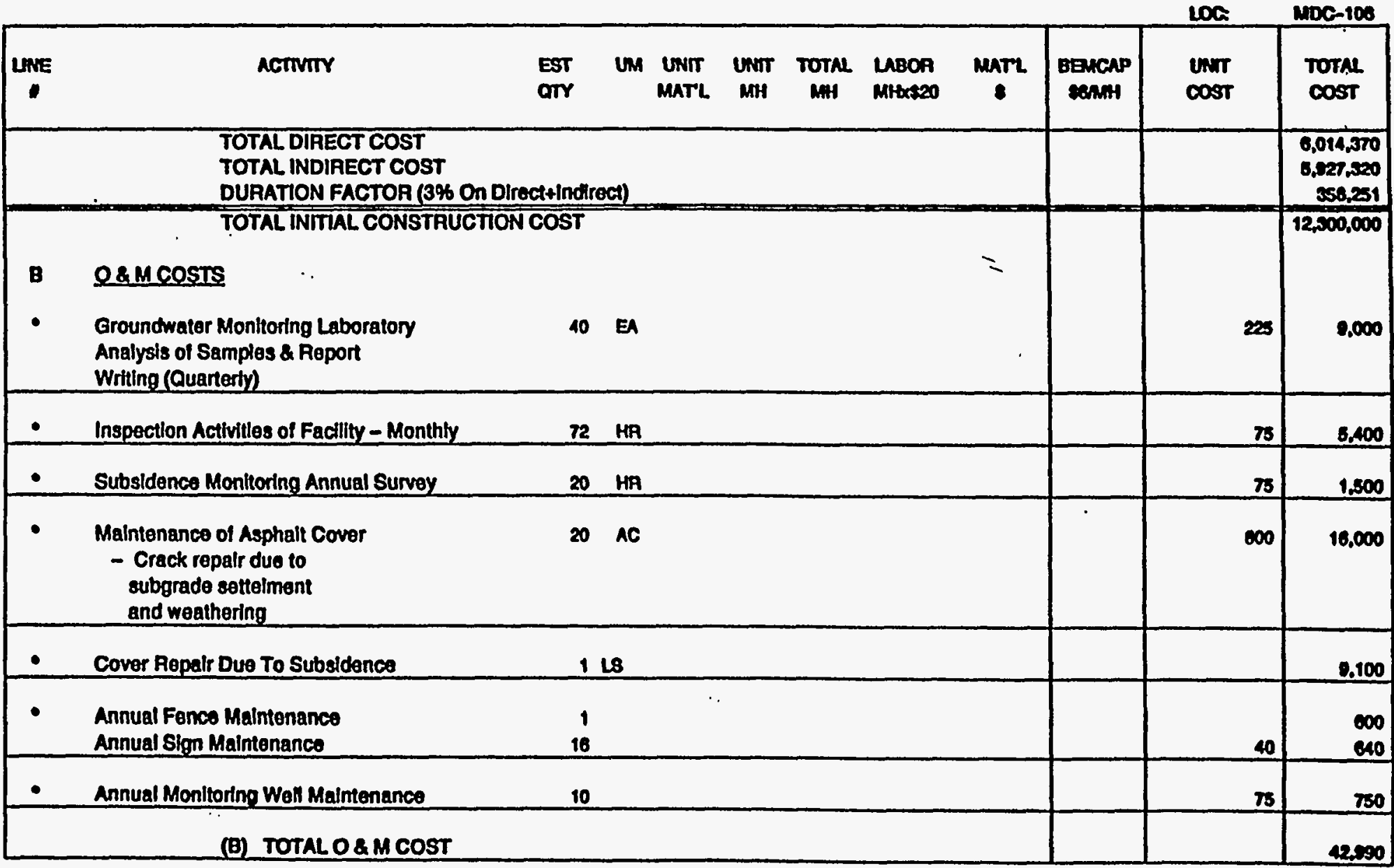

H-11. Cost Estimate for a Cover System Asphalt Concrete Hydraulic Barriet and a 30-mil FML (Cont'd) 
D B TAIL BSTIMATB

1001: $7-03$

ACTIVITY CODB: BSE2CON12

MROJ.NAME: ECON. BVALUATTON OP CLOSURB CAP BARRTER MATERSAL
CHKD EY: 8. MEAD

ESTIMATOR: TOMIX

PHONE: 7-5206

DATE: OARSSBS

LOC: $\quad$ MDC-105

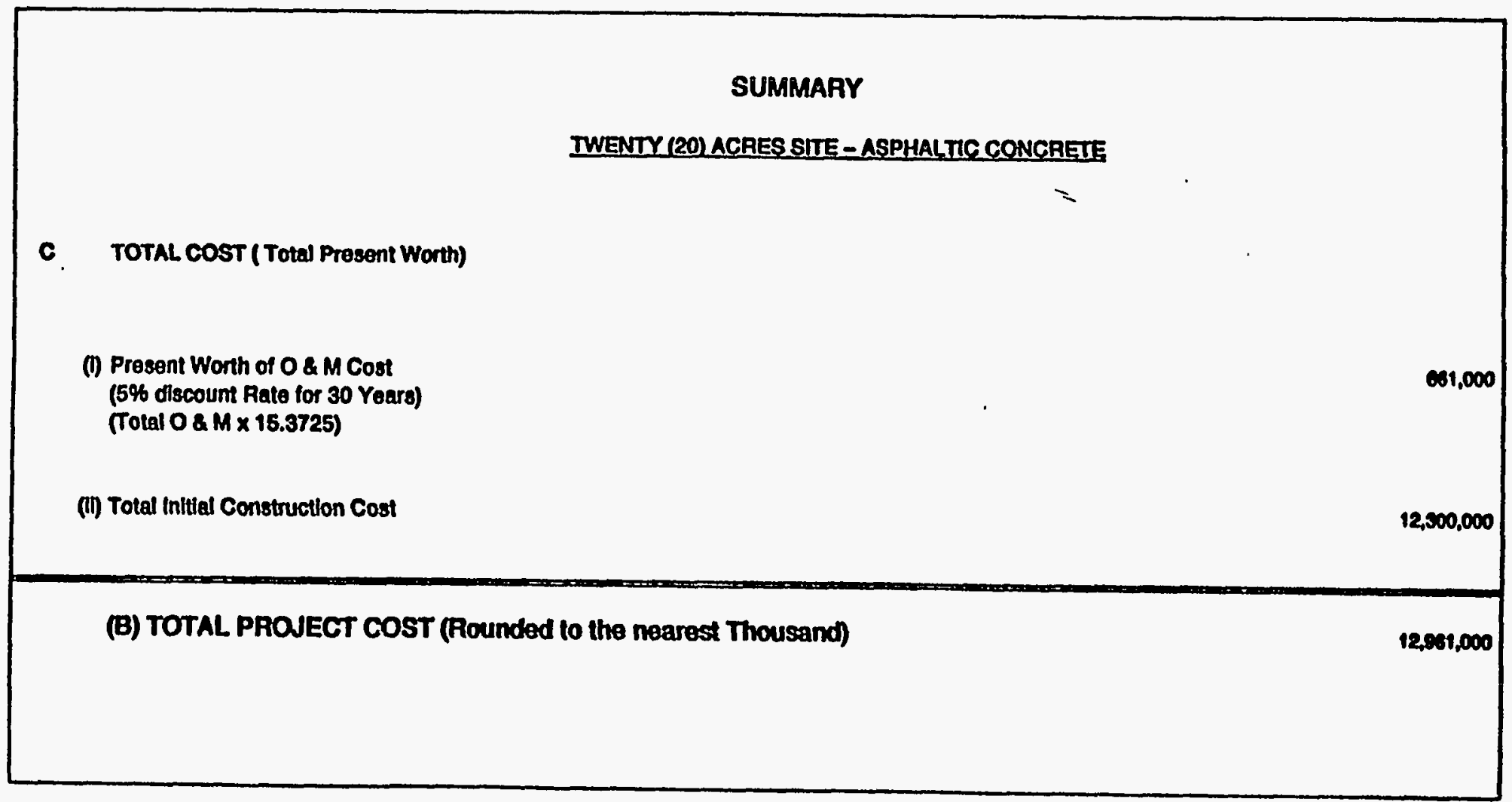

H-11. Cost Estimate for a Cover System Asphalt Concrete Hydraulic Barrier and a 30-mil FML (Cont'd) 
DETAIL ESTIMATE

LOQ: $7-03$

ACTIVTY CODE ESE200412

PFOU.NAME ECON. EVALUATION OF CLOSUHE CAP BAPRAER MATERAAL

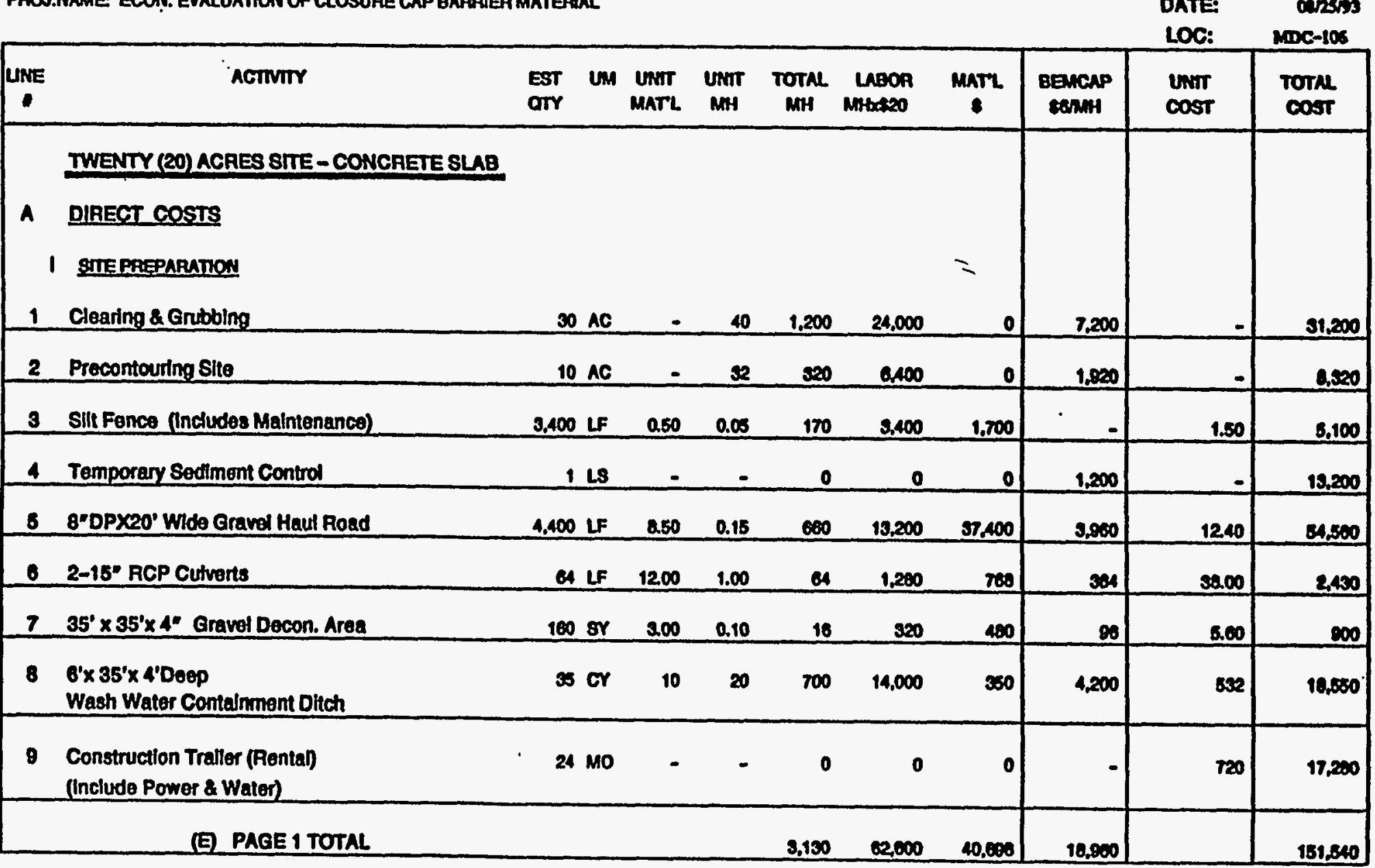

H-12. Cost Estimate for a Cover System Utilizing Poured-in Place Concrete Slab and a 30-mil FML 
Loo I: $7-03$

ACTIVITY CODE: ESE260412

PROS.NAME: ECON. BVALUATTON OF CLOSURB CAP BARRIER MATERUNL

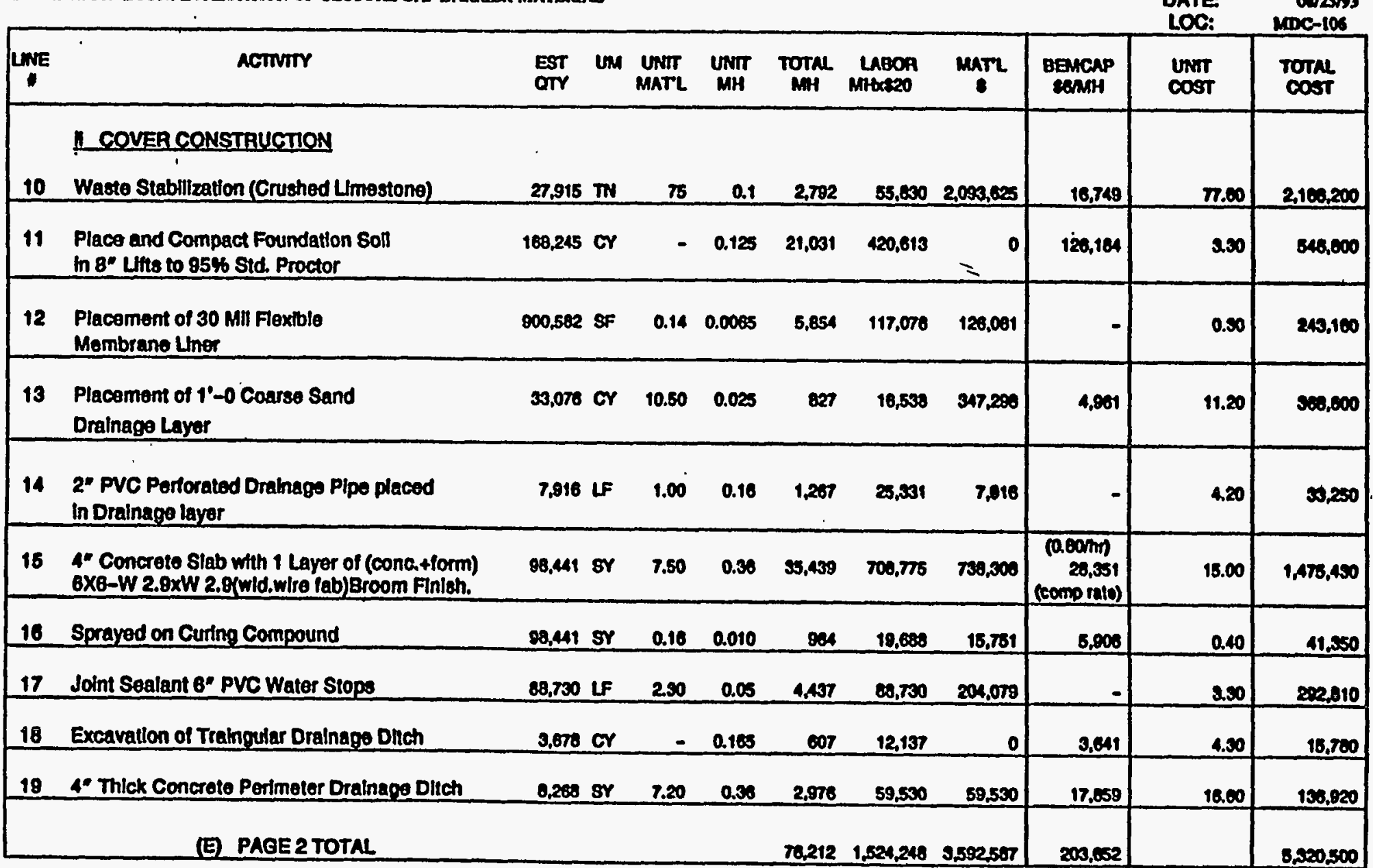

H-12. Cost Estimate for a Cover System Utilizing Poured-in Place Concrete Slab and a 30-mil FML (Cont'd) 


\section{B T A I L B S TIMAT B}

Lod: 7-03

NCTIVITY CODB: BSE200112

FROJ.KANE: ECON. BVALUATION OF CLOSURB CAP BARRIER MATRRIAL.

$\because i$

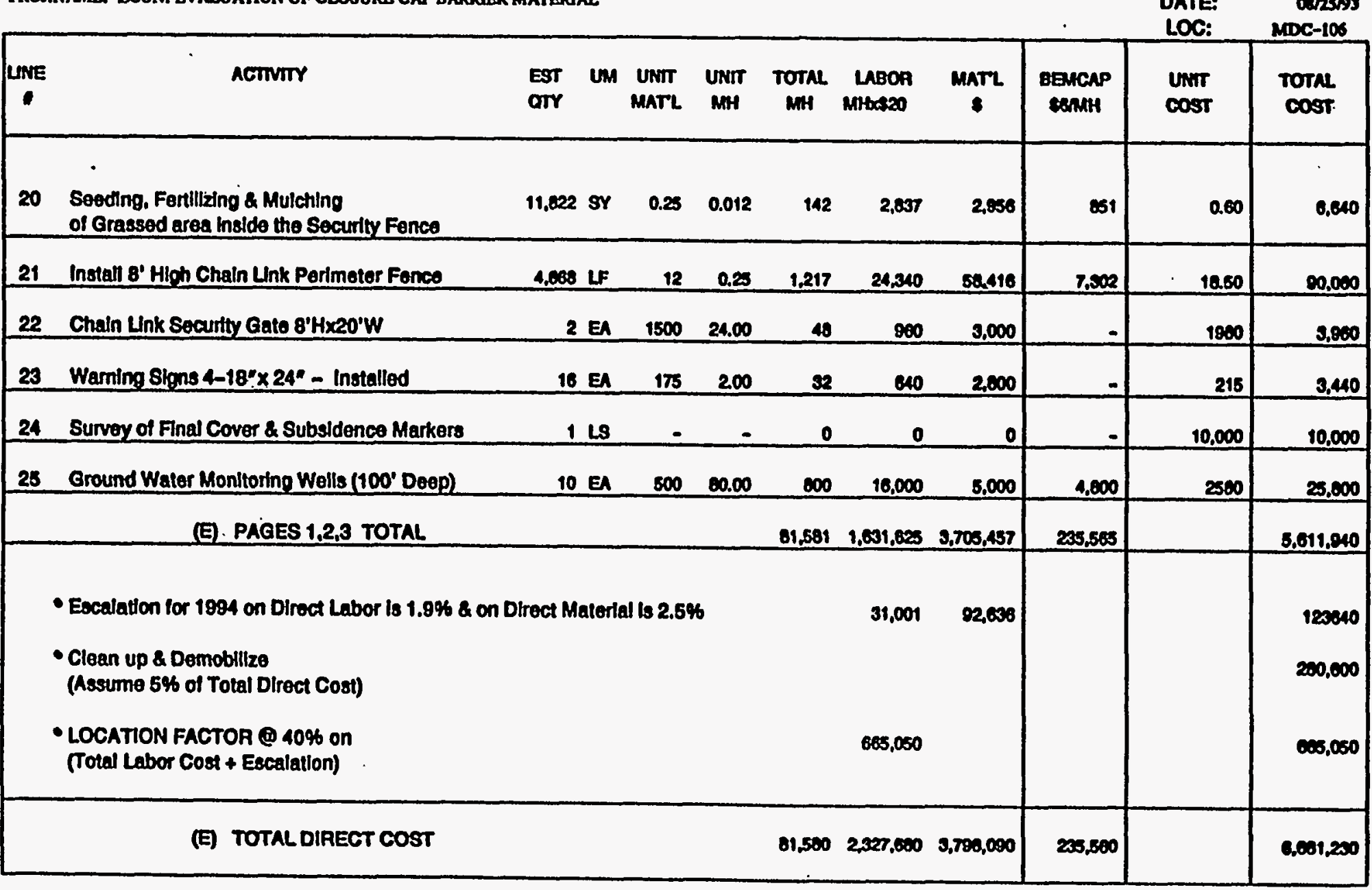

H-12. Cost Estimate for a Cover System Utilizing Poured-in Place Concrete Slab and a 30-mil FML (Cont'd) 


\section{B T A L B S T I M T B}

CHK'D BY: S. MEAD

Lo01: $7-03$

ACTTVTY CODE: BSE260112

PAOJ.NAMG: BCON. BVALUATION OP CLOSURB CAP BARRRER MATERIAL.

ESTIMATOR: Parrex

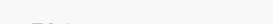

PHONE: T-5266

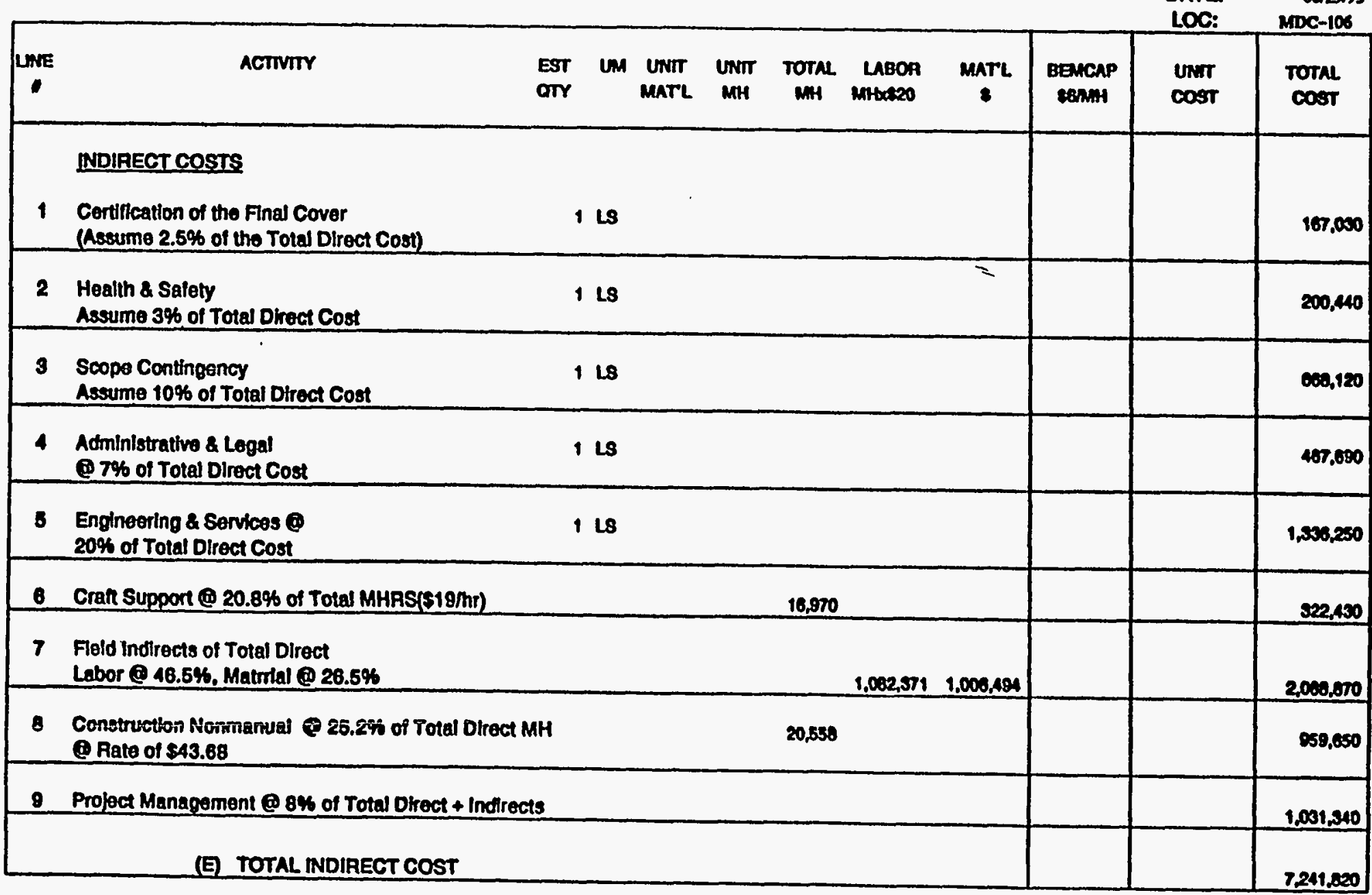

H-12. Cost Estimate for a Cover System Utilizing Poured-in Place Concrete Slab and a 30-mil FML (Cont'd) 
D B TAIL B S T I MATB

CHK'D BY: 8. MEAD

100: : 7-03

ACTIVITY CODR: BSEZOOA12

FROJ.NAMB: ECON. EVALUATION OF CLOSURB CAP BARRIRR MATERIAL

ESTIMATOR: ramx

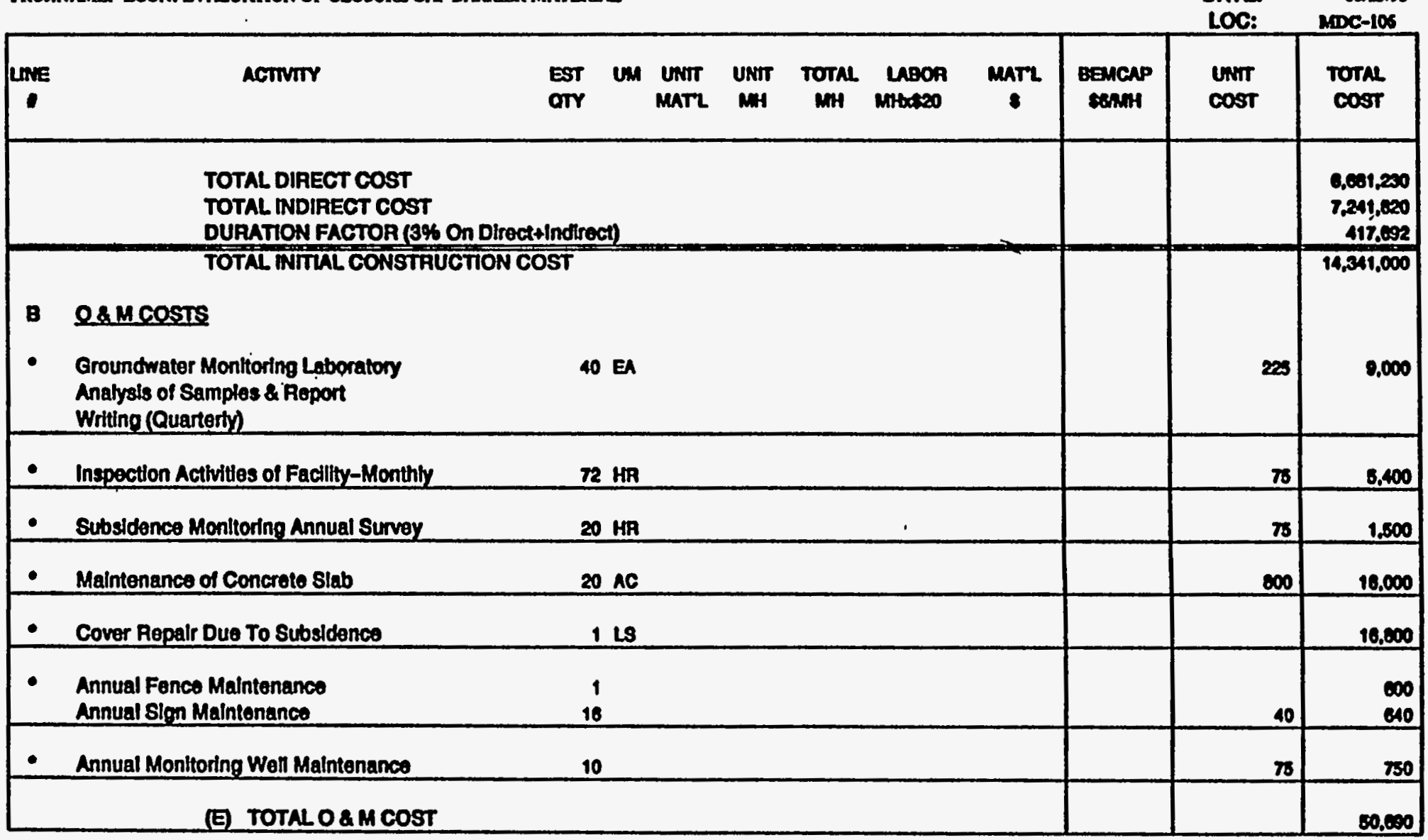

H-12. Cost Estimate for a Cover System Utilizing Poured-in Place Concrete Slab and a 30-mil FML (Cont'd) 
Lo01: $7-00$

ACTIVITY CODB: ESE260412

MOJ.NAMR: BCON. BVALUATION OF CLOSURB CAP BARRIRR MATERUAL

LOC:

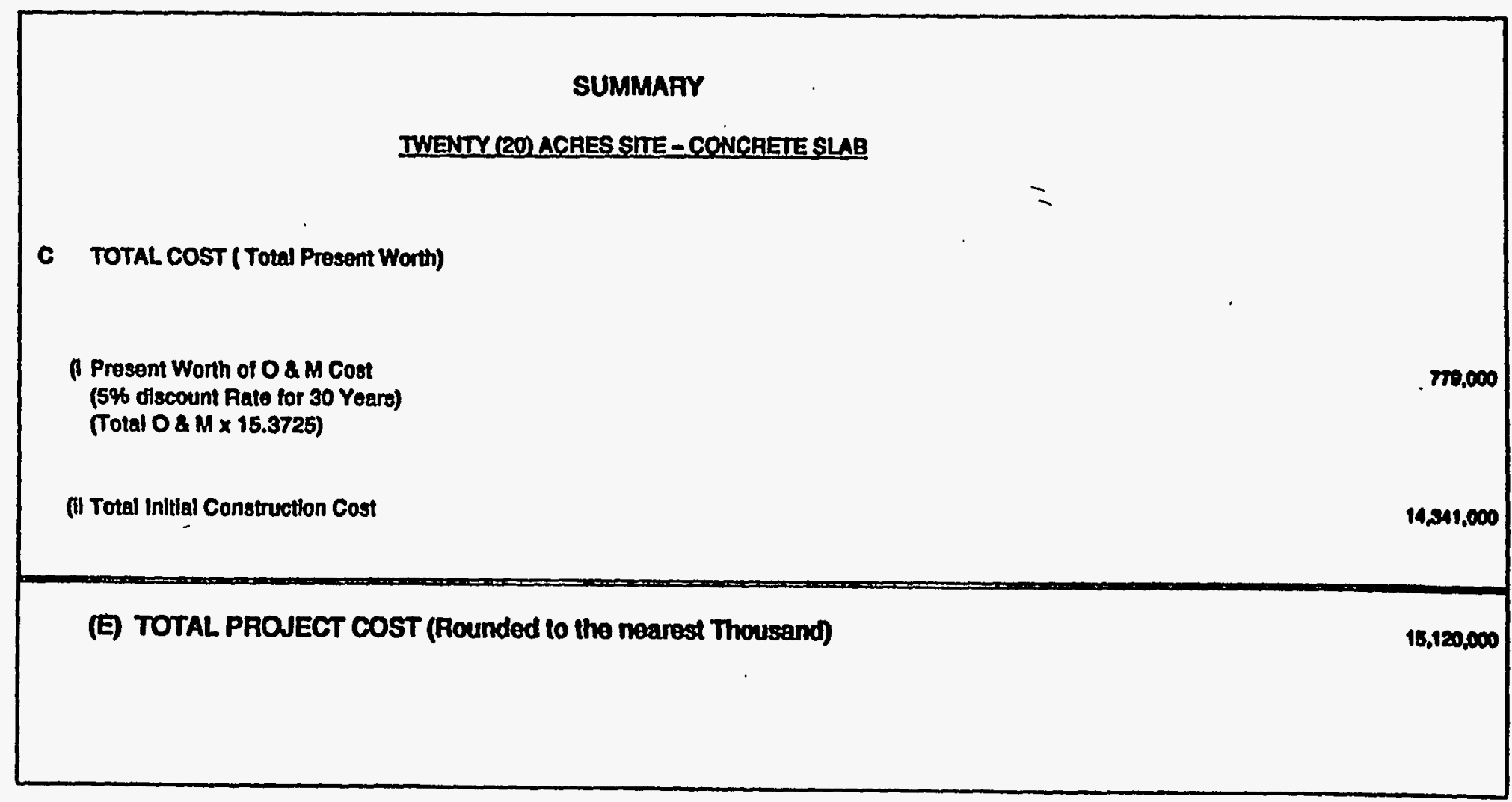

H-12. Cost Estimate for a Cover System Utilizing Poured-in Place Concrete Slab and a 30-mil FML (Cont'd) 


\section{Appendix I}

\section{Cost Estimates for 40-Acre Site}


L00 1: 7-03

ACTIVITY CODE: BSE2SOM12

PROJ.NAME: ECON. BVALUATION OP CLOSURE CAP BARRIER MATERIAL

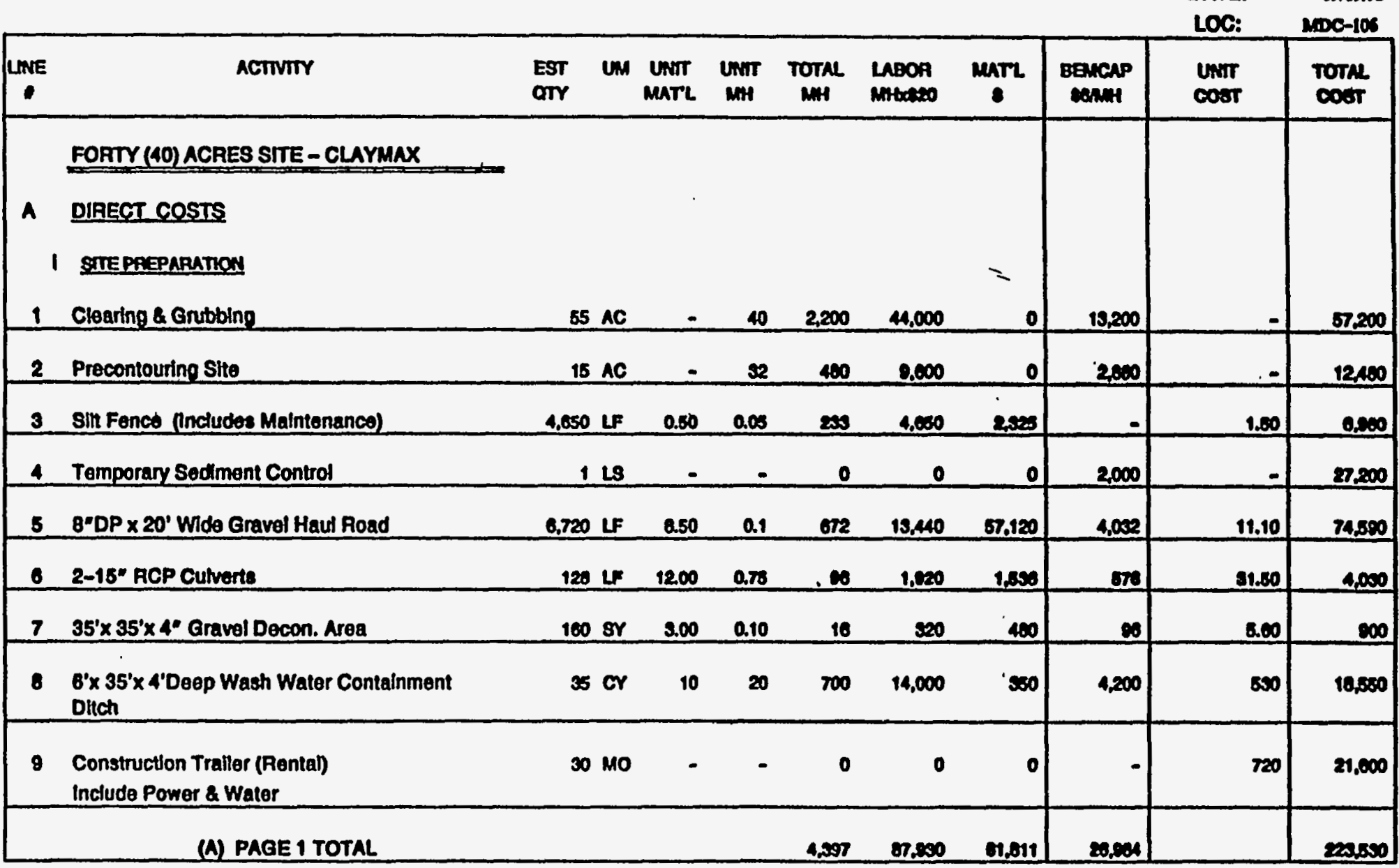

I-1. Cost Estimate for a Cover System Utilizing Claymax and a 30-mil FML 
DETAIL ESTIMATE

CHK'D QY: Q. MEAD

Lo0 1: 7-03

ACTIVTY CODE: BSERCOA12

PROJ.NAME: ECON. BVALUATION OF CLOSURB CAP BARRIER MATRRINL.

ESTIMATOR: ramx

\begin{tabular}{|c|c|c|c|c|c|c|c|c|c|c|c|}
\hline IME & ACTIVTY & $\begin{array}{l}\text { EST } \\
\text { OTY }\end{array}$ & un & $\begin{array}{l}\text { UNTT } \\
\text { MATL }\end{array}$ & $\begin{array}{l}\text { UNIT } \\
\mathbf{M H}\end{array}$ & TOTNL & $\begin{array}{l}\text { LABOR } \\
\text { MHCat20 }\end{array}$ & $\operatorname{marL}$ & $\begin{array}{l}\text { gevenp } \\
\text { senny }\end{array}$ & $\begin{array}{l}\text { Unirt } \\
\text { cost }\end{array}$ & $\begin{array}{l}\text { Total } \\
\cos \end{array}$ \\
\hline 10 & $\begin{array}{l}\text { D_ CQVER CONSTRUCTION } \\
\text { Wasto Stabilization (Crushod LImestone) }\end{array}$ & 55,757 & TN & 50 & 0.1 & 5,576 & 111,514 & $2,70 n, 050$ & $\mathbf{2 3 , 4 5 4}$ & 6260 & $2.032,0000$ \\
\hline 11 & $\begin{array}{l}\text { Place and Compact Foundation Soll } \\
\text { in } 8^{\prime \prime} \text { Utits to } 85 \% \text { sT0. Proctor }\end{array}$ & 395,858 & or & - & 0.106 & 41,565 & 891,502 & $=0$ & 249,301 & $2 \pi 0$ & $1,000,000$ \\
\hline 12 & Placement of Claymax Gootextlie Mat & $1,074,065$ & SF & 0.38 & 0.006 & 0.575 & 187,407 & 712.s75 & - & 0.40 & 00000 \\
\hline 13 & $\begin{array}{l}\text { Placoment of } 30 \text { Min Floxible } \\
\text { Membrane Lmer }\end{array}$ & $1,952,341$ & BF & 0.14 & 0.000 & 11.714 & 204,201 & 200,5es! & -1 & 0.30 & 807,010 \\
\hline 14 & $\begin{array}{l}\text { Placoment of 1'-0 Coures Sand } \\
\text { Drainage Layer }\end{array}$ & 66:126 & or & 10.80 & 0.025 & 1,053 & $\mathbf{s 0 , 0 0 0}$ & 004,520 & 0,010 & 11.10 & 797.500 \\
\hline 15 & $\begin{array}{l}\text { Placement of Geotexthe (TYPAR) Fliter on } \\
1^{\prime}-0^{\circ} \text { Coarse Sand Drainage Layer }\end{array}$ & 212,426 & 9r & 0.12 & 0.006 & 1,062 & 21,249 & 25,491 & - & 0.20 & 40,500 \\
\hline 16 & Placoment of 1'-6" Common Fill & 80.001 & or & 3.75 & 0.04 & 2,020 & 70,405 & 507,004 & 202630 & 4.00 & 40.010 \\
\hline 17 & Placement of $6^{*}$ Topsoll & 33.000 & or & 9.50 & 0.04 & 1,320 & 20,400 & 913,000 & 7.020 & 10.50 & 34,020 \\
\hline 18 & $\begin{array}{l}\text { Placement of Perimeter Exterfor } \\
\text { Drainage Layer } 3 / 4^{\prime \prime} \times 3^{\prime \prime} \text { DP (Crushed Stone) }\end{array}$ & 2,650 & SY & 1.50 & 0.16 & 424 & 8,460 & s,975 & 2544 & 8.70 & 15,000 \\
\hline 19 & Excavation of Trapezoldal Drainage Ditch & 39.024 & or & - & 0.15 & 5.974 & 119,472 & 0 & 30,842 & 2.00 & 155,\$10 \\
\hline
\end{tabular}

I-1. Cost Estimate for a Cover System Utilizing Claymax $\$$ and a 30-mil FML (Cont'd) 
DETAIL BSTIMATE

CHKD BY: MEAD

L001: 7-03

ACTIVTTY CODB: BSE26O412

PROJ.MAMB: BCON. GVALUATTON OP CLOSURB CAP BARRIER MATBRIAI

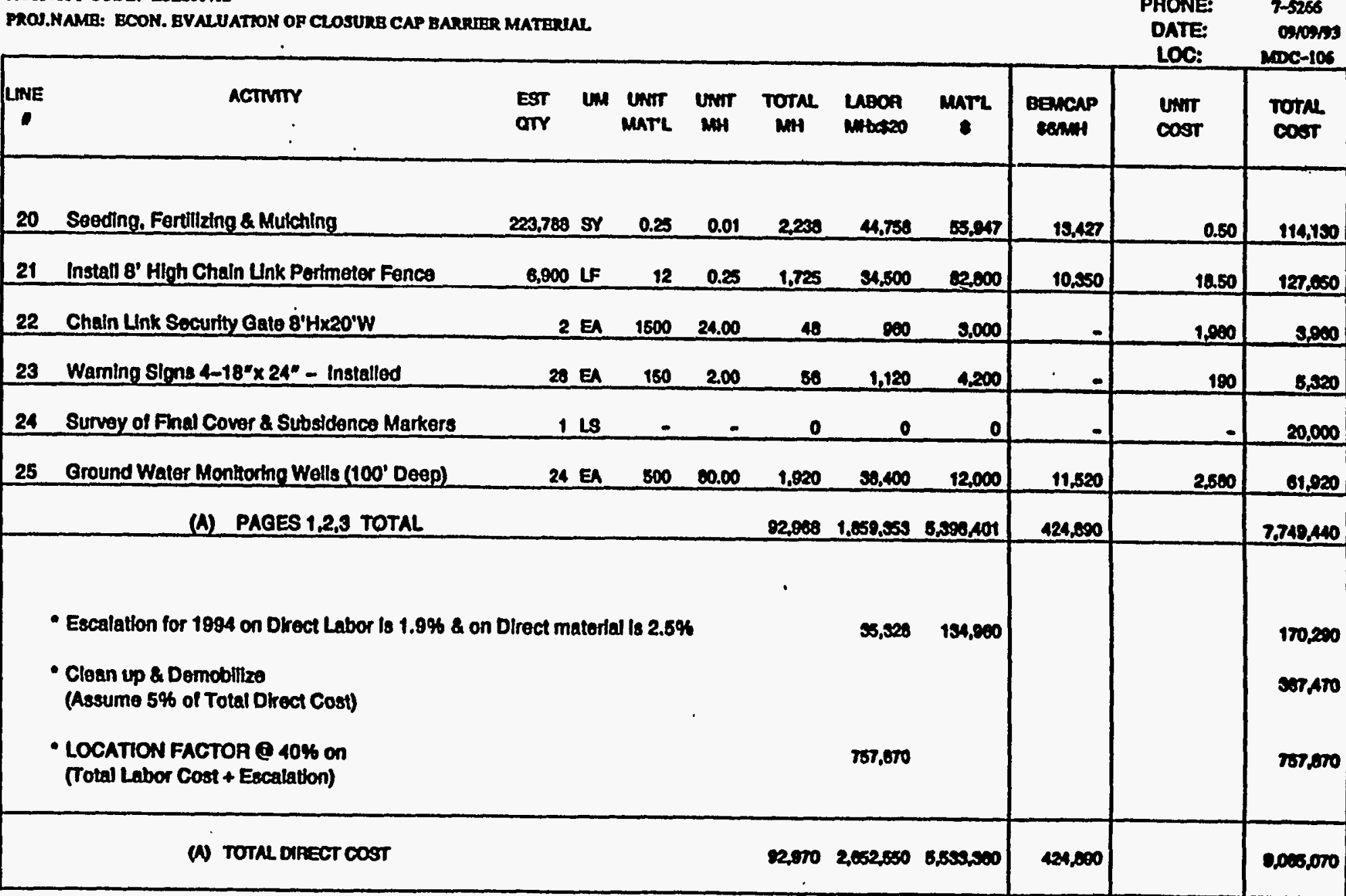

I-1. Cost Estimate for a Cover System Utilizing Claymax and a 30-mil FML (Cont'd) 
CHK'D BY: 8. MEAD

LOA I: 7-03

ACTIVTTY CODB: ESE260112

PROJ.NAME: ECON. EVALUATION OP CLOSURB CAP BARRTER MATERIAL

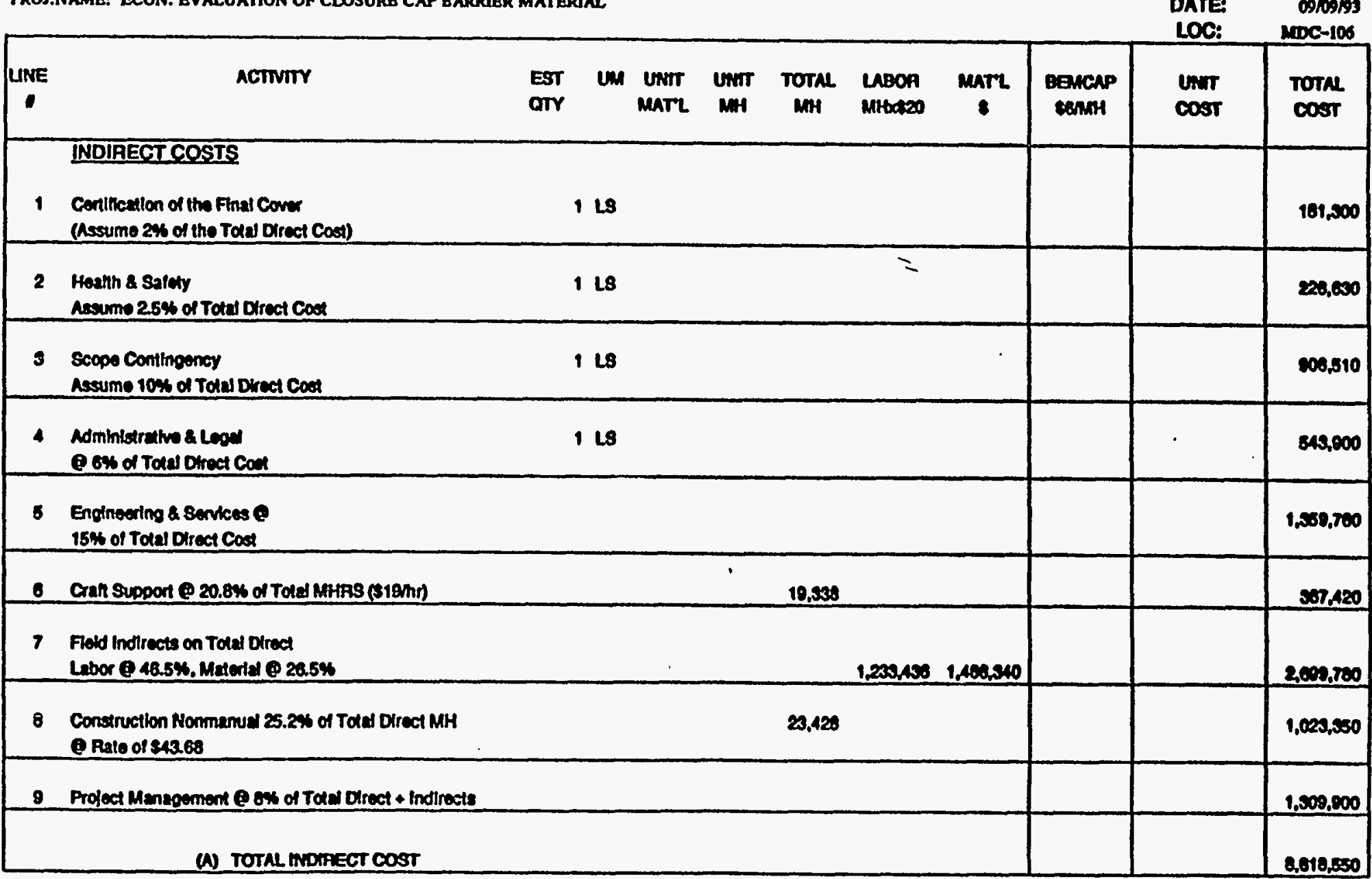

1-1. Cost Estimate for a Cover System Utilizing Claymax@ and a 30-mil FML (Cont'd) 
D B T A L B S T MAT B

CHK'D BY: S. MEAD

L00 1: 7-03

ACTTVITY CODE: ESE260412

PROJ.NAMB: ECON. EVALUATKON OF CLOSURB CAP BARRJER MATERIAL.

ESTIMATOF: NaWrK

PHONE: T-S250

DATE: 0909193

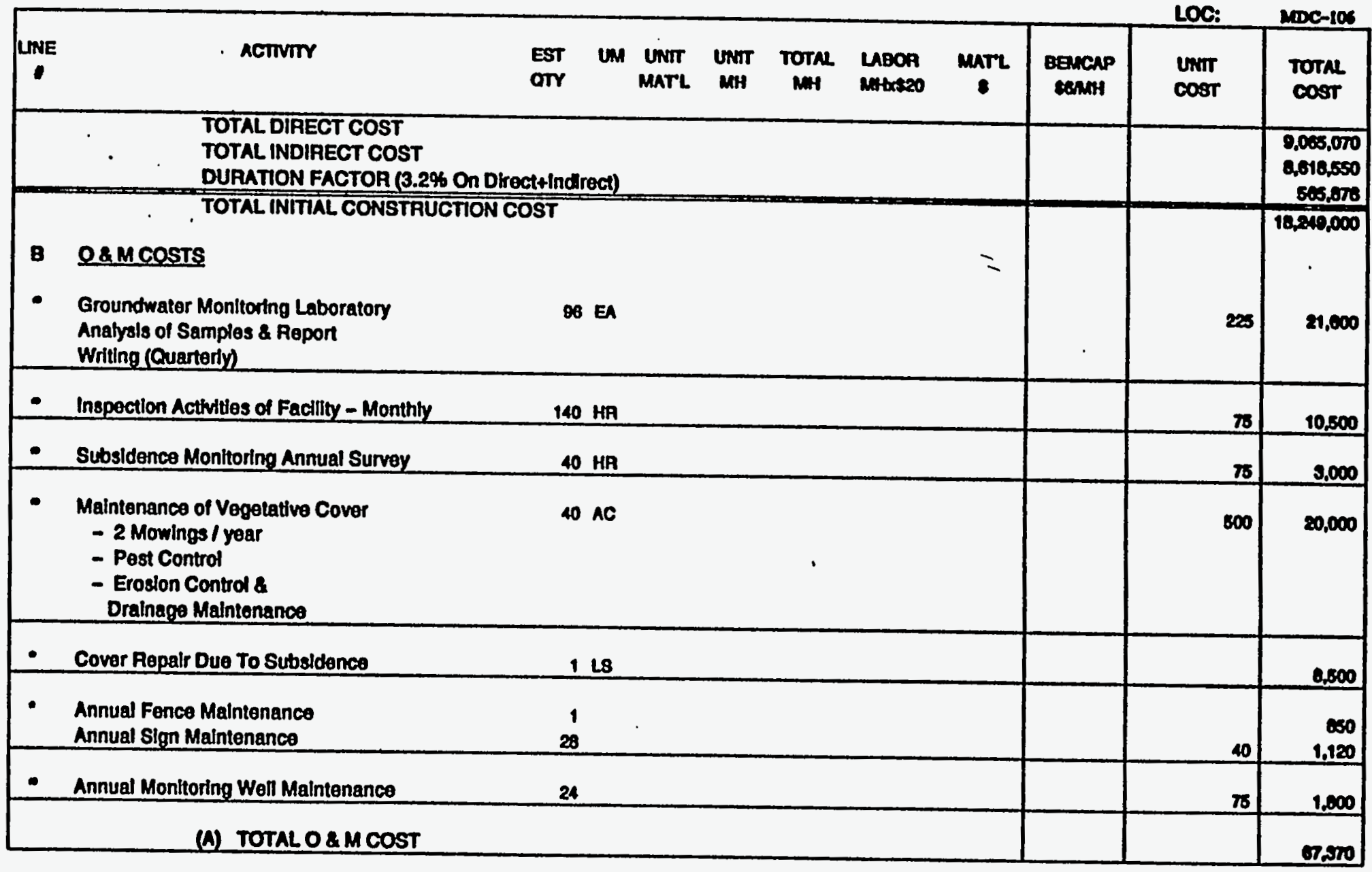

I-1. Cost Estimate for a Cover System Utilizing Claymax and a 30-mil FML (Cont'd) 
D B T I L B S T IMATB

CHKD BY: 8. MEAD

L001: 7-03

ACTIVITY CODB: ESE2CON12

Estimaton: ramix

MROS.NAMB: ECON. EVALUATION OP CLOSURE CAP SARRIER MATERIAL

PHONE: 7-5265

DATE: conss

LOC: $\quad$ NODC-106

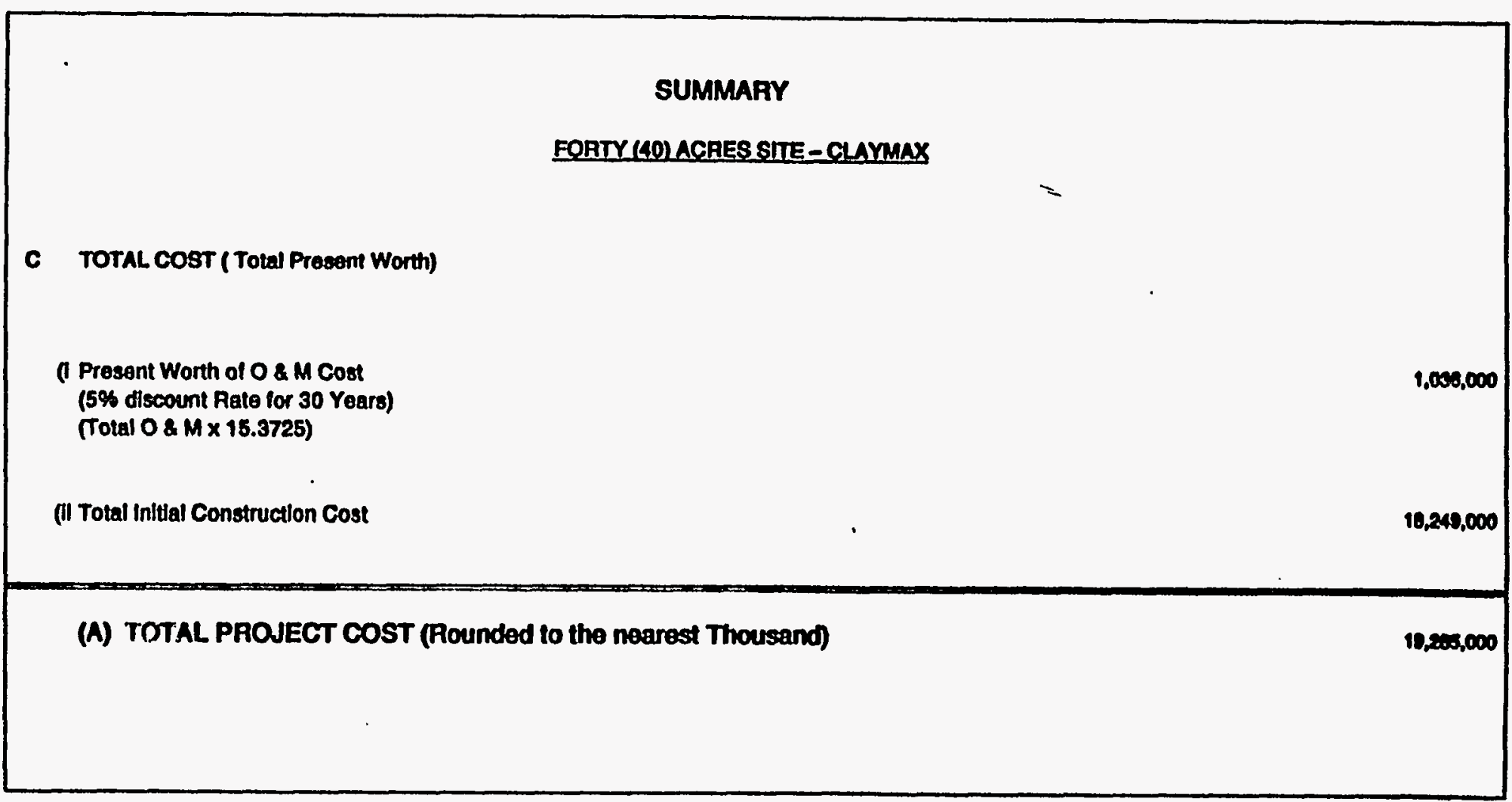

I-1. Cost Estimate for a Cover System Utilizing Claymax@ and a 30-mil FML (Cont'd) 
1001: 7-03

ACTIVTYY CODE: ESE2OOH12

PROS.NAMR: BCON. BVALUATION OF CLOSURB CAP BARRIER MATERHAL

CHK'D BY: 8. MEAD

ESTIMATOR: NaWIX

PHONE: 7-5065

DATE: OPOAPS

LOC: NDC-105

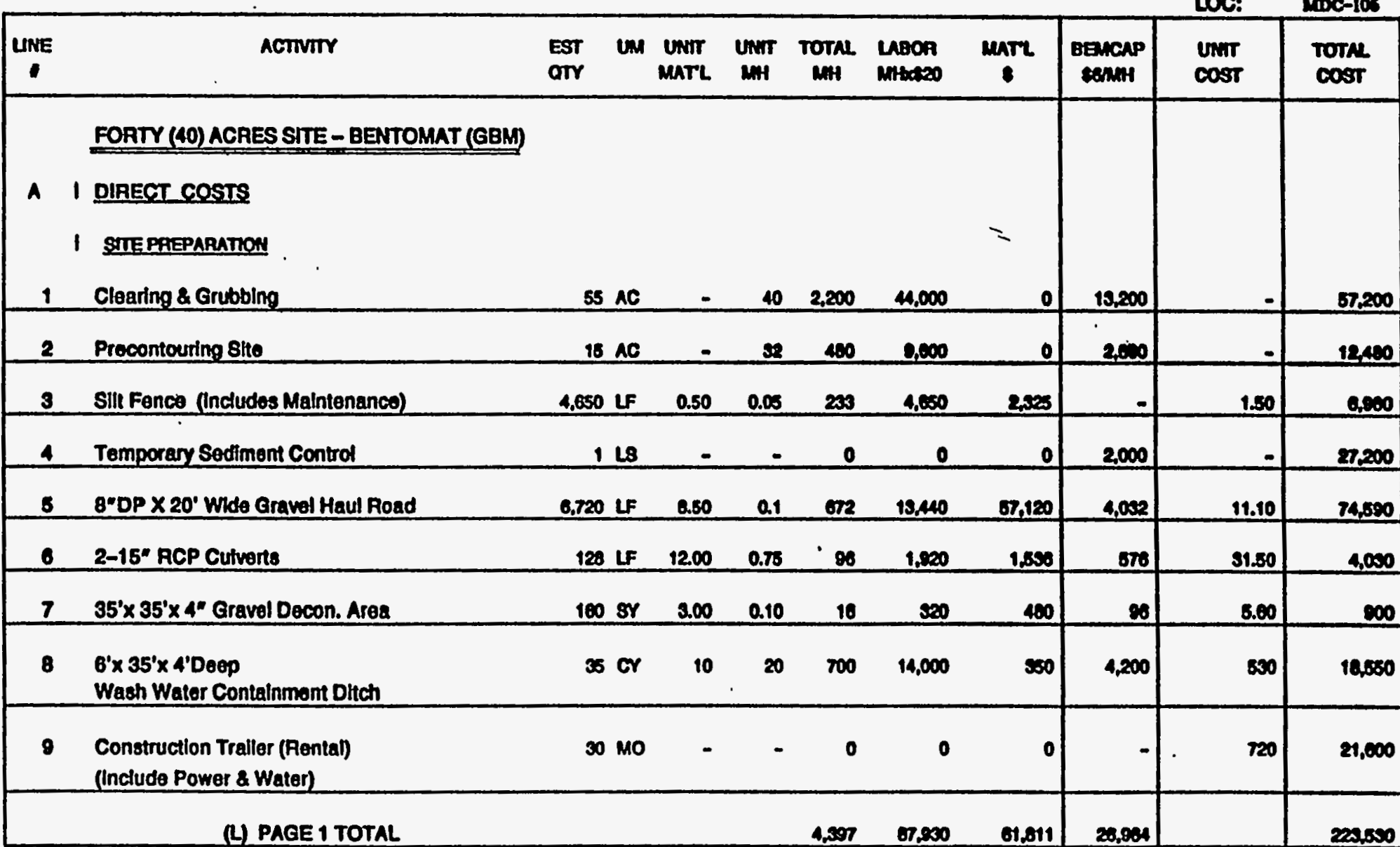

I-2. Cost Estimate for a Cover System Utilizing Bentomat@ and a 30-nil FML 
DETAIL ESTIMATE

CHKD BY: 8. MEAD

1001: $7-03$

ACTTVTYY CODE: BSE2SON12

PROJ.NAME: ECON. BVALUATTON OP CLOSURB CAP BARRIER MATERUAL

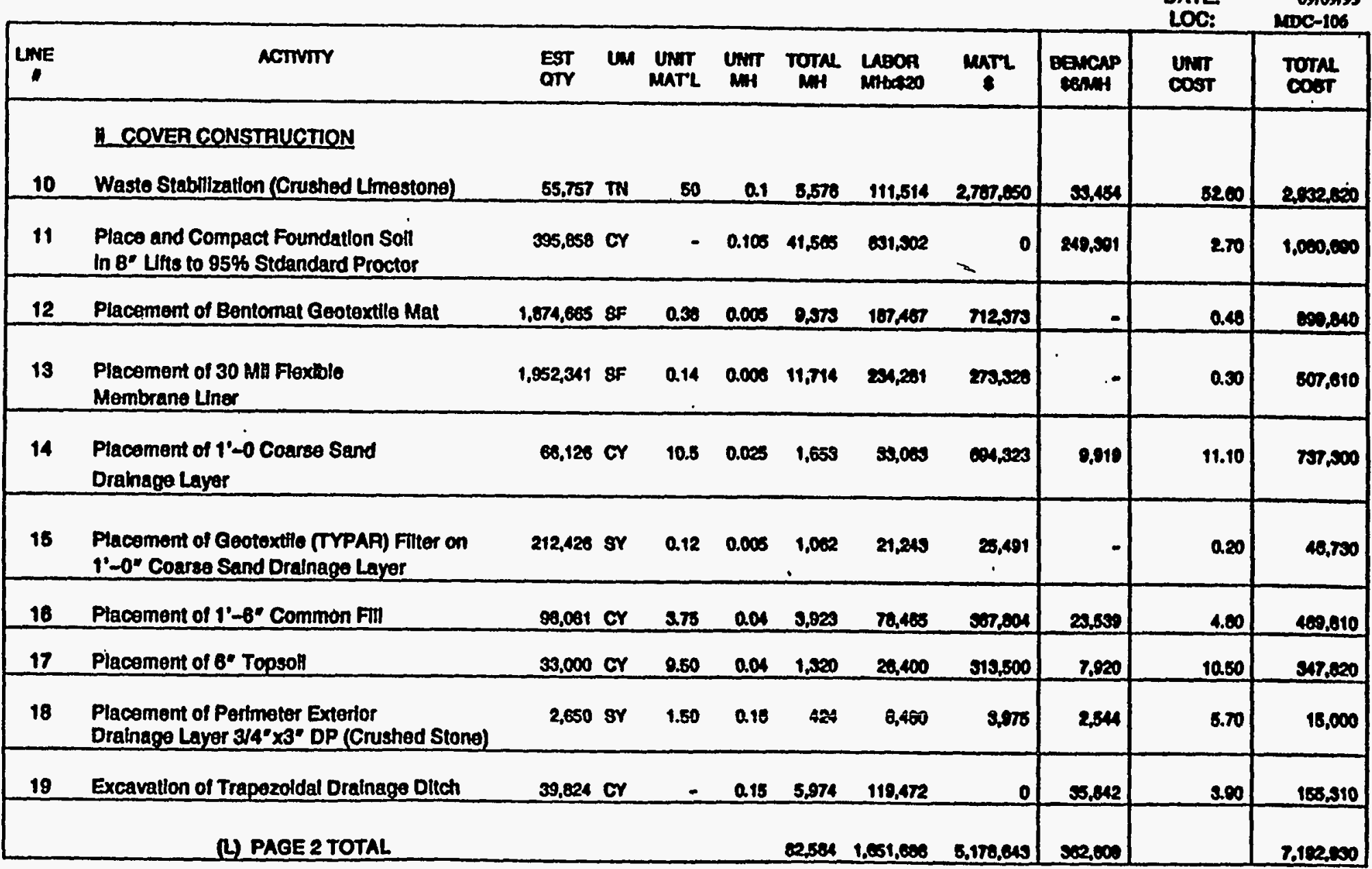

I-2. Cost Estimate for a Cover System Utilizing Bentomat@ and a 30-mil FML (Cont'd) 
CHKD QY: 8. MEND

L001: 7-03

ACTIVTYY CODH: BSB260412

PROS.NAME: BCON. EUALUATION OF CLOSURB CAP BARNRER MATERIAL

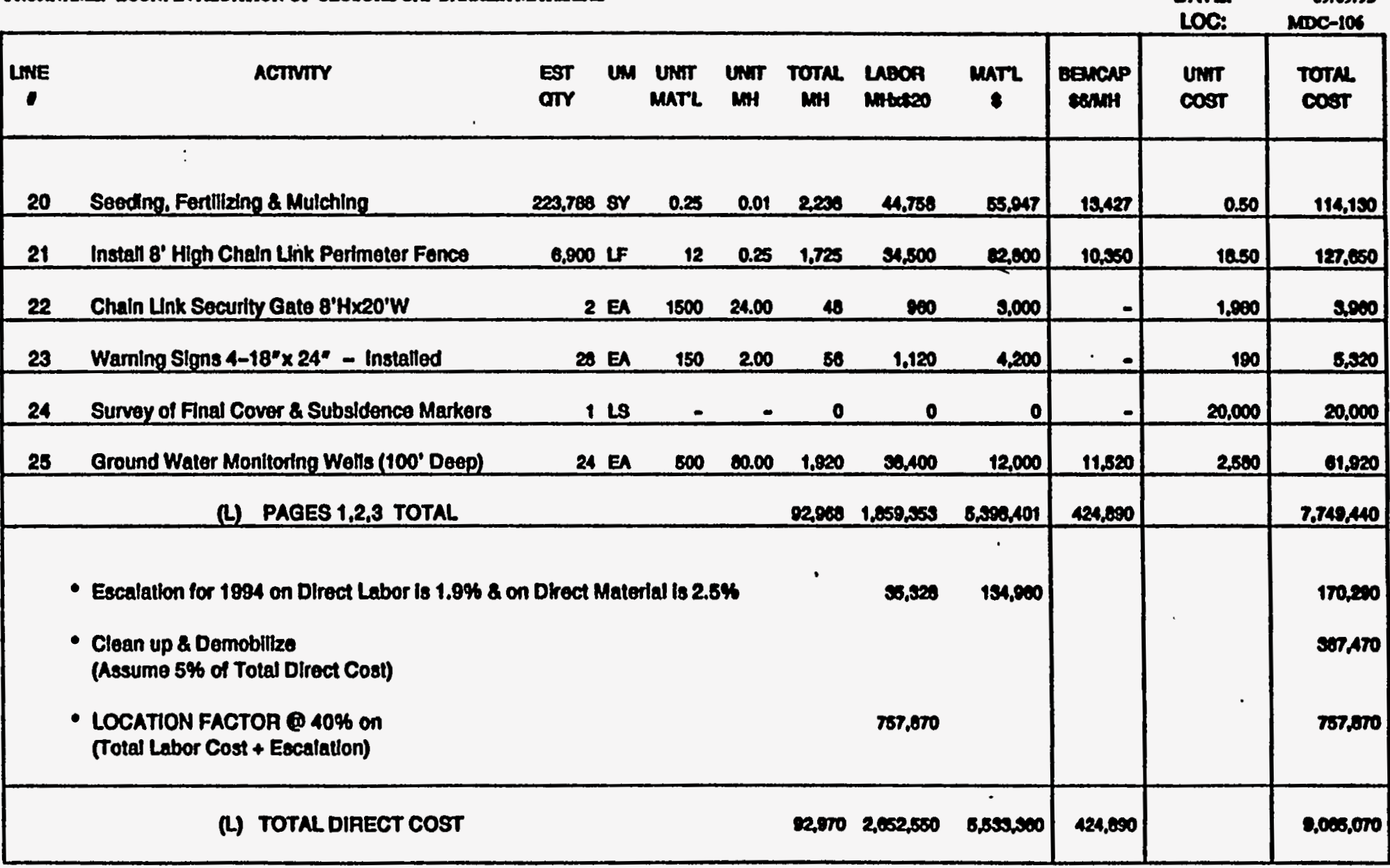

I-2. Cost Estimate for a Cover System Utilizing Bentomat 1 and a 30-mil FML (Cont'd) 
DETAIL BSTIMATE

CHK'D BY: 8. MEAD

LO0 : $7-03$

ACTTVITY CODE: ESE26ON12

PROJ.NAMR: ECON. EVALUATHON OF CLOSURB CAP BARRIER MATERIAL

PHONE:

nornax

DATE:

$7-5265$

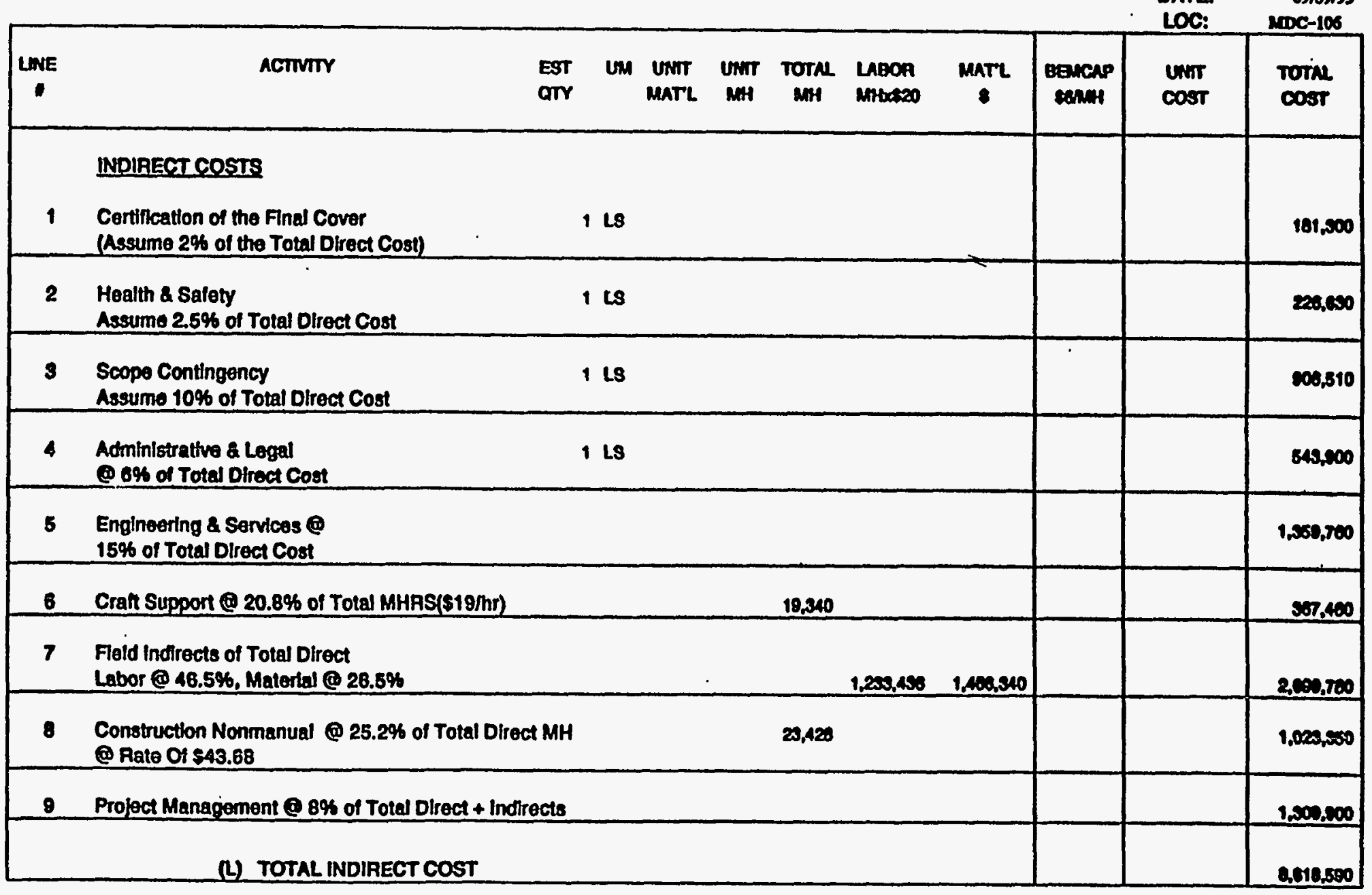

1-2. Cost Estimate for a Cover System Utilizing Bentomat(18 and a 30-mil FML (Cont'd) 
CHKD BY: 8. MEAD

LOOA: 7-03

ACTIVITY CODE: ESE260412

PROJ.NAME: ECON. BVALUATTON OF CLOSURB CAP BARRIER MATERINL

ESTIMATOR: MaWrK

PHONE: 7-S266

DATE:

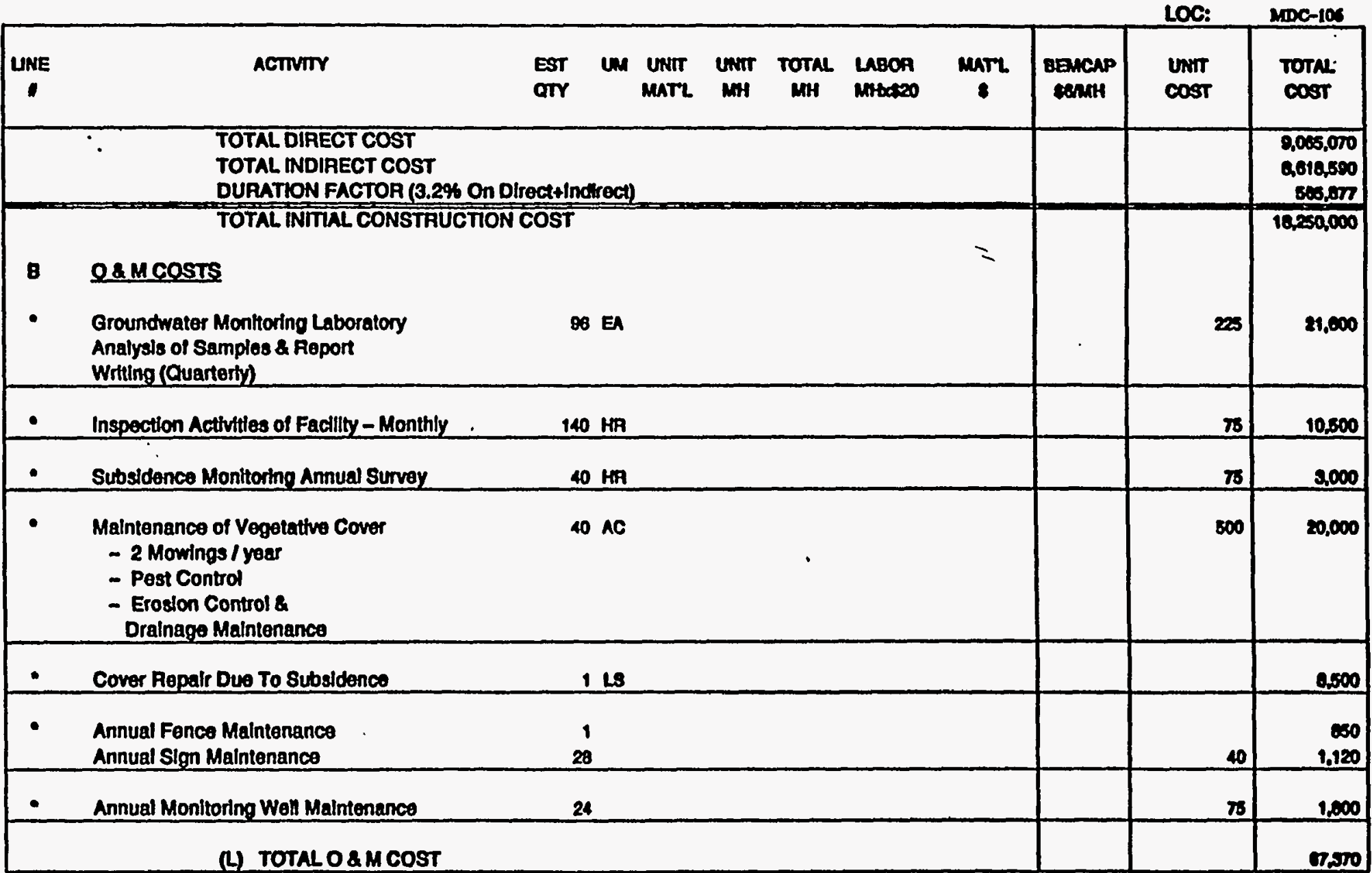

1-2. Cost Estimate for a Cover System Utilizing Bentomat and a 30-mil FML (Cont'd) 
DETAIL BSTIMATE

200 $1: 7-03$

ACTTVITY CODB: BSE2SON12

PROJ.MAME: ECON. BVALUATTON OP CLOSURE CAP BARRIER MATERIAL
CHK'D BY: 8. MEAD

E8TIMATOR: rame

PHONE: 7-5266

DATE: Oroess

LOC: $\quad$ MDC-106

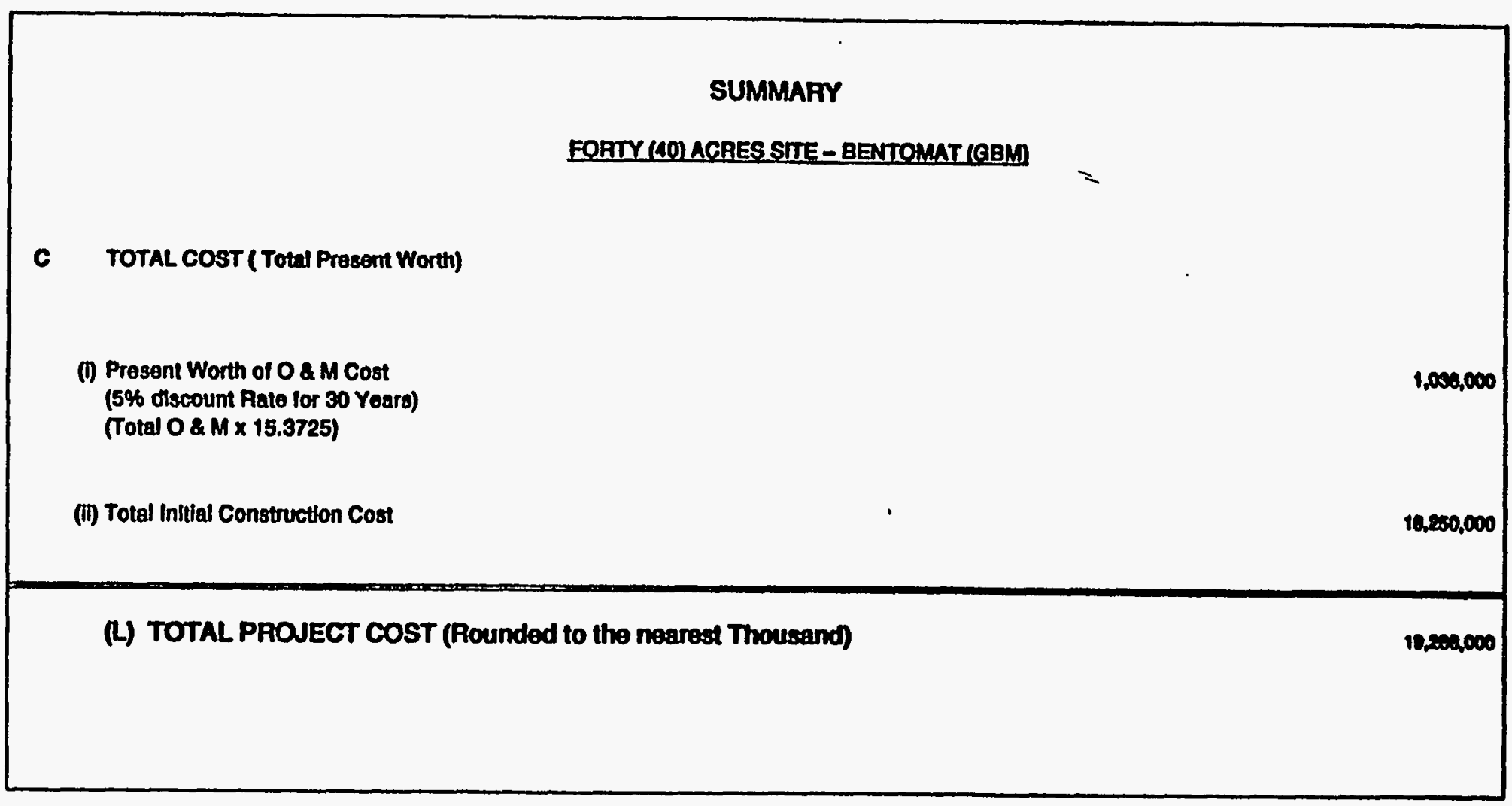

I-2. Cost Estimate for a Cover System Utilizing Bentomat $\circledast$ and a 30-mil FML (Cont'd) 
1001: $7-03$

ACTIVTTY CODE: ESE2SOS12

PROJ.NAME: ECON. EYALUATION OF CLOSURB CAP BARPIER MATERINL.

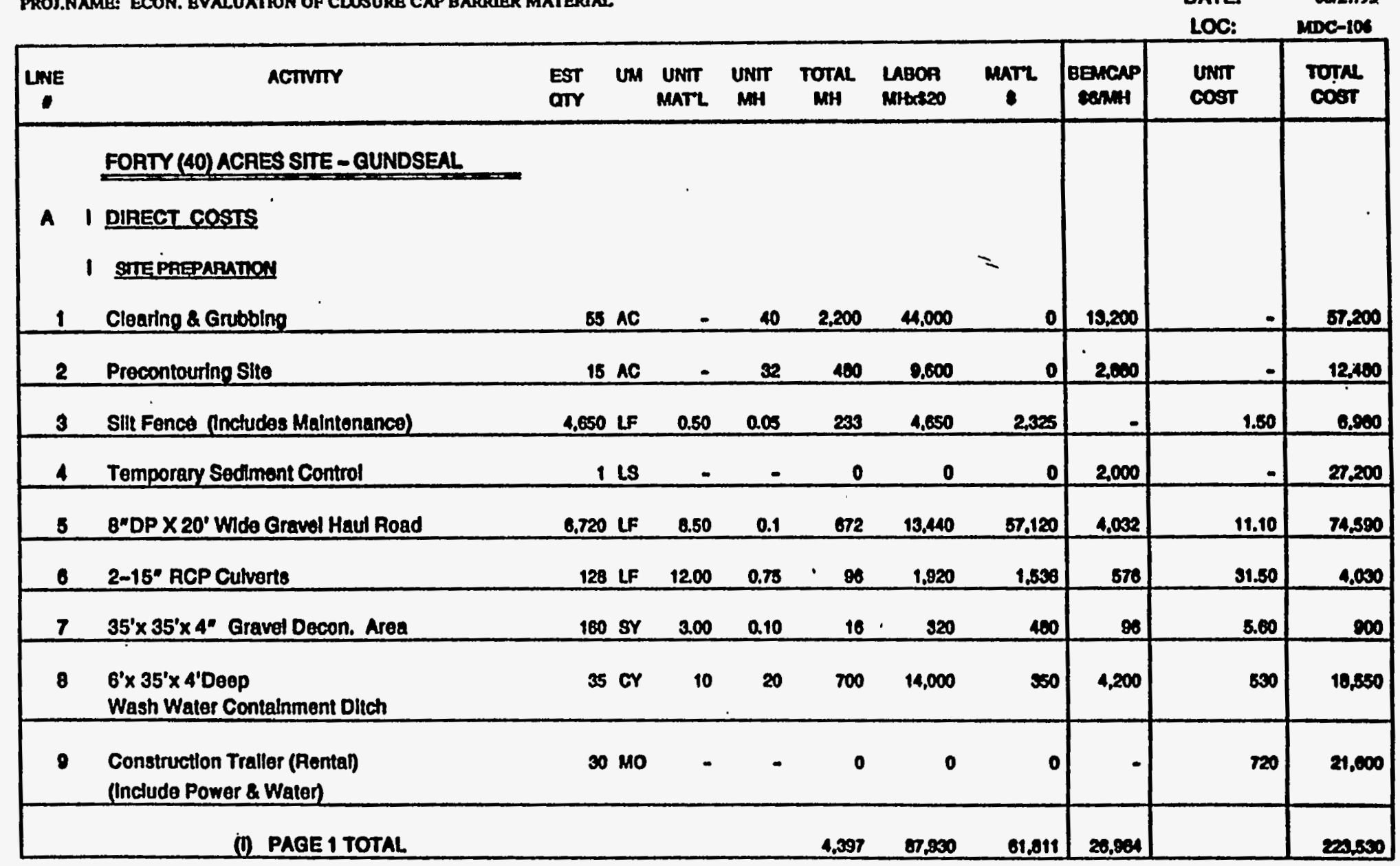

I-3. Cost Estimate for a Cover System Utilizing Gundseal(1) 
DETAIL ESTIMATE

CHKD BY: 8. MEAD

L001: 7-03

ACTIVITY CODB: HSE2COH12

PROS.NAME: BCON. EYALUATTON OF CLOSURB CAF BARRIER MATERUAL.

\begin{tabular}{|c|c|c|c|c|c|c|c|c|c|c|c|}
\hline LNE & ACTIVIV & $\begin{array}{l}\text { EST } \\
\text { OTY }\end{array}$ & un & $\begin{array}{l}\text { MnNT } \\
\text { MNTL }\end{array}$ & UMT & TOTAL & $\begin{array}{l}\text { LABOP } \\
\text { MAtreteO }\end{array}$ & $\operatorname{maTL}$ & $\begin{array}{l}\text { gencap } \\
\text { senuh }\end{array}$ & $\begin{array}{l}\ln _{\cos } \\
\text { cost }\end{array}$ & $\begin{array}{l}\text { TOTAL } \\
\text { COST }\end{array}$ \\
\hline & H COVER CONSTRUCTION & & & & & & & & & & \\
\hline 10 & Waste Stabillzation (Crushed Limestone) & 55,757 & $\mathrm{TN}$ & 50 & 0.1 & 8,576 & 111,514 & $2,707,050$ & 33,454 & 82.00 & 2,032020 \\
\hline 11 & $\begin{array}{l}\text { Placo and Compact Foundation Soll } \\
\text { In } 8^{\circ} \text { Llite to 95\% Std. Proctor }\end{array}$ & 395,050 & or & - & 0.105 & 41,565 & 001,302 & 0 & 240,391 & 270 & $1,000,000$ \\
\hline 12 & $\begin{array}{l}\text { Placement of Gundseal Goomembranel } \\
\text { Bentonite Mat }\end{array}$ & $1,074,685$ & SF & 0.30 & 0.005 & 8.573 & 187,407 & $712.5 n$ & - & 0.40 & 099,840 \\
\hline 13 & $\begin{array}{l}\text { Ptacoment of 1'-0 Coarse Sand } \\
\text { Orainago Layer }\end{array}$ & 68,128 & or & 10.50 & 0.023 & 1,653 & 33,063 & 604,320 & 0,018 & $\mathbf{9 1 . 1 0}$ & 797.300 \\
\hline 14 & $\begin{array}{l}\text { Placement of Geotextlle (TYPAR) Fitier on } \\
\text { 1'-0" Coarse Sand Drainage Layer }\end{array}$ & 212,428 & sr & 0.12 & 0.005 & 1,062 & 21,243 & 20.491 & - & 0.20 & 46,730 \\
\hline 15 & Placoment of $1^{\prime}-6^{*}$ Common Fil & 98,081 & or & 3.76 & 0.04 & 3,923 & 70,465 & 307.004 & 23.659 & 4.80 & 409,810 \\
\hline 18 & Placement of $6^{*}$ Topsoll & 33,000 & Cr & 9.50 & 0.04 & 1,320 & 26,400 & 313,500 & 7.920 & 10.50 & 347,020 \\
\hline 17 & $\begin{array}{l}\text { Placement of Perlmeler Exterlor } \\
\text { Dralnaga Layer } 3 / 4^{*} \times 3^{*} \text { DP (Crushed Stono) }\end{array}$ & 2,650 & Sr & 1.50 & 0.18 & 424 & 8,460 & 8,875 & 2544 & 8.70 & 18,000 \\
\hline 18 & Excavation of Trapezoldal Dralnage Ditch & 39.824 & or & $=$ & 0.15 & 5,974 & 118,472 & 0 & 35,642 & 290 & 185,910 \\
\hline & (1) PAGE 2 TOTAL & & & & & 70,870 & $1,417,405$ & A,905,310 & 362,000 & & $0,605.520$ \\
\hline
\end{tabular}

1-3. Cost Estimate for a Cover System Utilizing Gundseal $\AA_{(\text {Cont'd) }}$ 
D B T A I L B S TIMAT B

CHK'D BY: S. MEAD

2001: $7-03$

ACTIVTYY CODB: BSE260412

PROJ.NAME: ECON. BVALUATTON OP CLOSURB CAP BARRIER MATERIAL.

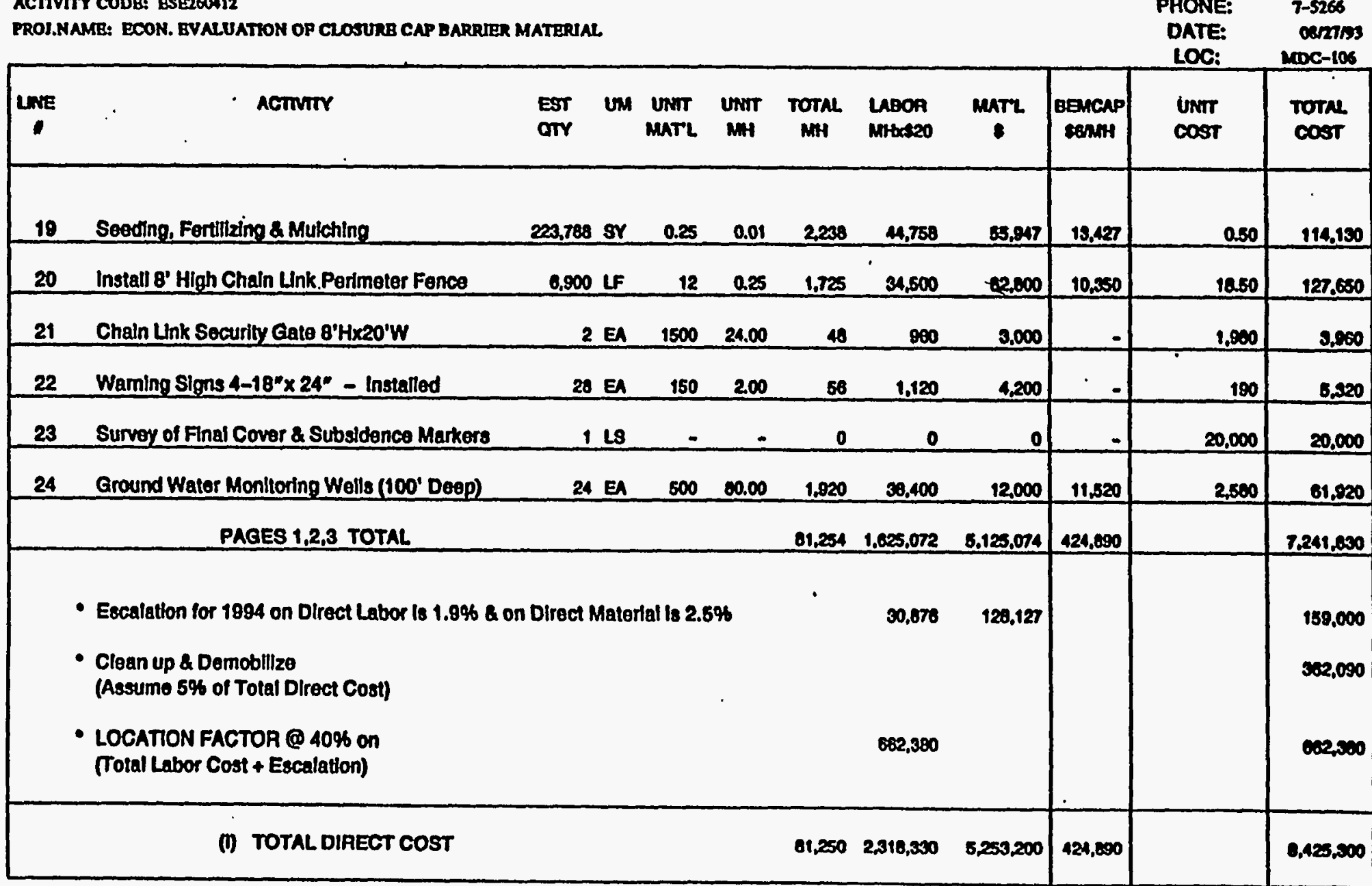

1-3. Cost Estimate for a Cover System Utilizing Gundseal@ (Cont'd) 
Loo : 1-03 ACTTVTY CODE: ESE260412

PROJ.NAME: BCON. EVALUATION OF CLOSURE CAP BARRIER MATERIAL

ESTIMATOR: roWLX

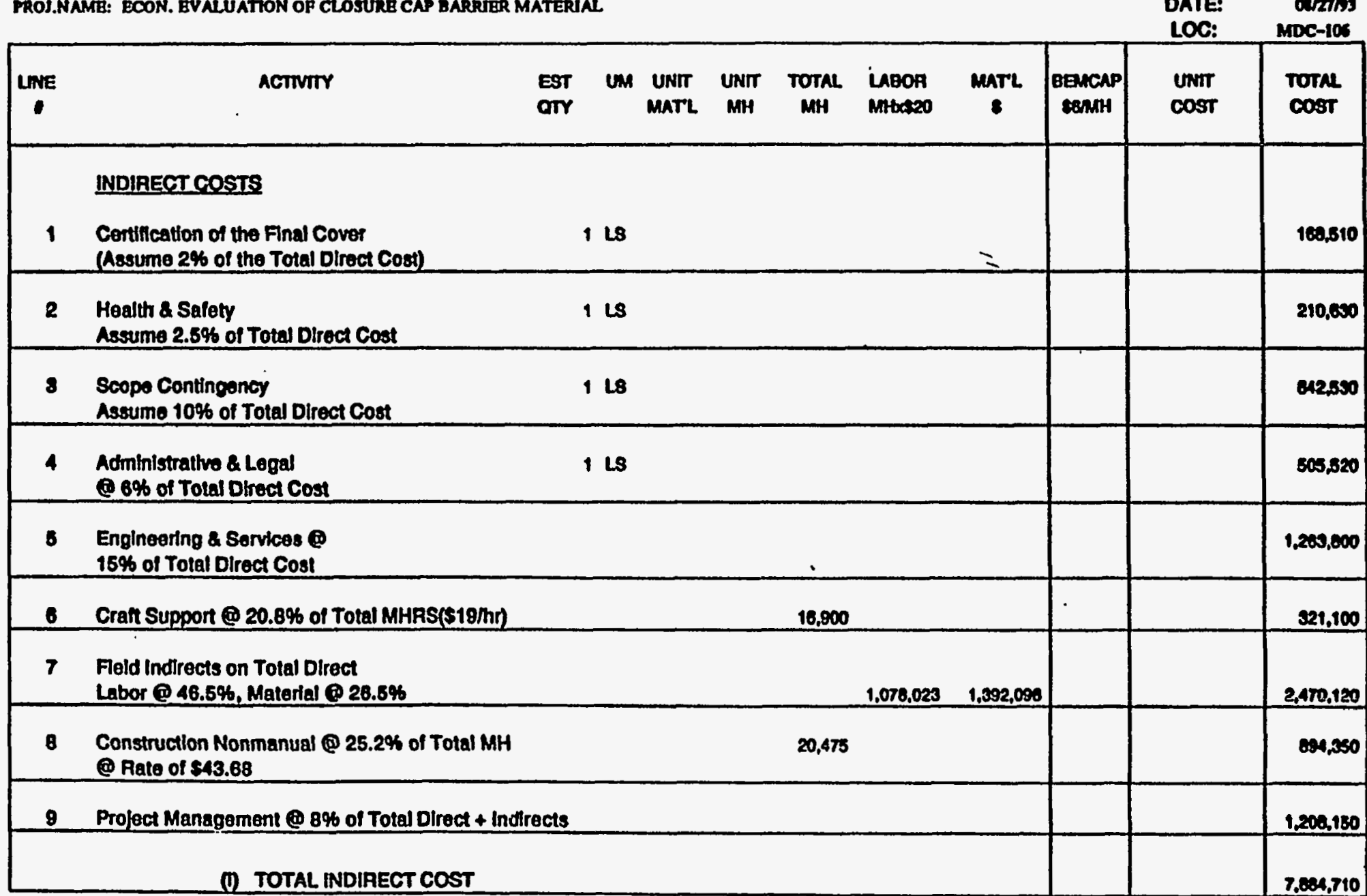

I-3. Cost Estimate for a Cover System Utilizing Gundseal@ (Cont'd) 


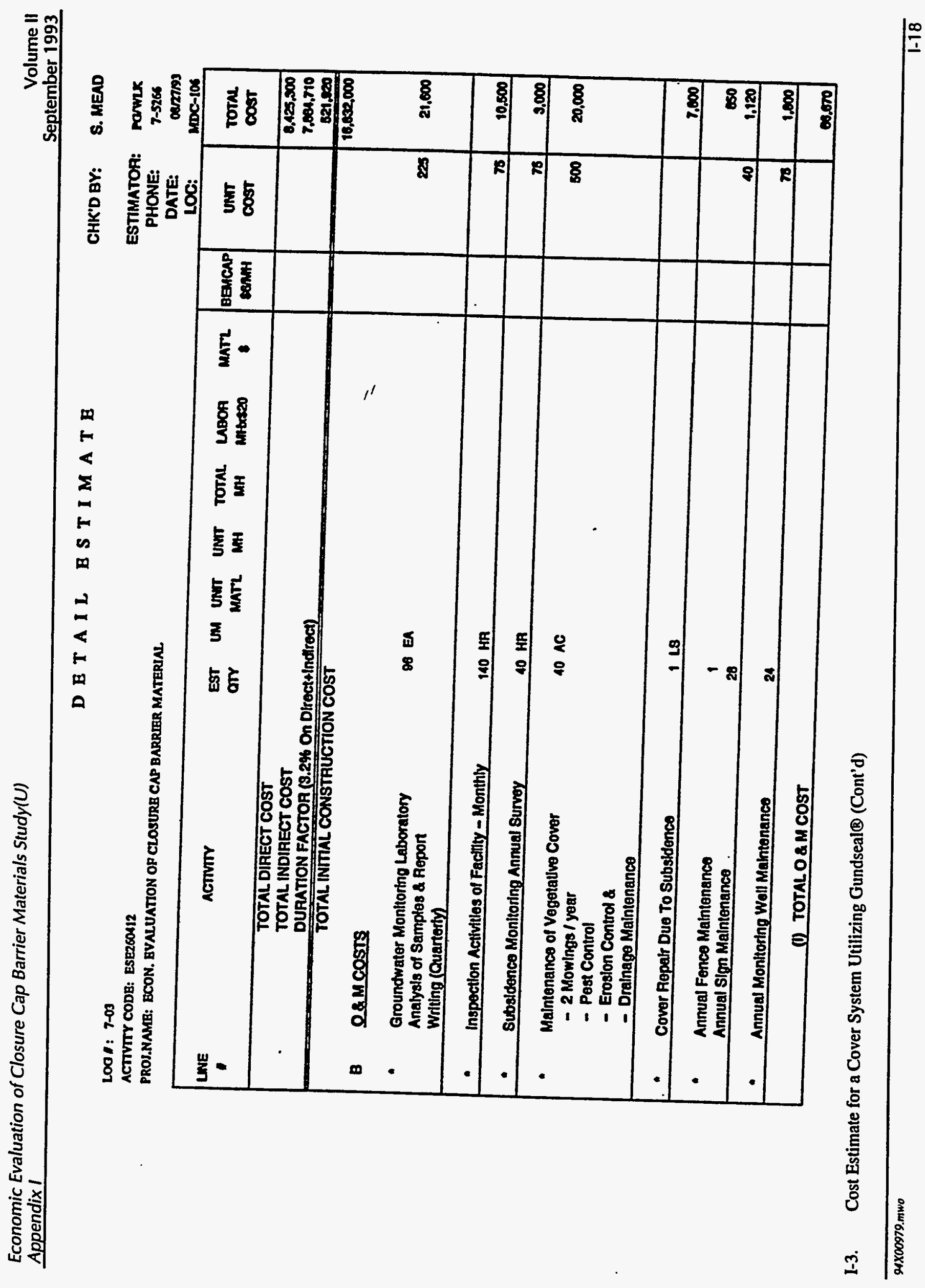


DBTAIL BSTIMATB

\begin{tabular}{|c|c|}
\hline & \\
\hline & \\
\hline $\begin{array}{l}\text { PHO } \\
\text { DAT }\end{array}$ & \\
\hline
\end{tabular}

L001: $7-03$

ACTIVITY CODB: BSE200412

MROS.NAMR: BCON. EVALUATTON OF CLOSURB CAP DARRIER MATRRINS

MiDe-106

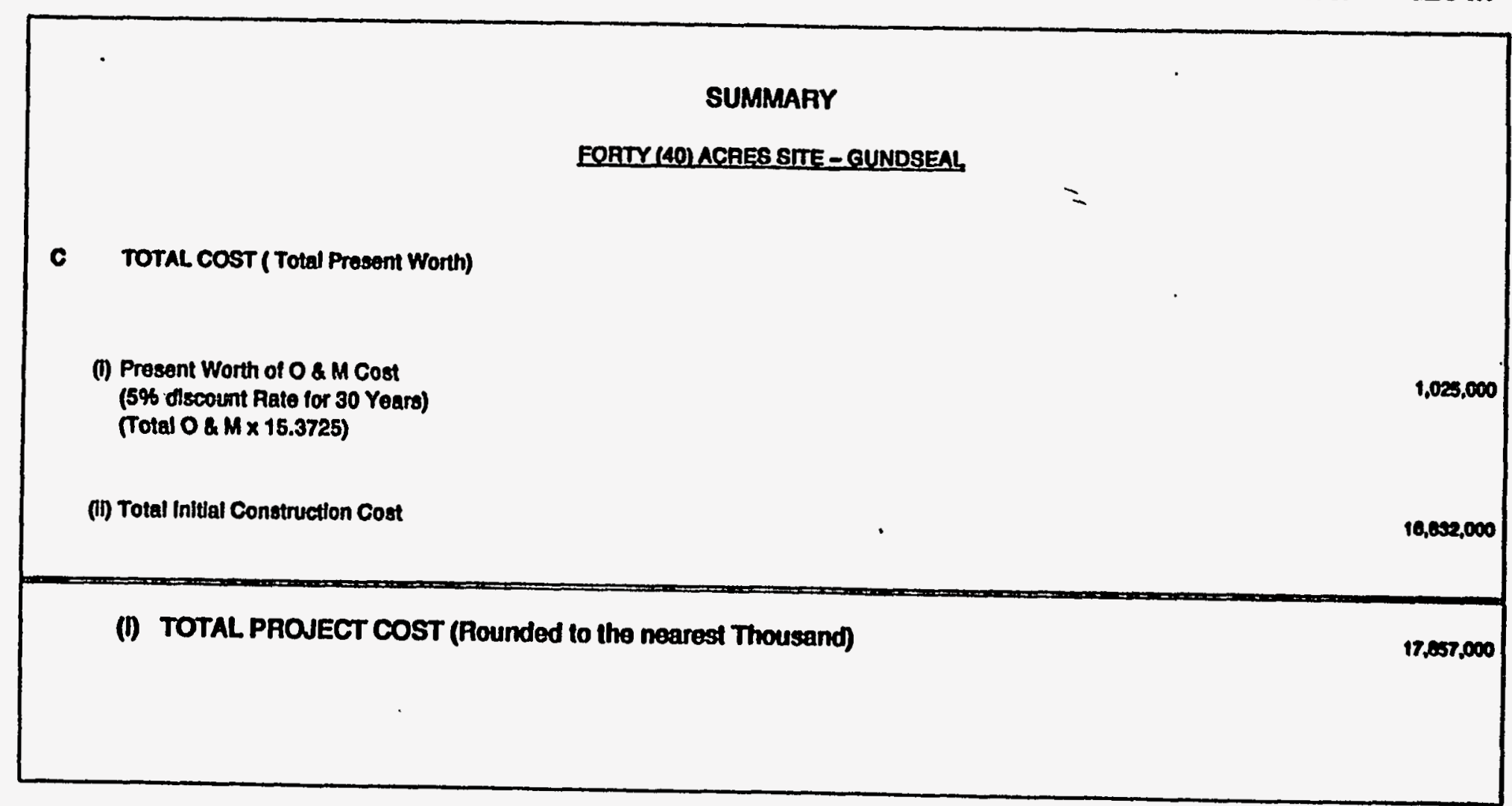

1-3. Cost Estimate for a Cover System Utilizing Gundseal@ (Cont'd) 
DETAIL ESTIMATE

L001: $7-03$

ACTIVITY CODB: ESE260412

PROS.NAME: ECON. EVALUATION OP CLOSURB CAP BARRIER MATERLAL

\begin{tabular}{|c|c|c|c|c|c|c|c|c|c|c|c|}
\hline UNE & ACTINTIS & $\begin{array}{l}\text { EST } \\
\text { aTr }\end{array}$ & un & $\begin{array}{l}\text { UNT } \\
\text { MATLL }\end{array}$ & $\begin{array}{l}\text { UNST } \\
\text { MH }\end{array}$ & $\begin{array}{c}\text { TOTRL } \\
\text { MHA }\end{array}$ & $\begin{array}{l}\text { LABOR } \\
\text { MAtXatzon }\end{array}$ & maTL & $\begin{array}{l}\text { Bancap } \\
\text { senny }\end{array}$ & $\begin{array}{l}\text { UnT } \\
\operatorname{cost}\end{array}$ & $\begin{array}{l}\text { TOTML } \\
\text { Cost }\end{array}$ \\
\hline $\begin{array}{l}1 \\
1\end{array}$ & $\begin{array}{l}\text { FORTY (40) ACRES SITE - (120 MII } \\
\text { DIRECT COSTS } \\
\text { SIEPREPARATON } \\
\text { Cloaring \& G Gubbing }\end{array}$ & 55 & $A C$ & - & 40 & 2,200 & 4.000 & $=$ & 13,200 & - & 57,200 \\
\hline 2 & Precontouring site & 15 & Ac & - & 32 & 400 & 2.000 & 0 & 2,000 & - & 12.400 \\
\hline 3 & Silt Fence (includes Maintenance) & 4,050 & LF & 0.50 & 0.05 & 233 & 4.650 & 2325 & - & 1.50 & 6,960 \\
\hline 4 & Temporary Sediment Control & 1 & LS & - & - & 0 & 0 & 0 & 2,000 & - & 27.200 \\
\hline 5 & 8"DP $\times 20^{\prime}$ Whde Graved Haul Road & 0,720 & $\mathbf{L}$ & 8.50 & 0.10 & 672 & 13,440 & 57,120 & $\begin{array}{l}1,032 \\
\end{array}$ & 11.10 & 74,590 \\
\hline 6 & 2-15" RCP Culverts & 128 & LF & 12.00 & 0.78 & 96 & 1,920 & 1,536 & 570 & 31.50 & 1,000 \\
\hline 7 & $35^{\prime} \times 35^{\prime} \times 4^{\prime \prime}$ Gravel Decon. Area & 160 & sr & 3.00 & 0.10 & 10 & 320 & 400 & $\theta$ & 8.00 & $\infty$ \\
\hline 8 & $\begin{array}{l}6^{\prime} \times 35^{\prime} \times 4^{\prime} \text { Deep } \\
\text { Wash Water Containment Dittch }\end{array}$ & 35 & or & 10 & 20 & 700 & 14,000 & 350 & 4,200 & BSO & 18,580 \\
\hline D & $\begin{array}{l}\text { Construction Traller (Rental) } \\
\text { (Include Power \& Water) }\end{array}$ & 30 & MO & - & - & - & 0 & 0 & - & $r 20$ & 21,000 \\
\hline & (F) PAGE TOTAL & & & & & 4.397 & 08,930 & 01,011 & 20,984 & & \\
\hline
\end{tabular}

1-4. Cost Estimate for a Cover System Utilizing 120-mil High Density Polyethylene (HDPE) Liner 
DETAIL ESTIMATE

CHKC BY: S. MEAD

Loof: 7-03

ACTIVTYY CODE: EsE200112

MOS.MAME: ECON. EVALUATION OF CLOSURB CAP DARRIER MATBRIAL

ESTIMATOR: POAMIX

PHONE: 7-5266

\begin{tabular}{|c|c|c|c|c|c|c|c|c|c|c|c|}
\hline UNE & Actrvitr & EST & M & $\begin{array}{l}\text { UNAT } \\
\text { MATL }\end{array}$ & $\underset{M H T}{U N T}$ & $\underset{M H 1}{\text { TOTM }}$ & $\begin{array}{l}\text { LABOR } \\
\text { MHBoteO }\end{array}$ & $\underset{:}{\operatorname{maTL}}$ & 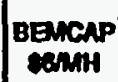 & $\lim _{\cos t}$ & $\begin{array}{l}\text { Total } \\
\operatorname{cost}\end{array}$ \\
\hline & II CQVER CONSTAUCTION & & & . & & & & & & & \\
\hline 10 & Waste Stabilization (Crushod Umestono) & 55,757 & $\mathrm{TN}$ & 50 & 0.1 & 5,578 & 111,514 & $2,707.0050$ & 23,454 & 52.00 & 2032,020 \\
\hline 11 & $\begin{array}{l}\text { Place and Compact Foundation Soll } \\
\text { in 8" Lits to } 95 \% \text { Std. Proctor }\end{array}$ & 395,050 & or & - & 0.105 & 41,565 & 831,302 & 0 & 249,391 & 270 & $1.000,090$ \\
\hline 12 & $\begin{array}{l}\text { Placement of } 120 \text { MII Floxhole } \\
\text { Membrane Lner }\end{array}$ & $1,674,665$ & BF & 0.36 & 0.000 & 11,240 & 224,000 & 712,373 & - & 0.50 & 037,330 \\
\hline 13 & $\begin{array}{l}\text { Placement of 1'-0 Coarse Sand } \\
\text { Drainage Layer }\end{array}$ & 68,126 & or & 10.50 & 0.025 & 1,653 & 33,063 & 004,320 & $\mathbf{9 , 9 1 8}$ & 11.10 & 757,500 \\
\hline 14 & $\begin{array}{l}\text { Placement of Geotextlle (TYPAR) Filter on } \\
1^{1}-0^{\prime \prime} \text { Coarse Sand Dralnage Layer }\end{array}$ & 212,428 & sr & 0.12 & 0.005 & 1,062 & 21,213 & 25,491 & - & 0.20 & 46,730 \\
\hline 15 & Placoment of 1'-6" Common Fil & 80,009 & or & 3.75 & 0.04 & 3,923 & 70,405 & 307,604 & 20.530 & 4.00 & 409,810 \\
\hline 16 & Placement of $6^{*}$ Topsoil & 33,000 & or & 9.50 & 0.04 & 1,320 & 26,400 & 319,500 & 7,920 & 10.50 & 347.620 \\
\hline 17 & $\begin{array}{l}\text { Placement of Pertmeter Exterlor } \\
\text { Drainage Layer 314" } \times 3^{3} \text { Stone }\end{array}$ & 2.650 & sr & 1.50 & 0.16 & 424 & 8.480 & 3,975 & 204 & 6.00 & $\$ 6,000$ \\
\hline 18 & Excavation of Trapezoldal Drainage Dilch & 39,824 & or & $=$ & 0.15 & 5,974 & 118,472 & 0 & 35,042 & 3.00 & 158,310 \\
\hline & (F) PAGE TOTAL & & & & & 22,743 & $1,454,000$ & $4,805,318$ & 502,000 & & $0,722,010$ \\
\hline
\end{tabular}

I-4. Cost Estimate for a Cover System Utilizing 120-mil High Density Polyethylene (HDPE) Liner (Cont'd) 
D BTAIL ESTIMATE

CHK'D BY: S. MEAD

2001: 7-03

ACTIVITY CODE: ESE260112

PROJ.NAME: BCON. BVALUATION OF CLOSURB CAP BARRIER MATERIAL.

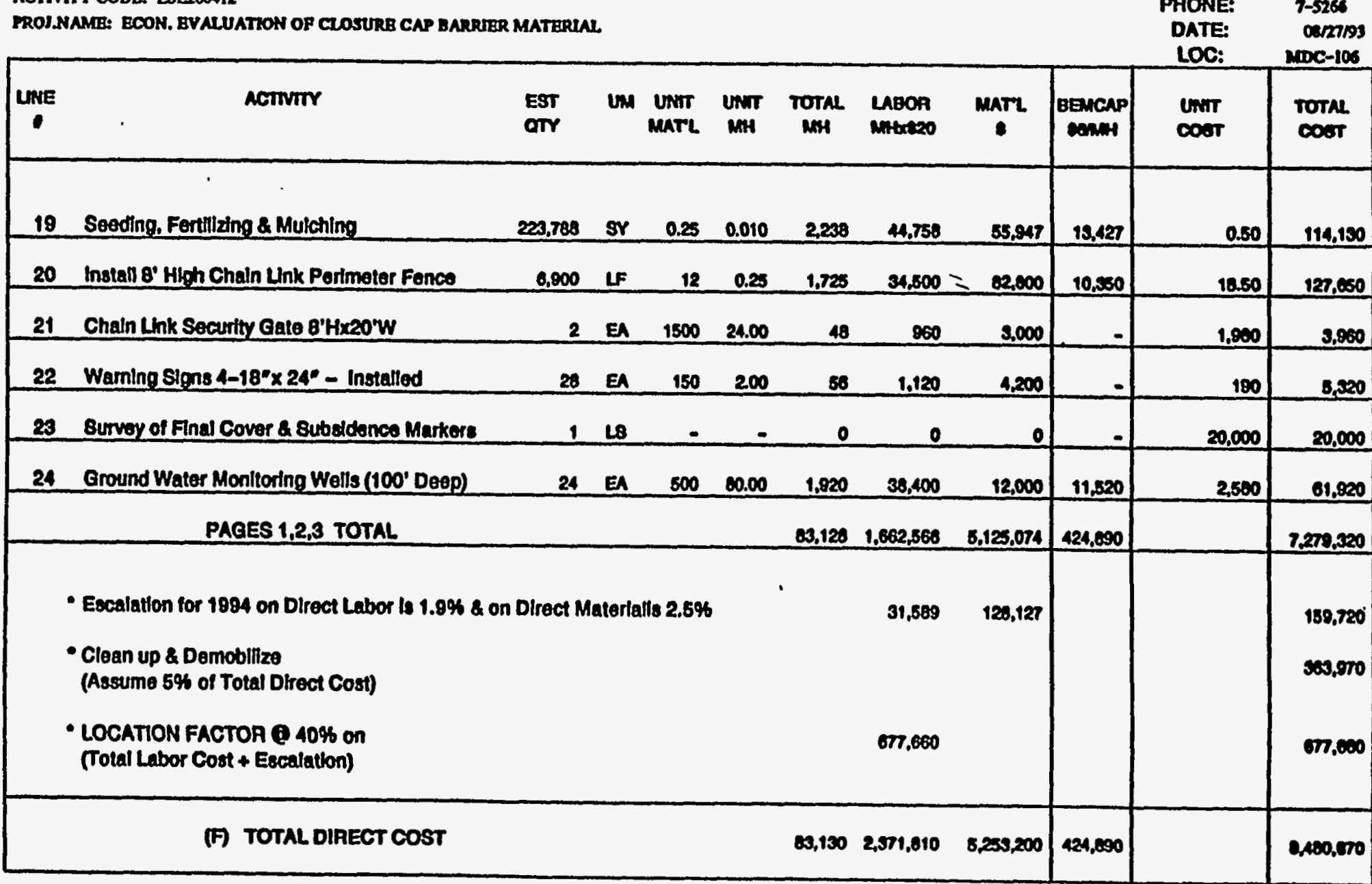

1-4. Cost Estimate for a Cover System Utilizing 120-mil High Density Polyethylene (HDPE) Liner (Cont'd) 
D B T A I L E S T I MA T B

CHK'D BY: S. MEAD

Lo0 1: 7-03

ACTTVTYY CODE: BSE260N12

PROJ.NAME: ECON. EVALUATION OP CLOSURR CAP BARRIER MATERIAL.

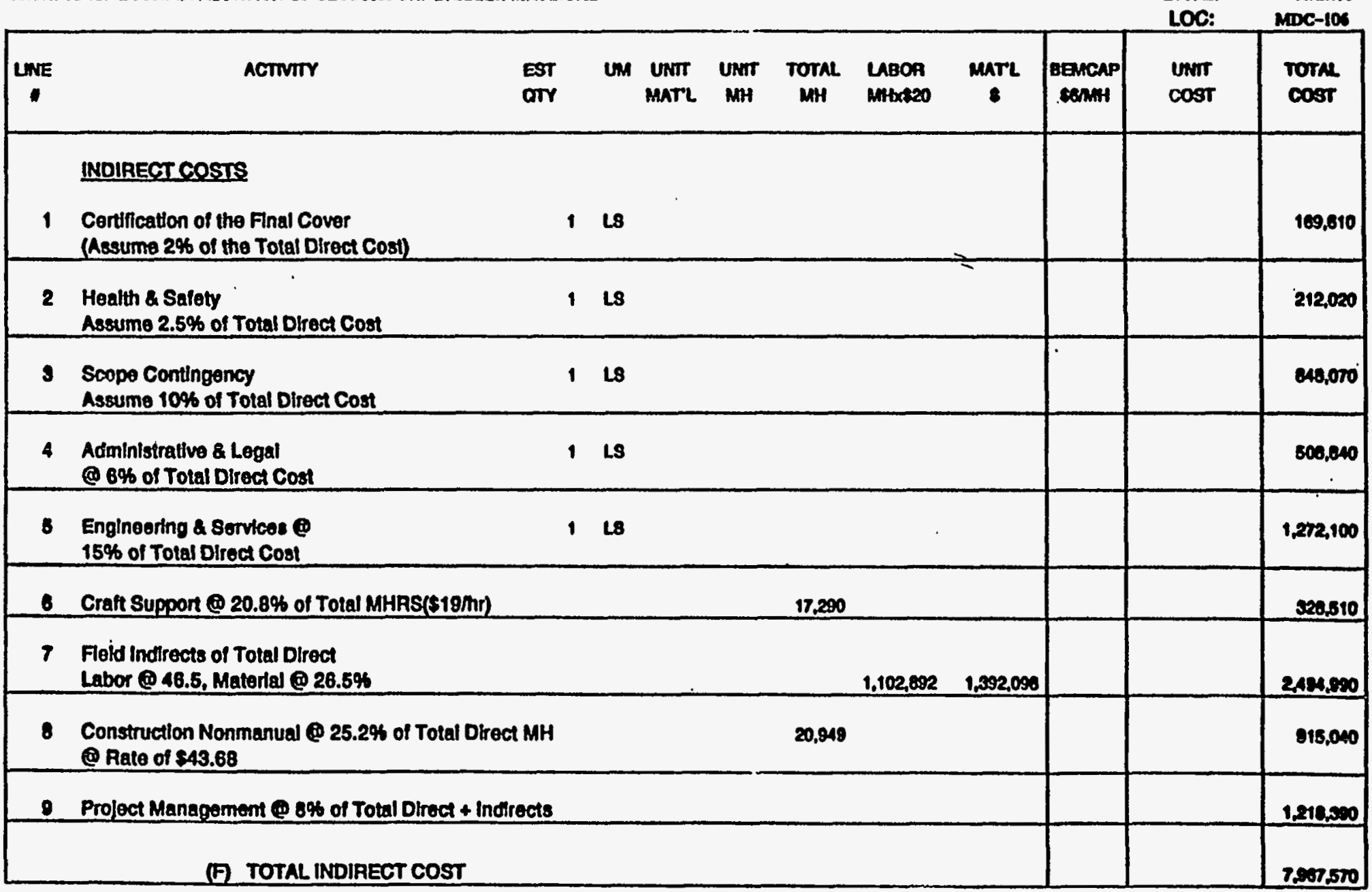

I-4. Cost Estimate for a Cover System Utilizing 120-mil High Density Polyethylene (HDPE) Liner (Cont'd) 
D B T A I L B S T I M T B

CHK'D BY: S. MEAD

1001: 7-03

ACTIVIT CODB: BSB260412

PROJ.KAME: ECON. BVALUATION OF CLOSURE CAP BARRIER MATERIAL

ESTIMATOA: ranux

PHONE: 7-5256

DATE: Oamistos

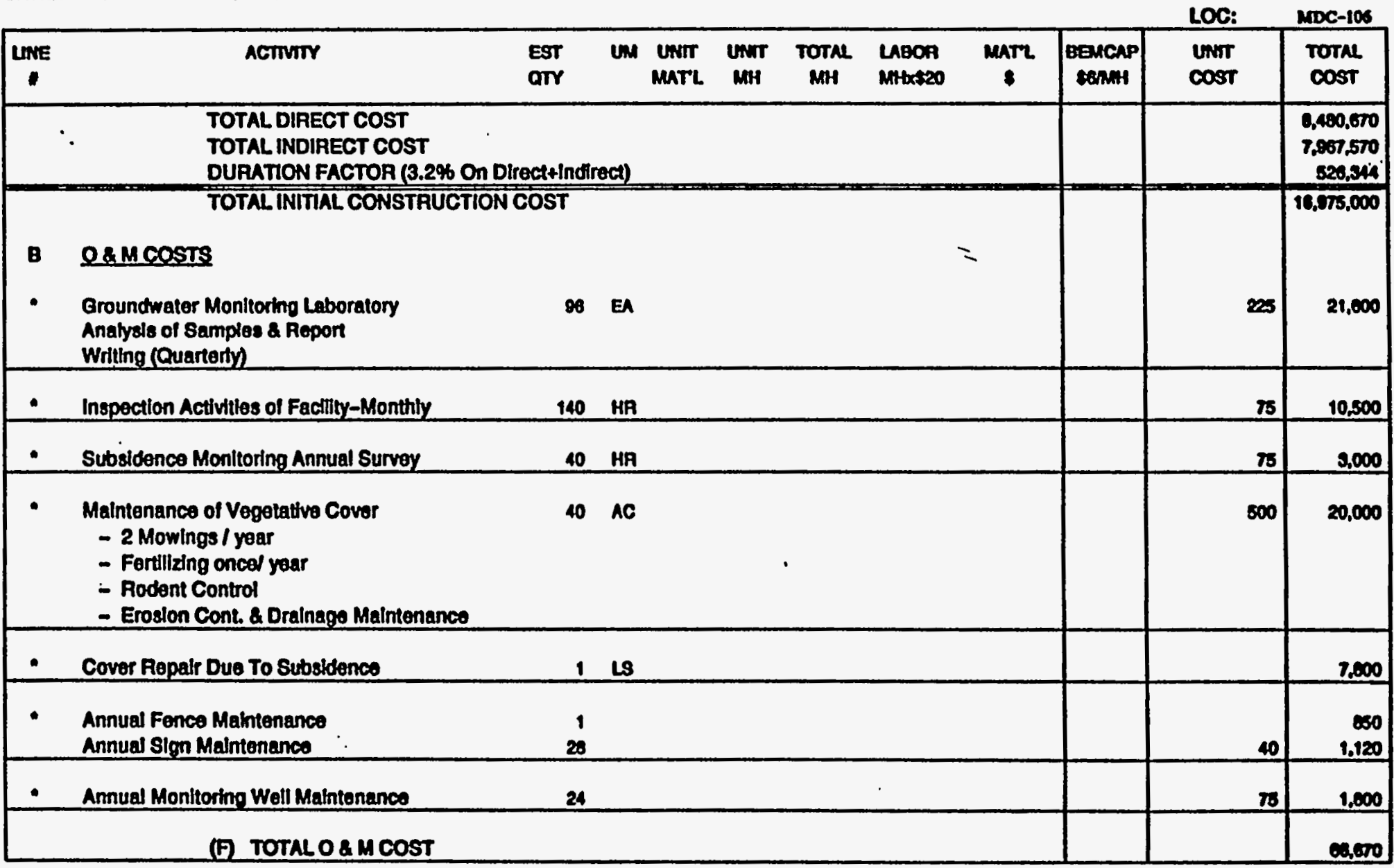

1-4. Cost Estimate for a Cover System Utilizing 120-mil High Density Polyethylene (HDPE) Liner (Cont'd) 
Lool: $1-03$

ACTIVITY CODE: ESE260N12

MROJ.NAME: BCON. EVALUATTON OF CLOSURB CAP BARRUER MATERLAL

LOC:

MDC-106

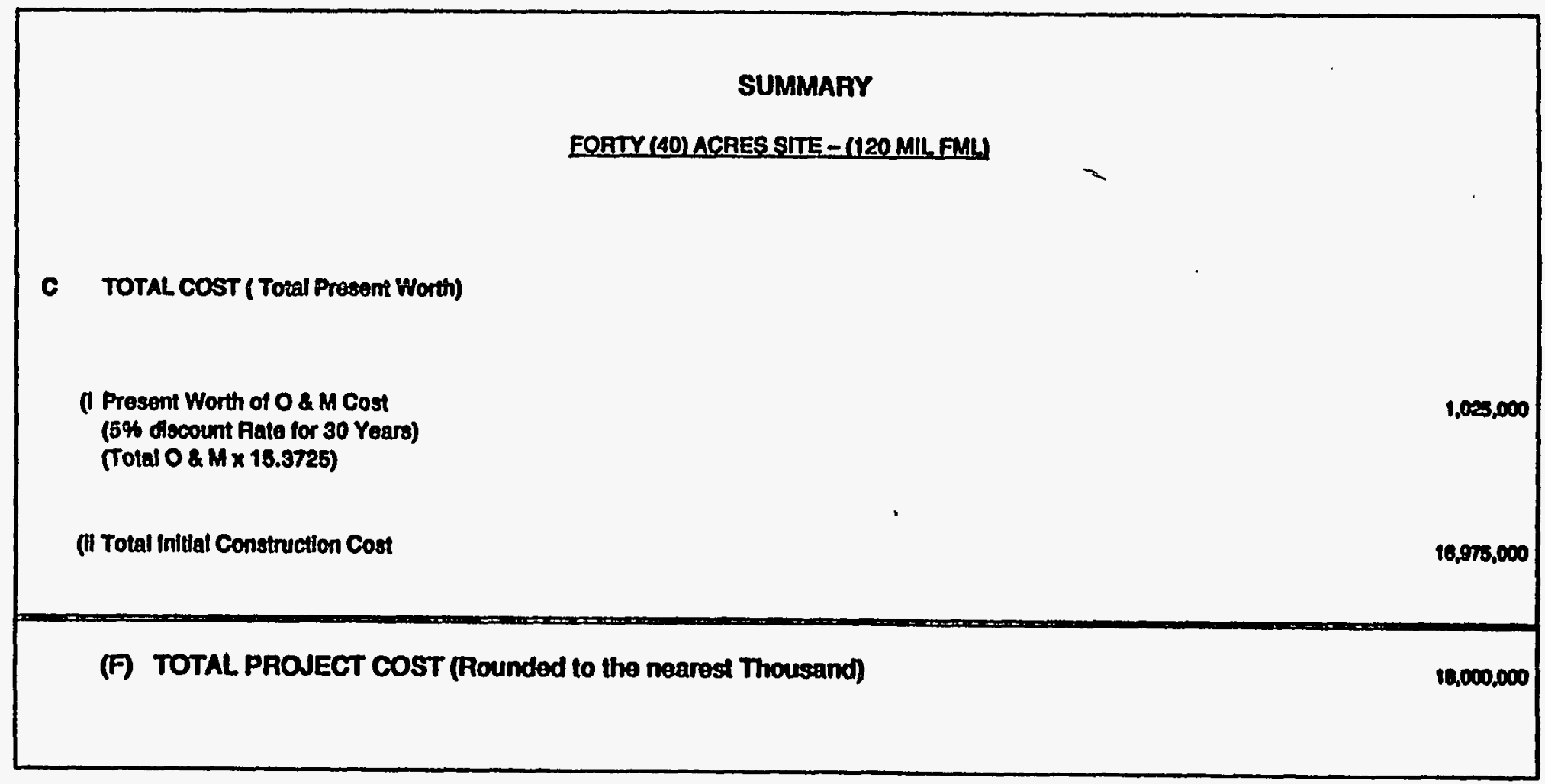

I-4. Cost Estimate for a Cover System Utilizing 120-mil High Density Polyethylene (HDPE) Liner (Cont'd) 


\section{DETAIL ESTIMATE}

$1001:$ :-03

ACTTVTY CODE: ESE26O412

PROJ.NAMB: ECON. EVALUATION CLOSURB CAP BARRIER MATERIAL

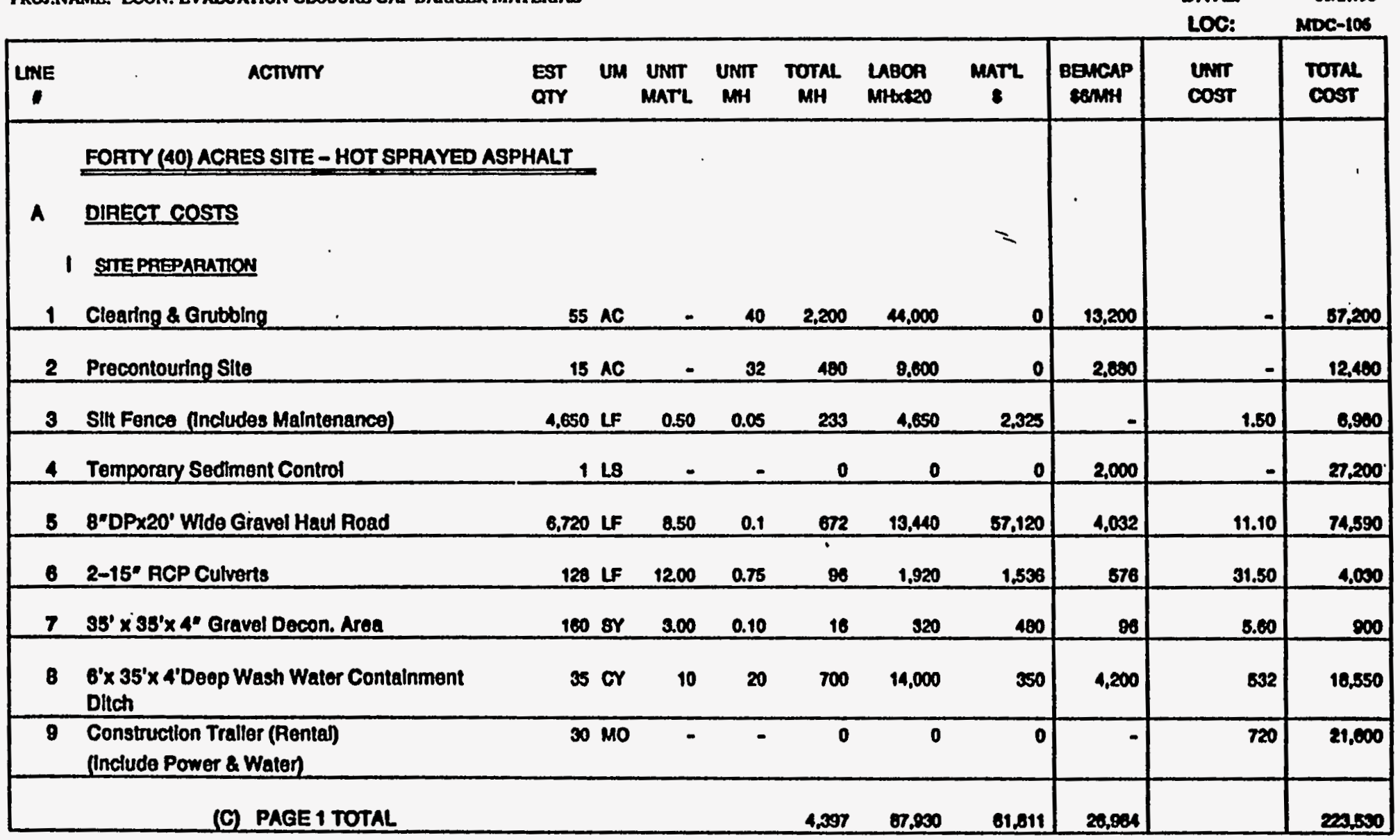

I-5. Cost Estimate for a Cover System Utilizing 1/4-inch Thick Hot Sprayed or Emulsified Asphalt 
DETAIL ESTIMATE

CHKD BY: 8. MEAD

2001: 7-03

ACTIVITY CODE: ESE260012

PROJ.NAME: ECON. BVALUATION CLOSURB CAP BARRIER MATERINL

ESTIMATOR: rawnx

PHONE: 7-5266

DATE: DUPTIS

\begin{tabular}{|c|c|c|c|c|c|c|c|c|c|c|c|}
\hline UNE & ACTVIr & $\begin{array}{l}\text { EST } \\
\text { OTr }\end{array}$ & um & $\begin{array}{l}\text { UNTr } \\
\text { MATL }\end{array}$ & UNT & ToTal & $\begin{array}{l}\text { LABOR } \\
\text { Mitatezo }\end{array}$ & $\operatorname{marL}$ & $\begin{array}{l}\text { egncap } \\
\text { sennt }\end{array}$ & $\operatorname{ung}_{\cos \pi}$ & $\begin{array}{l}\text { Total } \\
\cos t\end{array}$ \\
\hline 10 & $\begin{array}{l}\text { IL_COVER CONSTRUCTION } \\
\text { Wasto Stabilization (Crushod Limestono) }\end{array}$ & 55,757 & & 50 & 0.1 & 5,576 & 111.814 & 2,787,050 & 33,454 & 3200 & $2,002,020$ \\
\hline 11 & $\begin{array}{l}\text { Plece and Compact Foundation Soll } \\
\text { In 8* Lifts to } 95 \% \text { Std. Proctor }\end{array}$ & 395,858 & & - & 0.105 & \$1,565 & 631,302 & 0 & 249,391 & 2.70 & $1,000,090$ \\
\hline 12 & $\begin{array}{l}\text { Applled Hot Asphalt using } 3 \text { passes } \\
\text { at a rate of } 0.5 \text { gal / sY }\end{array}$ & $\begin{array}{l}196,377 \\
297,568\end{array}$ & ON & 2 & 0.13 & 20,769 & 815,700 & 396.754 & 154.734 & 8.40 & 1.007270 \\
\hline 13 & $\begin{array}{l}\text { Placement of } 30 \text { Mill Flextbte } \\
\text { Membrane Lner }\end{array}$ & $1,852,341$ & SF & 0.14 & 0.006 & 11,794 & 234,201 & 273,320 & - & 0.30 & 807,010 \\
\hline 14 & $\begin{array}{l}\text { Placement of } 1^{\prime}-0 \text { Coares Sand } \\
\text { Oralnage Layer }\end{array}$ & 60,126 & or & 10.50 & 0.023 & 1,653 & 30,060 & 094,323 & 9,918 & 11.10 & 751,300 \\
\hline 16 & $\begin{array}{l}\text { Piacement of Geotextile (TYPAF) Fiter on } \\
1^{\prime}-0^{*} \text { Coarse Sand Dralnage Layor }\end{array}$ & 212,426 & sr & 0.12 & 0.005 & 1,002 & 21,243 & 20,491 & - & 0.20 & $\$ 6,730$ \\
\hline 16 & Placement of $1^{\prime}-6^{\circ}$ Common Fill & 98,001 & or & 3.75 & 0.04 & 3,923 & 70,465 & 307,804 & 23.539 & 4.00 & 409,010 \\
\hline 17 & Placoment of 6" Topsoil & 33,000 & or & 9.50 & 0.04 & 1,320 & 20,400 & 315,500 & 7,920 & 10.50 & 347.020 \\
\hline 18 & $\begin{array}{l}\text { Placement of Perlmeter Extertor } \\
\text { Drainage Layer } 3 / 4^{\prime \prime} \times 3^{*} \text { Stone }\end{array}$ & 2,650 & gr & 1.50 & 0.16 & 424 & 0,460 & 0,976 & 2.544 & 8.70 & 18,000 \\
\hline 10 & Excavation of Trapezoldal Drainage Ditch & 39,024 & or & - & 0.15 & 8,974 & 119,472 & 0 & 35,812 & 2.00 & 155,310 \\
\hline & (C) PAGE 2 TOTAL & & & & & 99,000 & 1,079,099 & 4,003,0es & B17,345 & & $7300 \times 00$ \\
\hline
\end{tabular}

I-5. Cost Estimate for a Cover System Utilizing 1/4-inch Thick Hot Sprayed or Emulsified Asphalt (Cont'd) 
D B T A I B S T I M T B

CHKD BY: S. MEAD

Lo0 : : 7-03

ACTIVITY CODE: ESE26012

PROJ.NAME: ECON. BVALUATION CLOSURB CAP BARRIER MATERANL

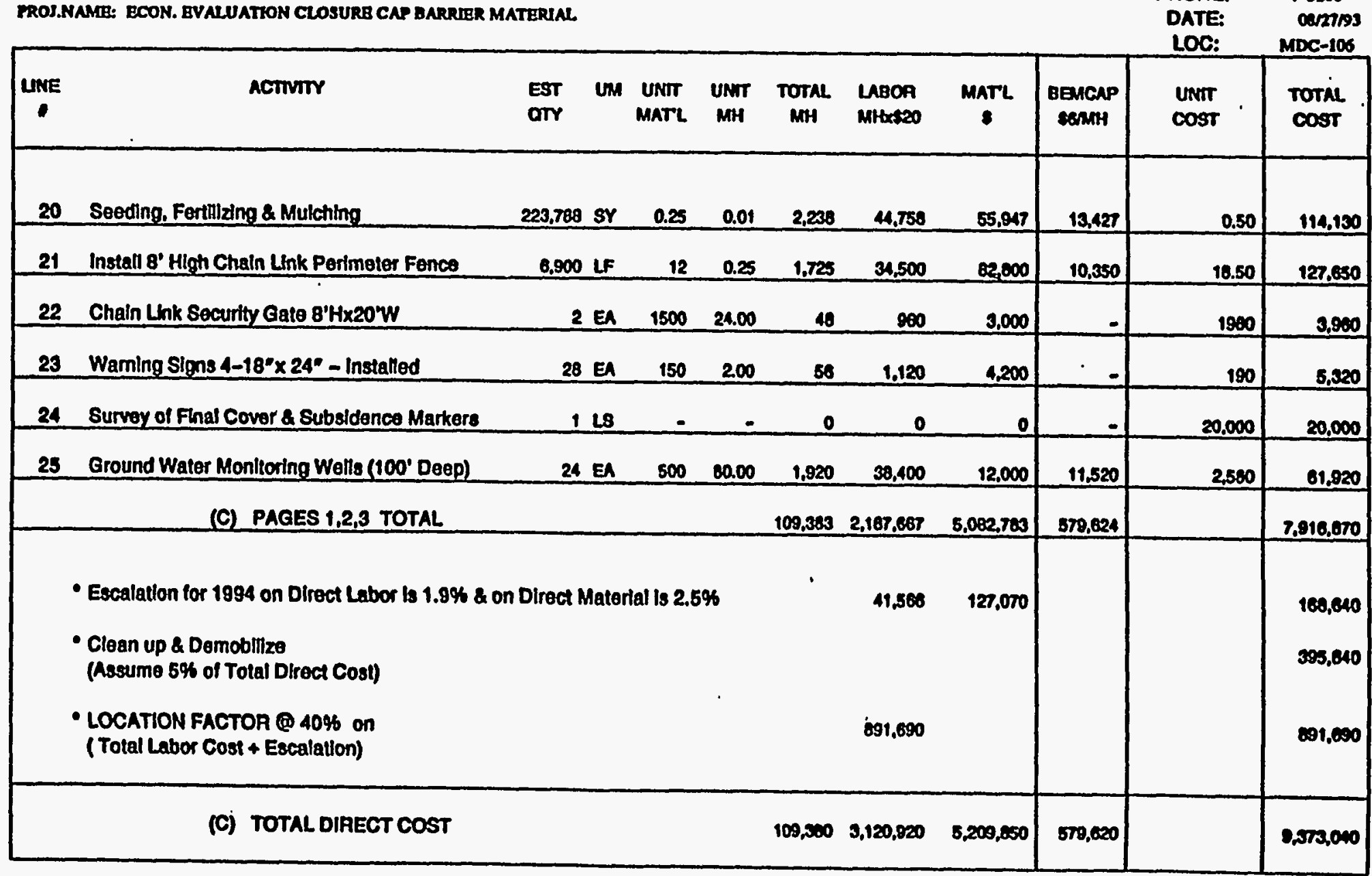

I-5. Cost Estimate for a Cover System Utilizing 1/4-inch Thick Hot Sprayed or Emulsified Asphalt (Cont'd) 


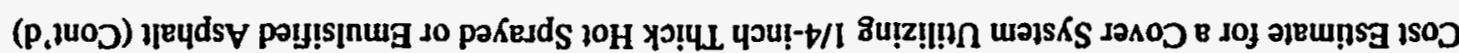

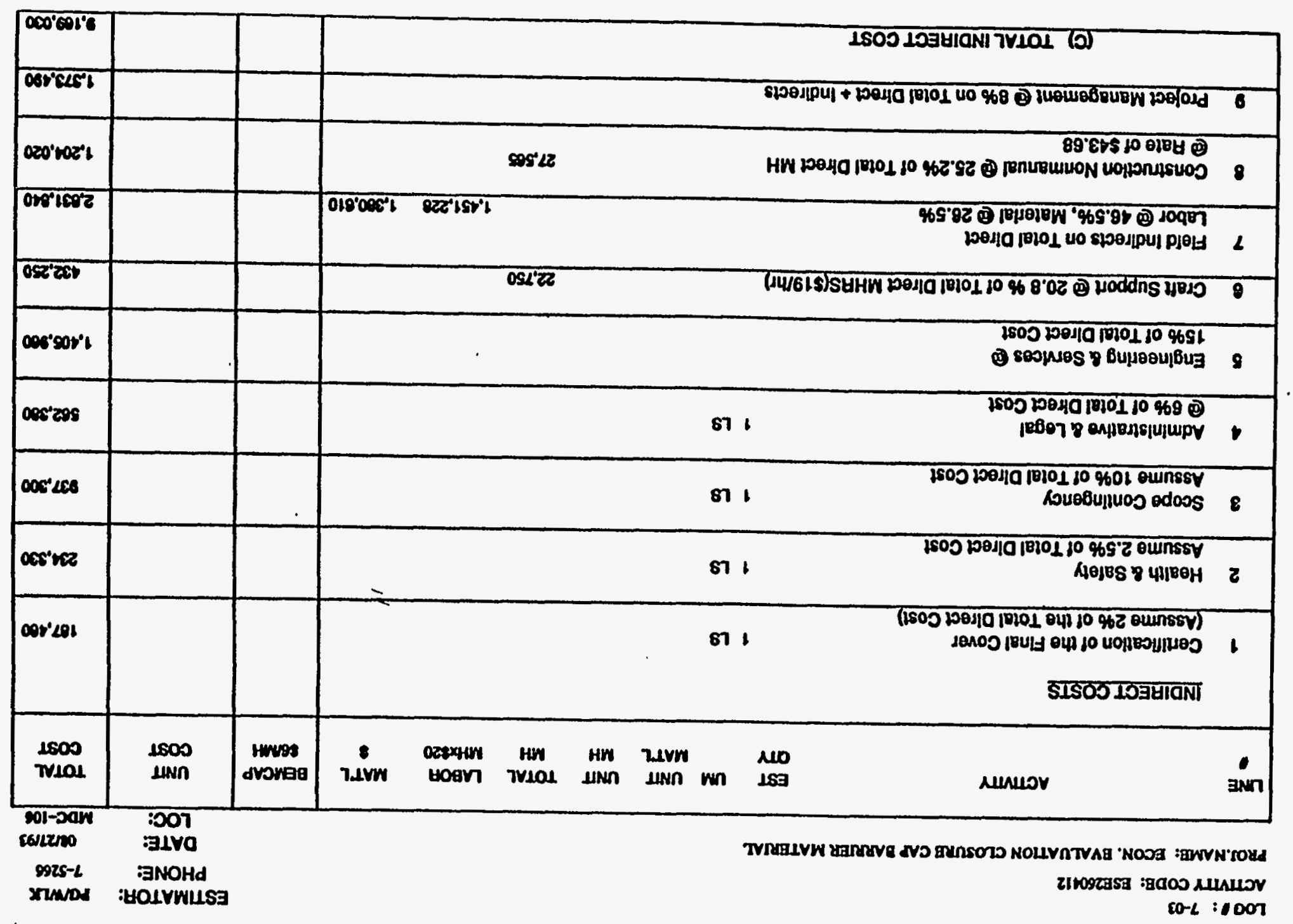

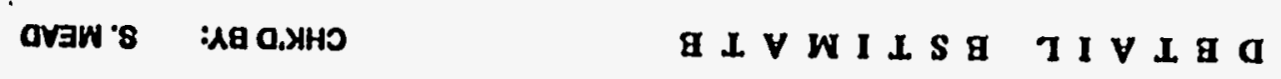




\section{B TAIL BSTIMATE}

\section{MEAD}

$1001: 7-03$

ACTIVTYY CODE: ESE260412

PROJ.NAME: ECON. EVALUATTON CLOSURB CAP BARRIER MATERIAL.

GHKD QY: S. MEAD

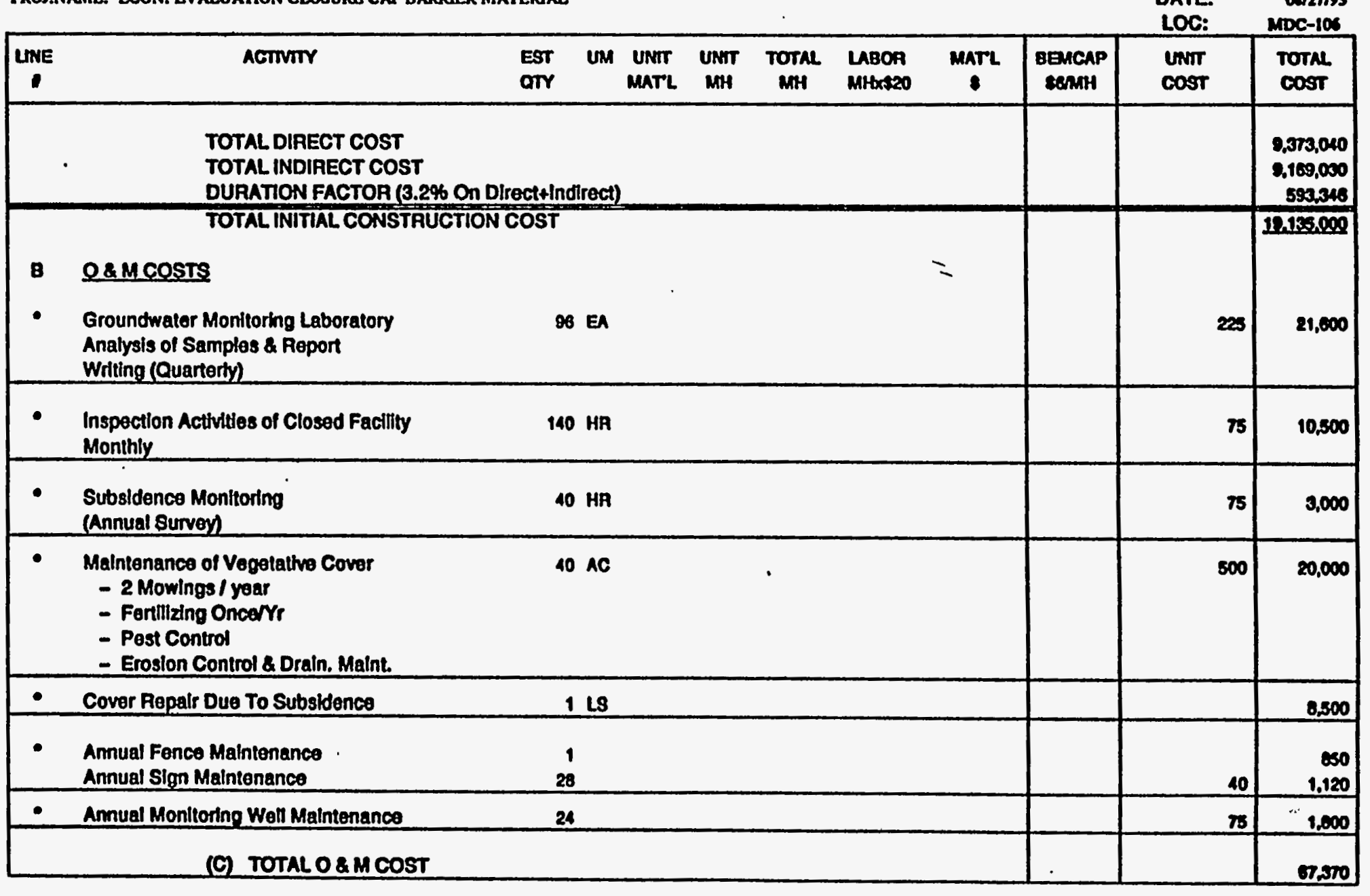

1-5. Cost Estimate for a Cover System Utilizing 1/4-inch Thick Hot Sprayed or Emulsified Asphalt (Cont'd) 
D B TAIL BSTIMAT B

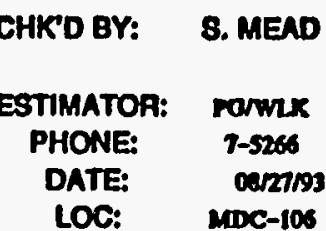

\section{Lo01: 7-03}

ACTTVITY CODE: ESE260N12

FROJ.NAME: ECON. EVALUATTON CLOSURB CAP BARRIER MATERIAL

LOC:

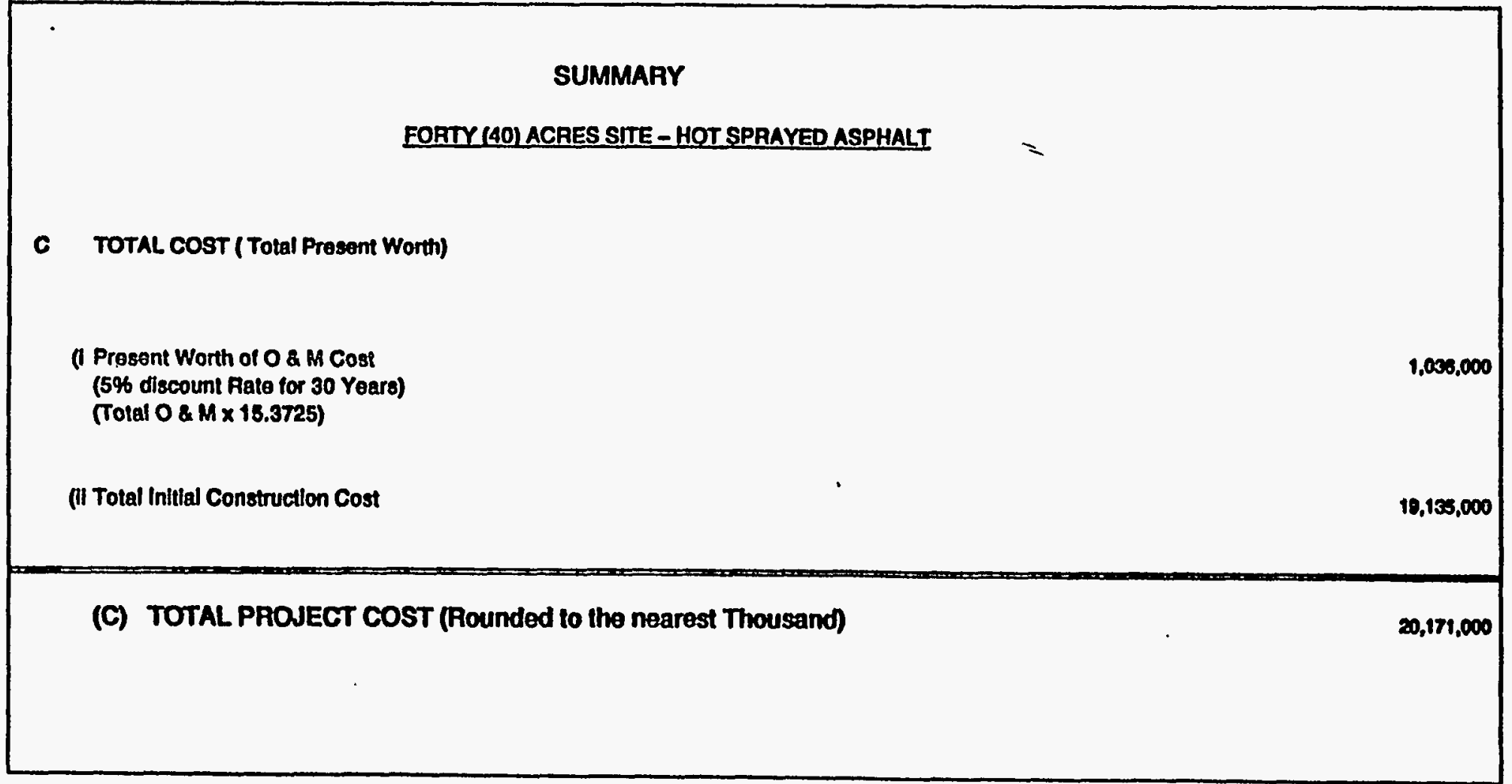

1-5. Cost Estimate for a Cover System Utilizing 1/4-inch Thick Hot Sprayed or Emulsified Asphalt (Cont'd) 
DETAIL ESTIMATE

LOG : : 7-03

CHK'D BY: S. MEAD

ACTIVITY CODE: ESE260412

PROJ.NAME: ECON. EVALUATION OF CLOSUAE CAP BARRIER MATERIAL

ESTIMATOR: PQMUKK

\begin{tabular}{|c|c|c|c|c|c|c|c|c|c|c|c|}
\hline WEE & - activir & $\begin{array}{l}\text { EST } \\
\text { OTY }\end{array}$ & & $\begin{array}{l}\text { UNTI } \\
\text { MATL }\end{array}$ & UNIT & $\begin{array}{c}\text { TOTM } \\
\text { MH }\end{array}$ & $\begin{array}{l}\text { LABOA } \\
\text { MHtrateO }\end{array}$ & $\stackrel{\text { MATL }}{?}$ & $\begin{array}{l}\text { Bencap } \\
\text { sennH }\end{array}$ & $\begin{array}{l}\text { Unist } \\
\text { cost }\end{array}$ & $\begin{array}{l}\text { TOTAL } \\
\text { COST }\end{array}$ \\
\hline & $\begin{array}{l}\text { FOATY (40) ACAES SITE - REINFORC } \\
\text { DIRECT COSTS } \\
\text { STIE PREPARATION } \\
\text { Clearng \& Grubbing }\end{array}$ & ED ASP & $\stackrel{\text { HALT }}{=}$ & - & 10 & 2,200 & 44,000 & $=$ & 13,200 & - & 37,200 \\
\hline & Procontouring Site & 15 & AC & - & 32 & 400 & 9.600 & 0 & 2.000 & - & 12,400 \\
\hline & Sili Fonce (includes Maintenance) & 4,650 & LF & 0.50 & 0.05 & 233 & 4.650 & 2,325 & - & 1.50 & 0.900 \\
\hline & Temporary Sediment Controt & 1 & LS & - & - & 0 & 0 & 0 & 2,000 & - & 27,200 \\
\hline 5 & 20' Whoe Gravel Haul Road X 8" Deep & 6.720 & LF & 0.50 & 0.1 & 672 & 13,440 & 87,120 & 4.032 & 11.10 & 74,590 \\
\hline 6 & 2-15" RCP Culverts & 128 & LLF & 1200 & 0.75 & e & 1.920 & 1.530 & 576 & 31.50 & 4.000 \\
\hline 7 & $35^{\prime} \times 35^{\prime} \times 4^{\prime \prime}$ Gravel Decon, Area & 160 & $8 Y$ & 3.00 & 0.10 & 16 & 320 & 400 & 80 & 8.00 & 000 \\
\hline 8 & $\begin{array}{l}8^{\prime} \times 35^{\prime} \times 4^{\prime} \text { Deep } \\
\text { Wash Water Containment Dilech }\end{array}$ & 35 & or & 10 & 20 & 700 & 14,000 & 350 & 4,200 & 630 & 10,550 \\
\hline & $\begin{array}{l}\text { Construction Traller (Rental) } \\
\text { (Include Power \& Water) }\end{array}$ & 30 & MO & - & - & 0 & 0 & 0 & - & $r 20$ & 21,000 \\
\hline & (D) PAGE 1 TOTAL & & & & & 4,397 & 87,930 & 01,811 & 20,904 & & 202500 \\
\hline
\end{tabular}

I-6. Cost Estimate for a Cover System Utilizing Reinforced Sprayed Asphalt 
DETAIL ESTIMATE

CHK'D BY: 8. MEAD

LOG : : 7-03

ACTIVTY CODE: ESE260412

PROU.NAME: ECON. EVALUATION OF CLOSURE CAP BARRIER MATERIAL

ESTIMATOR: PGMLK

PHONE: 7-5268

\begin{tabular}{|c|c|c|c|c|c|c|c|c|c|c|c|}
\hline LWE & ACTivitr & $\begin{array}{l}\text { EST } \\
\text { OTY }\end{array}$ & $\ln$ & $\begin{array}{l}\text { UNIT } \\
\text { MATL }\end{array}$ & $\underset{M H}{\text { UNT }}$ & TOTML & $\begin{array}{l}\text { LABOA } \\
\text { Mathet20 }\end{array}$ & MaTL & BENCAP & $\begin{array}{l}\text { UNT } \\
\cos \end{array}$ & $\begin{array}{l}\text { TOTAL } \\
\text { COST }\end{array}$ \\
\hline & II COVER CONSTAUCTION & & & & & & & & & & \\
\hline 10 & Wasto Stabllization (Crushed Limestone) & 55,757 & $\mathbf{T N}$ & so & 0.1 & 5,578 & 111.514 & $2,787,850$ & 33,454 & $\$ 2.00$ & $2,932,020$ \\
\hline 11 & $\begin{array}{l}\text { Place and Compact Foundation Soll } \\
\text { in 8* Litts to } 95 \% \text { 8td. Proctor }\end{array}$ & 395,058 & or & - & 0.105 & 41,563 & 031,302 & o & 249,391 & 270 & $1,000,690$ \\
\hline 12 & $\begin{array}{l}\text { Placement of } 30 \text { Mil Flexible } \\
\text { Membrane Lner }\end{array}$ & $1,952,341$ & SF & 0.14 & 0.006 & 11,714 & 234,281 & 273,328 & - & 0.30 & $\$ 07.010$ \\
\hline 13 & $\begin{array}{l}\text { Placement of Geotextile Relnforcement } \\
\text { on Foundation Layer }\end{array}$ & 198,377 & sr & 0.28 & 0.005 & 892 & 19,630 & 51,570 & - & 0.40 & 71,420 \\
\hline 14 & $\begin{array}{l}\text { Appiled Hol Asphat using } 3 \text { passes at a rate } \\
\text { of } 0.5 \text { gal / SY for a total of } 1.5 \mathrm{~g} \text { USy }\end{array}$ & $\begin{array}{l}198,377 \\
297,568\end{array}$ & $\begin{array}{l}\text { SY } \\
\text { OAL }\end{array}$ & 200 & 0.13 & 25,789 & 515,700 & 390,754 & 134,734 & 8.40 & $1,067,270$ \\
\hline 15 & $\begin{array}{l}\text { Placement of 1'-0 Coarse Sand } \\
\text { Drainage Layer }\end{array}$ & 66,126 & or & 10.50 & 0.025 & 1.053 & 33,063 & 694,323 & 0,919 & 11.10 & 737,300 \\
\hline 16 & $\begin{array}{l}\text { Placement of Geotextlie (TYPAR) Fitter on } \\
1^{1}-0^{*} \text { Coarse Sand Orainage Layer }\end{array}$ & 212,426 & 8r & 0.12 & $0.005^{\circ}$ & 1,062 & 21,213 & 25.491 & - & 0.20 & 46,730 \\
\hline 17 & Placement of $9^{\prime}-6^{*}$ Common Fil & 98,081 & or & 0.75 & 0.04 & 9,923 & 70,465 & 307,004 & 20,539 & 4.00 & 409,810 \\
\hline 18 & Placement of 6" Topsoil & 33,000 & or & 9.50 & 0.04 & 1,320 & 28.400 & 313,500 & 7,020 & 10.50 & 347,020 \\
\hline 19 & $\begin{array}{l}\text { Placement of Perimeter Exterlor } \\
\text { Oranage Layer } 3 / 4^{*} \times 3^{*} \text { DP (Crushod Stone) }\end{array}$ & 2.650 & sy & 1.50 & 0.10 & 424 & 8,400 & 3,975 & 2,544 & 8.70 & 15.000 \\
\hline 20 & Excavation of Trapezoldal Drainage Ditch & 39,824 & or & - & 0.15 & 5,974 & 818,472 & 0 & 35,042 & 3.00 & 155,310 \\
\hline & (D) PAGE TOTAL & & & & & 09.992 & $1,999,007$ & $4,914,003$ & 817,343 & & $7,431,700$ \\
\hline
\end{tabular}

I-6. Cost Estimate for a Cover System Utilizing Reinforced Sprayed Asphalt (Cont'd) 
DETAIL ESTIMATE CHKDBY: S.MEAD

LOQ: : 7-03

ACTIVITY COOE: ESE260412

PROJ.NAME: ECON. EVALUATION OF CLOSURE CAP BARRIER MATERIAL

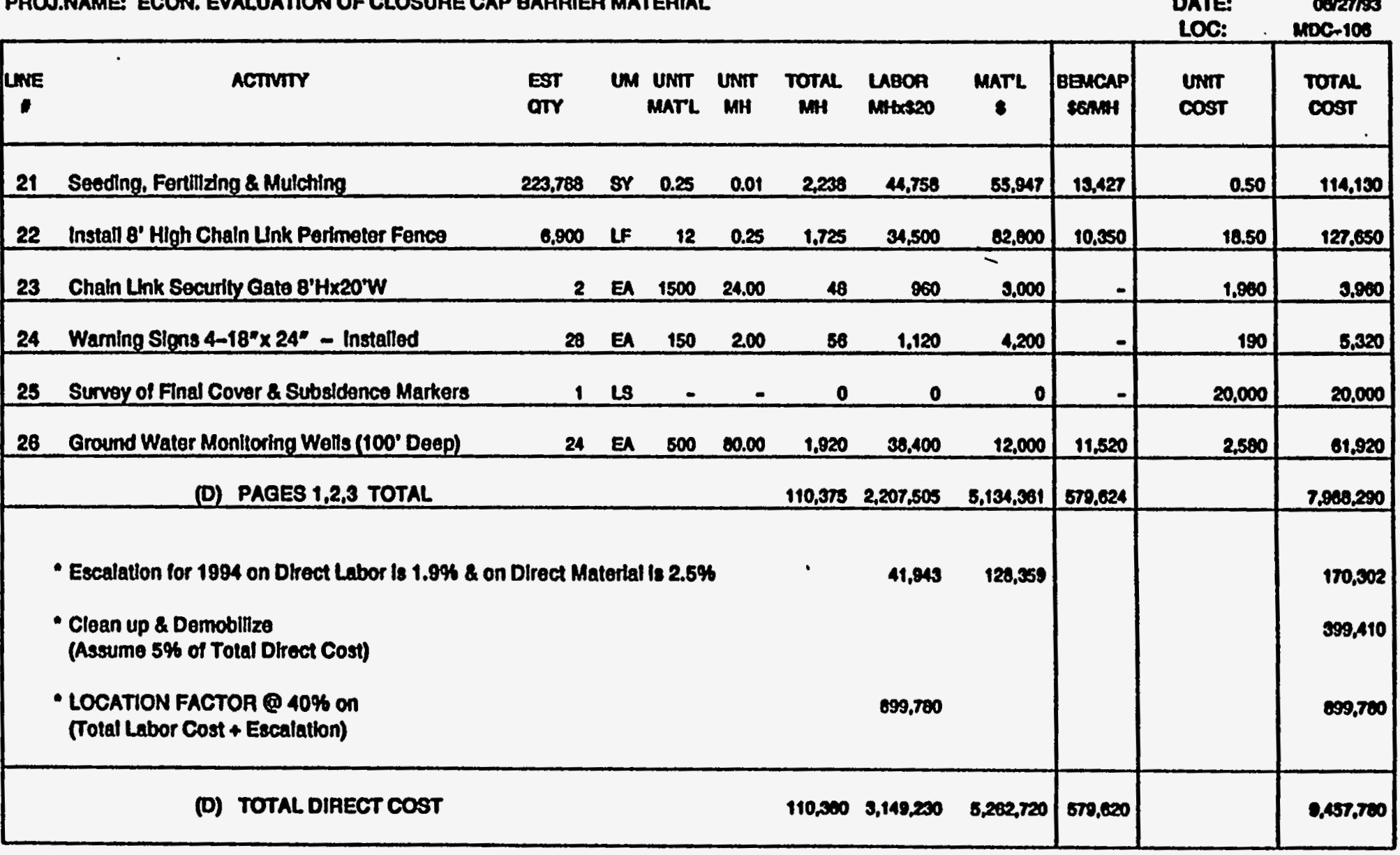

I-6. Cost Estimate for a Cover System Utilizing Reinforced Sprayed Asphalt (Cont'd) 
DETAIL ESTIMATE

CHK'D BY: 8. MEAD

LOQ : : 7-03

ACTIVITY CODE: ESE260412

PAOJ.NAME: ECON. EVALUATION OF CLOSURE CAP BARRIER MATERIAL

ESTIMATOR: PaMnK

PHONE: 7-5260

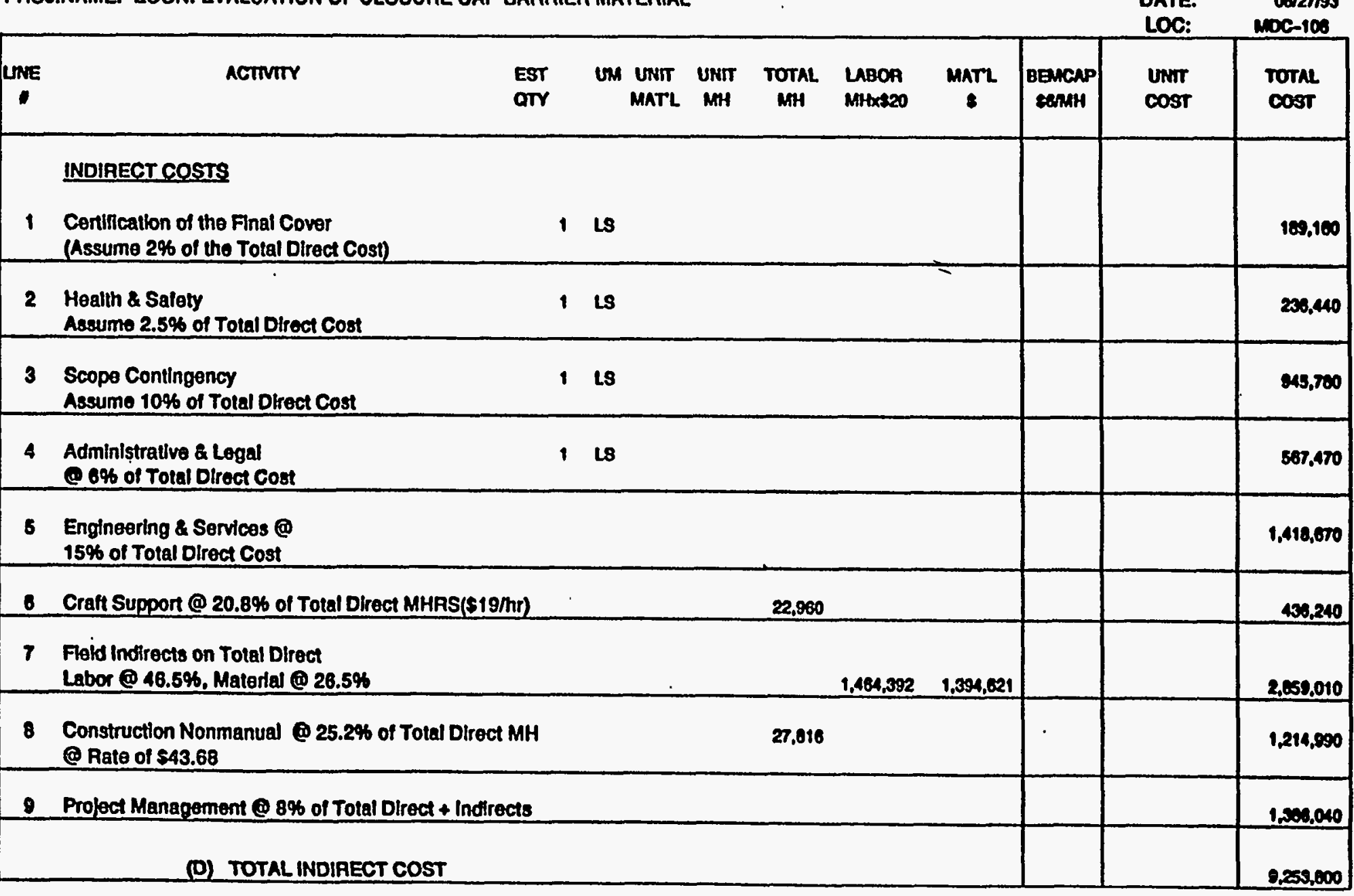

1-6. Cost Estimate for a Cover System Utilizing Reinforced Sprayed Asphalt (Cont'd) 
DETAIL ESTIMATE

8. MEAO

LOQ : : 7-03

ACTIVITY CODE: ESE260412

PROJ.NAME: ECON. EVALUATION OF CLOSUAE CAP BARAIEA MATERIAL

\begin{tabular}{|c|c|c|c|c|c|c|c|c|c|c|}
\hline LWE & Activitr & $\begin{array}{l}\text { EST } \\
\text { OTY }\end{array}$ & $\begin{array}{l}\text { UM UNT } \\
\text { MATL }\end{array}$ & $\begin{array}{l}\text { UNT } \\
\text { MHI }\end{array}$ & TOTAL & $\begin{array}{l}\text { LABOA } \\
\text { MH+x+20 }\end{array}$ & $\stackrel{\text { MATL }}{:}$ & $\begin{array}{l}\text { BEMCAP } \\
\text { senni }\end{array}$ & $\begin{array}{l}\text { LOG: } \\
\text { UnT } \\
\text { Cost }\end{array}$ & $\begin{array}{l}\text { MDC-106 } \\
\text { TOTAL } \\
\text { COST }\end{array}$ \\
\hline & $\begin{array}{l}\text { TOTALDIAECT COST } \\
\text { TOTAL INDIRECT COST } \\
\text { DURATION FACTOA } 13 .\end{array}$ & ct+indire & & & & & & & & $\begin{array}{r}9, .557,700 \\
9,255,000 \\
590, \pi 71\end{array}$ \\
\hline B & $\begin{array}{l}\text { TOTAL INITIAL CONSTF } \\
\text { O\&M COSTS } \\
\text { Groundwater Monitoring Laboratory } \\
\text { Analysis of Samples \& Report } \\
\text { Writting Quarterty) }\end{array}$ & SST & EA & & & & $=$ & & 223 & $19,310,000$ \\
\hline & $\begin{array}{l}\text { Inspection Activties of Closed Facillty } \\
\text { Monthly. }\end{array}$ & 140 & HR & & & & & & 75 & 10.500 \\
\hline - & $\begin{array}{l}\text { Maintenance of Vegetative Cover } \\
\text { - } 2 \text { Mowings / year } \\
\text { - Fertillzing OncerYear } \\
\text { - Pest Control } \\
\text { - Erosion Control \& Drain. Maint. }\end{array}$ & 10 & $A C$ & & & & & & 500 & 20,000 \\
\hline$\bullet$ & Cover Repalr Due To Subsidence & 1 & LS & & & & & & & 9,000 \\
\hline & $\begin{array}{l}\text { Annual Fence Maintenance } \\
\text { Annual Sign Maintenance }\end{array}$ & $\begin{array}{r}1 \\
28 \\
\end{array}$ & & & & & & & 40 & $\begin{array}{r}850 \\
1.120\end{array}$ \\
\hline - & Annual Monltoring Well Mainténance & 24 & & & & & & & 75 & 1,000 \\
\hline - & Subsidence Monitoring (Annual Survey) & 10 & HA & & & & & & 78 & 0,000 \\
\hline & (D) TOTAL O A M COST & & & & & & & & & ordere \\
\hline
\end{tabular}

1-6. Cost Estimate for a Cover System Utilizing Reinforced Sprayed Asphalt (Cont'd) 
DETAIL ESTIMATE

\section{LOG : : 7-03}

ACTIVITY CODE: ESE260412

PROS.NAME: ECON. EVALUATION OF CLOSUAE CAP BARRIER MATEAIAL

\begin{tabular}{|c|c|}
\hline CHKO BY: & 8. MEAD \\
\hline $\begin{array}{l}\text { ESTIMATOA: } \\
\text { PHONE: } \\
\text { DATE: } \\
\text { LOC: }\end{array}$ & $\begin{array}{c}\text { Pami } \\
7-5200 \\
00 / 27100 \\
\text { MDC-106 }\end{array}$ \\
\hline
\end{tabular}

\section{SUMMARY}

EORTY (40) ACRES SITE-REINFORCED SPAAYED ASPHALT

C TOTAL COST ( Total Present Worth)

(1 Present Worth of O \& $M$ Cost

(5\% discount Rate for 30 Years)

$1,045,000$

(Total O\&M $M$ 16.3725)

(il Total Initial Construction Cost

$10,310,000$

(D) TOTAL PROJECT COST (Rounded to the nearest Thousand)

$20,305,000$

1-6. Cost Estimate for a Cover System Utilizing Reinforced Sprayed Asphalt (Cont'd) 
DETAIL ESTIMATE

LOO I: 7-03

ACTIVTTY CODE: ESEESOA12

RROJ.NAMG: ECON. GVALUATTON OF CLOSURB CAP BARRIBR MATERIAL.

CHK'D BY: 8. MEAD

LINE

-

ACTIVIV

MAT

UNIT UNT
MATL

FORTY (40) ACAES SITE-2'-0' COMPACTED CLAY (OFF SITE

A I DIAECT COSTS

1 SIIEPEAPAPATION

1 Claaring \& Grubbing

85 AC

$40 \quad 2,200$

44,000

2 Procontouring Silte

$15 \wedge C$

3 Silt Fance (inctudes Maintenance)

4650

4 Temporary Sediment Control

$1 \mathrm{Lg}$

5 B"DP X 20' Wide Gravel Haul Road

\begin{tabular}{rrrrr}
8,720 LF & 8.50 & 0.1 & 672 & 19,440 \\
\hline
\end{tabular}

6 2-15* RCP Culverts

128 If $12.00 \quad 0 . \pi$

$7 \quad 35^{\prime} \times 35^{\prime} \times 4^{\prime \prime}$ Gravei Decon. Area

$\begin{array}{lllll}100 & 8 Y & 3.00 & 0.10 & 10\end{array}$

6 6'x 35'x 4'Deop

Wash Water Containment Ditch

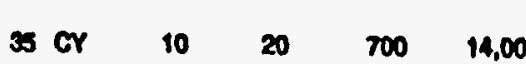

, 000

Construction Traller (Rental)

(Include Power \& Waten)

(6) PAge 1 TOTAL

30 MO

0

$4,397 \quad 87.950$

61,811 20,904

ESTIMATOR: rown

PHONE: 7-5205

DATE: Gerss

LOC: NDO-105

$\begin{array}{ll} & \end{array}$

\begin{tabular}{|l|l|l}
$\sin \mid \cos t$ & $\cos$ \\
\hline
\end{tabular}

1-7. Cost Estimate for a Cover System Utilizing Compacted Kaolin (Offsite) Clay and a 30-mil FML 
DETAIL ESTIMATE

CHK'D BY: 8. MEAO

Lo0: $1-03$

ACTIVITY CODB: ESB260412

PROJ.NAME: ECON. EVALUATTON OF CLOSURE CAP BARRIER MATERIAL. OSTOS/93

\section{ESTIMATOR: NownX}

PHONE: 7-5266

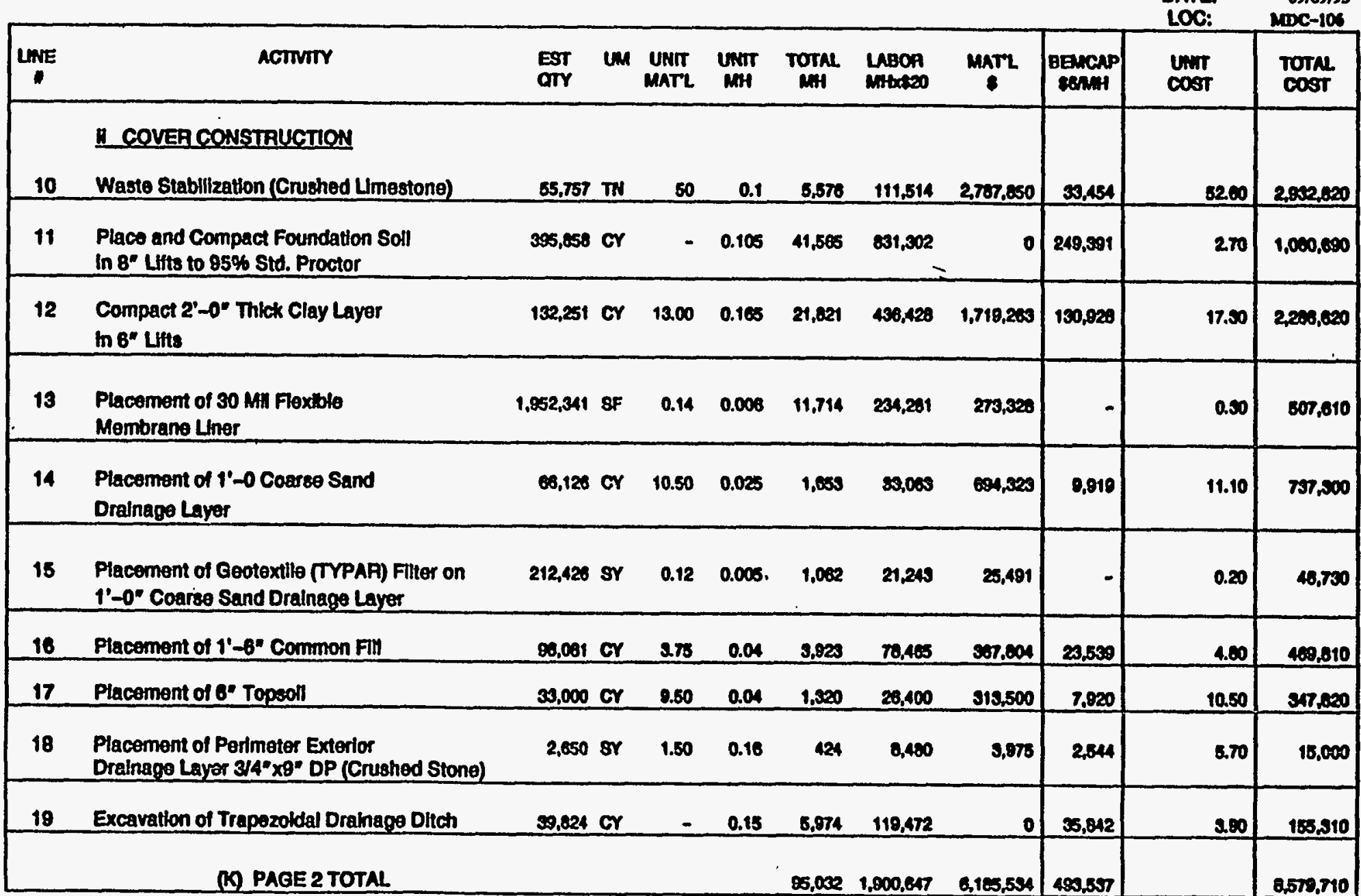

I-7. Cost Estimate for a Cover System Utilizing Compacted Kaolin (Offsite) Clay and a 30-mil FML (Cont'd) 
DETAIL ESTIMATE

CHKD BY: 8. MEAD

LO01: 7-03

ACTIVITY CODE: ESEZOON12

PROS.NAME: ECON. EVALUATTON OF CLOSURE CAP BARRIER MATERIAL

ESTIMATOA: MaNiLX

PHONE: 7-5250

DATE:

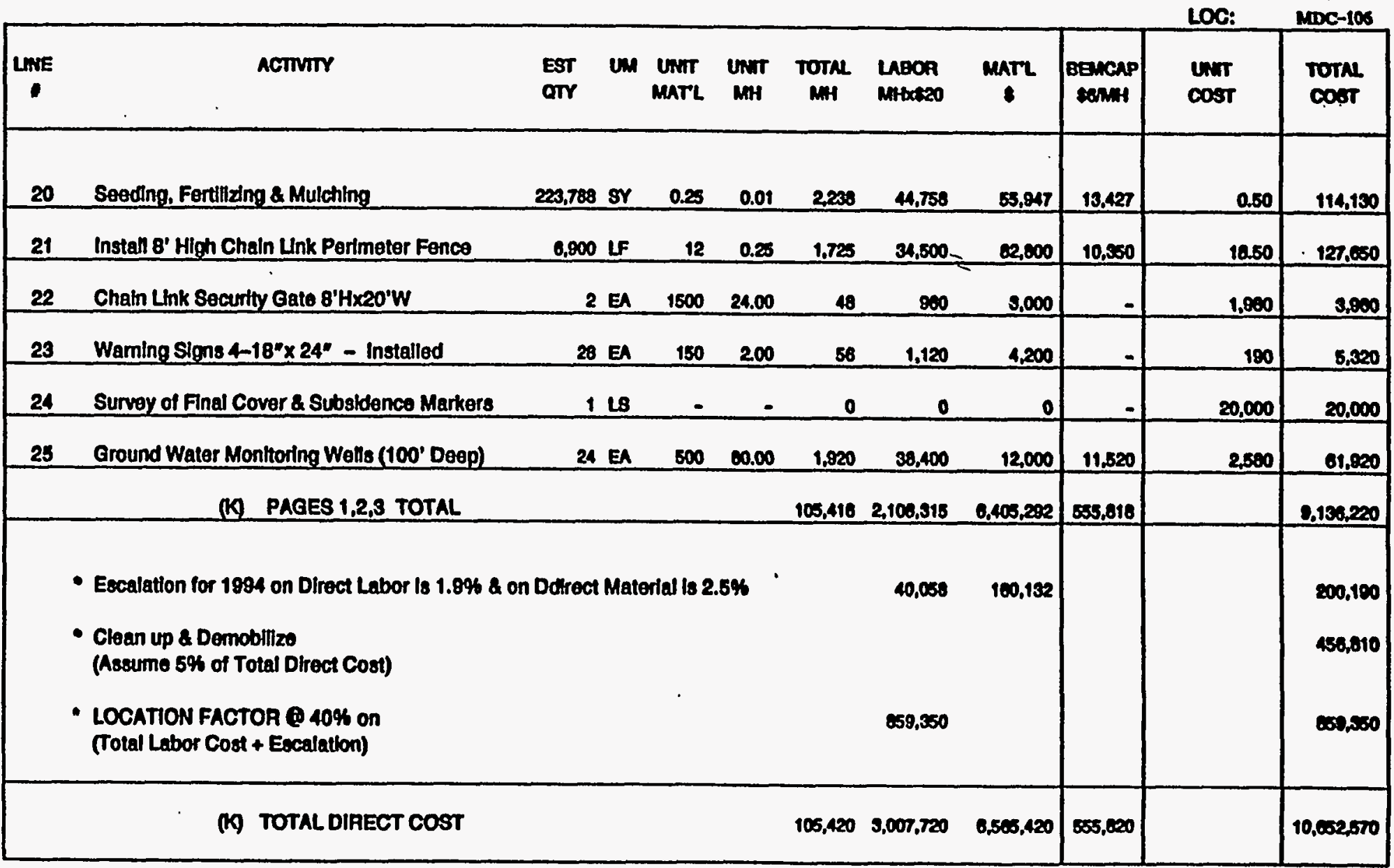

1-7. Cost Estimate for a Cover System Utilizing Compacted Kaolin (Offsite) Clay and a 30-mil FML (Cont'd) 
DETAIL ESTIMATE

\section{LOO 1: 7-03}

ACTTVITY CODF: PSE260112

FROI.NAME: ECON. EVALUATION OF CLOSURE CAP BARRIER MATERIAL

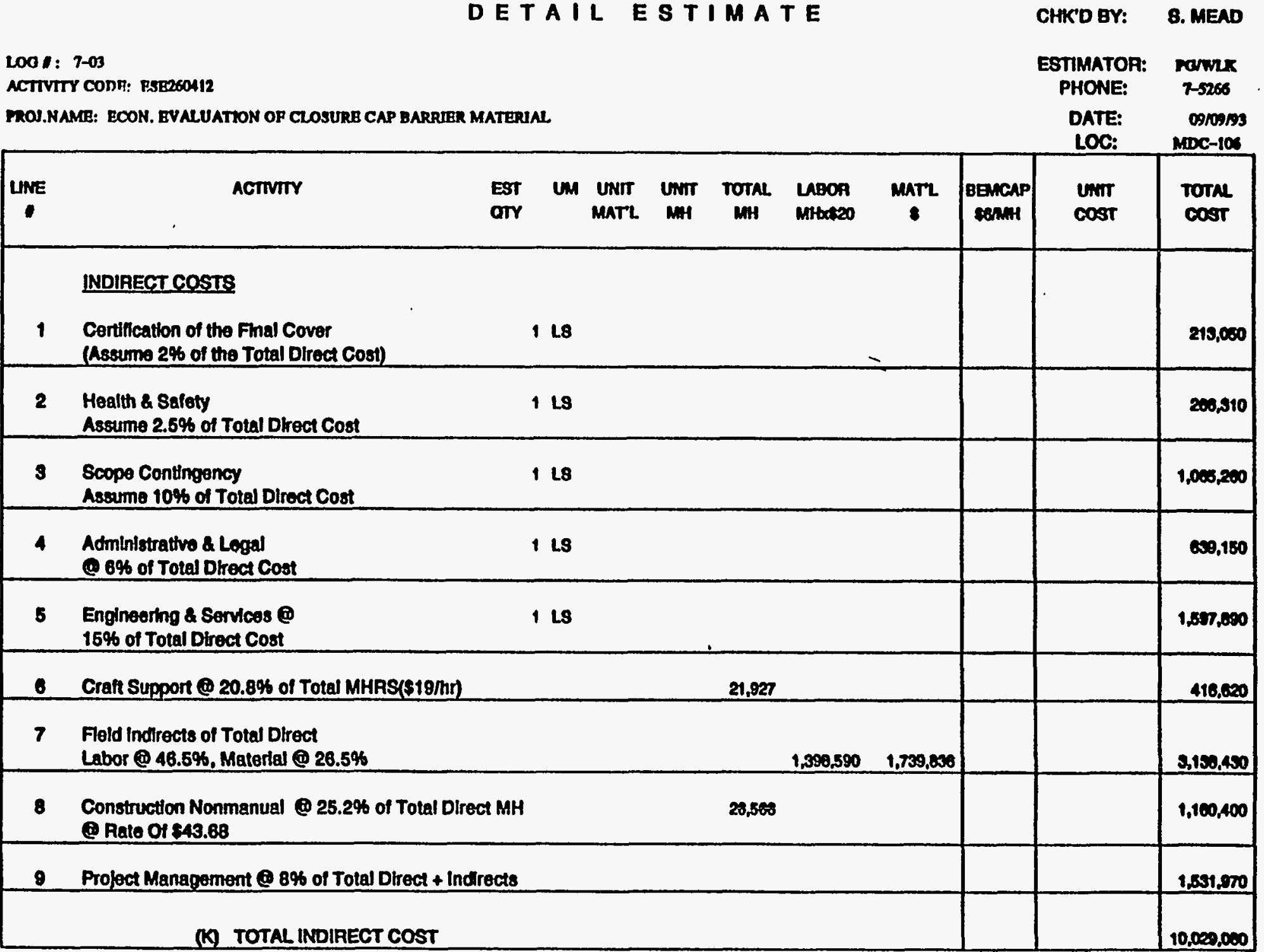

I-7. Cost Estimate for a Cover System Utilizing Compacted Kaolin (Offsite) Clay and a 30-mil FML (Cont'd)
CHKO BY: 8. MEAD

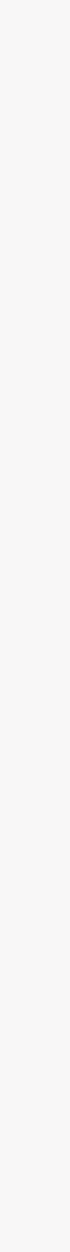


DETAIL ESTIMATE

CHK'D BY: S. MEAD

LoO I: $7-03$

ACTIVITY CODE: ESE260412

PROJ.NAME: BCON. EVALUATTON OR CLOSURB CAP BARRIER MATERIAL

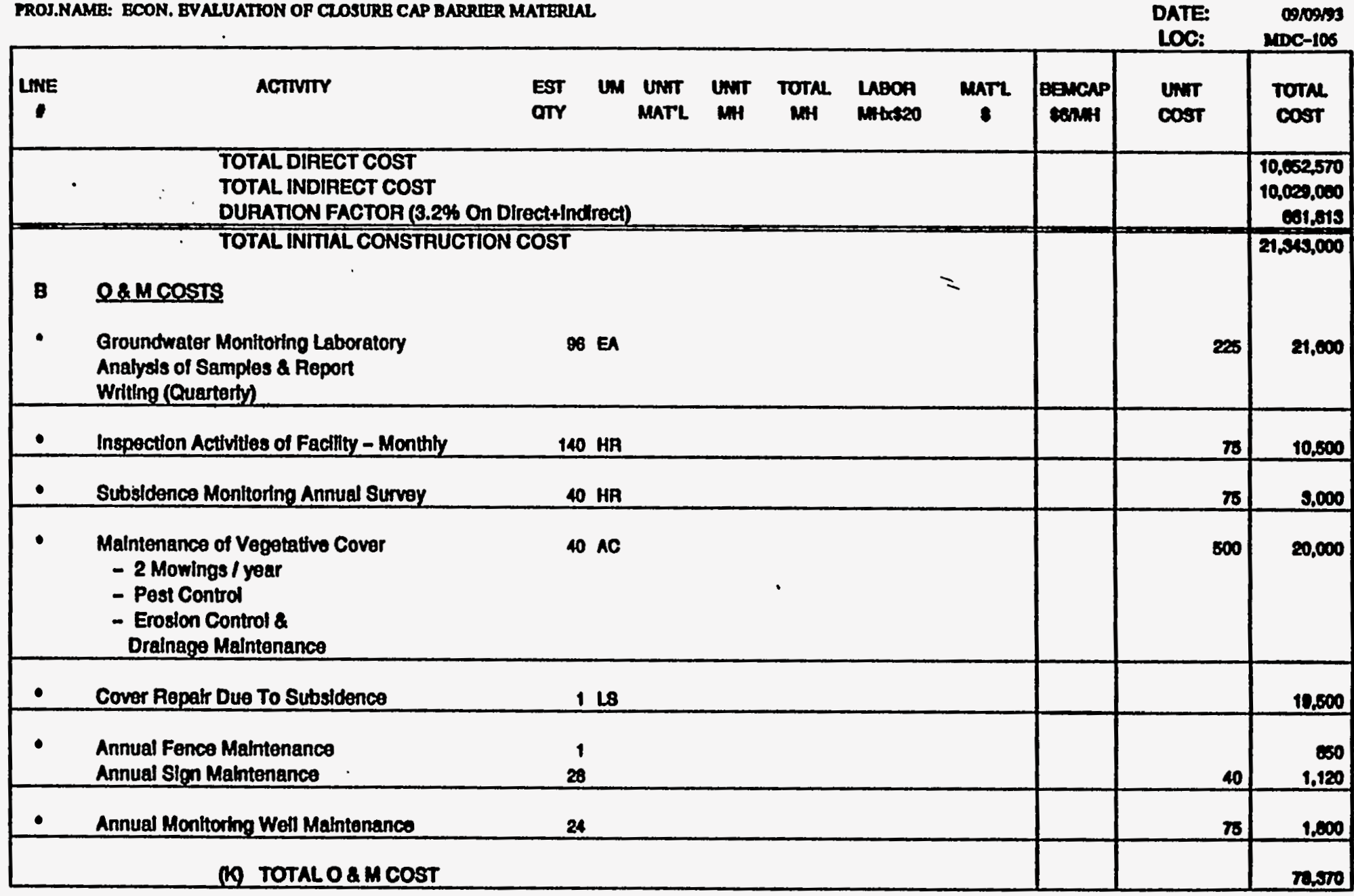

I-7. Cost Estimate for a Cover System Utilizing Compacted Kaolin (Offsite) Clay and a 30-mil FML (Cont'd) 
DETAIL ESTIMATE

\begin{tabular}{|c|c|}
\hline SHK'D BY: & 8. MEAD \\
\hline & \\
\hline $\begin{array}{l}\text { PHONE: } \\
\text { DATE: } \\
\text { LOC: }\end{array}$ & $\begin{array}{c}7-5266 \\
0905 / 93 \\
200 C-106\end{array}$ \\
\hline
\end{tabular}

\section{L001: 7-03}

ACTIVITY CODB: ESB260412

PROJ.NAMR: ECON. EVALUATION OF CLOSURB CAP BARRIER MATERIAL

MDC-100

\section{SUMMARY}

\section{FORTY (40) ACAES SITE - 2'-0' COMPACTED CLAY IOFF SITE}

C TOTAL COST ( Total Present Worth)

(I) Present Worth of O\& M Cost

(5\% olscount Pate for 30 Yeare)

$1,200,000$

(Total O\& M X 15.3725)

(ii) Total Inttial Construction Cost

(K) TOTAL PROJECT COST (Rounded to the nearest Thousand)

20580000

I-7. Cost Estimate for a Cover System Utilizing Compacted Kaolin (Offsite) Clay and a 30-mil FML (Cont'd) 
DETAIL ESTIMATE

Loo : : $7-03$

ACTIVITY CODE: ESE260412

PROJ.NAME: BCON. BYALUATION OF CLOSURE CAP BARRIBR MATERULL

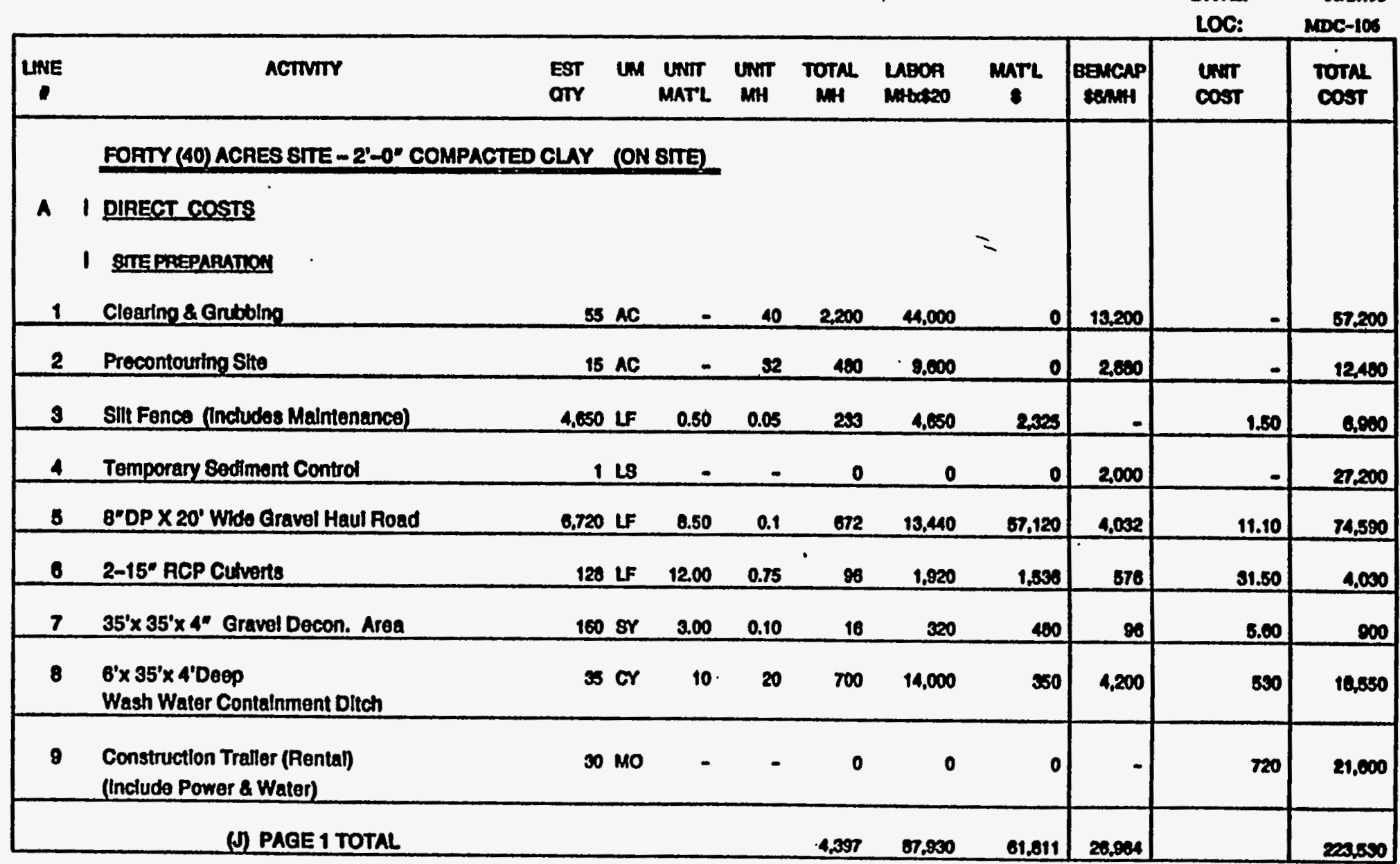

I-8. Cost Estimate a for Cover System Utilizing Compacted Onsite Sandy Clay and a 30-mil FML 
DETAIL ESTIMATE

CHK'D BY: 8. MEAD

Lo01: $7-03$

ACTVITY CODE: BSE2SOS12

PROS. NAME: ECON. BVALUATTON OF CLOSURB CAP BARRRER MATERUAL

\begin{tabular}{|c|c|c|c|c|c|c|c|c|c|c|c|}
\hline UNE & ACTVITY & $\begin{array}{l}\text { EST } \\
\text { OTr }\end{array}$ & um & $\begin{array}{l}\text { UNIT } \\
\text { MATL }\end{array}$ & $\operatorname{unmin}_{\text {nut }}$ & $\underset{M H}{\text { TOTAL }}$ & $\begin{array}{l}\text { LABOA } \\
\text { MHthetzon }\end{array}$ & $\underset{8}{\operatorname{marL}}$ & $\begin{array}{l}\text { Bencap } \\
\text { sennit }\end{array}$ & $\underset{\text { Cost }}{\operatorname{UnT}}$ & $\begin{array}{l}\text { TotAl } \\
\text { cost }\end{array}$ \\
\hline & I COVER CONSTRUCTION & & & & & & & & & & \\
\hline 10 & Waste Stabillzation (Crushod Limestone) & 55,757 & $\mathrm{TN}$ & 50 & 0.1 & 5.570 & 111,514 & $2,707,0000$ & 32,451 & 5200 & 2032000 \\
\hline 11 & $\begin{array}{l}\text { Place and Compact Foundation Soll } \\
\text { in } 8^{\circ} \text { Uns to } 95 \% \text { Std. Proctor }\end{array}$ & 395,850 & or & - & 0.105 & 41,565 & 831,302 & 0 & 249,291 & $2 \pi 0$ & $1,000,000$ \\
\hline 12 & $\begin{array}{l}\text { Compact 2'-0" Thick Clay Layer } \\
\text { In } 8^{*} \text { Lins }\end{array}$ & 132,251 & or & - & 0.165 & 21,021 & 430,428 & 0 & 180,028 & 4.30 & 807,300 \\
\hline 13 & $\begin{array}{l}\text { Placemont of } 30 \text { Mil Fiexible } \\
\text { Membrane Liner }\end{array}$ & $1,952,341$ & SF & 0.14 & 0.006 & 11,714 & 234,281 & 273,320 & - & 0.30 & 507,610 \\
\hline 14 & $\begin{array}{l}\text { Placement of 1'-0 Coaree Sand } \\
\text { Drainage Layer }\end{array}$ & 68,128 & or & 10.50 & 0.025 & 1,053 & 33,083 & 604,323 & 0.810 & 11.10 & 797.300 \\
\hline 16 & $\begin{array}{l}\text { Placement of Geotextlle (TYPAR) Filler on } \\
1^{\prime}-0^{\circ} \text { Coarse Sand Draingge Layer }\end{array}$ & 212,428 & sy & 0.12 & 0.005 . & 1,062 & 21,213 & 25.491 & - & 0.20 & 46,730 \\
\hline 10 & Piacement of $1^{\prime}-0^{\prime \prime}$ Common Fill & 90,001 & or & 3.78 & 0.04 & 9.023 & 70,460 & sor.e04 & 20,8se & 4.00 & $400.010^{\circ}$ \\
\hline 17 & Placement of 6" Topsoll & 33,000 & or & 9.50 & 0.09 & 1.320 & 20,400 & 313,500 & 7.020 & 10.50 & 347,820 \\
\hline 18 & $\begin{array}{l}\text { Placement of Perimeter Exterior } \\
\text { Draingge Layer } 34^{*} \times 3^{*} \text { DP (Crushed Stone) }\end{array}$ & 2.650 & sY & 1.50 & 0.16 & 424 & 8,400 & 3,875 & 2,544 & 6.70 & 15,000 \\
\hline 10 & Excavation of Trapezoldal Drainage Dilch & 39,824 & or & - & 0.15 & 5,974 & 110,472 & 0 & 35,842 & 200 & 153,210 \\
\hline & (V) PAGE 2 TOTAL & & & & & 95,032 & 1.000 .647 & $4,408,271$ & 490,537 & & $0,600,450$ \\
\hline
\end{tabular}

1-8. Cost Estimate a for Cover System Utilizing Compacted Onsite Sandy Clay and a 30-mil FML (Cont'd) 
DETAIL ESTIMATE

\section{CHK'D BY: 8. MEAD}

Lod: :-03

ACTTVITY CODB: ESE2SOL12

PROJ.NAMB: BCON. BVALUATION OF CLOSURB CAP BARRIBR MATERRAL.

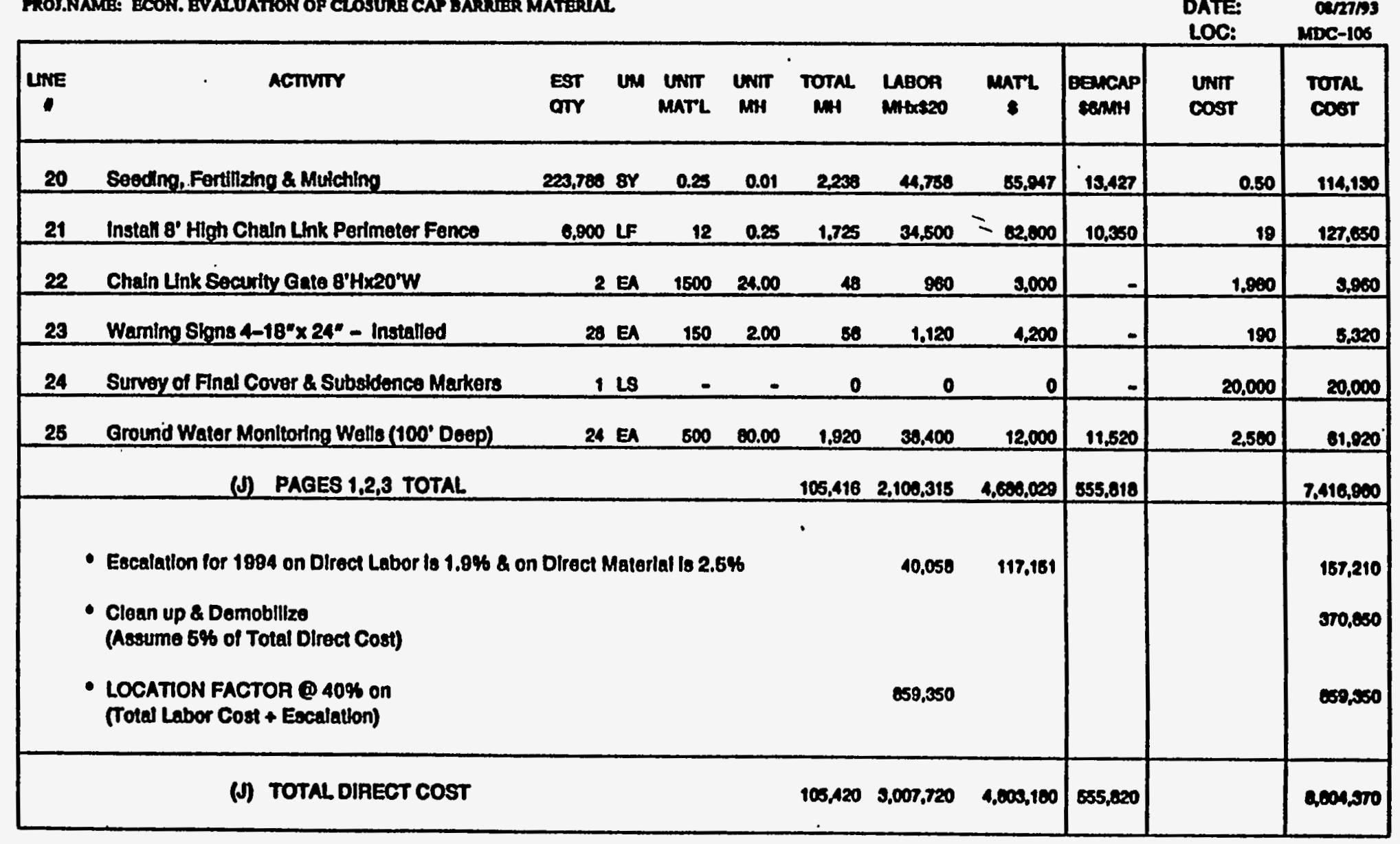

1-8. Cost Estimate a for Cover System Utilizing Compacted Onsite Sandy Clay and a 30-mil FML (Cont'd) 
Lo01: 7-03

ACTVITY CODE: ESE260+12

PROS.MAME: BCON. BVALUATION OF CLOSURB CAP QARRIER MATERINL

ESTIMATOR: ParmK

PHONE: 7-S260

DATE:

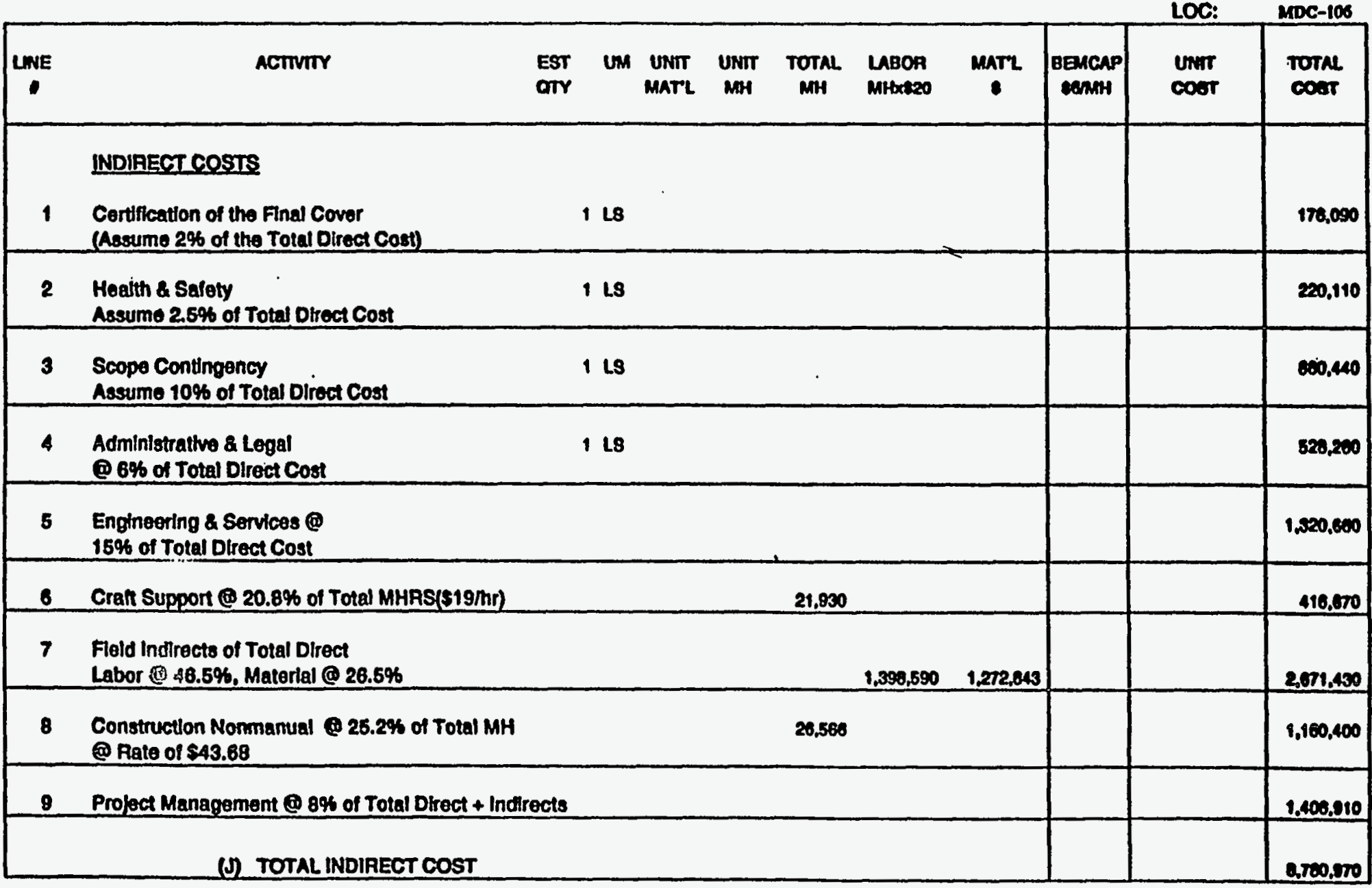

1-8. Cost Estimate a for Cover System Utilizing Compacted Onsite Sandy Clay and a 30-mil FML (Cont'd) 
DETAIL ESTIMATE

\begin{abstract}
Loof: 7-03
\end{abstract}
ACTTVTTY CODE: ESE260412

PROJ.NAME: ECON. BVALUATTON OP CLOSUNB CAP BARRIER MATERIAL.
CHKD BY: 8. MEAD

ESTIMATOR:

PHONE:

DATE:

LOC:

ronvix

7-5266

oarnss

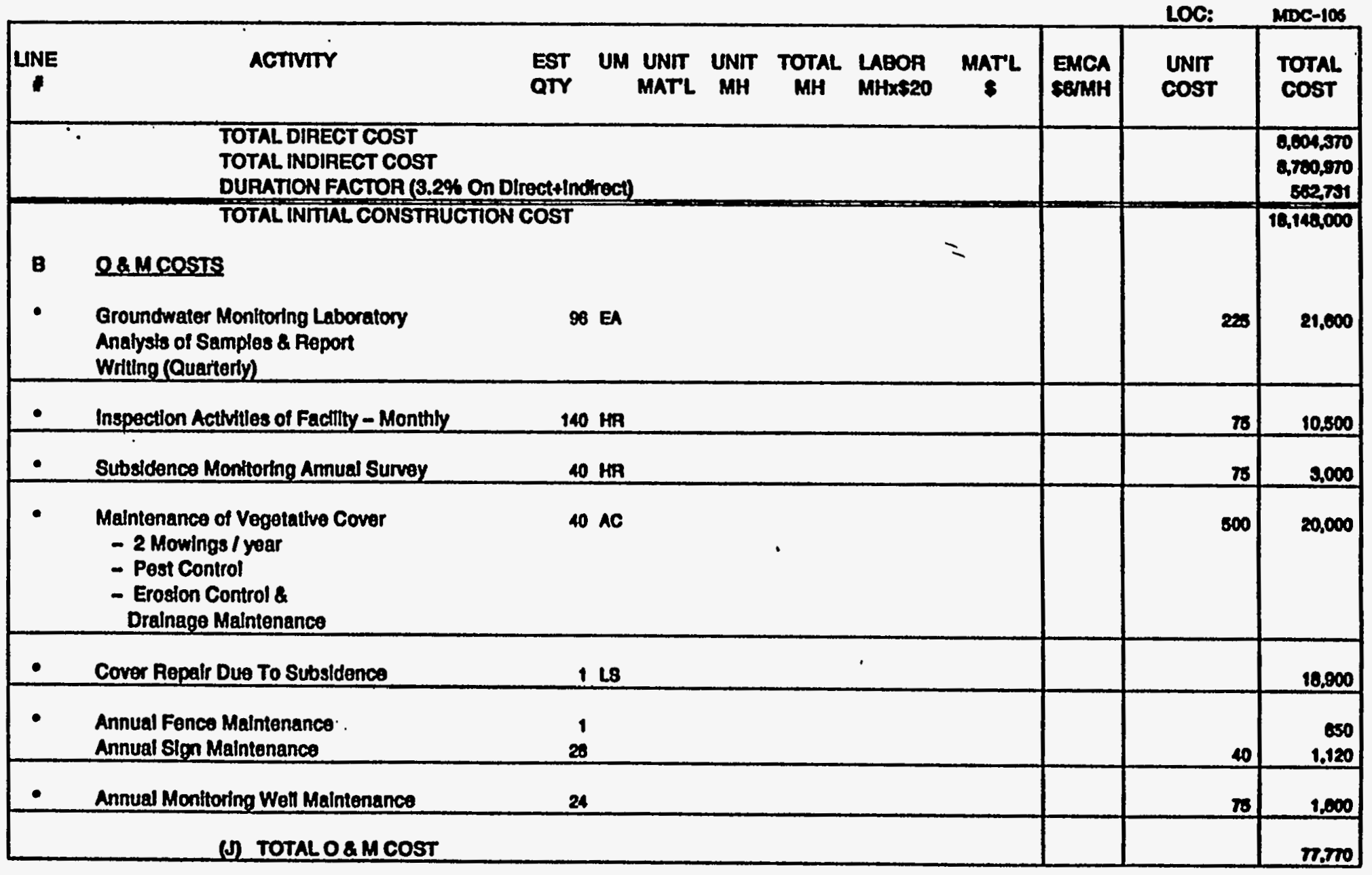

1-8. Cost Estimate a for Cover System Utilizing Compacted Onsite Sandy Clay and a 30-mil FML (Cont'd) 


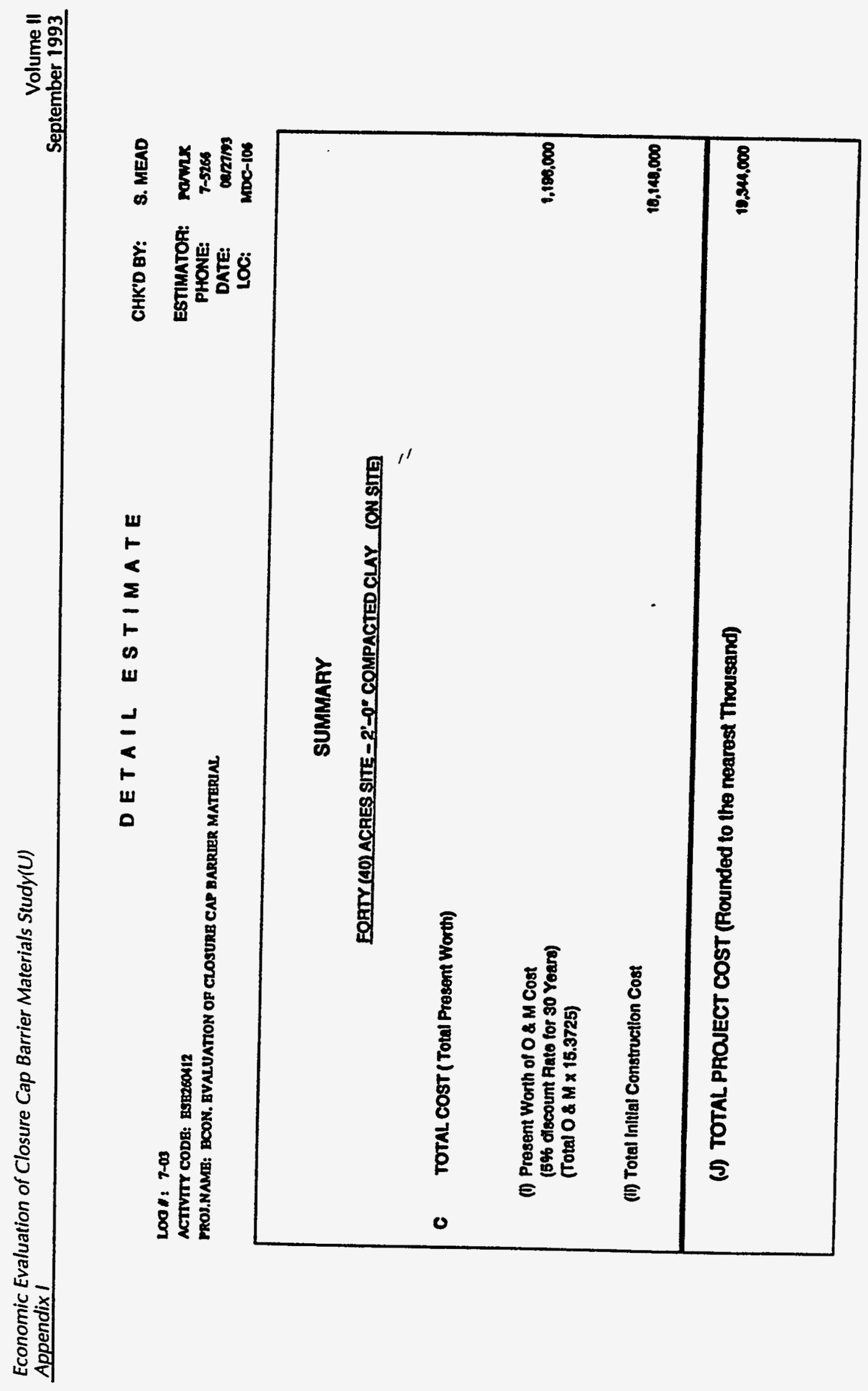

$\mid \frac{q}{i}$

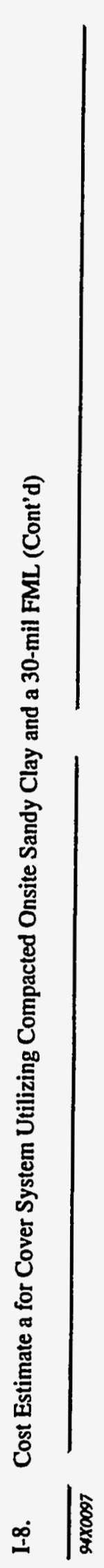


DETAIL ESTIMATE

LO0 I : 7-03

ACTIVITY CODE: ESEZ6ON12

PROJ.NAME: ECON. EVALUATTON OF CLOSURB CAP BARRIER MATERIAL.
CHKO BY: 8. MEAD

ESTMMATOF: rame

PHONE: 7-2006

DATE: OPOSPS

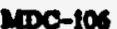

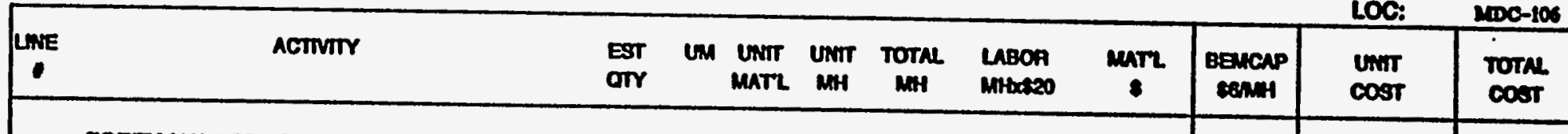

FORTY (40) ACAES SITE-SOIL CEMENT COVER

A DIAECT COSTS

1 SIIEPREPARATON

1 Clearing \& Gruboing

2 Procontouring Site $551 C \quad-\quad 10 \quad 2,200 \quad 44,000$

$16 \wedge$ $-5$

3 Sin Fence (Includes Maintenance) 4.650 LF $\quad 0.50 \quad 0.05 \quad 230 \quad 4050$

1 Tomporary Sooliment Control

12

$58^{\prime \prime D P} \times 20^{\prime}$ Wide Gravel Haut Road

$\begin{array}{lllll}6.720 \text { LF } & 0.50 & 0.1 & \text { or2 } & 15.440\end{array}$

6 2-15" RCP Cuverts 128 LF $\quad 12.00 \quad 0.75 \quad \infty 6 \quad 1.020$

$735^{\prime} \times 35^{\prime} \times 4^{\prime \prime}$ Gravol Deoon. Area

100 or $\quad 200 \quad 0.10 \quad 10 \quad 200$

$86^{\prime} \times 35^{\prime} \times 4^{\prime}$ Deep Wash Water Containment Ditch

$3 \mathbf{C Y}$

10. 20200

14,000

- Construction Traller (Rental) (Include Power \& Waten)

30 mo

$-$

(c) PAGE 1 TOTAL

07.000

I-9. Cost Estimate for a Cover System Utilizing Soil Cement and 30-mil FML 


\section{DETAIL ESTIMATE CHKDBY: 8.MEAD}

\section{1: 7-03}

ACTSVTY CODE: BSB260112

PROJ.NAMB: ECON. EVALUATION OF CLOSURE CAP I ARRIER MATEPIAL

\begin{tabular}{|c|c|c|c|c|c|c|c|c|c|c|c|}
\hline UNE & activin & $\begin{array}{l}\text { EST } \\
\text { aTY }\end{array}$ & un & $\begin{array}{l}\text { unvr } \\
\text { mart }\end{array}$ & $\underset{M H}{\text { UNTH }_{M}}$ & $\underset{\text { MHA }}{\text { rotal }}$ & $\begin{array}{l}\text { LABOP } \\
\text { Matation }\end{array}$ & $\operatorname{marl}_{:}$ & $\begin{array}{l}\operatorname{Bencap} \\
\operatorname{sanh}\end{array}$ & cosir & $\begin{array}{l}\text { Tothe } \\
\operatorname{cost}\end{array}$ \\
\hline 10 & $\begin{array}{l}\text { DCOVER CONSTRUCTION } \\
\text { Waate Stabilization (Crughod Limestono) }\end{array}$ & 55,757 & & so & 0.1 & 8.570 & 111,314 & 2707.850 & 30,454 & 52.00 & 2002000 \\
\hline 11 & $\begin{array}{l}\text { Place and Compact Foundation Soll } \\
\text { In } 8^{\circ} \text { Uifts to } 85 \% \text { Std. Proctor }\end{array}$ & 395,050 & & - & 0.105 & 41,586 & 001,302 & of & 249,391 & 270 & $1,000,000$ \\
\hline 12 & 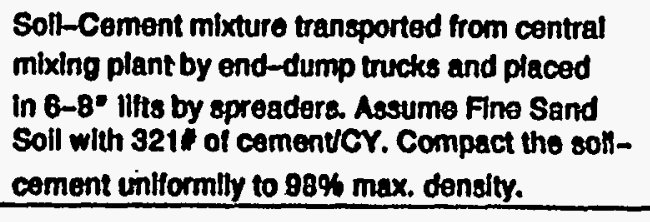 & 185,714 & & 16 & 0.12 & 23,406 & 400.714 & 3,522,062 & 140,014 & 21.10 & $4,158,400$ \\
\hline 13 & $\begin{array}{l}\text { Curing soll-cement. Assume liquidd Asphath } \\
\text { applled 0.4galloy for } 1 \text { St coat \& } 0.2 \text { for } 2 \text { nd. }\end{array}$ & $\begin{array}{l}190,362 \\
109,362 \\
\end{array}$ & & $\begin{array}{l}0.53 \\
0.17 \\
\end{array}$ & $\begin{array}{l}0.03 \\
0.02 \\
\end{array}$ & $\begin{array}{l}3,851 \\
3,007 \\
\end{array}$ & $\begin{array}{r}119.017 \\
70,345 \\
\end{array}$ & $\begin{array}{r}6,159 \\
30,722 \\
\end{array}$ & - & $\begin{array}{l}0.80 \\
0.60 \\
\end{array}$ & $\begin{array}{l}180,400 \\
118,000 \\
\end{array}$ \\
\hline 14 & $\begin{array}{l}\text { Placement of } 30 \text { Ma Floxible } \\
\text { Membrane Liner }\end{array}$ & $1,785,254$ & SF & 0.14 & 0.000 & 10,712 & 214,230 & 240.006 & - & 0.50 & 464,170 \\
\hline 18 & $\begin{array}{l}\text { Placement of } 1^{\prime}-0 \text { Coarse Sand } \\
\text { Dralnage Layer }\end{array}$ & 68,126 & & 10.50 & 0.025 & 1,650 & 30,005 & costses & 2.010 & 11.10 & 797,500 \\
\hline 16 & $\begin{array}{l}\text { Placement of Gootextile (TYPAF) Fitter on } \\
1^{1}-0^{*} \text { Coarse Sand Drainage Layer }\end{array}$ & 212,420 & sr & 0.12 & 0.005 & 1,062 & 21,240 & 20,491 & - & 0.20 & 40750 \\
\hline 17 & Placement of $1^{\prime}-6^{\prime \prime}$ Common Fill & 90,001 & cr & 2.76 & 0.04 & 3,023 & 70.465 & 507,004 & 23,539 & 4.00 & 409,810 \\
\hline 18 & Placoment of $6^{*}$ Topsoll & 39,000 & or & 2.5 & 0.04 & 1,520 & 200.400 & 315,500 & 7,020 & 10.30 & 347,020 \\
\hline & (Q) PAGE 2 TOTAL & & & & & $\infty$ & $1,000.427$ & 7.500 .000 & 453,670 & & $10,510,570$ \\
\hline
\end{tabular}

I-9. Cost Estimate for a Cover System Utilizing Soil Cement and 30-mil FML (Cont'd) 
Loo 1: $7-03$

DETAIL BSTIMATB

8. MEAD

ACTIVTY CODE: BSE2SOL12

PROJ.NAME: ECON. EVALUATTON OP CLOSURB CAP BARRIRR MATERUAL

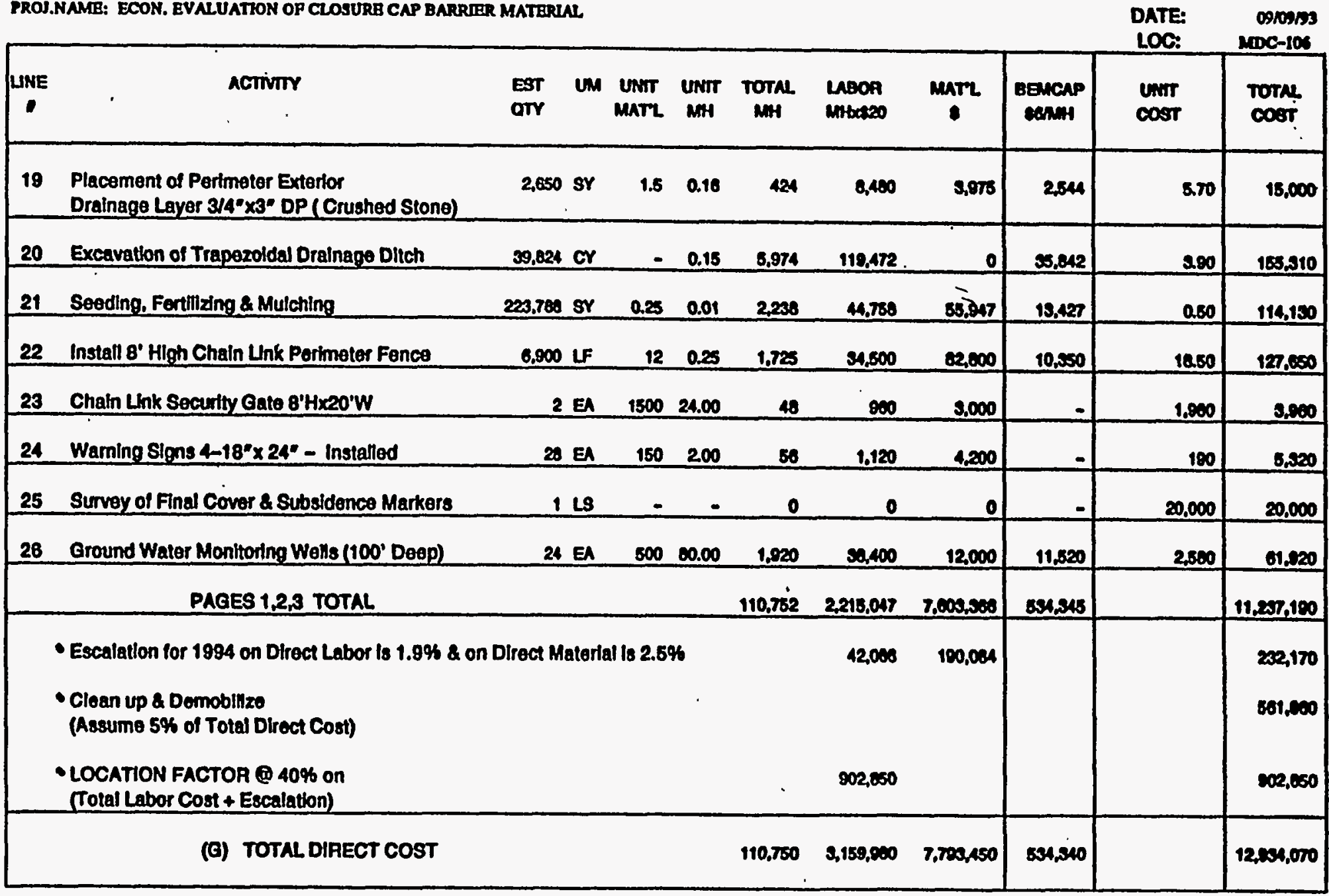

1-9. Cost Estimate for a Cover System Utilizing Soil Cement and 30-mil FML (Cont'd) 
D B T A I B T I M T B

\section{LOOA: $7-03$}

ACTIVTY CODE: PSE260112

PROI.NAME: ECON. EVALUATION OF CLOSURB CAP BARRIER MATERYAL

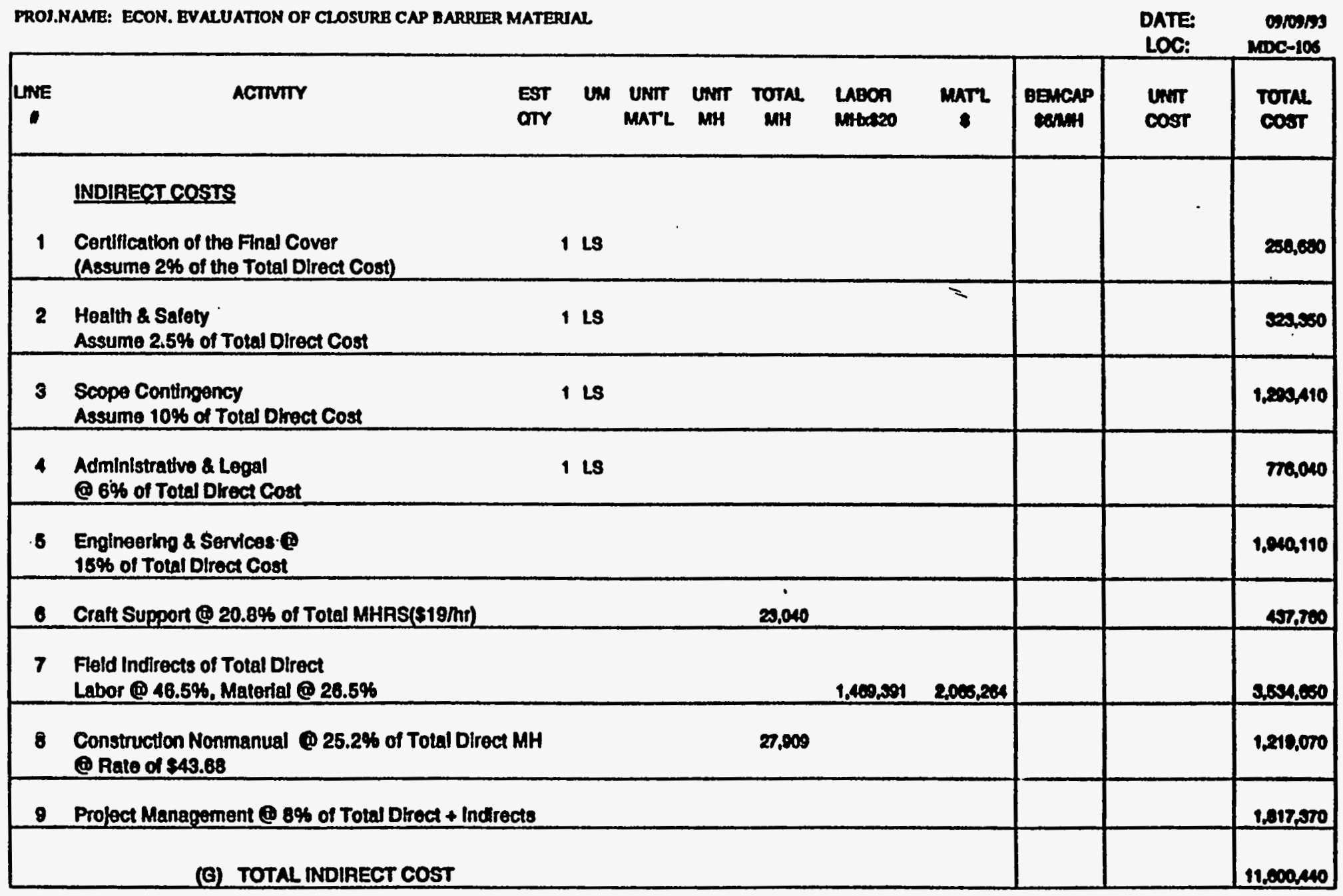

I-9. Cost Estimate for a Cover System Utilizing Soil Cement and 30-mil FML (Cont'd) 


\section{DETAIL B S T I MA T B}

LO0 : $7-03$

ACTIVITY CODE: ESE260412

TROJ.NAME: BCON. EVALUATION OR CLOSURB CAP BARRIER MATERLAL.

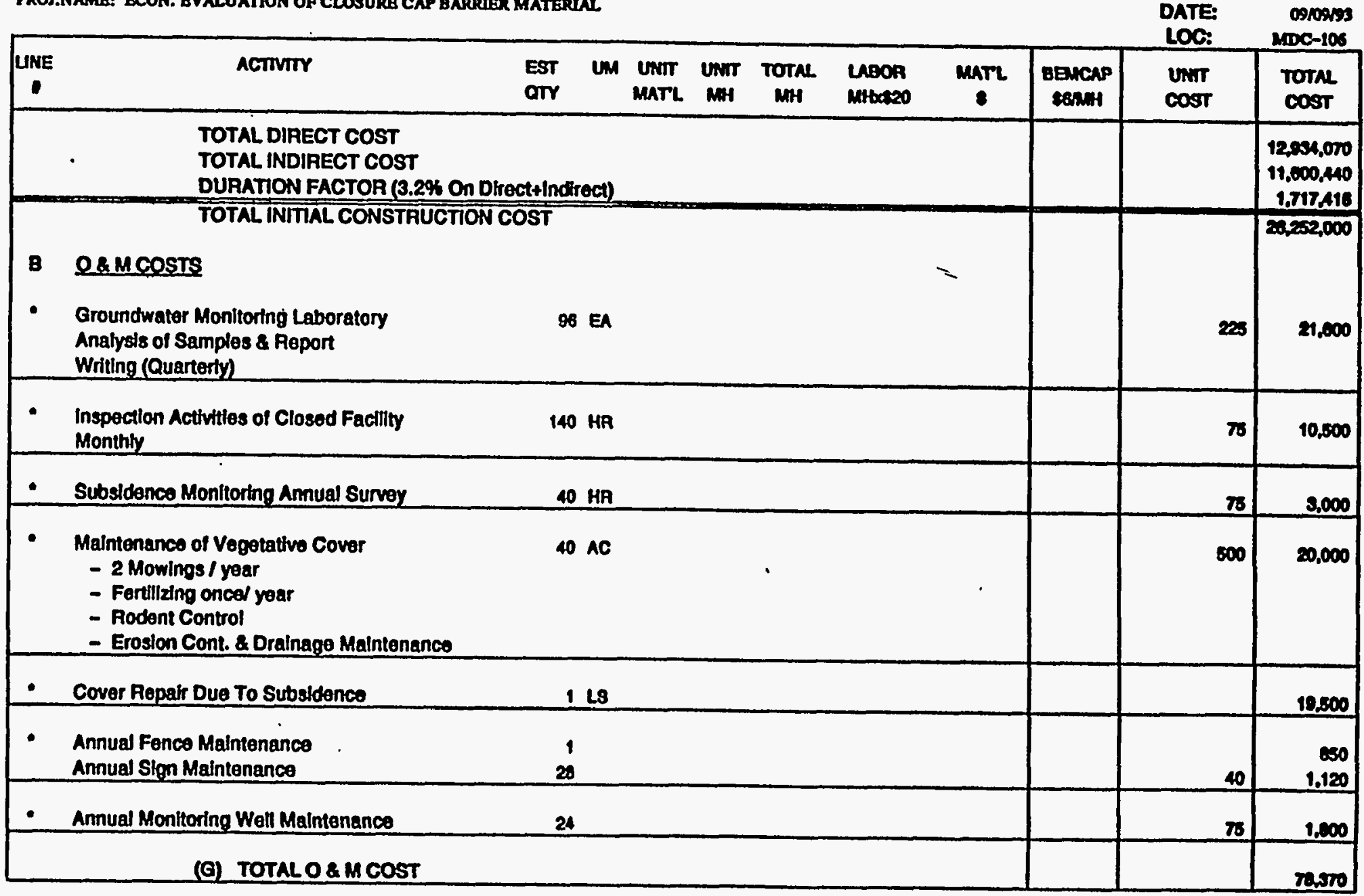

I-9. Cost Estimate for a Cover System Utilizing Soil Cement and 30-mil FML (Cont'd) 
D B TAI L BSTIMAT B

$\begin{array}{cc}\text { CHKD BY: } & \text { 8. MEAD } \\ \text { ESTIMATOA: } & \text { NOMIX } \\ \text { PHONE: } & 7-5256 \\ \text { OATE: } & 0910953 \\ \text { LOC: } & \text { MOC-106 }\end{array}$

Loo : $1-03$

ACTIVITY CODE: ESEZ60412

MOO.NAME: ECON. BVALUATTON OP CLOSURB CAP BARRIRR MATERIAL

LOC:

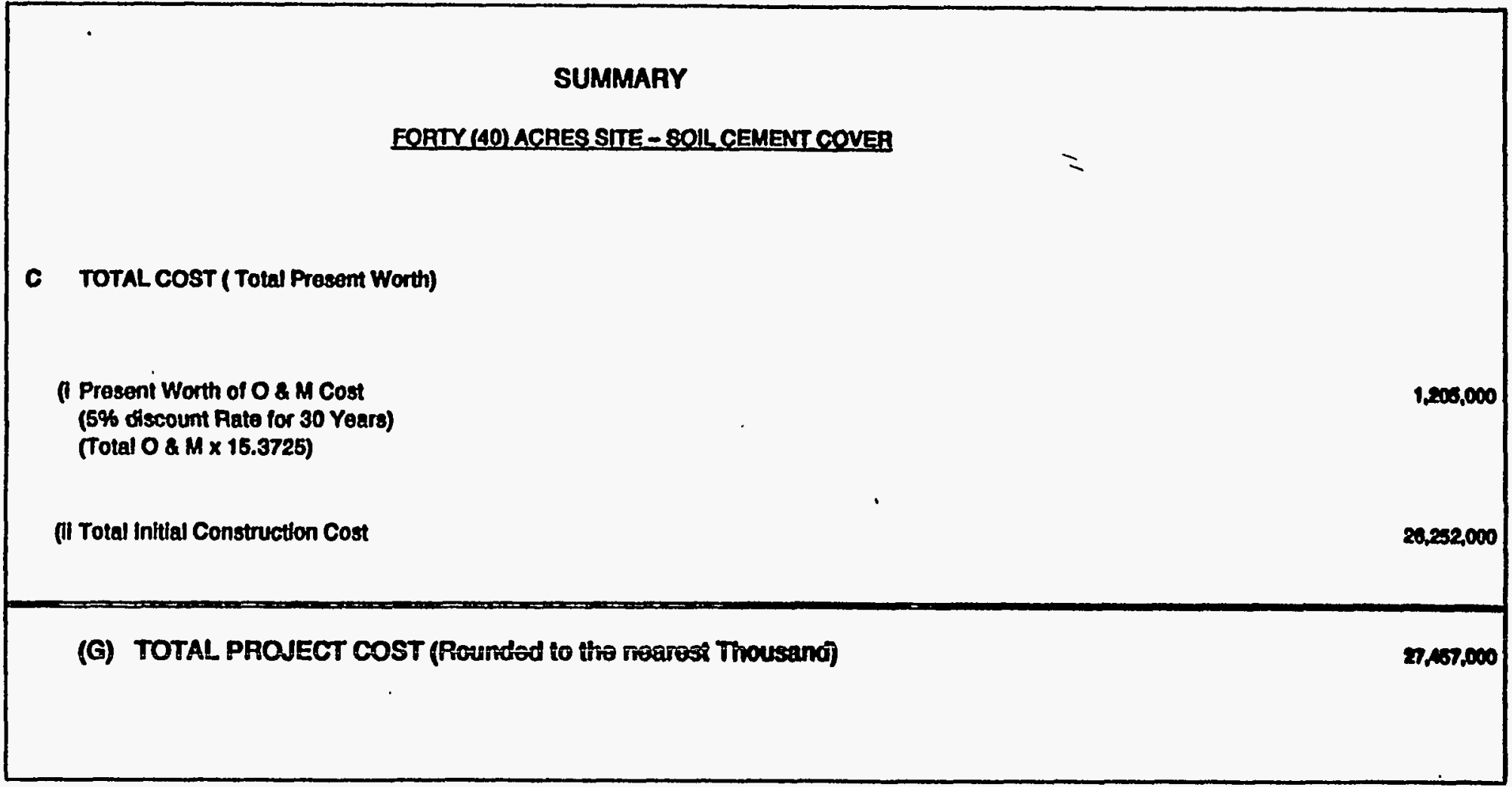

I-9. Cost Estimate for a Cover System Utilizing Soil Cement and 30-mil FML (Cont'd) 


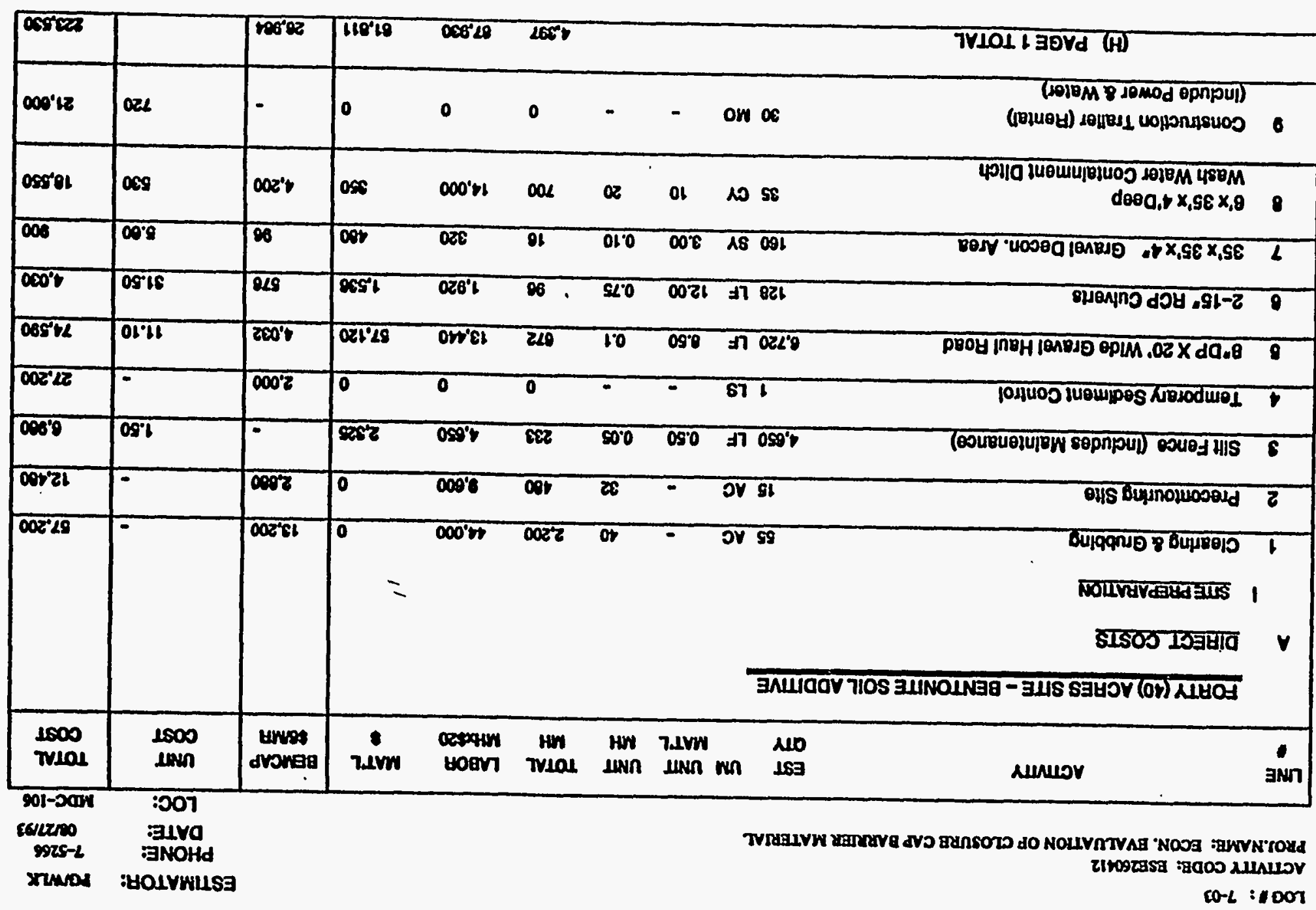

avaw's :Ав Q.XHO

$\exists \perp \forall W I S \exists 71 \forall \perp \exists a$ 
DETAIL ESTIMATE

CHKD BY: S. MEAD

L001: $7-03$

ACTIVITY CODE: ESH2604 12

PROJ.NAME: BCON. EVALUATTON OF CLOSURB CAP BARRRER MATERIAL

ESTIMATOR: ramix

PHONE: 7-5265

\begin{tabular}{|c|c|c|c|c|c|c|c|c|c|c|c|}
\hline UME & ACTVITY & $\begin{array}{l}\text { EST } \\
\text { QTT }\end{array}$ & UM & $\begin{array}{l}\text { UNIT } \\
\text { MATL }\end{array}$ & UNTH & TOTAL & $\begin{array}{l}\text { LABOR } \\
\text { MrtheteO }\end{array}$ & mart & $\begin{array}{c}\text { вencup } \\
\text { senmp }\end{array}$ & $\underset{\text { cosst }}{\text { covrs }}$ & $\begin{array}{l}\text { TOTAL } \\
\text { COAT }\end{array}$ \\
\hline & $\begin{array}{l}\angle \text { CONER CONSTRUCTION } \\
\text { Waste Stabillzallon (Crushod Limestono) }\end{array}$ & 85.757 & & so & 0.1 & 5,570 & 111,814 & $2,707,060$ & 32,454 & 82.00 & 2032020 \\
\hline 11 & $\begin{array}{l}\text { Piace and Compact Foundation Soll } \\
\text { in } \theta^{\circ} \text { Litts to } 95 \% \text { Sid. Proctor }\end{array}$ & 395,058 & & - & 0.105 & 41,565 & 631,302 & $=$ & 249,391 & 270 & $1,000,690$ \\
\hline 12 & 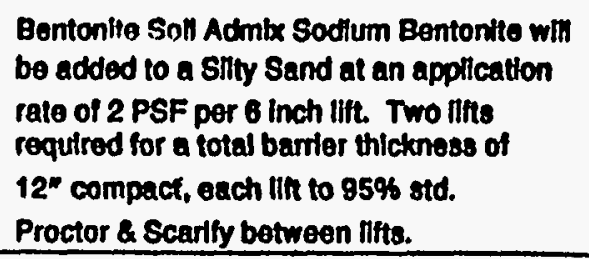 & $\begin{array}{c}1,756 \\
\text { Sodum } 80 n \\
69.857 \\
\text { Siny Sand \& }\end{array}$ & in & 146.50 & 0.15 & 8,594 & 191,071 & 859,253 & 57,561 & 100.50 & $\begin{array}{r}327,400 \\
1,200,700\end{array}$ \\
\hline 13 & $\begin{array}{l}\text { Placement of } 30 \text { Mil Flexible } \\
\text { Mombrane Liner }\end{array}$ & $1,758.102$ & SF & 0.14 & 0.006 & 10,557 & 210,732 & 245,084 & - & 0.30 & 450,580 \\
\hline 14 & $\begin{array}{l}\text { Placement of 1'-0 Coarse Sand } \\
\text { Dralnage Layer }\end{array}$ & 86.126 & Cr & 10.50 & 0.023 & 1,653 & 33,000 & 024,325 & 8.810 & 19.10 & 797,300 \\
\hline 15 & $\begin{array}{l}\text { Placement of Gootextile (TYPAR) Filter on } \\
1^{-}-0^{*} \text { Coarse Sand Drainage Layer }\end{array}$ & 212,426 & sr & 0.12 & 0.005 & 1,062 & 21,245 & 26,491 & - & 0.20 & 40,730 \\
\hline 16 & Placement of 1'-6" Common Fin & 80,081 & cr & 3.75 & 0.04 & 3,923 & 70,465 & 307.004 & 23,539 & 4.00 & s09210 \\
\hline 17 & Placement of $6^{\circ}$ Topsoll & 33,000 & or & 9.50 & 0.04 & 1,320 & 20,400 & 319,500 & 7,820 & 10.50 & 347820 \\
\hline & (H) PAGE 2 TOTAL & & & & & 73,490 & $1,469,963$ & $4,970,127$ & 350,025 & & 7.00000100 \\
\hline
\end{tabular}

I-10. Cost Estimate for a Cover System Utilizing Bentonite Soil Additive and a 30-mil FML (Cont'd) 
ACTIVITY CODE: BSE260412

DETAIL ESTIMATE

CHKD BY: S. MEAD

ROJ.NAMB: BCON. BVALUATHON OF CLOSURB CAP BARRIER MATENUAL.

ESTIMATOR: Tank

PHONE: 7-5265

DATE: OARTSS

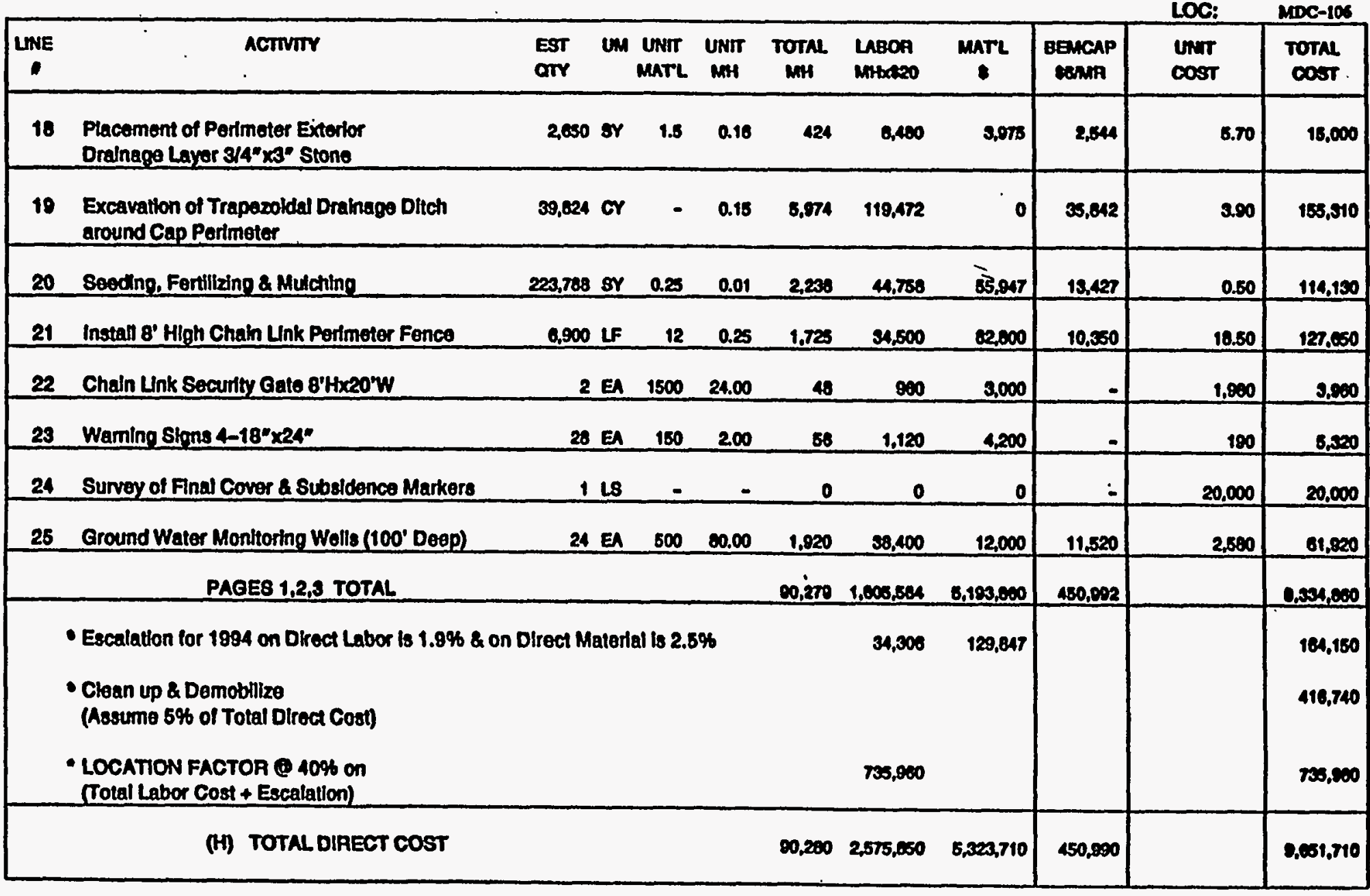

I-10. Cost Estimate for a Cover System Utilizing Bentonite Soil Additive and a 30-mil FML (Cont'd) 
L00 I: 7-03

ACTTVTYY CODE: BSE260A12

PROJ.NAME: ECON. EYALUATTON OP CLOSURE CAP BARRTER MATERSAL

8.MEAD

\section{ESTIMATOA: PaMrR}

PHONE: 7-5265

PROT.NAME

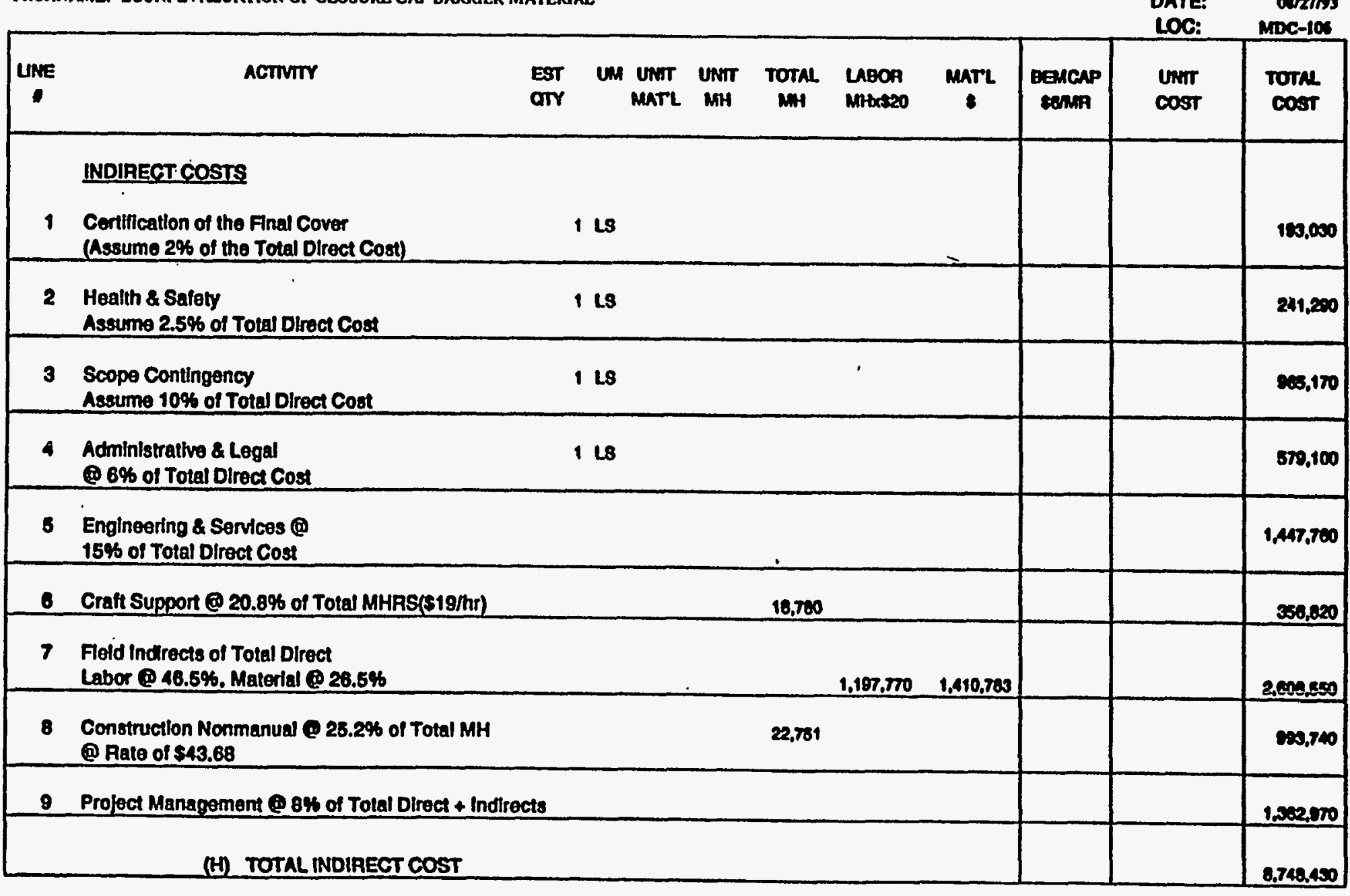

1-10. Cost Estimate for a Cover System Utilizing Bentonite Soil Additive and a 30-mil FML (Cont'd) 
Loo 1 : 7-03

ACTIVITY CODE: ESE26O112

RROJNAME: ECON. BVALUATKON OP CLOSURE CAP BARRIER MATERIAL

CHKD EY: S. MEAD

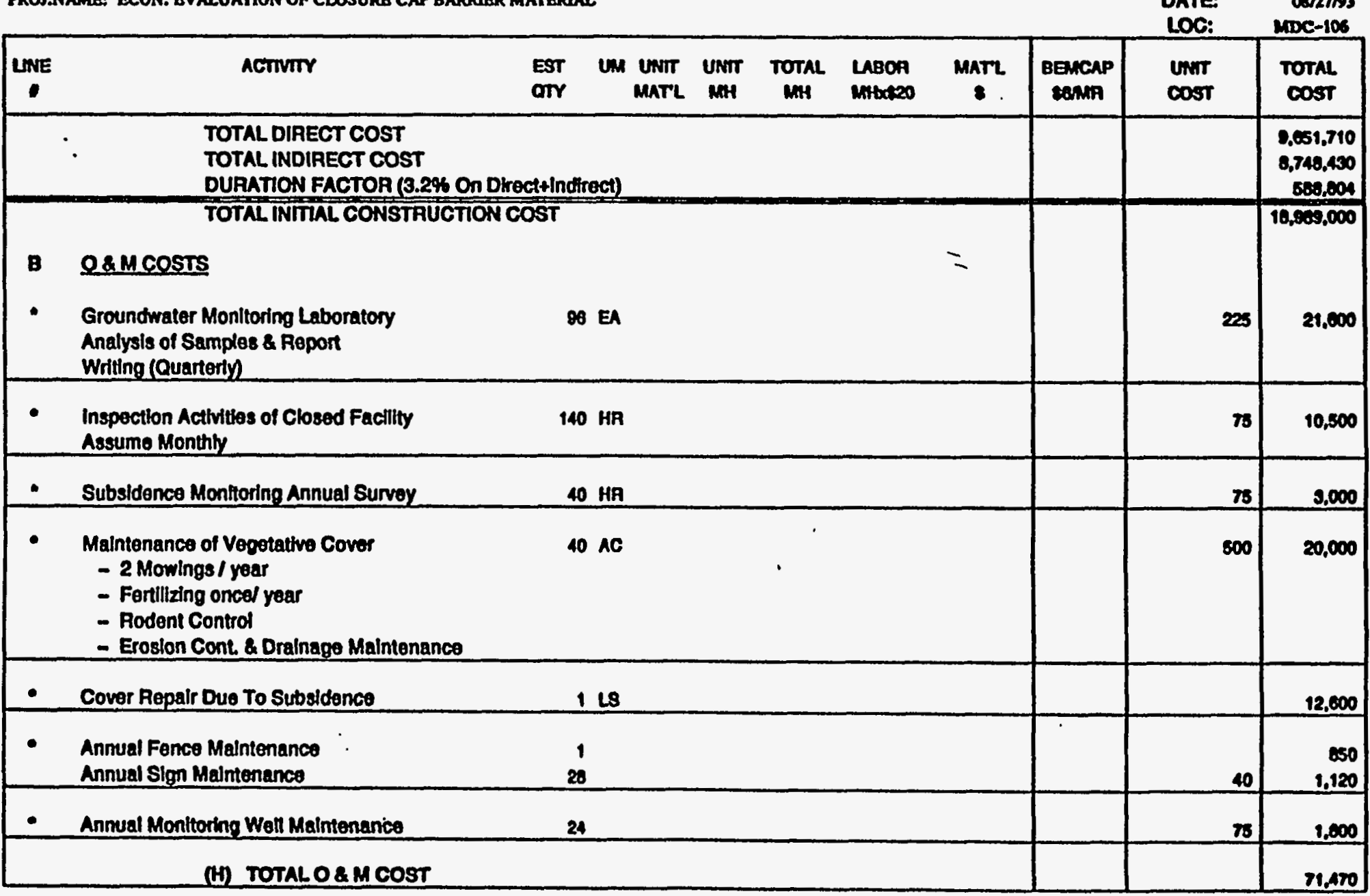

I-10. Cost Estimate for a Cover System Utilizing Bentonite Soil Additive and a 30-mil FML (Cont'd) 
DETAIL ESTIMATE

$\begin{array}{cc}\text { CHKD BY: } & \text { 8. MEAD } \\ \text { ESTIMATOR: } & \text { raMWK } \\ \text { PHONE: } & \text { 7-S266 } \\ \text { DATE: } & \text { DA27N3 } \\ \text { LOC: } & \text { MDC-106 }\end{array}$

2001: 7-03

ACTIVITY CODE: RSE200112

PROS.NAME: ECON. BVALUATION OP CLOSURB CAP BARRTER MATERRAL

LOC:

MDC-106

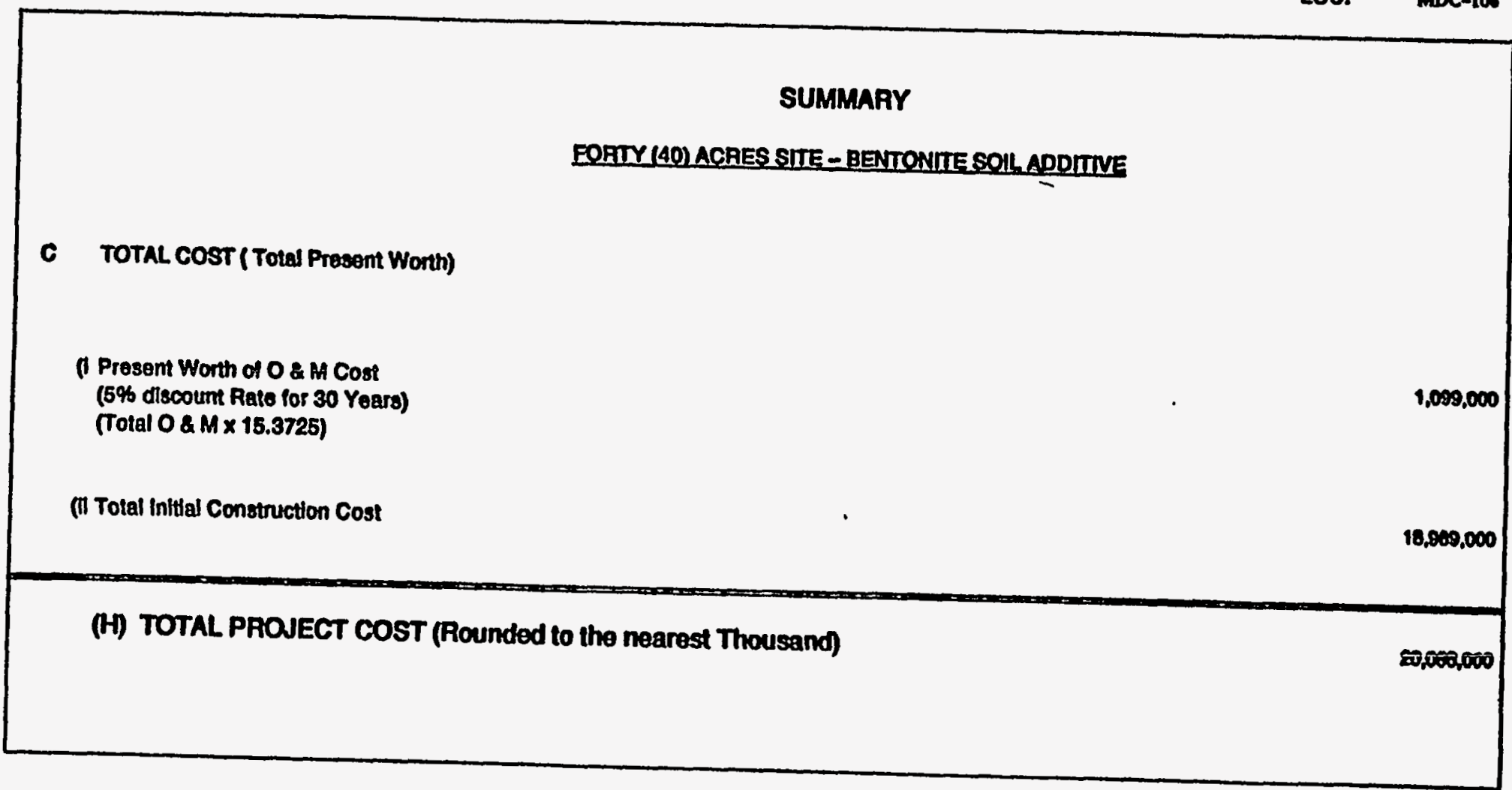

1-10. Cost Estimate for a Cover System Utilizing Bentonite Soil Additive and a 30-mil FML (Cont'd) 


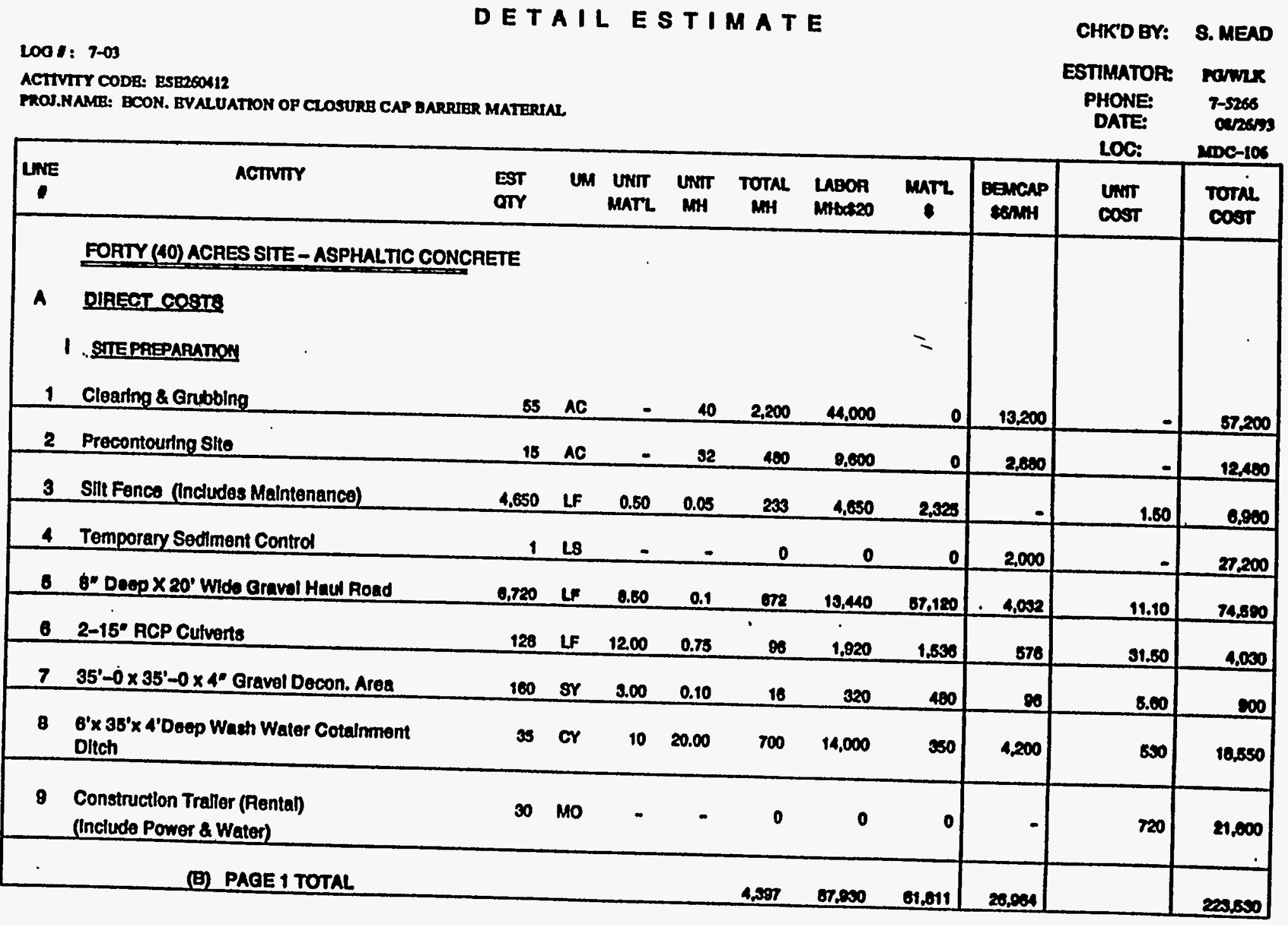

I-11. Cost Estimate for a Cover System Asphalt Concrete Hydraulic Barrier and a 30-mil FML 
DETAILESTIMATE

Loa 1: $7-03$

ACTIVITY CODB: BSB260412

FROJ.NAMGE: BCON. BVALUATTON OF CLOSURB CAP BARRIER MATEPIAI

\begin{tabular}{|c|c|c|c|c|c|c|c|c|c|c|c|}
\hline UNE & ACTINTYY & $\begin{array}{l}\text { EST } \\
\text { OTV }\end{array}$ & un & $\begin{array}{l}\operatorname{UniT} \\
\operatorname{maTL}\end{array}$ & Umin & $\underset{\text { MOTH }}{\text { TOTAL }}$ & $\begin{array}{l}\text { LABOR } \\
\text { Mtrated }\end{array}$ & $\operatorname{mar}^{\prime}$ & $\underset{\operatorname{mancap}}{\operatorname{manh}}$ & $\frac{\text { LOC: }}{\operatorname{cosir}}$ & $\frac{\text { MDC-106 }}{\text { TOTML }_{\text {COST }}}$ \\
\hline & 1 COVER CONSTRUCTION & & & & & & & & & & \\
\hline 10 & Waste Stabilization (Crushed Limestone) & 55,757 & $T N$ & 50 & 0.1 & 8,570 & 111,314 & 2707,850 & 32,454 & 82,00 & $2,032,020$ \\
\hline 11 & $\begin{array}{l}\text { Place and Compact Foundation Soll } \\
\text { In } 8^{\circ} \text { Unts to } 85 \% \text { Std. Proctor }\end{array}$ & 333,650 & cr & - & 0.105 & 35,054 & 701,083 & $=$ & 210,320 & 270 & 811,410 \\
\hline 12 & $\begin{array}{l}\text { Placement of } 30 \text { Mil Flextlole } \\
\text { Membrane Liner }\end{array}$ & $1,810,827$ & $8 F$ & 0.14 & 0.006 & 10,865 & 217,299 & 253,516 & - & 0.30 & 470,020 \\
\hline 13 & $\begin{array}{l}\text { Placement of } 1^{\prime}-0 \text { Coarse Sand } \\
\text { Dralnage Layer }\end{array}$ & 68,675 & or & 10.50 & 0.025 & 1,667 & 33,338 & 700,008 & 10,001 & 11.20 & 749,430 \\
\hline 14 & $\begin{array}{l}\text { 2" PVC Perforatod Drainago Pipe placed } \\
\text { in Drainaga layer }\end{array}$ & 14,600 & LF & 1.0 & 0.12 & 1.752 & 38,040 & 14,600 & -1 & 3.40 & 49.840 \\
\hline 15 & $21 / 2^{\prime \prime}$ Binder Course & 195,954 & sr & 4.15 & 0.055 & $10.7 m$ & 215,540 & 813,209 & 64,065 & 3.60 & $1.093,420$ \\
\hline 16 & 4" Hydraulle Asphati Conc. & 195,854 & SY & 6.60 & 0.03 & 8,079 & 117.572 & $1,293,2006$ & 35,272 & 7.40 & $1,446,140$ \\
\hline & Asphalt Mastlo Beal & 195,854 & sr & 0.50 & 0.018 & 2,959 & 80.700 & m,9n & - & 0.00 & 156,700 \\
\hline 18 & Sprayed Asphatilc Concrete Sealent & 185,554 & SY & 0.73 & 0.015 & 2,839 & 58,786 & 146,960 & 17,050 & 1.10 & 220,300 \\
\hline 19 & Excavation of Traingular Drainage Ditch & 22,148 & or & - & 0.15 & 3,322 & 68,444 & 0 & 10.933 & 3.90 & 00.300 \\
\hline 20 & 4" Thlek Asphaht Permoter Dralnage Ditah & 10.870 & sr & 7.6 & 0.144 & 2,717 & 84.548 & 149.412 & 16,304 & 11.30 & 214,000 \\
\hline & (B) PAGE 2 TOTAL & & & & & 83,408 & $1,500,700$ & $0.250,919$ & 400,500 & & $0,320,220$ \\
\hline
\end{tabular}

I-11. Cost Estimate for a Cover System Asphalt Concrete Hydraulic Barrier and a 30-mil FML (Cont'd) 


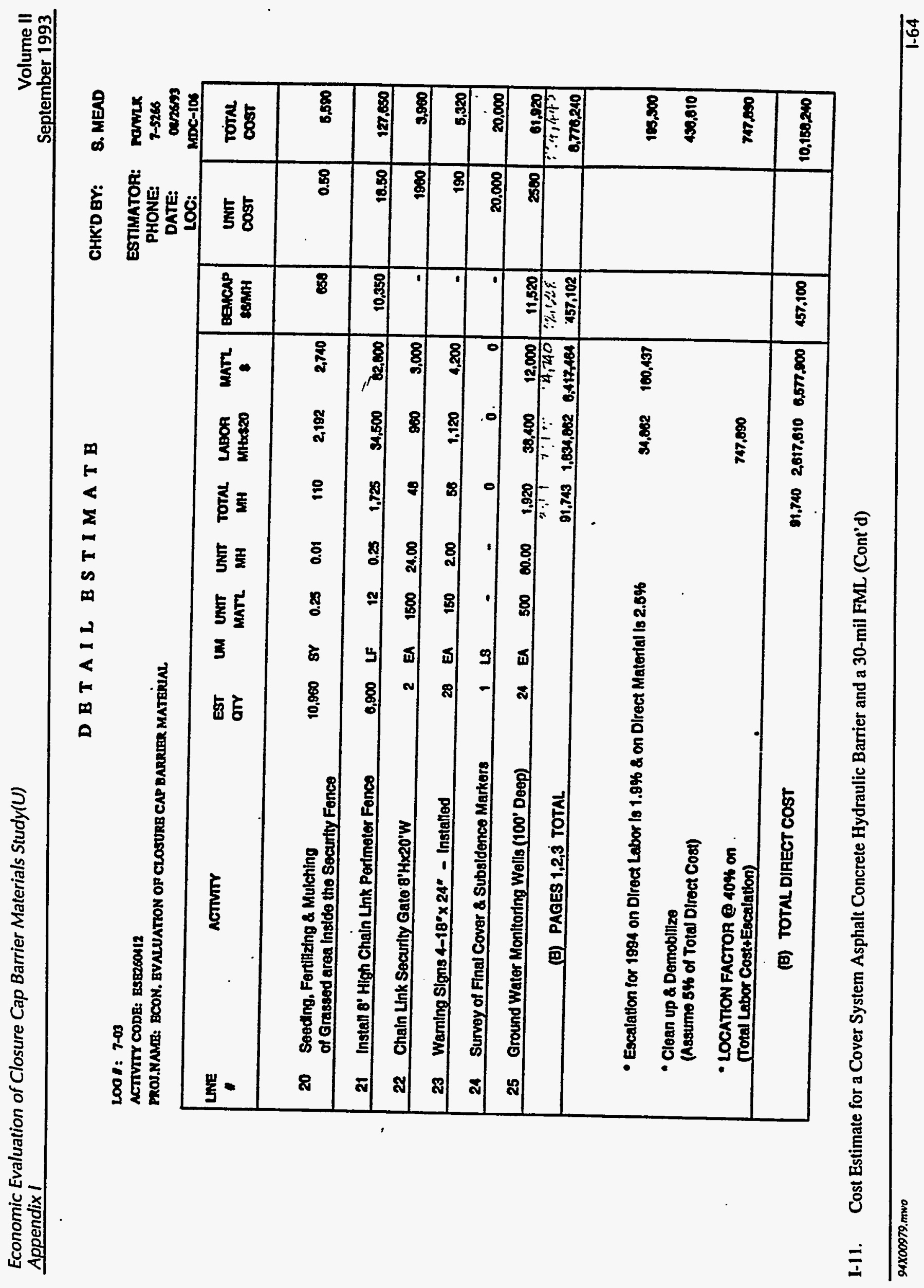


DBTAILESTIMATB

CHKD BY: 8. MEAD

Lo01: 7-03

ACTIVTYY CODH: ESE260112

PROJ.NAMBE: ECON. EVALUATTON OF CLOSURB CAP BARRIER MATERIAL.

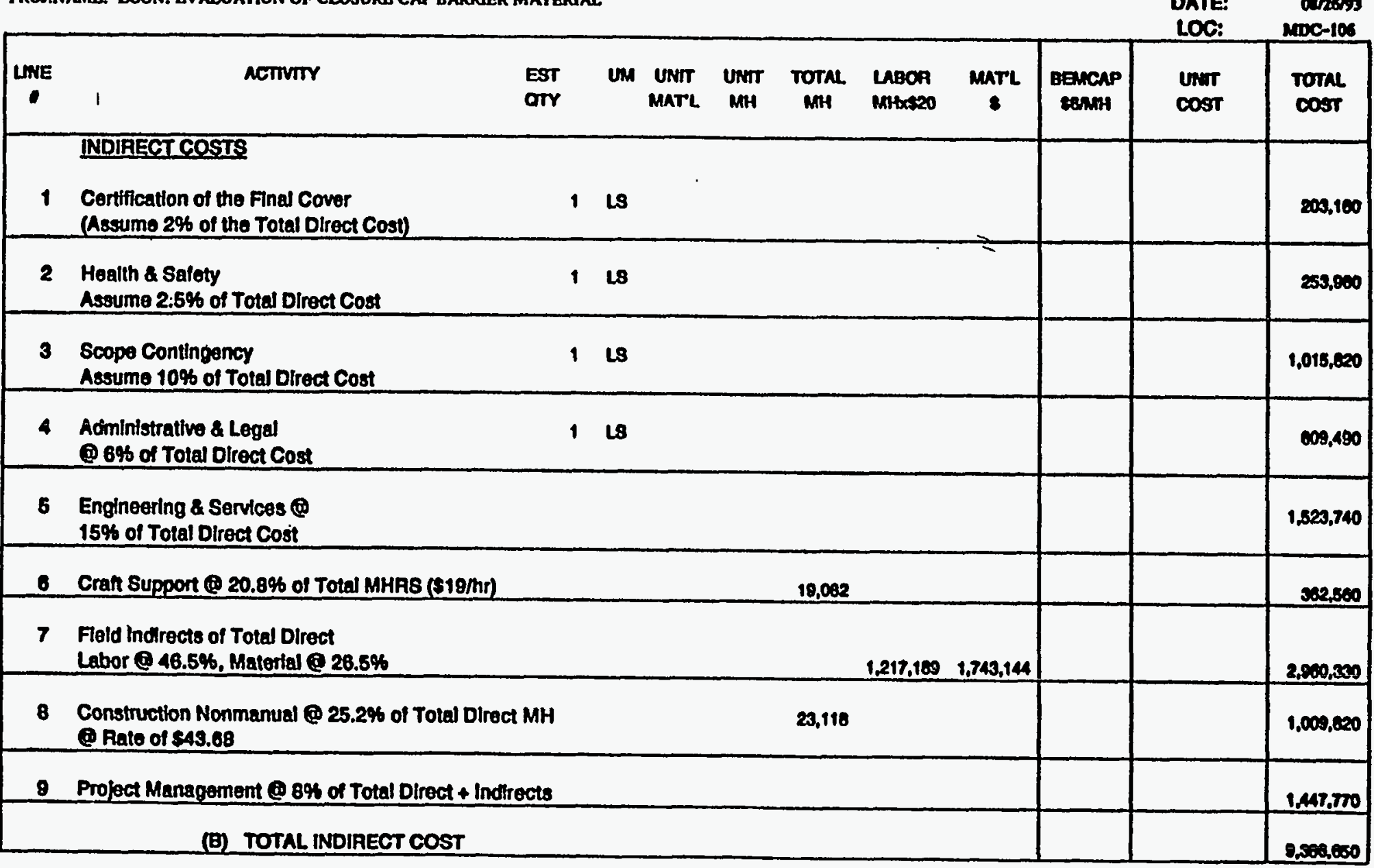

I-11. Cost Estimate for a Cover System Asphalt Concrete Hydraulic Barrier and a 30-mil FML (Cont'd) 
Economic Evaluation of Closure Cap Barrier Materials Study $(U)$

Volume II

Appendix 1

DETAILESTIMATE

September 1993

100 : 7-03

ACTIVTY CODB: BSE260412

PROJ.NAME: BCON. BVALUATION OP CLOSURR CAP BARRTRR MATERUAL.

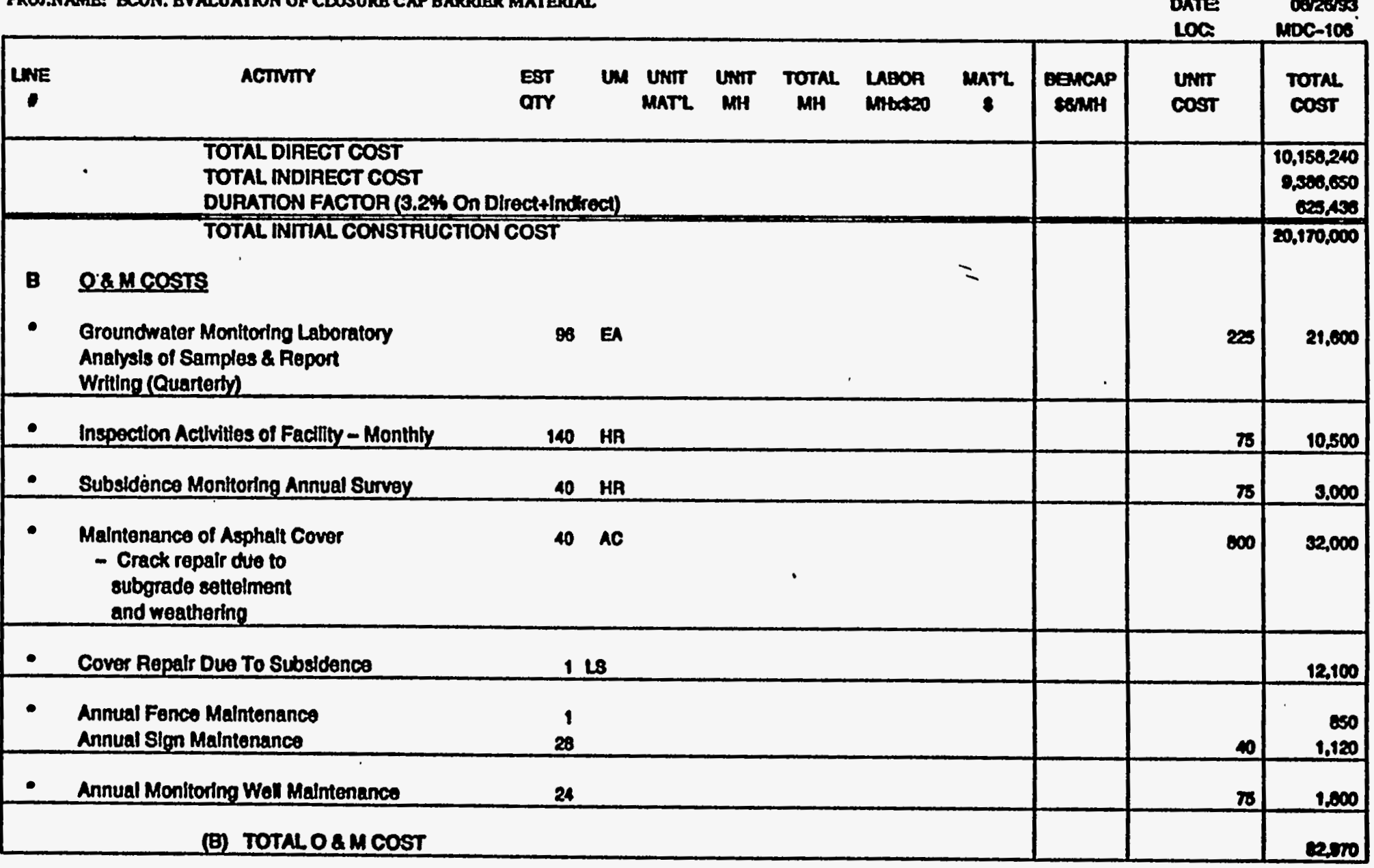

I-11. Cost Estimate for a Cover System Asphalt Concrete Hydraulic Barrier and a 30-mil FML (Cont'd) 


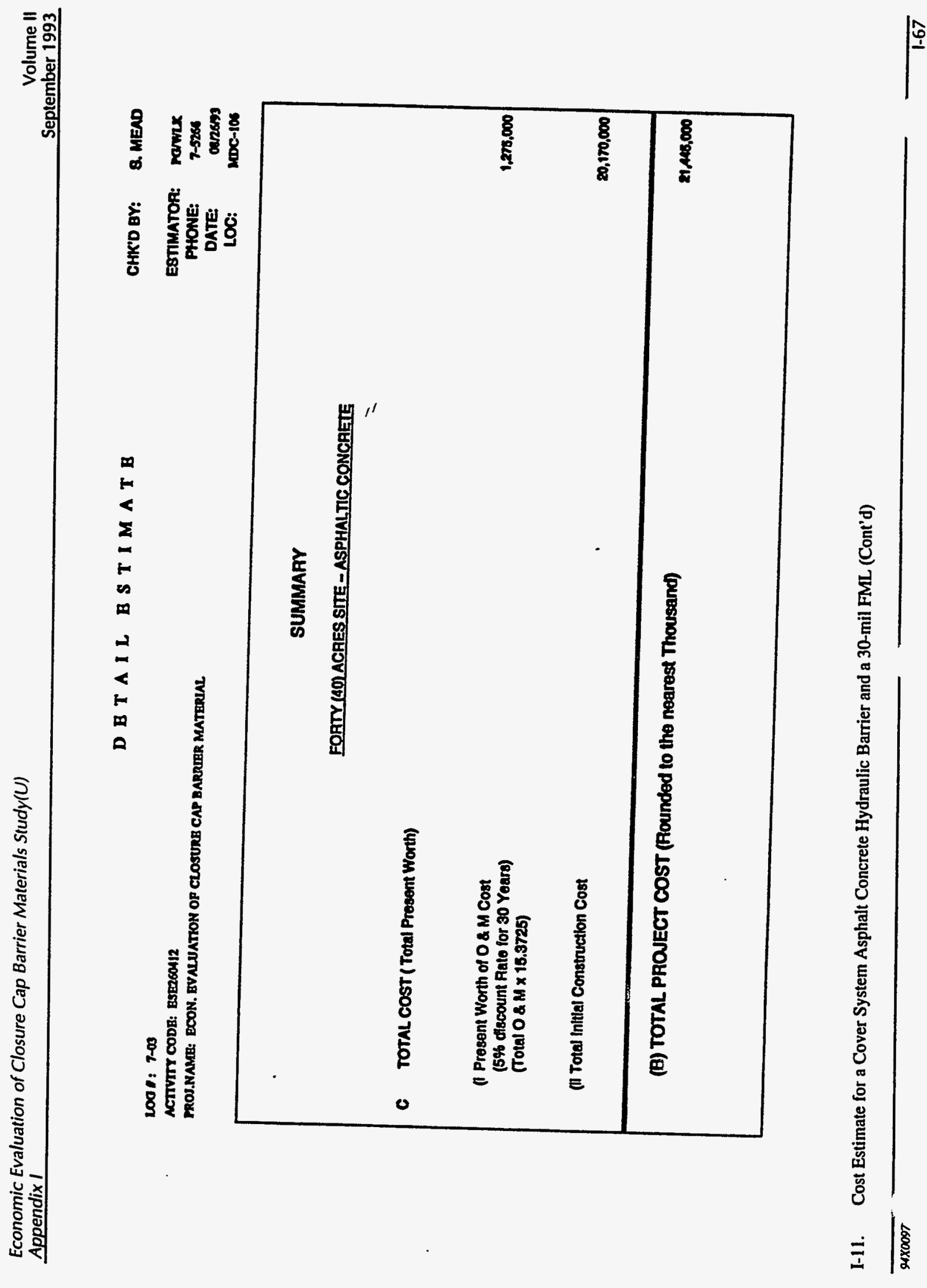




\section{DETAIL ESTIMATE}

LOQ: $7-03$

ACTVITY CODE: ESE2OOA12

PPOU.NAME: ECON. EVALUATION OF CLOSURE CAP BAPFIEAMATEFANL

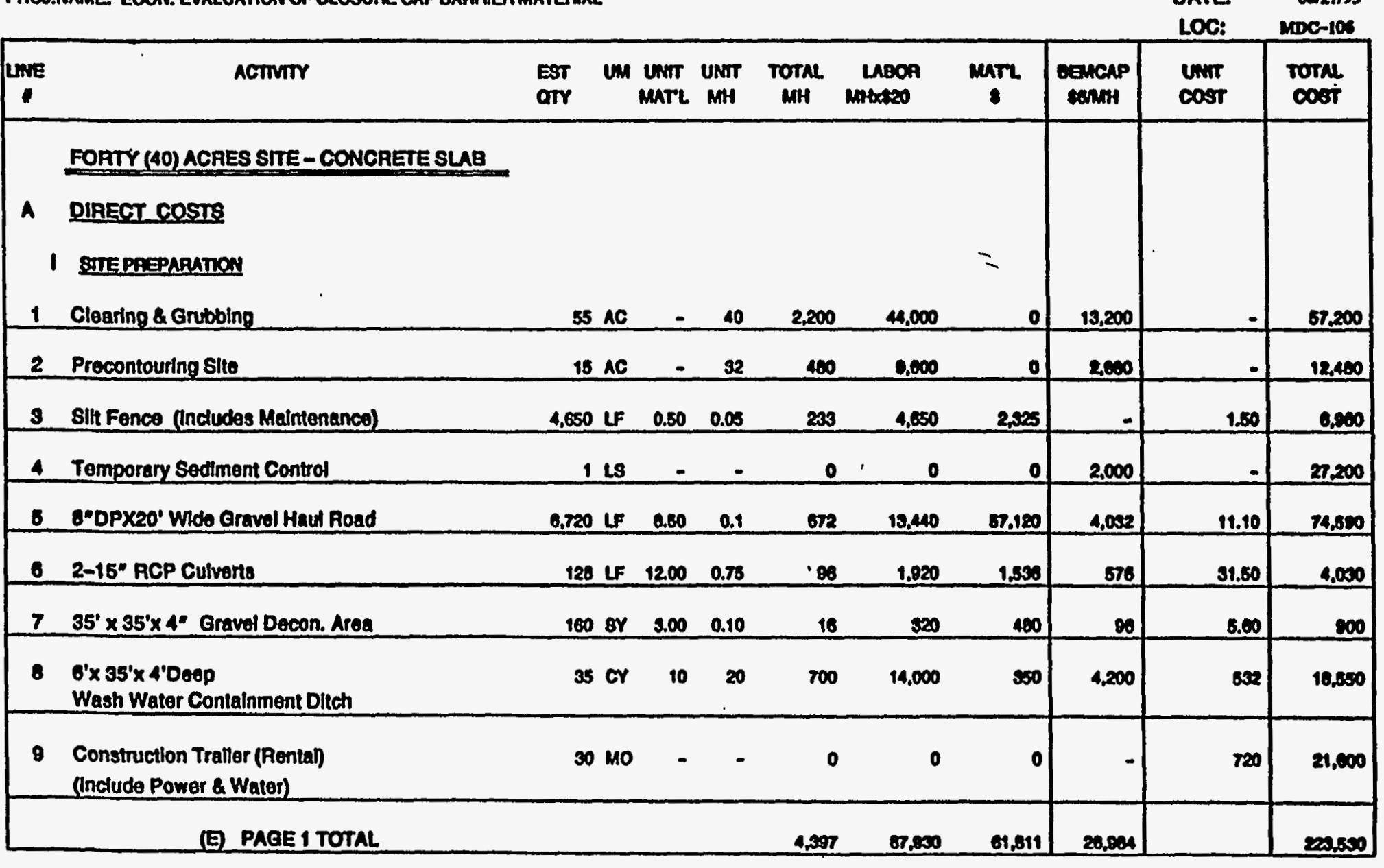

CHK'D EY: S. MEAD

ESTIMATOR: NOMWX

PHONE:

DATE: DARTSB

LOC: NADC-106

$$
\text { | }
$$

I-12. Cost Estimate for a Cover System Utilizing Poured-in Place Concrete Slab and a 30-mil FML 
DETAIL ESTIMATE

L00 I: $7-03$

ACTVITY CODR: ESE26011

PROS.NAME: ECON. BVALUATION OF CLOSURB CAP BARRIER MATERIAL

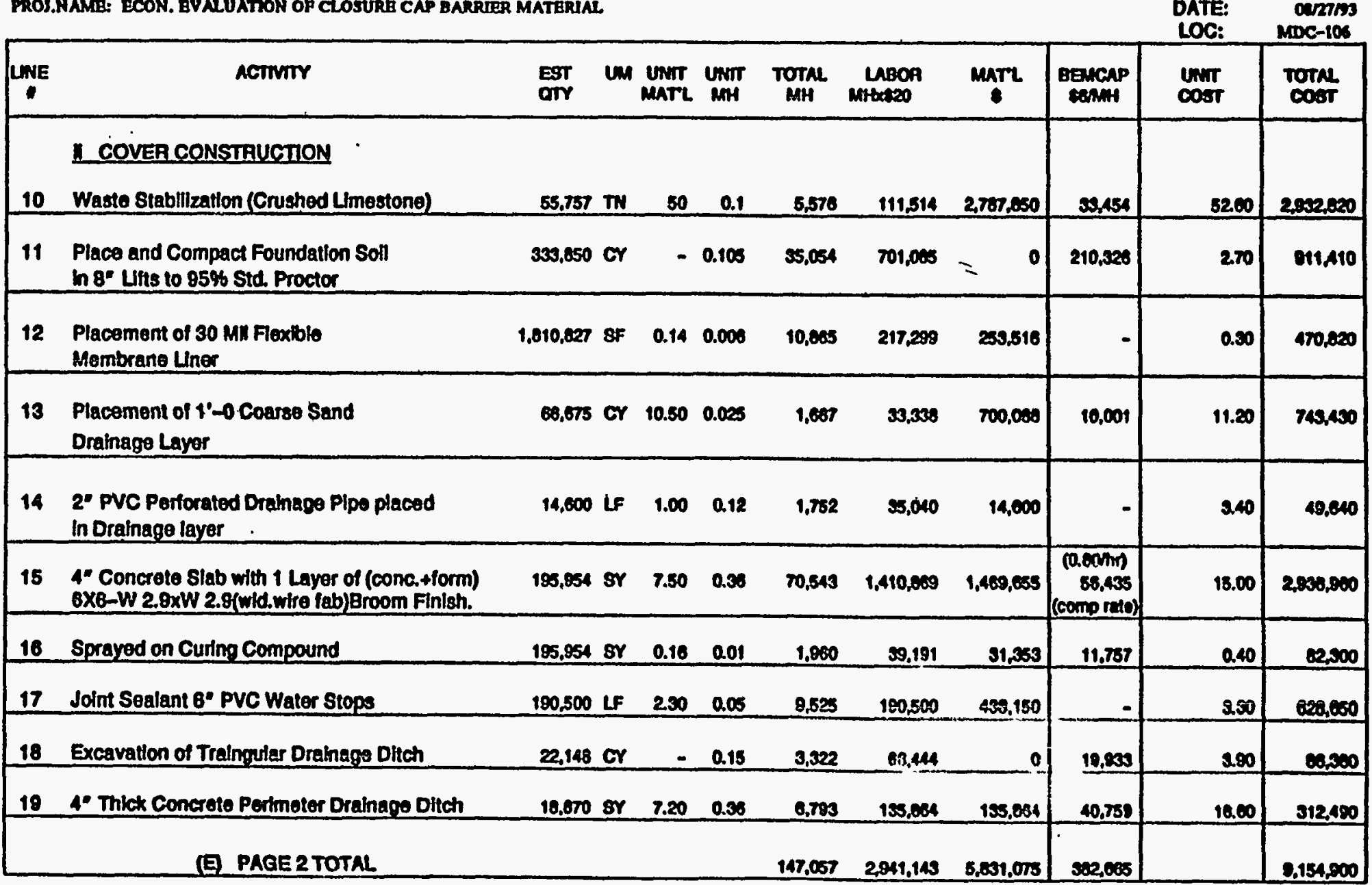

CHK'D BY: 8. MEAD

ESTIMATOR: POWIK

PHONE: $\quad 7-5266$

DATE: Danzins

OTRM.

I-12. Cost Estimate for a Cover System Utilizing Poured-in Place Concrete Slab and a 30-mil FML (Cont'd) 
$1001: 7-03$

ACTIVTTY CODE: ESE200412

PROJ.NAMB: ECON. BVALUATTON OF CLOSURB CAP BARUIER MATERUAL,

ESTIMATOR: ramLX

PHONE: 7-S26S

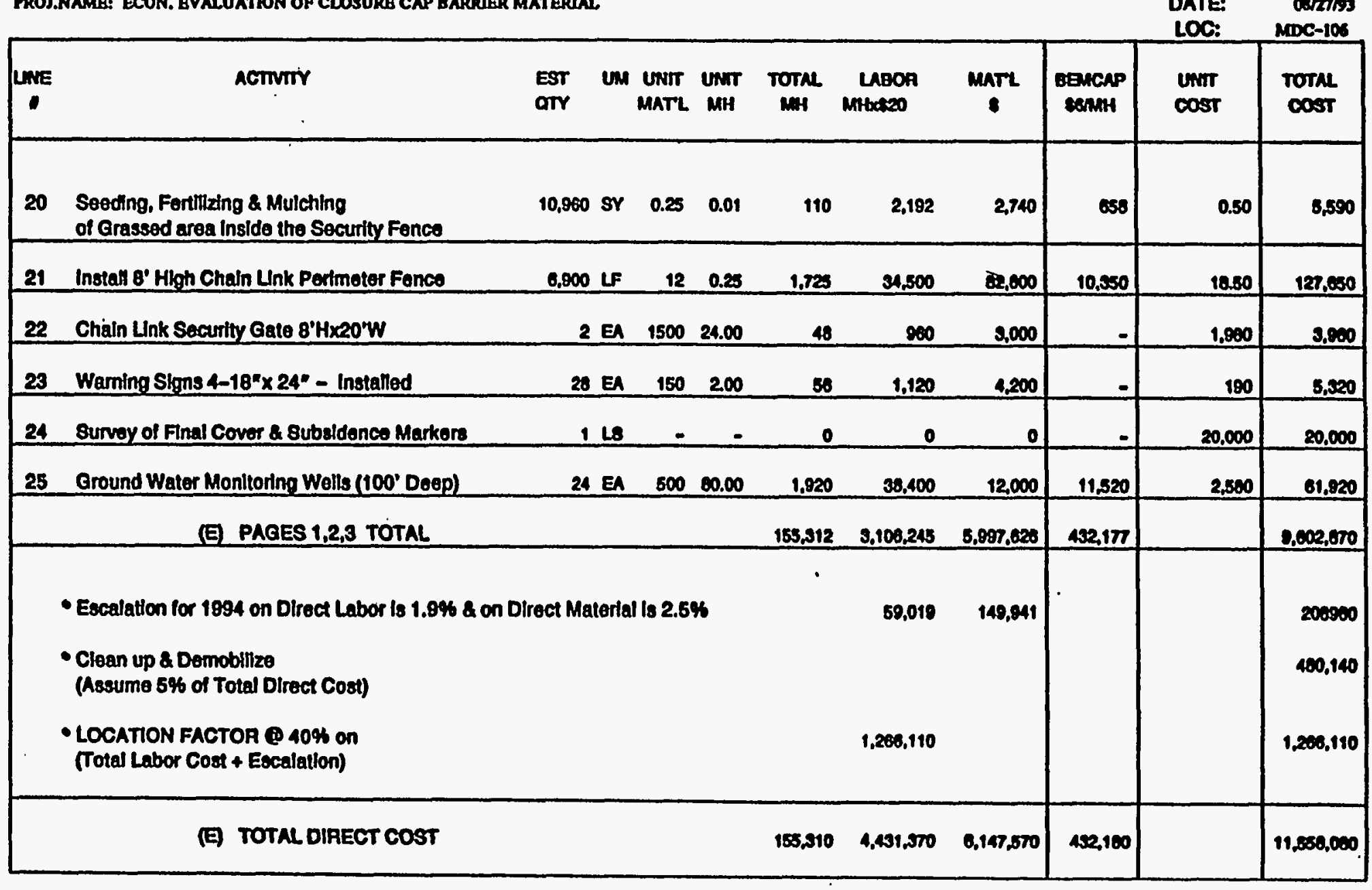

I-12. Cost Estimate for a Cover System Utilizing Poured-in Place Concrete Slab and a 30-mil FML (Cont'd) 
DETAIL B STIMATE

CHK'D BY: 8. MEAD

2001: 7-03

ACTVUTY CODB: ESEZCOA12

PROJ.NAME: ECON. EVALUATION OF CLOSURB CAP BARRIRR MATERIAL

\section{ESTIMATOA: ranix}

PHONE: 7-5266

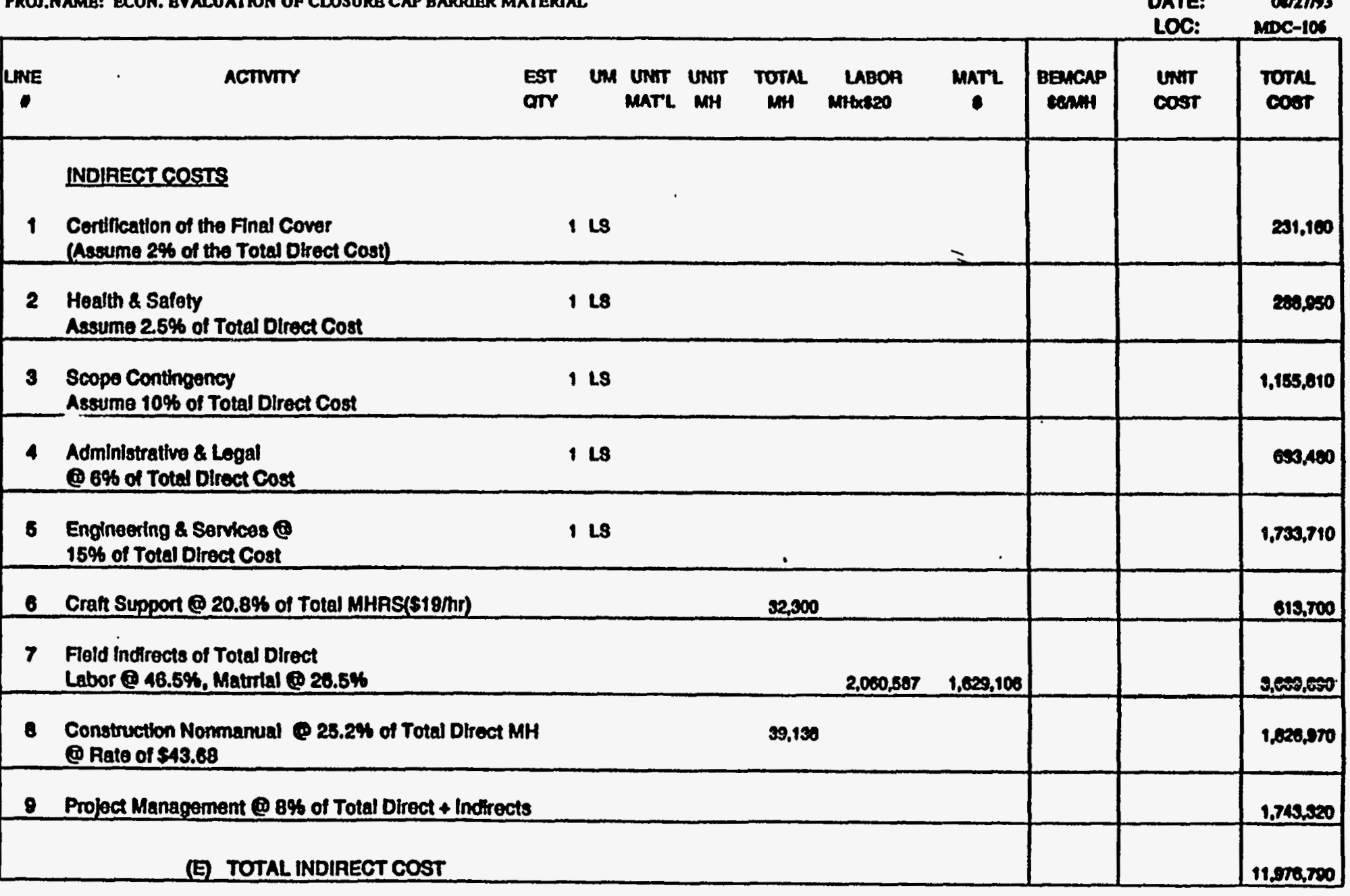

I-12. Cost Estimate for a Cover System Utilizing Poured-in Place Concrete Slab and a 30-mil FML (Cont'd) 


\section{0 : 7-03}

ACTIVIT CODE: ESE260412

MOS.NAME: ECON. EVALUATION OF CLOSURB CAP BARRIER MATERUAL.

ESTIMATOR: $\operatorname{ramx}$

PHONE: 7-526S

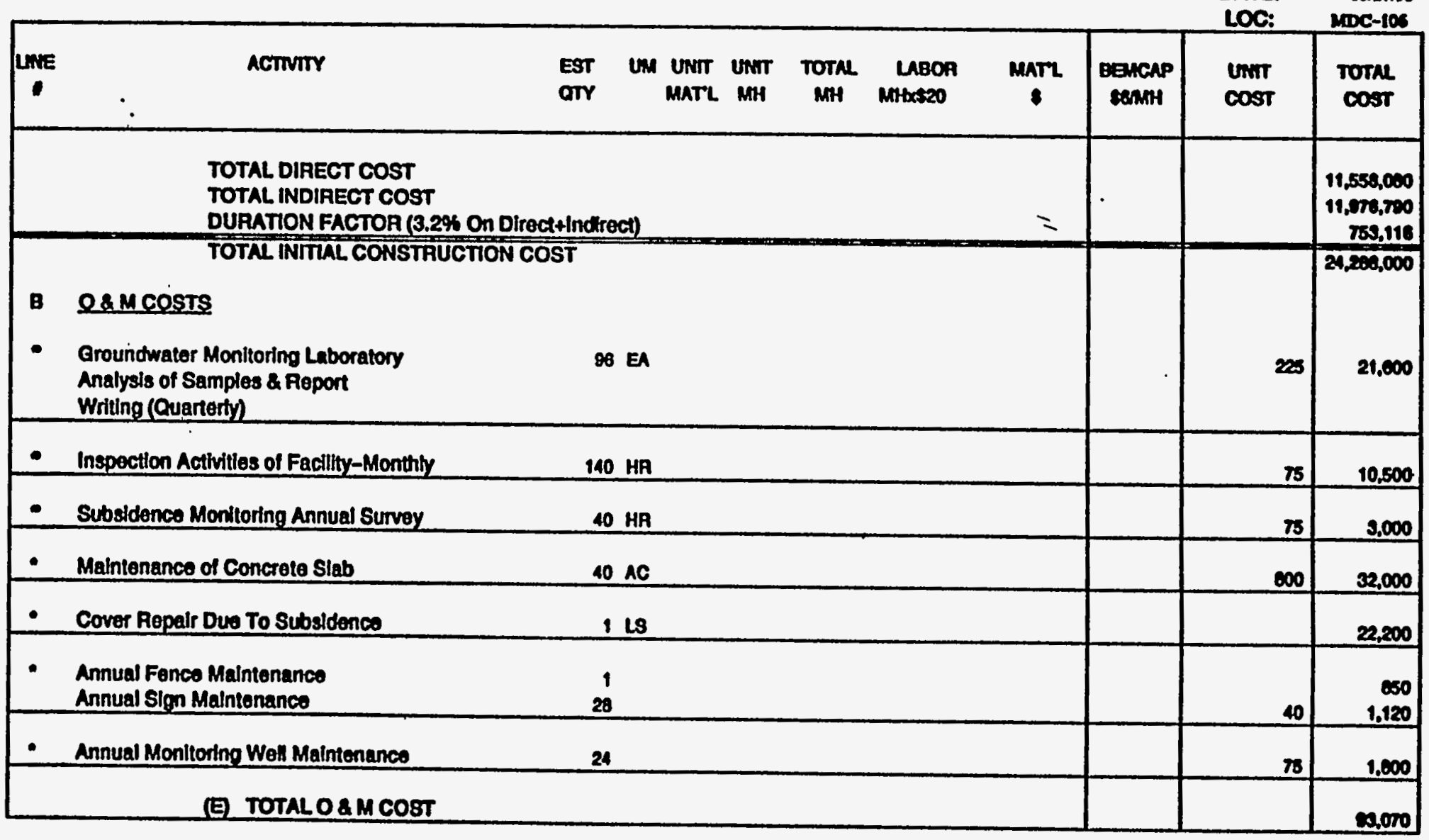

I-12. Cost Estimate for a Cover System Utilizing Poured-in Place Concrete Slab and a 30-mil FML (Cont'd) 
D R TAIL BSTIMATB

CHKD BY: 8. MEAD

L001: 7-03

ACTIVITY CODE: ESE2SOL12

MOJ.NAMB: BCON. BVALUATHON OR CLOSURE CAP BARRUER MATERIAL.

ESTIMATOA: ramx

PHONE: 7-5265

DATE: Damos

LOC:

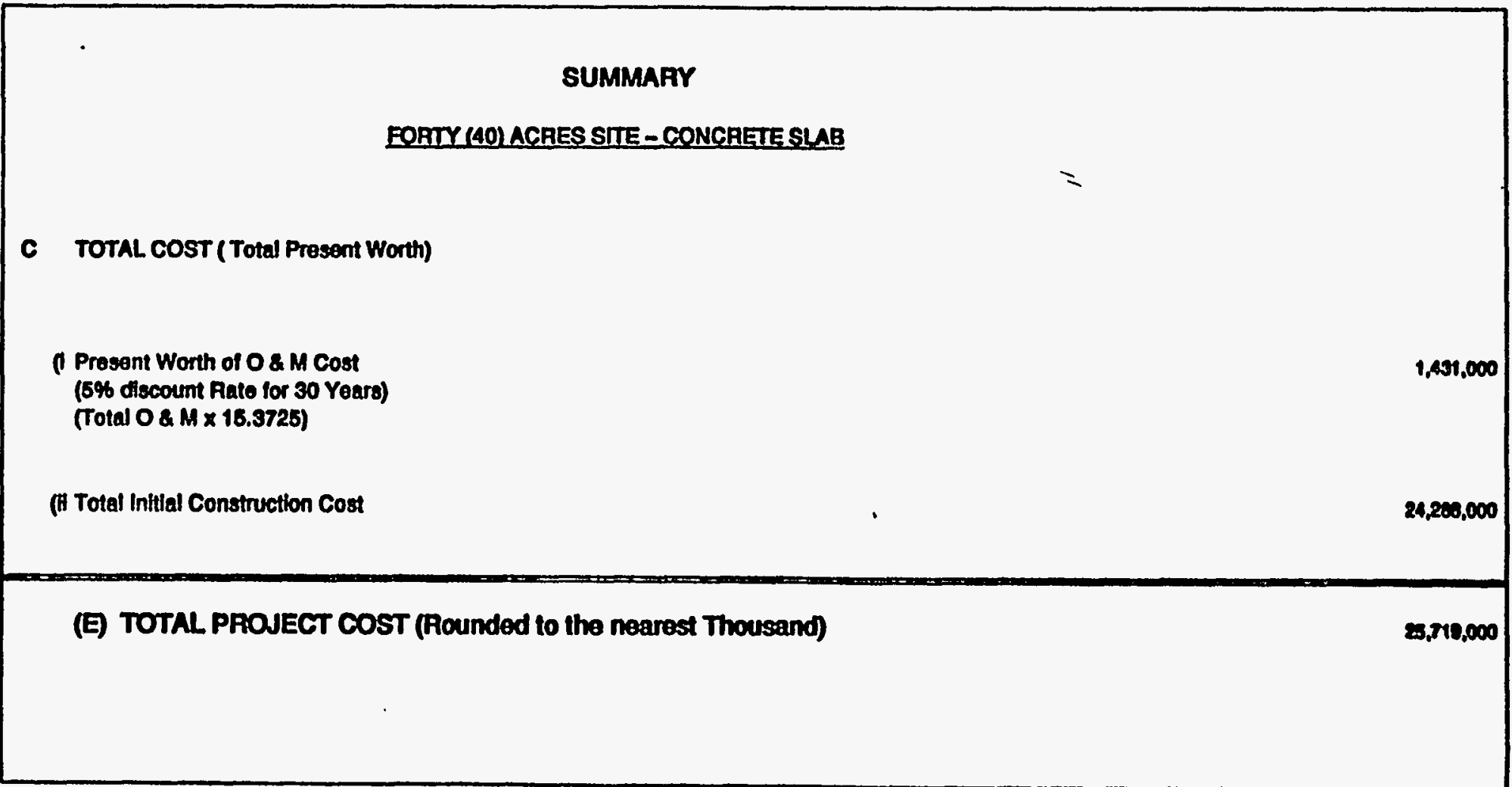

1-12. Cost Estimate for a Cover System Utilizing Poured-in Place Concrete Slab and a 30-mil FML (Cont'd) 


\section{Appendix J}

\section{Cost Estimates for 80-Acre Site}


Loof: 7-03

ACTIVITY CODE: ESEZSOH12

PROJ.NAME: ECON. EVALUATION OR CLOSURB CAP BARUIER MATERIAL

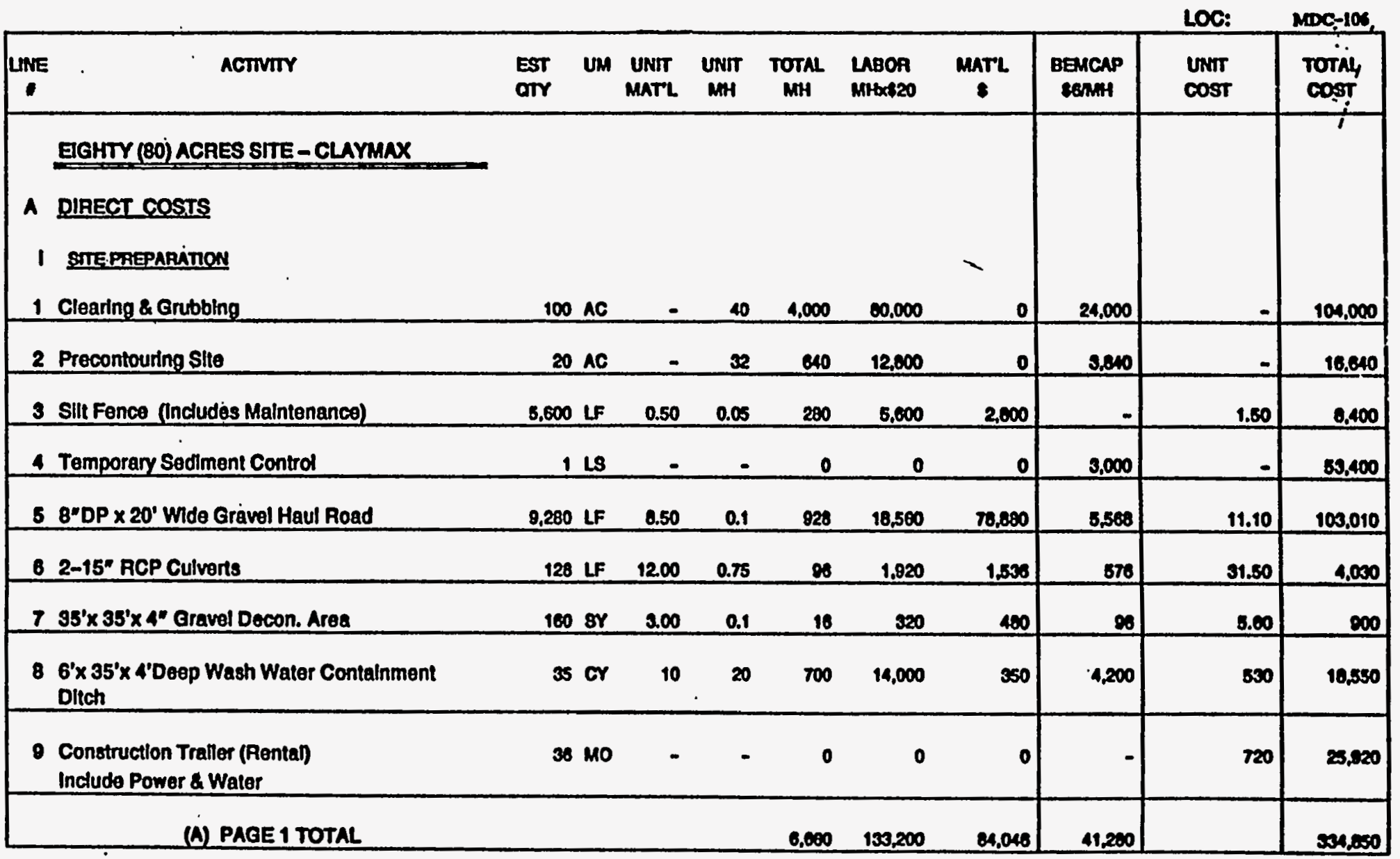

J-1. Cost Estimate for a Cover System Utilizing Claymax@ and a 30-mil FML 
DETAIL ESTIMATE

CHKO BY: 8. MEAD

200 N: 7-03

ACTIVTYY CODE: ESE2SOA12

PROJ.NAMB: ECON. EVALUATION OF CLOSURB CAP BARRUER MATERAAL

\section{ESTIMATOR: ramx}

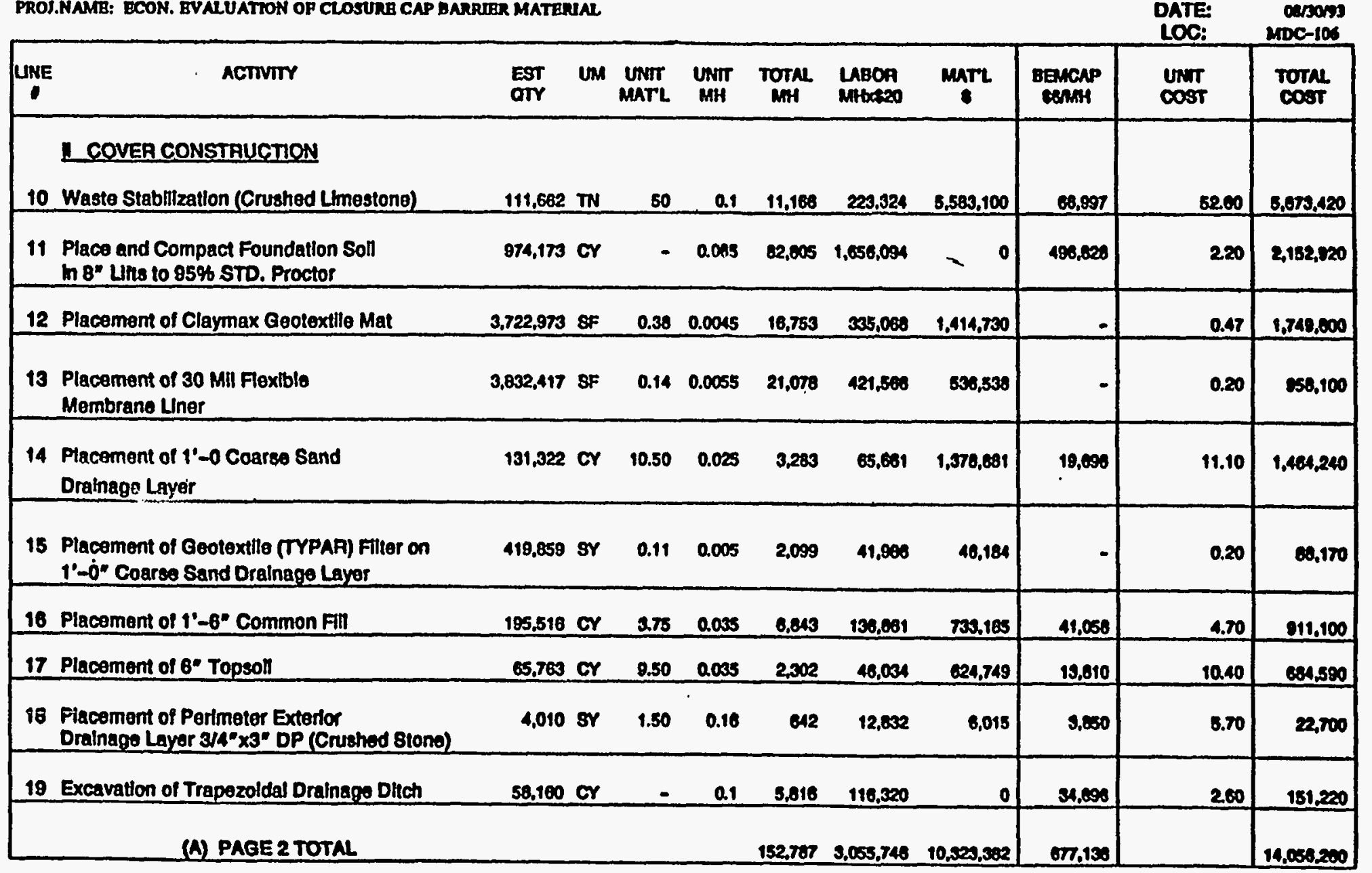

J-1. Cost Estimate for a Cover System Utilizing Claymax and a 30-mil FML (Cont'd) 
D B T A I E S T I MAT B

CHKD BY: S. MEAD

L001: 7-03

ACTIVTY CODE: ESE2OOH12

PROJ.NAMR: ECON. EYALUATION OF CLOSURB CAS BARRIER MATERIAR

ESTIMATOR: ramK

PHONE: 7-5266

DATE: OUSOSB

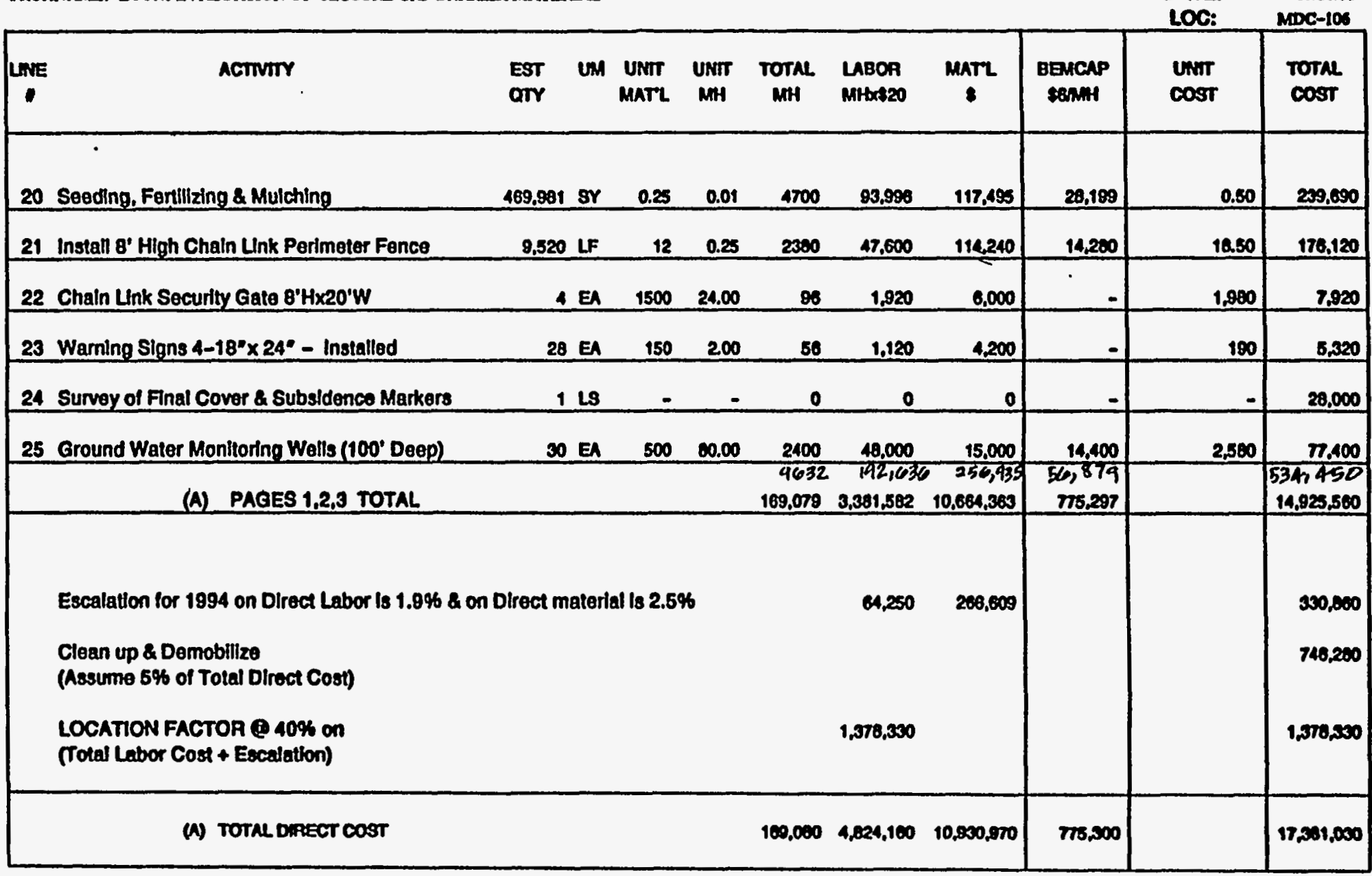

J-1. Cost Estimate for a Cover System Utilizing Claymax@ and a 30-mil FML (Cont'd) 
LOO I: $7-03$

ACTIVITY CODE; BSE200412

PROJ.NAME: ECON. BVALUATTON OP CLOSURB CAP BARRIER MATERIAL.

\section{ESTIMATOP: NaWIX}

PHONE: $\quad 7-5266$

DATE: arsors

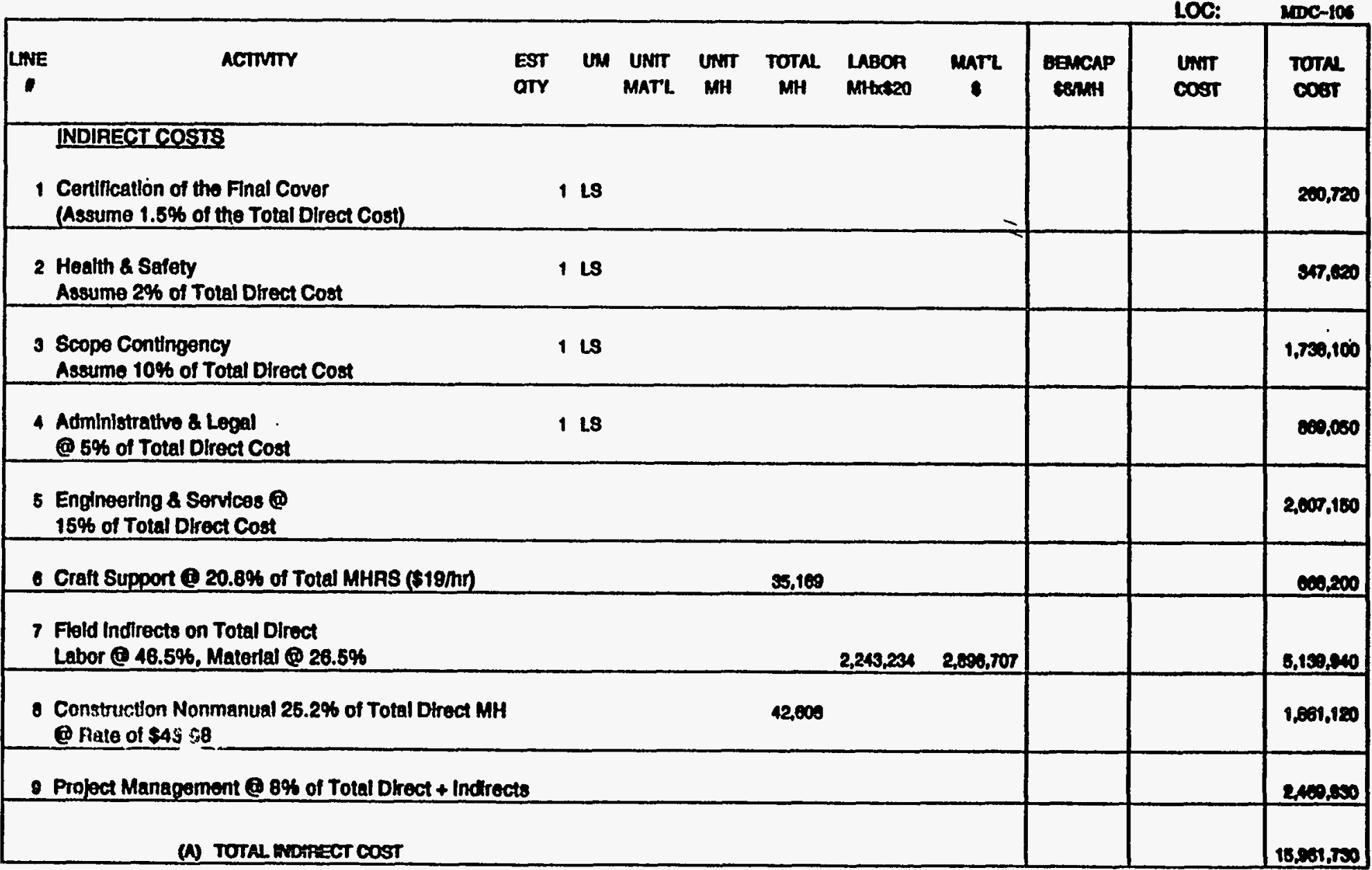

J-1. Cost Estimate for a Cover System Utilizing Claymax@ and a 30-mil FML (Cont'd) 


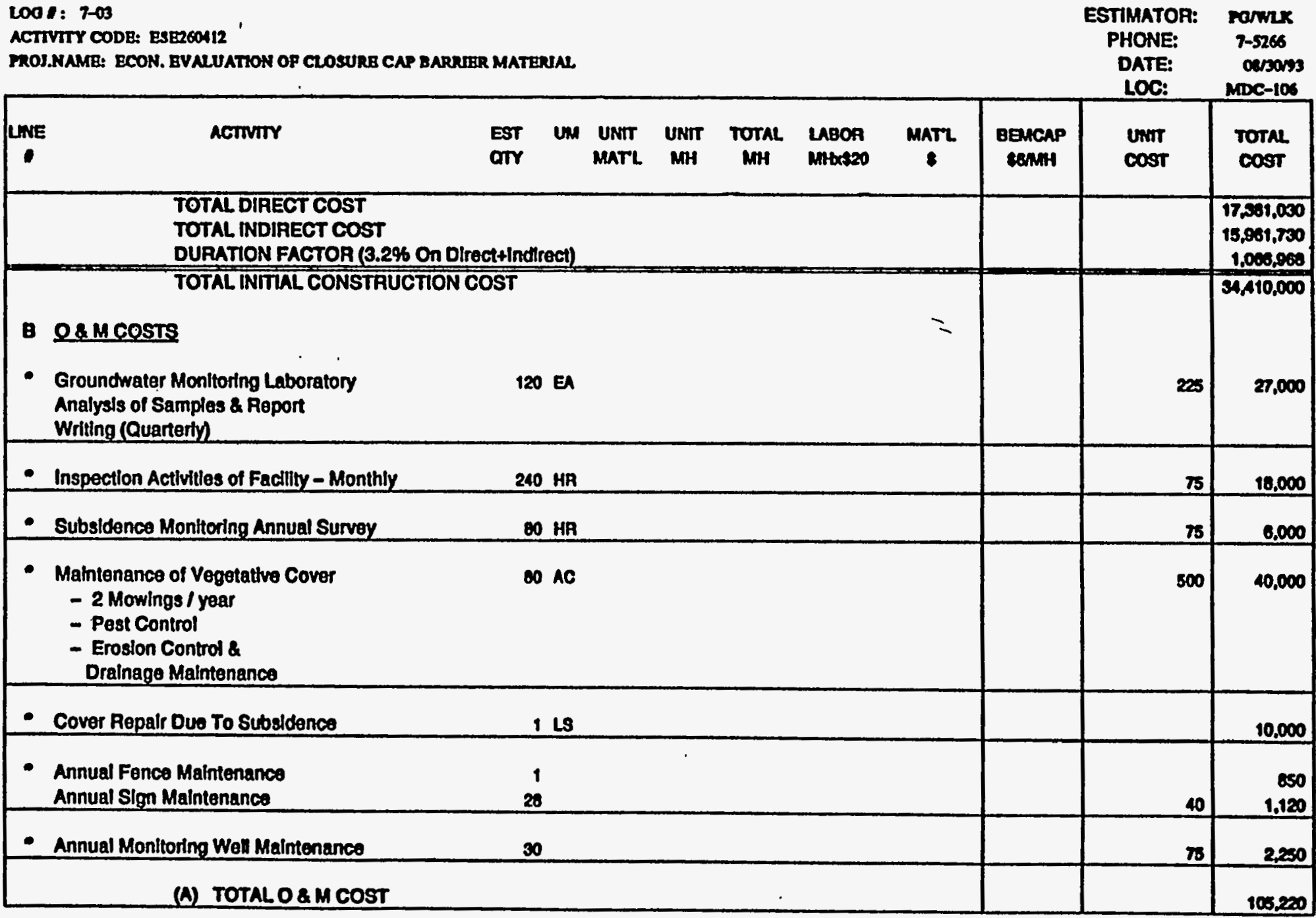

J-1. Cost Estimate for a Cover System Utilizing Claymax $ه$ and a 30-mil FML (Cont'd) 
D B TAIL B S T I A T B

\begin{tabular}{|c|c|}
\hline & \\
\hline & \\
\hline $\begin{array}{l}\text { PHON } \\
\text { DAT }\end{array}$ & \\
\hline
\end{tabular}

\section{L001: 7-03}

ACTIVITY CODA: ESE26ON12

PROJ.NAME: BCON. EVALUATION OP CLOSURB CAP BARPIER MATERIAL.

MDC-106

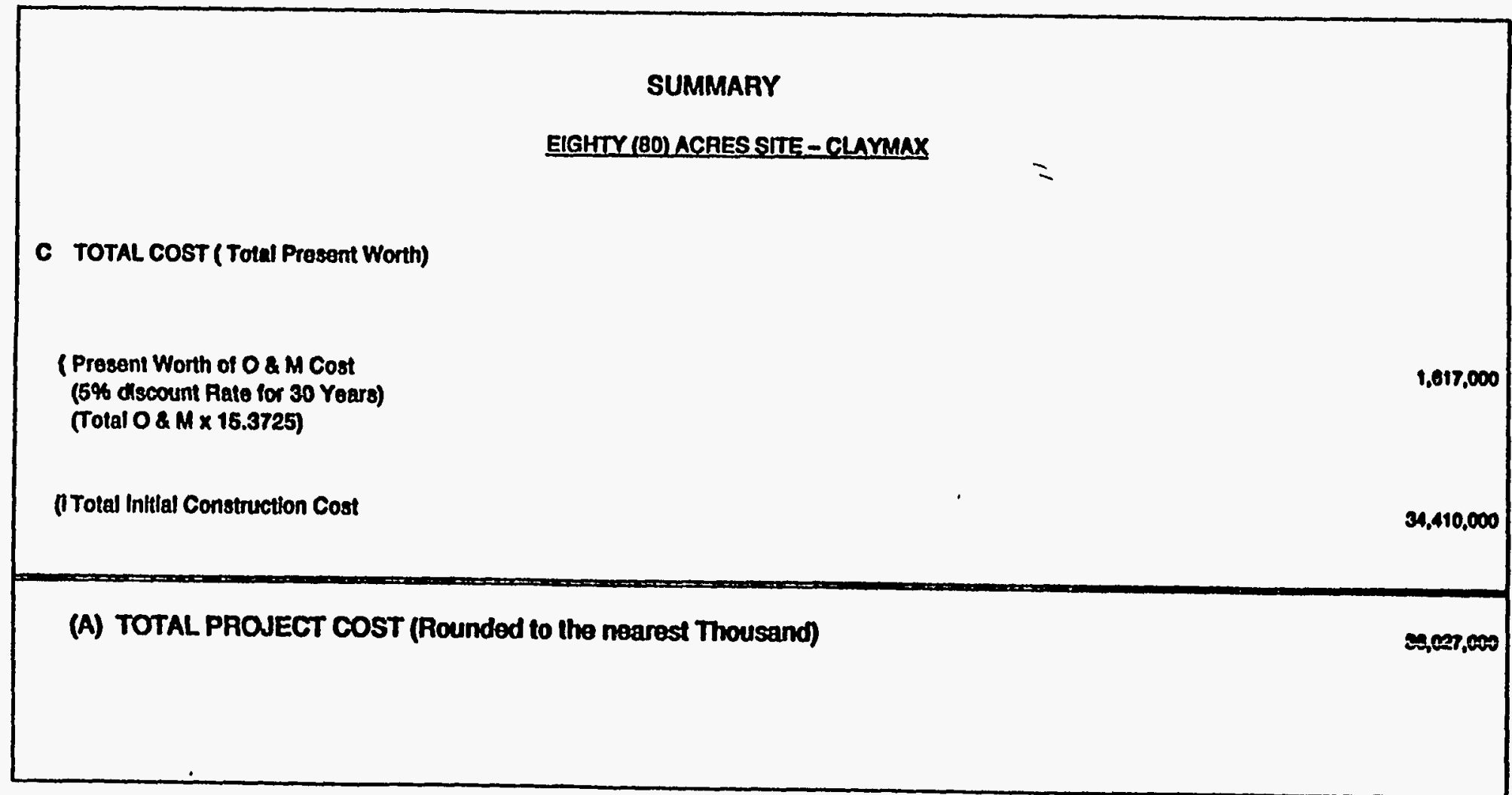

J-1. Cost Estimate for a Cover System Utilizing Claymax® and a 30-mil FML (Cont'd) 
DETAIL ESTIMATE

\section{Lo0 1: $7-03$}

ACTTVITY CODE: ESB260112

PROS.NAME: ECON. EVALUATTON OF CLOSURB CAY BARRIER MATERIAL

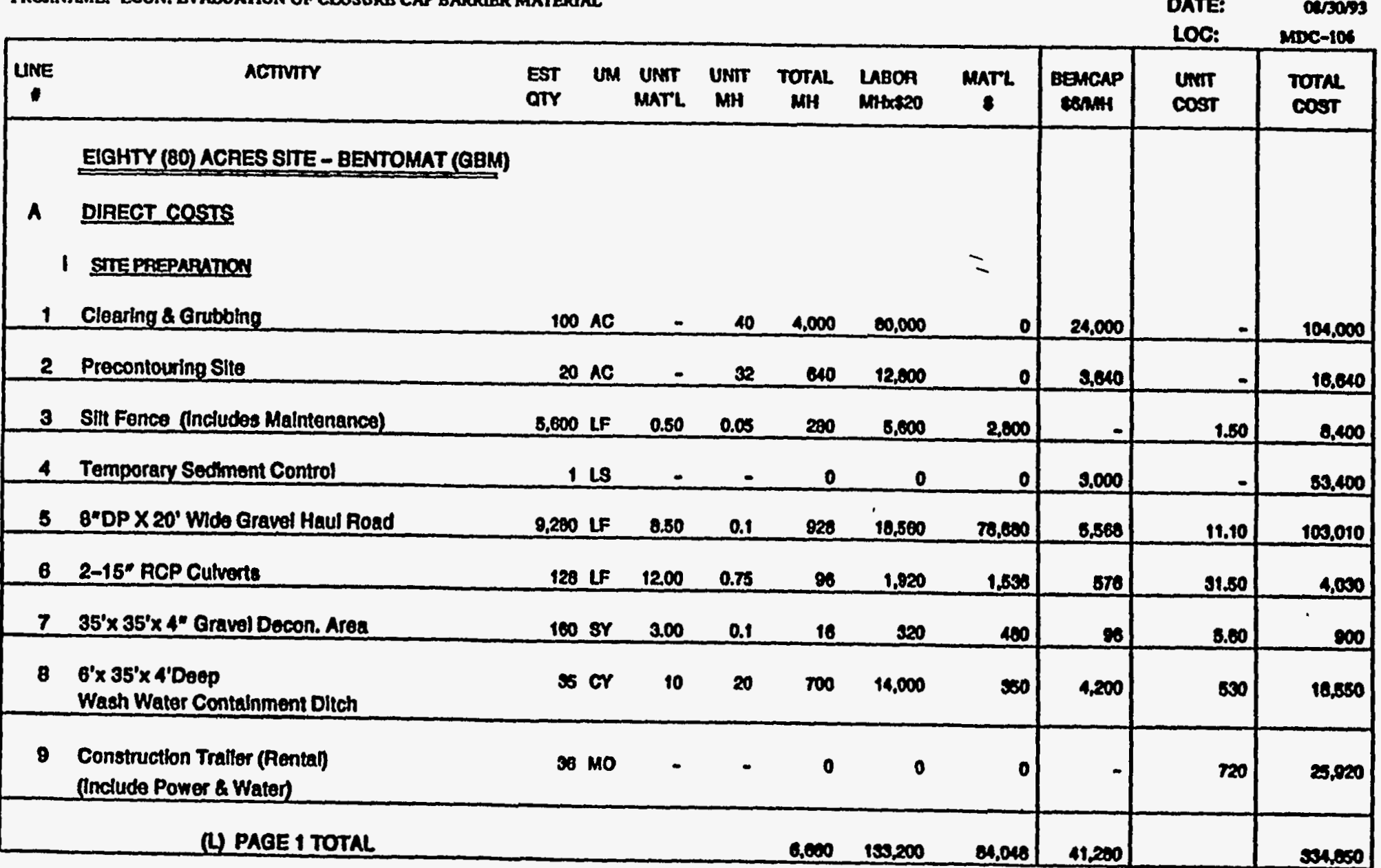

J-2. Cost Estimate for a Cover System Utilizing Bentomat@ and a 30-mil FML 
DETAILESTIMATE

CHK'D BY: 8. MEAD

Lo01: 7-03

ACTVITY CODB: ESE260112

PROI.NAME: ECON. BVALUATTON OF CLOSURE CAP BARRIER MATERIAL

\begin{tabular}{|c|c|c|c|c|c|c|c|c|c|c|c|}
\hline LNE & ACTIVITY & $\begin{array}{l}\text { EST } \\
\text { OTY }\end{array}$ & UM & $\begin{array}{l}\text { UNIT } \\
\text { MATL }\end{array}$ & $\begin{array}{l}\text { UNT } \\
\text { MH }\end{array}$ & $\underset{M H}{\text { TOTAL }}$ & $\begin{array}{l}\text { LABOR } \\
\text { MHXASZO }\end{array}$ & $\underset{8}{\operatorname{MnTL}}$ & $\begin{array}{l}\text { Bencenp } \\
\text { ERAMH }\end{array}$ & $\begin{array}{l}\operatorname{unn} \\
\cos t\end{array}$ & $\begin{array}{l}\text { Torne } \\
\text { cost }\end{array}$ \\
\hline & I_ COVERCONSTRUCTION & & & & & & & & & & \\
\hline 10 & Waste Stabilization (Crushed Limestone) & 111.662 & TN & so & 0.1 & 11,168 & 223,324 & $5,563,100$ & 00.097 & 3200 & $8,070,420$ \\
\hline 11 & $\begin{array}{l}\text { Place and Compact Foundation Soll } \\
\text { In } 8^{\prime \prime} \text { Litis to } 95 \% \text { Sidandard Proctor }\end{array}$ & 974.173 & & - & 0.065 & 82,005 & $1,656,094$ & $=$ & 196,628 & 2.20 & $2,162,020$ \\
\hline 12 & Placement of Bentomat Geotextlle Mat & $3,722,973$ & SF & 0.38 & 0.0045 & 16,755 & 335,060 & $1,414,730$ & - & 0.50 & $1,749,000$ \\
\hline 13 & $\begin{array}{l}\text { Placement of } 30 \text { Min Flexible } \\
\text { Membrane Lher }\end{array}$ & $3,632,419$ & sf & 0.14 & 0.0053 & 21,078 & 421,560 & 696,639 & - & 0.20 & 60,100 \\
\hline 14 & $\begin{array}{l}\text { Pracement of 1'-0 Coarse Sand } \\
\text { Oralnage Layer }\end{array}$ & 931,322 & or & 10.50 & 0.025 & $\mathbf{5 , 2 8 3}$ & 05,061 & $1,370,001$ & 19,600 & 11.10 & $1,404,240$ \\
\hline 18 & $\begin{array}{l}\text { Placement of Gootexille (TYPAF) Fither on } \\
1^{\prime}-0^{*} \text { Coarse Sand Dralnage Layer }\end{array}$ & 419,859 & 8Y & 0.11 & 0.005 & 2.099 & 41,960 & 46,184 & - & 0.20 & $\infty 0,120$ \\
\hline 16 & Placement of $1^{\prime}-6^{\circ}$ Common Fin & 185,516 & or & 3.76 & 0.005 & 0,243 & 136,061 & 703,106 & 41,050 & 4.70 & 011,100 \\
\hline 17 & Placement of $6^{*}$ Topsoll & 85,703 & or & 9.50 & 0.025 & 2,2002 & 46.034 & 024,749 & 15,810 & 10.40 & 604500 \\
\hline 18 & $\begin{array}{l}\text { Placement of Perimeter Extertor } \\
\text { Dralnaga Layer } 3 / 4^{\prime} \times 3^{*} \text { DP (Crushed Stono) }\end{array}$ & 4,010 & & 1.60 & 0.16 & 642 & 12,602 & 0,018 & 8,050 & 8.70 & 22,700 \\
\hline 10 & Excavation of Trapezoldal Drainage Ditch & 58,100 & or & $=$ & a. & 8,016 & 110.320 & 0 & 34,600 & 200 & 131,220 \\
\hline & (L) PAGE 2 TOTAL & & & & & 152,708 & $3.055,748$ & 10,3ea,se2 & $m, 130$ & & $14,050,200$ \\
\hline
\end{tabular}

J-2. Cost Estimate for a Cover System Utilizing Bentomat $($ and a 30-mil FML (Cont'd) 
D B T A I L B T I M A T B

CHK'D BY: 8. MEAD

LO01: $7-03$

ACTIVITY CODE: ESE260412

PROJ.NAME: ECON. BVALUATION OP CLOSURB CAP BARRIRR MATERLAI

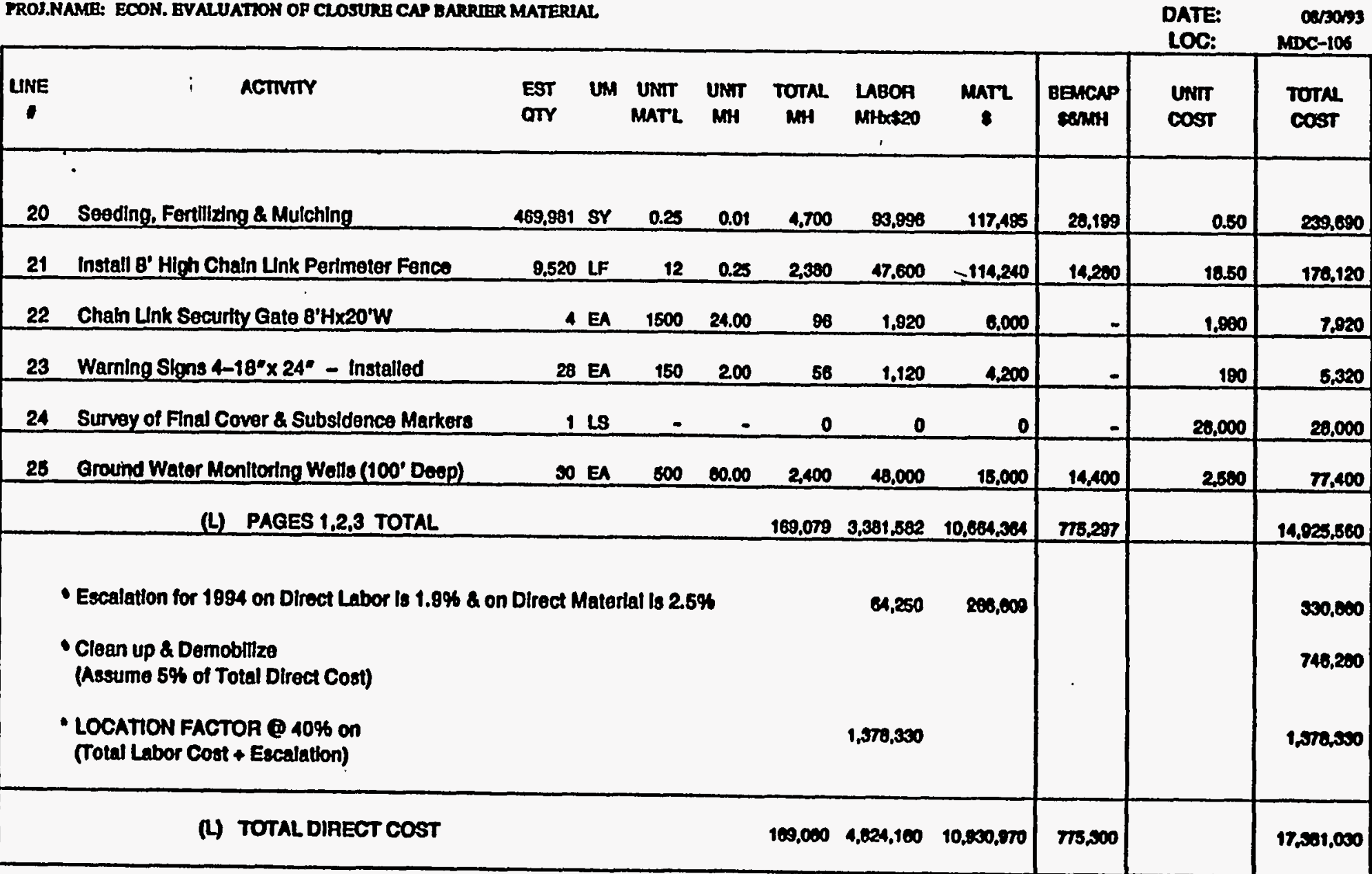

J-2. Cost Estimate for a Cover System Utilizing Bentomat $\$$ and a 30-mil FML (Cont'd) 
DETAIL BST I M A T

L00 I: $7-03$

ACTTVITY CODB: ESE260412

PROI.NAMB: ECON. BVALUATTON OF CLOSURB CAP BARRIER MATERJAL

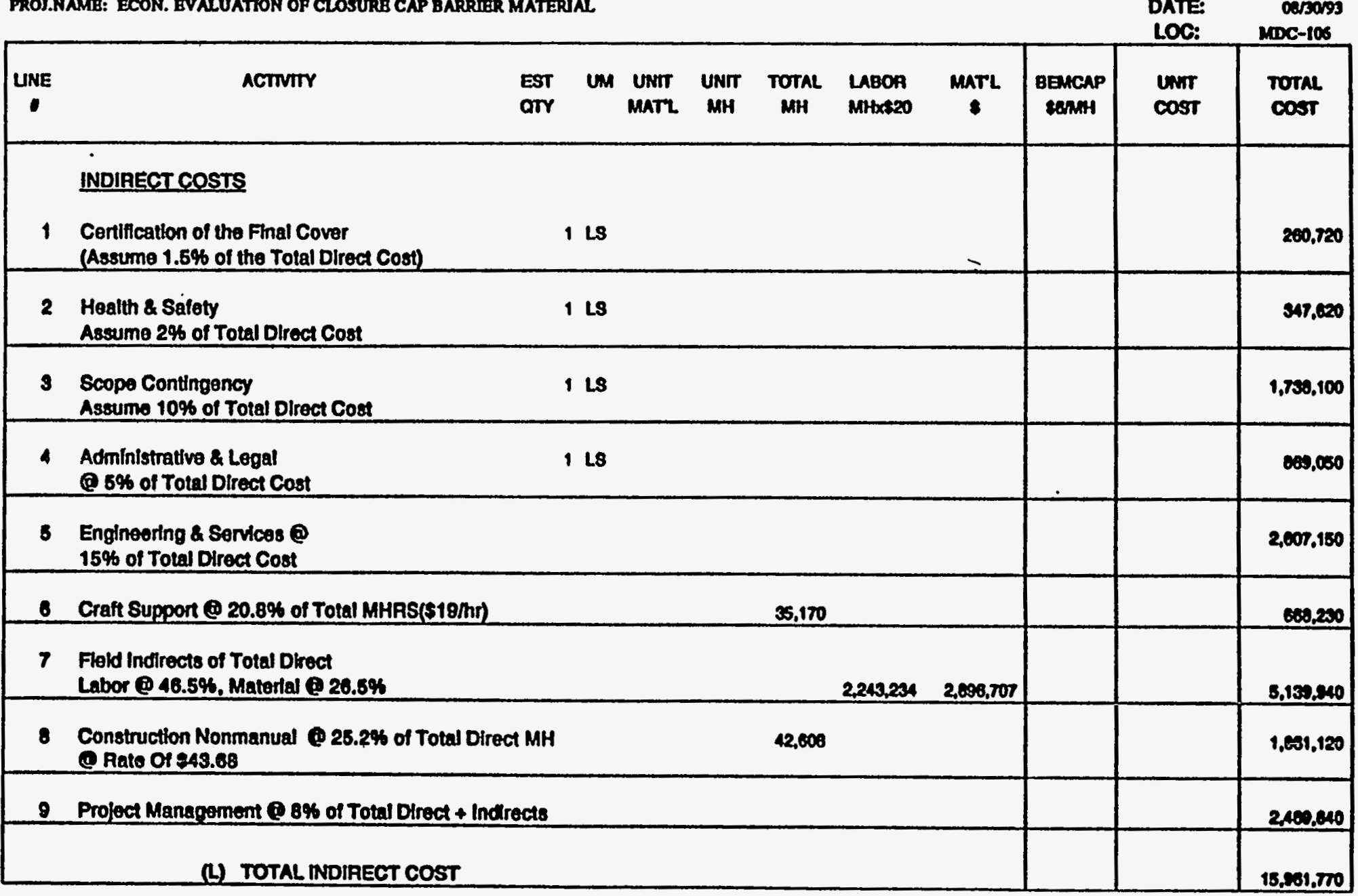

J-2. Cost Estimate for a Cover System Utilizing Bentomato and a 30-mil FML (Cont'd) 


\section{L001: 7-03}

ACTIVTY CODB: BSB260412

PROJ.NAME: ECON. EVALUATON OF CLOSURB CAP SARRIER MATERINL

\section{ESTIMATOR: ROMTK}

PHONE: 7-5266

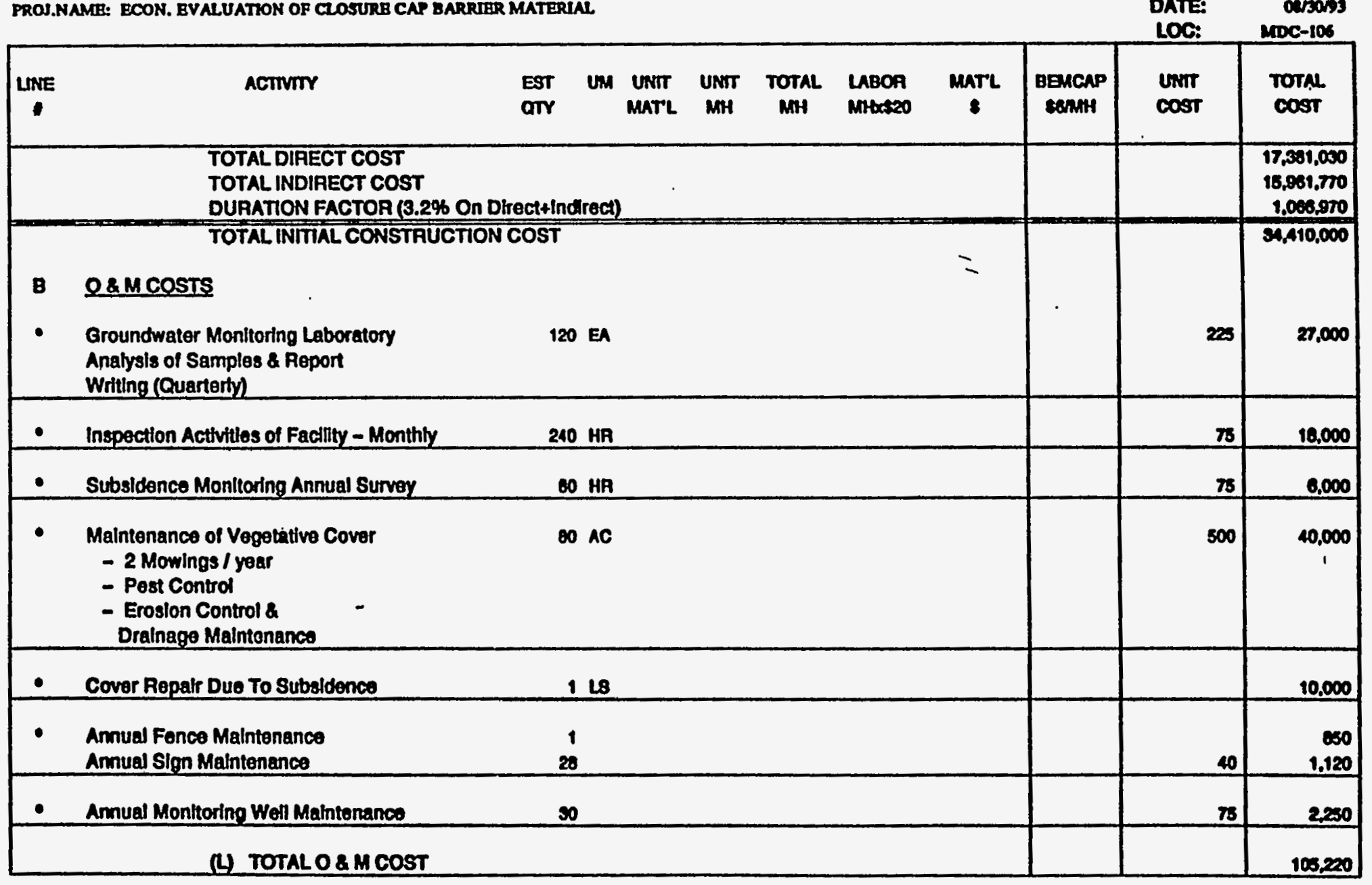

J-2. Cost Estimate for a Cover System Utilizing Bentomat@ and a 30-mil FML (Cont'd) 
D B T A I L B S T IMAT

1001: 7-03

ACTIVIT CODB: ESE260112

PROS.NAME: ECON. BUALUATHON OP CLOSURB CAP BARRIER MATERIAL
CHKD BY: 8. MEAD

ESTIMATOF: NaWMX

PHONE: 7-5265

DATE: Cors0rs

LOC: MDC-106

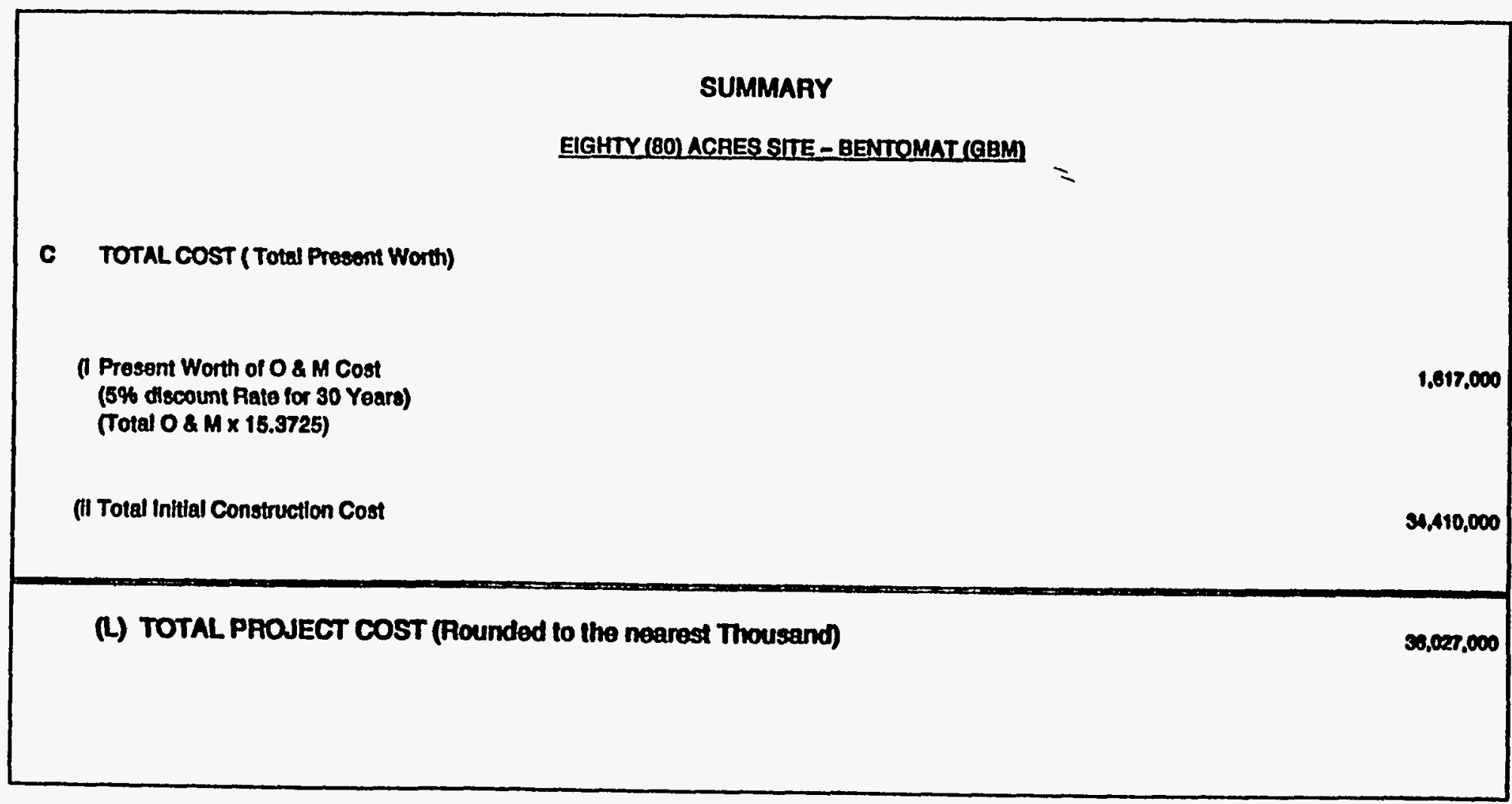

J-2. Cost Estimate for a Cover System Utilizing Bentomat $\circledast^{8}$ and a 30-mil FML (Cont'd) 
DETAIL ESTIMATE

L001: 7-03

ACTIVITY CODE: ESE260112

TROJ.NAME: ECON. EVALUATTON OF CLOSURB CAP BARRIER MATERIAL

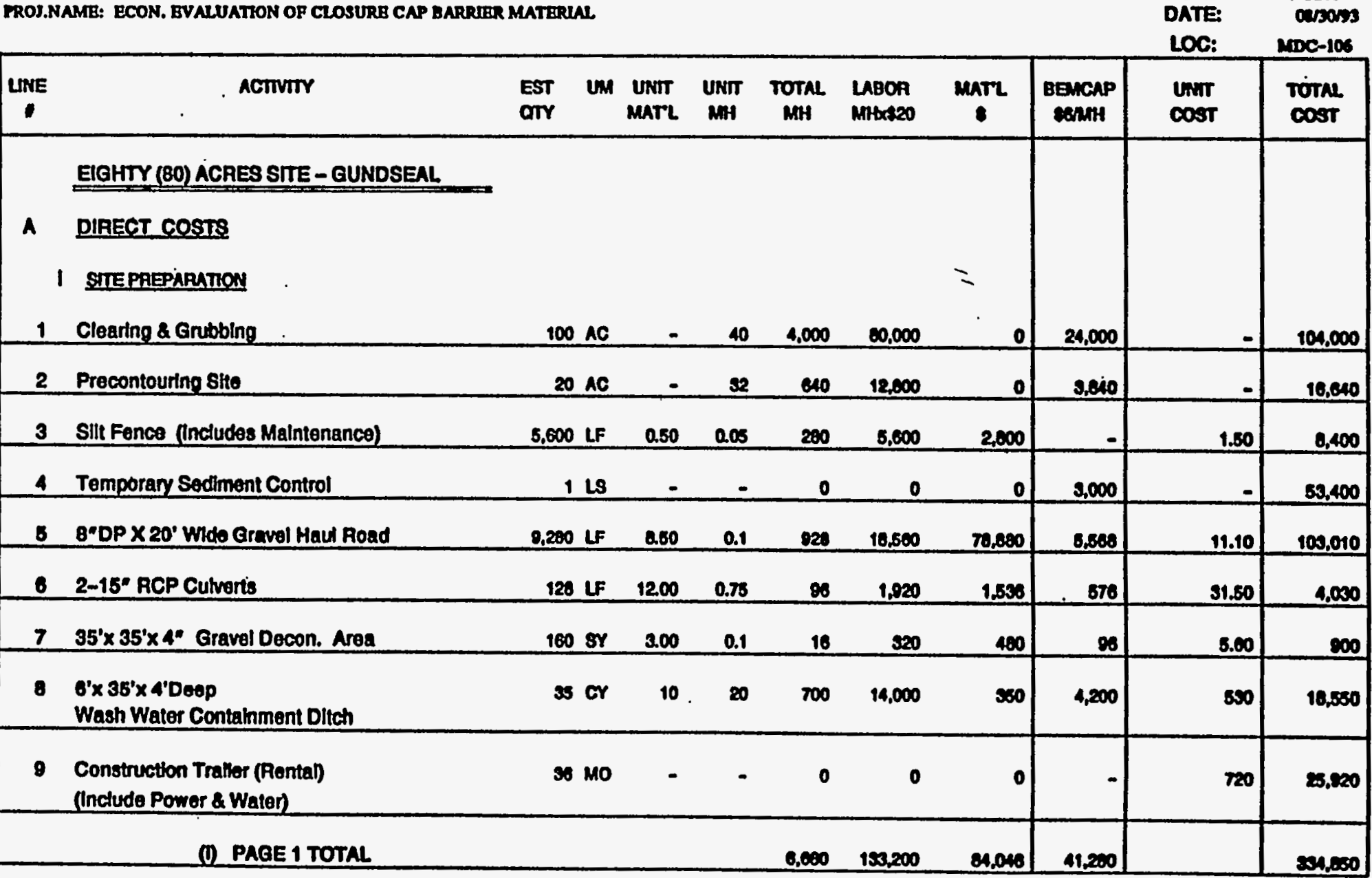

J-3. Cost Estimate for a Cover System Utilizing Gundseal(B) 
DETAIL ESTIMATE

CHK'D BY: S. MEAD

Lod : $7-03$

ACTIVTYY CODE: BSEZCOU12

PROS.NAME: ECON. BVALUATION OP CLOSURB CAP BARRUER MATERIAL

\begin{tabular}{|c|c|c|c|c|c|c|c|c|c|c|c|}
\hline UNE & ACTIVIT & $\begin{array}{l}\text { EST } \\
\text { OTY }\end{array}$ & UM & $\begin{array}{l}\text { UNIT } \\
\text { MATLL }\end{array}$ & $\begin{array}{l}\text { UniT } \\
\text { MH }\end{array}$ & $\begin{array}{c}\text { TOTAL } \\
\text { MH }\end{array}$ & $\begin{array}{l}\text { LABOA } \\
\text { MHBASE2O }\end{array}$ & $\underset{8}{\operatorname{maTh}}$ & ogncap & $\begin{array}{l}\text { Unit } \\
\text { Cost }\end{array}$ & $\begin{array}{l}\text { TOTAL } \\
\text { COST }\end{array}$ \\
\hline & I COVER CONSTAUCTION & & & & & & & & & & \\
\hline 10 & Waste Stablization (Crushed Limestone) & 111,662 & $\mathbf{m}$ & 80 & 0.1 & 11.168 & 223,324 & $8,589,100$ & 60,997 & 82.00 & $8,075,420$ \\
\hline 11 & $\begin{array}{l}\text { Place and Compact Foundation Soll } \\
\text { In } 8^{*} \text { Lits to } 95 \% \text { Std. Proctor }\end{array}$ & 974,173 & Cr & - & 0.085 & 82,605 & $1,656,094$ & $\mathbf{0}$ & 490,828 & 220 & $2,162,020$ \\
\hline 12 & $\begin{array}{l}\text { Placement of Gundseal Geomembranel } \\
\text { Bentonite Mat }\end{array}$ & $3,722,973$ & SF & 0.38 & 0.0048 & 10,763 & 535,000 & $1,414,750$ & - & 0.47 & $1,749,000$ \\
\hline 13 & $\begin{array}{l}\text { Placement of t'-0 Coarse Sand } \\
\text { Drainage Layer }\end{array}$ & 131,322 & Cr & 10.50 & 0.025 & 3,283 & 65,681 & $1,370,801$ & 19,600 & 11.10 & $1,484,240$ \\
\hline 14 & $\begin{array}{l}\text { Placoment of Geotextile (TYPAA) Filter on } \\
\text { 1'-0" Coarse Sand Dralnage Layer }\end{array}$ & 419,859 & sr & 0.11 & 0.005 & 2,099 & 41,906 & 40,184 & - & 0.20 & 60,170 \\
\hline 15 & Placoment of 1'-6. Common Fll & 195,516 & CY & 3.75 & 0.035 & 6,843 & 130,801 & 733,163 & 41,050 & 4.70 & 011,100 \\
\hline 16 & Placement of 6: Topsoll & 6,763 & Cr & 9.50 & 0.065 & 2,302 & 40,0034 & 624,749 & 13,010 & 10.40 & 604580 \\
\hline 17 & $\begin{array}{l}\text { Piacement of Perimeter Exterlor } \\
\text { Drainage Layar } 34^{*} \times 3^{*} \text { DP (Crushed Stono) }\end{array}$ & 4,010 & $8 Y$ & 1.50 & 0.18 & 642 & 12,032 & 0,016 & 3,000 & $\mathbf{8 . 7 0}$ & 22,700 \\
\hline 18 & Excavation of Trapezoldal Dralnape Ditch & 60,160 & Cr & - & 0.1 & 5,010 & 110,320 & 0 & 34,080 & 200 & 151,20 \\
\hline & (1) PAGE2 TOTAL & & & & & 131,700 & $2,634,180$ & $0.700,044$ & 67.130 & & $13,090,100$ \\
\hline
\end{tabular}

J-3. Cost Estimate for a Cover System Utilizing Gundseal@ (Cont'd) 
DBTAIL BSTIMATE

$1001: 7-03$

ACTIVITY CODE: ESB2SOH12

PROJ.NAMB: BCON: EVALUATTON OF CLOSURB CAP BARUIRR MATERIAL

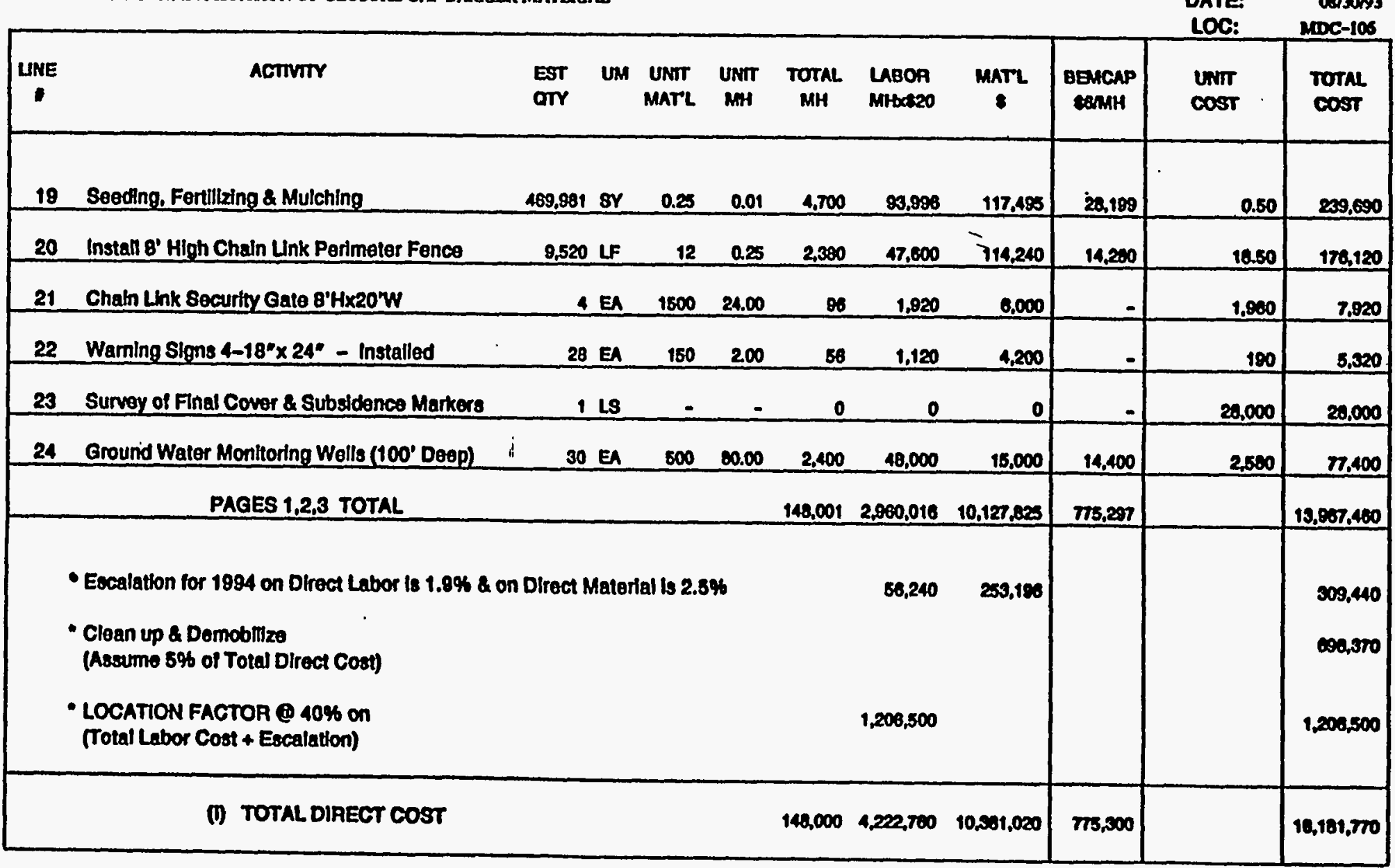

J-3. Cost Estimate for a Cover System Utilizing Gundseal@ (Cont'd) 
Economic Evaluation of Closure Cap Barrier Materials Study(U)

Volume II Appendix

DETAIL BSTIMAT B

LOO 1: 7-03

ACTTVITY CODB: ESE260112

PROJ.MAME: ECON. BYALUATION OF CLOSURB CAP BARRIER MATERUAL.

CHK'D BY: 8. MEAD

ESTIMATOR: ROMIX

PHONE: 7-5268

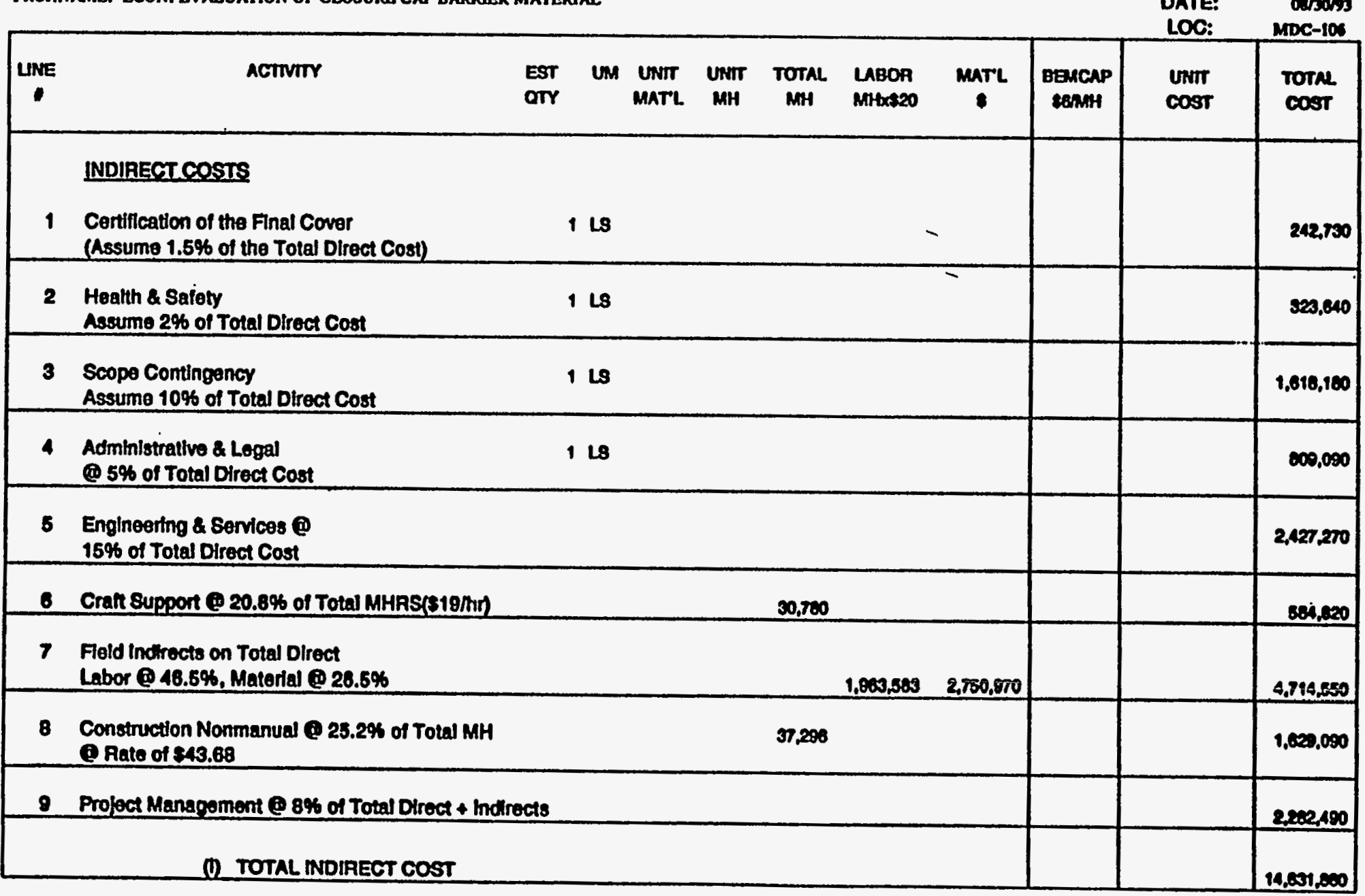

J-: Cost Estimate for a Cover System Utilizing Gundseal@ (Cont'd) 
D B T A L B S T I M A B

CHK'D BY: 8. MEAD

Loo I: $7-03$

ACTIVTTY CODB: BSE260112

PROJ.NAMRE: ECON. EVALUATION OF CLOSURB CAP BARRIRR MATERLAL

ESTIMATOR: rormx

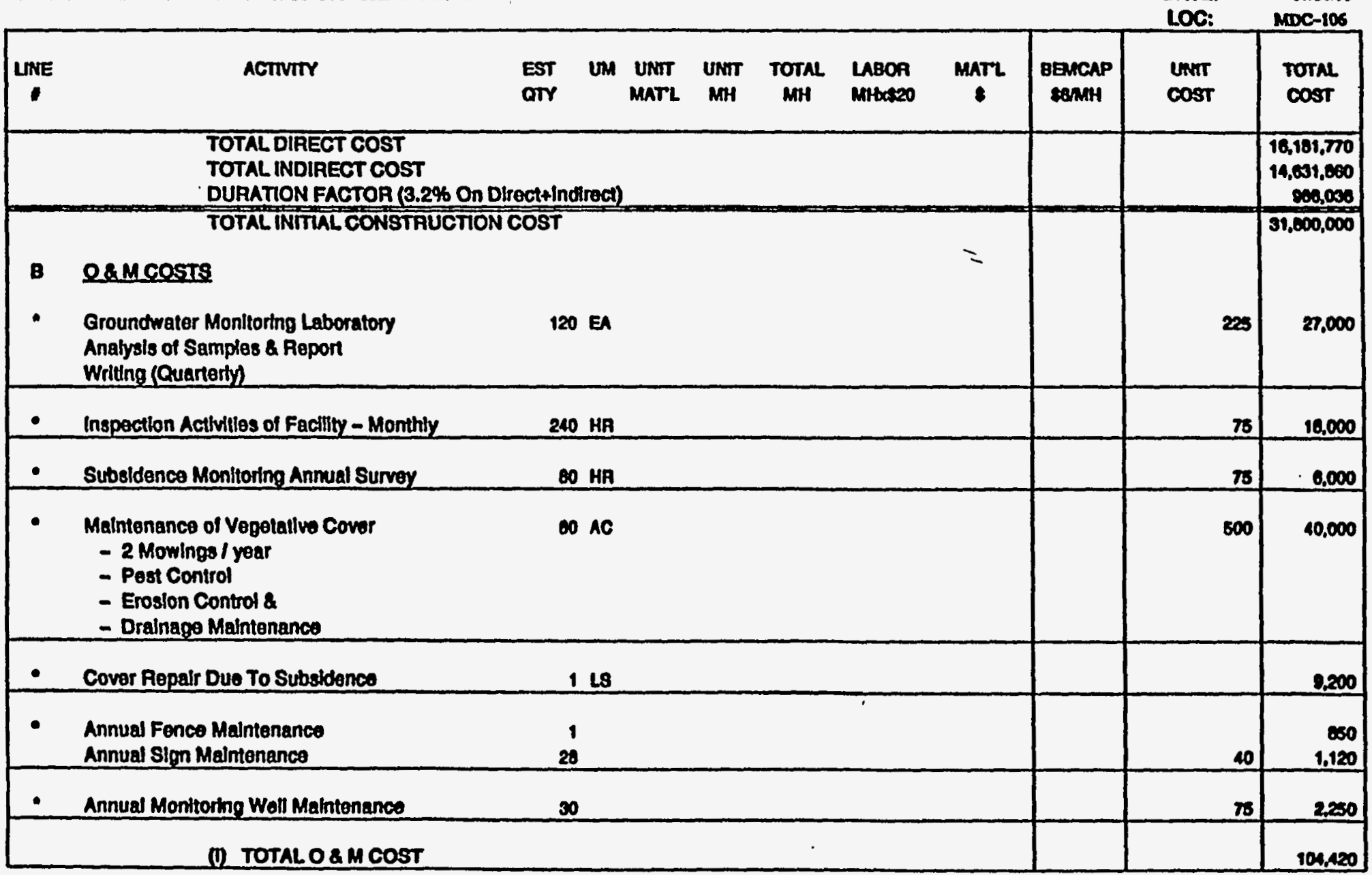

J-3. Cost Estimate for a Cover System Utilizing Gundseal@ (Cont'd) 
DETAIL BSTIMATB

$\begin{array}{cc}\text { CHK'D BY: } & \text { S. MEAD } \\ \text { ESTIMATOR: } & \text { ROMWK } \\ \text { PHONE: } & \text { 7-5266 } \\ \text { DATE: } & \text { OAMO193 } \\ \text { LOC: } & \text { MDC-106 }\end{array}$

ACTIVITY CODE: BSEQGOU12

MOJ.NAME: ECON. BVALUATTON OF CLOSURB CAP BARRIER MATERINL

Loci

MDC-106

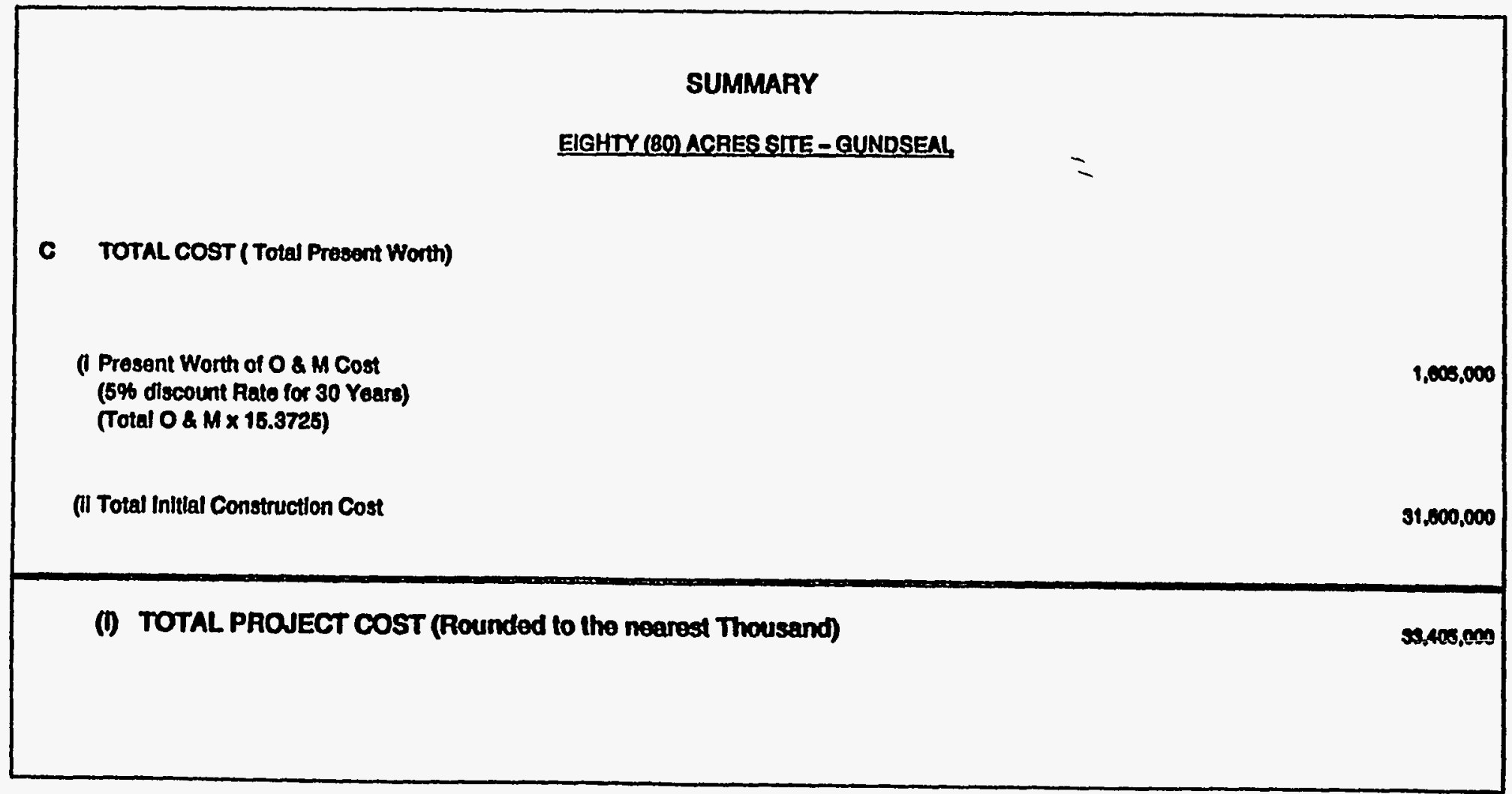

J-3. Cost Estimate for a Cover System Utilizing Gundseal@ (Cont'd) 


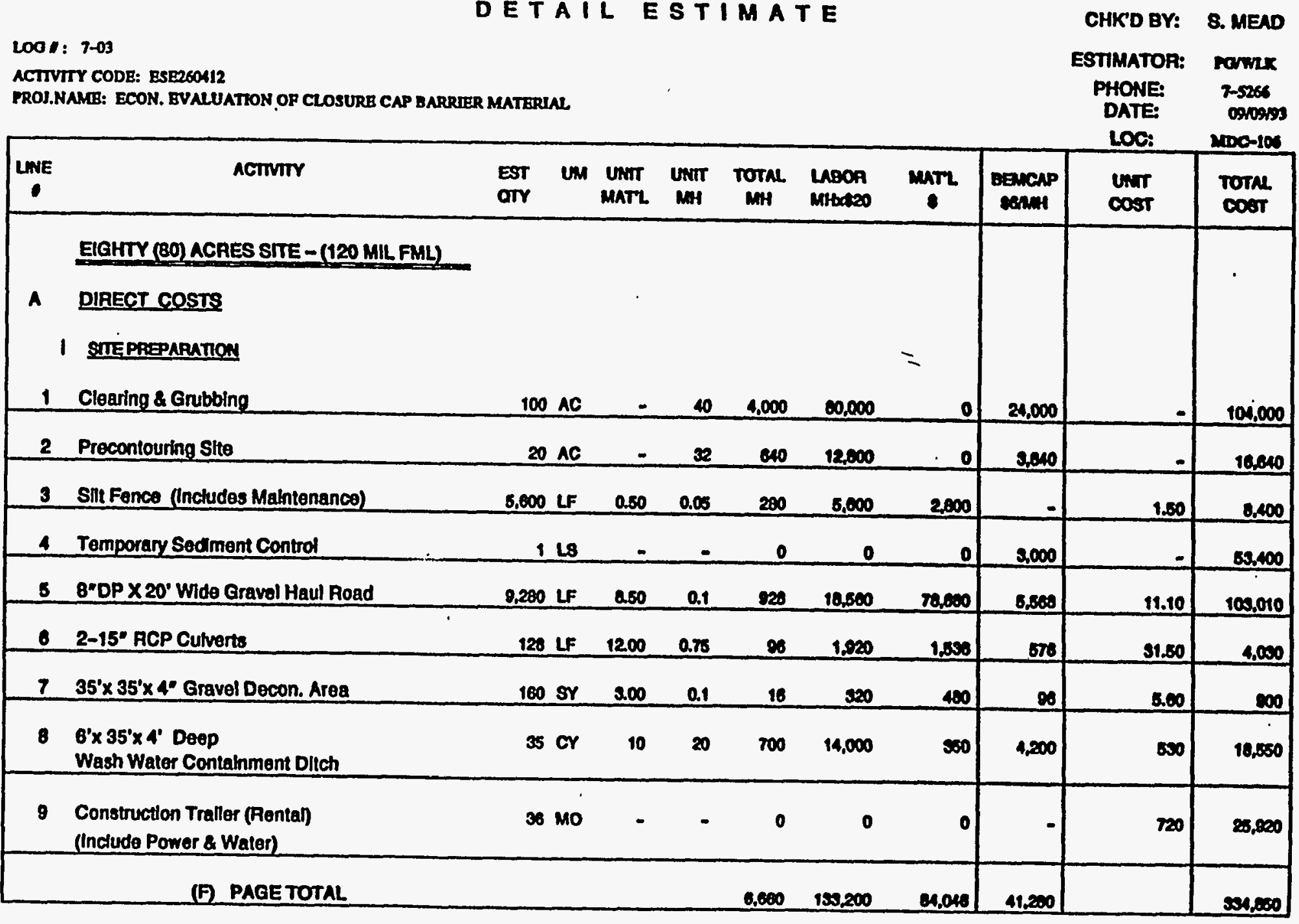

J-4. Cost Estimate for a Cover System Utilizing 120-mil High Density Polyethylene (HDPE) Liner 
DETAIL ESTIMATE

1001: $7-03$

NCTIVITY CODE: ESE260112

PROJ.NAME: ECON. EVALUATTON OF CLOSURB CAP BARRIER MATERUAL.

\begin{tabular}{|c|c|c|c|c|c|c|c|c|c|c|c|}
\hline & & & & & & & & & & LOC: & $\begin{array}{l}09009103 \\
M 0 C-106\end{array}$ \\
\hline UNE & ACTNTIY & $\begin{array}{l}\text { EST } \\
\text { OTr }\end{array}$ & un & $\begin{array}{c}\text { Unirr } \\
\text { MATL }\end{array}$ & ${ }_{\mathrm{MH}}^{\mathrm{UNH}}$ & $\begin{array}{l}\text { TOTRL } \\
\text { MH }\end{array}$ & $\begin{array}{l}\text { LABOA } \\
\text { Mtatzo }\end{array}$ & $\stackrel{\operatorname{marl}}{*}$ & $\begin{array}{l}\text { gencap } \\
\text { sennt }\end{array}$ & $\begin{array}{l}\ln \pi r \\
\text { costr }\end{array}$ & $\begin{array}{l}\text { TOTRL } \\
\text { Cosst }\end{array}$ \\
\hline & H. COVEA CONSTRUCTION & & & & & & & & & & \\
\hline 10 & Waste Stabillzatlon (Crushod Limestone) & 111,662 & $\pi$ & 50 & 0.1 & 11,168 & 2204.524 & $5,500,100$ & 60,997 & 52.00 & $8,070,420$ \\
\hline 11 & $\begin{array}{l}\text { Place and Compact Foundation Soll } \\
\text { In } 8^{\circ} \text { Lifts to } 95 \% \text { Sid. Procior }\end{array}$ & 974,173 & & - & 0.005 & 02,0005 & $1,606,004$ & 0 & 480,020 & 220 & $2,162,000$ \\
\hline 12 & $\begin{array}{l}\text { Placement of } 120 \text { MII Fexible } \\
\text { Membrane Uner }\end{array}$ & $3.722,973$ & & 0.37 & 0.008 & 18,018 & sment & $1,37,500$ & - & 0.50 & $1,740,000$ \\
\hline 13 & $\begin{array}{l}\text { Placoment of 1'-0 Coarse Sand } \\
\text { Drainage Layer }\end{array}$ & 191,322 & or & 10.50 & 0.025 & 3,203 & 65,001 & $1,570,001$ & 19,000 & 11.10 & $1,404,200$ \\
\hline 14 & 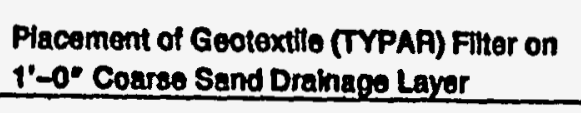 & A19,859 & SY & 0.11 & 0.005 & 2,099 & 41,000 & 46,164 & -1 & 0.20 & 00,170 \\
\hline 18 & Piacoment of $1^{\prime}-6^{*}$ Common FIII & 195,510 & or & 375 & 0.005 & 0,040 & 130,001 & 703,105 & 11,058 & 0.70 & 011,100 \\
\hline 16 & Placoment of $B^{\prime \prime}$ Topsoll & 65,763 & or & 0.50 & 0.005 & 2,302 & 46.034 & 024,749 & 19,810 & 10.40 & 604.650 \\
\hline 17 & $\begin{array}{l}\text { Placement of Perlmeter Exterlor } \\
\text { Drainage Layer } 3 / 4^{*} \times 3^{*} \text { Stone }\end{array}$ & 4,010 & OY & 1.50 & 0.18 & 842 & 12,602 & 8.015 & 3.000 & 8.70 & 22,700 \\
\hline 18 & Excavation of Trapezoldal Dralnape Ditch & 58,160 & cr & - & a. & 5,016 & 116.320 & 0 & 34,000 & 200 & 181,220 \\
\hline & (A) PAGE TOTAL & & & & & 133.570 & 2071,410 & $0.749,014$ & on.138 & & $18,000,100$ \\
\hline
\end{tabular}

J-4. Cost Estimate for a Cover System Utilizing 120-mil High Density Polyethylene (HDPE) Liner (Cont'd) 
D B TAIL BSTIMATB

CHK'D BY: S. MEAD

Loa I: 7-03

ACTTVTYY CODB: ESE260112

PROU.NAME: ECON. BVALUATON OF CLOSURB CAP BARRIER MATERLAL

ESTIHATOA: namx

PHONE: 7-5265

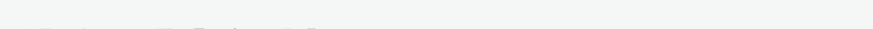

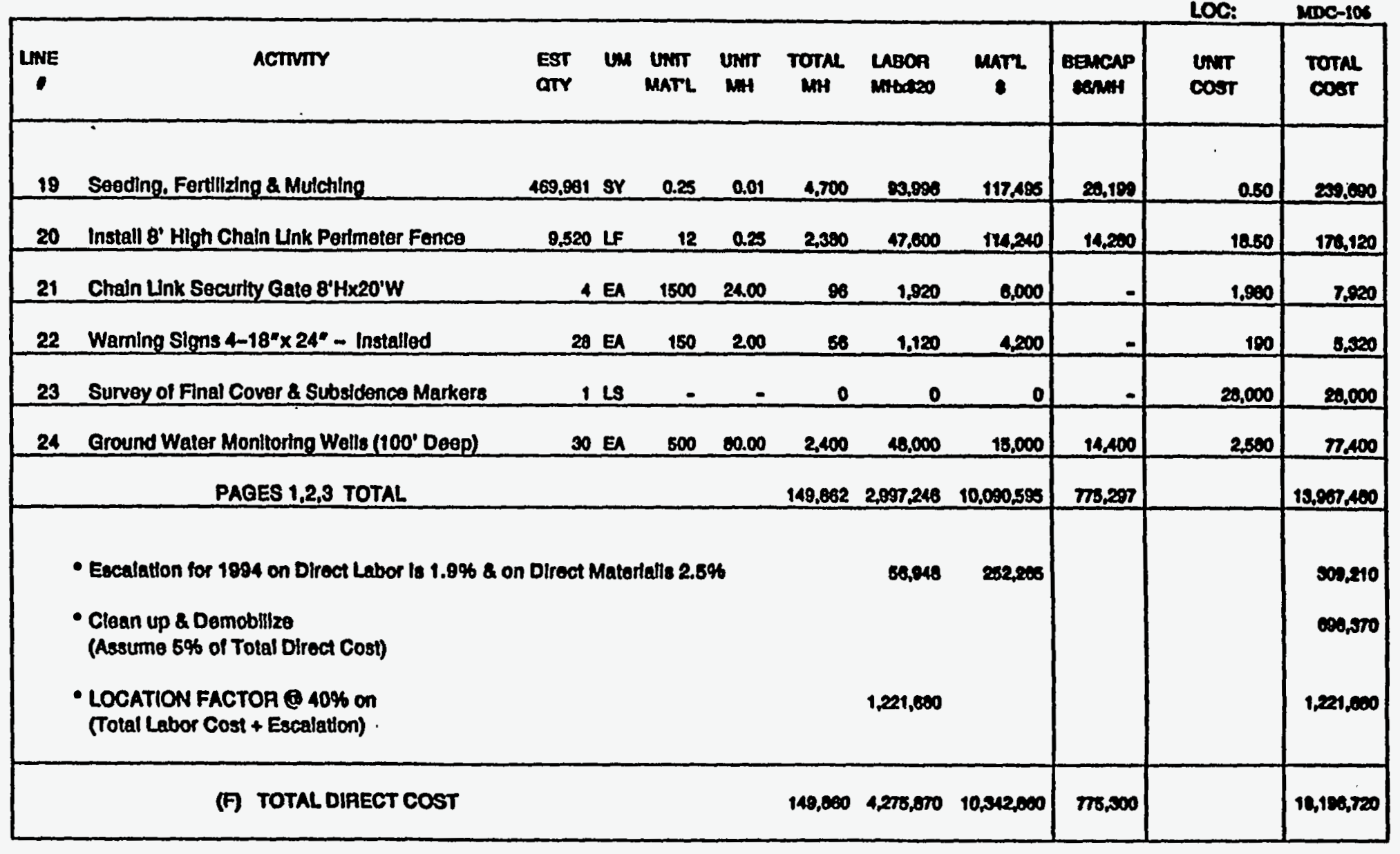

J-4. Cost Estimate for a Cover System Utilizing 120-mil High Density Polyethylene (HDPE) Liner (Cont'd) 
D E T A I L B S TIMATE

CHKD BY: 8. MEAD

LOOI: 7-03

ACTIVTY CODB: ESE260412

PROJ.NAMR: ECON. EYALUATION OP CLOSURB CAP BARRIER MATERUAL.

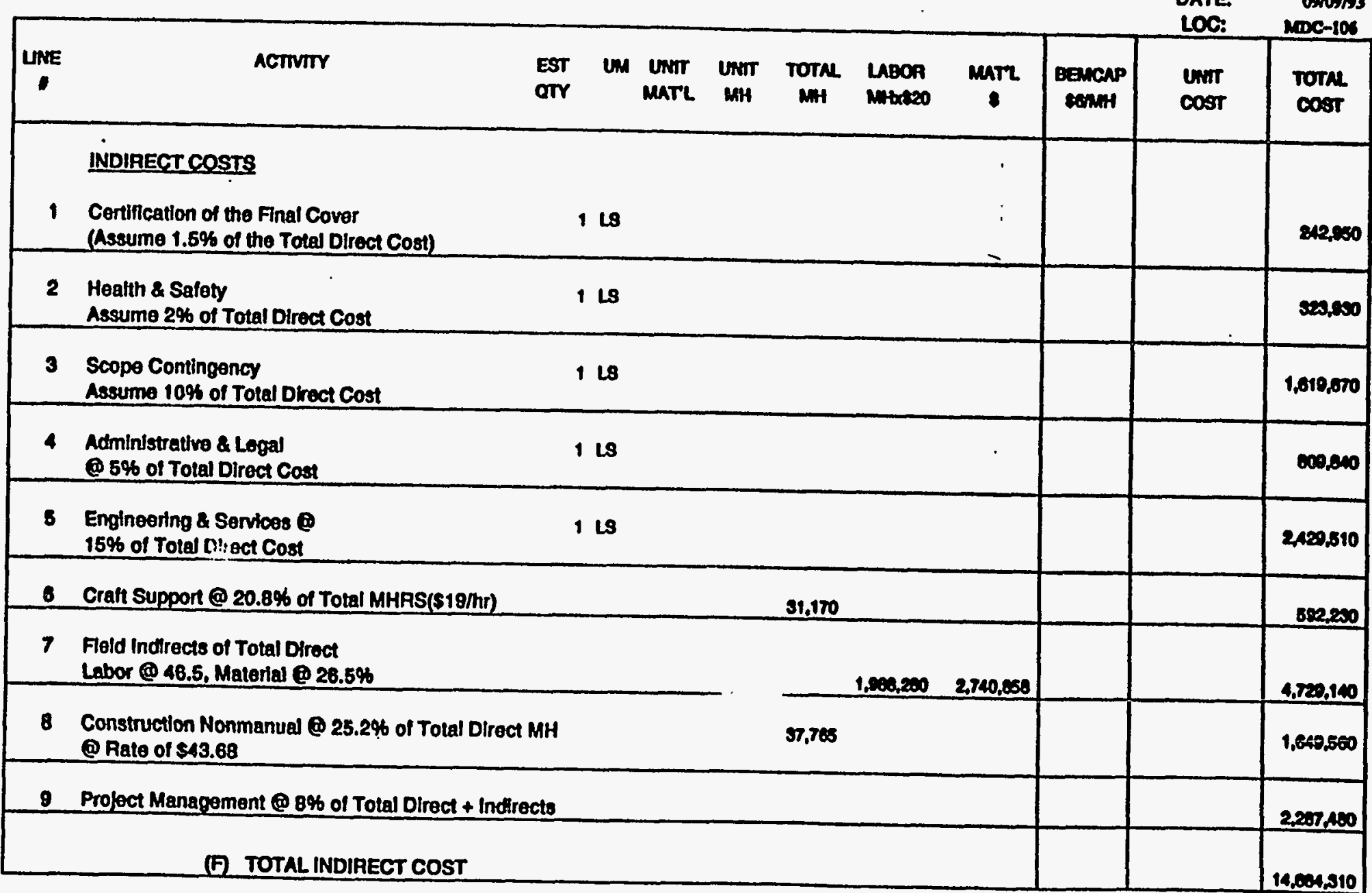

J-4. Cost Estimate for a Cover System Utilizing 120-mil High Density Polyethylene (HDPE) Liner (Cont'd) 
D BTAIL BSTIMATB

CHK'D BY: 8. MEAD

LOO : 7-03

ACTIVITY CODE: BSEOSOA12

PROI.MAMB: ECON. EVALUATION OF CLOSURB CAP BARRIER MATERLAL.

\begin{tabular}{|c|c|c|c|c|}
\hline 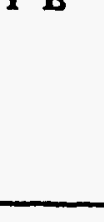 & & & $\begin{array}{l}\text { ESTIMATOR: } \\
\text { PHONE: } \\
\text { DATE: } \\
\text { LOC: } \\
\end{array}$ & 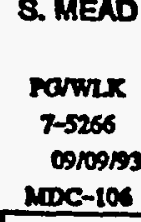 \\
\hline \multirow[t]{9}{*}{$\begin{array}{l}\text { LABOA } \\
\text { MBtrat20 }\end{array}$} & $\underset{?}{\operatorname{mar2}}$ & $\begin{array}{l}\text { Bencap } \\
\text { ranin }\end{array}$ & $\begin{array}{l}\text { Unm } \\
\text { cost }\end{array}$ & $\begin{array}{l}\text { Totnl } \\
\text { cost }\end{array}$ \\
\hline & & & & $\begin{array}{r}16,196,720 \\
14,064,010 \\
806,100\end{array}$ \\
\hline & $=$ & & 223 & $\begin{array}{r}51,000,000 \\
27,000\end{array}$ \\
\hline & & & 78 & \\
\hline & & & 78 & \\
\hline & & & 600 & 40,000 \\
\hline & & & & \\
\hline & & & 40 & $\begin{array}{r}850 \\
1.120\end{array}$ \\
\hline & & & $\pi$ & 22250 \\
\hline & & & & \\
\hline
\end{tabular}

J-4. Cost Estimate for a Cover System Utilizing 120-mil High Density Polyethylene (HDPE) Liner (Cont'd) 
D B T A L B S T IMAT B

\section{1: 7-03}

ACTVITY CODB: ES8260412

MROJ.NAME: BCON. EVALUATION OF CLOSURB CAP BARRIER MATERIAS.

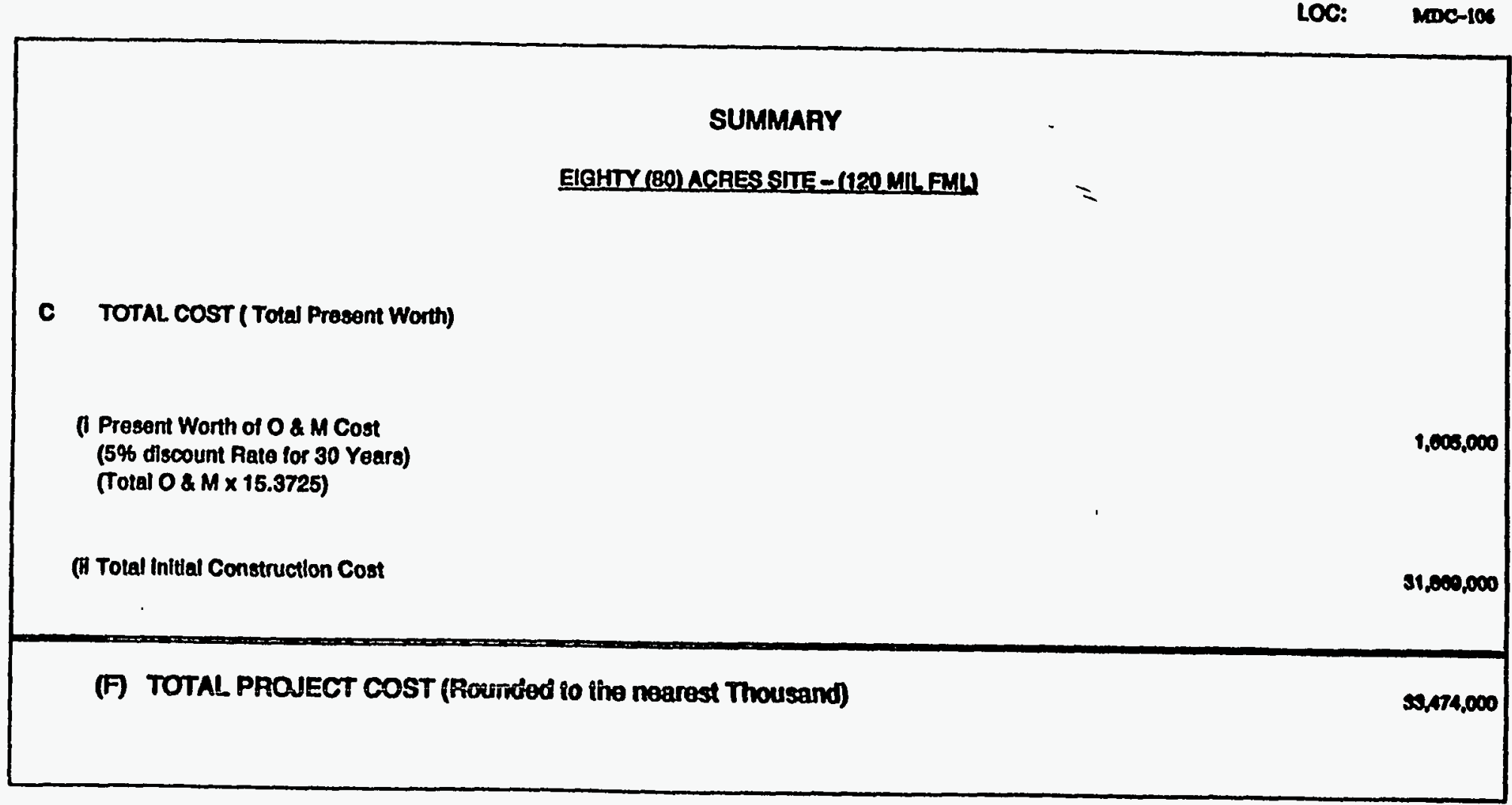

\begin{tabular}{|c|c|}
\hline CHK'D BY: & 8. MEAD \\
\hline $\begin{array}{l}\text { ESTMMATOR: } \\
\text { PHONE: } \\
\text { DATE: } \\
\text { LOC: }\end{array}$ & $\begin{array}{l}\text { rowitx } \\
\text { r-soss } \\
\text { osioses } \\
\text { noc-10s }\end{array}$ \\
\hline
\end{tabular}


DETAIL ESTIMATE

CHKO BY: 8. MEAD

Lod: $7-03$

ACTIVITY CODE: ESE2SON12

PROJ.NAME: ECON. EVALUATION CLOSURB CAP BARRIER MATERIAL

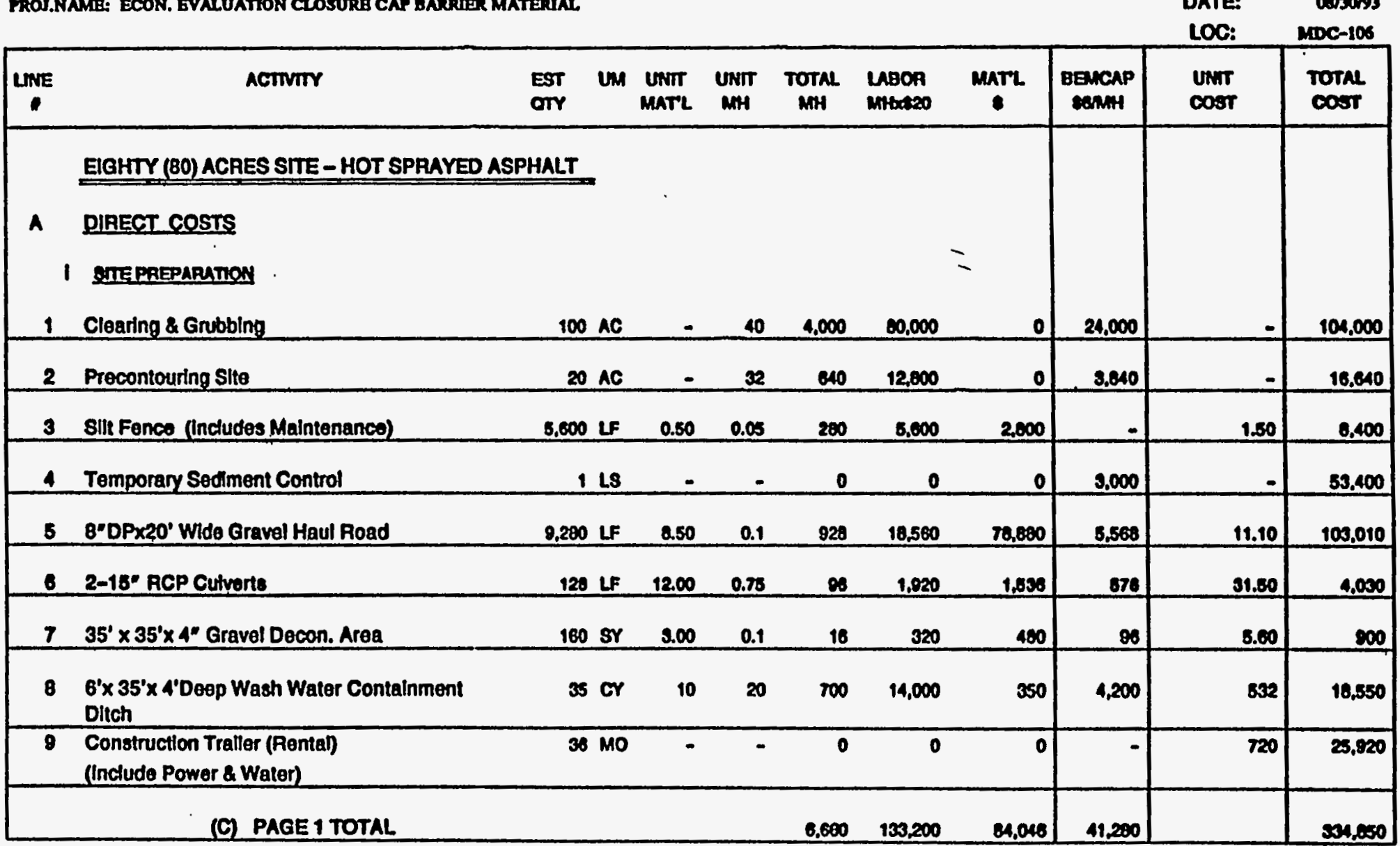

J-5. Cost Estimate for a Cover System Utilizing 1/4-inch Thick Hot Sprayed or Emulsified Asphalt 


\section{DETAIL ESTIMATE}

CHKD BY: 8. MEAD

2001: 7-03

ACTIVTYY CODB: ESE2SON12

PROI.NAMB: ECON, BVALUATTON CLOSURB CAP BARRIER MATERIAL

\begin{tabular}{|c|c|c|c|c|c|c|c|c|c|c|c|}
\hline UNE & ACTIVIT & $\begin{array}{l}\text { EST } \\
\text { oTr }\end{array}$ & un & UNAT & $\bigcup_{M H T}^{U N T}$ & TOTAL & $\begin{array}{l}\text { MABOR } \\
\text { MHateo }\end{array}$ & $\underset{:}{\operatorname{maTL}}$ & $\begin{array}{l}\text { gencup } \\
\operatorname{cosnn}\end{array}$ & $\begin{array}{l}\text { UnTा } \\
\text { cost }\end{array}$ & $\begin{array}{l}\text { TOTAL } \\
\text { COST }\end{array}$ \\
\hline 10 & $\begin{array}{l}\text { _COVER CONSTRUCTION } \\
\text { Waste Stabilization (Crushed Limestono) }\end{array}$ & 111,662 & & 50 & 0.1 & 11,168 & 223,324 & $5,583,100$ & 66,999 & 52.00 & $8,073,420$ \\
\hline 11 & $\begin{array}{l}\text { Place and Compact Foundation Soll } \\
\text { in 8* Uns to 95\% Sid. Proctor }\end{array}$ & 074,173 & or & - & 0.005 & 02,005 & $1,058,094$ & o) & 499,028 & 2.20 & $2,162,020$ \\
\hline 12 & $\begin{array}{l}\text { Applled Hot Asphall using } 3 \text { passes } \\
\text { at a rate of } 0.5 \text { gal / SY }\end{array}$ & $\begin{array}{l}413,664 \\
620,496 \\
\end{array}$ & $\begin{array}{l}\text { OY } \\
\text { OAL }\end{array}$ & 2 & 0.13 & 83,778 & 1,075,528 & 827,328 & 322,050 & 6.40 & $2,225,510$ \\
\hline 13 & $\begin{array}{l}\text { Placement of } 30 \text { Mill Flexiblo } \\
\text { Membrane Uner }\end{array}$ & $3,832,419$ & $8 \mathbf{F}$ & 0.14 & 0.0055 & 21,078 & 421,568 & 536,530 & - & 0.20 & 950,100 \\
\hline 14 & $\begin{array}{l}\text { Placement of 1'-0 Coarse Sand } \\
\text { Drainage Layer }\end{array}$ & 131,322 & or & 10.50 & 0.025 & 3,203 & 65,661 & $1,570,801$ & 10,000 & 11.10 & $1,464,240$ \\
\hline 15 & $\begin{array}{l}\text { Placement of Geotextlle (TYPAR) Filter on } \\
1^{1}-0^{*} \text { Coarse Sand Drahage Layer }\end{array}$ & 419,859 & sy & 0.11 & 0.005 & 2,099 & 41,989 & 46,184 & -1 & 0.20 & 60,170 \\
\hline 16 & Placement of $1^{\prime}-6^{\prime \prime}$ Common FHI & 195,510 & or & 3.75 & 0.035 & 0,043 & 136,061 & 733,185 & 41,058 & 4.70 & 911,100 \\
\hline 17 & Placement of 6" Topsoll & 65.703 & or & 9.50 & 0.035 & 2,302 & 46.034 & 624,749 & 13,010 & 10.40 & $\cos , 500$ \\
\hline 18 & $\begin{array}{l}\text { Piacoment of Perlmater Exterior } \\
\text { Drainage Layer } 3 / 4^{a} \times 3^{*} \text { "Stone }\end{array}$ & 4,010 & & 1.50 & 0.16 & 042 & 12,632 & 6.015 & 3,050 & 5.70 & 22,700 \\
\hline 19 & Excavation of Trapezoldal Dralnage Ditch & 58,160 & or & $=$ & 0.1 & 5,016 & 116,320 & 0 & 34,898 & 200 & 151,220 \\
\hline & (C) PAGE 2 TOTAL & & & & & 189.810 & $0,796,205$ & $0,735,001$ & 999,798 & & $14,531,070$ \\
\hline
\end{tabular}

J-5. Cost Estimate for a Cover System Utilizing 1/4-inch Thick Hot Sprayed or Emulsified Asphalt (Cont'd) 


\section{E T A L B S TIMATE}

CHK'D BY: S. MEAD

LOOA: 7-03

ACTIVTTY CODE: ESE260412

PROJ.NAME: ECON. BYALUATION CLOSURB CAP BARRIER MATERHAL

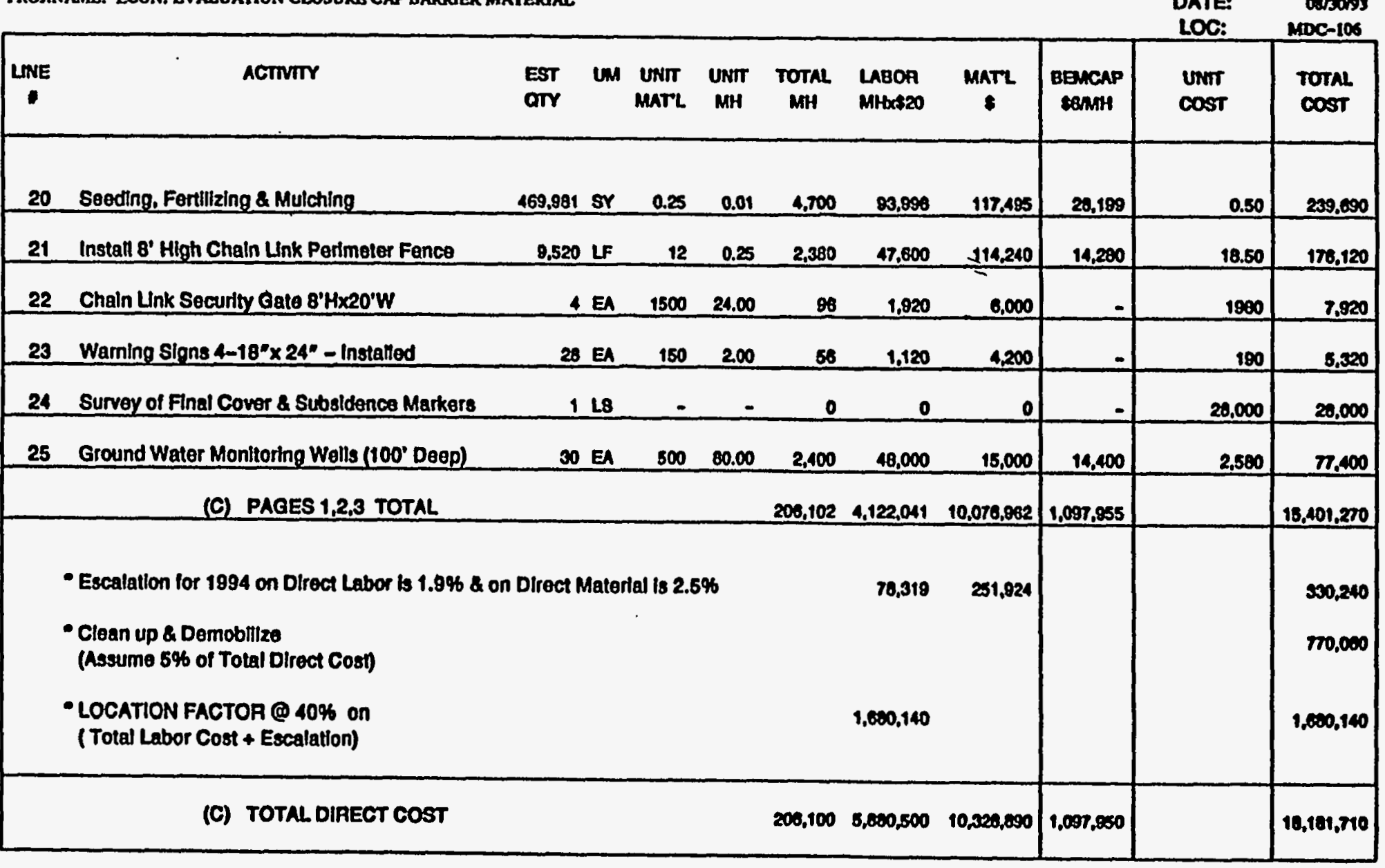

J-5. Cost Estimate for a Cover System Utilizing 1/4-inch Thick Hot Sprayed or Emulsified Asphalt (Cont'd) 
D B TAIL BSTIMATE

CHK'D BY: S. MEAD

\section{1: 7-03}

ACTIVTTY CODE: ESE2SOA12

PROJ.NAME: ECON. EVALUATION CLOSURB CAT BARRIER MATERIAL.

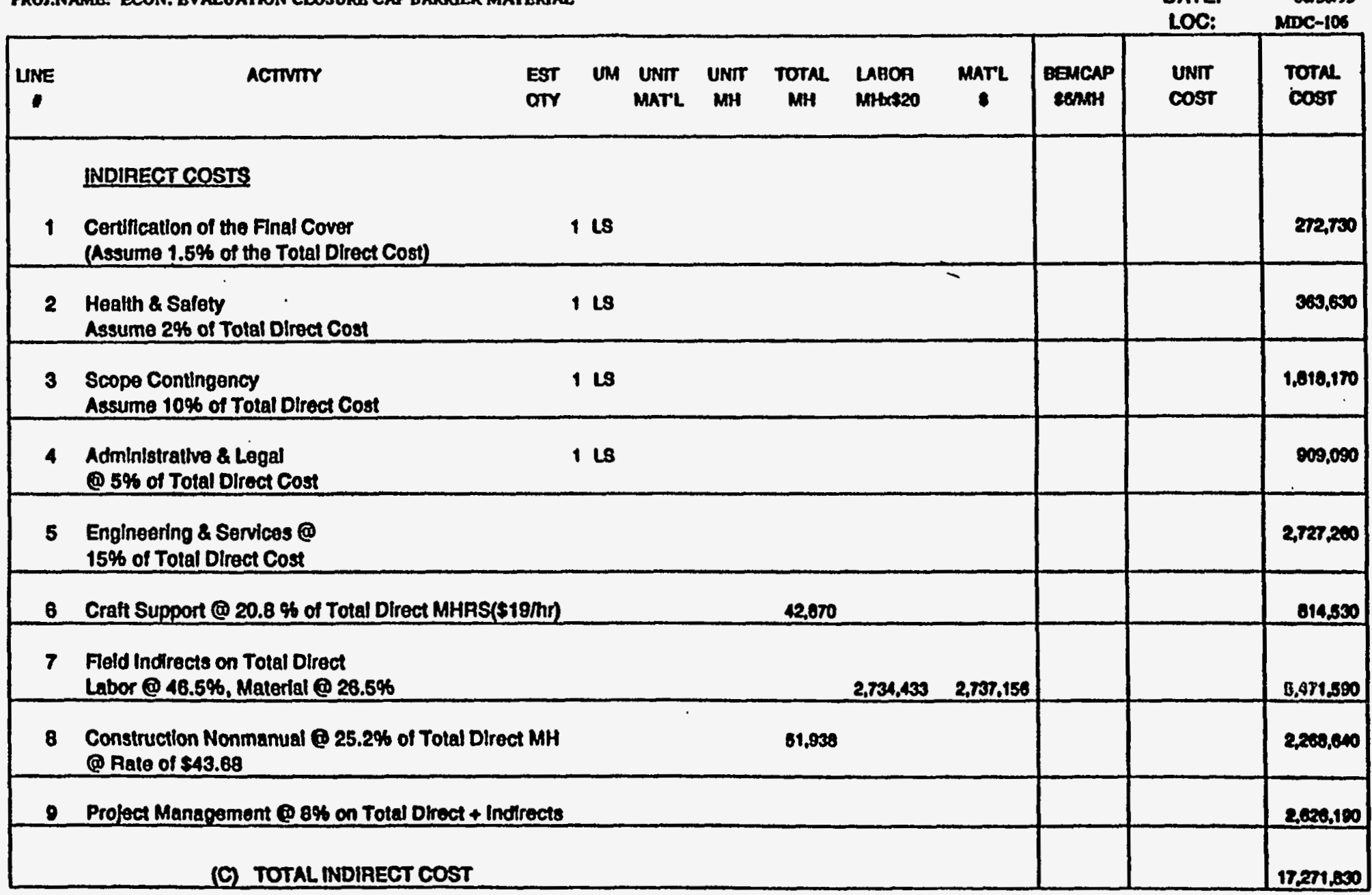

J-5. Cost Estimate for a Cover System Utilizing 1/4-inch Thick Hot Sprayed or Emulsified Asphalt (Cont'd) 
D E T A I L E S T I M A T B

CHK'D BY: 8. MEAD.

Loof: 7-03

ACTIVITY CODE: ESEZ6O412

PROJ.NAMB: ECON. BVALUATION CLOSURB CAP BARRIER MATEUAL

ESTIMATOR: ronwx

PHONE: T-5266

DATE: Darsors

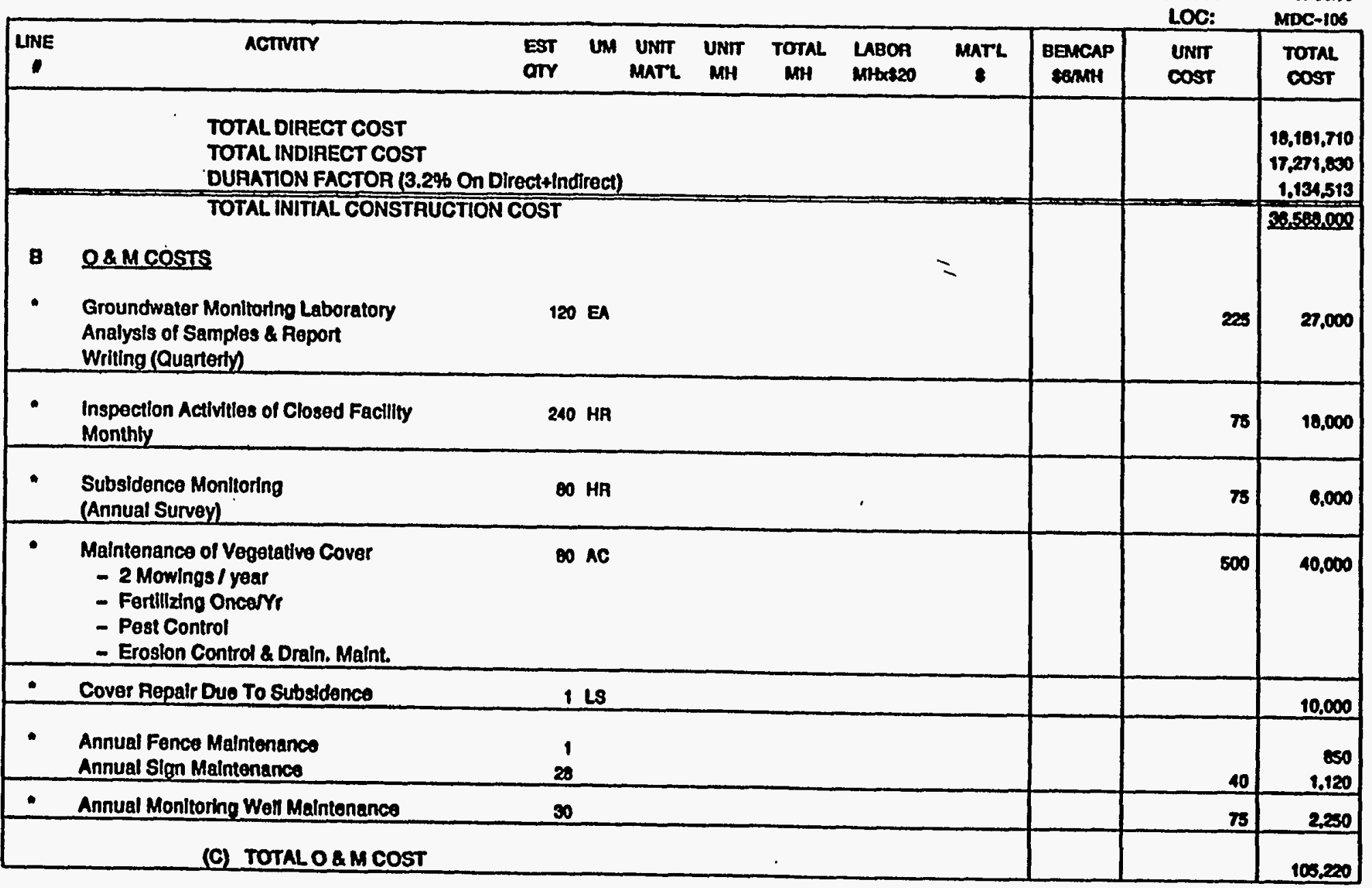

J-5. Cost Estimate for a Cover System Utilizing 1/4-inch Thick Hot Sprayed or Emulsified Asphalt (Cont'd) 
D B TAIL B S TIMATE

L001: 7-03

ACTTVITY CODE: ESE260112

PROJ.NAME: ECON. EVALUATTON CLOSURE CAP BARRIRR MATERLAL
CHK'D BY: 8. MEAD

ESTIMATOR: rarmK

PHONE: 7-5266

DATE:

LOC: MDC-10S

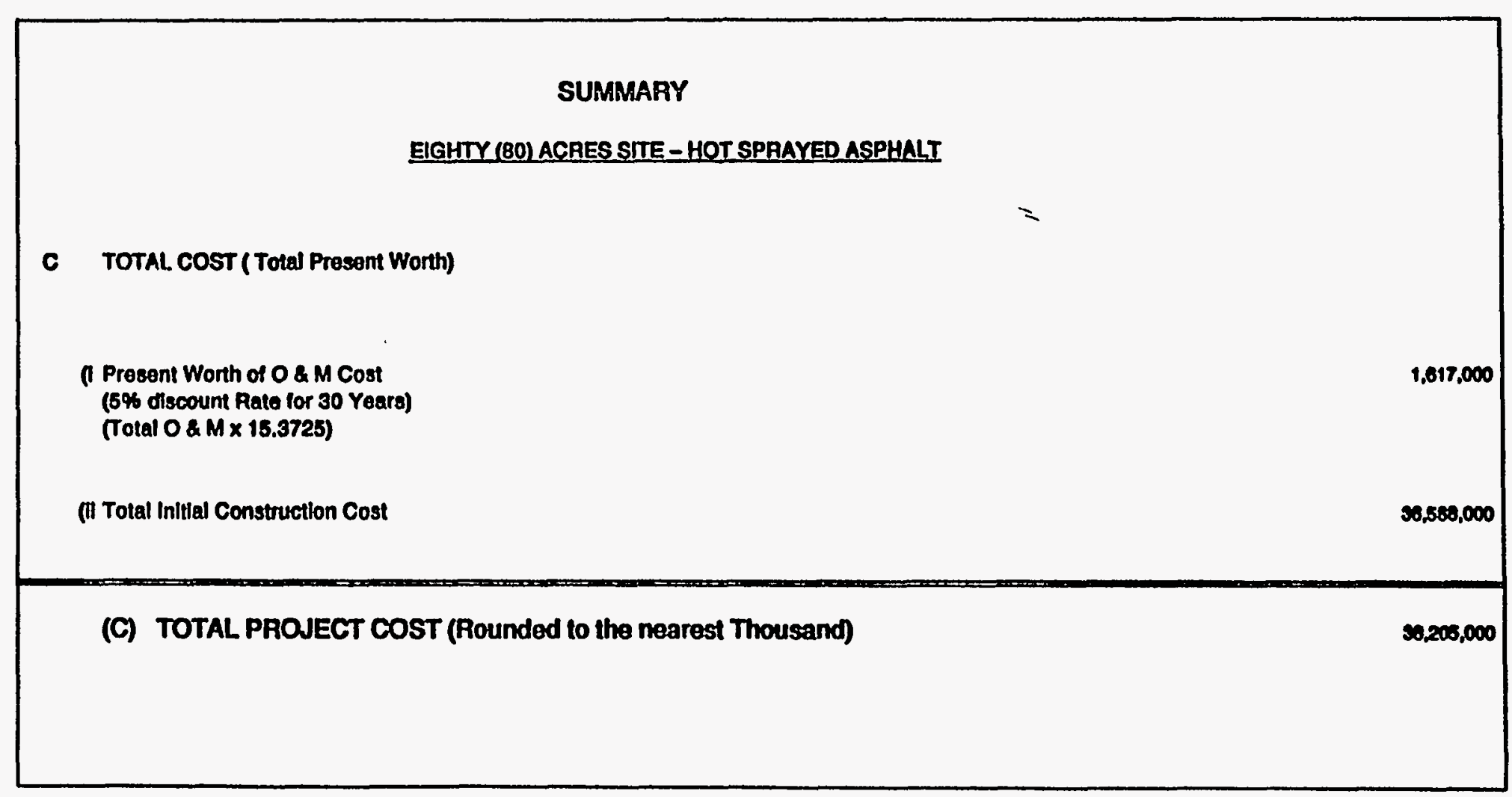

J-5. Cost Estimate for a Cover System Utilizing 1/4-inch Thick Hot Sprayed or Emulsified Asphalt (Cont'd) 
DETAIL ESTIMATE

CHK'D BY: S. MEAD

LOG : : 7-03

ACTIVTY CODE: ESE260412

PROINAME: ECON EVALUATION OF GLOSURE CAP BARAIER MATERIAL

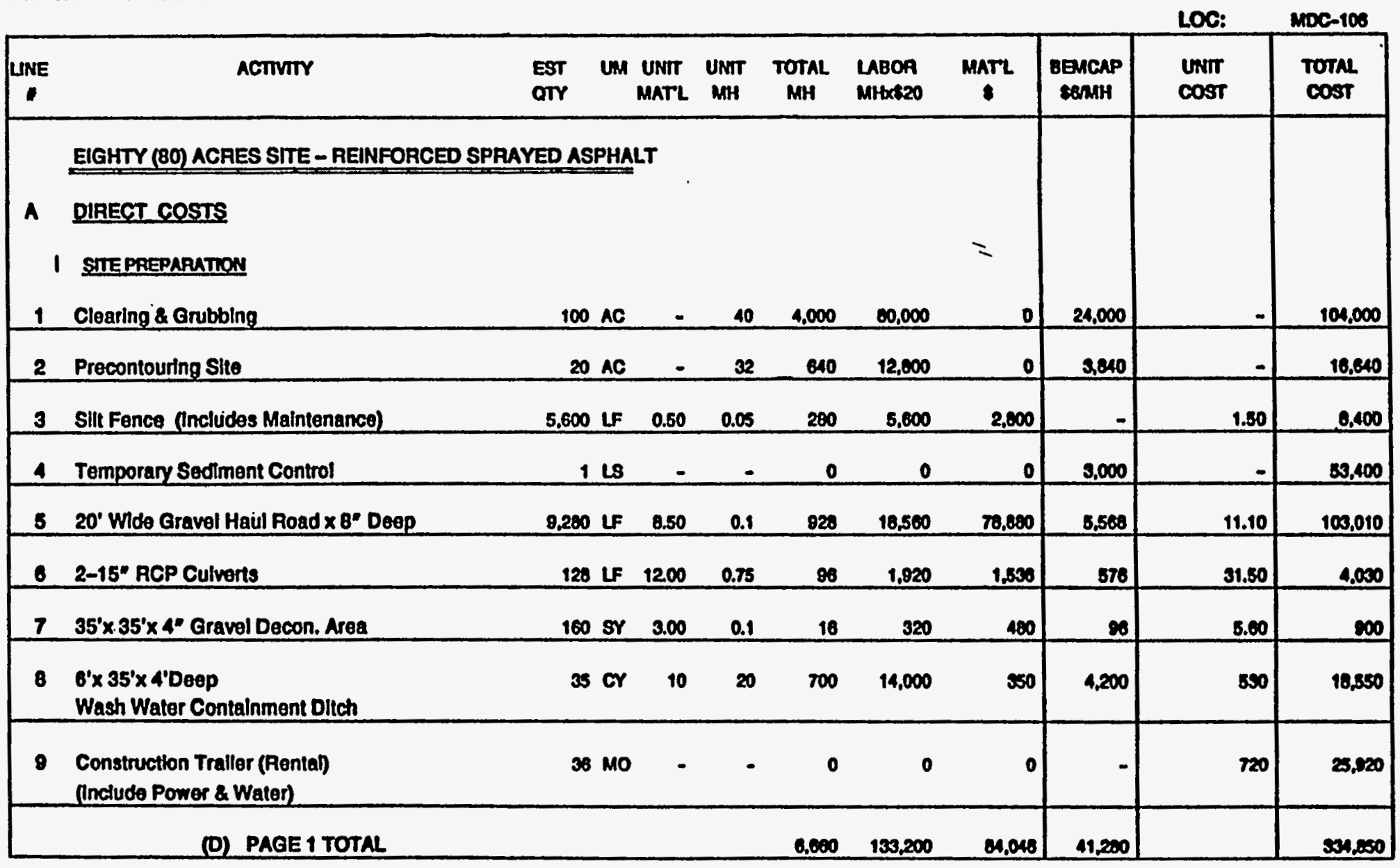

J-6. Cost Estimate for a Cover System Utilizing Reinforced Sprayed Asphalt 


\section{DETAIL ESTIMATE}

CHK'D BY: 8. MEAD

LOG : : 7-03

ACTIVITY CODE: ESE260412

PROJ.NAME: ECON. EVALUATION OF CLOSURE CAP BARPIER MATERIAL

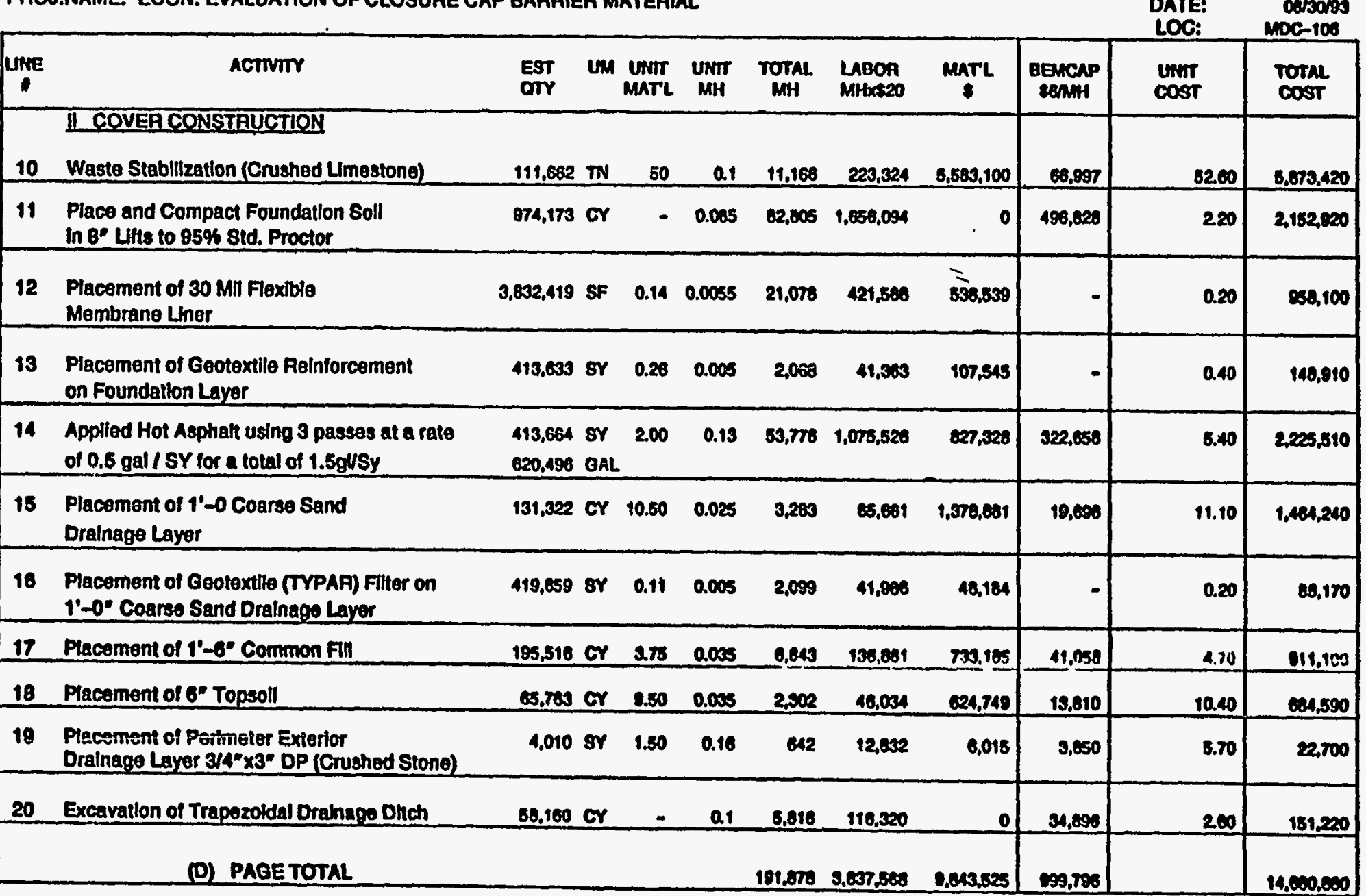

J-6. Cost Estimate for a Cover System Utilizing Reinforced Sprayed Asphalt (Cont'd) 


\section{DETAIL ESTIMATE}

S. MEAD

LOG: : 7-03

ACTIVITY CODE: ESE260412

PROJ.NAME: ECON. EVALUATION OF ClOSUAE CAP BAARIEA MATERIAL

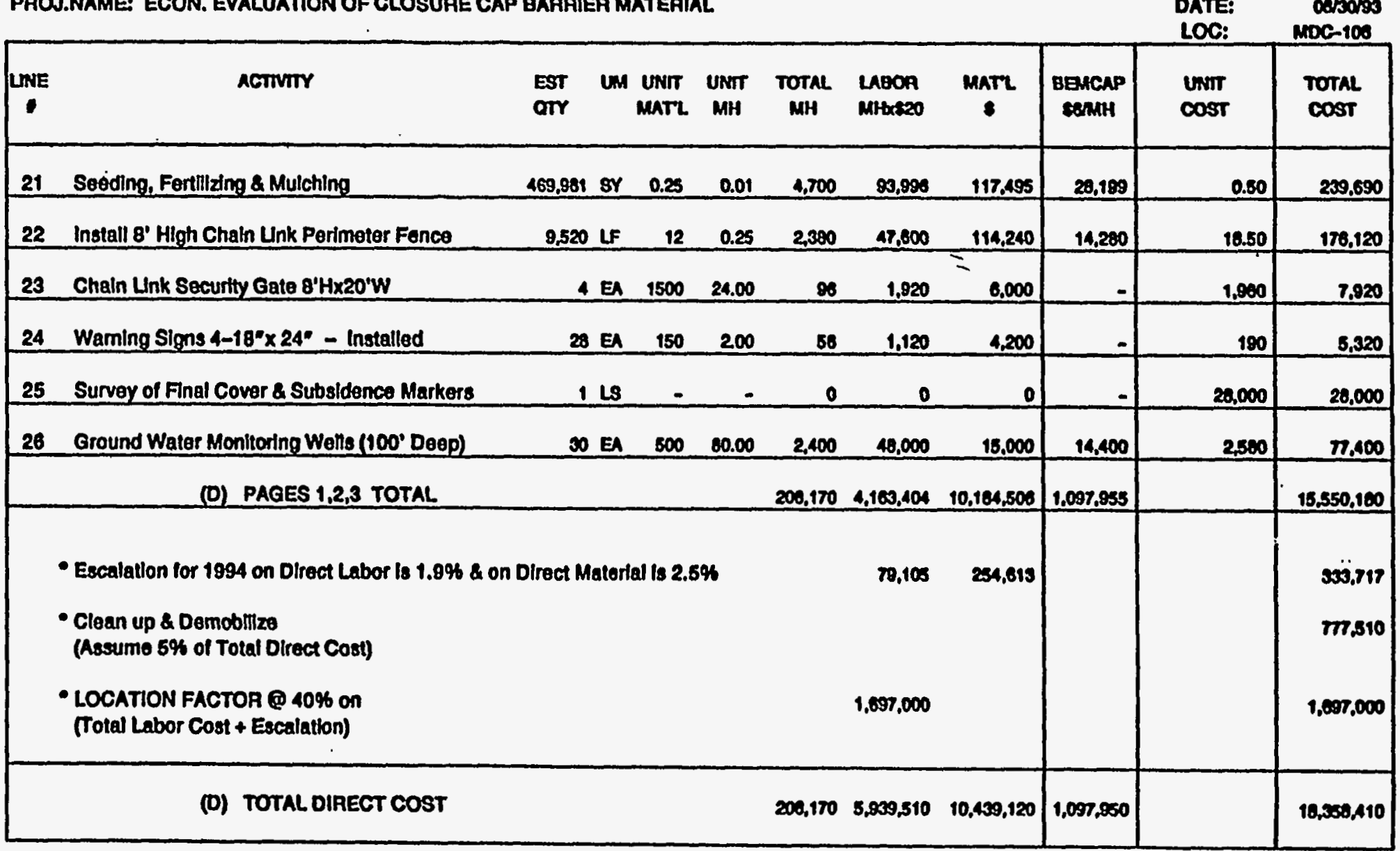

J-6. Cost Estimate for a Cover System Utilizing Reinforced Sprayed Asphalt (Cont'd) 
DETAIL ESTIMATE

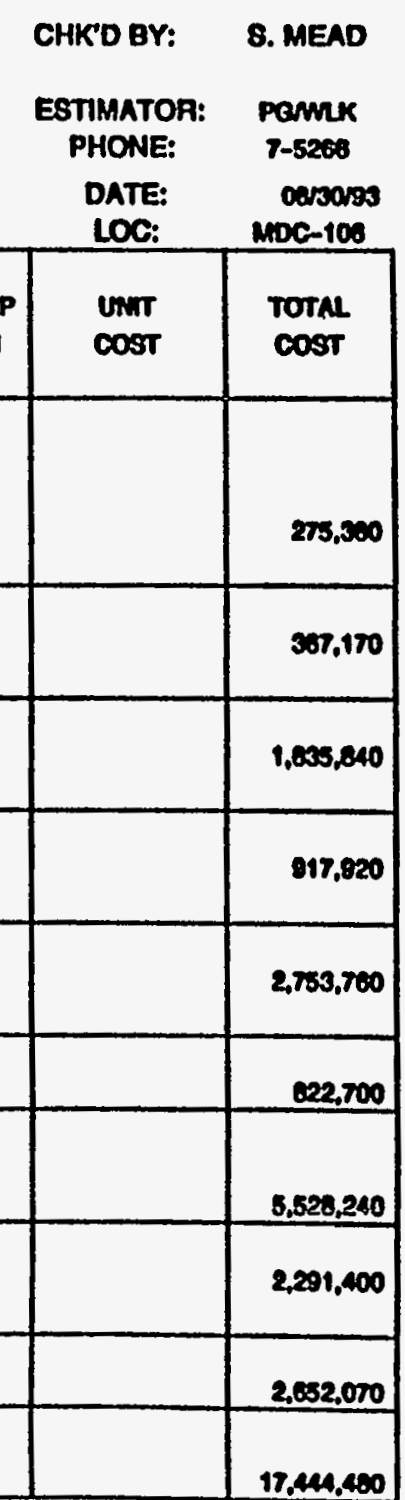

143
1 Cortification of the Final Cover (Assume 1.5\% of the Total Direct Cast)

2 Mealth \& Salety Assume 2\% of Total Direct Cost

3 Scope Contingeney Assume 10\% of Total Direct Cost

4 Administrative \& Lagal @ 596 of Total Direct Cost

149

$1 \mathrm{LS}$

$1 \mathrm{LS}$

- Englneering \& Services (1) $15 \%$ of Total Diroct Cost

- Craft Support \& $20.8 \%$ of Total Direct MHAS(519/m)

7 Field indirects on Total Direct Labor @ 46.5\%, Materlal @ 26.5\%

- Construction Nonmanual 25.240 of Total Direct MH (i) Rate of $\$ 43.68$

- Project Management a $8 \%$ of Total Direct + Indirects

\section{(D) TOTALINDIRECT COST}

J-6. Cost Estimate for a Cover System Utilizing Reinforced Sprayed Asphalt (Cont'd) 


\section{DETAIL ESTIMATE}

CHK'D BY: S. MEAD

LOG : : 7-03

ACTIVITY CODE: ESE260412

PROS.NAME: ECON. EVALUATION OF CLOSURE CAP BAFAIER MATERIAL

ESTIMATOR: POWWK

PHONE: 7-5268

DATE: DOSOSOS

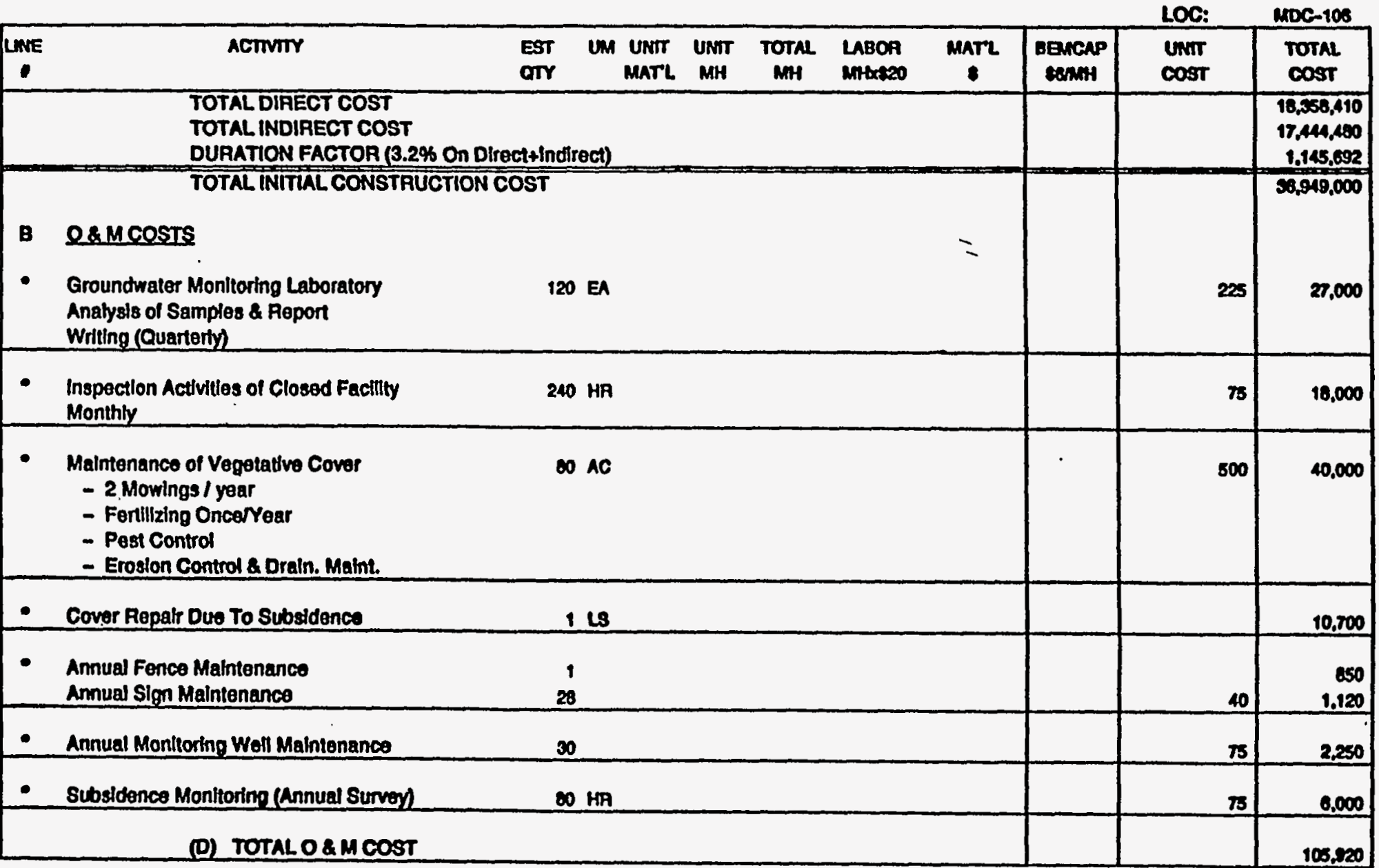

J-6. Cost Estimate for a Cover System Utilizing Reinforced Sprayed Asphalt (Cont'd) 
DETAIL ESTIMATE CHKDBY: 8.MEAD

LOG : : 7-03

ACTIVITY CODE: ESE260412

PROJ.NAME: ECON. EVALUATION OF CLOSURE CAP BARAIER MATERIAL

\begin{tabular}{|c|c|}
\hline $\begin{array}{l}\text { ESTIMATOR: } \\
\text { PHONE: } \\
\text { OATE: } \\
\text { LOG: }\end{array}$ & 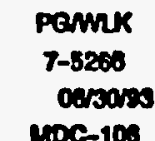 \\
\hline
\end{tabular}

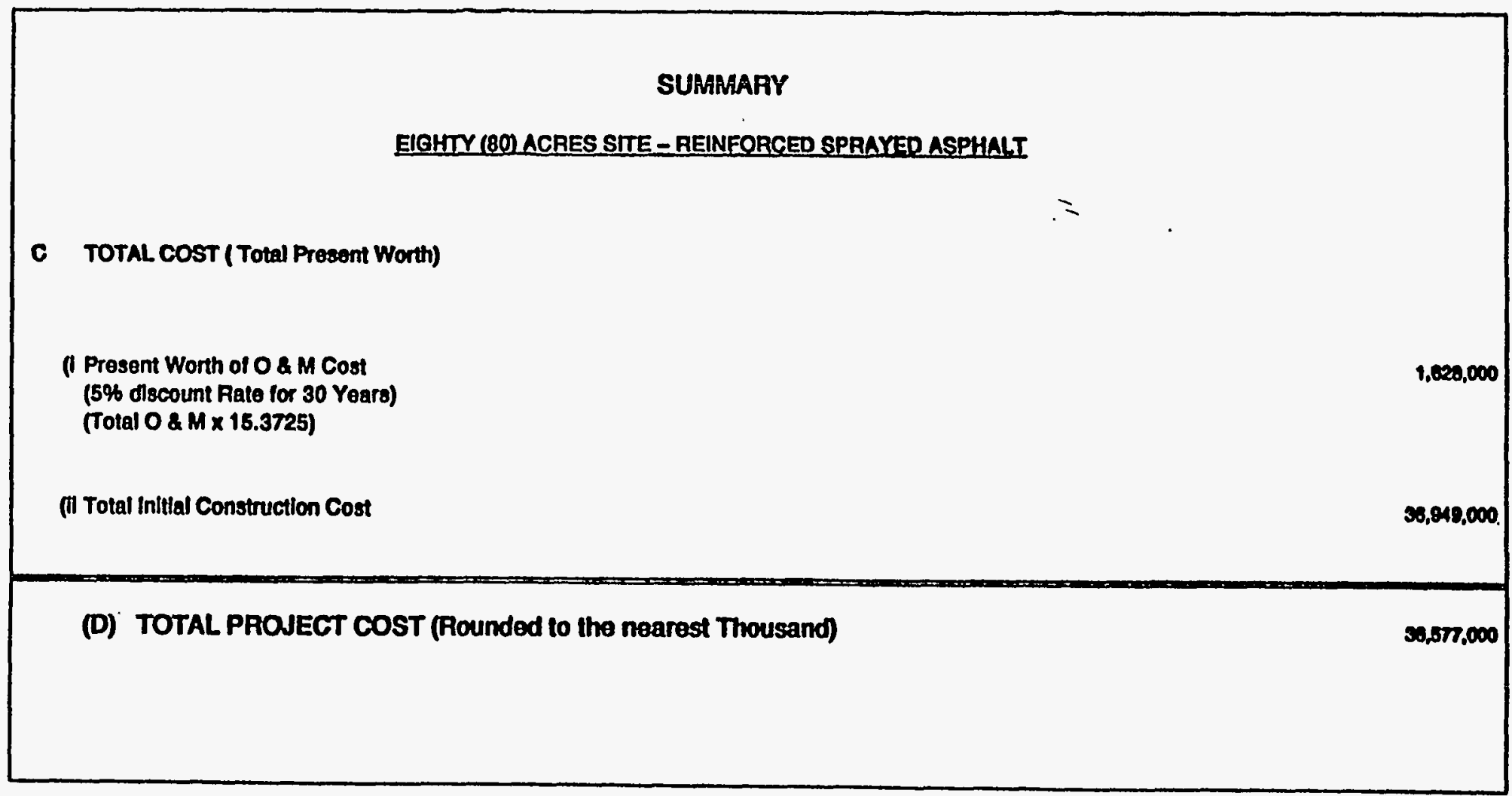

J-6. Cost Estimate for a Cover System Utilizing Reinforced Sprayed Asphalt (Cont'd) 
Loaf: 7-03

ACTTITY CODE: BSE260012

PROJ.NAME: ECON. EVALUATTON OF CLOSURB CAP BARRIBR MATERUAL.

\begin{tabular}{|c|c|c|c|c|c|c|c|c|c|}
\hline AGTVTr & $\begin{array}{l}\text { EST UM } \\
\text { OTY }\end{array}$ & $\begin{array}{l}\text { UNIT } \\
\text { MATL }\end{array}$ & UNI & $\begin{array}{l}\text { TOTAL } \\
M H\end{array}$ & $\begin{array}{l}\text { LABOA } \\
\text { MHtrP2O }\end{array}$ & $\stackrel{M A T L}{*}$ & $\begin{array}{l}\text { Bencap } \\
\text { sennt }\end{array}$ & $\begin{array}{l}\text { Unvrt } \\
\text { cost }\end{array}$ & $\begin{array}{l}\text { Totnl } \\
\cos \end{array}$ \\
\hline \multicolumn{3}{|c|}{ EIGHTY (BO) ACRES SITE - 2'-0' COMPACTED CLAY (OFF SITE) } & & & & & & & \\
\hline \multicolumn{10}{|l|}{ A DIRECT COSTS } \\
\hline I SMEPREPARATION & & & & & & $=$ & & & \\
\hline 1 Clearing \& Grubbing & $100 \wedge C$ & - & 10 & 4.000 & 00,000 & 0 & 24,000 & $=$ & 104,000 \\
\hline 2 Precontouring Sito & $20 \wedge C$ & - & 32 & 20 & 12,000 & 0 & 3,840 & - & 16.640 \\
\hline 3 Silt Fence (Inctudes Maintenanco) & $8.600 \mathrm{LF}$ & 0.50 & 0.05 & 200 & 5.600 & 2,000 & - & 1.50 & 0,400 \\
\hline 4 Temporary Sodimem Control & 1 LS & - & - & o & 0 & 0 & 3.000 & - & 83,400 \\
\hline $58^{\prime \prime} \mathrm{DP} \times 20^{\prime}$ Wide Gravei Haul Road & 0,280 LF & 8.50 & 0.1 & 820 & 10,560 & 70,600 & 8.560 & 11.10 & 103,010 \\
\hline 6 2-15" RCP Culverts & $128 \mathrm{LF}$ & 1200 & 0.75 & $\infty$ & 1.920 & 1.530 & 678 & 31.60 & 1.000 \\
\hline $795^{\prime} \times 35^{\prime} \times 4^{\prime \prime}$ Gravel Docon. Area & $160 \mathrm{gr}$ & 3.00 & 0.1 & 16 & 320 & 400 & $\infty$ & 8.60 & $\infty \infty 0$ \\
\hline 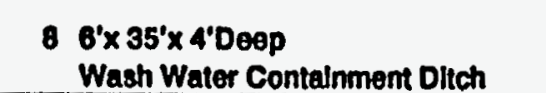 & $35 \mathrm{Cr}$ & 10 & 20 & 700 & 14,000 & $\mathbf{s 0 0}$ & 4,200 & 850 & 10,530 \\
\hline $\begin{array}{l}\text { - Construction Traner (Rental) } \\
\text { (Include Power \& Water) }\end{array}$ & 3 mo & - & - & o & $\mathbf{0}$ & 0 & - & $\mathbf{7 2 0}$ & 205,220 \\
\hline (M) PAGE I TOTAL & & & & 8.000 & 123,200 & 04,040 & 41,200 & & 304,850 \\
\hline
\end{tabular}

J-7. Cost Estimate for a Cover System Utilizing Compacted Kaolin (Orfsite) Clay and a 30-mil FML 
DETAIL ESTIMATE

L00 N: 7-03

ACTTVITY CODE: ESE260412

PROS.NAME: ECON. BVALUATION OP CLOSURB CAP BARBSBR MATENAL

\begin{tabular}{|c|c|c|c|c|c|c|c|c|c|c|}
\hline ACTIVIT & $\begin{array}{l}\text { EST } \\
\text { arr }\end{array}$ & Un & $\begin{array}{c}\text { UNTT } \\
\text { MATLL }\end{array}$ & $\underset{\text { UNIT }}{\text { UNIT }}$ & $\underset{M H}{\text { ToTAL }}$ & $\begin{array}{l}\text { LABOA } \\
\text { MHAar2O }\end{array}$ & $\underset{8}{\text { MATL }}$ & $\begin{array}{l}\text { Bancap } \\
\text { sennet }\end{array}$ & $\begin{array}{l}\text { unrt } \\
\text { cost }\end{array}$ & $\begin{array}{l}\text { Total } \\
\text {. COST }\end{array}$ \\
\hline $\begin{array}{l}\text { 1. COVER CONSTRUGTION } \\
\text { 10. Wasto Siablization (Crushed Limestono) }\end{array}$ & 111.662 & $I N$ & 80 & 0.1 & 11.168 & 223,324 & $5,583,100$ & 68,997 & 8200 & $8,073,420$ \\
\hline $\begin{array}{l}11 \text { Place and Compact Foundation Soll } \\
\text { in } 8^{\circ} \text { Lifts to } 95 \% \text { Std. Proctor }\end{array}$ & 974,173 & CY & - & 0.065 & 82,805 & $1,058,094$ & 0 & 498,028 & 220 & $2,152,020$ \\
\hline $\begin{array}{l}12 \text { Compact } 2^{\prime}-0^{\circ} \text { Thlck Clay Layor } \\
\text { In } 8^{-1} \text { Lifts }\end{array}$ & 259,653 & CY & 13.00 & 0.165 & 42.043 & 856,855 & $3,375,409$ & 257,058 & 17.30 & $4,400,400$ \\
\hline $\begin{array}{l}13 \text { Placement of } 30 \text { Mil Flexible } \\
\text { Membrane Liner }\end{array}$ & $3,632,419$ & SF & 0.14 & 0.0053 & 21,078 & 421,568 & 636,539 & $=$ & 0.20 & 938,100 \\
\hline $\begin{array}{l}14 \text { Piacement of f'-o Coarse Sand } \\
\text { Oralnage Layer. }\end{array}$ & 131,322 & Or & 10.50 & 0.025 & 3,203 & 65,681 & $1,370,001$ & 19,090 & 11.10 & $1, M 44,240$ \\
\hline $\begin{array}{l}15 \text { Placement of Geotextile (TYPAR) Filter on } \\
\text { 1'-0' Coarse Sand Dralnage Layer }\end{array}$ & 419.859 & $\mathbf{S r}$ & 0.11 & 0.005 & 2,099 & 41,890 & 19,184 & - & 0.20 & 80,170 \\
\hline 16 Placement of $1^{\prime}-6^{\prime}$ Common FII & 195,516 & or & 375 & 0.055 & 8,843 & 136,861 & 703.165 & 41,060 & 4.70 & 211.100 \\
\hline 17 Placement of 6" Topeoll & 65.763 & CY & 8.50 & 0.003 & 2302 & 46,034 & 024,740 & 18,010 & 10.40 & $\cos 590$ \\
\hline $\begin{array}{l}18 \text { Placsimant of Perimeior Extorior } \\
\text { Drainage Layer } 314^{\prime \prime} \times 0^{\circ} \text { DP (Crushed Stono) }\end{array}$ & 4.010 & $\mathbf{S Y}$ & 1.50 & 0.18 & 642 & 12,032 & 6,015 & 3,060 & 8.70 & 2,700 \\
\hline 19 Excavation of Trapezoldal Dralnage Ditch & 58.160 & or & 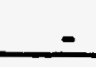 & 0.1 & 5,010 & 116,320 & $\underline{0}$ & 24,000 & 200 & 181.220 \\
\hline G PAge 2 TOTAL & & & & & ITo,on & $3.5 \pi, 553$ & $12,204,142$ & $\mathbf{2 4 , 1 8 4}$ & & $10,185,000$ \\
\hline
\end{tabular}

J-7. Cost Estimate for a Cover System Utilizing Compacted Kaolin (Offsite) Clay and a 30-mil FML (Cont'd) 
DETAILESTIMATE

Loof: $7-03$

ACTIVIT CODE: ESE260412

PROJ.NAMB: ECON. EVALUATION OF CLOSURE CAP BARRRER MATERIAL.

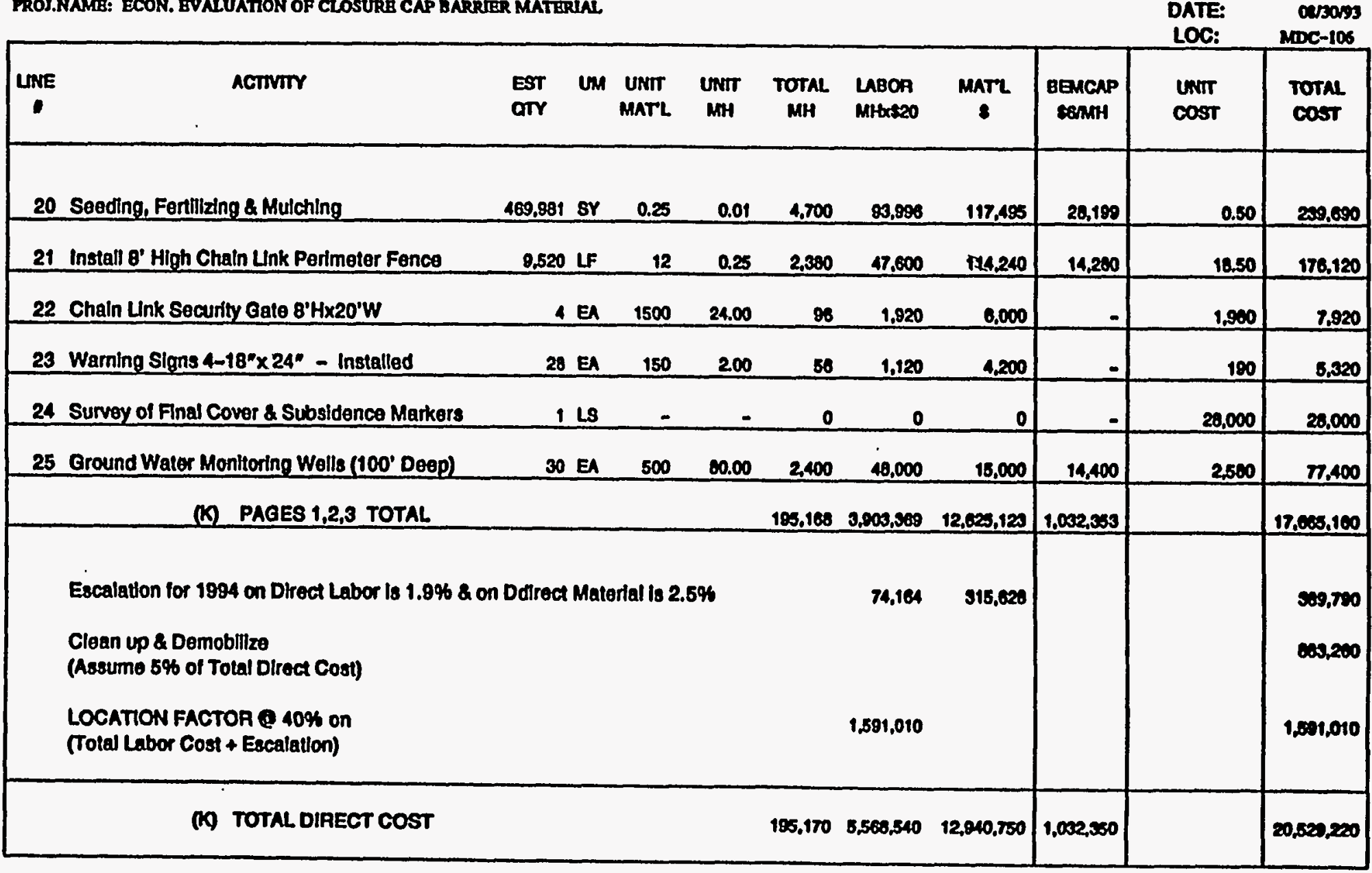

J-7. Cost Estimate for a Cover System Utilizing Compacted Kaolin (Offsite) Clay and a 30-mil FML (Cont'd) 
1001: 7-03

ACTTVITY CODE: ESE2SOS12

PROJ.NAME: ECON. EVALUATKON OF CLOSURB CAP BARRIER MATENJAL

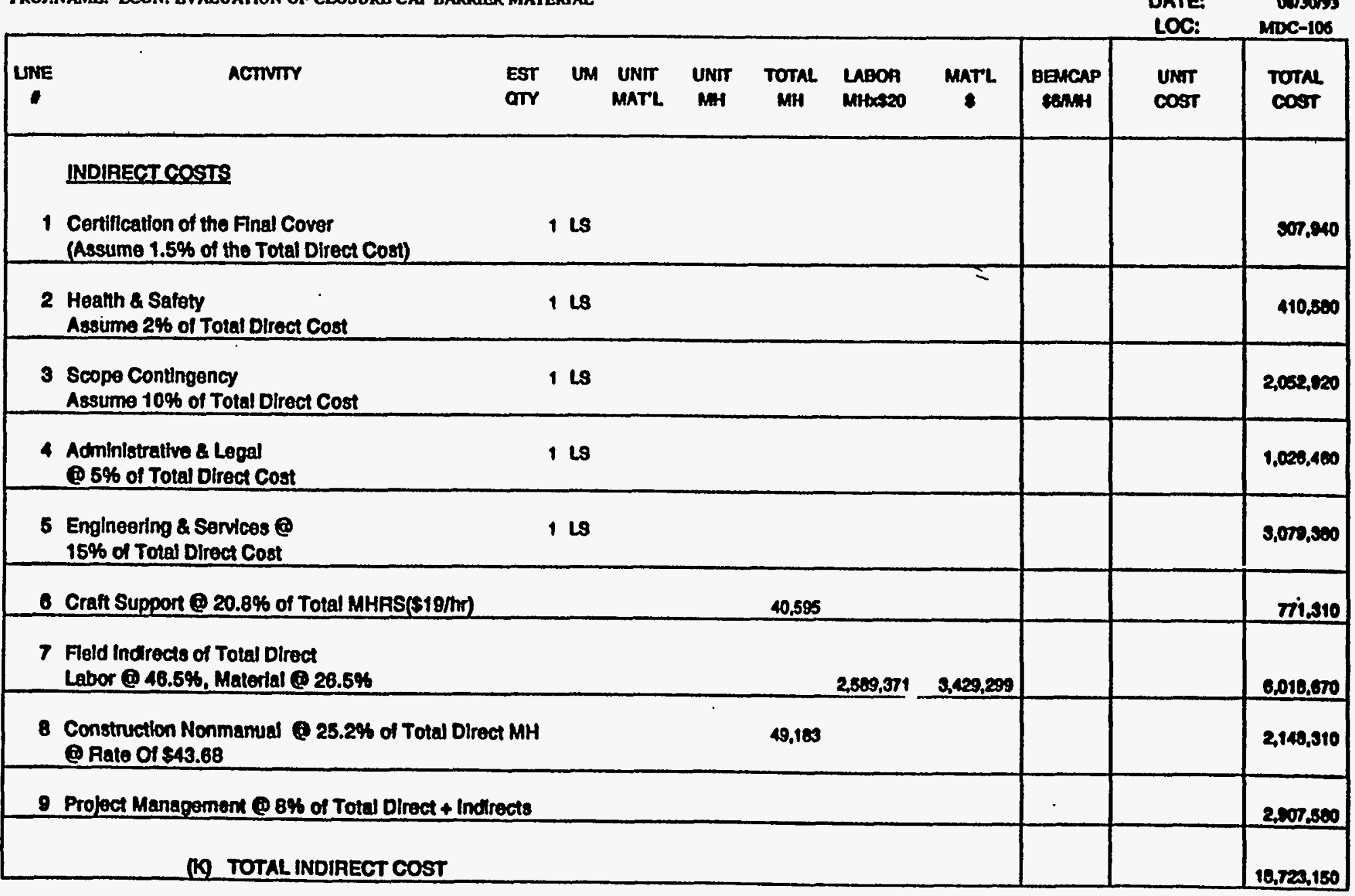

J-7. Cost Estimate for a Cover System Utilizing Compacted Kaolin (Offsite) Clay and a 30-mil FML (Cont'd) 
Lod A: 1-03

ACTTVITY CODE: ESEROO12

PROI.NAMB: ECON. BVALUATTON OP CLOSURB CAP BARRTER MATERJAL

CHKD BY: S. MEAD

ESTIMATOR: rom

PHONE: 7-5266

DATE:

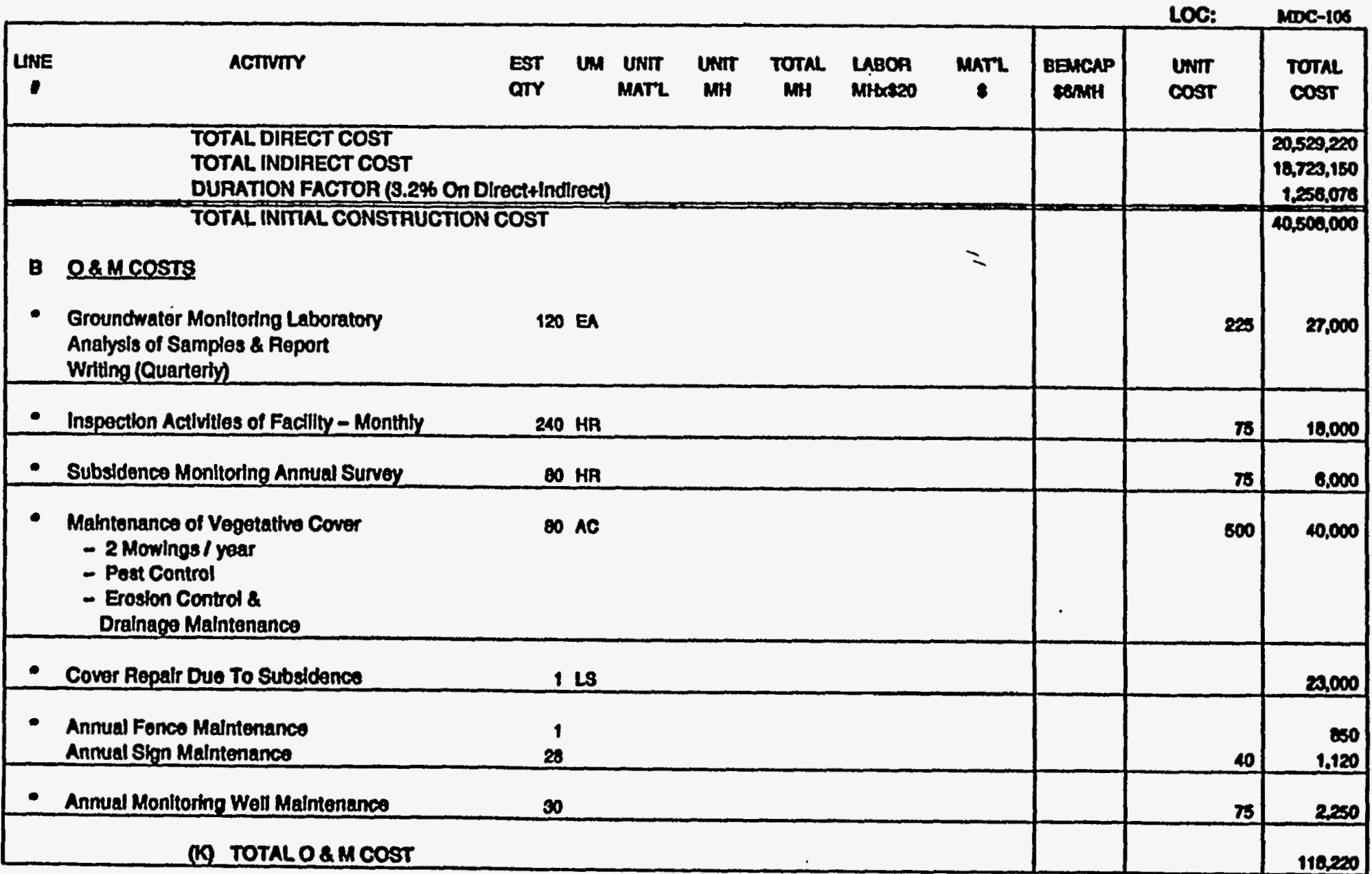

J-7. Cost Estimate for a Cover System Utilizing Compacted Kaolin (Offsite) Clay and a 30-mil FML (Cont'd) 
L00 11: 7-03

ACTIVITY CODB: ESB260412

PROS.MAMB: ECON. BVALUATTON OP CLOSURB CAP BARRIER MATERIAL

LOC: MNOC-106

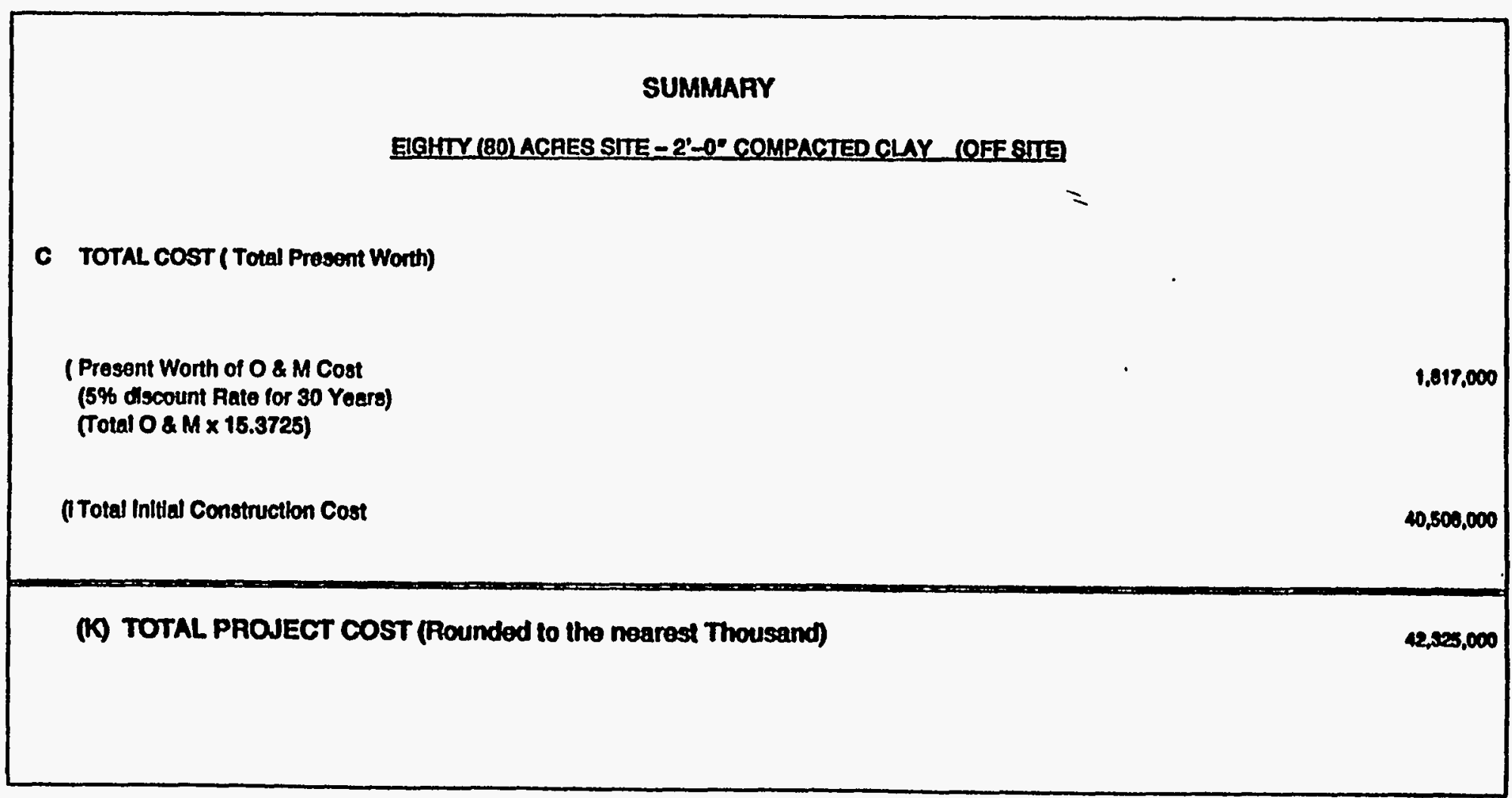

J-7. Cost Estimate for a Cover System Utilizing Compacted Kaolin (Offsite) Clay and a 30-mil FML (Cont'd) 
Loo : $7-03$

ACTIVITY CODE: ESE260112

PROJ.NAME: ECON. BYALUATION OF CLOSURB CAP BARRIER MATERIAL
DETAIL ESTIMATE

ber 1993

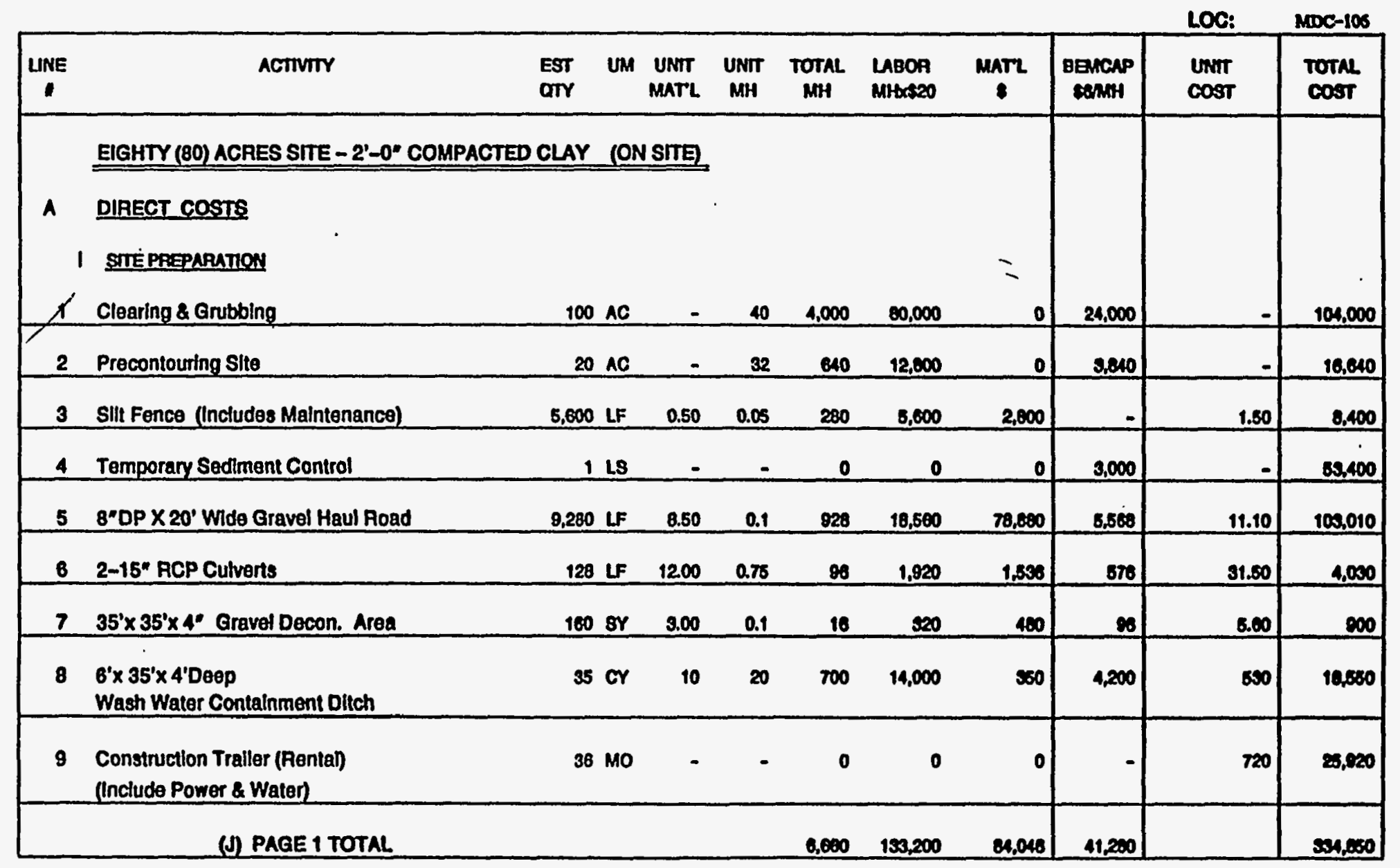

J-8. Cost Estimate a for Cover System Utilizing Compacted Onsite Sandy Clay and a 30-mil FML 


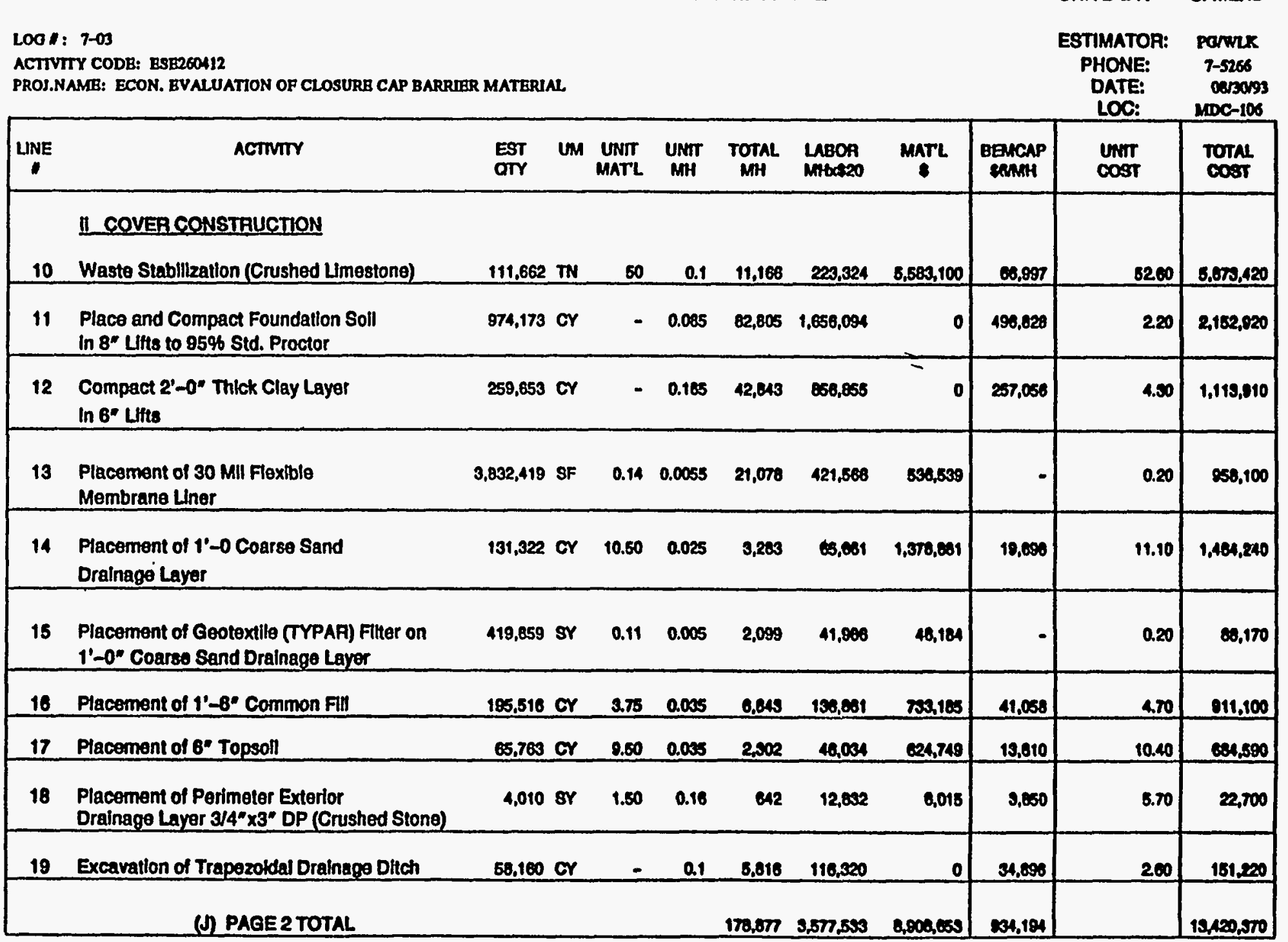

J-8. Cost Estimate a for Cover System Utilizing Compacted Onsite Sandy Clay and a 30-mil FML (Cont'd) 
DETAIL ESTIMATE

CHK'D BY: S. MEAD

LOO : $7-03$

ACTIVITY CODE: BSE260412

PROJ.NAME: ECON. EVALUATION OF CLOSURB CAP BARRIER MATERIAL

\section{ESTIMATOR: POWRX}

PHONE: 7-S266

DATE: Oarass

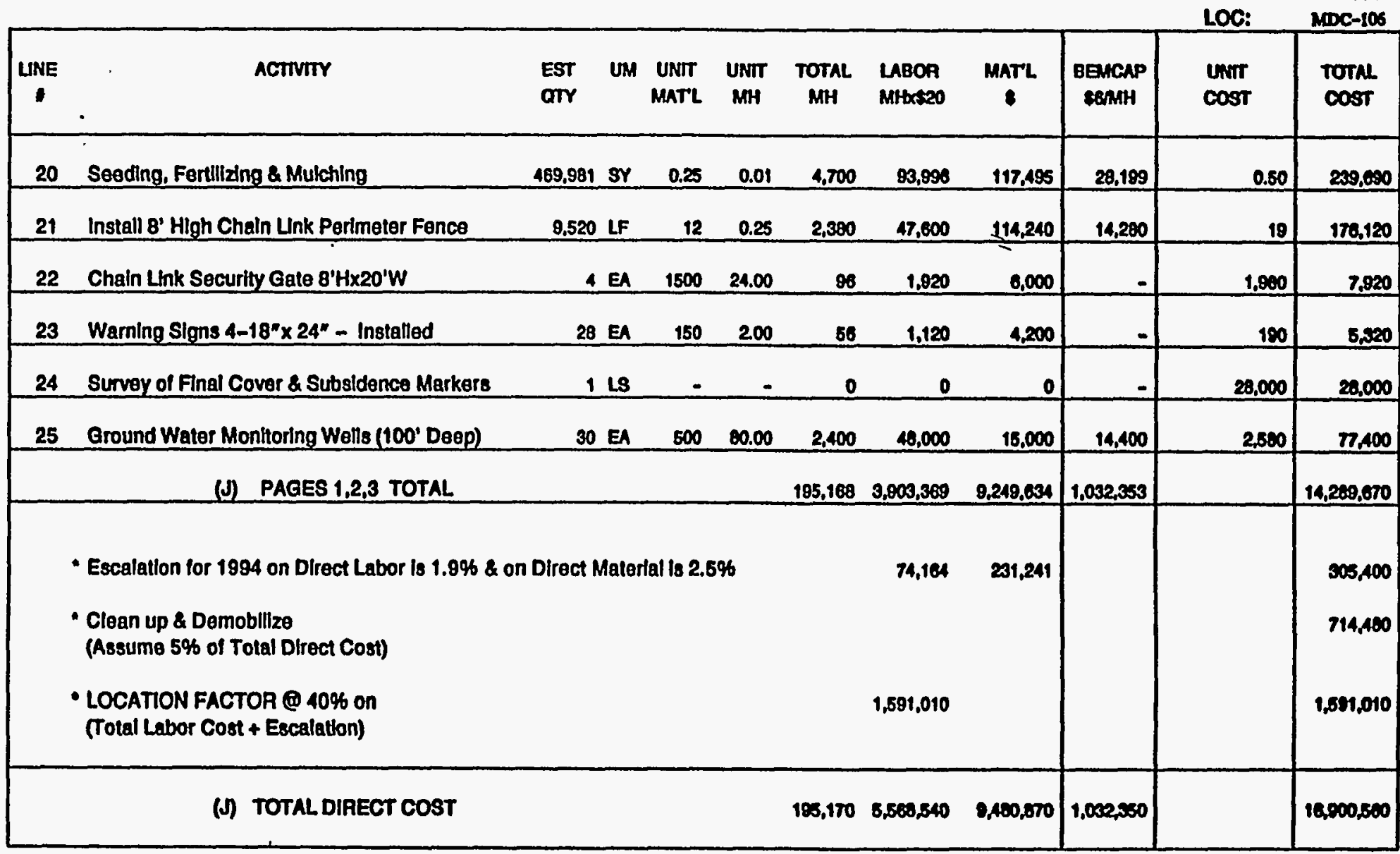

J-8. Cost Estimate a for Cover System Utilizing Compacted Onsite Sandy Clay and a 30-mil FML (Cont'd) 


\section{DETAIL ESTIMATE}

CHK'D BY: S. MEAD

LOO I: 7-03

ACTIVITY CODB: ESE260112

PROJ.NAME: ECON. EVALUATION OF CLOSURB CAP BARRIER MATERIAL

STIMATOR: PaWrK

PHONE: 7-5265

DATE:

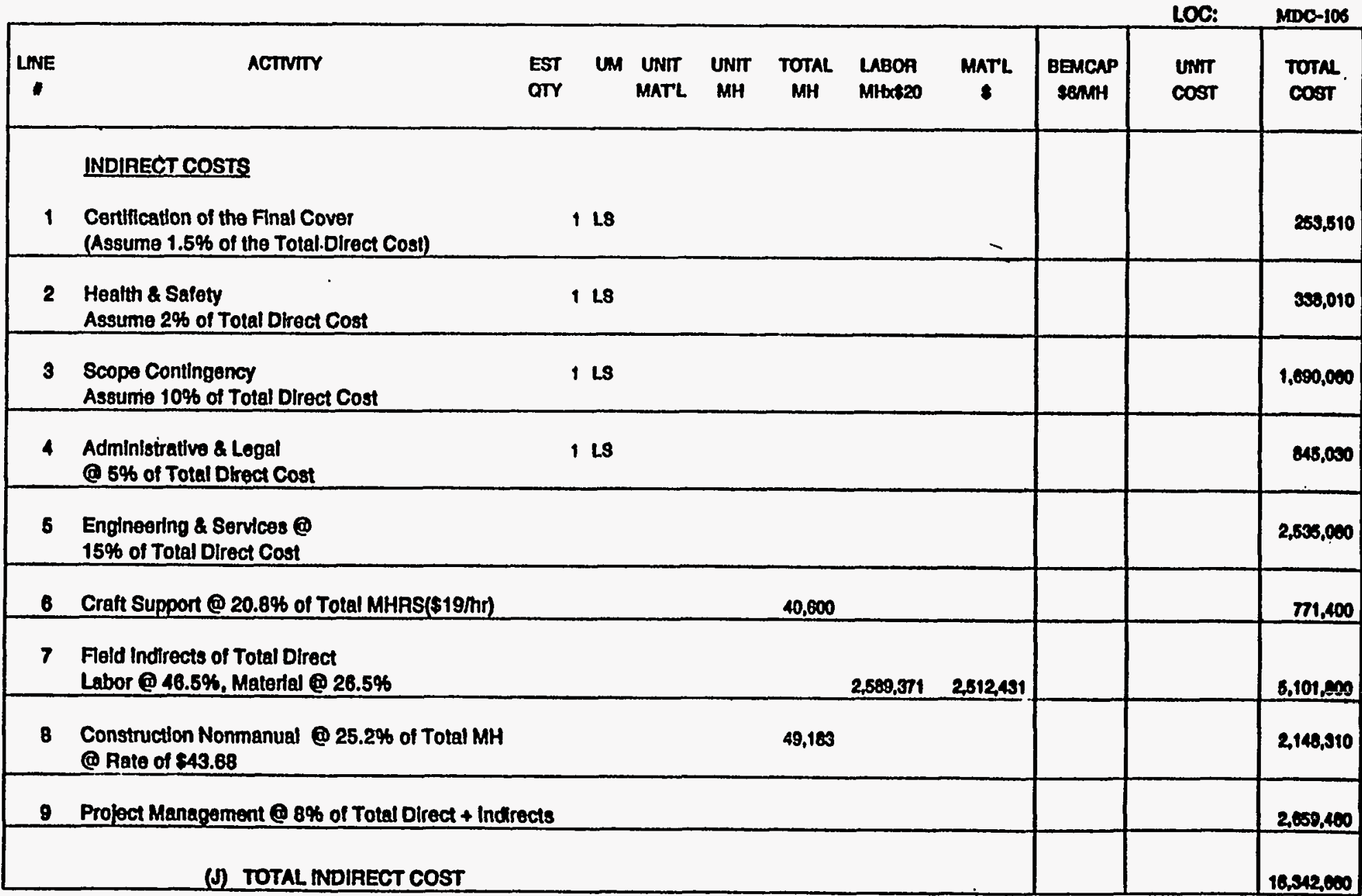

J-8. Cost Estimate a for Cover System Utilizing Compacted Onsite Sandy Clay and a 30-mil FML (Cont'd) 


\section{DETAIL ESTIMATE}

CHK'D BY: S. MEAD

Lo0 $1:$ 7-03

ACTIVTTY CODE: ESE260112

PROJ.NAME: ECON. BVALUATTON OR CLOSURB CAP BARRIIR MATERIAL

ESTIMATOR: TOWLX

PHONE: 7-5266

DATE: OOR3093

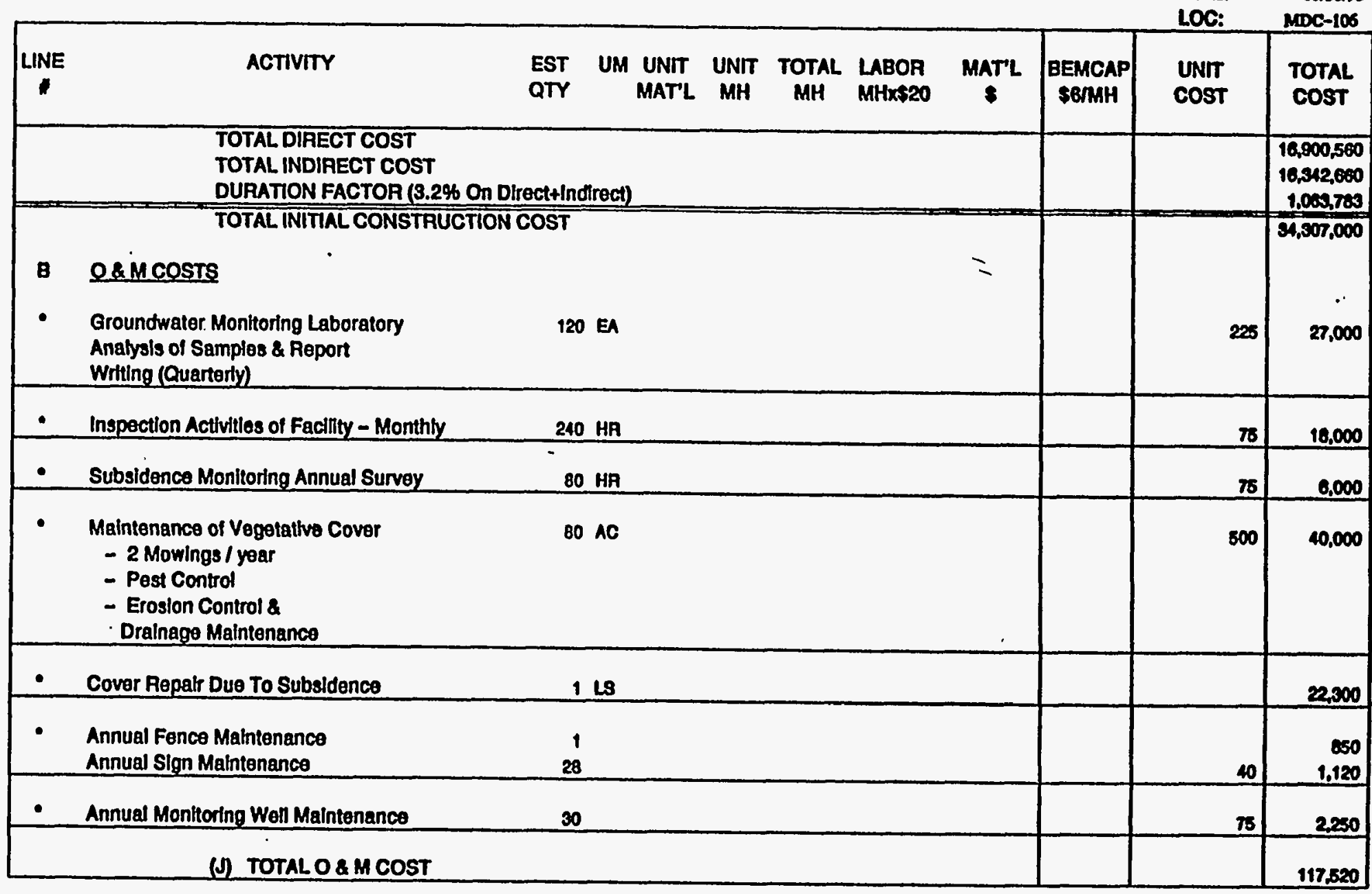

J-8. Cost Estimate a for Cover System Utilizing Compacted Onsite Sandy Clay and a 30-mil FML (Cont'd) 


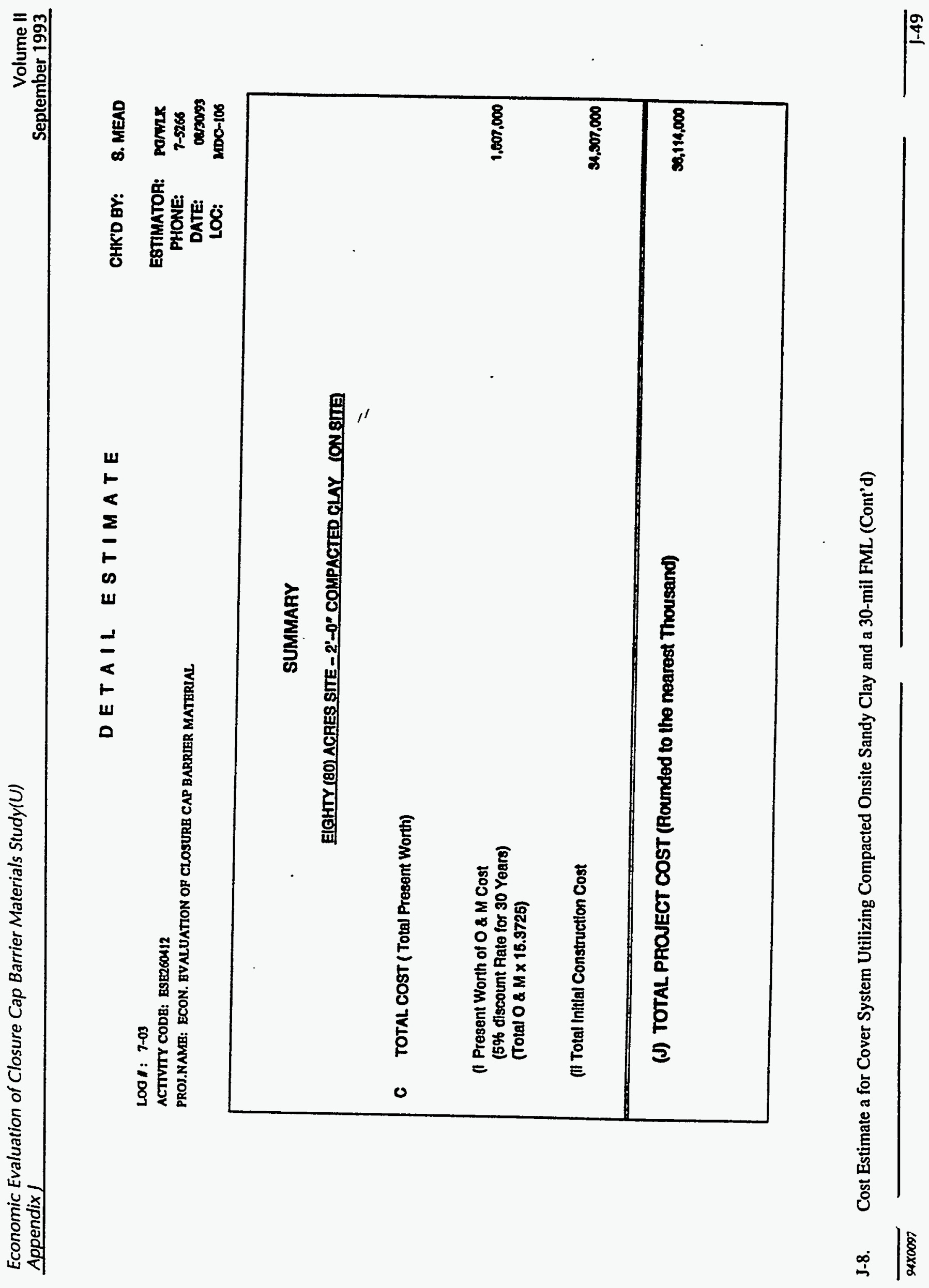




\section{DETAIL ESTIMATE}

$1008: 7-03$

ACTTVTY CODE: BSEQ60112

PROI.NAMB: ECON. EVALUATTON OF CLOSURB CAP BARUUER MATERUAL.

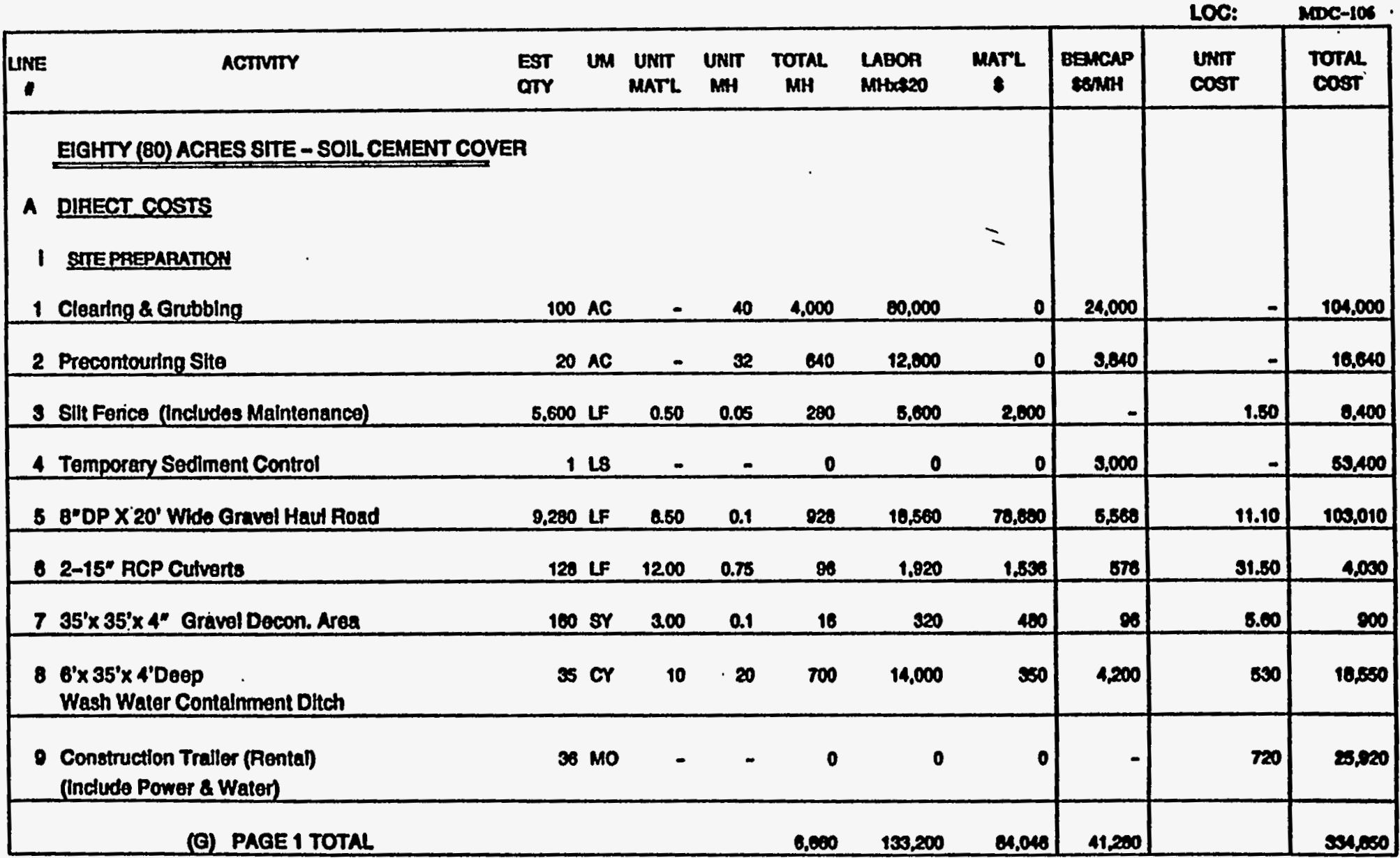

J-9. Cost Estimate for a Cover System Utilizing Soil Cement and 30-mil FML 


\section{DETAIL ESTIMATE}

CHK'D BY: S. MEAD

L001: 7-03

ACTIVITY CODE: ESE260112

PROS.NAMR: ECON. EVALUATION OP CLOSURE CAP BARRIER MATERIAL

ESTIMATOR: rorWRX

PHONE: 7-5266

DATE: DOPOSO

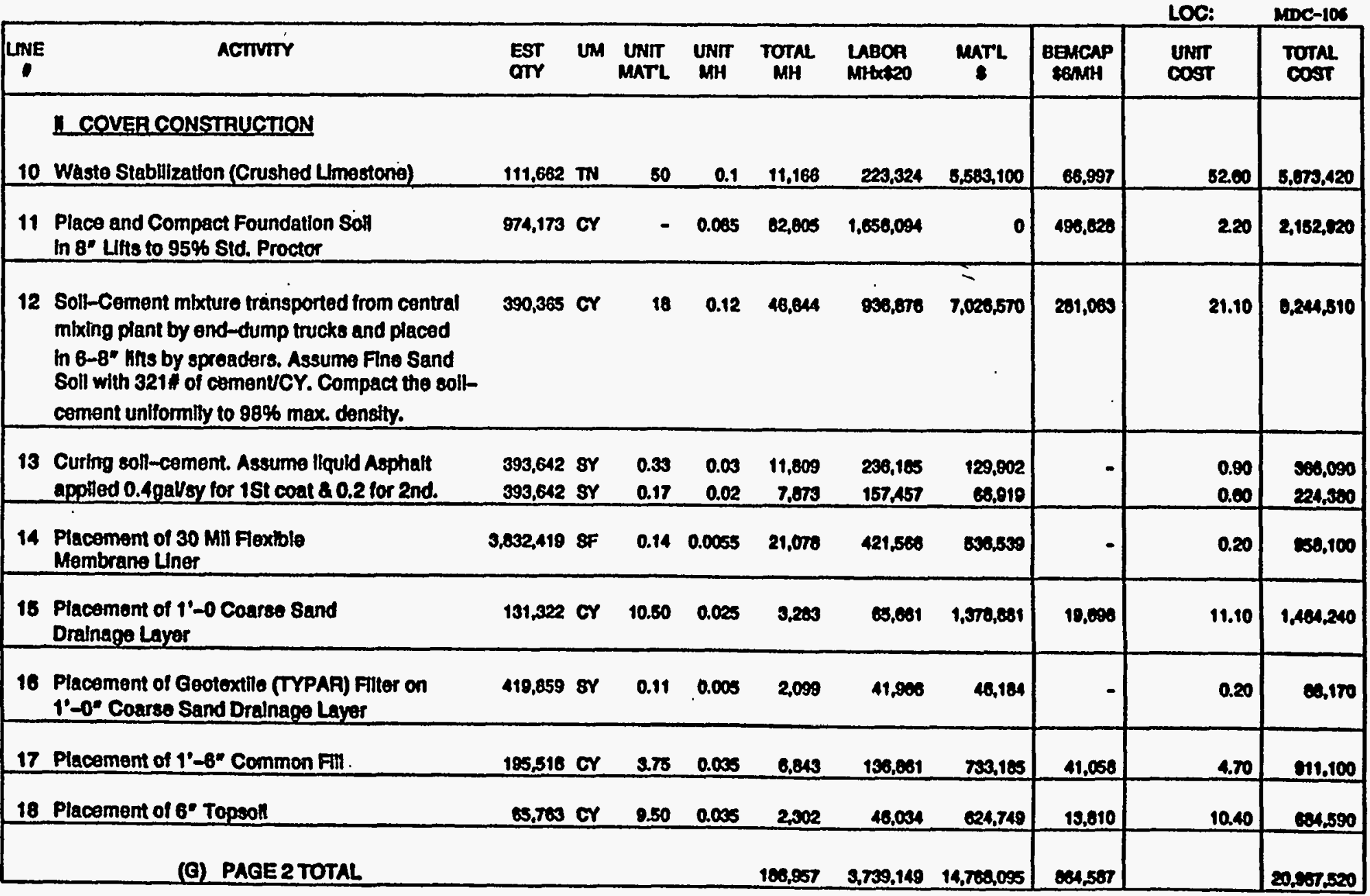

J-9. Cost Estimate for a Cover System Utilizing Soil Cement and 30-mil FML (Cont'd) 


\section{B T A L B S TIMATB}

Lo0 : :-03

ACTIVITY CODE: ESE260112

PROJ.NAMBB: ECON. BVALUATTON OF CLOSURB CAP BARRIER MATERIALL

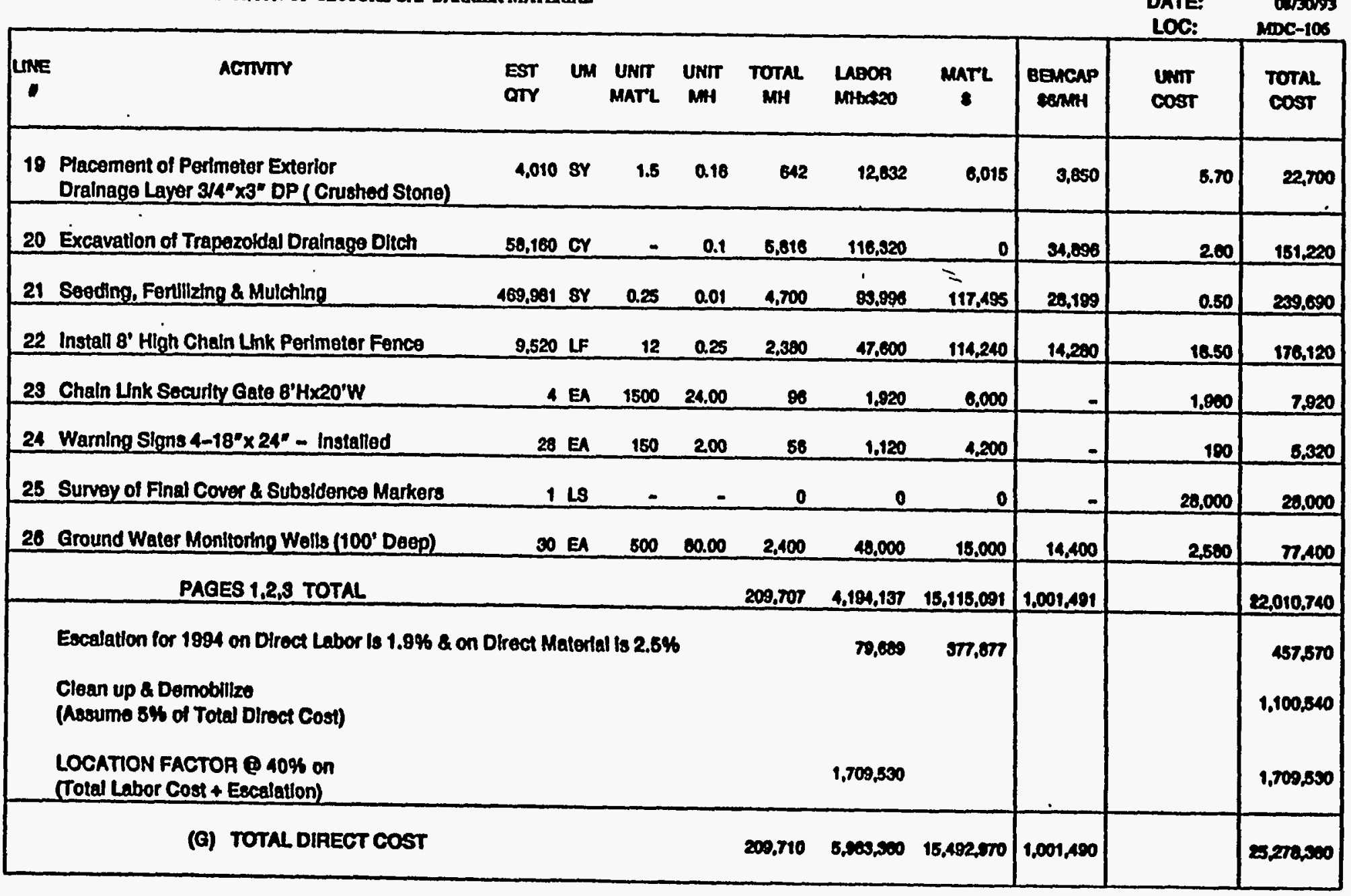

J-9. Cost Estimate for a Cover System Utilizing Soil Cement and 30-mil FML (Cont'd) 
D B TAIL B STIMATB

CHK'D BY: 8. MEAD

Loof: 7-03

ACTIVTY CODB: BsE260112

PROI.NAMB: ECON. BVALUATION OF CLOSTTR CAP DANRIRR MATERIAL.

ESTIMATOR: romK

PHONE: 7-5268

DATE: Darsons

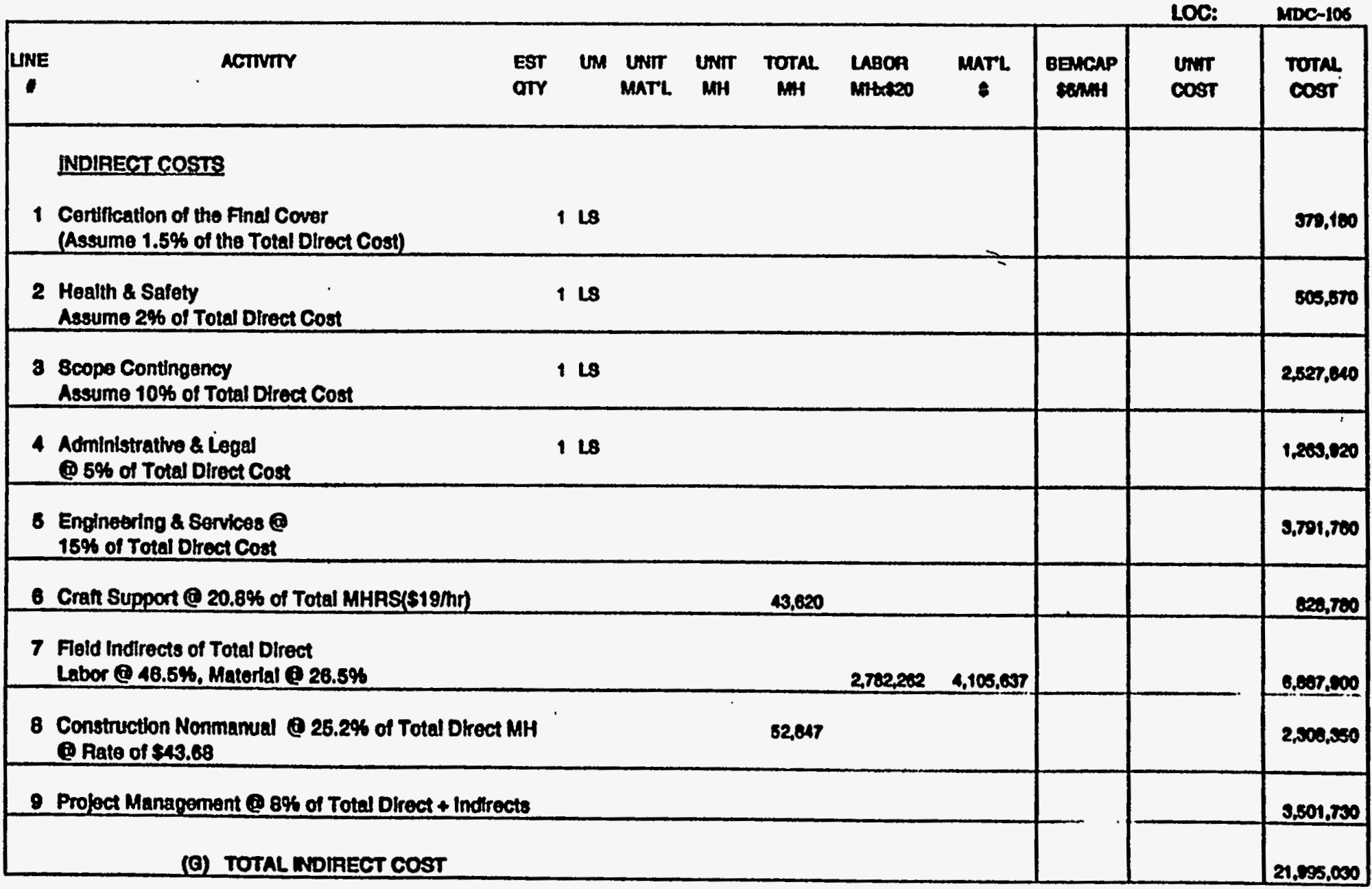

J-9. Cost Estimate for a Cover System Utilizing Soil Cement and 30-mil FML (Cont'd) 
D B T A I L B S TIMAT B

CHKD BY: 8. MEAD

L00 I: $7-03$

ACTIVITY CODE: BSE260412

PROJ.NAMR: BCON. EVALUATION OP CLOSURE CAP DARRIER MATERUAL

ESTIMATOR: ravin

PHONE: 7-5266

DATE: Dasors

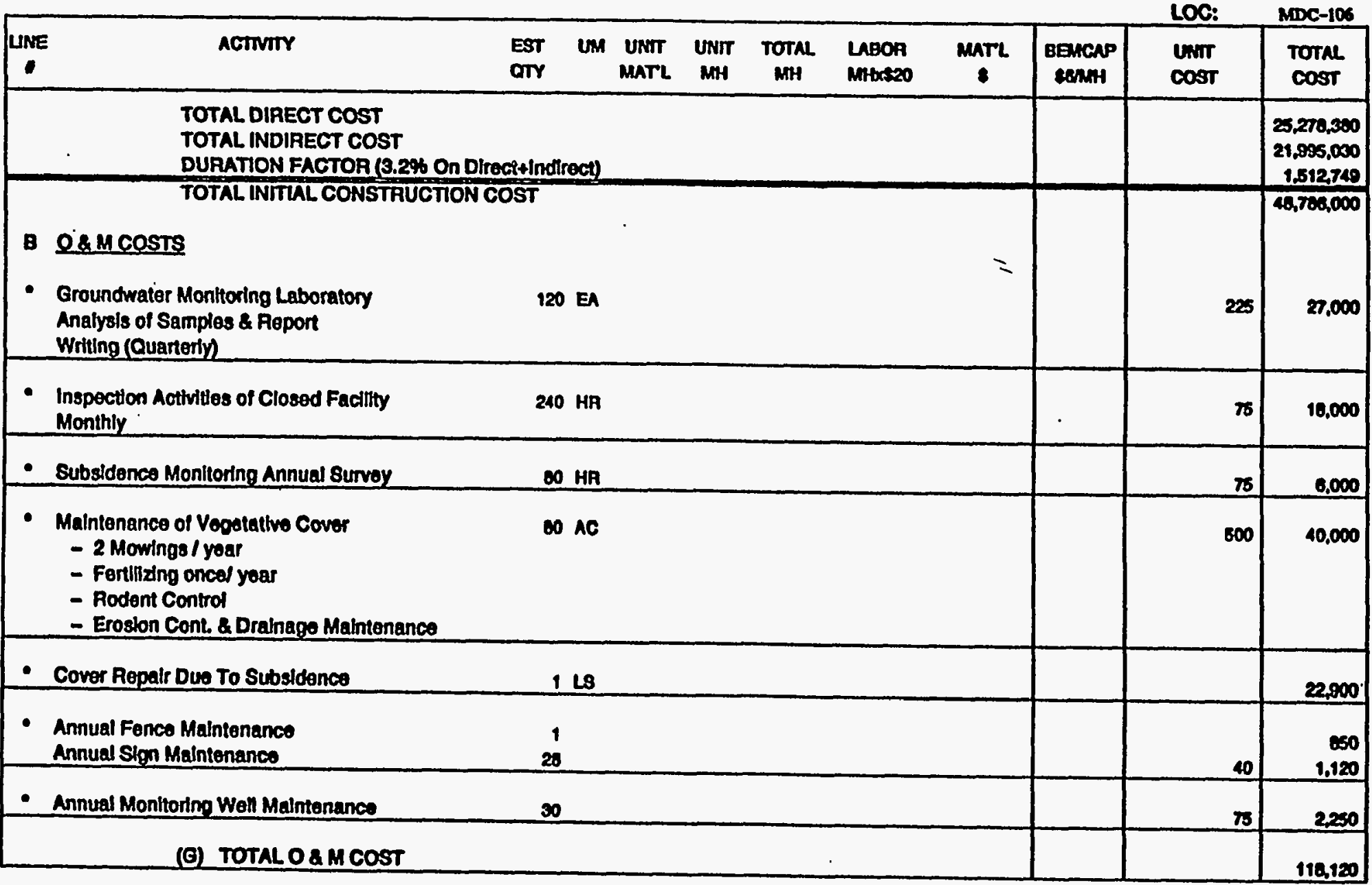

J-9. Cost Estimate for a Cover System Utilizing Soil Cement and 30-mil FML (Cont'd) 
D B T A I B S T IMAT B

Loof: $7-03$

ACTIVITY CODE: ESE260412

MOJ.YYMME: ECON. EVALUATION OF CLOSURB CAP BARRIER MATERIAL

\begin{tabular}{|c|c|}
\hline CHKD BY: & 8. MEAD \\
\hline $\begin{array}{l}\text { ESTIMATOA: } \\
\text { PHONE: } \\
\text { DATE: } \\
\text { LOC: }\end{array}$ & $\begin{array}{c}\text { ramix } \\
7-5266 \\
\text { porsoms } \\
\text { MDC-106 }\end{array}$ \\
\hline
\end{tabular}

\section{SUMMARY}

EQHTV (80) ACRES SIIE- SOIL CEMENT COVER

C TOTAL COST (Total Present Worth)

(Present Worth of O \& M Cost

(5\% discount Rato for 30 Yearo)

$1,016,000$

(Total O\& M X 15.3725)

(1Total Inittal Construction Cost

$40,700,000$

(G) TOTAL PROJECT COST (Rounded to the nearest Thousand)

$50,602,000$

J-9. Cost Estimate for a Cover System Utilizing Soil Cement and 30-mil FML (Cont'd) 
DETAIL ESTIMATE

CHKD BY: S. MEAD

1001: 7-03

ACTIVITY CODE: ESEZ6OA12

PROJ.NAME: ECON. EVALUATION OP CLOSURB CAP DARRIER MATERLAL.

\begin{tabular}{|c|c|c|c|c|c|c|c|c|c|c|}
\hline UNE & ACTIVIV & $\begin{array}{l}\text { EST Un } \\
\text { arr }\end{array}$ & $\begin{array}{l}\text { UnII } \\
\text { MATL }\end{array}$ & $\begin{array}{l}\text { UNIT } \\
\text { MNH }\end{array}$ & $\begin{array}{c}\text { TOTAL } \\
\text { MH }\end{array}$ & $\begin{array}{l}\text { LABOR } \\
\text { Mitrated }\end{array}$ & $\operatorname{mam}^{\prime}$ & $\begin{array}{c}\text { comanp } \\
\text { senmin }\end{array}$ & $\begin{array}{l}\text { und } \\
\cos \pi\end{array}$ & $\begin{array}{l}\text { TOTAL } \\
\text { COST }\end{array}$ \\
\hline $\begin{array}{r}A \\
1 \\
\end{array}$ & $\begin{array}{l}\text { EIGHTY (BO) ACRES SITE-BENTO } \\
\text { DIAECT COSTR } \\
\text { SMEPREPARAIOON } \\
\text { Clearing \& Grubbing }\end{array}$ & DITVE & - & 40 & 1,000 & 00,000 & $=$ & 24,000 & - & 104,000 \\
\hline 2 & Precontouring Slte & $20 \wedge C$ & - & 32 & 640 & 12800 & 2 & 2040 & - & 10040 \\
\hline 3 & Silt Fence (inctudes Maintenance) & $8,600 \mathrm{LF}$ & 0.60 & 0.05 & 200 & $\mathbf{B}, 000$ & 2,000 & - & 1.50 & 2400 \\
\hline 4 & Temporary Secilment Control & 148 & - & - & 0 & 0 & o & 2,000 & - & 82400 \\
\hline 6 & "OP X 20" Wodo Gravel Haul Rond & $0,200 \mathrm{LF}$ & 8.60 & 0.1 & $\infty 20$ & 10.500 & 70,000 & 8,500 & 11.10 & 108010 \\
\hline 6 & 2-15" RCP Culvents & 128 LF & 1200 & a.75 & $\boldsymbol{\infty}$ & 1,020 & 1.050 & 870 & 31.60 & 4.000 \\
\hline 7 & $35^{\prime} \times 35^{\prime} \times 4^{\prime \prime}$ Gravel Decon. Area & $160 \mathrm{SY}$ & 3.00 & 0.1 & 16 & 320 & 400 & $\boldsymbol{\infty}$ & $\mathbf{8 . 0 0}$ & 000 \\
\hline 8 & $\begin{array}{l}\text { 6'x 35'x 4'000p } \\
\text { Wash Water Containment Ditch }\end{array}$ & $35 \mathrm{Cr}$ & 10 & 20 & 700 & 14,000 & $\infty$ & 4,200 & 650 & 18080 \\
\hline 8 & $\begin{array}{l}\text { Constructlon Trailer (Aonial) } \\
\text { (include Power \& Water) }\end{array}$ & 36 MO & - & - & 0 & 0 & 0 & - & 720 & 20,620 \\
\hline & (H) PAGE I TOTAL & & & & 6,600 & 130,200 & Q4,043 & 41200 & & 204800 \\
\hline
\end{tabular}

J-10. Cost Estimate for a Cover System Utilizing Bentonite Soil Additive and a 30-mil FML 
DETAIL ESTIMATE

CHK'D BY: 8. MEAD

Lo01: $7-09$

ACTIVITY CODB: BSE26ON12

PROJ.NAMB: ECON. BVALUATHON OP CLOSURB CAP BARRIRR MATERIAL

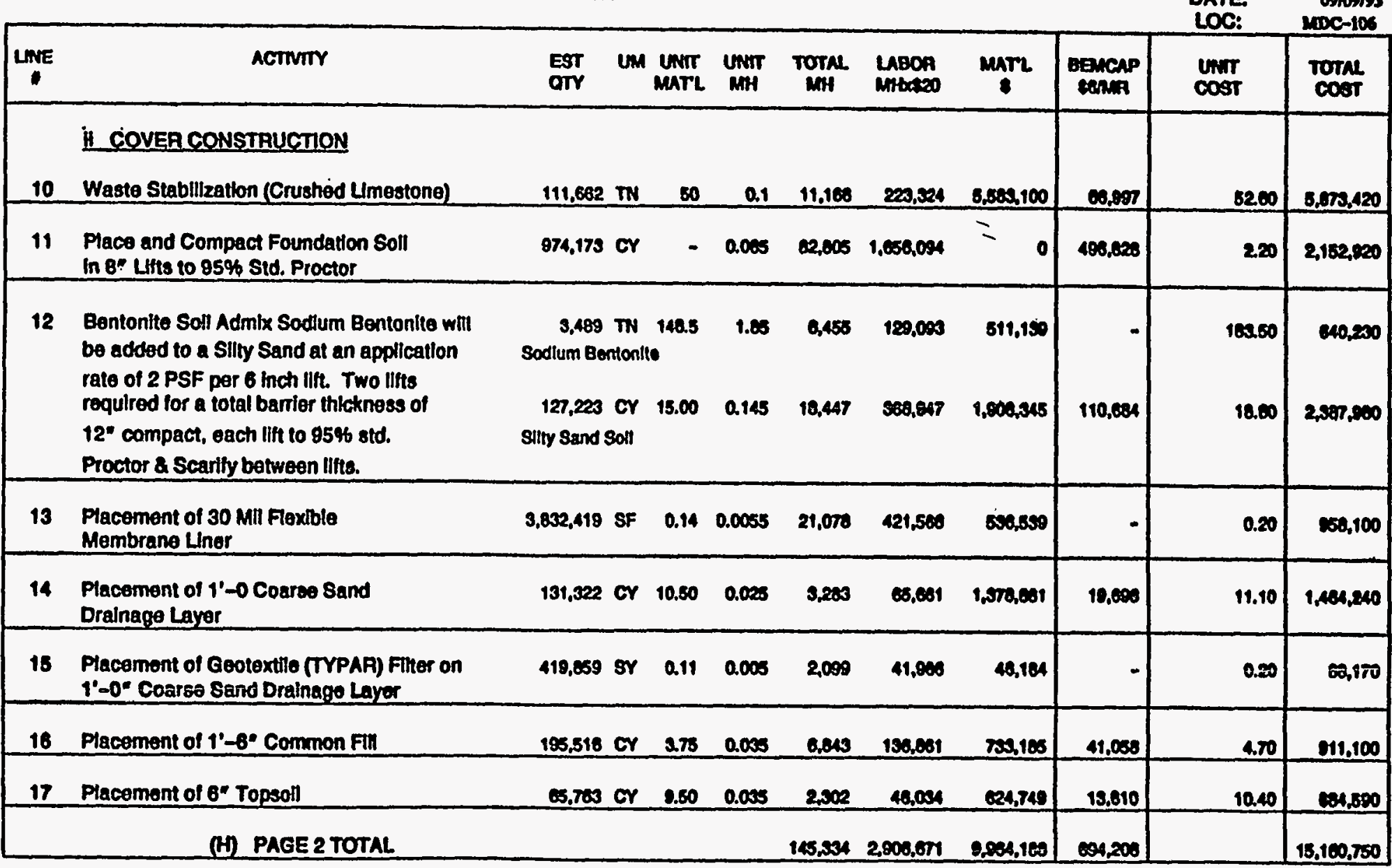

J-10. Cost Estimate for a Cover System Utilizing Bentonite Soil Additive and a 30-mil FML (Cont'd) 
DETAIL ESTIMATE

CHK'D BY: S. MEAD

2001: 7-03

NCTIVTTY CODE: BSE2SON12

FROI.NAMR: ECON. BVALUATION OF CLOSURB CAP BARRIER MATERLAL

ESTIMATOR: rarinx

PHONE: 7-5266

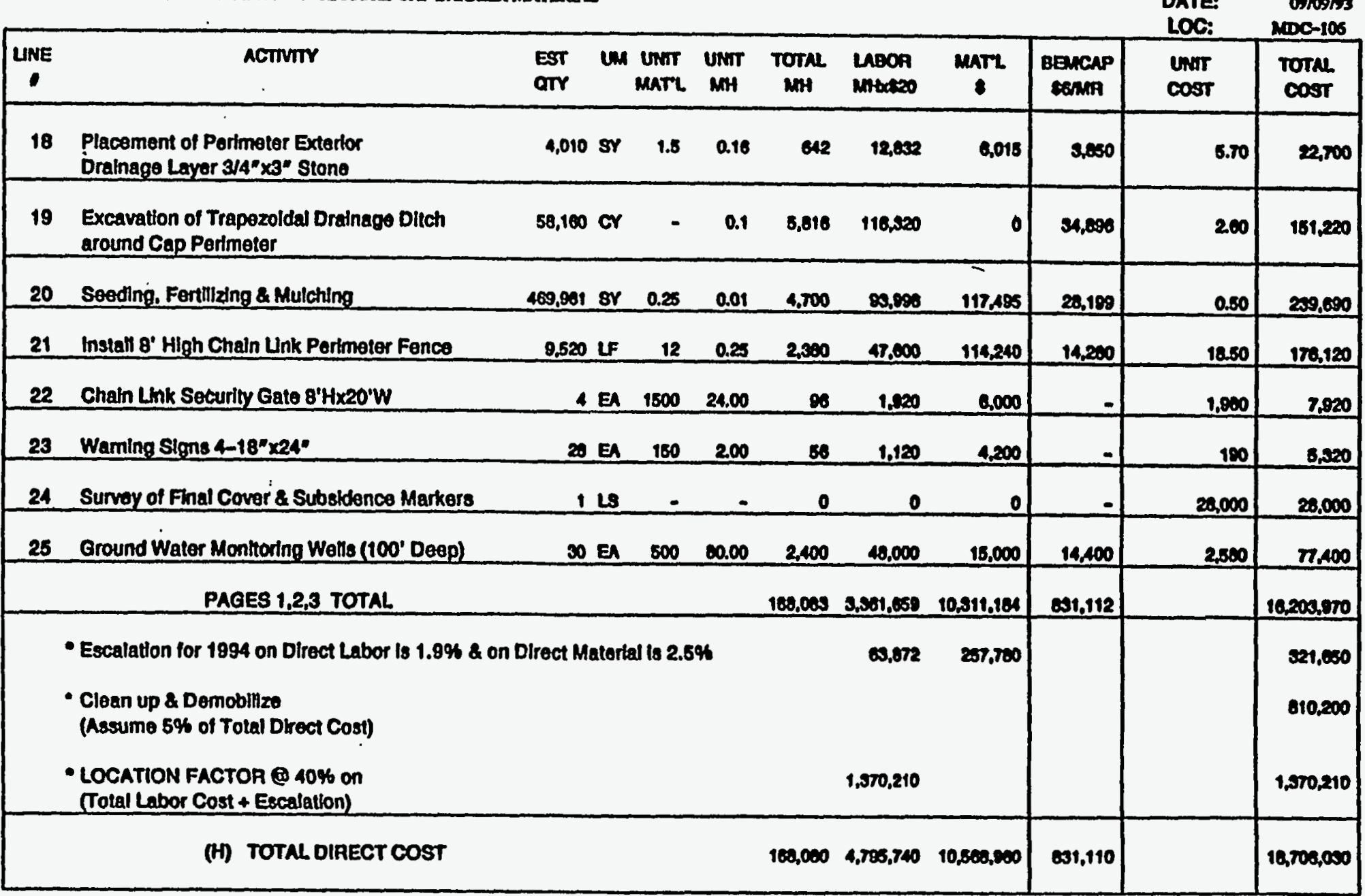

J-10. Cost Estimate for a Cover System Utilizing Bentonite Soil Additive and a 30-mil FML (Cont'd) 


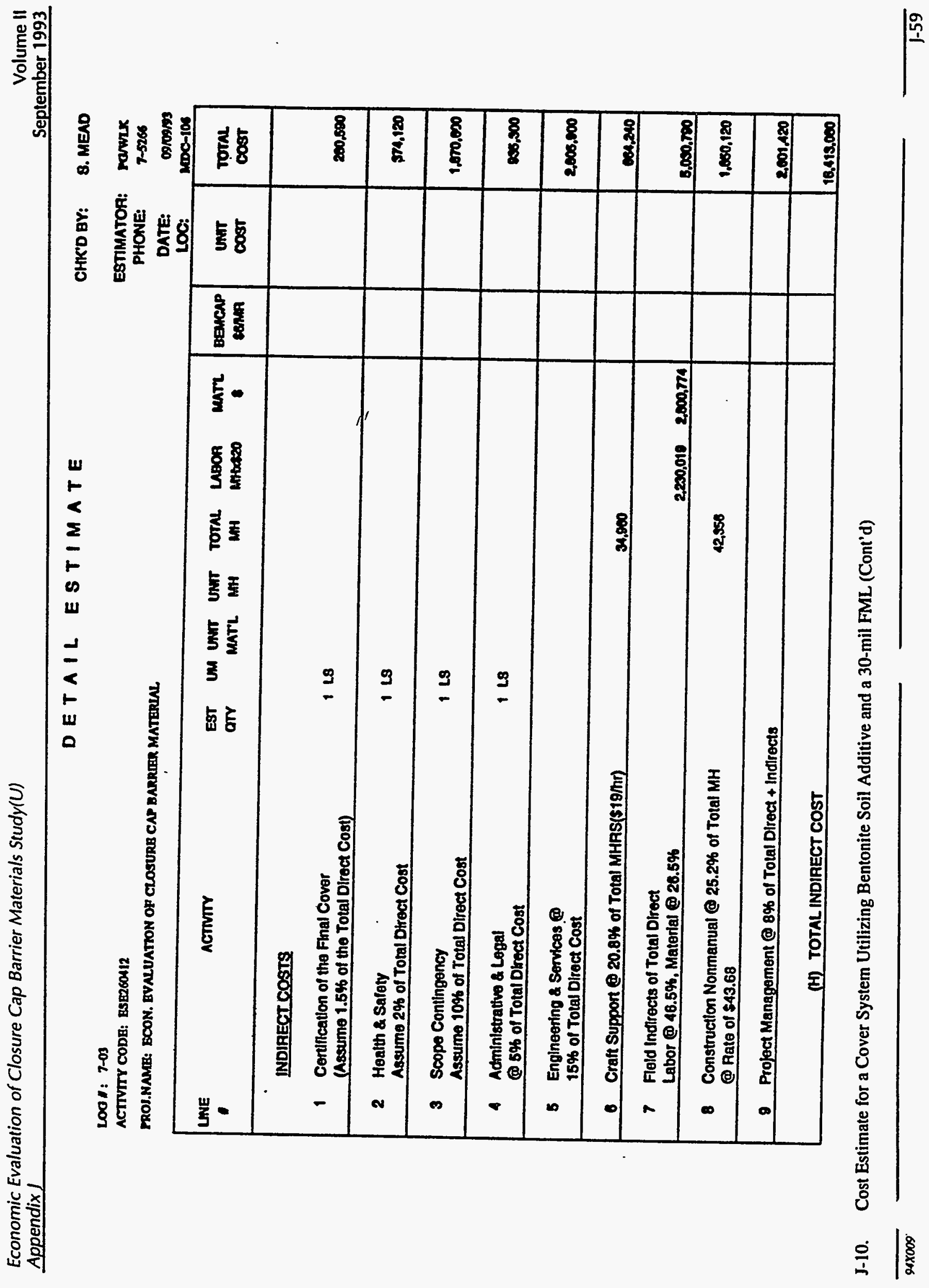


DETAIL ESTIMATE

CHKD BY: 8. MEAD

\section{1: $7-03$}

ACTIVITY CODE: BSEZ6O412

PROJ.NAMB: ECON. EVALUATTON OF CLOSURB CAP BARRTER MATEREAL

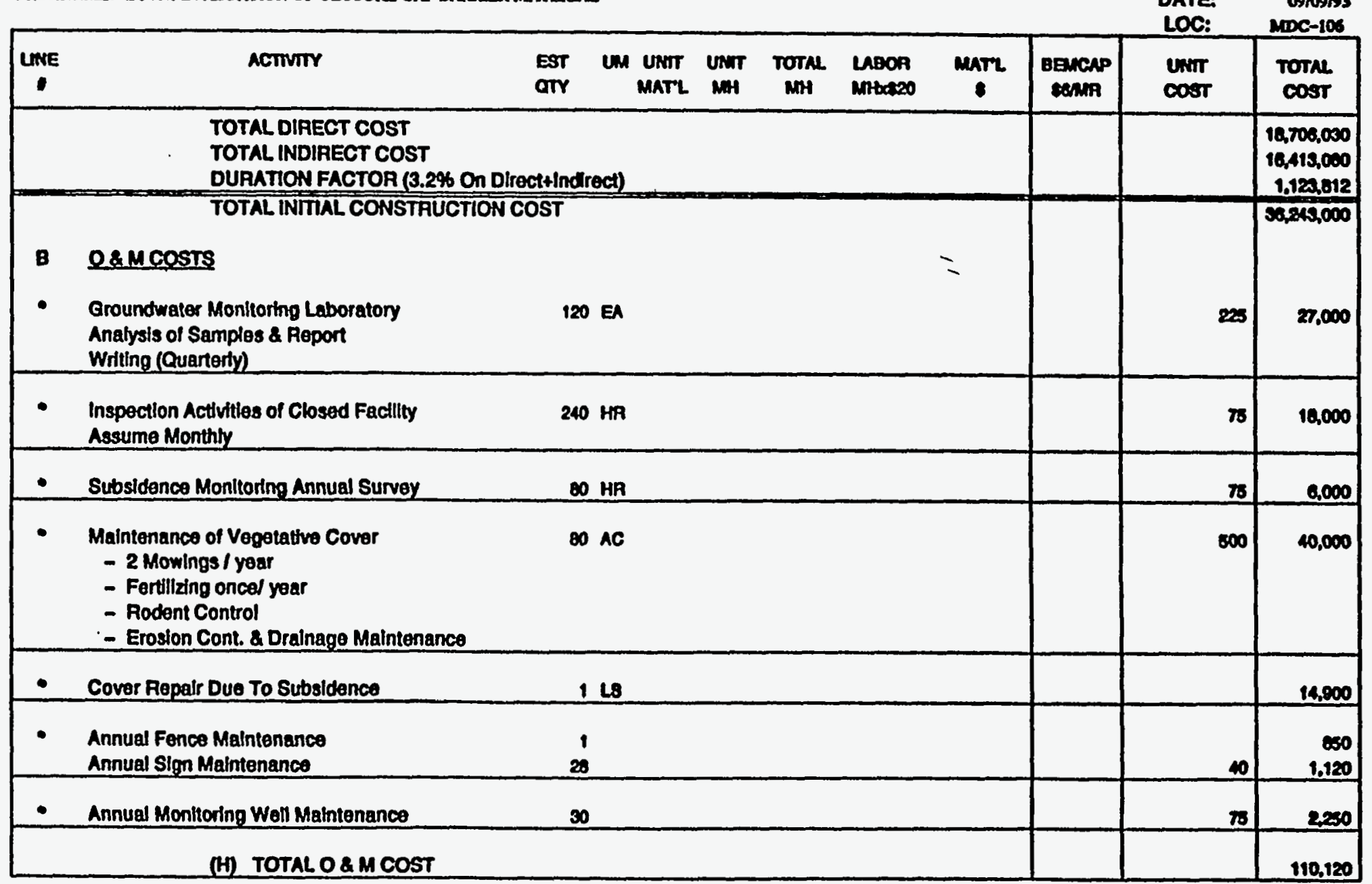

J-10. Cost Estimate for a Cover System Utilizing Bentonite Soil Additive and a 30-mil FML (Cont'd) 
DETAIL ESTIMATE

Loo: : 7-03

ACTTVTT CODE: ESEZOOA12

MROJ.NAME: ECON. EVALUATION OP CLOSURB CAP BARREER MATERIAL

\begin{tabular}{|c|c|}
\hline CHKD BY: & B. MEAD \\
\hline $\begin{array}{l}\text { ESTIMATOR: } \\
\text { PHONE: } \\
\text { DATE: } \\
\text { LOC: }\end{array}$ & $\begin{array}{c}\text { rownx } \\
7-5265 \\
\text { 0xwess } \\
\text { moc-10s }\end{array}$ \\
\hline
\end{tabular}

\section{SUMMARY}

\section{EIGHTY (BO) ACRES SITE - BENTONITESOQL ADOITNE}

$=$

C TOTAL COST (Total Presem Worth)

(1) Present Worth of O \& M Cost

(6\% discoumt Rate for 30 Years)

$1,695,000$

(Total O \& $M \times 15.3725)$

(il Total Initial Construction Cost

$30,240,000$

(H) TOTAL PROJECT COST (Rounded to the nearest Thousand)

spsesa,000

J-10. Cost Estimate for a Cover System Utilizing Bentonite Soil Additive and a 30-mil FML (Cont'd) 
DETAIL ESTIMATE

Loo: $7-03$

ACTIVITY CODE: ESE260112

PROS.AAMR: ECON. BVALUATION OP CLOSURE CAP BARRUER MATERIAL,

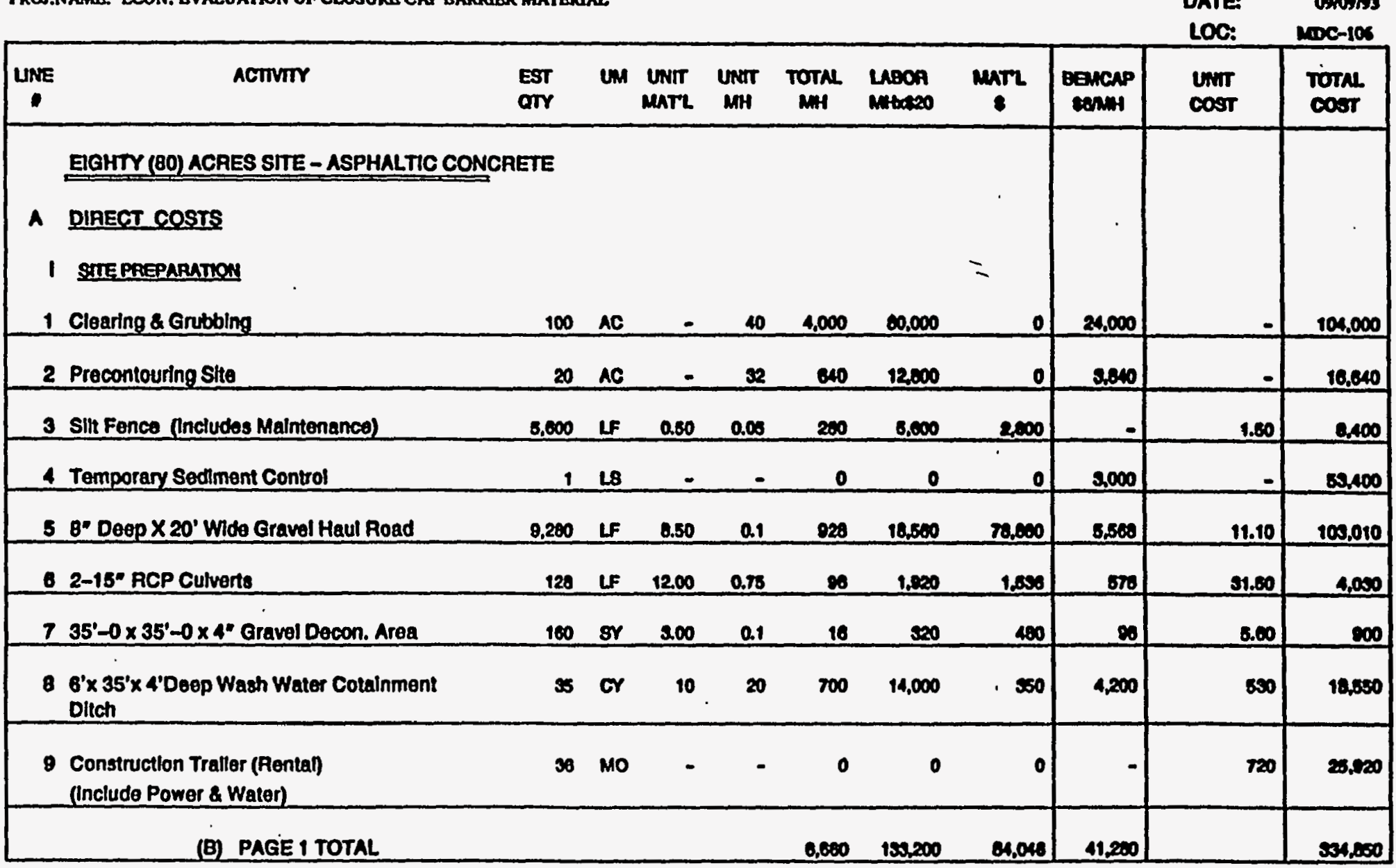

J-11. Cost Estimate for a Cover System Asphalt Concrete Hydraulic Barrier and a 30-mil FML 
DETAILESTIMATE

CHK'D BY: 8. MEAD

Loo : : 7-03

ACTIVITY CODE: BSER6OS12

PROI.NAMR: ECYH. BYALUATION OP CLOSURB CAP BARRIER MATERLAL

ESTIMATOR: rownX

PHONE: 7-5265

\begin{tabular}{|c|c|c|c|c|c|c|c|c|c|c|}
\hline ACTMITY & $\begin{array}{l}\text { EST } \\
\text { OTY }\end{array}$ & Un & $\begin{array}{l}\text { Unirt } \\
\text { MANTL }\end{array}$ & $\begin{array}{l}\text { Unim } \\
\text { Mint }\end{array}$ & rotre & $\begin{array}{l}\text { LABOA } \\
\text { Mitated }\end{array}$ & mart & $\begin{array}{l}\text { Beacenp } \\
\text { sennit }\end{array}$ & $\begin{array}{l}\ln _{\cos } \\
\cos 1\end{array}$ & $\begin{array}{l}\text { Totne } \\
\text { cost }\end{array}$ \\
\hline $\begin{array}{l}\text { D COVER CONSTAUCTION } \\
10 \text { Wasto Stabillzation (Crushed Limestono) }\end{array}$ & 111.662 & $\mathbf{T N}$ & 50 & 0.1 & 11,166 & 223,324 & $8,802,100$ & 0,097 & 62.00 & $8,073,420$ \\
\hline $\begin{array}{l}11 \text { Place and Compact Foundation Soll } \\
\text { In } 8^{\circ} \text { Lilts to } 95 \% \text { Sid. Proctor }\end{array}$ & 687,000 & Cr & - & 0.065 & 56,056 & $1,133,900$ & 0 & 340,170 & 2.20 & $1,974,070$ \\
\hline $\begin{array}{l}12 \text { Placement of } 30 \text { Mil Flexible } \\
\text { Membrane Lher }\end{array}$ & $3,619,600$ & $8 F$ & 0.14 & 0.0055 & 18.909 & 500,170 & 800,77 & - & 0.50 & 204,860 \\
\hline $\begin{array}{l}13 \text { Placement of } 1^{\prime}-0 \text { Coarse Sand } \\
\text { Dralnage Layer }\end{array}$ & 133,350 & or & 10.60 & 0.025 & 3,334 & 68,075 & $1,400,175$ & 20,000 & 11.10 & $1,400,060$ \\
\hline $\begin{array}{l}14 \text { 2" Pvic Perforated Oralnage Plpe placed } \\
\text { In Dralnage layer }\end{array}$ & 29,200 & $\mathbf{L}$ & 1.0 & 0.00 & 2,356 & 40,720 & 20,200 & - & 2.00 & $\pi, 600$ \\
\hline $1521 / 2^{\prime}$ Bindar Courso & 391,900 & SY & 4.15 & 0.055 & 21,555 & 451.009 & $1,028, A 18$ & 120.230 & 8.00 & $2,100,050$ \\
\hline 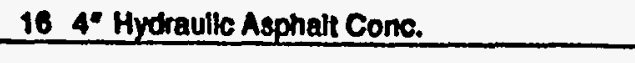 & 391,906 & SY & 200 & 0.00 & 11,707 & 200,145 & 2600,503 & roses & 7.40 & $2,002,200$ \\
\hline 17 Asphaft Mastic Seal & 391,900 & sr & 0.50 & 0.015 & 8,070 & 117,572 & 16.054 & - & 0.00 & sis.s50 \\
\hline 18 Sprayod Asphattic Concrete Sealant & 391,806 & 8r & a.rs & 0.018 & $8,6 \pi$ & \$17,572 & 209031 & $\mathbf{3 0 , 2 2 0}$ & 1.10 & ma,nog \\
\hline 19 Excavation of Tralnqular Dralnage Ditch & 14,290 & CY & - & 0.1 & 4,400 & 60,592 & 0 & 20,570 & 2.00 & 116,170 \\
\hline 20 4" Thlck Asphait Perlmoter Drainage Ottch & $\mathbf{3 7 . 7 4 0}$ & sr & 7.60 & 0.144 & 8,45 & 100.091 & 200,024 & 20,007 & 11.20 & 120,120 \\
\hline (B) PAGE 2 TOTAL & & & & & 146,575 & $2,807,400$ & 12500807 & 721,499 & & $16,107,540$ \\
\hline
\end{tabular}

J-11. Cost Estimate for a Cover System Asphalt Concrete Hydraulic Barrier and a 30-mil FML (Cont'd) 
Economic Evaluation of Closure Cap Barrier Materials Study(U)

Volume II Appendix I

DETAIL BSTIMATB

CHKD BY: 8. MEAD

Lo01: $7-03$

ACTIVTTY CODE: BSE26012

PROJ.NAME: ECON. BVALUATTON OF CLOSURB CAP BARRRER MATBRIAL.

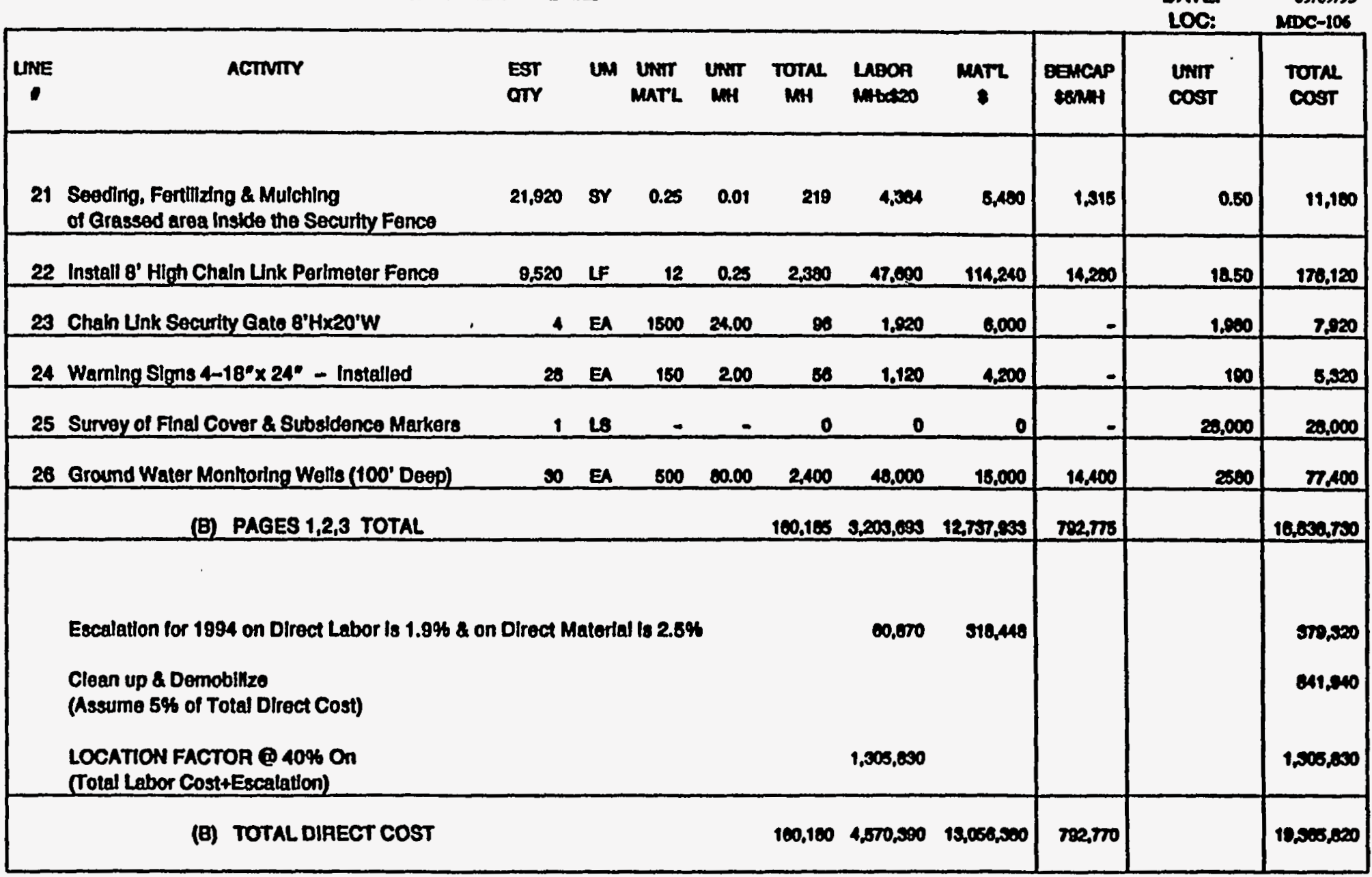

J-11. Cost Estimate for a Cover System Asphalt Concrete Hydraulic Barrier and a 30-mil FML (Cont'd) 
DETAIL B S T IMAT B

CHKD BY: 8. MEAD

L001: 7-03

ACTIVITY CODB: BSE2CON12

MROJ.NAME: ECON. EVALUATION OF CLOSURB CAP BARRIRR MATERIAL

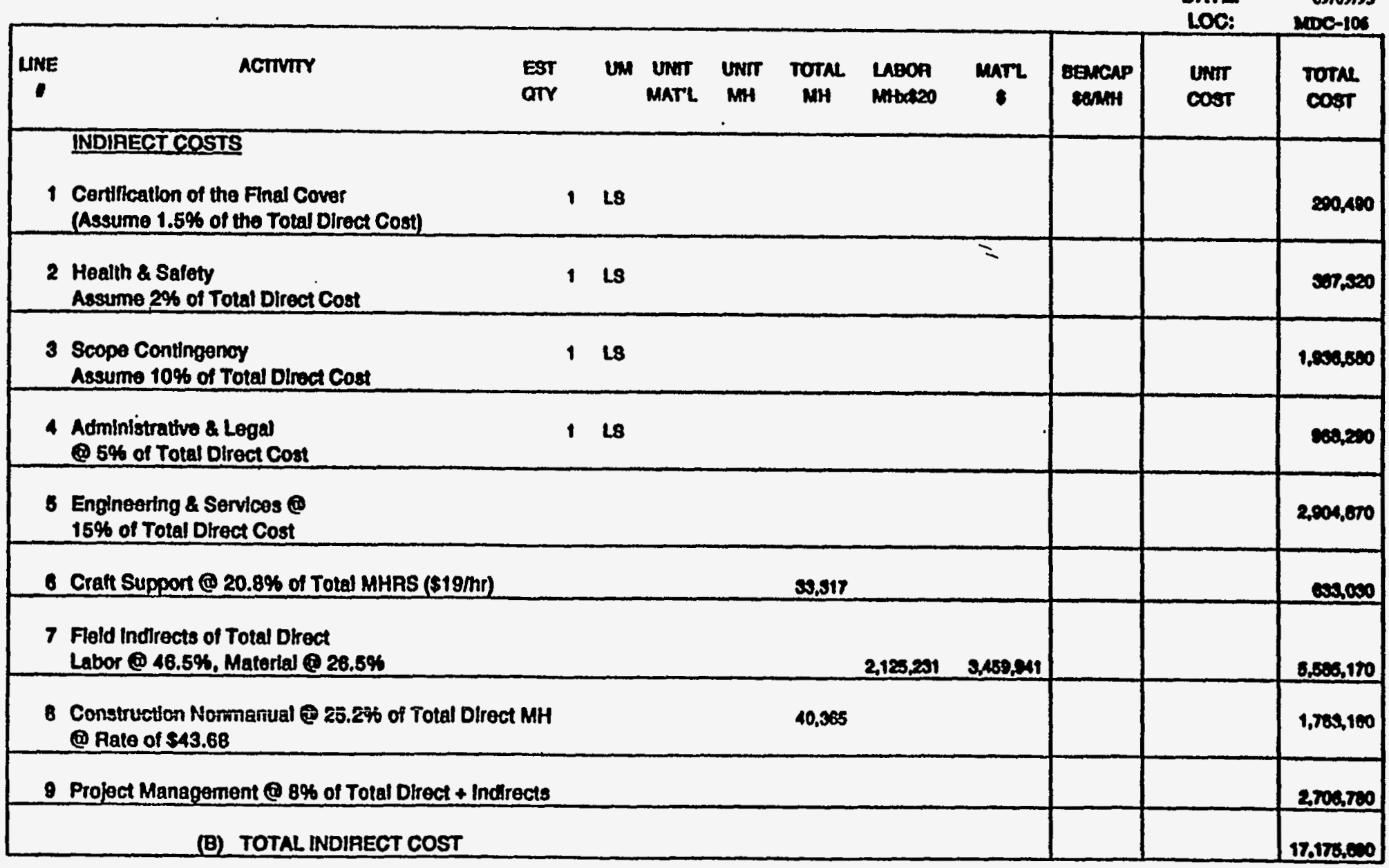

J-11. Cost Estimate for a Cover System Asphalt Concrete Hydraulic Barrier and a 30-mil FML (Cont'd) 
DETAILESTIMATE

L00 I: $7-03$

ACTVITY CODE: ESE26012 12

PROJ.NAME: BCON. BVALUATKON OF CLOSURB CAP BARRIER MATERIAL.

\begin{tabular}{|c|c|c|c|c|}
\hline 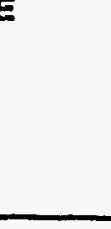 & & & $\begin{array}{l}\text { CHKO BV: } \\
\text { ESTmuntort } \\
\text { PHONE } \\
\text { DATE } \\
\text { LOC: }\end{array}$ & 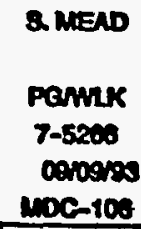 \\
\hline \multirow[t]{9}{*}{ 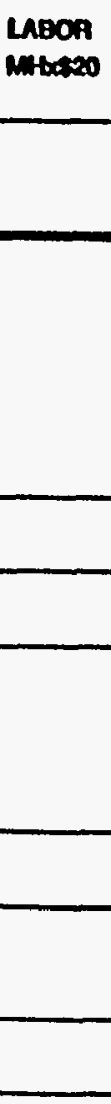 } & MATL & $\begin{array}{l}\text { Benchs } \\
\text { BenMH }\end{array}$ & $\begin{array}{l}\text { Unim } \\
\text { cost }\end{array}$ & $\begin{array}{l}\text { TOTAL } \\
\text { cost }\end{array}$ \\
\hline & & & & $\begin{array}{r}10,505,020 \\
17,175,690 \\
1,100,520\end{array}$ \\
\hline & $=$ & & 205 & $\begin{array}{r}31.71,000 \\
27,000\end{array}$ \\
\hline & & & $\pi$ & 10,000 \\
\hline & & & 73 & 0.000 \\
\hline & & & 800 & 04,000 \\
\hline & & & & 19,300 \\
\hline & & & 40 & $\begin{array}{r}800 \\
8.120 \\
\end{array}$ \\
\hline & & & 73 & 2.250 \\
\hline & & & & 100.52 \\
\hline
\end{tabular}

J-11. Cost Estimate for a Cover System Asphalt Concrete Hydraulic Barrier and a 30-mil FML (Cont'd) 


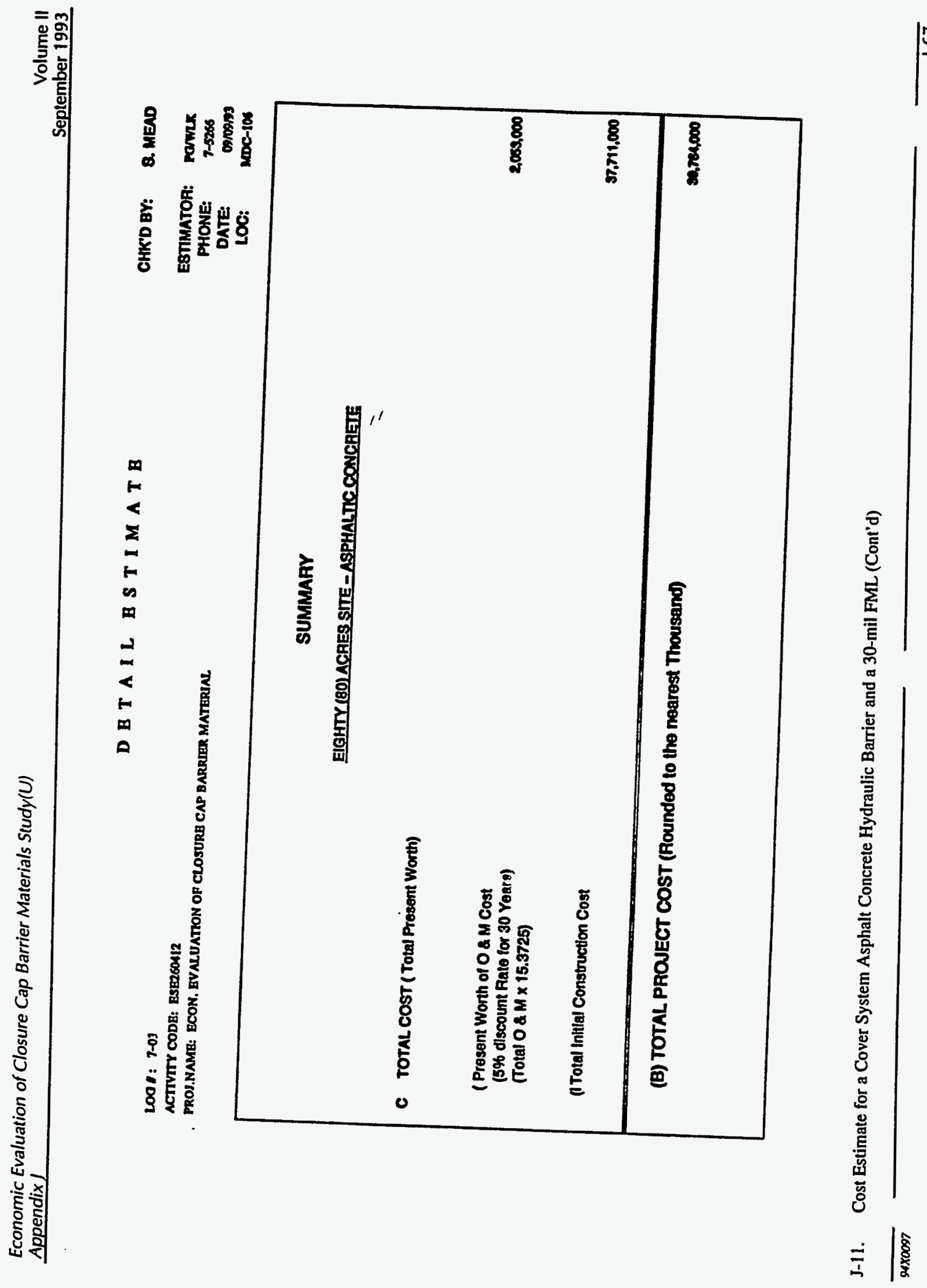


DETAIL ESTIMATE

LOQ : : 7-03

ACTIVTY CODE: ESEZSOM12 PPOU.NAMEE ECON. EVALUATION OF CLOSURE CAP BARPTER MATEEAAL

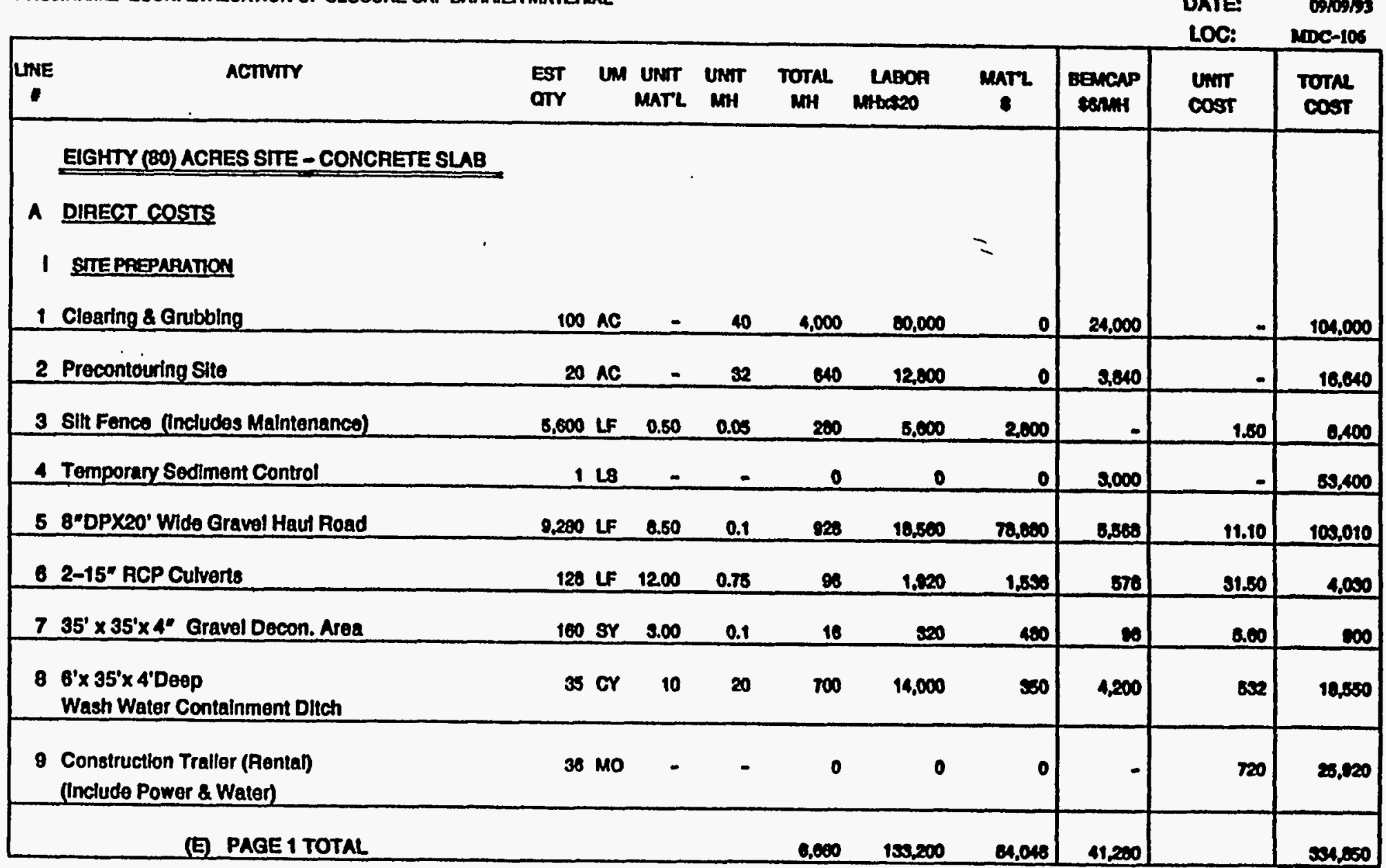

J-12. Cost Estimate for a Cover System Utilizing Poured-in Place Concrete Slab and a 30-mil FML 


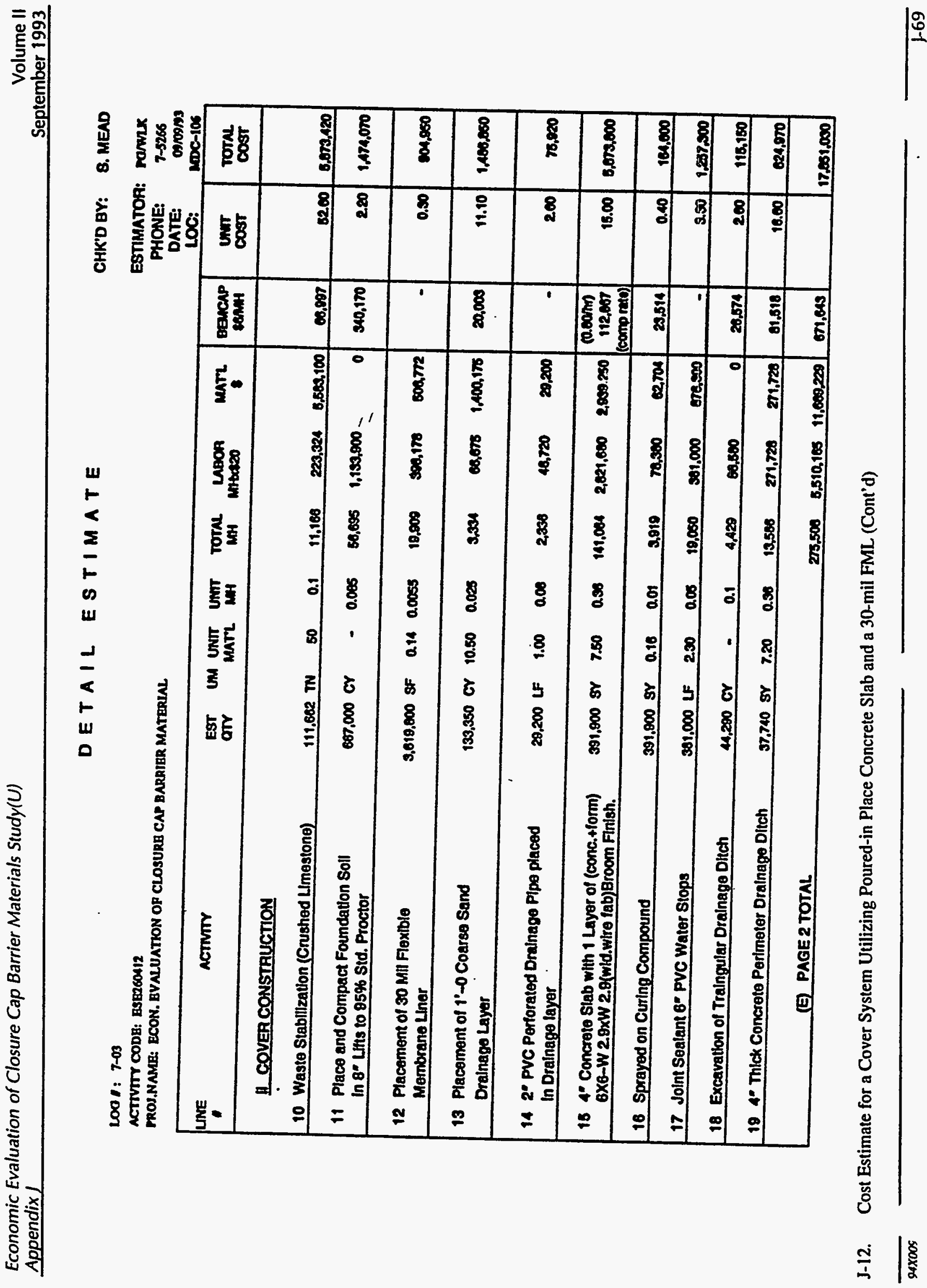


DBTAIL BSTIMATB

LOO I: 7-03

ACTIVTYY CODE: ESE260112

PROU.NAMB: ECON. BVALUATION OF CLOSURB CAP BARRRER MATERLAL.

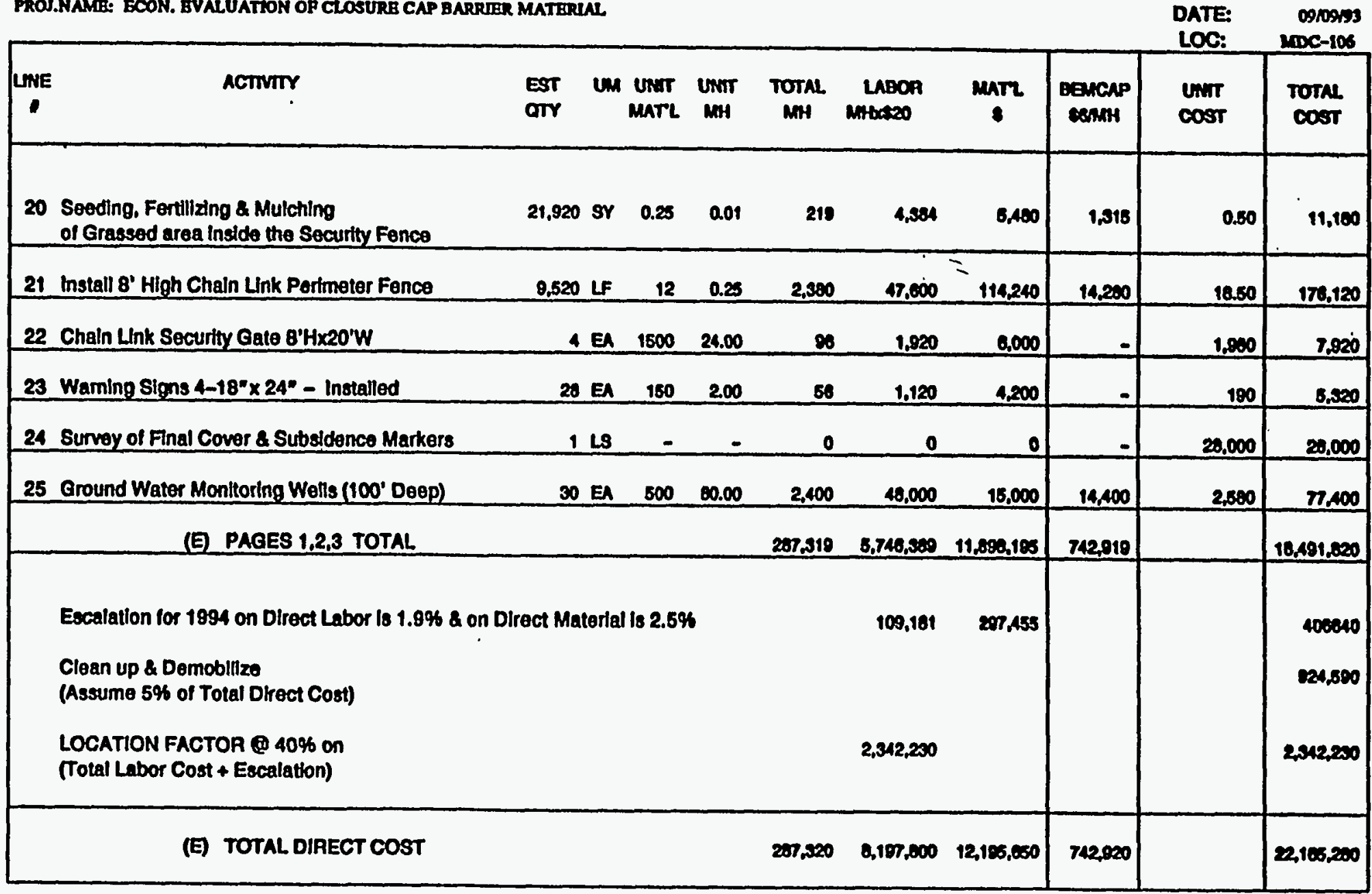

CHK'D QY: 8. MEAD

ESTIMATOR: rame

PHONE: 7-5265

PHONE: 7-5266

unde-106

cotras 
D B T A I L B S T I A T B

\section{L001: 7-03}

ACTIVITY CODB: ESE260412

RROS.NAMB: ECON. BVALUATTON OF CLOSURB CAP BARRRER MATERIAL.

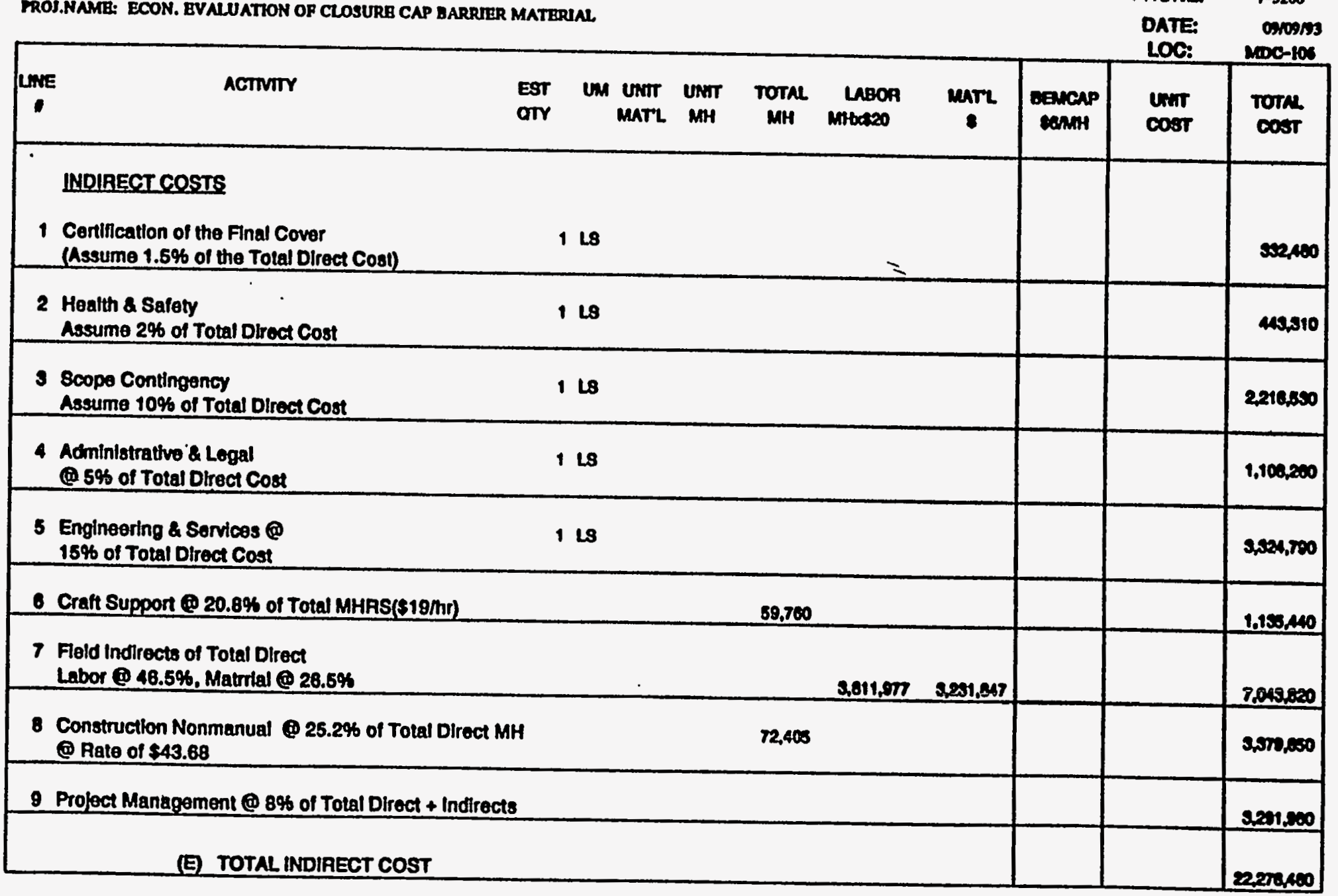

CHK'D BY: 8. MEAD

ESTMATOA: Panix

7-5266

torn.

$\cos 1$

100

210

18850

200,200

00,700

19,400

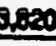

200000

1000

202000

J-12. Cost Estimate for a Cover System Utilizing Poured-in Place Concrete Slab and a 30-mil FML (Cont'd) 


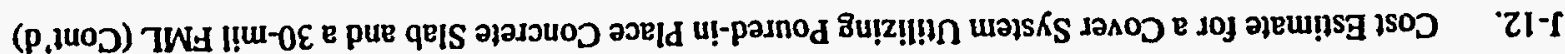

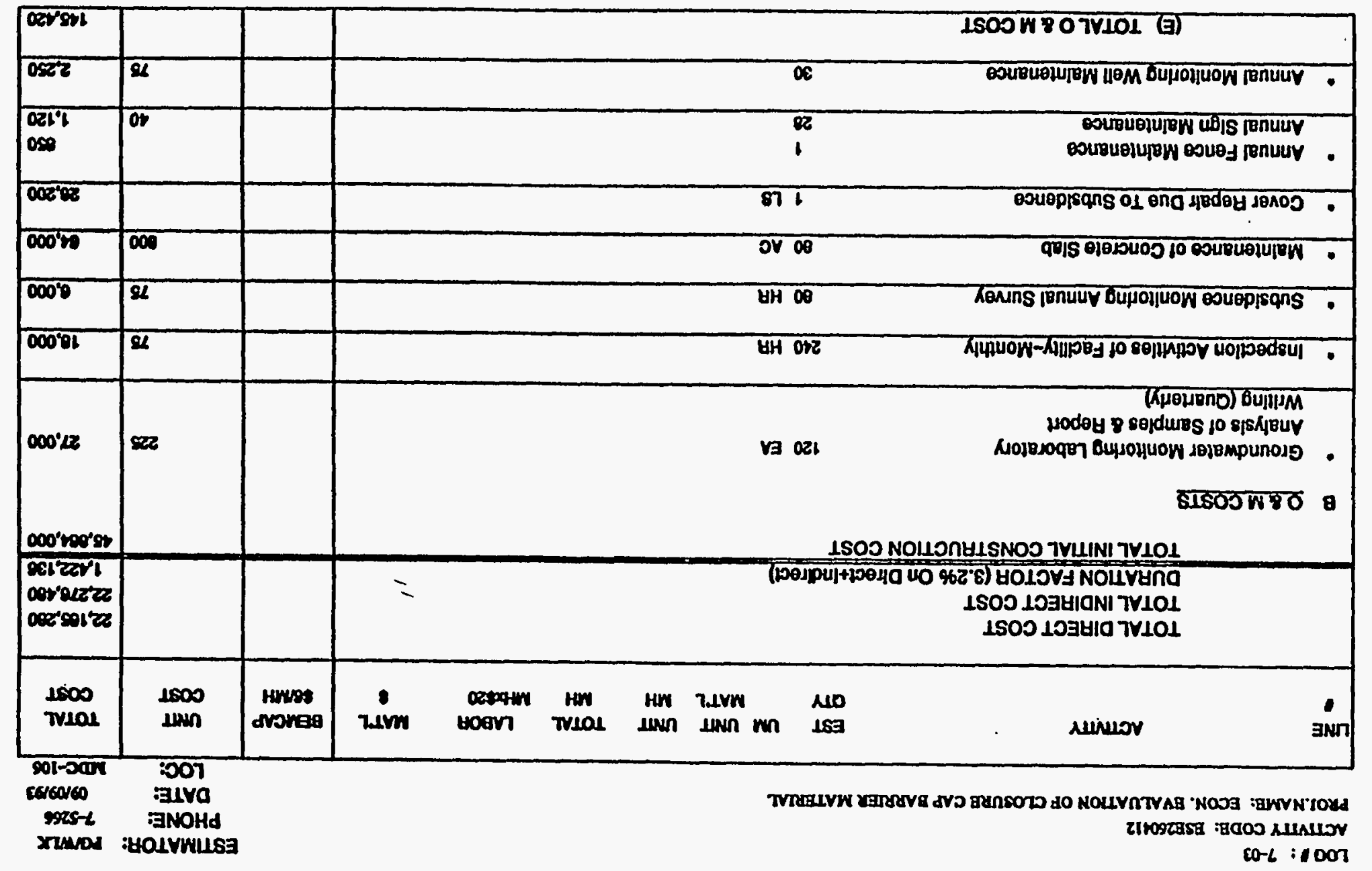

Gvaw 's :AB OSHHO

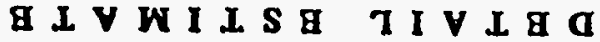




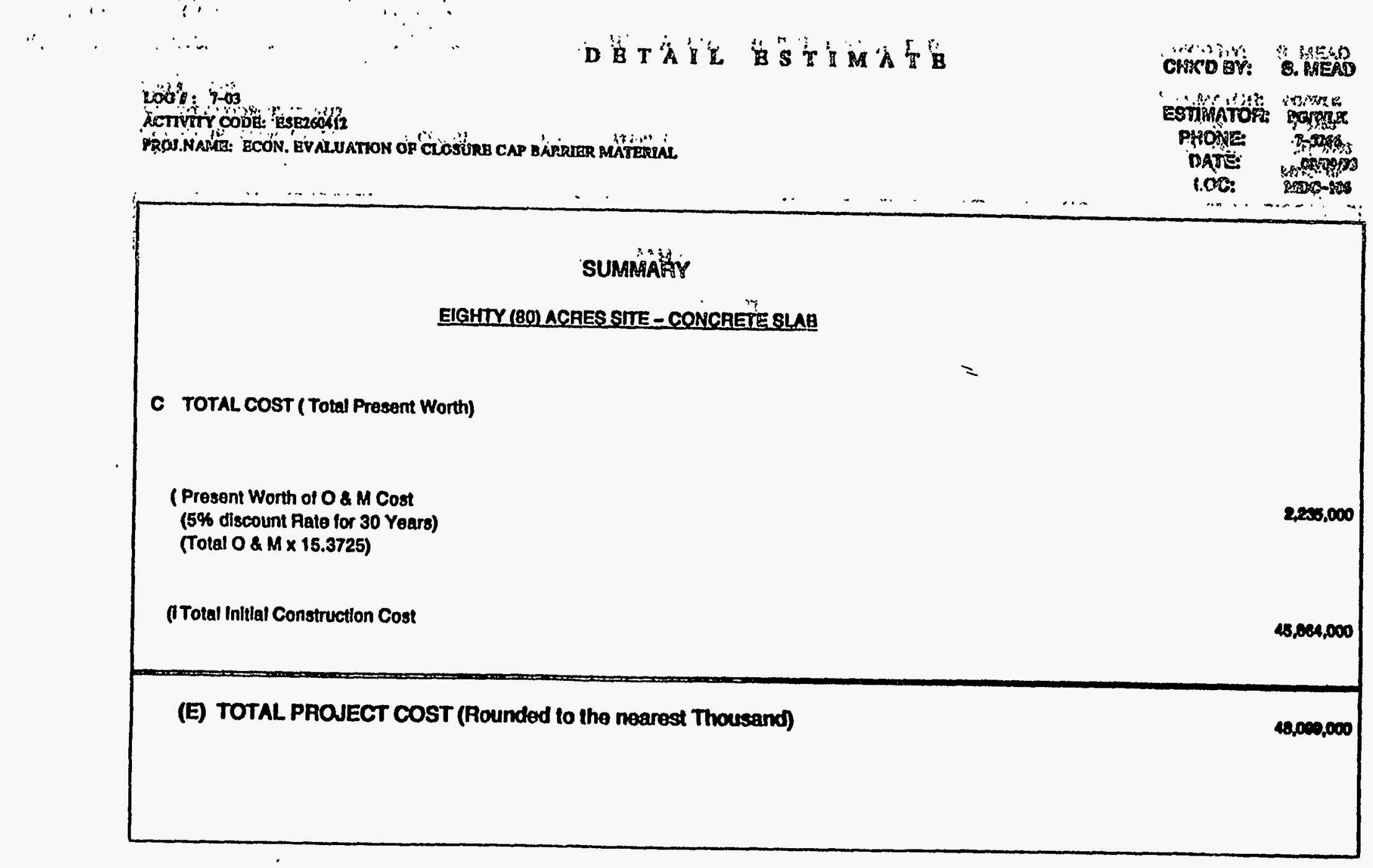

J-12. Cost Estimate for a Cover System Utilizing Poured-in Place Concrete Slab and a 30-mil FML (Cont'd) 